\title{
Erzeugung von Radikalen aus teilhalogenierten Methan-, Alkohol- und Etherderivaten und deren Reaktionen sowie thermische und chemische Aktivierung von 1-Ethinyl-1-methylcyclopropan in der Gasphase
}

\author{
Dissertation \\ zur Erlangung des Doktorgrades \\ der Mathematisch-Naturwissenschaftlichen Fakultäten \\ der Georg-August-Universität zu Göttingen
}

vorgelegt von

Markus Hold

aus Kassel 
D 7

Referent: Prof. Dr. K. Hoyermann

Korreferent: Prof. Dr. M. Buback

Tag der mündlichen Prüfung: 30. Januar 2002 
„Nur das Unbekannte ängstigt die Menschen;

wenn sie erst in den Ereignissen drinstehen,

fürchten sie sich nicht mehr"

(Antoine de Saint-Exupery) 
Für

Ina und Nikola

sowie

für

Michael,

der leider vor Fertigstellung seiner eigenen Dissertation durch einen Unfall verstarb. 
Bei Herrn Prof. Dr. K. Hoyermann bedanke ich mich für die Ermöglichung dieser Dissertation und die Übernahme des Referats, bei Herrn Prof. Dr. M. Buback für die Übernahme des Korreferats.

Natürlich ist die vorliegende Arbeit nicht das Werk nur einer einzelnen Person.

Daher gilt zudem mein ganz besonderer Dank Herrn Prof. Dr. K. Hoyermann für seine wertvollen Ratschläge und die anregenden Diskussionen sowie für die stete Hilfsbereitschaft zur Lösung sowohl experimenteller als auch theoretischer Probleme.

Mein Dank gilt weiterhin Herrn Prof. Dr. I. Morozov für die gute Kooperation bei der Untersuchung der teilfluorierten Ether und Alkohole sowie für die herzliche Aufnahme in seine Arbeitsgruppe während des Forschungsaufenthalts in Moskau.

Herrn Dr. U. Schmitt sowie Herrn Prof. Dr. M. Suhm danke ich für die Einweisung in das Computerprogramm „Gaussian98“.

Bedanken möchte ich mich auch bei allen Mitgliedern der Arbeitsgruppe für ihre kollegiale Hilfe und das überaus freundschaftliche Arbeitsklima, namentlich bei Frau W. Heerdt und Frau G. Gnoyke sowie den Herren M. Fügener, Dr. C. Kersten, Dr. F. Nacke, J. Nothdurft, Dr. U. Tröger, J. Wehmeyer und T. Zeuch.

Hervorzuheben ist dabei die tatkräftige Hilfe bei dem Aufbau des GC-MS-Gerätes und die Unterstützung bei den Messungen am REMPI-TOF durch Herrn J. Wehmeyer sowie die gute Kooperation mit Herrn T. Zeuch und dessen Unterstützung bei den Messungen am FT-IR. Den Herren Wehmeyer und Zeuch bin ich ferner sehr zu Dank verpflichtet für die Hilfe bei der Lösung experimenteller Probleme sowie für die fruchtbaren Diskussionen über die Planungen und Auswertungen vieler Messungen. Außerdem danke ich den Herren Dr. Nacke und Wehmeyer für die Überlassung ihrer Abbildungen der REMPI-TOF-Apparatur.

Für die Hilfe bei allen Problemen des Laboralltags danke ich Herrn D. Heinen und besonders Herrn W. Noack sowie den Mitarbeitern der Werkstätten unter der Leitung von V. Meyer, A. Knorr und H. Nolte.

Meiner lieben Frau Ina danke ich ganz herzlich für all ihre Hilfe und Unterstützung (insbesondere für die Durchsicht großer Teile des Manuskripts) sowie das Verständnis während der Anfertigung dieser Arbeit; gleiches gilt für unsere Familien. 



\section{Kurzgliederung}

Inhaltsverzeichnis

Abbildungsverzeichnis

Tabellenverzeichnis

Symbol- und Abkürzungsverzeichnis

1 Einleitung

1.1 Problemstellung

1.2 Herstellung und Verwendung der untersuchten Substanzen

1.3 Aufbau der Arbeit

2 Theoretische Grundlagen

2.1 Chemische Kinetik

2.2 Bestimmung von Produktverteilungen

2.3 Strömungsmechanik

3 Beschreibung der experimentellen Bedingungen

3.1 Apparaturen

3.2 Erzeugung der Fluor-, Sauerstoff- und Wasserstoffatome

3.3 Dosierung der Gase und Flüssigkeiten

3.4 Reinheit der verwendeten Chemikalien

3.5 Ausführung und Auswertung der Messungen

4 Meßergebnisse und Diskussion

4.1 Untersuchung von 1-Ethinyl-1-methylcyclopropan

4.2 Untersuchungen teilfluorierter Ether

4.3 Untersuchungen teilfluorierter Alkohole

4.4 Untersuchungen von Methan-Derivaten

5 Zusammenfassung

Anhang

Literaturverzeichnis

Beschreibung des selbsterstellten Programms „GCMS-CP 1.4“

Beschreibung des selbsterstellten Programms „WinSiccon 1.7“

Beschreibung des selbsterstellten Programms „FFCP 1.2“ 


\section{Inhaltsverzeichnis}

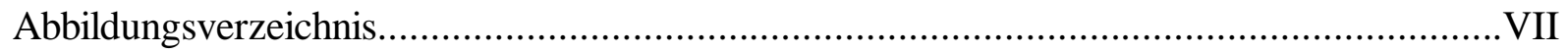

Tabellenverzeichnis............................................................................................ XII

Symbol- und Abkürzungsverzeichnis.....................................................................

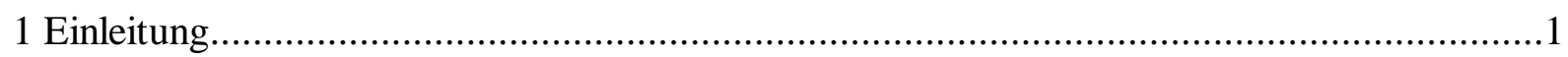

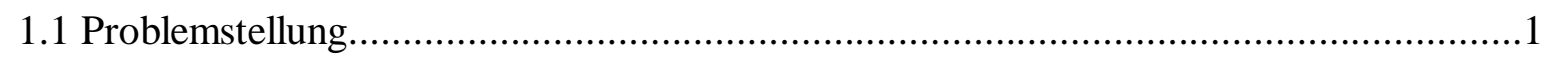

1.2 Herstellung und Verwendung der untersuchten Substanzen......................................4

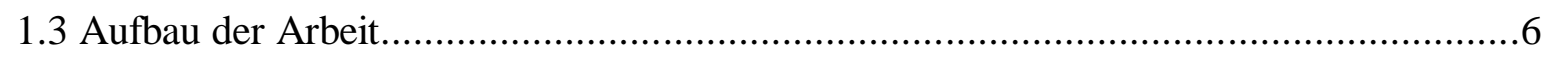

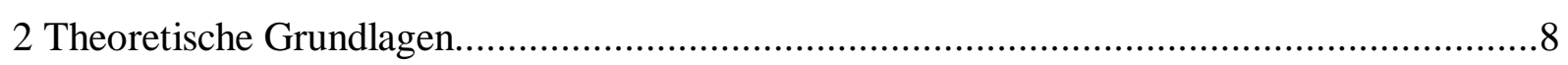

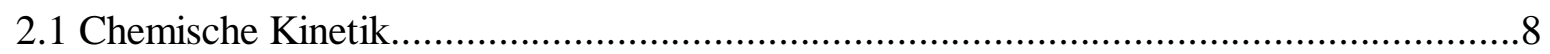

2.1.1 Bestimmung von Geschwindigkeitskoeffizienten ............................................ 8

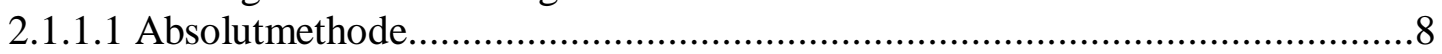

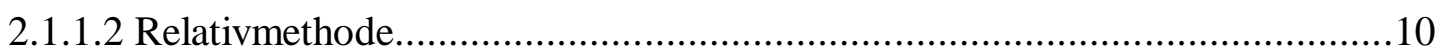

2.1.2 Bestimmung der Temperaturabhängigkeit von Geschwindigkeitskoeffizienten......10

2.2 Bestimmung von Produktverteilungen......................................................... 12

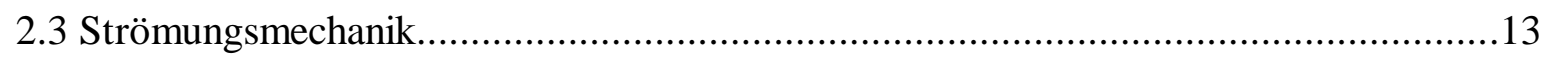

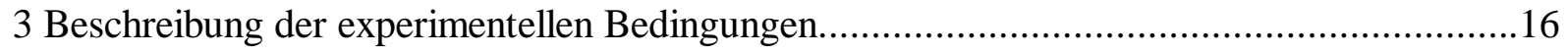

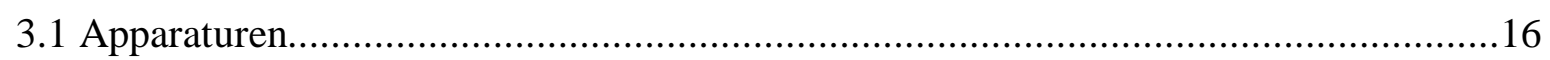

3.1.1 EI-Massenspektrometer mit Probennahme aus dem Molekularstrahl....................16

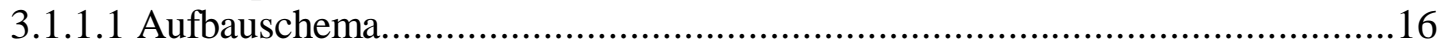

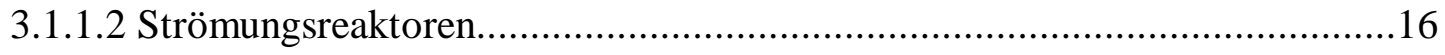

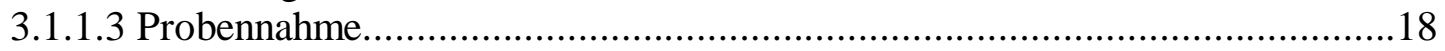

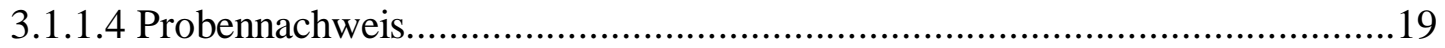

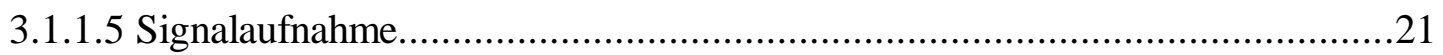

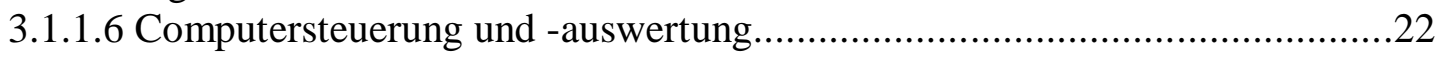

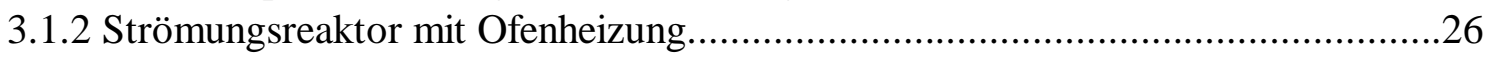

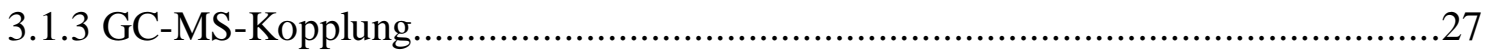

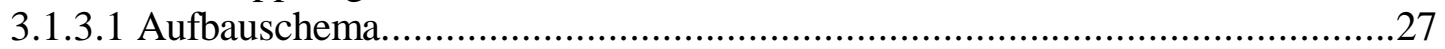

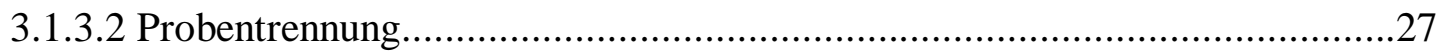

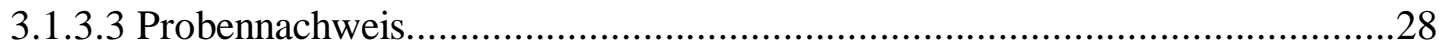

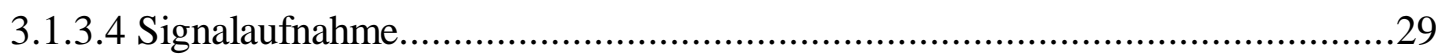

3.1.3.5 Computersteuerung und -auswertung...................................................29

3.1.4 REMPI-Flugzeitmassenspektrometer mit Molekularstrahlprobennahme..............33

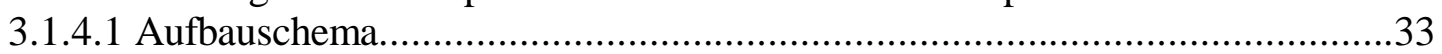

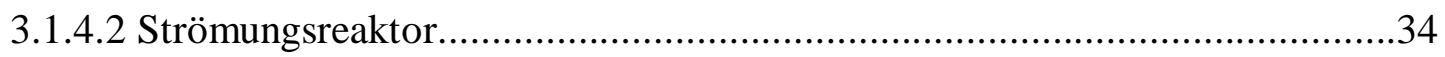

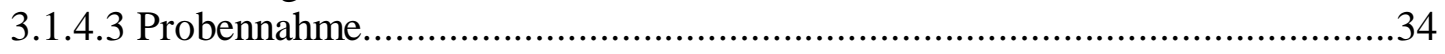

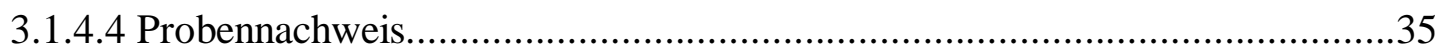

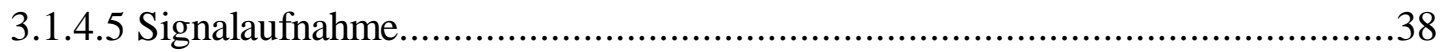

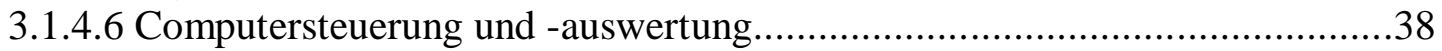

3.1.5 FT-IR-Spektrometer mit Multireflektionskugelzelle....................................42

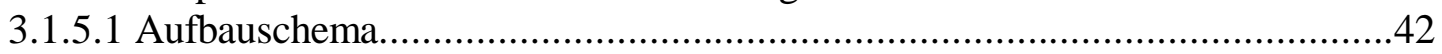

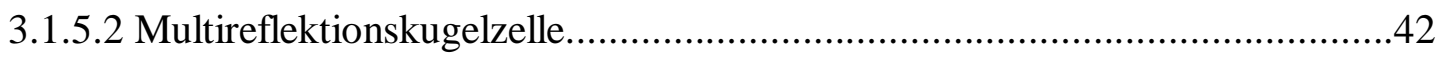

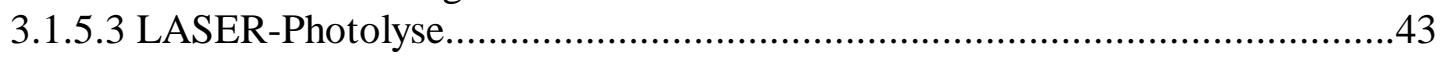

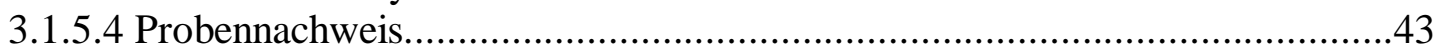

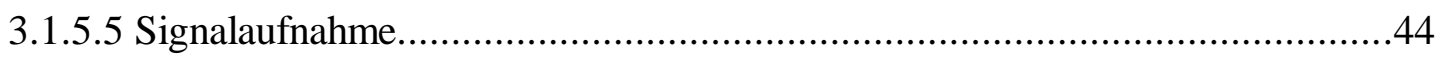




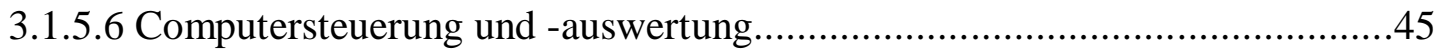

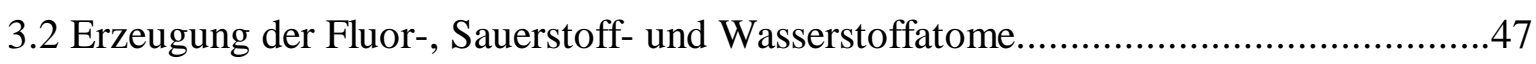

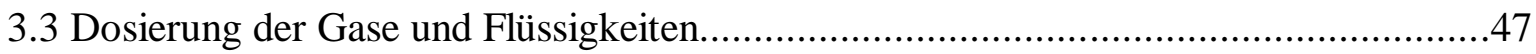

3.3.1 Messungen in der Multireflektionszelle an der FT-IR-Apparatur.......................47

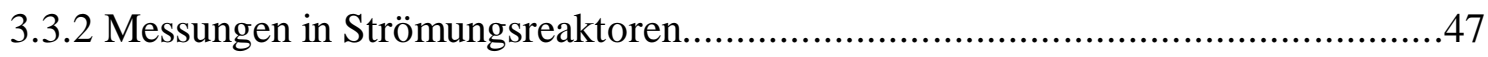

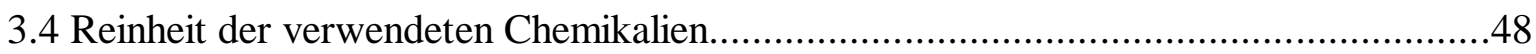

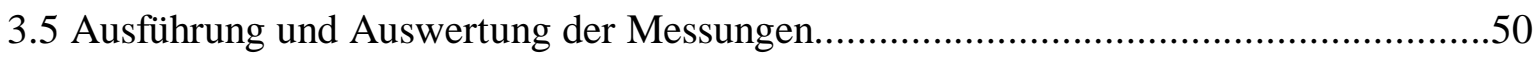

3.5.1 Messungen im Strömungsreaktor mit dem EI-Massenspektrometer......................50

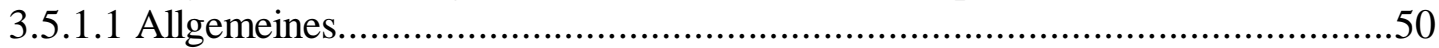

3.5.1.2 Durchführung der Produktuntersuchungen.............................................50

3.5.1.3 Ermittlung der Geschwindigkeitskoeffizienten nach der Relativmethode.......50

3.5.1.4 Ermittlung der Geschwindigkeitskoeffizienten nach der Absolutmethode......55

3.5.2 Verwendung des Strömungsreaktors mit Ofenheizung.......................................56

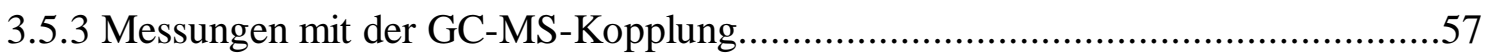

3.5.4 Messungen mit dem REMPI-Flugzeitmassenspektrometer.............................57

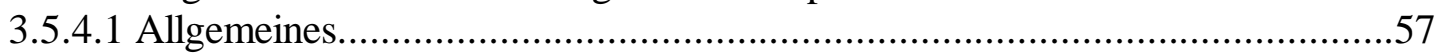

3.5.4.2 Aufnahmen mit dem automatischen Wellenlängendurchlauf........................58

3.5.4.3 Ermittlung der Geschwindigkeitskoeffizienten nach der Relativmethode.......58

3.5.5 Messungen mit dem FT-IR-Spektrometer..................................................59

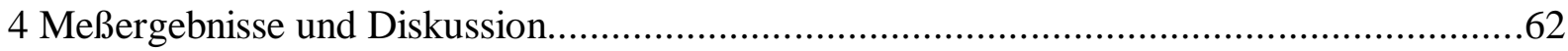

4.1 Untersuchung von 1-Ethinyl-1-methylcyclopropan............................................62

4.1.1 Reaktionen nach thermischer Aktivierung................................................62

4.1.2 Kinetik der radikalerzeugenden Reaktion mit Fluor-Atomen............................. 88

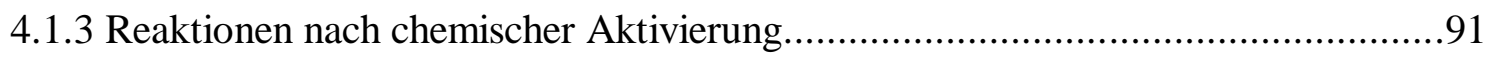

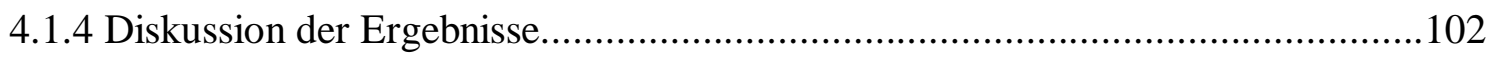

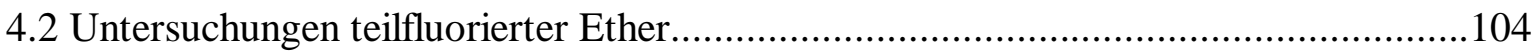

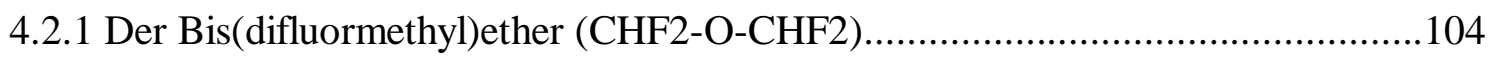

4.2.1.1 Produkte der radikalerzeugenden Reaktion mit Fluor-Atomen....................104

4.2.1.2 Kinetik der radikalerzeugenden Reaktion mit Fluor-Atomen......................105

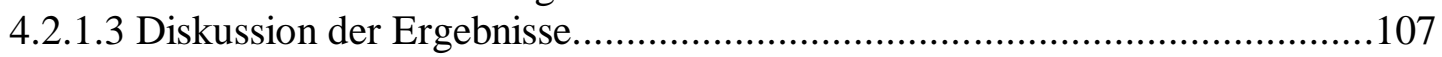

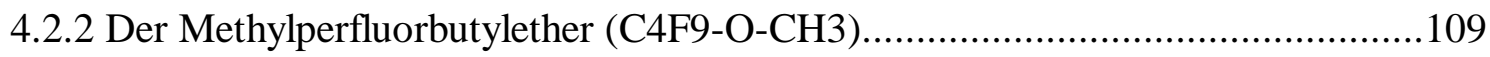

4.2.2.1 Produkte der radikalerzeugenden Reaktion mit Fluor-Atomen....................109

4.2.2.2 Kinetik der radikalerzeugenden Reaktion mit Fluor-Atomen.......................110

4.2.2.3 Produkte der Reaktion des erzeugten Radikals mit molekularem Sauerstoff

4.2.2.4 Kinetik der Reaktion des erzeugten Radikals mit molekularem Sauerstoff...119

4.2.2.5 REMPI-Nachweis des Radikals.............................................................127

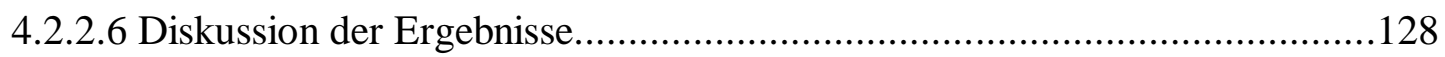

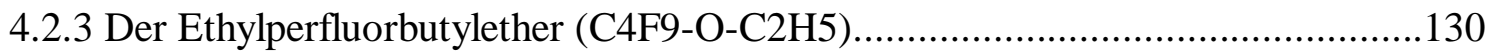

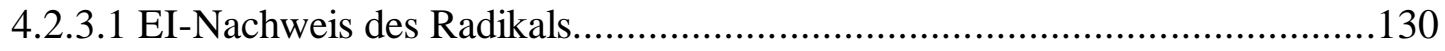

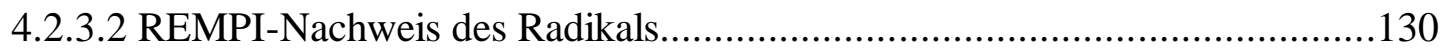

4.3 Untersuchungen teilfluorierter Alkohole............................................................132

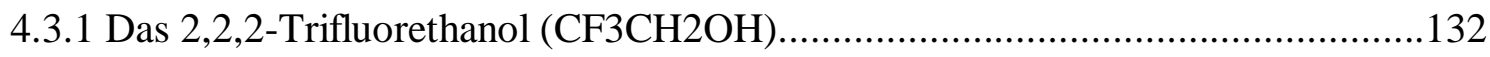

4.3.1.1 Produkte der radikalerzeugenden Reaktion mit Fluor-Atomen....................132

4.3.1.2 Kinetik der radikalerzeugenden Reaktion mit Fluor-Atomen.......................134

4.3.1.3 Produkte der Reaktion des erzeugten Radikals mit molekularem Sauerstoff 
4.3.1.4 Kinetik der Reaktion des erzeugten Radikals mit molekularem Sauerstoff...143

4.3.1.5 Kinetik der Reaktion des erzeugten Radikals mit Stickstoffmonoxid...........151

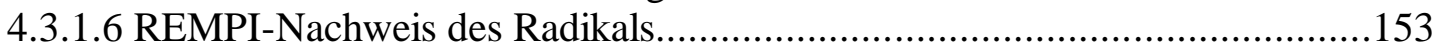

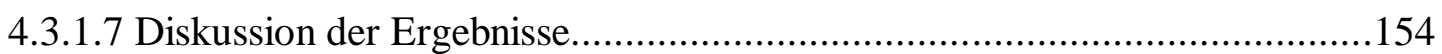

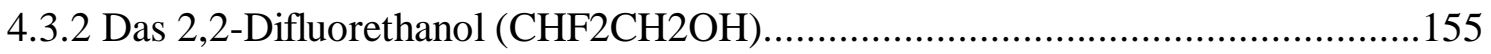

4.3.2.1 Produkte der radikalerzeugenden Reaktion mit Fluor-Atomen....................155

4.3.2.2 Kinetik der radikalerzeugenden Reaktion mit Fluor-Atomen.......................157

4.3.2.3 Produkte der Reaktion des erzeugten Radikals mit molekularem Sauerstoff

4.3.2.4 Kinetik der Reaktion des erzeugten Radikals mit molekularem Sauerstoff...160

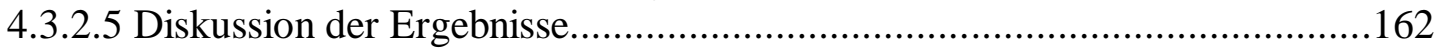

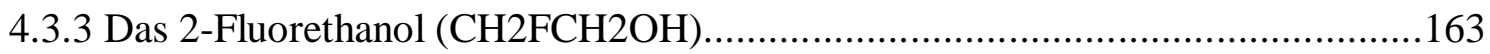

4.3.3.1 Produkte der radikalerzeugenden Reaktion mit Fluor-Atomen.....................163

4.3.3.2 Kinetik der radikalerzeugenden Reaktion mit Fluor-Atomen.......................163

4.3.3.3 Produkte der Reaktion des erzeugten Radikals mit molekularem Sauerstoff

4.3.3.4 Kinetik der Reaktion des erzeugten Radikals mit molekularem Sauerstoff...173

4.3.3.5 Produkte der Reaktion des erzeugten Radikals mit Stickstoffmonoxid........181

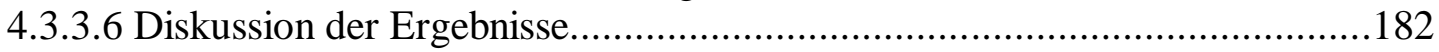

4.3.4 Das 2,2,3,3,4,4,4-Heptafluorbutanol (CF3CF2CF2CH2OH)...........................183

4.3.4.1 Kinetik der radikalerzeugenden Reaktion mit Fluor-Atomen.......................183

4.3.4.2 Kinetik der Reaktion des erzeugten Radikals mit molekularem Sauerstoff...189

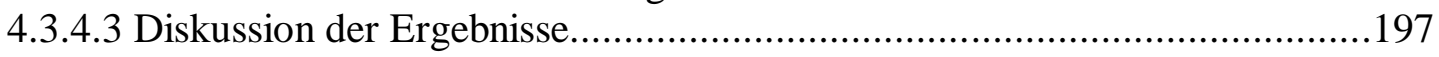

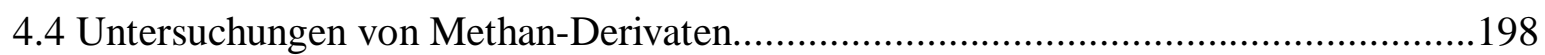

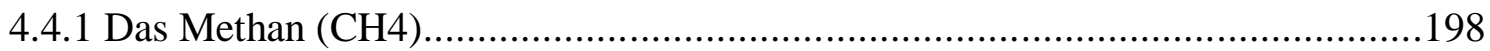

4.4.1.1 Produkte der radikalerzeugenden Reaktion mit Fluor-Atomen....................198

4.4.1.2 Voruntersuchung: Nachweisempfindlichkeit von CO relativ zu Ar im EI-MS

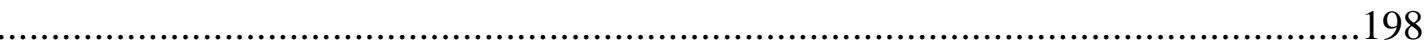

4.4.1.3 Produkte der Reaktion des erzeugten Radikals mit Sauerstoff-Atomen.......199

4.4.1.4 Diskussion der Ergebnisse ......................................................................203

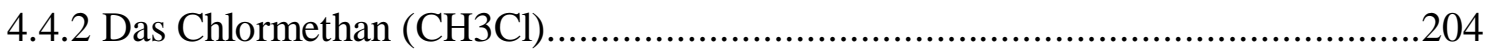

4.4.2.1 Produkte der radikalerzeugenden Reaktion mit Fluor-Atomen....................204

4.4.2.2 Kinetik der radikalerzeugenden Reaktion mit Fluor-Atomen......................204

4.4.2.3 Produkte der Reaktion des erzeugten Radikals mit Sauerstoff-Atomen.......204

4.4.2.4 Kinetik der Reaktion des erzeugten Radikals mit Sauerstoff-Atomen...........209

4.4.2.5 REMPI-Nachweis des erzeugten Radikals.............................................209

4.4.2.6 Diskussion der Ergebnisse................................................................210

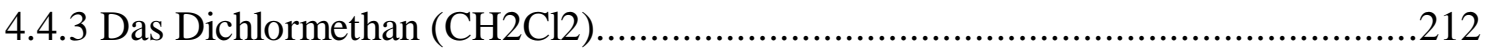

4.4.3.1 Produkte der radikalerzeugenden Reaktion mit Fluor-Atomen....................212

4.4.3.2 Kinetik der radikalerzeugenden Reaktion mit Fluor-Atomen......................212

4.4.3.3 Produkte der Reaktion des erzeugten Radikals mit Sauerstoff-Atomen.......212

4.4.3.4 Kinetik der Reaktion des erzeugten Radikals mit Sauerstoff-Atomen..........218

4.4.3.5 REMPI-Nachweis des erzeugten Radikals..............................................219

4.4.3.6 Kinetik der Reaktion des erzeugten Radikals mit Stickstoffmonoxid...........220

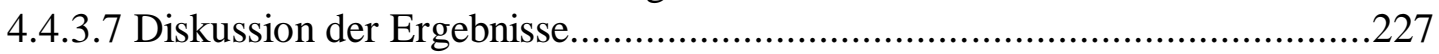

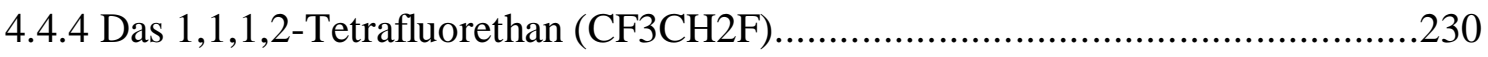

4.4.4.1 Produkte der radikalerzeugenden Reaktion mit Fluor-Atomen....................230

4.4.4.2 Kinetik der radikalerzeugenden Reaktion mit Fluor-Atomen......................231

4.4.4.3 Kinetik der Kombinationsreaktion des erzeugten Radikals........................241

4.4.4.4 Produkte der Reaktion des erzeugten Radikals mit Sauerstoff-Atomen.......243 
4.4.4.5 Kinetik der Reaktion des erzeugten Radikals mit Sauerstoff-Atomen .244 4.4.4.6 Kinetik der Reaktion des erzeugten Radikals mit molekularem Sauerstoff...246 4.4.4.7 Diskussion der Ergebnisse.....................................................................2251

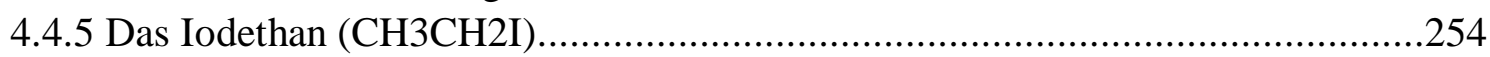

4.4.5.1 Erzeugung von Ethyl-Radikalen durch LASER-Photolyse........................254

4.4.5.2 Kinetik der Reaktion des erzeugten Radikals mit molekularem Sauerstoff...254

4.4.5.3 Diskussion der Ergebnisse.....................................................................256

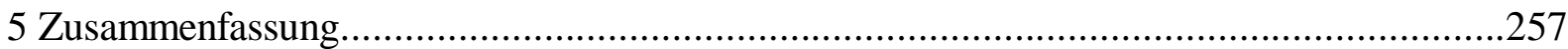

5.1 Allgemeines zur Messung der Geschwindigkeitskoeffizienten...............................257

5.2 Ergebnisse der Analyse von 1-Ethinyl-1-methylcyclopropan.....................................257

5.3 Ergebnisse der Analysen der teilfluorierten Ether................................................259

5.4 Ergebnisse der Analysen der teilfluorierten Alkohole...........................................260

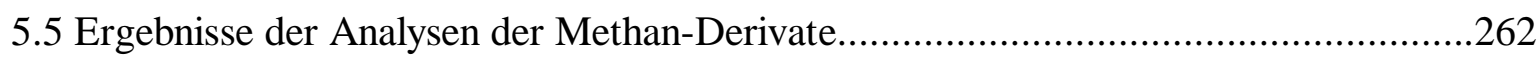

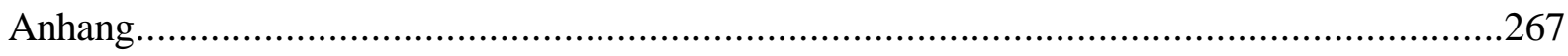

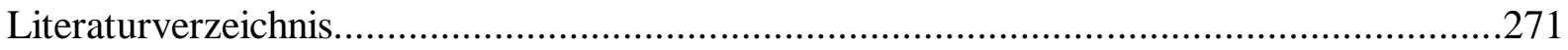

Beschreibung des selbsterstellten Programms „GCMS-CP 1.4“ $\quad$ (eigene Seitennumerierung)

Beschreibung des selbsterstellten Programms „WinSiccon 1.7“ $\quad$ (eigene Seitennumerierung)

Beschreibung des selbsterstellten Programms „FFCP 1.2“_ (eigene Seitennumerierung) 


\section{Abbildungsverzeichnis}

Abbildung 1: Stark vereinfachtes Schema des katalysierten Ozon-Abbaus.............................2

Abbildung 2: Verkürztes Reaktionsschema der Abbaureaktionen in der Troposphäre ..............2

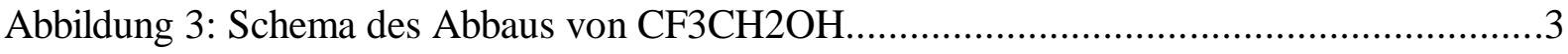

Abbildung 4: Aufbauschema Massenspektrometer mit Molekularstrahlprobennahme..............16

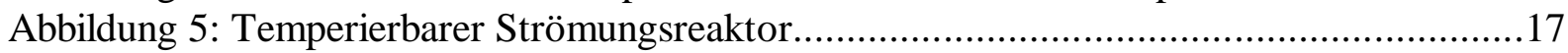

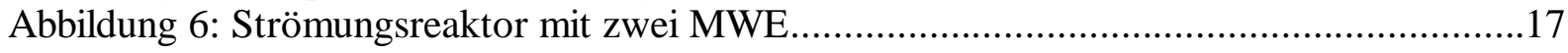

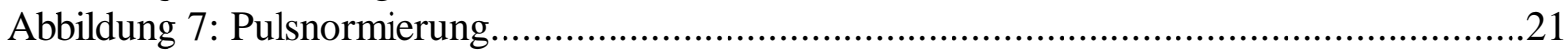

Abbildung 8: Schema Computersteuerung MS mit Molekularstrahlprobennahme..................23

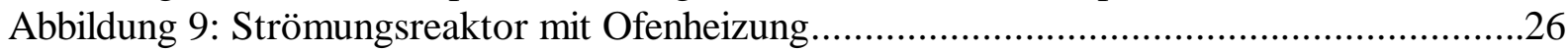

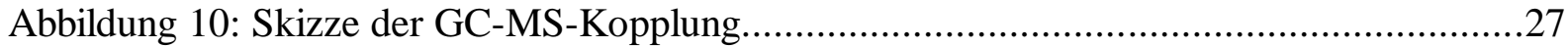

Abbildung 11: Schema Computersteuerung der GC-MS-Kopplung........................................30

Abbildung 12: Skizze des REMPI-Flugzeitmassenspektrometers [Quelle: Wehmeyer, J.

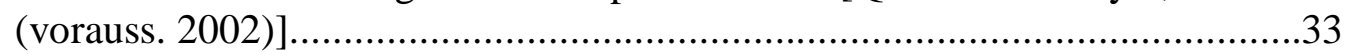

Abbildung 13: Mechanismen der Photoionisation [Quelle: Nacke, F. (1998), S. 6]................36

Abbildung 14: Schema der Computersteuerung des Flugzeitmassenspektrometers [Nacke, F.

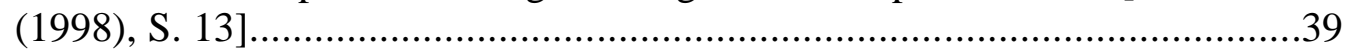

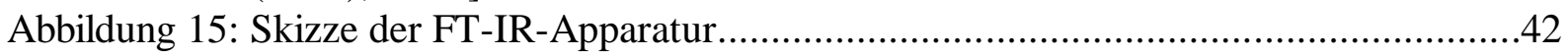

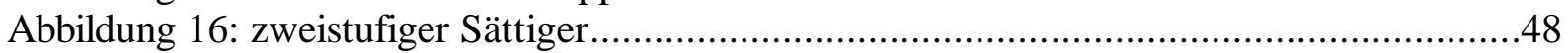

Abbildung 17:Beispiel zur Illustration der Umsatzkorrektur..........................................52

Abbildung 18: Denkbare Reaktionsprodukte nach thermischer Aktivierung .........................62

Abbildung 19: EI-Massenspektren vor und nach Pyrolyse ............................................63

Abbildung 20: Vergleich IR-Spektren mit/ohne Pyrolyse bei 683 K...................................64

Abbildung 21: Ausschnitte aus hochaufgelöstem IR-Spektrum nach Pyrolyse bei $683 \mathrm{~K} . . . . . .64$

Abbildung 22: FT-IR-Spektrum von 1-Ethinyl-1-methylcyclopropan...................................65

Abbildung 23: Berechnetes IR-Spektr. 1-Ethinyl-1-methylcyclopropans...........................65

Abbildung 24: Berechnetes IR-Spektrum von (Z)-3-Methyl-pent-3-en-1-in..........................66

Abbildung 25: Berechnetes IR-Spektrum von (E)-3-Methyl-pent-3-en-1-in........................66

Abbildung 26: Berechn. IR-Spekt. von 3-Methyl-pent-4-en-1-in.......................................67

Abbildung 27: Berechn. IR-Spekt. von Ethinylcyclobutan.............................................67

Abbildung 28: Berechn. IR-Spekt. von 3-Methylpent-1,2,4-trien.......................................67

Abbildung 29: Berechn. IR-Spekt. von 2-Methylpent-1-en-4-in........................................68

Abbildung 30: Berechnetes IR-Spekt. von Verbindung 10.............................................68

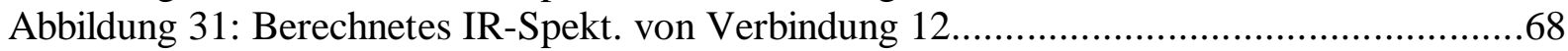

Abbildung 32: Berechnetes IR-Spekt. von 1,3-Cyclopentadien......................................69

Abbildung 33: Tatsächlich gemessenes IR-Spektrum von 1,3-Cyclopentadien [Quelle:

Schrader, B. (1995)]............................................................................69

Abbildung 34: IR-Spektren vor/nach Pyrolyse bei 853 K................................................ 70

Abbildung 35: IR-Spektren vor/nach Pyrolyse bei 905 K...............................................73

Abbildung 36: Vergleich IR-Spektren nach Pyrolyse bei $853 \mathrm{~K}$ und $905 \mathrm{~K}$ (Ausschnitt).........74

Abbildung 37: Vergleich IR-Spektren nach Pyrolyse bei $853 \mathrm{~K}$ und $905 \mathrm{~K}$ (Ausschnitt).........74

Abbildung 38: Reaktionsmechanismus zur Bildung des Allen-Derivats................................75

Abbildung 39: Reaktionsmechanismus zur Bildung des 1-Methyl-1,4-cyclopentadiens...........75

Abbildung 40: Umlagerung einer Alkenyl-Gruppe..........................................................76

Abbildung 41: GC- und Massenspektrum von reinem 1-Ethinyl-1-methylcyclopropan............77

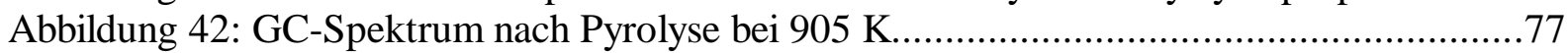

Abbildung 43: GC-Spektrum nach Pyrolyse bei 905 K........................................................78

Abbildung 44: Temperaturverlauf und Massenspektrum Nr. 68 des GC-Spektrums aus

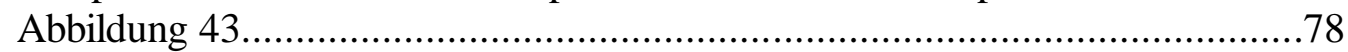

Abbildung 45: Massenspektren Nr. 71 und 74 des GC-Spektrums aus Abbildung 43.............79 
Abbildung 46: Massenspektren Nr. 76 und 80 des GC-Spektrums aus Abbildung 43.............79

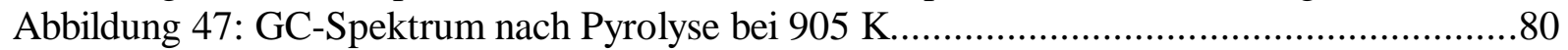

Abbildung 48: Massenspektren Nr. 124 und 128 des GC-Spektrums aus Abbildung 47.........81

Abbildung 49: Massenspektren Nr. 134 und 135 des GC-Spektrums aus Abbildung 47..........81

Abbildung 50: Massenspektren Nr. 139 und 142 des GC-Spektrums aus Abbildung 47.........82

Abbildung 51: Massenspektren Nr. 145 und 152 des GC-Spektrums aus Abbildung 47.........82

Abbildung 52: Literatur-Massenspektren der Verbindungen 3/4 sowie von 1-Methyl-1,3-

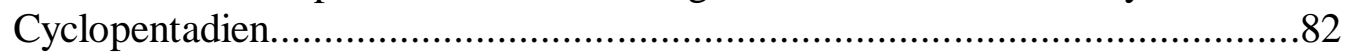

Abbildung 53: FT-IR-Spektrum vor/nach Pyrolyse bei 953 K..........................................83

Abbildung 54: Vergleich IR-Spektren nach Pyrolyse bei $905 \mathrm{~K}$ und $953 \mathrm{~K}$ (Ausschnitt).........84

Abbildung 55: Vergleich IR-Spektren nach Pyrolyse bei $905 \mathrm{~K}$ und $953 \mathrm{~K}$ (Ausschnitt)........84

Abbildung 56: GC-Spektrum nach Pyrolyse bei 953 K........................................................85

Abbildung 57: GC-Spektrum nach Pyrolyse bei 953 K (Ausschnitt)...................................85

Abbildung 58: Temperaturverlauf und Massenspektrum Nr. 85 des GC-Spektrums in

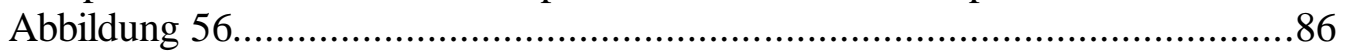

Abbildung 59: Massenspektren Nr. 88 und 92 des GC-Spektrums in Abbildung 56...............86

Abbildung 60: Massenspektren Nr. 96 und 98 des GC-Spektrums in Abbildung 56................87

Abbildung 61: Massenspektren Nr. 100 und 106 des GC-Spektrums in Abbildung 56...........87

Abbildung 62: Reaktionskanäle der Reaktion 1-Ethinyl-1-methylcyclopropan + F-Atome......88

Abbildung 63: Kinetik 1-Ethinyl-1-methylcyclopropan + F ; 298 K.................................89

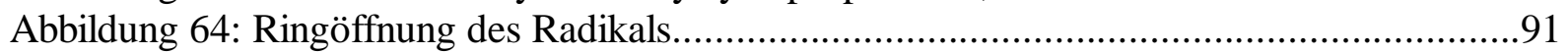

Abbildung 65: Ringöffnung unmittelbar nach Radiaklerzeugung.....................................92

Abbildung 66: Denkbare Reaktionskanäle bei chemischer Aktivierung...............................93

Abbildung 67: Denkbare Produkte aus Kanal (b) der Abb. 66..........................................93

Abbildung 68: Denkbare Produkte aus Kanal (c) der Abb. 66.............................................94

Abbildung 69: Denkbare Produkte aus Kanal (d) der Abb. 66.............................................94

Abbildung 70: Denkbare Produkte aus Kanal (e) der Abb. 66............................................94

Abbildung 71: Einzelzählung mit EI-MS für 1-Ethinyl-1-methylcyclopropan + F +/- D.........95

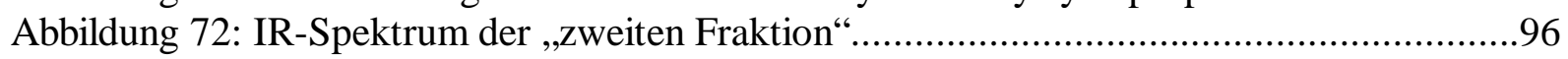

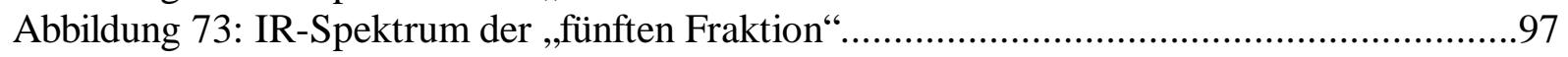

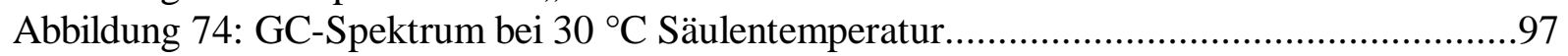

Abbildung 75: Massenspektrum von GC-Peak Nr. 147................................................98

Abbildung 76: Massenspektrum von GC-Peak Nr. 158................................................... 98

Abbildung 77: Massenspektrum von GC-Peak Nr. 176..................................................99

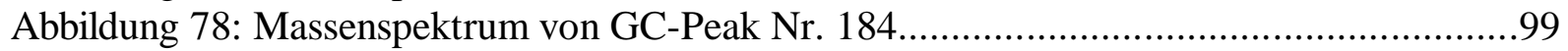

Abbildung 79: EI-MS für 1-Ethinyl-1-methylcyclopropan + F +/- H..............................100

Abbildung 80: IR-Spektrum nach chemischer Aktivierung mit H-Atomen...........................100

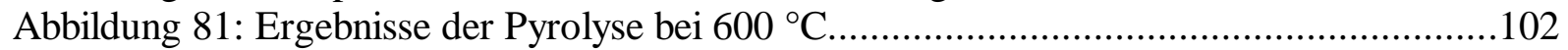

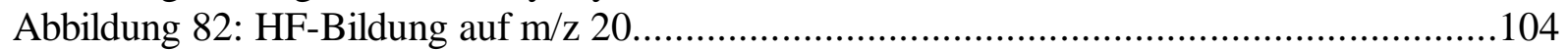

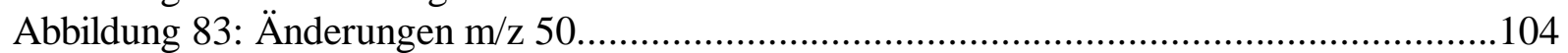

Abbildung 84: MS CHF2OCHF2 +/- F................................................................... 104

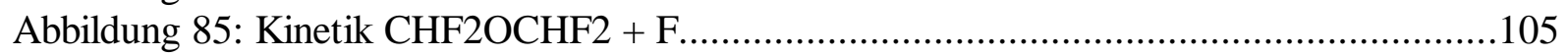

Abbildung 86: Kinetik CHF2OCHF2 + F............................................................... 106

Abbildung 87: Resonanzstrukturen des Übergangszustands.........................................108

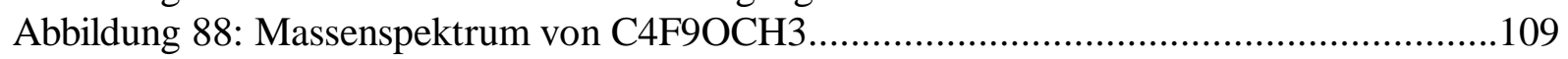

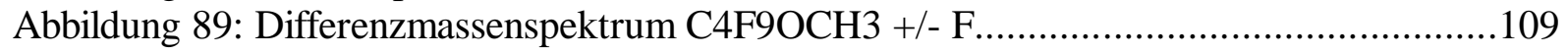

Abbildung 90: Kinetik C4F9OCH3 + F rel. zu CH4; 298 K.............................................111

Abbildung 91: Kinetik C4F9OCH3 + F rel. zu CH2Cl2; 268 K........................................112

Abbildung 92: Kinetik C4F9OCH3 + F rel. zu CH2Cl2; 298 K.....................................113

Abbildung 93: Kinetik C4F9OCH3 + F rel. zu CH2Cl2; 328 K.....................................114

Abbildung 94: Kinetik C4F9OCH3 + F rel. zu CH2Cl2; 358 K.......................................115 
Abbildung 95: Arrhenius-Auftragung $\mathrm{C} 4 \mathrm{~F} 9 \mathrm{OCH} 3+\mathrm{F}$ rel. zu CH2Cl2 ...........................116

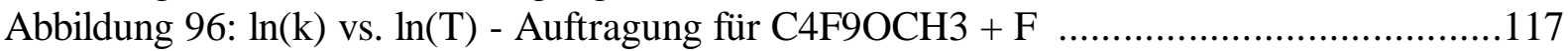

Abbildung 97: Differenzmassenspektrum C4F9OCH3 + F +/- O2 ................................118

Abbildung 98: EI-MS Einzelzählung für Produkte C4F9OCH3 + F +/- O2 ......................118

Abbildung 99: Kinetik C4F9OCH2 + 2 rel. zu CHCl2; 298 K.....................................120

Abbildung 100: Kinetik C4F9OCH2 + O2 rel. zu CHCl2; 318 K....................................121

Abbildung 101: Kinetik C4F9OCH2 + O2 rel. zu CHCl2; 338 K.....................................122

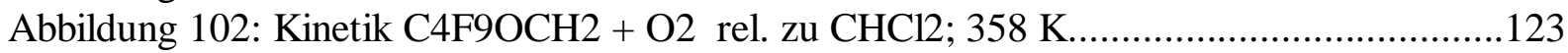

Abbildung 103: Arrhenius-Auftragung C4F9OCH2 + O2 rel. zu CHCl2 ..........................125

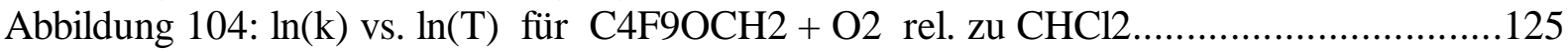

Abbildung 105: REMPI-Nachweis des C4F9OCH2-Radikals bei verschiedenen Wellenlängen

127

Abbildung 106: REMPI-Nachweis des C4F9OCH2-Radikals bei verschiedenen Wellenlängen

127

Abbildung 107: REMPI-Nachweis des C4F9OC2H4-Radikals bei verschiedenen Wellenlängen

Abbildung 108: REMPI-Nachweis des C4F9OC2H4-Radikals bei verschiedenen Wellenlängen

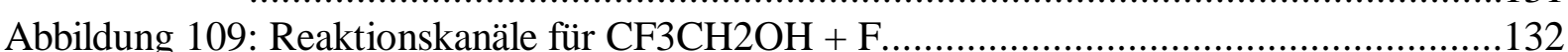

Abbildung 110: EI-MS-Produktuntersuchung CF3CH2OH +/- F................................133

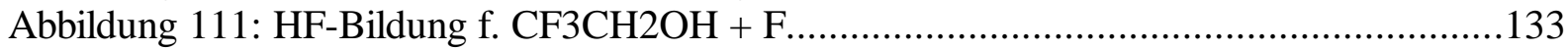

Abbildung 112: I(m/z 98, 99) vs. Reaktionsstrecke und IP f. CF3CH2OH + F................133

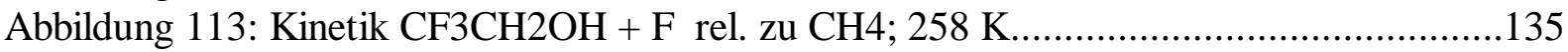

Abbildung 114: Kinetik CF3CH2OH + F rel. zu CH4; 278 K.....................................136

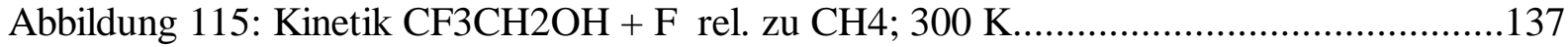

Abbildung 116: Kinetik CF3CH2OH + F rel. zu CH4; 328 K........................................138

Abbildung 117: Kinetik CF3CH2OH + F rel. zu CH4; 358 K.........................................139

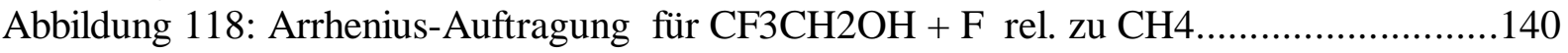

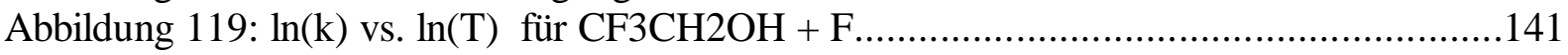

Abbildung 120: EI-MS-Produktuntersuchung $\mathrm{CF} 3 \mathrm{CH} 2 \mathrm{OH}+/-\mathrm{F}+/-\mathrm{O} 2 \ldots \ldots \ldots \ldots \ldots \ldots \ldots \ldots . . . . . . . . . . . . .142$

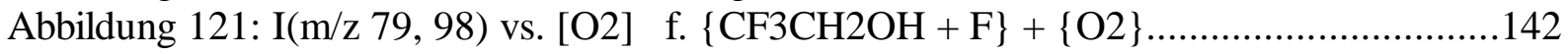

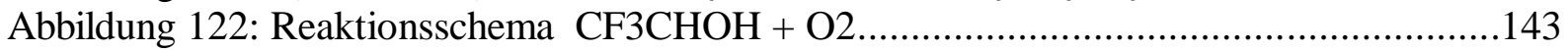

Abbildung 123: Kinetik $\{\mathrm{CF} 3 \mathrm{CH} 2 \mathrm{OH}+\mathrm{F}\}+\{\mathrm{O} 2\}$ rel. zu CH2Cl2; $258 \mathrm{~K} \ldots \ldots \ldots \ldots \ldots \ldots \ldots . . . . . . . . .144$

Abbildung 124: Kinetik $\{\mathrm{CF} 3 \mathrm{CH} 2 \mathrm{OH}+\mathrm{F}\}+\{\mathrm{O} 2\}$ rel. zu CH2Cl2; $278 \mathrm{~K} \ldots \ldots \ldots \ldots \ldots \ldots \ldots . . . . . . . . . . .145$

Abbildung 125: Kinetik $\{\mathrm{CF} 3 \mathrm{CH} 2 \mathrm{OH}+\mathrm{F}\}+\{\mathrm{O} 2\}$ rel. zu CH2Cl2; $298 \mathrm{~K} \ldots \ldots \ldots \ldots \ldots \ldots \ldots . . . . . . . . . .146$

Abbildung 126: Kinetik $\{\mathrm{CF} 3 \mathrm{CH} 2 \mathrm{OH}+\mathrm{F}\}+\{\mathrm{O} 2\}$ rel. zu CH2Cl2; $328 \mathrm{~K} \ldots \ldots \ldots \ldots \ldots \ldots \ldots . . . . . . . . . .147$

Abbildung 127: Kinetik $\{\mathrm{CF} 3 \mathrm{CH} 2 \mathrm{OH}+\mathrm{F}\}+\{\mathrm{O} 2\}$ rel. zu CH2Cl2; $358 \mathrm{~K} \ldots \ldots \ldots \ldots \ldots \ldots \ldots . . . \ldots 148$

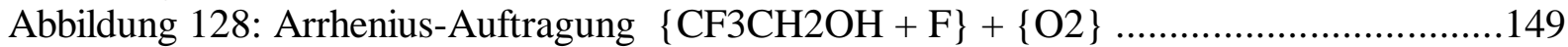

Abbildung 129: $\ln (\mathrm{k})$ vs. $\ln (\mathrm{T} /)$ f. $\{\mathrm{CF} 3 \mathrm{CH} 2 \mathrm{OH}+\mathrm{F}\}+\{\mathrm{O} 2\}$ rel. zu CH2Cl2 ...................150

Abbildung 130: Kinetik $\{\mathrm{CF} 3 \mathrm{CH} 2 \mathrm{OH}+\mathrm{F}\}+\{\mathrm{NO}\}$ rel. zu CH2Cl2; $298 \mathrm{~K} \ldots \ldots \ldots \ldots \ldots \ldots \ldots . . . . . . . . . . .152$

Abbildung 131: REMPI-Nachweis des aus $\mathrm{CF} 3 \mathrm{CH} 2 \mathrm{OH}+\mathrm{F}$ erzeugten Radikals..................153

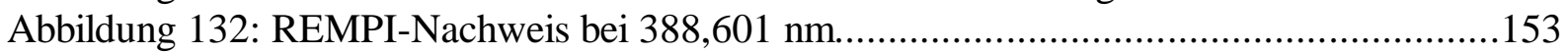

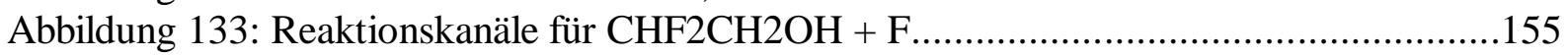

Abbildung 134: EI-MS Produktuntersuchung CHF2CH2OH +/- F..................................156

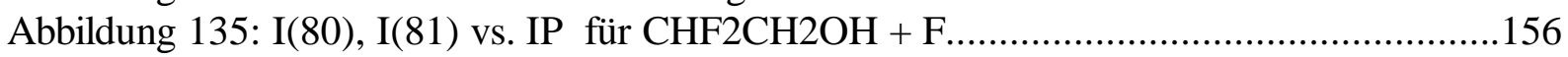

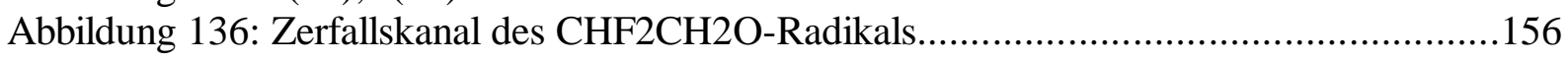

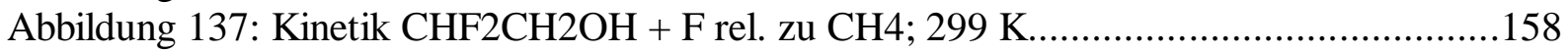

Abbildung 138: EI-MS Produktuntersuchung $\{\mathrm{CHF} 2 \mathrm{CH} 2 \mathrm{OH}+\mathrm{F}\}+/-\{\mathrm{O} 2\} \ldots \ldots \ldots \ldots \ldots \ldots . . . . . . .159$

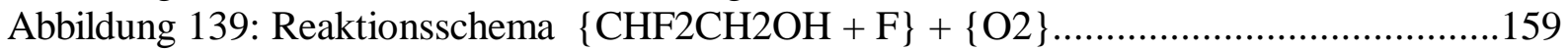

Abbildung 140: Kinetik $\{\mathrm{CHF} 2 \mathrm{CH} 2 \mathrm{OH}+\mathrm{F}\}+\{\mathrm{O} 2\}$ rel. zu CH $3 \mathrm{Cl} ; 300 \mathrm{~K} \ldots \ldots \ldots \ldots \ldots \ldots \ldots . . . . . . . . . .161$

Abbildung 141: EI-MS-Übersichtsspektrum CH2FCH2OH +/- F....................................163 


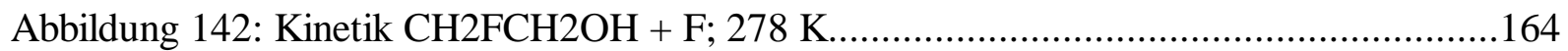

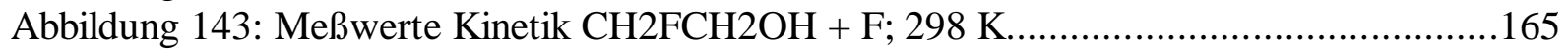

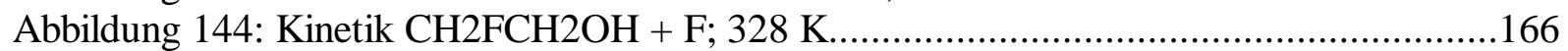

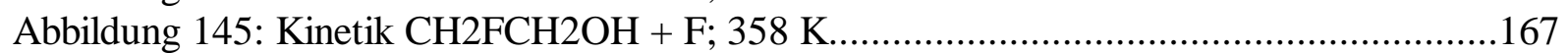

Abbildung 146: Arrhenius-Auftragung f. CH2FCH2OH + F rel. zu CH4 ...........................168

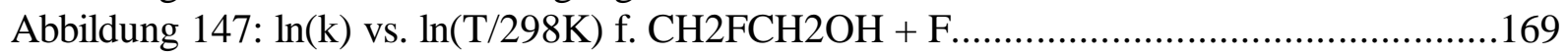

Abbildung 148: Einzelzählung mit EI-MS für $\{\mathrm{CH} 2 \mathrm{FCH} 2 \mathrm{OH}+\mathrm{F}\}+/-\{\mathrm{O} 2\} \ldots \ldots \ldots \ldots \ldots \ldots \ldots \ldots \ldots \ldots \ldots . . . . . .170$

Abbildung 149: Denkbare Reaktionskanäle für $\{\mathrm{CH} 2 \mathrm{FCH} 2 \mathrm{OH}+\mathrm{F}\}+\{\mathrm{O} 2\} \ldots \ldots \ldots \ldots \ldots \ldots \ldots . . . . . . . .170$

Abbildung 150: IR-Spektren für Produktuntersuchung von $\{\mathrm{CH} 2 \mathrm{FCH} 2 \mathrm{OH}+\mathrm{F}\}+\{\mathrm{O} 2\} \ldots .171$

Abbildung 151: IR-Spektren für Produktuntersuchung von $\{\mathrm{CH} 2 \mathrm{FCH} 2 \mathrm{OH}+\mathrm{F}\}+\{\mathrm{O} 2\} \ldots 172$

Abbildung 152: Berechnete IR-Spektren von HCHO und FCHO...................................172

Abbildung 153: Kinetik $\{\mathrm{CH} 2 \mathrm{FCH} 2 \mathrm{OH}+\mathrm{F}\}+\{\mathrm{O} 2\}$ rel. zu 1,4-Dioxan;258K....................174

Abbildung 154: Kinetik $\{\mathrm{CH} 2 \mathrm{FCH} 2 \mathrm{OH}+\mathrm{F}\}+\{\mathrm{O} 2\}$ rel. zu 1,4-Dioxan;278K....................175

Abbildung 155: Kinetik $\{\mathrm{CH} 2 \mathrm{FCH} 2 \mathrm{OH}+\mathrm{F}\}+\{\mathrm{O} 2\}$ rel. zu 1,4-Dioxan;298K....................176

Abbildung 156: Kinetik $\{\mathrm{CH} 2 \mathrm{FCH} 2 \mathrm{OH}+\mathrm{F}\}+\{\mathrm{O} 2\}$ rel. zu 1,4-Dioxan; 328K...................177

Abbildung 157: Kinetik $\{\mathrm{CH} 2 \mathrm{FCH} 2 \mathrm{OH}+\mathrm{F}\}+\{\mathrm{O} 2\}$ rel. zu 1,4-Dioxan; 358K...................178

Abbildung 158: Arrhenius $\{\mathrm{CH} 2 \mathrm{FCH} 2 \mathrm{OH}+\mathrm{F}\}+\{\mathrm{O} 2\}$ rel. zu 1,4-Dioxan.......................179

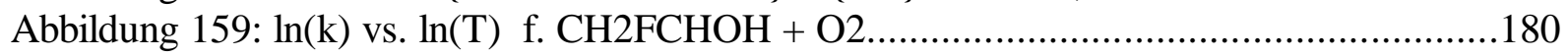

Abbildung 160: EI-MS Produktuntersuchung CH2FCH2OH +/- F +/- NO.......................181

Abbildung 161: EI-MS von reinem CF3CF2CF2CH2OH sowie Differenzen bei Zugabe von F-

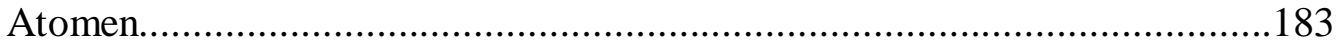

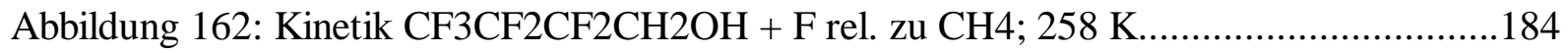

Abbildung 163: Kinetik CF3CF2CF2CH2OH + F rel. zu CH4; 298 K...............................185

Abbildung 164: Kinetik CF3CF2CF2CH2OH + F rel. zu CH4; 338 K...............................186

Abbildung 165: Arrhenius-Auftragung f. CF3CF2CF2CH2OH + F rel. zu CH4 .................187

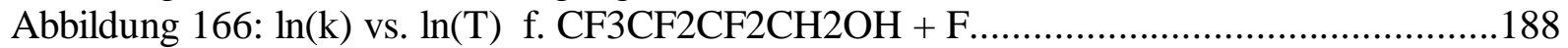

Abbildung 167: $\{\mathrm{CF} 3 \mathrm{CF} 2 \mathrm{CF} 2 \mathrm{CH} 2 \mathrm{OH}+\mathrm{F}\}+\{\mathrm{O} 2\}$ rel. zu CH2Cl2; $258 \mathrm{~K} \ldots \ldots \ldots \ldots \ldots \ldots \ldots . . . . . . . . . . . .190$

Abbildung 168: $\{\mathrm{CF} 3 \mathrm{CF} 2 \mathrm{CF} 2 \mathrm{CH} 2 \mathrm{OH}+\mathrm{F}\}+\{\mathrm{O} 2\}$ rel. zu CH2Cl2; $298 \mathrm{~K} \ldots \ldots \ldots \ldots \ldots \ldots \ldots . . . . . . . . . . . .191$

Abbildung 169: $\{\mathrm{CF} 3 \mathrm{CF} 2 \mathrm{CF} 2 \mathrm{CH} 2 \mathrm{OH}+\mathrm{F}\}+\{\mathrm{O} 2\}$ rel. zu CH2Cl2; $299 \mathrm{~K} \ldots \ldots \ldots \ldots \ldots \ldots \ldots . . . . . . . . . .192$

Abbildung 170: $\{\mathrm{CF} 3 \mathrm{CF} 2 \mathrm{CF} 2 \mathrm{CH} 2 \mathrm{OH}+\mathrm{F}\}+\{\mathrm{O} 2\}$ rel. zu CH2Cl2; $338 \mathrm{~K} \ldots \ldots \ldots \ldots \ldots \ldots \ldots . . . . . . . . . . .193$

Abbildung 171: $\{\mathrm{CF} 3 \mathrm{CF} 2 \mathrm{CF} 2 \mathrm{CH} 2 \mathrm{OH}+\mathrm{F}\}+\{\mathrm{O} 2\}$ rel. zu CH2Cl2; $358 \mathrm{~K} \ldots \ldots \ldots \ldots \ldots \ldots \ldots . . . . . . . . . . . .194$

Abbildung 172: Arrhenius-Auftr. f. CF3CF2CF2CHOH + O2 ......................................195

Abbildung 173: $\ln (\mathrm{k})$ vs. $\ln (\mathrm{T})$ f. $\mathrm{CF} 3 \mathrm{CF} 2 \mathrm{CF} 2 \mathrm{CHOH}+\mathrm{O} 2$ rel. zu CHCl2 .........................196

Abbildung 174: Massenspektrometrische Empfindlichkeit von CO rel. zu Ar......................199

Abbildung 175: Literatur-EI-MS von HCHO sowie EI-MS für CH4+ F +/- O..................200

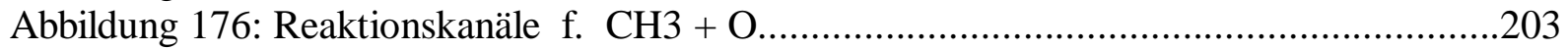

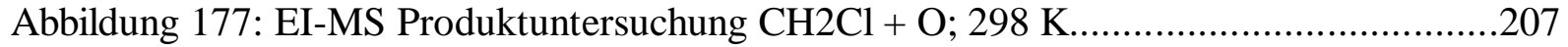

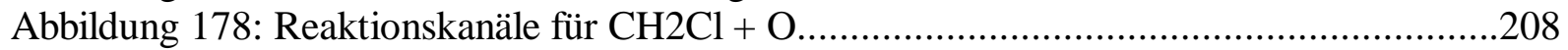

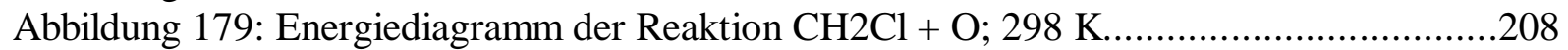

Abbildung 180: REMPI-Wellenlängendurchlauf 368-402 nm f. CH3Cl + F.......................209

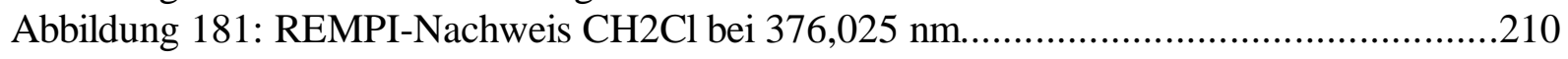

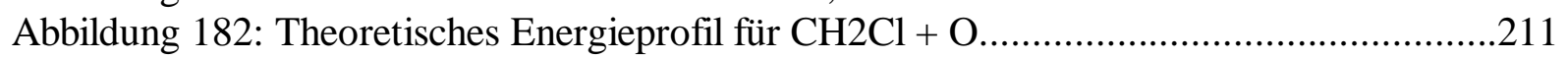

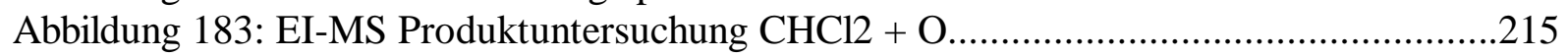

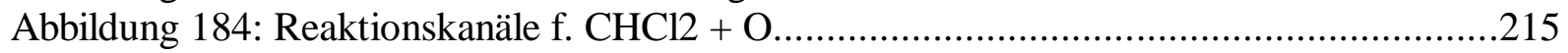

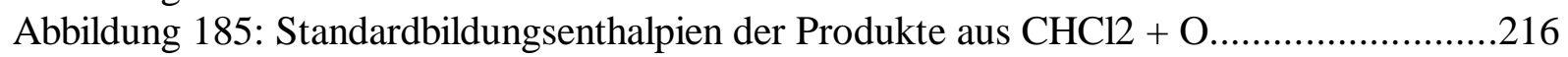

Abbildung 186: Abschätzung k(E) für unimolekularen Zerfall von $(\mathrm{CHCl} 2 \mathrm{O})^{*}$...................218

Abbildung 187: REMPI-Nachweis des CHCl2-Radikals bei verschiedenen Wellenlängen.....219

Abbildung 188: REMPI-Nachweis des CHCl2-Radikals bei verschiedenen Wellenlängen.....219

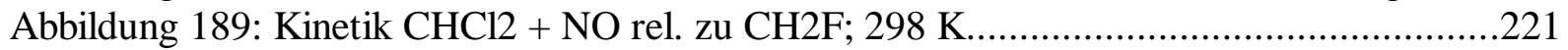

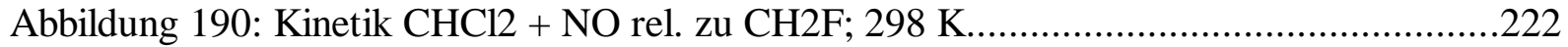

Abbildung 191: Kinetik CHCl2 + NO rel. zu CH2F; 298 K..............................................223 


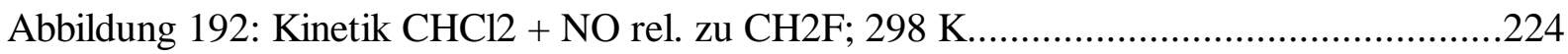

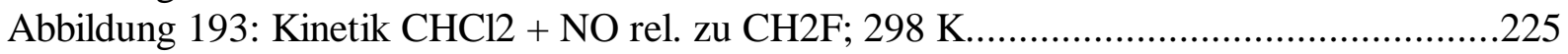

Abbildung 194: Kinetik CHCl2 + NO rel. zu CH2F; 298 K............................................226

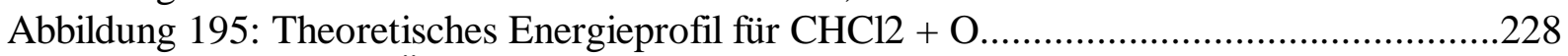

Abbildung 196: EI-MS Übersichtsspektren CF3CH2F +/- F........................................230

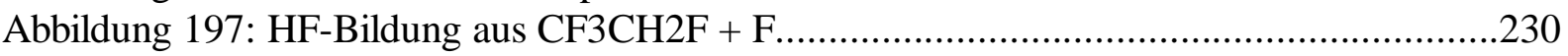

Abbildung 198: Kinetik CF3CH2F + F rel. zu CHCl3; 298 K.........................................231

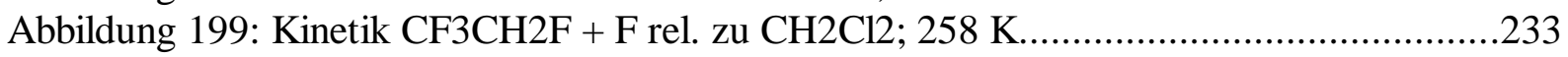

Abbildung 200: Kinetik CF3CH2F + F rel. zu CH2Cl2; 278 K........................................2234

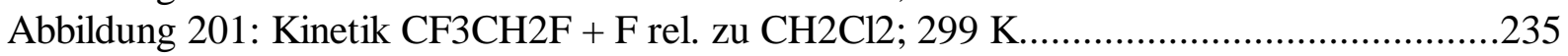

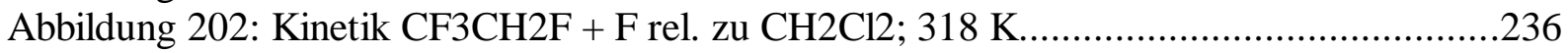

Abbildung 203: Kinetik CF3CH2F + F rel. zu CH2Cl2; 338 K......................................237

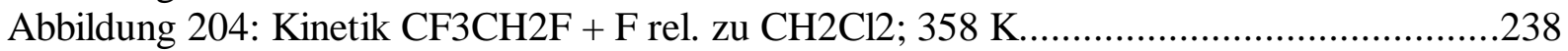

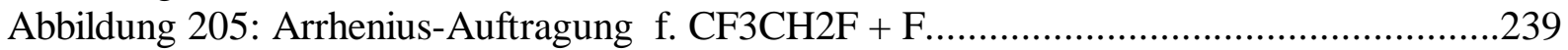

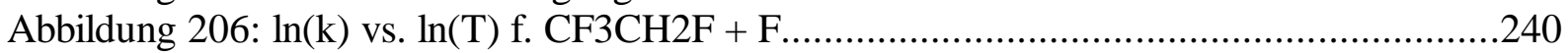

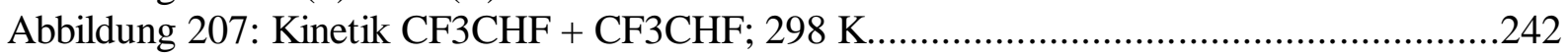

Abbildung 208: Einzelzählung mit EI-MS für CF3CH2F + F +/- O...............................243

Abbildung 209: Reaktionskanäle f. CF3CHF + O.....................................................243

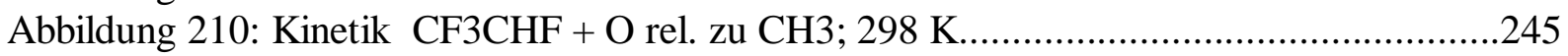

Abbildung 211: Kinetik CF3CHF + O rel. zu CH3; 298 K..........................................246

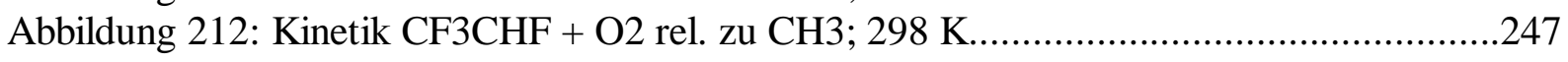

Abbildung 213: Kinetik CF3CHF + O2 (Absolutmessung); 298 K....................................249

Abbildung 214: Tatsächlich gemessener und simulierter zeitlicher Verlauf der Radikal-

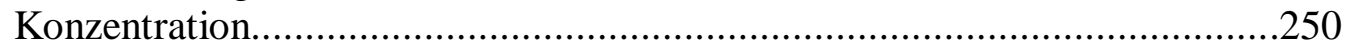

Abbildung 215: Zeitliche Entwicklung der Absorbanz f. CH3CH2OO (1040-1070 1/cm)....255

Abbildung 216: Zeitliche Entwicklung der Absorbanz f. CH3CH2OO (1040-1070 1/cm)....255

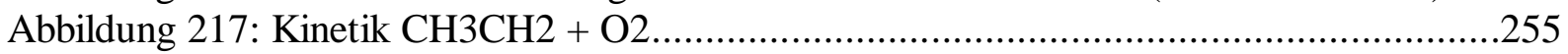




\section{Tabellenverzeichnis}

Tabelle 1: Reinheit der verwendeten Chemikalien.........................................................49

Tabelle 2: Meßwerte der Kinetik 1-Ethinyl-1-methylcyclopropan + F ; 298 K.......................88

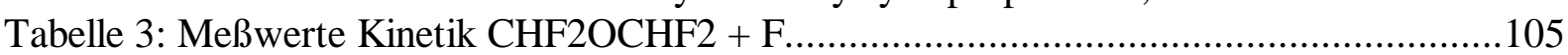

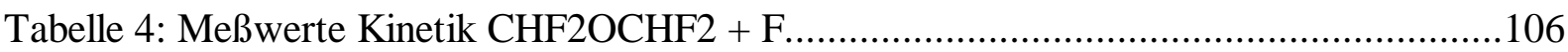

Tabelle 5: Meßwerte Kinetik C4F9OCH3 + F rel. zu CH4; 298 K...................................110

Tabelle 6: Meßwerte Kinetik C4F9OCH3 + F rel. zu CH2C12; 268 K................................111

Tabelle 7: Meßwerte Kinetik C4F9OCH3 + F rel. zu CH2Cl2; 298 K................................112

Tabelle 8: Meßwerte Kinetik C4F9OCH3 + F rel. zu CH2Cl2; 328 K...............................113

Tabelle 9: Meßwerte Kinetik C4F9OCH3 + F rel. zu CH2Cl2; 358 K..............................114

Tabelle 10: Werte für Arrhenius-Auftragung C4F9OCH3 + F rel. zu CH2Cl2 ...................115

Tabelle 11: Meßwerte Kinetik C4F9OCH2 + O2 rel. zu CHCl2; 298 K.............................119

Tabelle 12: Meßwerte Kinetik C4F9OCH2 + O2 rel. zu CHCl2; 318 K.............................120

Tabelle 13: Meßwerte Kinetik C4F9OCH2 + O2 rel. zu CHCl2; 338 K.............................121

Tabelle 14: Meßwerte Kinetik C4F9OCH2 + O2 rel. zu CHC12; 358 K............................122

Tabelle 15: Werte für Arrhenius-Auftragung C4F9OCH2 + O2 rel. zu CHCl2 ...................123

Tabelle 16: Meßwerte Kinetik CF3CH2OH + F rel. zu CH4; 258 K.................................135

Tabelle 17: Meßwerte Kinetik CF3CH2OH + F rel. zu CH4; 278 K................................136

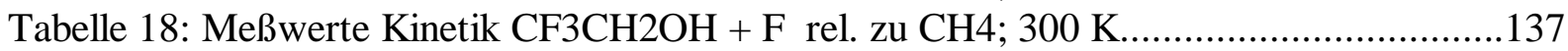

Tabelle 19: Meßwerte Kinetik CF3CH2OH + F rel. zu CH4; 328 K..................................138

Tabelle 20: Meßwerte Kinetik CF3CH2OH + F rel. zu CH4; 358 K...............................139

Tabelle 21: Werte für Arrhenius-Auftragung sowie $\mathrm{k}(\mathrm{T})$ für $\mathrm{CF} 3 \mathrm{CH} 2 \mathrm{OH}+\mathrm{F}$ rel. zu CH4. 140

Tabelle 22: Meßwerte Kinetik $\{\mathrm{CF} 3 \mathrm{CH} 2 \mathrm{OH}+\mathrm{F}\}+\{\mathrm{O} 2\}$ rel. zu CH2Cl2; $258 \mathrm{~K} \ldots \ldots \ldots \ldots . . . . .144$

Tabelle 23: Meßwerte Kinetik $\{\mathrm{CF} 3 \mathrm{CH} 2 \mathrm{OH}+\mathrm{F}\}+\{\mathrm{O} 2\}$ rel. zu CH2Cl2; $278 \mathrm{~K} \ldots \ldots \ldots \ldots . . . . .145$

Tabelle 24: Meßwerte Kinetik $\{\mathrm{CF} 3 \mathrm{CH} 2 \mathrm{OH}+\mathrm{F}\}+\{\mathrm{O} 2\}$ rel. zu CH2Cl2; $298 \mathrm{~K} \ldots \ldots \ldots \ldots . . . .146$

Tabelle 25: Meßwerte Kinetik $\{\mathrm{CF} 3 \mathrm{CH} 2 \mathrm{OH}+\mathrm{F}\}+\{\mathrm{O} 2\}$ rel. zu CH2Cl2; $328 \mathrm{~K} \ldots \ldots \ldots \ldots . . . .147$

Tabelle 26: Meßwerte Kinetik $\{\mathrm{CF} 3 \mathrm{CH} 2 \mathrm{OH}+\mathrm{F}\}+\{\mathrm{O} 2\}$ rel. zu CH2Cl2; $358 \mathrm{~K} \ldots \ldots \ldots \ldots . . . .148$

Tabelle 27: Werte für Arrhenius-Auftragung $\{\mathrm{CF} 3 \mathrm{CH} 2 \mathrm{OH}+\mathrm{F}\}+\{\mathrm{O} 2\}$ rel. zu CH2Cl2...149

Tabelle 28: Meßwerte Kinetik $\{\mathrm{CF} 3 \mathrm{CH} 2 \mathrm{OH}+\mathrm{F}\}+\{\mathrm{NO}\}$ rel. zu CH2Cl2; $298 \mathrm{~K} \ldots \ldots \ldots \ldots . . . . .151$

Tabelle 29: Meßwerte Kinetik CHF2CH2OH + F rel. zu CH4; 299 K.................................157

Tabelle 30: Meßwerte Kinetik $\{\mathrm{CHF} 2 \mathrm{CH} 2 \mathrm{OH}+\mathrm{F}\}+\{\mathrm{O} 2\}$ rel. zu CH3Cl; $300 \mathrm{~K} \ldots \ldots \ldots \ldots . . . . .160$

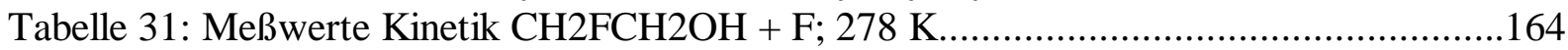

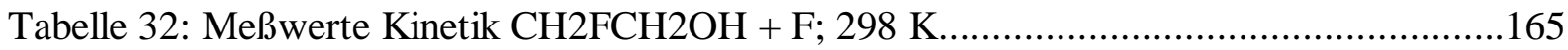

Tabelle 33: Meßwerte Kinetik CH2FCH2OH + F; 328 K..............................................166

Tabelle 34: Meßwerte Kinetik CH2FCH2OH + F; 358 K...............................................167

Tabelle 35: Absolute k sowie Werte der Arrhenius-Auftragung f. CH2FCH2OH + F.........168

Tabelle 36: Meßwerte Kinetik $\{\mathrm{CH} 2 \mathrm{FCH} 2 \mathrm{OH}+\mathrm{F}\}+\{\mathrm{O} 2\}$ rel. zu 1,4-Dioxan; $258 \mathrm{~K} \ldots \ldots . .174$

Tabelle 37: Meßwerte Kinetik $\{\mathrm{CH} 2 \mathrm{FCH} 2 \mathrm{OH}+\mathrm{F}\}+\{\mathrm{O} 2\}$ rel. zu 1,4-Dioxan; $278 \mathrm{~K} \ldots \ldots . .175$

Tabelle 38: Meßwerte Kinetik $\{\mathrm{CH} 2 \mathrm{FCH} 2 \mathrm{OH}+\mathrm{F}\}+\{\mathrm{O} 2\}$ rel. zu 1,4-Dioxan; $298 \mathrm{~K} \ldots . . . .176$

Tabelle 39: Meßwerte Kinetik $\{\mathrm{CH} 2 \mathrm{FCH} 2 \mathrm{OH}+\mathrm{F}\}+\{\mathrm{O} 2\}$ rel. zu 1,4-Dioxan; $328 \mathrm{~K} \ldots \ldots . .177$

Tabelle 40: Meßwerte Kinetik $\{\mathrm{CH} 2 \mathrm{FCH} 2 \mathrm{OH}+\mathrm{F}\}+\{\mathrm{O} 2\}$ rel. zu 1,4-Dioxan; $358 \mathrm{~K} \ldots \ldots . .178$

Tabelle 41: Werte für Arrhenius-Auftragung sowie $k(\mathrm{~T})$ für $\{\mathrm{CH} 2 \mathrm{FCH} 2 \mathrm{OH}+\mathrm{F}\}+\{\mathrm{O} 2\}$ rel.

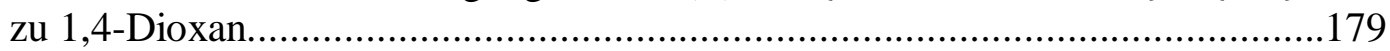

Tabelle 42: Meßwerte Kinetik CF3CF2CF2CH2OH + F rel. zu CH4; 258 K.....................184

Tabelle 43: Meßwerte Kinetik CF3CF2CF2CH2OH + F rel. zu CH4; 298 K......................185

Tabelle 44: : Meßwerte Kinetik CF3CF2CF2CH2OH + F rel. zu CH4; 338 K....................186

Tabelle 45: $\mathrm{k}(\mathrm{T})$ sowie Werte der Arrhenius-Auftragung f. CF3CF2CF2CH2OH + F.......187

Tabelle 46: Meßwerte Kinetik $\{\mathrm{CF} 3 \mathrm{CF} 2 \mathrm{CF} 2 \mathrm{CH} 2 \mathrm{OH}+\mathrm{F}\}+\{\mathrm{O} 2\}$ rel. zu CH2Cl2; $258 \mathrm{~K} . .189$

Tabelle 47: Meßwerte Kinetik $\{\mathrm{CF} 3 \mathrm{CF} 2 \mathrm{CF} 2 \mathrm{CH} 2 \mathrm{OH}+\mathrm{F}\}+\{\mathrm{O} 2\}$ rel. zu CH2Cl2; $298 \mathrm{~K} .190$

Tabelle 48: Meßwerte Kinetik $\{\mathrm{CF} 3 \mathrm{CF} 2 \mathrm{CF} 2 \mathrm{CH} 2 \mathrm{OH}+\mathrm{F}\}+\{\mathrm{O} 2\}$ rel. zu CH2Cl2; $299 \mathrm{~K} . .191$ 
Tabelle 49: Meßwerte Kinetik $\{\mathrm{CF} 3 \mathrm{CF} 2 \mathrm{CF} 2 \mathrm{CH} 2 \mathrm{OH}+\mathrm{F}\}+\{\mathrm{O} 2\}$ rel. zu CH2Cl2; $338 \mathrm{~K} . .192$ Tabelle 50: Meßwerte Kinetik $\{\mathrm{CF} 3 \mathrm{CF} 2 \mathrm{CF} 2 \mathrm{CH} 2 \mathrm{OH}+\mathrm{F}\}+\{\mathrm{O} 2\}$ rel. zu CH2Cl2; $358 \mathrm{~K} . .193$ Tabelle 51: $\mathrm{k}(\mathrm{T})$ sowie Werte f. Arrhenius-Auftr. f. $\{\mathrm{CF} 3 \mathrm{CF} 2 \mathrm{CF} 2 \mathrm{CH} 2 \mathrm{OH}+\mathrm{F}\}+\{\mathrm{O} 2\} \ldots . .194$ Tabelle 52: Massenspektrometrische Empfindlichkeit von CO rel. zu Ar.............................198

Tabelle 53: Abschätzung k(E) für unimolekularen Zerfall von $(\mathrm{CHCl} 2 \mathrm{O})^{*}$.........................217

Tabelle 54: Meßwerte Kinetik CHCl2 + NO rel. zu CH2F; 298 K.....................................221

Tabelle 55: Meßwerte Kinetik CHCl2 + NO rel. zu CH2F; 298 K.....................................222

Tabelle 56: Meßwerte Kinetik CHCl2 + NO rel. zu CH2F; 298 K......................................223

Tabelle 57: Meßwerte Kinetik CHCl2 + NO rel. zu CH2F; 298 K.....................................224

Tabelle 58: Meßwerte Kinetik CHCl2 + NO rel. zu CH2F; 298 K.....................................22

Tabelle 59: Meßwerte Kinetik CHCl2 + NO rel. zu CH2F; 298 K...................................226

Tabelle 60: Meßwerte Kinetik CF3CH2F + F rel. zu CHCl3; 298 K.....................................231

Tabelle 61: Meßwerte Kinetik CF3CH2F + F rel. zu CH2Cl2; 258 K................................233

Tabelle 62: Meßwerte Kinetik CF3CH2F + F rel. zu CH2Cl2; 278 K.................................234

Tabelle 63: Meßwerte Kinetik CF3CH2F + F rel. zu CH2Cl2; 299 K..................................235

Tabelle 64: Meßwerte Kinetik CF3CH2F + F rel. zu CH2Cl2; 318 K..................................236

Tabelle 65: Meßwerte Kinetik CF3CH2F + F rel. zu CH2Cl2; 338 K.................................237

Tabelle 66: Meßwerte Kinetik CF3CH2F + F rel. zu CH2Cl2; 358 K.................................238

Tabelle 67: $\mathrm{k}(\mathrm{T})$ sowie Werte f. Arrhenius-Auftr. f. CF3CH2F + F..................................239

Tabelle 68: Meßwerte Kinetik CF3CHF + CF3CHF; 298 K.............................................241

Tabelle 69: Meßwerte Kinetik CF3CHF + O rel. zu CH3; 298 K....................................244

Tabelle 70: Meßwerte Kinetik CF3CHF + O rel. zu CH3; 298 K......................................245

Tabelle 71: Meßwerte Kinetik CF3CHF + O2 rel. zu CH3; 298 K...................................247

Tabelle 72: Meßwerte Kinetik CF3CHF + O2 (Absolutmessung); 298 K...........................249

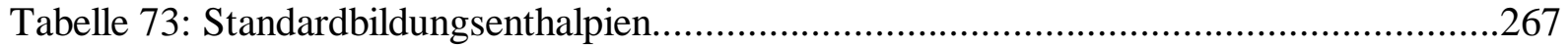

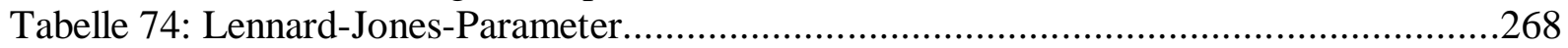




\section{Symbol- und Abkürzungsverzeichnis}

\section{1.) Schreibweisen:}

$\mathrm{I}(15)$

$\Delta \mathrm{I}(15)$

$+\mathrm{F}$

$-\mathrm{F}$

2,14E-12

$\exp (\mathrm{x})$

[B]

[m/z 16]

$(\mathrm{B})^{*}$

./.

$\left\{\mathrm{CH}_{4}+\mathrm{F}\right\}+\left\{\mathrm{O}_{2}\right\}$
Intensität bei $\mathrm{m} / \mathrm{z}=15$ (bei EI-MS ist dies der DIFF-Wert = DATA ./. BKG) Mittlerer Fehler des Mittelwertes von I(15) für Konfidenzintervall von $95 \%$ deutet an, daß die Messung unter Anwesenheit von F-Atomen erfolgte deutet an, daß die Messung unter Abwesenheit von F-Atomen erfolgte andere Schreibweise für: $2,14 \cdot 10^{-12}$

andere Schreibweise für $\mathrm{e}^{\mathrm{x}}$; e: Eulersche Zahl

Konzentration der Substanz B; Einheit: $\mathrm{mol} / \mathrm{cm}^{3}$

Intensität ( $\propto$ Konzentration) im MS gemessen bei m/z 16

Teilchen B im angeregten Zustand andere Schreibweise für das Minuszeichen ,,““

Reaktion des aus $\mathrm{CH}_{4}+\mathrm{F}$ erzeugten Radikals mit $\mathrm{O}_{2}$ (d.h. hier: $\cdot \mathrm{CH}_{3}+\mathrm{O}_{2}$ )

\section{2.) Symbole:}

$\phi$

$\vartheta$

A

$\mathrm{E}_{\mathrm{a}}$

I(15)

$\mathrm{k}$

$\mathrm{k}$ oder $\mathrm{k}_{\mathrm{B}}$

$\mathrm{k}_{\text {rel }}$

$\mathrm{k}_{\mathrm{abs}}$

$\mathrm{m} / \mathrm{z}$

$\mathrm{M}_{\mathrm{r}}$

$\mathrm{n}$

$\mathrm{n}(\mathrm{F} 2)$

$\mathrm{p}$

$\mathrm{p}_{\mathrm{StrR}}$

$\mathrm{p}_{\text {vor }}$

$\mathrm{R}$

R

$\mathrm{S}$

$\mathrm{t}$

$\mathrm{T}$
Gasfluß bezogen auf $101325 \mathrm{~Pa}$ und 273,15 K; Einheit: $\mathrm{cm}^{3} / \mathrm{min}$

Temperatur; Einheit: ${ }^{\circ} \mathrm{C}$

Präexponentieller Faktor (auch als „Arrhenius-Vorfaktor“ bezeichnet)

Aktivierungsenergie

Intensität bei $\mathrm{m} / \mathrm{z}=15$ (bei EI-MS ist dies der DIFF-Wert $=$ DATA.$/$. BKG)

Geschwindigkeitskoeffizient

Boltzmann-Konstante $\left(1,38066 \cdot 10^{-23} \mathrm{~J} / \mathrm{K}\right)$

relativer Geschwindigkeitskoeffizient (siehe Gleichung (10), S. 10)

(absoluter) Geschwindigkeitskoeffizient (entspricht $\mathrm{k}_{\mathrm{a}}$ in Gleichung (10)

Masse/Ladung-Verhältnis; $\mathrm{z}=\mathrm{n} \cdot \mathrm{e}=$ Ladung des Ions

relative Molekülmasse (früher als Molekulargewicht bezeichnet)

Stoffmenge

Anzahl Umdrehungen am Hoke-Ventil zur Dosierung des F2-Flusses

Druck

Druck im Strömungsreaktor

Vordruck Helium im Sättiger bei Zugabe von flüssigen Substanzen

nicht näher charakterisierter (ggf. halogenierter) Kohlenwasserstoffrest

Gaskonstante $(8,31441 \mathrm{~J} /(\mathrm{mol} \cdot \mathrm{K}))$

Reaktionsstrecke im Strömungsreaktor

Zeit

Temperatur (in K)

\section{3.) Abkürzungen:}

abs.

$\mathrm{AC}$

$\mathrm{AD}$

ASCII

Aufl.

berechn. absolut

anlternating current (engl.) $=$ Wechselstrom

Analog-Digital

American Standard Code for Information Interchange

Auflage

berechnetes 


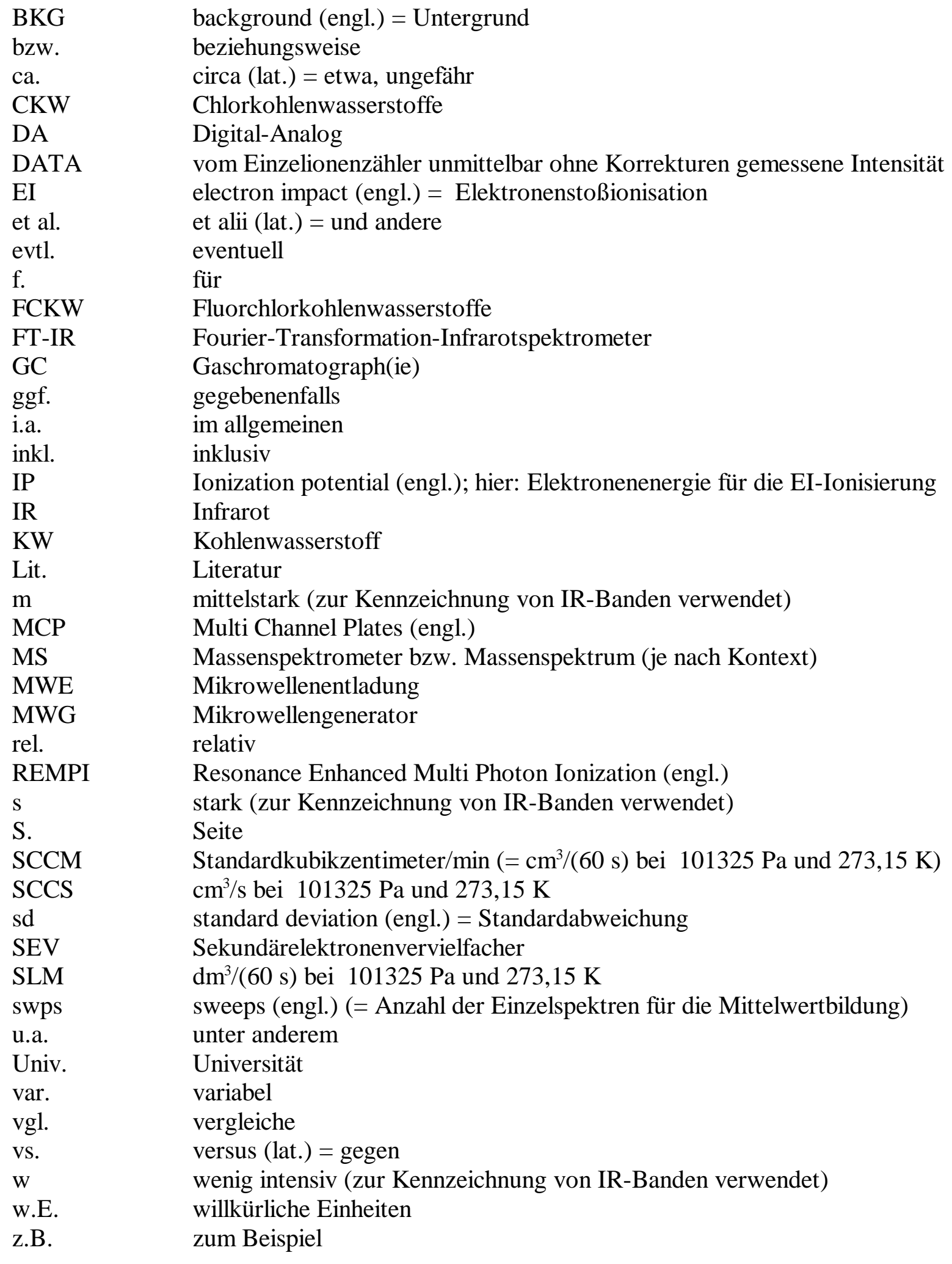





\section{Einleitung}

\subsection{Problemstellung}

Aufgrund ihrer hohen Ringspannung (für Cyclopropan beträgt diese ${ }^{1}$ ca. $121 \mathrm{~kJ} / \mathrm{mol}$ ) können Cyclopropanderivate durch Zuführung von Energie (,Aktivierung“) Reaktionen eingehen, die unter Öffnung des dreigliedrigen Rings verlaufen. Die thermische Aktivierung (bei $400-500{ }^{\circ} \mathrm{C}$ ) von Cyclopropan bewirkt beispielsweise die Isomerisierung zu Propen, wobei überwiegend angenommen wird, daß diese Reaktion über eine diradikalische Zwischenstufe verläuft ${ }^{2}$ :

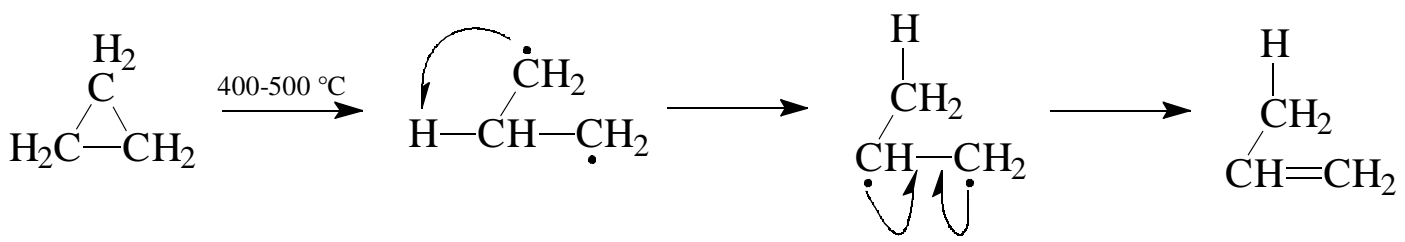

Einen ersten Schwerpunkt in dieser Arbeit bildet die Untersuchung der (unimolekularen) Reaktionen von 1-Ethinyl-1-methylcyclopropan in der Gasphase nach dessen Aktivierung. Von anderen Arbeitsgruppen wurden bislang nur die Reaktionen nach thermischer Aktivierung (Pyrolyse) einiger ähnlicher hochgespannter Kohlenwasserstoffe (Cyclopropan-Derivate) beschrieben $^{3}$. Eine solche thermische Aktivierung des 1-Ethinyl-1-methylcyclopropans wird hier ebenfalls durchgeführt, um die hierbei entstehenden Reaktionsprodukte zu identifizieren. Darüber hinaus soll überprüft werden, ob auch die chemische Aktivierung dieser Substanz möglich ist und welche Produkte ggf. dabei auftreten.

Als zweiter Schwerpunkt werden Reaktionen von Substanzklassen untersucht, die für die Atmosphärenchemie von Bedeutung sind bzw. sein könnten. Die Ozon-Schicht in der Atmosphäre dient für viele Lebewesen als Schutzschild vor dem Anteil der „harten“ UVStrahlung der Sonne. Seit den dreißiger Jahren gibt es bereits kontinuierlich Messungen der Ozonschichtdicke, deren Gesamtauswertung 1991 bestätigte, daß sich mit Ausnahme der tropischen Breiten die Ozonsäulendichten in allen Regionen deutlich negativ entwickeln ${ }^{4}$, so daß dieses Phänomen von vielen Arbeitsgruppen untersucht wurde und noch immer Gegenstand aktueller Forschung ist. In diesem Zusammenhang wurde 1995 auch der Nobelpreis für Chemie an die drei Wissenschaftler F.S. Rowland, P. Crutzen und M.J. Molina verliehen und als Konsequenz nicht zuletzt auch aus deren Arbeiten die Produktion von

1 Carey, F.; Sundberg, R. (1995); S. 153.

2 March, J. (1992); S. 1076.

3 Vgl. Hopf, H.; Wachholz, G. (1987); S. 1259-1261 / Dalacker, V.; Hopf, H. (1974) / Hopf, H. et. al. (1989); S. 377-382 / Wachholz, G. (1987) /

Hellwig, J. (1999); S. 14 ff.

4 Vgl. Zellner, R. (1993); S. 230-231. 
Fluorchlorkohlenwassertoffen (FCKW) in den Industrieländern seit 1996 eingestellt ${ }^{5}$.

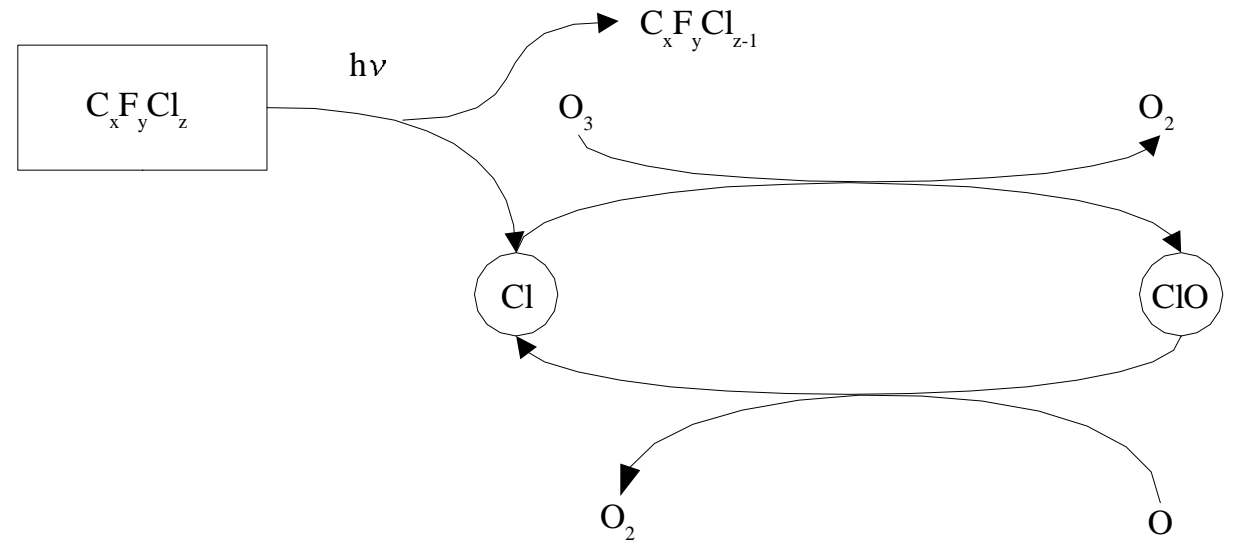

Abbildung 1: Stark vereinfachtes Schema des katalysierten Ozon-Abbaus

In Abbildung 1 ist ein stark vereinfachtes Schema des katalytischen Ozon-Abbaus ${ }^{6}$, der durch photolytische Spaltung der FCKW eingeleitet wird, dargestellt; dies ist nur möglich, weil die C-Cl-Bindung mit ca. $335 \mathrm{~kJ} / \mathrm{mol}$ relativ zur C-F- und auch zur C-H-Bindung um etwa 20 bis $25 \%$ schwächer ist ${ }^{7}$ und deshalb in der Stratosphäre schon durch den Anteil des Sonnenlichtes (hv) mit einer Wellenlänge kleiner als ca. $350 \mathrm{~nm}$ gespalten werden kann.

Die Problematik der FCKW wurde erst relativ spät erkannt, weil zwischen der Troposphäre (unterster Teil der Atmosphäre, bis ca. 12 km Höhe) und der Stratosphäre (Bereich oberhalb der Troposphäre, 12-30 km Höhe) nur ein sehr langsamer Stoffaustausch stattfindet ${ }^{4}$. Viele der anthropogen emittierten Gase werden deshalb bereits in der Troposphäre abgebaut, bevor sie in die Stratosphäre gelangen können; dies erfolgt meistens über eine $\mathrm{H}$-Abstraktion durch $\mathrm{OH}-$ Radikale (aus Wasserdampf im Sonnenlicht gebildet) und einer Oxidation des entstehenden Kohlenwasserstoffradikales (siehe Abbildung 2), wobei die Reaktionsprodukte dann oft abregnen, bevor sie die Stratosphäre erreichen ${ }^{8}$.

$$
\begin{aligned}
& \mathrm{R}-\mathrm{H}+\mathrm{OH} \rightarrow \mathrm{H}_{2} \mathrm{O}+\mathrm{R} \quad ; \mathrm{R}: \text { Kohlenwasserstoff-Rest (Radikal) } \\
& \mathrm{R}+\mathrm{O}_{2} \rightarrow \mathrm{RO}_{2} \\
& \mathrm{RO}_{2}+\mathrm{NO} \rightarrow \mathrm{RO}+\mathrm{NO}_{2} \\
& \mathrm{RO}+\left(\mathrm{O}_{2}\right)+\ldots \rightarrow \text { Oxidationsprodukte } \\
& \text { Abbildung 2: Verkürztes Reaktionsschema der Abbaureaktionen in der Troposphäre }
\end{aligned}
$$

Genau dieser Abbau erfolgt mit den chemisch äußerst inerten FCKW aber nur sehr langsam, so

5 Vgl. Zellner, R. (1995); S. 322-323.

6 Vgl. dazu Reaktionsschema in: Karger, G. (1993); S. 174

7 Vgl. Lide, D. (1991), Kapitel 9, S. 121, Tabelle 5, Zeile 3.

8 Pilling, M.J.; Seakins, P.W. (1995); S. 208. 
daß z.B. $\mathrm{CCl}_{2} \mathrm{~F}_{2}$ eine Lebensdauer von $120 \mathrm{Jahren}^{9}$, das in dieser Arbeit untersuchte Chlormethan $\left(\mathrm{CH}_{3} \mathrm{Cl}\right)$ aber lediglich von $1 \mathrm{Jahr}$ aufweist ${ }^{10}$.

Aus den geschilderten Vorgängen läßt sich also entnehmen, daß CKW- und FCKWErsatzstoffe neben den erforderlichen physikalischen Eigenschaften

1. möglichst keine ,labilen“ Bindungen wie $\mathrm{C}-\mathrm{Cl}$ oder $\mathrm{C}-\mathrm{Br}$ (Verhinderung der HalogenAbspaltung in der Stratosphäre), dafür aber

2. möglichst C-H-Bindungen (Erleichterung des Abbaus in der Troposphäre) enthalten sollten. Als solche scheinen einige teilfluorierte Ether sowie teilfluorierte Alkohole geeignet ${ }^{11}$ und werden daher im Rahmen dieser Arbeit untersucht. Dazu soll insbesondere die Kinetik der Abbaureaktion in der Troposphäre nachgestellt werden, indem durch Zugabe von F-Atomen ein H-Atom abstrahiert (dies soll die Reaktion mit $\mathrm{OH}$ ersetzen) und das entstandene Radikal mit $\mathrm{O}_{2}$ umgesetzt wird. Ferner wird auch die analoge Reaktion mit NO statt $\mathrm{O}_{2}$ durchgeführt; diese erscheint interessant, weil bei dem Einsatz von hochfliegenden SST-Flugzeugen ${ }^{12}$ ein hoher NO-Ausstoß erwartet wird ${ }^{13}$, dessen Folgen besser vor der Weiterentwicklung dieses Flugzeugtyps abgeschätzt sein sollten.

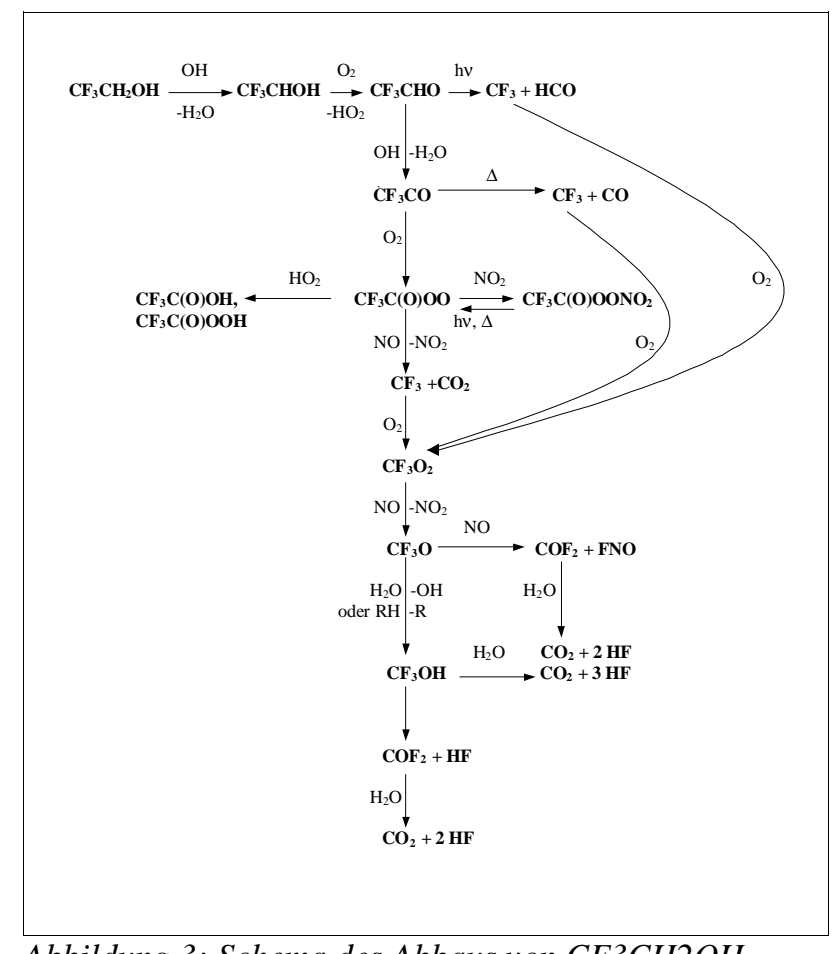

Abbildung 3: Schema des Abbaus von $\mathrm{CF} 3 \mathrm{CH} 2 \mathrm{OH}$

9 Zellner, R. (1995); S. 322.

10 Crutzen, P.; GDCh-Vortrag an der Univ. Göttingen vom 29.05.1997

11 Morozov, I. et al. (2000) / Dhooge, P. et al. (2000).

12 SST: SuperSonic Transport.

13 Vgl. Zellner, R. (1995); S. 322. 
Abbildung 3 (S. 3) zeigt das wahrscheinliche Reaktionsschema ${ }^{14}$ für den Abbau des industriell bedeutsamen 2,2,2-Trifluorethanols. Man erkennt darin, daß die Reaktion des gebildeten Radikals mit molekularem Sauerstoff von entscheidender Bedeutung ist. Daher wurde diese Reaktion eingehender untersucht und unter anderem die Temperaturabhängigkeit ihres Geschwindigkeitskoeffizienten ermittelt.

Die Kenntnis der kinetischen Daten für die oben genannten Reaktionen der teilfluorierten Ether und Alkohole ist notwendig, um Modellrechnungen zur genaueren Charakterisierung der komplexen Atmosphärenchemie durchführen zu können.

Den dritten Schwerpunkt dieser Arbeit bilden die Reaktionen einiger (teilweise teilhalogenierter) Radikale, die aus Methan-Derivaten erzeugt wurden, mit Sauerstoff-Atomen. Hier soll u.a. überprüft werden, ob Methanal HCHO (so wie es die herrschende Meinung in der Literatur sieht) für die Reaktion von Methyl-Radikalen $\mathrm{CH}_{3}$ mit atomarem Sauerstoff wirklich der einzige Produktkanal ist oder ob auch Kohlenmonoxid gebildet wird. Die Reaktionen mit atomarem Sauerstoff sind zum einen von theoretischem Interesse, um die mittels quantenmechanischer Rechnungen ermittelten Produktverteilungen im Experiment überprüfen zu können, zum anderen könnten aber auch sie für die Atmosphärenchemie von Belang sein; durch die Einwirkung von Sonnenlicht (UV-Anteil) entstehen in geringer Konzentration stets O-Atome $\left(\mathrm{O}_{2}+\mathrm{h} v \rightarrow 2 \mathrm{O} ; \mathrm{O}_{3}+\mathrm{hv} \rightarrow \mathrm{O}+\mathrm{O}_{2}\right)$, die mit gebildeten Halogenkohlenwasserstoff-Radikalen reagieren könnten. Ferner wird für einige dieser Radikale auch die für die Atmosphärenchemie noch bedeutendere Reaktion mit molekularem Sauerstoff untersucht.

\subsection{Herstellung und Verwendung der untersuchten Substanzen}

Der hochgespannte Kohlenwasserstoff 1-Ethinyl-1-methylcyclopropan wird zwar nicht industriell hergestellt, jedoch wurde im Arbeitskreis de Meijere eine Synthesemethode entwickelt, um diese Substanz immerhin im Multigrammaßstab synthetisieren zu können ${ }^{15}$. Ihre Verwendung dürfte ausschließlich zu Forschungszwecken erfolgen.

Methylnonafluorbutylether $\left(\mathrm{C}_{4} \mathrm{~F}_{9}-\mathrm{O}-\mathrm{CH}_{3}\right)$ und Ethylnonafluorbutylether $\left(\mathrm{C}_{4} \mathrm{~F}_{9}-\mathrm{O}-\mathrm{C}_{2} \mathrm{H}_{5}\right)$ werden von der Firma „3M Deutschland GmbH, Neuss“ in großem Maßstab unter den Produktnamen „Novec HFE-7100“ bzw. „Novec HFE-7200“ vertrieben. Sie werden als Flüssigkeit für (leichte) Reinigungsarbeiten, für Formulierungen und als Wärmeübertragungsmedien 
verwendet. Dabei sollen sie insbesondere früher verwendete die Ozonschicht schädigende FCKW bzw. CKW ersetzen ${ }^{16}$. Insbesondere Methylnonafluorbutylether dient als Ersatzprodukt für FCKW, HFCKW, Perchlorethylen und Trichlorethan in speziellen Lösungsmittel- und Industrieanwendungen ${ }^{17}$.

Das 2,2,2-Trifluorethanol $\left(\mathrm{CF}_{3} \mathrm{CH}_{2} \mathrm{OH}\right)$ ist ein wichtiges Synthese-Zwischenprodukt für die heute in der Medizin vorwiegend verwendeten Inhalationsanästhetika (Isofluran, Desfluran) sowie für die Agrochemie ${ }^{18}$. Ferner kann es aufgrund seiner thermodynamischen Eigenschaften als Flüssigkeit in Kompressions-Wärmepumpen eingesetzt werden ${ }^{18}$. 2,2,2-Trifluorethanol wird auch als Lösungsmittel für organische Substanzen wie Nylon, Polymethacrylat, Ethylzellulose und Polyvinylacetat verwendet. Letztlich ist es auch ein wichtiges Material für optische Polymere und Elektronik-Bauteile ${ }^{18}$. Auf Grund dieser vielfältigen Einsatzbereiche dürfte 2,2,2-Trifluorethanol in erheblichen Mengen industriell hergestellt werden und könnte damit auch in die Atmospäre gelangen.

Über eine großtechnische Produktion bzw. eine industrielle Verwendung der anderen teilfluorierten Ether und Alkohole lagen keinerlei Angaben vor.

Im Jahr 1981 wurden in West-Europa etwa 265000 t/a (100000 t/a in der BR-Deutschland) Chlormethan und 410000 t/a (170000 t/a in der BR-Deutschland) Dichlormethan hergestellt ${ }^{19}$. Chlorkohlenwasserstoffe werden auch in großem Maß von der Natur synthetisiert; so gibt es jährlich etwa fünf Millionen Tonnen biogenes Chlormethan ${ }^{20}$. Der größte Teil hiervon (70 \%) wird von marinen Organismen produziert; für die Atmosphärenchemie dürften insbesondere die durch terrestrische Holzabbauprozesse und Holzverbrennung (Waldbrände, Brandrodungen) ${ }^{20}$ freigesetzten Mengen relevant sein.

Chlormethan wird heute hauptsächlich durch die Hydrochlorierung von Methanol hergestellt; Dichlormethan durch Chlorierung von Methan $^{21}$.

Industrielle Verwendung ${ }^{21}$ findet Chlormethan zur Herstellung höherchlorierter Methane, als Methylierungsmittel in der organischen Chemie, zur Herstellung quartärer Ammonium-Salze und früher von Tetramethylblei als Kraftstoffadditiv. Wegen seiner hohen Verdampfungswärme wird es auch als Kühlmittel eingesetzt. Es ist ferner ein wichtiges Edukt zur Silikonherstellung durch die Rochow-Synthese, bei dem es in Gegenwart eines Kupfer-

16 Siehe Internet: „www.3M.com/fluids“ (Stand: Februar 2000)

17 Sicherheitsdatenblatt der Firma 3M zu „Novec HFE-7100“.

18 F-TECH INC, Tosoh Kyobashi, Chuo-ku, Tokyo, 104-0031, Japan (http://www.f-techinc.co.jp/pages.etfeain.html).

19 Ullmann's Encyclopedia of industrial chemistry (1986); S. 255

20 Naumann, K. (1993); S. 34

21 Römpp Chemie Lexikon (1991); S. 2746 und 2750 
Katalysators mit Silizium umgesetzt wird: $2 \mathrm{CH}_{3} \mathrm{Cl}+\mathrm{Si} \rightarrow\left(\mathrm{CH}_{3}\right)_{2} \mathrm{SiCl}_{2}$. Diese Methylchlorsilane werden dann hydrolysiert (heute mit Methanol, um das Edukt zurückzugewinnen) und die anschließende Kondensation liefert das Silikon.

Dichlormethan dient als Lösungs- und Extraktionsmittel, ist Bestandteil von Abbeiz-, Metallentfettungs- und Fleckenreinigungsmitteln und wird als Treibmittelzusatz für Polyurethan-Schaum und als Treibgaskomponente in Spraydosen verwendet. Die Verwendung in kosmetischen Artikeln ist auf $35 \%$ beschränkt.

\subsection{Aufbau der Arbeit}

In Kapitel 2 (S. 8) werden zunächst die theoretischen Grundlagen erläutert, welche für das Verständnis der vorliegenden Arbeit notwendig sind und einen engen Bezug zu den durchgeführten Experimenten haben. Natürlich existieren neben den dort erwähnten noch eine Vielzahl weiterer Methoden zur Bestimmung von Reaktionskinetiken, deren Beschreibung jedoch an dieser Stelle zu umfangreich sein würde.

Anschließend erfolgt im Experimentellen Teil (Kapitel 3, S. 16) die Beschreibung der verwendeten Apparaturen sowie die der praktischen Durchführung der Experimente. Weil einige Apparaturen im Rahmen dieser Arbeit wesentlich erweitert bzw. neu aufgebaut wurden, erfolgt hierfür eine entsprechend detaillierte Schilderung.

Die Meßergebnisse werden in Kapitel 4 (S. 62) dargestellt. Im Interesse einer leichteren Lesbarkeit wurde von einer strengen Aufteilung der reinen Meßwerte und deren weiterer Auswertung in verschiedene Kapitel abgesehen; die weitere Auswertung schließt sich daher stets unmittelbar an die zuvor dargestellten Meßwerte an. Am Ende der Untersuchung einer jeden Substanz findet eine Diskussion der Ergebnisse statt, die (sofern möglich) auch einen Vergleich mit den Resultaten anderer Arbeitsgruppen beinhaltet.

Im letzten Kapitel werden die wichtigsten Ergebnisse kurz zusammengefaßt.

Der Anhang enthält u.a. Angaben zur Durchführung der Fehlerrechnung.

Den Abschluß dieser Arbeit bilden die ausführlichen Beschreibungen der selbsterstellten Computerprogramme, die als eigenständige Werke jeweils über ein separates Inhalts- und Stichwortverzeichnis verfügen. 
Teile dieser Arbeit wurden bereits publiziert oder sind dafür vorgesehen:

1.)W. Hack, M. Hold, K. Hoyermann; Erzeugungs- und Oxidationsreaktionen der Radikale $\mathrm{CH}_{2} \mathrm{Cl}$ und $\mathrm{CHCl}_{2}$ in der Gasphase, Bunsentagung Dortmund, 13.-15. Mai 1999.

2.) S. Belotcerkovetc, W. Hack, M. Hold, K. Hoyermann, I. Morozov, and E. Vasil'ev; Atmospheric Processes of Partially Fluorinated Ethers, Chemical Mechanisms of Atmospheric Processes, EC Workshop Copenhagen (Denmark), August 24-25, 1998.

3.)W. Hack, M. Hold, K. Hoyermann, I. Morozov, E. Vasiliev; Mechanism and Rate of the Reactions of Fluorinated Oxygen-Containing Organic Radicals with Molecular Oxygen in the Gas Phase, International Discussion Meeting "Competetive processes in Vibrationally Highly Excited Molecules”; Göttingen, 4-7 October, 2000.

4.) W. Hack, M. Hold, K. Hoyermann, I. Morozov, E. Vasiliev; Mechanism and Rate of the Reactions of Fluorinated Oxygen-Containing Organic Radicals with Molecular Oxygen in the Gas Phase. Atmospheric Chemistry Meeting, NOAA, Boulder, Colorado (USA), July $23-25,2001$.

5.) W. Hack, M. Hold, K. Hoyermann, I. Morozov, E. Vasiliev; Mechanism and Rate of the Reactions of Fluorinated Oxygen-Containing Organic Radicals with Molecular Oxygen in the Gas Phase. 8th Eurpoean Symposium on Physico-Chemical Behaviour of Air Pollutants; Turin, 17-20 September 2001.

(http://www.ei.jrc.it/ap/events/torino2001/)

6.) Y. Dorofeev, W. Hack, M. Hold, K. Hoyermann, I. Morozov, E. Vasiliev; Atmospheric reactions of 2,2,2-Trifluorethanol; to be published in Russian „Doclady Physical Chemistry“ 2001. 


\section{Theoretische Grundlagen}

\subsection{Chemische Kinetik}

\subsubsection{Bestimmung von Geschwindigkeitskoeffizienten}

\subsubsection{Absolutmethode}

Für eine Elementarreaktion der Art $\mathrm{A}+\mathrm{X} \rightarrow$ Produkte gilt folgendes Zeitgesetz:

$$
\frac{\mathrm{d}[\mathrm{A}]}{\mathrm{dt}}=-\mathrm{k}_{\mathrm{a}}[\mathrm{A}][\mathrm{X}]
$$

Trennung der Variablen und Integration führt zu

$$
\int_{[\mathrm{A}]_{0}}^{[\mathrm{A}]_{\mathrm{t}}} \frac{\mathrm{d}[\mathrm{A}]}{[\mathrm{A}]}=-\mathrm{k}_{\mathrm{a}} \int_{\mathrm{t}_{0}}^{\mathrm{t}}[\mathrm{X}] \mathrm{dt}^{\prime}
$$

Mit der Anfangsbedingung $[\mathrm{A}]=[\mathrm{A}]_{0}$ bei $\mathrm{t}_{0}$ erhält man:

$$
\ln \left(\frac{[\mathrm{A}]_{0}}{[\mathrm{~A}]_{\mathrm{t}}}\right)=\mathrm{k}_{\mathrm{a}} \int_{\mathrm{t}_{0}}^{\mathrm{t}}[\mathrm{X}] \mathrm{dt}^{\prime}
$$

Und unter der Bedingung pseudo-erster Ordnung ([X] $>[\mathrm{A}]$ ) ergibt sich für $\mathrm{t}_{0}=0$ aus Gleichung (3) folgender analytischer Ausdruck, weil sich dann die Konzentration [X] während der Reaktion nur in kaum meßbarer Weise verringert und somit als konstant angesehen wird:

$$
\ln \left(\frac{[\mathrm{A}]_{0}}{[\mathrm{~A}]_{\mathrm{t}}}\right)=\mathrm{k}_{\mathrm{a}}[\mathrm{X}] \mathrm{t}
$$

Durch eine Auftragung der linken Seite von Gleichung (4) gegen die Zeit t erhält man aus der Geradensteigung, sofern die Konzentration des Reaktanten $\mathrm{X}$ bekannt ist, den gesuchten Geschwindigkeitskoeffizienten $\mathrm{k}_{\mathrm{a}}$.

Experimentell ergeben sich bei dieser Art der Messung jedoch eine Vielzahl von Problemen. Die Bestimmung der Konzentration $[\mathrm{X}]$ aus der massenspektrometrischen Intensität bedarf aufwendiger Kalibrierungsmessungen und im Fall vieler hier ausgeführter Messungen entspricht der Stoff $\mathrm{X}$ den Fluor bzw. Sauerstoffatomen, deren massenspektrometrische Empfindlichkeiten ohnehin sehr gering sind; bei der Konzentrationsbestimmung über eine bekannte Zuflußrate in Verbindung mit der Messung des Dissoziationsgrades ergeben sich als Fehlerquelle die Reaktionen mit Verunreinigungen in der Apparatur, z.B. mit Wasser, die nur 
schwer quantifizierbar sind; eine „Titration“ wäre mit den gleichen Problemen behaftet.

Ein weiteres Problem stellt die Umrechnung der Reaktionsstrecke in die Reaktionszeit dar, die man üblicherweise unter Annahme einer laminaren Strömung nach Poiseuille (vgl. Kapitel 2.3) durchführt; diese laminare Strömung stellt sich aber nach Zumischen des 2. Reaktanten nicht sofort ein und man erhält in diesem Zusammenhang gleich das nächste Problem, nämlich die Mischzeit. Bei extrem schnellen Reaktionen ist die Mischzeit im Verhältnis zur Reaktionszeit nicht zu vernachlässigen, so daß die Zeitbestimmung mit einem hohen Fehler behaftet ist. Jedoch können, um diesen Fehler gering zu halten, nicht beliebig lange Reaktionsstrecken gewählt werden, da dann insbesondere auch zu große Umsätze und unerwünschte Folgereaktionen auftreten könnten; um dann noch hinreichend genau die Endkonzentration $[\mathrm{A}]_{\mathrm{t}}$ messen zu können, wäre evtl. eine hohe Ausgangskonzentration $[\mathrm{A}]_{0}$ erforderlich, die aber zu einer Verletzung der Bedingungen pseudo-erster Ordnung führen würde.

Abschließend soll noch ein für die Auswertungen in dieser Arbeit erforderlicher Spezialfall, nämlich derjenige der Radikalkombination $(\mathrm{R}+\mathrm{R})$, behandelt werden.

Für diese Elementarreaktion der Art $\mathrm{R}+\mathrm{R} \rightarrow$ Produkte gilt folgendes Zeitgesetz:

$$
\frac{\mathrm{d}[\mathrm{R}]}{\mathrm{dt}}=-2 \mathrm{k}_{\mathrm{r}}[\mathrm{R}]^{2}
$$

Trennung der Variablen und Integration führt zu

$$
\int_{[\mathrm{R}]_{0}}^{[\mathrm{R}]_{\mathrm{t}}} \frac{\mathrm{d}[\mathrm{R}]}{[\mathrm{R}]^{2}}=-2 \mathrm{k}_{\mathrm{r}} \int_{\mathrm{t}_{0}}^{\mathrm{t}} \mathrm{dt}^{\prime}
$$

Mit der Anfangsbedingung $[\mathrm{R}]=[\mathrm{R}]_{0}$ bei $\mathrm{t}=\mathrm{t}_{0}=0$ erhält man:

$$
\frac{1}{[\mathrm{R}]_{\mathrm{t}}}-\frac{1}{[\mathrm{R}]_{0}}=2 \mathrm{k}_{\mathrm{r}} \mathrm{t}
$$

Gleichung (7) läßt sich umformen zu:

$$
\frac{[\mathrm{R}]_{0}}{[\mathrm{R}]_{\mathrm{t}}}=1+2[\mathrm{R}]_{0} \mathrm{k}_{\mathrm{r}} \mathrm{t}
$$

Durch eine Auftragung der linken Seite von Gleichung (8) gegen die Reaktionszeit t erhält man aus der Geradensteigung, sofern die Anfangskonzentration des Reaktanten $\mathrm{R}$ bei $\mathrm{t}=\mathrm{t}_{0}$ bekannt ist, den gesuchten Geschwindigkeitskoeffizienten $\mathrm{k}_{\mathrm{r}}$. 


\subsubsection{Relativmethode}

Zur Vermeidung der im vorhergehenden Kapitel (Absolutmethode) genannten Probleme wird für die Bestimmung der Geschwindigkeitskoeffizienten extrem schneller Reaktionen oft die sogenannte Methode der Relativmessung verwendet. Dazu benötigt man eine (Referenz-) Substanz B, deren Geschwindigkeitskoeffizient $\mathrm{k}_{\mathrm{b}}$ bezüglich der Reaktion mit dem selben Reaktanten $\mathrm{X}$ bereits bekannt ist. Für diese Elemtarreaktion $\mathrm{B}+\mathrm{X} \rightarrow$ Produkte läßt sich dann analog zu Gleichung (3) folgende Gleichung aufstellen:

$$
\ln \left(\frac{[\mathrm{B}]_{0}}{[\mathrm{~B}]_{\mathrm{t}}}\right)=\mathrm{k}_{\mathrm{b}} \int_{\mathrm{t}_{0}}^{\mathrm{t}}[\mathrm{X}] \mathrm{dt}^{\prime}
$$

Läßt man nun beide Reaktionen gleichzeitig im Strömungsreaktor ablaufen und geht man davon aus, daß sie sich gegenseitig nicht beeinflussen und daß die Konzentration [X] für die beiden Reaktanten A und B immer gleich ist, d.h. also $\int[X] \mathrm{dt}^{\prime}$ in Gleichung (3) und (9) identisch sind, so erhält man:

$$
\frac{\ln \left(\frac{[\mathrm{A}]_{0}}{[\mathrm{~A}]_{\mathrm{t}}}\right)}{\ln \left(\frac{[\mathrm{B}]_{0}}{[\mathrm{~B}]_{\mathrm{t}}}\right)}=\frac{\mathrm{k}_{\mathrm{a}}^{\mathrm{t}}[\mathrm{X}] \mathrm{dt}}{\mathrm{t}_{0}}=\frac{\mathrm{k}_{\mathrm{a}}}{\mathrm{k}_{\mathrm{b}} \int_{\mathrm{t}_{0}}^{\mathrm{t}}[\mathrm{X}] \mathrm{dt}^{\prime}} \equiv \mathrm{k}_{\mathrm{rel}}
$$

Es genügt also, das Verhältnis (!) der Konzentrationen zu Beginn der Reaktion ([A $]_{0}$ bzw. $[\mathrm{B}]_{0}$ bei $\left.t_{0}=0\right)$ und am Ende der Reaktion $\left([A]_{t}\right.$ bzw. $[B]_{t}$ bei $\left.t_{\text {ende }}=t\right)$ für jeweils Stoff $A$ und $B$ zu messen und man erhält dann aus der Steigung der doppelt logarithmischen Auftragung von $\ln \left([\mathrm{A}]_{0} /[\mathrm{A}]_{\mathrm{t}}\right)$ gegen $\ln \left([\mathrm{B}]_{0} /[\mathrm{B}]_{\mathrm{t}}\right)$ den Wert $\mathrm{k}_{\text {rel }}$ und mit dem bekannten Geschwindigkeitskoeffizienten $\mathrm{k}_{\mathrm{b}}$ unmittelbar den gesuchten $\mathrm{k}_{\mathrm{a}}$.

\subsubsection{Bestimmung der Temperaturabhängigkeit von}

\section{Geschwindigkeitskoeffizienten}

Von Arrhenius wurde bereits 1889 publiziert, daß für eine Vielzahl von Geschwindigkeitskoeffizienten folgende Temperaturabhängigkeit gilt ${ }^{22}$ :

$$
\mathrm{k}=\mathrm{A} \cdot \exp \left(-\frac{\mathrm{E}_{\mathrm{a}}}{\mathrm{RT}}\right)
$$

Hierbei wird $A$ als präexponentieller Faktor und $E_{a}$ als (experimentelle) Aktivierungsenergie 
bezeichnet; $\mathrm{R}$ ist die universelle Gaskonstante und $\mathrm{T}$ die absolute Temperatur.

Der A-Faktor kann als Stoßzahl bei unendlich hoher Temperatur interpretiert werden und wird in dieser Gleichung als temperaturunabhängig angesehen.

Bei der Herleitung des bimolekularen Geschwindigkeitskoeffizienten aus der Stoßtheorie mit der Annahme, daß der Stoßquerschnitt eine Funktion der Relativgeschwindigkeit ist, wird der präexponentielle Faktor allerdings sehr wohl temperaturabhängig: Er enthält die relative Geschwindigkeit der Stoßpartner und daraus ergibt sich eine $\mathrm{T}^{1 / 2}$-Abhängigkeit ${ }^{23}$. Aus der Theorie des aktivierten Komplexes erhält man ebenfalls eine Temperaturabhängigkeit, so daß die empirischen Daten allgemein besser mittels folgender Gleichung angegeben werden sollten:

$$
k=A T^{n} \exp \left(-\frac{E_{a}}{R T}\right)
$$

mit $\mathrm{n} \in \mathrm{P}$ (reelle Zahlen).

Für Reaktionen mit hoher Aktivierungsenergie, die in einem geringen Temperaturintervall beschrieben werden sollen, ist Gleichung (11) aber völlig ausreichend, da vorwiegend der Exponentialterm den Temperatureinfluß bewirkt; betrachtet man z.B. eine Reaktion mit $100 \mathrm{~kJ} / \mathrm{mol}$ Aktivierungsenergie, dann erhöht sich der präexponentielle Faktor bei einem Temperaturanstieg von 300 auf $310 \mathrm{~K}$ nur um 1,7 \% (bei $\mathrm{T}^{1 / 2}$-Abhängigkeit), während sich der Exponentialterm um den Faktor 364 \% erhöht. Im Rahmen der Meßgenauigkeit gibt es für solche Reaktionen also keinen offensichtlichen Widerspruch zwischen der theoretischen Gleichung aus der Stoßtheorie und der empirischen Arrheniusgleichung.

Bei Reaktionen mit sehr geringer Aktivierungsenergie werden die Meßwerte häufig durch Gleichung (13) beschrieben ${ }^{24}$ :

$$
\mathrm{k}=\mathrm{AT}^{\mathrm{n}}
$$

mit $\mathrm{n} \in \mathrm{P}$ (reelle Zahlen).

Die Stoßzahl einer Reaktion läßt sich (genauer als in der oben erwähnten Stoßtheorie) bei einer beliebigen Temperatur mit Hilfe folgender Formel berechnen:

$$
\mathrm{Z}_{\mathrm{AB}}=\mathrm{N}_{\mathrm{A}} \pi\left(\sigma_{\mathrm{AB}}\right)^{2} \sqrt{\frac{8 \mathrm{RT}}{\pi \mathrm{M}_{\mathrm{AB}}}} \Omega_{\mathrm{AB}}
$$

mit $\mathrm{N}_{\mathrm{A}}$ : Avogadrokonstante; $\sigma_{\mathrm{AB}}:$ Lennard-Jones-Länge; $\mathrm{M}_{\mathrm{AB}}:$ reduzierte Molmasse

23 Atkins, P.W. (1990), S. 665 ff.

24 Führt man eine Reihenentwicklung von Gleichung (12) durch, erhält man:

$\mathrm{k}=\mathrm{A} \mathrm{T}^{\mathrm{n}}\left(1+\{\mathrm{E} /(\mathrm{RT})\} / 1 !+\{\mathrm{E} /(\mathrm{RT})\}^{2} / 2 !+\{\mathrm{E} /(\mathrm{RT})\}^{3} / 3 !+\ldots\right)$

allgemein gilt Gleichung (13) also nur, wenn $\mathrm{E} /(\mathrm{RT})<<1$ ist. 


$$
\sigma_{\mathrm{AB}}=\frac{\sigma_{\mathrm{A}}+\sigma_{\mathrm{B}}}{2} \quad \mathrm{M}_{\mathrm{AB}}=\frac{\mathrm{M}_{\mathrm{A}} \cdot \mathrm{M}_{\mathrm{B}}}{\mathrm{M}_{\mathrm{A}}+\mathrm{M}_{\mathrm{B}}}
$$

Für das Stoßintegral $\Omega_{\mathrm{AB}}$ gilt $^{25}$ :

$$
\Omega_{\mathrm{AB}}=\frac{1,16145}{\left(\mathrm{~T}^{\prime}\right)^{0,1474}}+\frac{0,52487}{\exp \left(0,77320 \cdot \mathrm{T}^{\prime}\right)}+\frac{2,16178}{\exp \left(2,43787 \cdot \mathrm{T}^{\prime}\right)}
$$

mit: $\mathrm{T}^{\prime}=\mathrm{kT} / \varepsilon_{\mathrm{AB}} ; \mathrm{k}$ : Boltzmann-Konstante $\varepsilon_{\mathrm{AB}}:$ Lennard-Jones-Potential: $\epsilon_{\mathrm{AB}}=\sqrt{\epsilon_{\mathrm{A}} \cdot \epsilon_{\mathrm{B}}}$

\subsection{Bestimmung von Produktverteilungen}

Unterstellt sei folgende unimolekulare Reaktion ${ }^{26}$, bei der ein Edukt $\mathrm{A}$ in die Produkte $\mathrm{P}_{1}$ bis $\mathrm{P}_{\mathrm{n}}$ zerfällt: $\quad \mathrm{A} \rightarrow \mathrm{P}_{1}+\mathrm{P}_{2}+. .+\mathrm{P}_{\mathrm{n}}$

Wenn A mit einem Anteil $\alpha_{i}($ mol- $\%)$ zu $P_{i}$ reagiert, dann gilt allgemein für die Konzentrationsänderungen:

$-\alpha_{i} d[\mathrm{~A}]=\mathrm{d}\left[\mathrm{P}_{\mathrm{i}}\right] \quad$ und $\quad \sum \alpha_{\mathrm{i}}=1 \quad$; für $\mathrm{i}=1 . . \mathrm{n}$

Man hat theoretisch also $n+1$ Gleichungen, um die $n$ Variablen $\alpha_{i}$ zu bestimmen. Wie schon erwähnt wurde, ist die am Massenspektrometer gemessene Intensität individuell für jeden untersuchten Stoff in dessen Konzentration umzurechnen. Dabei gehen neben stoffspezifischen auch gerätespezifische Parameter ein, so daß die Verwendung von tabellierten Umrechnungsfaktoren (Eichkonstanten) schwierig und mit vielen Fehlern behaftet sein kann. Vereinfachend sei ein linearer Zusammenhang zwischen der Intensität und der Konzentration unterstellt, dann gilt:

$$
\mathrm{I}\left(\mathrm{P}_{\mathrm{i}}\right)=\varepsilon_{\mathrm{i}}\left[\mathrm{P}_{\mathrm{i}}\right] \quad ; \text { mit } \mathrm{I}\left(\mathrm{P}_{\mathrm{i}}\right) \text { : Intensität des Produktes } \mathrm{i} ; \varepsilon_{\mathrm{i}} \text { : Eichkonstante von } \mathrm{i}
$$

Setzt man dies in obige Gleichungen ein, dann erhält man Gleichung (15) :

$$
\alpha_{\mathrm{i}}=-\frac{\epsilon_{\mathrm{A}}}{\epsilon_{\mathrm{i}}} \frac{\mathrm{dI}\left(\mathrm{P}_{\mathrm{i}}\right)}{\mathrm{dI}(\mathrm{A})}
$$

Sofern also das Verhältnis der Eichkonstanten des Produktes i und des Eduktes A bekannt ist, können gemäß Gleichung (15) aus der Steigung einer Auftragung von den Intensitäten der Produkte gegen diejenigen des Eduktes die gesuchten Anteil-Parameter $\alpha_{i}$ bestimmt werden.

25 ReId / Prausnitz/Sherwood (1977); Gleichung 9-43

26 Die Formulierung für eine bimolekulare Reaktion ist nur ein formales Problem; daher lassen sich die weiteren Ergebnisse auch auf diesen Reaktionstyp anwenden. 
Weil lediglich das Verhältnis der Eichkonstanten eingeht, kann man davon ausgehen, daß sich die gerätespezifischen Anteile rauskürzen, so daß man näherungsweise auch tabellierte Werte aus der Literatur verwenden kann. Dabei ist aber unbedingt zu beachten, daß eine Änderung der EI-Elektronenenergie am Massenspektrometer einen stark unterschiedlichen Einfluß auf die Intensitäten bei den verschiedenen Substanzen haben kann; insbesondere wenn in der Nähe der Ionisierungsenergie einer Substanz gearbeitet wird.

\subsection{Strömungsmechanik}

Welche Strömungsart im System vorherrscht, kann mit Hilfe der Reynoldszahl $R_{\mathrm{e}}$ abgeschätzt werden; sie wird folgendermaßen berechnet:

$$
\mathrm{R}_{\mathrm{e}}=\frac{2 \mathrm{rv} \rho}{\eta}
$$

mit r: Radius; v: Strömungsgeschwindigkeit; $\varrho$ : Dichte; $\eta$ : Viskosität

Liegt ihr Wert unter 2300 (bei Strömung in einem Kreisrohr), dann handelt es sich um eine laminare Strömung. Unter den üblichen Meßbedingungen $(\mathrm{v} \approx 50 \mathrm{~m} / \mathrm{s})$ ergibt sich für Helium ( $\left.\varrho=1,36 \cdot 10^{-7} \mathrm{~g} / \mathrm{cm}^{3} ; \eta=1,96 \cdot 10^{-5} \mathrm{~Pa} \cdot \mathrm{s}\right)$, das stets über 90 Vol-\% des Gesamtgasflusses ausmachte, eine Reynoldszahl von etwa 6,6; damit lag also ein laminares Strömungsprofil vor.

Diese laminare Strömung bildet sich jedoch erst nach der Einlauflänge $L_{e}=0,116 \cdot r \cdot R_{e} \quad$ aus; sie betrug in der vorliegenden Arbeit (mit $\mathrm{r}=0,95 \mathrm{~cm}$ ) etwa $0,7 \mathrm{~cm}$.

Für die lineare Strömungsgeschwindigkeit gilt:

$$
\mathrm{v}=\frac{\Phi_{\text {ges }}}{\pi \mathrm{r}^{2}} \frac{\mathrm{T} \mathrm{p}_{0}}{\mathrm{~T}_{0} \mathrm{p}}
$$

mit $\Phi_{\text {ges }}: \sum \Phi_{\mathrm{i}}$; gesamter Gasfluß bei $\mathrm{p}=101325 \mathrm{~Pa}$ und $\mathrm{T}=273,15 \mathrm{~K}$

r: Radius des Strömungsreaktors

p: Druck im Strömungssystem

T: absolute Temperatur im Strömungssystem; die Einheit ist $K$

$\mathrm{T}_{0}=273,15 \mathrm{~K} ; \mathrm{p}_{0}=101325 \mathrm{~Pa}$

Die Verweilzeit im Strömungssystem (Reaktionszeit) ergibt sich daraus zu

$$
\mathrm{t}=\frac{\mathrm{s}}{\mathrm{v}}
$$

mit $\quad \mathrm{s}$ : Reaktionsstrecke (Sondenstellung)

Die Konzentration c der Substanz i im Strömungssystem kann man folgendermaßen berechnen: 


$$
\mathrm{c}_{\mathrm{i}}=[\mathrm{i}]=\frac{\Phi_{\mathrm{i}}}{\Phi_{\mathrm{ges}}} \frac{\mathrm{p}}{\mathrm{T} \cdot \mathrm{R}}
$$

mit $\Phi_{\mathrm{i}}$ : Gasfluß der Substanz i bei $\mathrm{p}=101325 \mathrm{~Pa}$ und $\mathrm{T}=273,15 \mathrm{~K}$

Die Wechselwirkung zwischen Strömung, Diffusion und chemischer Reaktion wird unter Vernachlässigung von Druck- und Temperaturgradienten und bei Vorliegen eines PoiseuilleProfils durch die Kontinuitätsgleichung angegeben ${ }^{27}$ :

$$
2 \mathrm{v}\left(1-\frac{\mathrm{r}^{2}}{\mathrm{R}^{2}}\right) \frac{\partial \mathrm{c}}{\partial \mathrm{z}}=\mathrm{D}\left(\frac{\partial^{2} \mathrm{c}}{\partial \mathrm{r}^{2}}+\frac{1}{\mathrm{r}} \frac{\partial \mathrm{c}}{\partial \mathrm{r}}+\frac{\partial^{2} \mathrm{c}}{\partial \mathrm{z}^{2}}\right)+\frac{\partial \mathrm{c}}{\partial \mathrm{t}}
$$

mit $\quad \mathrm{r}, \mathrm{z} \quad$ : Zylinderkoordinaten

$\mathrm{v} \quad$ : mittlere Flußgeschwindigkeit

R : Reaktorradius

c : Konzentration

D : binärer Diffusionskoeffizient

t : Zeit

Gleichung (20) ist analytisch nicht geschlossen lösbar, es existieren jedoch einige Näherungen, so z.B. eine Abschätzung bei Relativmessungen für die axiale Diffusion unter Vernachlässigung von radialer Diffusion und von Wandreaktionen ${ }^{28}$ :

$$
\frac{\mathrm{k}_{\mathrm{A}}}{\mathrm{k}_{\mathrm{B}}}=\frac{\ln \frac{[\mathrm{A}]_{0}}{[\mathrm{~A}]_{\mathrm{t}}}\left(1+\frac{\mathrm{D}}{\mathrm{v}^{2}} \ln \frac{[\mathrm{A}]_{0}}{[\mathrm{~A}]_{\mathrm{t}}}\right)}{\ln \frac{[\mathrm{B}]_{0}}{[\mathrm{~B}]_{\mathrm{t}}}\left(1+\frac{\mathrm{D}}{\mathrm{v}^{2}} \ln \frac{[\mathrm{B}]_{0}}{[\mathrm{~B}]_{\mathrm{t}}}\right)}
$$

mit $\quad \mathrm{v}$ : lineare Flußgeschwindigkeit

D: binärer Diffusionskoeffizient

Der binäre Diffusionskoeffizient läßt sich näherungsweise mit Hilfe der Chapman-EnskogGleichung für ideale Gase unter Annahme eines Lennard-Jones-Potentials ausrechnen ${ }^{29}$ :

$$
\mathrm{D}_{\mathrm{AB}}=2,6610^{-3} \frac{\sqrt{\mathrm{T}^{3}}}{\mathrm{p} \sqrt{\mathrm{M}_{\mathrm{AB}}} \sigma_{\mathrm{AB}}^{2} \Omega_{\mathrm{D}}}
$$


mit

$$
\mathrm{M}_{\mathrm{AB}}=\frac{2}{\frac{1}{\mathrm{M}_{\mathrm{A}}}+\frac{1}{\mathrm{M}_{\mathrm{B}}}} \quad \sigma_{\mathrm{AB}}=\frac{\sigma_{\mathrm{A}}+\sigma_{\mathrm{B}}}{2}
$$

$\mathrm{D}_{\mathrm{AB}} \quad$ : binärer Diffusionskoeffizient $\left[\mathrm{cm}^{2} / \mathrm{s}\right]$

$\mathrm{T}$ : Temperatur $[\mathrm{K}]$

$\mathrm{p}$ : Druck [bar]

$\mathrm{M}_{\mathrm{A}}, \mathrm{M}_{\mathrm{B}} \quad$ : Molmasse von Trägergas A bzw. Gas B [g/mol]

$\sigma_{\mathrm{A}}, \sigma_{\mathrm{B}} \quad$ : Lennard-Jones-Länge von Substanz A bzw. B $\left[10^{-10} \mathrm{~m}\right]$

$\Omega_{\mathrm{D}} \quad$ : Diffusions-Stoßintegral [dimensionslos]

Für das Stoßintegral läßt sich folgende Näherung angeben ${ }^{30}$ :

$$
\begin{gathered}
\Omega_{\mathrm{D}}=\frac{1,06036}{\mathrm{~T}^{\prime 0,15610}}+\frac{0,19300}{\exp \left(0,47635 \mathrm{~T}^{\prime}\right)}+\frac{1,03587}{\exp \left(1,52996 \mathrm{~T}^{\prime}\right)}+\frac{1,76474}{\exp \left(3,89411 \mathrm{~T}^{\prime}\right)} \\
\text { mit } \quad \mathrm{T}^{\prime}=\frac{\mathrm{k}_{\mathrm{B}} \mathrm{T}}{\epsilon_{\mathrm{AB}}} \quad \text { und } \epsilon_{\mathrm{AB}}=\sqrt{\epsilon_{\mathrm{A}} \epsilon_{\mathrm{B}}}
\end{gathered}
$$

$\mathrm{k}_{\mathrm{B}} \quad$ : Boltzmann-Konstante

$\mathrm{T}$ : absolute Temperatur

$\varepsilon_{\mathrm{A}}, \varepsilon_{\mathrm{B}}:$ Lennard-Jones-Energie von Substanz A bzw. B 


\section{Beschreibung der experimentellen Bedingungen}

\subsection{Apparaturen}

\subsubsection{El-Massenspektrometer mit Probennahme aus dem Molekularstrahl}

\subsubsection{Aufbauschema}

Zur Untersuchung der Kinetik und der Mechanismen schneller Reaktionen, die homogen in der Gasphase ablaufen, hat sich die nachstehend beschriebene Anordnung (vgl. Abbildung 4) als geeignet erwiesen. Die Apparatur bestand aus einem Strömungssystem, das die Transformation von der Zeit- in die Streckenskala erlaubt, und einem Massenspektrometer, welches eine kontinuierliche und sehr empfindliche Probenanalyse gestattet. Sie läßt sich in die drei Bereiche Reaktor, Probennahme und Nachweis unterteilen. Da dieser Aufbau schon ausführlich beschrieben wurde ${ }^{31}$, erfolgt hier in den folgenden Unterkapiteln nur eine verkürzte Darstellung, so wie sie für das Verständnis der vorliegenden Arbeit notwendig ist.

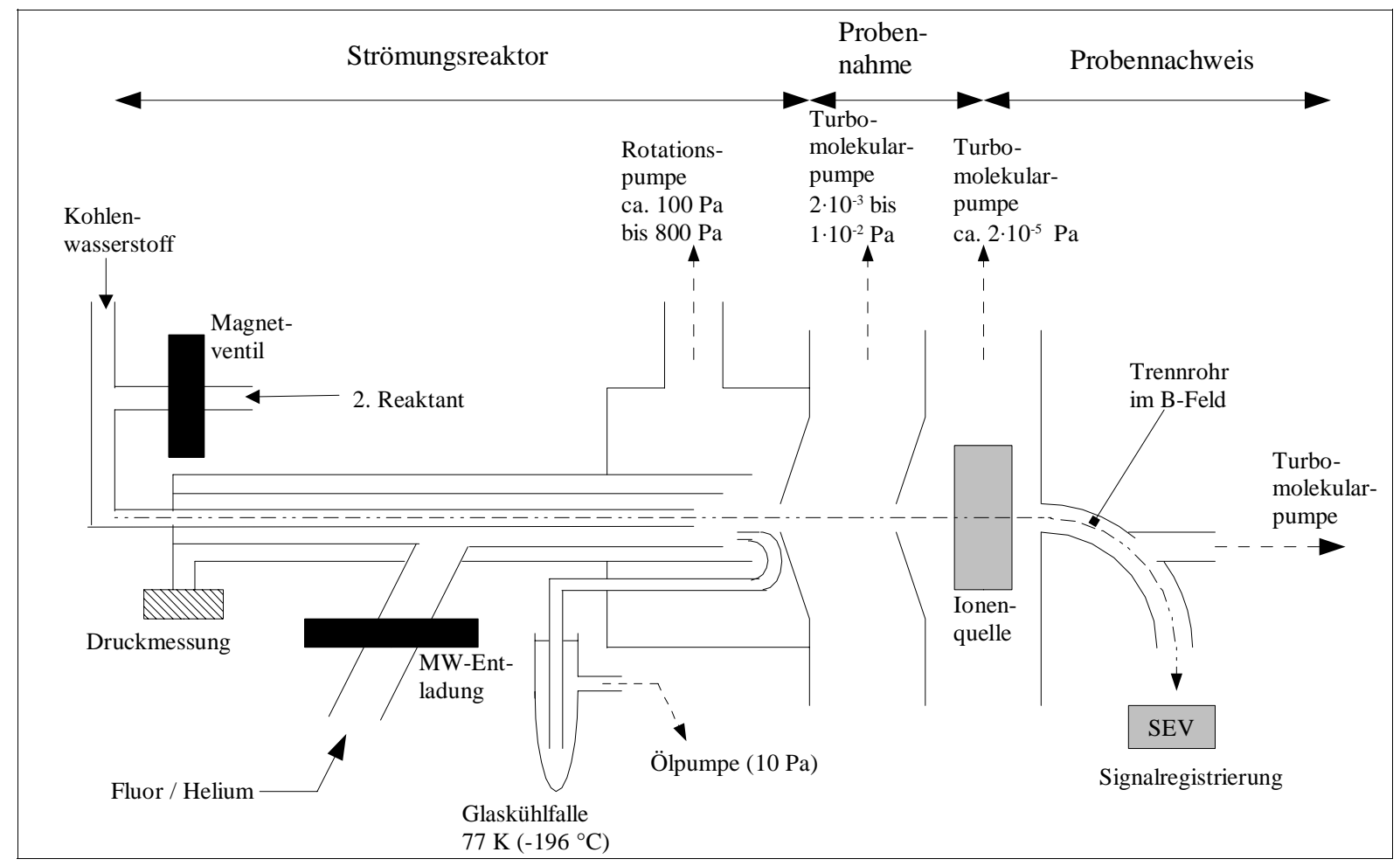

Abbildung 4: Aufbauschema Massenspektrometer mit Molekularstrahlprobennahme

\subsubsection{Strömungsreaktoren}

Es wurden im wesentlichen nur zwei verschiedene Arten an Strömungsreaktoren verwendet. Die erste Art hatte lediglich einen seitlichen Einlaß, an dem die Mikrowellenentladung angebracht war, dafür jedoch Raum für den Durchfluß einer Temperierflüssigkeit. Die zweite

31 Hoyermann, K. (1979). 
Art konnte zwar nicht temperiert werden, hatte aber zwei seitliche Einlässe, an denen jeweils eine Mikrowellenentladung angebracht war.

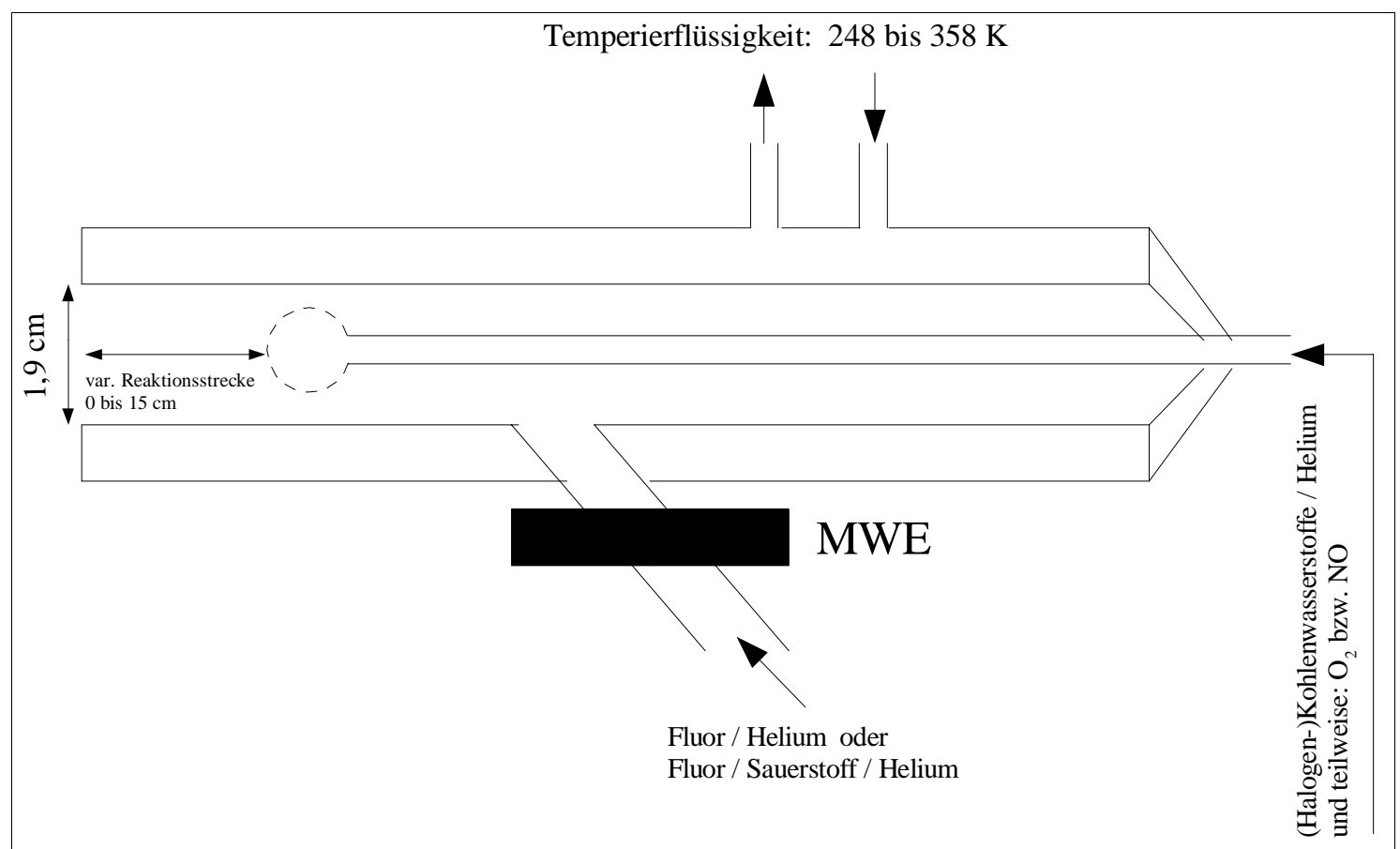

Abbildung 5: Temperierbarer Strömungsreaktor

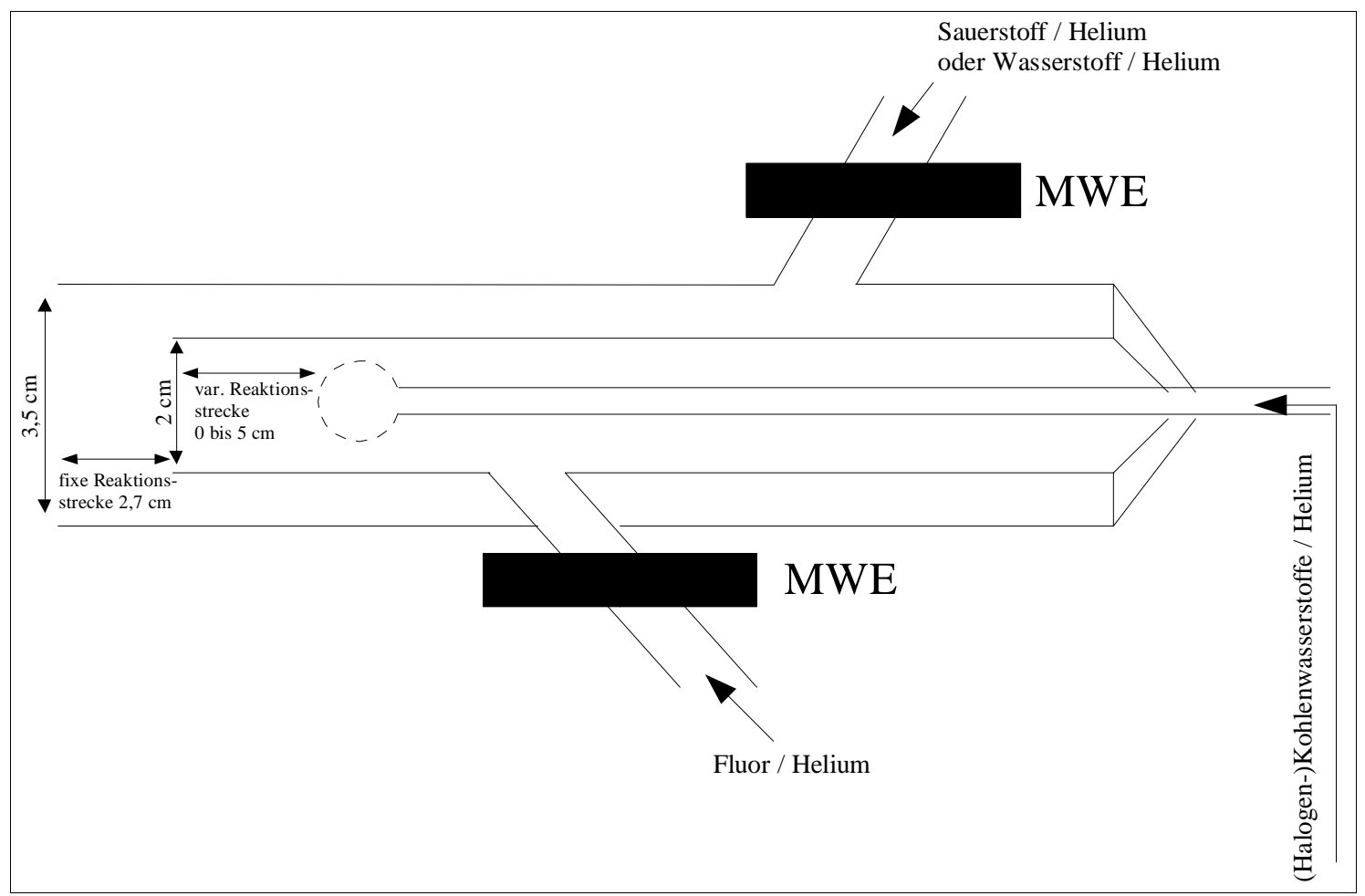

Abbildung 6: Strömungsreaktor mit zwei MWE

Die Reaktoren bestanden aus einem Pyrexglasrohr (Strömungssystem), in dessen Inneren sich eine verschiebbare Sonde befand, die zur besseren Durchmischung in eine Lochkugel von ca. 
$1 \mathrm{~cm}$ Durchmesser endete. Durch diese Sonde wurde die zu untersuchende Substanz zugeführt; sie war relativ zum äußeren Rohr verschiebbar, so daß verschiedene Strecken und damit Reaktionszeiten eingestellt werden konnten. An dem seitlichen Einlaß war über eine Metallverschraubung ein Quarzröhrchen angebracht, in dem durch elektrodenlose Mikrowellenentladung die jeweiligen Atome, z.B. F-Atome aus $\mathrm{F}_{2}$, erzeugt werden konnten.

Durch eine Sperrschiebereinblockpumpe (Leybold Heraeus, E 250; $250 \mathrm{~m}^{3} / \mathrm{h}$ Saugleistung) wurde im Strömungsreaktor ein Druck von 100 bis 800 Pa (0,8-6 Torr) erzeugt, der durch die hohe Saugleistung der Pumpe auch bei hohem Gasfluß aufrecht erhalten werden konnte.

Der Druck im Strömungsreaktor wurde mit einem Druckaufnehmer (BARATRON MKS 622; 0-10 mbar) gemessen.

An das Strömungssystem war ein Kryostat (Julabo F40, Ultratemp 2000) angeschlossen, mit dem die Kühlflüssigkeit (Ethylenglykol/Wasser-Mischung) im Strömungssystem auf Temperaturen zwischen ca. $248 \mathrm{~K}$ und $368 \mathrm{~K}\left(-25\right.$ bis $\left.+95^{\circ} \mathrm{C}\right)$ gebracht werden konnte. Die Schläuche zwischen dem Kryostaten und dem Strömungssystem waren der besseren Isolierung wegen aus Perbunan, weshalb der Temperaturgradient als vernachlässigbar angesehen und die Temperatur direkt am Kryostaten abgelesen wurde. Eine Testmessung mit einem Thermoelement im Innern des Strömungsreaktors bestätigte, daß diese Temperatur im Innern des Strömungsreaktors identisch war mit der der Temperierflüssigkeit.

\subsubsection{Probennahme}

Durch eine Expansionsdüse erreichte ein kleiner Teil des Reaktionsgemisches das Zwischenvakuum. Das Strömungssystem wurde mittels eines Tombakkörpers so justiert, daß die Nachweisempfindlichkeit maximal war.

Der Druck von $10^{-3}$ bis $10^{-4} \mathrm{~Pa}$ (ca. $10^{-5}$ bis $10^{-6}$ Torr) im Zwischenvakuum wurde durch eine Turbomolekularpumpe (Leybold Heraeus, Turbovac 450) mit vorgeschalteter Ölrotationspumpe (Leybold Heraeus, Trivac D 60 A) erzeugt.

Vom Zwischenvakuumbereich wurde durch einen Abschäler (Skimmer) ein Molekularstrahl in den Hochvakuumbereich ausgeblendet; der Skimmer hatte eine Höhe von $17 \mathrm{~mm}$, einen Innenwinkel von $17^{\circ}$ und eine Öffnung mit einem Durchmesser von 0,8 mm. Diese Werte ergaben sich aus Berechnungen ${ }^{32}$ und sind ein Kompromiß zwischen geringen Strahl-SkimmerWechselwirkungen und einem gut fokussiertem Molekularstrahl. Dadurch war auch die Detektion kurzlebiger Spezies (z.B. Radikale) möglich, denn die Reaktion wurde nach der Probennahme quasi „eingefroren“, weil Stöße zwischen den Molekülen auf Grund der geringen

32 Hoyermann, K. (1979) 
Konzentrationen nun sehr unwahrscheinlich waren. Die relativen Konzentrationen entsprachen dabei prinzipiell den Verhältnissen am Ende des Strömungsreaktors.

Um weitere Reaktionen (Folgereaktionen) im Probennahme- und Nachweisteil zu vermeiden, war ein sehr gutes Vakuum $\left(<10^{-4} \mathrm{~Pa}\right)$ erforderlich; dieses wurde durch eine Turbomolekularpumpe (Pfeiffer Balzers, TPH 270) mit vorgeschalteter Ölrotationspumpe (Edwards E30) erzeugt. Der Druck im Hochvakuumteil lag zwischen $7 \cdot 10^{-5}$ und $7 \cdot 10^{-6} \mathrm{~Pa}$ $\left(5 \cdot 10^{-7}\right.$ bis $5 \cdot 10^{-8}$ Torr $)$.

Für ein ölfreies Hochvakuum im Trennteil sorgte eine Turbomolekularpumpe (Leybold Heraeus Turbovac 150), deren Vorvakuum von einer Ölrotationspumpe (Brand RD 8) erzeugt wurde.

Der Druck im Zwischen- und Hochvakuumteil wurde mit Ionisationsvakuumetern nach Penning (Leybold Heraeus, Penningvac PM 41) gemessen.

Zusätzlich bestand aber auch die Möglichkeit, unmittelbar am Ende des Strömungsreaktors einen Teil der Reaktionsmischung in einer Glaskühlfalle oder die gesamte Reaktionsmischung (abgesehen von dem Teil, der als Molekularstrahl abgenommen wurde) in einer Metallkühlfalle bei $-196{ }^{\circ} \mathrm{C}$ auszufrieren. Dadurch konnten (insbesondere bei komplexen Produktmischungen) ergänzend FT-IR- und GC-MS-Analysen durchgeführt werden.

\subsubsection{Probennachweis}

Im Hochvakuumteil der Apparatur befand sich ein einfachfokussierendes Sektorfeldmassenspektrometer (VARIAN MAT, CH5). Der Ionenquellenraum war zur besseren Evakuierung und besseren Ausbildung eines Molekularstrahls vergrößert worden. In der Ionenquelle wurden durch Wechselwirkungen zwischen aus einer Rheniumdraht-Kathode emittierten Elektronen und den Molekülen (überwiegend) positiv geladene Molekülionen erzeugt; dieses Verfahren wird als electron impact (EI) bezeichnet. Die so erzeugten Molekülionen wurden mit einer Spannung von $3 \mathrm{kV}$ beschleunigt und über ein elektrooptisches Linsensystem auf den Eintrittsspalt eines sich zwischen zwei Magnetpolen befindenden, im Winkel von $90^{\circ}$ gebogenen, Trennrohres fokussiert. Im Inneren des Trennrohres wurden die Ionen durch das Magnetfeld auf eine Kreisbahn gezwungen und es passierten nur solche den Austrittsspalt, für die folgende Beziehung gilt ${ }^{33}$ :

$$
\frac{\mathrm{m}}{\mathrm{z}}=\frac{\mathrm{r}^{2} \cdot \mathrm{B}^{2}}{2 \cdot \mathrm{U}}
$$

mit m: Masse; z: Ladung;

33 Hesse, M.; Meier, H.; Zeeh, B. (1995); S. 221. 
r: Radius; B: magnetische Flußdichte; U: Beschleunigungsspannung Gleichung (23) wird häufig als massenspektrometrische Grundgleichung bezeichnet und läßt sich leicht durch das Gleichsetzen der Lorenzkraft und der Zentripetalkraft, die auf ein bewegtes Ion im Magnetfeld wirken, herleiten.

Durch Variation der Magnetfeldstärke, bei konstantem Ablenkradius und konstanter Beschleunigungsspannung, ist es also möglich, nur Ionen mit einem bestimmten Masse/Ladungsverhältnis ( $\mathrm{m} / \mathrm{z}$ ) zu registrieren.

Es hat sich gezeigt, daß bei Ionisierungsenergien kleiner als $70 \mathrm{eV}$ hauptsächlich einfach positiv geladene Ionen entstehen ${ }^{22}$ und damit das registrierte Verhältnis $\mathrm{m} / \mathrm{z}$ identisch mit der Masse des Ions ist.

Durch eine wohldefinierte zeitliche Änderung des Magnetstromes können mehrere Massen hintereinander durchlaufen und somit ein Spektrum registriert werden. Wird am Massenspektrometer hingegen ein konstanter Magnetstrom eingestellt, so kann selektiv die Intensität einer bestimmten Masse registriert werden. Die dem Magnetfeld proportionale Hall-Spannung wird über ein Teslameter registriert und zur Berechnung des $\mathrm{m} / \mathrm{z}$-Verhältnisses verwendet.

Bei der EI-Ionisierung werden nicht nur Ionen mit der Masse gebildet, die dem Muttermolekül (d.h. der zu registrierenden Substanz) entsprechen, sondern infolge der hohen Überschußenergie auch Fragmente, welche bei einer bestimmten EI-Elektronenenergie eine für die jeweilige Substanz charakteristische Intensitätsverteilung aufweisen. Die Ionisierungsenergien ${ }^{34}$ organischer Substanzen liegen meistens in der Größenordnung 8-15 eV, während die Bindungsenergien ${ }^{35}$ der C-C-Bindungen organischer Moleküle häufig in der Größenordnung 290-340 kJ/mol (= 3,0-3,5 eV) liegen; man erkennt durch diese Abschätzung, daß selbst bei niedrigen EI-Elektronenenergien von 17 bis $20 \mathrm{eV}$ schon genug Überschußenergie zur Spaltung einer C-C-Bindung und damit zur Fragmentierung vorliegt. Diese Fragmentierungsspektren sind für eine Vielzahl von Substanzen bekannt (meistens für 70 eV) und erleichtern damit ihre Identifizierung. Für Radikale existieren jedoch keine solchen Spektrensammlungen, weil der Anregungszustand des Radikales aus verschiedenen radikalerzeugenden Reaktionen und damit die Fragmentierung unterschiedlich sein kann. Bei dem verwendeten Massenspektrometer konnte die EI-Elektronenenergie entweder fest auf 70 $\mathrm{eV}$ oder stufenlos zwischen 4,5 und 29,5 eV eingestellt werden. Bei einer Verringerung der Energie nimmt der Fragmentierungsanteil ab und man kann ggf. aufgrund einer relativen Intensitätszunahme auf der interessierenden Masse erkennen, ob ein bestimmtes Molekül bzw. 
Radikal bei der Reaktion gebildet wird oder ob es sich nur um ein Fragment des Muttermoleküls handelt.

Die durch den Austrittsspalt austretenden Ionen treffen entweder auf einen Faraday-Auffänger oder auf einen aus 17 Dynoden bestehenden Sekundärelektronenvervielfacher (SEV), der mit einer Spannung zwischen 1,5 und 2,0 kV betrieben wurde. Die Ladungsverstärkung des SEV betrug dabei etwa $10^{5}$ bis $10^{6}$. Alle in der vorliegenden Arbeit gemessenen Werte wurden über den SEV aufgenommen; der Faraday-Auffänger wurde lediglich zur Messung der Ladungsverstärkung des SEV genutzt.

\subsubsection{Signalaufnahme}

Das vom SEV verstärkte Signal wurde über eine dreiteilige Baugruppe (SSR Instruments; Model 1120 Amplifier/Diskriminator/Pulsformer) in einen Zweikanalzähler (SSR Instruments; Model 1110 Digital Syncronous Computer) gegeben. Dabei floß die durch ein einzelnes Ion am SEV erzeugte Ladung über einen $50 \Omega$ Widerstand schnell ab und der dabei erzeugte Spannungspuls wurde im ersten Teil der Baugruppe (Amplifier) um den Faktor 2300 verstärkt; im zweiten Teil (Diskriminator) wurden zur Störsignalunterdrückung alle Signale unter $50 \mu \mathrm{V}$ „ausgeschieden“ und im dritten Teil schließlich aus den verbleibenden Signalen ein normierter Puls (vgl. Abbildung 7) geformt.

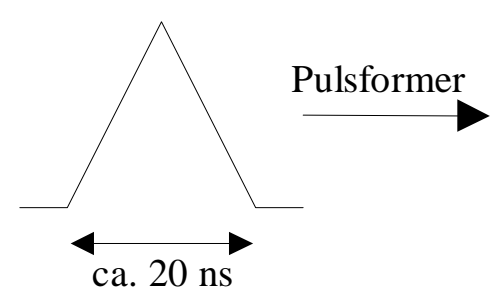

Abbildung 7: Pulsnormierung

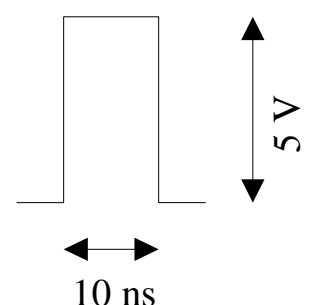

$10 \mathrm{~ns}$

Diese normierten Pulse wurden nun auf den Zweikanalzähler gegeben, der sie über eine voreingestellte Zeit aufsummierte; anschließend wurde diese Summe (,DATA-Wert“) dann an einen angeschlossenen Personal Computer weitergeleitet. Parallel dazu wurde das Signal auch auf einen Vielkanalanalysator (Tracor Northern NS-575 A Digital Signal Analyser; 15 MHz) gegeben, auf dem es optisch verfolgt werden konnte.

Da die Anzahl der pro Zeiteinheit auf den SEV auftreffenden Ionen etwa proportional der Konzentration der jeweiligen Substanz am Ort der Probennahme ist, führt eine Konzentrationsänderung durch Variation der Reaktionsparameter zu einer Intensitätsänderung des jeweiligen Peaks, welche mittels des Zweikanalzählers recht empfindlich nachgewiesen werden konnte. Unter Umständen vereinzelt auftretende Störpulse, die oberhalb der 
Diskriminatorschwelle lagen, wurden als einzelner Puls registriert und dürften gegenüber der Gesamtzahl der Pulse zu vernachlässigen sein.

Das für eine bestimmte Konzentration registrierte Signal ist stoffspezifisch und auch von experimentellen Parametern (Art der Probennahme, Einstellung der Ionenoptik) abhängig. Daher können (unter sonst gleichen Bedingungen) aus unterschiedlichen Signalen für ein und denselben Stoff zwar die relativen, jedoch nicht die absoluten Konzentrationen angegeben werden; letztere müßten durch eine Kalibrierung bestimmt werden. Für unterschiedliche Stoffe ist nicht einmal die Ermittlung der relativen Konzentrationen möglich, was insbesondere bei der Bestimmung von Produktverzweigungsverhältnissen zu beachten ist.

\subsubsection{Computersteuerung und -auswertung}

Der an die Apparatur angeschlossene Personal Computer (Typ: 80386) war mit drei Schnittstellen (Appli Data, PA110, PA150 und PA300) ausgerüstet:

- PA110: Interface mit 64 digitalen optoentkoppelten Eingängen

- PA150: Interface mit 16 digitalen Ausgängen

- PA300: 12 Bit A/D-Wandler mit 16 Kanälen und 2 Logik-Treibern

Im Rahmen dieser Arbeit wurde ein bereits während der Anfertigung der Diplomarbeit ${ }^{36}$ entwickeltes Programm zur Steuerung und Auswertung der Messungen wesentlich erweitert; es wird nachfolgend mit „WinSiccon 1.7“ bezeichnet. Es wurde in der Programmiersprache „Object-Pascal“ geschrieben und als 16-Bit-Programm mit „Delphi 1.02“ der Firma Borland compiliert. Es wurde auf dem Betriebssystem Windows 3.1 ausgeführt.

Zunächst sollen kurz die unten in Abbildung 8 erwähnten Funktionen beschrieben werden:

Über die PA150 wurde vom Programm „WinSiccon 1.7“ ein Startimpuls gegeben, der den Zweikanalzähler veranlaßte, eine vom Benutzer voreingestellte Zeit zu zählen. Im Programm konnte man die Anzahl der Messungen zwischen 1 und 10 beliebig vorgeben. Über die PA110 wurden die Kanäle DATA, BKG und DIFF des Zweikanalzählers im BCD-Format eingelesen, wobei der DATA-Wert unmittelbar die Anzahl der während einer voreingestellten Zählzeit registrierten normierten Pulse (vgl. Abbildung 7, S. 21) angab und BKG ein ggf. vorher aufgenommenes Hintergrundsignal; DIFF war die Differenz aus DATA und BKG. Diese Werte wurden sofort auf dem Bildschirm ausgegeben und auf die Festplatte geschrieben. Sofern man die Option, mehrfach hintereinander zu messen, genutzt hat, wurden auch der arithmetische Mittelwert und der mittlere Fehler des Mittelwertes für ein vorgegebenes Konfidenzintervall (in 
der vorliegenden Arbeit $95 \%$ ) berechnet und ausgegeben, so daß man starke Schwankungen sofort erkennen und eine Wiederholung der Messung durchführen konnte; bei Relativmessungen wurden zudem noch jeweils die gemessenen Logarithmenverhältnisse grafisch dargestellt und eine lineare Regression mit allen bisher gemessenen Werten durchgeführt.

Über die PA300 wurde eine vom Teslameter ausgegebene Spannung digitalisiert und in den Rechner eingelesen; das m/z-Verhältnis ist dieser Spannung proportional und wurde daher vom Programm über eine Eichkurve ${ }^{37}$ berechnet und angezeigt.

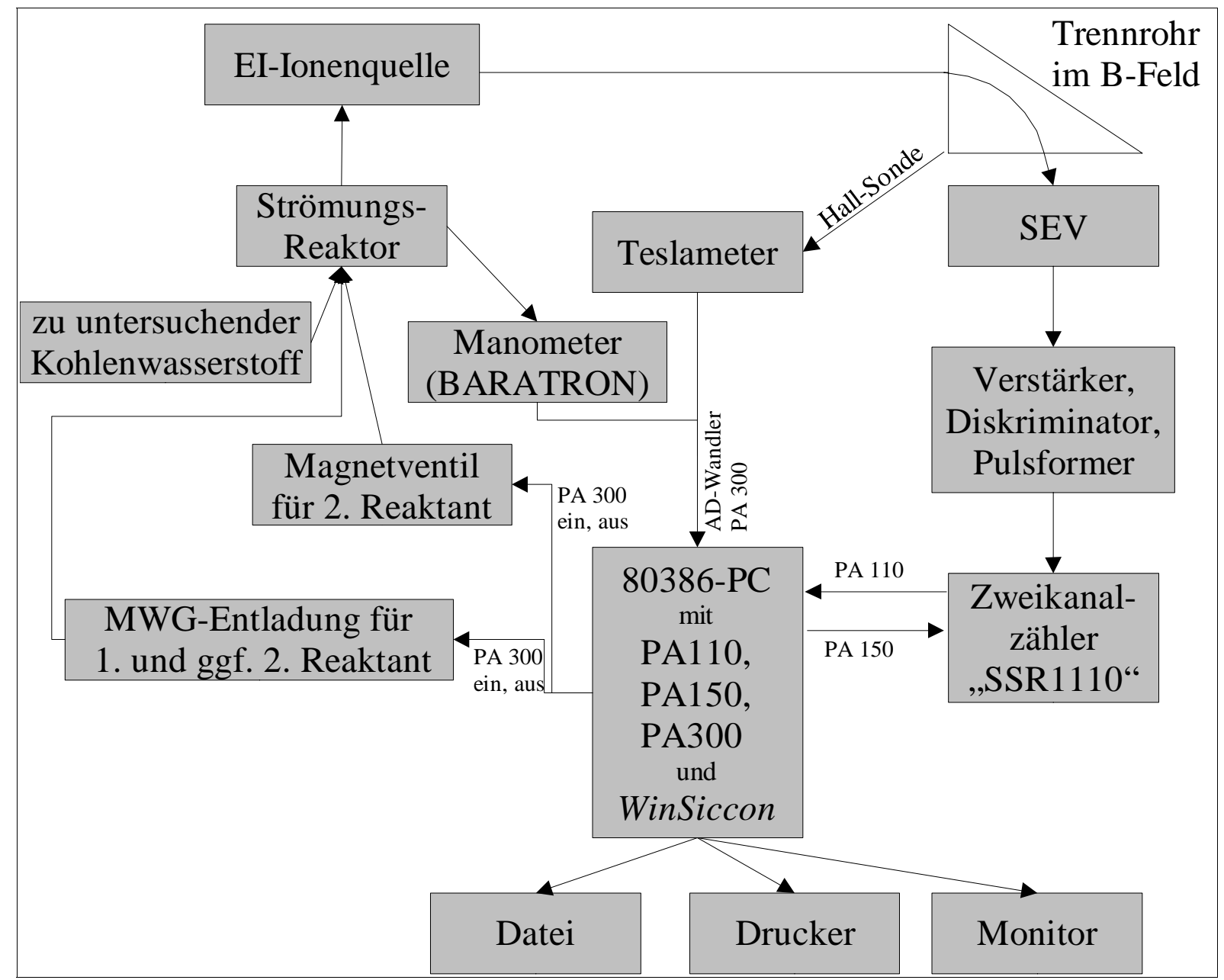

Abbildung 8: Schema Computersteuerung MS mit Molekularstrahlprobennahme

Weiterhin konnte mit dem 5 V-Signal des 1. Logik-Treibers der PA300 über einen Optokoppler der Mikrowellengenerator vom Programm automatisch ein- bzw. ausgeschaltet werden. Diese Funktion ermöglichte es, schnell hintereinander, mit einer dem Programm vorgegebenen Pause nach dem Ein- bzw. Ausschalten (um Anstiegs- bzw. Abfallflanken abzuschneiden), zu zählen und damit weitgehend konstante Versuchsbedingungen zu erlangen.

37 Die Eichkurve wurde mit Perfluorkerosin bis zu m/z 430 aufgenommen. 
Im Meßprotokoll wurde automatisch der jeweilige Zustand (,ein“/,,aus“) des MWG sowie die Pause-Zeit eingetragen.

Mittels des 2. Logik-Treibers der PA300 konnte entweder ein zweiter Mikrowellengenerator oder aber ein TTL-Schalter und mit letzterem ein Magnetventil zur Zugabe eines weiteren Reaktanten vom Programm angesteuert werden. Auch hierbei konnten analog zum 1. LogikTreiber Pausezeiten vor und nach dem Ein- bzw. Abschalten vorgegeben werden.

Da die detaillierte Beschreibung von „WinSiccon 1.7“ recht umfangreich ist, wurde im Rahmen der vorliegenden Arbeit auch eine ausführliche Programmdokumentation ${ }^{38}$ erstellt, die alle Programmteile erklärt sowie die im Programm verwendeten Formeln angibt und auch den Inhalt aller für die Auswertung erzeugten Dateien beschreibt.

Die wichtigsten Fähigkeiten des Programms sollen kurz stichpunktartig erwähnt werden:

- m/z-Anzeige kann als eigenes Fenster ständig angezeigt werden.

- Meßprotokoll kann vollständig am Bildschirm eingesehen werden.

- Meßtexte beliebiger Länge können vor jedem Meßzyklus eingegeben werden und erscheinen dann automatisch im Meßprotokoll.

- Bei Messung gegen eine Referenzsubstanz werden die aufgenommenen Meßwertpaare unmittelbar nach jedem Meßzyklus grafisch dargestellt und auch eine lineare Regression angezeigt. Dies erfolgt sowohl bei den radikalerzeugenden Reaktionen als auch bei den Reaktionen der Radikale mit einem 2. Reaktant.

- Die Fehlerrechnung erfolgt nun für ein Konfidenzintervall, das den Mittelwert wahlweise mit 50, 68, 75, 95 oder $99 \%$ Wahrscheinlichkeit überstreicht.

- Evtl. vorhandene Hintergrundwerte einer bestimmten $\mathrm{m} / \mathrm{z}-$ Zahl können getrennt für die eigentliche Messung und die Referenz-Messung inkl. Fehler eingegeben werden und werden bei der Auswertung (DIFF-Werte) berücksichtigt; die erfaßten Fehler gehen über Fehlerfortpflanzung in die weiteren Berechnungen ein.

- Bei der Messung der Reaktion eines Radikals mit einem Reaktant kann bei Überlagerung mit Fragmenten aus den Muttermolekülen durch WinSiccon unterstützt umsatzkorrigiert gemessen werden. Die grafisch angezeigte lineare Regression erfolgt dann bereits unter Berücksichtigung dieser Umsatzkorrekturen und stellt daher unmittelbar das Ergebnis dar.

- Die Zugabe eines Reaktanten (in WinSiccon mit „2. Reaktant“ bezeichnet) zu erzeugten Radikalen kann auch computergesteuert mittels Magnetventil erfolgen. Wahlweise kann aber auch computergesteuert eine zweite Mikrowellenentladung gestartet werden.

38 Hold, M. (2001); Programmbeschreibung für WinSiccon Version 1.7. 
- Die gesamten Ergebnisse (inkl. ggf. durchgeführter BKG- und/oder Umsatzkorrektur) werden sinnvoll aufbereitet in verschiedene Dateien geschrieben, die einen unmittelbaren Import und somit eine rasche (Nach-)Auswertung mittels Tabellenkalkulation ermöglichen.

- Durch einmalig hinterlegte Eichkurven bzw. Konversionsfaktoren können äußerst bequem die Gasflüsse durch „Hoke“-Dosierventile bzw. „Tylan“-Massenflußregler ermittelt werden; aus diesen Werten werden dann die Konzentrationen und Reaktionszeiten im Strömungsreaktor berechnet.

- Es können Massenspektren aufgenommen werden, wobei das Programm im Anschluß an die Registrierung die in den Rohmeßdaten erkennbar zusammengehörenden Peaks zu jeweils einem Meßpunkt (m/z-Mittelwert und aufsummierter DATA-Wert) zusammenfaßt und gesondert in einer Datei abspeichert. Diese „Peak-Dateien“ können nun problemlos voneinander subtrahiert werden, um automatisch ein Differenzspektrum zu erzeugen.

Mittels der hier beschriebenen Apparatur und des dafür entwickelten Programms „WinSiccon“ wurden außer den in dieser Arbeit dargestellten auch eine Reihe anderer Substanzen erfolgreich untersucht; es erfolgte damit z.B. die Ermittlung der Produkte und Kinetik von Reaktionen verschiedener Xylole (Dimethylbenzol) mit Fluor-Atomen und der dadurch erzeugten Radikale mit Sauerstoff-Atomen ${ }^{39}$, die analoge Untersuchung von methylsubstituierten Alkenen ${ }^{40}$ sowie diejenige von chlorsubstituierten Alkenen ${ }^{41,42}$.

39 Gieseking, T. (1998).

40 Zeuch, T. (1999).

41 Heerdt, W. (2001).

42 Fügener, M. (2001). 


\subsubsection{Strömungsreaktor mit Ofenheizung}

Für die thermische Aktivierung, bei der die Aufheizung über die Reaktorwand erfolgt, wurde die in Abbildung 9 skizzierte Apparatur aufgebaut.

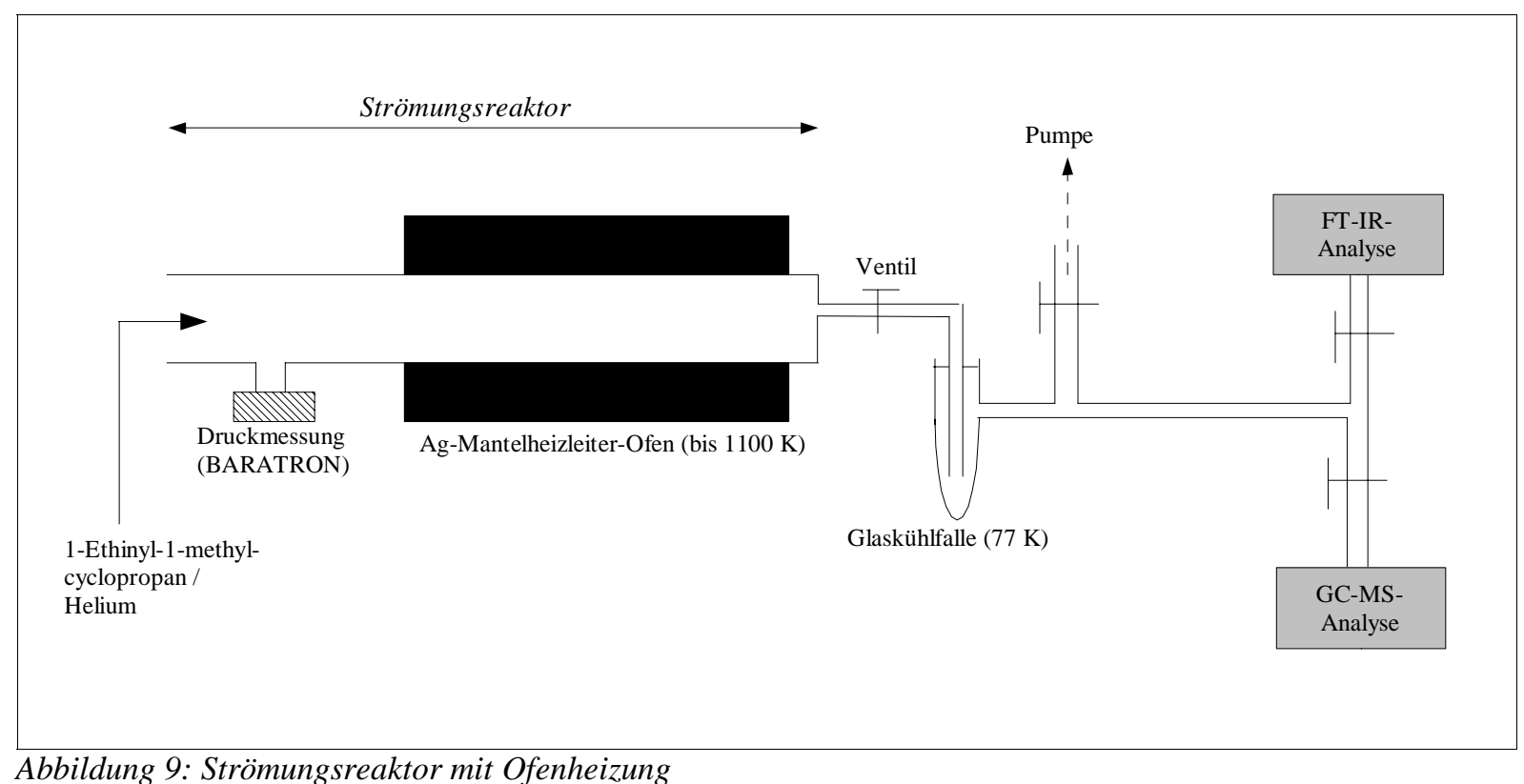

Ein Strömungsreaktor aus Quartzgut (Innendurchmesser: $20 \mathrm{~mm}$ ) war auf einer Länge von $200 \mathrm{~mm}$ mit einem elektrisch beheizbaren Silber-Mantelheizleiterofen (Wanddicke: $7 \mathrm{~mm}$ ) umgeben. Mit dieser Ofenheizung konnten Temperaturen bis $1100 \mathrm{~K}$ mit einem genau definierten Temperaturprofil $( \pm 1 \mathrm{~K})$ erreicht werden. Um „Wandreaktionen“ zu vermindern, wurde ein Glasreaktor gegenüber einem Metallreaktor bevorzugt.

Der Druck im Strömungsreaktor wurde mittels eines Druckmessers (MKS, BARATRON 122A, 0-100 mbar) kontrolliert und konnte über das Ventil zur Pumpe (Leybold Heraeus, D12A) in einem Bereich von etwa 100 bis $5000 \mathrm{~Pa}$ (1-50 mbar) eingestellt werden.

Die Temperatur innerhalb des Ofens wurde mit einem NiCr-Ni-Thermoelement gegen $0{ }^{\circ} \mathrm{C}$ (Eis/Wasser-Mischung) als Referenztemperatur gemessen.

Die Reaktionsprodukte wurden in einer mit flüssigem Stickstoff auf $77 \mathrm{~K}$ gekühlten Glaskühlfalle gesammelt und konnten anschließend im FT-IR-Spektrometer oder im Massenspektrometer bzw. der GC-MS-Kopplung analysiert werden. 


\subsubsection{GC-MS-Kopplung}

\subsubsection{Aufbauschema}

Die Analyse komplexer Produktmischungen kann häufig vorteilhaft mit einer GC-MSKopplung durchgeführt werden.

Dazu wurde ein Gaschromatograph so an ein Sektorfeldmassenspektrometer angebaut, daß die Kapillarsäule direkt in der Ionenquelle des Massenspektrometers endete (vgl. Abbildung 10).

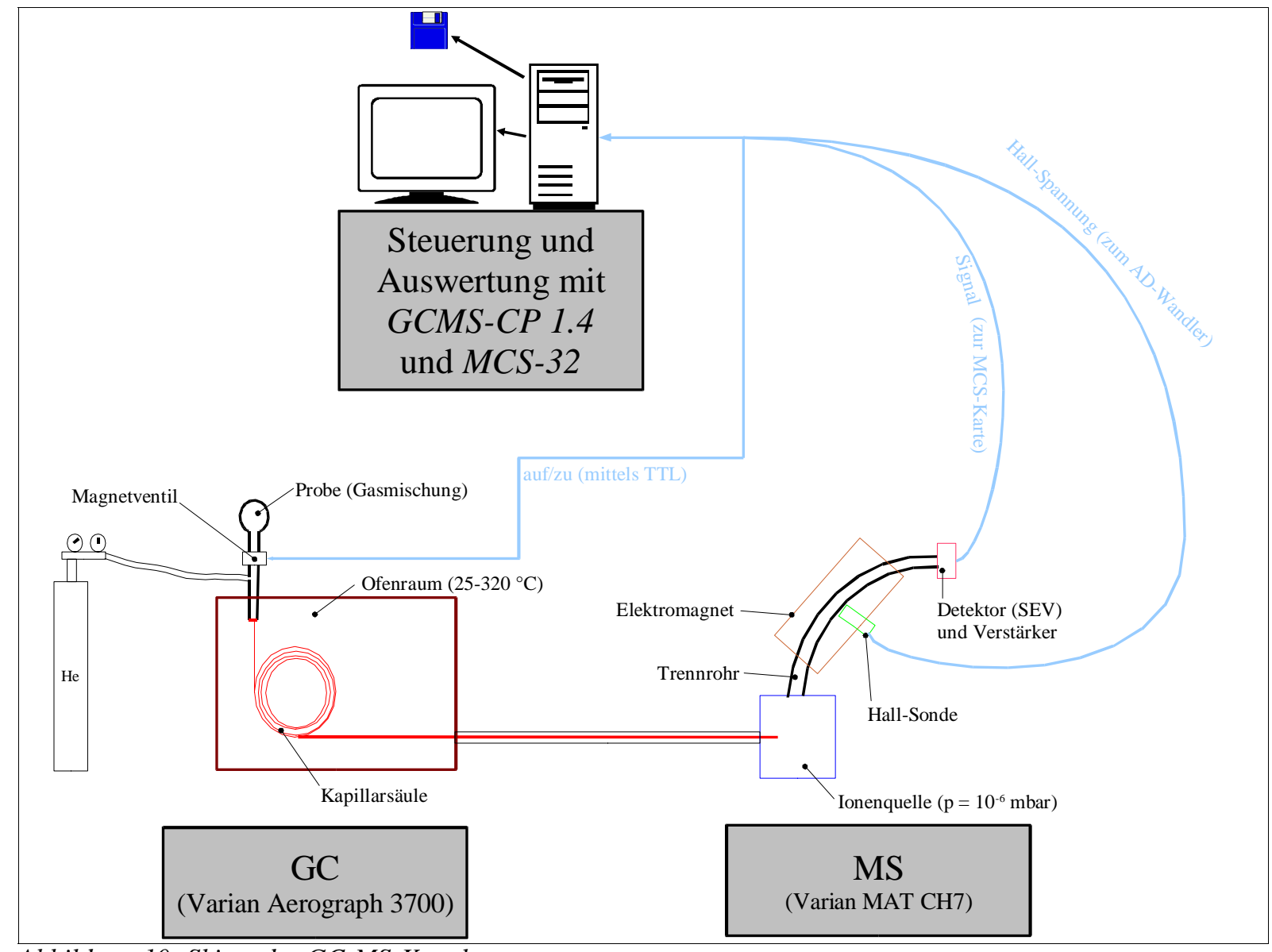

Abbildung 10: Skizze der GC-MS-Kopplung

\subsubsection{Probentrennung}

Die Trennung der einzelnen Komponenten einer zu analysierenden Produktmischung erfolgte im Gaschromatograph (VARIAN, Aerograph 3700). Die Mischung wurde in die heizbare Injektionskammer injiziert und dort ggf. in die Gasphase überführt. Von dort gelangte die Gasmischung in eine Kapillarsäule, in der ihre Komponenten (in der Regel) eine jeweils unterschiedlich gute „Wechselwirkung“ mit der stationären Phase eingingen und somit die Säule auch nach jeweils unterschiedlichen Durchlaufzeiten verließen ${ }^{43}$. Zur Optimierung dieser Trennung konnte die Temperatur im GC-Ofenraum und damit die Temperatur der

43 Detailliert dazu: Metzner, K. in: Leibnitz, E.; Struppe, H. (1984); S. 30 ff. 
Kapillarsäule zwischen $25{ }^{\circ} \mathrm{C}$ und $320{ }^{\circ} \mathrm{C}$ verändert werden ${ }^{44}$. Auch ein linearer Temperaturanstieg während der Analyse konnte programmiert werden. Beim Injizieren wurde ein Split-Ventil zu einer zweistufigen Drehschieberpumpe (Leybold Heraeus, D6) geöffnet, um ein „Überladen“ der Kapillarsäule zu verhindern; ein großer Teil der injizierten Substanz wurde damit gleich abgepumpt und nur der verbleibende Rest gelangte in die Kapillarsäule. Durch ein Feindosierventil zwischen der Injektionskammer und dieser Pumpe konnte der Fluß durch den Split variiert werden.

Als Trägergas, d.h. als mobile Phase, diente Helium. Dieses wurde über ein Dosierventil (Hoke, Mikro-Mite) in die Injektionskammer geleitet, so daß dort ein Druck von ca. 1,2 bar abs. herrschte.

Für alle in dieser Arbeit durchgeführten Analysen kam eine 60 m lange Kapillarsäule mit einem Innendurchmesser von $0,25 \mathrm{~mm}$ und einer Schichtdicke der stationären Phase von 1,0 $\mu \mathrm{m}$ (Macherey-Nagel, Optima 5) zum Einsatz. Ihre stationäre Phase bestand im Mittel zu 95\% aus Polydimethylsiloxan und 5\% Polydiphenylsiloxan.

\subsubsection{Probennachweis}

Der Nachweis der die Kapillarsäule verlassenden Substanzen erfolgte in einem Sektorfeldmassenspektrometer (VARIAN MAT, CH7). Letzteres war quasi der „Detektor“ des Gaschromatographen.

Durch die Möglichkeit der Feld-Regelung konnte mit diesem Gerät eine sehr hohe Durchlaufgeschwindigkeit des magnetischen Massendurchlaufs von bis zu einer Sekunde pro Massendekade erreicht werden. Bzgl. der Massenauftrennung im Magnetfeld gelten analog die Ausführungen oben in Kap. 3.1.1.4 (S. 19).

Die Ionisierung erfolgte nach dem EI-Verfahren, wobei die Energie der EI-Elektronen stufenlos zwischen 5 und $105 \mathrm{eV}$ eingestellt werden konnte. Um den Vergleich mit Literaturspektren $\mathrm{zu}$ ermöglichen, wurde jedoch stets mit $70 \mathrm{eV}$ gearbeitet. Eine hohe Nachweisempfindlichkeit wurde durch einen Emissionsstrom von 2 mA erreicht.

Die durch den Austrittsspalt austretenden Ionen trafen auf einen aus 17 Dynoden bestehenden Sekundärelektronenvervielfacher (SEV), der mit einer Spannung zwischen 2,5 und 3,2 kV betrieben wurde.

Das zum massenspektrometrischen Nachweis erforderliche Hochvakuum in der Ionenquelle und im Trennrohr wurde durch zwei Turbomolekuklarpumpen (Pfeiffer, TPH 200) erzeugt,

44 Detailliert dazu: Struppe, H. in: Leibnitz, E.; Struppe, H. (1984); S. 97 ff. 
denen eine Drehschieberpumpe (Edwards E2M28) vorgeschaltet war. Damit konnte der Druck auch bei eingeschaltetem Trägergasfluß durch den GC auf ca. $5 \cdot 10^{-4} \mathrm{~Pa}\left(5 \cdot 10^{-6} \mathrm{mbar}\right)$ gesenkt werden.

\subsubsection{Signalaufnahme}

Zur Datenaufnahme wurde zunächst die Spannung der Hall-Sonde über eine AD-Wandlerkarte (ComputerBoards; CIO-DAS802/16) in einem Personalcomputer (Pentium I) erfaßt, um die Massenzahl zu ermitteln. Das Signal, das ein am SEV auftreffendes Ion erzeugte, wurde direkt an einen schnellen Vorverstärker (EG\&G ORTEC; VT120A) mit einer Eingangsimpedanz von $50 \Omega$ sowie einer Ansprechzeit kleiner als 1 ns geleitet und dort um den Faktor 200 verstärkt. Dadurch erzeugte ein einzelnes Ion einen NIM-Puls in einer Höhe von ca. -800 mV. Die Anzahl an Pulsen wurde von einer $100 \mathrm{MHz}-Z$ ählerkarte (EG\&G ORTEC; multichannel scaling card: MCS-plus) jeweils für eine programmierte Zeit in einem einzelnen Kanal aufsummiert. Diese Zeit konnte zwischen $2 \mu$ s und 30 min variiert werden. Insgesamt standen bis zu 8192 Kanäle zur Erfassung eines Massenspektrums zur Verfügung. Die Zählerkarte war ebenfalls in dem Personalcomputer installiert.

Die Programmierung der Zählerkarte erfolgte über ein mitgeliefertes Programm (EG\&G ORTEC; MCS-32 Operating Software Version 2). Dieses Programm wurde seinerseits jedoch von einem selbsterstellten Programm (das nachfolgend mit „GCMS-CP“ bezeichnet wird) gesteuert, das auch die Errechnung der Massenzahl sowie die Injektion der (Gas-)Probe in das GC-MS-Gerät übernahm.

Ferner konnte die Temperatur im GC-Ofen mittels eines Thermoelements ausgelesen und gespeichert werden.

\subsubsection{Computersteuerung und -auswertung}

Im Rahmen der vorliegenden Arbeit wurde ein Programm („GCMS-CP“) geschrieben, das die Registrierung von GC-MS-Daten und deren weitere Auswertung komfortabel ermöglichte.

Weil das Massenspektrometer der „Detektor“ des Gaschromatographen war, mußten nach Injektion der Probe ständig in rascher Folge einzelne Massenspektren registriert werden.

Aus diesem Grund wurde am Massenspektrometer ein zyklischer Massendurchlauf mit schnellem Rücksprung eingestellt. Das Massenspektrometer fuhr also den Magnetstrom beginnend bei der gewünschten Start-m/z bis zu einer eingestellten Stop-m/z hoch und fiel dann sehr rasch auf die Start-m/z zurück, um dann den Zyklus von neuem zu beginnen.

Sobald der Benutzer dem Programm den Startbefehl erteilte, öffnete das Programm zunächst 
das Split-Ventil an der GC-Injektionskammer (um den Druck in der Kammer herabzusetzen), dann öffnete es ganz kurz (üblicherweise für $15 \mathrm{~ms}$ ) das Ventil zur Injektion der Substanzmischung und schloß danach wieder das Split-Ventil.

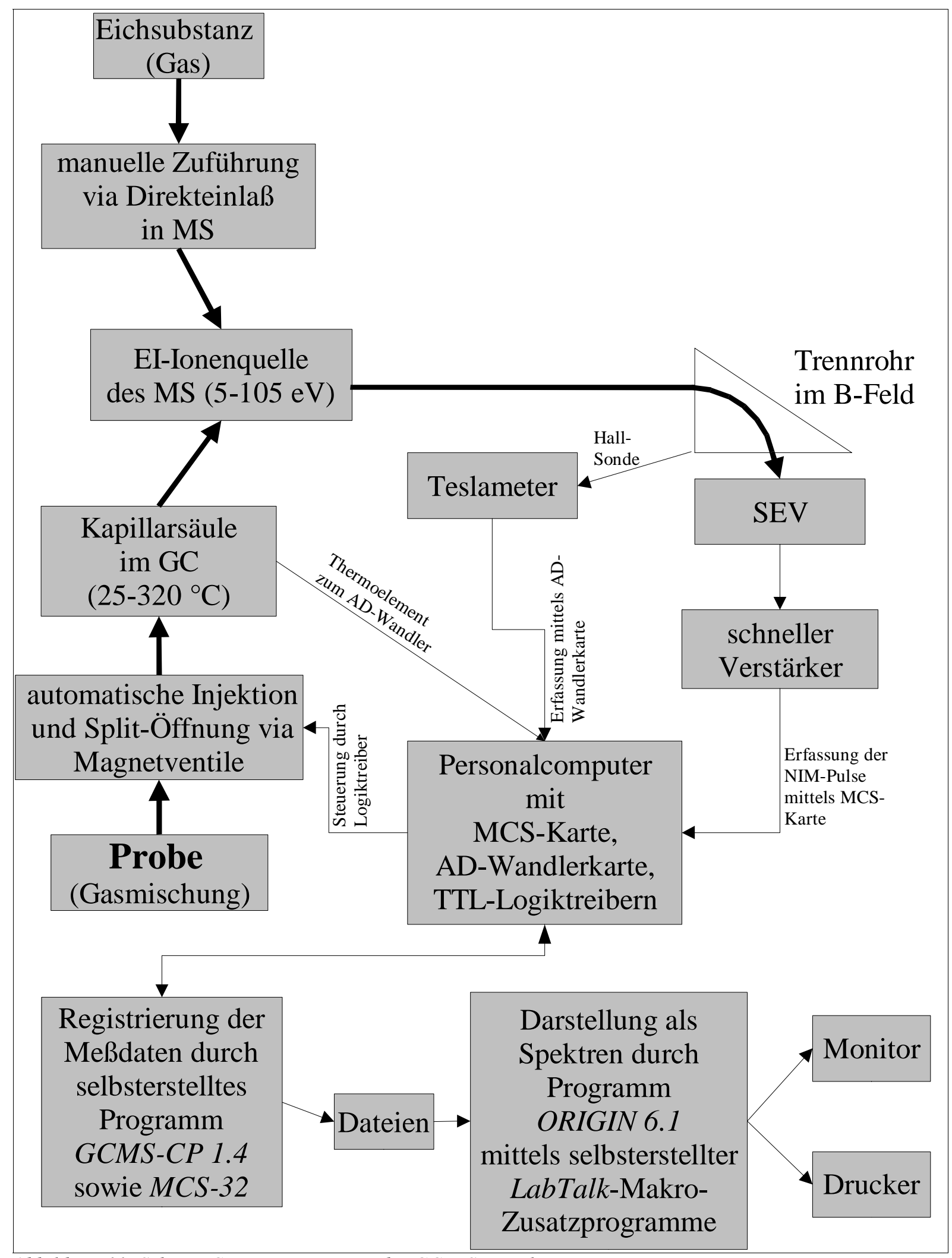

Abbildung 11: Schema Computersteuerung der GC-MS-Kopplung 
Nun wurden automatisch in rascher Folge einzelne Massenspektren registriert.

Dazu wurde die AD-Karte vom „GCMS-CP“-Programm auf externe Triggerung etc. programmiert und zunächst nur in eine Aufnahmebereitschaft versetzt. Gleichzeitig startete es das „MCS-32“-Programm, teilte ihm die vom Benutzer gewünschte Aufnahmezeit pro Kanal und die Anzahl aufzunehmender Kanäle (die bis zum Erreichen der gewünschten Stop-m/z notwendig ist) mit und versetzte dann auch dieses zunächst nur in eine Aufnahmebereitschaft.

Das Programm prüfte nun ständig, ob die m/z-Zahl unter die vom Benutzer gewünschte Start$\mathrm{m} / \mathrm{z}$ fiel und gab beim nächsten Erreichen der Start-m/z das Signal zur Datenaufnahme. Letzteres bestand aus einem kurzen 5V-TTL-Puls als Startsignal an die MCS-Karte, welche daraufhin mit der Zählung der ankommenden NIM-Pulse in die einzelnen Kanäle begann. Bei jedem Wechsel zwischen den einzelnen Kanälen gab die MCS-Karte ihrerseits einen 5V-TTLPuls aus, der als Triggersignal der AD-Karte diente. Bei jedem Triggersignal erfaßte die ADKarte nun die Hall-Spannung sowie die Spannung des Thermoelements.

Dieses aufwendig erscheinende Vorgehen mit den TTL-Pulsen hat den Vorteil, daß sowohl die AD- als auch die MCS-Karte nur einmal vor Beginn der Messung von der Software Befehle zur Erzeugung der Meßbereitschaft entgegennehmen mußte; danach aber konnten die Karten unabhängig von der Software und damit zeitlich echt parallel Daten aufnehmen. Alle so registrierten Daten wurden zunächst auf der jeweiligen Karte selbst zwischengespeichert.

Das Problem war jedoch, daß der Speicherplatz auf der AD- und MCS-Karte lediglich für ein einzelnes Spektrum ausreichte. Daher mußten zwischen den einzelnen Massenspektren die Rohdaten (d.h. die digitalisierten Werte) vom „MCS-32“- und vom „GCMS-CP“-Programm aus den Karten ausgelesen und auf der Festplatte zwischengespeichert werden. Dieser Vorgang erforderte jedoch etwas Rechenzeit, weil die Karten nicht echt-, sondern nur quasi-parallel ausgelesen werden konnten. Da die Zeit für den Rücksprung von der Stop-m/z auf die Start$\mathrm{m} / \mathrm{z}$ alleine nicht ausreichte, mußte man dem Programm eine zusätzliche (empirisch zu bestimmende) Verzögerungszeit zugestehen. Sie betrug bei dem hier verwendeten Personalcomputer ca. $500 \mathrm{~ms}$.

Erst am Ende der gesamten Datenaufnahme wurden die Rohdaten der einzelnen Massenspektren in die eigentlichen Werte konvertiert und in fortlaufend numerierten Dateien gespeichert. Dabei wurde auch die Summe der DATA-Werte über alle $\mathrm{m} / \mathrm{z}$-Werte in jedem einzelnen Massenspektrum ermittelt. Letztere wurde für jedes Massenspektrum zusammen mit der Nummer dieses Spektrums und der Zeit seit Injektion in die Kapillarsäule sowie der Temperatur im GC-Ofen in einer separaten Datei gespeichert; sie stellt das GC-Spektrum dar. 
Die Konvertierung der Rohdaten durch das „GCMS-CP“-Programm umfaßte folgende Schritte: Die Hall-Spannung war zunächst nur als 16-Bit digitalisierter Wert gespeichert und mußte daher in den analogen Spannungswert umgerechnet werden und erst danach konnte aus einem (vom Benutzer erstellten) m/z-Kalibrierungspolynom der eigentliche $\mathrm{m} / \mathrm{z}$-Wert errechnet werden. Ebenso waren die Zählwerte der einzelnen Kanäle (hier als DATA-Werte bezeichnet) zunächst nur als 32-Bit digitalisierte Werte gespeichert und mußten noch in den Dezimalwert umgewandelt werden. Schließlich wurden die Ergebnisse dieser Konvertierungen in einer Datei zusammengefaßt, so daß zu jedem DATA-Wert eines Kanals auch die zugehörige m/z-Zahl angegeben war. Diese Massenspektren-Dateien wurden fortlaufend numeriert.

Um die aufgenommenen Daten mühelos als Spektren darstellen und ausdrucken zu können, wurden für das Programm „Origin 6.1“ in dessen Programmiersprache „LabTalk“ einige Zusatzprogramme entwickelt.

Die genaue Beschreibung des „GCMS-CP“-Programms und der erstellten „LabTalk“Zusatzprogramme ist recht umfangreich. Deshalb wurde im Rahmen dieser Arbeit auch eine ausführliche Dokumentation für diese Programme erstellt ${ }^{45}$.,GCMS-CP“ wurde in der Programmiersprache „Object-Pascal“ geschrieben und als 32-Bit-Programm mit „Delphi 2.01“ der Firma Borland compiliert. Es ist unter den Betriebssystemen Windows 95/98 und NT lauffähig. Für die Messungen in dieser Arbeit wurde es unter Windows NT 4.0 ausgeführt.

Seit dem Sommersemester 2001 wird mittels der hier beschriebenen GC-MS-Kopplung und der dazu erstellten Programme auch ein Versuch im Rahmen des Fortgeschrittenenpraktikums für LAK Chemie durchgeführt.

45 Hold, M. (2001); Programmbeschreibung für GCMS-CP Version 1.4. 


\subsubsection{REMPI-Flugzeitmassenspektrometer mit Molekularstrahlprobennahme}

\subsubsection{Aufbauschema}

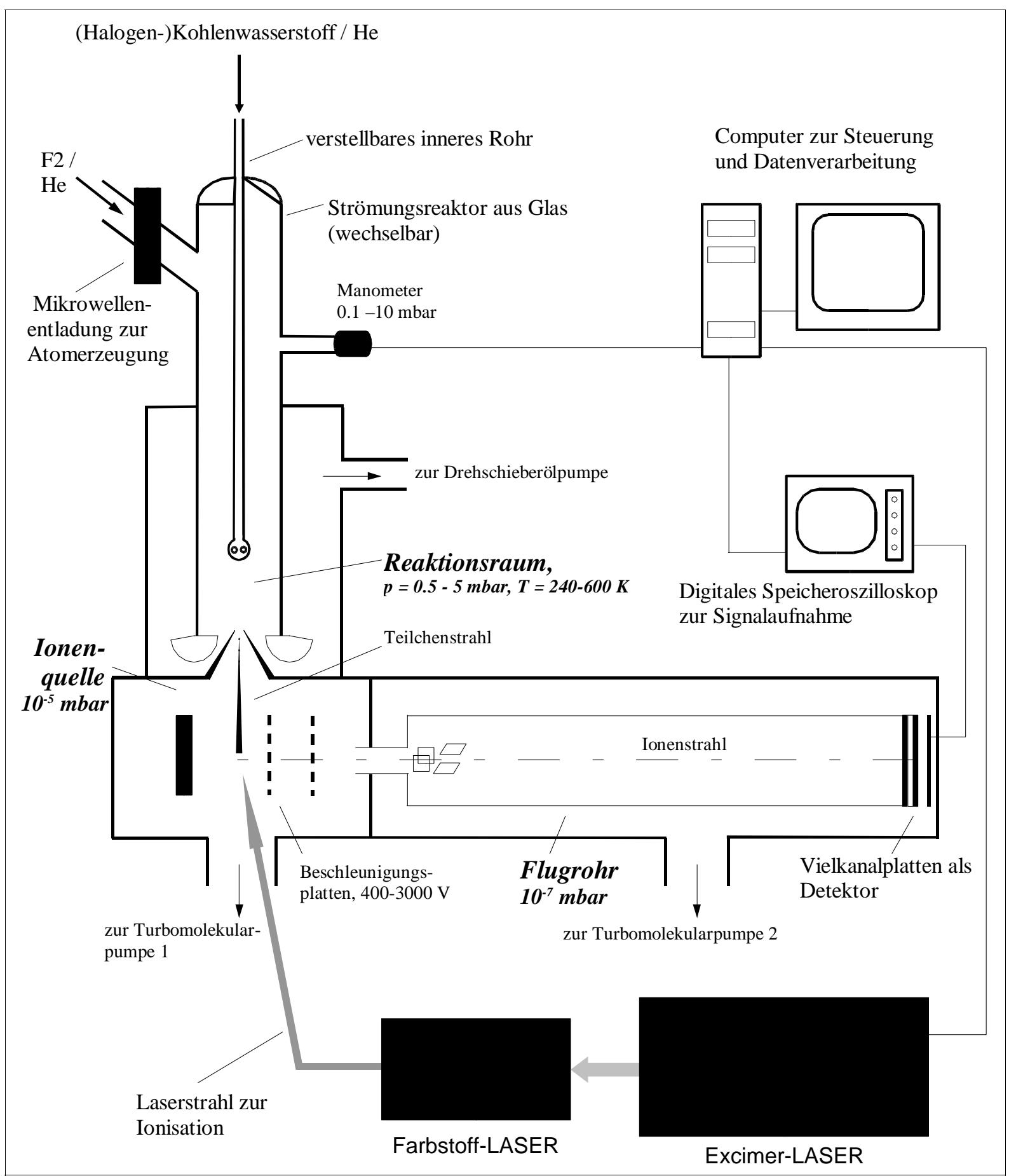

Abbildung 12: Skizze des REMPI-Flugzeitmassenspektrometers [Quelle: Wehmeyer, J. (vorauss. 2002)]

In Bezug auf den Strömungsreaktor und die Molekularstrahlprobennahme gleicht das REMPIFlugzeitmassenspektrometer im Prinzip dem oben in Kap. 3.1.1 (S. 16) beschriebenen EISektorfeldmassenspektrometer. Jedoch erfolgt hier die Ionisierung durch die Photonen eines LASERs und die Massenauftrennung findet in einem Flugrohr statt. Da dieser Aufbau schon 
mehrfach ausführlich beschrieben wurde ${ }^{46}$, erfolgt hier in den folgenden Unterkapiteln nur eine stark verkürzte Darstellung, so wie sie für das Verständnis der vorliegenden Arbeit notwendig ist.

Die Apparatur eignet sich sowohl für einen (teilweise spezifischen) Radikal-Nachweis als auch für die Messung von Geschwindigkeitskoeffizienten für die Reaktion eines Radikals mit einem anderen Reaktanten.

\subsubsection{Strömungsreaktor}

Für die in dieser Arbeit durchgeführten Messungen wurde ein Strömungsreaktor verwendet, der im Prinzip dem oben in Abbildung 6 (S. 17) beschriebenen Reaktor gleicht. Abweichend davon war jedoch für die radikalerzeugende Reaktion des (Halogen-)Kohlenwasserstoffs mit Fluor-Atomen eine Reaktionsstrecke von $1,0 \mathrm{~cm}$ und für die weitere Reaktion des Radikals mit dem zweiten Reaktanten (in dieser Arbeit: NO) eine fixe Reaktionsstrecke von 3,0 cm eingestellt. Ferner betrug der Innendurchmesser lediglich 2,2 cm. Auch war an dem Einlaß für den zweiten Reaktanten keine Mikrowellenentladung angebracht, da dies für die Reaktion des Radikals mit NO nicht notwendig war.

An dem anderen seitlichen Einlaß (für Fluor/Helium) war über eine Metallverschraubung ein Quarzröhrchen angebracht, in dem durch elektrodenlose Mikrowellenentladung die FluorAtome erzeugt werden konnten.

Durch eine zweistufige Pumpe (Leybold Heraeus, DK 100; $100 \mathrm{~m}^{3} / \mathrm{h}$ Saugleistung) wurde im Strömungsreaktor ein Druck von 50 bis $500 \mathrm{~Pa}$ (0,5-5,0 mbar) erzeugt, der durch die hohe Saugleistung der Pumpe auch bei hohem Gasfluß aufrecht erhalten werden konnte.

Der Druck im Strömungsreaktor wurde mit einem Druckaufnehmer (BARATRON MKS 122; 0-10 mbar) gemessen.

\subsubsection{Probennahme}

Durch eine Expansionsdüse (Skimmer) erreichte ein kleiner Teil des Reaktionsgemisches als Teilchenstrahl die Ionenquelle. Der Strömungsreaktor wurde mittels eines Tombakkörpers so justiert, daß die Nachweisempfindlichkeit maximal war.

Der Skimmer hatte eine Höhe von $20 \mathrm{~mm}$, einen Innenwinkel von $34^{\circ}$ und eine Öffnung mit einem Durchmesser von 0,8 mm. Durch diese Probennahme war auch die Detektion kurzlebiger Spezies (z.B. Radikale) möglich, denn die Reaktion wurde nach dem Skimmer quasi „eingefroren“, weil Stöße zwischen den Molekülen auf Grund der geringen Konzentrationen nur noch sehr unwahrscheinlich waren. Die relativen Konzentrationen 
entsprachen dabei prinzipiell den Verhältnissen am Ende des Strömungsreaktors.

Um weitere Reaktionen (Folgereaktionen) im Probennahme- und Nachweisteil zu vermeiden, war ein sehr gutes Vakuum erforderlich; der Druck von $10^{-3}$ bis $10^{-4} \mathrm{~Pa}\left(10^{-5}\right.$ bis $10^{-6}$ mbar $)$ in der Ionenquelle wurde durch eine Turbomolekularpumpe (Leybold Heraeus, Turbovac 360) mit vorgeschalteter Drehschieberölpumpe (Leybold Heraeus, Trivac D 25 B) erreicht.

Für ein ölfreies Hochvakuum im Flugrohr (Trennteil) sorgte eine Turbomolekularpumpe (Leybold Heraeus Turbovac 150), deren Vorvakuum von einer zweistufigen Drehschieberpumpe (Leybold Heraeus, D6) erzeugt wurde.

Der Druck in der Ionenquelle und im Flugrohr wurde mit Ionisationsvakuumetern nach Penning (Leybold Heraeus, Penningvac PM 41) gemessen.

\subsubsection{Probennachweis}

Im Hochvakuumteil der Apparatur befand sich zum massenspektrometrischen Nachweis ein modifiziertes Bendix-MA2-Flugzeitmassenspektrometer.

Die Beschleunigungsstrecke für die Ionen bestand aus 3 Platten mit kreisförmigen Öffnungen, an denen unterschiedliche Spannungen variabler Höhe anliegen. An der dritten Platte lag die Hauptbeschleunigung von $-2.7 \mathrm{kV}$ bis $-3.1 \mathrm{kV}$ (bezogen auf das Ionenquellengehäuse, das auf Masse liegt) an. An den Beschleunigungsbereich, in dem alle Ionen die gleiche kinetische Energie erhalten, schloss sich die feldfreie Driftstrecke $(145 \mathrm{~cm})$ an. Hier spalteten sich die Ionen je nach Masse-Ladungs-Verhältnis in eine unterschiedliche Flugzeit $t$ auf, die sich durch folgende Gleichung beschreiben läßt:

$$
\begin{aligned}
t & =\left(\frac{s}{\sqrt{2 \cdot e \cdot U}}\right) \cdot \sqrt{m} \\
s & =\text { Driftstrecke } \\
e & =\text { Ladung des Ions } \\
U & =\text { Beschleunigungsspannung } \\
m & =\text { Masse des Ions }
\end{aligned}
$$

Diese Flugzeit t ist die Zeit, die ein Ion nach Durchlaufen des Beschleunigungsbereichs bis zum Erreichen des Detektors benötigt.

Zur Erhöhung der Nachweisempfindlichkeit wurden die Ionen über ein Linsensystem (bestehend aus horizontalen und vertikalen Platten sowie einer kreisrunden Ionenlinse, deren Potentiale jeweils unterschiedlich eingestellt werden konnten) auf den Detektor fokussiert. Letzterer bestand aus zwei durch einen Kupferring getrennte Vielkanal-Verstärkerplatten (Multi Channel Plates, MCP) (Varian 8946, Durchmesser 25 mm) und einem Auffänger. Die 
MCP wurden gemäß ihrer Spezifikation mit einer Spannung von jeweils $1 \mathrm{kV}$ betrieben, was im Tandembetrieb einen Verstärkungsfaktor von $10^{6}$ ergab. Die Ladung floß über einen $50-\Omega$ Widerstand gegen Masse ab und erzeugte einen kurzen Spannungspuls, der anschließend weiterverarbeitet wird (siehe unten Signalaufnahme). Mit dieser Anordnung wurde innerhalb ca. $50 \mu$ s ein gesamtes Massenspektrum erhalten. Die Massenauflösung beträgt $\Delta \mathrm{m} / \mathrm{m} \approx 300$ (10\%-Tal-Definition).

Bei der Trennung in einem Flugrohr darf die Ionisierung naturgemäß nicht kontinuierlich erfolgen, sondern sie muß gepulst sein. Je kürzer diese Pulse zur Ionisierung sind, je höher ist das Auflösungsvermögen. In der hier verwendeten Anordnung wurden kurze LASER-Pulse zur Ionisierung verwendet.

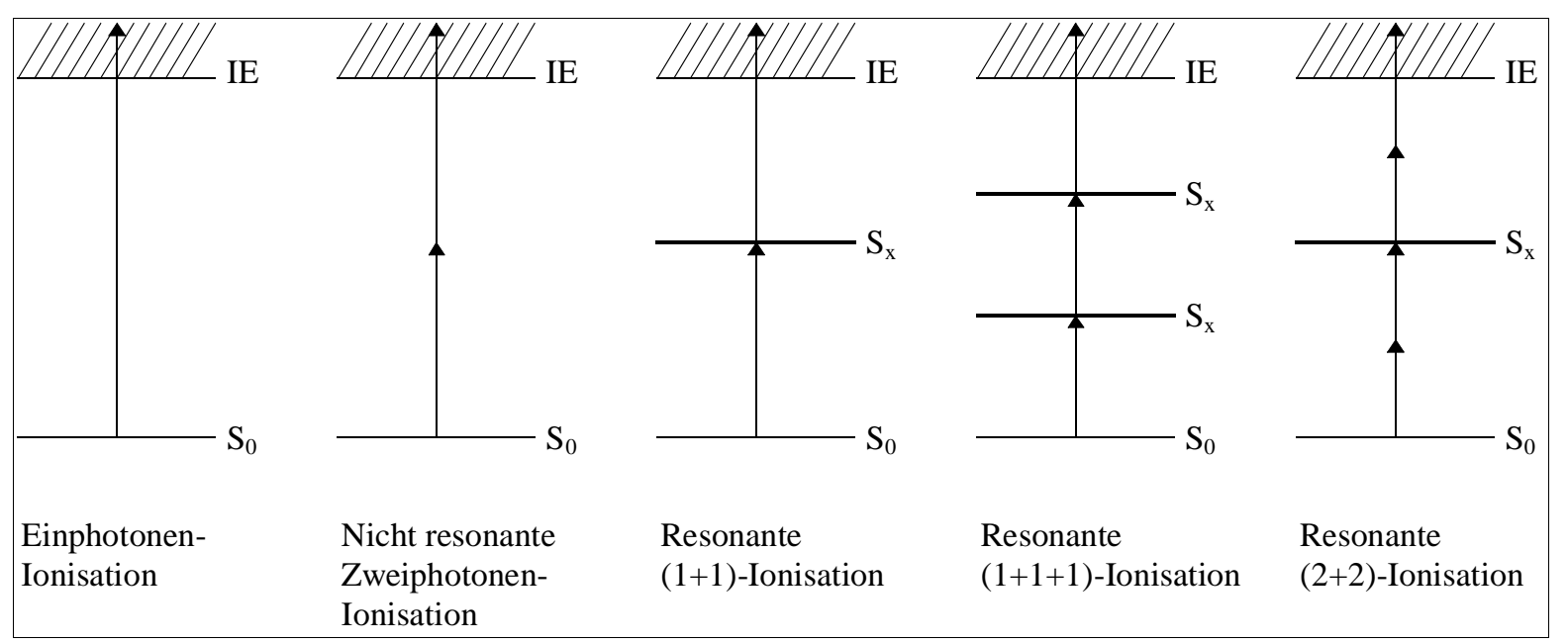

Abbildung 13: Mechanismen der Photoionisation [Quelle: Nacke, F. (1998), S. 6]

Dieses Verfahren nennt sich Mehrphotonenionisation (bzw. REMPI = Resonance Enhanced Multi Photon Ionization) $)^{47}$. Es beruht auf der Wechselwirkung mit Licht. Um ein Molekül mit einem Ionisierungspotential von $10 \mathrm{eV}$ ionisieren $\mathrm{zu}$ können, benötigt man Licht einer Wellenlänge von $124 \mathrm{~nm}$ (Vakuum-UV). Die Wahrscheinlichkeit dafür, daß bei höheren Wellenlängen mehrere Photonen absorbiert werden, ist sehr gering. Erst durch den Einsatz von Laserlicht, bedingt durch die hohe Lichtintensität $\left(10^{6}-10^{9} \mathrm{~W} / \mathrm{cm}^{2}\right)$, wird die simultane Aufnahme mehrerer Photonen wahrscheinlicher. Die Ionenausbeute nimmt dabei mit der Zahl der benötigten Photonen ab. Spezifischer und empfindlicher gelingt die Ionisation, wenn die Absorption über angeregte Molekül- oder Rydbergzustände verläuft. Dabei wird zunächst ein elektronisch angeregter Zustand durch Absorption eines oder mehrerer Photonen bevölkert, von dem aus durch Aufnahme eines weiteren Photons das Ionisationskontinuum erreicht wird. Abbildung 13 zeigt die grundsätzlichen Mechanismen der Photoionisation.

47 Vgl. zu den gesamten Ausführungen hierzu: Nacke, F. (1998), S. 5-8 und die dort zitierte Literatur. 
Die Aufnahme weiterer Photonen zum Erreichen des Ionisationskontinuums steht in Konkurrenz mit möglichen Verlustprozessen. Das kann Fluoreszenz in niedrigere Zustände, strahlungslose innere Konversion in Schwingungszustände niedriger liegender elektronischer Zustände oder Prädissoziation in kleinere neutrale Moleküle sein.

Eine Beeinträchtigung des Ionensignals durch Verlustprozesse ist vernachlässigbar, wenn die Ionisation mit $10^{-11}-10^{-12} \mathrm{~s}$ schnell gegenüber der Fluoreszenzlebensdauer mit $10^{-8}-10^{-9} \mathrm{~s}$ oder einer Stoßdesaktivierung im Molekularstrahl ist.

Die Multiphotonenionisation von Radikalen verläuft oft über besondere elektronische Zustände, den Rydbergzuständen. Dies sind elektronische Niveaus, die durch wasserstoffähnliche Orbitale beschrieben werden können. Für die Energieterme der Rydbergorbitale gilt ${ }^{47}$ :

$$
\begin{aligned}
E_{R} & =I P-\frac{R}{(n-\delta)^{2}} \\
I P & =\text { adiabatisches Ionisierungspotential } \\
R & =\text { Rydbergkonstante } \\
n & =\text { Hauptquantenzahl } \\
\delta & =\text { Quantendefekt }
\end{aligned}
$$

Eine Ionisation aus diesen Zuständen ist aufgrund des großen Absorptionsquerschnitts leicht möglich.

Neben den Mutterionen treten infolge der hohen Energiedichte auch oft Fragmentionen auf, und zwar um so mehr, je kürzer die Wellenlänge des Laserlichts ist. Da für die Photofragmentierung die simultane Aufnahme weiterer Photonen ein sehr unwahrscheinlicher

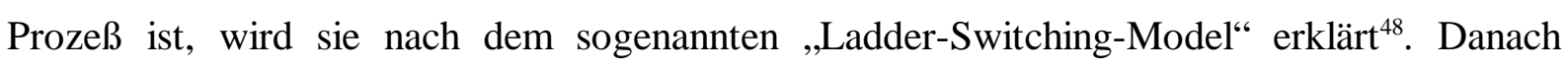
werden erst nach der Ionisation weitere Photonen aufgenommen, die zu einer Fragmentierung führen. Dies kann stufenweise bis hin $\mathrm{zu} \mathrm{C}_{1}$-Fragmenten erfolgen. Für einen überlagerungsfreien Nachweis der Radikale muß die Energiedichte des Lasers soweit abgesenkt werden, daß keine Fragmentierung mehr auftritt.

Das Licht für die Mehrphotonenionisation wurde von einem durchstimmbaren Farbstofflaser (Lambda Physik FL 2002) erzeugt, der von einem Excimerlaser (Lambda Physik LPX 205 iCC) mit einer mittleren Pulsenergie von $400 \mathrm{~mJ}$ bei $308 \mathrm{~nm}(\mathrm{XeCl})$ und einer Pulslänge von 28 ns gepumpt wurde. Mit Hilfe verschiedener Farbstoffe ließ sich ein Wellenlängenbereich von $\lambda=320-970 \mathrm{~nm}$ kontinuierlich abdecken. Je nach Konversionsrate des verwendeten

48 Dietz, W.; Neusser, H.J.; Bosel, U.; Schlag, E.W.; Lin, S.N. (1982) 
Farbstoffs betrug die Pulsenergie 1-50 mJ bei einer Pulslänge von 20 ns. Der Laserstrahl wurde durch ein Quarzprisma um $90^{\circ}$ umgelenkt und mit einer Quarzlinse ( $\mathrm{f}=40 \mathrm{~mm}$ ) in das Zentrum der Ionenquelle fokussiert, wo er senkrecht auf den Molekularstrahl traf. Zur gleichzeitigen Energiemessung wurde ein Teil des Strahls durch eine Quarzplatte ausgekoppelt und von einem Piezokopf gemessen, der mit einem Joulemeter der Firma Gentec ED 200 geeicht wurde.

\subsubsection{Signalaufnahme}

Beim Flugzeitmassenspektrometer erfordert die Verarbeitung der am Detektor anfallenden Signale wegen der geringen Laufzeitunterschiede der Ionen im Flugrohr eine schnelle Meßelektronik (z.B. für $\mathrm{m} / \mathrm{z}=50$ und 51: $\Delta \mathrm{t} \approx 150 \mathrm{~ns}$ ). Diese bestand aus einem digitalen Speicheroszilloskop (LeCroy DSO 9350 AL), das über eine IEEE488-Schnittstelle (GPIB) verfügte. Über letztere fand die Kommunikation mit einem Personalcomputer (Pentium I) statt, in den eine GPIB-Schnittstellen-Karte (National Instruments, GPIB-PCII) eingebaut war. Die Synchronisation zwischen der Ionenerzeugung und der Datenaufnahme geschah durch den Lichtstrahl des Farbstofflasers, der durch einen Photomultiplier (Hamamatsu 1P28) verstärkt, in einen TTL-Puls umgewandelt und dann auf den externen Triggereingang des digitalen Speicheroszilloskops gegeben wurde. Nach Eingang dieses Trigger-Pulses nahm das digitale Speicheroszilloskop mit einer Abtastrate von $500 \mathrm{MHz}$ das Ausgangssignal (Spannung) der Vielkanal-Verstärkerplatten (MCP) auf, bis ein komplettes Massenspektrum registriert war (typischerweise nach $50 \mu \mathrm{s}$ ). Jeder einzelne Spannungswert wurde dabei in 8 Bit digitalisiert. Um eine höhere statistische Zuverlässigkeit zu erhalten, konnte das Oszilloskop aus mehreren hintereinander aufgenommenen Spektren selbständig den Mittelwert bilden. Dieses Mittelwertspektrum wurde in 12 Bit digitalisiert, d.h. in einer beträchtlich höheren Auflösung als jedes Einzelspektrum. Es hat sich gezeigt, daß erst nach etwa 300-600 Einzelspektren ein reproduzierbares Mittelwertspektrum entsteht. Durch die schnelle Aufnahmegeschwindigkeit und eine Wiederholfrequenz der LASER-Pulse von 3-6 Hz ließ sich dieses Erfordernis jedoch leicht realisieren.

\subsubsection{Computersteuerung und -auswertung}

Sowohl für die Aufnahme von Multiphotonenionisations-Spektren als auch für die kinetischen Messungen stand das von F. Nacke ${ }^{49}$ entwickelte Programm „TofShell“ zu Verfügung, das es ermöglichte, sich wiederholende Meßprozeduren automatisch ablaufen zu lassen. Damit war es auch möglich, in kurzer Zeit eine größere Anzahl von Meßpunkten und damit eine höhere

49 Nacke, F. (1998); S. 12-13. 
statistische Zuverlässigkeit zu erlangen.

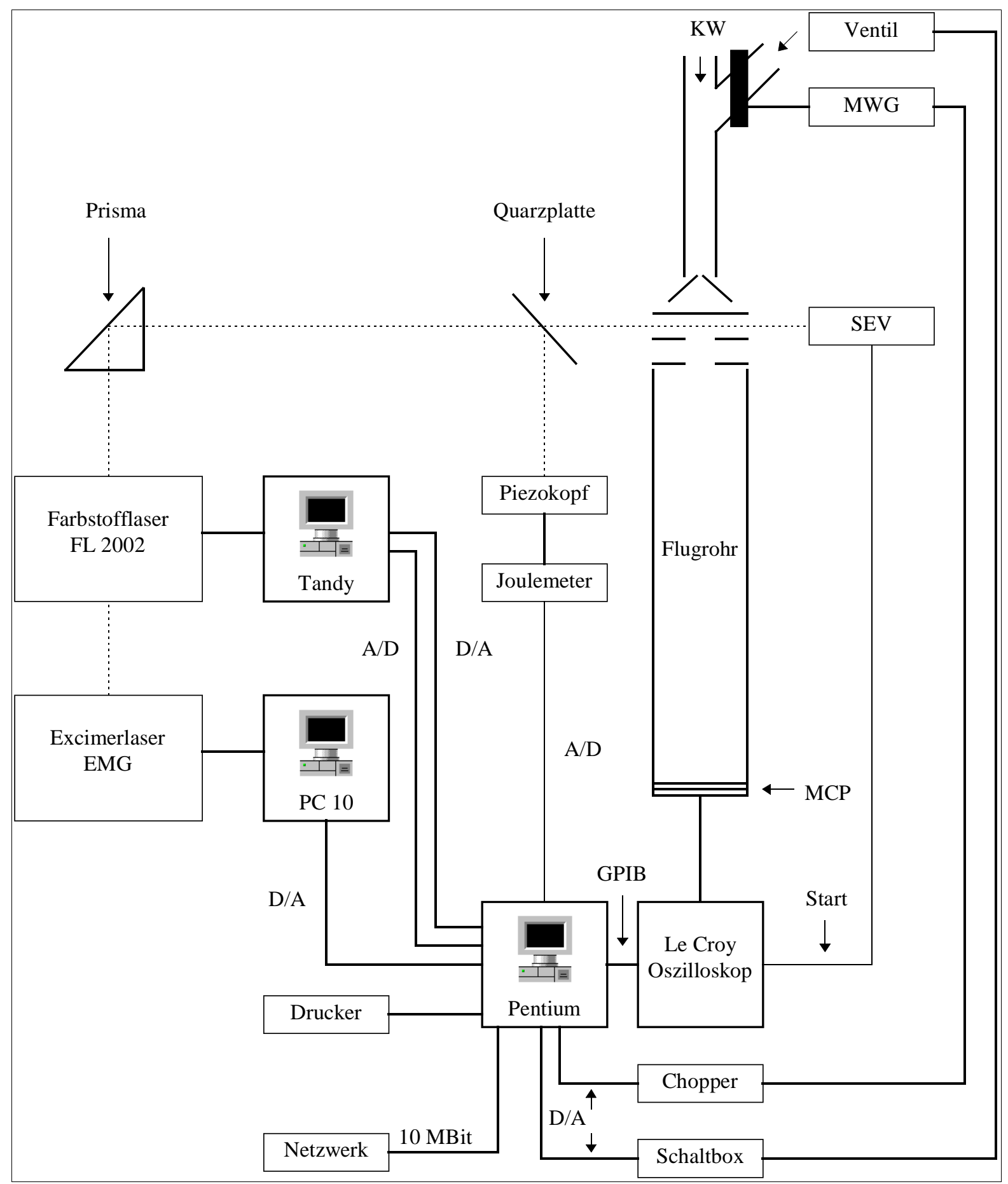

Abbildung 14: Schema der Computersteuerung des Flugzeitmassenspektrometers [Nacke, F. (1998), S. 13]

Der Personalcomputer war mit einer AD-DA-Wandlerkarte von Addi-Data (PA 310, 16 ADEingänge 0-10 V, 6 DA-Ausgänge 0-10 V, 2 Logiktreiber 5V) ausgerüstet. Der Excimerlaser wurde durch einen Commodore PC-10, der Farbstofflaser über einen Tandy TRS-80 angesteuert. 
Die schematischen Verknüpfungen ${ }^{50}$ zeigt oben Abbildung 14.

Der prinzipielle Ablauf soll im folgenden kurz vorgestellt werden.

a) Automatischer Wellenlängendurchlauf:

Das Programm triggerte den Excimerlaser über einen Softwareinterrupt und laß die mit dem Joulemeter (Baumbach We019, JM) gemessene Energie des Farbstofflasers über einen A/DEingang ein. Gleichzeitig wurde das Oszilloskop über den GPIB-Bus abgefragt, ob die vorher eingestellte Zahl an Spektren akkumuliert ist. War dies der Fall, so hörte das Programm auf, den Excimerlaser anzutriggern, übertrug und verarbeitete das gemittelte Spektrum und gab dem Tandy des Farbstofflasers einen Puls. Dieser erhöhte durch ein BASIC-Programm die Wellenlänge des Farbstofflasers um einen vorher eingestellten Wert (Scanning Step), indem ein Schrittmotor das Gitter um einen bestimmten Winkel veränderte und gab anschließend eine Bestätigung in Form eines Spannungssignals ab. Dieses wurde über einen A/D-Eingang der Karte des Pentiums eingelesen und das Programm fuhr weiter fort, bis die Endwellenlänge erreicht war.

Die Verarbeitung der Spektren bestand darin, daß in bis zu zwei vorher festgelegten Bereichen des Flugzeitmassenspektrums das Intensitätsmaximum oder Peak-Integral ermittelt und abgespeichert wurde.

b) Kinetikmessung:

Bei einer kinetischen Messung (Relativmessung) wurde über einen D/A-Ausgang eine 5-VSpannung auf die Schaltbox gegeben, woraufhin sich ein Magnetventil öffnete und den Zufluß für einen Reaktanten freigab. Anschließend triggerte das Programm den Excimerlaser, bis die vorher eingestellte Spektrenzahl am Oszilloskop erreicht war. Dann hörte das Programm auf, den Excimerlaser anzutriggern, übertrug und verarbeitete das gemittelte Spektrum und schloß das Magnetventil. Danach lief die gleiche Prozedur ohne Reaktantenzugabe ab. Dieser Vorgang wurde wiederholt, bis die vorher festgelegte Zahl an Messungen erreicht war. Aus den vorangegangenen Messungen resultierte ein Wertepaar, das gesondert abgespeichert und dessen Ergebnis in einer Vorauswertung (lineare Regression) graphisch angezeigt wurde. Die Meßprozedur konnte nun mit einer veränderten Reaktantenkonzentration wiederholt werden.

Das Programm „TofShell“ wurde in Borland Pascal 7.0 mit Hilfe des objektorientierten

50 Das Schema wurde entnommen aus: Nacke, F. (1998), S. 13. 
Zusatzpaketes Turbo Vision geschrieben ${ }^{51}$. Da der Quelltext des Programms zur Verfügung stand, konnten im Rahmen der vorliegenden Arbeit noch einige Änderungen bzw. Erweiterungen vorgenommen werden. Hervorzuheben ist, daß nunmehr die im Oszilloskop gebildeten Mittelwertspektren mit ihrer vollen Auflösung von 12 Bit (zuvor nur 8 Bit) vom Oszilloskop an den Personalcomputer bzw. an „TofShell“ übertragen wurden. Dadurch konnten bereits nach wenigen Meßzyklen (oft bereits nach einem) reproduzierbare Meßwerte erreicht werden. Eine weitere Verbesserung mit der gleichen Zielrichtung war die Möglichkeit, den Signal-Peak zu integrieren. Der Benutzer erhielt die Möglichkeit, entweder nur das Peakmaximum oder aber das Integral des Peaks als Meßwert von „TofShell“ auswerten zu lassen. Für die Berechnung des Integrals konnte der Benutzer die Integrationsgrenzen als \%Wert des Peakmaximums festlegen, d.h. der Computer integrierte den Peak ausgehend vom Peakmaximum nacheinander in beide Richtungen, bis jeweils das Signal unter den gewünschten $\%$-Wert des Peakmaximums fiel. Das Programm führte dazu eine numerische Intergration nach der Trapezregel durch. Falls die Grundlinie einen von null verschiedenen Wert hatte, konnte der Benutzer einen Korrekturwert für die Grundlinie eingeben (was im üblicherweise gewählten AC-Meßmodus des Oszilloskops eigentlich nur bei sehr schwachen Signalen relevant war). Die Möglichkeit zur Auswertung mittels des Integrals stand sowohl bei dem automatischen Wellenlängendurchlauf als auch bei den Kinetikmessungen zur Verfügung. Schließlich wurde auch einprogrammiert, die vom Programm aufgenommenen Werte und durchgeführten Vorauswertungen in solchen ASCII-Textdateien speichern zu lassen, die zur Nachauswertung unmittelbar in jede gängige Tabellenkalkulation unter „Windows“ importiert werden konnten. 


\subsubsection{FT-IR-Spektrometer mit Multireflektionskugelzelle}

\subsubsection{Aufbauschema}

Das FT-IR-Spektrometer mit Multireflektionskugelzelle konnte einerseits einfach zur Messung von IR-Spektren beliebiger Substanzen in der Gasphase, andererseits in Kombination mit dem Photolyse-LASER zur quantitativen Endproduktanalyse und schließlich sogar zur zeitaufgelösten Messung ( $5 \mu \mathrm{s})$ von Reaktionskinetiken in Absorption verwendet werden.

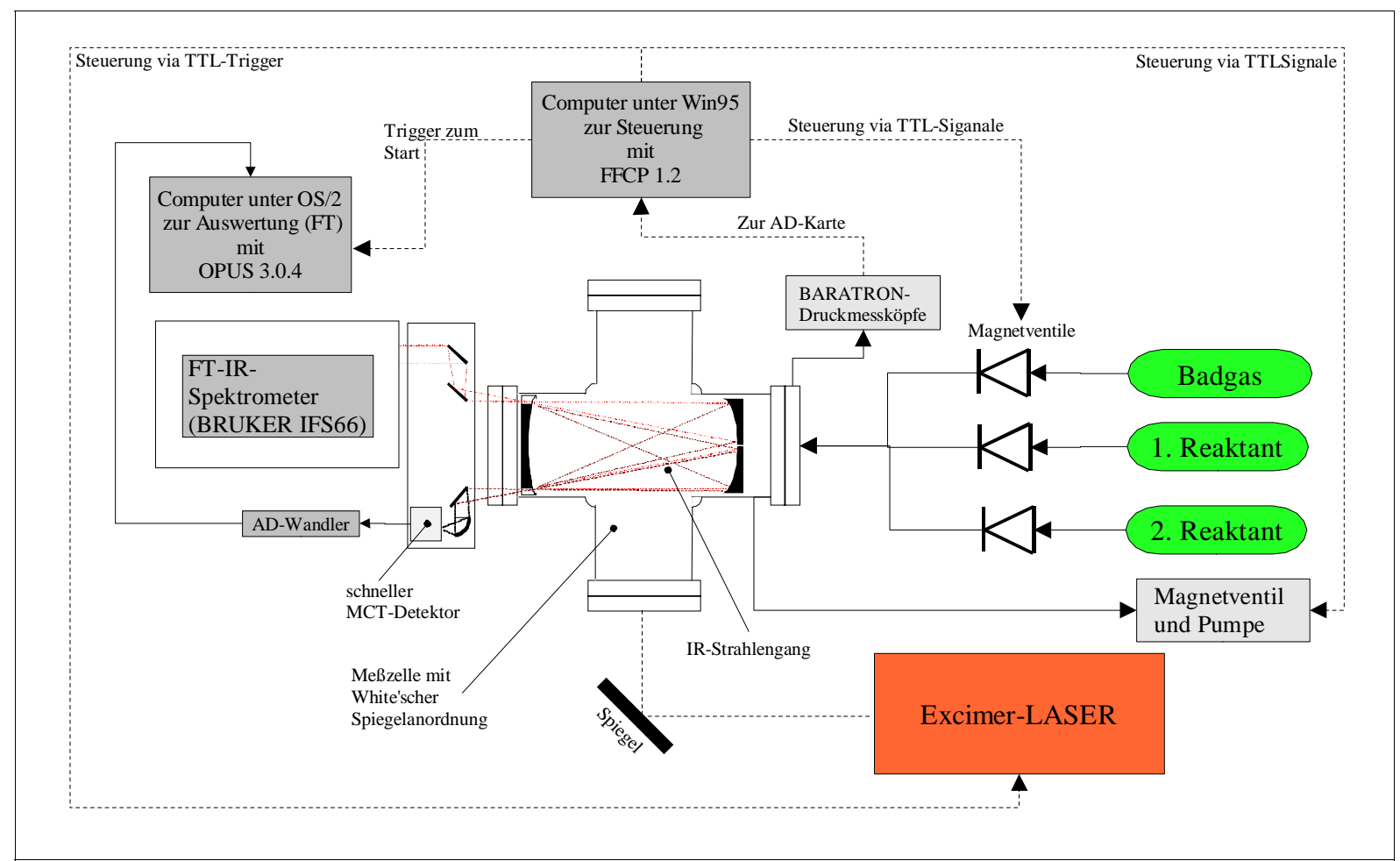

Abbildung 15: Skizze der FT-IR-Apparatur

Die einzelnen Komponenten werden in den nachfolgenden Unterkapiteln näher beschrieben. Weil die Anordnung jedoch schon früher ausführlich dargestellt wurde ${ }^{52}$, erfolgt hier nur eine sehr verkürzte Schilderung, so wie sie für das Verständnis dieser Arbeit notwendig ist.

\subsubsection{Multireflektionskugelzelle}

Die Multireflektionskugelzelle, die als Nachweis- und Photolysezelle diente, bestand aus einer Edelstahlkugel aus VA-Stahl (VAB Elmshorn VA 1.4311) mit sechs CF 100 Flanschöffnungen. Das Zellvolumen ${ }^{53}$ betrug $4,98 \mathrm{dm}^{3}$. Der untere Flansch war über ein Eckventil und eine Kühlfalle mit einer Vakuumpumpe (Brand RD 15) verbunden. Oberhalb des Eckventils befanden sich zwei Druckmeßköpfe (MKS, BARATRON 0-100 mbar und 0-1000 mbar), um den Gesamtdruck in der Zelle zu ermittlen. Von den vier horizontal liegenden Flanschen dienten jeweils zwei gegenüberliegende zur Justage der Spiegeloptik 
(Spiegelanordnung nach White) und zwei als Ein- und Austrittsfenster für den Strahl des Excimer-LASERs. Der vordere Flansch war mit zwei Gaseinlaßhähnen, der obere mit einem Gaseinlaßhahn versehen. Zur computergesteuerten Befüllung der Zelle waren vor den Gaseinlaßhähnen Magnetventile angebaut. Die Zelle war auf einer optischen Bank aufgebaut. Über einen Parabolspiegel $(\mathrm{f}=153,7 \mathrm{~mm}$ ) und einen Planspiegel wurde der IR-Strahl durch ein $\mathrm{KBr}-F e n s t e r$ in die Zelle fokussiert. In der Zelle befanden sich drei goldbeschichtete Spiegel, die im Abstand ihrer Brennweite $(f=200 \mathrm{~mm}$ ) aufgebaut waren. Mittels dieser White'schen Anordnung konnte ein Lichtweg bis zu $8 \mathrm{~m}$ durch bis zu über 40 Reflexionen erreicht werden. Das Nachweisvolumen zwischen diesen Spiegeln betrug ${ }^{54} 400 \mathrm{~cm}^{3}$. Der die Photolysezelle verlassende IR-Strahl wurde über einen Planspiegel und mit einem Ellipsoidspiegel ( $f=250 / 40$ $\mathrm{mm}$ ) auf das Detektorfenster fokussiert.

\subsubsection{LASER-Photolyse}

Zur Photolyse der Vorläufer der Reaktionspartner wurde ein Excimer-LASER (Lambda Physik, Compex 102) verwendet. Die Wellenlänge des LASER-Lichts betrug mit der in dieser Arbeit verwendeten LASER-Füllung 193 nm (ArF). Die Energie eines LASER-Pulses lag bei 100-120 mJ; sie sank jedoch nach Passieren einiger Umlenkspiegel auf 30-50 mJ bei Eintritt in die Photolysezelle. Die Repititionsrate konnte mittels eines selbsterstellten Programms (siehe unten) zwischen 0,1 und $20 \mathrm{~Hz}$ eingestellt werden. Das im Nachweisvolumen liegende Reaktionsvolumen betrug ${ }^{54}$ ca. $12,3 \mathrm{~cm}^{3}$.

\subsubsection{Probennachweis}

Der Probennachweis erfolgte IR-spektrometrisch. Für die Aufnahme der IR-Spektren stand ein FT-IR-Spektrometer (BRUKER; Typ: IFS 66) zur Verfügung. Die Fourier-Transformation ${ }^{55}$ wurde dabei unmittelbar nach der Registrierung des Interferogramms auf einem Personalcomputer (Pentium III mit OS/2) durch die mitgelieferte Software (BRUKER, OPUS 3.0.4) durchgeführt. Mit diesem Gerät war eine sehr hohe Auflösung (bis zu 0,12 $\mathrm{cm}^{-1}$ ) möglich. Durch die Fourier-Transformation-Technik betrug die Aufnahmezeit für ein komplettes IR-Spektrum lediglich wenige Sekunden.

Das Gerät kann bis zu 60 Interferogrammpunkte pro Sekunde aufnehmen, was bei einer Auflösung von $32 \mathrm{~cm}^{-1}$ zu einer maximalen Zeitauflösung von $20 \mathrm{~ms}$ führt ${ }^{56}$. Ist eine höhere Zeitauflösung erwünscht, so kann dies im sogenannten „Step Scan“-Modus realisiert werden; je nach verwendetem Detektor läßt sich damit im mitteleren IR-Bereich unter Verwendung

54 Tröger, U. (1997); S. 35.

55 Vgl. hierzu: Tröger, U. (1997); S. 12-18.

56 Tröger, U. (1997); S. 18. 
einer im Personalcomputer eingebauten Transientenrekorderkarte (12 Bit Digitalisierung) eine Zeitauflösung bis etwa 40 ns erreichen ${ }^{57}$.

Bei der „Step Scan“-Meßtechnik ${ }^{58}$ wird im Unterschied zum „,normalen“ Meßverfahren der bewegliche Spiegel des „Michelson-Interfermeters“ fest positioniert und in dieser Position zeitaufgelöst die jeweiligen Interferogrammpunkte ${ }^{59}$ der verschiedenen Zeitscheiben registriert. Dann wird der Spiegel auf die für die Aufnahme des nächsten Interferogrammpunkts erforderliche Position bewegt und dort wiederum zeitaufgelöst die jeweiligen Interferogrammpunkte der verschiedenen Zeitscheiben registriert. Dieser Vorgang wird bis zur vollständigen Aufnahme aller Interferogrammpunkte wiederholt. Aus diesen Punkten kann nun mittels der FT für jede Zeitscheibe ein IR-Spektrum berechnet werden.

Zur Durchführung einer „Step Scan“-Messung ist es jedoch erforderlich, für jeden zu messenden Interferogrammpunkt eine absolut identische Probensituation zu schaffen, d.h. die Reaktanten müssen jedesmal in identischen Konzentrationen vorliegen und der PhotolyseLASER-Puls muß identische Energie aufweisen sowie zur selben Zeit in die Zelle eintreten. Ferner müssen auch sonst alle experimentellen Parameter identisch sein (z.B. Verzögerungszeit zwischen LASER-Puls und Datenaufnahme, Empfindlichkeit des Detektors, Temperatur, etc.). Um ein komplettes Absorptionsspektrum bei einer Auflösung von $16 \mathrm{~cm}^{-1}$ aufzunehmen, benötigt man fast 2000 Interferogrammpunkte ${ }^{60}$. Zur Erhöhung der statistischen Sicherheit ist es sinnvoll, jeden Interferogrammpunkt wiederholt aufzunehmen und daraus einen Mittelwert zu bilden, so daß mindestens 4000 oder 6000 identische Einzelexperimente notwendig sind. Es bietet sich daher an, einen möglichst kleinen, aber für die gewünschte Reaktion charakteristischen Bereich des Spektrums auszuwählen und durch geeignete Filter den IRStrahl auf diesen Bereich zu begrenzen. Dadurch läßt sich die Zahl der aufzunehmenden Interferogrammpunkte und damit die der notwendigen identischen Einzelexperimente drastisch reduzieren.

\subsubsection{Signalaufnahme}

Wie bereits oben beschrieben wurde, trat der vom Spektrometer mittels eines Globars erzeugte IR-Strahl nach einigen Reflexionen in der Nachweiszelle aus dieser durch ein KBr-Fenster wieder aus und wurde auf einen Detektor fokussiert. Für alle Messungen in dieser Arbeit wurde stets ein mit flüssigem Stickstoff gekühlter MCT-Detektor $(\mathrm{Hg} / \mathrm{Cd} / \mathrm{Te})$ verwendet. Mit

57 persönliche Mitteilung während des „IR PD - Praxiskurses“ bei der Fa. BRUKER am 06.10.1999.

58 Vgl. hierzu: Tröger, U. (1997); S. 18-20.

59 Vgl. hierzu: Tröger, U. (1997); S. 13 ff.

60 Tröger, U. (1997); S. 20. 
diesem Detektor waren zeitaufgelöste Messungen bis $5 \mu$ s möglich; er konnte im Wellenzahlbereich von 600 bis $5000 \mathrm{~cm}^{-1}$ eingesetzt werden. Das Ausgangssignal des Detektors wurde von einem AD-Wandler des FT-IR-Spektrometers aufgenommen und zusammen mit den anderen Geräteparametern von der mitgelieferten Software (BRUKER, OPUS 3.0.4) zu einem IR-Spektrum verarbeitet.

\subsubsection{Computersteuerung und -auswertung}

Das FT-IR-Spektrometer konnte durch die mitgelieferte Software (BRUKER, OPUS 3.0.4) vom Personalcomputer aus gesteuert werden. Mit dieser Software war auch die weitere Bearbeitung der registrierten IR-Spektren möglich (Erzeugung von Transmissionsspektren aus den Einkanalspektren, Umwandlung von Transmissionsspektrum in Absorptionsspektrum, Subtraktion von Spektren, Drucken, Export in ASCII-Datei, etc.).

Wie bereits oben erwähnt wurde, war zur Aufnahme von zeitaufgelösten Absorptionsspektren im „Step Scan“-Modus die Durchführung vieler absolut identischer Einzelexperimente erforderlich. $\mathrm{Zu}$ diesem Zweck wurde im Rahmen der vorliegenden Dissertation ein Programm erstellt, das mit „FFCP Version 1.2“ bezeichnet wird. Es wurde in der Programmiersprache „Object-Pascal“ geschrieben und als 32-Bit-Programm mit „Delphi 2.01“ der Firma Borland compiliert. Es ist unter den Betriebssystemen Windows 95/98 und NT lauffähig. Für die Messungen in dieser Arbeit wurde es unter Windows 95 ausgeführt. Das Programm steuert die wiederholte identische Füllung der Photolysezelle und sorgt für den Start der „Step Scan“Messung.

Quasi als Nebenprodukt dieser Zielvorgaben für eine „Step Scan“-Messung ermöglicht das Programm auch das automatische Einfüllen eines bestimmten Reaktanten in die Zelle bis zu einem vorgegebenen Druck, die Triggerung des LASERs (und ggf. der FT-IR-Software) sowie die Steuerung der Pumpe.

Die gesamte Beschreibung des „FFCP“-Programms ist recht umfangreich und daher wurde im Rahmen dieser Arbeit auch eine ausführliche Programmbeschreibung erstellt ${ }^{61}$.

Der prinzipielle Programmablauf läßt sich folgendermaßen beschreiben:

Zur Vorbereitung einer Messung müssen die beiden Reaktanten und das Badgas mit einem geeigneten Vordruck bzw. einer geeigneten Einstellung des Dosierventils an die Zelle angeschlossen werden. Ferner muß das Programm „Opus“ im „Step Scan“-Modus mit den gewünschten Parametern (insbesondere externer Triggerung) gestartet und in Aufnahmebereitschaft versetzt werden.

61 Hold, M. (2001); Programmbeschreibung für FFCP Version 1.2. 
Nun übernimmt das Programm „FFCP“ die weitere Steuerung/Kontrolle.

Bei der automatischen „Step-Scan“-Messung wird zunächst die Pumpe gestartet, dann erst nach einer kurzen Verzögerungszeit (um eine Verunreinigung der Zelle zu vermeiden) das Magnetventil zwischen Pumpe und Reaktionszelle geöffnet und die Zelle so lange evakuiert, bis der Druck unter einen vom Benutzer vorgegebenen Wert gesunken ist. Dann schließt dieses Magnetventil und die Pumpe wird abgeschaltet (um Vibrationen während der Messung zu vermeiden).

Jetzt wird das entsprechende Magnetventil geöffnet, um den 1. Reaktanten in die Zelle einzufüllen. Dabei wird ständig der Druck gemessen und das Magnetventil bei Erreichen des gewünschten Fülldruckes geschlossen. Die Druckmessung erfolgt dabei automatisch mit dem Druckmeßgerät, das die höchst mögliche Genauigkeit bietet. Auf diese Weise werden nacheinander die drei Gase in den gewünschten Mengen eingefüllt.

Nach Füllung der Zelle wartet das Programm eine vorgegebene Zeit bis zur vollständigen Durchmischung und gibt dann zeitgleich einen Trigger-TTL-Puls zum Start sowohl an den LASER als auch an „Opus“. Sofern mehrere Photolysen derselben Füllung gewünscht sind, wartet das Programm eine vorgegebene Zeit und gibt dann erneut die Trigger-Pulse an den LASER und „Opus“.

Danach wird die Reaktionsmischung abgepumpt und die Füllprozedur startet wie oben beschrieben von neuem. 


\subsection{Erzeugung der Fluor-, Sauerstoff- und Wasserstoffatome}

Die für die Reaktionen benötigten Atome wurden durch eine elektrodenlose Mikrowellenentladung ( $\lambda / 4$-Resonator; Bosch Radarmed) mit einer mittleren Ausgangsleistung von 70 Watt erzeugt. Das Zünden der Mikrowelle wurde durch ein schwaches elektrisches Wechselfeld $(15 \mathrm{kV} / \mathrm{cm} ; 10 \mathrm{kHz})$ erleichtert. Fluor und Sauerstoff wurden als hochverdünnte Gasmischungen mit Helium eingesetzt und vor der Entladung noch weiter mit Helium verdünnt. Bei der Erzeugung der Fluor-Atome befand sich im Entladungsröhrchen eine $\mathrm{Al}_{2} \mathrm{O}_{3^{-}}$ Keramik, um die Reaktion mit dem Quarzröhrchen (Bildung von Siliziumfluorid) zu vermindern.

Der Dissoziationsgrad von Fluor (gemessen auf $\mathrm{m} / \mathrm{z}=38$ ) lag bei 75 bis $95 \%$, derjenige von Sauerstoff (untergrundkorrigiert gemessen auf $\mathrm{m} / \mathrm{z}=32$ ) in Abwesenheit von Fluor bei $20 \%$. Bei gleichzeitiger Zugabe von Sauerstoff und Fluor, so wie es bei der Durchführung der Messungen der Fall war, blieb der Dissoziationsgrad von Fluor fast unverändert, während der von Sauerstoff auf 40-50 \% anstieg.

\subsection{Dosierung der Gase und Flüssigkeiten}

\subsubsection{Messungen in der Multireflektionszelle an der FT-IR-Apparatur}

Weil man auf Grund der hohen Nachweisempfindlichkeit für Messungen in der Multireflektionszelle nur eine sehr geringe Konzentration (einen Partialdruck im 1 mbarBereich oder darunter) benötigt, gelang auch bei allen untersuchten flüssigen Substanzen das Einfüllen in die Zelle mittels des eigenen Dampfdrucks dieser Substanzen. Die Konzentration in der Zelle konnte durch Messung des Druckanstiegs beim Einfüllen nach dem idealen Gasgesetz berechnet werden.

Bei flüssigen Substanzen mit hohem Dampfdruck oder bei Gasen aus Druckgasflaschen wurde ein Feindosierventil (Hoke Mikro-Mite) zwischengeschaltet, um einen langsamen und damit kontrollierbaren Druckanstieg in der Zelle zu gewährleisten.

Für die in dieser Arbeit durchgeführten Messungen war es nicht erforderlich, während der Aufnahme der IR-Spektren weitere Substanzen in die Zelle einströmen zu lassen.

\subsubsection{Messungen in Strömungsreaktoren}

Alle in einen Strömungsreaktor gegebenen Substanzen wurden so weit mit Helium verdünnt, daß letzteres mehr als 90 Vol-\% des gesamten Gasflusses ausmachte. Helium eignet sich 
besonders gut als Trägergas, weil es eine hohe Wärmeleitfähigkeit hat und damit die gleichmäßige und schnelle Temperierung der Reaktionsmischung gewährleistet ist (isotherme Bedingungen im Strömungssystem); dies ist eine wichtige Voraussetzung für die Messung der Temperaturabhängigkeiten der Geschwindigkeitskoeffizienten.

Die Dosierung von nicht aggressiven Gasen bzw. Flüssiggasen (z.B. Helium, Argon, Chlormethan, Methan sowie Ethan) erfolgte mit geeichten Flußmessern (Tylan FC 260).

Die aggressiven Gase (z.B. Fluor, Stickstoffmonoxid) wurden über ein Feindosierventil (Hoke Mikro-Mite) zugegeben.

Die untersuchten Flüssigkeiten wurden in einen Sättiger (siehe unten Abbildung 16) gegeben, dann mit Helium als Trägergas ein Druck von 3,0·10 $\mathrm{Pa}$ (absolut) eingestellt und diese Gasmischungen ebenfalls über ein selbst kalibriertes Feindosierventil (Hoke, Mikro-Mite) dem Strömungsreaktor zugeführt.

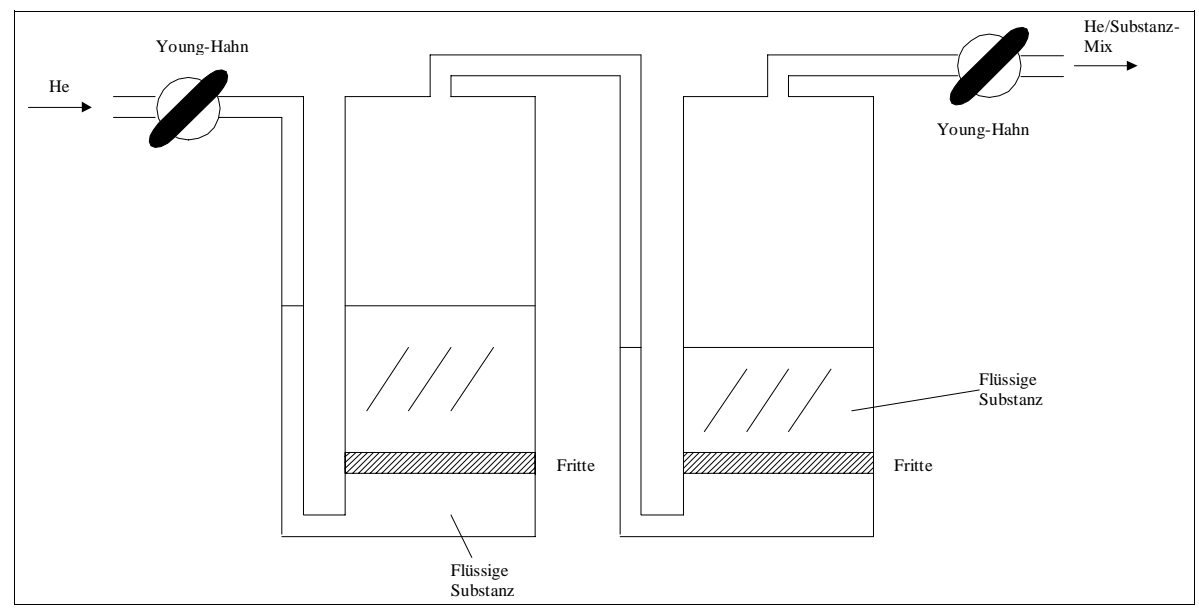

Abbildung 16: zweistufiger Sättiger

\subsection{Reinheit der verwendeten Chemikalien}

Die folgenden Chemikalien wurden ohne weitere Reinigung direkt eingesetzt:

\begin{tabular}{|l|l|l|}
\hline \multicolumn{1}{|c|}{ Substanz } & \multicolumn{1}{|c|}{ Reinheit } & \multicolumn{1}{c|}{ Hersteller/Verkäufer } \\
\hline Helium & $99,996 \%$ & $\begin{array}{l}\text { Messer-Griesheim, } \\
\text { Frankfurt a. M. }\end{array}$ \\
\hline Argon & $99,998 \%$ & Messer-Griesheim \\
\hline $\begin{array}{l}\text { Methan } \\
\mathrm{CH}_{4}\end{array}$ & $99,995 \%$ & Messer-Griesheim \\
\hline
\end{tabular}




\begin{tabular}{|c|c|c|}
\hline Substanz & Reinheit & Hersteller/Verkäufer \\
\hline $\begin{array}{l}\text { Ethan } \\
\mathrm{C}_{2} \mathrm{H}_{6}\end{array}$ & $99,5 \%$ & Messer-Griesheim \\
\hline $\begin{array}{l}\text { Helium/Fluor-Gasmischung } \\
\text { (0,5 Vol-\% Fluor) }\end{array}$ & 99,996\% (Helium) & Messer-Griesheim \\
\hline $\begin{array}{l}\text { Helium/Fluor-Gasmischung } \\
\text { (5,0 Vol-\% Fluor) }\end{array}$ & 99,996\% (Helium) & Messer-Griesheim \\
\hline $\begin{array}{l}\text { Dichlormethan } \\
\mathrm{CH}_{2} \mathrm{Cl}_{2}\end{array}$ & $\begin{array}{l}99,5 \% \\
\text { (enthält 20 ppm 2- } \\
\text { Methyl-2-buten als } \\
\text { Stabilisator) }\end{array}$ & $\begin{array}{l}\text { Merck, } \\
\text { Darmstadt }\end{array}$ \\
\hline $\begin{array}{l}\text { Chlormethan } \\
\mathrm{CH}_{3} \mathrm{Cl}\end{array}$ & $99,5 \%$ & $\begin{array}{l}\text { Prax-Air, } \\
\text { Oevel (Belgien) }\end{array}$ \\
\hline $\begin{array}{l}\text { Bis(difluormethyl)ether } \\
\mathrm{CHF}_{2} \mathrm{OCHF}_{2}\end{array}$ & $97 \%$ & $\begin{array}{l}\text { Fluorochem LTD, } \\
\text { Wesley Street, Old Glossop, } \\
\text { Derbyshire SK13 9RY }\end{array}$ \\
\hline Sauerstoff & $99,995 \%$ & Messer Griesheim \\
\hline $\begin{array}{l}\text { Stickstoffmonoxid } \\
\text { NO }\end{array}$ & $99,5 \%$ & Messer Griesheim \\
\hline 1-Ethinyl-1-methylcyclopropan & & $\begin{array}{l}\text { Inst. f. Organische Chemie, } \\
\text { Arbeitskreis de Meijere, } \\
\text { Universität Göttingen }\end{array}$ \\
\hline $\begin{array}{l}\text { Kohlenmonoxid } \\
\text { CO }\end{array}$ & $>99 \%$ & \\
\hline $\begin{array}{l}\text { Ethylnonafluorbutylether } \\
\mathrm{C}_{4} \mathrm{~F}_{9} \mathrm{OC}_{2} \mathrm{H}_{5}\end{array}$ & & $\begin{array}{l}\text { 3M Deutschland GmbH, } \\
\text { Carl-Schurz-Str. 1, } \\
41453 \text { Neuss }\end{array}$ \\
\hline $\begin{array}{l}\text { Methylnonafluorbutylether } \\
\mathrm{C}_{4} \mathrm{~F}_{9} \mathrm{OCH}_{3}\end{array}$ & & 3M Deutschland GmbH \\
\hline $\begin{array}{l}\text { 2,2,3,3,4,4,4-Heptafluor-1-butanol } \\
\mathrm{CF}_{3} \mathrm{CF}_{2} \mathrm{CF}_{2} \mathrm{CH}_{2} \mathrm{OH}\end{array}$ & $98 \%$ & $\begin{array}{l}\text { Aldrich, Milwaukee, } \\
\text { WI } 53201 \text { USA }\end{array}$ \\
\hline $\begin{array}{l}\text { 2-Fluorethanol } \\
\mathrm{CH}_{2} \mathrm{FCH}_{2} \mathrm{OH}\end{array}$ & $95 \%$ & Aldrich \\
\hline 1,1,1,2-Tetrafluorethan & $99+\%$ & Aldrich \\
\hline $\begin{array}{l}\text { 2,2,2-Trifluorethanol } \\
\mathrm{CF}_{3} \mathrm{CH}_{2} \mathrm{OH}\end{array}$ & $99+\%$ & Lancaster \\
\hline
\end{tabular}

Tabelle 1: Reinheit der verwendeten Chemikalien 


\subsection{Ausführung und Auswertung der Messungen}

\subsubsection{Messungen im Strömungsreaktor mit dem El-Massenspektrometer}

\subsubsection{Allgemeines}

Alle untersuchten Reaktionen wurden in einem isothermen Strömungssystem durchgeführt. In diesem lag eine laminare Strömung mit konstanter Strömungsgeschwindigkeit vor (vgl. dazu Kapitel 2.3, S.13). Eine genaue Beschreibung der Apparatur findet sich in Kap. 3.1.1 (S. 16).

Das Ventil zwischen der Pumpe E 250 und dem Strömungssystem war während der Messungen stets vollständig geöffnet, um einen möglichst geringen Druck zu erreichen; dies war wichtig, um Folgereaktionen gering zu halten.

Bei EI-Elektronenenergien (IP) im Bereich von 4,5 bis $29,5 \mathrm{eV}$ wurde stets ein Emissionsstrom von $22 \mu \mathrm{A}$, bei IP $=70 \mathrm{eV}$ einer von $30 \mu \mathrm{A}$ eingestellt.

Alle untersuchten Radikale wurden durch Zugabe von Fluor-Atomen (= erster zugegebener Reaktant) zu den Kohlenwasserstoffen ${ }^{62}$ erzeugt:

$$
\mathrm{R}-\mathrm{H}+\mathrm{F} \cdot \rightarrow \mathrm{R} \cdot+\mathrm{HF} \quad \text {; mit R-H: Kohlenwasserstoff }
$$

\subsubsection{Durchführung der Produktuntersuchungen}

$\mathrm{Zu}$ Beginn einer Meßreihe wurden zunächst Massenspektren unter den verschiedenen Reaktionsbedingungen aufgenommen, um einen Überblick über die ablaufenden Reaktionen zu bekommen. Alle Übersichtsspektren wurden mit dem Zweikanalzähler bei 100 bzw. 200 ms Zählzeit pro Meßpunkt und linearem Massendurchlauf registriert.

Anschließend wurden nacheinander am Massenspektrometer gezielt die m/z-Zahlen für die sich im Übersichtsspektrum ändernden Signale eingestellt und letztere nochmals mit längeren Einzelionen-Zählzeiten (üblicherweise 5-10 Messungen mit je 10 Sekunden) gemessen. Auf diese Weise wurden die qualitativen Produktuntersuchungen durchgeführt.

Für quantitative Produktuntersuchungen war die Ermittlung der massenspektrometrischen Empfindlichkeiten erforderlich (vgl. Kap. 2.2, S. 12). Dazu wurden die als Produkte entstehenden Substanzen separat in verschiedenen bekannten Konzentrationen (berechnet nach Gleichung (19), S. 14) in die Apparatur gegeben und diese Konzentration dann als Funktion des DATA-Werts aus der Einzelionenzählung aufgenommen.

\subsubsection{Ermittlung der Geschwindigkeitskoeffizienten nach der Relativmethode}

Zur Bestimmung der Geschwindigkeitskoeffizienten wurde überwiegend die Relativmethode

62 In den Begriff Kohlenwasserstoff sind hier auch die teilhalogenierten Kohlenwasserstoffe, Ether und Alkohole mit einbezogen. 
gemäß Kapitel 2.1.1.2 (S. 10) eingesetzt.

Dabei wurde jeweils die Reaktion unter gleichen Bedingungen mit und ohne Umsatz untersucht. Die Reaktionsstrecke blieb konstant. Bei der Radikalerzeugung wurde dazu die Änderung der Intensität des Kohlenwasserstoffs gemessen und eine Variation der Umsätze durch Verändern der zugegebenen Fluor-Atom-Konzentration erreicht. Bei der Umsetzung der Radikale wurde entsprechend die Änderung der Intensität des Radikales gemessen und die Konzentration des zweiten Reaktanten variiert, während die Fluor-Atom-Konzentration konstant blieb. Die für die Auswertung verwendeten Werte sind Mittelwerte aus 3 bis 10 Messungen bei Zählzeiten von 5 bis 30 Sekunden; die genauen Versuchsbedingungen sind jeweils bei den Meßwerttabellen vermerkt.

Für die Messung der Umsetzung eines Kohlenwasserstoffs mit einem anderen Reaktanten wurde praktisch so vorgegangen, daß der zu untersuchende Kohlenwasserstoff und die Referenzsubstanz gleichzeitig unter großer Verdünnung mit Helium $([\mathrm{He}]>90$ Vol-\%) in das Strömungssystem geleitet und sodann die interessierende m/z-Zahl des Kohlenwasserstoffs am Massenspektrometer eingestellt wurde; nun erfolgte unter Abwesenheit des anderen Reaktanten die wiederholte Messung der Intensität (meistens 5 Messungen bei je 10 Sekunden Zählzeit), dann wurde der andere Reaktant zugegeben und die Messung in gleicher Weise wiederholt. Die zwischen der Zugabe des anderen Reaktanten und dem Beginn der (zweiten) Zählung liegende Pause wurde so gewählt, daß zum Start der Zählung ein konstantes Signal vorlag. Analog wurden auch die Messungen auf dem m/z-Wert der Referenzsubstanz durchgeführt. Aus den so erhaltenen vier Mittelwerten konnte nun ein Wert für die doppeltlogarithmische Auftragung gemäß Gleichung (10) (S. 10) berechnet werden (vgl. Kap. 2.1.1.2, S. 10). Dabei wurde für die Konzentration zur Zeit $t^{\prime}=0$ der DATA-Wert ohne Zugabe des anderen Reaktanten und für diejenige zur Zeit $t^{\prime}=t$ derjenige mit Zugabe des anderen Reaktanten eingesetzt. Die gesamte soeben beschriebene Prozedur wurde bei verschiedenen Konzentrationen des anderen Reaktanten wiederholt. Die Steigung der doppeltlogarithmischen Auftragung wurde mittels linearer Regression (Methode der kleinsten Quadratsumme) berechnet.

Sofern ein meßbarer Untergrund (BKG) bei einer Masse vorlag, wurde dieser unter den gleichen Bedingungen, unter denen die Messung durchgeführt werden sollte, registriert und für die Auswertung vom späteren Meßwert (DATA) subtrahiert; das Steuerungsprogramm „WinSiccon“ führte letztere Korrektur dann automatisch durch.

Sofern bei den Reaktionen der erzeugten Radikale der interessierende m/z-Wert des Radikals 
noch mit einem Fragment des Ausgangsmoleküls (Vorläufer des Radikales) überlagert war, erfolgte eine Umsatzkorrektur dieses Wertes. Das Verfahren sei am Beispiel von Methan erklärt:

Soll die Reaktion des (aus Zugabe von Fluor-Atomen zu Methan-Gas erzeugten) Methylradikals $\left(\mathrm{M}_{\mathrm{r}}=15\right)$ mit $\mathrm{O}$-Atomen $\left(\mathrm{CH}_{3}+\mathrm{O} \rightarrow\right.$ Produkte $)$ gemessen werden, so ist auf m/z 15 auch bei nicht sehr hoher EI-Elektronenenergie noch ein Fragment von Methan $\left(M_{r}=\right.$ 16) $\mathrm{zu}$ erkennen. Die für die weitere Auswertung verwendeten (umsatz-)korrigierten Intensitäten I $^{\text {korr }}$ wurden dann folgendermaßen berechnet:

$$
\begin{aligned}
& \mathrm{I}^{\text {korr }}(15+\mathrm{F}-\mathrm{O})=\mathrm{I}^{\text {überlagert }}(15+\mathrm{F}-\mathrm{O})-\mathrm{I}(15-\mathrm{F}-\mathrm{O}) \frac{\mathrm{I}(16+\mathrm{F}-\mathrm{O})}{\mathrm{I}(16-\mathrm{F}-\mathrm{O})} \\
& \mathrm{I}^{\text {korr }}(15+\mathrm{F}+\mathrm{O})=\mathrm{I}^{\text {überlagert }}(15+\mathrm{F}+\mathrm{O})-\mathrm{I}(15-\mathrm{F}-\mathrm{O}) \frac{\mathrm{I}(16+\mathrm{F}-\mathrm{O})}{\mathrm{I}(16-\mathrm{F}-\mathrm{O})}
\end{aligned}
$$

Dabei bedeuten:

$$
\begin{aligned}
& \mathrm{I}(16-\mathrm{F}-\mathrm{O})=\text { Intensität auf } \mathrm{m} / \mathrm{z} 16 \text {, ohne Zugabe von } \mathrm{F} \text { - und O-Atomen } \\
& \mathrm{I}(16+\mathrm{F}-\mathrm{O})=\text { Intensität auf } \mathrm{m} / \mathrm{z} 16 \text {, bei Zugabe von F-, aber ohne die von O-Atomen } \\
& \mathrm{I}^{\text {übrlagert }}(15+\mathrm{F}+\mathrm{O})=\text { tatsächlich gemessene Intensität auf } \mathrm{m} / \mathrm{z} 15 \text { bei Zugabe von } \mathrm{F} \text { u. O }
\end{aligned}
$$

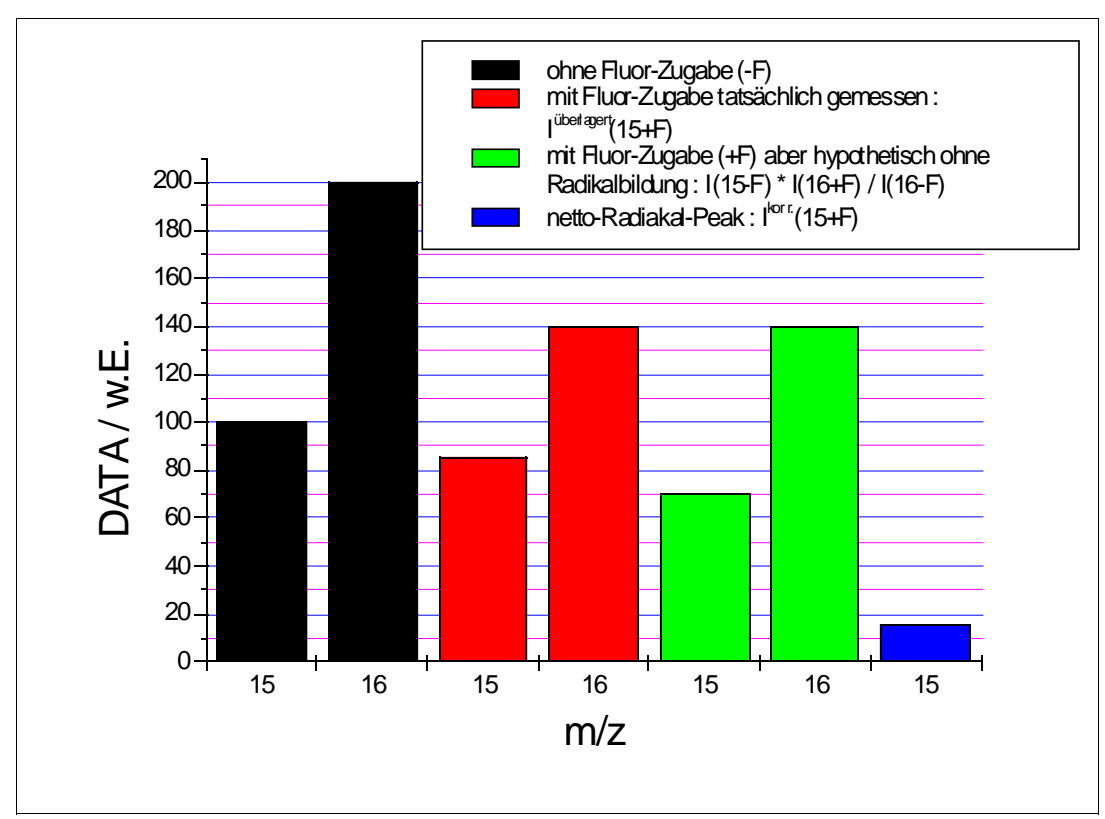

Abbildung 17:Beispiel zur Illustration der Umsatzkorrektur

Zur Berechnung von Gleichung (24) muß zunächst also auf m/z 16 das Verhältnis der Intensitäten I(16+F-O) zu I(16-F-O) bestimmt werden, d.h. die relative Abnahme des Molekülionen-Peaks bei Zugabe von Fluor-Atomen (= Umsatz der radikalerzeugenden Reaktion). Der reine Fragmentanteil auf m/z 15 wird durch diese Reaktion natürlich in gleicher 
Höhe vermindert; wenn man also (hypothetisch) annimmt, das Radikal selbst würde auf m/z 15 kein Signal zeigen, würde man die in Abbildung 17 grün gezeichneten Peaks erwarten, die beide bezogen auf die schwarzen Peaks um den selben Prozentsatz (in diesem Beispiel: 30 \%) vermindert sind. Das Verhältnis der Intensitäten auf m/z 16 zu dem auf m/z 15 in Höhe von 200/100=2,00 sollte also auch nach Flur-Atom-Zugabe erhalten bleiben.

Faktisch sinkt dieses Verhältnis aber auf 1,65, denn tatsächlich wird auf m/z 15 nicht eine Intensität von 70 (hypothetischer grüner Peak in Abbildung 17), sondern von 85 (roter Peak) gemessen. Die Differenz aus dem grünen hypothetischen und dem roten tatsächlichen Wert auf m/z 15 ergibt die umsatzkorrigierte (d.h. die wahre) Signalintensität des Radikals. Nur dieser umsatzkorrigierte (in Abbildung 17 blau dargestellte) Wert wird vom Programm „WinSiccon“ zur weiteren Auswertung (Erstellung der doppelt-logarithmische Auftragung gemäß Gleichung (10), S. 10) herangezogen.

Das Computerprogramm ermöglicht auch eine konsequente Fehlerrechnung mit Fehlerfortpflanzung, so daß alle in der vorliegenden Arbeit angegebenen Fehler der Mittelwerte für ein Konfidenzintervall von $95 \%$ berechnet sind. Eine ausführliche Erläuterung der Fehlerrechnung findet man in der Programmbeschreibung ${ }^{63}$.

Als Fehler der Steigungen und Achsenabschnitte aus den linearen Regressionen sind die jeweiligen Standardabweichungen angegeben.

Aufgrund der endlichen Bandbreite des $85-\mathrm{MHz}-Z$ weikanalzählers treten bei Ionenströmen größer als ca. $10^{6} \mathrm{~s}^{-1}$ Sättigungserscheinungen (,,pulse pile up“) auf, die zu einer Verfälschung der Zählung führen; im Experiment wurde daher immer weit unterhalb dieses Wertes gezählt.

Zur Bestimmung des Geschwindigkeitskoeffizienten der radikalerzeugenden Reaktion des Kohlenwasserstoffs mit Fluor-Atomen fand der Strömungsreaktor in Abbildung 5 (S. 17) Verwendung. Die Kohlenwasserstoffe wurden durch die innere Sonde zugegeben und die durch Mikrowellenentladung aus Fluor-Molekülen erzeugten Fluor-Atome durch den seitlichen Einlaß zugeführt. Die Reaktionsstrecke lag typischerweise bei 1-3 cm. Die Messung der Intensität ohne Umsatz erfolgte durch Abschalten der Mikrowellenentladung, wobei der $\mathrm{F}_{2^{-}}$ Fluß in den Strömungsreaktor aber beibehalten wurde. Durch Voruntersuchungen war sichergestellt, daß der Kohlenwasserstoff unter den relevanten Bedingungen keine meßbare Reaktion mit Fluor-Molekülen $\left(\mathrm{F}_{2}\right)$ einging.

Zur Bestimmung des Geschwindigkeitskoeffizienten der Reaktion des erzeugten Kohlenwasserstoffradikals mit molekularem Sauerstoff oder mit Stickstoffmonoxid kam 
ebenfalls der Strömungsreaktor in Abbildung 5 (S. 17) zum Einsatz. Wie soeben beschrieben, wurden zur radikalerzeugenden Reaktion Fluor-Atome durch den seitlichen MWE-Einlaß zugeführt. Zusätzlich zum Kohlenwasserstoff wurde durch die innere Sonde noch molekularer Sauerstoff bzw. Stickstoffmonoxid zugeführt, um die Reaktion mit dem Radikal zu ermöglichen. Die Reaktionsstrecken lagen typischerweise bei 2-5 cm. Zur Messung der Intensität des Radikals ohne Umsatz wurde der Zufluß des molekularen Sauerstoffs bzw. Stickstoffmonoxids computergesteuert durch ein Magnetventil abgeschaltet. Die Zugabe von Helium kompensierte den Druck im Strömungsreaktor, sofern dieser durch das Schließen des Magnetventils für den zweiten Reaktanten meßbar abnahm.

Zur Bestimmung des Geschwindigkeitskoeffizienten der Reaktion des erzeugten Kohlenwasserstoffradikals mit atomarem Sauerstoff fand häufig ebenfalls der Strömungsreaktor in Abbildung 5 (S. 17) Verwendung. Wie oben beschrieben, wurden zur radikalerzeugenden Reaktion durch den seitlichen MWE-Einlaß Fluor-Atome und zusätzlich zu diesen noch atomarer Sauerstoff zugeführt (welcher durch Mikrowellenentladung aus molekularem Sauerstoff erzeugt worden war). Die Reaktionsstrecken lagen typischerweise bei 2-4 cm. Zur Messung der Intensität des Radikals ohne Umsatz wurde der Zufluß des molekularen und damit auch der des atomaren Sauerstoffs computergesteuert durch ein Magnetventil abgeschaltet. Weil es sich dabei um einen vergleichsweise geringen Teilgasfluß handelte, war eine Druck-Kompensation mit Helium nicht erforderlich.

Zur Bestimmung des Geschwindigkeitskoeffizienten der Reaktion des erzeugten Kohlenwasserstoffradikals mit atomarem Sauerstoff wurde zum Teil auch der Strömungsreaktor in Abbildung 6 (S. 17) verwendet und ebenso für die Untersuchung der Reaktion des Radikals mit Wasserstoffatomen genutzt. Im Gegensatz zu dem oben Beschriebenen, konnten hiermit jedoch keine Messungen zur Temperaturabhängig durchgeführt werden. Auch hierbei wurden zur radikalerzeugenden Reaktion Fluor-Atome durch den ersten seitlichen MWE-Einlaß zugeführt. Durch den zweiten seitlichen MWE-Einlaß strömte atomarer Sauerstoff bzw. atomarer Wasserstoff (welcher durch Mikrowellenentladung aus molekularem Sauerstoff bzw. molekularem Wasserstoff erzeugt worden war), um die Reaktion mit dem Radikal zu ermöglichen. Die Reaktionsstrecken lagen typischerweise bei 2-4 cm. Die Messung der Intensität ohne Umsatz erfolgte durch Abschalten der Mikrowellenentladung, wobei der $\mathrm{O}_{2}$-Fluß bzw. $\mathrm{H}_{2}$-Fluß in den Strömungsreaktor aber beibehalten wurde. 
Auf eine Diffusions-Korrektur nach Gleichung (21) (S. 14) wurde verzichtet, da die dafür zu berücksichtigenden Änderungen weit geringer als die Meßfehler waren ${ }^{64}$.

\subsubsection{Ermittlung der Geschwindigkeitskoeffizienten nach der Absolutmethode}

Aus den in Kap. 2.1.1.1 (S. 8) genannten Gründen ist die Methode der Absolutmessung mit einigen Schwierigkeiten verbunden, so daß sie in der vorliegenden Arbeit nur für einige ausgewählte Reaktionen Verwendung fand. Alle Reaktionen wurden isotherm in dem in Abbildung 5 (S. 17) beschriebenen Strömungsreaktor ausgeführt.

Zum einen erfolgte die Ermittlung der Geschwindigkeitskoeffizienten für die Kombinationsreaktion von Radikalen mittels der Absolutmethode. Dazu wurden durch die Reaktion eines geeigneten Vorläufer-Kohlenwasserstoffs mit Fluor-Atomen die Radikale erzeugt, dann das Signal auf dem m/z-Wert des Radikals als Funktion der Reaktionsstrecke (durch Verschiebung der inneren Sonde) aufgenommen. Für die Sondenstellung, für die das Signal maximale Intensität aufwies, wurde unter der Annahme, daß die Radikalerzeugung beendet war und hier die Kombination begann, die Reaktionszeit der Kombination $t_{0}=0$ gesetzt. Nun mußten die nötigen Werte für eine Auftragung gemäß Gleichung (8) (S. 9) registriert werden. Dazu wurde jeweils für verschiedene Reaktionsstrecken (und damit für verschiedene Reaktionszeiten) das Signal auf dem m/z-Wert des Radikals mit und ohne Zugabe von Fluor-Atomen gemessen. Die Umrechnung der Reaktionsstrecken in die Reaktionszeiten erfolgte nach Gleichung (18) (S. 13). Die Ausgangskonzentration des Radikals wurde aus der Konzentration des Vorläufer-Kohlenwasserstoffs (berechnet nach Gleichung (19), S. 14) und dessen Umsatz nach Zugabe von Fluor-Atomen berechnet. Dieser Umsatz wurde auf der m/zZahl des Molekülionenpeaks des Vorläufer-Kohlenwasserstoffs bei derjenigen Reaktionsstrecke gemessen, die die maximale Signalintensität auf der m/z-Zahl des Radikals aufwies. Weil in dem Ausdruck auf der linken Seite der Gleichung (8) (S. 9) lediglich das Verhältnis von Konzentrationen eingeht, ist eine Kalibrierung und Umrechnung der DATAWerte für die Reaktionszeiten $\mathrm{t}>0$ in die entsprechenden Konzentrationen nicht erforderlich. Statt der Konzentrationen konnte dort direkt die Differenz der DATA-Werte mit Zugabe und ohne Zugabe von Fluor-Atomen (= DATA-Wert des reinen Radikals ohne BKG) eingesetzt werden.

Zum anderen wurde der Geschwindigkeitskoeffizient für die Reaktion von Radikalen mit molekularem Sauerstoff mittels der Absolutmethode analog ermittelt. Zur Auswertung fand hierzu jedoch die Gleichung(4) (S. 8) Verwendung; die Messungen wurden mit einem

64 Dies ergab eine exemplarische Rechnung für $\mathrm{CH}_{2} \mathrm{Cl}_{2}+\mathrm{F}$ bei $298 \mathrm{~K}$ mit v $=5750 \mathrm{~cm} / \mathrm{s}$ und $\mathrm{D}_{\mathrm{AB}}=317 \mathrm{~cm}^{2} / \mathrm{s}$. 
Überschuß an molekularem Sauerstoff, d.h. unter der Bedingung pseudo-erster Ordnung durchgeführt. Die Konzentration des molekularen Sauerstoffs wurde gemäß Gleichung (19) (S. 14) berechnet, wobei der Fluß durch einen geeichten Flußmesser bestimmt worden war.

\subsubsection{Verwendung des Strömungsreaktors mit Ofenheizung}

In dem in Kapitel 3.1.2 (S. 26) beschriebenen Strömungsreaktor mit Ofenheizung wurde die thermische Aktivierung des 1-Ethinyl-1-methylcyclopropans durchgeführt. Die Zuführung der Substanz fand über einen Sättiger (Abbildung 16, S. 48) statt (vgl. Kap. 3.3, S. 47), wobei sie stark mit Helium verdünnt wurde $([\mathrm{He}] \approx 90-95$ Vol-\%). Über das Ventil zur Pumpe konnte der gewünschte Druck im Strömungsreaktor eingestellt werden. Die Konzentrationen ergaben sich aus Gleichung (19) (S. 14). Die Reaktionszeiten wurden gemäß Gleichung (18) (S. 13) für $\mathrm{s}=200 \mathrm{~mm}$ berechnet. Soweit Temperatur, Aktivierungsenergie und Vorfaktor bekannt waren, konnte aus Gleichung (11) (S. 10) der Geschwindigkeitskoeffizient k berechnet werden. Mit diesem und mit der Reaktionszeit $\mathrm{t}$ konnte für die Isomerisierungsreaktion der Umsatz berechnet werden. Für eine solche Reaktion vom Typ KW $\rightarrow$ Produkte gilt folgendes Zeitgesetz:

$$
\frac{\mathrm{d}[\mathrm{KW}]}{\mathrm{dt}}=-\mathrm{k} \cdot[\mathrm{KW}]
$$

Durch Lösung dieser Differentialgleichung erhält man den Bruchteil, auf den die Konzentration des Edukts KW nach Ablauf der Reaktion (d.h. am Ende des Reaktorofens) abgesunken ist:

$$
\frac{[\mathrm{KW}]_{\mathrm{t}_{\text {ende }}}}{[\mathrm{KW}]_{\mathrm{t}_{0}}}=\exp \left\{-\mathrm{k} \cdot\left(\mathrm{t}_{\text {ende }}-\mathrm{t}_{0}\right)\right\}
$$

Umgekehrt kann man nach der Messung dieses Bruchteils in einem Experiment mit Hilfe der Gleichung (25) in Verbindung mit der Gleichung (11) (S. 10) auch eine grobe Abschätzung der (Arrhenius-)Aktivierungsenergie und des Vorfaktors vornehmen.

Unter Fluß von Helium wurde zunächst die Stabilisierung der gewünschten Ofentemperatur abgewartet, dann das 1-Ethinyl-1-methylcyclopropan zugegeben und anschließend erst die Produkte in der Glaskühlfalle bei $77 \mathrm{~K}$ ausgefrorenen. Danach konnte die Kühlfalle an die evakuierte Multireflektionskugelzelle des FT-IR-Spektrometers angeschlossen und dort ganz langsam bis auf $25^{\circ} \mathrm{C}$ erwärmt werden. Weil mit dem FT-IR-Gerät in schneller Folge Spektren aufgenommen werden konnten, war so eine gewisse Stofftrennung (eine Art „fraktionierende Destillation“) mit anschließender IR-Charakterisierung möglich. Durch das Programm „Opus“ konnten Spektren erstellt werden, die nur die jeweils neu hinzugekommenen Banden zeigten. 
Alternativ wurde die ausgefrorene Produktmischung mit der GC-MS-Kopplung analysiert.

\subsubsection{Messungen mit der GC-MS-Kopplung}

Die GC-MS-Apparatur wurde bereits oben in Kap. 3.1.3 (S. 27) eingehend beschrieben; dort ist auch die verwendete Kapillarsäule und das Trägergas, etc. angegeben.

Für alle in dieser Arbeit durchgeführten qualitativen Analysen wurden die Substanzen als Gasmischung in den Gaschromatographen injiziert, d.h bei flüssigen Substanzmischungen wurde die auf Grund ihres Dampfdrucks über ihr befindliche Gasphase verwendet.

Die EI-Elektronenenergie betrug stets $70 \mathrm{eV}$, um den Vergleich mit Literaturmassenspektren zu ermöglichen.

\subsubsection{Messungen mit dem REMPI-Flugzeitmassenspektrometer}

\subsubsection{Allgemeines}

Durch das REMPI-Ionisierungsverfahren können Radikale ionisiert und dann im Flugzeitmassenspektrometer nachgewiesen werden. Oft werden selektiv sogar nur Radikale ionisiert und der Radikalvorläufer-Kohlenwasserstoff erscheint nicht im Massenspektrum.

Alle untersuchten Reaktionen wurden in einem isothermen Strömungssystem durchgeführt. In diesem lag eine laminare Strömung mit konstanter Strömungsgeschwindigkeit vor (vgl. dazu Kapitel 2.3, S.13). Eine genaue Beschreibung der Apparatur findet sich in Kap. 3.1.4 (S. 33).

Das Ventil zwischen der Pumpe DK 100 und dem Strömungsreaktor war während der Messungen stets vollständig geöffnet, um einen möglichst geringen Druck zu erreichen; dies war wichtig, um Folgereaktionen gering zu halten.

Alle untersuchten Radikale wurden durch Zugabe von Fluor-Atomen (= erster zugegebener Reaktant) zu den Kohlenwasserstoffen ${ }^{65}$ erzeugt:

$$
\mathrm{R}-\mathrm{H}+\mathrm{F} \cdot \rightarrow \mathrm{R} \cdot+\mathrm{HF} \quad ; \text { mit R-H: Kohlenwasserstoff }
$$

Die Gasflüsse wurden in der Regel so eingestellt (vgl. dazu Kap. 3.3, S. 47), daß der Umsatz des Kohlenwasserstoffs bei 10-30 \% lag.

Die Energie des LASERs war so niedrig gewählt, daß keine Fragmente des VorläuferKohlenwasserstoffs auf der m/z-Zahl des erwarteten Radikals zu messen waren.

Die Umrechnung der am Oszilloskop gemessenen Flugzeiten in die m/z-Zahlen erfolgte durch

65 In den Begriff Kohlenwasserstoff sind hier auch die teilhalogenierten Kohlenwasserstoffe, Ether und Alkohole mit einbezogen. 
Vermessung von Substanzen mit bekanntem REMPI-Massenspektrum und Extra- bzw. Interpolation mittels des Zusammenhangs: $\mathrm{t} \propto \mathrm{m}^{1 / 2}$.

\subsubsection{Aufnahmen mit dem automatischen Wellenlängendurchlauf}

Mit dem automatischen Wellenlängendurchlauf konnte auf bequeme Art und Weise festgestellt werden, bei welchen Wellenlängen des Farbstoff-LASERs das Signal des erzeugten Radikals maximal wurde, bzw. ob überhaupt ein REMPI-Signal existiert.

Das prinzipielle Verfahren ist bereits oben in Kap. 3.1.4 (S. 33) dargestellt worden.

Dem Computerprogramm wurden eine oder zwei Flugzeiten (und damit m/z-Zahlen) angegeben, für die ein Radikal-Signal erwartet wurde. Ausgehend von diesen Flugzeiten sucht das Programm nun in dem registrierten Massenspektrum in einem zuvor festgelegten kleinen Zeitintervall nach dem Signal-Maximum und speichert dieses zusammen mit der erfaßten Energie des Farbstoff-LASERs. Diese Prozedur wird für verschiedene Wellenlängen wiederholt, wobei der Computer automatisch die Wellenlänge in einer vom Benutzer festgelegten Schrittweite ändert.

\subsubsection{Ermittlung der Geschwindigkeitskoeffizienten nach der Relativmethode}

Zur Bestimmung der Geschwindigkeitskoeffizienten wurde ausschließlich die Relativmethode gemäß Kapitel 2.1.1.2 (S. 10) eingesetzt.

Dabei wurde jeweils die Reaktion unter gleichen Bedingungen mit und ohne Umsatz untersucht. Die Reaktionsstrecke blieb konstant. Für die Reaktion der Radikale mit einem weiteren Reaktanten wurde die Änderung der Intensität des Radikales gemessen und die Konzentration des zweiten Reaktanten variiert, während die Fluor-Atom-Konzentration konstant blieb. Für die Auswertung wurden überwiegend die Peak-Integrale verwendet, die sich mit einer Integrationsgrenze von $10 \%$ des Peak-Maximums ergaben. Diese sind fast ausschließlich aus Mittelwertspektren berechnet worden, die aus 600 Einzelspektren gebildet wurden. Die genauen Versuchsbedingungen sind jeweils bei den Meßwerttabellen vermerkt.

Für die Ermittlung des Geschwindigkeitskoeffizienten der Reaktion eines Radikals mit einem anderen Reaktanten wurde praktisch so vorgegangen, daß der $\mathrm{zu}$ untersuchende Radikalvorläufer-Kohlenwasserstoff und die Radikalvorläufer-Referenzsubstanz sowie die zur Radikalerzeugung notwendigen Fluor-Atome gleichzeitig unter großer Verdünnung mit Helium ([He] > 90 Vol-\%) in das Strömungssystem geleitet wurden. Dem SteuerungsProgramm wurden nun die Flugzeiten für das zu untersuchende und das Referenz-Radikal angegeben sowie ein kleines Zeitintervall, in dem es jeweils automatisch das Maximum des 
Signals suchte. Nun erfolgte unter Anwesenheit des anderen Reaktanten die Messung der Signal-Intensität, dann wurde der Zufluß des anderen Reaktanten mittels Magnetventil abgeschaltet und die Messung in gleicher Weise wiederholt. Die zwischen der Abschaltung des anderen Reaktanten und dem Beginn der (zweiten) Zählung liegende Pause wurde so gewählt, daß zum Start der Zählung ein konstantes Signal vorlag.

Aus den so erhaltenen vier Meßwerten konnte nun ein Wert für die doppelt-logarithmische Auftragung gemäß Gleichung (10) (S. 10) berechnet werden (vgl. Kapitel 2.1.1.2, S. 10). Dabei wurde für die Konzentration zur Zeit $\mathrm{t}^{\prime}=0$ die Signalintensität ohne Zugabe des anderen Reaktanten und für die Konzentration zur Zeit $\mathrm{t}^{\prime}=\mathrm{t}$ diejenige mit Zugabe des anderen Reaktanten eingesetzt.

Die gesamte soeben beschriebene Prozedur wiederholte sich bei verschiedenen Konzentrationen des anderen Reaktanten.

Die Steigung der doppelt-logarithmischen Auftragung wurde mittels linearer Regression (Methode der kleinsten Quadratsumme) berechnet.

\subsubsection{Messungen mit dem FT-IR-Spektrometer}

Eine ausführliche Beschreibung der gesamten Apparatur findet sich in Kap. 3.1.5 (S. 42).

Die Reaktanten wurden als Gase mit Hilfe von Magnetventilen, die vom Programm „FFCP“ gesteuert wurden, in die Reaktionszelle eingefüllt, wobei der Zufluß ggf. durch ein Dosierventil (HOKE, Mikro-Mite) vor dem Magnetventil vermindert wurde.

Die Radikalerzeugung durch den Photolyse-LASER bei $193 \mathrm{~nm}$ konnte auf dreierlei Art erfolgen:

1. Durch Photolyse von Iod-Kohlenwasserstoffen gemäß R-I + hv $\rightarrow$ R + + I

2. Durch Photolyse von $\mathrm{CFCl}_{3}$ gemäß $\mathrm{CFCl}_{3}+\mathrm{hv} \rightarrow \mathrm{CFCl}_{2} \cdot \mathrm{Cl}$.

und anschließende Reaktion des Cl-Atoms mit dem Radikalvorläufer-Kohlenwasserstoff gemäß $\mathrm{R}-\mathrm{H}+\mathrm{Cl} \cdot \rightarrow \mathrm{R} \cdot+\mathrm{HCl}$

3. Durch Photolyse von Ketonen gemäß $\mathrm{RC}(=\mathrm{O}) \mathrm{R}+\mathrm{h} v \rightarrow 2 \mathrm{R} \cdot+\mathrm{CO}$

In der vorliegenden Arbeit wurden lediglich die ersten beiden Möglichkeiten genutzt.

In die Zelle wurde der gewünschte Radikalvorläufer-Kohlenwasserstoff sowie der zweite Reaktant bzw. dessen Vorläufer eingefüllt. Für die Untersuchung der Reaktion eines Radikals 
mit molekularem Sauerstoff konnte letzterer direkt eingefüllt werden, weil er auf Grund des sehr geringen Absorptionskoeffizienten $\left(0,21 / \mathrm{mol}^{*} \mathrm{~cm}\right)$ in kaum meßbarer Weise photolytisch in atomaren Sauerstoff gespalten wurde ${ }^{66}$. Die Untersuchung der Reaktion eines Radikals mit atomarem Sauerstoff wurde durch Zugabe von $\mathrm{SO}_{2}$ zur Reaktionsmischung und anschließende Cophotolyse gemäß $\mathrm{SO}_{2}+\mathrm{h} v \rightarrow \mathrm{SO}+\mathrm{O}$ ermöglicht ${ }^{66}$.

Vor Beginn der Reaktion und nach einer bestimmten Anzahl von Pulsen des PhotolyseLASERs wurden IR-Spektren aufgenommen und die Veränderung der Banden ausgewertet. Die zur Auswertung herangezogenen FT-IR-Spektren wurden vom Programm „Opus“ zur Verbesserung des Signal/Rausch-Verhältnisses als Mittelwertspektren aus vielen (üblicherweise 20-100) Einzelspektren berechnet.

Für quantitative Auswertungen wurden die interessierenden Bereiche integriert.

Die zeitaufgelöste Messung zur Bestimmung des Geschwindigkeitskoeffizienten der Reaktion eines Radikals mit molekularem Sauerstoff wurde nach der Absolutmethode unter der Bedingung pseudo-erster Ordnung ausgeführt (siehe Kap. 2.1.1.1, S. 8). Für die Auswertung ist jedoch zu beachten, daß nicht die Abnahme des Radikals, sondern die Zunahme des PeroxyKomplexes zeitaufgelöst verfolgt wurde. Gleichung(4) (S. 8) läßt sich jedoch unter Einbeziehung der Stöchiometrie und der Massenbilanz auch hierfür heranziehen. Für die Elementarreaktion $\mathrm{A}+\mathrm{X} \rightarrow \mathrm{P}$ läßt sich Gleichung (4) umstellen zu

$$
[\mathrm{A}]_{\mathrm{t}}=[\mathrm{A}]_{0} \cdot \exp \left\{-\mathrm{k}_{\mathrm{a}}[\mathrm{X}] \mathrm{t}\right\} \quad ; \quad \text { für: }[\mathrm{X}] \gg[\mathrm{A}]
$$

Für $\mathrm{t}=0$ gilt: $[\mathrm{A}]=[\mathrm{A}]_{0}$ sowie $[\mathrm{P}]_{0}=0$.

Für $\mathrm{t}>0$ gilt: $[\mathrm{P}]_{\mathrm{t}}=[\mathrm{A}]_{0}-[\mathrm{A}]_{\mathrm{t}}$.

Für $\mathrm{t} \rightarrow \infty$ gilt: $[\mathrm{P}]_{\infty}=[\mathrm{A}]_{0}$

Damit folgt:

$$
\begin{aligned}
& {[\mathrm{P}]_{\mathrm{t}}=[\mathrm{A}]_{0}-[\mathrm{A}]_{\mathrm{t}}=[\mathrm{A}]_{0}-[\mathrm{A}]_{0} \cdot \exp \left\{-\mathrm{k}_{\mathrm{a}}[\mathrm{X}] \mathrm{t}\right\} } \\
\Rightarrow \quad[\mathrm{P}]_{\mathrm{t}}=[\mathrm{A}]_{0} \cdot\left(1-\exp \left\{-\mathrm{k}_{\mathrm{a}}[\mathrm{X}] \mathrm{t}\right\}\right) & \ln \left\{1-\frac{[\mathrm{P}]_{\mathrm{t}}}{[\mathrm{A}]_{0}}\right\}=-\mathrm{k}_{\mathrm{a}}[\mathrm{X}]_{\mathrm{t}} \Rightarrow \ln \left\{1-\frac{[\mathrm{P}]_{\mathrm{t}}}{[\mathrm{P}]_{\infty}}\right\}=-\mathrm{k}_{\mathrm{a}}[\mathrm{X}] \mathrm{t}
\end{aligned}
$$

66 Vgl. Tröger, U. (1997); S. 28. 
Aus der Steigung der $\Rightarrow \ln \left\{\frac{[\mathrm{P}]_{\infty}-[\mathrm{P}]_{\mathrm{t}}}{[\mathrm{P}]_{\infty}}\right\}=-\mathrm{k}_{\mathrm{a}}[\mathrm{X}] \mathrm{t}$

Seite von Gleichung (26) gegen die Reaktionszeit $\mathrm{t}$ läßt sich also bei bekannter Konzentration des Reaktanten X der Geschwindigkeitskoeffizient $\mathrm{k}_{\mathrm{a}}$ ermitteln.

Weil auf der linken Seite von Gleichung (26) lediglich das Verhältnis der Konzentrationen des Produktes P eingeht, ist es möglich, dort die Absorbanzen einzusetzen. Die Konzentrationen sind letzteren nämlich gemäß dem Lambert-Beer-Gesetz ${ }^{67}$ direkt proportional. Die Absorbanz (auch als Extinktion E bezeichnet) ist der dekadische Logarithmus des Kehrwerts der Transmission $\mathrm{T}\left(\mathrm{T}=\mathrm{I} / \mathrm{I}_{0}\right)$; letztere ergibt sich unmittelbar aus dem IR-Spektrum.

Für die Absorbanz E gilt also:

$$
\begin{aligned}
\mathrm{E}=\log _{10} \frac{\mathrm{I}_{0}}{\mathrm{I}}=\epsilon \cdot \mathrm{c} \cdot 1 \quad ; \quad & \text { mit I: Intensität, } \mathrm{I}_{0} \text { : Intensität vor Durchtritt durch die Probe, } \\
& \text { c: Konzentration, l: Länge der Absorptionsstrecke } \\
& \varepsilon: \text { molarer dekadischer Absorptionskoeffizient }
\end{aligned}
$$

Die Software „Opus“ des FT-IR-Geräts ermöglicht die automatische Umwandlung der gemessenen Transmissionsspektren in Absorptionsspektren und die anschließende Integration der interessierenden Banden.

Letztlich wurden in den Term auf der linken Seite von Gleichung (26) statt der Konzentrationen also die entsprechenden Integralwerte des Absorptionsspektrums eingesetzt. Mit dem gemessenen Druck bzw. Druckanstieg konnte die Konzentration einer eingefüllten Substanz über das ideale Gasgesetz gemäß $c=p /(R \cdot T)$ berechnet werden. Auf diese Art wurde die Konzentration des im Überschuß vorliegenden Reaktanten X berechnet.

67 Vgl. dazu: Wedler, G. (1987); S. 532 f. 


\section{Meßergebnisse und Diskussion}

\subsection{Untersuchung von 1-Ethinyl-1-methylcyclopropan}

\subsubsection{Reaktionen nach thermischer Aktivierung}

Wie in der Einleitung (Kap. 1.1, S. 1) bereits erwähnt wurde, bewirkt die thermische Aktivierung von Cyclopropan-Derivaten häufig eine Öffnung des dreigliedrigen Rings und sich daran anschließende Isomerisierungsreaktionen, die wahrscheinlich über eine diradikalische Zwischenstufe verlaufen.

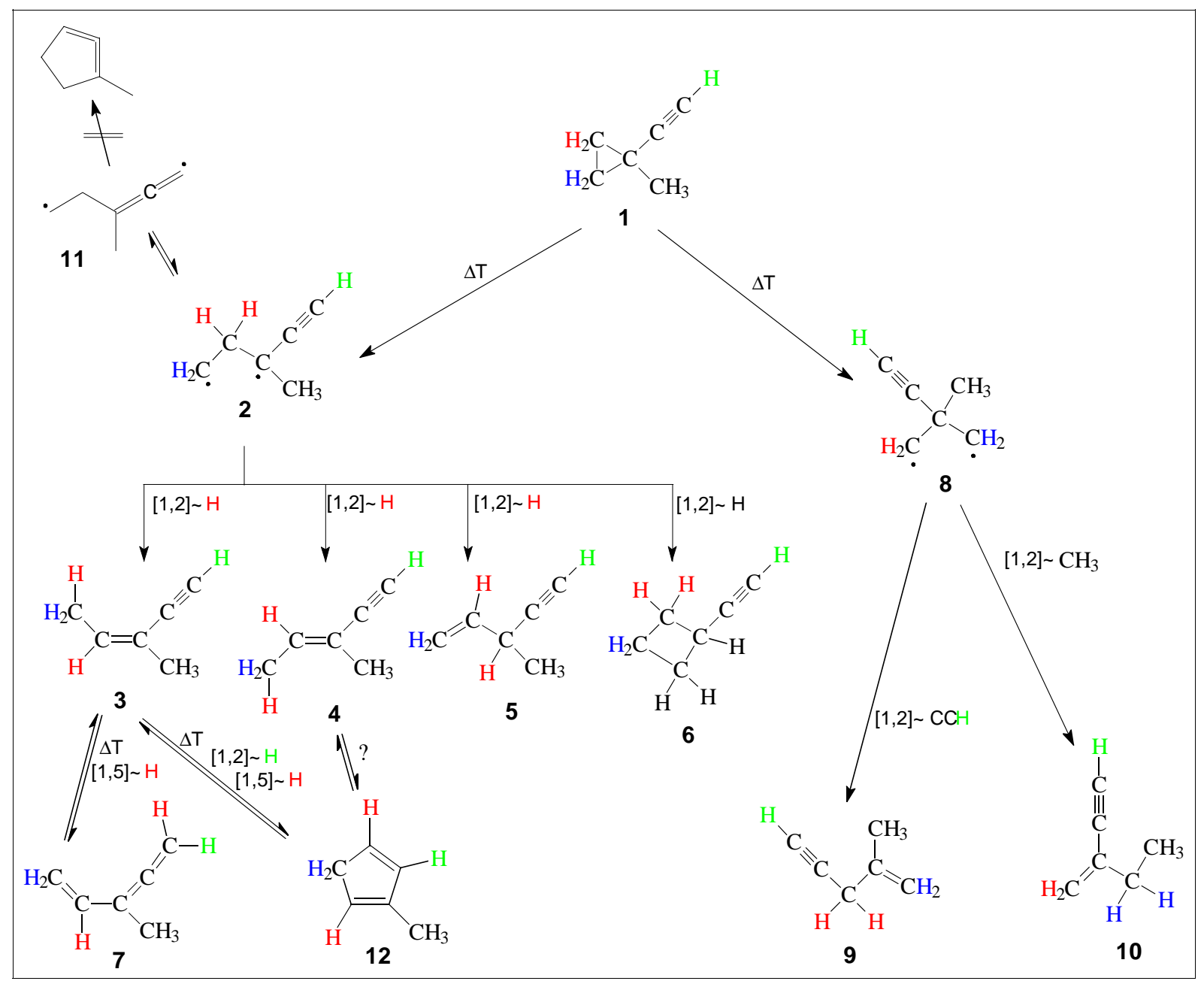

Abbildung 18: Denkbare Reaktionsprodukte nach thermischer Aktivierung

Basierend auf diesem Reaktionsmechanismus wären die in Abbildung 18 dargestellten Reaktionsprodukte bei der Pyrolyse von 1-Ethinyl-1-methylcyclopropan zu erwarten.

In der Experimentplanung wurde angestrebt, zunächst unter „milden“ Reaktionsbedingungen möglichst nur die Primärprodukte 3 bis 6 zu erzeugen. Dazu wurde einerseits eine hohe Verdünnung mit Helium (98 Vol-\% im Reaktor) gewählt, um Kombinationsprodukte zweier Diradikale $\mathbf{2}$ oder $\mathbf{8}$ zu verhindern; andererseits sollte eine möglichst niedrige Temperatur sowie 
eine kurze Aufenthaltszeit im Reaktor die Bildung von Folgeprodukten (z.B. 7) schmälern. Weil kinetische Daten für die Ringöffnung, d.h. die Reaktion von 1 zu 2, nicht vorlagen, wurde aus Experimenten bei höheren Temperaturen (siehe unten) eine Aktivierungsenergie von 189 $\mathrm{kJ} / \mathrm{mol}$ und ein Arrhenius-Vorfaktor von $10^{12} \mathrm{~s}^{-1}$ abgeschätzt ${ }^{68}$. Damit errechnet man für $40 \mathrm{~ms}$ Reaktionszeit und einer Temperatur von $583 \mathrm{~K}$ keinen signifikanten Umsatz, bei $683 \mathrm{~K}$ aber immerhin einen von etwa 1 Promille (berechnet mittels Gleichung (25), S. 56).

Erste Experimente wurden in der in Kap. 3.1.1 (S. 16) beschriebenen Apparatur bei $555 \mathrm{~K}$ und einem Druck von $15 \mathrm{hPa}$ mit einer Reaktionszeit von $50 \mathrm{~ms}$ durchgeführt. Erwartungsgemäß war unter diesen Bedingungen kein Umsatz bzw. keine Produktbildung meßbar.

Experimente bei $683 \mathrm{~K}$ und einem Druck von $10 \mathrm{hPa}$ (10 mbar) mit einer Reaktionszeit von 40 ms wurden in der in Kap. 3.1.1 (S. 16) beschriebenen Apparatur wiederholt, wobei sich in den Spektren Anzeichen für einen äußerst geringen Umsatz zeigten.
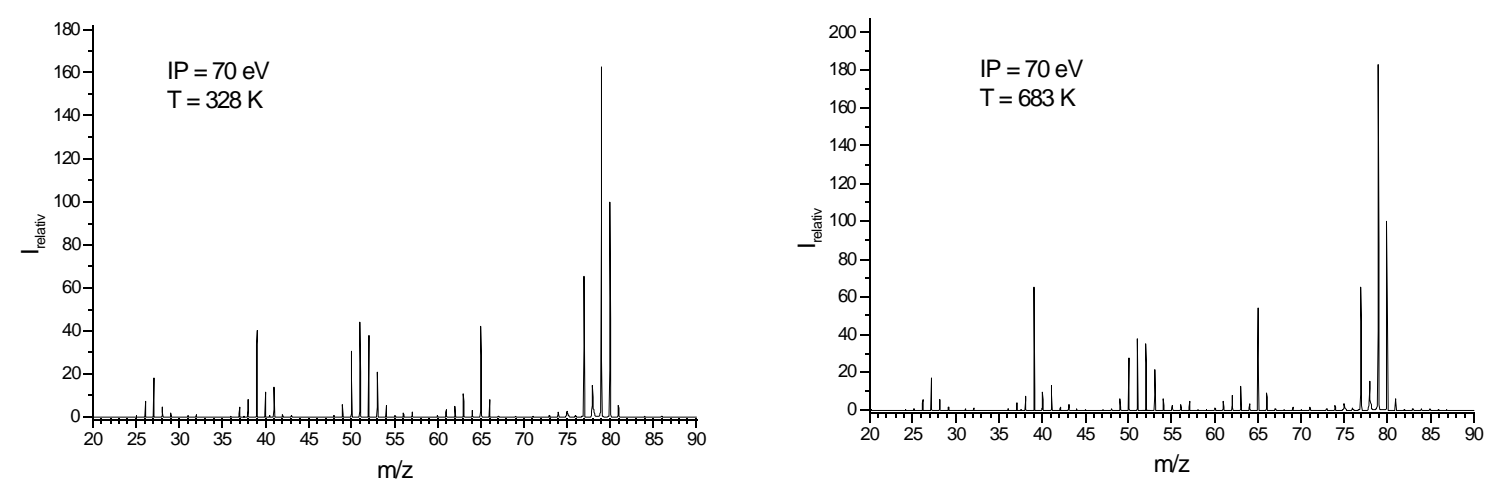

Abbildung 19: EI-Massenspektren vor und nach Pyrolyse

In den Massenspektren der Abbildung 19 lassen sich geringe Veränderungen der relativen Intensitäten erkennen; dieses Phänomen wurde auch bei einer niedrigeren EI-Elektronenenergie von $\mathrm{IP}=22 \mathrm{eV}$ beobachtet, bei der üblicherweise die Fragment-Ionen-Bildung herabgesetzt wird. Das Auftauchen neuer Peaks hingegen war nicht zu erwarten, da die potentiellen Reaktionsprodukte $\mathbf{3}$ bis $\mathbf{5}$ alle in etwa das gleiche Fragmentierungsmuster aufweisen dürften ${ }^{69}$. Die IR-Analyse zeigt in den Spektren mit einer Auflösung von $4 \mathrm{~cm}^{-1}$ eine Zunahme der Banden bei 756, 850, 902, 949, 1032, 1150, 1209, 1264, 1392, 1451, 1717, 2886 und 2940 $\mathrm{cm}^{-1}$ (vgl. unten Abbildung 20).

Die Interpretation wird allerdings dadurch erschwert, daß für die potentiellen

68 Vgl. dazu auch die tabellierten Werte für andere Cyclopropan-Derivate in: Hopf, H. et al. (1989); S. 380 sowie in: Pilling, M.; Seakins, P. (1995); S. 123.

69 So jedenfalls 3-Methyl-pent3-en-1-in; vgl. NIST (1990), Mass Spectral Database. 
Reaktionsprodukte 3 bis 7 weder Spektren aus der Literatur vorlagen, noch diese Substanzen (kommerziell) beschafft werden konnten; daher mußte auf Tabellen mit charakteristischen IRGruppenfrequenzen sowie auf Vergleichsspektren strukturell ähnlicher Verbindungen zurückgegriffen werden.

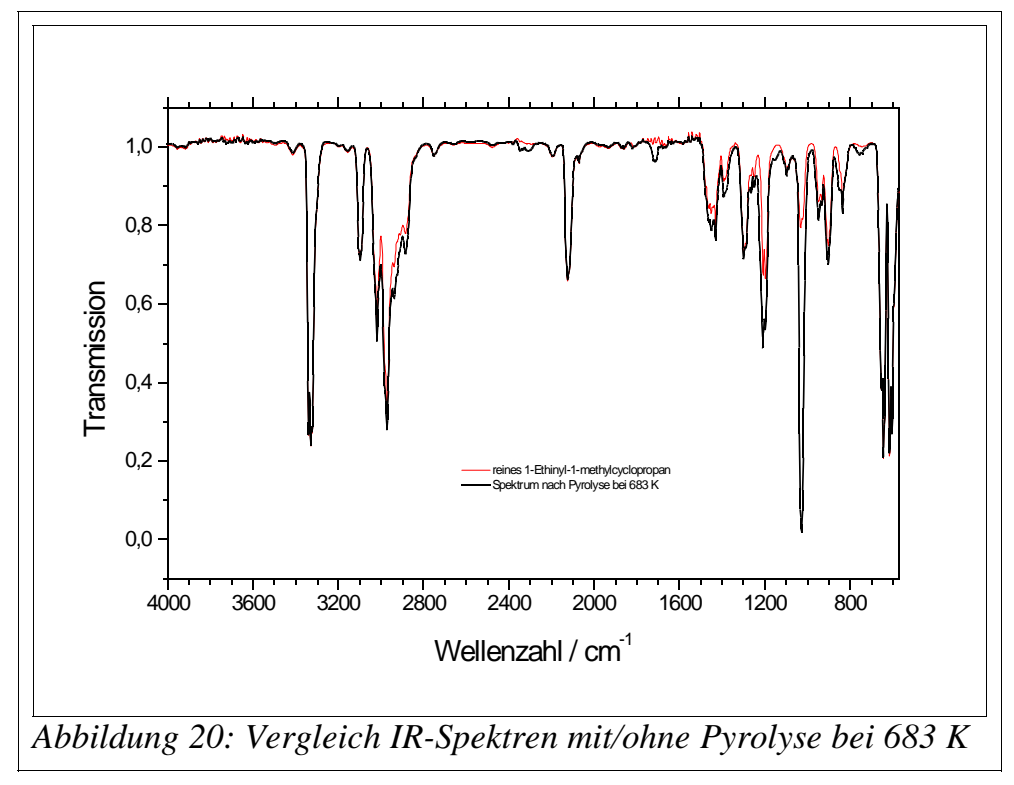

Ein hochaufgelöstes $\left(0,15 \mathrm{~cm}^{-1}\right)$ IR-Spektrum zeigt einige schwache neue Banden (vgl. Abbildung 21). Diejenigen bei $652 \mathrm{~cm}^{-1}$ und $1029 \mathrm{~cm}^{-1}$ sprechen für die Bildung von Verbindung 3 und/oder 4 (vgl. dazu das Spektrum ${ }^{70}$ des strukturell ähnlichen 3-Penten-1-in mit den Hauptbanden bei $656 \mathrm{~cm}^{-1}$ und $1024 \mathrm{~cm}^{-1}$ ). Die Banden bei $881 \mathrm{~cm}^{-1}$ und $1013 \mathrm{~cm}^{-1}$ sprechen für die Bildung von Verbindung 5 (vgl. dazu das Spektrum ${ }^{71}$ des strukturell ähnlichen 2-Methylbut-1-en-3-in mit den Banden bei $890 \mathrm{~cm}^{-1}$ und $1010 \mathrm{~cm}^{-1}$ ).
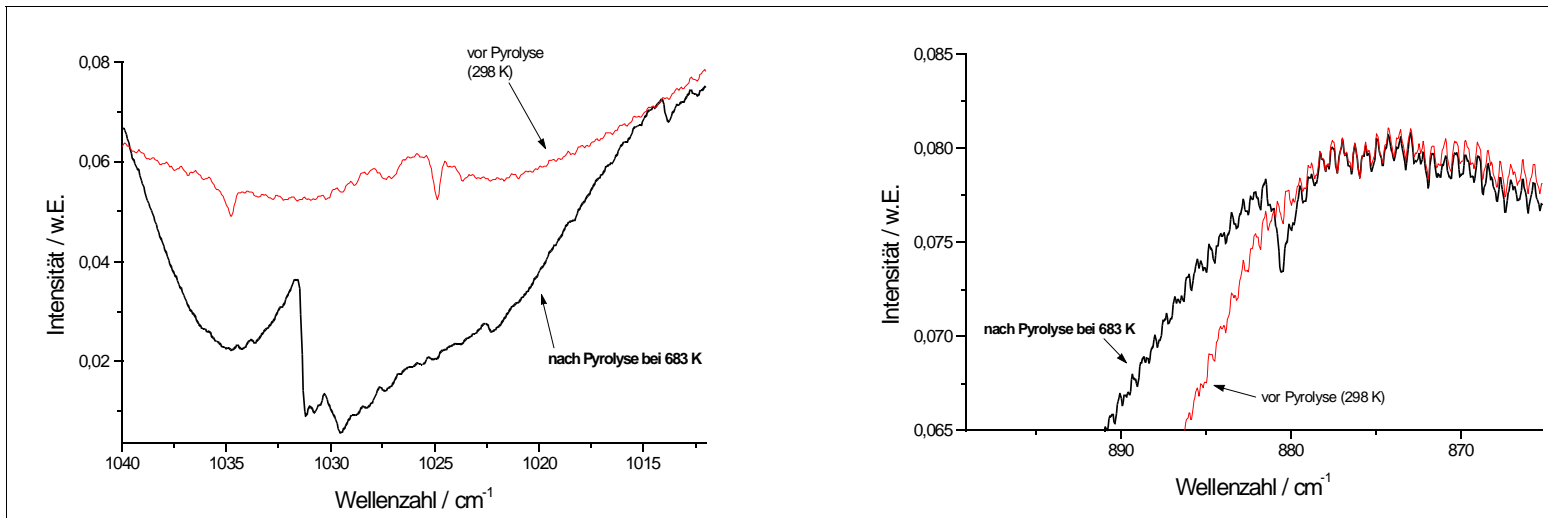

Abbildung 21: Ausschnitte aus hochaufgelöstem IR-Spektrum nach Pyrolyse bei $683 \mathrm{~K}$

Um die Folgerungen aus dem Vergleich mit den charakteristischen IR-Gruppenfrequenzen 70 NIST Chemistry WebBook (2001).

71 Pouchert, Ch. (1978). 
bzw. Vergleichsspektren ähnlicher Substanzen untermauern zu können, wurden zudem die IRSpektren einiger potentieller Isomerisierungsprodukte in Abbildung 18 (S. 62) mit dem Programmpaket „Gaussian98“ nach der Methode „B3LYP/6-31G*“ berechnet. Zuvor fand mit diesem Programm eine Geometrieoptimierung statt. Die so erhaltenen Wellenzahlen mußten jedoch zur Anpassung an experimentelle Werte noch mit einem Korrekturfaktor versehen werden. Durch Vergleich zwischen dem gemessenen (Abbildung 22) und dem berechneten IRSpektrum (Abbildung 23) des Edukts 1-Ethinyl-1-methylcyclopropan zeigte sich, daß ein Korrekturfaktor von 0,953 zu einer recht guten Anpassung führt. Unterhalb von $1500 \mathrm{~cm}^{-1}$ gibt es jedoch generell größere Abweichungen, so daß leider gerade in diesem wichtigen „Fingerprintbereich“ ein Vergleich mit den berechneten Spektren nur unter Vorbehalt möglich ist.

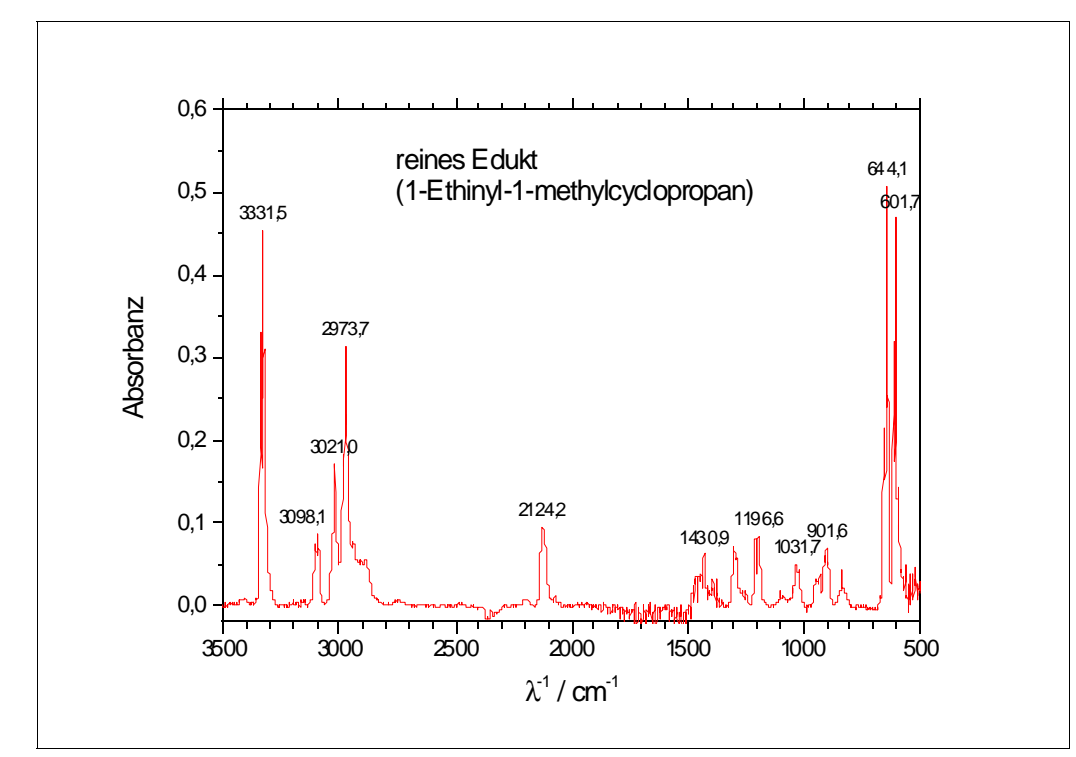

Abbildung 22: FT-IR-Spektrum von 1-Ethinyl-1-methylcyclopropan

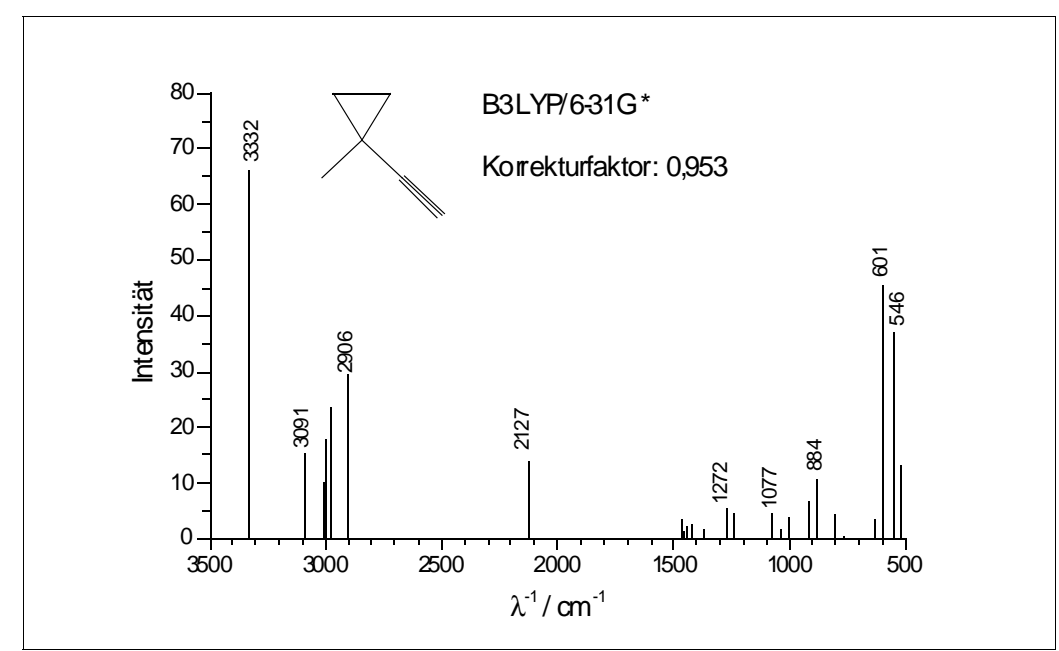

Abbildung 23: Berechnetes IR-Spektr. 1-Ethinyl-1-methylcyclopropans 
Auch die berechneten IR-Spektren der übrigen potentiellen Produkte wurden einheitlich mit dem Korrekturfaktor 0,953 angepaßt; die Übereinstimmung mit den experimentellen Werten läßt sich anhand der CH-Schwingung der Ethin-Gruppe kontrollieren, die nach der Skalierung stets zutreffend bei $3300 \mathrm{~cm}^{-1}$ liegt.

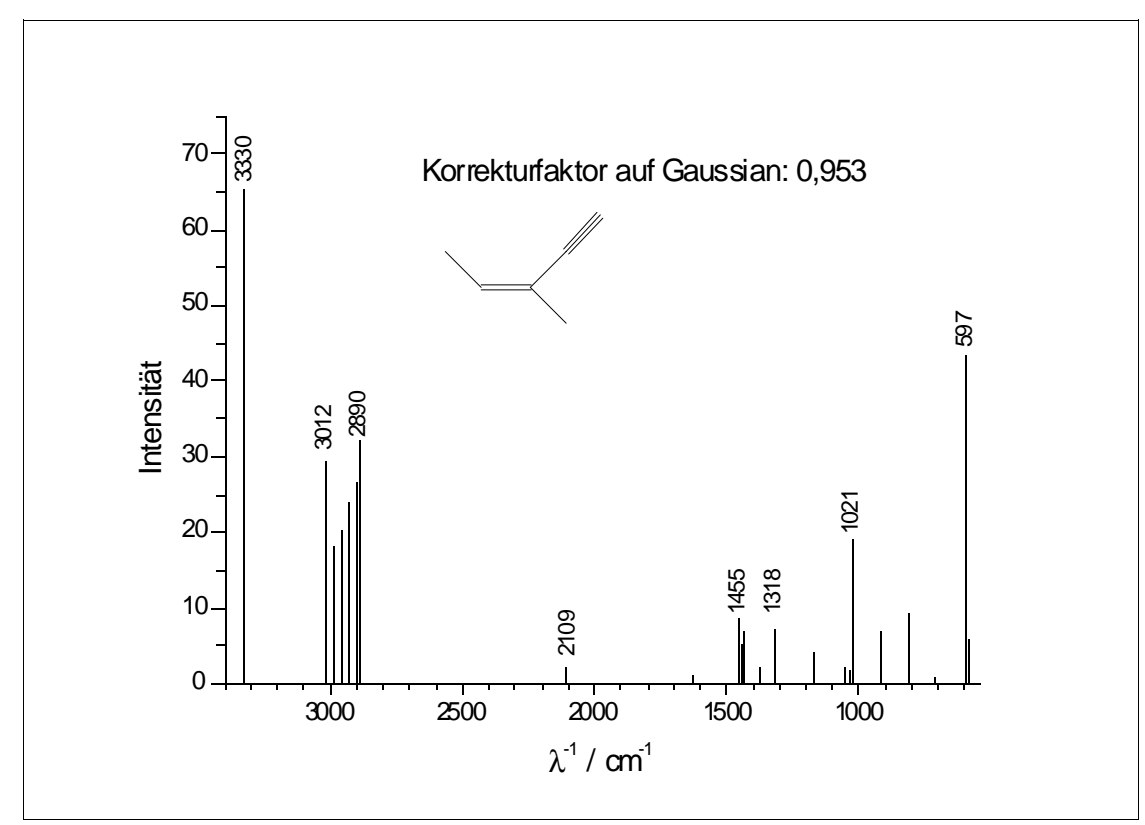

Abbildung 24: Berechnetes IR-Spektrum von (Z)-3-Methyl-pent-3-en-1-in

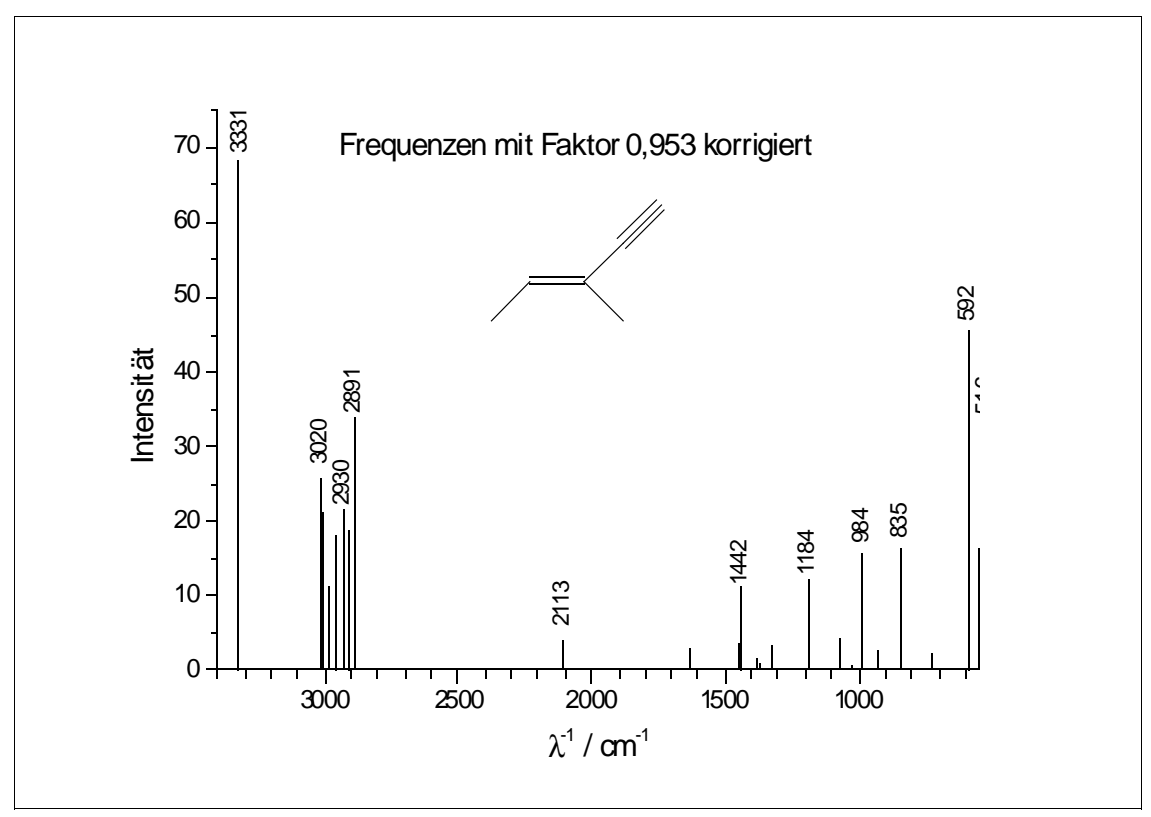

Abbildung 25: Berechnetes IR-Spektrum von (E)-3-Methyl-pent-3-en-1-in 


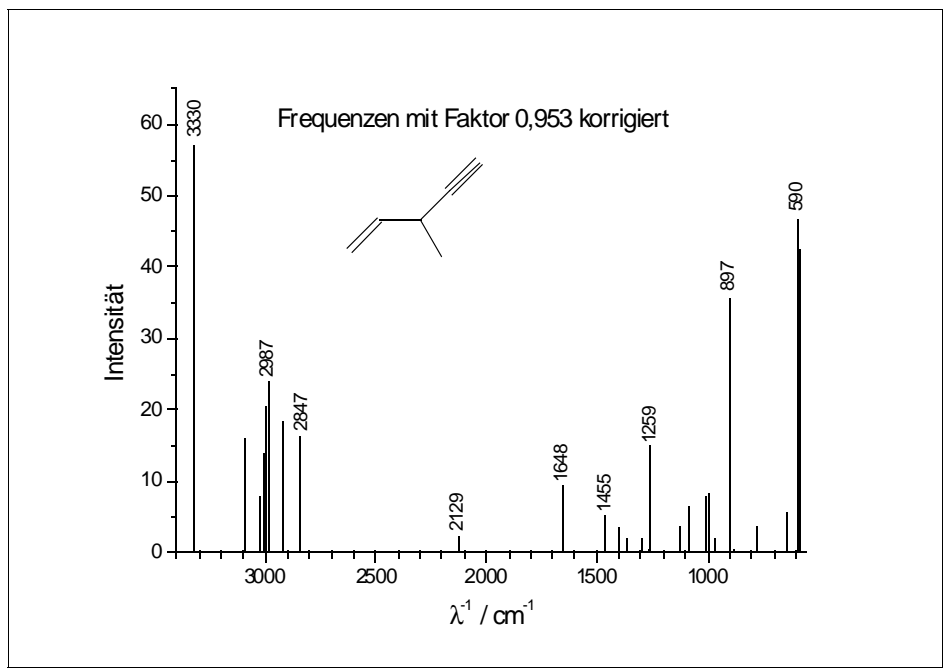

Abbildung 26: Berechn. IR-Spekt. von 3-Methyl-pent-4-en-1-in

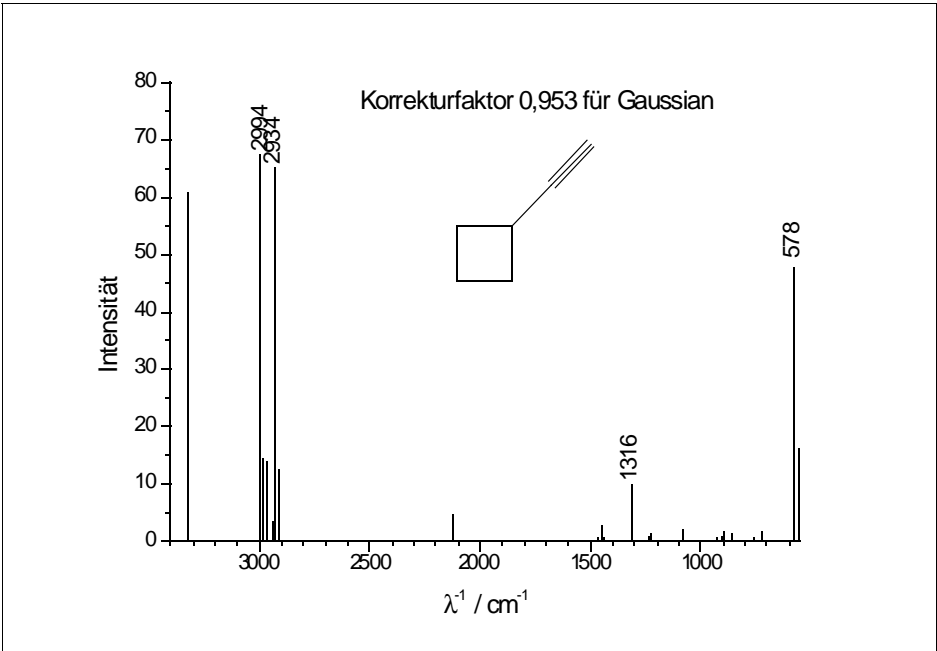

Abbildung 27: Berechn. IR-Spekt. von Ethinylcyclobutan

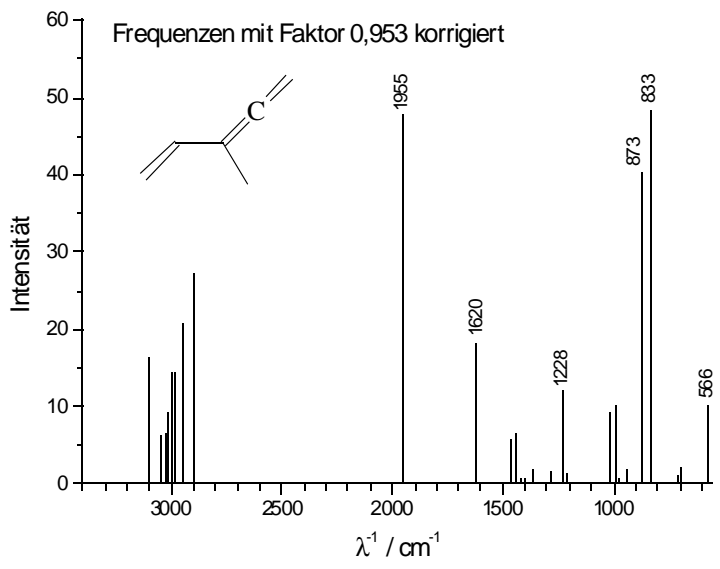

Abbildung 28: Berechn. IR-Spekt. von 3-Methylpent-1,2,4-trien 


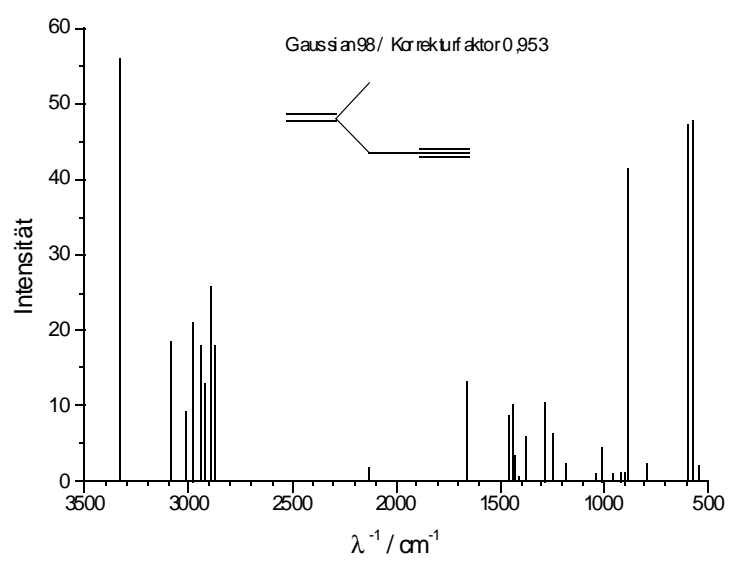

Abbildung 29: Berechn. IR-Spekt. von 2-Methylpent-1-en-4-in

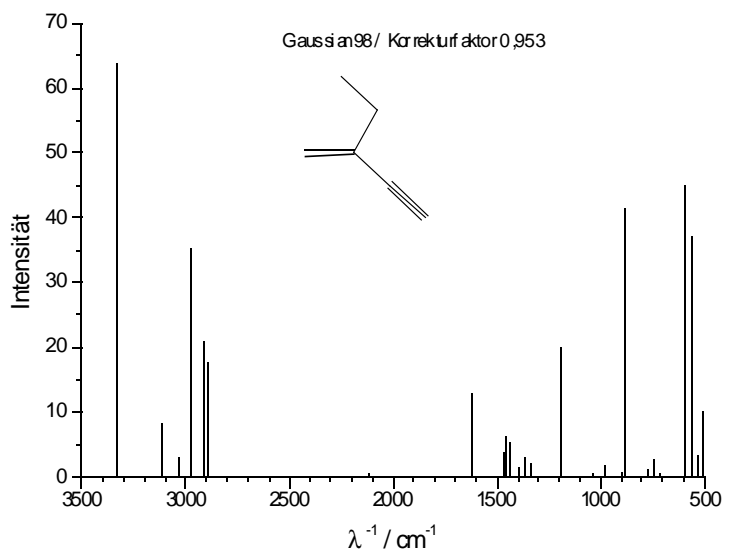

Abbildung 30: Berechnetes IR-Spekt. von Verbindung 10

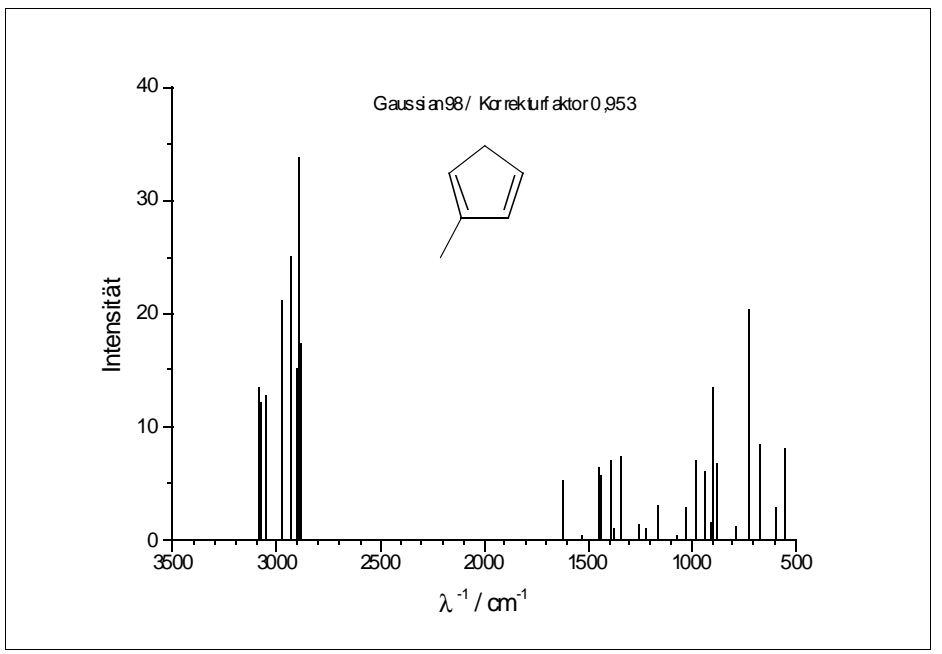

Abbildung 31: Berechnetes IR-Spekt. von Verbindung 12

Der Vergleich des berechneten IR-Spektrums von 1-Methyl-1,4-cyclopentadien (Verbindung 
12 der Abbildung 18), das oben in Abbildung 31 abgebildet ist, mit dem tatsächlich gemessenen Spektrum von 1,3-Cyclopentadien läßt jedoch Zweifel an der Vollständigkeit des berechneten Spektrums aufkommen, daher wurde auch das IR-Spektrum von 1,3Cyclopentadien berechnet.

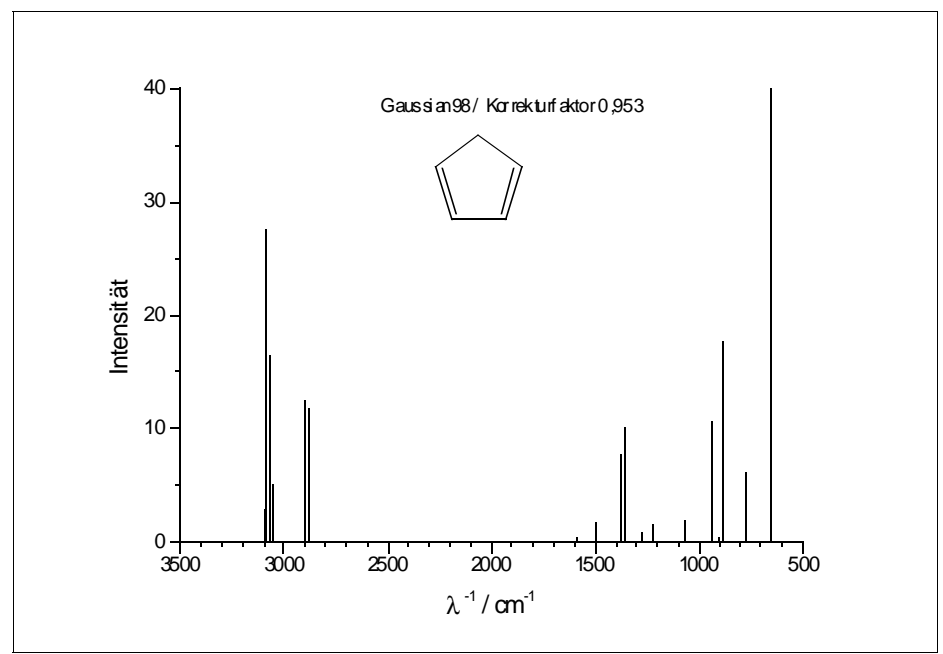

Abbildung 32: Berechnetes IR-Spekt. von 1,3-Cyclopentadien

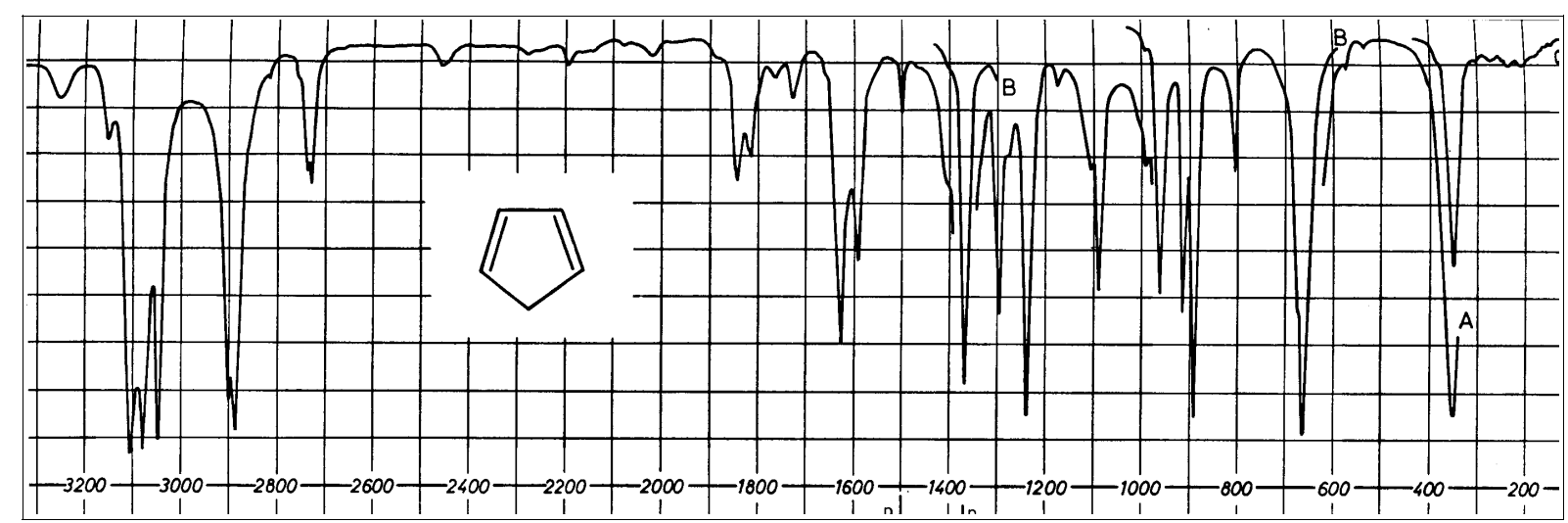
Abbildung 33: Tatsächlich gemessenes IR-Spektrum von 1,3-Cyclopentadien [Quelle: Schrader, B. (1995)]

Der direkte Vergleich von Abbildung 32 mit Abbildung 33 macht deutlich, daß in dem berechneten IR-Spektrum die Banden bei 1730, 1810, 1850 und $2750 \mathrm{~cm}^{-1}$ vollständig fehlen. Es ist daher wahrscheinlich, daß diese Banden in dem berechneten Spektrum in Abbildung 31 des strukturell sehr ähnlichen 1-Methyl-1,4-cyclopentadien (Verbindung 12 der Abbildung 18) ebenfalls fehlen.

Die weiteren Experimente zur thermischen Aktivierung wurden nunmehr in der in Kap. 3.1.2 (S. 26) beschriebenen Apparatur ausgeführt, um bei höheren Temperaturen einen höheren Umsatz erzielen zu können. Die Ausführung und Auswertung dieser Messungen ist in Kap. 3.5.2 (S. 56) beschrieben. 
Das erste Experiment in dieser Apparatur wurde bei $805 \mathrm{~K}\left(532{ }^{\circ} \mathrm{C}\right)$ realisiert. Im Strömungsreaktor herrschte dabei ein Druck von 33 mbar. Der gesamte Fluß betrug 219 SCCM. Daraus ließ sich eine Reaktionszeit von $191 \mathrm{~ms}$ berechnen. Unter diesen Bedingungen konnte anhand einer FT-IR-Analyse kein nennenswerter Umsatz festgestellt werden.

Daher wurde das Experiment bei $853 \mathrm{~K}\left(580^{\circ} \mathrm{C}\right)$ wiederholt. Im Strömungsreaktor herrschte hierbei ein Druck von 23 mbar. Der Fluß an Helium betrug 91,3 SCCM, derjenige an 1Ethinyl-1-methylcyclopropan 3,5 SCCM. Daraus ließ sich eine Konzentration in Höhe von $1,2 \cdot 10^{-8} \mathrm{~mol} / \mathrm{cm}^{3}$ sowie eine Reaktionszeit von $287 \mathrm{~ms}$ berechnen. Es zeigte sich in der FT-IRAnalyse (vgl. dazu Kap. 3.1.5, S. 42 sowie Kap. 3.5.5, S. 59), daß dabei ein größerer Teil des Edukts umgesetzt worden sein mußte. Abbildung 34 zeigt den direkten Vergleich der FT-IRSpektren des Edukts 1-Ethinyl-1-methylcyclopropan und der Substanz-Mischung nach thermischer Aktivierung bei $853 \mathrm{~K}$.

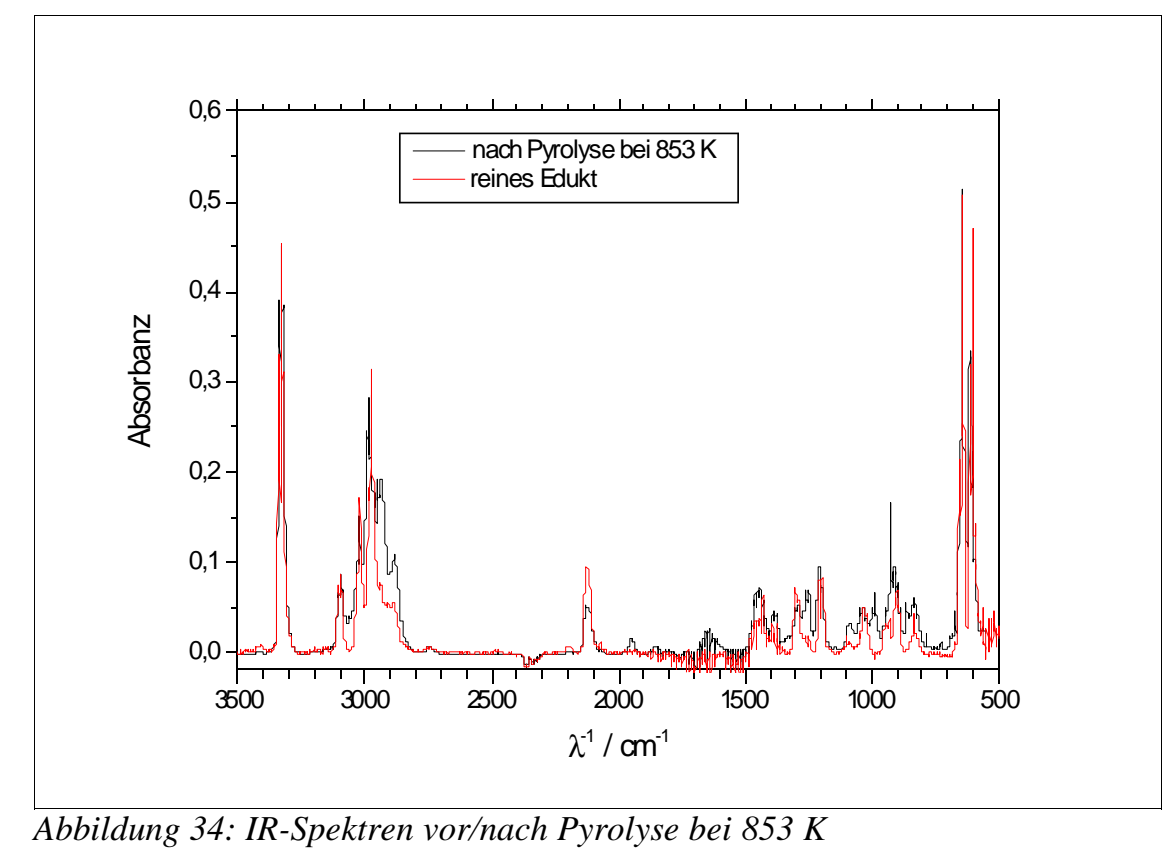

Die Abnahme des Edukts erkennt man in Abbildung 34 anhand der Bande bei $2128 \mathrm{~cm}^{-1}$, wobei letztere nicht sehr charakteristisch sein dürfte, weil sich aus den berechneten Spektren ergibt, daß auch einige potentielle Produkte hier eine schwache Bande zeigen (vgl. oben die Abbildungen 24 bis 28). Quantitative Auswertungen kommen somit nicht in Betracht.

Die im Kap. 3.5.5 (S. 59) beschriebene „fraktionierende Destillation“ durch langsames Erwärmen der Kühlfalle wurde zwar durchgeführt, brachte aber keine brauchbare Trennung, so daß die Auswertung mit dem IR-Spektrum der gesamten ausgefrorenen Substanz-Mischung vorgenommen werden soll. 
Insgesamt zeigt die Produktmischung in Abbildung 34 Banden bei $^{72} 568$ (w), 597 (w), 605, 610, 617, 669 (w), 674, 729, 734 (w), 787, 808 (w), 821, 833, 837, 842, 845 (w), 857 (w), 863, 898, 915, 927, 950, 987, 995, 1020, 1022, 1035, 1037, 1049, 1070, 1090, 1200, 1207, 1251, 1263, 1287, 1302, 1327 (w), 1378, 1442, 1450, 1458, 1461, 1466, 1475, 1478, 1633, 1642, 1650, 1842, 1850, 1950, 2128, 2882, 2890 (w), 2937, 2950, 2962, 2974, 2977, 2983, $2990,3020,3030,3093,3332 \mathrm{~cm}^{-1}$.

Für die Bildung von Verbindung 3 der Abbildung 18 (S. 62) sprechen die Banden bei 597, 808, 915, 1022, 1302, 1378, 1450, 1458, 1633, 2128, 2890 und $3332 \mathrm{~cm}^{-1}$. Dies zeigt ein Vergleich mit dem berechneten Spektrum der Abbildung 24 (S. 66). Die Bande bei $808 \mathrm{~cm}^{-1}$ rührt von der C-H -Schwingung aus $\mathrm{R}_{2} \mathrm{C}=\mathrm{CRH}$ her; weil diese üblicherweise stark ${ }^{73}$, im gemessenen Spektrum aber nur sehr schwach ist, dürfte Verbindung 3 bei dieser Temperatur (noch) kein Hauptprodukt sein. Die Banden bei 1378, 1450 und $1458 \mathrm{~cm}^{-1}$ können den Methylgruppen zugeordnet werden. Diejenige bei $1633 \mathrm{~cm}^{-1}$ resultiert aus der $\mathrm{C}=\mathrm{C}$-Bindung. Die $\mathrm{C} \equiv \mathrm{C}$ -Bindung bewirkt die Bande bei 2128, die $\mathrm{CH}$-Schwingung in $\mathrm{C} \equiv \mathrm{C}-\mathrm{H}$ diejenige bei 1302 und $3332 \mathrm{~cm}^{-1}$.

Für die Entstehung von Verbindung 4 der Abbildung 18 (S. 62) sprechen die Banden bei 597, 837, 987, 1442, 2128, 2890 und $3332 \mathrm{~cm}^{-1}$. Dies zeigt ein Vergleich mit dem berechneten Spektrum der Abbildung 25 (S. 66). Problematisch ist, daß die Banden bei 592 und $1184 \mathrm{~cm}^{-1}$, die als starke Banden berechnet wurden, im gemessenen Spektrum praktisch nicht auffindbar sind. Bezüglich derjenigen bei $592 \mathrm{~cm}^{-1}$ könnte die in diesem Bereich nur noch geringe Empfindlichkeit des Detektors verantwortlich sein, denn lt. Hersteller ist dieser erst oberhalb $600 \mathrm{~cm}^{-1}$ einsetzbar. Die Bande bei $1184 \mathrm{~cm}^{-1}$ wurde möglicherweise fehlerhaft berechnet, zumal in den Spektren des strukturell ähnlichen 2-Methylbut-1-en-3-in ${ }^{74}$ und des trans-3Penten-1-in ${ }^{75}$ keinerlei Banden in diesem Bereich vorkommen.

Auch Verbindung 5 der Abbildung 18 (S. 62) dürfte nach der thermischen Aktivierung bei 853 K gebildet worden sein. Hierfür sprechen die Banden bei 617, 787, 898, 987, 995, 1020, 1090, 1263, 1458, 1650, 2128, 2990, 3093 und $3332 \mathrm{~cm}^{-1}$. Dies zeigt ein Vergleich mit dem berechneten Spektrum der Abbildung 26 (S. 67). Die Bande bei 898 in Verbindung mit der bei $987 \mathrm{~cm}^{-1}$ ist charakteristisch ${ }^{76}$ für R-CH= $\mathrm{CH}_{2}$. Diese Struktureinheit wird ferner durch Banden

72 Zur Kennzeichnung der Intensitäten der IR-Banden wird die Abkürzung „,(s)“ für starke, (m) für mittelstarke und ,(w)“ für wenig intensive Banden verwendet.

73 Lide, D. (1991); Abschnitt 9 S. 126.

74 Pouchert, Ch. (1978).

75 NIST Chemistry WebBook (2001).

76 Hesse, M.; Meier, H.; Zeeh, B. (1995); S. 45 Tabelle 2.3. 
bei 1458, 1650 und $1850 \mathrm{~cm}^{-1}$ bestätigt ${ }^{80}$. Charakteristisch für Verbindung $\mathbf{5}$ ist auch diejenige bei $2882 \mathrm{~cm}^{-1}$, die aus der C-H -Schwingung der Struktureinheit $\mathrm{R}_{3} \mathrm{C}-\mathrm{H}$ herrührt ${ }^{77}$.

Für die Bildung von Verbindung 6 der Abbildung 18 (S. 62), deren berechnetes IR-Spektrum in Abbildung 27 (S. 67) zu sehen ist, könnte allenfalls die Bande bei $1327 \mathrm{~cm}^{-1}$ im gemessenen Spektrum sprechen. Allerdings enthält das berechnete Spektrum keine Banden, die nicht auch in den Spektren der anderen potentiellen Produkte auftreten, so daß eine sichere Identifizierung mittels der IR-Spektroskopie sehr schwer bzw. unmöglich ist.

Sehr wohl dürfte jedoch Verbindung 7 der Abbildung 18 (S. 62) gebildet worden sein, was sich aus den Banden bei 568, 833, 863, 995, 1020, 1442, 1466, 1633, 1950, 2990 und $3093 \mathrm{~cm}^{-1}$ durch Vergleich mit dem berechneten Spektrum in Abbildung 28 (S. 67) ergibt. Besonders charakteristisch ist die Bande bei $1950 \mathrm{~cm}^{-1}$, die aus der $\mathrm{C}=\mathrm{C}=\mathrm{C}$-Struktureinheit herrührt, weil in diesem Bereich praktisch keine anderen starken Banden auftreten ${ }^{78}$. Ein Mechanismus zur Bildung dieses Allen-Derivats wird unten in Abbildung 38 (S. 75) vorgeschlagen.

Unter den bei $853 \mathrm{~K}$ noch relativ milden Reaktionsbedingungen ist es eher unwahrscheinlich, daß Verbindung 12 der Abbildung 18 (S. 62) gebildet wird; denn der unten in Abbildung 39 (S. 75) vorgeschlagene Bildungsmechanismus verläuft über eine energetisch sehr ungünstige „carbenartige“ Zwischenstufe. Im IR-Spektrum (Abbildung 34, S. 70) finden sich zudem auch keine der für Verbindung 12 mutmaßlich charakteristischen Banden, so wie sie gemäß den Erläuterungen direkt unterhalb von Abbildung 33 (S. 69) zu erwarten wären.

Den bisher vorgeschlagenen Produkten (Verbindungen 3 bis 7) ist gemeinsam, daß sie alle vermutlich durch eine [1,2]-H-Umlagerung aus der diradikalischen Zwischenstufe 2 der Abbildung 18 (S. 62) gebildet werden. Diese Zwischenstufe 2 dürfte energetisch deutlich niedriger liegen als die Zwischenstufe 8, weil erstere zum einen durch Mesomerie (vgl. die als zweite Grenzstruktur formulierte Struktur 11) und zum anderen ihre Radikalstelle am C3Atom durch die Methylgruppe stabilisiert wird ${ }^{79}$. Die Radikalstellen der Zwischenstufe 8 liegen hingegen beide an primären C-Atomen und damit energetisch sehr hoch. Aus diesen Gründen ist $\mathrm{zu}$ erwarten, daß die Verbindungen $\mathbf{9}$ und $\mathbf{1 0}$ unter den gewählten relativ milden Reaktionsbedingungen nicht entstehen. Für diese ist die Struktureinheit $\mathrm{R}_{2} \mathrm{C}=\mathrm{CH}_{2}$ maßgeblich, für die Banden bei 885-895, 1640-1660, 1740-1780 und 3000-3100 cm-1 zu erwarten wären ${ }^{80}$. Im Spektrum fehlen aber zumindest diejenigen bei $885-895$ und $1740-1780 \mathrm{~cm}^{-1}$, die gemäß

77 Hesse, M.; Meier, H.; Zeeh, B. (1995); S. 44 Tabelle 2.1.

78 Hesse, M.; Meier, H.; Zeeh, B. (1995); S. 48 sowie S. 48 Tabelle 2.9.

79 Vgl. dazu: Carey, F.A.; Sundberg, R.J. (1995); S. 656-660.

80 Lide, D. (1991); Abschnitt 9 S. 126. 
den Tabellen für charakteristische IR-Frequenzen ${ }^{80}$ aber als starke bzw. mittelstarke Banden auftreten müßten. Somit dürften die Verbindungen $\mathbf{9}$ und $\mathbf{1 0}$ tatsächlich nicht entstanden sein. Zusammenfassend läßt sich festhalten, daß durch die thermische Aktivierung bei $853 \mathrm{~K}$ die Verbindungen 3 bis 7 der Abbildung 18 (S. 62) gebildet worden sein dürften, wobei Verbindung 6 weder bestätigt noch ausgeschlossen werden kann.

Um einen höheren Umsatz zu erzielen und ggf. Zugang zu den energetisch ungünstigeren Produkten $\mathrm{zu}$ erlangen, wurde ein weiteres Experiment in der in Kap.3.1.2 (S. 26) beschriebenen Apparatur bei $905 \mathrm{~K}\left(632^{\circ} \mathrm{C}\right)$ durchgeführt. Die Ausführung und Auswertung dieser Messung ist in Kap. 3.5.2 (S. 56) beschrieben. Im Strömungsreaktor herrschte dabei ein Druck von 22,9 mbar. Der Fluß an Helium betrug 91,3 SCCM, derjenige an 1-Ethinyl-1methylcyclopropan 3,5 SCCM. Daraus ließ sich eine Konzentration von $1,1 \cdot 10^{-8} \mathrm{~mol} / \mathrm{cm}^{3}$ sowie eine Reaktionszeit von $271 \mathrm{~ms}$ berechnen.

Mit der in der Glaskühlfalle ausgefrorenen Produkt-Mischung wurde zunächst eine FT-IRAnalyse (vgl. dazu Kap. 3.1.5, S. 42 sowie Kap. 3.5.5, S. 59) vorgenommen.

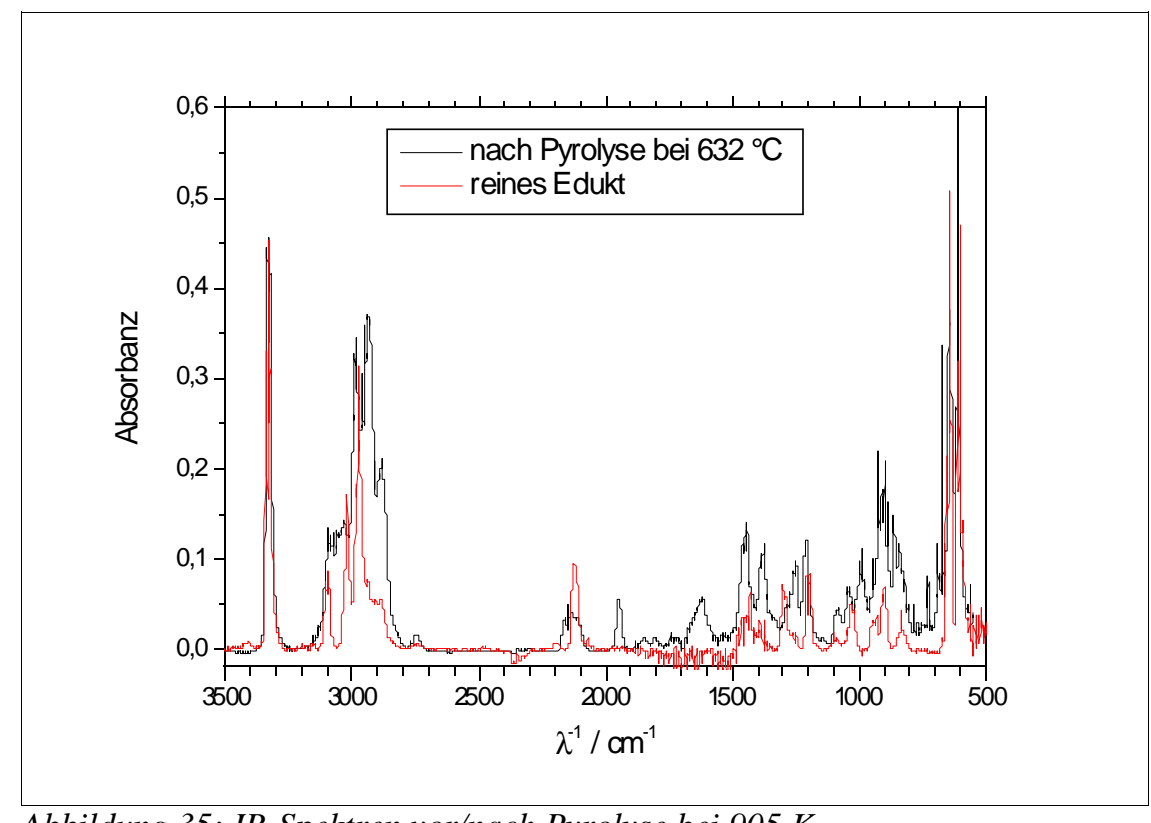

Abbildung 35: IR-Spektren vor/nach Pyrolyse bei $905 \mathrm{~K}$

Zum besseren Vergleich werden nachfolgend Ausschnitte aus den IR-Spektren des Edukts und der Produkt-Mischungen nach den Pyrolysen bei 853 und 905 K dargestellt. 

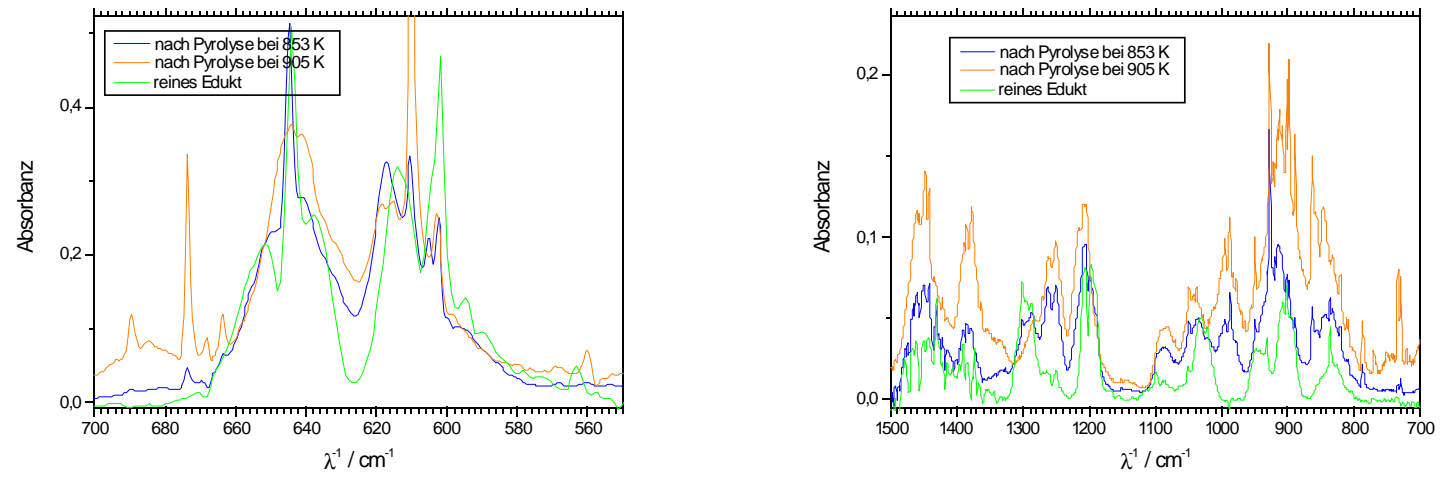

Abbildung 36: Vergleich IR-Spektren nach Pyrolyse bei 853 K und 905 K (Ausschnitt)

Aus Abbildung 36 ist erkennbar, daß nahezu alle nach der Pyrolyse bei $853 \mathrm{~K}$ im IR-Spektrum entstandenen Banden nach der Pyrolyse bei $905 \mathrm{~K}$ noch verstärkt wurden. Dies betrifft die Banden bei ${ }^{72}$ 560, 598, 605, 610 (s), 615, 668, 674 (s), 729 (s), 734 (s), 787 (s), 808 (w), 821 , 833, 837 (w), 846 (s), 857 (w), 863 (s), 898 (s), 915, 927 (s), 950 (s), 987 (s), 995 (s), 1037 (s), 1049 (s), 1070, 1090, 1208 (s), 1251 (s), 1262 (s), 1378 (s), 1443 (s), 1448 (s), 1457, 1461 (s) $\mathrm{cm}^{-1}$. Neu hinzugekommen sind Banden bei 618, 641, 664, 685 (w), 689, 750, 770 (s), 811, 889, 902, 912, 923, 979, 1028 (w), 1083, 1153 (w), 1215, 1270 (w), 1282 (w), 1335 (w), 1357 (w), 1364 (w), 1386 (s), 1422, 1436 (w), 1493 (w) cm². Nach der Pyrolyse bei $905 \mathrm{~K}$ nicht mehr zu erkennen waren im Gegensatz zur Pyrolyse bei $853 \mathrm{~K}$ die Banden bei 842, $1020,1022,1035,1200,1287,1302,1327,1466,1475$ und $1478 \mathrm{~cm}^{-1}$.
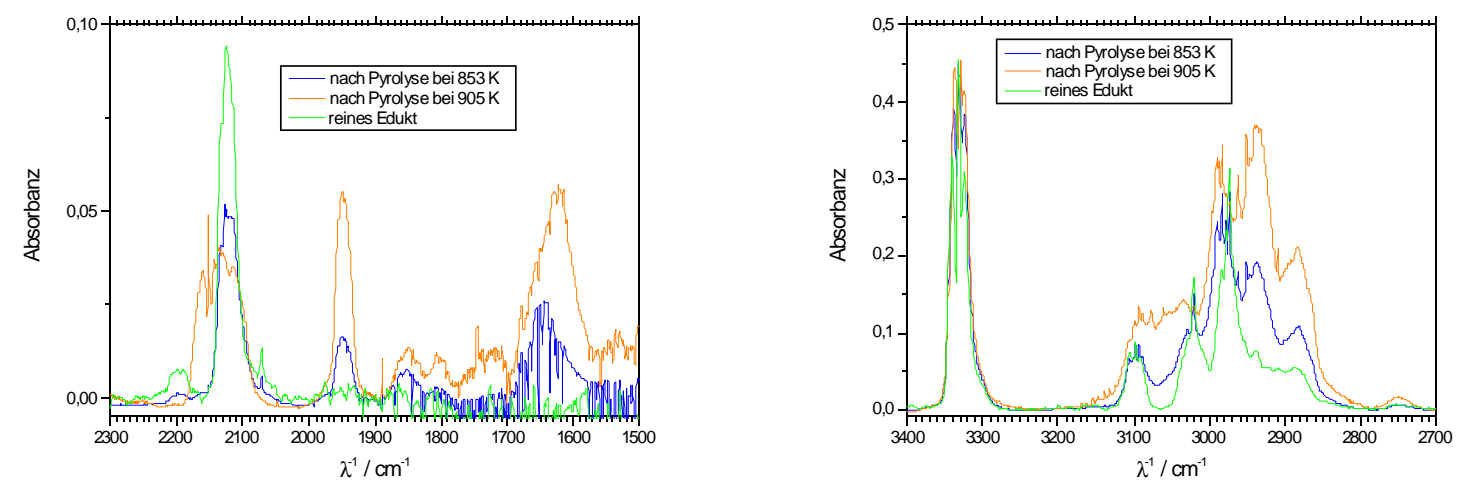

Abbildung 37: Vergleich IR-Spektren nach Pyrolyse bei $853 \mathrm{~K}$ und $905 \mathrm{~K}$ (Ausschnitt)

Auch aus Abbildung 37 ist erkennbar, daß nahezu alle nach der Pyrolyse bei $853 \mathrm{~K}$ im IRSpektrum entstandenen Banden nach der Pyrolyse bei $905 \mathrm{~K}$ noch verstärkt wurden. Dies betrifft die Banden bei 1615, 1622, 1630, 1639, 1842, 1849, 1945, 1950 (s), 2115, 2134, , 2883 (s), 2893 (w), 2938 (s), 2950 (s), 2962 (s), 2974 (w), 2977 (w), 2983 (s), 2932, 2941, 
2947 (w), 2988, 2990 (s), 2996, 3088, 3093, 3099, 3325 und $3337 \mathrm{~cm}^{-1}$. Neu hinzugekommen sind Banden bei 1647, 1656, 1665, 1677, 1708 (w), 1745, 1800 (w), 1807 (w), 1889, 2142 (w), 2151 (s), 2160, 2752 (w), 2910 (s), 3036, 3061, 3077 und $3330 \mathrm{~cm}^{-1}$. Nach der Pyrolyse bei $905 \mathrm{~K}$ nicht mehr zu erkennen waren im Gegensatz zur Pyrolyse bei $853 \mathrm{~K}$ die Banden bei 3020,3030 und $3332 \mathrm{~cm}^{-1}$.

Die IR-Spektren zeigen somit, daß die Pyrolyse bei $905 \mathrm{~K}$ gegenüber der bei $853 \mathrm{~K}$ prinzipiell die gleichen Produkte hervorbringt, wobei der Umsatz des Edukts deutlich gestiegen ist. Auffallend ist die überproportionale Zunahme der Bande bei $1950 \mathrm{~cm}^{-1}$; daraus kann gefolgert werden, daß Verbindung 7 in Abbildung 18 (S. 62) tatsächlich als Folgeprodukt bei höheren Temperaturen entsteht. Ein plausibler Reaktionsmechanismus für diese Folgereaktion ist in Abbildung 38 dargestellt ${ }^{81}$.

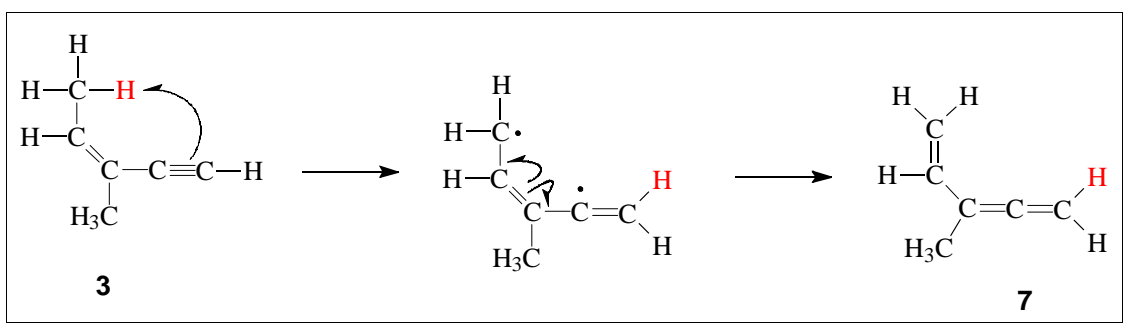

Abbildung 38: Reaktionsmechanismus zur Bildung des Allen-Derivats

Insbesondere die neu hinzugekommenen Banden bei 1745, 1807 (w), 1889 und 2752 (w) $\mathrm{cm}^{-1}$ sprechen nunmehr aber auch für eine zumindest geringe Bildung von Verbindung 12 (vgl. die Erläuterungen direkt unterhalb von Abbildung 33, S. 69). Für diese Folgereaktion ist in Abbildung 39 ein denkbarer Reaktionsmechanismus dargestellt ${ }^{82}$, wobei die Ringschließung und die [1,5]-H-Umlagerung auch in einem Schritt erfolgen könnten.

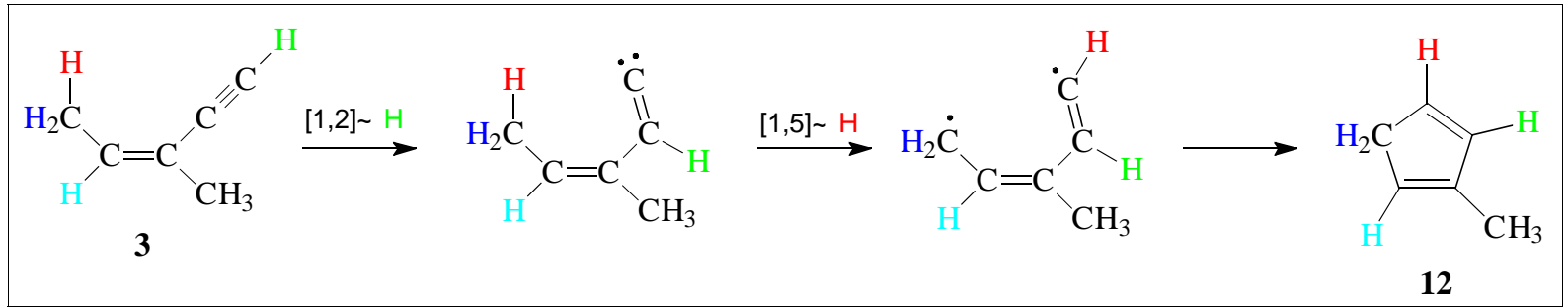

Abbildung 39: Reaktionsmechanismus zur Bildung des 1-Methyl-1,4-cyclopentadiens

Insbesondere die neu hinzugekommenen Banden bei 889, 1647, 1656 und $1745 \mathrm{~cm}^{-1}$ sprechen für die Entstehung der Verbindungen 9 und/oder 10. Für diese ist die Struktureinheit $\mathrm{R}_{2} \mathrm{C}=\mathrm{CH}_{2}$ maßgeblich, für die Banden bei 885-895, 1640-1660, 1740-1780 und 3000-3100 $\mathrm{cm}^{-1} \mathrm{zu}$ erwarten sind ${ }^{83}$. Die neue Bande bei 1436 sowie die starken Banden bei 729 und $734 \mathrm{~cm}^{-1}$

81 Vgl. den ähnlich formulierten Mechanismus in: Hopf, H.; Wachholz, G. (1987); S. 1259.

82 Vgl. den ähnlich formulierten Mechanismus in: Hopf, H.; Wachholz, G. (1987); S. 1260.

83 Lide, D. (1991); Abschnitt 9, S. 126. 
dürften der Methylengruppe $\left(\mathrm{CH}_{2}\right)$ der Verbindung 9 und/oder 10 zuzurechnen sein und deren Bildung somit bestätigen, denn diese Gruppe ist nur in diesen Verbindungen enthalten. Die Verbindung 10 wird vermutlich aus der Zwischenstufe 8 nach einer [1,2]-Umlagerung der Methyl-Gruppe gebildet, während Verbindung 9 nach einer [1,2]-Umlagerung der EthinylGruppe entsteht. Es hat sich gezeigt, daß in Radikalen die Wanderung von Phenyl- oder Alkenyl-Gruppen beträchtlich schneller als die von Alkyl-Gruppen ist, weil erstere vermutlich über einen in Abbildung 40 dargestellten dreigliedrigen Ring als Zwischenstufe verläuft ${ }^{84}$.

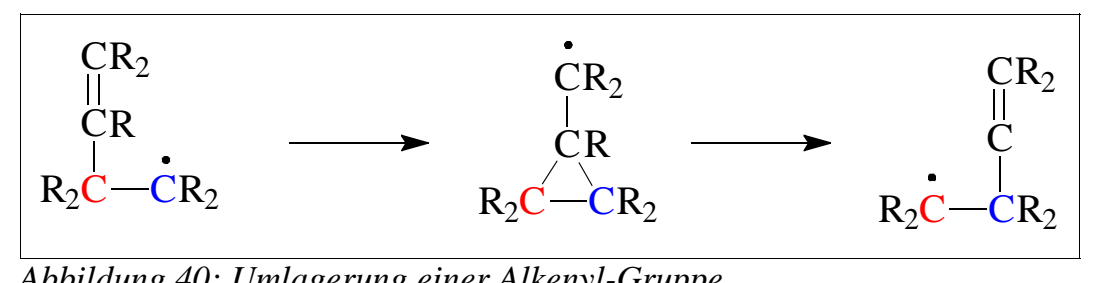

Abbildung 40: Umlagerung einer Alkenyl-Gruppe

Sicherlich fungiert in dem in Abbildung 40 dargestellten Mechanismus eine Alkinyl-Gruppe in gleicher Weise wie die Alkenyl-Gruppe. Daher ist zu erwarten, daß aus der Zwischenstufe 8 bevorzugt die Verbindung 9 der Abbildung 18 (S. 62) entsteht, weil die [1,2]-Umlagerung der Ethinyl-Gruppe deutlich schneller verlaufen müßte als die der Methyl-Gruppe. Die berechneten IR-Spektren der Verbindung 9 und 10 (Abbildung 29 bzw. 30, S. 68) unterscheiden sich aber nur unwesentlich, so daß diese Vermutung anhand der Spektren nicht untermauert werden kann.

Aus der FT-IR-Analyse läßt sich also entnehmen, daß die Spektren der nach thermischer Aktivierung bei $905 \mathrm{~K}$ erhaltenen Produkt-Mischung genügend Anhaltspunkte dafür bieten, um die Entstehung aller der in Abbildung 18 (S. 62) formulierten Produkte anzunehmen.

Um eine Vorstellung davon zu erhalten, in welchen Mengen die Produkte entstehen, wurde ferner eine GC-MS-Analyse (siehe dazu Kap. 3.1.3, S. 27 sowie Kap. 3.5.3, S. 57) vorgenommen. Natürlich wurde die dazu in der Glaskühlfalle ausgefrorene Produkt-Mischung unter den gleichen Reaktionsbedingungen gewonnen, wie die oben zur FT-IR-Analyse verwendete. Die thermische Aktivierung erfolgte daher ebenfalls bei $905 \mathrm{~K}$; die Durchführung und Auswertung ist in Kap. 3.5.2 (S. 56) beschrieben. Im Strömungsreaktor herrschte ein Druck von 21 mbar. Der Fluß an Helium betrug 91,3 SCCM, derjenige an 1-Ethinyl-1methylcyclopropan 3,5 SCCM. Daraus läßt sich eine Konzentration von $1,0 \cdot 10^{-8} \mathrm{~mol} / \mathrm{cm}^{3}$ sowie eine Reaktionszeit von 249 ms berechnen.

Vor der eigentlichen Analyse der Produktmischung wurde zunächst eine GC-MS-Analyse nur

84 Vgl. March, J. (1992); S. 1064-1066. 
mit dem Edukt (1-Ethinyl-1-methylcyclopropan) durchgeführt, um ein authentisches Massenspektrum dieser Verbindung und die Retentionszeit $\mathrm{zu}$ erhalten. Es wurde ein Temperaturprogramm für die Heizung des GC-Ofens (und damit der Kapillarsäule) gewählt, das die Temperatur zunächst 12 Minuten bei $100{ }^{\circ} \mathrm{C}$ hielt und danach mit einer Heizrate von $5{ }^{\circ} \mathrm{C}$ pro Minute bis auf $290{ }^{\circ} \mathrm{C}$ erhöhte; die Spektren sind in Abbildung 41 dargestellt.
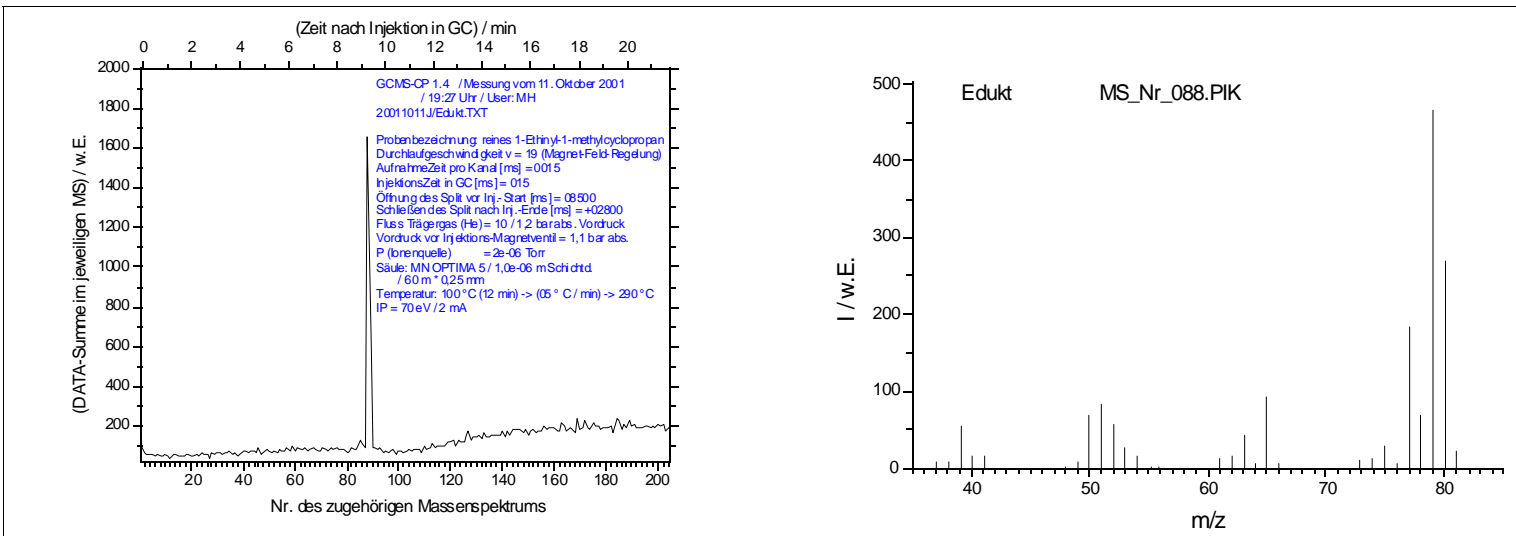

Abbildung 41: GC-und Massenspektrum von reinem 1-Ethinyl-1-methylcyclopropan

Die erste Analyse der Produkt-Mischung erfolgte bei einer Injektor-Temperatur von $110{ }^{\circ} \mathrm{C}$ und einem Temperaturprogramm für die Heizung des GC-Ofens (und damit der Kapillarsäule), das die Temperatur zunächst eine Minute bei $90{ }^{\circ} \mathrm{C}$ hielt und danach mit einer Heizrate von $5{ }^{\circ} \mathrm{C}$ pro Minute bis auf $260{ }^{\circ} \mathrm{C}$ erhöhte; das GC-Spektrum ist in Abbildung 42 dargestellt.

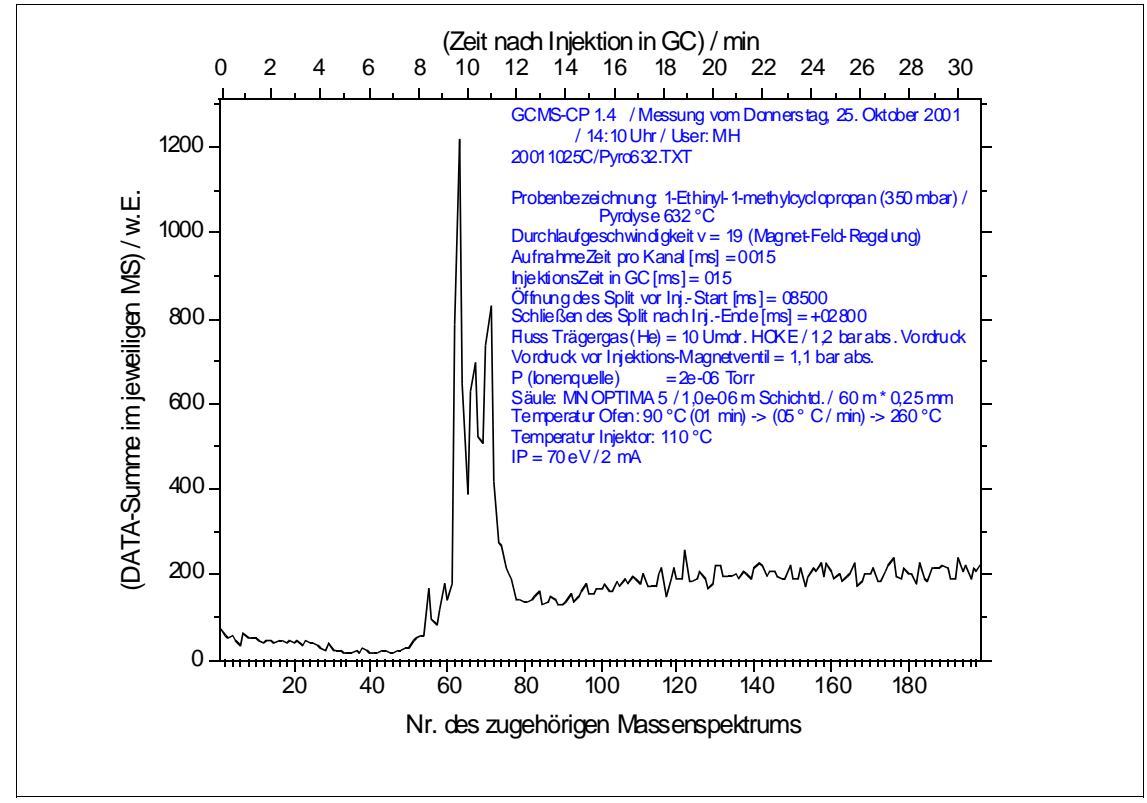

Abbildung 42: GC-Spektrum nach Pyrolyse bei $905 \mathrm{~K}$

In dem GC-Spektrum der Abbildung 42 läßt sich erkennen, daß unter diesen AnalyseBedingungen noch keine vollständige Trennung in die einzelnen Komponenten der 
Produktmischung möglich war. Daher wurde dieselbe Mischung nochmals einer GC-MSAnalyse unterzogen, jedoch wurde nun ein Temperaturprogramm gewählt, das bei einer Säulen-Temperatur von $70{ }^{\circ} \mathrm{C}$ statt $90{ }^{\circ} \mathrm{C}$ startete; die Heizrate von $5{ }^{\circ} \mathrm{C} / \mathrm{min}$ wurde beibehalten. Das GC-Spektrum ist in Abbildung 43 abgebildet.

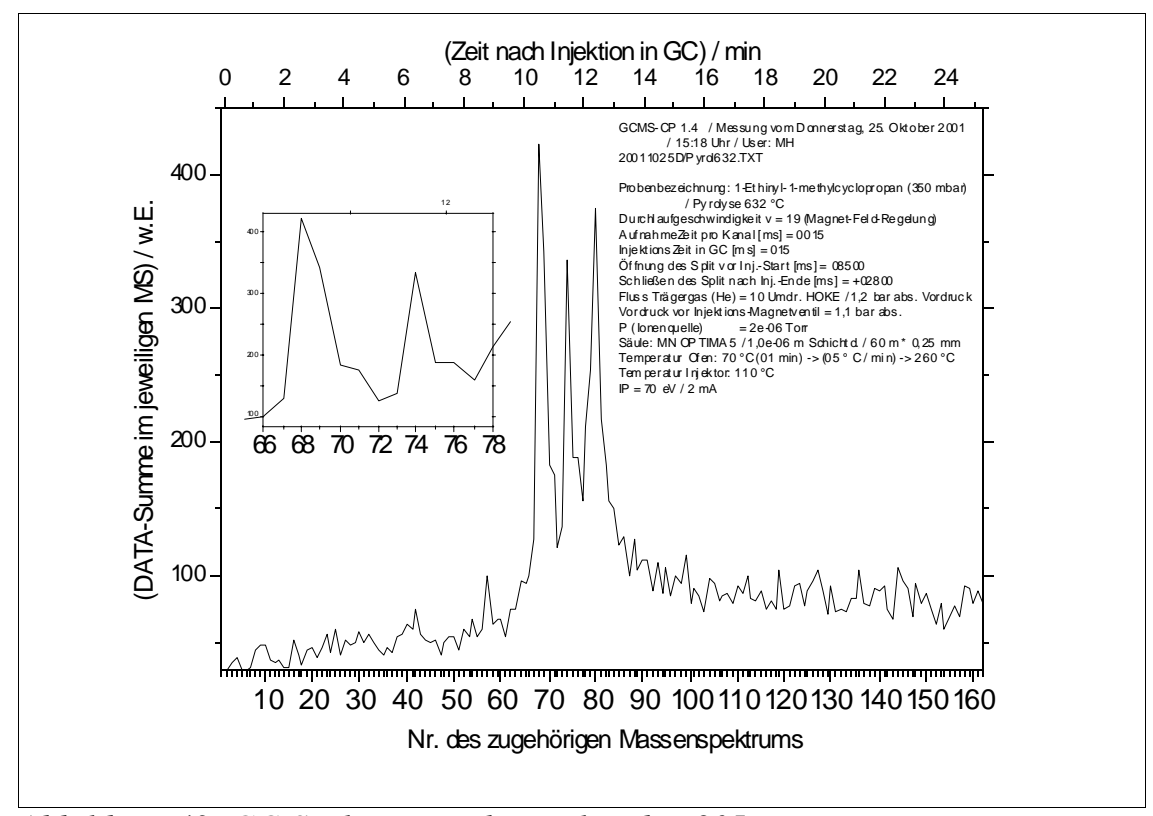

Abbildung 43: GC-Spektrum nach Pyrolyse bei $905 \mathrm{~K}$

Das GC-Spektrum in Abbildung 43 zeigt schon eine deutlich bessere Trennung und läßt erkennen, daß mindestens drei Hauptprodukte entstanden sind. Ein genauerer Blick auf die ersten beiden großen GC-Peaks zeigt jedoch, daß diese möglicherweise noch nicht vollständig getrennt waren (vgl. die Ausschnittvergrößerung in Abbildung 43). Bestätigt wird diese Vermutung durch das unterschiedliche Aussehen der Massenspektren Nr. 74 und 76 (vgl. Abbildungen 45 und 46).
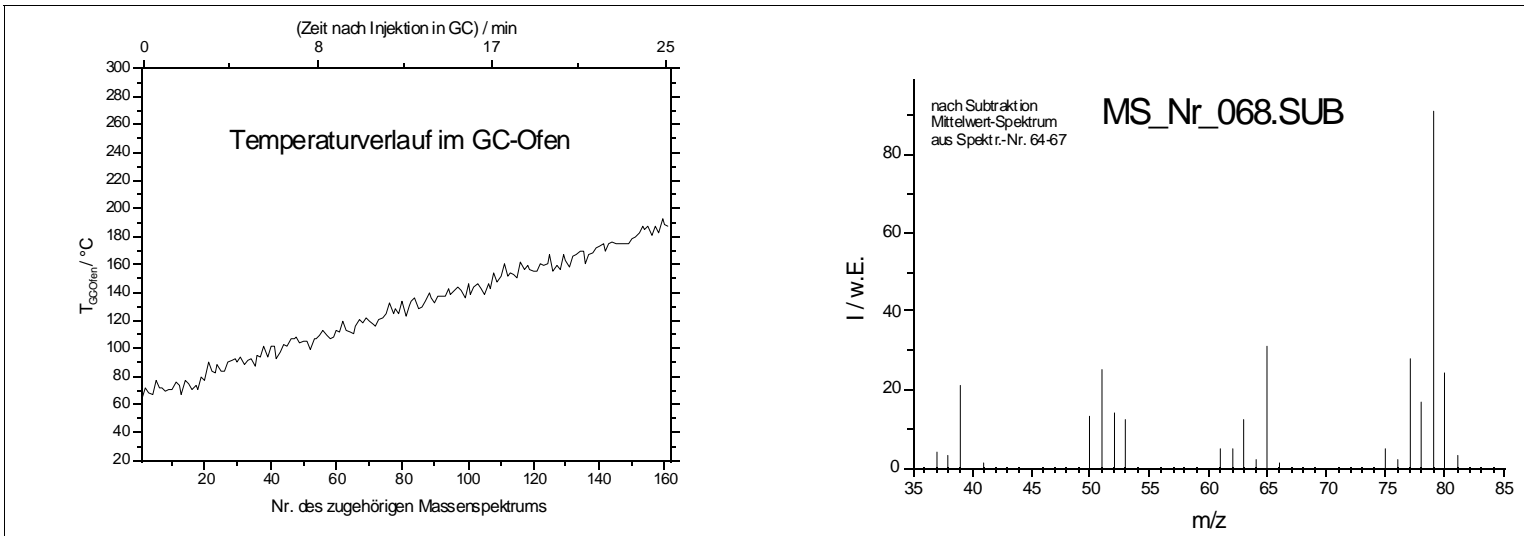

Abbildung 44: Temperaturverlauf und Massenspektrum Nr. 68 des GC-Spektrums aus Abbildung 43

Die Massenspektren Nr. 68, 74 und 80 (Abbildungen 44 bis 46) der Hauptkomponenten des 
GC-Spektrums zeigen keine Übereinstimmung mit dem des Edukts (Abbildung 41), so daß man von einem sehr hohen Umsatz ausgehen kann.
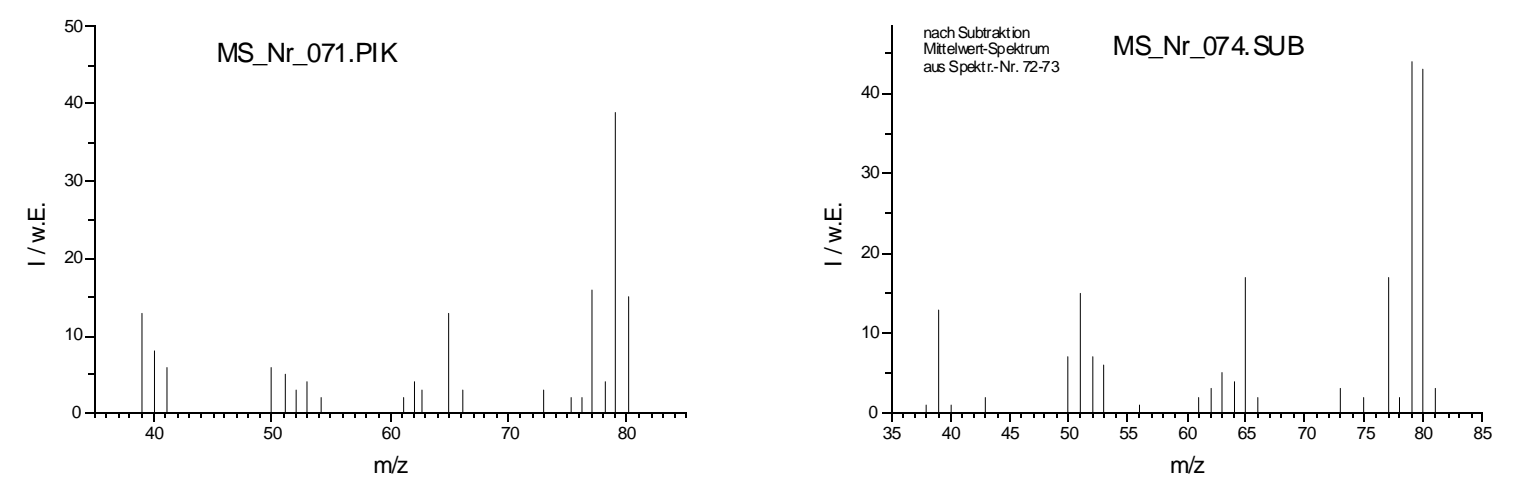

Abbildung 45: Massenspektren Nr. 71 und 74 des GC-Spektrums aus Abbildung 43

Das Massenspektrum Nr. 76 (Abbildung 46) des kleinen nicht vollständig getrennten GCPeaks dürfte vom Edukt stammen (vgl. das authentische Spektrum in Abbildung 41). Setzt man die Grundlinie in dem GC-Spektrum der Abbildung 43 auf 100 und führt damit eine numerische Integration über alle Peaks (Massenspektren Nr. 66 bis 87) durch, ergibt sich eine Fläche von 2169 w.E.; die Integration des verbliebenen Edukt-GC-Peaks (Massenspektrum Nr. 75 bis 77) ergibt eine Fläche von 89 w.E. Damit berechnet man einen Anteil von lediglich $89 / 2169=4 \%$ des Edukts in der Produktmischung. Unter der Voraussetzung, daß der Arrhenius-Vorfaktor wie bei vielen ähnlichen Cyclopropan-Derivaten in der Größenordnung von $10^{12} \mathrm{~s}^{-1}$ liegt ${ }^{85}$, ergibt sich aus Gleichung (25) (S. 56) mit einer Aktivierungsenergie von $189 \mathrm{~kJ} / \mathrm{mol}$ ein Rest des Edukts in Höhe von 5 \%, also in gleicher Höhe wie soeben in dieser GC-MS-Analyse ermittelt (vgl. zur Berechnung Kap. 3.5.2, S. 56).
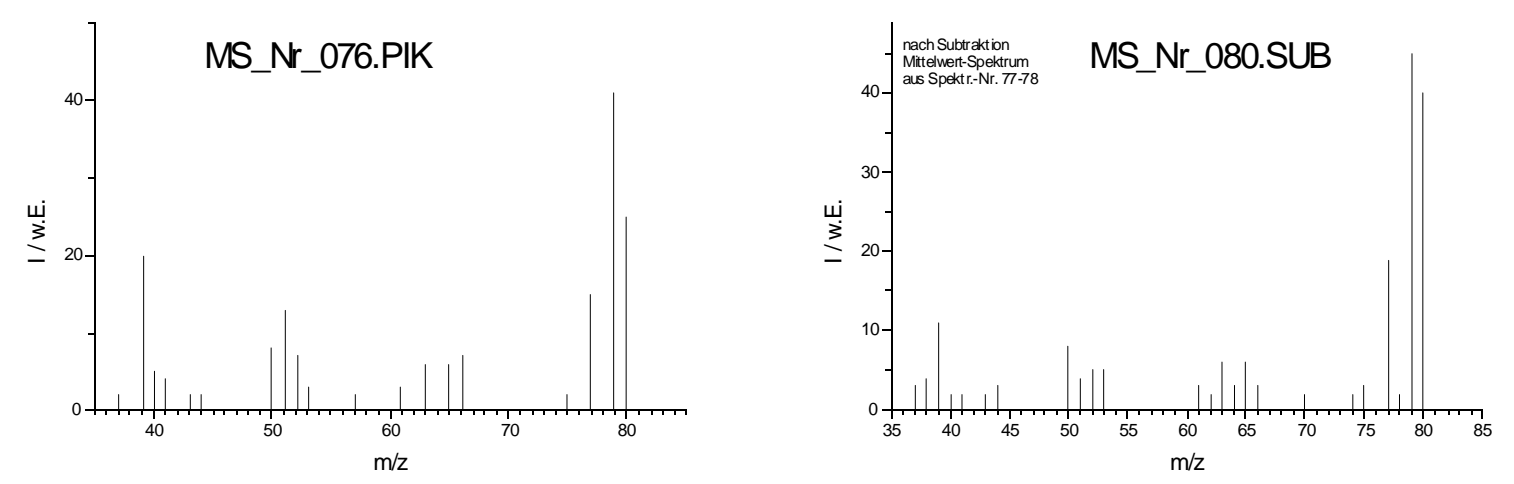

Abbildung 46: Massenspektren Nr. 76 und 80 des GC-Spektrums aus Abbildung 43

Zur besseren Trennung der Produkte wurde versucht, die Temperatur der Kapillarsäule noch

85 Vgl. dazu die tabellierten Werte für andere Cyclopropan-Derivate in: Hopf, H. et al. (1989); S. 380. 
weiter abzusenken, jedoch zeigte das GC-Spektrum bei einer Säulen-Temperatur von $50{ }^{\circ} \mathrm{C}$ keine vernünftige Trennung mehr, sondern nur noch einen sehr breiten Peak. Da die Siedetemperatur des Edukts ${ }^{86} 57^{\circ} \mathrm{C}$, diejenige der Verbindungen 3 und $\mathbf{4}$ aus Abbildung 18 (S. 62) $66-67{ }^{\circ} \mathrm{C}$ beträgt ${ }^{87}$, ist zu vermuten daß die Säulentemperatur nicht wesentlich unter die Siedetemperaturen abgesenkt werden darf. Für die Massenspektren des GC-Spektrums in Abbildung 43 ist der Bereich m/z 25 bis m/z 220 registriert worden, wobei sich aber herausstellte, daß oberhalb m/z 80 keine nennenswerten Peaks auftraten. Durch Reduzierung des aufzunehmenden Massenbereichs auf m/z 25 bis m/z 110 konnte bei gleicher Durchlaufgeschwindigkeit eine etwa doppelt so hohe Auflösung des GC-Spektrums erreicht werden.

Daher wurde die GC-MS-Analyse mit dem gleichen Temperaturprogramm (Start bei $70{ }^{\circ} \mathrm{C}$ ), jedoch der erhöhten Auflösung durch doppelte Zahl registrierter Massenspektren innerhalb der gleichen Analysenzeit, wiederholt. Das so erhaltene GC-Spektrum ist in Abbildung 47 dargestellt.

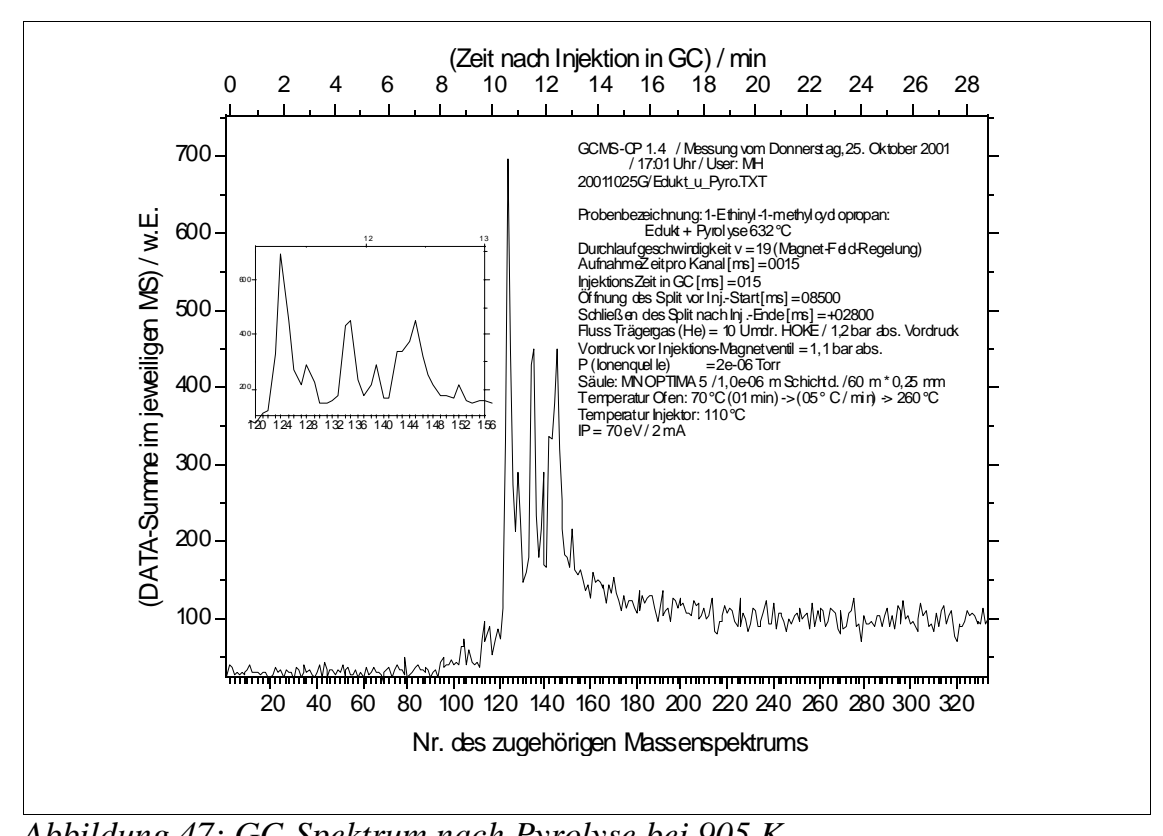

Abbildung 47: GC-Spektrum nach Pyrolyse bei $905 \mathrm{~K}$

In der Ausschnittvergrößerung der Abbildung 47 kann man nun erkennen, daß unmittelbar nach dem ersten großen GC-Peak (MS Nr. 124) ein kleinerer, nicht vollständig getrennter, GC-Peak (MS Nr. 128) vorhanden ist, dem ein Nebenprodukt zugerechnet wird. Möglicherweise besteht auch der mittlere große GC-Peak (MS Nr. 134 und 135) aus zwei nicht getrennten Komponenten, denn die zugehörigen Massenspektren sind etwas unterschiedlich. Deutlich ist nun der Peak des nicht umgesetzten Edukts (MS Nr. 139) zu 
erkennen. Mit großer Wahrscheinlich besteht auch der letzte große GC-Peak aus zwei nicht getrennten Komponenten (Massenspektren Nr. 142 und 145). Ein weiteres Nebenprodukt dürfte dem letzten kleinen GC-Peak (MS Nr. 152) zuzuordnen sein.

Die Identifizierung der Komponenten anhand der Massenspektren wird dadurch erschwert, daß lediglich für Verbindung 3 bzw. 4 der Abbildung 18 (S. 62) ein Massenspektrum vorlag (zu sehen in Abbildung 52, S. 82) ${ }^{88}$. Für die Identifizieierung der Verbindung 12 kann nur das Massenspektrum des nahezu identischen 1-Methyl-1,3-Cyclopentadien herangezogen werden (dargestellt in Abbildung 52, S. 82) ${ }^{89}$. Für alle übrigen potentiellen Produkte lagen jedoch keine Massenspektren vor.
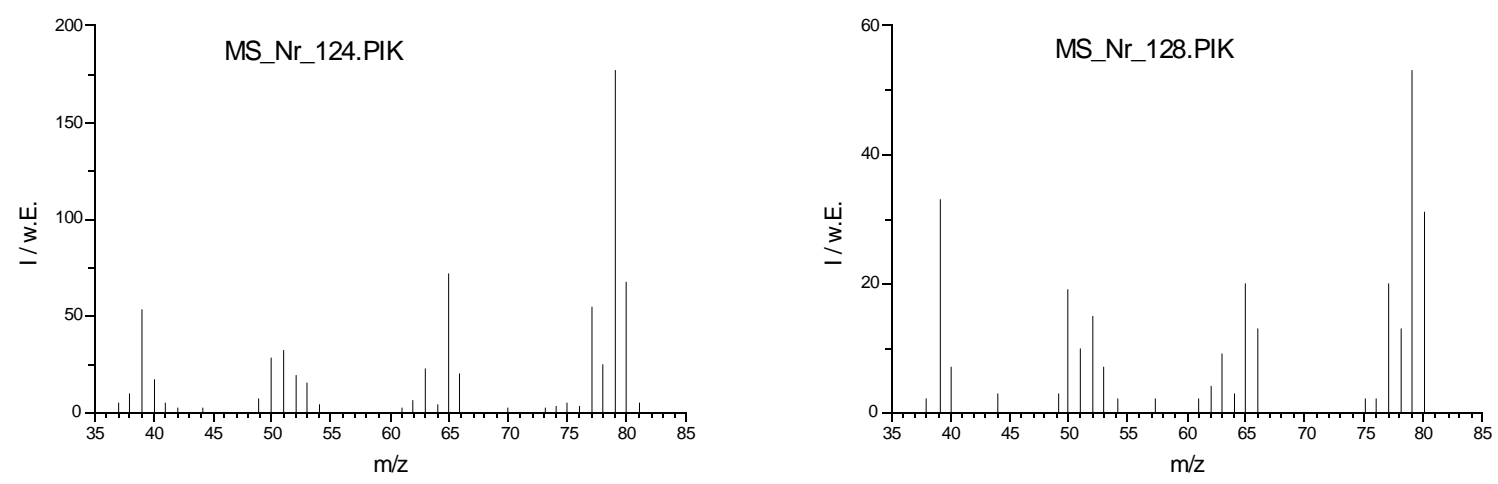

Abbildung 48: Massenspektren Nr. 124 und 128 des GC-Spektrums aus Abbildung 47

Es ist anzunehmen, daß das Massenspektrum Nr. 128 (Abbildung 48) der Verbindung 12 entstammt, weil diese aus energetischen Gründen (vgl. Abbildung 39, S. 75) nur als Nebenprodukt in Betracht kommen dürfte. Die Übereinstimmung mit dem Literaturspektrum des ähnlichen 1-Methyl-1,3-Cyclopentadiens (Abbildung 52, S. 82) ist akzeptabel.
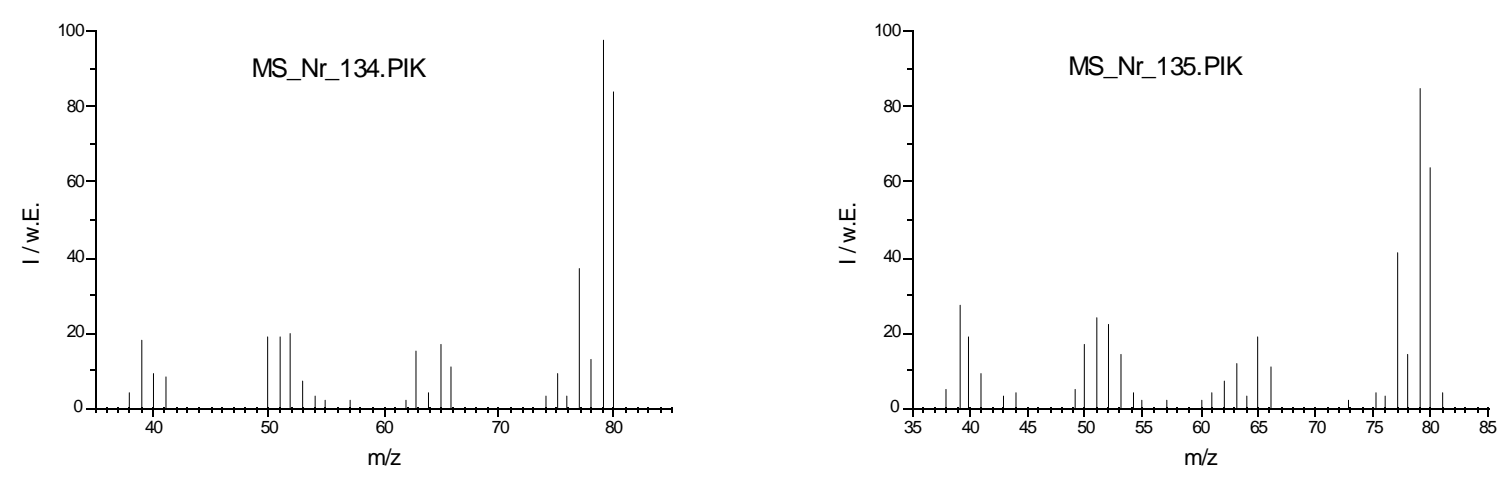

Abbildung 49: Massenspektren Nr. 134 und 135 des GC-Spektrums aus Abbildung 47

88 NIST Chemistry WebBook (2001); in dieser Datenbank war nicht angegebenen, ob es sich um das (E)- oder (Z)-Isomere handelt oder eine Mischung beider.

89 NIST Mass Spectral Database (1990). 

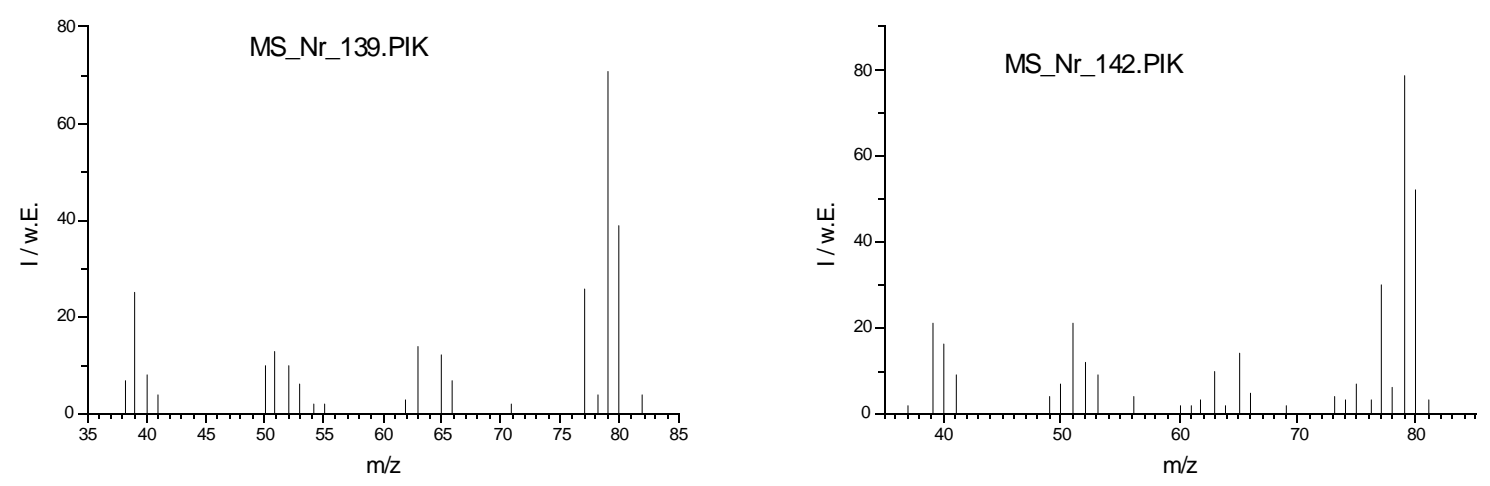

Abbildung 50: Massenspektren Nr. 139 und 142 des GC-Spektrums aus Abbildung 47

Das Massenspektrum Nr. 139 müßte von nicht umgesetztem Edukt stammen.
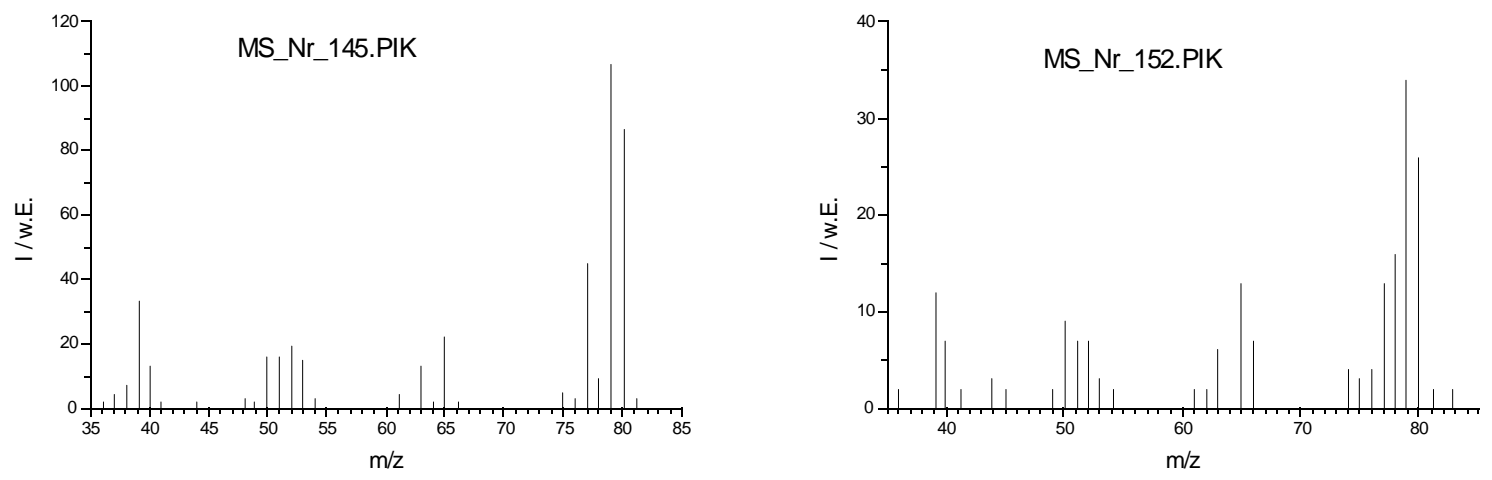

Abbildung 51: Massenspektren Nr. 145 und 152 des GC-Spektrums aus Abbildung 47

Nach Vergleich mit dem Literaturspektrum (Abbildung 52) dürfte das Massenspektrum Nr. 145 (Abbildung 51) der Verbindung 3 oder 4 der Abbildung 18 (S. 62) zuzuordnen sein. Letzteres ist auch im Einklang mit der vorangegangenen GC-MS-Analyse (Abbildung 43, S. 78), bei der das Massenspektrum (MS-Nr. 80 in Abbildung 46, S. 79) des letzten der großen GC-Peaks ebenfalls mit diesem Literaturspektrum übereinstimmt.
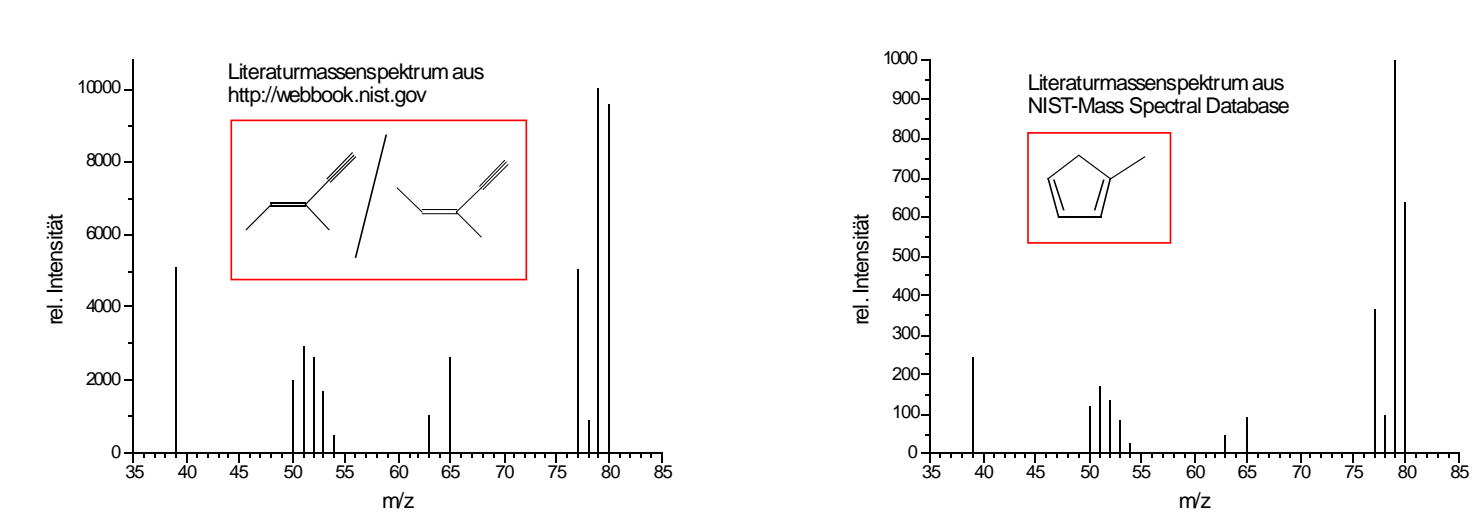

Abbildung 52: Literatur-Massenspektren der Verbindungen 3/4 sowie von 1-Methyl-1,3-Cyclopentadien 
Als Ergebnis der GC-MS-Analyse der Produkt-Mischung aus der Pyrolyse bei $905 \mathrm{~K}$ ist also festzuhalten, daß diese aus 4 oder 5 Hauptprodukten und 2 Nebenprodukten sowie etwas Edukt besteht. Verbindung 3 bzw. 4 der Abbildung 18 (S. 62) ist wahrscheinlich als Hauptprodukt und Verbindung 12 als Nebenprodukt anzusehen; die Aktivierungsenergie kann mit einem Arrhenius-Vorfaktor von $10^{12} \mathrm{~s}^{-1}$ auf $189 \mathrm{~kJ} / \mathrm{mol}$ geschätzt werden.

Ohne einen Vergleich mit den authentischen IR-, GC- und Massenspektren der potentiellen Produkte aus Abbildung 18 (S. 62) ist eine weitergehende Zuordnung zu den GC-Peaks bzw. überhaupt die sichere Identifizierung der Produkte nicht möglich. Keine dieser Substanzen konnte jedoch kommerziell beschafft werden

Den Abschluß der Untersuchungen zur thermischen Aktivierung bildete ein Experiment bei $953 \mathrm{~K}\left(680{ }^{\circ} \mathrm{C}\right)$. Im Strömungsreaktor herrschte hierbei ein Druck von 23,7 mbar. Der Fluß an Helium betrug 129,7 SCCM, derjenige an 1-Ethinyl-1-methylcyclopropan 8,3 SCCM. Daraus ließ sich eine Konzentration in Höhe von $1,8 \cdot 10^{-8} \mathrm{~mol} / \mathrm{cm}^{3}$ sowie eine Reaktionszeit von 183 ms berechnen. Aus der FT-IR-Analyse (vgl. dazu Kap. 3.1.5, S. 42 sowie Kap. 3.5.5, S. 59) erhielt man das in Abbildung 53 blau eingezeichnete Spektrum.

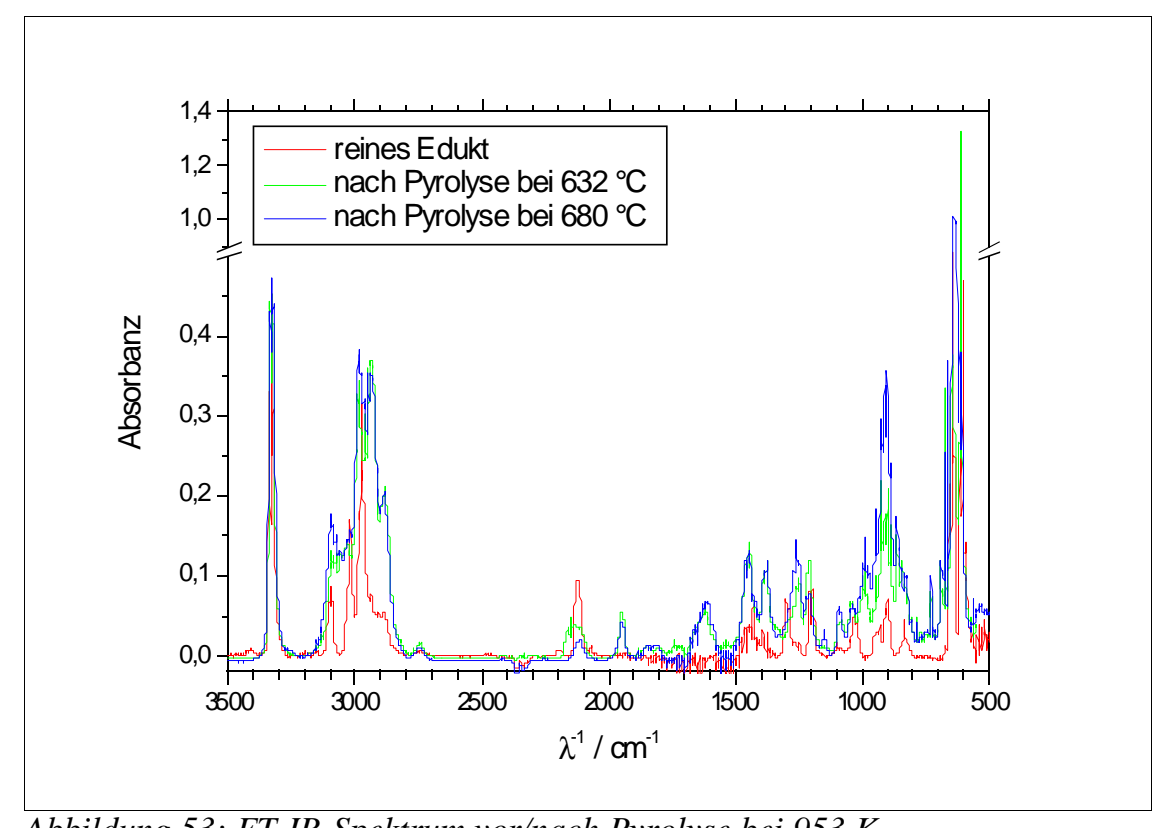

Abbildung 53: FT-IR-Spektrum vor/nach Pyrolyse bei $953 \mathrm{~K}$

Man erkennt in Abbildung 53, daß die Banden der Pyrolyse bei $953 \mathrm{~K}$ gegenüber der bei $905 \mathrm{~K}$ großenteils gleich geblieben, viele stärker geworden und auch einige ganz neu sind; nur wenige haben an Intensität verloren. 

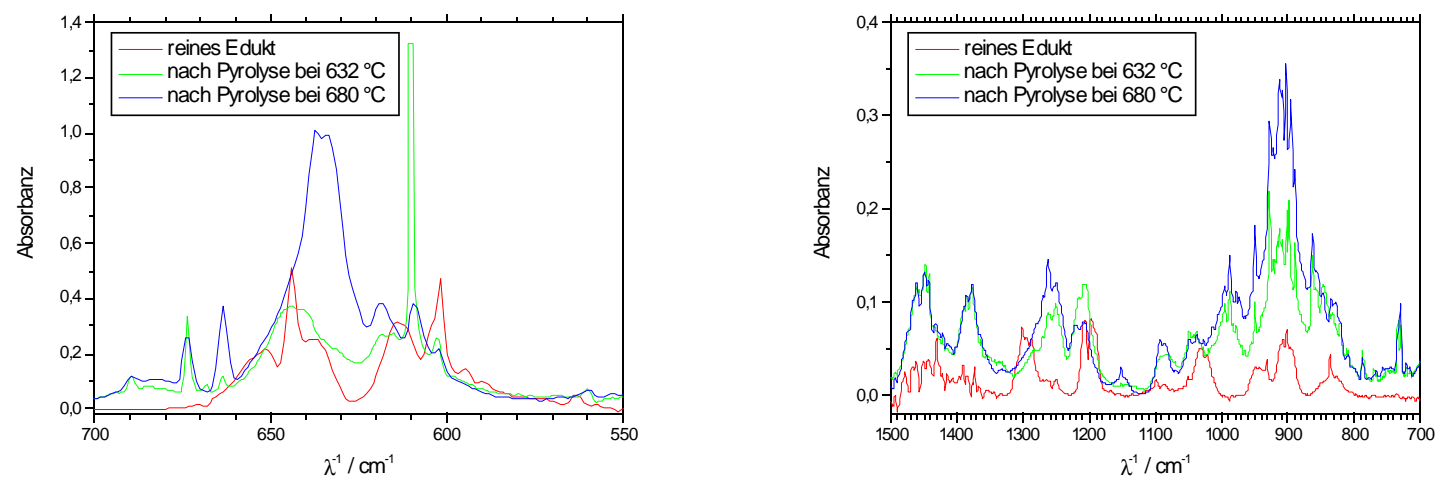

Abbildung 54: Vergleich IR-Spektren nach Pyrolyse bei 905 K und 953 K (Ausschnitt)

Den Ausschnittvergrößerungen in Abbildung 54 kann man entnehmen, daß die Banden bei 673, 674, 730 (m), $787(\mathrm{~m}), 1378(\mathrm{~m}), 1386(\mathrm{~m}), 1443(\mathrm{~m}), 1449(\mathrm{~m})$ und $1461(\mathrm{~m}) \mathrm{cm}^{-1}$ in etwa die gleichen sind, die bei 663, 863, 890, 896 (s), 903 (s), 912 (s), 927 (s), 950 (m), 987 (m), 996 (m), 1087 (w), 1093 (w), 1250 (m) und 1262 (m) cm'-1 stärker wurden, diejenigen bei 619 (m), 634 (s), 637 (s), 979 (w) und 1152 (w) $\mathrm{cm}^{-1}$ neu hinzukamen und die bei 609, 1208 und $1222 \mathrm{~cm}^{-1}$ gegenüber der Pyrolyse bei $905 \mathrm{~K}$ abnahmen.
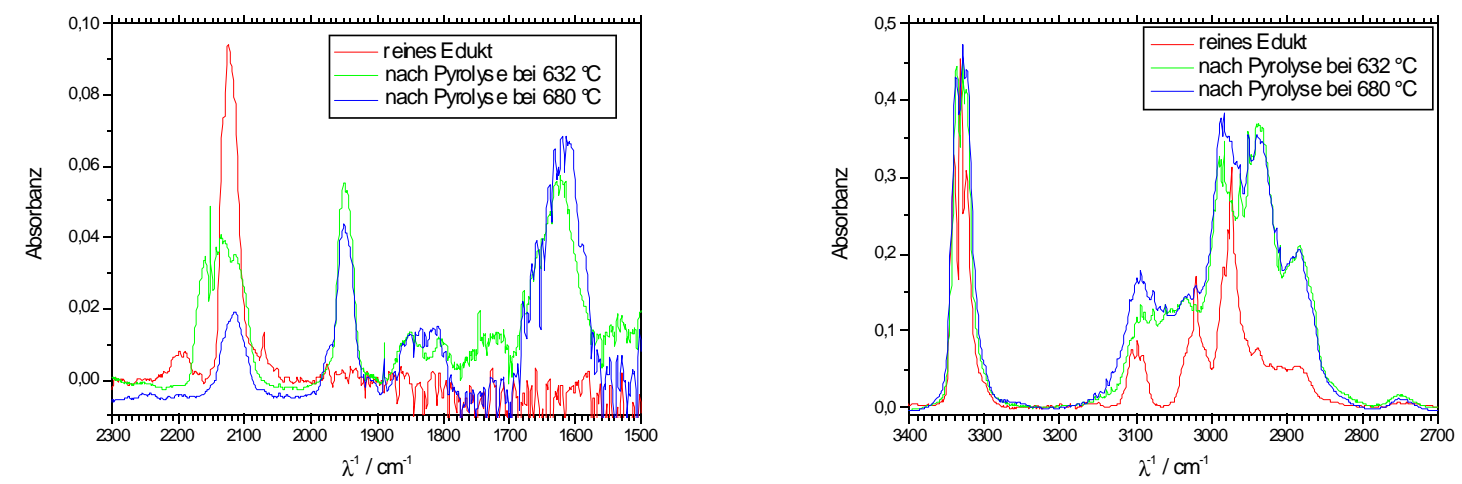

Abbildung 55: Vergleich IR-Spektren nach Pyrolyse bei 905 K und 953 K (Ausschnitt)

In den Ausschnittvergrößerungen in Abbildung 55 erkennt man, daß die Banden bei 1650, 1657, 1678, 1850, 2750, 2884, 2940, 2951 und $2962 \mathrm{~cm}^{-1}$ in etwa die gleichen sind, die bei 1614, 1620, 1632, 1639, 1807, 2967, 2983, 2988, 3077, 3093 und $3099 \mathrm{~cm}^{-1}$ stärker wurden, diejenigen bei 1589 (m), 1814 (w), 1827 (w), 1833 (w), 1969 (m), 3021 und $3061 \mathrm{~cm}^{-1}$ neu hinzukamen und die bei 1888, 1945, 1950 und $2115 \mathrm{~cm}^{-1}$ abnahmen.

Ergänzend zur FT-IR-Analyse wurde wieder eine GC-MS-Analyse (vgl. dazu Kap. 3.5.3, S. 57) mit der ausgefrorenen Produkt-Mischung vorgenommen (Abbildung 56). 


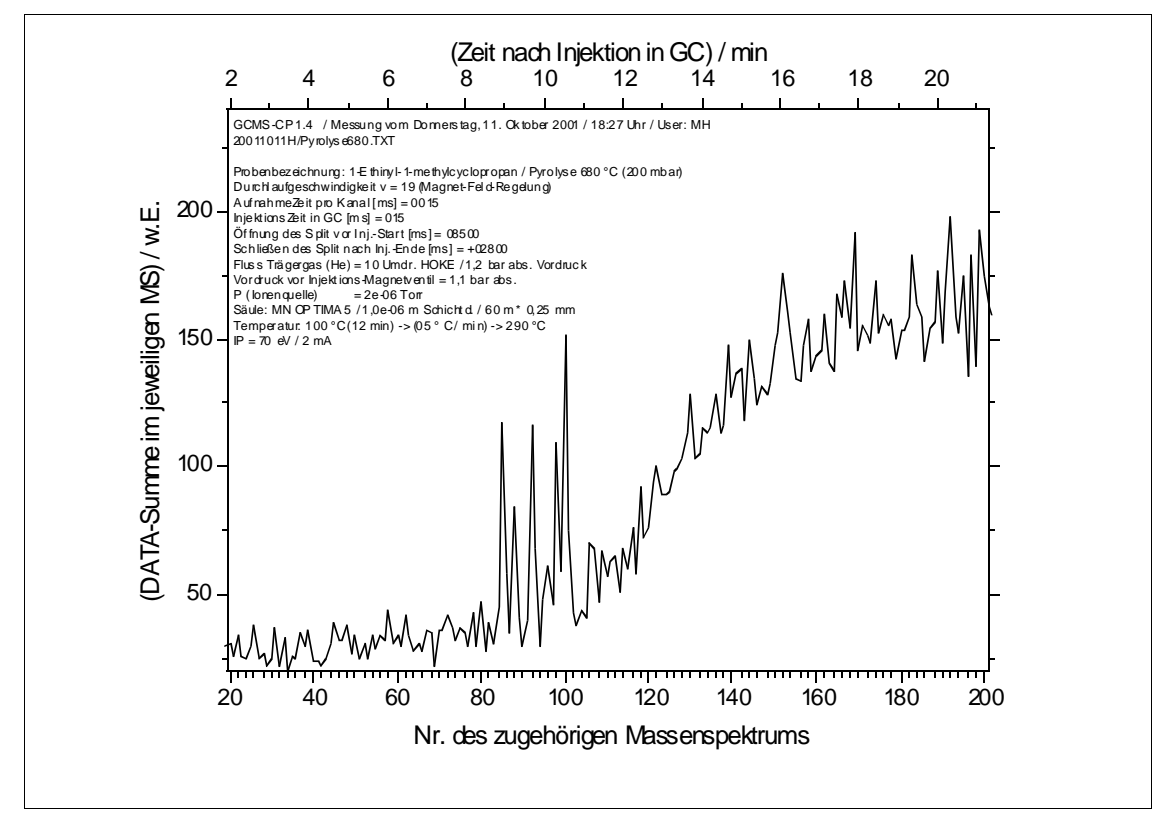

Abbildung 56: GC-Spektrum nach Pyrolyse bei $953 \mathrm{~K}$

Obwohl das gewählte Temperaturprogramm die Analyse mit $100{ }^{\circ} \mathrm{C}$ Säulentemperatur startete und diese nach $12 \mathrm{~min}$ mit einer Heizrate von $5{ }^{\circ} \mathrm{C} / \mathrm{min}$ auf $290{ }^{\circ} \mathrm{C}$ steigerte, kam es ca. $10 \mathrm{~min}$ nach der Injektion zu einem starken Anstieg der Grundlinie. Möglicherweise sind dafür höher siedende Produkte verantwortlich, die durch Kombination zweier oder mehrerer temporär entstandener Radikale gebildet worden sein könnten. Die Untersuchung soll hier jedoch auf die Isomerisierungsprodukte des Edukts $\left(\mathrm{M}_{\mathrm{r}}=80\right)$ beschränkt bleiben; daher werden nachfolgend nur die GC-Peaks bis MS-Nr. 106 ausgewertet, welche keine Signale oberhalb m/z 80 zeigen.

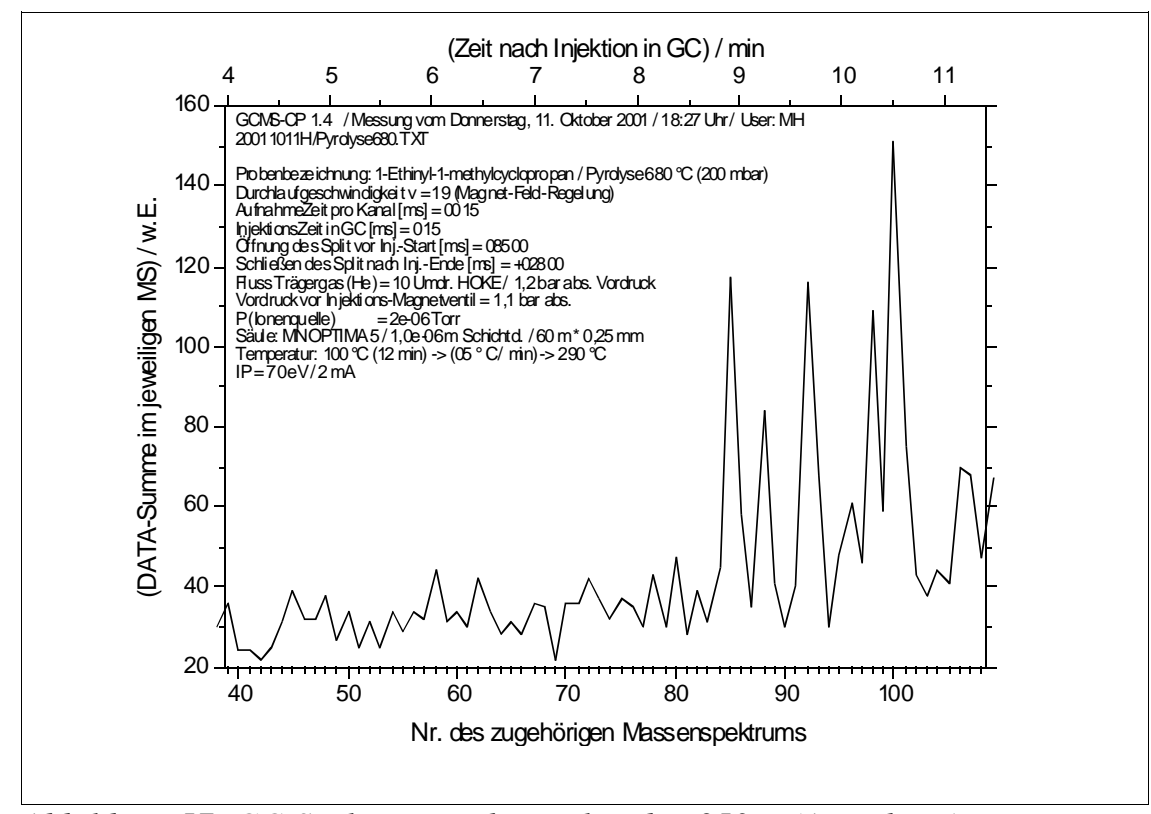

Abbildung 57: GC-Spektrum nach Pyrolyse bei 953 K (Ausschnitt) 
Abbildung 57 enthält den nachfolgend näher untersuchten Ausschnitt des GC-Spektrums.
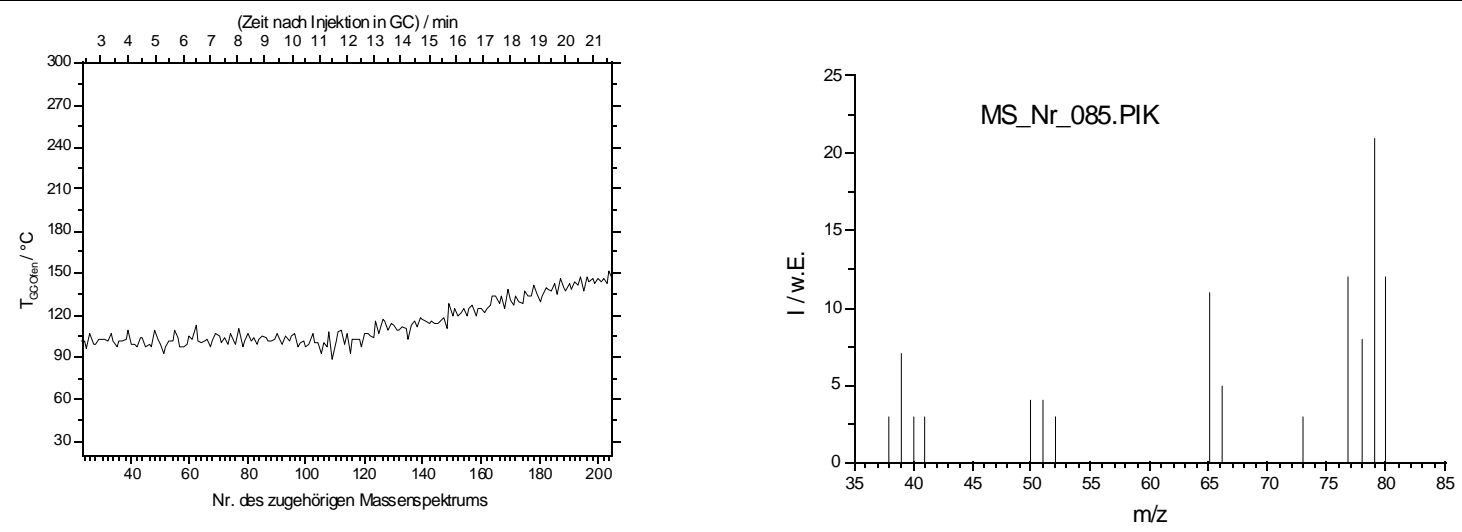

Abbildung 58: Temperaturverlauf und Massenspektrum Nr. 85 des GC-Spektrums in Abbildung 56
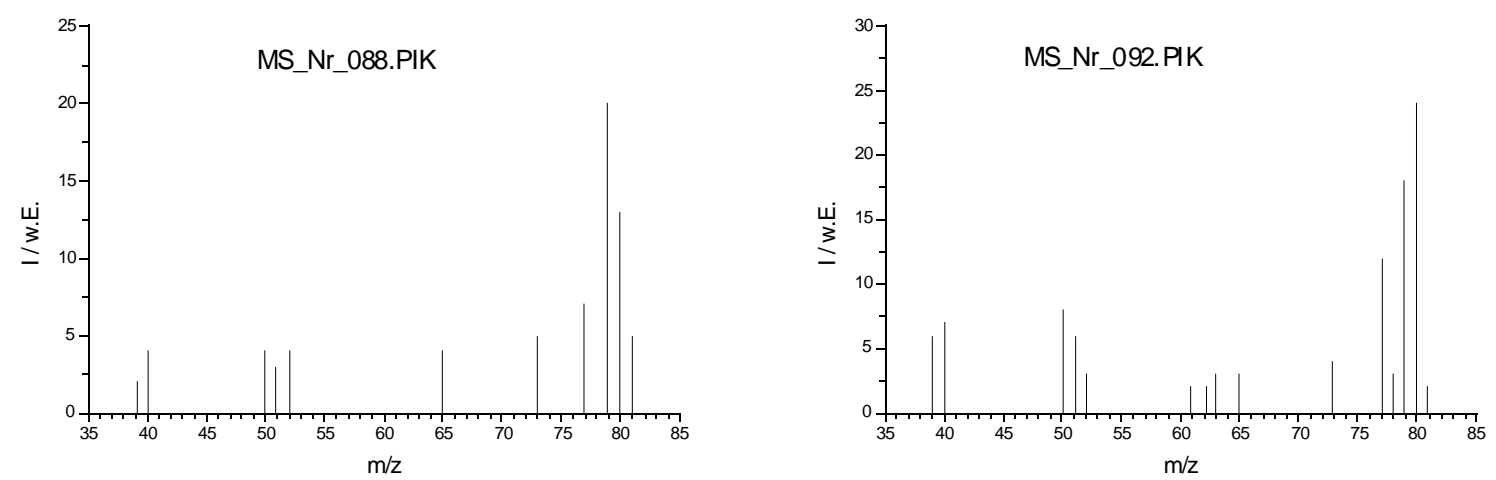

Abbildung 59: Massenspektren Nr. 88 und 92 des GC-Spektrums in Abbildung 56

Das Massenspektrum Nr. 88 könnte Verbindung 12 der Abbildung 18 (S. 62) zuzuordnen sein, wie der Vergleich mit dem Literaturspektrum der sehr ähnlichen Verbindung 1-Methyl-1,3cyclopentadien (Abbildung 52, S. 82) nahelegt. Aus der Größe des zugehörigen GC-Peaks in Abbildung 57 läßt sich folgern, daß ihr Anteil in der Produkt-Mischung gegenüber der Pyrolyse bei $905 \mathrm{~K}$ deutlich erhöht ist. 

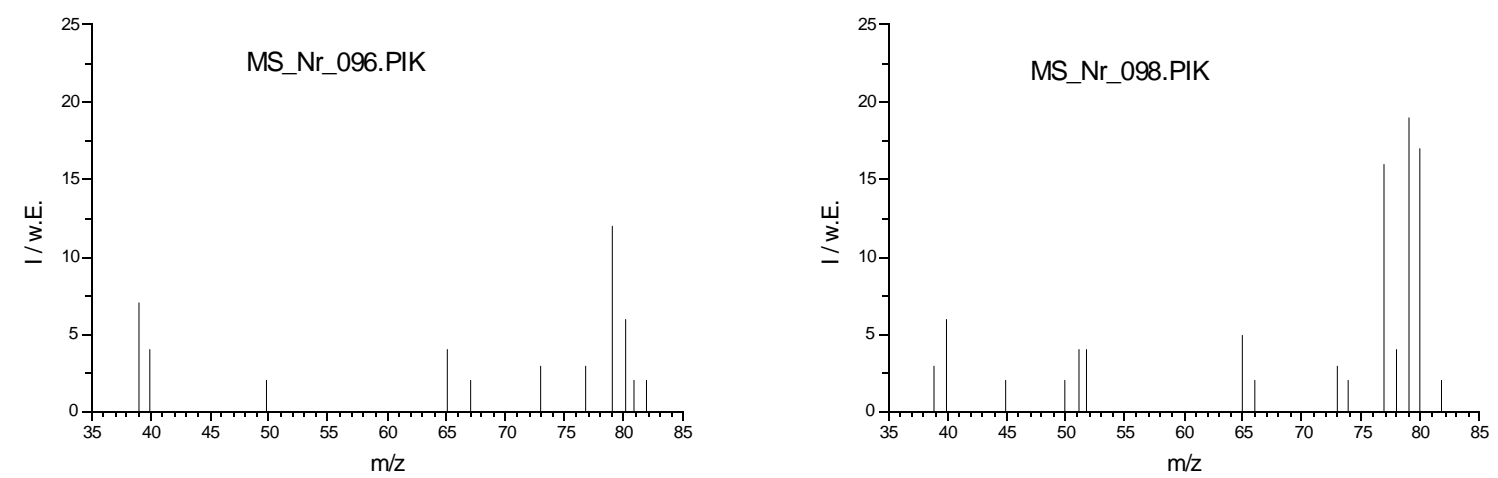

Abbildung 60: Massenspektren Nr. 96 und 98 des GC-Spektrums in Abbildung 56

Das Massenspektrum Nr. 100 in Abbildung 64 dürfte von Verbindung 3 oder 4 der Abbildung 18 (S. 62) stammen, wie der Vergleich mit dem Literaturspektrum (Abbildung 52, S. 82) zeigt. Aus der Größe des zugehörigen GC-Peaks in Abbildung 57 (S. 85) läßt sich folgern, daß diese Verbindung(en), wie bei der Pyrolyse bei $905 \mathrm{~K}$, als Hauptprodukt(e) anzusehen ist (sind).
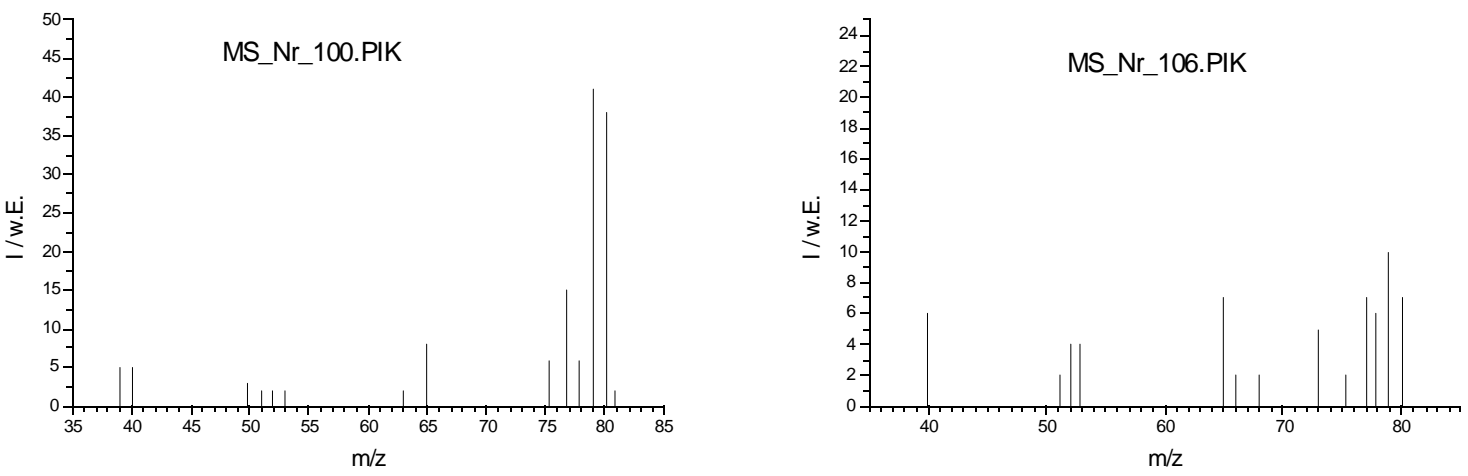

Abbildung 61: Massenspektren Nr. 100 und 106 des GC-Spektrums in Abbildung 56

Weitergehende Zuordnungen können ohne einen Vergleich mit den authentischen Spektren der potentiellen Produkte nicht vorgenommen werden. 


\subsubsection{Kinetik der radikalerzeugenden Reaktion mit Fluor-Atomen}

Zur chemischen Aktivierung von 1-Ethinyl-1-methylcyclopropan über die Reaktion des 1Ethinyl-1-methylcyclopropyl-Radikals mit atomarem Wasserstoff ist die Erzeugung des entsprechenden Radikals erforderlich, das dann mit H-Atomen wieder zum angeregten Edukt umgesetzt werden kann.

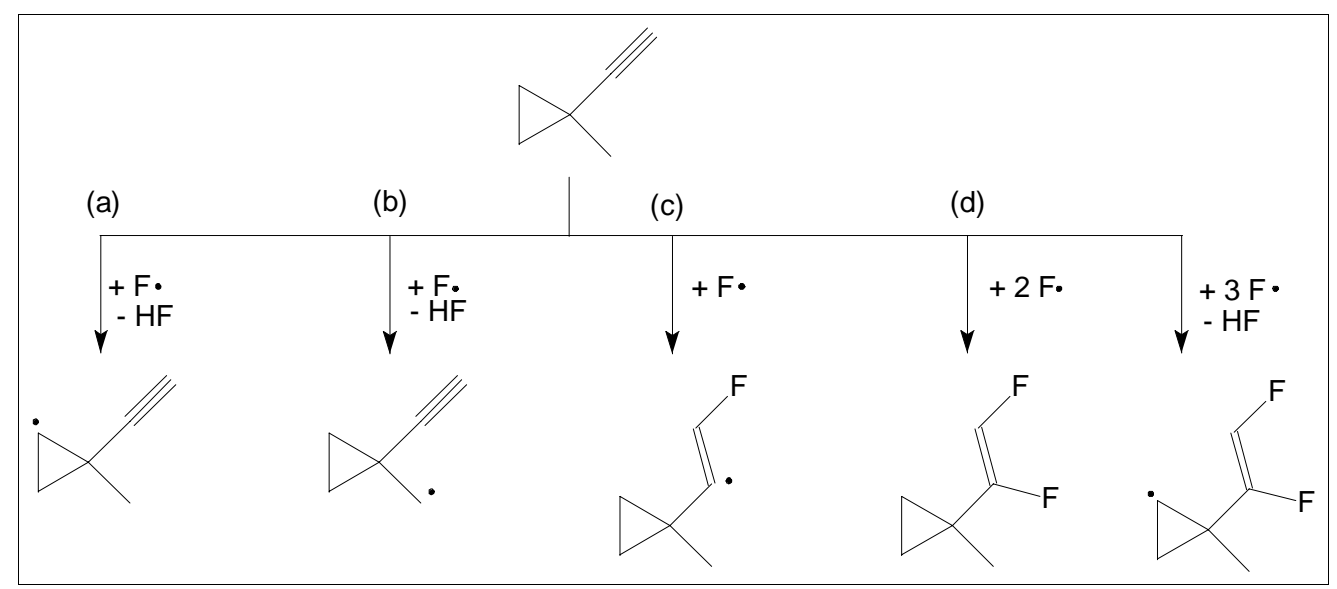

Abbildung 62: Reaktionskanäle der Reaktion 1-Ethinyl-1-methylcyclopropan + F-Atome

Oft ist die Reaktion von Fluor-Atomen mit Kohlenwasserstoffen recht gut zur Radikalerzeugung geeignet, weil Fluor-Atome mittels Mikrowellenentladung aus $F_{2}$ fast quantitativ erzeugt werden können und zudem sehr schnell mit dem Kohlenwasserstoff reagieren. In der in Kap. 3.1.1 (S. 16) beschriebenen Apparatur wurde daher zunächst die Geschwindigkeit der Reaktion von Fluor-Atomen mit 1-Ethinyl-1-methylcyclopropan bestimmt (vgl. dazu Kap. 3.5.1, S. 50).

\begin{tabular}{|c|c|c|c|c|c|c|c|}
\hline $\begin{array}{c}\mathbf{I}(\mathbf{8 0}) \\
-\mathrm{F} / \mathrm{w.E.}\end{array}$ & $\begin{array}{c}\Delta \mathbf{I}(\mathbf{8 0}) \\
-\mathrm{F} / \mathrm{w.E} .\end{array}$ & $\begin{array}{c}\mathbf{I}(\mathbf{8 0}) \\
+\mathrm{F} / \text { w.E. }\end{array}$ & $\begin{array}{c}\Delta \mathrm{I}(\mathbf{8 0}) \\
+\mathrm{F} / \mathrm{w.E.} .\end{array}$ & $\begin{array}{c}\mathbf{I}(16) \\
-\mathrm{F} / \mathrm{w.E} .\end{array}$ & $\begin{array}{c}\Delta \mathrm{I}(16) \\
-\mathrm{F} / \mathrm{w.E} .\end{array}$ & $\begin{array}{c}\mathrm{I}(16) \\
+\mathrm{F} / \mathrm{w.E.}\end{array}$ & $\begin{array}{c}\Delta \mathrm{I}(16) \\
+\mathrm{F} / \mathrm{w.E} .\end{array}$ \\
\hline 185000 & 1758 & 175800 & 1362 & 48580 & 283,5 & 46900 & 1051 \\
\hline 183600 & 4942 & 161400 & 2258 & 48900 & 527,5 & 44320 & 492,6 \\
\hline 180200 & 2224 & 146800 & 1040 & 47320 & 1397 & 40880 & 579,1 \\
\hline 183800 & 2548 & 132800 & 2043 & 48940 & 985,2 & 40860 & 712 \\
\hline 172400 & 1418 & 126600 & 1668 & 47260 & 335,9 & 39920 & 515,6 \\
\hline 176600 & 3472 & 136000 & 2637 & 48980 & 976,6 & 41240 & 1100 \\
\hline 177000 & 2487 & 152800 & 1040 & 46880 & 826,6 & 43140 & 996,9 \\
\hline 175200 & 2968 & 160200 & 1362 & 42200 & 1253 & 39040 & 1653 \\
\hline 184400 & 2258 & 167800 & 1040 & 50000 & 1073 & 47740 & 712 \\
\hline 155800 & 2968 & 139800 & 1844 & 48140 & 749,1 & 45480 & 1323 \\
\hline 155200 & 1040 & 99400 & 2748 & 50320 & 598,8 & 38940 & 643,6 \\
\hline
\end{tabular}

Tabelle 2: Meßwerte der Kinetik 1-Ethinyl-1-methylcyclopropan + F;298 K 


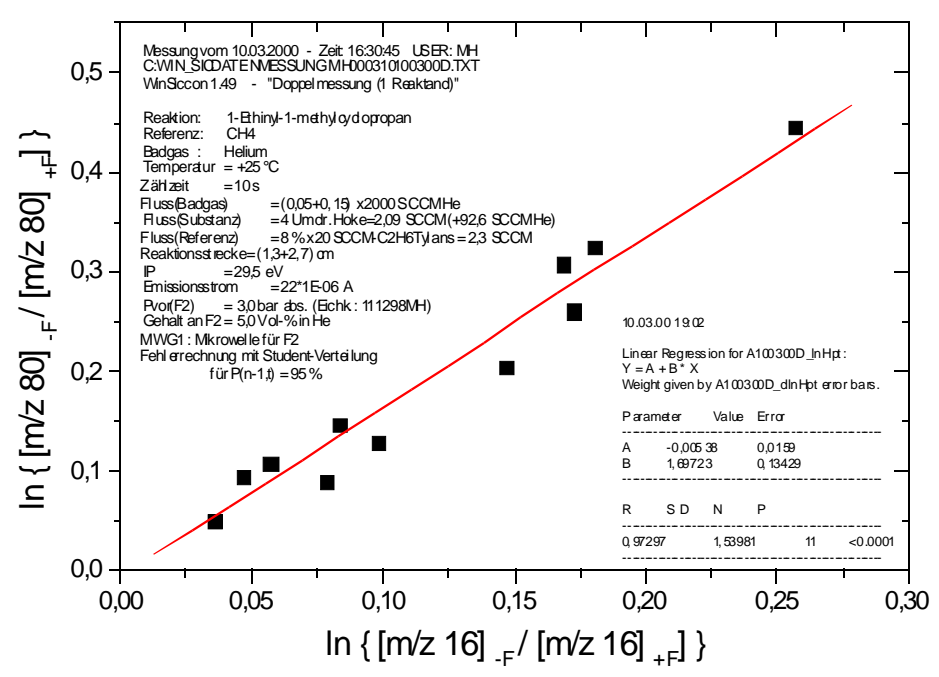

Abbildung 63: Kinetik 1-Ethinyl-1-methylcyclopropan + F; $298 \mathrm{~K}$

Hierzu wurde die Relativmethode (vgl. Kap. 2.1.1.2, S. 10) mit Methan $\left(\mathrm{M}_{\mathrm{r}}=16\right)$ als Referenz verwendet. Mit den gemessenen Intensitäten (DIFF-Werte ${ }^{90}$ ) aus Tabelle 2 konnte eine doppelt-logarithmische Auftragung gemäß Gleichung (10) (S. 10) angefertigt werden, die in Abbildung $63 \mathrm{zu}$ sehen ist. Man kann der Steigung b dieser Auftragung ( $b=1,7 \pm 0,1)$ entnehmen, daß der relative Geschwindigkeitskoeffizient $k_{\text {rel }}$ der Reaktion des 1-Ethinyl-1methylcyclopropans bezogen auf die Referenzreaktion (die Reaktion von Methan mit FAtomen) bei $298 \mathrm{~K}$

$\mathrm{k}_{\mathrm{rel}}=\mathrm{b}=(1,7 \pm 0,1)$ ist.

Der Geschwindigkeitskoeffizient für die Referenzreaktion $\left(\mathrm{CH}_{4}+\mathrm{F} \rightarrow \mathrm{CH}_{3}+\mathrm{HF}\right)$ beträgt ${ }^{91}$ bei $298 \mathrm{~K}: \mathrm{k}_{\mathrm{ref}}=4,72 \cdot 10^{13} \mathrm{~cm}^{3} /(\mathrm{mol} \cdot \mathrm{s})$.

Damit kann man den absoluten Geschwindigkeitskoeffizienten k der Reaktion des 1-Ethinyl-1methylcyclopropans wie folgt berechnen:

$\mathrm{k}=\mathrm{k}_{\mathrm{rel}} \cdot \mathrm{k}_{\mathrm{ref}}=(1,7 \pm 0,1) \cdot 4,72 \cdot 10^{13} \mathrm{~cm}^{3} /(\mathrm{mol} \cdot \mathrm{s})=(8,0 \pm 0,5) \cdot 10^{13} \mathrm{~cm}^{3} /(\mathrm{mol} \cdot \mathrm{s})$.

Damit ist diese Reaktion zwar schnell genug, um unter unseren Reaktionsbedingungen in weniger als $1 \mathrm{~cm}$ Reaktionsstrecke im Strömungsreaktor nahezu vollständig mit 1-Ethinyl-1methylcyclopropan zu reagieren, jedoch stehen mehrere Reaktionskanäle zur Verfügung (vgl. oben Abbildung 62, S. 88).

90 Vgl. dazu die Ausführungen zur Computerauswertung in Kap. 3.1.1 (S. 16 ff.).

91 Atkinson,R.; et al. (1997). 
Im Massenspektrum wurde eine Zunahme auf den Massenzahlen 99 und 118 registriert (bei $\mathrm{T}=298 \mathrm{~K}, \quad \mathrm{IP}=29,5 \mathrm{eV}, \mathrm{s}=4 \mathrm{~cm}, \mathrm{p}_{\mathrm{StrR}}=140 \mathrm{~Pa}$ ), was die Reaktionskanäle (c) und (d) in Abbildung 62 bestätigt. Eine direkte Zunahme auf der Massenzahl 79 konnte selbst bei einer Ionisierungsenergie von $17 \mathrm{eV}$ nicht beobachtet werden, weil dort noch ein sehr großer Fragment-Peak des Edukts lag. Dennoch ließ sich das Radikal auf m/z 79 mittels der in Kap. 3.5.1 (S. 50 ff.) beschriebenen Methode der Umsatzkorrektur nachweisen (bei T=298 K, $\left.\mathrm{IP}=17 \mathrm{eV}, \mathrm{s}=4 \mathrm{~cm}, \mathrm{p}_{\mathrm{StrR}}=145 \mathrm{~Pa}, \phi_{\mathrm{He}}=400 \mathrm{SCCM}, \phi_{\mathrm{Kw}}=2,1 \mathrm{SCCM}\right)$ : Durch die Reaktion mit F-Atomen sank die Intensität des Molekül-Peaks (m/z 80) von $1,700 \cdot 10^{4}$ w.E. auf $1,116 \cdot 10^{4}$ w.E., was einem Umsatz von 34,35 \% entspricht. Die Intensität des Fragment-Peaks (m/z 79)

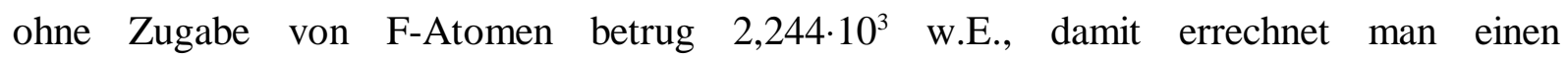
„hypothetischen“ Fragment-Peak bei Zugabe von F-Atomen in Höhe von (1 - 0,3435) . $2,244 \cdot 10^{3}$ w.E. $=1,473 \cdot 10^{3}$ w.E.; tatsächlich auf $\mathrm{m} / \mathrm{z} 79$ gemessen wurde jedoch eine Intensität von $1,739 \cdot 10^{3}$ w.E., so daß die Differenz in Höhe von $1,739 \cdot 10^{3}$ w.E. ./. $1,473 \cdot 10^{3}$ w.E. = 2,66 $10^{2}$ w.E. auf die Bildung des Radikals zurückgeführt werden kann (Gleichung (24), S. 52). Damit sind auch die Reaktionskanäle (a) und/oder (b) in Abbildung 62 (S. 88) bestätigt. Für das strukturell ähnliche 1-Methylcyclopropan läßt sich für den analogen Reaktionskanal (a) eine Standardreaktionsenthalpie bei $298 \mathrm{~K}$ von $-159,3 \mathrm{~kJ} / \mathrm{mol}$ und für Reaktionskanal (b) eine solche von -144,9 kJ/mol ermitteln ${ }^{92}$. Weil darüber hinaus für Kanal (a) vier gleichwertige HAtome zur Abstraktion verfügbar sind, für Kanal (b) jedoch nur drei, ist die Radikalbildungsreaktion statistisch und thermodynamisch bevorzugt (aber keinesfalls ausschließlich) nach Kanal (a) der Abbildung 62 zu erwarten.

Das H-Atom der Ethinyl-Gruppe dürfte hingegen nicht angegriffen werden, weil seine Bindungsenergie mit etwa $552 \mathrm{~kJ} / \mathrm{mol}$ deutlich höher als diejenige der H-Atome in der MethylGruppe $(\approx 438 \mathrm{~kJ} / \mathrm{mol})$ bzw. in dem Cyclopropan-Ring $(\approx 445 \mathrm{~kJ} / \mathrm{mol})$ ist $^{93}$.

Bei der Reaktion mit Chlor- statt Fluor-Atomen beträgt die Standardreaktionsenthalpie bei 298 $\mathrm{K}$ für 1-Methylcyclopropan analog nach Kanal (a) lediglich -21,2 kJ/mol und nach Kanal (b) $-6,8$ kJ/mol. Dadurch könnte die Radikalbildung selektiv nach nur einem Kanal erfolgen. Problematisch dürfte dabei allerdings der sehr geringe Dissoziationsgrad von $\mathrm{Cl}_{2}$ in Höhe nur einiger mol-\% werden, weil dadurch $\mathrm{Cl}_{2}$ im Überschuß zurückbleibt und im hinteren Teil des Reaktors mit Wasserstoff-Atomen in einer offenen Reaktionsfolge reagieren kann, die noch genauer charakterisiert werden muß. 


\subsubsection{Reaktionen nach chemischer Aktivierung}

Das Grundprinzip der hier geplanten chemischen Aktivierung besteht darin, am Kohlenwasserstoff durch F-Atome im vorderen Teil des Strömungsreaktors (Reaktionsstrecke: $1,3 \mathrm{~cm}$ ) ein $\mathrm{H}$-Atom zu abstrahieren und im hinteren Teil (Reaktionsstrecke: $2,7 \mathrm{~cm}$ ) ein $\mathrm{H}-$ oder D-Atom wieder zu addieren (vgl. dazu die in Kap. 3.1.1 beschriebene Apparatur mit dem in Abbildung 6 (S. 17) dargestellten Strömungsreaktor). Dadurch wird der Kohlenwasserstoff wieder zurückgebildet, jedoch verfügt er relativ zu seinem Grundzustand über eine hohe „Überschußenergie“, die eine unimolekulare Reaktion bei niedriger Badgastemperatur ermöglichen kann.

Es ist jedoch möglich, daß die Ringöffnung bereits im Zusammenhang mit der Radikalerzeugung stattfindet, denn diese Reaktion erfolgt z.B. für das Radikal, das aus Methylcyclopropan (welches dem 1-Ethinyl-1-methylcyclopropan strukturell ähnlich ist) erzeugt wird, bereits bei Temperaturen oberhalb $-100{ }^{\circ} \mathrm{C} \quad$ (Abbildung 64) ${ }^{94}$; der Geschwindigkeitskoeffizient dieser Ringöffnungsreaktion beträgt ${ }^{95} \mathrm{k}=2,1 \cdot 10^{8} \mathrm{~s}^{-1}$, ihre Aktivierungsenergie ${ }^{95}$ hat eine Höhe von $32 \mathrm{~kJ} / \mathrm{mol}$.

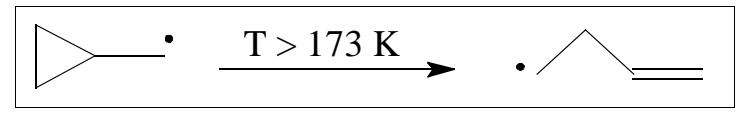

Abbildung 64: Ringöffnung des Radikals

Die chemische Aktivierung wurde typischerweise bei folgenden Reaktionsbedingungen durchgeführt: T $=298 \mathrm{~K}, \mathrm{p}_{\mathrm{StrR}}=138 \mathrm{~Pa}, \phi_{\mathrm{He}}=569 \mathrm{SCCM}, \phi_{\mathrm{KW}}=3,1 \mathrm{SCCM}, \phi_{\mathrm{H} 2}=6,4 \mathrm{SCCM}$. Mit einem Umsatz des Kohlenwasserstoffs bei Zugabe der Fluor-Atome von $45 \%$ ergibt sich ein Fluß des Radikals von $\phi_{\text {Radikal }}=1,4$ SCCM und mit einer Dissoziation des $\mathrm{H}_{2}$ in der MWE von $16 \%$ ergibt sich ein Fluß der H-Atome von $\phi_{\mathrm{H}-\mathrm{Atome}}=2$ SCCM. Für diese Bedingungen soll nun abgeschätzt werden, ob der Geschwindigkeitskoeffizient für die Reaktion des Radikals mit H-Atomen in vergleichbarer Größenordnung wie der für die Ringöffnungsreaktion gemäß Abbildung 64 liegt. Ersterer wird näherungsweise aus Gleichung (14) (S. 11) unter Vernachlässigung des Stoßintegrals (d.h.: $\Omega_{\mathrm{AB}}=1$ ) berechnet. Hierfür wird die Lennard-JonesLänge $\sigma$ für das H-Atom auf $\sigma=260 \mathrm{pm}$ und für den KW 1-Ethinyl-1-methylcyclopropan auf $\sigma=550 \mathrm{pm}$ geschätzt ${ }^{96}$. Aus den oben genannten Reaktionsbedingungen berechnet man gemäß Gleichung (19) (S. 14) eine Konzentration der H-Atome in Höhe von 1,9·10-10 $\mathrm{mol} / \mathrm{cm}^{3}$. Mit all

94 Lowry, T.; Richardson, K. (1980); S. 548 und 562.

95 Carey, F.A.; Sundberg, R.J. (1995); S. 653 Tabelle 12.2 / siehe auch: Mathew, L.; Warkentin, J. (1986).

96 Vgl. dazu die Werte von 258 pm für He, 297 pm für Wasserstoff-Moleküle und 527 pm für Benzol, die entnommen sind aus: Atkins, P. (1990); Tabelle 24-4, S. 872. 
diesen Angaben kann mittels der vereinfachten Gleichung (14) (S. 11) die Stoßzahl Z* von einem Teilchen mit allen H-Atomen errechnet werden: $\mathrm{Z}^{*}=\mathrm{Z} \cdot[\mathrm{H}]=1,5 \cdot 10^{5} \mathrm{~s}^{-1}$. Dieser Wert ist etwa einen Faktor 1000 kleiner als der Geschwindigkeitskoeffizient für die Ringöffnungsreaktion in Abbildung 64.

Somit ist es in der Tat sehr wahrscheinlich, daß auch für das hier untersuchte 1-Ethinyl-1methylcyclopropan die Öffnung des Rings unmittelbar nach der Radikalerzeugung und noch vor Reaktion des cyclo-KW-Radikals mit H-Atomen stattfindet. In diesem Fall würde sich die chemische Aktivierung quasi aus zwei Schritten zusammensetzten (Abbildung 65): (1.) die Radikalerzeugung mit anschließender Ringöffnung und (2.) die Reaktion des nunmehr offenkettigen Radikals mit H-Atomen.

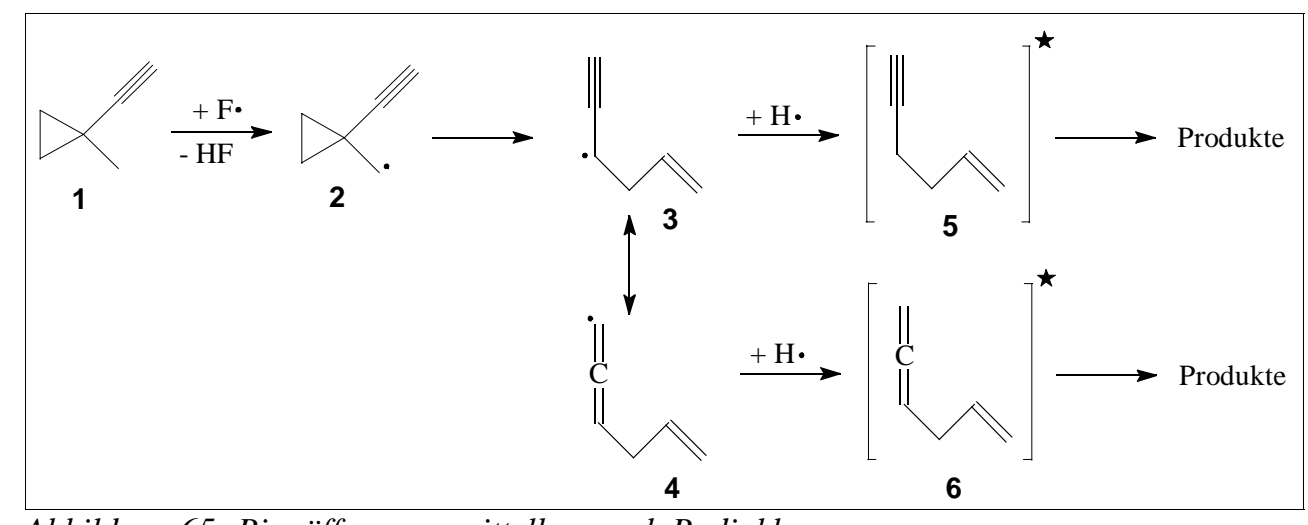

Abbildung 65: Ringöffnung unmittelbar nach Radiaklerzeugung

Falls obige Annahmen aber nicht zutreffen, könnte die chemische Aktivierung auch zu einem energetisch angeregten 1-Ethinyl-1-methylcyclopropan führen, das dann ähnliche Reaktionen wie bei der thermischen Aktivierung eingehen kann.

Das entspricht den Reaktionskanälen (a) und (b) in Abbildung 66.

Vorversuche in Abwesenheit von Fluor haben bei Zugabe von Deuterium-Atomen eine Zunahme auf der Massenzahl 84 gezeigt, wodurch Kanal (f) bestätigt wurde. Es kommt also auch zu einer Addition an die Dreifachbindung; aus diesem Grund muß ebenfalls Kanal (c) bei der Auswertung in Betracht gezogen werden.

Gleiches gilt für Fluor-Atome, die wegen ihrer hohen Reaktivität ebenfalls die Dreifachbindung der Ethinyl-Gruppe angreifen können; dadurch sind die Kanäle (d) und (e) möglich, was im vorangegangenen Unterkapitel auch nachgewiesen wurde.

Es ist denkbar, daß die in Abbildung 66 mit einem $\star$ gekennzeichneten angeregten Kohlenwasserstoffe aus den Kanälen (a) bis (d) sowie (e1) analog dem diradikalisch formulierten Mechanismus bei der thermischen Aktivierung reagieren (vgl. Abbildung 
Abbildung 18, S. 62).

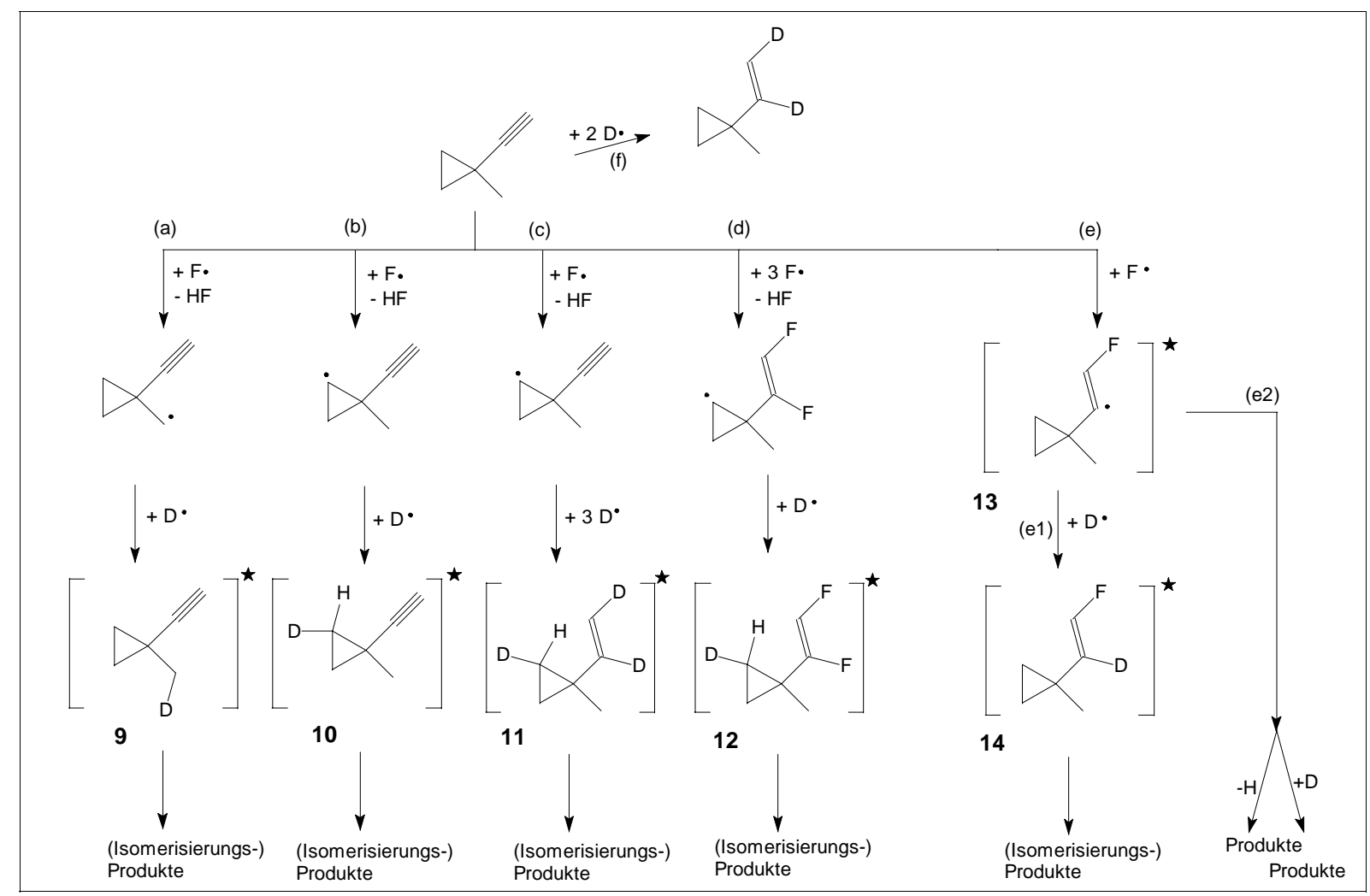

Abbildung 66: Denkbare Reaktionskanäle bei chemischer Aktivierung

Abbildung 67 zeigt die theoretisch möglichen weiteren Reaktionen für $\mathbf{1 0}$ aus Kanal (b).

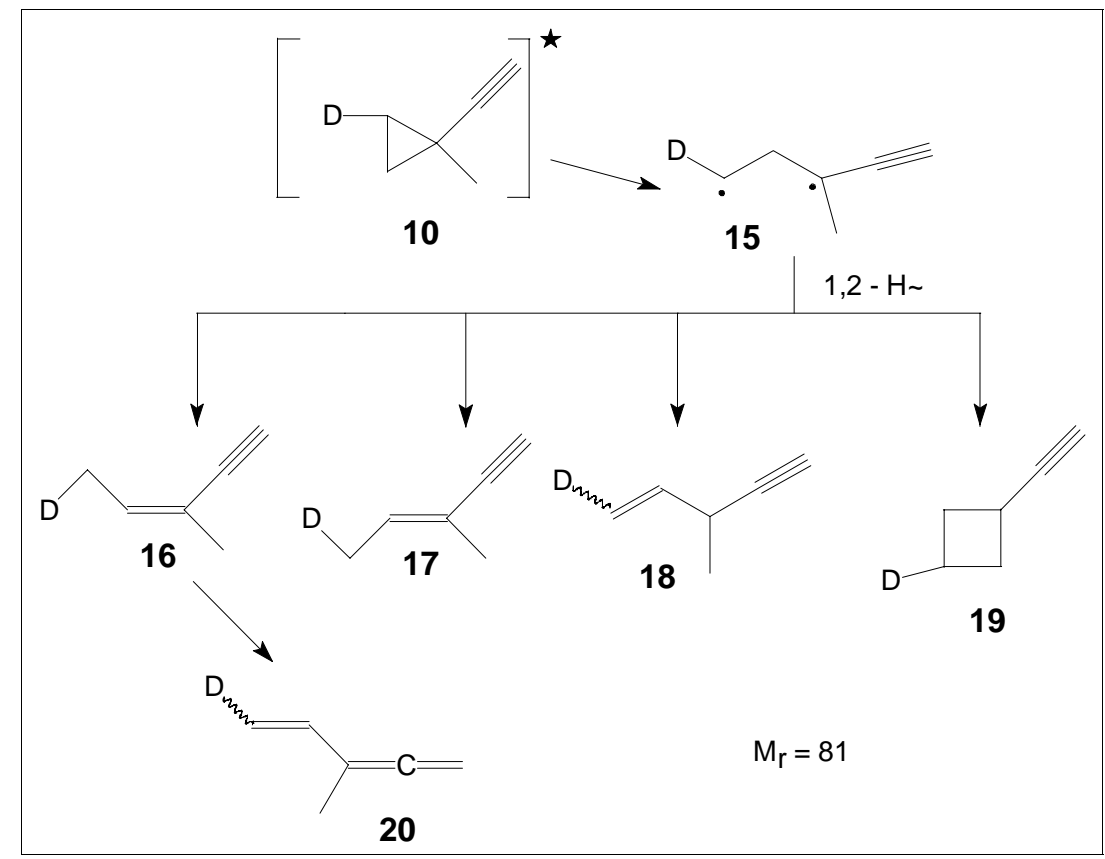

Abbildung 67: Denkbare Produkte aus Kanal (b) der Abb. 66

In Abbildung 68 sind die theoretisch möglichen weiteren Reaktionen für Verbindung $\mathbf{1 1}$ aus 
Kanal (c) dargestellt.

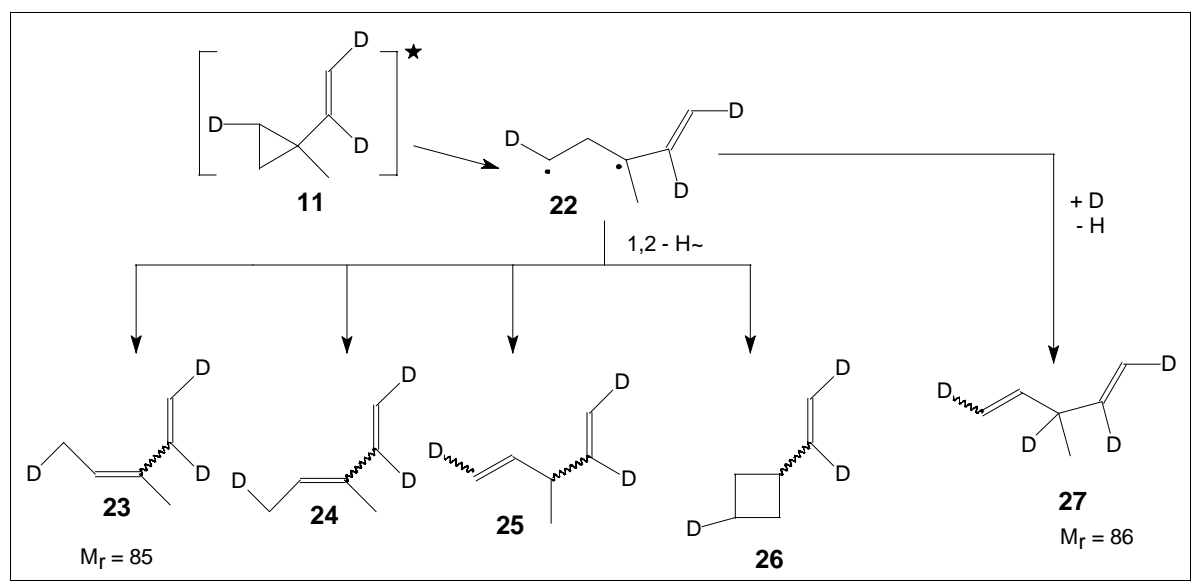

Abbildung 68: Denkbare Produkte aus Kanal (c) der Abb. 66

Abbildung 69 zeigt die theoretisch möglichen weiteren Reaktionen für 12 aus Kanal (d).

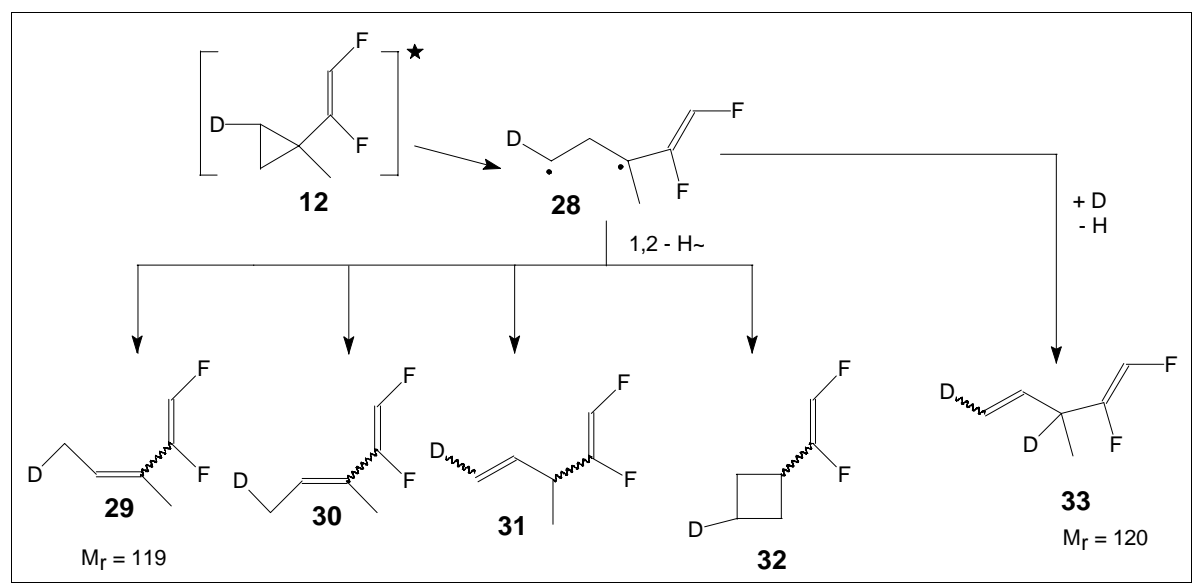

Abbildung 69: Denkbare Produkte aus Kanal (d) der Abb. 66

Abbildung 70 zeigt die theoretisch möglichen weiteren Reaktionen für $\mathbf{1 3}$ aus Kanal (e).

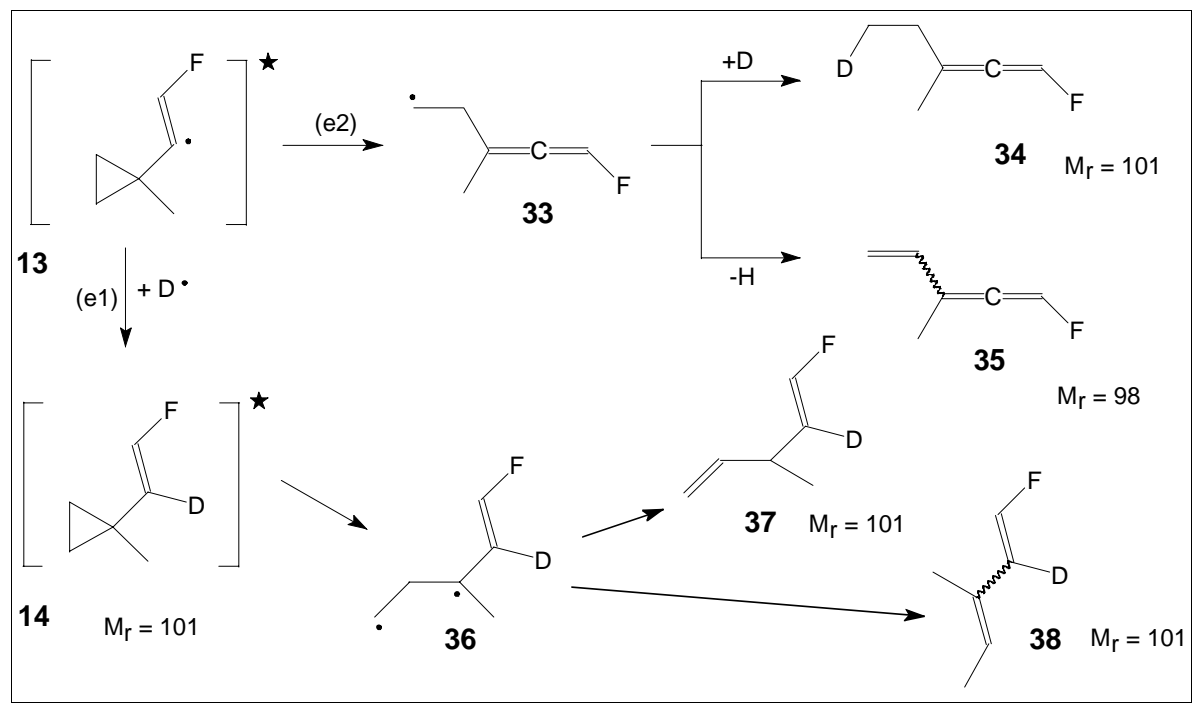

Abbildung 70: Denkbare Produkte aus Kanal (e) der Abb. 66 
Die chemische Aktivierung von 1-Ethinyl-1-methylcyclopropan wurde sowohl mit Wasserstoff- als auch mit Deuteriumatomen durchgeführt. Weil Deuterium durch die Isotopenmarkierung oft einen besseren Einblick in die ablaufenden Mechanismen gestattet, sollen hier jedoch vorwiegend die Reaktionen mit diesem behandelt werden.

Die Arbeiten wurden bei einem Druck im Strömungsreaktor von 1,5 bis 2,5 hPa durchgeführt, wobei zur Vermeidung von Folgereaktionen in hoher Verdünnung $([\mathrm{He}] \approx 98$ Vol-\%) gearbeitet wurde. Da der Dissoziationsgrad von $\mathrm{D}_{2}$ lediglich bei $10-15$ mol- $\%$ lag, mußte eine entsprechend große Menge zugesetzt werden, um den potentiell gebildeten Radikalen ausreichend Reaktionspartner anbieten zu können. Alle Untersuchungen wurden bei $298 \mathrm{~K}$ in dem in Abbildung 6 (S. 17) dargestellten Strömungsreaktor durchgeführt.

Bei den Massenspektren mit Probennahme direkt aus dem Molekularstrahl (vgl. Kap. 3.1.1, S. 16 sowie Kap. 3.5.1, S. 50) zeigten sich mit EI-Elektronenenergien von IP $=29,5 \mathrm{eV}$ Zunahmen auf den Massenzahlen 84 und 81, mit IP = 19 eV zudem auf 40, 41, 67, 68 und 69 (Abbildung 71). Weil aber die meisten mutmaßlichen Produkte ähnlich dem immerhin noch zu 60 bis 80 mol-\% enthaltenen Edukt fragmentieren, ist eine Interpretation ohne vorherige GCTrennung nur begrenzt möglich. Insbesondere die Zunahme auf den Massenzahlen 81 und (als Fragment) 40 dürfte aber die Kanäle (a) und/oder (b) aus Abbildung 66 (S. 93) bestätigen (vgl. dazu Abbildung 67, S. 93).

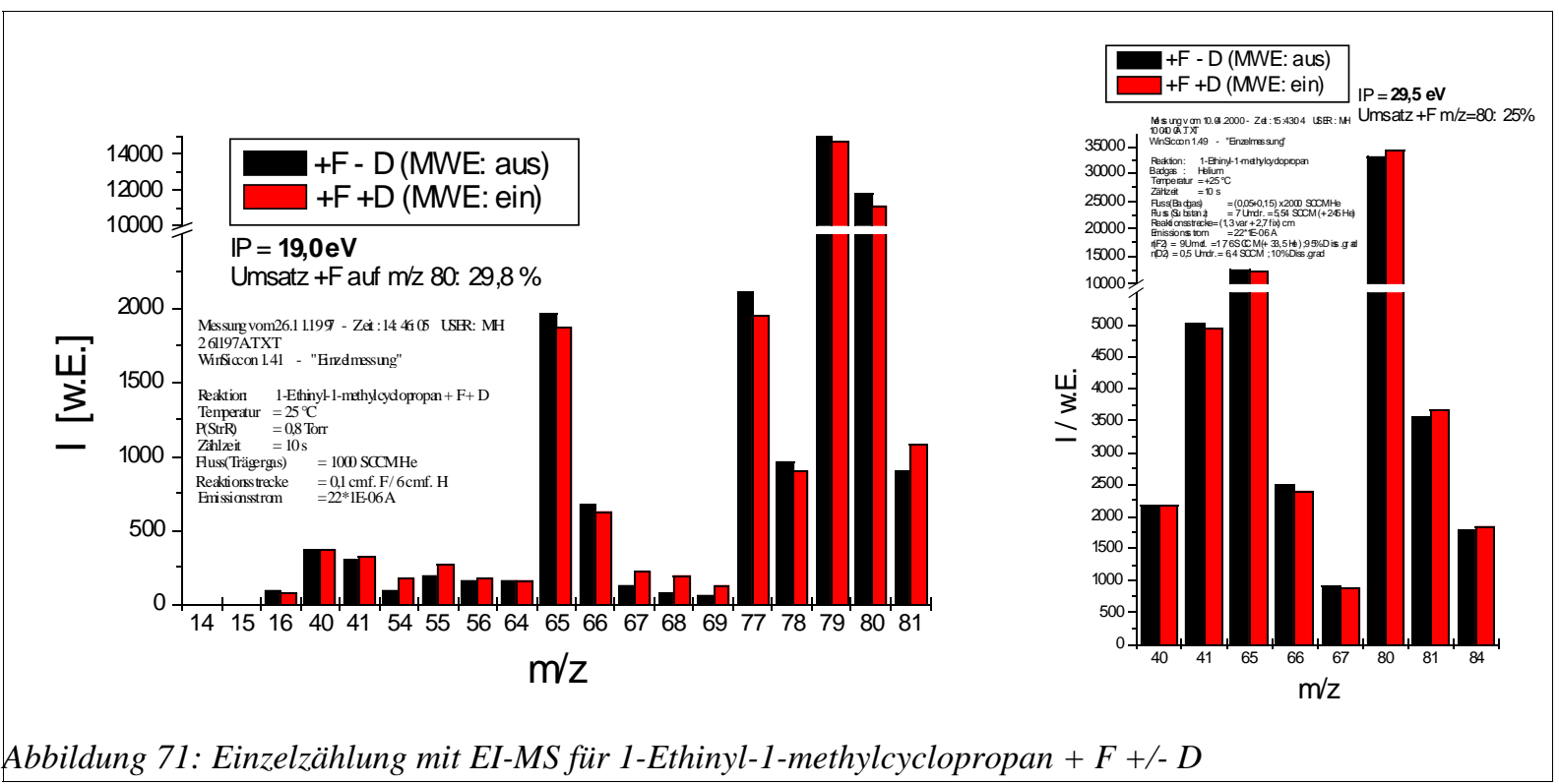

Ergänzend wurden die in der Kühlfalle ausgefrorenen Produkte im FT-IR-Spektrometer untersucht. Dazu wurde die Kühlfalle beginnend bei $77 \mathrm{~K}$ langsam auf $298 \mathrm{~K}$ erwärmt und 
quasi als fraktionierende Destillation die in die Gasphase übertretenden Produkte nacheinander in der Multireflektionskugekzelle mittels FT-IR untersucht (vgl. dazu auch Kap. 3.5.5, S. 59). Selbstverständlich konnte damit nicht die Trennqualität einer GC-Trennung erreicht werden, aber die ,ersten Fraktionen“ sind noch frei vom Edukt und können daher recht gut interpretiert werden.

In diesem Spektrum in Abbildung 72 deutet die Bande bei $3034 \mathrm{~cm}^{-1}$ auf die $\mathrm{CH}-$ Valenzschwingung eines Alkens $(\mathrm{C}=\mathrm{CH})$ oder einer $\mathrm{CH}_{\mathrm{x}} \mathrm{F}-\mathrm{Gruppe}$ hin. Die beiden Banden bei 1957 und $1928 \mathrm{~cm}^{-1}$ sprechen für das Vorliegen eines Allens $(\mathrm{C}=\mathrm{C}=\mathrm{C})$, wobei die Doppelbande entweder ein terminales Allen oder aber das Vorhandensein einer elektronenziehenden Gruppe (z.B. F-Atom) indiziert ${ }^{97}$. Aus Vergleichsspektren diverser Allen-Derivate ergibt sich, daß die Doppelbande bei 1740 und $1728 \mathrm{~cm}^{-1}$ von einem (ggf. auch F-substituierten) Allen herrühren könnte. Die Banden bei 1667 und $1637 \mathrm{~cm}^{-1}$ sprechen für eine nicht konjugierte Doppelbindung; in Verbindung mit der Bande bei $802 \mathrm{~cm}^{-1}$ könnte es sich einerseits um ein dreifach substituiertes Alken $\left(\mathrm{R}_{2} \mathrm{C}=\mathrm{CHR}\right)$ handeln, in Verbindung mit den Banden bei 730 und $700 \mathrm{~cm}^{-1}$ kommen andererseits zweifach cis-substituierte Alkene in Betracht (RHC=CHR). Die starke Bande bei $1151 \mathrm{~cm}^{-1}$ deutet schließlich auf ein Fluor-Atom an einer Alken-Gruppe hin. Die starke Bande bei $1028 \mathrm{~cm}^{-1}$ stammt wahrscheinlich von $\mathrm{SiF}_{4}$, das durch die Reaktion von F-Atomen mit der Wand des Glasreaktors entsteht ${ }^{98}$.

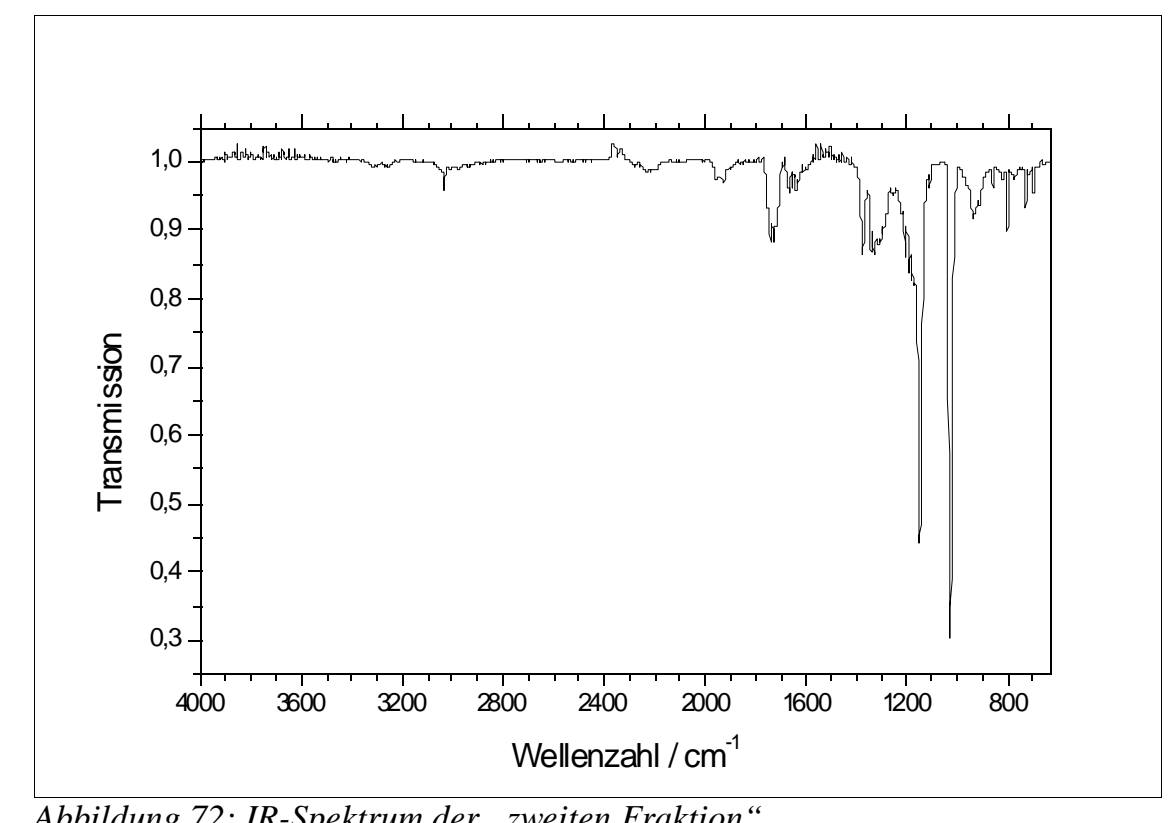

In den weiteren „Fraktionen“ vermindern sich diese Banden kontinuierlich und es treten solche hervor, die identisch mit denen im Spektrum des Edukts sind (Abbildung 73).

97 Hesse, M.; Meier, H.; Zeeh, B. (1995); S. 48.

98 Vgl. das IR-Spektrum im NIST Chemistry WebBook (2001), das nur eine Bande bei $1025 \mathrm{~cm}^{-1}$ aufweist. 


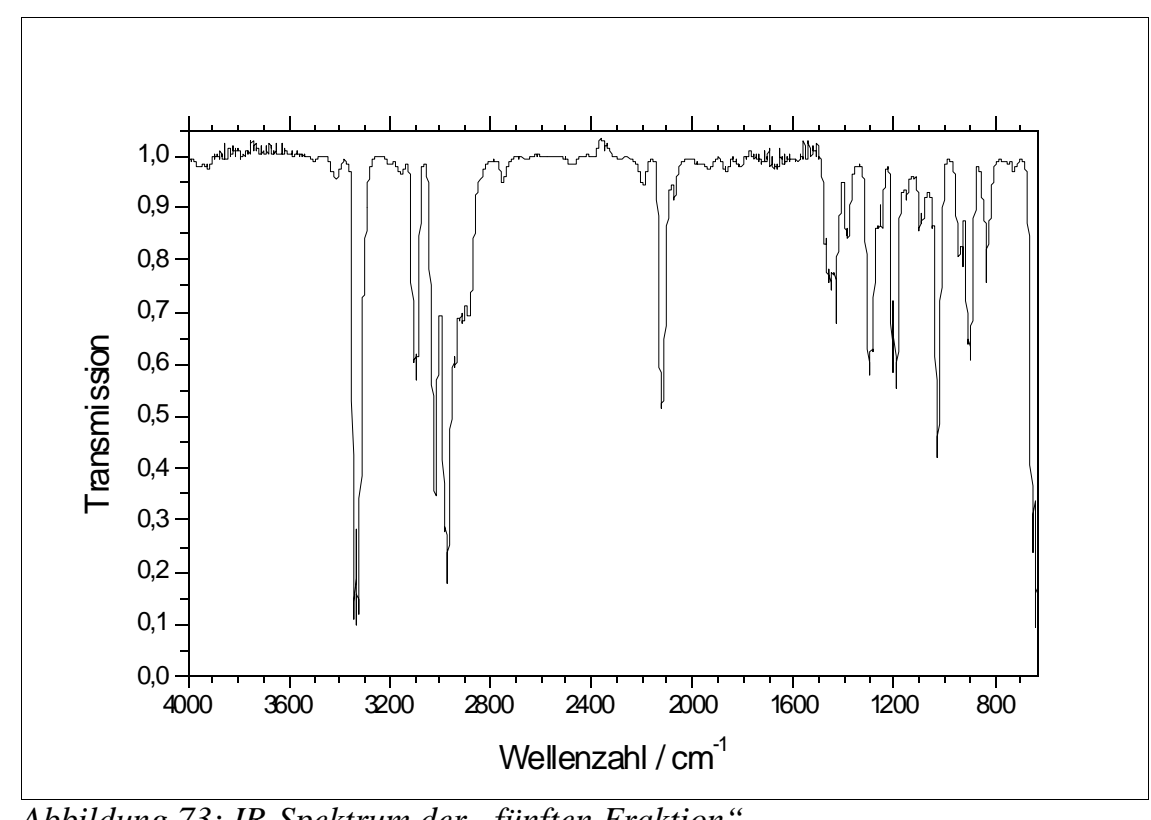

Abbildung 73: IR-Spektrum der „fünften Fraktion“

Um einen Überblick über die Anzahl der Reaktionsprodukte und deren Massenspektren zu erhalten, wurde eine GC-MS-Analyse vorgenommen.

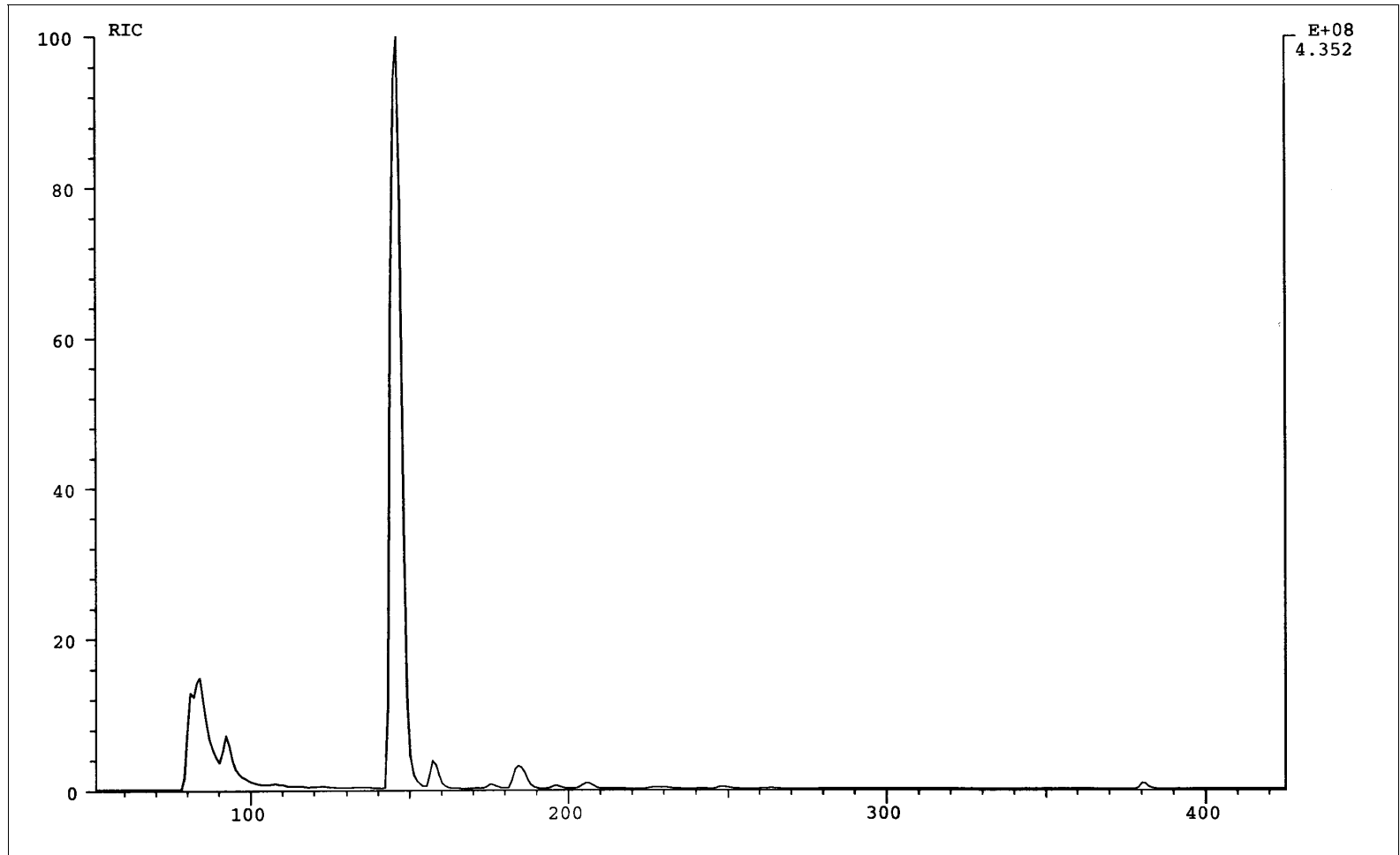

Abbildung 74: GC-Spektrum bei $30^{\circ} \mathrm{C}$ Säulentemperatur

Jedoch mußte auf eine Kapillarsäule vom Typ SE 54 (25 m) zurückgegriffen werden, die nach den Ergebnissen für unser Trennproblem nur bedingt geeignet scheint. Bei einer Säulentemperatur über $100{ }^{\circ} \mathrm{C}$ konnten im wesentlichen nur 2-3 GC-Fraktionen erhalten 
werden und auch die Absenkung auf $30{ }^{\circ} \mathrm{C}$ zeigt noch einige offensichtlich nicht vollständig getrennte Fraktionen (vgl. Abbildung 74).

Die erste Fraktion im GC-Peak Nr. 85 (Abbildung 21) hat einen Hauptpeak bei der Massenzahl 85 und daher dürfte sie neben anderen unidentifizierten Substanzen $\mathrm{SiF}_{4}$ enthalten, das durch die Reaktion von F-Atomen mit der Reaktorwand gebildet worden sein dürfte.

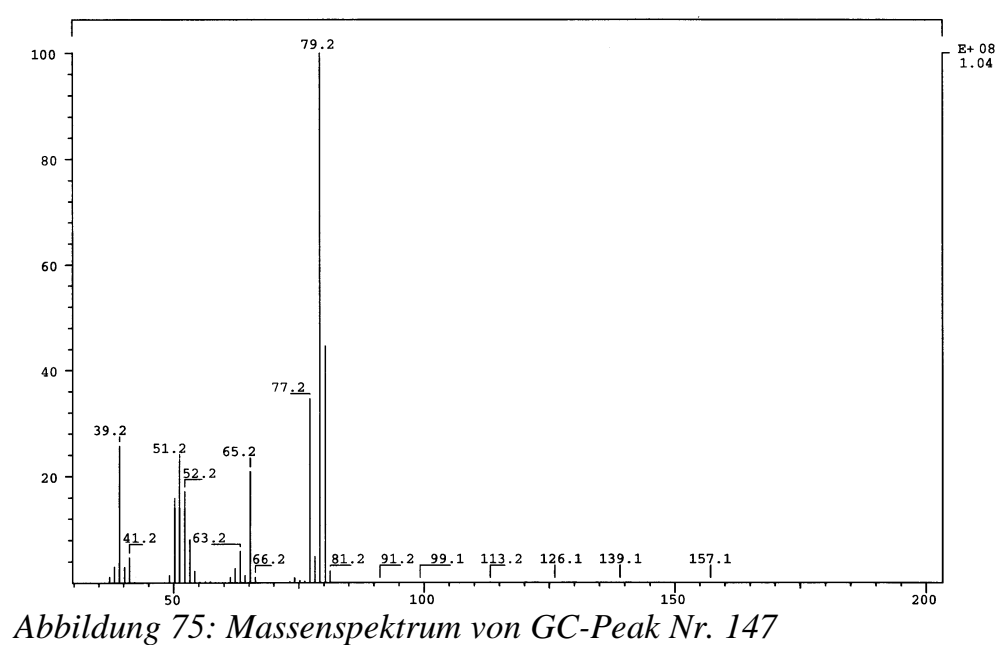

Der größte GC-Peak Nr. 147 zeigt im wesentlichen die Massenzahlen, die auch im Spektrum des Edukts vorhanden sind und dürfte daher hauptsächlich aus dem Edukt bestehen. Es wäre jedoch möglich, daß auch ungetrennte Produkte mit gleichem Fragmentierungsmuster in dieser Fraktion enthalten sind. Dieses Problem wurde bereits im Zusammenhang mit der thermischen Aktivierung diskutiert.

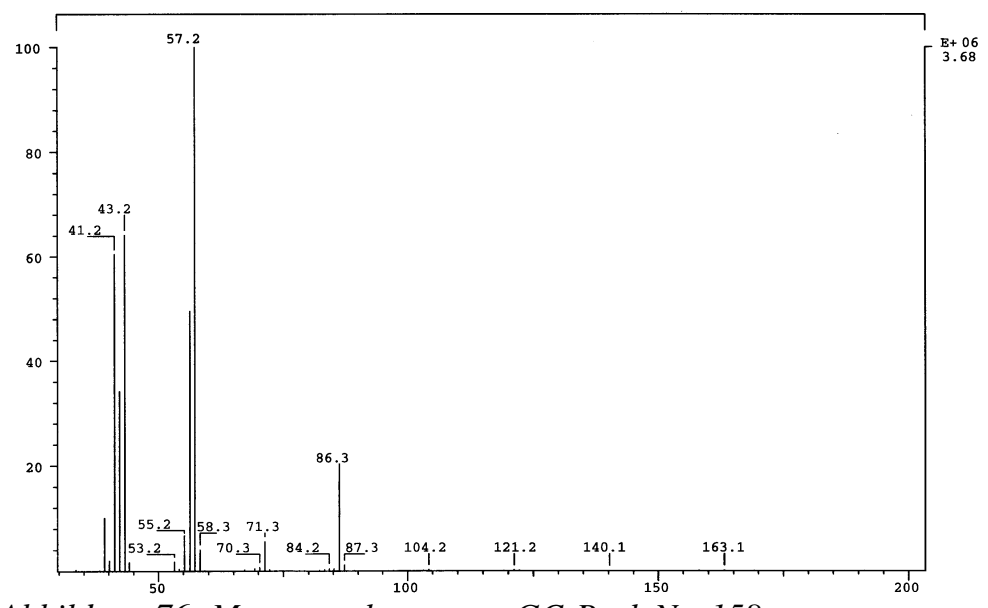

Abbildung 76: Massenspektrum von GC-Peak Nr. 158

Das Massenspektrum von GC-Peak Nr. 158 (Abbildung 76) könnte durch Verbindung 27 in Abbildung 68 (S. 94) erklärt werden. Dies wäre zwar durch die IR-Analyse gedeckt, jedoch fehlen entsprechende Isotopen-Peaks, die bei diesem Mechanismus wahrscheinlich auftreten würden; auch zeigt das Spektrum keine gute Übereinstimmung mit einem Literaturspektrum 
der nicht deuterierten Verbindung.

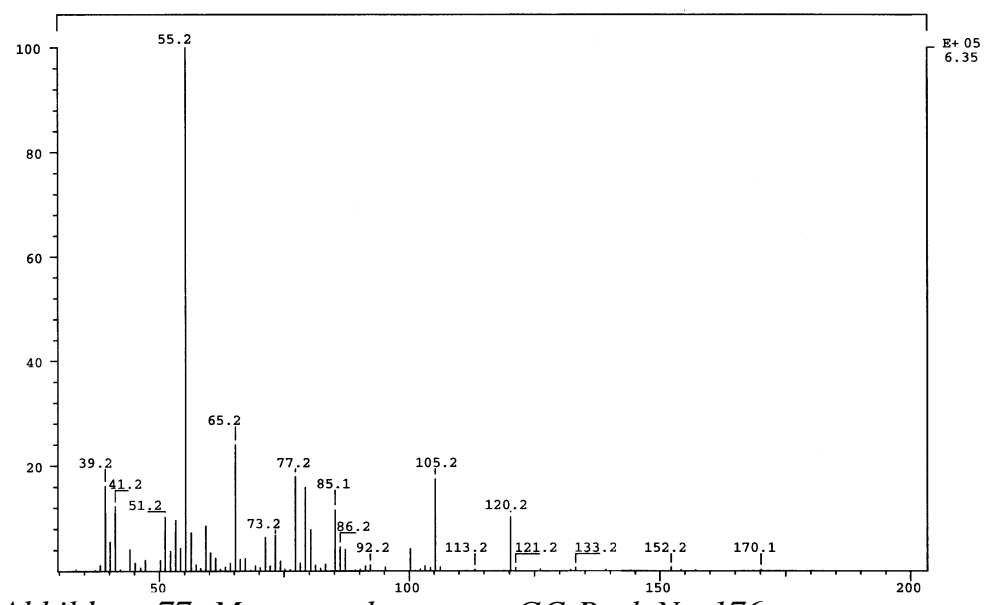

Abbildung 77: Massenspektrum von GC-Peak Nr. 176

Das zuletzt Gesagte gilt analog auch für das Spektrum von GC-Peak Nr. 176 (Abbildung 77), das sich abgesehen von diesen Einwänden durch Verbindung 33 in Abbildung 69 (S. 94) erklären ließe.

Das Massenspektrum von GC-Peak Nr. 184 (Abbildung 78) könnte durch Verbindung 34 in Abbildung 70 (S. 94) erklärt werden.

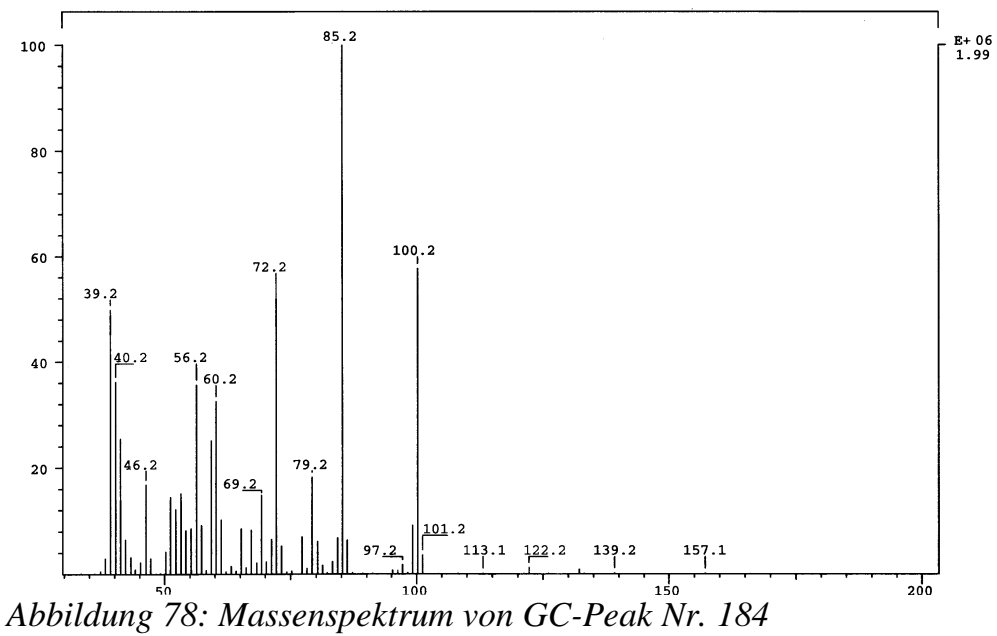

Für die Verbindungen der GC-Peaks Nr. 196 (Hauptpeak auf Massenzahl 151) und 206 können keine Vorschläge gemacht werden; möglicherweise handelt es sich um das Kombinationsprodukt zweier angeregter Moleküle. Der letzte GC-Peak hat einen intensiven Peak auf der Massenzahl 98; dafür käme prinzipiell Verbindung 35 in Abbildung 70 (S. 94) in Betracht.

Die chemische Aktivierung mit H- statt D-Atomen führt (erwartungsgemäß) zu den gleichen 
Resultaten (vgl. Abbildungen 79 und 80).

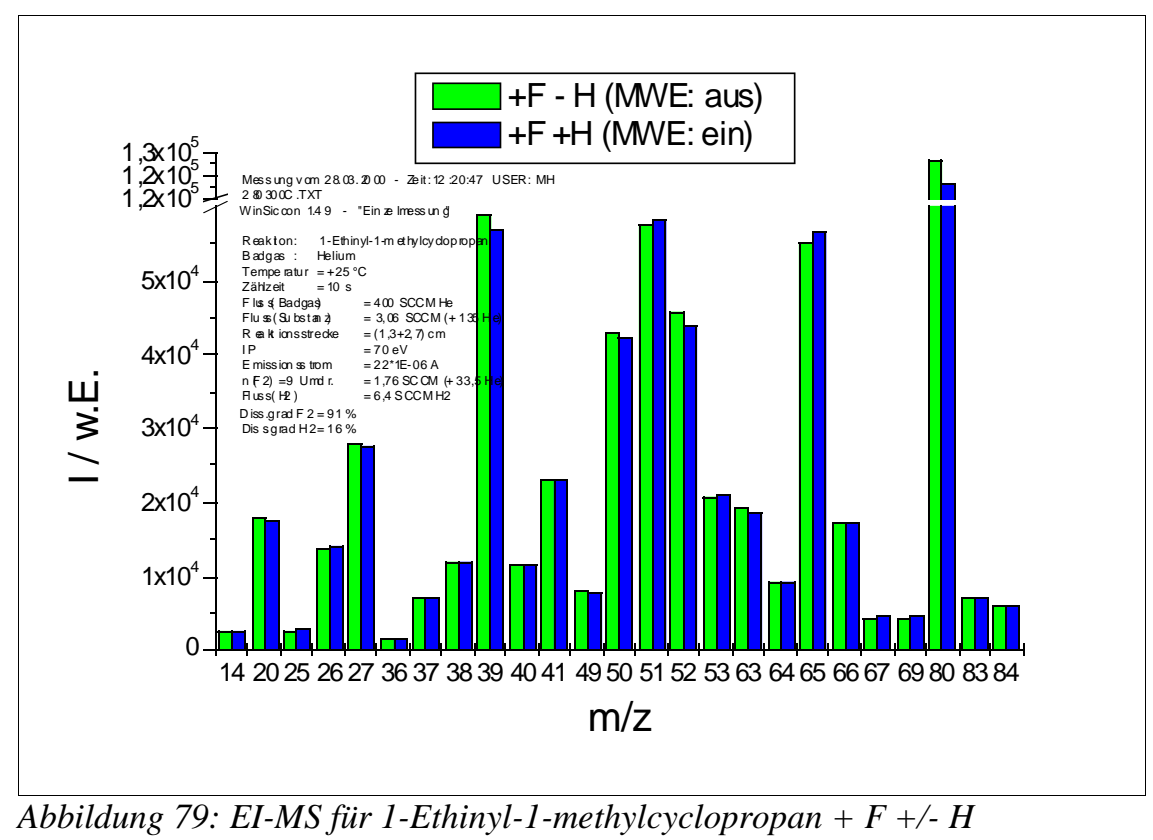

Von der ausgefrorenen Produkt-Mischung aus dem in Abbildung 79 beschriebenen Experiment wurde das in Abbildung 80 gezeigte FT-IR-Spektrum registriert.

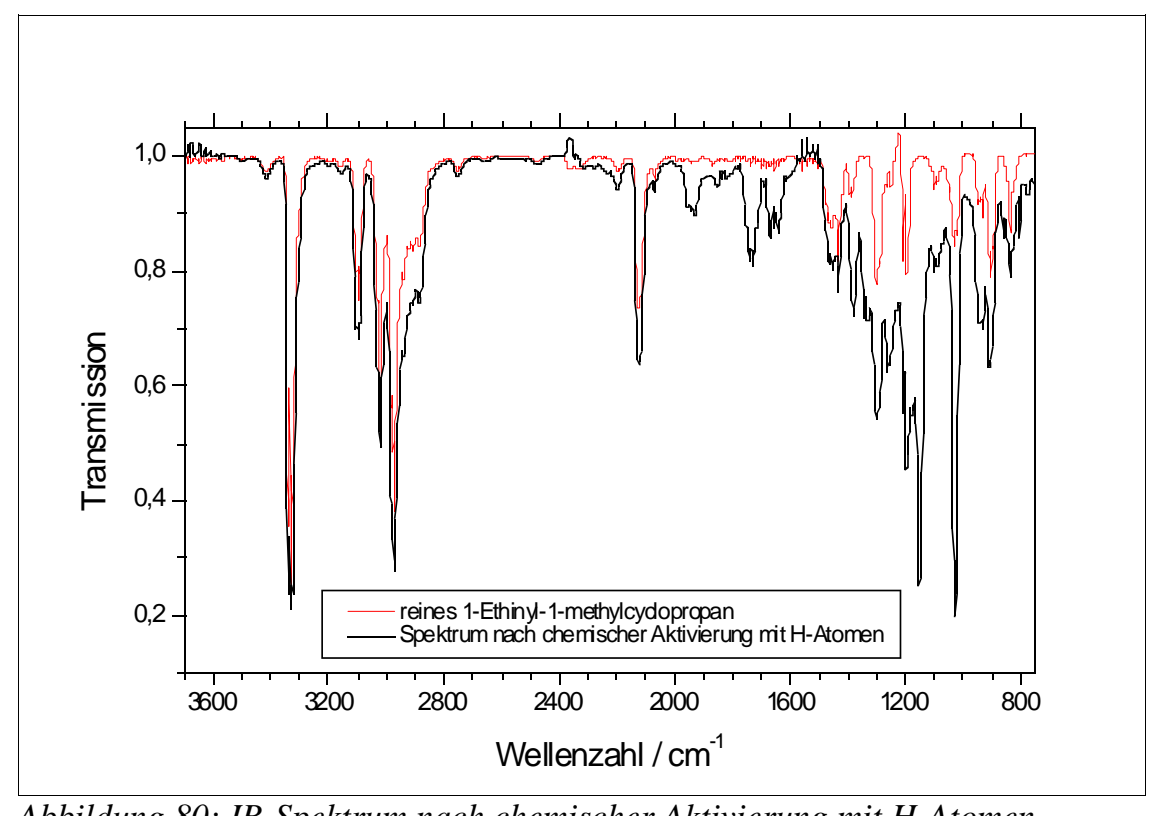

Abbildung 80: IR-Spektrum nach chemischer Aktivierung mit H-Atomen

Das IR-Spektrum nach chemischer Aktivierung mit H-Atomen in Abbildung 80 zeigt neue Banden bzw. Zunahmen auf den Banden bei 773 (w), 802 (m), 837 (m), 862 (w), 902 (s), 910 (s), 931 (s), 941 (s), 987 (w), 1028 (s), 1088 (w), 1099 (w), 1151 (s), 1176 (w), 1196 (s), 1207 (m), 1251 (w), 1261 (w), 1288 (m), 1302 (s), 1331 (w), 1342 (w), 1377 (m), 1431 (m), 1452 (w), 1462 (w), 1610 (w), 1641 (s), 1655 (s), 1666 (s), 1691 (w), 1728 (s), 1739 (s), $1819(\mathrm{w}), 1836$ (w), 1851-1860 (m), 1875 (w), 1928 (s), 1944 (s), 1956 (s), 2071 (w), 2124 
(s), 2197 (m), 2885 (m), 2912 (w), 2139 (w), 2971 (s), 3020 (s), 3097 (s), 3157 (w), 3325 (s), $3330(\mathrm{~s})$ und $3340(\mathrm{~s}) \mathrm{cm}^{-1}$.

Die Diskussion dieser Banden erfolgte im wesentlichen bereits im Zusammenhang mit den Ergebnissen aus den Abbildungen 72 und 73 (S. 96). Weitergehende Zuordnungen können auch hier nicht getroffen werden.

Es bleibt zu erwähnen, daß die Banden bei 1431, 2071, 2973, 3020, 3097, 3325, 3330 und $3340 \mathrm{~cm}^{-1}$ identisch mit denen des Edukts bzw. nur geringfügig stärker sind. 


\subsubsection{Diskussion der Ergebnisse}

In der Literatur wurde lediglich eine Untersuchung der thermischen Aktivierung von der strukturell sehr ähnlichen Verbindung Ethinylcyclopropan gefunden ${ }^{99}$. Die Ergebnisse der dort beschriebenen Gasphasenpyrolyse bei $600{ }^{\circ} \mathrm{C}$ sind in Abbildung 81 zusammengestellt.

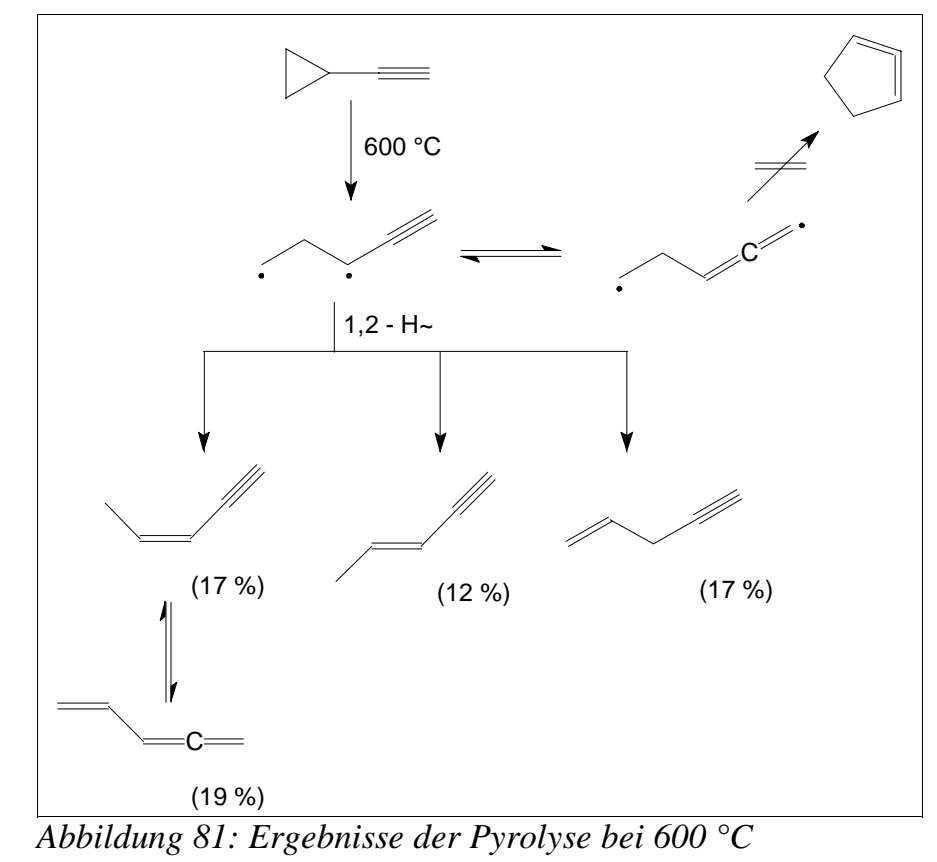

Aus den FT-IR- und GC-MS-Analysen der Produktmischungen der hier durchgeführten thermischen Aktivierungen von 1-Ethinyl-1-methylcyclopropan bei 580, 632 und $680{ }^{\circ} \mathrm{C}$ ergaben sich zahlreiche Hinweise darauf, daß dabei die zur Abbildung 81 analogen Produkte entstanden sind. Insofern sind diese Ergebnisse im Einklang mit der Literatur. Bedingt durch das Vorhandensein der Methylgruppe eröffnen sich für 1-Ethinyl-1-methylcyclopropan jedoch noch weitere Reaktionskanäle, für die es bei höheren Temperaturen Anzeichen gab.

Weil aber die authentischen GC-, MS- und IR-Spektren der mutmaßlichen Produkte aus Abbildung 18 (S. 62) nicht vorlagen und im Rahmen der Untersuchung auch einige Schwächen der berechneten IR-Spektren zu Tage traten, kann eine quantitative Auswertung nicht erfolgen. Aus der Tatsache, daß für die Pyrolyse bei $532{ }^{\circ} \mathrm{C}$ ein Umsatz des Edukts kaum meßbar war, dieser bei $632{ }^{\circ} \mathrm{C}$ aber auf ca. $96 \%$ gestiegen war und bei $680{ }^{\circ} \mathrm{C}$ wohl nahezu $100 \%$ betrug, konnte eine Aktivierungsenergie für die Isomerisierungsreaktion des 1-Ethinyl-1methylcyclopropans von $189 \mathrm{~kJ} / \mathrm{mol}$ abgeschätzt werden. Für Ethinylcyclopropan war eine Aktivierungsenergie nicht angegeben. Bei anderen Ethinyl- oder Ethenyl-substituierten Cyclopropanderivaten liegt sie deutlich niedriger $(130-150 \mathrm{~kJ} / \mathrm{mol})^{100}$; jedoch dürfte dies auf 
das Vorhandensein von Methylgruppen am relativ zur Ethinyl- oder Ethenylgruppe benachbarten Ring-C-Atom zurückzuführen sein, die einen anderen Reaktionsmechanismus ermöglichen ${ }^{100}$. Methylcyclopropan hingegen hat eine wesentlich höhere Aktivierungsenergie ${ }^{101}$ von $272 \mathrm{~kJ} / \mathrm{mol}$. Auch für die Isomerisierung von Cyclopropan zu Propen liegt sie bei ${ }^{102} 274$ $\mathrm{kJ} / \mathrm{mol}$. Es ist also davon auszugehen, daß die Ethinyl-Gruppe die Aktivierungsenergie für eine Isomerisierungsreaktion wesentlich herabsetzt ${ }^{103}$.

Der gemessene Geschwindigkeitskoeffizient für die radikalerzeugende Reaktion mit F-Atomen in Höhe von $(8,0 \pm 0,5) \cdot 10^{13} \mathrm{~cm}^{3} /(\mathrm{mol} \cdot \mathrm{s})$ liegt etwas unter dem des Cyclopropans mit $1,3 \cdot 10^{14}$ $\mathrm{cm}^{3} /(\mathrm{mol} \cdot \mathrm{s})$. Dies erscheint aber plausibel, weil für den wahrscheinlich thermodynamisch bevorzugten Kanal (a) der Abbildung 62 (S. 88) durch die Methyl- und Ethinyl-Substituenten relativ zum Cyclopropan statistisch $2 \mathrm{H}$-Atome weniger zur Verfügung stehen.

Bezüglich der chemischen Aktivierung bestand schon Unsicherheit darüber, ob die Öffnung des Cyclopropan-Rings bereits unmittelbar nach der Radikalerzeugung mit F-Atomen und noch vor Reaktion dieses Radikals mit Wasserstoff-Atomen erfolgt. Insofern würde ein Teil der Energie aus der chemischen Aktivierung erst der bereits azyklisch gewordenen Verbindung zugeführt, was den Vergleich mit der thermischen Aktivierung unmöglich macht.

Ferner haben die Analysen gezeigt, daß (unter anderem auch auf Grund der reaktiven EthinGruppe) eine sehr hohe Zahl potentieller Reaktionskanäle zu beachten ist, deren systematische Untersuchung fast unmöglich erscheint. Hierfür müßten quantenchemische Berechnungen den Experimenten quasi den Weg weisen, indem sie Voraussagen treffen, welche Kanäle bevorzugt zu erwarten sind. Dann müßten die authentischen Verbindungen der potentiellen Produkte synthetisiert werden, um deren Existenz im Experiment gezielt überprüfen zu können. Ohne solch eine theoretische Begleitung scheint die chemische Aktivierung allerdings keine sinnvolle Ergänzung zur thermischen zu sein. Das prinzipielle Problem der chemischen Aktivierung für eine GC-Analyse ist, daß etwa 60-80 \% des Edukts in der Reaktionsmischung zurückbleiben und lediglich die umgesetzten 20-40 \% zu den Produkten reagieren. Bei der GC-MS-Analyse im Rahmen der thermischen Aktivierung hatte sich jedoch die Schwierigkeit gezeigt, eine vollständige Trennung zu erzielen, obwohl dort kaum noch Edukt in der Mischung war. Es ist zu befürchten, daß deshalb einige Produkte im großen GC-Peak des Edukts ,untergehen“; dies war vermutlich auch bei der hier im Rahmen der chemischen Aktivierung durchgeführten GCMS-Analyse der Fall.

101 Chesick, J.P. (1960).

102 Rickborn,S.F.; et al. (1986).

103 Vgl. dazu auch: Hellwig, J. (1999); S. 15. 


\subsection{Untersuchungen teilfluorierter Ether}

\subsubsection{Der Bis(difluormethyl)ether $\left(\mathrm{CHF}_{2}-\mathrm{O}-\mathrm{CHF}_{2}\right)$}

\subsubsection{Produkte der radikalerzeugenden Reaktion mit Fluor-Atomen}

Eine ausführliche Produktuntersuchung für die radikalerzeugende Reaktion des $\mathrm{CHF}_{2} \mathrm{OCHF}_{2}$ mit F-Atomen wurde zwar nicht vorgenommen, jedoch zeigt die HF-Bildung auf m/z 20 als Funktion der Konzentration des Ethers bei sonst konstanten Bedingungen in Abbildung 82, daß die Abstraktion eines H-Atoms des Ethers durch F-Atome sehr wahrscheinlich ist. Die Intensität

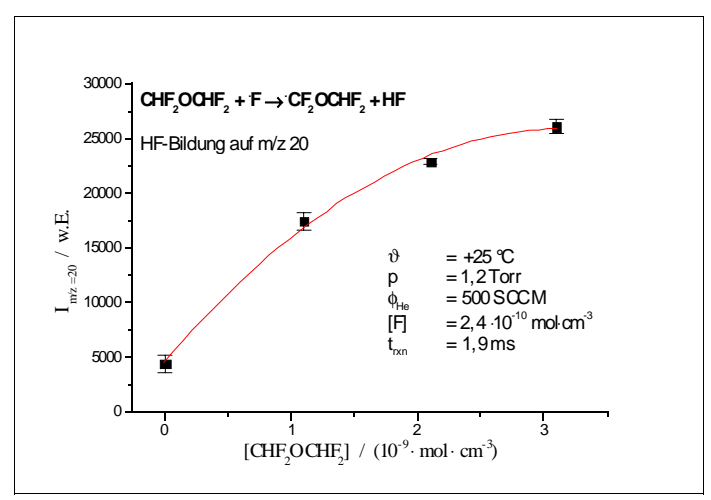

Abbildung 82: HF-Bildung auf $\mathrm{m} / \mathrm{z} 20$ des Molekül-Peaks des Ethers auf m/z 118 nahm bei Zugabe von F-Atomen ab (Abbildung 84).

Eine Zunahme auf m/z 117 (der Masse des zu erwartenden $\cdot \mathrm{CF}_{2} \mathrm{OCHF}_{2}$-Radikals) bei Zugabe von F-Atomen zu dem Ether konnte nicht beobachtet werden, wohl aber eine deutliche Steigerung der Intensität auf $\mathrm{m} / \mathrm{z} 50$ (vgl. Abbildung 83). Weil letztere bei Zugabe von $\mathrm{O}_{2}$ (Radikalfänger !) wieder stark abnahm, könnte es sich auf m/z 50 um ein Fragment des Radikals

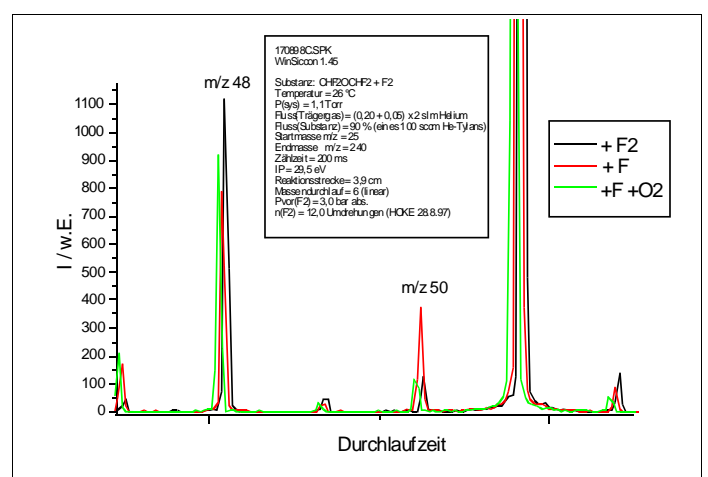

Abbildung 83: Änderungen $\mathrm{m} / \mathrm{z} 50$ handeln.

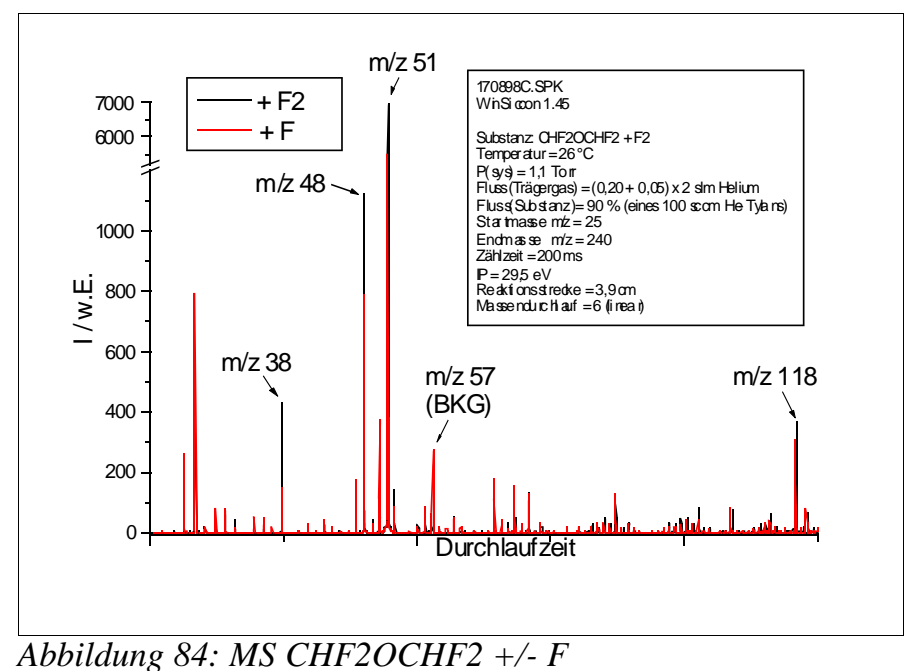




\subsubsection{Kinetik der radikalerzeugenden Reaktion mit Fluor-Atomen}

Der Geschwindigkeitskoeffizient für die Reaktion von $\mathrm{CHF}_{2} \mathrm{OCHF}_{2}\left(\mathrm{M}_{\mathrm{r}}=118\right)$ mit F-Atomen wurde in der in Kap. 3.1.1 (S. 16) beschriebenen Apparatur nach der Relativmethode (vgl. Kap. 2.1.1.2, S. 10) durchgeführt. Die Ausführung der Messungen ist in Kap. 3.5.1 (S. 50) dargestellt. In der ersten Messung diente $\mathrm{CHF}_{3}\left(\mathrm{M}_{\mathrm{r}}=70\right)$ als Referenzsubstanz. Die Umsätze konnten jeweils auf den Molekül-Peaks der Substanzen (m/z 70 bzw. 118) gemessen werden:

$\vartheta=27^{\circ} \mathrm{C} ; \phi_{\mathrm{He}}=500 \mathrm{SCCM} ; \mathrm{p}_{(\mathrm{StrR})}=160 \mathrm{~Pa} ; \mathrm{s}=4,2 \mathrm{~cm} ; \mathrm{IP}=70 \mathrm{eV} ;$ Zählzeit $=10 \mathrm{~s}$

\begin{tabular}{|c|c|c|c|c|c|c|c|}
\hline $\begin{array}{c}\text { I(118) } \\
\text {-F / w.E. }\end{array}$ & $\begin{array}{c}\Delta \mathbf{I}(\mathbf{1 1 8}) \\
-\mathrm{F} / \mathrm{w.E} .\end{array}$ & $\begin{array}{c}I(118) \\
+F / \text { w.E. }\end{array}$ & $\begin{array}{c}\Delta \mathrm{I}(118) \\
+\mathrm{F} / \mathrm{w.E} .\end{array}$ & $\begin{array}{c}\mathbf{I}(\mathbf{7 0}) \\
-\mathrm{F} / \mathrm{w.E.}\end{array}$ & $\begin{array}{c}\Delta \mathbf{I}(\mathbf{7 0}) \\
-\mathbf{F} / \mathbf{w . E} .\end{array}$ & $\begin{array}{c}\mathrm{I}(\mathbf{7 0}) \\
+\mathrm{F} / \mathrm{w.E.}\end{array}$ & $\begin{array}{c}\Delta \mathbf{I}(\mathbf{7 0}) \\
+\mathbf{F} / \mathbf{w} . \mathbf{E} .\end{array}$ \\
\hline 24000 & 224,9 & 21450 & 304,5 & 11580 & 437,9 & 11030 & 200,1 \\
\hline 23480 & 301 & 19550 & 205,3 & 11450 & 205,3 & 10930 & 238,5 \\
\hline 23830 & 238,5 & 19150 & 205,3 & 11180 & 79,5 & 10630 & 152,2 \\
\hline 23230 & 238,5 & 17950 & 159 & 10680 & 271,5 & 10010 & 209,9 \\
\hline 22450 & 668,3 & 17950 & 331 & 9072 & 342,5 & 8497 & 196,2 \\
\hline 24000 & 430,6 & 19750 & 400,1 & 9862 & 217,2 & 9437 & 154,9 \\
\hline 26180 & 79,5 & 22330 & 79,5 & 10360 & 284,4 & 9937 & 154,9 \\
\hline 27250 & 159 & 24480 & 152,2 & 11140 & 398,5 & 10810 & 174,1 \\
\hline 26230 & 152,2 & 24300 & 224,9 & 11390 & 316 & 10960 & 338,5 \\
\hline
\end{tabular}

Tabelle 3: Meßwerte Kinetik CHF2OCHF2 + F

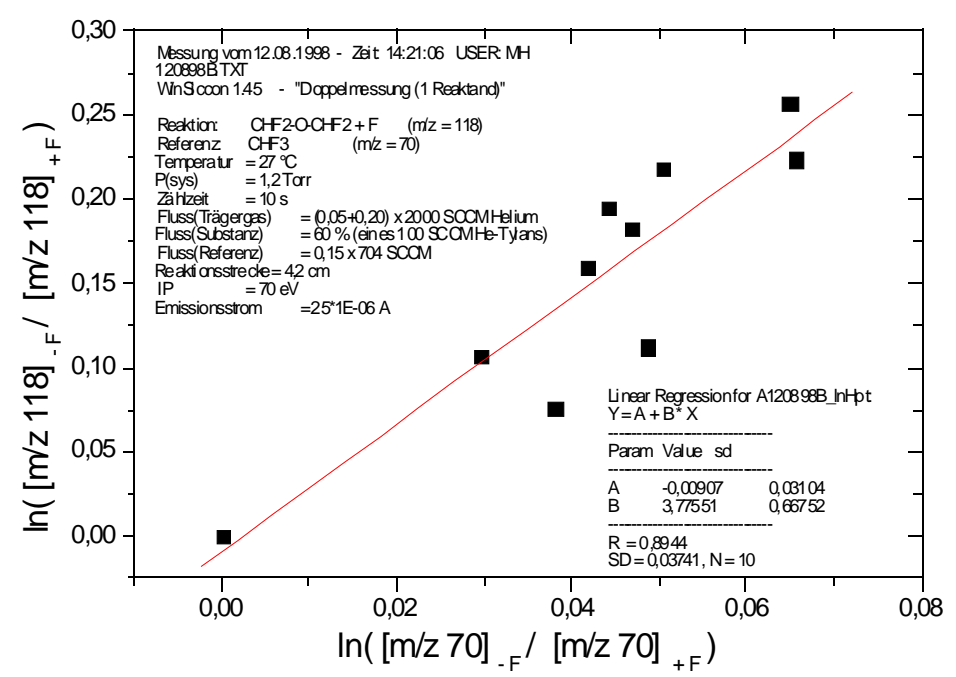

Abbildung 85: Kinetik CHF2OCHF2 +F

Aus der Steigung der Auftragung in Abbildung 85 erhält man gemäß Gleichung (10) (S. 10) den relativen Geschwindigkeitskoeffizienten: $\mathrm{k}_{\mathrm{rel}, 1}=(3,78 \pm 0,67)$. 
In der zweiten Messung diente $\mathrm{CF}_{3} \mathrm{CH}_{2} \mathrm{~F}\left(\mathrm{M}_{\mathrm{r}}=102\right)$ als Referenzsubstanz. Die Umsätze konnten jeweils auf den Molekül-Peaks der Substanzen (m/z 102 bzw. 118) gemessen werden: $\vartheta=25^{\circ} \mathrm{C} ; \phi_{\mathrm{He}}=500 \mathrm{SCCM} ; \mathrm{p}_{(\mathrm{StrR})}=173 \mathrm{~Pa} ; \mathrm{s}=3,0 \mathrm{~cm} ; \mathrm{IP}=29,5 \mathrm{eV} ;$ Zählzeit $=10 \mathrm{~s}$

\begin{tabular}{|c|c|c|c|c|c|c|c|}
\hline $\begin{array}{c}\mathbf{I}(\mathbf{1 1 8}) \\
-\mathrm{F} / \mathrm{w.E} .\end{array}$ & $\begin{array}{c}\Delta \mathrm{I}(118) \\
-\mathrm{F} / \mathrm{w.E} .\end{array}$ & $\begin{array}{c}\text { I(118) } \\
+\mathrm{F} / \mathrm{w.E} .\end{array}$ & $\begin{array}{c}\Delta \mathrm{I}(118) \\
+\mathrm{F} / \mathrm{w} . \mathrm{E} .\end{array}$ & $\begin{array}{c}\text { I(102) } \\
\text {-F / w.E. }\end{array}$ & $\begin{array}{c}\Delta \mathrm{I}(102) \\
-\mathrm{F} / \mathrm{w} . \mathrm{E} .\end{array}$ & $\begin{aligned} & \mathbf{I}(\mathbf{1 0 2}) \\
&+\mathrm{F} / \text { w.E. }\end{aligned}$ & $\begin{array}{r}\Delta \mathrm{I}(102) \\
+\mathrm{F} / \mathrm{w} . \mathrm{E} .\end{array}$ \\
\hline 14230 & 573,3 & 13600 & 248,3 & 10600 & 656,8 & 8483 & 186,3 \\
\hline 14030 & 79,5 & 13430 & 397,5 & 10430 & 238,5 & 8908 & 266,2 \\
\hline 14230 & 271,5 & 13780 & 397,5 & 10330 & 327,8 & 9615 & 252,2 \\
\hline 14330 & 200,1 & 14180 & 152,2 & 10350 & 91,8 & 9818 & 217,4 \\
\hline 14500 & 224,9 & 14280 & 79,5 & 10930 & 238,5 & 10350 & 275,4 \\
\hline 13580 & 301 & 13530 & 327,8 & 10550 & 205,3 & 10070 & 171 \\
\hline 13800 & 259,6 & 13400 & 290,3 & 10750 & 91,8 & 9110 & 119,7 \\
\hline 14380 & 352,6 & 13980 & 418,2 & 10700 & 183,6 & 9165 & 419,2 \\
\hline 14880 & 271,5 & 14650 & 275,4 & 10500 & 290,3 & 9745 & 166,5 \\
\hline 14700 & 502,8 & 14450 & 527,3 & 10550 & 159 & 9848 & 173,9 \\
\hline 14730 & 271,5 & 14230 & 271,5 & 10850 & 205,3 & 9718 & 224,6 \\
\hline 15100 & 290,3 & 14650 & 91,8 & 10950 & 91,8 & 9873 & 177,7 \\
\hline 15000 & 129,8 & 14430 & 152,2 & 10700 & 259,6 & 8698 & 295,6 \\
\hline 14930 & 397,5 & 14150 & 275,4 & 10500 & 389,5 & 8405 & 160,3 \\
\hline
\end{tabular}

Tabelle 4: Meßwerte Kinetik CHF2OCHF2 + F

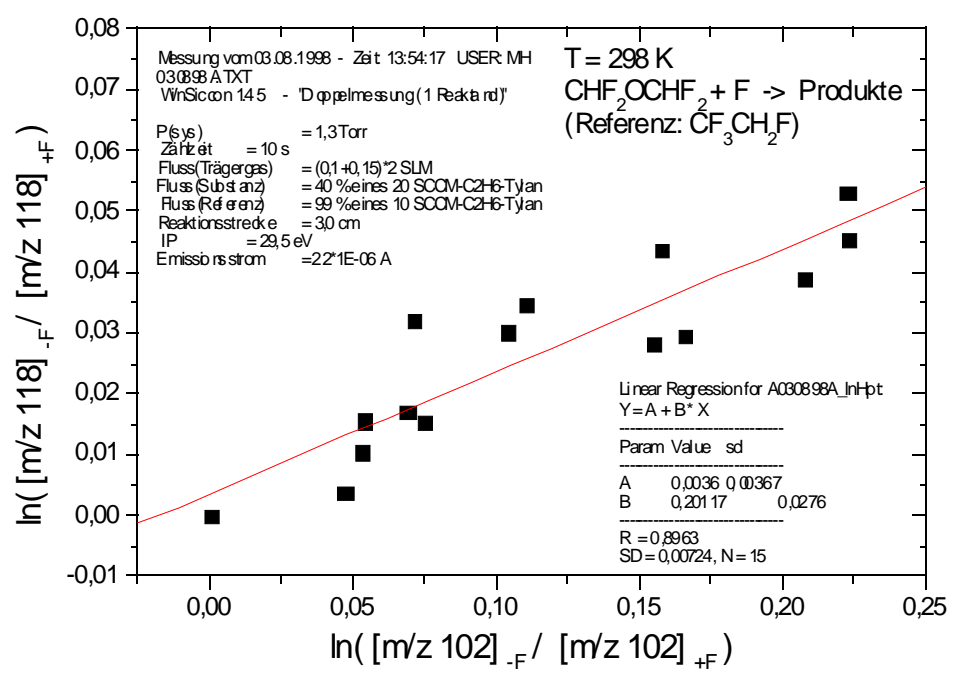

Abbildung 86: Kinetik CHF2OCHF2 + F

Aus der Steigung der Auftragung in Abbildung 86 erhält man gemäß Gleichung (10) (S. 10) den relativen Geschwindigkeitskoeffizienten: $\mathrm{k}_{\mathrm{rel}, 2}=(0,20 \pm 0,03)$. 
Mit den bekannten Geschwindigkeitskoeffizienten für die Referenzreaktionen kann aus den relativen auch der absolute Geschwindigkeitskoeffizient berechnet werden.

Für die in der ersten Messung als Referenz verwendete Reaktion $\mathrm{CHF}_{3}+\mathrm{F} \rightarrow$ Produkte sind in der Literatur verschiedene Werte angegeben; diejenigen, die unter vergleichbaren Reaktionsbedingungen wie hier ermittelt wurden, liegen alle bei $9 \cdot 10^{10} \mathrm{~cm}^{3} /(\mathrm{mol} \cdot \mathrm{s})$, so daß hier der jüngst publizierte Wert ${ }^{104}$ von $\mathrm{k}_{\mathrm{ref}, 1}=9,11 \cdot 10^{10} \mathrm{~cm}^{3} /(\mathrm{mol} \cdot \mathrm{s})$ verwendet werden soll.

Mit diesem errechnet man folgenden absoluten Geschwindigkeitskoeffizienten $\mathrm{k}_{1}$ :

$\mathrm{k}_{1}=\mathrm{k}_{\mathrm{rel}, 1} \cdot \mathrm{k}_{\mathrm{ref}, 1}=(3,78 \pm 0,67) \cdot 9,11 \cdot 10^{10} \mathrm{~cm}^{3} /(\mathrm{mol} \cdot \mathrm{s})=(3,4 \pm 0,6) \cdot 10^{11} \mathrm{~cm}^{3} /(\mathrm{mol} \cdot \mathrm{s})$.

Die in der zweiten Messung als Referenz verwendete Reaktion $\mathrm{CF}_{3} \mathrm{CH}_{2} \mathrm{~F}+\mathrm{F} \rightarrow$ Produkte ist im Rahmen der vorliegenden Arbeit untersucht worden (siehe Kapitel 4.4.4, S. 230), wobei ein Geschwindigkeitskoeffizient von $\mathrm{k}_{\mathrm{ref}, 2}=2,8 \cdot 10^{12} \mathrm{~cm}^{3} /(\mathrm{mol} \cdot \mathrm{s})$ ermittelt wurde.

Damit errechnet man folgenden absoluten Geschwindigkeitskoeffizienten $\mathrm{k}_{2}$ :

$\mathrm{k}_{2}=\mathrm{k}_{\mathrm{rel}, 2} \cdot \mathrm{k}_{\mathrm{ref}, 2}=(0,20 \pm 0,03) \cdot 2,8 \cdot 10^{12} \mathrm{~cm}^{3} /(\mathrm{mol} \cdot \mathrm{s})=(5,6 \pm 0,8) \cdot 10^{11} \mathrm{~cm}^{3} /(\mathrm{mol} \cdot \mathrm{s})$.

Die beiden Ergebnisse stimmen nur mäßig gut überein, was jedoch auf die Unsicherheiten bei der Wahl des korrekten Referenzwertes zurückzuführen ist. Für $k_{\text {ref, } 2}$ ist auch ein wesentlich kleinerer Wert von $9,77 \cdot 10^{11} \mathrm{~cm}^{3} /(\mathrm{mol} \cdot \mathrm{s})$ veröffentlicht ${ }^{105}$, der $\mathrm{zu}$ einem Geschwindigkeitskoeffizienten von $1,9 \cdot 10^{11} \mathrm{~cm}^{3} /(\mathrm{mol} \cdot \mathrm{s})$ für $\mathrm{k}_{2}$ führen würde. Hier soll jedoch der eigene Wert verwendet und als Gesamtergebnis der arithmetische Mittelwert von $\mathrm{k}_{1}$ und $\mathrm{k}_{2}$ mit der zugehörigen Standardabweichung angegeben werden:

$\mathbf{k}(\mathbf{2 9 8 K})=(\mathbf{4 , 5} \pm \mathbf{1 , 6}) \cdot \mathbf{1 0}^{11} \mathrm{~cm}^{3} /(\mathbf{m o l} \cdot \mathbf{s}) \quad ;$ für: $\mathrm{CHF}_{2} \mathrm{OCHF}_{2}+\mathrm{F} \rightarrow$ Produkte

\subsubsection{Diskussion der Ergebnisse}

Untersuchungen anderer Arbeitsgruppen bezüglich der Reaktion $\mathrm{CHF}_{2} \mathrm{OCHF}_{2}+\mathrm{F} \rightarrow$ Produkte lagen nicht vor. Daher soll der hier ermittelte Geschwindigkeitskoeffizient mit denen des strukturell ähnlichen $\mathrm{Bis}\left(2,2,2\right.$-trifluorethyl)ethers $\left(\mathrm{CF}_{3} \mathrm{CH}_{2} \mathrm{OCH}_{2} \mathrm{CF}_{3}\right)$ sowie Bis(fluormethyl) ethers $\left(\mathrm{CH}_{2} \mathrm{FOCH}_{2} \mathrm{~F}\right)$ verglichen werden.

Für $\mathrm{CF}_{3} \mathrm{CH}_{2} \mathrm{OCH}_{2} \mathrm{CF}_{3}$ wurde bei $298 \mathrm{~K}$ ein Wert von $1,1 \cdot 10^{13} \mathrm{~cm}^{3} /(\mathrm{mol} \cdot \mathrm{s})$ und für $\mathrm{CH}_{2} \mathrm{FOCH}_{2} \mathrm{~F}$ einer von $9,9 \cdot 10^{12} \mathrm{~cm}^{3} /(\mathrm{mol} \cdot \mathrm{s})$ gemessen ${ }^{106}$.

104 Louis, F.; Sawerysyn, J. (1998).

105 Louis,F.; et al. (1997).

106 Hold, M. (1997); S. 103 bzw. 105. 
Das hier analysierte $\mathrm{CHF}_{2} \mathrm{OCHF}_{2}$ besitzt nur zwei H-Atome, die zu vergleichenden Ether jedoch jeweils vier, was zu einem statistischen Korrekturfaktor von $2 / 4=0,5$ führt. Danach wäre für $\mathrm{CHF}_{2} \mathrm{OCHF}_{2}$ also ein Geschwindigkeitskoeffizient von etwa $0,5 \cdot 1 \cdot 10^{13} \mathrm{~cm}^{3} /(\mathrm{mol} \cdot \mathrm{s}) \mathrm{zu}$ erwarten. Der tatsächlich gemessene Wert ist aber um den Faktor 10 kleiner, was elektronische Ursachen haben muß.

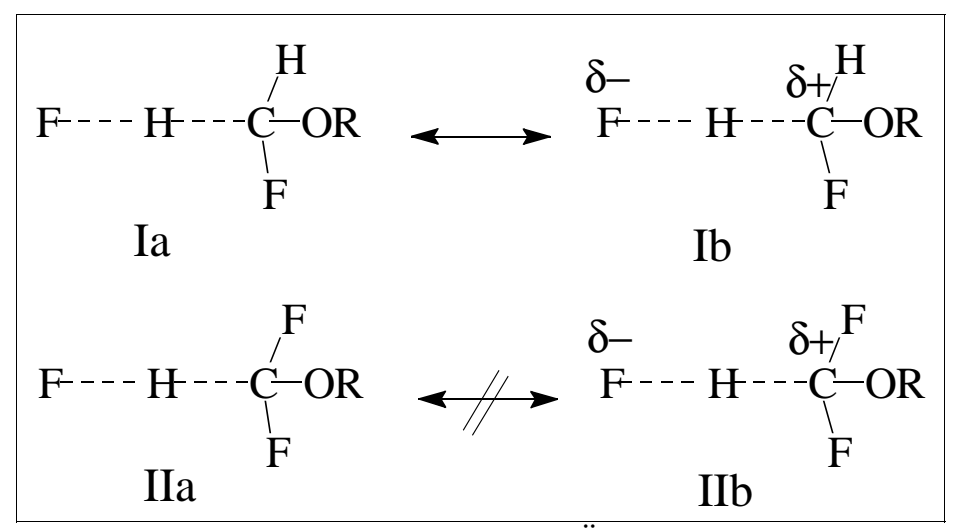

Abbildung 87: Resonanzstrukturen des Übergangszustands

In Abbildung 87 trägt die Resonanzstruktur I b zur Stabilisierung des Übergangszustands für die Abstraktion eines H-Atoms aus dem Ether bei, weil dem elektronegativen angreifenden FAtom eine negative Partialladung zukommt. Wird nun an dem C-Atom, das zwangsläufig die positive Partialladung trägt, ein zusätzlicher elektronegativer Substituent eingeführt, so erhält man die Struktur II a. In diesem Fall trägt die hypothetische Resonanzstruktur II b jedoch nicht mehr zur Stabilisierung des Übergangszustands bei, weil der stark elektronegative zweite F-Substituent keine positive Partialladung in seiner Umgebung duldet ${ }^{107}$; dadurch ist nun die Energiebarriere höher und somit der Geschwindigkeitskoeffizient geringer als bei Struktur I.

Aus den geschilderten Gründen ist es also durchaus plausibel, daß der höher fluorsubstituierte Ether $\left(\mathrm{CHF}_{2} \mathrm{OCHF}_{2}\right)$ einen wesentlich geringeren Geschwindigkeitskoeffizienten als $\mathrm{CF}_{3} \mathrm{CH}_{2} \mathrm{OCH}_{2} \mathrm{CF}_{3}$ oder $\mathrm{CH}_{2} \mathrm{FOCH}_{2} \mathrm{~F}$ aufweist. 


\subsubsection{Der Methylperfluorbutylether $\left(\mathrm{C}_{4} \mathrm{~F}_{9}-\mathrm{O}-\mathrm{CH}_{3}\right)$}

\subsubsection{Produkte der radikalerzeugenden Reaktion mit Fluor-Atomen}

Bei dem hier untersuchten Ether handelt es sich laut Hersteller um eine Mischung von Methylperfluorisobutylether (50-70\%) und Methylnonafluorbutylether (30-50\%).

Die Analysen wurden in der in Kap. 3.1.1 (S. 16) beschriebenen Apparatur durchgeführt, wobei der Strömungsreaktor aus Abbildung 5 (S. 17) zum Einsatz kam.

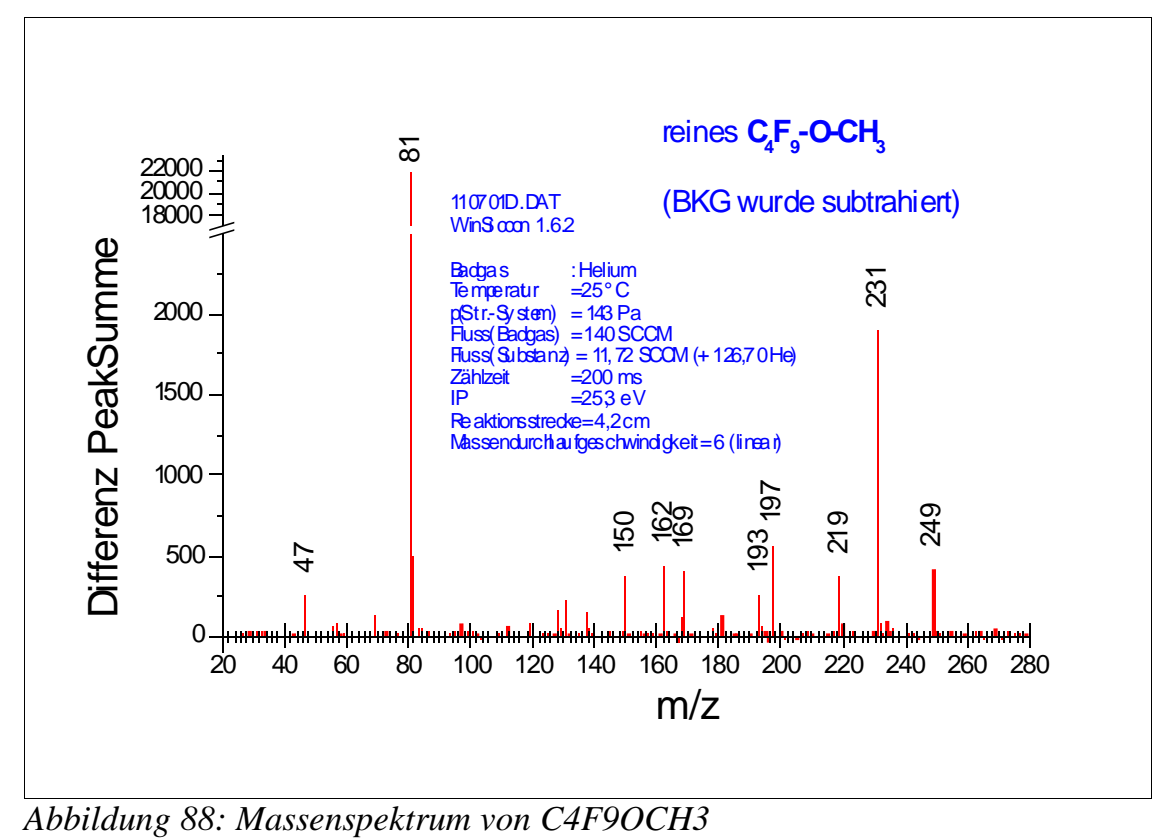

Das Massenspektrum (Abbildung 88) zeigt, daß die Substanz selbst bei IP $=25,3 \mathrm{eV}$ keinen Molekül-Peak (m/z 250) aufweist. Jedoch sind insbesondere die Fragmente $\mathrm{C}_{4} \mathrm{~F}_{9}-\mathrm{O}-\mathrm{CH}_{2}(\mathrm{~m} / \mathrm{z}$ 249) und $\mathrm{C}_{4} \mathrm{~F}_{8}-\mathrm{O}-\mathrm{CH}_{3}(\mathrm{~m} / \mathrm{z} 231)$ deutlich zu erkennen.

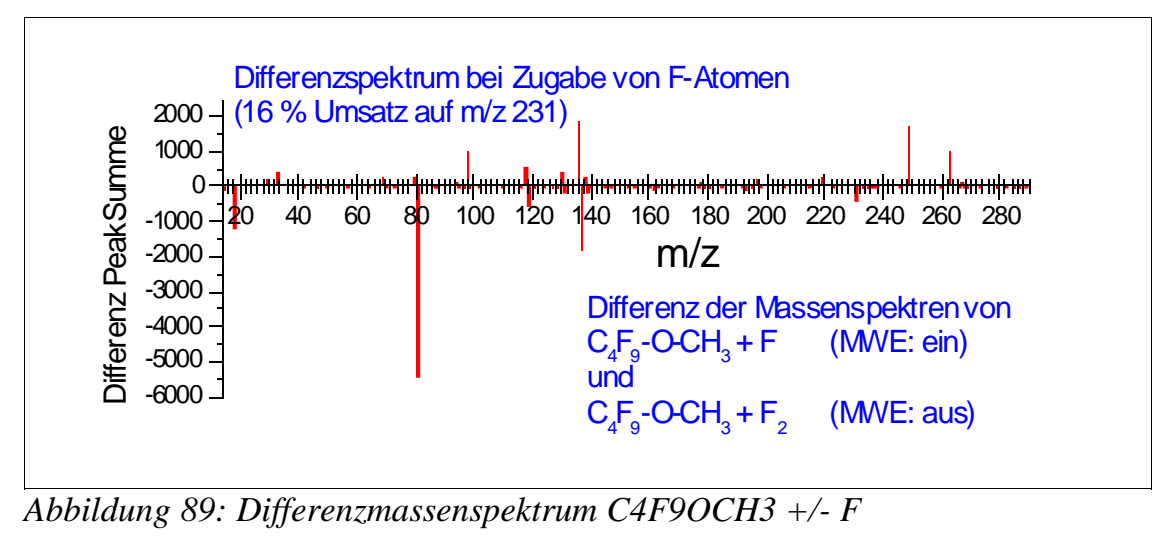

Das Differenzmassenspektrum nach/vor Zugabe von Fluor-Atomen (Abbildung 89) zeigt eine Abnahme insbesondere auf dem Fragment-Peak m/z 231 des Ethers, der zur Bestimmung seines Umsatzes herangezogen wird, weil das gemäß $\mathrm{C}_{4} \mathrm{~F}_{9}-\mathrm{O}-\mathrm{CH}_{3}+\mathrm{F} \rightarrow \mathrm{C}_{4} \mathrm{~F}_{9}-\mathrm{O}-\mathrm{CH}_{2}+\mathrm{HF}$ 
potentiell gebildete Radikal hier kein Fragment haben kann. Die Bildung des Radikals wird durch die starke Zunahme auf m/z 249 bestätigt. Die damit einhergehende Bildung von HF $(\mathrm{m} / \mathrm{z} 20)$ ist in den Spektren bei IP $=25,3 \mathrm{eV}$ nicht $\mathrm{zu}$ sehen, weil HF eine zu hohe Ionisierungsenergie hat; bei IP $=70 \mathrm{eV}$ konnte die Zunahme auf $\mathrm{m} / \mathrm{z} 20$ bestätigt werden. Die Zunahme auf m/z 263 dürfte von einem Kombinationsprodukt zweier Radikale stammen (vgl. das Fragment $\mathrm{C}_{4} \mathrm{~F}_{9}-\mathrm{O}-\mathrm{CH}_{2}-\mathrm{CH}_{2}$ ), denn bei sinkendem Umsatz des Ethers (auf $\mathrm{m} / \mathrm{z} 231$ gemessen) nimmt dieser Peak relativ zu dem auf m/z 249 überproportional ab. Der MolekülPeak (m/z 498) des Kombinationsprodukts oder ein Fragment auf m/z 479 konnten jedoch nicht nachgewiesen werden.

Es bleibt damit festzuhalten, daß die Reaktion des Ethers mit Fluor-Atomen gemäß

$$
\mathrm{C}_{4} \mathrm{~F}_{9}-\mathrm{O}-\mathrm{CH}_{3}+\mathrm{F} \rightarrow \mathrm{C}_{4} \mathrm{~F}_{9}-\mathrm{O}-\mathrm{CH}_{2}+\mathrm{HF}
$$

abläuft, der Umsatz des Ethers auf seinem Fragment-Peak m/z 231 ermittelt werden kann und das gebildete Radikal einen hohen Molekül-Peak auf m/z 249 zeigt.

\subsubsection{Kinetik der radikalerzeugenden Reaktion mit Fluor-Atomen}

Die Messungen wurden in der in Kap. 3.1.1 (S. 16) beschriebenen Apparatur unter Einsatz des Strömungsreaktors aus Abbildung 5 (S. 17) durchgeführt. Die Ausführung und Auswertung nach der Relativmethode ist in Kap. 3.5.1 (S. 50) beschrieben.

In einer ersten Messung diente Methan $\left(\mathrm{M}_{\mathrm{r}}=16\right)$ als Referenzsubstanz. Der Umsatz des Ethers konnte anhand der Intensitätsänderung seines Fragment-Peaks m/z 231, derjenige von Methan anhand des Molekül-Peaks (m/z 16) bestimmt werden.

$\vartheta=25^{\circ} \mathrm{C} ; \phi_{\mathrm{He}}=414 \mathrm{SCCM} ; \phi_{\mathrm{Ether}}=4,5 \mathrm{SCCM} ; \phi_{\mathrm{CH} 4}=4,4 \mathrm{SCCM} ; \mathrm{p}_{(\mathrm{StrR})}=109 \mathrm{~Pa} ; \mathrm{s}=2,5 \mathrm{~cm}$; $\mathrm{IP}=24,5 \mathrm{eV} ;$ Zählzeit $=5 \mathrm{~s}$

\begin{tabular}{|c|c|c|c|c|c|c|c|}
\hline $\begin{array}{c}\mathrm{I}(231) \\
-\mathrm{F} / \mathrm{w.E} .\end{array}$ & $\begin{array}{c}\Delta \mathbf{I}(231) \\
-\mathrm{F} / \mathrm{w.E} .\end{array}$ & $\begin{array}{c}\mathrm{I}(231) \\
+\mathrm{F} / \mathrm{w.E} .\end{array}$ & $\begin{array}{c}\Delta \mathbf{I}(231) \\
+\mathbf{F} / \text { w.E. }\end{array}$ & $\begin{array}{c}\text { I(16) } \\
-F / w . E .\end{array}$ & $\begin{array}{c}\Delta \mathrm{I}(16) \\
-\mathrm{F} / \mathrm{w.E} .\end{array}$ & $\begin{array}{c}\mathrm{I}(16) \\
+\mathrm{F} / \mathrm{w.E.}\end{array}$ & $\begin{array}{c}\Delta \mathrm{I}(16) \\
+\mathrm{F} / \mathrm{w.E.}\end{array}$ \\
\hline 5879 & 46 & 5681 & 41 & 28680 & 308 & 22870 & 182 \\
\hline 5908 & 68 & 5638 & 40 & 39030 & 415 & 30740 & 140 \\
\hline 5984 & 69 & 5706 & 57 & 36550 & 262 & 26450 & 274 \\
\hline 6045 & 58 & 5750 & 79 & 42390 & 209 & 31470 & 151 \\
\hline 6154 & 77 & 5974 & 70 & 43340 & 268 & 37480 & 310 \\
\hline 6024 & 67 & 5942 & 66 & 44530 & 126 & 39140 & 194 \\
\hline
\end{tabular}

Tabelle 5: Meßwerte Kinetik C4F9OCH3 + F rel. zu CH4; 298 K 
Mit den Werten aus Tabelle 5 konnte eine doppelt-logarithmische Auftragung gemäß Gleichung (10) (S. 10) vorgenommen werden (Abbildung 90).

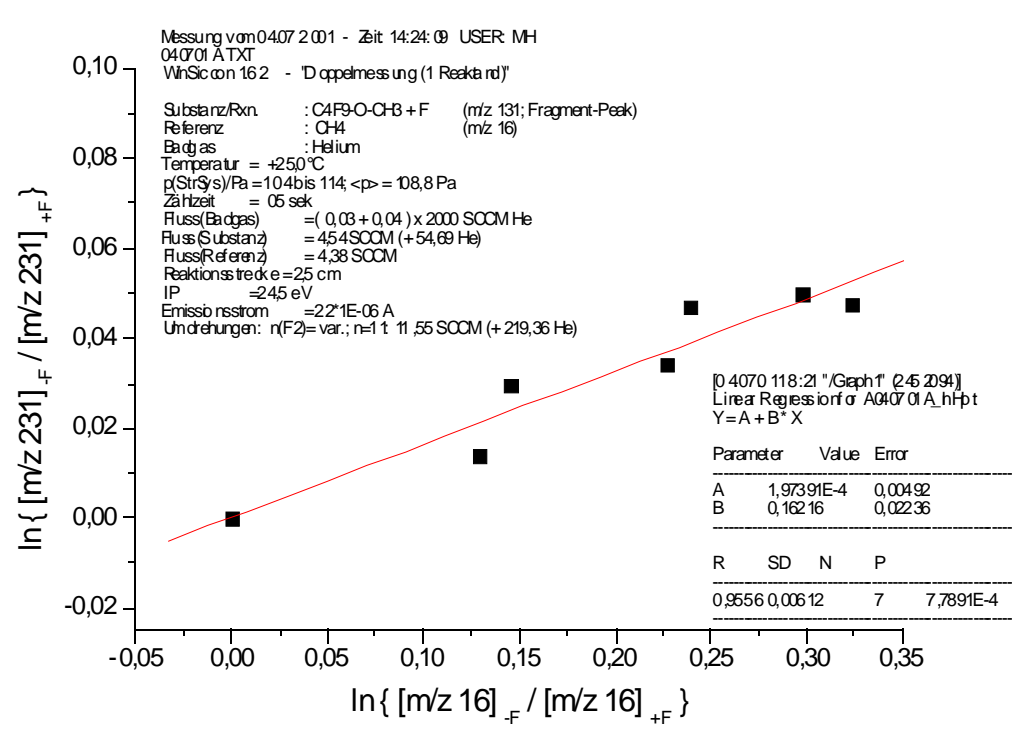

Abbildung 90: Kinetik C4F9OCH3 + F rel. zu CH4; 298 K

Aus Abbildung 90 erhält man den relativen Geschwindigkeitskoeffizienten: $k_{\text {rel }}=(0,16 \pm 0,02)$.

Da die Reaktion mit der Referenzsubstanz Methan offensichtlich wesentlich schneller ist als die des Ethers, wurden die weiteren Messungen mit Dichlormethan als Referenz durchgeführt. Letzteres hat einen vergleichbaren Geschwindigkeitskoeffizienten wie der Ether, was präzisere Messungen ermöglicht; seine Umsatzbestimmung erfolgte auf dem Molekül-Peak m/z 84.

$\vartheta=-5{ }^{\circ} \mathrm{C} ; \phi_{\mathrm{He}}=465 \mathrm{SCCM} ; \phi_{\text {Ether }}=6,4 \mathrm{SCCM} ; \phi_{\mathrm{CH} 2 \mathrm{Cl} 2}=6,2 \mathrm{SCCM} ; \mathrm{p}_{(\mathrm{StrR})}=91 \mathrm{~Pa} ; \mathrm{s}=2,5$ $\mathrm{cm} ; \mathrm{IP}=29,5 \mathrm{eV} ;$ Zählzeit $=5 \mathrm{~s}$

\begin{tabular}{|r|r|r|r|r|r|r|r|}
\hline $\begin{array}{r}\mathbf{I}(\mathbf{2 3 1}) \\
\text {-F / w.E. }\end{array}$ & $\begin{array}{c}\Delta \mathbf{I}(\mathbf{2 3 1}) \\
\mathbf{- F} / \mathbf{w . E} .\end{array}$ & $\begin{array}{c}\mathbf{I}(\mathbf{2 3 1}) \\
+\mathbf{+ F} / \mathbf{w . E} .\end{array}$ & $\begin{array}{c}\Delta \mathbf{I}(\mathbf{2 3 1}) \\
+\mathbf{+ F} / \mathbf{w . E} .\end{array}$ & $\begin{array}{c}\mathbf{I}(\mathbf{8 4}) \\
\mathbf{- F} / \mathbf{w . E} .\end{array}$ & $\begin{array}{c}\Delta \mathbf{I}(\mathbf{8 4}) \\
\mathbf{- F} / \mathbf{w . E} .\end{array}$ & $\begin{array}{c}\mathbf{I}(\mathbf{8 4}) \\
+\mathbf{+ F} / \mathbf{w . E} .\end{array}$ & $\begin{array}{c}\Delta \mathbf{I}(\mathbf{8 4}) \\
+\mathbf{F} / \mathbf{w . E} .\end{array}$ \\
\hline 2289 & 33 & 1967 & 30 & 57910 & 212 & 49990 & 173 \\
\hline 2298 & 55 & 1962 & 18 & 57170 & 330 & 49260 & 194 \\
\hline 2171 & 31 & 2076 & 25 & 57190 & 186 & 55010 & 239 \\
\hline 2173 & 52 & 2130 & 40 & 56880 & 267 & 55970 & 298 \\
\hline 2203 & 38 & 2073 & 31 & 56550 & 255 & 52750 & 232 \\
\hline 2123 & 39 & 1961 & 22 & 54900 & 265 & 50350 & 203 \\
\hline 2222 & 44 & 2018 & 35 & 53380 & 323 & 49340 & 327 \\
\hline 2265 & 45 & 1939 & 37 & 55300 & 292 & 48710 & 145 \\
\hline 2229 & 45 & 1970 & 31 & 54940 & 206 & 48380 & 196 \\
\hline
\end{tabular}

Tabelle 6: Meßwerte Kinetik $\mathrm{C} 4 \mathrm{~F} 9 \mathrm{OCH} 3+\mathrm{F}$ rel. zu CH2Cl2; $268 \mathrm{~K}$ 


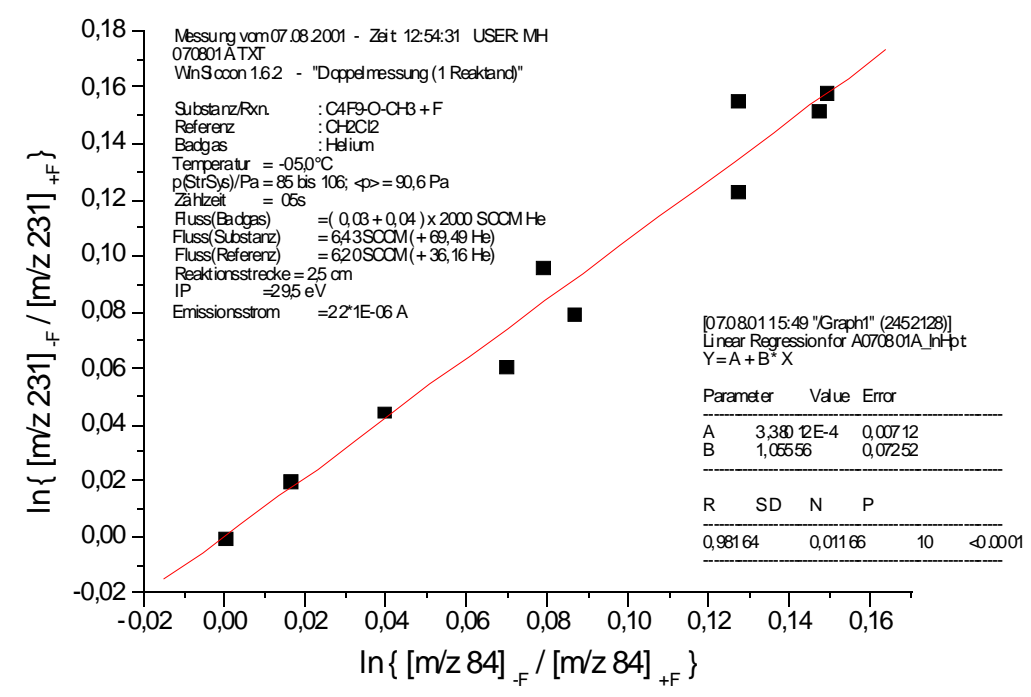

Abbildung 91: Kinetik C4F9OCH3 + F rel. zu CH2Cl2; $268 \mathrm{~K}$

Bei $\mathrm{T}=268 \mathrm{~K}$ erhält man aus Abbildung 91 für die Reaktion des Ethers relativ zur Reaktion des Dichlormethans mit Fluor-Atomen einen relativen Geschwindigkeitskoeffizienten von $\mathrm{k}_{\mathrm{rel}}=(1,06 \pm 0,07)$.

$\vartheta=25^{\circ} \mathrm{C} ; \phi_{\mathrm{He}}=395 \mathrm{SCCM} ; \phi_{\mathrm{Ether}}=2,7 \mathrm{SCCM} ; \phi_{\mathrm{CH} 2 \mathrm{Cl} 2}=2,5 \mathrm{SCCM} ; \mathrm{p}_{(\mathrm{StrR})}=111 \mathrm{~Pa} ; \mathrm{s}=2,5$ $\mathrm{cm} ; \mathrm{IP}=70 \mathrm{eV} ;$ Zählzeit $=5 \mathrm{~s}$

\begin{tabular}{|c|c|c|c|c|c|c|c|}
\hline $\begin{array}{c}\text { I(231) } \\
-\mathrm{F} / \mathrm{w.E} .\end{array}$ & $\begin{array}{c}\Delta \mathbf{I}(231) \\
-\mathrm{F} / \mathrm{w.E} .\end{array}$ & $\begin{aligned} & \mathrm{I}(231) \\
+ & \mathrm{F} / \mathrm{w.E} .\end{aligned}$ & $\begin{array}{c}\Delta \mathbf{I}(231) \\
+\mathrm{F} / \mathrm{w} . \mathrm{E} .\end{array}$ & $\begin{array}{c}\mathrm{I}(84) \\
-\mathrm{F} / \mathrm{w.E.}\end{array}$ & $\begin{array}{c}\Delta \mathbf{I}(84) \\
-\mathbf{F} / \mathbf{w . E} .\end{array}$ & $\begin{array}{c}\mathrm{I}(84) \\
+\mathrm{F} / \mathrm{w.E.}\end{array}$ & $\begin{array}{c}\Delta \mathbf{I}(\mathbf{8 4}) \\
+\mathrm{F} / \mathrm{w.E} .\end{array}$ \\
\hline 57420 & 220,4 & 49260 & 155,1 & 344700 & 482,4 & 298600 & 602,7 \\
\hline 63940 & 131,3 & 55080 & 225,5 & 345500 & 505,4 & 300000 & 583,5 \\
\hline 64230 & 378,2 & 57410 & 176,5 & 345500 & 967,7 & 309600 & 369,1 \\
\hline 64790 & 194,8 & 60580 & 228 & 349400 & 768,3 & 323800 & 656,7 \\
\hline 66970 & 250 & 62170 & 150,9 & 360000 & 673,8 & 335100 & 786,5 \\
\hline 67000 & 258,8 & 64860 & 197 & 360900 & 625,8 & 352000 & 753,3 \\
\hline 66990 & 250,9 & 65170 & 265,4 & 363700 & 678 & 350800 & 999,4 \\
\hline
\end{tabular}

Tabelle 7: Meßwerte Kinetik C4F9OCH3 + F rel. zu CH2Cl2; $298 \mathrm{~K}$ 


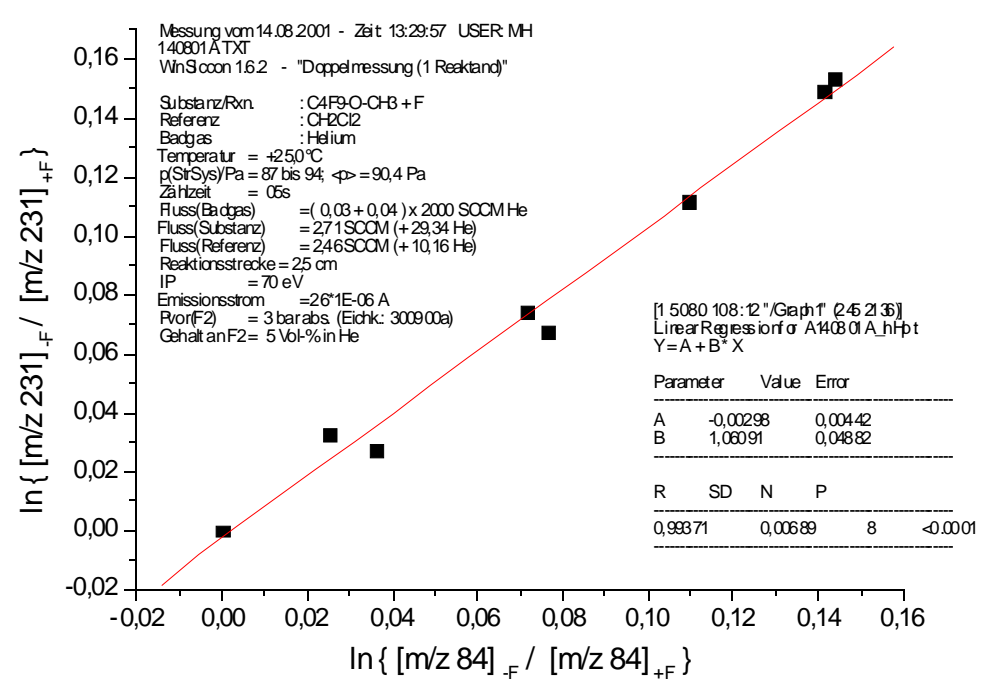

Abbildung 92: Kinetik C4F9OCH3 + F rel. zu CH2Cl2; 298 K

Bei T = $298 \mathrm{~K}$ erhält man aus Abbildung 92 einen relativen Geschwindigkeitskoeffizienten von $\mathrm{k}_{\mathrm{rel}}=(1,06 \pm 0,05)$.

$\vartheta=55{ }^{\circ} \mathrm{C} ; \phi_{\mathrm{He}}=481 \mathrm{SCCM} ; \phi_{\mathrm{Ether}}=8,0 \mathrm{SCCM} ; \phi_{\mathrm{CH} 2 \mathrm{Cl} 2}=8,3 \mathrm{SCCM} ; \mathrm{p}_{(\mathrm{StrR})}=95 \mathrm{~Pa} ; \mathrm{s}=2,5$ $\mathrm{cm} ; \mathrm{IP}=70 \mathrm{eV} ;$ Zählzeit $=5 \mathrm{~s}$

\begin{tabular}{|r|r|r|r|r|r|r|r|}
\hline $\begin{array}{c}\mathbf{I}(\mathbf{2 3 1}) \\
\text {-F / w.E. }\end{array}$ & $\begin{array}{c}\Delta \mathbf{I}(\mathbf{2 3 1}) \\
\text {-F / w.E. }\end{array}$ & $\begin{array}{c}\mathbf{I}(\mathbf{2 3 1}) \\
\text { +F / w.E. }\end{array}$ & $\begin{array}{c}\Delta \mathbf{I}(\mathbf{2 3 1}) \\
\text { +F / w.E. }\end{array}$ & $\begin{array}{c}\mathbf{I}(\mathbf{8 4}) \\
\text {-F / w.E. }\end{array}$ & $\begin{array}{c}\Delta \mathbf{I}(\mathbf{8 4}) \\
\text {-F / w.E. }\end{array}$ & $\begin{array}{c}\mathbf{I}(\mathbf{8 4}) \\
\text { +F / w.E. }\end{array}$ & $\begin{array}{c}\Delta \mathbf{I}(\mathbf{8 4}) \\
\text { +F / w.E. }\end{array}$ \\
\hline 64160 & 321 & 55230 & 298 & 356900 & 1040 & 312900 & 940 \\
\hline 66540 & 141 & 57910 & 211 & 354900 & 827 & 314400 & 432 \\
\hline 66530 & 243 & 58900 & 256 & 345500 & 5712 & 310500 & 1892 \\
\hline 66590 & 372 & 61600 & 184 & 364300 & 1069 & 341400 & 621 \\
\hline 63890 & 554 & 56210 & 341 & 352000 & 1261 & 308800 & 1529 \\
\hline 66850 & 214 & 62340 & 227 & 367700 & 895 & 343800 & 1055 \\
\hline
\end{tabular}

Tabelle 8: Meßwerte Kinetik C4F9OCH3 + F rel. zu CH2Cl2; $328 \mathrm{~K}$ 


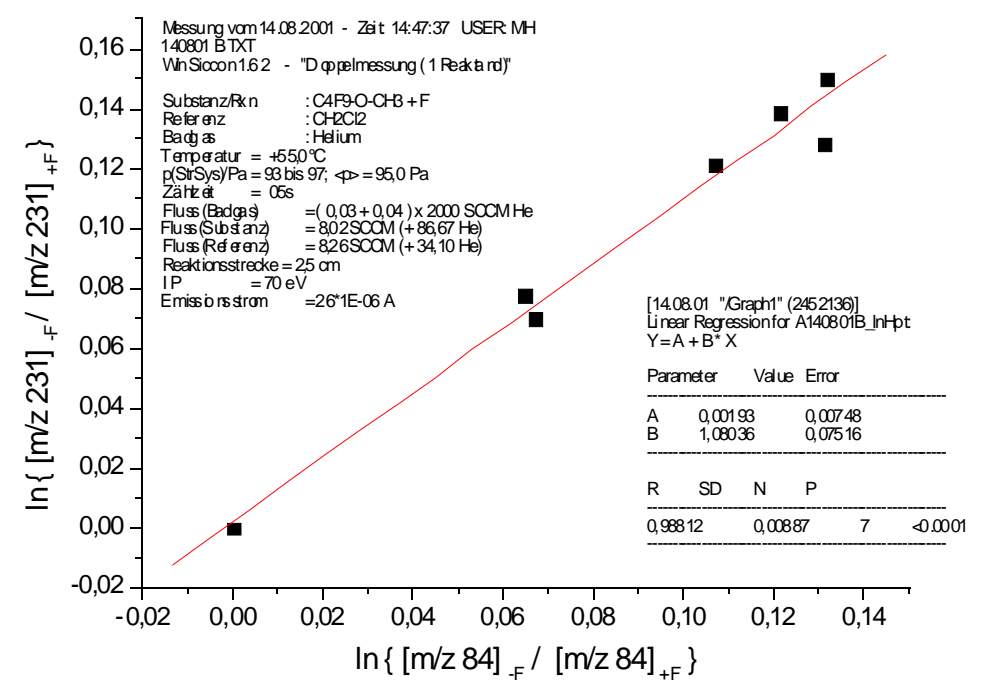

Abbildung 93: Kinetik C4F9OCH3 + F rel. zu CH2Cl2; $328 \mathrm{~K}$

Bei $\mathrm{T}=328 \mathrm{~K}$ erhält man aus Abbildung 93 einen relativen Geschwindigkeitskoeffizienten von $\mathrm{k}_{\mathrm{rel}}=(1,08 \pm 0,08)$.

$\vartheta=85^{\circ} \mathrm{C} ; \phi_{\mathrm{He}}=465 \mathrm{SCCM} ; \phi_{\mathrm{Ether}}=6,4 \mathrm{SCCM} ; \phi_{\mathrm{CH} 2 \mathrm{Cl} 2}=6,2 \mathrm{SCCM} ; \mathrm{p}_{(\mathrm{StrR})}=99 \mathrm{~Pa} ; \mathrm{s}=2,5$ $\mathrm{cm} ; \mathrm{IP}=29,5 \mathrm{eV} ;$ Zählzeit $=5 \mathrm{~s}$

\begin{tabular}{|c|c|c|c|c|c|c|c|}
\hline $\begin{array}{c}\text { I(231) } \\
-F / \text { w.E. }\end{array}$ & $\begin{array}{c}\Delta \mathbf{I}(\mathbf{2 3 1}) \\
-\mathrm{F} / \mathrm{w.E} .\end{array}$ & $\begin{array}{c}I(231) \\
+F / \text { w.E. }\end{array}$ & $\begin{array}{c}\Delta \mathrm{I}(231) \\
+\mathrm{F} / \mathrm{w} . \mathrm{E} .\end{array}$ & $\begin{array}{c}\text { I(84) } \\
-F / \text { w.E. }\end{array}$ & $\begin{array}{c}\Delta \mathbf{I}(\mathbf{8 4}) \\
-\mathrm{F} / \mathbf{w . E} .\end{array}$ & $\begin{array}{c}\mathbf{I}(\mathbf{8 4 )} \\
+\mathbf{F} / \mathbf{w . E .}\end{array}$ & $\begin{array}{c}\Delta \mathrm{I}(\mathbf{8 4}) \\
+\mathrm{F} / \mathrm{w.E} .\end{array}$ \\
\hline 1897 & 36 & 1623 & 33 & 48780 & 168 & 42590 & 156 \\
\hline 1853 & 43 & 1600 & 11 & 49120 & 150 & 42730 & 151 \\
\hline 2133 & 35 & 1919 & 25 & 49340 & 151 & 44600 & 169 \\
\hline 2106 & 35 & 1924 & 28 & 49250 & 309 & 44490 & 119 \\
\hline 2127 & 17 & 2001 & 32 & 49800 & 188 & 46810 & 128 \\
\hline 2173 & 40 & 2076 & 23 & 46970 & 447 & 45490 & 258 \\
\hline 2109 & 40 & 2032 & 47 & 46200 & 175 & 44320 & 238 \\
\hline 2133 & 28 & 1823 & 37 & 49880 & 168 & 43280 & 196 \\
\hline 2048 & 45 & 1852 & 34 & 50310 & 183 & 46260 & 188 \\
\hline
\end{tabular}

Tabelle 9: Meßwerte Kinetik C4F9OCH3 + F rel. zu CH2Cl2; 358 K 


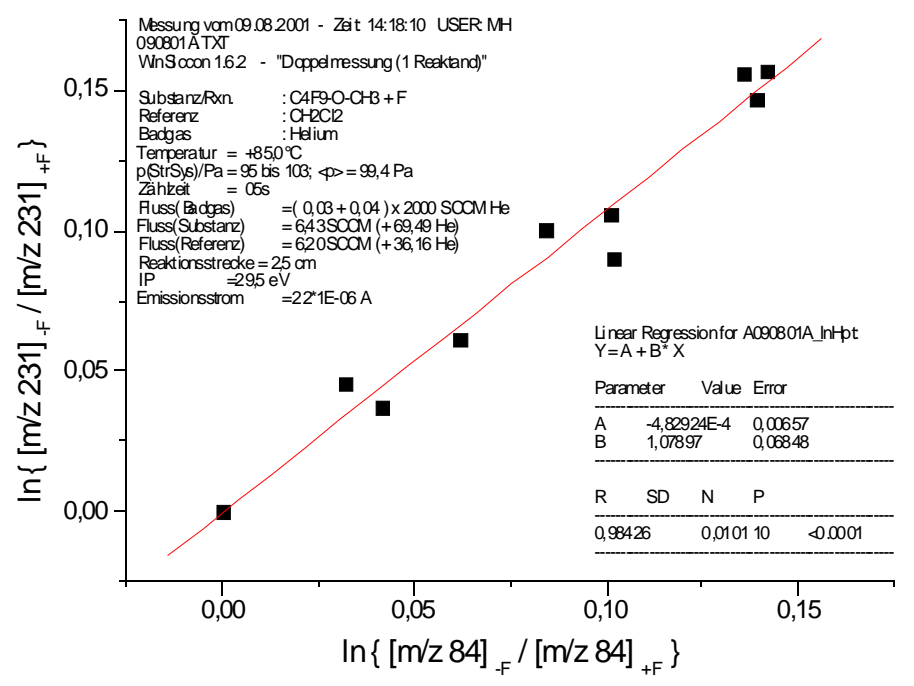

Abbildung 94: Kinetik C4F9OCH3 + F rel. zu CH2Cl2; $358 \mathrm{~K}$

Bei $\mathrm{T}=358 \mathrm{~K}$ erhält man aus Abbildung 94 einen relativen Geschwindigkeitskoeffizienten von $\mathrm{k}_{\mathrm{rel}}=(1,08 \pm 0,07)$.

Aus den ermittelten relativen Geschwindigkeitskoeffizienten können nun mit der bekannten Temperaturabhängigkeit des Geschwindigkeitskoeffizienten für die Referenzreaktion ${ }^{108} k_{\text {ref }}(T)$ die absoluten Geschwindigkeitskoeffizienten $\mathrm{k}_{\mathrm{Ether}}$ für die Reaktion des Methylperfluorbutylethers mit F-Atomen berechnet werden (Tabelle 10).

$\mathrm{k}_{\mathrm{ref}}(\mathrm{T})=(8,4 \pm 1,0) \cdot 10^{12} \cdot \exp \{(0,12 \pm 0,29) \mathrm{kJ} / \mathrm{mol} /(\mathrm{R} \cdot \mathrm{T})\} \mathrm{cm}^{3} /(\mathrm{mol} \cdot \mathrm{s})$

Ferner kann mit diesen Werten eine Arrhenius-Auftragung gemäß Gleichung (11) (S. 10) vorgenommen werden.

\begin{tabular}{|r|r|r|r|r|r|}
\hline$\vartheta /{ }^{\circ} \mathbf{C}$ & \multicolumn{1}{|c|}{$\mathbf{T}^{-1} / \mathbf{K}^{-1}$} & \multicolumn{1}{|c|}{$\mathbf{k}_{\text {rel }}$} & \multicolumn{1}{c|}{$\mathbf{l n}\left(\mathbf{k}_{\text {rel }}\right)$} & \multicolumn{1}{|c|}{$\begin{array}{c}\mathbf{k}_{\text {ref }} / \\
\mathbf{c m}^{\mathbf{3}} /(\mathbf{m o l ~ s})\end{array}$} & \multicolumn{1}{c|}{$\begin{array}{c}\mathbf{k}_{\text {Ether }} / \\
\mathbf{c m}^{\mathbf{3}} /(\mathbf{m o l} \mathbf{~ s})\end{array}$} \\
\hline-5 & $3,73 \mathrm{E}-03$ & 1,06 & $5,41 \mathrm{E}-02$ & $8,86 \mathrm{E}+12$ & $9,36 \mathrm{E}+12$ \\
\hline 25 & $3,35 \mathrm{E}-03$ & 1,06 & $5,91 \mathrm{E}-02$ & $8,82 \mathrm{E}+12$ & $9,35 \mathrm{E}+12$ \\
\hline 55 & $3,05 \mathrm{E}-03$ & 1,08 & $7,73 \mathrm{E}-02$ & $8,78 \mathrm{E}+12$ & $9,48 \mathrm{E}+12$ \\
\hline 85 & $2,79 \mathrm{E}-03$ & 1,08 & $7,60 \mathrm{E}-02$ & $8,75 \mathrm{E}+12$ & $9,44 \mathrm{E}+12$ \\
\hline
\end{tabular}

Tabelle 10: Werte für Arrhenius-Auftragung C4F9OCH3 + F rel. zu CH2Cl2

108 Hold, M. (1997); S. 115. Dieser Wert ist auch in Kap. 4.4 .3 (S. 212) zu finden. 


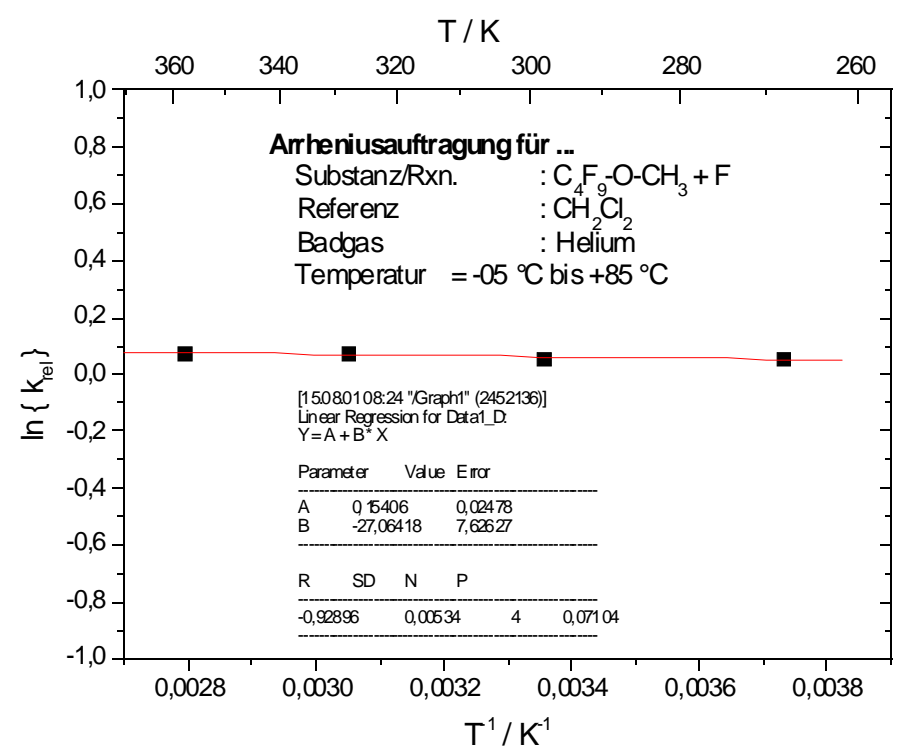

Abbildung 95: Arrhenius-Auftragung C4F9OCH3 + F rel. zu CH2Cl2

In Abb. 95 beträgt die Steigung $b=(-27,06 \pm 7,63)$, der Achsenabschnitt $a=(0,154 \pm 0,025)$.

Bildet man den Quotienten aus der Arrhenius-Gleichung (11) (S. 10) für Reaktion und Referenzreaktion, dann ergibt sich:

$$
\ln \left(\mathrm{k}_{\text {rel }}\right)=\ln \left(\frac{\mathrm{k}}{\mathrm{k}_{\text {Referenz }}}\right)=-\frac{\mathrm{E}_{\mathrm{a}}-\mathrm{E}_{\mathrm{a}, \text { Referenz }}}{\mathrm{R}} \cdot \frac{1}{\mathrm{~T}}+\ln \left(\frac{\mathrm{A}}{\mathrm{A}_{\text {Referenz }}}\right)
$$

Die Aktivierungsenergie der hier untersuchten Reaktion erhält man durch Vergleich der Steigung aus Abbildung 95 mit dem Ausdruck in Gleichung (27):

$\mathrm{E}_{\mathrm{a}}=\mathrm{E}_{\mathrm{a}, \text { Referenz }}-($ Steigung $) \cdot \mathrm{R}=(-120-(-27,06 \cdot 8,314)) \mathrm{J} / \mathrm{mol}=105 \mathrm{~J} / \mathrm{mol}$.

Bezüglich des präexponentiellen Faktors wird der Achsenabschnitt aus Abbildung 95 herangezogen:

$\mathrm{A}=\exp ($ Achsenabschnitt $) \cdot \mathrm{A}_{\text {Referenz }}=\exp (0,154) \cdot 8,4 \cdot 10^{12} \mathrm{~cm}^{3} \mathrm{~mol}^{-1} \mathrm{~s}^{-1}$

$$
=9,8 \cdot 10^{12} \mathrm{~cm}^{3} \mathrm{~mol}^{-1} \mathrm{~s}^{-1} \text {. }
$$

Im Ergebnis beträgt der gesuchte Geschwindigkeitskoeffizient damit:

$$
\begin{aligned}
& \mathbf{k}(\mathbf{T})=(9,8 \pm 1,2) \cdot 10^{12} \cdot \exp \{-(0,105 \pm 0,297) \mathrm{kJ} / \mathrm{mol} /(\mathbf{R} \cdot \mathbf{T})\} \mathrm{cm}^{3} /(\mathbf{m o l} \cdot \mathbf{s}) \\
& \text { für: } \mathrm{C}_{4} \mathrm{~F}_{9}-\mathrm{O}-\mathrm{CH}_{3}+\mathrm{F} \rightarrow \mathrm{C}_{4} \mathrm{~F}_{9}-\mathrm{O}-\mathrm{CH}_{2}+\mathrm{HF}
\end{aligned}
$$

Mit $\mathrm{T}=298 \mathrm{~K}$ errechnet man daraus: $\mathbf{k}(298 \mathrm{~K})=(\mathbf{9 , 4} \pm \mathbf{1 , 6}) \cdot \mathbf{1 0}^{\mathbf{1 2}} \mathbf{c m}^{\mathbf{3}} \mathrm{mol}^{-1} \mathrm{~s}^{-1}$.

Mit einer Aktivierungsenergie von lediglich $105 \mathrm{~J} / \mathrm{mol}$ ist der Geschwindigkeitskoeffizient für diese Reaktion praktisch temperaturunabhängig. 
Auf Grund der geringen Aktivierungsenergie empfiehlt sich eher eine Auftragung gemäß Gleichung (13) (S. 11). Aus der logarithmierten Version dieser Gleichung läßt sich leicht der Parameter n ermitteln (Abbildung 96).

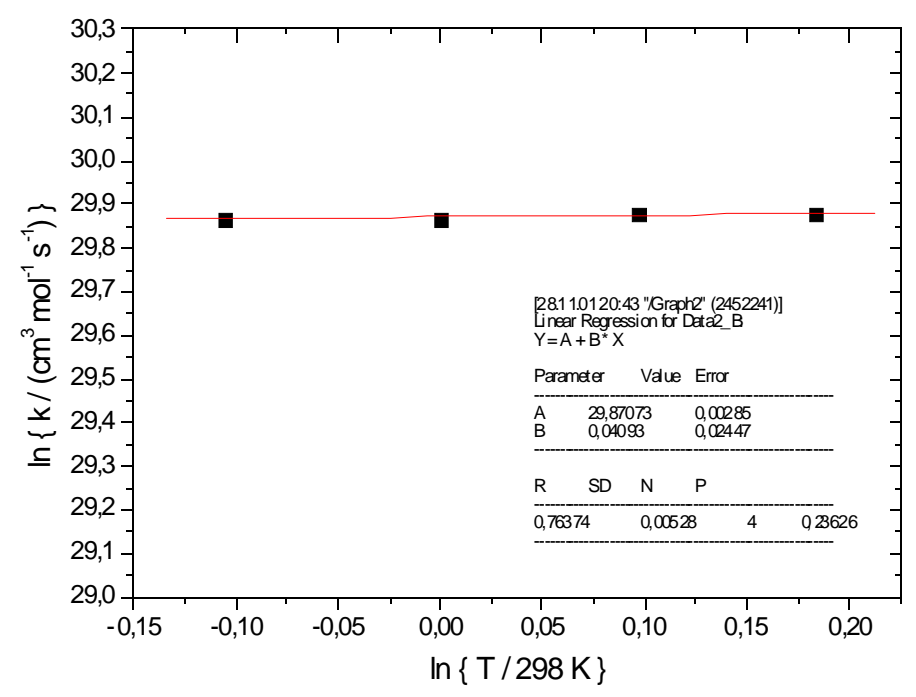

Abbildung 96: $\ln (k)$ vs. $\ln (T)$ - Auftragung für C4F9OCH3 $+F$

Der gesuchte Parameter $\mathrm{n}$ ist die Steigung b der Auftragung in Abbildung 96, aus dem Achsenabschnitt a ergibt sich der Parameter $A=\exp (\mathrm{a})$ :

$\mathrm{n}=(0,04 \pm 0,02) ; \mathrm{A}=\exp (29,87073)=(9,39 \pm 0,03) \cdot 10^{12} \mathrm{~cm}^{3} /(\mathrm{mol} \cdot \mathrm{s})$.

Damit ergibt sich für den gesuchten Geschwindigkeitskoeffizienten:

$$
\begin{aligned}
& \mathbf{k}(\mathbf{T})=(9,39 \pm 0,03) \cdot 10^{12} \cdot(\mathrm{T} / 298 \mathrm{~K})^{(0,04 \pm 0,02)} \mathrm{cm}^{3} /(\mathbf{m o l} \cdot \mathbf{s}) \\
& \text { für: } \mathrm{C}_{4} \mathrm{~F}_{9}-\mathrm{O}-\mathrm{CH}_{3}+\mathrm{F} \rightarrow \mathrm{C}_{4} \mathrm{~F}_{9}-\mathrm{O}-\mathrm{CH}_{2}+\mathrm{HF} .
\end{aligned}
$$

Mit $\mathrm{T}=298 \mathrm{~K}$ errechnet man daraus: $\mathbf{k}(298 \mathrm{~K})=(\mathbf{9 , 3 9} \pm \mathbf{0 , 0 3}) \cdot \mathbf{1 0}^{\mathbf{1 2}} \mathbf{c m}^{\mathbf{3}} \mathbf{m o l}^{-1} \mathbf{s}^{-1}$.

\subsubsection{Produkte der Reaktion des erzeugten Radikals mit molekularem Sauerstoff}

Wie oben dargelegt, entsteht bei der Reaktion des Ethers mit Fluor-Atomen das $\mathrm{C}_{4} \mathrm{~F}_{9}-\mathrm{O}-\mathrm{CH}_{2}$ Radikal. Im EI-Massenspektrum ist der Molekül-Peak dieses Radikals auf m/z $249 \mathrm{zu}$ erkennen, welcher durch Zugabe von molekularem Sauerstoff abnimmt. Um herauszufinden, welche Produkte bei dieser Reaktion des Radikals entstehen, wurden Massenspektren mit und ohne Zugabe von Sauerstoff aufgenommen; der nach Abschalten des $\mathrm{O}_{2}$-Flusses gesunkene Druck im Strömungsreaktor mußte durch He-Zugabe ausgeglichen werden, um eine Verfälschung der Peakhöhen zu verhindern. Die gewählten Reaktionsbedingungen führten zu 
einem Umsatz des Ethers bei Zugabe von Fluor-Atomen in Höhe von 16 \% (gemessen auf m/z 231). Die Zugabe von molekularem Sauerstoff führte umsatzkorrigiert (vgl. dazu Kap. 3.5.1, S. 50 ff.) zu einem Umsatz des Radikals in Höhe von 39 \% (gemessen auf m/z 249). Die EIElektronenenergie betrug IP $=25,3 \mathrm{eV}$, der Druck im Reaktor $143 \mathrm{~Pa}$, die Reaktionsstrecke 4,2 cm. Es wurde der in Abbildung 5 (S. 17) dargestellte Reaktor verwendet und die Spektren mit der in Kap. 3.1.1 (S. 16) beschriebenen Apparatur aufgenommen. In Abbildung 97 ist die Differenz der beiden registrierten Übersichtsspektren zu sehen.

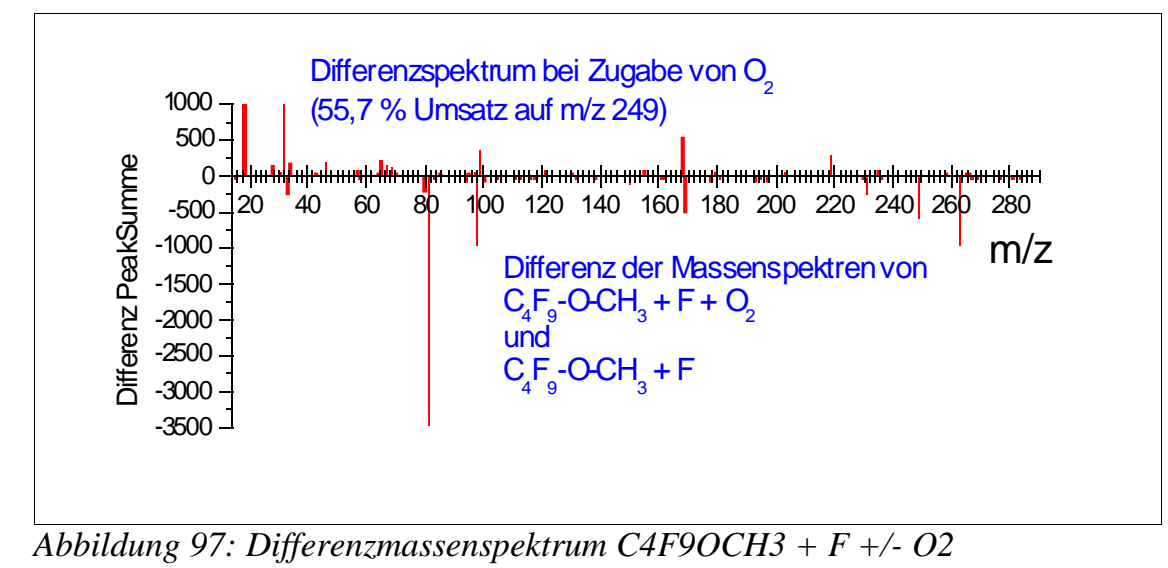

Basierend auf den Veränderungen in diesen Übersichtsspektren konnten Einzelmessungen mit längeren Zählzeiten zur Überprüfung durchgeführt werden (Abbildung 98). Die Reaktionsbedingungen sind in der Grafik vermerkt; der Umsatz des Ethers mit F-Atomen betrug 9,6\% und derjenige des Radikals $55,7 \%$ bei Zugabe von $\mathrm{O}_{2}$.

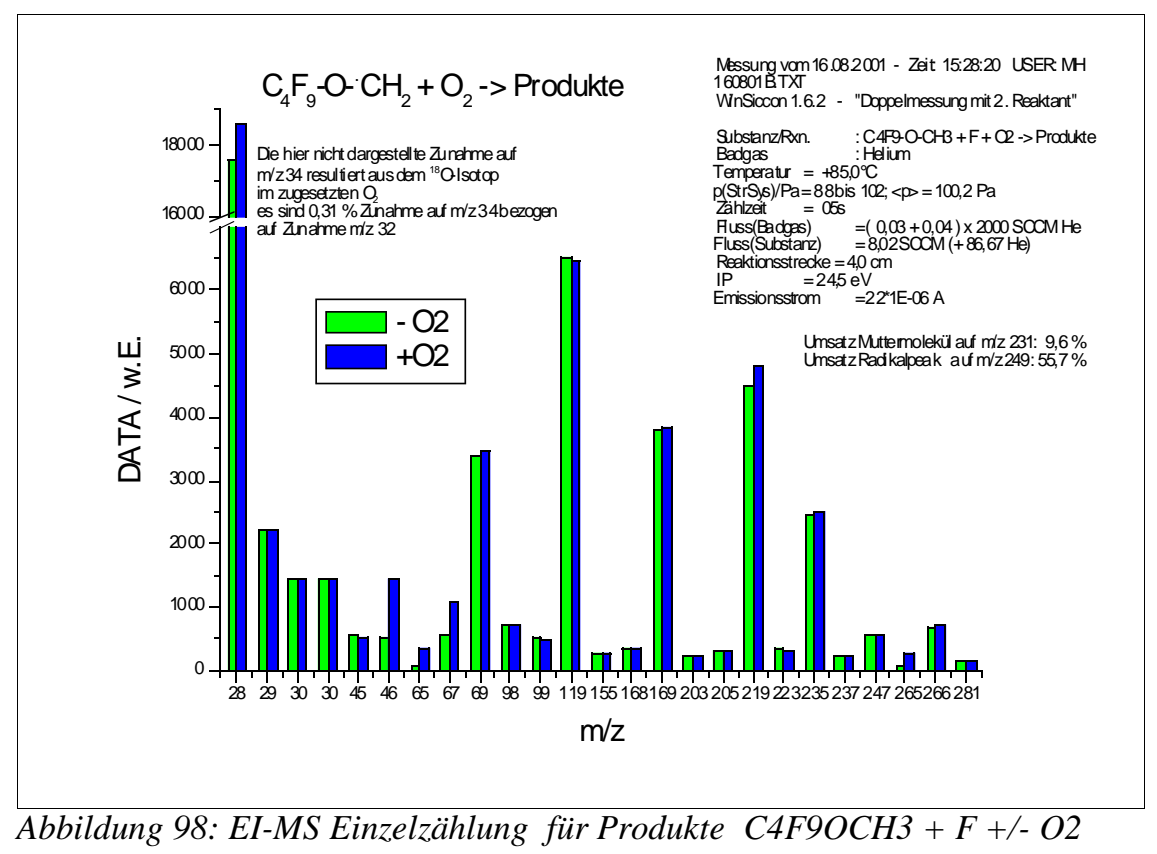

Auffallend in Abbildung 98 sind die starken Zunahmen auf m/z 28, 46, 65, 67 und 219 sowie 
die schwächeren auf m/z 69, 169, 235 und 266. Die zunächst auf m/z 30 beobachtete Zunahme konnte in einer zweiten Messung nicht bestätigt werden. Zusammen mit dem fehlenden Anstieg auf m/z 29 kann also Formaldehyd (Methanal) als Produkt ausgeschlossen werden. Auch Fluormethanal (FCHO, $\left.\mathrm{M}_{\mathrm{r}}=48\right)$ und Difluormethanal $\left(\mathrm{FCFO}, \mathrm{M}_{\mathrm{r}}=66\right)$ scheinen nicht gebildet worden zu sein. Es ist denkbar, daß der Anstieg auf m/z 46 auf der Bildung von Ameisensäure (Methansäure, $\mathrm{M}_{\mathrm{r}}=46$ ) beruht. Weitere Vorschläge bzgl. der gebildeten Produkte können nicht gemacht werden.

\subsubsection{Kinetik der Reaktion des erzeugten Radikals mit molekularem Sauerstoff}

Die Messungen wurden in der in Kap. 3.1.1 (S. 16) beschriebenen Apparatur unter Einsatz des Strömungsreaktors aus Abbildung 5 (S. 17) durchgeführt.

Das Dichlormethyl-Radikal diente als Referenzsubstanz. Der Umsatz des Ether-Radikals konnte anhand der Intensitätsänderung seines Molekül-Peaks $\mathrm{m} / \mathrm{z} 249$, derjenige des Dichlormethyl-Radikals ebenfalls anhand seines Molekül-Peaks (m/z 83) bestimmt werden.

Die Messungen wurden umsatzkorrigiert nach der Relativmethode durchgeführt; die genaue Ausführung und Auswertung ist in Kap. 3.5.1 (S. 50) beschrieben.

Der Umsatz bei Zugabe von Fluor-Atomen betrug 17,3\% auf m/z 231 und 11,5\% auf m/z 84 .

$\vartheta=25{ }^{\circ} \mathrm{C} ; \phi_{\mathrm{He}}=392 \mathrm{SCCM} ; \phi_{\mathrm{Ether}}=6,4 \mathrm{SCCM} ; \phi_{\mathrm{CH} 2 \mathrm{Cl} 2}=6,0 \mathrm{SCCM} ; \mathrm{p}_{(\mathrm{StrR})}=123 \mathrm{~Pa} ; \mathrm{s}=4,2$ $\mathrm{cm} ; \mathrm{IP}=24,5 \mathrm{eV}$; Zählzeit $=5 \mathrm{~s}$

\begin{tabular}{|c|c|c|c|c|c|c|c|}
\hline $\begin{array}{c}I(249) \\
-O_{2} / \text { w.E. }\end{array}$ & $\begin{array}{c}\Delta \mathrm{I}(249) \\
-\mathrm{O}_{2} / \mathrm{w} . \mathrm{E}\end{array}$ & $\begin{array}{c}\mathrm{I}(249) \\
+\mathrm{O}_{2} / \text { w.E. }\end{array}$ & $\begin{array}{r}\Delta \mathrm{I}(249) \\
+\mathrm{O}_{2} / \text { w.E. } \\
\end{array}$ & $\begin{array}{c}\mathrm{I}(\mathbf{8 3}) \\
-\mathrm{O}_{2} / \mathrm{w.E.}\end{array}$ & $\begin{array}{c}\Delta \mathbf{I}(\mathbf{8 3}) \\
-\mathbf{O}_{2} / \mathbf{w . E} .\end{array}$ & $\begin{array}{c}\mathrm{I}(83) \\
+\mathrm{O}_{2} / \mathrm{w} . \mathrm{E} .\end{array}$ & $\begin{aligned} & \Delta \mathrm{I}(\mathbf{8 3}) \\
+ & \mathrm{O}_{2} / \mathrm{w} . \mathrm{E}\end{aligned}$ \\
\hline 8274 & 128 & 3230 & 74 & 2734 & 87 & 1298 & 85 \\
\hline 8254 & 90 & 3184 & 43 & 2778 & 91 & 1336 & 90 \\
\hline 8355 & 127 & 3223 & 53 & 2570 & 81 & 1221 & 73 \\
\hline 7817 & 81 & 3427 & 44 & 2261 & 98 & 1367 & 97 \\
\hline 7937 & 114 & 3380 & 40 & 2009 & 147 & 921 & 87 \\
\hline 8453 & 152 & 3884 & 67 & 2225 & 80 & 1220 & 100 \\
\hline 8498 & 133 & 3907 & 35 & 2207 & 93 & 1339 & 100 \\
\hline 8512 & 111 & 4901 & 70 & 1805 & 114 & 1152 & 98 \\
\hline 8225 & 135 & 5912 & 74 & 1740 & 89 & 1394 & 88 \\
\hline 8366 & 108 & 6982 & 81 & 1554 & 105 & 1340 & 87 \\
\hline 8384 & 170 & 6990 & 71 & 1777 & 116 & 1443 & 93 \\
\hline
\end{tabular}

Tabelle 11: Meßwerte Kinetik C4F9OCH2 + O2 rel. zu CHCl2; $298 \mathrm{~K}$ 
Aus den Meßwerten der Tabelle 11 kann gemäß Gleichung (10) (S. 10) eine Auftragung zur Bestimmung des relativen Geschwindigkeitskoeffizienten angefertigt werden (Abb. 99).

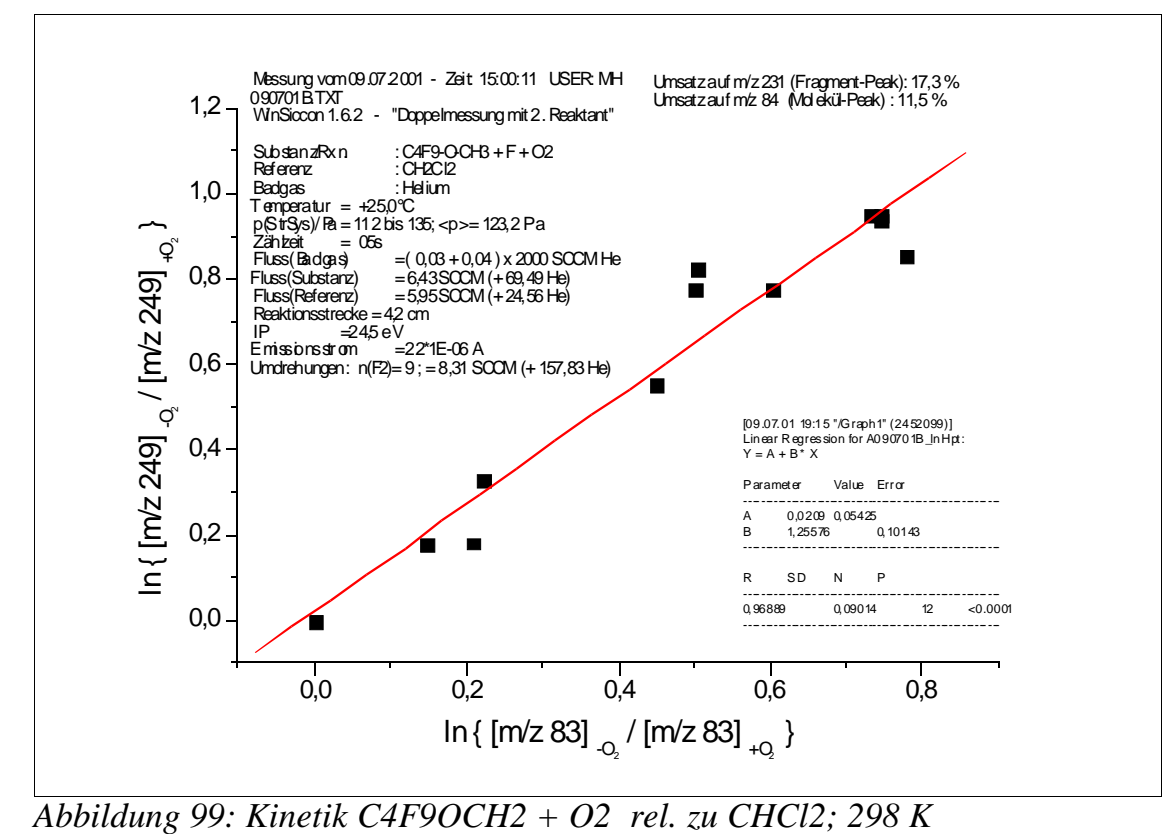

Bei T = 298 K erhält man aus Abbildung 99 einen relativen Geschwindigkeitskoeffizienten von $\mathrm{k}_{\mathrm{rel}}=(1,26 \pm 0,02)$.

$\vartheta=45^{\circ} \mathrm{C} ; \phi_{\mathrm{He}}=392 \mathrm{SCCM} ; \phi_{\text {Ether }}=6,4 \mathrm{SCCM} ; \phi_{\mathrm{CH} 2 \mathrm{Cl} 2}=6,0 \mathrm{SCCM} ; \mathrm{p}_{(\mathrm{StrR})}=106 \mathrm{~Pa} ; \mathrm{s}=4,2$ $\mathrm{cm} ; \mathrm{IP}=29,5 \mathrm{eV} ;$ Zählzeit $=5 \mathrm{~s}$

\begin{tabular}{|c|c|c|c|c|c|c|c|}
\hline $\begin{array}{c}\mathrm{I}(249) \\
-\mathrm{O}_{2} / \text { w.E. }\end{array}$ & $\begin{array}{c}\Delta \mathrm{I}(249) \\
-\mathrm{O}_{2} / \text { w.E. }\end{array}$ & $\begin{array}{c}\mathrm{I}(249) \\
+\mathrm{O}_{2} / \text { w.E. }\end{array}$ & $\begin{array}{r}\Delta \mathrm{I}(249) \\
+\mathrm{O}_{2} / \text { w.E. } \\
\end{array}$ & $\begin{array}{c}\mathbf{I}(\mathbf{8 3}) \\
-\mathrm{O}_{2} / \mathbf{w . E} .\end{array}$ & $\begin{array}{c}\Delta \mathbf{I}(\mathbf{8 3}) \\
-\mathbf{O}_{2} / \mathbf{w . E .}\end{array}$ & $\begin{array}{c}\mathrm{I}(83) \\
+\mathrm{O}_{2} / \mathrm{w} . \mathrm{E} .\end{array}$ & $\begin{array}{c}\Delta \mathrm{I}(\mathbf{8 3}) \\
+\mathrm{O}_{2} / \mathrm{w} . \mathrm{E} .\end{array}$ \\
\hline 27470 & 141 & 13570 & 92 & 4900 & 182 & 3010 & 118 \\
\hline 27250 & 265 & 13570 & 136 & 4681 & 144 & 2811 & 153 \\
\hline 26940 & 213 & 13300 & 134 & 3755 & 222 & 2225 & 258 \\
\hline 7455 & 155 & 4152 & 168 & 4422 & 139 & 2932 & 159 \\
\hline 27210 & 151 & 16410 & 122 & 4263 & 173 & 3123 & 151 \\
\hline 25680 & 173 & 17230 & 308 & 4403 & 237 & 3433 & 146 \\
\hline 28890 & 275 & 27310 & 141 & 2566 & 116 & 2286 & 261 \\
\hline
\end{tabular}

Tabelle 12: Meßwerte Kinetik C4F9OCH2 + O2 rel. zu CHCl2; $318 \mathrm{~K}$ 


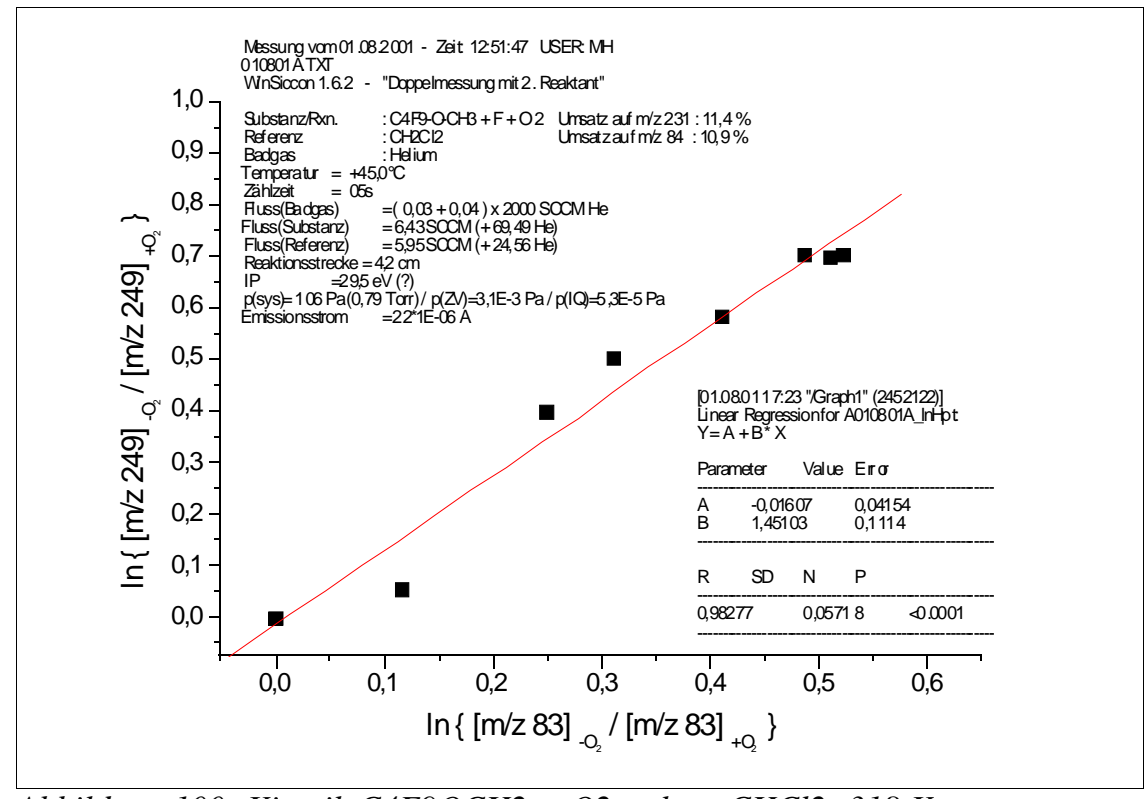

Abbildung 100: Kinetik C4F9OCH2 + O2 rel. zu CHCl2; $318 \mathrm{~K}$

Bei $\mathrm{T}=318 \mathrm{~K}$ erhält man aus Abbildung 100 einen relativen Geschwindigkeitskoeffizienten von $\mathrm{k}_{\mathrm{rel}}=(1,45 \pm 0,11)$.

$\vartheta=65{ }^{\circ} \mathrm{C} ; \phi_{\mathrm{He}}=392 \mathrm{SCCM} ; \phi_{\mathrm{Ether}}=6,4 \mathrm{SCCM} ; \phi_{\mathrm{CH} 2 \mathrm{C} 12}=6,0 \mathrm{SCCM} ; \mathrm{p}_{(\mathrm{StrR})}=135 \mathrm{~Pa} ; \mathrm{s}=4,2$ $\mathrm{cm} ; \mathrm{IP}=24,5 \mathrm{eV} ;$ Zählzeit $=5 \mathrm{~s}$

\begin{tabular}{|c|c|c|c|c|c|c|c|}
\hline $\begin{array}{c}\mathrm{I}(249) \\
-\mathrm{O}_{2} / \text { w.E. }\end{array}$ & $\begin{array}{c}\Delta \mathrm{I}(\mathbf{2 4 9}) \\
-\mathrm{O}_{2} / \text { w.E. }\end{array}$ & $\begin{array}{c}\mathrm{I}(249) \\
+\mathrm{O}_{2} / \text { w.E. }\end{array}$ & $\begin{array}{c}\Delta \mathbf{I}(249) \\
+\mathrm{O}_{2} / \text { w.E. }\end{array}$ & $\begin{array}{c}\mathbf{I}(\mathbf{8 3}) \\
-\mathbf{O}_{2} / \mathbf{w . E .}\end{array}$ & $\begin{array}{c}\Delta \mathrm{I}(\mathbf{8 3}) \\
-\mathrm{O}_{2} / \mathrm{w} . \mathrm{E} .\end{array}$ & $\begin{array}{c}\mathrm{I}(83) \\
+\mathrm{O}_{2} / \text { w.E. }\end{array}$ & $\begin{array}{c}\Delta \mathrm{I}(\mathbf{8 3}) \\
+\mathbf{O}_{2} / \text { w.E. }\end{array}$ \\
\hline 13020 & 86 & 5670 & 57 & 2833 & 107 & 1705 & 92 \\
\hline 12730 & 97 & 5942 & 84 & 2955 & 88 & 1836 & 101 \\
\hline 12620 & 108 & 7144 & 63 & 2620 & 78 & 1870 & 83 \\
\hline 12530 & 101 & 7772 & 103 & 2461 & 81 & 1871 & 112 \\
\hline 12600 & 74 & 8734 & 82 & 2449 & 100 & 2003 & 125 \\
\hline 12610 & 98 & 10100 & 120 & 2138 & 120 & 1902 & 101 \\
\hline 12560 & 72 & 10010 & 106 & 2234 & 101 & 1964 & 139 \\
\hline 12350 & 92 & 11260 & 88 & 2324 & 92 & 2165 & 100 \\
\hline 12970 & 134 & 5937 & 96 & 2741 & 133 & 1779 & 97 \\
\hline 13170 & 89 & 6104 & 69 & 2762 & 102 & 1706 & 96 \\
\hline
\end{tabular}

Tabelle 13: Meßwerte Kinetik C4F9OCH2 + O2 rel. zu CHCl2; $338 \mathrm{~K}$ 


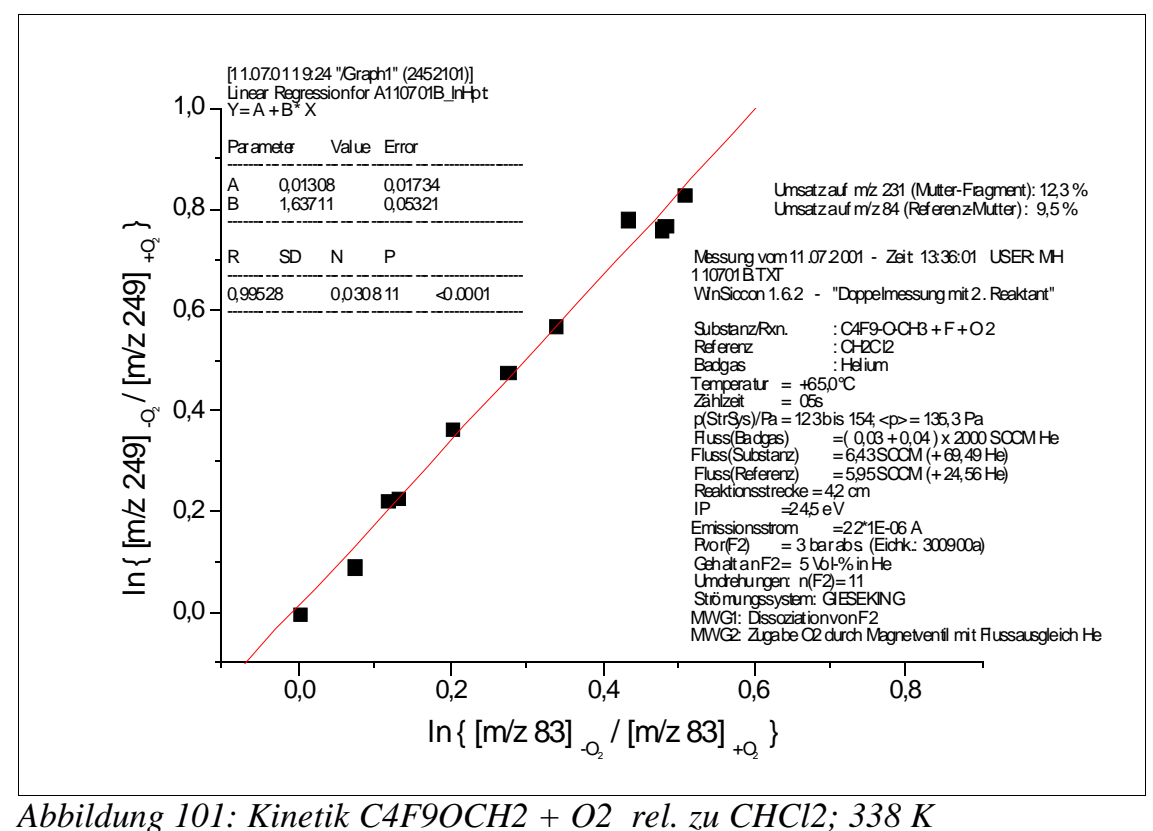

Bei $\mathrm{T}=338 \mathrm{~K}$ erhält man aus Abbildung 101 einen relativen Geschwindigkeitskoeffizienten von $\mathrm{k}_{\mathrm{rel}}=(1,64 \pm 0,05)$.

$\vartheta=85^{\circ} \mathrm{C} ; \phi_{\mathrm{He}}=392 \mathrm{SCCM} ; \phi_{\mathrm{Ether}}=8,0 \mathrm{SCCM} ; \phi_{\mathrm{CH} 2 \mathrm{Cl} 2}=8,2 \mathrm{SCCM} ; \mathrm{p}_{(\mathrm{StrR})}=102 \mathrm{~Pa} ; \mathrm{s}=4,0$ $\mathrm{cm} ; \mathrm{IP}=24,5 \mathrm{eV}$; Zählzeit $=5 \mathrm{~s}$

\begin{tabular}{|c|c|c|c|c|c|c|c|}
\hline $\begin{array}{c}\mathrm{I}(249) \\
-\mathrm{O}_{2} / \text { w.E. }\end{array}$ & $\begin{array}{c}\Delta \mathbf{I}(249) \\
-\mathbf{O}_{2} / \mathbf{w . E}\end{array}$ & $\begin{array}{c}\mathrm{I}(249) \\
+\mathrm{O}_{2} / \text { w.E. }\end{array}$ & $\begin{array}{c}\Delta \mathbf{I}(249) \\
+\mathrm{O}_{2} / \text { w.E. }\end{array}$ & $\begin{array}{c}\mathbf{I}(\mathbf{8 3}) \\
-\mathrm{O}_{2} / \mathbf{w . E .}\end{array}$ & $\begin{array}{c}\Delta \mathbf{I}(\mathbf{8 3}) \\
-\mathbf{O}_{2} / \mathbf{w . E} .\end{array}$ & $\begin{array}{c}\mathrm{I}(83) \\
+\mathrm{O}_{2} / \text { w.E. }\end{array}$ & $\begin{array}{c}\Delta \mathrm{I}(\mathbf{8 3}) \\
+\mathrm{O}_{2} / \mathrm{w} . \mathrm{E} .\end{array}$ \\
\hline 18140 & 157 & 6210 & 97 & 3017 & 117 & 1760 & 108 \\
\hline 17980 & 143 & 6398 & 130 & 2930 & 100 & 1756 & 107 \\
\hline 17550 & 198 & 7462 & 102 & 2732 & 105 & 1721 & 85 \\
\hline 17620 & 84 & 7548 & 95 & 2772 & 182 & 1896 & 126 \\
\hline 18120 & 153 & 9018 & 99 & 2571 & 132 & 1863 & 105 \\
\hline 18100 & 119 & 10590 & 90 & 2480 & 131 & 1909 & 133 \\
\hline 17410 & 109 & 12520 & 108 & 2697 & 113 & 2421 & 112 \\
\hline 17090 & 118 & 15130 & 162 & 2322 & 109 & 2129 & 122 \\
\hline
\end{tabular}

Tabelle 14: Meßwerte Kinetik C4F9OCH2 + O2 rel. zu CHCl2; $358 \mathrm{~K}$ 


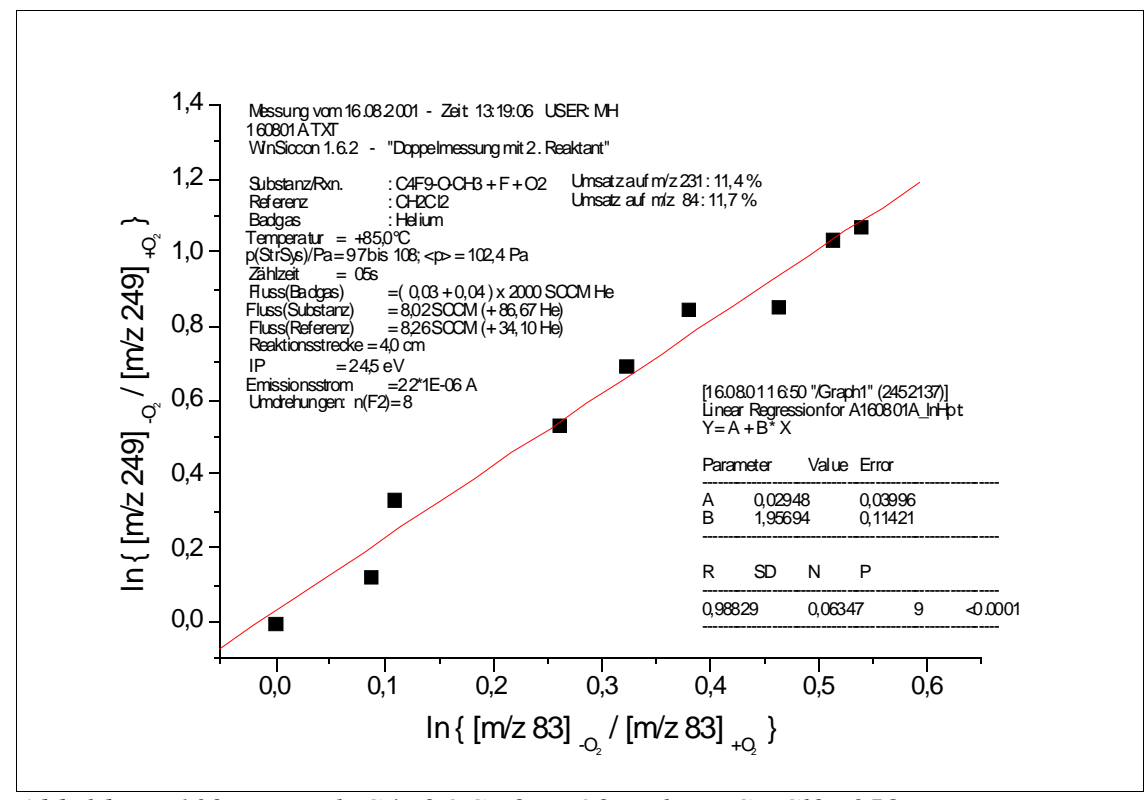

Abbildung 102: Kinetik C4F9OCH2 + O2 rel. zu CHCl2; $358 \mathrm{~K}$

Bei $\mathrm{T}=358 \mathrm{~K}$ erhält man aus Abbildung 102 einen relativen Geschwindigkeitskoeffizienten von $\mathrm{k}_{\mathrm{rel}}=(1,96 \pm 0,11)$.

Aus den ermittelten relativen Geschwindigkeitskoeffizienten können nun mit der bekannten Temperaturabhängigkeit des Geschwindigkeitskoeffizienten für die Referenzreaktion ${ }^{109} \mathrm{k}_{\mathrm{ref}}(\mathrm{T})$ die absoluten Geschwindigkeitskoeffizienten $\mathrm{k}_{\text {Ether }}$ für die Reakation des Perfluorbutoxymethyl-Radikals mit $\mathrm{O}_{2}$ berechnet werden (Tabelle 15).

$\mathrm{k}_{\mathrm{ref}}(\mathrm{T})=(1,56 \pm 0,08) \cdot 10^{17} \cdot(\mathrm{T} / 298 \mathrm{~K})^{-(6,7 \pm 0,7)} \cdot[\mathrm{He}] \mathrm{cm}^{6} /\left(\mathrm{mol}^{2} \cdot \mathrm{s}\right)$.

Ferner kann mit diesen Werten eine Arrhenius-Auftragung gemäß Gleichung (11) (S. 10) angefertigt werden, welche in Abbildung 103 (S. 125) zu sehen ist.

\begin{tabular}{|r|r|r|r|r|r|r|}
\hline$\vartheta /{ }^{\circ} \mathbf{C}$ & \multicolumn{1}{|l|}{$\mathbf{l n}(\mathbf{T} / \mathbf{K})$} & \multicolumn{1}{|c|}{$\mathbf{T}^{-1} / \mathbf{K}^{-1}$} & \multicolumn{1}{|c|}{$\mathbf{k}_{\text {rel }}$} & \multicolumn{1}{|c|}{$\mathbf{l n}\left(\mathbf{k}_{\text {rel }}\right)$} & $\begin{array}{c}\mathbf{k}_{\text {ref }} / \\
\mathbf{c m}^{6} /\left(\mathbf{m o l}^{\mathbf{2}} \mathbf{s}\right)\end{array}$ & $\begin{array}{l}\mathbf{k}_{\text {Ether-Radikal }} / \\
\mathbf{c m}^{\mathbf{6}} /\left(\mathbf{m o l}^{\mathbf{2}} \mathbf{s}\right)\end{array}$ \\
\hline 25 & 5,7 & $3,35 \mathrm{E}-03$ & 1,25576 & $5,41 \mathrm{E}-02$ & $1,55 \mathrm{E}+17$ & $1,95 \mathrm{E}+17$ \\
\hline 45 & 5,76 & $3,14 \mathrm{E}-03$ & 1,45103 & $5,91 \mathrm{E}-02$ & $1,01 \mathrm{E}+17$ & $1,46 \mathrm{E}+17$ \\
\hline 65 & 5,82 & $2,96 \mathrm{E}-03$ & 1,63711 & $7,73 \mathrm{E}-02$ & $6,69 \mathrm{E}+16$ & $1,10 \mathrm{E}+17$ \\
\hline 85 & 5,88 & $2,79 \mathrm{E}-03$ & 1,95694 & $7,60 \mathrm{E}-02$ & $4,55 \mathrm{E}+16$ & $8,91 \mathrm{E}+16$ \\
\hline
\end{tabular}

Tabelle 15: Werte für Arrhenius-Auftragung C4F9OCH2 + O2 rel. zu CHCl2 
Bei der hier untersuchten Reaktion mit $\mathrm{O}_{2}$ dürfte es sich vom Typ her um eine Assoziationsreaktion handeln, für die als vereinfachtes Kinetik-Schema angegeben werden kann:

$$
\begin{aligned}
& \mathrm{C}_{4} \mathrm{~F}_{9} \mathrm{OC} \mathrm{CH}_{2}+\mathrm{O}_{2} \underset{\mathrm{k}_{\mathrm{diss}}}{\stackrel{\mathrm{k}_{\mathrm{a}}}{=}}\left(\begin{array}{c}
\dot{\mathrm{O}}_{2} \\
\mathrm{C}_{4} \mathrm{~F}_{9} \mathrm{OCH}_{2}
\end{array}\right)^{\star} \\
& \left(\begin{array}{c}
\dot{\mathrm{O}}_{2} \\
\mathrm{C}_{4} \mathrm{~F}_{9} \mathrm{OCH}_{2}
\end{array}\right)^{\star}+\mathrm{M} \stackrel{\mathrm{k}_{\mathrm{d}}}{\longrightarrow} \underset{\mathrm{C}_{4} \mathrm{~F}_{9} \mathrm{OCH}_{2}}{\stackrel{\dot{\mathrm{O}}_{2}}{\longrightarrow}}+\mathrm{M}^{\star}
\end{aligned}
$$

Wendet man auf die Konzentration des angeregten Komplexes das Konzept der Quasistationarität (steady-state) an, so gilt für das Zeitgesetz:

$\mathrm{d}\left[\mathrm{CH}_{2} \mathrm{FOCHFO}_{2} *\right] / \mathrm{dt}=+\mathrm{k}_{\mathrm{a}}\left[\mathrm{CH}_{2} \mathrm{FOCHF}\right]\left[\mathrm{O}_{2}\right]-\mathrm{k}_{\text {diss }}\left[\mathrm{CH}_{2} \mathrm{FOCHFO}_{2} *\right]-\mathrm{k}_{\mathrm{d}}\left[\mathrm{CH}_{2} \mathrm{FOCHFO}_{2} *\right][\mathrm{M}] \approx 0$

Damit ergibt sich für die Bildung des stabilisierten Komplexes:

$$
\frac{\mathrm{d}\left[\mathrm{CH}_{2} \text { FOCHFO }_{2}\right]}{\mathrm{dt}}=\frac{\mathrm{k}_{\mathrm{d}} \mathrm{k}_{\mathrm{a}}\left[\mathrm{CH}_{2} \text { FOCHF }\right]\left[\mathrm{O}_{2}\right][\mathrm{M}]}{\mathrm{k}_{\text {diss }}+\mathrm{k}_{\mathrm{d}}[\mathrm{M}]}
$$

Die Versuche wurden bei sehr geringem Druck (100-130 Pa) ausgeführt, so daß $k_{d}[M]<<k_{\text {diss }}$ ist und damit näherungsweise für das Zeitgesetz der Bildung des stabilisierten Komplexes gilt:

$$
\left(\frac{\mathrm{d}\left[\mathrm{CH}_{2} \mathrm{FOCHFO}_{2}\right]}{\mathrm{dt}}\right)^{\mathrm{p} \rightarrow 0}=\frac{\mathrm{k}_{\mathrm{d}} \mathrm{k}_{\mathrm{a}}}{\mathrm{k}_{\text {diss }}}\left[\mathrm{CH}_{2} \mathrm{FOCHF}\right]\left[\mathrm{O}_{2}\right][\mathrm{M}]
$$

Aus Gleichung (28) erkennt man, daß es sich um eine Kinetik 3. Ordnung handelt, bei der die (Stoß-)Deaktivierung des gebildeten angeregten Komplexes geschwindigkeitsbestimmend für die Bildung des stabilisierten Komplexes ist. Im durchgeführten Experiment wurde Helium in großem Überschuß als Badgas verwendet, so daß (fast) nur dieses als Stoßpartner M fungierte. Offensichtlich kommt den Eigenschaften des verwendeten Badgases eine große Bedeutung für den Wert des zu messenden Geschwindigkeitskoeffizienten zu. So hat sich z.B. herausgestellt, daß Argon pro Stoß etwa $75 \%$ mehr Energie aufnehmen kann als Helium, andererseits aber die Stoßzahl etwa $35 \%$ geringer ist ${ }^{110}$. Eine signifikante Temperaturabhängigkeit für die Energieübertragung wurde nicht gefunden ${ }^{111}$. Aus diesen Gründen wurde eine Referenzreaktion gewählt, die ebenfalls in Helium als Badgas untersucht wurde.

Weil die Reaktion wahrscheinlich im Niederdruckbereich ablief, sind die berechneten absoluten Geschwindigkeitskoeffizienten in Tabelle 15 termolekular angegeben.

110 Vgl. HIPPLER, H. et. al. (1983); S. 6712. Die Versuche wurden mit Toluol in diversen Badgasen durchgeführt, wobei die Ergebnisse auch gut auf andere Stoffe übertragbar sein sollen.

111 Vgl. HIPPLER, H. et. al. (1983); S. 6709 


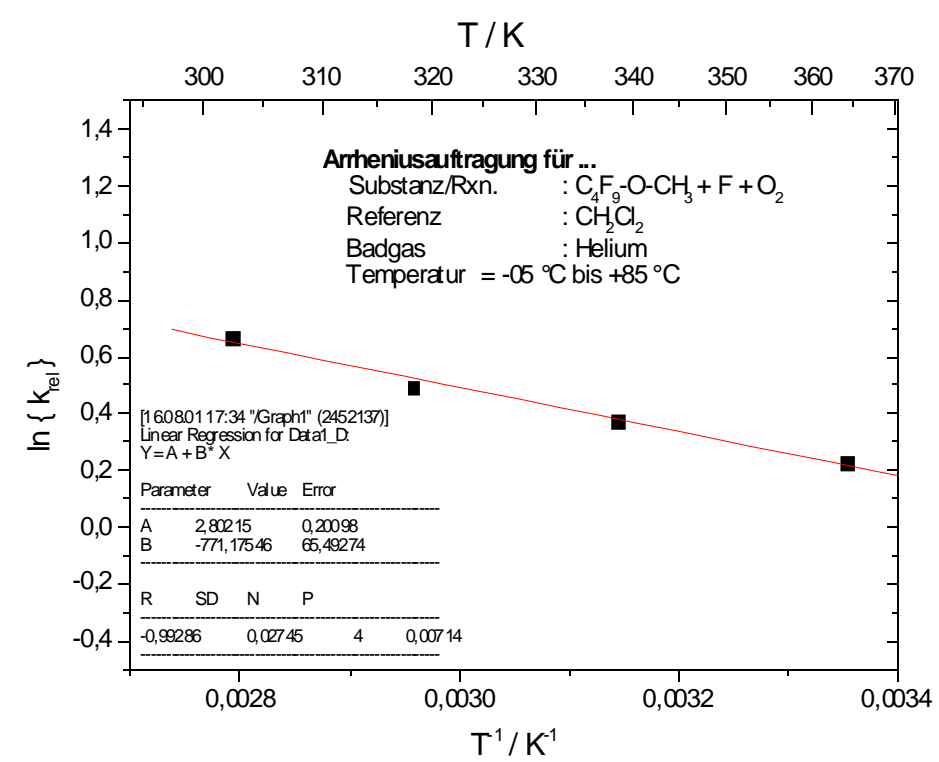

Abbildung 103: Arrhenius-Auftragung C4F9OCH2 + O2 rel. zu CHCl2

Die Steigung der Arrhenius-Auftragung in Abbildung 103 beträgt $b=(771,1 \pm 65,5) \mathrm{K}$, der Achsenabschnitt $\mathrm{a}=(2,802 \pm 0,201)$.

Zur Ermittlung der Temperaturabhängigkeit des Geschwindigkeitskoeffizienten wird ferner mit den Werten aus Tabelle 15 eine Auftragung $\ln (\mathrm{k})$ vs. $\ln (\mathrm{T})$ angefertigt (Abbildung 104).

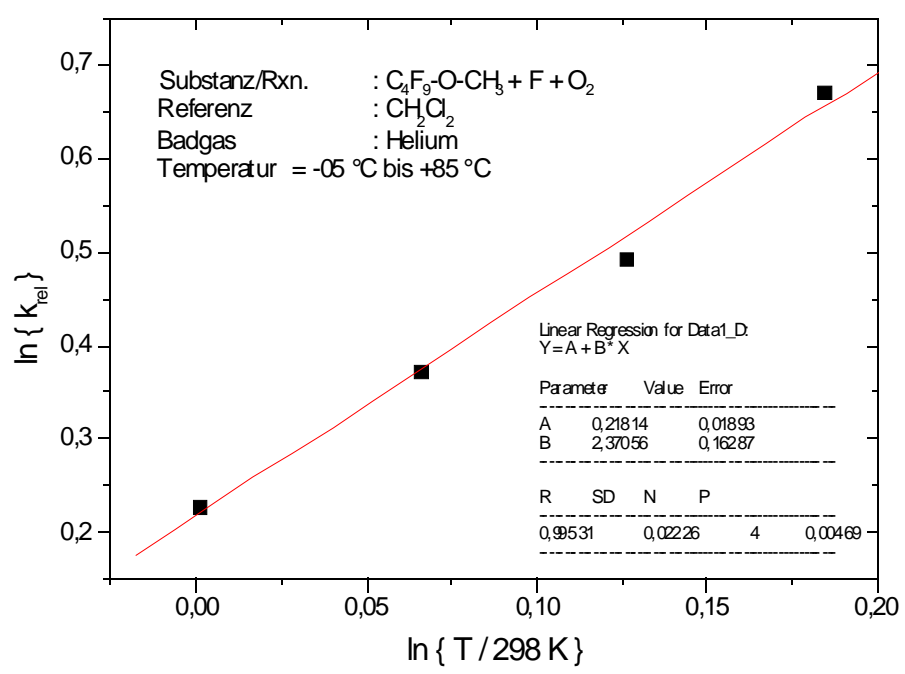

Abbildung 104: $\ln (k)$ vs. $\ln (\mathrm{T})$ für $\mathrm{C} 4 \mathrm{~F} 9 \mathrm{OCH} 2+\mathrm{O} 2$ rel. zu CHCl2

Die Steigung der Auftragung in Abbildung 104 beträgt $b=(0,22 \pm 0,02)$, der Achsenabschnitt $a=(2,37 \pm 0,16)$. 
Bildet man den Quotienten aus der Gleichung (13) (S. 11) für Reaktion und Referenzreaktion, dann ergibt sich:

$$
\mathrm{k}_{\mathrm{rel}}:=\frac{\mathrm{k}}{\mathrm{k}_{\mathrm{ref}}}=\frac{\mathrm{A} \cdot \mathrm{T}^{\mathrm{n}}}{\mathrm{A}_{\mathrm{ref}} \cdot \mathrm{T}^{\mathrm{n}_{\mathrm{ref}}}}
$$

Nach Logarithmieren erhält man daraus:

$$
\ln \left(\mathrm{k}_{\text {rel }}\right)=\ln \left(\frac{\mathrm{A}}{\mathrm{A}_{\text {ref }}}\right)+\left(\mathrm{n}-\mathrm{n}_{\text {ref }}\right) \cdot \ln (\mathrm{T})
$$

Mittels Gleichung (29) kann nun aus dem Achsenabschnitt a der Abbildung 104 der Vorfaktor A berechnet werden:

$\mathrm{A}=\mathrm{A}_{\mathrm{ref}} \cdot \exp (\mathrm{a})=1,56 \cdot 10^{17} \mathrm{~cm}^{6} /\left(\mathrm{mol}^{2} \cdot \mathrm{s}\right) \cdot \exp (0,21814)=1,94 \cdot 10^{17} \mathrm{~cm}^{6} /\left(\mathrm{mol}^{2} \cdot \mathrm{s}\right)$.

Den Parameter n kann man gemäß Gleichung (29) folgendermaßen aus der Steigung b der Abbildung 104 ermittlen:

$\mathrm{n}=\left(\mathrm{b}+\mathrm{n}_{\mathrm{ref}}\right)=(2,37+(-6,70))=-4,33$.

Somit ergibt sich als Temperaturabhängigkeit des Geschwindigkeitskoeffizienten:

$$
\begin{aligned}
& \mathbf{k}(\mathbf{T})=(1,94 \pm 0,10) \cdot 10^{17} \cdot(\mathbf{T} / 298 \mathrm{~K})^{-(4,33 \pm 0,69)} \cdot[\mathrm{He}] \mathbf{c m}^{6} /\left(\mathbf{m o l}^{2} \cdot \mathbf{s}\right) \\
& \text { für: } \mathrm{C}_{4} \mathrm{~F}_{9}-\mathrm{O}-\mathrm{CH}_{2}+\mathrm{O}_{2} \rightarrow \mathrm{C}_{4} \mathrm{~F}_{9}-\mathrm{O}-\mathrm{CH}_{2} \mathrm{O}_{2}
\end{aligned}
$$

Mit $\mathrm{T}=298 \mathrm{~K}$ berechnet man daraus:

$$
\mathrm{k}(298 \mathrm{~K})=(1,94 \pm 0,10) \cdot 10^{17} \cdot[\mathrm{He}] \mathrm{cm}^{6} /\left(\mathrm{mol}^{2} \cdot \mathrm{s}\right)
$$

Während der Messung bei $\mathrm{T}=298 \mathrm{~K}$ herrschte ein (mittlerer) Druck von $123 \mathrm{~Pa}$ im Reaktor, was bei einem Gesamtgasfluß von $\phi_{\mathrm{ges}}=412$ SCCM und den anderen oberhalb der Tabelle 11 (S. 119) angegebenen Reaktionsbedingungen gemäß Gleichung (19) (S. 14) zu folgender Konzentration des Badgases Helium führt:

$$
[\mathrm{He}]=\frac{392 \mathrm{SCCM}}{412 \mathrm{SCCM}} \cdot \frac{123 \mathrm{~Pa}}{298 \mathrm{~K} \cdot 8,314 \mathrm{~J} /(\mathrm{mol} \cdot \mathrm{K})}=4,72 \cdot 10^{-08} \mathrm{~mol} / \mathrm{cm}^{3}
$$

Damit kann man den bimolekularen Geschwindigkeitskoeffizienten berechnen:

$$
\mathrm{k}(298 \mathrm{~K}, 123 \mathrm{~Pa})=9,16 \cdot 10^{09} \mathrm{~cm}^{3} /(\mathrm{mol} \cdot \mathrm{s})
$$




\subsubsection{REMPI-Nachweis des Radikals}

Die bisherigen Messungen wurden sämtlich in einem Sektorfeldmassenspektrometer mit EIIonisierung durchgeführt. Es soll nun überprüft werden, ob das Radikal auch mit dem REMPIVerfahren (möglichst selektiv) ionisiert werden kann. Dieses Verfahren sowie die dafür eingesetzte Apparatur ist eingehend in Kap. 3.1.4 (S. 33 ff.) beschrieben. Die Meßwerte sind mit dem automatischen Wellenlängendurchlauf registriert worden (vgl. Kap. 3.5.4, S. 57), wobei zur Auswertung das Integral (mit den Integrationsgrenzen von $\pm 10 \%$ des Peakmaximums) des Peaks bei m/z 249 (bzw. der entsprechenden Flugzeit) herangezogen wurde.

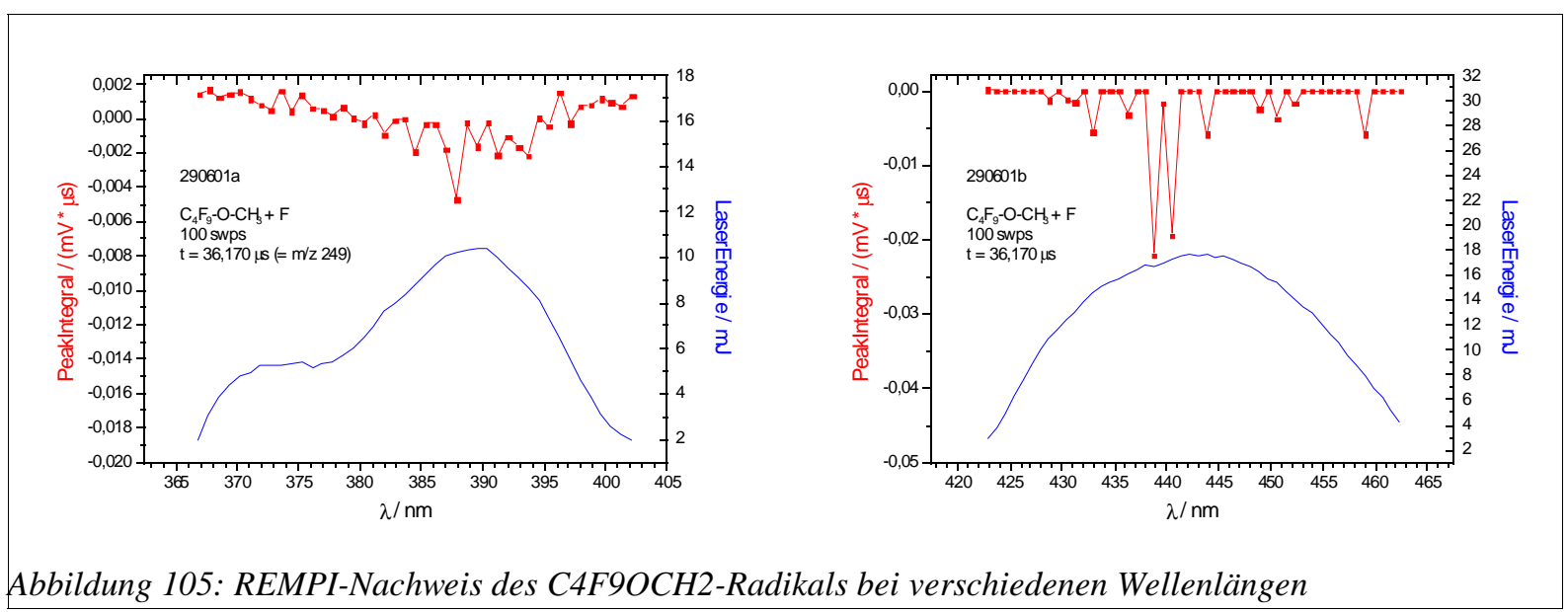

Das Radikal konnte bei der Wellenlänge $\lambda=387,8 ; 438,8$ und 440,5 nm nachgewiesen werden.
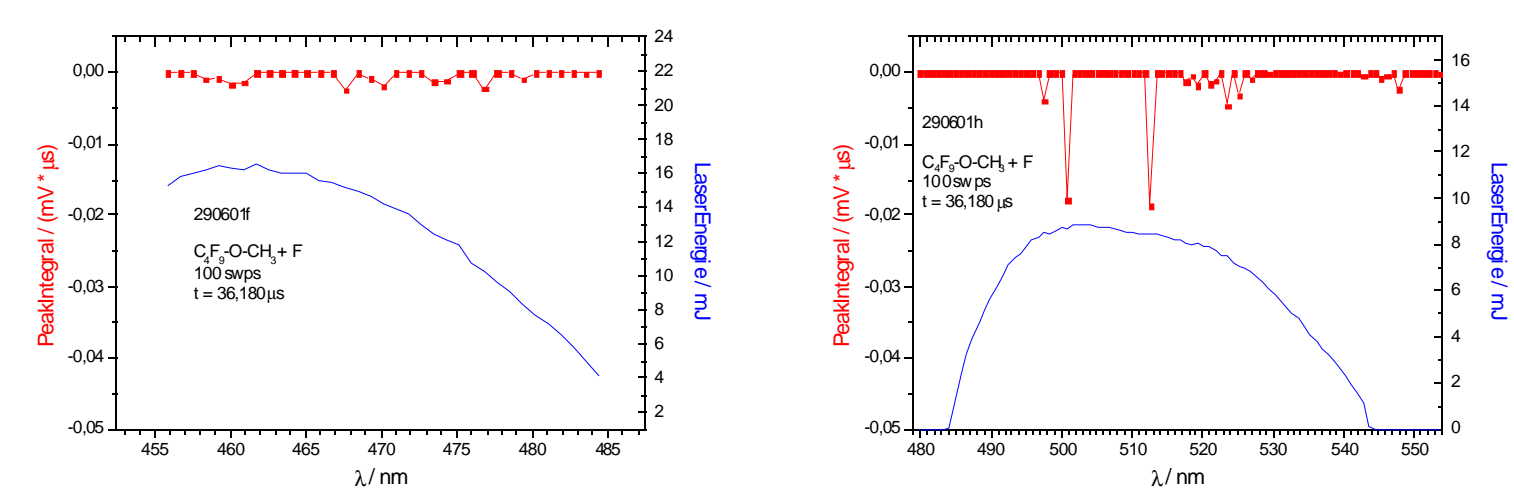

Abbildung 106: REMPI-Nachweis des C4F9OCH2-Radikals bei verschiedenen Wellenlängen

Das Radikal konnte bei der Wellenlänge $\lambda=508,8$ und 512,6 nm nachgewiesen werden.

Auch wenn der Radikalnachweis in den Abbildungen 105 und 106 eindeutig erbracht werden konnte, muß jedoch festgestellt werden, daß die Signalintensität bei allen Wellenlängen sehr gering ist. Die REMPI-Methode ist für Kinetik-Messungen daher nur bedingt geeignet. 


\subsubsection{Diskussion der Ergebnisse}

Untersuchungen anderer Arbeitsgruppen bezüglich der Reaktion $\mathrm{C}_{4} \mathrm{~F}_{9} \mathrm{OCH}_{3}+\mathrm{F} \rightarrow$ Produkte lagen nicht vor. Daher soll der hier ermittelte Geschwindigkeitskoeffizient mit dem des strukturell ähnlichen Dimethylethers $\left(\mathrm{CH}_{3} \mathrm{OCH}_{3}\right)$ verglichen werden.

Für $\mathrm{CH}_{3} \mathrm{OCH}_{3}$ ist ein Wert von $1,1 \cdot 10^{14}(\mathrm{~T} / 298 \mathrm{~K})^{0,65} \mathrm{~cm}^{3} /(\mathrm{mol} \cdot \mathrm{s})$ angegeben ${ }^{112}$.

Der Vergleich mit dem hier ermittelten Wert von $\mathrm{k}(\mathrm{T})=9,39 \cdot 10^{12} \cdot(\mathrm{T} / 298 \mathrm{~K})^{(0,04)} \mathrm{cm}^{3} /(\mathrm{mol} \cdot \mathrm{s})$ zeigt, daß der Austausch einer Methyl- gegen die Perfluorbutyl-Gruppe zu einer Herabsetzung um den Faktor 10 führt. Aus der Verringerung der zur Abstraktion verfügbaren H-Atome hätte man lediglich den Faktor 2 erwarten können. Der Grund dafür dürfte weniger in der Größe des Substituenten (sterisch anspruchsvoller), sondern eher in der hohen Elektronegativität der neun F-Atome zu suchen sein. Diese Annahme wird auch durch den Vergleich der Reaktion von $\mathrm{CH}_{3} \mathrm{OCH}_{3}$ und $\mathrm{CF}_{3} \mathrm{OCH}_{3}$ mit $\mathrm{OH}$ gestützt, wo der Geschwindigkeitskoeffizient für den Methyltrifluormethylether ${ }^{113}$ relativ zum Dimethylether ${ }^{114}$ etwa um den Faktor 100 geringer ist, obwohl $\mathrm{CF}_{3}$ und $\mathrm{CH}_{3}$ ungefähr die gleiche Größe haben.

Untersuchungen anderer Arbeitsgruppen bezüglich der Reaktion $\mathrm{C}_{4} \mathrm{~F}_{9} \mathrm{OCH}_{2}+\mathrm{O}_{2} \rightarrow$ Produkte lagen ebenfalls nicht vor.

Der negative Wert des Parameters $n$ ist typisch für Assoziationsreaktionen; er läßt sich damit erklären, daß keine nennenswerte Energiebarriere für die Komplexbildung vorhanden ist und somit bei höherer Temperatur eine größere Überschußenergie im angeregten Komplex vorliegt $^{115}$, die zunehmend zu einer raschen Rückreaktion (Zerfall in die Edukte) führt, bevor eine Stoßdeaktivierung mit einem dritten Stoßpartner (Badgas) erfolgen kann. Dies wird in der analogen Reaktion mit Methoxymethyl-Radikalen bestätigt, deren Aktivierungsenergie auch deutlich negativ ist ${ }^{116}$. Die Reaktion von $\mathrm{CH}_{2} \mathrm{FOCHF}$ mit $\mathrm{O}_{2}$ hat mit $\mathrm{n}=-5,51$ ebenfalls einen negativen Wert für den Paramter n, der zudem ähnlich hoch wie der hier bestimmte ist ${ }^{117}$.

Der hier ermittelte Geschwindigkeitskoeffizient soll nunmehr mit dem des strukturell ähnlichen Methoxymethyl- $\left(\mathrm{CH}_{3} \mathrm{OCH}_{2}\right)$ und tButoxymethyl-Radikals $\left(\mathrm{C}_{4} \mathrm{H}_{9} \mathrm{OCH}_{2}\right.$ bzw. $\left.\mathrm{C}_{4} \mathrm{H}_{8} \mathrm{OCH}_{3}\right)$ verglichen werden.

112 Hoyermann, K.; Nacke, F. (1996).

113 Zhang, Z.; Saini, R.D.; Kurylo, M.J.; Huie, R.E. (1992).

114 Atkinson,R.; et al. (1997).

$115 \mathrm{k}(\mathrm{E})$ für den Zerfall ist bei höherer Temperatur größer, weil die Geschwindigkeit der Stoßpartner und somit die Energie E im gebildeten Komplex höher ist.

116 Nacke, F. (1998), S. 72.

117 Vgl. Hold, M. (1997), S. 110. 
Es zeigt sich, daß der hier gemessene Wert um den Faktor 100 relativ zum tButoxymethyl- und um den Faktor 200 relativ zum Methoxymethyl-Radikal geringer ist ${ }^{118}$. Hierfür sind einerseits vermutlich wieder elektronische Gründe (Elektronegativität der F-Atome) verantwortlich, zum anderen ist aber auch ein Mechanismuswechsel denkbar. Der für das Methoxymethyl- und analog für das Fluor(fluormethoxy)methyl-Radikal plausible nachstehende Mechanismus ${ }^{119}$ ist mit dem Perfluorbutyl-Substituent nämlich nicht möglich (weil er keine H-Atome hat), was die oben beschriebene Produktuntersuchung auch bestätigt hat.

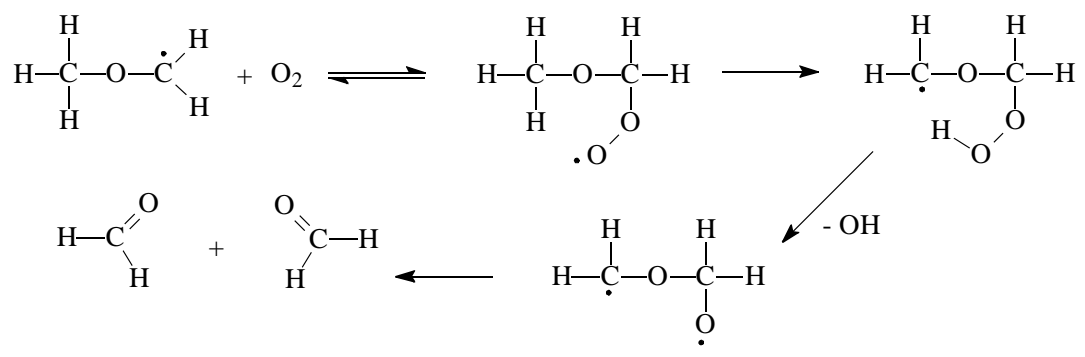

Eine Teilursache für den geringeren Geschwindigkeitskoeffizienten könnte auch die höhere Molekülmasse sein, die gemäß Gleichung (14) (S. 11) zu einer Verringerung der Stoßzahlen sowohl für die Bildung des Komplexes als auch für die Stoßdeaktivierung führt (dies kann jedoch maximal einen 10-20\% geringeren Wert erklären).

118 vgl. Nacke, F. (1998), S. 167.

119 vgl. Nacke, F. (1998), S. 69-70 sowie: Hold, M. (1997), S. 106. 


\subsubsection{Der Ethylperfluorbutylether $\left(\mathrm{C}_{4} \mathrm{~F}_{9}-\mathrm{O}-\mathrm{C}_{2} \mathrm{H}_{5}\right)$}

\subsubsection{EI-Nachweis des Radikals}

Völlig analog dem $\mathrm{C}_{4} \mathrm{~F}_{9}-\mathrm{O}-\mathrm{CH}_{3}$ weist auch $\mathrm{C}_{4} \mathrm{~F}_{9}-\mathrm{O}-\mathrm{C}_{2} \mathrm{H}_{5}$ im EI-Massenspektrum bei IP $=27 \mathrm{eV}$ keinen Molekül-Peak auf m/z 264 auf, wohl aber große Fragmente auf m/z 263, 249 und 219.

Bei Zugabe von F-Atomen vergrößert sich der Peak auf m/z 263 ganz erheblich, was auf die Bildung des $\mathrm{C}_{4} \mathrm{~F}_{9}-\mathrm{O}-\mathrm{C}_{2} \mathrm{H}_{4}$-Radikals hindeutet; gleichzeitig vermindern sich die Intensitäten bei $\mathrm{m} / \mathrm{z} 249$ und 219.

Es konnte jedoch kein Fragment spezifisch für die Messung des Umsatzes $\mathrm{C}_{4} \mathrm{~F}_{9}-\mathrm{O}-\mathrm{C}_{2} \mathrm{H}_{5}+\mathrm{F}$ benannt werden, ferner kann nicht zwischen $\mathrm{C}_{4} \mathrm{~F}_{9}-\mathrm{O}-\mathrm{CH}_{2} \mathrm{CH}_{2}$ und $\mathrm{C}_{4} \mathrm{~F}_{9}-\mathrm{O}-\mathrm{CHCH}_{3}-\mathrm{Radikalen}$ unterschieden werden. Aus diesen Gründen eignet sich das EI-Massenspektrometer (siehe Kapitel 3.1.1, S. 16) zur Messung der Geschwindigkeitskoeffizienten des Radikals mit anderen Reaktanten nicht (denn eine Umsatzkorrektur gemäß Kap. 3.5.1 (S. 50) ist so unmöglich).

\subsubsection{REMPI-Nachweis des Radikals}

Weil eine Kinetik-Messung mit EI-Ionisierung nicht durchgeführt werden kann, soll nun überprüft werden, ob das Radikal mit dem REMPI-Verfahren (möglichst selektiv) zu ionisieren ist. Dieses Verfahren sowie die dafür eingesetzte Apparatur ist eingehend in Kap. 3.1 .4 (S. 33 ff.) beschrieben. Die Meßwerte sind mit dem automatischen Wellenlängendurchlauf registriert worden (vgl. Kap. 3.5.4, S. 57), wobei zur Auswertung das Integral (mit den Integrationsgrenzen von $\pm 10 \%$ des Peakmaximums) des Peaks bei m/z 263 (bzw. der entsprechenden Flugzeit) herangezogen wurde.
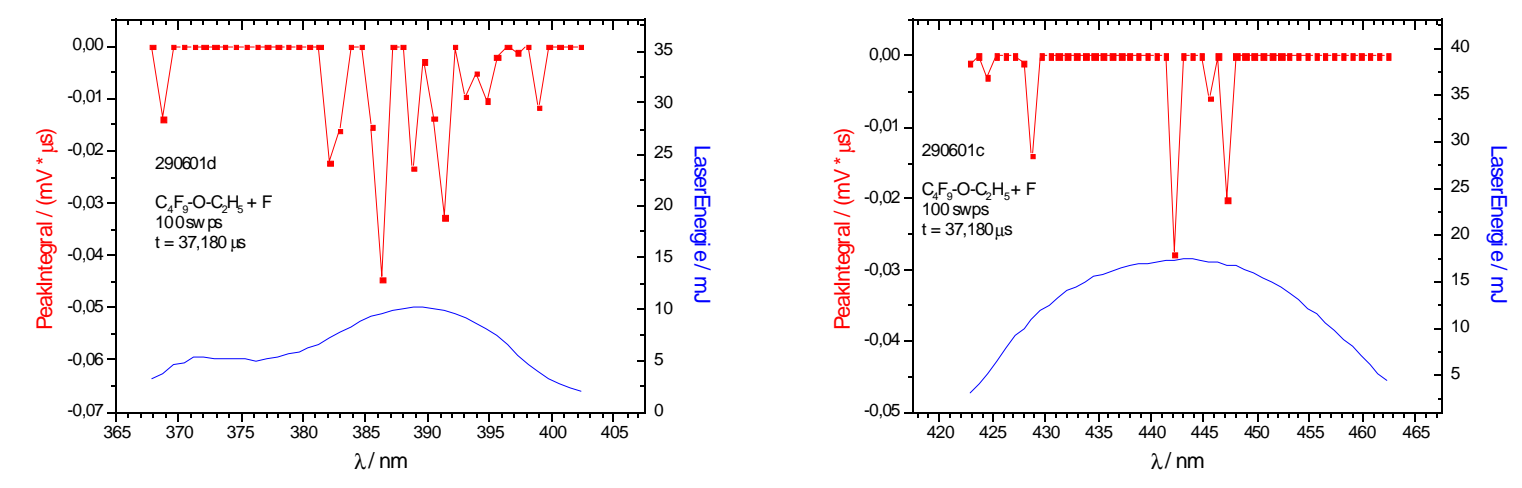

Abbildung 107: REMPI-Nachweis des C4F9OC2H4-Radikals bei verschiedenen Wellenlängen

Das Radikal konnte bei den Wellenlängen $\lambda=382,1 ; 386,3 ; 388,8 ; 391,4 ; 442,2$ und 447,2 nm nachgewiesen werden. 

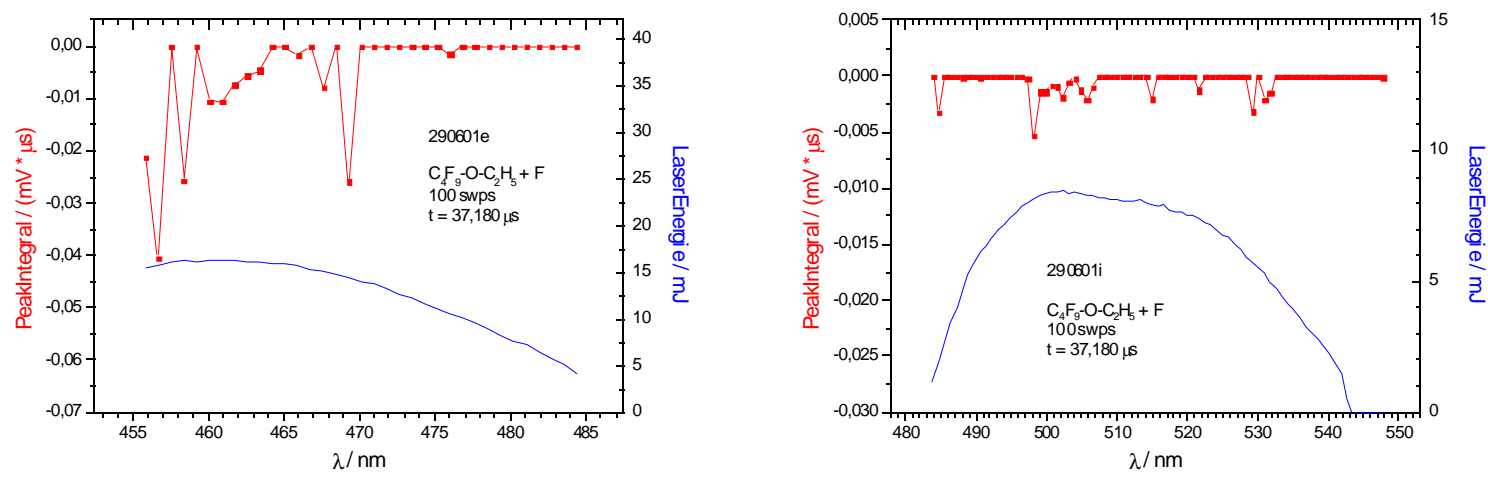

Abbildung 108: REMPI-Nachweis des C4F9OC2H4-Radikals bei verschiedenen Wellenlängen

Das Radikal konnte ferner bei den Wellenlängen $\lambda=456,7 ; 458,4$ und 469,3 nm nachgewiesen werden.

Insbesondere die Peaks bei den Wellenlängen $\lambda=386,3 ; 391,4$ und 456,7 nm sollten zur Bestimmung von Geschwindigkeitskoeffizienten ausreichend hohe Intensitäten haben

Zur Unterscheidung zwischen dem $\mathrm{C}_{4} \mathrm{~F}_{9} \mathrm{OCH}_{2} \mathrm{CH}_{2}$ - und dem $\mathrm{C}_{4} \mathrm{~F}_{9} \mathrm{OCHCH}_{3}$-Radikal könnten (sofern verfügbar) mit einer deuterierten Verbindung (z.B. $\mathrm{C}_{4} \mathrm{~F}_{9}-\mathrm{O}-\mathrm{CH}_{2} \mathrm{CD}_{3}$ ) die obigen Messungen wiederholt werden, denn oft ist bei bestimmten Wellenlängen selektiv eines von mehreren gebildeten Radikalen mit REMPI nachweisbar ${ }^{120}$. 


\subsection{Untersuchungen teilfluorierter Alkohole}

\subsubsection{Das 2,2,2-Trifluorethanol $\left(\mathrm{CF}_{3} \mathrm{CH}_{2} \mathrm{OH}\right)$}

\subsubsection{Produkte der radikalerzeugenden Reaktion mit Fluor-Atomen}

In der Reaktion des 2,2,2-Trifluorethanols mit Fluor-Atomen sind zwei verschiedene Arten von H-Atomen verfügbar, wobei dasjenige an der $\mathrm{OH}$-Gruppe mit $436 \mathrm{~kJ} / \mathrm{mol}$ eine höhere Bindungsdissoziationsenergie ${ }^{121}$ aufweist als das der $\mathrm{CH}_{2}$-Gruppe mit $389 \mathrm{~kJ} / \mathrm{mol}$. In der Reaktion mit $\mathrm{Cl}$-Atomen wird daher selektiv nur letzteres abstrahiert ${ }^{122}$. Fluor ist jedoch wesentlich reaktiver als Chlor, so daß hierfür auch die Abstraktion an der OH-Gruppe in Betracht gezogen werden muß (siehe Abbildung 109).

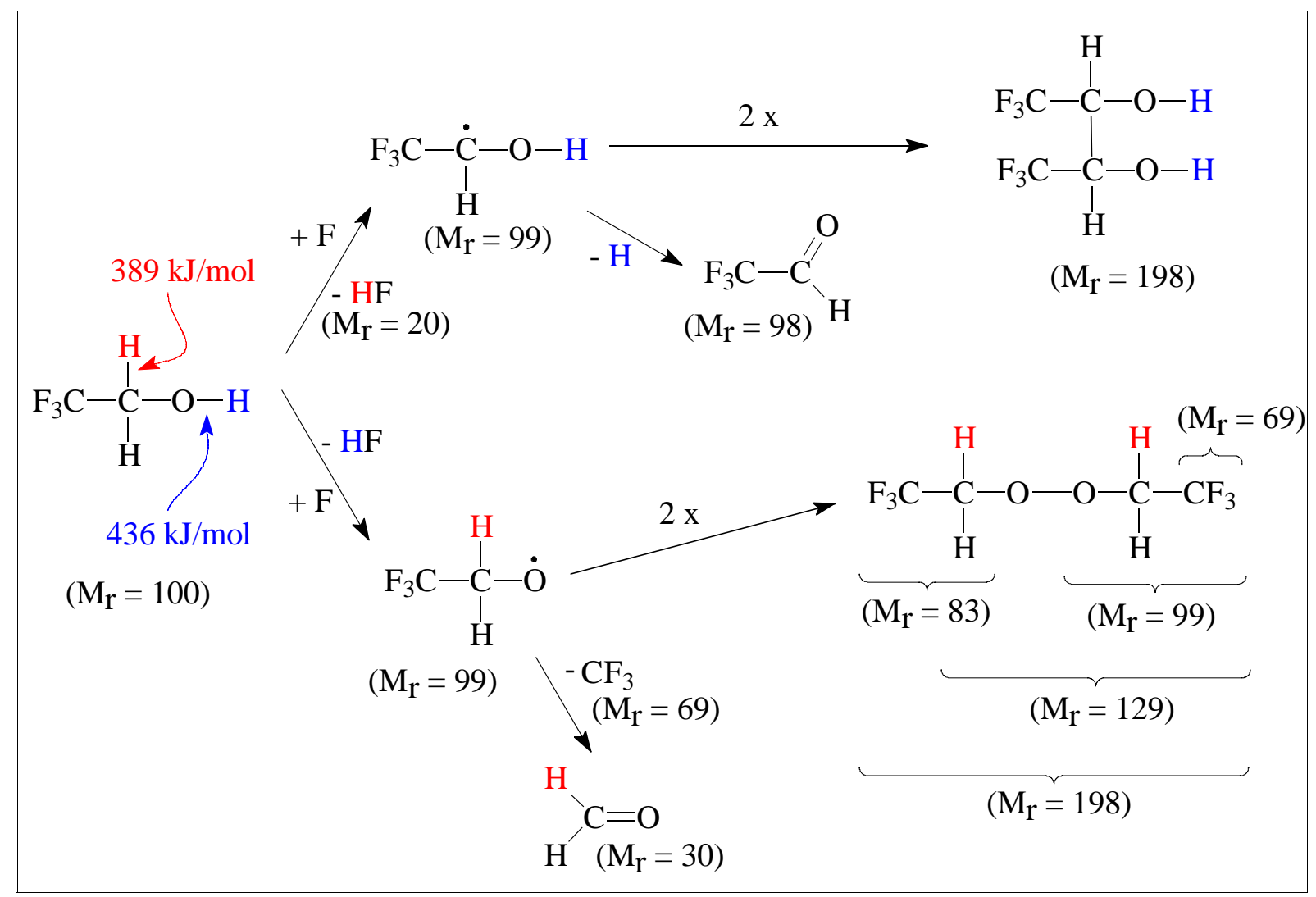

Abbildung 109: Reaktionskanäle für $\mathrm{CF} 3 \mathrm{CH} 2 \mathrm{OH}+\mathrm{F}$

In EI-Übersichtsmassenspektren, die mit der in Kapitel 3.1.1 (S. 16) beschriebenen Apparatur registriert wurden, zeigte sich bei Zugabe von Fluor-Atomen eine Abnahme auf dem MolekülPeak m/z 100 sowie u.a. eine Zunahme auf m/z 99, d.h. dem Molekül-Peak des Radikals. Die Durchführung der Messungen ist in Kapitel 3.5.1 (S. 50) beschrieben.

Es wurden anschließend gezielt in Einzelzählung (6 sek Zählzeit, IP = 29,5 eV) die m/z-Werte überprüft, die im Übersichtsspektrum größere Veränderungen zeigten. Die Bedingungen waren 
dazu so gewählt, daß sich bei Zugabe der Fluor-Atome auf dem Molekül-Peak m/z 100 eine Abnahme um $34 \%$ ergab. Die Ergebnisse sind in Abbildung 110 zu sehen.

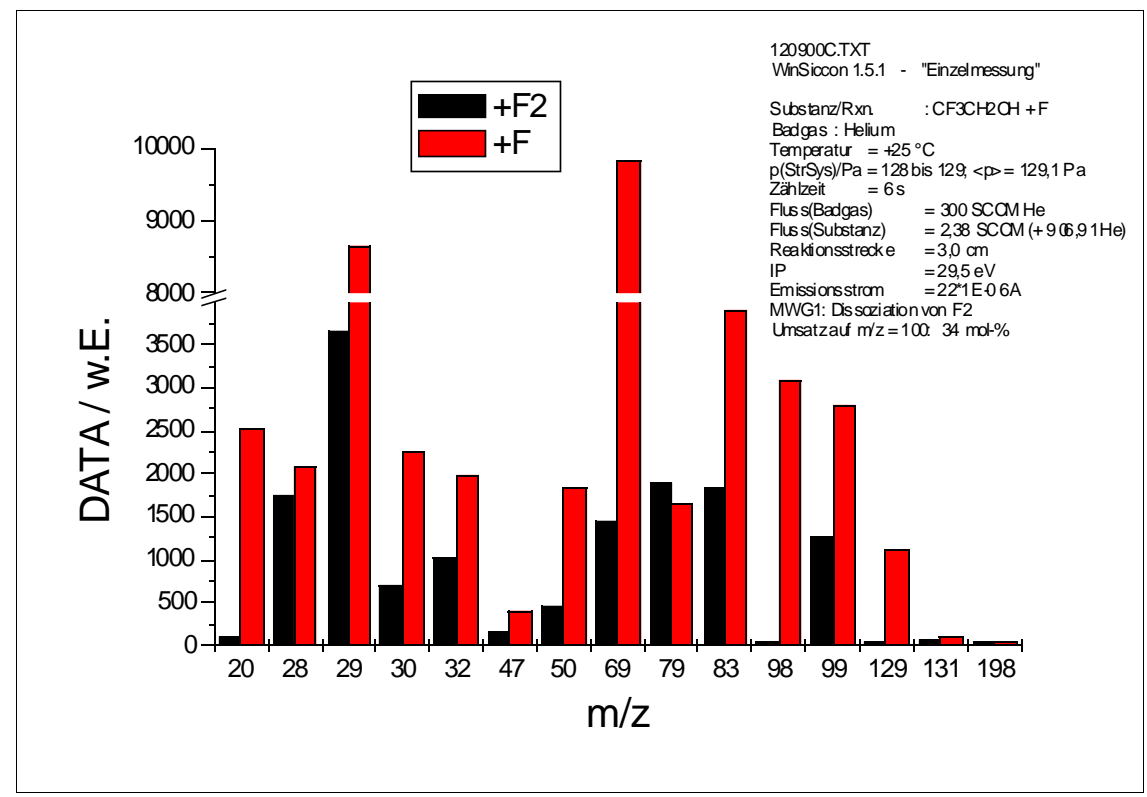

Abbildung 110: EI-MS-Produktuntersuchung CF3CH2OH +/- F

Die Zunahme auf $\mathrm{m} / \mathrm{z} 20$ ist der HF-Bildung aus der Abstraktion vom $\mathrm{CF}_{3} \mathrm{CH}_{2} \mathrm{OH}$ mit F-Atomen zuzurechnen. Dies bestätigt Abbildung 111, die die Änderung der Intensität auf $\mathrm{m} / \mathrm{z} 20$ in Abhängigkeit vom Umsatz auf m/z 100 als linearen Zusammenhang zeigt.

Die Zunahmen auf $\mathrm{m} / \mathrm{z} 29$ und 30 dürften aus der Bildung von $\mathrm{HCHO}$ resultieren, was eine

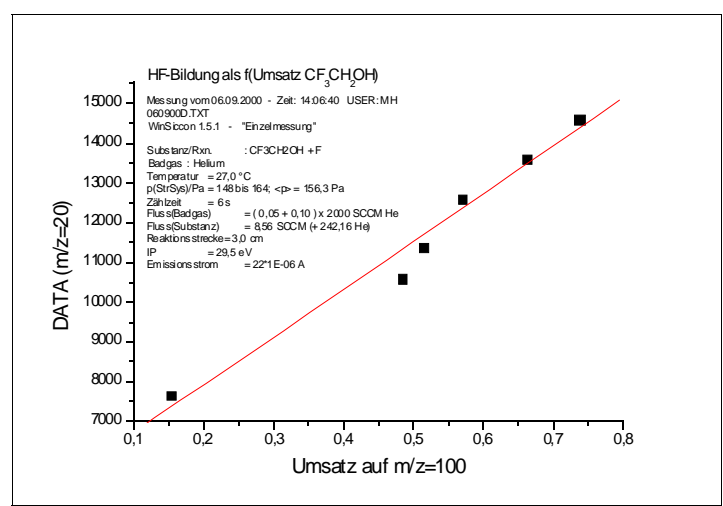

Abbildung 111: $\mathrm{HF}$-Bildung f. CF3CH2OH + F Abstraktion auch von der OH-Gruppe nahelegt (vgl. Schema in Abbildung 109).
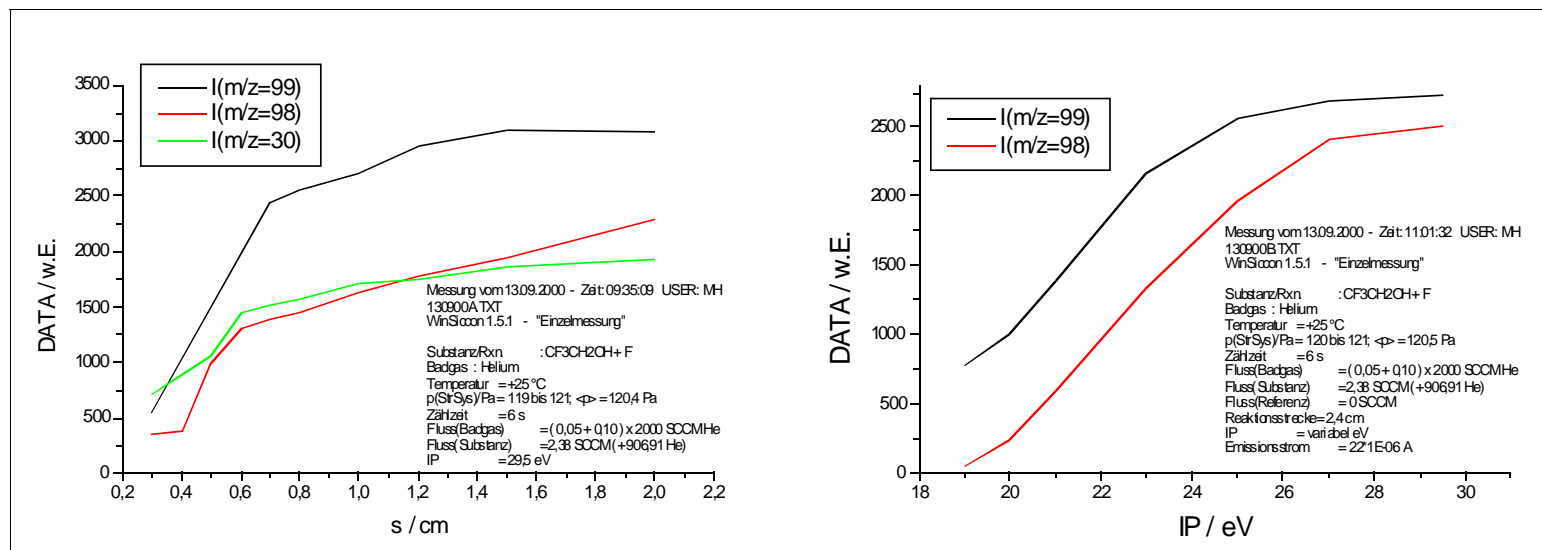

Abbildung 112: I(m/z, 98, 99) vs. Reaktionsstrecke und IP f. $\mathrm{CF} 3 \mathrm{CH} 2 \mathrm{OH}+\mathrm{F}$ 
Durch die Messungen in Abbildung 112 sollte untersucht werden, ob die starken Zunahmen auf $\mathrm{m} / \mathrm{z} 30$ und insbesondere auf m/z 98 auf die Bildung von Folgereaktionen gemäß dem Schema in Abbildung 109 zurückzuführen sind. In diesem Fall sollte das Signal auf m/z 30 und 98 später ansteigen als das auf m/z 99, d.h. erst bei längeren Reaktionsstrecken s. Der linke Graph in Abbildung 112 scheint diese These zu bestätigen. Die Zunahme auf m/z 98 könnte jedoch auch ein Fragment eines der gebildeten Radikale $\left(\mathrm{M}_{\mathrm{r}}=99\right)$ sein. Der rechte Graph in Abbildung 112 zeigt die beiden Intensitäten auf m/z 98 und 99 in Abhängigkeit von der EIElektronenenergie (IP), wobei diejenige auf m/z 98 bei Absenkung der EI-Elektronenenergie früher auf Null sinkt. Letzteres würde für ein Radikal-Fragment sprechen. Andererseits spricht die Zunahme auf m/z 47 (Fragment von $\mathrm{CF}_{3} \mathrm{CHO}$ ) ${ }^{123}$ in Verbindung mit der auf $\mathrm{m} / \mathrm{z} 98$ für die Entstehung des Aldehyds $\mathrm{CF}_{3} \mathrm{CHO}$, wobei die fehlende Zunahme auf m/z 79 (Fragment von $\left.\mathrm{CF}_{3} \mathrm{CHO}\right)^{123}$ nicht dagegen spricht, weil hier ebenfalls ein Fragment des Edukts $\mathrm{CF}_{3} \mathrm{CH}_{2} \mathrm{OH}$ liegt, dessen Abnahme die Zunahme kompensieren könnte.

Die leichte Zunahme auf m/z 198 in Verbindung mit denjenigen auf m/z 83 und 129 dokumentieren die Bildung eines Kombinationsproduktes zweier gebildeter Radikale, wobei $\mathrm{m} / \mathrm{z} 83$ ein Fragment dieses Kombinationsproduktes ist, bei dem eine $\mathrm{CH}_{2} \mathrm{CF}_{3}$-Gruppe und $\mathrm{m} / \mathrm{z}$ 129 eines ist, bei dem eine $\mathrm{CF}_{3}$-Gruppe abgespalten wurde (vgl. Abb. 109).

Es kann nicht ausgeschlossen werden, daß sich HCHO (m/z 29 und 30) auch nach einer [1,2]$\mathrm{H}$-Umlagerung mit anschließender $\mathrm{CF}_{3}$-Abspaltung aus dem thermodynamisch günstiger $\mathrm{zu}$ erzeugenden $\mathrm{CF}_{3} \mathrm{CHOH}$-Radikal bildet. Abgesehen von diesem Einwand sind Indizien für alle in Abbildung 109 aufgezeigten Reaktionskanäle vorhanden.

Festzuhalten bleibt, daß der Umsatz des Alkohols $\mathrm{CF}_{3} \mathrm{CH}_{2} \mathrm{OH}$ auf seinem Molekül-Peak m/z 100 und derjenige des (bzw. der) gebildeten Radikals (bzw. Radikale) umsatzkorrigiert ebenfalls auf dessen (bzw. deren) Molekül-Peak(s) m/z 99 gemessen werden kann.

\subsubsection{Kinetik der radikalerzeugenden Reaktion mit Fluor-Atomen}

Die Messungen wurden in der in Kap. 3.1.1 (S. 16) beschriebenen Apparatur unter Einsatz des Strömungsreaktors aus Abbildung 5 (S. 17) durchgeführt. Die Ausführung und Auswertung nach der Relativmethode ist in Kap. 3.5.1 (S. 50) beschrieben.

Methan $\left(\mathrm{M}_{\mathrm{r}}=16\right)$ diente als Referenzsubstanz. Der Umsatz des Alkohols $\mathrm{CF}_{3} \mathrm{CH}_{2} \mathrm{OH}$ konnte anhand der Intensitätsänderung seines Molekül-Peaks m/z 100, derjenige von Methan anhand des Molekül-Peaks (m/z 16) bestimmt werden.

123 Vgl. das Massenspektrum von CF3CHO in: NIST Mass Spectral Database (1990). 
$\vartheta=-15{ }^{\circ} \mathrm{C} ; \phi_{\mathrm{He}}=640 \mathrm{SCCM} ; \phi_{\mathrm{CF} 3 \mathrm{CH} 2 \mathrm{OH}}=8,6 \mathrm{SCCM} ; \phi_{\mathrm{CH} 4}=8,6 \mathrm{SCCM} ; \mathrm{p}_{(\mathrm{StrR})}=150 \mathrm{~Pa} ;$ $\mathrm{s}=3,0 \mathrm{~cm} ; \mathrm{IP}=25 \mathrm{eV}$; Zählzeit $=6 \mathrm{~s}$

\begin{tabular}{|r|r|r|r|r|r|r|r|}
\hline $\begin{array}{c}\mathbf{I}(\mathbf{1 0 0}) \\
\text {-F / w.E. }\end{array}$ & $\begin{array}{c}\Delta \mathbf{I}(\mathbf{1 0 0}) \\
\text {-F / w.E. }\end{array}$ & $\begin{array}{c}\mathbf{I}(\mathbf{1 0 0}) \\
\text { +F / w.E. }\end{array}$ & $\begin{array}{c}\Delta \mathbf{I}(\mathbf{1 0 0}) \\
\text { +F / w.E. }\end{array}$ & $\begin{array}{c}\text { I(16) } \\
\text {-F / w.E. }\end{array}$ & $\begin{array}{c}\Delta \mathbf{I}(\mathbf{1 6}) \\
\text {-F / w.E. }\end{array}$ & $\begin{array}{c}\mathbf{I}(\mathbf{1 6}) \\
+\mathbf{F} / \mathbf{w . E} .\end{array}$ & $\begin{array}{c}\Delta \mathbf{I}(\mathbf{1 6}) \\
+\mathbf{F} / \mathbf{w . E} .\end{array}$ \\
\hline 10470 & 419 & 6950 & 66 & 11560 & 184 & 9163 & 126 \\
\hline 11300 & 100 & 7798 & 222 & 11710 & 83 & 9723 & 130 \\
\hline 11030 & 116 & 7304 & 227 & 11250 & 109 & 9073 & 110 \\
\hline 10930 & 116 & 6783 & 156 & 11090 & 175 & 8825 & 115 \\
\hline 10940 & 118 & 8193 & 132 & 11450 & 118 & 9501 & 126 \\
\hline 10630 & 116 & 8278 & 175 & 11290 & 151 & 9666 & 132 \\
\hline 10510 & 83 & 9335 & 116 & 11350 & 100 & 10430 & 86 \\
\hline 11100 & 126 & 9740 & 89 & 11350 & 63 & 10280 & 86 \\
\hline 11050 & 77 & 10510 & 94 & 10940 & 118 & 10460 & 76 \\
\hline
\end{tabular}

Tabelle 16: Meßwerte Kinetik $\mathrm{CF} 3 \mathrm{CH} 2 \mathrm{OH}+\mathrm{F}$ rel. zu $\mathrm{CH} 4 ; 258 \mathrm{~K}$

Mit den Werten aus Tabelle 16 kann eine Auftragung gemäß Gleichung (10) (S. 10) zur Bestimmung des relativen Geschwindigkeitskoeffizienten angefertigt werden (Abb. 113)

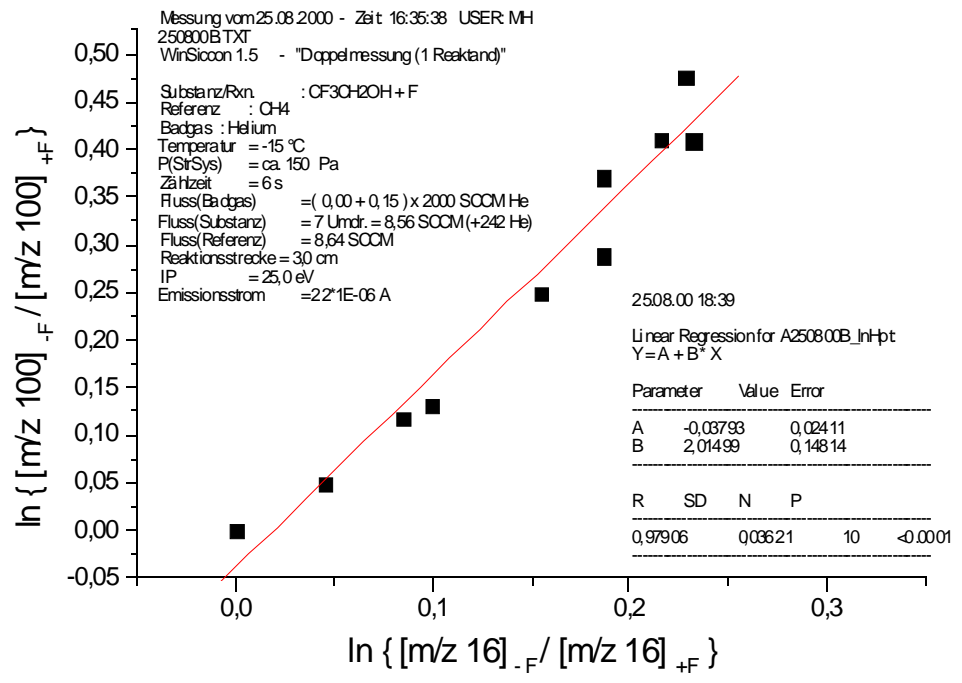

Abbildung 113: Kinetik CF3CH2OH + F rel. zu CH4; $258 \mathrm{~K}$

Aus der Steigung in Abbildung 113 läßt sich der relative Geschwindigkeitskoeffizient bei $\mathrm{T}=258 \mathrm{~K}$ ermitteln: $\mathrm{k}_{\mathrm{rel}}=(2,01 \pm 0,15)$. 
$\vartheta=+5{ }^{\circ} \mathrm{C} ; \phi_{\mathrm{He}}=640 \mathrm{SCCM} ; \phi_{\text {СF3Сн2OH }}=8,6 \mathrm{SCCM} ; \phi_{\mathrm{CH} 4}=8,6 \mathrm{SCCM} ; \mathrm{p}_{(\mathrm{StrR})}=148 \mathrm{~Pa}$;

$\mathrm{s}=3,0 \mathrm{~cm} ; \mathrm{IP}=25 \mathrm{eV}$; Zählzeit $=6 \mathrm{~s}$

\begin{tabular}{|r|r|r|r|r|r|r|r|}
\hline $\begin{array}{c}\mathbf{I}(\mathbf{1 0 0}) \\
\text {-F / w.E. }\end{array}$ & $\begin{array}{c}\Delta \mathbf{I}(\mathbf{1 0 0}) \\
\text {-F / w.E. }\end{array}$ & $\begin{array}{c}\mathbf{I}(\mathbf{1 0 0}) \\
\text { +F / w.E. }\end{array}$ & $\begin{array}{c}\Delta \mathbf{I}(\mathbf{1 0 0}) \\
\text { +F / w.E. }\end{array}$ & $\begin{array}{c}\mathbf{I}(\mathbf{1 6}) \\
\text {-F / w.E. }\end{array}$ & $\begin{array}{c}\Delta \mathbf{I}(\mathbf{1 6}) \\
\text {-F / w.E. }\end{array}$ & $\begin{array}{c}\mathbf{I}(\mathbf{1 6}) \\
+\mathbf{+ F} / \mathbf{w . E} .\end{array}$ & $\begin{array}{c}\Delta \mathbf{I}(\mathbf{1 6}) \\
+\mathbf{F} / \mathbf{w . E} .\end{array}$ \\
\hline 8804 & 75 & 6163 & 184 & 50830 & 159 & 38380 & 139 \\
\hline 8430 & 95 & 5925 & 91 & 51750 & 219 & 38690 & 144 \\
\hline 8521 & 138 & 6464 & 119 & 50990 & 94 & 39850 & 118 \\
\hline 8810 & 82 & 6494 & 139 & 51300 & 299 & 40290 & 94 \\
\hline 9163 & 83 & 7774 & 107 & 52630 & 217 & 45390 & 202 \\
\hline 9820 & 65 & 8759 & 132 & 52990 & 181 & 47710 & 164 \\
\hline
\end{tabular}

Tabelle 17: Meßwerte Kinetik $\mathrm{CF} 3 \mathrm{CH} 2 \mathrm{OH}+\mathrm{F}$ rel. zu CH4; $278 \mathrm{~K}$

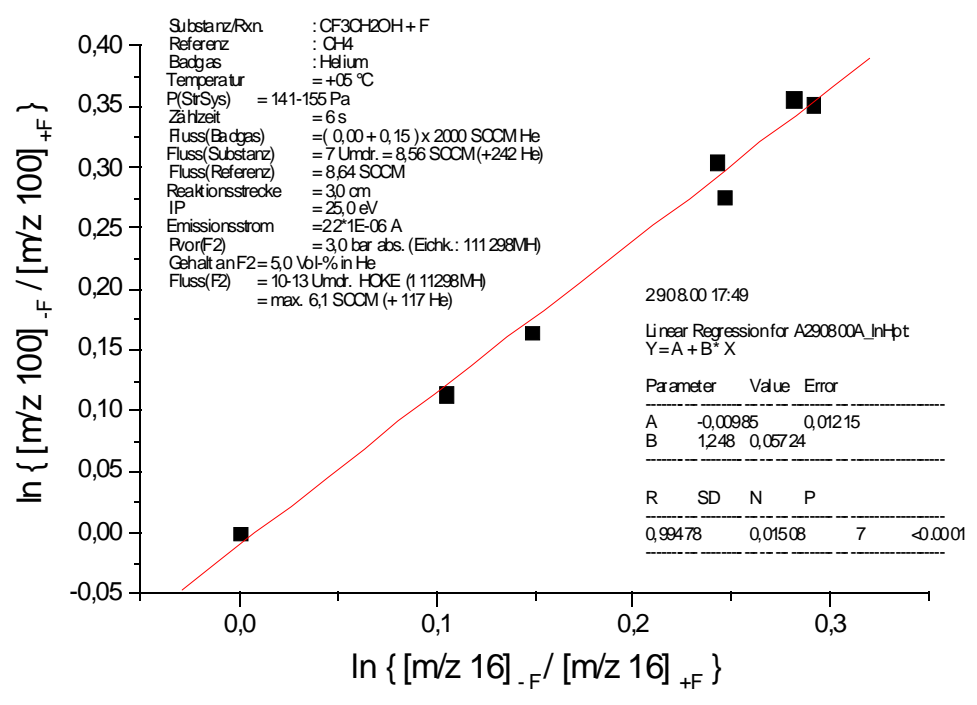

Abbildung 114: Kinetik CF3CH2OH + F rel. zu CH4; $278 \mathrm{~K}$

Aus der Steigung in Abbildung 114 läßt sich der relative Geschwindigkeitskoeffizient bei $\mathrm{T}=278 \mathrm{~K}$ ermitteln: $\mathrm{k}_{\mathrm{rel}}=(1,25 \pm 0,06)$. 
$\vartheta=+27^{\circ} \mathrm{C} ; \phi_{\mathrm{He}}=640 \mathrm{SCCM} ; \phi_{\mathrm{CF} 3 \mathrm{CH} 2 \mathrm{OH}}=8,6 \mathrm{SCCM} ; \phi_{\mathrm{CH} 4}=8,6 \mathrm{SCCM} ; \mathrm{p}_{(\mathrm{StrR})}=148 \mathrm{~Pa} ;$ $\mathrm{s}=3,0 \mathrm{~cm} ; \mathrm{IP}=25 \mathrm{eV}$; Zählzeit $=6 \mathrm{~s}$

\begin{tabular}{|r|r|r|r|r|r|r|r|}
\hline $\begin{array}{c}\mathbf{I}(\mathbf{1 0 0}) \\
\text {-F / w.E. }\end{array}$ & $\begin{array}{c}\Delta \mathbf{I}(\mathbf{1 0 0}) \\
\text {-F / w.E. }\end{array}$ & $\begin{array}{c}\mathbf{I}(\mathbf{1 0 0}) \\
\text { +F / w.E. }\end{array}$ & $\begin{array}{c}\Delta \mathbf{I}(\mathbf{1 0 0}) \\
\text { +F / w.E. }\end{array}$ & $\begin{array}{c}\text { I(16) } \\
\text {-F / w.E. }\end{array}$ & $\begin{array}{c}\Delta \mathbf{I}(\mathbf{1 6}) \\
\text {-F / w.E. }\end{array}$ & $\begin{array}{c}\mathbf{I}(\mathbf{1 6}) \\
+\mathbf{+ F} / \mathbf{w . E} .\end{array}$ & $\begin{array}{c}\Delta \mathbf{I}(\mathbf{1 6}) \\
+\mathbf{F} / \mathbf{w . E} .\end{array}$ \\
\hline 6455 & 62 & 4480 & 115 & 56040 & 354 & 42840 & 722 \\
\hline 6184 & 108 & 4225 & 112 & 59180 & 566 & 45490 & 175 \\
\hline 6104 & 105 & 4354 & 150 & 67000 & 497 & 51590 & 456 \\
\hline 6166 & 58 & 5484 & 181 & 66980 & 461 & 61450 & 741 \\
\hline 6174 & 80 & 5318 & 169 & 72290 & 443 & 64960 & 798 \\
\hline 5246 & 61 & 4460 & 91 & 72930 & 292 & 64850 & 668 \\
\hline
\end{tabular}

Tabelle 18: Meßwerte Kinetik $\mathrm{CF} 3 \mathrm{CH} 2 \mathrm{OH}+\mathrm{F}$ rel. zu CH4; $300 \mathrm{~K}$

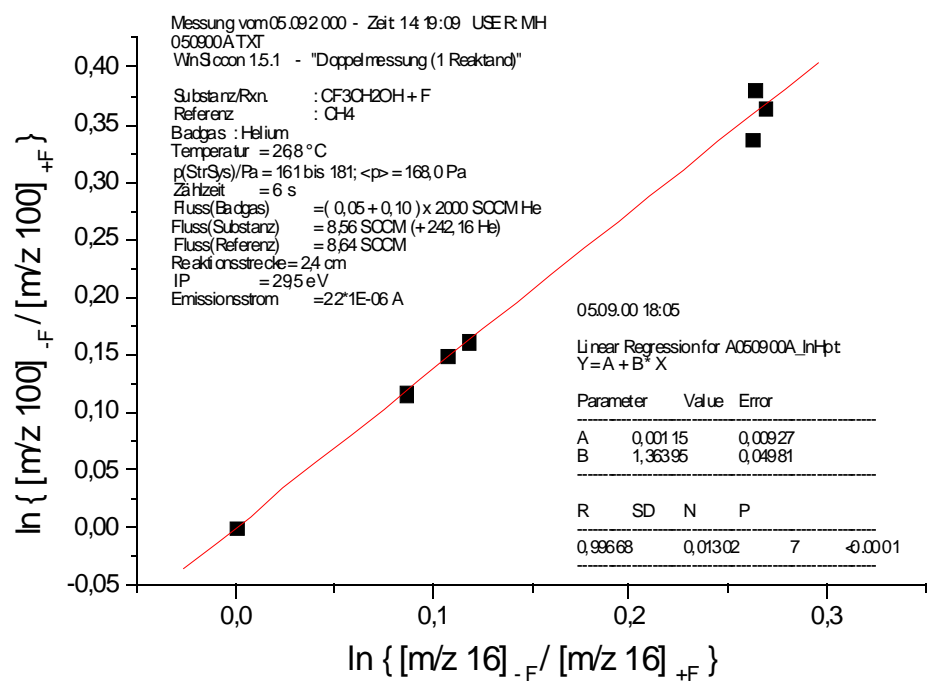

Abbildung 115: Kinetik $\mathrm{CF} 3 \mathrm{CH} 2 \mathrm{OH}+\mathrm{F}$ rel. zu CH4; $300 \mathrm{~K}$

Aus der Steigung in Abbildung 115 läßt sich der relative Geschwindigkeitskoeffizient bei $\mathrm{T}=300 \mathrm{~K}$ ermitteln: $\mathrm{k}_{\mathrm{rel}}=(1,36 \pm 0,05)$. 
$\vartheta=+55^{\circ} \mathrm{C} ; \phi_{\mathrm{He}}=640 \mathrm{SCCM} ; \phi_{\mathrm{CF} 3 \mathrm{CH} 2 \mathrm{OH}}=8,6 \mathrm{SCCM} ; \phi_{\mathrm{CH} 4}=8,6 \mathrm{SCCM} ; \mathrm{p}_{(\mathrm{StrR})}=148 \mathrm{~Pa}$; $\mathrm{s}=3,0 \mathrm{~cm}$; IP = $25 \mathrm{eV}$; Zählzeit $=6 \mathrm{~s}$

\begin{tabular}{|r|r|r|r|r|r|r|r|}
\hline $\begin{array}{c}\mathbf{I}(\mathbf{1 0 0}) \\
\text {-F / w.E. }\end{array}$ & $\begin{array}{c}\Delta \mathbf{I}(\mathbf{1 0 0}) \\
\text {-F / w.E. }\end{array}$ & $\begin{array}{c}\mathbf{I}(\mathbf{1 0 0}) \\
\text { +F / w.E. }\end{array}$ & $\begin{array}{c}\Delta \mathbf{I}(\mathbf{1 0 0}) \\
\text { +F / w.E. }\end{array}$ & $\begin{array}{c}\mathbf{I}(\mathbf{1 6}) \\
\text {-F / w.E. }\end{array}$ & $\begin{array}{c}\Delta \mathbf{I}(\mathbf{1 6}) \\
\text {-F / w.E. }\end{array}$ & $\begin{array}{c}\mathbf{I}(\mathbf{1 6}) \\
\text { +F / w.E. }\end{array}$ & $\begin{array}{c}\Delta \mathbf{I}(\mathbf{1 6}) \\
+\mathbf{+ F} / \text { w.E. }\end{array}$ \\
\hline 5545 & 79 & 4471 & 85 & 23040 & 236 & 18800 & 167 \\
\hline 5539 & 67 & 5084 & 101 & 22760 & 178 & 20930 & 188 \\
\hline 5296 & 34 & 3629 & 48 & 22990 & 186 & 16430 & 107 \\
\hline 5254 & 52 & 3656 & 40 & 23260 & 160 & 16780 & 146 \\
\hline 5756 & 62 & 4440 & 53 & 23380 & 171 & 18580 & 153 \\
\hline 5913 & 85 & 5349 & 72 & 24250 & 167 & 21930 & 74 \\
\hline
\end{tabular}

Tabelle 19: Meßwerte Kinetik $\mathrm{CF} 3 \mathrm{CH} 2 \mathrm{OH}+\mathrm{F}$ rel. zu CH4; $328 \mathrm{~K}$

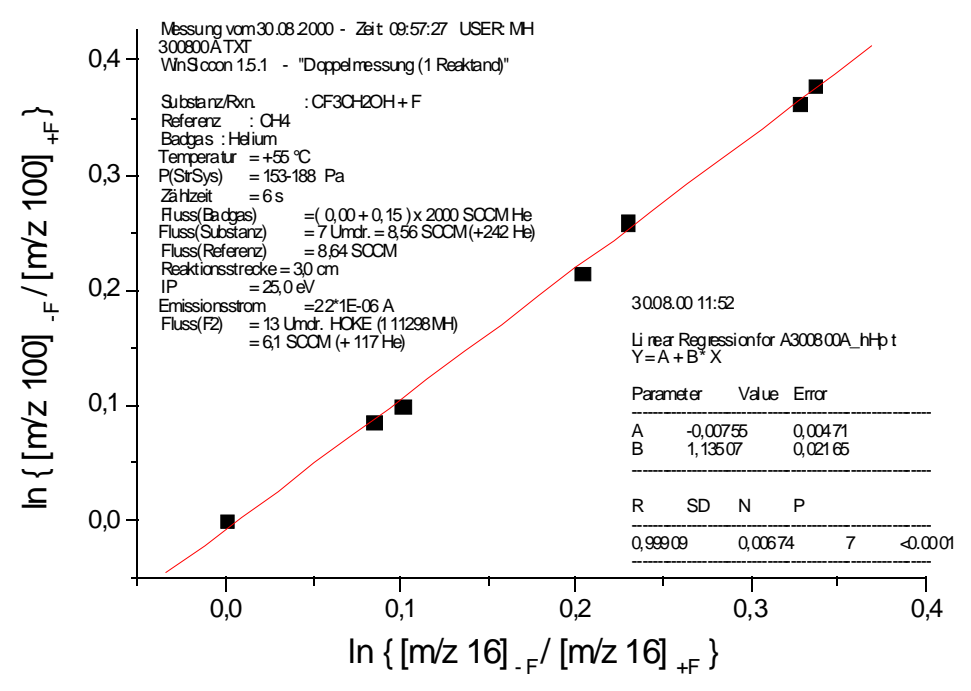

Abbildung 116: Kinetik CF3CH2OH + F rel. zu CH4; $328 \mathrm{~K}$

Aus der Steigung in Abbildung 116 läßt sich der relative Geschwindigkeitskoeffizient bei $\mathrm{T}=328 \mathrm{~K}$ ermitteln: $\mathrm{k}_{\mathrm{rel}}=(1,135 \pm 0,022)$. 
$\vartheta=+85^{\circ} \mathrm{C} ; \phi_{\mathrm{He}}=640 \mathrm{SCCM} ; \phi_{\mathrm{CF} 3 \mathrm{CH} 2 \mathrm{OH}}=8,6 \mathrm{SCCM} ; \phi_{\mathrm{CH} 4}=8,6 \mathrm{SCCM} ; \mathrm{p}_{(\mathrm{StrR})}=159 \mathrm{~Pa}$; $\mathrm{s}=3,0 \mathrm{~cm} ; \mathrm{IP}=25 \mathrm{eV}$; Zählzeit $=6 \mathrm{~s}$

\begin{tabular}{|r|r|r|r|r|r|r|r|}
\hline \begin{tabular}{|}
$\mathbf{I}(\mathbf{1 0 0})$ \\
-F / w.E.
\end{tabular} & $\begin{array}{r}\Delta \mathbf{I}(\mathbf{1 0 0}) \\
\text {-F / w.E. }\end{array}$ & $\begin{array}{c}\mathbf{I}(\mathbf{1 0 0}) \\
\text { +F / w.E. }\end{array}$ & $\begin{array}{c}\Delta \mathbf{I}(\mathbf{1 0 0}) \\
\text { +F / w.E. }\end{array}$ & $\begin{array}{c}\text {-F } / \mathbf{1 6} \mathbf{w . E} . \\
\text {-F }\end{array}$ & $\begin{array}{c}\Delta \mathbf{I}(\mathbf{1 6}) \\
\text {-F.E. }\end{array}$ & $\begin{array}{c}\mathbf{I}(\mathbf{1 6}) \\
\text { +F / w.E. }\end{array}$ & $\begin{array}{c}\Delta \mathbf{I}(\mathbf{1 6}) \\
\text { +F / w.E. }\end{array}$ \\
\hline 5611 & 78 & 4624 & 85 & 37840 & 99 & 30600 & 126 \\
\hline 5548 & 76 & 4566 & 48 & 37890 & 216 & 30440 & 141 \\
\hline 5551 & 70 & 4370 & 83 & 38100 & 289 & 29810 & 202 \\
\hline 5556 & 43 & 4360 & 80 & 38400 & 134 & 30180 & 97 \\
\hline 5015 & 64 & 4465 & 58 & 38830 & 183 & 34410 & 170 \\
\hline 5055 & 56 & 4571 & 53 & 38860 & 148 & 34540 & 218 \\
\hline 4916 & 83 & 4180 & 41 & 30010 & 137 & 24950 & 148 \\
\hline
\end{tabular}

Tabelle 20: Meßwerte Kinetik $\mathrm{CF} 3 \mathrm{CH} 2 \mathrm{OH}+\mathrm{F}$ rel. zu CH4; $358 \mathrm{~K}$

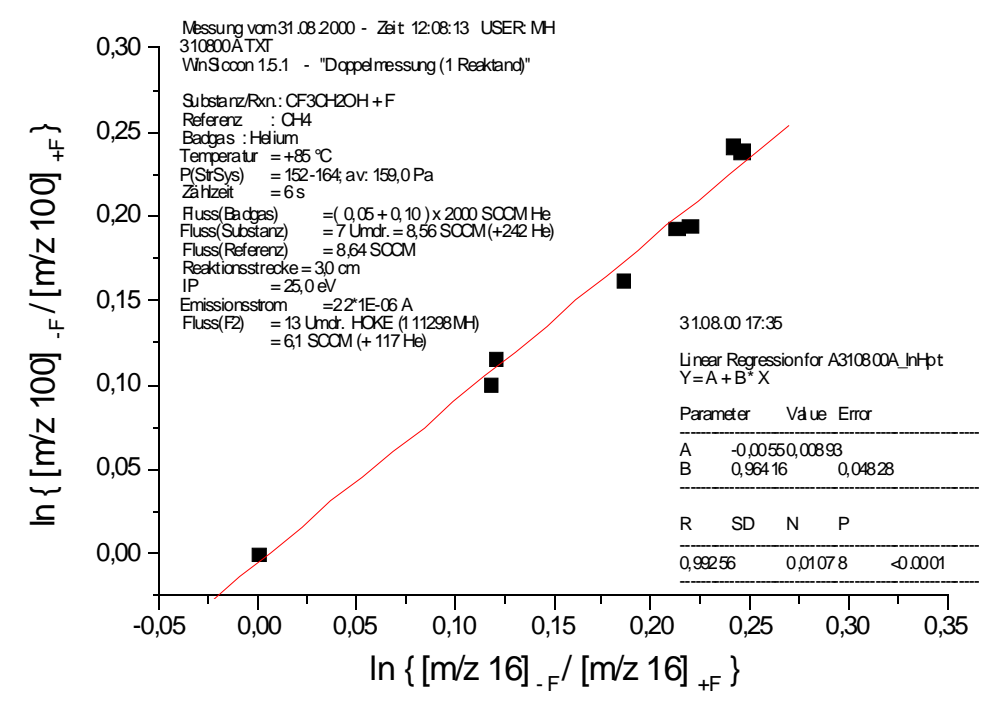

Abbildung 117: Kinetik CF3CH2OH + F rel. zu CH4; $358 \mathrm{~K}$

Aus der Steigung in Abbildung 117 läßt sich der relative Geschwindigkeitskoeffizient bei $\mathrm{T}=358 \mathrm{~K}$ ermitteln: $\mathrm{k}_{\mathrm{rel}}=(0,964 \pm 0,048)$. 
Aus den Ergebnissen der Abbildungen 113 bis 117 kann eine Arrhenius-Auftragung gemäß Gleichung (11) (S. 10) angefertigt und mit den Geschwindigkeitskoeffizienten der bekannten Referenzreaktion ${ }^{124} \mathrm{k}_{\text {ref }}(\mathrm{T})$ die absoluten Geschwindigkeitskoeffizienten berechnet werden.

$\mathrm{k}_{\mathrm{ref}}(\mathrm{T})=(1,81) \cdot 10^{14} \cdot \exp \{-(3,33) \mathrm{kJ} / \mathrm{mol} /(\mathrm{R} \cdot \mathrm{T})\} \mathrm{cm}^{3} /(\mathrm{mol} \cdot \mathrm{s})$

Die Ergebnisse sind in Tabelle 21 eingetragen, die Auftragung ist in Abbildung 118 zu sehen.

\begin{tabular}{|c|c|c|c|c|c|}
\hline$\vartheta /{ }^{\circ} \mathrm{C}$ & $\mathbf{T}^{-1} / \mathbf{K}^{-1}$ & $\mathbf{k}_{\text {rel }}$ & $\ln \left\{\mathbf{k}_{\text {rel }}\right\}$ & $\begin{array}{l}\mathbf{k}_{\mathrm{CF} 3 \mathrm{CH} 2 \mathrm{OH}} / \\
\mathrm{cm}^{3} /(\mathbf{m o l ~ s})\end{array}$ & $\begin{array}{l}\ln \left\{\mathbf{k}_{\mathrm{CF3CH} 2 \mathrm{OH}} /\right. \\
\left.\left(\mathbf{c m}^{3} /(\mathbf{m o l ~ s})\right)\right\}\end{array}$ \\
\hline-15 & 0,00387 & 2,015 & 0,70100 & $7,73 \mathrm{E}+13$ & 31,97900 \\
\hline 5 & 0,00360 & 1,248 & 0,22200 & $5,35 \mathrm{E}+13$ & 31,61100 \\
\hline 25 & 0,00333 & 1,364 & 0,31000 & $6,50 \mathrm{E}+13$ & 31,80500 \\
\hline 55 & 0,00305 & 1,135 & 0,12700 & $6,06 \mathrm{E}+13$ & 31,73600 \\
\hline 85 & 0,00279 & 0,964 & $-0,03600$ & $5,70 \mathrm{E}+13$ & 31,67500 \\
\hline
\end{tabular}

Tabelle 21: Werte für Arrhenius-Auftragung sowie $k(T)$ für $\mathrm{CF} 3 \mathrm{CH} 2 \mathrm{OH}+\mathrm{F}$ rel. zu CH4

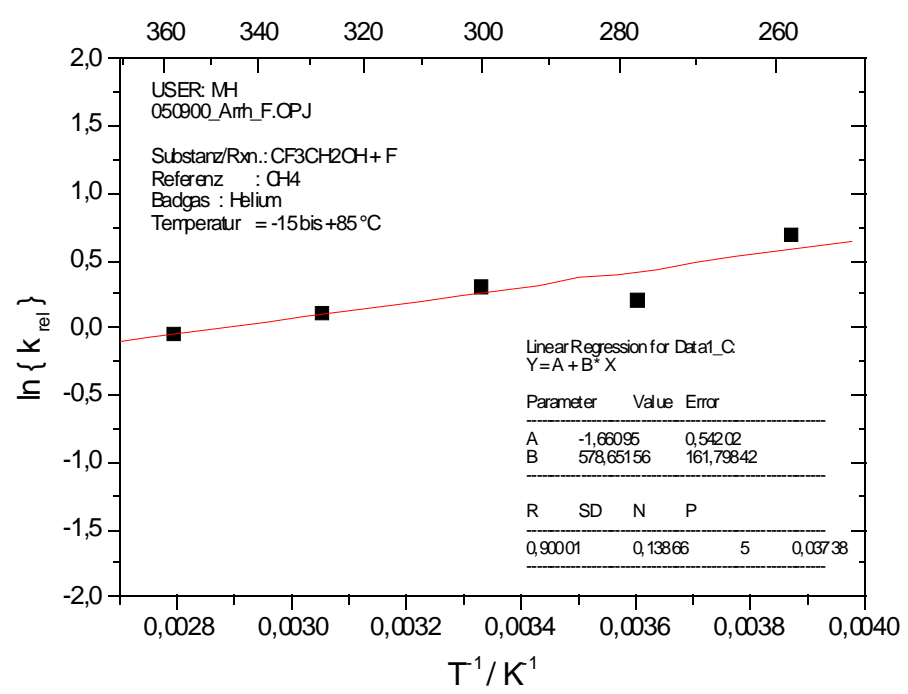

Abbildung 118: Arrhenius-Auftragung für $\mathrm{CF} 3 \mathrm{CH} 2 \mathrm{OH}+\mathrm{F}$ rel. zu $\mathrm{CH} 4$

Die Aktivierungsenergie der hier untersuchten Reaktion erhält man durch Vergleich der Steigung aus Abbildung 118 mit dem Ausdruck in Gleichung (27) (S. 116):

$\mathrm{E}_{\mathrm{a}}=\mathrm{E}_{\mathrm{a}, \text { Referenz }}-($ Steigung $) \cdot \mathrm{R}=(3330-(578,65 \cdot 8,314)) \mathrm{J} / \mathrm{mol}=-1480,9 \mathrm{~J} / \mathrm{mol}$.

Bezüglich des präexponentiellen Faktors wird der Achsenabschnitt aus Abbildung 118 herangezogen: 
$\mathrm{A}=\exp ($ Achsenabschnitt $) \cdot \mathrm{A}_{\text {Referenz }}=\exp (-1,66095) \cdot 1,81 \cdot 10^{14} \mathrm{~cm}^{3} \mathrm{~mol}^{-1} \mathrm{~s}^{-1}$

$$
=3,44 \cdot 10^{13} \mathrm{~cm}^{3} \mathrm{~mol}^{-1} \mathrm{~s}^{-1} \text {. }
$$

Im Ergebnis beträgt der gesuchte Geschwindigkeitskoeffizient damit:

$$
\begin{aligned}
& \mathbf{k}(\mathbf{T})=(3,4 \pm 1,9) \cdot 10^{13} \cdot \exp \{-(-1,48 \pm 1,35) \mathrm{kJ} / \mathrm{mol} /(\mathbf{R} \cdot \mathbf{T})\} \mathrm{cm}^{3} /(\mathbf{m o l} \cdot \mathbf{s}) \\
& \text { für: } \mathrm{CF}_{3} \mathrm{CH}_{2} \mathrm{OH}+\mathrm{F} \rightarrow \text { Produkte }
\end{aligned}
$$

Mit $\mathrm{T}=298 \mathrm{~K}$ errechnet man daraus: $\mathbf{k}(298 \mathrm{~K})=(\mathbf{6 , 3} \pm \mathbf{4 , 8}) \cdot \mathbf{1 0}^{\mathbf{1 3}} \mathbf{c m}^{\mathbf{3}} \mathrm{mol}^{-1} \mathrm{~s}^{-1}$.

Weil die Reaktion im Rahmen ihrer Fehlergrenzen nur eine schwache Temperaturabhängigkeit zeigt, wird zudem eine Auftragung gemäß Gleichung (13) (S. 11) angefertigt (Abb. 119).

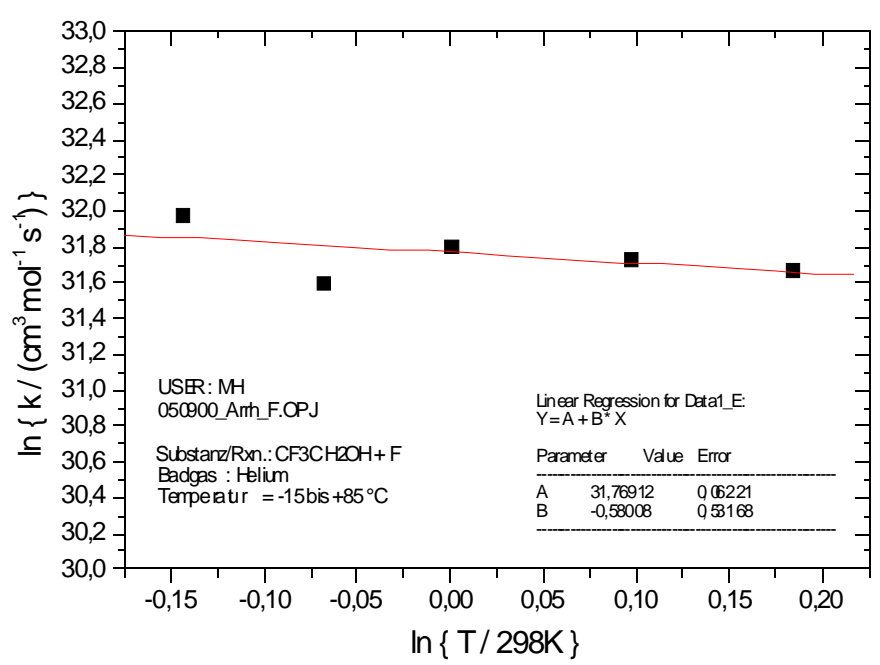

Abbildung 119: $\ln (k)$ vs. $\ln (\mathrm{T})$ für $\mathrm{CF} 3 \mathrm{CH} 2 \mathrm{OH}+\mathrm{F}$

Aus den Ergebnissen der Abbildung 119 berechnet man:

$$
\begin{aligned}
& \mathbf{k}(\mathbf{T})=(6,27 \pm \mathbf{0 , 3 9}) \cdot 10^{13} \cdot(\mathrm{T} / 298 \mathrm{~K})^{-(0,58 \pm 0,53)} \mathrm{cm}^{3} /(\mathbf{m o l} \cdot \mathbf{s}) \\
& \text { für: } \mathrm{CF}_{3} \mathrm{CH}_{2} \mathrm{OH}+\mathrm{F} \rightarrow \text { Produkte }
\end{aligned}
$$

Mit $\mathrm{T}=298 \mathrm{~K}$ errechnet man daraus: $\mathbf{k}(298 \mathrm{~K})=(\mathbf{6 , 2 7} \pm \mathbf{0 , 3 9}) \cdot \mathbf{1 0}^{\mathbf{1 3}} \mathbf{c m}^{\mathbf{3}} \mathbf{m o l}^{-\mathbf{1}} \mathbf{s}^{-1}$ 


\subsubsection{Produkte der Reaktion des erzeugten Radikals mit molekularem Sauerstoff}

Um einen Überblick über die Änderungen im Massenspektrum zu erhalten, sind mit der in Kapitel 3.1.1 (S. 16) beschriebenen Apparatur unmittelbar nacheinander EI-Übersichtsspektren unter Zugabe und Abwesenheit von molekularem Sauerstoff (Fluor-Atome wurden stets gleichermaßen zugegeben) aufgenommen worden.

Es wurden anschließend gezielt in Einzelzählung (5 sek Zählzeit, IP = 29,5 eV) die m/z-Werte überprüft, die im Übersichtsspektrum größere Veränderungen zeigten. Die Durchführung der Messungen ist in Kapitel 3.5.1 (S. 50) beschrieben. Die Reaktionsbedingungen und Ergebnisse sind in Abbildung 120 dargestellt.

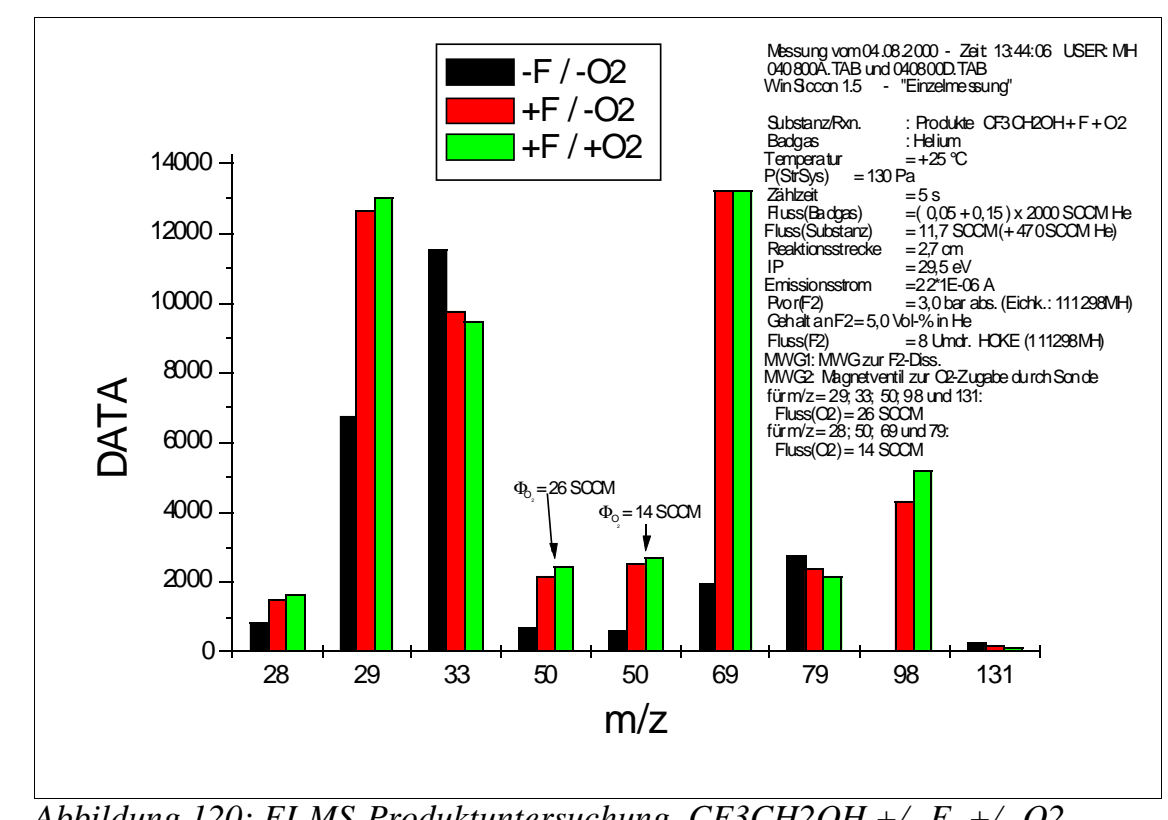

Abbildung 120: EI-MS-Produktuntersuchung $\mathrm{CF} 3 \mathrm{CH} 2 \mathrm{OH}+/-\mathrm{F}+/-\mathrm{O} 2$

Die Zunahme auf m/z 98 könnte auf die Bildung eines Produktes hindeuten, denn als Fragment des Radikals $\left(\mathrm{M}_{\mathrm{r}}=99\right)$ müßte die Intensität bei Zugabe von $\mathrm{O}_{2}$ gleichermaßen Abnehmen wie auf $\mathrm{m} / \mathrm{z} 99$ (letzteres ist hier nicht gezeigt, im Übersichtsspektrum aber beobachtet worden).

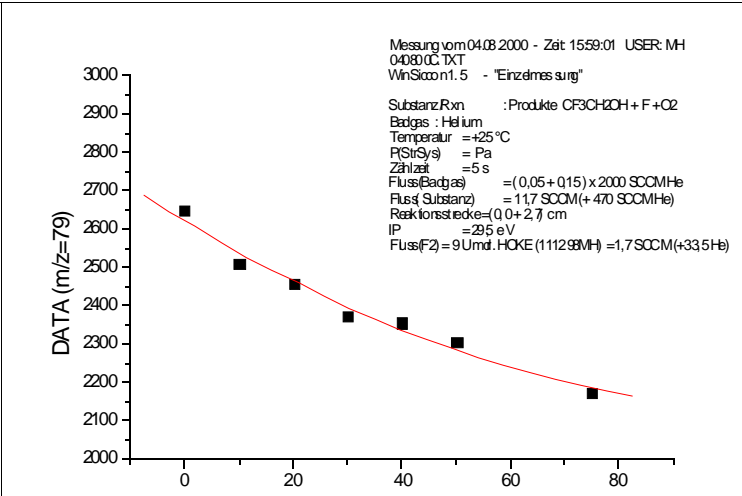

$\left[\mathrm{O}_{2}\right]$ / (\% eines 100-SCCM-He-TYLAN)

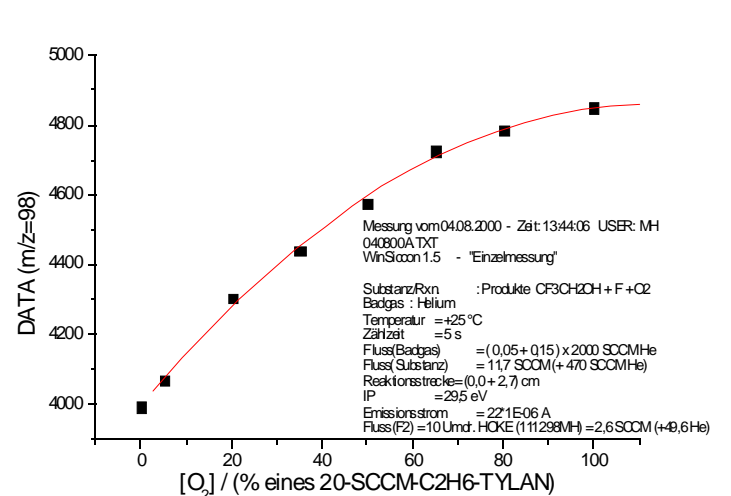

$\left[\mathrm{O}_{2}\right] /(\%$ eines 20-SCCM-C2HG-TYLAN) 
Für die analoge Reaktion $\mathrm{CH}_{3} \mathrm{CHOH}+\mathrm{O}_{2}$ sind $\mathrm{CH}_{3} \mathrm{CHO}$ und $\mathrm{HO}_{2}$ als Produkte $(91,4 \%)$ angegeben ${ }^{125}$. Davon ausgehend wäre für $\left\{\mathrm{CF}_{3} \mathrm{CH}_{2} \mathrm{OH}+\mathrm{F}\right\}+\left\{\mathrm{O}_{2}\right\}$ die Reaktion in Abbildung $122 \mathrm{zu}$ erwarten.

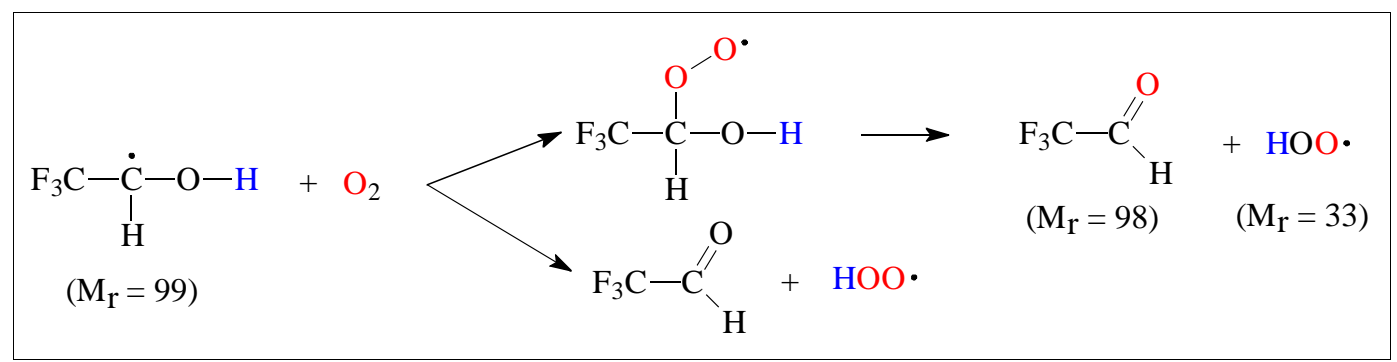

Abbildung 122: Reaktionsschema $\mathrm{CF} 3 \mathrm{CHOH}+\mathrm{O} 2$

Zwar konnte auf m/z 33 keine Zunahme festgestellt werden (vgl. Abb. 120), jedoch ist diese Masse auch stark mit anderen Fragmenten überlagert, was eine Zunahme gemäß der Reaktion in Abb. 122 kompensieren könnte. Gleiches gilt für m/z 79, das als Fragment des gebildeten $\mathrm{CF}_{3} \mathrm{CHO}$ zu erwarten wäre. Die rechte Grafik in Abb. 121 zeigt auf m/z 98 den für ein Produkt $\mathrm{zu}$ erwartenden Verlauf in Abhängigkeit der $\mathrm{O}_{2}$-Konzentration. In der linken Grafik ist die gleiche Messung für m/z 79 (mutmaßliches Fragment von $\mathrm{CF}_{3} \mathrm{CHO}$ ) dargestellt, bei der sich hingegen tendenziell eine Abnahme mit steigender $\mathrm{O}_{2}$-Konzentration ergibt. Letzteres ist aber wahrscheinlich auch auf eine Überlagerung mit anderen Fragmenten zurückzuführen.

Gestützt auf die Zunahme bei m/z 98 und die steigende Intensität bei Erhöhung der $\mathrm{O}_{2^{-}}$ Konzentration ist davon auszugehen, daß $\mathrm{CF}_{3} \mathrm{CHO}$ gemäß Abb. 122 das Hauptprodukt der hier untersuchten Reaktion darstellt.

\subsubsection{Kinetik der Reaktion des erzeugten Radikals mit molekularem Sauerstoff}

Die Messungen wurden in der in Kap. 3.1.1 (S. 16) beschriebenen Apparatur unter Einsatz des Strömungsreaktors aus Abbildung 5 (S. 17) durchgeführt.

Das Dichlormethyl-Radikal diente als Referenzsubstanz. Der Umsatz des Alkohol-Radikals konnte anhand der Intensitätsänderung seines Molekül-Peaks m/z 99 und der des Dichlormethyl-Radikals anhand seines Molekül-Peaks m/z 85 bestimmt werden.

Die Messungen wurden umsatzkorrigiert nach der Relativmethode durchgeführt, wobei zur Messung des Umsatzes des Alkohols bei Zugabe der Fluor-Atome die Intensitätsänderung auf $\mathrm{m} / \mathrm{z} 100$, und für den des Dichlormethans diejenige auf m/z 86 diente; die genaue Ausführung und Auswertung ist in Kap. 3.5.1 (S. 50) beschrieben. 
$\vartheta=-15^{\circ} \mathrm{C} ; \phi_{\mathrm{He}}=802 \mathrm{SCCM} ; \phi_{\mathrm{CF} 3 \mathrm{CH} 2 \mathrm{OH}}=8,6 \mathrm{SCCM} ; \phi_{\mathrm{CH} 2 \mathrm{Cl} 2}=11,7 \mathrm{SCCM} ; \mathrm{p}_{(\mathrm{StrR})}=167 \mathrm{~Pa} ;$ $\mathrm{s}=3,0 \mathrm{~cm} ; \mathrm{IP}=25 \mathrm{eV}$; Zählzeit $=6 \mathrm{~s}$

\begin{tabular}{|c|c|c|c|c|c|c|c|}
\hline $\begin{array}{c}I(99) \\
-O_{2} / \text { w.E. }\end{array}$ & $\begin{array}{c}\Delta \mathrm{I}(99) \\
-\mathrm{O}_{2} / \mathrm{w.E}\end{array}$ & $\begin{array}{c}I(99) \\
+O_{2} / \text { w.E. }\end{array}$ & $\begin{array}{c}\Delta \mathrm{I}(\mathbf{9 9}) \\
+\mathrm{O}_{2} / \text { w.E. }\end{array}$ & $\begin{array}{c}\mathbf{I}(\mathbf{8 5}) \\
-\mathrm{O}_{2} / \mathrm{w.E.}\end{array}$ & $\begin{array}{c}\Delta \mathbf{I}(\mathbf{8 5}) \\
-\mathrm{O}_{2} / \mathbf{w . E .}\end{array}$ & $\begin{array}{c}I(85) \\
+O_{2} / \text { w.E. }\end{array}$ & $\begin{array}{c}\Delta \mathrm{I}(\mathbf{8 5}) \\
+\mathrm{O}_{2} / \mathrm{w} . \mathrm{E} .\end{array}$ \\
\hline 3995 & 75 & 3393 & 84 & 4425 & 118 & 4113 & 119 \\
\hline 3923 & 77 & 3291 & 91 & 4430 & 200 & 4155 & 178 \\
\hline 3873 & 82 & 3273 & 77 & 4478 & 153 & 4065 & 131 \\
\hline 3755 & 78 & 3172 & 81 & 4352 & 172 & 3977 & 161 \\
\hline 4097 & 82 & 3259 & 58 & 4013 & 175 & 3588 & 157 \\
\hline 4039 & 68 & 3241 & 80 & 4248 & 152 & 3911 & 173 \\
\hline 4032 & 72 & 3158 & 76 & 4382 & 167 & 3845 & 172 \\
\hline 4009 & 89 & 3145 & 78 & 4066 & 159 & 3554 & 154 \\
\hline 3999 & 96 & 3495 & 82 & 4076 & 206 & 3726 & 151 \\
\hline 3997 & 97 & 3582 & 71 & 4343 & 158 & 4055 & 183 \\
\hline 4040 & 101 & 3681 & 81 & 4321 & 163 & 4071 & 169 \\
\hline 4038 & 92 & 3769 & 95 & 4125 & 163 & 3912 & 198 \\
\hline
\end{tabular}

Tabelle 22: Meßwerte Kinetik $\{\mathrm{CF} 3 \mathrm{CH} 2 \mathrm{OH}+\mathrm{F}\}+\{\mathrm{O} 2\}$ rel. zu CH2Cl2; $258 \mathrm{~K}$

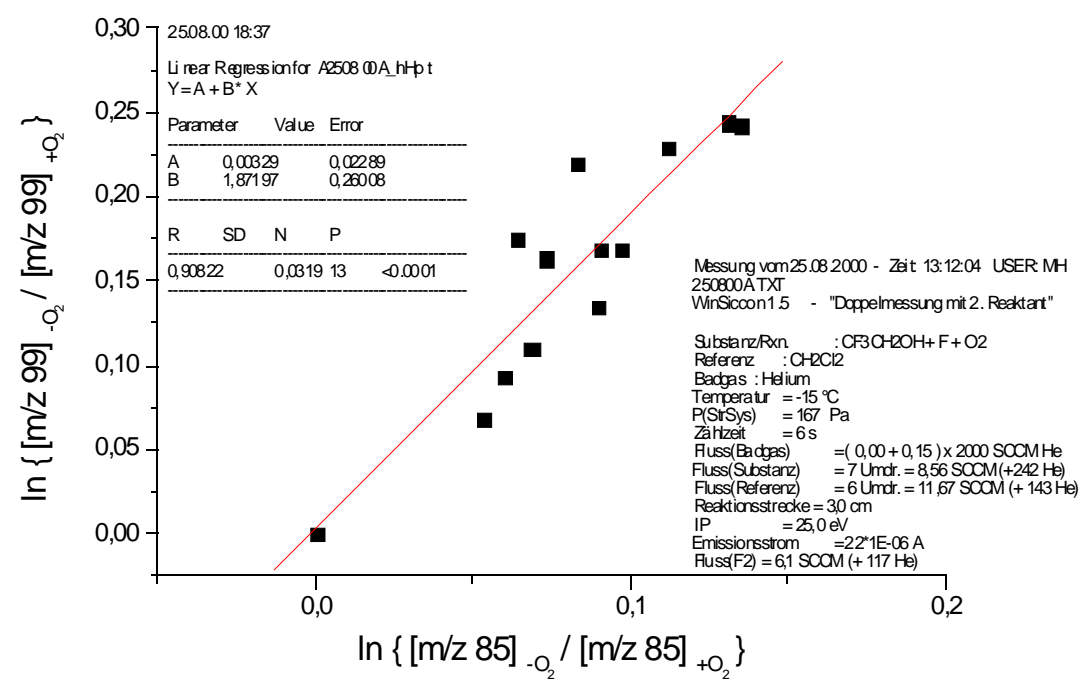

Abbildung 123: Kinetik $\{\mathrm{CF} 3 \mathrm{CH} 2 \mathrm{OH}+\mathrm{F}\}+\{\mathrm{O} 2\}$ rel. zu CH2Cl2; $258 \mathrm{~K}$

Aus der Steigung in Abbildung 123 läßt sich der relative Geschwindigkeitskoeffizient bei $\mathrm{T}=258 \mathrm{~K}$ ermitteln: $\mathrm{k}_{\mathrm{rel}}=(1,87 \pm 0,26)$. 
$\vartheta=+5^{\circ} \mathrm{C} ; \phi_{\mathrm{He}}=802 \mathrm{SCCM} ; \phi_{\mathrm{CF} 3 \mathrm{CH} 2 \mathrm{OH}}=8,6 \mathrm{SCCM} ; \phi_{\mathrm{CH} 2 \mathrm{Cl} 2}=11,7 \mathrm{SCCM} ; \mathrm{p}_{(\mathrm{StrR})}=166 \mathrm{~Pa} ;$ $\mathrm{s}=3,0 \mathrm{~cm} ; \mathrm{IP}=25 \mathrm{eV}$; Zählzeit $=6 \mathrm{~s}$

\begin{tabular}{|c|c|c|c|c|c|c|c|}
\hline $\begin{array}{c}\mathbf{I}(\mathbf{9 9 )} \\
-\mathrm{O}_{2} / \text { w.E. }\end{array}$ & $\begin{array}{c}\Delta \mathrm{I}(\mathbf{( 9 9 )} \\
-\mathbf{O}_{2} / \text { w.E. }\end{array}$ & $\begin{array}{c}\mathbf{I}(\mathbf{9 9 )} \\
+\mathrm{O}_{2} / \text { w.E. }\end{array}$ & $\begin{array}{c}\Delta \mathrm{I}(\mathbf{I 9 9 )} \\
+\mathrm{O}_{2} / \text { w.E. }\end{array}$ & $\begin{array}{c}\mathbf{I}(\mathbf{8 5}) \\
-\mathrm{O}_{2} / \text { w.E. }\end{array}$ & $\begin{array}{c}\Delta \mathbf{I}(\mathbf{8 5}) \\
-\mathbf{O}_{2} / \text { w.E. }\end{array}$ & $\begin{array}{c}\mathrm{I}(\mathbf{8 5}) \\
+\mathrm{O}_{2} / \text { w.E. }\end{array}$ & $\begin{array}{c}\Delta \mathrm{I}(\mathbf{8 5}) \\
+\mathbf{O}_{2} / \text { w.E. }\end{array}$ \\
\hline 3477 & 67 & 2864 & 54 & 4237 & 184 & 3950 & 123 \\
\hline 3431 & 66 & 2804 & 48 & 4460 & 175 & 4148 & 126 \\
\hline 3322 & 61 & 2637 & 46 & 4395 & 123 & 4007 & 145 \\
\hline 3416 & 52 & 2737 & 72 & 4357 & 136 & 4057 & 136 \\
\hline 3372 & 74 & 2653 & 58 & 4505 & 135 & 4155 & 127 \\
\hline 3446 & 70 & 3043 & 70 & 4303 & 117 & 4065 & 139 \\
\hline 3423 & 68 & 2938 & 84 & 4268 & 149 & 4055 & 167 \\
\hline 3417 & 52 & 2964 & 42 & 3774 & 206 & 3612 & 142 \\
\hline
\end{tabular}

Tabelle 23: Meßwerte Kinetik $\{\mathrm{CF} 3 \mathrm{CH} 2 \mathrm{OH}+\mathrm{F}\}+\{\mathrm{O} 2\}$ rel. zu CH2Cl2; $278 \mathrm{~K}$

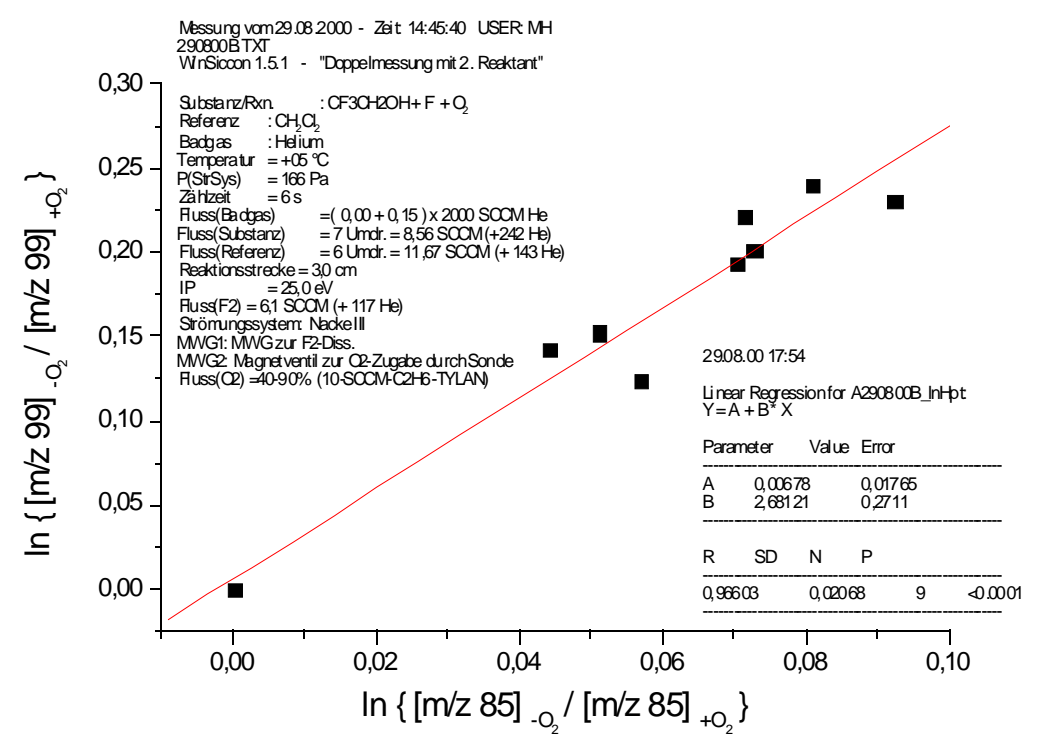

Abbildung 124: Kinetik $\{\mathrm{CF} 3 \mathrm{CH} 2 \mathrm{OH}+\mathrm{F}\}+\{\mathrm{O} 2\}$ rel. zu CH2Cl2; $278 \mathrm{~K}$

Aus der Steigung in Abbildung 124 läßt sich der relative Geschwindigkeitskoeffizient bei $\mathrm{T}=278 \mathrm{~K}$ ermitteln: $\mathrm{k}_{\text {rel }}=(2,68 \pm 0,27)$. 
$\vartheta=+25^{\circ} \mathrm{C} ; \phi_{\mathrm{He}}=800 \mathrm{SCCM} ; \phi_{\mathrm{CF} 3 \mathrm{CH} 2 \mathrm{OH}}=7,8 ; \mathrm{SCCM} ; \mathrm{p}_{(\mathrm{StrR})}=131 \mathrm{~Pa} ; \mathrm{s}=2,7 \mathrm{~cm}$; IP $=29,5$ eV; Zählzeit $=5 \mathrm{~s}$

\begin{tabular}{|c|c|c|c|c|c|c|c|}
\hline $\begin{array}{c}\mathbf{I}(\mathbf{9 9 )} \\
-\mathrm{O}_{2} / \text { w.E. }\end{array}$ & $\begin{array}{c}\Delta \mathbf{I}(\mathbf{9 9}) \\
-\mathrm{O}_{2} / \mathbf{w . E}\end{array}$ & $\begin{array}{c}\mathrm{I}(99) \\
+\mathrm{O}_{2} / \text { w.E. }\end{array}$ & $\begin{aligned} & \Delta \mathrm{I}(\mathbf{9 9}) \\
+ & \mathrm{O}_{2} / \text { w.E. }\end{aligned}$ & $\begin{array}{c}\mathbf{I}(\mathbf{8 5}) \\
-\mathbf{O}_{2} / \text { w.E. }\end{array}$ & $\begin{array}{c}\Delta \mathbf{I}(\mathbf{8 5}) \\
-\mathbf{O}_{2} / \mathbf{w} . \mathbf{E} .\end{array}$ & $\begin{array}{c}\mathrm{I}(\mathbf{8 5}) \\
+\mathrm{O}_{2} / \text { w.E. }\end{array}$ & $\begin{array}{c}\Delta \mathbf{I}(\mathbf{8 5}) \\
+\mathbf{O}_{2} / \text { w.E. }\end{array}$ \\
\hline 3621 & 67 & 2138 & 100 & 3755 & 119 & 3203 & 49 \\
\hline 3603 & 69 & 2167 & 62 & 3489 & 131 & 2959 & 121 \\
\hline 3510 & 84 & 2283 & 58 & 3795 & 143 & 3426 & 140 \\
\hline 3135 & 70 & 2228 & 86 & 3653 & 89 & 3321 & 80 \\
\hline 1912 & 49 & 1534 & 48 & 3568 & 90 & 3367 & 103 \\
\hline 1908 & 62 & 1678 & 55 & 3625 & 131 & 3532 & 119 \\
\hline 2130 & 50 & 1772 & 47 & 3650 & 149 & 3453 & 143 \\
\hline 2157 & 50 & 1352 & 91 & 3688 & 100 & 3198 & 109 \\
\hline
\end{tabular}

Tabelle 24: Meßwerte Kinetik $\{\mathrm{CF} 3 \mathrm{CH} 2 \mathrm{OH}+\mathrm{F}\}+\{\mathrm{O} 2\}$ rel. zu $\mathrm{CH} 2 \mathrm{Cl2} ; 298 \mathrm{~K}$

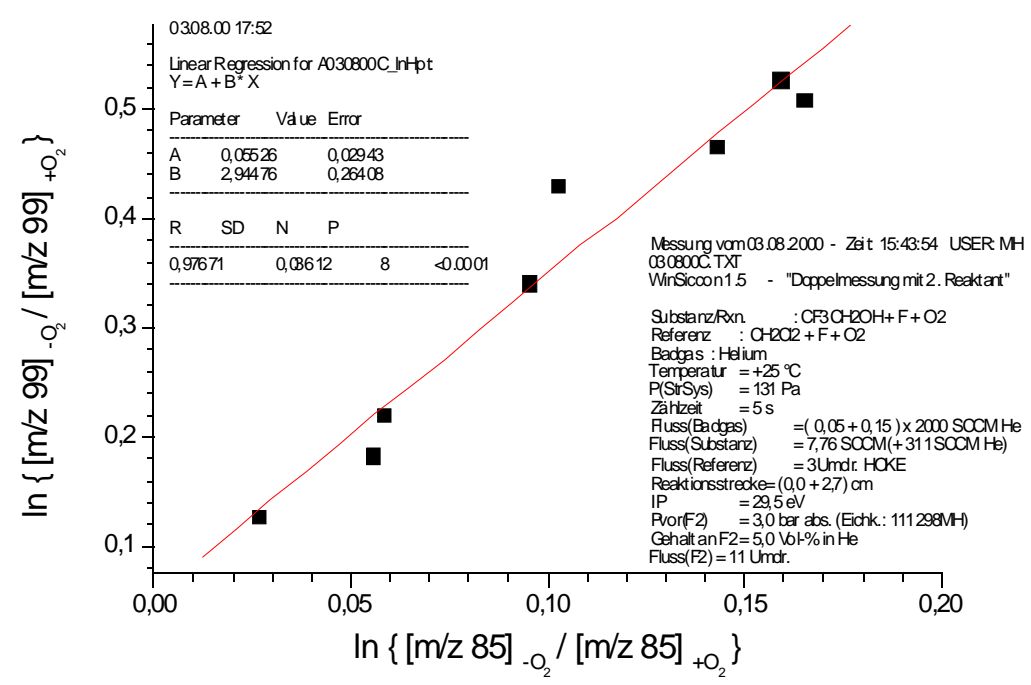

Abbildung 125: Kinetik $\{\mathrm{CF} 3 \mathrm{CH} 2 \mathrm{OH}+\mathrm{F}\}+\{\mathrm{O} 2\}$ rel. $z$ u CH2Cl2; $298 \mathrm{~K}$

Aus der Steigung in Abbildung 125 läßt sich der relative Geschwindigkeitskoeffizient bei $\mathrm{T}=298 \mathrm{~K}$ ermitteln: $\mathrm{k}_{\mathrm{rel}}=(2,94 \pm 0,26)$. 
$\vartheta=+55^{\circ} \mathrm{C} ; \phi_{\mathrm{He}}=802 \mathrm{SCCM} ; \phi_{\mathrm{CF} 3 \mathrm{CH} 2 \mathrm{OH}}=8,6 \mathrm{SCCM} ; \phi_{\mathrm{CH} 2 \mathrm{Cl} 2}=11,7 \mathrm{SCCM} ; \mathrm{p}_{(\mathrm{StrR})}=196 \mathrm{~Pa} ;$ $\mathrm{s}=3,0 \mathrm{~cm} ; \mathrm{IP}=25 \mathrm{eV}$; Zählzeit $=6 \mathrm{~s}$

\begin{tabular}{|c|c|c|c|c|c|c|c|}
\hline $\begin{array}{c}\mathrm{I}(\mathbf{9 9 )} \\
-\mathrm{O}_{2} / \mathrm{w.E} .\end{array}$ & $\begin{array}{c}\Delta \mathrm{I}(\mathbf{9 9}) \\
-\mathrm{O}_{2} / \mathrm{w} . \mathrm{E} .\end{array}$ & $\begin{array}{c}\mathrm{I}(\mathbf{9 9 )} \\
+\mathrm{O}_{2} / \text { w.E. }\end{array}$ & $\begin{array}{c}\Delta \mathrm{I}(\mathbf{9 9}) \\
+\mathrm{O}_{2} / \text { w.E. }\end{array}$ & $\begin{array}{c}\mathrm{I}(\mathbf{8 5}) \\
-\mathrm{O}_{2} / \mathrm{w.E} .\end{array}$ & $\begin{array}{c}\Delta \mathbf{I}(\mathbf{8 5}) \\
-\mathrm{O}_{2} / \mathbf{w . E} .\end{array}$ & $\begin{array}{c}\mathrm{I}(85) \\
+\mathrm{O}_{2} / \text { w.E. }\end{array}$ & $\begin{array}{c}\Delta \mathrm{I}(\mathbf{8 5}) \\
+\mathrm{O}_{2} / \mathrm{w} . \mathrm{E} .\end{array}$ \\
\hline 3534 & 93 & 2856 & 61 & 4890 & 181 & 4690 & 181 \\
\hline 3797 & 78 & 2824 & 60 & 6530 & 156 & 6055 & 122 \\
\hline 3648 & 53 & 2770 & 33 & 6810 & 148 & 6285 & 162 \\
\hline 3739 & 60 & 2308 & 73 & 7003 & 128 & 6128 & 106 \\
\hline 3770 & 78 & 2241 & 70 & 6932 & 146 & 6144 & 129 \\
\hline 3697 & 69 & 2355 & 36 & 6716 & 150 & 6079 & 157 \\
\hline 3762 & 67 & 2318 & 50 & 6956 & 109 & 6169 & 151 \\
\hline 3622 & 70 & 2374 & 44 & 6983 & 140 & 6145 & 166 \\
\hline 3528 & 65 & 2378 & 33 & 7056 & 132 & 6456 & 116 \\
\hline 3569 & 63 & 2568 & 32 & 6975 & 125 & 6488 & 143 \\
\hline 3575 & 60 & 2718 & 48 & 6866 & 128 & 6454 & 134 \\
\hline
\end{tabular}

Tabelle 25: Meßwerte Kinetik $\{\mathrm{CF} 3 \mathrm{CH} 2 \mathrm{OH}+\mathrm{F}\}+\{\mathrm{O} 2\}$ rel. zu $\mathrm{CH} 2 \mathrm{Cl} 2 ; 328 \mathrm{~K}$

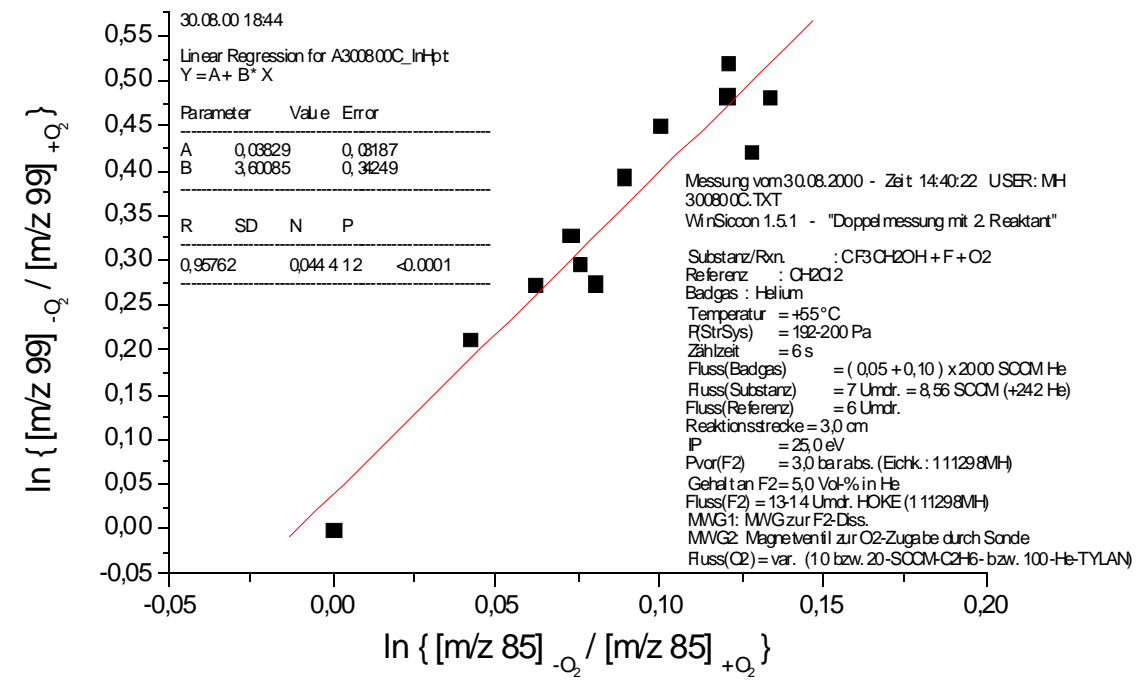

Abbildung 126: Kinetik $\{\mathrm{CF} 3 \mathrm{CH} 2 \mathrm{OH}+\mathrm{F}\}+\{\mathrm{O} 2\}$ rel. zu CH2Cl2; $328 \mathrm{~K}$

Aus der Steigung in Abbildung 126 läßt sich der relative Geschwindigkeitskoeffizient bei $\mathrm{T}=328 \mathrm{~K}$ ermitteln: $\mathrm{k}_{\text {rel }}=(3,60 \pm 0,34)$. 
$\vartheta=+85^{\circ} \mathrm{C} ; \phi_{\mathrm{He}}=802 \mathrm{SCCM} ; \phi_{\mathrm{CF} 3 \mathrm{CH} 2 \mathrm{OH}}=8,6 \mathrm{SCCM} ; \phi_{\mathrm{CH} 2 \mathrm{Cl} 2}=11,7 \mathrm{SCCM} ; \mathrm{p}_{(\mathrm{StrR})}=204 \mathrm{~Pa} ;$ $\mathrm{s}=3,0 \mathrm{~cm} ; \mathrm{IP}=25 \mathrm{eV}$; Zählzeit $=6 \mathrm{~s}$

\begin{tabular}{|c|c|c|c|c|c|c|c|}
\hline $\begin{array}{c}\mathbf{I}(\mathbf{9 9 )} \\
-\mathbf{O}_{2} / \text { w.E. }\end{array}$ & $\begin{array}{c}\Delta \mathbf{I}(\mathbf{9 9}) \\
-\mathbf{O}_{2} / \mathbf{w . E}\end{array}$ & $\begin{array}{c}\mathrm{I}(99) \\
+\mathrm{O}_{2} / \text { w.E. }\end{array}$ & $\begin{aligned} & \Delta \mathrm{I}(\mathbf{9 9}) \\
+ & \mathrm{O}_{2} / \text { w.E. }\end{aligned}$ & $\begin{array}{c}\mathbf{I}(\mathbf{8 5}) \\
-\mathbf{O}_{2} / \mathbf{w . E .}\end{array}$ & $\begin{array}{c}\Delta \mathbf{I}(\mathbf{8 5}) \\
-\mathbf{O}_{2} / \mathbf{w . E}\end{array}$ & $\begin{array}{c}\mathrm{I}(\mathbf{8 5}) \\
+\mathrm{O}_{2} / \text { w.E. }\end{array}$ & $\begin{array}{c}\Delta \mathbf{I}(\mathbf{8 5}) \\
+\mathbf{O}_{2} / \text { w.E. }\end{array}$ \\
\hline 3832 & 48 & 2226 & 36 & 10020 & 208 & 9217 & 98 \\
\hline 3885 & 75 & 2274 & 63 & 9570 & 126 & 8732 & 129 \\
\hline 3778 & 83 & 2225 & 54 & 10360 & 429 & 9498 & 263 \\
\hline 3883 & 53 & 2420 & 69 & 10210 & 83 & 9484 & 89 \\
\hline 3896 & 75 & 2376 & 41 & 10230 & 95 & 9295 & 141 \\
\hline 3838 & 88 & 2496 & 68 & 10290 & 123 & 9436 & 105 \\
\hline 3815 & 75 & 2504 & 55 & 10240 & 144 & 9523 & 109 \\
\hline 3806 & 47 & 2461 & 40 & 10240 & 169 & 9439 & 103 \\
\hline 3788 & 67 & 2579 & 36 & 9875 & 119 & 9125 & 164 \\
\hline 3672 & 76 & 2676 & 50 & 10030 & 174 & 9576 & 153 \\
\hline 3689 & 48 & 2714 & 66 & 10180 & 190 & 9703 & 129 \\
\hline 3496 & 382 & 2740 & 379 & 9863 & 128 & 9426 & 133 \\
\hline 3556 & 81 & 2905 & 64 & 9558 & 213 & 9220 & 143 \\
\hline
\end{tabular}

Tabelle 26: Meßwerte Kinetik $\{\mathrm{CF} 3 \mathrm{CH} 2 \mathrm{OH}+\mathrm{F}\}+\{\mathrm{O} 2\}$ rel. zu CH2Cl2; $358 \mathrm{~K}$

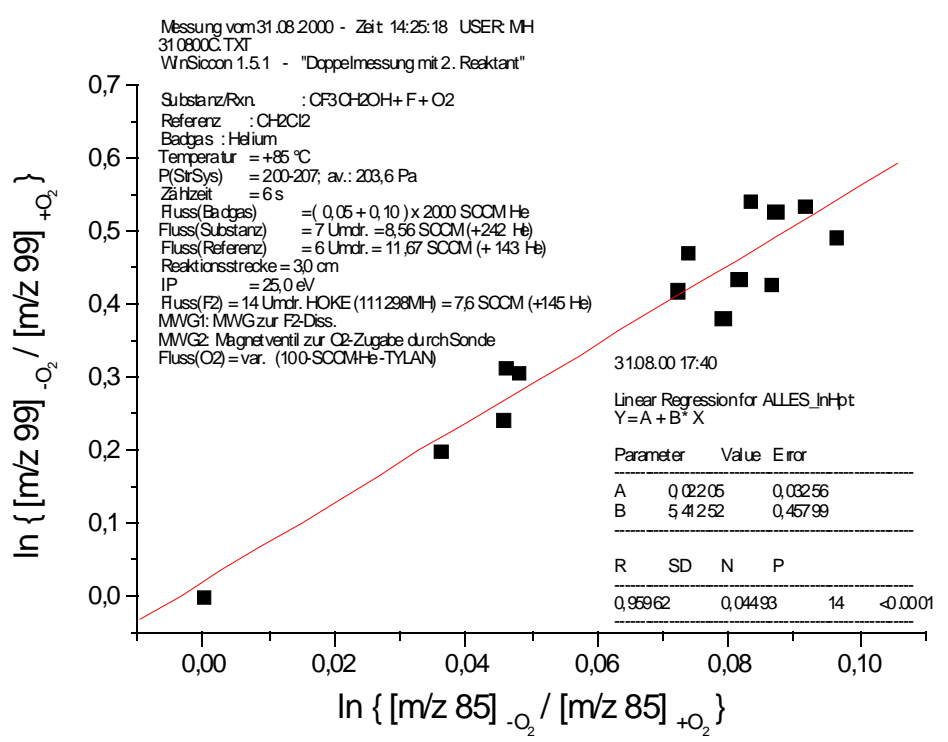

Abbildung 127: Kinetik $\{\mathrm{CF} 3 \mathrm{CH} 2 \mathrm{OH}+\mathrm{F}\}+\{\mathrm{O} 2\}$ rel. zu CH2Cl2; $358 \mathrm{~K}$

Aus der Steigung in Abbildung 127 läßt sich der relative Geschwindigkeitskoeffizient bei $\mathrm{T}=328 \mathrm{~K}$ ermitteln: $\mathrm{k}_{\mathrm{rel}}=(5,41 \pm 0,46)$. 
Aus den ermittelten relativen Geschwindigkeitskoeffizienten der Abbildungen 123 bis 127 können nun mit der bekannten Temperaturabhängigkeit des Geschwindigkeitskoeffizienten für die Referenzreaktion ${ }^{126} \mathrm{k}_{\text {ref }}(\mathrm{T})$ die absoluten Geschwindigkeitskoeffizienten $\mathrm{k}_{\text {Alkohol }}$ für die Reakation des aus dem Alkohol erzeugten Radikals mit $\mathrm{O}_{2}$ berechnet werden (Tabelle 27).

$\mathrm{k}_{\mathrm{ref}}(\mathrm{T})=(1,56 \pm 0,08) \cdot 10^{17} \cdot(\mathrm{T} / 298 \mathrm{~K})^{-(6,7 \pm 0,7)} \cdot[\mathrm{He}] \mathrm{cm}^{6} /\left(\mathrm{mol}^{2} \cdot \mathrm{s}\right)$.

Ferner kann mit diesen Werten eine Arrhenius-Auftragung gemäß Gleichung (11) (S. 10) angefertigt werden, welche in Abbildung $128 \mathrm{zu}$ sehen ist.

\begin{tabular}{|c|c|c|c|c|c|c|c|c|}
\hline $\begin{array}{l}\vartheta / \\
{ }^{\circ} \mathbf{C}\end{array}$ & $\mathbf{T} / \mathbf{K}$ & $\mathbf{T}^{-1} / \mathbf{K}^{-1}$ & $\mathbf{k}_{\text {rel }}$ & $\ln \left(k_{\mathrm{rel}}\right)$ & $\begin{array}{c}\mathbf{k}_{\text {termolek. }} / \\
\left\{\mathbf{c m}^{6} /\right. \\
\left.\left(\mathrm{mol}^{2} \mathbf{s}\right)\right\}\end{array}$ & $\mathbf{p}_{\text {StrR }} / \mathbf{P a}$ & $\begin{array}{c}{[\mathrm{He}] /} \\
\mathrm{mol} / \mathrm{cm}^{3}\end{array}$ & $\begin{array}{c}\mathbf{k}_{\text {bimolek. }} / \\
\left\{\mathrm{cm}^{3} /\right. \\
(\mathrm{mol} \mathrm{s})\}\end{array}$ \\
\hline-15 & 258,15 & 0,00387 & 1,872 & 0,627 & $7,64 \mathrm{E}+17$ & 167 & $7,78 \mathrm{E}-08$ & $5,95 \mathrm{E}+10$ \\
\hline 5 & 278,15 & 0,00360 & 2,681 & 0,986 & $6,64 \mathrm{E}+17$ & 166 & $7,18 \mathrm{E}-08$ & $4,76 \mathrm{E}+10$ \\
\hline 25 & 298,15 & 0,00335 & 2,945 & 1,080 & $4,58 \mathrm{E}+17$ & 131 & $5,28 \mathrm{E}-08$ & $2,42 \mathrm{E}+10$ \\
\hline 55 & 328,15 & 0,00305 & 3,601 & 1,281 & $2,95 \mathrm{E}+17$ & 196 & $7,18 \mathrm{E}-08$ & $2,12 \mathrm{E}+10$ \\
\hline 85 & 358,15 & 0,00279 & 5,413 & 1,689 & $2,46 \mathrm{E}+17$ & 204 & $6,85 \mathrm{E}-08$ & $1,69 \mathrm{E}+10$ \\
\hline
\end{tabular}

Tabelle 27: Werte für Arrhenius-Auftragung $\{\mathrm{CF} 3 \mathrm{CH} 2 \mathrm{OH}+\mathrm{F}\}+\{\mathrm{O} 2\}$ rel. zu $\mathrm{CH} 2 \mathrm{Cl} 2$

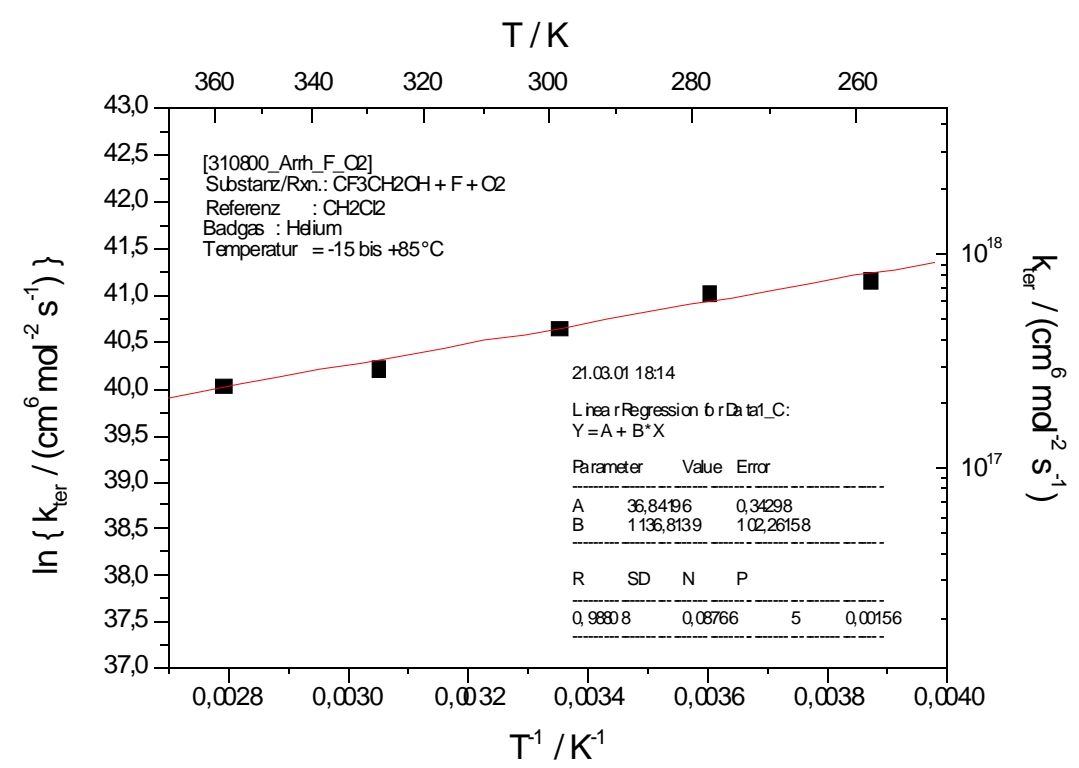

Abbildung 128: Arrhenius-Auftragung $\{\mathrm{CF} 3 \mathrm{CH} 2 \mathrm{OH}+\mathrm{F}\}+\{\mathrm{O} 2\}$

Die Steigung der Arrhenius-Auftragung in Abb. 128 beträgt $b=(1136,8 \pm 102,3) \mathrm{K}$ und der Achsenabschnitt $\mathrm{a}=(36,84 \pm 0,34)$. 
Weil Assoziationsreaktionen mit $\mathrm{O}_{2}$ üblicherweise ohne Energiebarriere ablaufen, sollte besser eine Auftragung $\ln (\mathrm{k})$ vs. $\ln (\mathrm{T})$ gemäß Gleichung (13) (S. 11) angefertigt werden (Abb. 129).

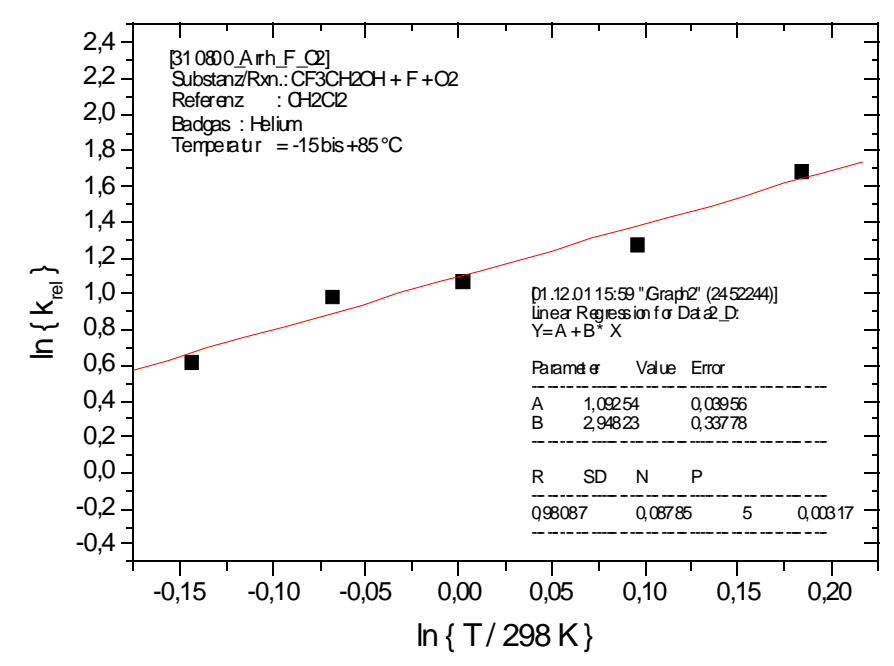

Abbildung 129: $\ln (k)$ vs. $\ln (\mathrm{T} /)$ f. $\{\mathrm{CF} 3 \mathrm{CH} 2 \mathrm{OH}+\mathrm{F}\}+\{\mathrm{O} 2\}$ rel. zu $\mathrm{CH} 2 \mathrm{Cl} 2$

Mittels Gleichung (29) (S. 126) kann nun aus dem Achsenabschnitt a der Abbildung 129 der Vorfaktor A berechnet werden:

$\mathrm{A}=\mathrm{A}_{\mathrm{ref}} \cdot \exp (\mathrm{a})=1,56 \cdot 10^{17} \mathrm{~cm}^{6} /\left(\mathrm{mol}^{2} \cdot \mathrm{s}\right) \cdot \exp (1,09254)=4,65 \cdot 10^{17} \mathrm{~cm}^{6} /\left(\mathrm{mol}^{2} \cdot \mathrm{s}\right)$.

Den Parameter n kann man gemäß Gleichung (29) (S. 126) folgendermaßen aus der Steigung b der Abbildung 129 ermittlen:

$\mathrm{n}=\left(\mathrm{b}+\mathrm{n}_{\mathrm{ref}}\right)=(2,948+(-6,70))=-3,75$.

Somit ergibt sich als Temperaturabhängigkeit des Geschwindigkeitskoeffizienten:

$$
k(T)=(4,65 \pm 0,30) \cdot 10^{17} \cdot(T / 298 K)^{-(3,75 \pm 0,75)} \cdot[\mathrm{He}] \mathrm{cm}^{6} /\left(\mathrm{mol}^{2} \cdot \mathrm{s}\right)
$$

für: $\left\{\right.$ Radikal aus der Reaktion $\left.\mathrm{CF}_{3} \mathrm{CH}_{2} \mathrm{OH}+\mathrm{F}\right\}+\left\{\mathrm{O}_{2}\right\} \rightarrow$ Produkte

Mit T $=298 \mathrm{~K}$ berechnet man daraus:

$$
\mathrm{k}(298 \mathrm{~K})=(4,65 \pm 0,30) \cdot 10^{17} \cdot[\mathrm{He}] \mathrm{cm}^{6} /\left(\mathrm{mol}^{2} \cdot \mathrm{s}\right)
$$

Aus dem Druck im Reaktor und dem Gesamtgasfluß sowie den anderen angegebenen Reaktionsbedingungen können gemäß Gleichung (19) (S. 14) die Konzentration des Badgases Helium und damit die bimolekularen Geschwindigkeitskoeffizienten berechnet werden. Diese Werte sind als $\mathrm{k}_{\text {bimolek. }}$ in Tabelle 27 eingetragen. 


\subsubsection{Kinetik der Reaktion des erzeugten Radikals mit Stickstoffmonoxid}

Die Messungen wurden in der in Kap. 3.1.1 (S. 16) beschriebenen Apparatur unter Einsatz des Strömungsreaktors aus Abbildung 6 (S. 17) durchgeführt. Dabei wurden 2,2,2-Trifluorethanol und NO zusammen durch die innere Sonde zugegeben und der zweite seitliche MWE-Einlaß blieb ungenutzt.

Das Dichlormethyl-Radikal diente als Referenzsubstanz. Der Umsatz des Alkohol-Radikals konnte anhand der Intensitätsänderung seines Molekül-Peaks $\mathrm{m} / \mathrm{z} 99$, derjenige des Dichlormethyl-Radikals ebenfalls anhand seines Molekül-Peaks (m/z 85) bestimmt werden.

Die Messungen wurden umsatzkorrigiert nach der Relativmethode durchgeführt, wobei zur Messung des Umsatzes des Alkohols bei Zugabe der Fluor-Atome die Intensitätsänderung auf $\mathrm{m} / \mathrm{z} 100$, und für den des Dichlormethans diejenige auf m/z 86 diente; die genaue Ausführung und Auswertung ist in Kap. 3.5.1 (S. 50) beschrieben.

$\vartheta=+25{ }^{\circ} \mathrm{C} ; \phi_{\mathrm{He}}=913 \mathrm{SCCM} ; \phi_{\mathrm{CF} 3 \mathrm{CH} 2 \mathrm{OH}}=9,6 \mathrm{SCCM} ; \phi_{\mathrm{CH} 2 \mathrm{Cl} 2}=14,5 \mathrm{SCCM} ; \mathrm{p}_{(\mathrm{StrR})}=145 \mathrm{~Pa}$; $\mathrm{s}=2,7 \mathrm{~cm} ; \mathrm{IP}=25 \mathrm{eV} ;$ Zählzeit $=5 \mathrm{~s}$

\begin{tabular}{|r|r|r|r|r|r|r|r|}
\hline $\begin{array}{r}\mathbf{I}(\mathbf{9 9}) \\
\text {-NO / w.E. }\end{array}$ & $\begin{array}{c}\Delta \mathbf{I}(\mathbf{9 9}) \\
\text {-NO / w.E. }\end{array}$ & $\begin{array}{c}\mathbf{I}(\mathbf{9 9}) \\
\text { +NO /w.E. }\end{array}$ & $\begin{array}{c}\Delta \mathbf{I}(\mathbf{9 9}) \\
\text { +NO /w.E. }\end{array}$ & $\begin{array}{c}\mathbf{I}(\mathbf{8 5}) \\
\text {-NO / w.E. }\end{array}$ & $\begin{array}{c}\Delta \mathbf{I}(\mathbf{8 5}) \\
\text {-NO / w.E. }\end{array}$ & $\begin{array}{c}\mathbf{I}(\mathbf{8 5}) \\
\text { +NO/ w.E. }\end{array}$ & $\begin{array}{c}\Delta \mathbf{I}(\mathbf{8 5}) \\
\text { +NO/w.E. }\end{array}$ \\
\hline 1827 & 17,43 & 1118 & 28,81 & 4204 & 217,3 & 2950 & 127,9 \\
\hline 1756 & 45,75 & 1129 & 49,14 & 4312 & 146,7 & 3099 & 106,7 \\
\hline 1910 & 46,76 & 1504 & 29,87 & 4080 & 117,4 & 3560 & 116,6 \\
\hline 1905 & 33,17 & 1675 & 44,89 & 4237 & 129,5 & 3875 & 112,6 \\
\hline 1716 & 65,88 & 1495 & 35,09 & 4214 & 161,3 & 3952 & 134,8 \\
\hline 1713 & 32,77 & 1309 & 50,67 & 4257 & 117,9 & 3633 & 116,8 \\
\hline 1699 & 61,12 & 1035 & 41,38 & 4365 & 210 & 3250 & 112,4 \\
\hline 2003 & 20,3 & 1422 & 39,14 & 4240 & 88 & 3500 & 101,5 \\
\hline 1998 & 38,19 & 1441 & 33,47 & 4455 & 90,98 & 3525 & 108,7 \\
\hline 2120 & 53,27 & 1935 & 54,29 & 4305 & 102,6 & 4080 & 89,69 \\
\hline
\end{tabular}

Tabelle 28: Meßwerte Kinetik $\{\mathrm{CF} 3 \mathrm{CH} 2 \mathrm{OH}+\mathrm{F}\}+\{\mathrm{NO}\}$ rel. zu CH2Cl2; $298 \mathrm{~K}$

Mit den Meßwerten aus Tabelle 28 kann nun mittels einer Auftragung gemäß Gleichung (10) (S. 10) der relative Geschwindigkeitskoeffizient bestimmt werden. 


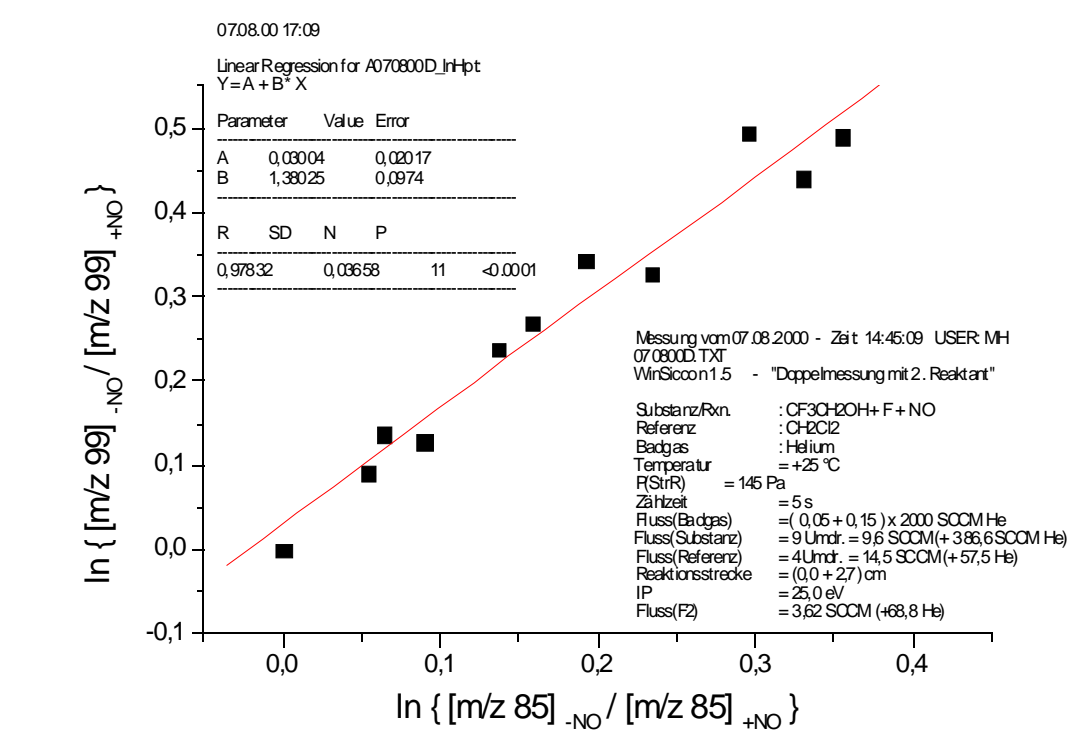

Abbildung 130: Kinetik $\{\mathrm{CF} 3 \mathrm{CH} 2 \mathrm{OH}+\mathrm{F}\}+\{\mathrm{NO}\}$ rel. zu CH2Cl2; $298 \mathrm{~K}$

Aus der Steigung in Abbildung 130 läßt sich der relative Geschwindigkeitskoeffizient bei $\mathrm{T}=298 \mathrm{~K}$ ermitteln: $\mathrm{k}_{\mathrm{rel}}=(1,38 \pm 0,10)$.

Mit dem bekannten Geschwindigkeitskoeffizienten der Referenzreaktion $\mathrm{k}_{\mathrm{ref}}$ (welcher in Kap. 4.4.3, S. 212 bestimmt wird) läßt sich der absolute Geschwindigkeitskoeffizient k berechnen. $\mathrm{k}_{\mathrm{ref}}(298 \mathrm{~K})=(1,21 \pm 0,28) \cdot 10^{17} \cdot[\mathrm{He}] \mathrm{cm}^{6} /\left(\mathrm{mol}^{2} \cdot \mathrm{s}\right)$

Man erhält als Ergebnis (termolekular):

$$
\begin{aligned}
& \mathbf{k}(298 \mathrm{~K})=(1,67 \pm \mathbf{0 , 3 0}) \cdot 10^{17} \cdot[\mathrm{He}] \mathbf{c m}^{6} /\left(\mathbf{m o l}^{2} \cdot \mathbf{s}\right) \\
& \text { für: }\left\{\mathrm{CF}_{3} \mathrm{CH}_{2} \mathrm{OH}+\mathrm{F}\right\}+\{\mathrm{NO}\} \rightarrow \text { Produkte }
\end{aligned}
$$

Mit $[\mathrm{He}]=5,8 \cdot 10^{-08} \mathrm{~mol} / \mathrm{cm}^{3}$ berechnet man daraus den bimolekularen Koeffizienten:

$$
\mathbf{k}(298 \mathrm{~K})=9,7 \cdot 10^{9} \mathrm{~cm}^{3} /(\mathrm{mol} \cdot \mathrm{s})
$$




\subsubsection{REMPI-Nachweis des Radikals}

Abschließend soll nun überprüft werden, ob das durch Zugabe von F-Atomen erzeugte Radikal auch mit dem REMPI-Verfahren (möglichst selektiv) zu ionisieren ist. Dieses Verfahren sowie die dafür eingesetzte Apparatur ist eingehend in Kap. 3.1.4 (S. 33 ff.) beschrieben. Die Meßwerte sind mit dem automatischen Wellenlängendurchlauf registriert worden (vgl. Kap. 3.5.4, S. 57), wobei zur Auswertung das Integral (mit den Integrationsgrenzen von $\pm 10 \%$ des Peakmaximums) des Peaks bei m/z 99 (bzw. der entsprechenden Flugzeit) herangezogen wurde.
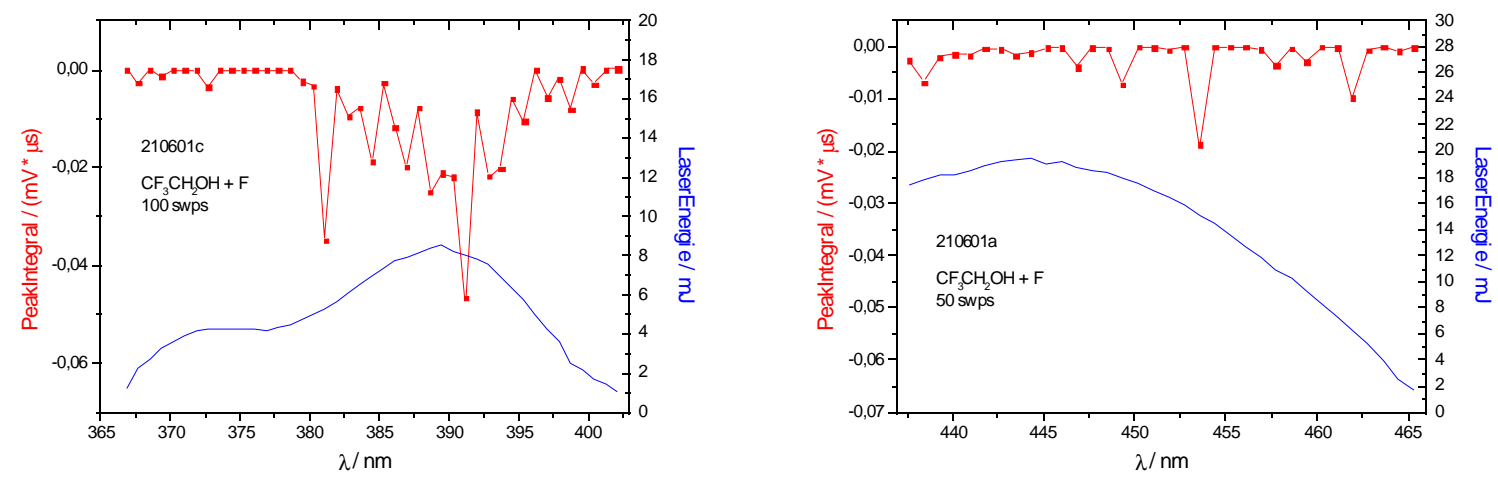

Abbildung 131: REMPI-Nachweis des aus $\mathrm{CF} 3 \mathrm{CH} 2 \mathrm{OH}+\mathrm{F}$ erzeugten Radikals

Das Radikal konnte bei den Wellenlängen $\lambda=381,1$ und 391,2 nm nachgewiesen werden.

In der Umgebung dieser beiden Peaks wurde anschließend mit einer feineren Auflösung das Maximum (bzw. mathematisch gesehen: Minimum) exakter gesucht und bei $\lambda=\mathbf{3 8 8 , 6 0 1} \mathbf{n m}$ gefunden (vgl. Abbildung 132).

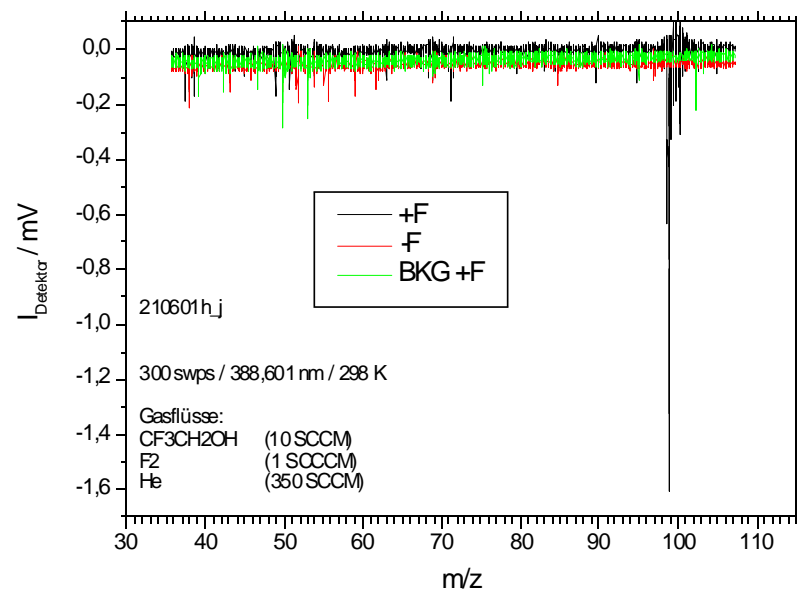

Abbildung 132: REMPI-Nachweis bei 388,601 nm 
In Abbildung 132 erkennt man, daß bei Zugabe von Fluor-Atomen zu $\mathrm{CF}_{3} \mathrm{CH}_{2} \mathrm{OH}$ selektiv ein Peak bei m/z 99 (d.h. der Masse des erzeugten Radikals) entsteht (schwarze Linie) und dieser vollständig verschwindet, wenn der $\mathrm{Zufluß}$ von $\mathrm{CF}_{3} \mathrm{CH}_{2} \mathrm{OH}$ oder derjenige der Fluor-Atome abgeschaltet wird (grüne bzw. rote Linie in Abb. 132).

\subsubsection{Diskussion der Ergebnisse}

Der ermittelte Geschwindigkeitskoeffizient für die Reaktion des Alkohols $\mathrm{CF}_{3} \mathrm{CH}_{2} \mathrm{OH}$ mit Fluor-Atomen ist mit $\mathrm{k}(298 \mathrm{~K})=6,27 \cdot 10^{13} \mathrm{~cm}^{3} \mathrm{~mol}^{-1} \mathrm{~s}^{-1}$ ähnlich hoch wie derjenige für die analoge Reaktion des Ethanols, welcher mit $\mathrm{k}=7,69 \cdot 10^{13} \mathrm{~cm}^{3} \mathrm{~mol}^{-1} \mathrm{~s}^{-1}$ angegeben wird ${ }^{127}$. Bemerkenswert ist allerdings der negative Parameter $n=(-0,58 \pm 0,53)$ zur Beschreibung der Temperaturabhängigkeit. Es ist kein Grund ersichtlich, warum für eine Abstraktionsreaktion eine solche „negative Temperaturabhängigkeit“ vorliegen sollte, denn man würde eine positive Barriere dafür erwarten. Im Hinblick auf die Fehlergrenzen ist es daher wahrscheinlich, daß die Reaktion gar nicht temperaturabhängig ist $(\mathrm{n} \approx 0)$. Ein ganz ähnliches Ergebnis wird auch bei dem strukturell ähnlichen $\mathrm{CH}_{2} \mathrm{FCH}_{2} \mathrm{OH}$ gefunden (vgl. Kap. 4.3.3, S. 163).

Bei der Reaktion des erzeugten Radikals mit molekularem Sauerstoff wurde ein Geschwindigkeitskoeffizient von $\mathrm{k}(298 \mathrm{~K})=2,4 \cdot 10^{10} \mathrm{~cm}^{3} \mathrm{~mol}^{-1} \mathrm{~s}^{-1}$ gefunden, der erheblich kleiner als derjenige der analogen Reaktion mit $\mathrm{CH}_{3} \mathrm{CHOH}$ ist, welcher mit $\mathrm{k}=1,15 \cdot 10^{13} \mathrm{~cm}^{3} \mathrm{~mol}^{-1} \mathrm{~s}^{-1}$ angegeben wird $^{128}$. Der gleiche Befund hatte sich auch beim Übergang des Dimethylethers hin zu fluorierten Ethern ergeben und scheint auf die elektronegativen Fluor-Substituenten zurückzuführen zu sein. Der negative Wert für den Parameter $n=(-3,75 \pm 0,75)$ zur Beschreibung der Temperaturabhängigkeit ist für eine Assoziationsreaktion mit $\mathrm{O}_{2}$ typisch und war daher auch so zu erwarten (vgl. die Diskussion am Ende von Kap. 4.2.2).

Fraglich bleibt, ob die Reaktion des Radikals mit $\mathrm{O}_{2}$ bimolekular oder aus den im Zusammenhang mit Gleichung (28) (S. 124) erörterten Gründen termolekular ist. Für die analoge Reaktion des $\mathrm{CH}_{3} \mathrm{CHOH}$-Radikals ist sie bimolekular angegeben ${ }^{128}$. Weil in dieser Arbeit keine Untersuchungen zur Druckabhängigkeit vorgenommen wurden, wird das Ergebnis vorsichtshalber für die termolekulare Reaktion formuliert. Die bimolekularen Werte sind jedoch in Tabelle 27 (S. 149) aufgeführt.

127 Khatoon, T.; Edelbuttel-Einhaus, J.; Hoyermann, K.; Wagner, H.Gg. (1989).

128 Atkinson, R.; et al. (1997). 


\subsubsection{Das 2,2-Difluorethanol $\left(\mathrm{CHF}_{2} \mathrm{CH}_{2} \mathrm{OH}\right)$}

\subsubsection{Produkte der radikalerzeugenden Reaktion mit Fluor-Atomen}

Im Prinzip sind für die Reaktion des 2,2,-Difluorethanols mit Fluor-Atomen die gleichen Produkte möglich wie für 2,2,2-Trifluorethanol (siehe dazu auch Kap. 4.3.1, S. 132). Diese sind in Abbildung 133 dargestellt.

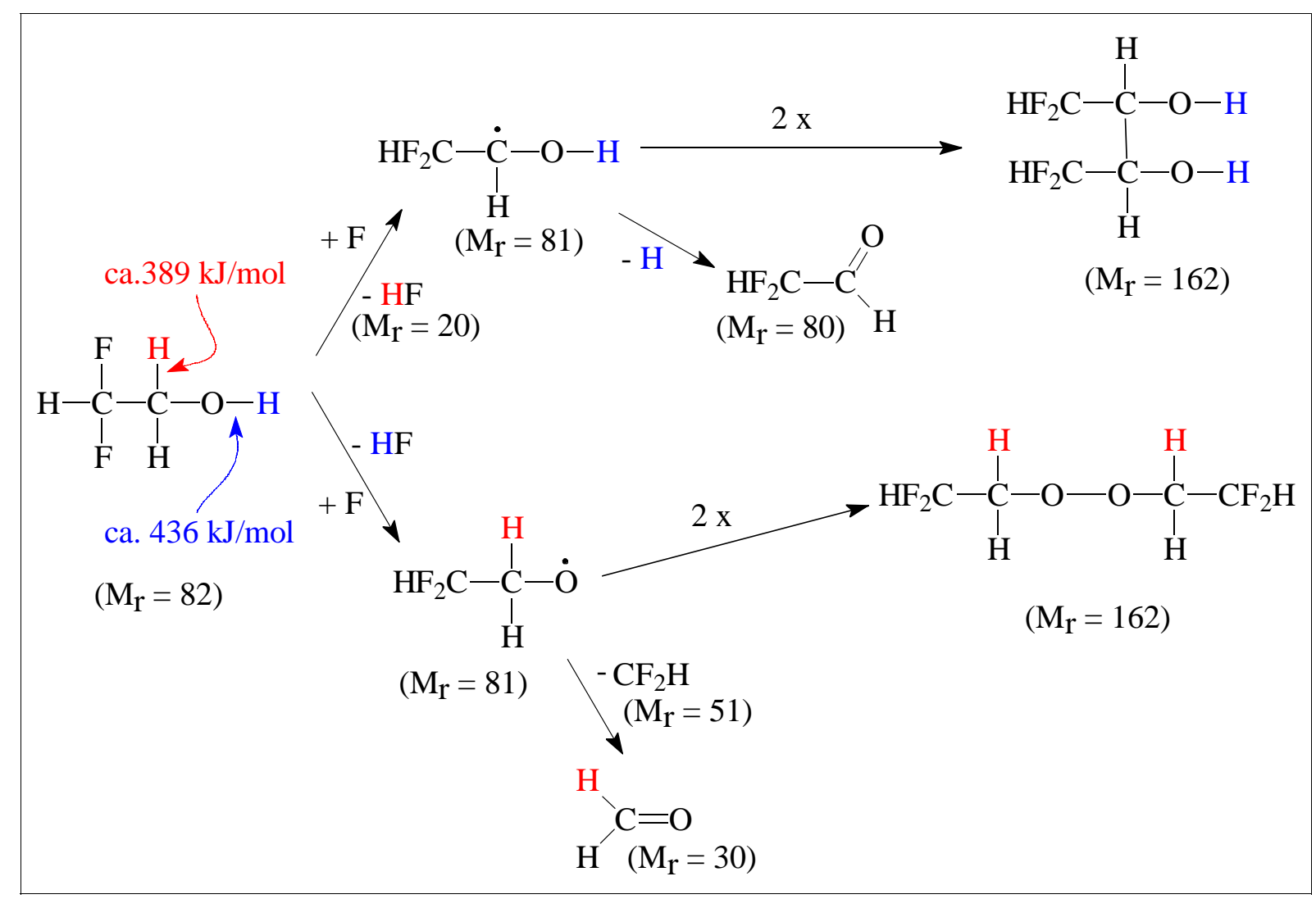

Abbildung 133: Reaktionskanäle für $\mathrm{CHF} 2 \mathrm{CH} 2 \mathrm{OH}+\mathrm{F}$

In EI-Übersichtsmassenspektren, die mit der in Kapitel 3.1.1 (S. 16) beschriebenen Apparatur registriert wurden, zeigte sich bei Zugabe von Fluor-Atomen eine Abnahme auf dem MolekülPeak m/z 82 sowie u.a. eine Zunahme auf m/z 81, d.h. dem Molekül-Peak des Radikals. Die Durchführung der Messungen ist in Kapitel 3.5.1 (S. 50) beschrieben.

Es wurden anschließend gezielt in Einzelzählung (6 sek Zählzeit, IP = $20 \mathrm{eV}$ ) die m/z-Werte überprüft, die im Übersichtsspektrum größere Veränderungen zeigten. Die Bedingungen waren dazu so gewählt, daß sich bei Zugabe der Fluor-Atome auf dem Molekül-Peak m/z 82 eine Abnahme um $67 \%$ ergab. Die Ergebnisse sowie die genauen Reaktionsbedingungen sind unten in Abbildung $134 \mathrm{zu}$ sehen.

Durch die in Abbildung 134 gemessenen Zunahmen auf m/z 30; 51; 80; 81 und 162 kommen praktisch alle in Abbildung 133 vorgeschlagenen Reaktionskanäle in Betracht. 


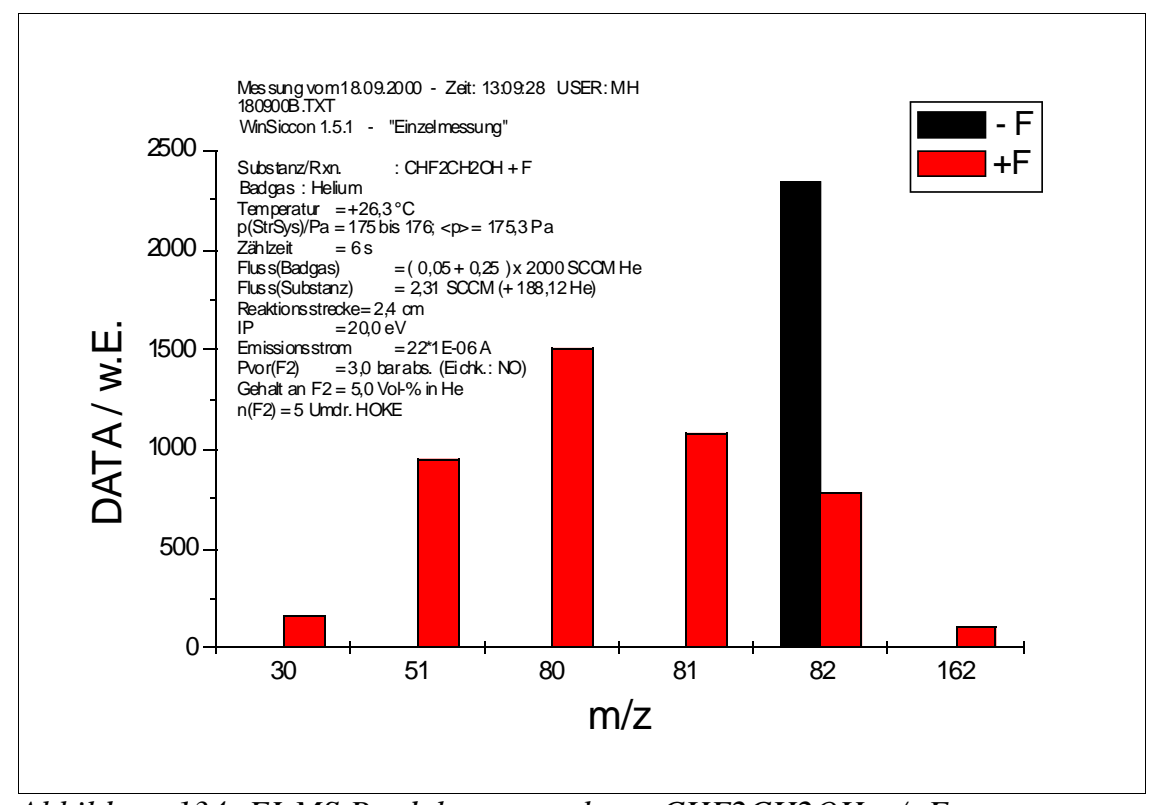

Abbildung 134: EI-MS Produktuntersuchung CHF2CH2OH +/- F

Die Zunahme auf $\mathrm{m} / \mathrm{z} \quad 80$ könnte einerseits durch 2,2-Difluorethanal $\left(\mathrm{CHF}_{2} \mathrm{CHO}\right)$ hervorgerufen werden, welches nach H-Abspaltung aus einem Radikal gebildet werden kann. Andererseits könnte aber auch ein Fragment von einem (oder beiden) der gebildeten Radikale dafür verantwortlich sein. Aus diesem Grund wurden die Intensitäten auf m/z 80 und 81 in Abhängigkeit der EI-Elektronenenergie (IP) aufgenommen (Abb. 135).

Man erkennt, daß bei verminderter EIElektronenenergie das Signal auf $\mathrm{m} / \mathrm{z}$ 80 deutlich schneller sinkt als dasjenige auf $\mathrm{m} / \mathrm{z} 81$ und ersteres bei einer um etwa $1,5 \mathrm{eV}$ höheren EI-Elektronenenergie als letzteres völlig verschwindet. Häufig haben Radikale eine geringere Ionisierungsenergie als die Moleküle, aus denen sie hervorgingen. Aus diesen Gründen ist anzunehmen,

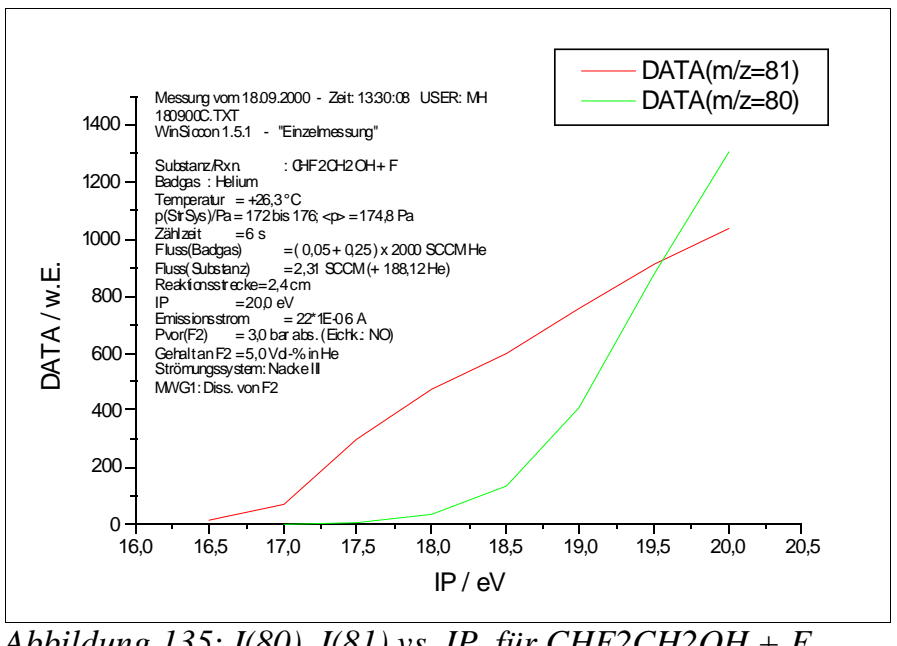
Abbildung 135: I(80), I(81) vs. IP für CHF2CH2OH + F daß m/z 80 von einem Radikal-Fragment und nicht einem Produkt herrührt.

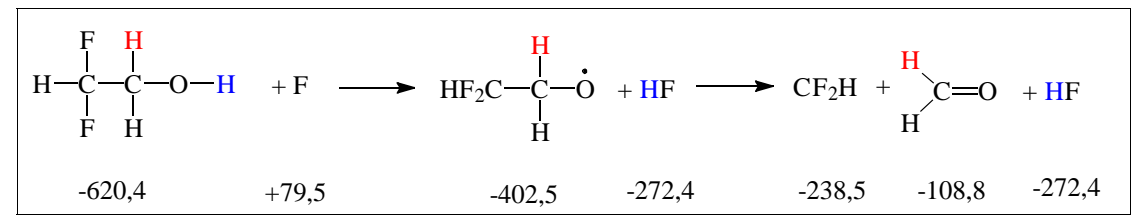


In Abbildung 136 ist nochmals der Kanal für die Bildung des Oxy-Radikals mit der Weiterreaktion zu Formaldehyd aufgezeigt, wobei unter jeder Verbindung die Standardbildungsenthalpie $^{129}$ für $298 \mathrm{~K}$ in der Einheit $\mathrm{kJ} / \mathrm{mol}$ vermerkt ist. Mit einer Standardreaktionsenthalpie für die gesamte Reaktion von $-78,8 \mathrm{~kJ} / \mathrm{mol}$ ist diese stark exotherm. Es ist also sehr gut möglich, daß das Oxy-Radikal $\left(\mathrm{CHF}_{2} \mathrm{CH}_{2} \mathrm{O} \cdot\right)$ zwar gebildet wird, aber sehr viel Energie in sich birgt, um schnell weiter zu den nachgewiesenen Produkten $\left(\mathrm{HCHO}\right.$ und $\left.\mathrm{CHF}_{2}\right)$ zu zerfallen. In diesem Fall könnte dieses Radikal kaum für weitere Reaktionen mit einem weiteren Reaktanten (z.B. molekularem Sauerstoff) verfügbar sein. Genauere Angaben (insbesondere über den Geschwindigkeitskoeffizienten für diesen Zerfallskanal) lagen jedoch nicht vor.

\subsubsection{Kinetik der radikalerzeugenden Reaktion mit Fluor-Atomen}

Die Messungen wurden in der in Kap. 3.1.1 (S. 16) beschriebenen Apparatur unter Einsatz des Strömungsreaktors aus Abbildung 5 (S. 17) durchgeführt. Die Ausführung und Auswertung nach der Relativmethode ist in Kap. 3.5.1 (S. 50) beschrieben.

Methan $\left(\mathrm{M}_{\mathrm{r}}=16\right)$ diente als Referenzsubstanz. Der Umsatz des Alkohols $\mathrm{CHF}_{2} \mathrm{CH}_{2} \mathrm{OH}$ konnte anhand der Intensitätsänderung seines Molekül-Peaks m/z 82, derjenige von Methan anhand des Molekül-Peaks (m/z 16) bestimmt werden.

$\vartheta=+26^{\circ} \mathrm{C} ; \phi_{\mathrm{He}}=600 \mathrm{SCCM} ; \phi_{\mathrm{CHF} 2 \mathrm{CH} 2 \mathrm{OH}}=2,3 \mathrm{SCCM} ; \phi_{\mathrm{CH} 4}=2,3 \mathrm{SCCM} ; \mathrm{p}_{(\mathrm{StrR})}=115 \mathrm{~Pa}$; $\mathrm{s}=2,4 \mathrm{~cm} ; \mathrm{IP}=29,5 \mathrm{eV}$; Zählzeit $=6 \mathrm{~s}$

\begin{tabular}{|r|r|r|r|r|r|r|r|}
\hline $\begin{array}{c}\text { I(82) } \\
\text {-F / w.E. }\end{array}$ & $\begin{array}{c}\Delta \mathbf{I}(\mathbf{8 2}) \\
\text {-F / w.E. }\end{array}$ & $\begin{array}{c}\mathbf{I}(\mathbf{8 2}) \\
\text { +F / w.E. }\end{array}$ & $\begin{array}{c}\Delta \mathbf{I}(\mathbf{8 2}) \\
\text { +F / w.E. }\end{array}$ & $\begin{array}{c}\mathbf{I}(\mathbf{1 6}) \\
\text {-F / w.E. }\end{array}$ & $\begin{array}{c}\Delta \mathbf{I}(\mathbf{1 6}) \\
\text {-F / w.E. }\end{array}$ & $\begin{array}{c}\mathbf{I}(\mathbf{1 6}) \\
\text { +F / w.E. }\end{array}$ & $\begin{array}{c}\Delta \mathbf{I}(\mathbf{1 6}) \\
\text { +F / w.E. }\end{array}$ \\
\hline 16680 & 107,4 & 11410 & 109,3 & 14560 & 99,1 & 11200 & 118 \\
\hline 16050 & 109,7 & 11810 & 109,3 & 15930 & 212,7 & 12730 & 230,7 \\
\hline 16000 & 134,2 & 12720 & 132,5 & 23930 & 182,5 & 20190 & 157,3 \\
\hline 16350 & 89,75 & 13640 & 104,7 & 25560 & 117,5 & 22110 & 201,6 \\
\hline 16430 & 165,7 & 14090 & 109,3 & 29400 & 118 & 25810 & 163,5 \\
\hline 16480 & 107,4 & 15460 & 122,2 & 30000 & 99,73 & 28060 & 133,3 \\
\hline 16830 & 132,3 & 15700 & 134,3 & 30980 & 177 & 29200 & 141 \\
\hline
\end{tabular}

Tabelle 29: Meßwerte Kinetik $\mathrm{CHF} 2 \mathrm{CH} 2 \mathrm{OH}+\mathrm{F}$ rel. zu CH4; $299 \mathrm{~K}$

129 Werte entnommen oder abgeschätzt mit: NIST Structures and Properties (1994). 
Mit den Meßwerten aus Tabelle 29 kann eine Auftragung gemäß Gleichung (10) (S. 10) angefertigt werden (Abbildung 137).

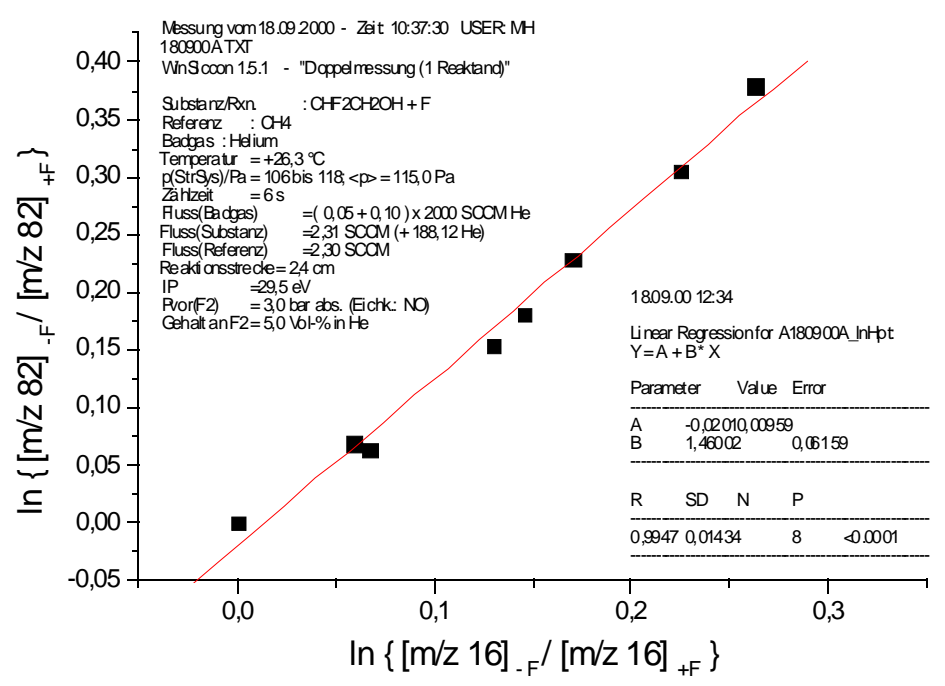

Abbildung 137: Kinetik CHF2CH2OH + F rel. zu CH4; 299 K

Aus der Steigung der Auftragung in Abbildung 137 erhält man den relativen

Geschwindigkeitskoeffizienten $\mathrm{k}_{\mathrm{rel}}=(1,46 \pm 0,06)$.

Mit dem bekannten Geschwindigkeitskoeffizienten der Referenzreaktion ${ }^{130} \mathrm{k}_{\text {ref }}$ läßt sich gemäß Gleichung (10) (S. 10) der absolute Geschwindigkeitskoeffizient k berechnen.

$\mathrm{k}_{\mathrm{ref}}(299 \mathrm{~K})=4,74 \cdot 10^{13} \mathrm{~cm}^{3} /(\mathrm{mol} \cdot \mathrm{s})$

Man erhält als Ergebnis:

$\mathrm{k}(299 \mathrm{~K})=(6,92 \pm 0,28) \cdot 10^{13} \mathrm{~cm}^{3} /(\mathrm{mol} \cdot \mathrm{s})$

für: $\mathrm{CHF}_{2} \mathrm{CH}_{2} \mathrm{OH}+\mathrm{F} \rightarrow$ Produkte 


\subsubsection{Produkte der Reaktion des erzeugten Radikals mit molekularem Sauerstoff}

Um einen Überblick über die Änderungen im Massenspektrum zu erhalten, sind mit der in Kapitel 3.1.1 (S. 16) beschriebenen Apparatur unmittelbar nacheinander EI-Übersichtsspektren unter Zugabe und Abwesenheit von molekularem Sauerstoff (Fluor-Atome wurden stets gleichermaßen zugegeben) aufgenommen worden.

Es wurden anschließend gezielt in Einzelzählung (10 sek Zählzeit, IP = 20,5 eV) die m/zWerte überprüft, die im Übersichtsspektrum größere Veränderungen zeigten. Die Durchführung der Messungen ist in Kapitel 3.5.1 (S. 50) beschrieben. Die Reaktionsbedingungen und Ergebnisse sind in Abbildung 138 dargestellt.

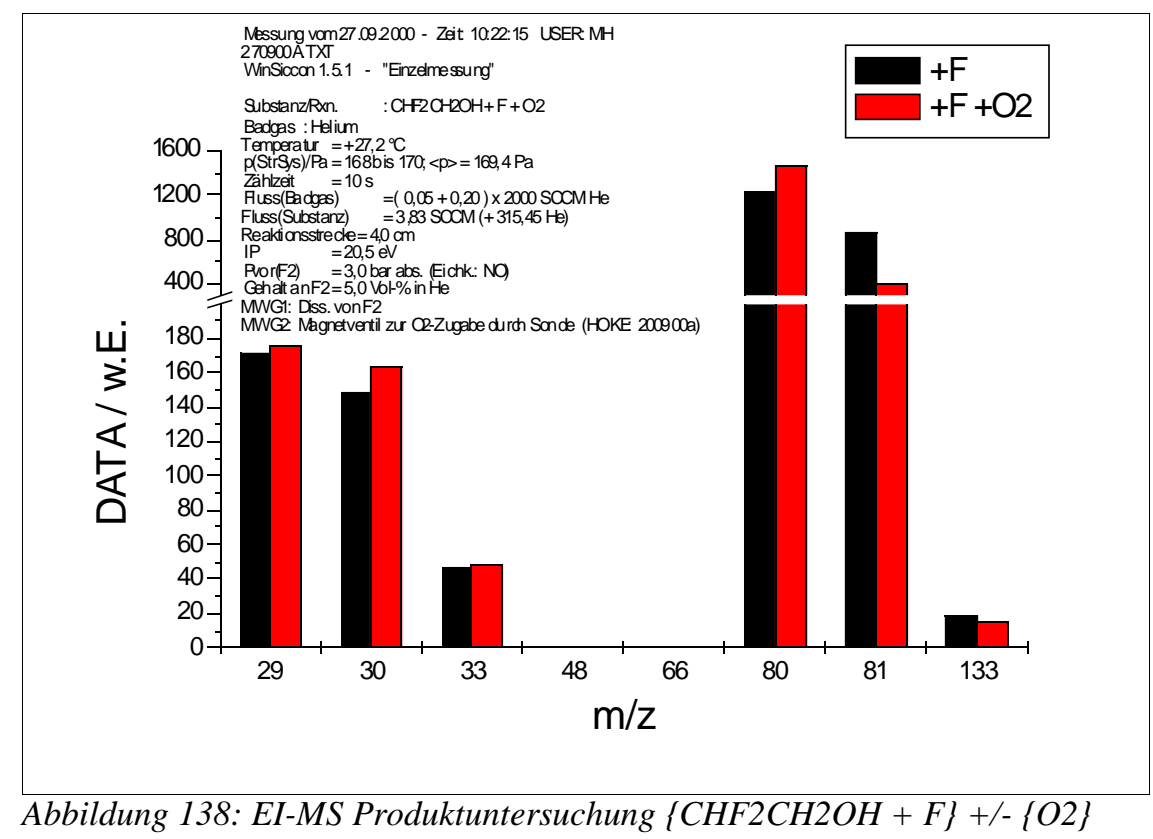

Auf m/z 81 (Molekül-Peak des Radikals) ergibt sich bei Zugabe von molekularem Sauerstoff eine Abnahme in Höhe von 54 \%, während die Intensität auf m/z 80 von 1230 auf 1450 w.E. ansteigt.

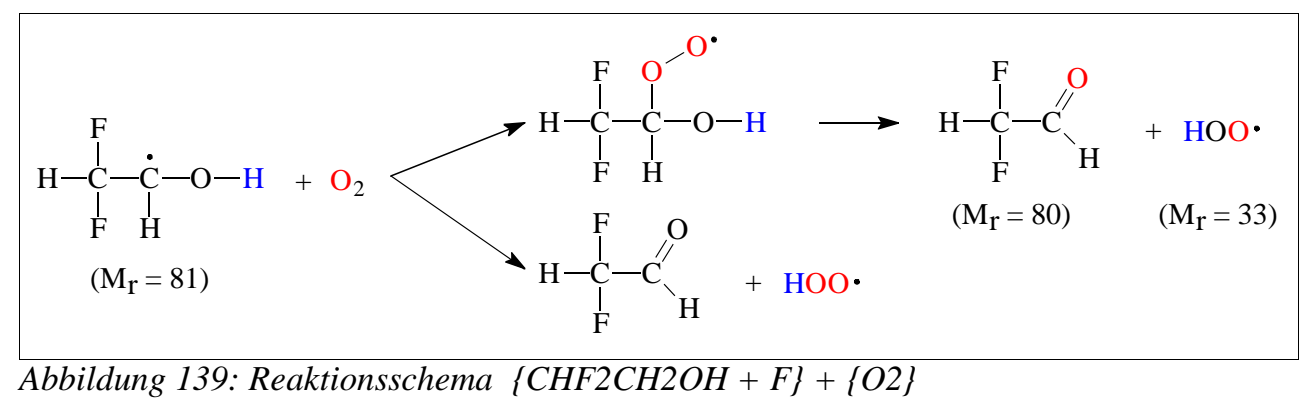

Oben bei der Untersuchung der Produkte aus der Reaktion des Alkohols mit Fluor-Atomen wurde herausgearbeitet, daß m/z 80 bei jener Reaktion wahrscheinlich ein Fragment des Radikals ist. Folgt man dem, dann würde umsatzkorrigiert (vgl. dazu Kap. 3.5.1, S. 50) eine 
Intensität des Radikal-Fragments auf m/z 80 in Höhe von $(1-0,54) \cdot 1230$ w.E. $=566$ w.E. resultieren, so daß der wahre Anstieg durch die Zugabe von $\mathrm{O}_{2}$ auf m/z 80 sogar (1450-566) w.E. $=884$ w.E. beträgt. In Verbindung mit dem hier deutlich zu erkennenden Anstieg auf $\mathrm{m} / \mathrm{z}$ 33 dürfte die in Abbildung 139 gezeigte Reaktion der Hauptreaktionskanal sein, der zu 2,2Difluorethanal $\left(\mathrm{CHF}_{2} \mathrm{CHO}\right)$ führt. Die Zunahmen auf $\mathrm{m} / \mathrm{z} 29$ und 30 sprechen ferner für die Bildung von Methanal (Formaldehyd, HCHO) als Nebenprodukt.

\subsubsection{Kinetik der Reaktion des erzeugten Radikals mit molekularem Sauerstoff}

Die Messungen wurden in der in Kap. 3.1.1 (S. 16) beschriebenen Apparatur unter Einsatz des Strömungsreaktors aus Abbildung 5 (S. 17) durchgeführt.

Das Chlormethyl-Radikal diente als Referenzsubstanz. Der Umsatz des Alkohol-Radikals konnte anhand der Intensitätsänderung seines Molekül-Peaks $\mathrm{m} / \mathrm{z} 81$, derjenige des Chlormethyl-Radikals ebenfalls anhand seines Molekül-Peaks (m/z 49) bestimmt werden.

Die Messungen wurden umsatzkorrigiert nach der Relativmethode durchgeführt, wobei zur Messung des Umsatzes des Alkohols bei Zugabe der Fluor-Atome die Intensitätsänderung auf $\mathrm{m} / \mathrm{z} 82$, und für den des Chlormethans diejenige auf m/z 50 diente; die genaue Ausführung und Auswertung ist in Kap. 3.5.1 (S. 50) beschrieben.

$\vartheta=27{ }^{\circ} \mathrm{C} ; \phi_{\mathrm{He}}=950 \mathrm{SCCM} ; \phi_{\mathrm{CHF} 2 \mathrm{CH} 2 \mathrm{OH}}=3,8 \mathrm{SCCM} ; \phi_{\mathrm{CH} 3 \mathrm{Cl}}=7,6 \mathrm{SCCM} ; \mathrm{p}_{(\mathrm{StrR})}=182 \mathrm{~Pa} ;$ $\mathrm{s}=4,0 \mathrm{~cm} ; \mathrm{IP}=20,5 \mathrm{eV} ;$ Zählzeit $=10 \mathrm{~s}$

\begin{tabular}{|c|c|c|c|c|c|c|c|}
\hline $\begin{array}{c}\mathbf{I}(\mathbf{8 1}) \\
-\mathrm{O}_{2} / \text { w.E. }\end{array}$ & $\begin{array}{c}\Delta \mathbf{I}(\mathbf{8 1}) \\
-\mathbf{O}_{2} / \mathbf{w . E .}\end{array}$ & $\begin{array}{c}\mathrm{I}(81) \\
+\mathrm{O}_{2} / \text { w.E. }\end{array}$ & $\begin{aligned} & \Delta \mathrm{I}(\mathbf{8 1}) \\
+ & \mathrm{O}_{2} / \text { w.E. }\end{aligned}$ & $\begin{array}{c}\mathbf{I}(49) \\
-\mathbf{O}_{2} / \text { w.E. }\end{array}$ & $\begin{array}{c}\Delta \mathbf{I}(49) \\
-\mathbf{O}_{2} / \text { w.E. }\end{array}$ & $\begin{array}{c}I(49) \\
+O_{2} / \text { w.E. }\end{array}$ & $\begin{array}{c}\Delta \mathbf{I}(49) \\
+\mathbf{O}_{2} / \text { w.E. }\end{array}$ \\
\hline 513,4 & 13,06 & 380,3 & 10,3 & 1929 & 32,86 & 1830 & 14,48 \\
\hline 439,2 & 9,63 & 358,1 & 20,94 & 1988 & 41,83 & 1907 & 27,4 \\
\hline 430,3 & 19,38 & 311,1 & 13,97 & 1972 & 44,34 & 1877 & 41,47 \\
\hline 473,5 & 13,95 & 367 & 12,7 & 2046 & 37,55 & 1930 & 51,43 \\
\hline 473,3 & 18,7 & 341,8 & 14,29 & 2140 & 31,9 & 2000 & 31,73 \\
\hline 482,9 & 19,64 & 357,2 & 14,84 & 1622 & 44,74 & 1519 & 26,35 \\
\hline 511,9 & 14,3 & 366,3 & 13,28 & 1681 & 32,73 & 1570 & 48,39 \\
\hline 515,1 & 13,16 & 493,7 & 14,42 & 2106 & 36,15 & 2105 & 24,69 \\
\hline 420,2 & 14 & 386 & 16,59 & 2353 & 258,3 & 2291 & 35,85 \\
\hline
\end{tabular}

Tabelle 30: Meßwerte Kinetik $\{\mathrm{CHF} 2 \mathrm{CH} 2 \mathrm{OH}+\mathrm{F}\}+\{\mathrm{O} 2\}$ rel. zu $\mathrm{CH} 3 \mathrm{Cl} ; 300 \mathrm{~K}$ 
Mit den Meßwerten aus Tabelle 30 kann eine Auftragung gemäß Gleichung (10) (S. 10) angefertigt werden (Abb. 140).

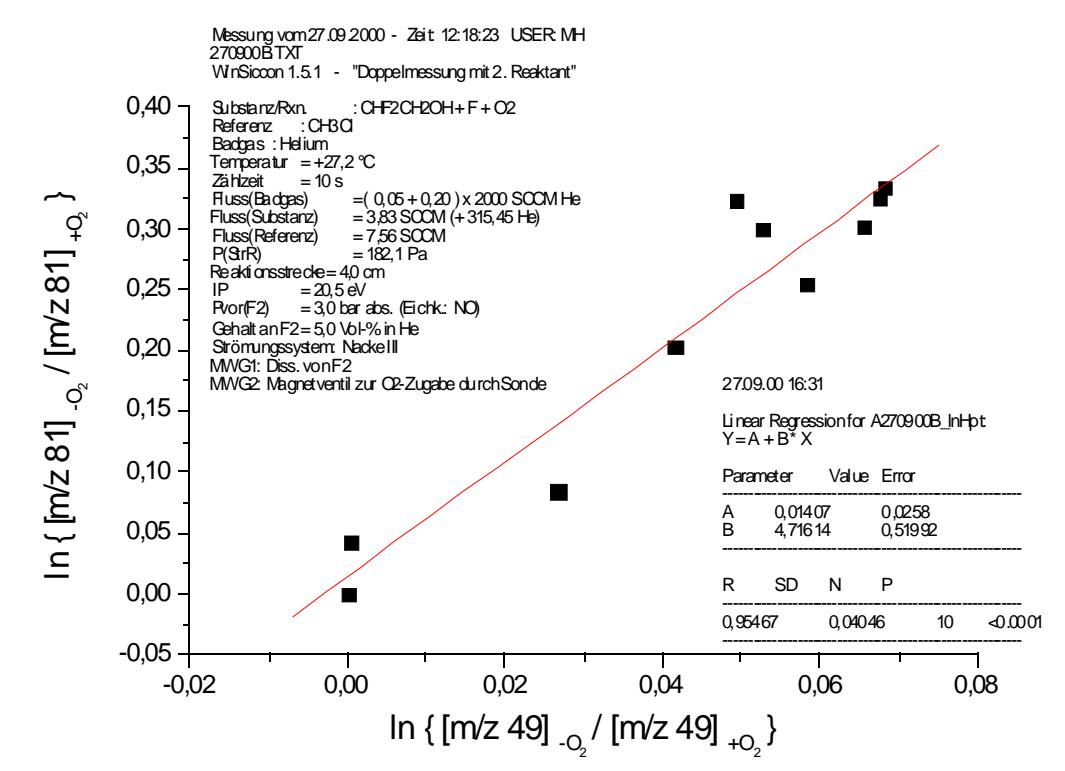

Abbildung 140: Kinetik $\{\mathrm{CHF} 2 \mathrm{CH} 2 \mathrm{OH}+\mathrm{F}\}+\{\mathrm{O} 2\} \mathrm{rel}$. zu CH3Cl; $300 \mathrm{~K}$

Aus der Steigung in Abbildung 141 läßt sich der relative Geschwindigkeitskoeffizient bei $\mathrm{T}=298 \mathrm{~K}$ ermitteln: $\mathrm{k}_{\mathrm{rel}}=(4,72 \pm 0,52)$.

Mit dem bekannten Geschwindigkeitskoeffizienten der Referenzreaktion ${ }^{131} \mathrm{k}_{\text {ref }}$ läßt sich der absolute Geschwindigkeitskoeffizient k berechnen.

$\mathrm{k}_{\mathrm{ref}}(\mathrm{T})=(6,89 \pm 0,34) \cdot 10^{17} \cdot(\mathrm{T} / 298 \mathrm{~K})^{(-3,20 \pm 0,19)} \cdot[\mathrm{He}] \mathrm{cm}^{6} /\left(\mathrm{mol}^{2} \cdot \mathrm{s}\right)$

Man erhält als Ergebnis (termolekular):

$$
\begin{aligned}
& \mathbf{k}(300 \mathrm{~K})=(\mathbf{3 , 1 8} \pm \mathbf{0 , 3 9}) \cdot \mathbf{1 0}^{18} \cdot[\mathrm{He}] \mathbf{c m}^{6} /\left(\mathbf{m o l}^{2} \cdot \mathbf{s}\right) \\
& \text { für: }\left\{\text { Radikal aus der Reaktion } \mathrm{CHF}_{2} \mathrm{CH}_{2} \mathrm{OH}+\mathrm{F}\right\}+\left\{\mathrm{O}_{2}\right\} \rightarrow \text { Produkte }
\end{aligned}
$$

Mit $[\mathrm{He}]=7,3 \cdot 10^{-08} \mathrm{~mol} / \mathrm{cm}^{3}$ berechnet man daraus den bimolekularen Koeffizienten:

$$
\mathbf{k}(300 \mathrm{~K})=2,3 \cdot 10^{11} \mathrm{~cm}^{3} /(\mathrm{mol} \cdot \mathrm{s})
$$




\subsubsection{Diskussion der Ergebnisse}

Der ermittelte Geschwindigkeitskoeffizient für die Reaktion des Alkohols $\mathrm{CHF}_{2} \mathrm{CH}_{2} \mathrm{OH}$ mit Fluor-Atomen ist mit $\mathrm{k}(299 \mathrm{~K})=6,92 \cdot 10^{13} \mathrm{~cm}^{3} \mathrm{~mol}^{-1} \mathrm{~s}^{-1}$ ähnlich hoch wie derjenige für die analoge Reaktion des Ethanols, welcher mit $\mathrm{k}=7,69 \cdot 10^{13} \mathrm{~cm}^{3} \mathrm{~mol}^{-1} \mathrm{~s}^{-1}$ angegeben wird ${ }^{132}$. Er ist etwas höher als für die Reaktion des strukturell sehr ähnlichen Alkohols $\mathrm{CF}_{3} \mathrm{CH}_{2} \mathrm{OH}$, für den $\mathrm{k}(298 \mathrm{~K})=6,27 \cdot 10^{13} \mathrm{~cm}^{3} \mathrm{~mol}^{-1} \mathrm{~s}^{-1}$ bestimmt worden war, was als Indiz für die Herabsetzung der Geschwindigkeitskoeffizienten bei zunehmender Zahl Fluor-Substituenten angesehen werden kann.

Bei der Reaktion des erzeugten Radikals mit molekularem Sauerstoff wurde ein Geschwindigkeitskoeffizient von $\mathrm{k}(298 \mathrm{~K})=2,3 \cdot 10^{11} \mathrm{~cm}^{3} \mathrm{~mol}^{-1} \mathrm{~s}^{-1}$ gefunden, der deutlich kleiner als derjenige der analogen Reaktion mit $\mathrm{CH}_{3} \mathrm{CHOH}$ ist, welcher mit $\mathrm{k}=1,15 \cdot 10^{13} \mathrm{~cm}^{3} \mathrm{~mol}^{-1} \mathrm{~s}^{-1}$ angegeben wird ${ }^{133}$. Allerdings ist er größer als derjenige für die analoge Reaktion mit $\mathrm{CF}_{3} \mathrm{CH}_{2} \mathrm{OH}$, der mit $\mathrm{k}(298 \mathrm{~K})=2,4 \cdot 10^{10} \mathrm{~cm}^{3} \mathrm{~mol}^{-1} \mathrm{~s}^{-1}$ ermittelt worden war (vgl. Kap. 4.3.1, S. 132). Der gleiche Befund hatte sich auch beim Übergang des Dimethylethers hin zu fluorierten Ethern ergeben und scheint auf die elektronegativen Fluor-Substituenten zurückzuführen zu sein.

Bei den Produktuntersuchungen konnten im wesentlichen die zum $\mathrm{CF}_{3} \mathrm{CH}_{2} \mathrm{OH}$ analogen Produkte identifiziert werden, was bei einer strukturell so ähnlichen Verbindung auch plausibel ist.

Eine weitergehende Diskussion soll erst nach Untersuchung der übrigen fluorierten Alkohole am Ende von Kapitel 4.3.4 erfolgen.

132 Khatoon, T.; Edelbuttel-Einhaus, J.; Hoyermann, K.; Wagner, H.Gg. (1989).

133 Atkinson, R.; et al. (1997). 


\subsubsection{Das 2-Fluorethanol $\left(\mathrm{CH}_{2} \mathrm{FCH} \mathrm{CH}_{2} \mathrm{OH}\right.$}

\subsubsection{Produkte der radikalerzeugenden Reaktion mit Fluor-Atomen}

In EI-Übersichtsmassenspektren, die mit der in Kapitel 3.1.1 (S. 16) beschriebenen Apparatur registriert wurden, zeigte sich bei Zugabe von Fluor-Atomen eine Abnahme auf dem MolekülPeak m/z 64. Die Durchführung der Messungen ist in Kapitel 3.5.1 (S. 50) beschrieben.

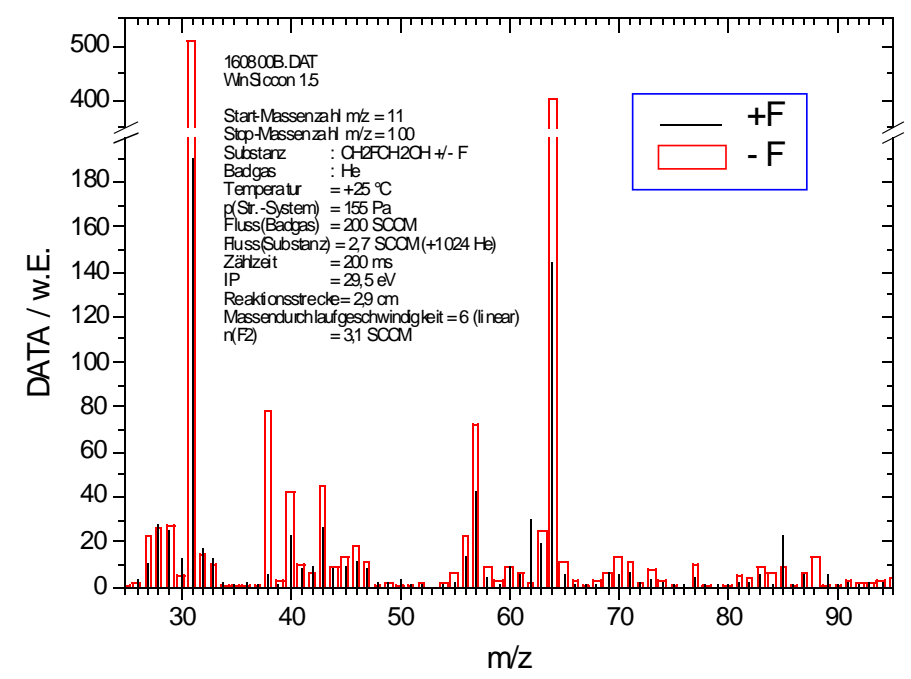

Abbildung 141: EI-MS-Übersichtsspektrum $\mathrm{CH} 2 \mathrm{FCH} 2 \mathrm{OH}+/-\mathrm{F}$

Die Abnahme auf m/z 64 (Molekül-Peak von $\mathrm{CH}_{2} \mathrm{FCH}_{2} \mathrm{OH}$ ) bei Zugabe von Fluor-Atomen beträgt $64 \%$, die auf dem Fragment-Peak des Edukts m/z 63 aber lediglich $25 \%$. Nach Subtraktion dieses Fragmentanteils im Rahmen einer Umsatzkorrektur (vgl. dazu Kap. 3.5.1, S. 50) erhält man daher auf $\mathrm{m} / \mathrm{z} 63$ eine Zunahme. Somit ist die Bildung eines Radikals $\left(\mathrm{M}_{\mathrm{r}}=\right.$ 63) nachgewiesen, welches durch Abstraktion eines $\mathrm{H}$-Atoms vom $\mathrm{CH}_{2} \mathrm{FCH}_{2} \mathrm{OH}$ entsteht.

Bzgl. der Zunahmen auf m/z 30 und 62 kann auf die Ausführungen zu den strukturell ähnlichen Alkoholen $\mathrm{CF}_{3} \mathrm{CH}_{2} \mathrm{OH}$ sowie $\mathrm{CHF}_{2} \mathrm{CH}_{2} \mathrm{OH}$ verwiesen werden, die hierfür analog gelten dürften (Vgl. Kap. 4.3.1 f.). Es handelt sich damit bei diesen Produkten wahrscheinlich um Methanal ( $\mathrm{HCHO})$ und 2-Fluorethanal $\left(\mathrm{CH}_{2} \mathrm{FCHO}\right)$.

\subsubsection{Kinetik der radikalerzeugenden Reaktion mit Fluor-Atomen}

Die Messungen wurden in der in Kap. 3.1.1 (S. 16) beschriebenen Apparatur unter Einsatz des Strömungsreaktors aus Abbildung 5 (S. 17) durchgeführt. Die Ausführung und Auswertung nach der Relativmethode ist in Kap. 3.5.1 (S. 50) beschrieben.

Methan $\left(\mathrm{M}_{\mathrm{r}}=16\right)$ diente als Referenzsubstanz. Der Umsatz des Alkohols $\mathrm{CH}_{2} \mathrm{FCH}_{2} \mathrm{OH}$ konnte 
anhand der Intensitätsänderung seines Molekül-Peaks m/z 64, derjenige von Methan ebenfalls anhand seines Molekül-Peaks (m/z 16) bestimmt werden.

$\vartheta=+5{ }^{\circ} \mathrm{C} ; \phi_{\mathrm{He}}=1250 \mathrm{SCCM} ; \phi_{\mathrm{CHF} 2 \mathrm{CH} 2 \mathrm{OH}}=2,4 \mathrm{SCCM} ; \phi_{\mathrm{CH} 4}=2,4 \mathrm{SCCM} ; \mathrm{p}_{(\mathrm{StrR})}=175 \mathrm{~Pa} ;$ $\mathrm{s}=2,4 \mathrm{~cm} ; \mathrm{IP}=29,5 \mathrm{eV} ;$ Zählzeit $=6 \mathrm{~s}$

\begin{tabular}{|r|r|r|r|r|r|r|r|}
\hline \multicolumn{1}{|c|}{$\mathbf{I}(\mathbf{6 4})$} & \multicolumn{1}{c|}{$\begin{array}{c}\Delta \mathbf{I}(\mathbf{6 4}) \\
\text {-F / w.E. }\end{array}$} & $\begin{array}{c}\mathbf{I}(\mathbf{6 4}) \\
\text {-F / w.E. }\end{array}$ & $\begin{array}{c}\Delta \mathbf{I}(\mathbf{6 4}) \\
\text { +F / w.E. }\end{array}$ & $\begin{array}{c}\mathbf{I}(\mathbf{1 6}) \\
\text { +F / w.E. }\end{array}$ & $\begin{array}{c}\Delta \mathbf{I}(\mathbf{1 6}) \\
\text {-F / w.E. }\end{array}$ & $\begin{array}{c}\mathbf{I}(\mathbf{1 6}) \\
\text {-F / w.E. }\end{array}$ & $\begin{array}{c}\Delta \mathbf{I}(\mathbf{1 6}) \\
\text { +F.E. } / \text { w.E. }\end{array}$ \\
\hline 42450 & 248,3 & 32810 & 265,5 & 11730 & 97,2 & 10710 & 144,1 \\
\hline 37900 & 248,3 & 23150 & 345,5 & 10990 & 93,95 & 9264 & 93,33 \\
\hline 42490 & 163,5 & 34480 & 165,4 & 10930 & 86,37 & 10200 & 126,1 \\
\hline 42250 & 223 & 36350 & 141 & 11290 & 93,95 & 10640 & 76,44 \\
\hline 42640 & 244 & 34010 & 175,2 & 11280 & 59 & 10180 & 139,3 \\
\hline 42590 & 265,5 & 32180 & 177 & 9784 & 99,52 & 8686 & 120,9 \\
\hline 42350 & 189,2 & 28540 & 183,6 & 9500 & 79,78 & 8304 & 143,1 \\
\hline
\end{tabular}

Tabelle 31: Meßwerte Kinetik $\mathrm{CH} 2 \mathrm{FCH} 2 \mathrm{OH}+\mathrm{F} ; 278 \mathrm{~K}$

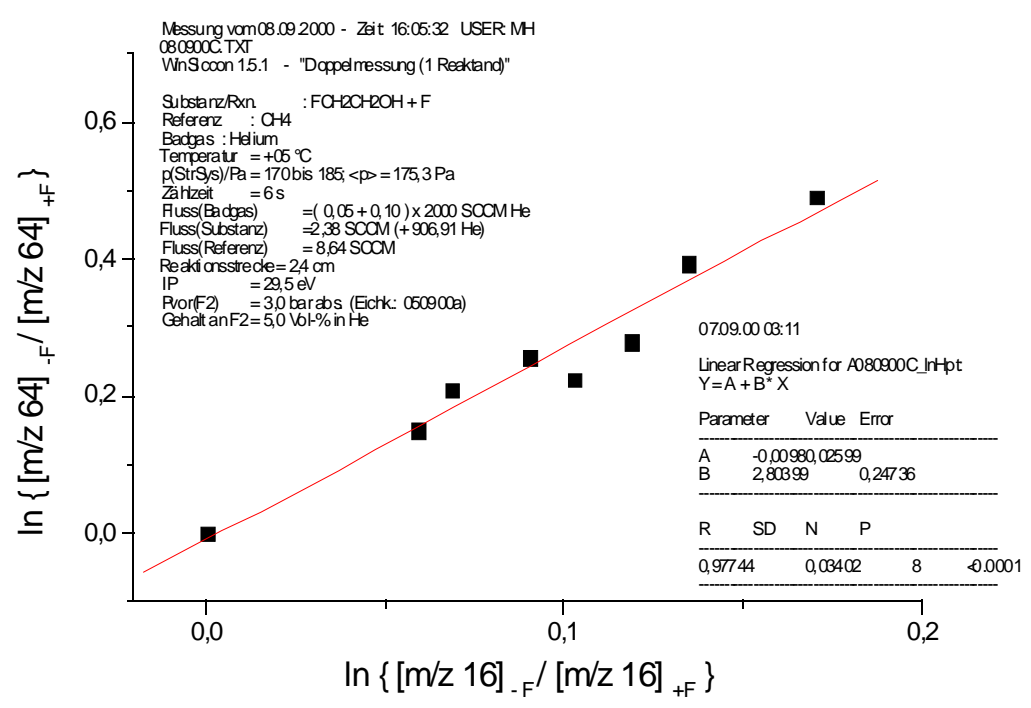

Abbildung 142: Kinetik $\mathrm{CH} 2 \mathrm{FCH} 2 \mathrm{OH}+\mathrm{F} ; 278 \mathrm{~K}$

Aus der Steigung der Auftragung in Abbildung 142 erhält man den relativen

Geschwindigkeitskoeffizienten $\mathrm{k}_{\mathrm{rel}}=(2,80 \pm 0,25)$. 
$\vartheta=+25^{\circ} \mathrm{C} ; \phi_{\mathrm{He}}=1150 \mathrm{SCCM} ; \phi_{\mathrm{CHF} 2 \mathrm{CH} 2 \mathrm{OH}}=2,4 \mathrm{SCCM} ; \phi_{\mathrm{CH} 4}=2,4 \mathrm{SCCM} ; \mathrm{p}_{(\mathrm{StrR})}=174 \mathrm{~Pa}$; $\mathrm{s}=2,4 \mathrm{~cm} ; \mathrm{IP}=29,5 \mathrm{eV}$; Zählzeit $=6 \mathrm{~s}$

\begin{tabular}{|c|c|c|c|c|c|c|c|}
\hline $\begin{array}{c}\mathbf{I}(64) \\
-\mathrm{F} / \mathrm{w.E} .\end{array}$ & $\begin{array}{c}\Delta \mathbf{I}(64) \\
-\mathrm{F} / \mathrm{w.E} .\end{array}$ & $\begin{array}{c}\mathrm{I}(64) \\
+\mathrm{F} / \mathrm{w.E.}\end{array}$ & $\begin{array}{c}\Delta \mathbf{I}(\mathbf{6 4}) \\
+\mathbf{F} / \mathbf{w . E .}\end{array}$ & $\begin{array}{c}\text { I(16) } \\
-F / \text { w.E. }\end{array}$ & $\begin{array}{c}\Delta \mathbf{I}(16) \\
-\mathrm{F} / \mathrm{w.E} .\end{array}$ & $\begin{array}{c}\mathrm{I}(16) \\
+\mathrm{F} / \mathrm{w} . \mathrm{E} .\end{array}$ & $\begin{array}{c}\Delta \mathrm{I}(\mathbf{1 6}) \\
+\mathrm{F} / \mathrm{w.E} .\end{array}$ \\
\hline 72150 & 406,3 & 56490 & 332,1 & 21480 & 106,9 & 19510 & 169,5 \\
\hline 42750 & 160,8 & 29790 & 224,9 & 24100 & 166,9 & 20810 & 144,1 \\
\hline 41690 & 220,5 & 31150 & 172,7 & 24350 & 218,5 & 21450 & 147,9 \\
\hline 41510 & 332,1 & 33610 & 137 & 24730 & 139,3 & 22850 & 209,2 \\
\hline 41400 & 99,73 & 35050 & 126,1 & 25040 & 256 & 23430 & 217,4 \\
\hline 41610 & 121,6 & 35830 & 131,9 & 25650 & 126,1 & 24530 & 139,3 \\
\hline 40400 & 218,5 & 35380 & 131,9 & 26430 & 171,3 & 24900 & 204,4 \\
\hline 40410 & 229,3 & 35830 & 277,6 & 25310 & 180,8 & 23890 & 163,5 \\
\hline
\end{tabular}

Tabelle 32: Meßwerte Kinetik $\mathrm{CH} 2 \mathrm{FCH} 2 \mathrm{OH}+\mathrm{F} ; 298 \mathrm{~K}$

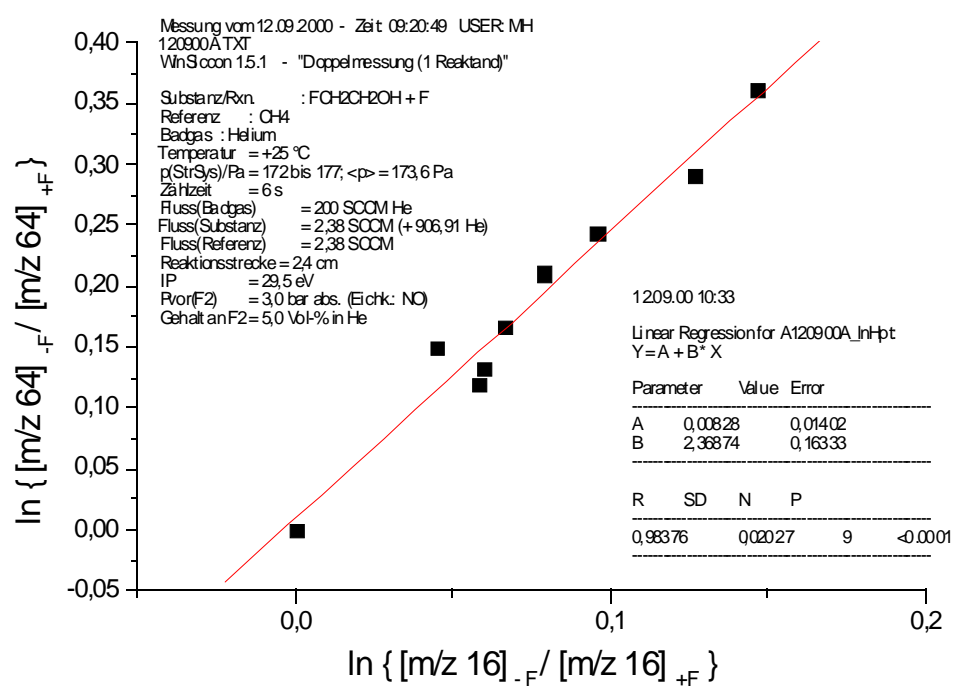

Abbildung 143: Meßwerte Kinetik CH2FCH2OH + F; $298 \mathrm{~K}$

Aus der Steigung der Auftragung in Abbildung 143 erhält man den relativen Geschwindigkeitskoeffizienten $\mathrm{k}_{\text {rel }}=(2,37 \pm 0,16)$. 
$\vartheta=+55^{\circ} \mathrm{C} ; \phi_{\mathrm{He}}=1150 \mathrm{SCCM} ; \phi_{\mathrm{CHF} 2 \mathrm{CH} 2 \mathrm{OH}}=2,4 \mathrm{SCCM} ; \phi_{\mathrm{CH} 4}=2,4 \mathrm{SCCM} ; \mathrm{p}_{(\mathrm{StrR})}=182 \mathrm{~Pa} ;$ $\mathrm{s}=2,4 \mathrm{~cm} ; \mathrm{IP}=29,5 \mathrm{eV}$; Zählzeit $=6 \mathrm{~s}$

\begin{tabular}{|r|r|r|r|r|r|r|r|}
\hline $\begin{array}{c}\text { I(64) } \\
\text {-F / w.E. }\end{array}$ & $\begin{array}{c}\Delta \mathbf{I}(\mathbf{6 4 )} \\
\text {-F / w.E. }\end{array}$ & $\begin{array}{c}\mathbf{I}(\mathbf{6 4}) \\
\text { +F / w.E. }\end{array}$ & $\begin{array}{c}\Delta \mathbf{I}(\mathbf{6 4}) \\
\text { +F / w.E. }\end{array}$ & $\begin{array}{c}\mathbf{I}(\mathbf{1 6}) \\
\text {-F / w.E. }\end{array}$ & $\begin{array}{c}\Delta \mathbf{I}(\mathbf{1 6}) \\
\text {-F / w.E. }\end{array}$ & $\begin{array}{c}\mathbf{I}(\mathbf{1 6}) \\
+\mathbf{+ F} / \mathbf{w . E} .\end{array}$ & $\begin{array}{c}\Delta \mathbf{I}(\mathbf{1 6}) \\
+\mathbf{F} / \mathbf{w . E} .\end{array}$ \\
\hline 36250 & 236 & 31280 & 217,4 & 11080 & 97,2 & 10290 & 104 \\
\hline 35580 & 124,2 & 27290 & 283,6 & 10270 & 174,1 & 9068 & 169 \\
\hline 30090 & 343,8 & 23780 & 212,7 & 12540 & 88,5 & 11350 & 99,73 \\
\hline 35390 & 233,6 & 30590 & 250,1 & 11710 & 421,8 & 10880 & 124,2 \\
\hline 35180 & 146,2 & 31350 & 126,1 & 11610 & 113,2 & 11040 & 117,5 \\
\hline 34100 & 446 & 32100 & 231,7 & 11830 & 59 & 11410 & 82,69 \\
\hline
\end{tabular}

Tabelle 33: Meßwerte Kinetik $\mathrm{CH} 2 \mathrm{FCH} 2 \mathrm{OH}+\mathrm{F} ; 328 \mathrm{~K}$

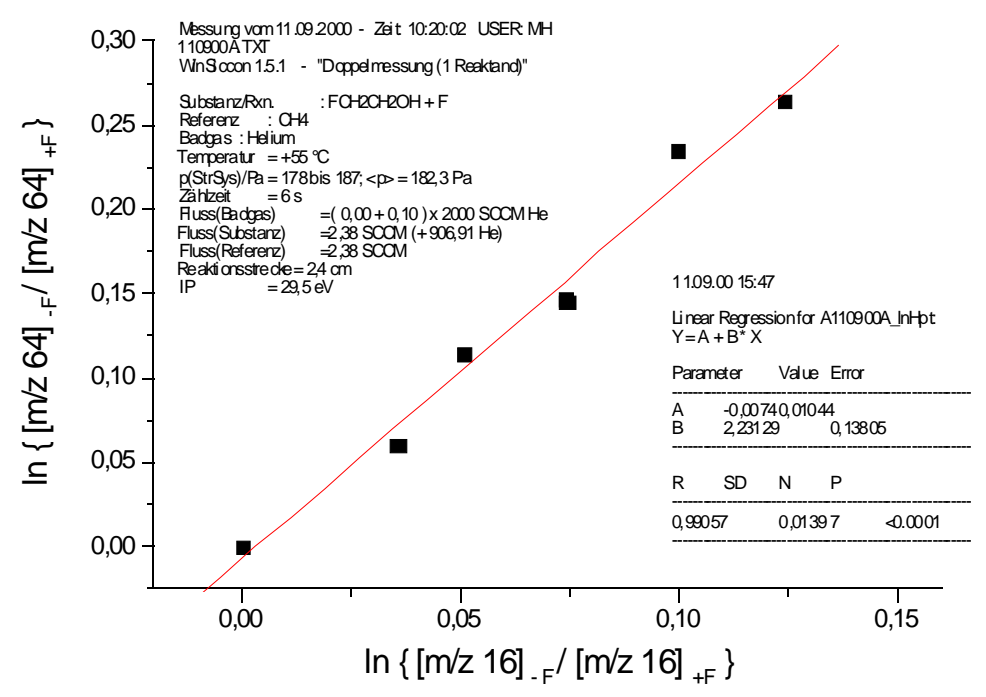

Abbildung 144: Kinetik $\mathrm{CH} 2 \mathrm{FCH} 2 \mathrm{OH}+\mathrm{F} ; 328 \mathrm{~K}$

Aus der Steigung der Auftragung in Abbildung 144 erhält man den relativen

Geschwindigkeitskoeffizienten $\mathrm{k}_{\mathrm{rel}}=(2,23 \pm 0,14)$. 
$\vartheta=+85^{\circ} \mathrm{C} ; \phi_{\mathrm{He}}=1150 \mathrm{SCCM} ; \phi_{\mathrm{CHF} 2 \mathrm{CH} 2 \mathrm{OH}}=2,4 \mathrm{SCCM} ; \phi_{\mathrm{CH} 4}=2,4 \mathrm{SCCM} ; \mathrm{p}_{(\mathrm{StrR})}=187 \mathrm{~Pa} ;$ $\mathrm{s}=2,4 \mathrm{~cm} ; \mathrm{IP}=29,5 \mathrm{eV} ;$ Zählzeit $=6 \mathrm{~s}$

\begin{tabular}{|c|c|c|c|c|c|c|c|}
\hline $\begin{array}{c}\text { I(64) } \\
-F / w . E .\end{array}$ & $\begin{array}{c}\Delta \mathbf{I}(64) \\
\text {-F / w.E. }\end{array}$ & $\begin{array}{c}\mathrm{I}(64) \\
+\mathrm{F} / \mathrm{w.E} .\end{array}$ & $\begin{array}{c}\Delta \mathrm{I}(64) \\
+\mathrm{F} / \mathrm{w.E} .\end{array}$ & $\begin{array}{c}\text { I(16) } \\
\text {-F / w.E. }\end{array}$ & $\begin{array}{c}\Delta \mathbf{I}(\mathbf{1 6}) \\
\text {-F / w.E. }\end{array}$ & $\begin{array}{c}\mathrm{I}(16) \\
+\mathrm{F} / \mathrm{w.E} .\end{array}$ & $\begin{array}{c}\Delta \mathrm{I}(16) \\
+\mathrm{F} / \mathrm{w.E} .\end{array}$ \\
\hline 16890 & 82,69 & 11840 & 194,1 & 32140 & 312 & 27230 & 159,3 \\
\hline 16930 & 230,7 & 14280 & 86,37 & 37740 & 305,6 & 33930 & 388,2 \\
\hline 17730 & 177 & 15380 & 146,2 & 35340 & 736,8 & 33260 & 235,7 \\
\hline 17590 & 196,6 & 15700 & 99,73 & 40180 & 243,3 & 37850 & 154,5 \\
\hline 18550 & 77,25 & 16750 & 118 & 39890 & 335,1 & 38280 & 146,2 \\
\hline 18140 & 160,4 & 14630 & 124,2 & 40980 & 187,9 & 37530 & 177 \\
\hline 18500 & 172,7 & 13260 & 133,3 & 40750 & 154,5 & 34040 & 271,1 \\
\hline 18310 & 191,5 & 14240 & 166,5 & 40950 & 223 & 35810 & 215,9 \\
\hline 18480 & 243,3 & 13880 & 139,3 & 40550 & 218,5 & 35080 & 193,1 \\
\hline
\end{tabular}

Tabelle 34: Meßwerte Kinetik $\mathrm{CH} 2 \mathrm{FCH} 2 \mathrm{OH}+\mathrm{F} ; 358 \mathrm{~K}$

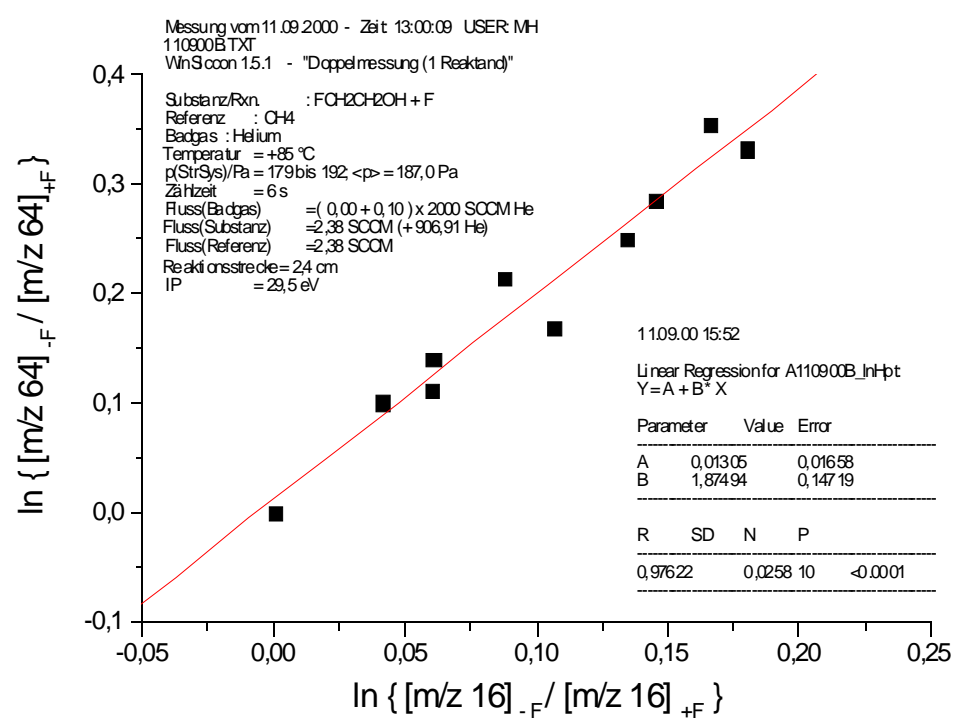

Abbildung 145: Kinetik $\mathrm{CH} 2 \mathrm{FCH} 2 \mathrm{OH}+\mathrm{F} ; 358 \mathrm{~K}$

Aus der Steigung der Auftragung in Abbildung 145 erhält man den relativen

Geschwindigkeitskoeffizienten $\mathrm{k}_{\mathrm{rel}}=(1,87 \pm 0,15)$. 
Aus den Ergebnissen der Abbildungen 142 bis 145 kann eine Arrhenius-Auftragung gemäß Gleichung (11) (S. 10) angefertigt und mit den Geschwindigkeitskoeffizienten der bekannten Referenzreaktion ${ }^{134} \mathrm{k}_{\text {ref }}(\mathrm{T})$ die absoluten Geschwindigkeitskoeffizienten berechnet werden.

$\mathrm{k}_{\mathrm{ref}}(\mathrm{T})=(1,81) \cdot 10^{14} \cdot \exp \{-(3,33) \mathrm{kJ} / \mathrm{mol} /(\mathrm{R} \cdot \mathrm{T})\} \mathrm{cm}^{3} /(\mathrm{mol} \cdot \mathrm{s})$

Die Ergebnisse sind in Tabelle 35 eingetragen, die Auftragung ist in Abbildung 146 zu sehen.

\begin{tabular}{|c|c|c|c|c|c|c|}
\hline $\begin{array}{l}\vartheta / \\
{ }^{\circ} \mathbf{C}\end{array}$ & $\mathbf{T} / \mathbf{K}$ & $\mathbf{T}^{-1} / \mathbf{K}^{-1}$ & $\mathbf{k}_{\text {rel }}$ & $\ln \left\{\mathbf{k}_{\text {rel }}\right\}$ & $\begin{array}{c}\mathbf{k}_{\mathrm{CH} 2 \mathrm{FCH} 2 \mathrm{OH}+\mathrm{F}} / \\
\mathbf{c m}^{3} /(\mathbf{m o l ~ s})\end{array}$ & $\begin{array}{c}\ln \left\{\mathbf{k}_{\text {CH2Fсн2ОН + F }} /\right. \\
\left.\left(\mathbf{c m}^{3} /(\mathbf{m o l ~ s})\right)\right\}\end{array}$ \\
\hline 5 & 278,15 & $3,595 \mathrm{E}-03$ & 2,804 & 1,031 & $1,202 \mathrm{E}+14$ & 32,4206 \\
\hline 25 & 298,15 & $3,354 \mathrm{E}-03$ & 2,369 & 0,862 & $1,119 \mathrm{E}+14$ & 32,3485 \\
\hline 55 & 328,15 & 3,047E-03 & 2,231 & 0,803 & $1,192 \mathrm{E}+14$ & 32,4115 \\
\hline 85 & 358,15 & 2,792E-03 & 1,875 & 0,629 & $1,109 \mathrm{E}+14$ & 32,3398 \\
\hline
\end{tabular}

Tabelle 35: Absolute $k$ sowie Werte der Arrhenius-Auftragung f. $\mathrm{CH} 2 \mathrm{FCH} 2 \mathrm{OH}+\mathrm{F}$

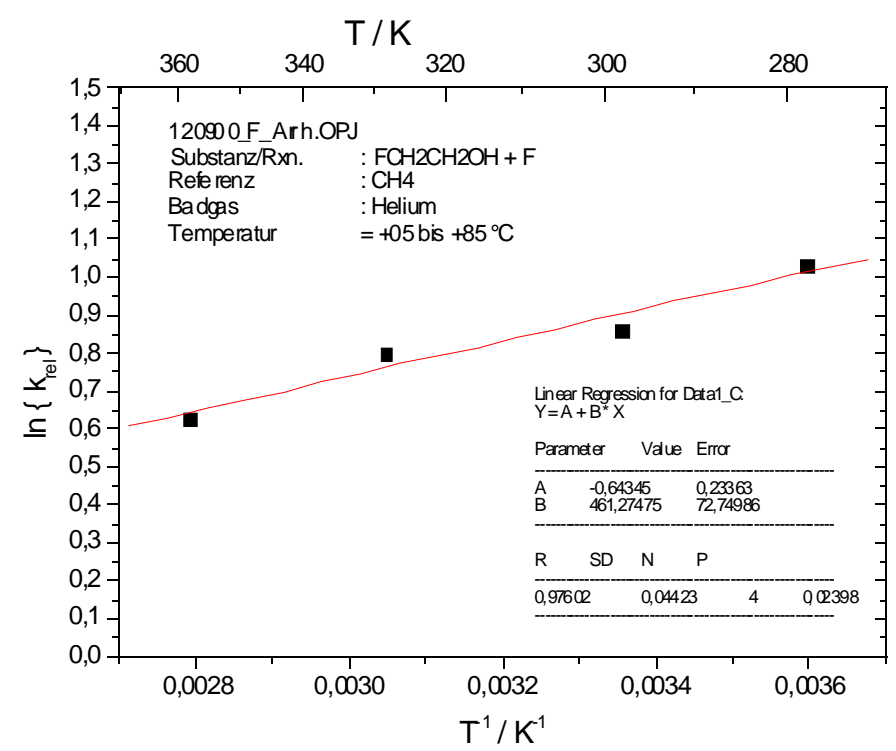

Abbildung 146: Arrhenius-Auftragung f. $\mathrm{CH} 2 \mathrm{FCH} 2 \mathrm{OH}+\mathrm{F}$ rel. zu CH4

Die Aktivierungsenergie der hier untersuchten Reaktion erhält man durch Vergleich der Steigung aus Abbildung 146 mit dem Ausdruck in Gleichung (27) (S. 116):

$\mathrm{E}_{\mathrm{a}}=\mathrm{E}_{\mathrm{a}, \text { Referenz }}-($ Steigung $) \cdot \mathrm{R}=(3330-(461,27 \cdot 8,314)) \mathrm{J} / \mathrm{mol}=-505,0 \mathrm{~J} / \mathrm{mol}$.

Bezüglich des präexponentiellen Faktors wird der Achsenabschnitt aus Abbildung 146 
herangezogen:

$\mathrm{A}=\exp ($ Achsenabschnitt $) \cdot \mathrm{A}_{\text {Referenz }}=\exp (-0,64345) \cdot 1,81 \cdot 10^{14} \mathrm{~cm}^{3} \mathrm{~mol}^{-1} \mathrm{~s}^{-1}$

$$
=9,51 \cdot 10^{13} \mathrm{~cm}^{3} \mathrm{~mol}^{-1} \mathrm{~s}^{-1} \text {. }
$$

Im Ergebnis beträgt der gesuchte Geschwindigkeitskoeffizient damit:

$$
\begin{aligned}
& \mathbf{k}(\mathbf{T})=(9,5 \pm 2,2) \cdot 10^{13} \cdot \exp \{-(-0,50 \pm 0,60) \mathbf{k J} / \mathbf{m o l} /(\mathbf{R} \cdot \mathbf{T})\} \mathbf{c m}^{3} /(\mathbf{m o l} \cdot \mathbf{s}) \\
& \text { für: } \mathrm{CH}_{2} \mathrm{FCH}_{2} \mathrm{OH}+\mathrm{F} \rightarrow \text { Produkte }
\end{aligned}
$$

Mit $\mathrm{T}=298 \mathrm{~K}$ errechnet man daraus: $\mathbf{k}(298 \mathrm{~K})=(\mathbf{1 , 2} \pm \mathbf{0 , 4}) \cdot \mathbf{1 0}^{\mathbf{1 4}} \mathbf{c m}^{\mathbf{3}} \mathbf{m o l}^{-1} \mathrm{~s}^{-\mathbf{1}}$.

Weil die Reaktion im Rahmen ihrer Fehlergrenzen kaum eine Temperaturabhängigkeit zeigt, wird mit den absoluten Geschwindigkeitskoeffizienten aus Tabelle 35 zudem eine Auftragung gemäß Gleichung (13) (S. 11) angefertigt (Abb. 147).

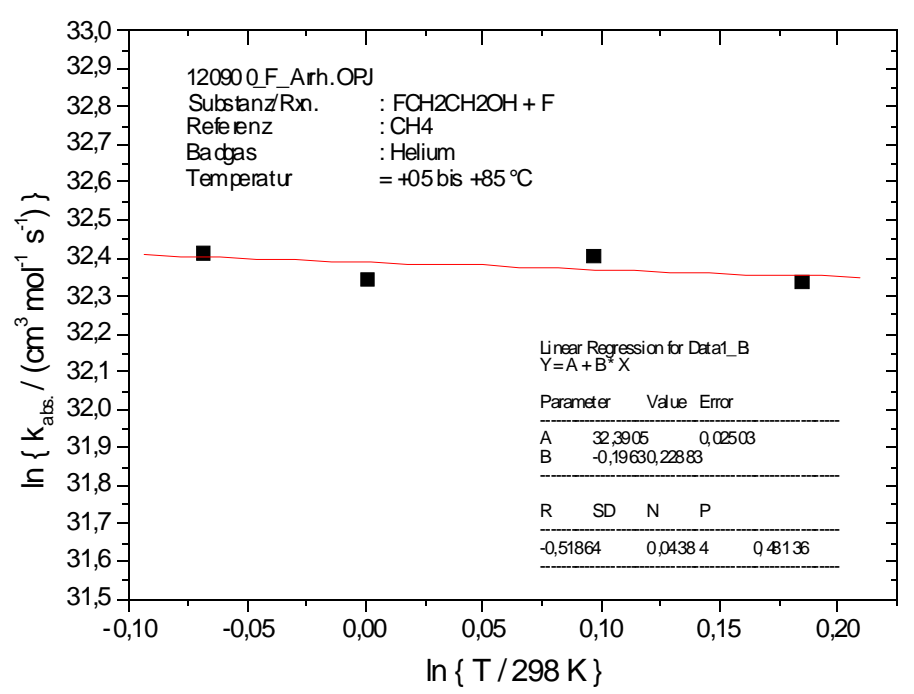

Abbildung 147: $\ln (k) v s \cdot \ln (T / 298 K) f . C H 2 F C H 2 O H+F$

Aus den Ergebnissen der Abbildung 147 berechnet man:

$$
\begin{aligned}
& \mathbf{k}(\mathbf{T})=(1,17 \pm \mathbf{0 , 0 3}) \cdot 10^{14} \cdot(\mathrm{T} / 298 \mathrm{~K})^{-(0,20 \pm 0,23)} \mathbf{c m}^{3} /(\mathbf{m o l} \cdot \mathbf{s}) \\
& \text { für: } \mathrm{CH}_{2} \mathrm{FCH}_{2} \mathrm{OH}+\mathrm{F} \rightarrow \text { Produkte }
\end{aligned}
$$

Mit $\mathrm{T}=298 \mathrm{~K}$ errechnet man daraus: $\mathbf{k}(298 \mathrm{~K})=(\mathbf{1 , 1 7} \pm \mathbf{0 , 0 3}) \cdot \mathbf{1 0} \mathbf{c m}^{\mathbf{1 4}} \mathbf{m o l}^{-1} \mathbf{s}^{-1}$ 


\subsubsection{Produkte der Reaktion des erzeugten Radikals mit molekularem Sauerstoff}

Um einen Überblick über die Änderungen im Massenspektrum zu erhalten, sind mit der in Kapitel 3.1.1 (S. 16) beschriebenen Apparatur unmittelbar nacheinander EI-Übersichtsspektren unter Zugabe und Abwesenheit von molekularem Sauerstoff (Fluor-Atome wurden stets gleichermaßen zugegeben) aufgenommen worden.

Es wurden anschließend gezielt in Einzelzählung (6 sek Zählzeit, IP = 29,5 eV) die m/z-Werte überprüft, welche im Übersichtsspektrum größere Veränderungen zeigten. Die Durchführung der Messungen ist in Kapitel 3.5.1 (S. 50) beschrieben. In Abbildung 148 sind die Reaktionsbedingungen und Ergebnisse dargestellt.

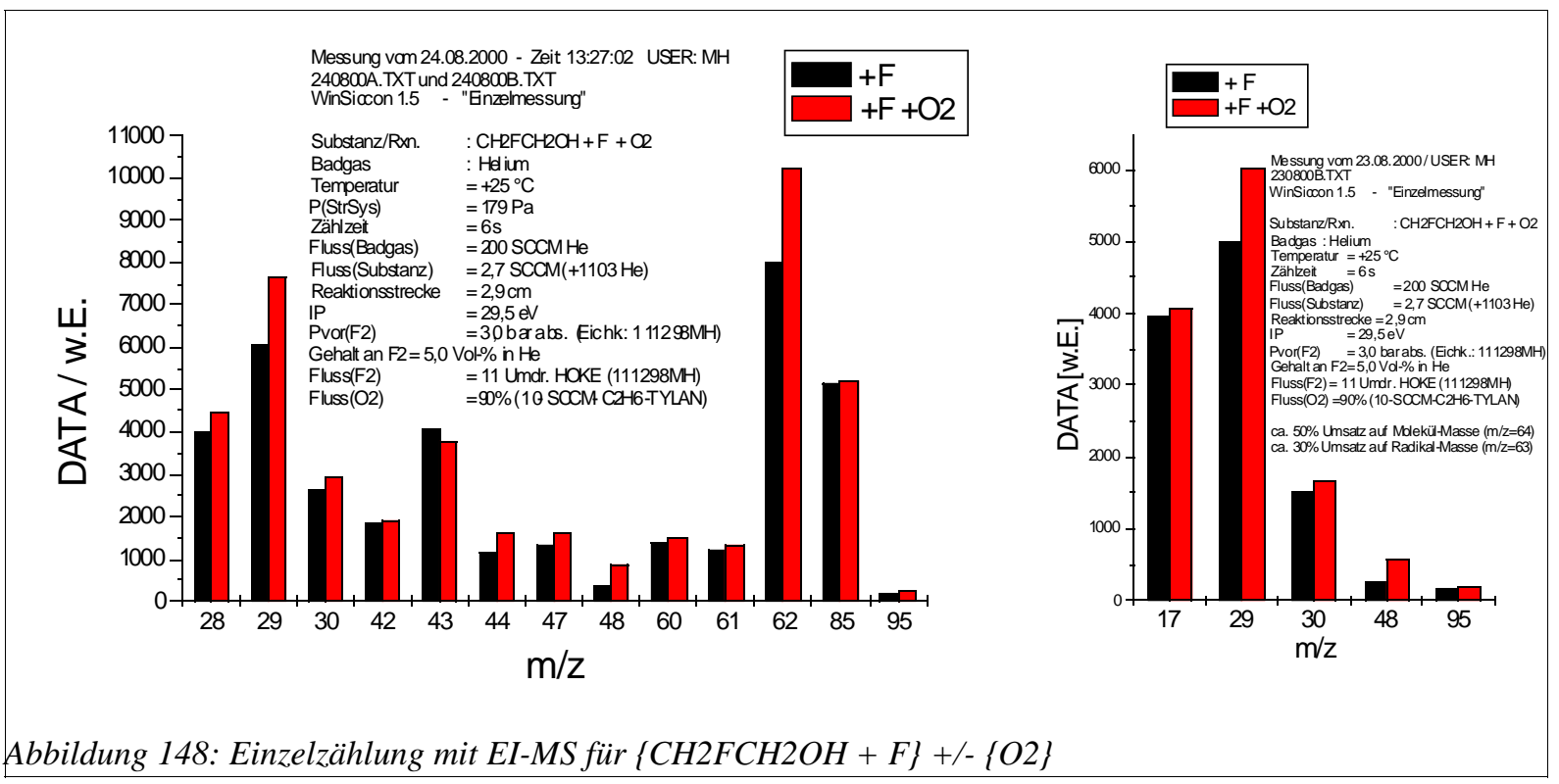

In Abb. Abbildung 149 sind einige denkbare Reaktionskanäle aufgezeigt.

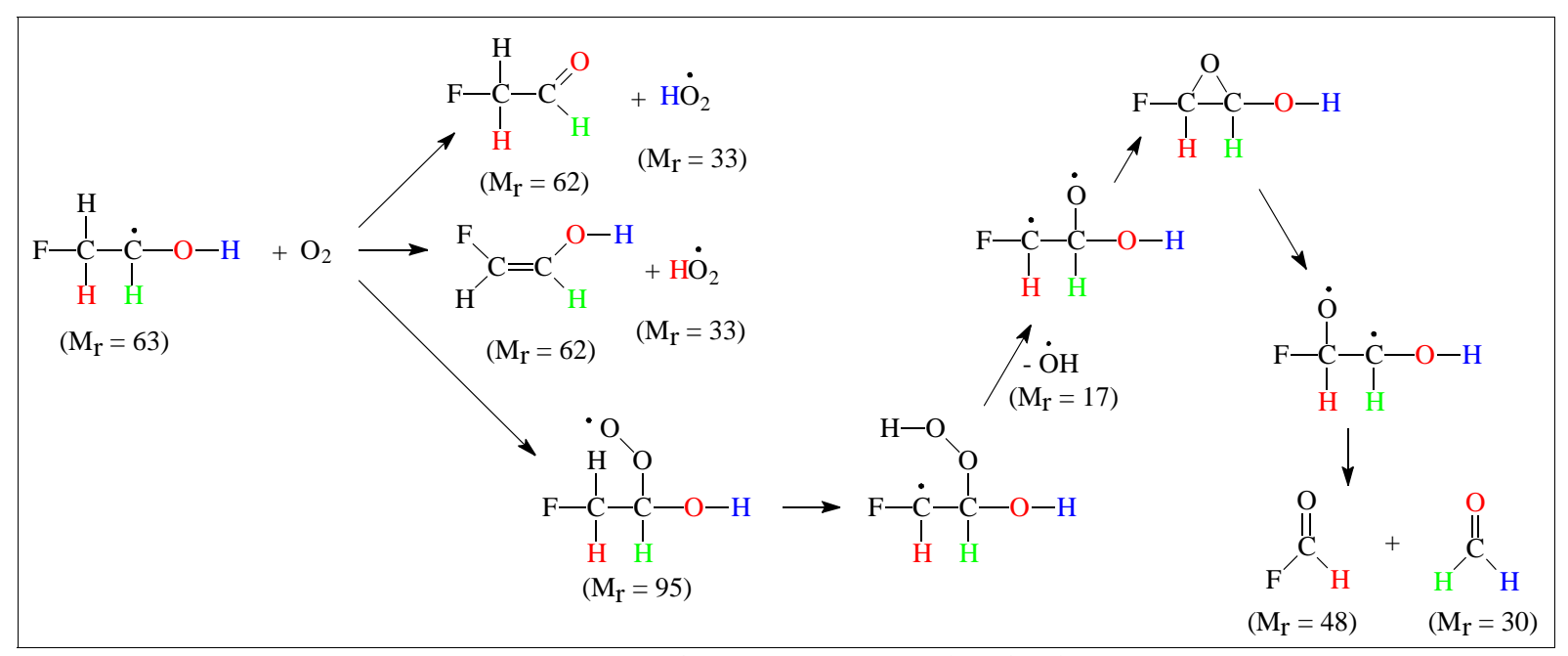

Abbildung 149: Denkbare Reaktionskanäle für $\{\mathrm{CH} 2 \mathrm{FCH} 2 \mathrm{OH}+\mathrm{F}\}+\{\mathrm{O} 2\}$

Die leichte Zunahme auf m/z 95 spricht für die Bildung des Assoziationskomplexes mit $\mathrm{O}_{2}$, die 
Zunahme auf m/z 62 und m/z 33 (letzteres wurde im Übersichtsspektrum beobachtet) deutet auf die Bildung von 2-Fluorethanal $\left(\mathrm{CH}_{2} \mathrm{FCHO}\right)$ hin. Denkbar ist jedoch auch die Bildung des in Abb. 149 gezeigten Alkenols mit $\mathrm{M}_{\mathrm{r}}=62$. In Lösung ist es üblich, daß Aldehyde und Ketone $\mathrm{zu}$ einem gewissen Teil als Enol-Tautomere vorliegen ${ }^{135}$, daher scheint dieser Strukturvorschlag auch hier nicht sehr abwegig. Die Zunahmen auf m/z 28; 29 und 30 sind ein Indiz für die Entstehung von Methanal (Formaldehyd, HCHO), welches im MS entsprechend fragmentiert. Es ist jedoch möglich, daß zudem CO entsteht, welches ebenfalls einen Peak bei m/z 28 hat. Der starke Anstieg der Intensität auf m/z 48 spricht für die Bildung von Fluormethanal (FCHO). Der aufgezeigte Mechanismus in Abb. 149 wird durch eine Zunahme auf $\mathrm{m} / \mathrm{z} 17$ plausibel gemacht, der durch die Abspaltung von $\mathrm{OH}$ zu erklären ist. Der Anstieg auf $\mathrm{m} / \mathrm{z} 44$ scheint auf die Bildung von $\mathrm{CO}_{2}$ zurückzuführen sein, das meistens als finales Oxidationsprodukt entsteht. Die Zunahmen auf m/z 42, 60 und 61 sind vermutlich Fragmente des Produkts mit $\mathrm{M}_{\mathrm{r}}=62$.

Unter den gleichen Reaktionsbedingungen, die während der Analyse im EIMassenspektrometer herrschten, wurden in einer Glaskühlfalle bei $77 \mathrm{~K}$ die Stoffe nach Verlassen des Strömungsreaktors aufgefangen (vgl. Abb. 4, S. 16).

Ergänzend zur EI-MS-Analyse konnten die ausgefrorenen Produkte nun im FT-IRSpektrometer untersucht werden. Dazu wurde die Kühlfalle beginnend bei $77 \mathrm{~K}$ langsam auf 298 K erwärmt und quasi als fraktionierende Destillation von den in die Gasphase übertretenden Produkte nacheinander in der Multireflektionskugekzelle FT-IR-Spektren registriert (vgl. dazu auch Kap. 3.1.5, S. 42 sowie Kap. 3.5.5, S. 59). Die Spektren sind kumulativ, d.h. die Banden der jeweils vorangegangenen Fraktionen sind auch in den nachfolgenden Spektren enthalten.
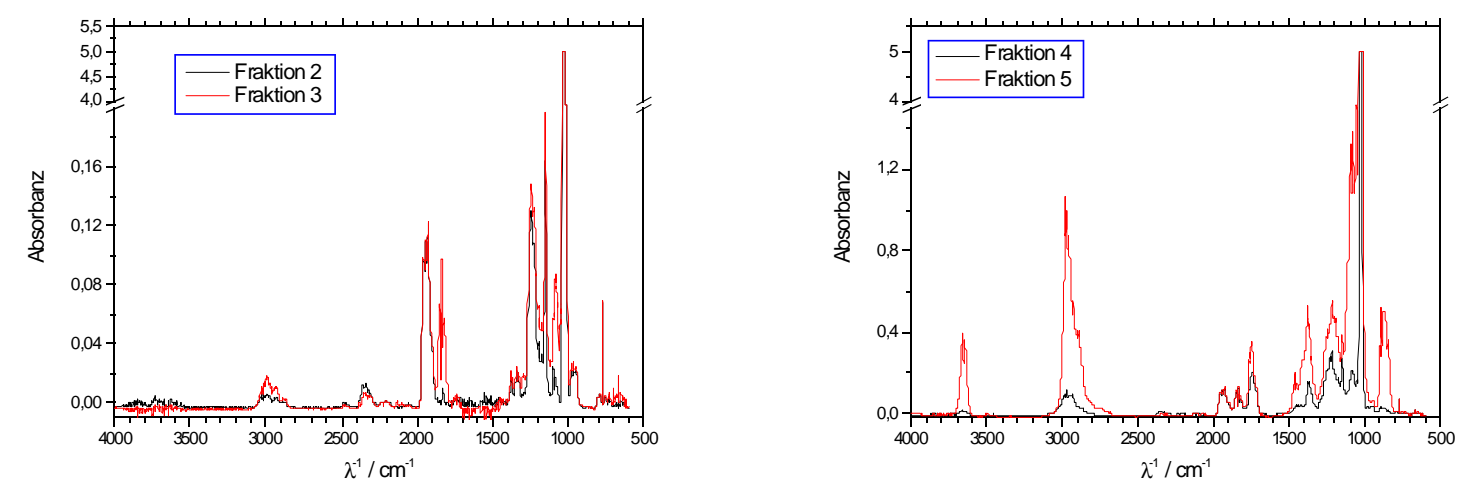

Abbildung 150: IR-Spektren für Produktuntersuchung von $\{\mathrm{CH} 2 \mathrm{FCH} 2 \mathrm{OH}+\mathrm{F}\}+\{\mathrm{O} 2\}$

135 Vgl. Streitwieser, A.; Heathcock, C. (1990), S. 433 ff. 
In Abb. 150 sind die Spektren der „Fraktionen“ 2 bis 5 dargestellt. Da das Edukt eine charakteristische Bande bei $\lambda^{-1}=3660$ und $3646 \mathrm{~cm}^{-1}$ (OH-Gruppe des Alkohols, vgl. Abb. 151 ) hat, kann man feststellen, daß die Fraktionen 2 und 3 völlig und die Fraktion 4 weitgehend frei vom Edukt sind. Die starke Bande bei $1028 \mathrm{~cm}^{-1}$ ist auf die Bildung von $\mathrm{SiF}_{4}$ durch die Reaktion der Fluor-Atome mit dem Glasreaktor (enthält $\mathrm{SiO}_{\mathrm{x}}$ ) zurückzuführen.
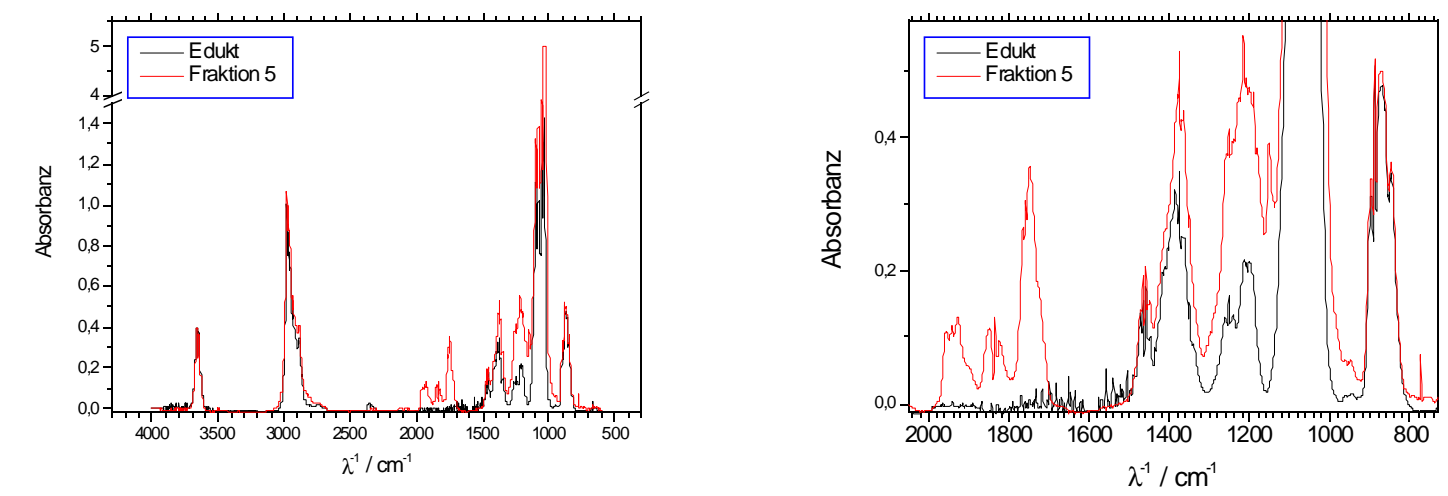

Abbildung 151: IR-Spektren für Produktuntersuchung von $\{\mathrm{CH} 2 \mathrm{FCH} 2 \mathrm{OH}+\mathrm{F}\}+\{\mathrm{O} 2\}$

Zur Erleichterung der Argumentation wurden mit dem Computerprogramm „Gaussian98“ nach der Methode „B3LYP/6-31G*“ die IR-Spektren von HCHO und FCHO berechnet. Zuvor fand mit diesem Programm eine Geometrieoptimierung statt. Die so erhaltenen Wellenzahlen mußten jedoch zur Anpassung an experimentelle Werte noch mit einem Korrekturfaktor versehen werden. Durch Vergleich mit dem gemessenen Spektrum ${ }^{136}$ von HCHO (Hauptbanden bei 2896, 2801 und $1745 \mathrm{~cm}^{-1}$ ) ergab sich ein Korrekturfaktor von 0,9437.
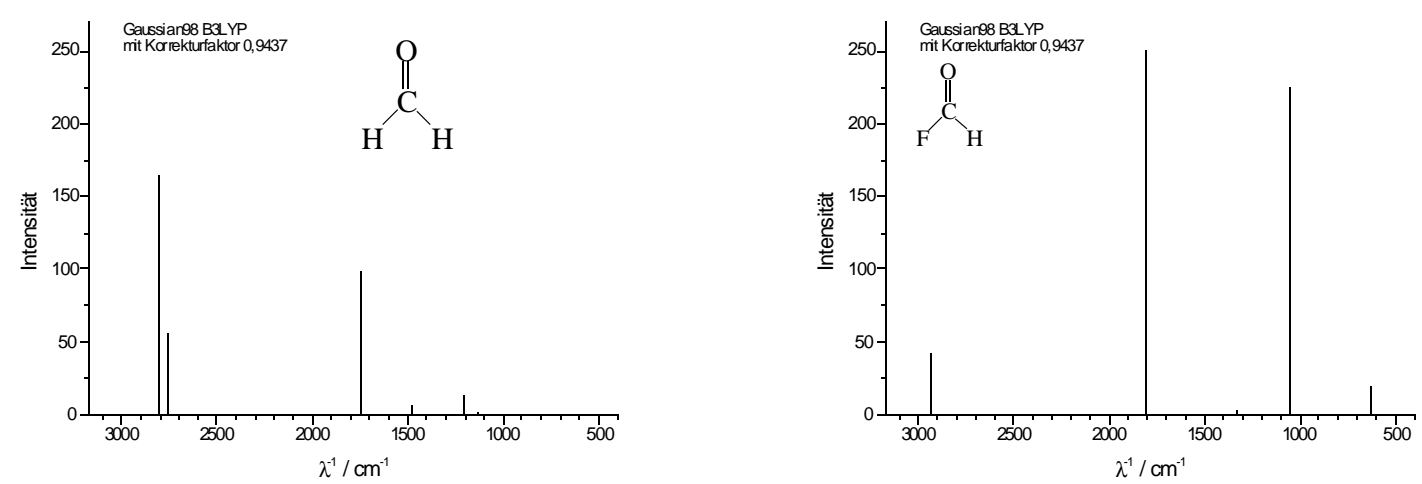

Abbildung 152: Berechnete IR-Spektren von HCHO und FCHO

In der Fraktion 2 weisen die beiden Banden bei 1927 und $1943 \mathrm{~cm}^{-1}$ eindeutig ein Allen nach, wobei die Doppelbande entweder ein terminales Allen oder aber einen elektronegativen Substituenten (z.B. C=C=C-F) identifiziert. Ein konkreter Vorschlag für eine Struktur bzw. 
einen Reaktionsmechanismus mit der Bildung eines entsprechenden Allen-Produkts kann nicht gemacht werden. Die Bande bei $774 \mathrm{~cm}^{-1}$ deutet auf eine Doppelbindung mit der Struktureinheit $\mathrm{XHC}=\mathrm{CHY}$ (in der cis-Konformation) oder $\mathrm{XYC}=\mathrm{CH}_{2}$ (X, Y: Nicht-AlkylReste) hin. Die Bande bei $1152 \mathrm{~cm}^{-1}$ spricht für die Struktureinheit C=C-F. Beides zusammen könnte mit der in Abbildung 149 vorgeschlagenen Alkenol-Verbindung $\mathrm{HFC}=\mathrm{C}(\mathrm{OH}) \mathrm{H}$ erklärt werden; da die Banden der OH-Gruppe bei $3650 \mathrm{~cm}^{-1}$ bzgl. der zu erwartenden Intensität sehr variabel sind, muß das Fehlen einer solchen Bande im IR-Spektrum in Abb. 150 kein Ausschluß-Kriterium sein. Die Bande bei $2345 \mathrm{~cm}^{-1}$ beweist das Entstehen von $\mathrm{CO}_{2}$.

In der Fraktion 3 treten $\lambda^{-1}=1835$ und $1083 \mathrm{~cm}^{-1}$ als neue starke Banden hinzu, die in Verbindung mit der Bande bei 2930-2950 $\mathrm{cm}^{-1}$ das Fluormethanal (FCHO) nachweisen; das ergibt sich durch Vergleich mit dem berechneten IR-Spektrum in Abb. 152.

Fraktion 4 zeigt insbesondere starke Zunahmen bei 1217, 1730-1750, mittelstarke bei 2886, 2897, 2928 sowie schwache bei 2824 und $2824 \mathrm{~cm}^{-1}$. Damit läßt sich die Bildung von Methanal (HCHO) erklären (vgl. Abb. 152 sowie die experimentell gefundenen Hauptbanden ${ }^{136}$ bei 2896, 2801 und $1745 \mathrm{~cm}^{-1}$ ). Die Bande bei $1217 \mathrm{~cm}^{-1}$ weist auf die Struktureinheit C-F hin, was zusammen mit den anderen typischen Carbonyl-Banden bei $1730-1750 \mathrm{~cm}^{-1}$ gut mit der Bildung von 2-Fluorethanal $\left(\mathrm{CH}_{2} \mathrm{FCHO}\right)$ erklärt werden kann.

Die Fraktion 5 dürfte im wesentlichen aus Edukt bestehen, wie der direkte Vergleich der Spektren der kumulierten Fraktionen 1-5 mit dem Spektrum des reinen Edukts 2-Fluorethanol in Abbildung 151 zeigt. Dort ist zur besseren Ablesbarkeit auch eine Ausschnittsvergrößerung für den Bereich 2000-800 $\mathrm{cm}^{-1}$ abgebildet.

Die Ergebnisse der IR-Analyse sind demnach im Einklang mit denen der EI-MS-Untersuchung und bestätigen die in Abbildung 149 vorgeschlagenen Reaktionskanäle.

\subsubsection{Kinetik der Reaktion des erzeugten Radikals mit molekularem Sauerstoff}

Die Messungen wurden in der in Kap. 3.1.1 (S. 16) beschriebenen Apparatur unter Einsatz des Strömungsreaktors aus Abbildung 5 (S. 17) durchgeführt.

Das 1,4-Dioxanyl-Radikal diente als Referenzsubstanz. Der Umsatz des Alkohol-Radikals konnte anhand der Intensitätsänderung seines Molekül-Peaks m/z 63 und der des 1,4Dioxanyl-Radikals anhand seines Molekül-Peaks m/z 87 bestimmt werden.

Die Messungen wurden umsatzkorrigiert nach der Relativmethode durchgeführt, wobei zur Messung des Umsatzes des Alkohols bei Zugabe der Fluor-Atome die Intensitätsänderung auf 
m/z 64, und für den des 1,4-Dioxans diejenige auf m/z 88 diente; die genaue Ausführung und Auswertung ist in Kap. 3.5.1 (S. 50) beschrieben.

$\vartheta=-15^{\circ} \mathrm{C} ; \phi_{\mathrm{He}}=1513 \mathrm{SCCM} ; \phi_{\mathrm{CH} 2 \mathrm{FCH} 2 \mathrm{OH}}=2,7 \mathrm{SCCM} ; \phi_{1,4 \text {-Dioxan }}=2,7 \mathrm{SCCM} ; \mathrm{p}_{(\mathrm{StrR})}=170 \mathrm{~Pa} ;$ $\mathrm{s}=2,9 \mathrm{~cm} ; \mathrm{IP}=29,5 \mathrm{eV} ;$ Zählzeit $=6 \mathrm{~s}$

\begin{tabular}{|c|c|c|c|c|c|c|c|}
\hline $\begin{array}{c}\mathbf{I}(\mathbf{6 3 )} \\
-\mathbf{O}_{2} / \text { w.E. }\end{array}$ & $\begin{array}{c}\Delta \mathbf{I}(\mathbf{6 3}) \\
-\mathrm{O}_{2} / \mathbf{w . E}\end{array}$ & $\begin{array}{c}\mathrm{I}(63) \\
+\mathrm{O}_{2} / \text { w.E. }\end{array}$ & $\begin{aligned} & \Delta \mathrm{I}(63) \\
+ & \mathrm{O}_{2} / \text { w.E. }\end{aligned}$ & $\begin{array}{c}\mathbf{I}(\mathbf{8 7}) \\
-\mathbf{O}_{2} / \text { w.E. }\end{array}$ & $\begin{array}{c}\Delta \mathrm{I}(\mathbf{8 7}) \\
-\mathbf{O}_{2} / \mathbf{w . E}\end{array}$ & $\begin{array}{c}\mathrm{I}(87) \\
+\mathrm{O}_{2} / \text { w.E. }\end{array}$ & $\begin{array}{c}\Delta \mathrm{I}(\mathbf{8 7}) \\
+\mathrm{O}_{2} / \mathrm{w.E} .\end{array}$ \\
\hline 1174 & 67,19 & 918,8 & 41,94 & 4938 & 93,3 & 3831 & 88,56 \\
\hline 1104 & 64,51 & 826,5 & 64,36 & 4962 & 66,72 & 3813 & 85,1 \\
\hline 1142 & 41,6 & 889 & 48,6 & 4827 & 90,95 & 3778 & 109,2 \\
\hline 1127 & 62,17 & 856,6 & 72,78 & 4865 & 68,03 & 3927 & 90,6 \\
\hline 1088 & 69,91 & 871,8 & 53,88 & 4744 & 70,31 & 4051 & 84,04 \\
\hline 1133 & 65,81 & 997,1 & 58,71 & 4878 & 99,56 & 4239 & 99,96 \\
\hline 1159 & 56,78 & 1058 & 67,7 & 4982 & 79,34 & 4607 & 103,7 \\
\hline 1143 & 69,09 & 982,9 & 59,84 & 4890 & 71,4 & 4183 & 89,29 \\
\hline
\end{tabular}

Tabelle 36: Meßwerte Kinetik $\{\mathrm{CH} 2 \mathrm{FCH} 2 \mathrm{OH}+\mathrm{F}\}+\{\mathrm{O} 2\}$ rel. zu 1,4-Dioxan; $258 \mathrm{~K}$

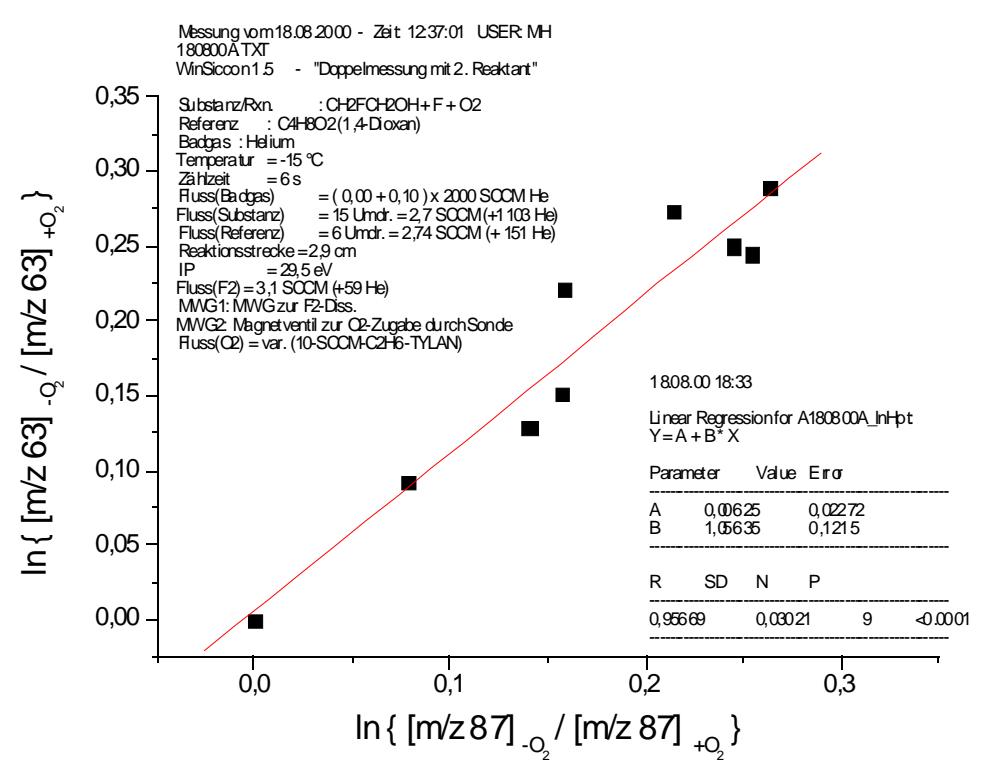

Abbildung 153: Kinetik $\{C H 2 F C H 2 O H+F\}+\{O 2\}$ rel. zu 1,4-Dioxan; $258 K$

Aus der Steigung der Auftragung in Abbildung 153 läßt sich der relative Geschwindigkeitskoeffizient bei $\mathrm{T}=258 \mathrm{~K}$ ermitteln: $\mathrm{k}_{\mathrm{rel}}=(1,06 \pm 0,12)$. 
$\vartheta=+5{ }^{\circ} \mathrm{C} ; \phi_{\mathrm{He}}=1513 \mathrm{SCCM} ; \phi_{\text {Сн2FCH2OH }}=2,7 \mathrm{SCCM} ; \phi_{1,4-\mathrm{Dioxan}}=2,7 \mathrm{SCCM} ; \mathrm{p}_{(\mathrm{StrR})}=177 \mathrm{~Pa} ;$ $\mathrm{s}=2,9 \mathrm{~cm} ; \mathrm{IP}=29,5 \mathrm{eV} ;$ Zählzeit $=6 \mathrm{~s}$

\begin{tabular}{|c|c|c|c|c|c|c|c|}
\hline $\begin{array}{c}\mathrm{I}(63) \\
-\mathrm{O}_{2} / \text { w.E. }\end{array}$ & $\begin{array}{c}\Delta \mathbf{I}(63) \\
-\mathbf{O}_{2} / \text { w.E. }\end{array}$ & $\begin{array}{c}\mathrm{I}(63) \\
+\mathrm{O}_{2} / \text { w.E. }\end{array}$ & $\begin{array}{c}\Delta \mathrm{I}(63) \\
+\mathrm{O}_{2} / \text { w.E. } \\
\end{array}$ & $\begin{array}{c}\mathbf{I}(87) \\
-O_{2} / \text { w.E. }\end{array}$ & $\begin{array}{c}\Delta \mathbf{I}(87) \\
-\mathbf{O}_{2} / \text { w.E. }\end{array}$ & $\begin{array}{c}\mathrm{I}(87) \\
+\mathrm{O}_{2} / \text { w.E. }\end{array}$ & $\begin{array}{c}\Delta \mathrm{I}(\mathbf{8 7}) \\
+\mathrm{O}_{2} / \mathrm{w} . \mathrm{E} .\end{array}$ \\
\hline 1139 & 68,08 & 847 & 65,51 & 4625 & 111 & 3445 & 102,8 \\
\hline 1126 & 53,52 & 899,6 & 56,91 & 4791 & 73,4 & 3649 & 89,35 \\
\hline 1148 & 66,09 & 974,1 & 57,51 & 4590 & 73,25 & 3905 & 118,1 \\
\hline 1048 & 83,61 & 904,4 & 61,03 & 4610 & 118,8 & 3992 & 86,73 \\
\hline 1046 & 71,86 & 949,8 & 81,39 & 4685 & 99,75 & 4331 & 110,9 \\
\hline 1086 & 63,68 & 991 & 59,48 & 4662 & 89,29 & 4194 & 84,19 \\
\hline 1016 & 68,84 & 867,3 & 69,73 & 4506 & 106,5 & 3595 & 107,2 \\
\hline 1086 & 44,53 & 854,6 & 41,84 & 4760 & 95,7 & 3686 & 91,85 \\
\hline 1064 & 59,34 & 838,5 & 53,43 & 4607 & 66,62 & 3716 & 57,88 \\
\hline
\end{tabular}

Tabelle 37: Meßwerte Kinetik $\{\mathrm{CH} 2 \mathrm{FCH} 2 \mathrm{OH}+\mathrm{F}\}+\{\mathrm{O} 2\}$ rel. zu 1,4-Dioxan; $278 \mathrm{~K}$

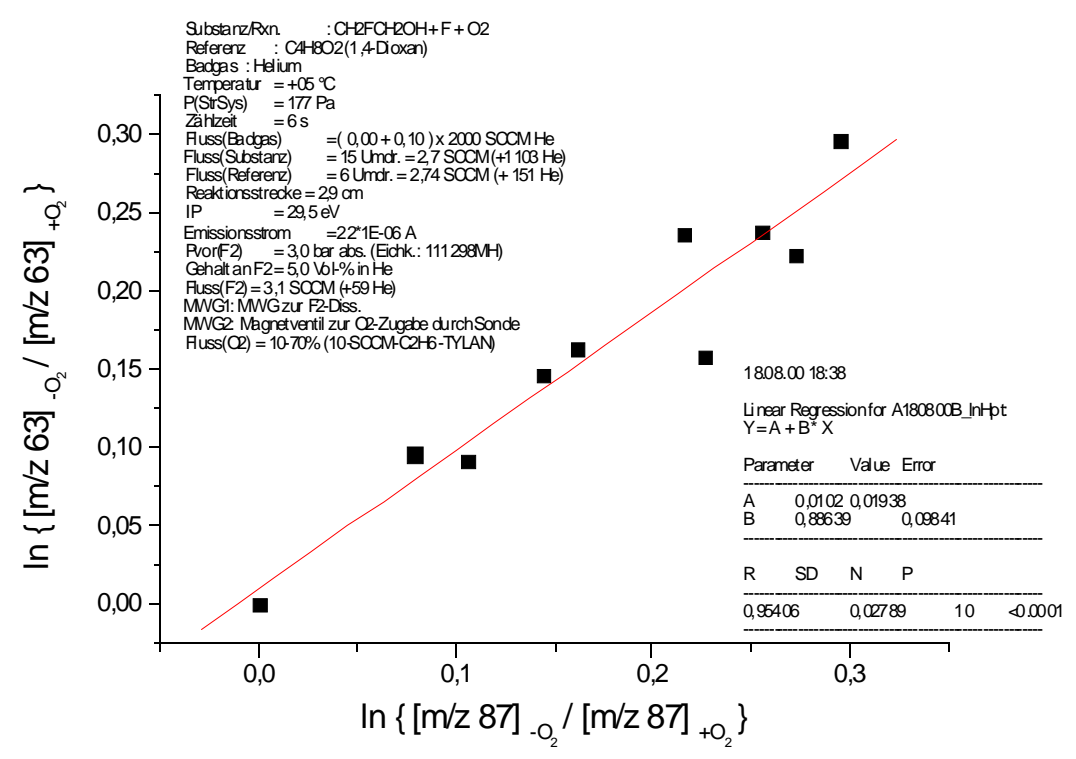

Abbildung 154: Kinetik $\{\mathrm{CH} 2 \mathrm{FCH} 2 \mathrm{OH}+\mathrm{F}\}+\{\mathrm{O} 2\}$ rel. zu 1,4-Dioxan; $278 \mathrm{~K}$

Aus der Steigung der Auftragung in Abbildung 154 läßt sich der relative Geschwindigkeitskoeffizient bei $\mathrm{T}=278 \mathrm{~K}$ ermitteln: $\mathrm{k}_{\mathrm{rel}}=(0,89 \pm 0,10)$. 
$\vartheta=+25^{\circ} \mathrm{C} ; \phi_{\mathrm{He}}=1332 \mathrm{SCCM} ; \phi_{\text {Сн2FCH2OH }}=2,7 \mathrm{SCCM} ; \phi_{1,4 \text {-Dioxan }}=1,7 \mathrm{SCCM} ; \mathrm{p}_{(\mathrm{StrR})}=169 \mathrm{~Pa} ;$ $\mathrm{s}=3,1 \mathrm{~cm} ; \mathrm{IP}=29,5 \mathrm{eV}$; Zählzeit $=6 \mathrm{~s}$

\begin{tabular}{|c|c|c|c|c|c|c|c|}
\hline $\begin{array}{c}\mathbf{I}(\mathbf{6 3}) \\
-\mathbf{O}_{2} / \mathbf{w . E .}\end{array}$ & $\begin{array}{c}\Delta \mathbf{I}(\mathbf{6 3}) \\
-\mathrm{O}_{2} / \mathbf{w . E .}\end{array}$ & $\begin{array}{c}\mathrm{I}(63) \\
+\mathrm{O}_{2} / \text { w.E. }\end{array}$ & $\begin{array}{c}\Delta \mathrm{I}(63) \\
+\mathrm{O}_{2} / \text { w.E. }\end{array}$ & $\begin{array}{c}\mathbf{I}(\mathbf{8 7}) \\
-\mathbf{O}_{2} / \text { w.E. }\end{array}$ & $\begin{array}{c}\Delta \mathrm{I}(\mathbf{I} 7) \\
-\mathrm{O}_{2} / \text { w.E. }\end{array}$ & $\begin{array}{c}\mathrm{I}(\mathbf{8 7}) \\
+\mathrm{O}_{2} / \text { w.E. }\end{array}$ & $\begin{array}{c}\Delta \mathbf{I}(\mathbf{8 7}) \\
+\mathbf{O}_{2} / \mathbf{w . E .}\end{array}$ \\
\hline 1667 & 47,34 & 1233 & 46,29 & 2362 & 48,92 & 1710 & 66,64 \\
\hline 1635 & 76,4 & 1358 & 49,35 & 2765 & 54,36 & 2274 & 62,84 \\
\hline 1605 & 60,31 & 1340 & 37,41 & 2607 & 53,17 & 2153 & 50,01 \\
\hline 1697 & 44,49 & 1327 & 60,91 & 2646 & 53,9 & 2186 & 76,09 \\
\hline 1688 & 49,54 & 1465 & 55,91 & 2646 & 59,24 & 2387 & 75,59 \\
\hline 1585 & 56,95 & 1507 & 45,93 & 2799 & 65,03 & 2574 & 56,87 \\
\hline 1579 & 63,84 & 1371 & 46,68 & 2737 & 69,98 & 2300 & 83,91 \\
\hline 1628 & 55,46 & 1357 & 64,08 & 2558 & 66,73 & 2160 & 77,46 \\
\hline 1644 & 59,76 & 1290 & 49,61 & 2511 & 83,52 & 2066 & 77,6 \\
\hline 1668 & 53,7 & 1271 & 55,52 & 2511 & 69,37 & 2010 & 83,06 \\
\hline
\end{tabular}

Tabelle 38: Meßwerte Kinetik $\{\mathrm{CH} 2 \mathrm{FCH} 2 \mathrm{OH}+\mathrm{F}\}+\{\mathrm{O} 2\}$ rel. zu 1,4-Dioxan; $298 \mathrm{~K}$

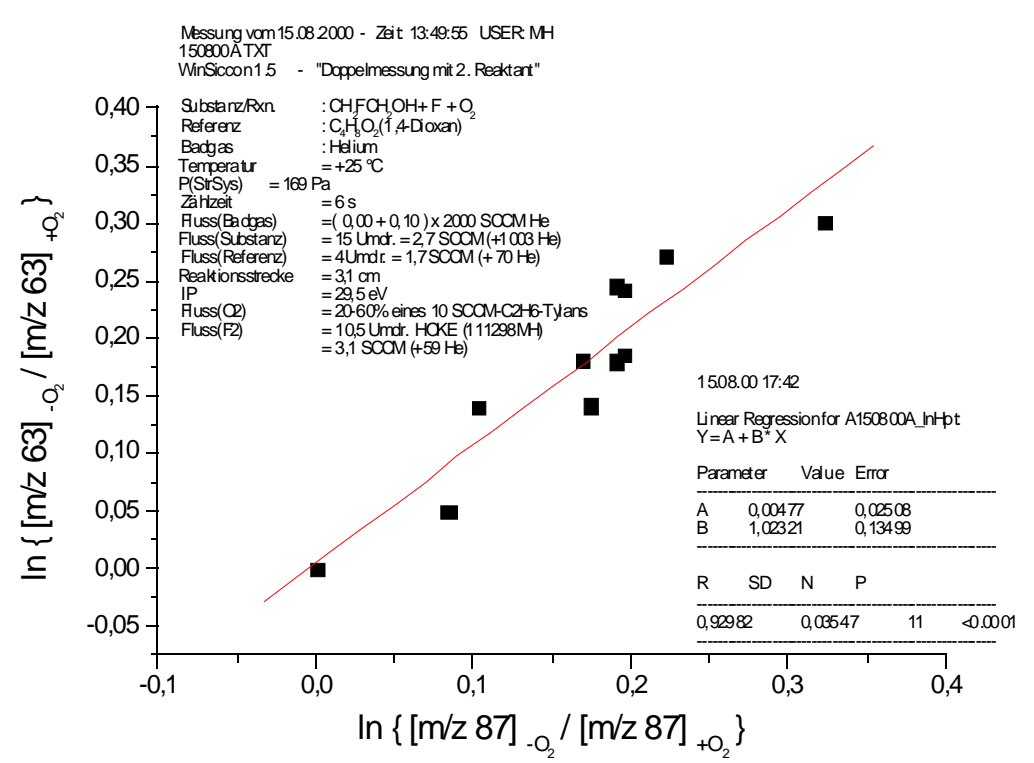

Abbildung 155: Kinetik $\{\mathrm{CH} 2 \mathrm{FCH} 2 \mathrm{OH}+\mathrm{F}\}+\{$ O2\} rel. zu 1,4-Dioxan;298K

Aus der Steigung der Auftragung in Abbildung 155 läßt sich der relative Geschwindigkeitskoeffizient bei $\mathrm{T}=298 \mathrm{~K}$ ermitteln: $\mathrm{k}_{\mathrm{rel}}=(0,89 \pm 0,10)$. 
$\vartheta=+55^{\circ} \mathrm{C} ; \phi_{\mathrm{He}}=1345 \mathrm{SCCM} ; \phi_{\mathrm{CH} 2 \mathrm{FCH} 2 \mathrm{OH}}=2,7 \mathrm{SCCM} ; \phi_{1,4-\text { Dioxan }}=2,7 \mathrm{SCCM} ; \mathrm{p}_{(\mathrm{StrR})}=180 \mathrm{~Pa} ;$ $\mathrm{s}=2,9 \mathrm{~cm} ; \mathrm{IP}=29,5 \mathrm{eV} ;$ Zählzeit $=6 \mathrm{~s}$

\begin{tabular}{|c|c|c|c|c|c|c|c|}
\hline $\begin{array}{c}\mathrm{I}(63) \\
-\mathrm{O}_{2} / \text { w.E. }\end{array}$ & $\begin{array}{c}\Delta \mathrm{I}(63) \\
-\mathrm{O}_{2} / \mathrm{w} . \mathrm{E} .\end{array}$ & $\begin{array}{c}\mathrm{I}(63) \\
+\mathrm{O}_{2} / \text { w.E. }\end{array}$ & $\begin{array}{c}\Delta \mathrm{I}(63) \\
+\mathrm{O}_{2} / \text { w.E. }\end{array}$ & $\begin{array}{c}\mathrm{I}(\mathbf{8 7}) \\
-\mathrm{O}_{2} / \text { w.E. }\end{array}$ & $\begin{array}{c}\Delta \mathrm{I}(\mathbf{8 7}) \\
-\mathrm{O}_{2} / \text { w.E. }\end{array}$ & $\begin{array}{c}\mathrm{I}(87) \\
+\mathrm{O}_{2} / \text { w.E. }\end{array}$ & $\begin{array}{c}\Delta \mathrm{I}(87) \\
+\mathrm{O}_{2} / \mathrm{w} . \mathrm{E} .\end{array}$ \\
\hline 1101 & 50,32 & 861 & 51,3 & 4910 & 99,31 & 3926 & 95,99 \\
\hline 1023 & 63,67 & 860,7 & 45,93 & 4890 & 116,5 & 3968 & 87,04 \\
\hline 1613 & 59,46 & 1333 & 64,28 & 5852 & 181,3 & 4639 & 102,8 \\
\hline 1627 & 70,33 & 1402 & 71,17 & 5850 & 85,7 & 5075 & 180,7 \\
\hline 1585 & 55,27 & 1403 & 64,33 & 6178 & 155,1 & 5406 & 67,52 \\
\hline 1599 & 62,03 & 1366 & 41,48 & 6079 & 96,84 & 5316 & 113,6 \\
\hline 1532 & 60,57 & 1475 & 46,05 & 6481 & 76,36 & 5992 & 80,75 \\
\hline 1530 & 78,34 & 1394 & 64,12 & 6366 & 72,49 & 5980 & 107 \\
\hline 1529 & 48,51 & 1406 & 73,12 & 6181 & 79,06 & 5824 & 98,2 \\
\hline 1574 & 75,16 & 1326 & 69,99 & 6278 & 110,6 & 5030 & 118,6 \\
\hline 1513 & 87,51 & 1201 & 44,69 & 6293 & 112,5 & 5057 & 90,09 \\
\hline 1543 & 57,61 & 1484 & 54,66 & 6253 & 109,5 & 6038 & 72,66 \\
\hline
\end{tabular}

Tabelle 39: Meßwerte Kinetik $\{\mathrm{CH} 2 \mathrm{FCH} 2 \mathrm{OH}+\mathrm{F}\}+\{\mathrm{O} 2\}$ rel. zu 1,4-Dioxan; $328 \mathrm{~K}$

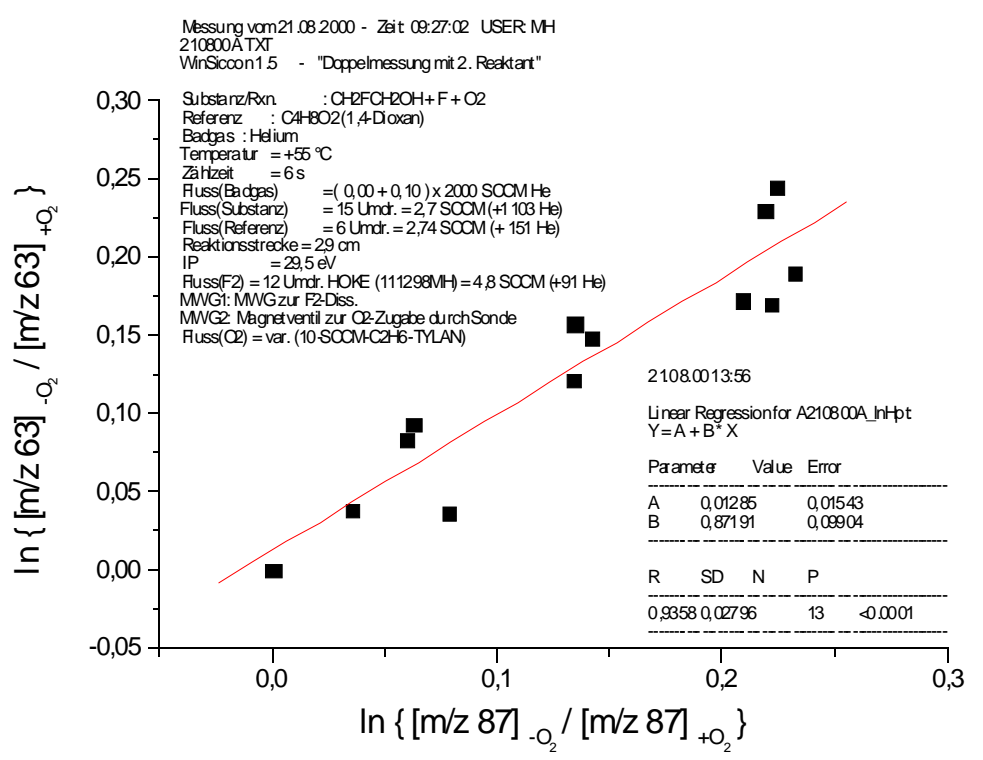

Abbildung 156: Kinetik $\{\mathrm{CH} 2 \mathrm{FCH} 2 \mathrm{OH}+\mathrm{F}\}+\{\mathrm{O} 2\}$ rel. zu 1,4-Dioxan; $328 \mathrm{~K}$

Aus der Steigung der Auftragung in Abbildung 156 läßt sich der relative Geschwindigkeitskoeffizient bei $\mathrm{T}=328 \mathrm{~K}$ ermitteln: $\mathrm{k}_{\mathrm{rel}}=(0,87 \pm 0,10)$. 
$\vartheta=+85^{\circ} \mathrm{C} ; \phi_{\mathrm{He}}=1600 \mathrm{SCCM} ; \phi_{\mathrm{CH} 2 \mathrm{FCH} 2 \mathrm{OH}}=2,7 \mathrm{SCCM} ; \phi_{1,4 \text {-Dioxan }}=2,7 \mathrm{SCCM} ; \mathrm{p}_{(\mathrm{StrR})}=221 \mathrm{~Pa}$; $\mathrm{s}=2,9 \mathrm{~cm} ; \mathrm{IP}=29,5 \mathrm{eV} ;$ Zählzeit $=6 \mathrm{~s}$

\begin{tabular}{|c|c|c|c|c|c|c|c|}
\hline $\begin{array}{c}\mathbf{I}(\mathbf{6 3}) \\
-\mathrm{O}_{2} / \text { w.E. }\end{array}$ & $\begin{array}{c}\Delta \mathbf{I}(\mathbf{6 3}) \\
-\mathrm{O}_{2} / \text { w.E. }\end{array}$ & $\begin{array}{c}\mathbf{I}(63) \\
+\mathrm{O}_{2} / \text { w.E. }\end{array}$ & $\begin{array}{c}\Delta \mathrm{I}(\mathbf{6 3}) \\
+\mathrm{O}_{2} / \text { w.E. }\end{array}$ & $\begin{array}{c}\mathbf{I}(\mathbf{8 7}) \\
-\mathbf{O}_{2} / \text { w.E. }\end{array}$ & $\begin{array}{c}\Delta \mathbf{I}(\mathbf{8 7}) \\
-\mathrm{O}_{2} / \mathbf{w . E .}\end{array}$ & $\begin{array}{c}\mathbf{I}(87) \\
+\mathrm{O}_{2} / \text { w.E. }\end{array}$ & $\begin{array}{c}\Delta \mathrm{I}(\mathbf{8 7}) \\
+\mathrm{O}_{2} / \text { w.E. }\end{array}$ \\
\hline 1716 & 54,62 & 1425 & 69,78 & 5350 & 74,96 & 4401 & 100,1 \\
\hline 1737 & 31,61 & 1455 & 48,19 & 5439 & 125,1 & 4403 & 103,4 \\
\hline 1721 & 40,97 & 1457 & 46,26 & 5340 & 104,9 & 4355 & 81,25 \\
\hline 1731 & 54,6 & 1518 & 69,48 & 5039 & 85,83 & 4299 & 80,2 \\
\hline 1705 & 46,52 & 1570 & 52,92 & 4842 & 108,1 & 4459 & 87,44 \\
\hline 1672 & 63,32 & 1562 & 51,55 & 4852 & 164,1 & 4491 & 70,63 \\
\hline 1683 & 52,79 & 1557 & 66,77 & 4860 & 111,7 & 4524 & 80,9 \\
\hline 1627 & 39,95 & 1387 & 58,7 & 4672 & 93,59 & 4039 & 109,6 \\
\hline 1496 & 69,79 & 1391 & 68,71 & 4518 & 94,57 & 4139 & 69,63 \\
\hline
\end{tabular}

Tabelle 40: Meßwerte Kinetik $\{\mathrm{CH} 2 \mathrm{FCH} 2 \mathrm{OH}+\mathrm{F}\}+\{\mathrm{O} 2\}$ rel. zu 1,4-Dioxan; $358 \mathrm{~K}$

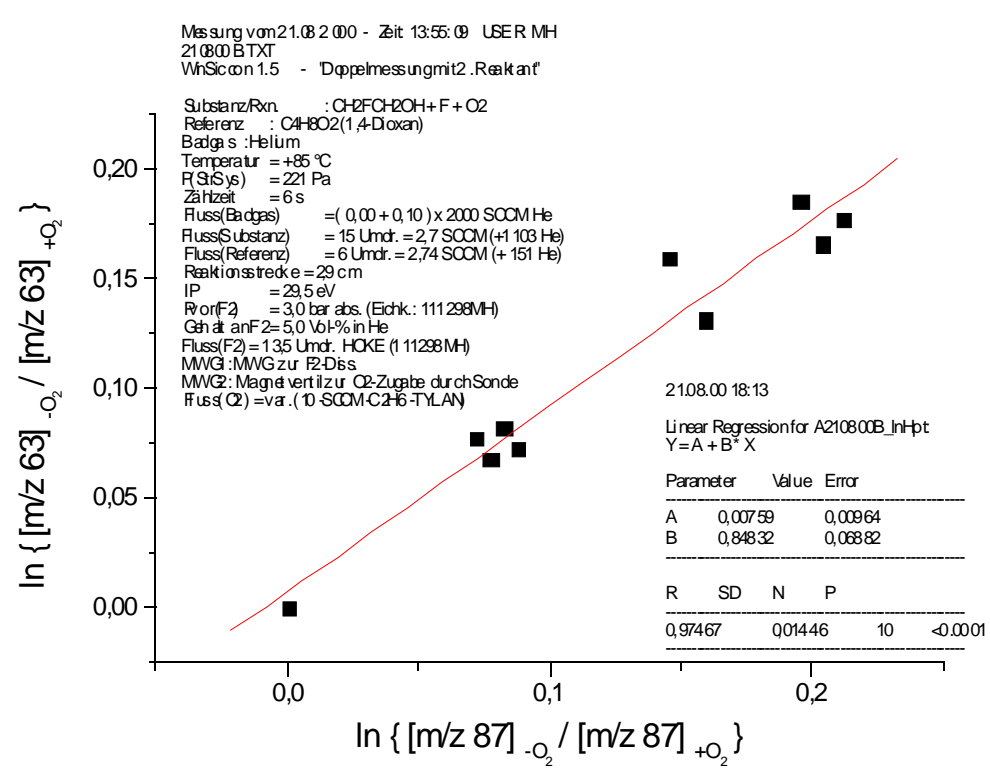

Abbildung 157: Kinetik $\{C H 2 F C H 2 O H+F\}+\{O 2\}$ rel. zu 1,4-Dioxan; $358 K$

Aus der Steigung der Auftragung in Abbildung 157 läßt sich der relative Geschwindigkeitskoeffizient bei $\mathrm{T}=358 \mathrm{~K}$ ermitteln: $\mathrm{k}_{\mathrm{rel}}=(0,87 \pm 0,10)$. 
Aus den Ergebnissen der Abbildungen 153 bis 157 kann eine Arrhenius-Auftragung gemäß Gleichung (11) (S. 10) angefertigt und mit den Geschwindigkeitskoeffizienten der bekannten Referenzreaktion ${ }^{137} \mathrm{k}_{\mathrm{ref}}(\mathrm{T})$ die absoluten Geschwindigkeitskoeffizienten berechnet werden.

$\mathrm{k}_{\mathrm{ref}}(\mathrm{T})=(2,5 \pm 1,0) \cdot 10^{11} \cdot \exp \{-(-3,9 \pm 1,2) \mathrm{kJ} / \mathrm{mol} /(\mathrm{R} \cdot \mathrm{T})\} \mathrm{cm}^{3} /(\mathrm{mol} \cdot \mathrm{s})$

Die Ergebnisse sind in Tabelle 41 eingetragen, die Auftragung ist in Abbildung 158 zu sehen.

\begin{tabular}{|c|c|c|c|c|c|c|c|}
\hline $\begin{array}{l}\vartheta / \\
{ }^{\circ} \mathbf{C}\end{array}$ & $\mathbf{T}^{-1} / \mathbf{K}^{-1}$ & $\mathbf{k}_{\text {rel }}$ & $\ln \left\{k_{\text {rel }}\right\}$ & $\begin{array}{c}\mathbf{k}_{\text {bimolek. }} / \\
\mathbf{c m}^{3} /(\mathbf{m o l ~ s})\end{array}$ & $\begin{array}{c}\ln \left\{\mathbf{k}_{\text {bimolek. }} /\right. \\
\left.\left(\mathbf{c m}^{3} /(\mathbf{m o l} \mathbf{s})\right)\right\}\end{array}$ & $\begin{array}{c}{[\mathrm{He}] /} \\
\mathrm{mol} / \mathrm{cm}^{3}\end{array}$ & $\begin{array}{c}\mathbf{k}_{\text {termolek. }} / \\
\mathbf{c m}^{6} /\left(\mathbf{m o l}^{2} \mathbf{s}\right)\end{array}$ \\
\hline-15 & 0,00387 & 1,0564 & 0,0548 & $1,63 \mathrm{E}+012$ & 28,1177 & 7,9E-08 & $2,05 \mathrm{E}+19$ \\
\hline 5 & 0,00360 & 0,8864 & $-0,1206$ & $1,20 \mathrm{E}+012$ & 27,8115 & 7,7E-08 & $1,56 \mathrm{E}+19$ \\
\hline 25 & 0,00333 & 1,0232 & 0,0229 & $1,23 \mathrm{E}+012$ & 27,8418 & $6,8 \mathrm{E}-08$ & $1,81 \mathrm{E}+19$ \\
\hline 55 & 0,00305 & 0,8719 & $-0,1371$ & $9,11 \mathrm{E}+011$ & 27,5378 & $6,6 \mathrm{E}-08$ & $1,38 \mathrm{E}+19$ \\
\hline 85 & 0,00279 & 0,8483 & $-0,1645$ & $7,86 \mathrm{E}+011$ & 27,3905 & $7,4 \mathrm{E}-08$ & $1,06 \mathrm{E}+19$ \\
\hline
\end{tabular}

Tabelle 41: Werte für Arrhenius-Auftragung sowie $k(T)$ für $\{C H 2 F C H 2 O H+F\}+\{O 2\}$ rel. zu 1,4-Dioxan

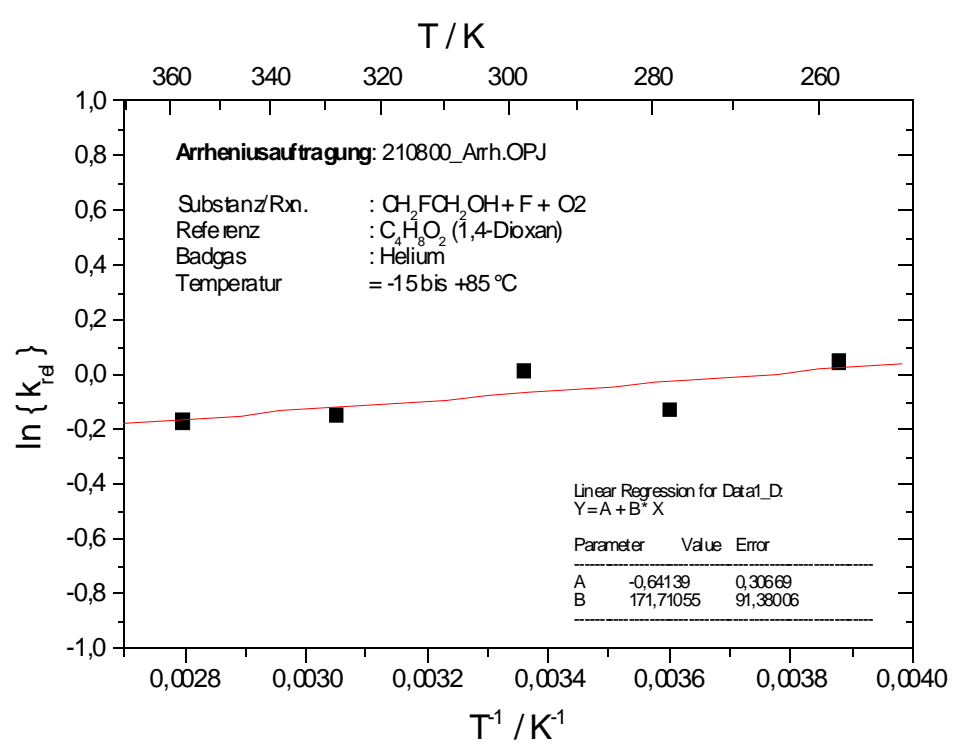

Abbildung 158: Arrhenius $\{\mathrm{CH} 2 \mathrm{FCH} 2 \mathrm{OH}+\mathrm{F}\}+\{\mathrm{O} 2\}$ rel. zu 1,4-Dioxan

Die Aktivierungsenergie der hier untersuchten Reaktion erhält man durch Vergleich der Steigung aus Abbildung 158 mit dem Ausdruck in Gleichung (27) (S. 116):

$E_{a}=E_{a, \text { Referenz }}-($ Steigung $) \cdot R=(-3900-(171,71 \cdot 8,314)) \mathrm{J} / \mathrm{mol}=-5328 \mathrm{~J} / \mathrm{mol}$.

Bezüglich des präexponentiellen Faktors wird der Achsenabschnitt aus Abbildung 118 herangezogen: 
$\mathrm{A}=\exp ($ Achsenabschnitt $) \cdot A_{\text {Referenz }}=\exp (-0,64139) \cdot 2,5 \cdot 10^{11} \mathrm{~cm}^{3} \mathrm{~mol}^{-1} \mathrm{~s}^{-1}$

$$
=1,32 \cdot 10^{11} \mathrm{~cm}^{3} \mathrm{~mol}^{-1} \mathrm{~s}^{-1} \text {. }
$$

Im Ergebnis beträgt der gesuchte Geschwindigkeitskoeffizient damit:

$$
k(T)=(1,3 \pm 0,6) \cdot 10^{11} \cdot \exp \{-(-5,3 \pm 1,4) \mathrm{kJ} / \mathrm{mol} /(\mathrm{R} \cdot \mathrm{T})\} \mathrm{cm}^{3} /(\mathrm{mol} \cdot \mathrm{s}) .
$$

für: $\left\{\right.$ Radikal aus der Reaktion $\left.\mathrm{CH}_{2} \mathrm{FCH}_{2} \mathrm{OH}+\mathrm{F}\right\}+\left\{\mathrm{O}_{2}\right\} \rightarrow$ Produkte

Mit $\mathrm{T}=298 \mathrm{~K}$ errechnet man daraus: $\mathbf{k}(298 \mathrm{~K})=(\mathbf{1 , 1} \pm \mathbf{0 , 9}) \cdot \mathbf{1 0}^{\mathbf{1 2}} \mathbf{c m}^{\mathbf{3}} \mathbf{m o l}^{-\mathbf{1}} \mathbf{s}^{-1}$.

Weil Assoziationsreaktionen mit $\mathrm{O}_{2}$ üblicherweise keine Energiebarriere aufweisen, wird zudem eine Auftragung gemäß Gleichung (13) (S. 11) angefertigt ( Abbildung 159)

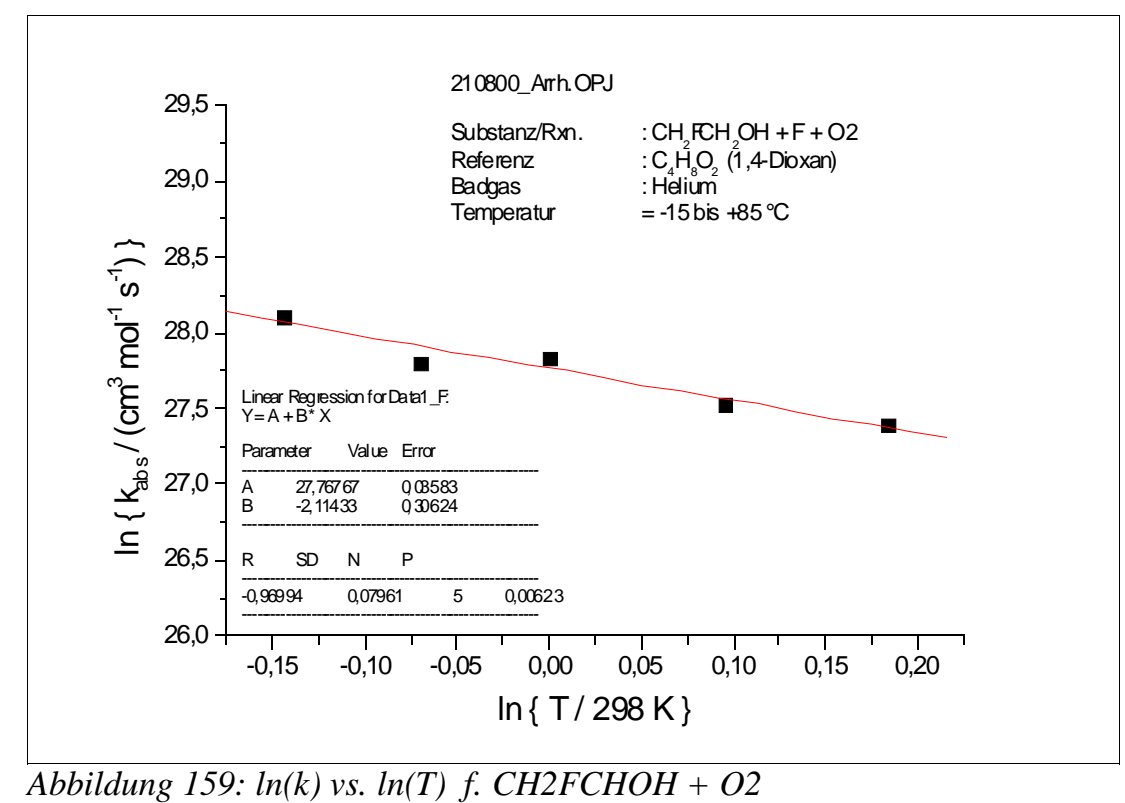

Aus den Ergebnissen der Abbildung 159 berechnet man:

$$
\begin{aligned}
& \mathbf{k}(\mathbf{T})=(\mathbf{1}, 15 \pm \mathbf{0 , 0 4}) \cdot 10^{12} \cdot(\mathbf{T} / 298 \mathrm{~K})^{-(2,1 \pm 0,3)} \mathbf{c m}^{3} /(\mathbf{m o l} \cdot \mathbf{s}) \\
& \text { für: }\left\{\text { Radikal aus der Reaktion } \mathrm{CH}_{2} \mathrm{FCH}_{2} \mathrm{OH}+\mathrm{F}\right\}+\left\{\mathrm{O}_{2}\right\} \rightarrow \text { Produkte }
\end{aligned}
$$

Mit $\mathrm{T}=298 \mathrm{~K}$ errechnet man daraus: $\mathbf{k}(298 \mathrm{~K})=(\mathbf{1 , 1 5} \pm \mathbf{0 , 0 4}) \cdot \mathbf{1 0}^{\mathbf{1 2}} \mathbf{c m}^{\mathbf{3}} \mathbf{m o l}^{-1} \mathbf{s}^{-1}$.

Das Ergebnis wird hier (entsprechend der Referenzreaktion) als Geschwindigkeitskoeffizient einer bimolekularen Reaktion angegeben; für eine termolekulare ist es in Tabelle 41 vermerkt. 


\subsubsection{Produkte der Reaktion des erzeugten Radikals mit Stickstoffmonoxid}

Um einen Überblick über die Änderungen im Massenspektrum zu erhalten, sind mit der in Kapitel 3.1.1 (S. 16) beschriebenen Apparatur unmittelbar nacheinander EI-Übersichtsspektren unter Zugabe und Abwesenheit von Stickstoffmonoxid (Fluor-Atome wurden stets gleichermaßen zugegeben) aufgenommen worden. Dabei zeigten sich insbesondere starke Zunahmen auf $\mathrm{m} / \mathrm{z}$ 30, 31, 62 und 63.

Es wurden anschließend gezielt in Einzelzählung (6 sek Zählzeit, IP = 29,5 eV) die m/z-Werte 63 und 93 untersucht. Die Durchführung der Messungen ist in Kapitel 3.5.1 (S. 50) beschrieben. Die Reaktionsbedingungen und Ergebnisse sind in Abbildung 160 dargestellt.

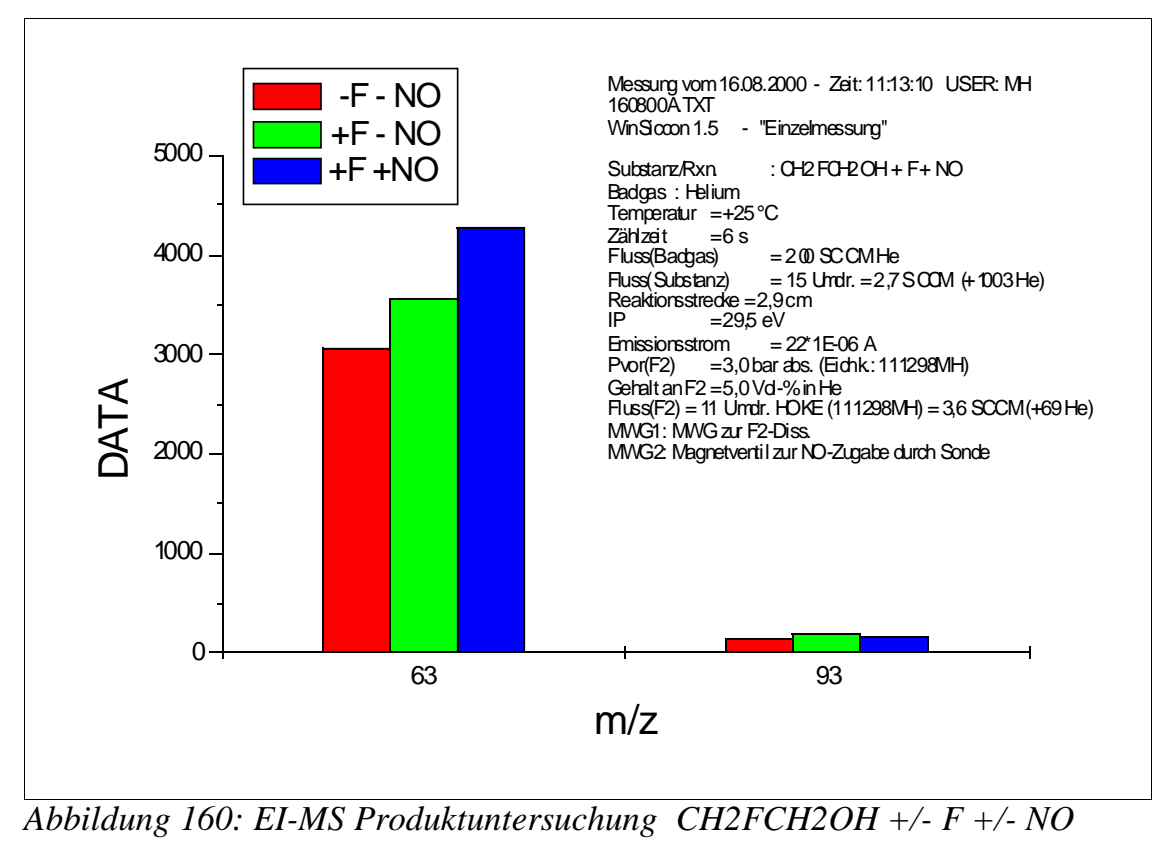

Auch in der Einzelmessung (Abb. 160) ergab sich ein Anstieg der Intensität auf m/z 63, d.h. der Molekülmasse des Radikals. Es wird vermutet, daß in der Reaktion des Radikals mit NO ein Komplex gebildet wird, der in der EI-Ionenquelle besser ionisiert werden kann als das Alkohol-Radikal und als Fragment m/z 63 ansteigen läßt. Auf der Molekülmasse m/z 93 des mutmaßlich gebildeten Komplexes wurde keine Zunahme beobachtet.

Aus diesen Gründen war eine Kinetik-Messung für die Reaktion des Alkohol-Radikals mit NO im EI-Massenspektrometer nicht möglich. 


\subsubsection{Diskussion der Ergebnisse}

Der ermittelte Geschwindigkeitskoeffizient für die Reaktion des Alkohols $\mathrm{CFH}_{2} \mathrm{CH}_{2} \mathrm{OH}$ mit Fluor-Atomen ist mit $\mathrm{k}(298 \mathrm{~K})=1,17 \cdot 10^{14} \mathrm{~cm}^{3} \mathrm{~mol}^{-1} \mathrm{~s}^{-1}$ höher als die der übrigen bisher untersuchten strukturell sehr ähnlichen fluorierten Alkohole. Für $\mathrm{CF}_{3} \mathrm{CH}_{2} \mathrm{OH}$ hatte sich $\mathrm{k}(298$ $\mathrm{K})=6,27 \cdot 10^{13} \mathrm{~cm}^{3} \mathrm{~mol}^{-1} \mathrm{~s}^{-1}$ und für $\mathrm{CHF}_{2} \mathrm{CH}_{2} \mathrm{OH} \mathrm{k}(299 \mathrm{~K})=6,92 \cdot 10^{13} \mathrm{~cm}^{3} \mathrm{~mol}^{-1} \mathrm{~s}^{-1}$ ergeben. Dies kann als Indiz für die Herabsetzung der Geschwindigkeitskoeffizienten bei zunehmender Zahl von Fluor-Substituenten angesehen werden. Allerdings ist der Geschwindigkeitskoeffizient damit auch höher als für die analoge Reaktion des Ethanols, welcher mit $\mathrm{k}=7,69 \cdot 10^{13} \mathrm{~cm}^{3} \mathrm{~mol}^{-1} \mathrm{~s}^{-1}$ angegeben wird ${ }^{138}$. Dafür kann keine befriedigende Erklärung gegeben werden.

Die Abstraktionsreaktion ist praktisch temperaturunabhängig (ähnlich wie bei der Reaktion $\left.\mathrm{CF}_{3} \mathrm{CH}_{2} \mathrm{OH}+\mathrm{F}\right)$.

Bei der Reaktion des erzeugten Radikals mit molekularem Sauerstoff wurde ein Geschwindigkeitskoeffizient von $\mathrm{k}(298 \mathrm{~K})=1,15 \cdot 10^{12} \mathrm{~cm}^{3} \mathrm{~mol}^{-1} \mathrm{~s}^{-1}$ gefunden. Dieser ist deutlich kleiner als derjenige der analogen Reaktion mit $\mathrm{CH}_{3} \mathrm{CHOH}$, welcher mit $\mathrm{k}=1,15 \cdot 10^{13} \mathrm{~cm}^{3} \mathrm{~mol}^{-1} \mathrm{~s}^{-1}$ angegeben wird ${ }^{139}$. Allerdings ist er größer als derjenige für die analoge Reaktion mit $\mathrm{CF}_{3} \mathrm{CH}_{2} \mathrm{OH}$, der als $\mathrm{k}(298 \mathrm{~K})=2,4 \cdot 10^{10} \mathrm{~cm}^{3} \mathrm{~mol}^{-1} \mathrm{~s}^{-1}$ bestimmt und derjenige für $\mathrm{CHF}_{2} \mathrm{CH}_{2} \mathrm{OH}$, für den $\mathrm{k}(298 \mathrm{~K})=2,3 \cdot 10^{11} \mathrm{~cm}^{3} \mathrm{~mol}^{-1} \mathrm{~s}^{-1}$ gefunden worden war. Der gleiche Befund hatte sich auch beim Übergang des Dimethylethers hin $\mathrm{zu}$ fluorierten Ethern ergeben. Der Geschwindigkeitskoeffizient korreliert mit der Anzahl an elektronegativen Fluor-Substituenten; mit steigender Zahl Fluor-Atome in den hier untersuchten Alkoholen bzw. Ethern nimmt er ab.

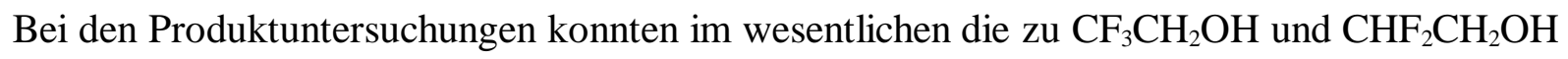
analogen Produkte identifiziert werden, was bei einer strukturell so ähnlichen Verbindung auch plausibel ist. Zusätzlich kam jedoch ein neuer Reaktionskanal für die Reaktion des Radikals mit $\mathrm{O}_{2}$ hinzu, der bedingt durch den Mechanismus zumindest für $\mathrm{CF}_{3} \mathrm{CH}_{2} \mathrm{OH}$ aber auch nicht denkbar ist. Er führt zu HCHO und FCHO. 


\subsubsection{Das 2,2,3,3,4,4,4-Heptafluorbutanol $\left(\mathrm{CF}_{3} \mathrm{CF}_{2} \mathrm{CF}_{2} \mathrm{CH}_{2} \mathrm{OH}\right)$}

\subsubsection{Kinetik der radikalerzeugenden Reaktion mit Fluor-Atomen}

Zu Beginn der Meßreihe wurden mit der in Kapitel 3.1.1 (S. 16) beschriebenen Apparatur EIÜbersichtsmassenspektren registriert.
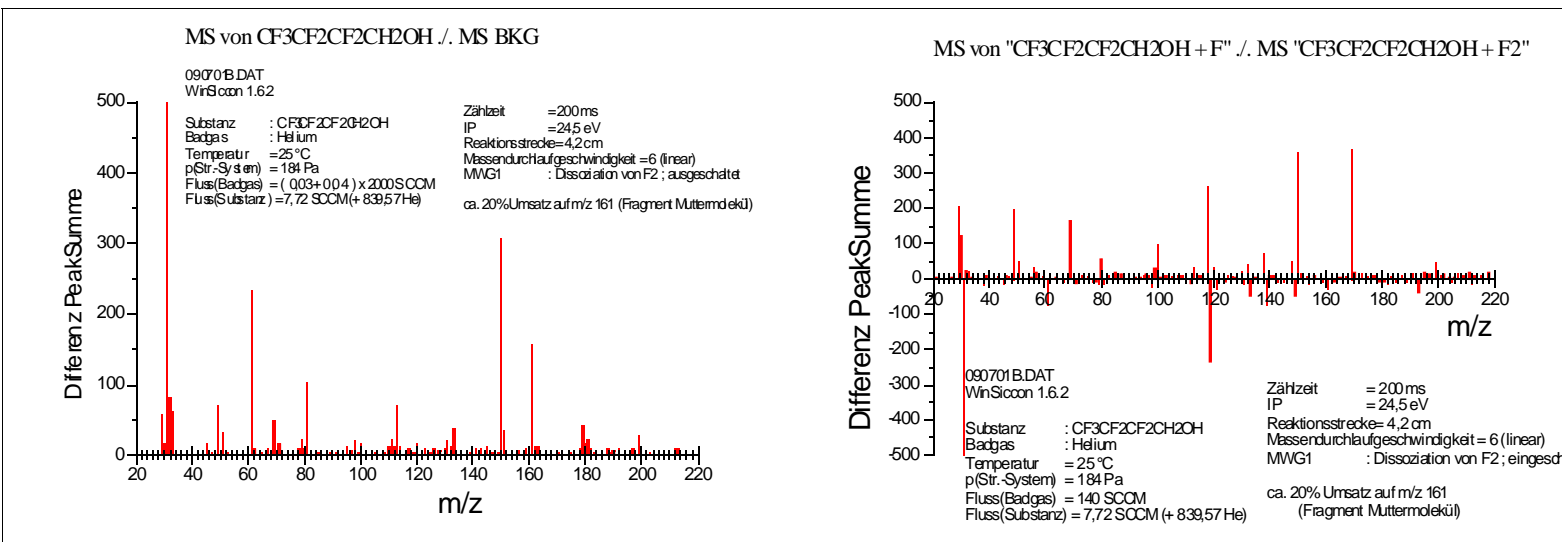

Abbildung 161: EI-MS von reinem CF3CF2CF2CH2OH sowie Differenzen bei Zugabe von F-Atomen

Die linke Grafik in Abbildung 161 zeigt das Massenspektrum von reinem $\mathrm{CF}_{3} \mathrm{CF}_{2} \mathrm{CF}_{2} \mathrm{CH}_{2} \mathrm{OH}$ $\left(M_{r}=200\right)$. Obwohl die EI-Elektronenenergie in diesem Spektrum auf IP $=24,5 \mathrm{eV}$ vermindert war, zeigte sich auf m/z 200 kein Molekül-Peak. Man erkennt jedoch kleinere Fragmente auf m/z 199 und 179 sowie größere auf m/z 161 und 150. In der rechten Grafik in Abbildung 161 ist das Differenz-Massenspektrum für die Bedingungen , $+\mathrm{F}^{“}$ abzüglich , $+\mathrm{F}_{2}{ }^{\text {“ }}$ abgebildet, das auf m/z 199 eine Zunahme zeigt. Letzteres ist auf die Bildung des Radikals $\mathrm{CF}_{3} \mathrm{CF}_{2} \mathrm{CF}_{2} \mathrm{CHOH}\left(\mathrm{M}_{\mathrm{r}}=199\right)$ oder $\mathrm{CF}_{3} \mathrm{CF}_{2} \mathrm{CF}_{2} \mathrm{CH}_{2} \mathrm{O}\left(\mathrm{M}_{\mathrm{r}}=199\right)$ zurückzuführen. Ferner sind Abnahmen auf m/z 161 und 150 zu erkennen. Dem Fragment des Edukts $\mathrm{CF}_{3} \mathrm{CF}_{2} \mathrm{CF}_{2} \mathrm{CH}_{2} \mathrm{OH}$ (Summenformel: $\mathrm{C}_{4} \mathrm{H}_{3} \mathrm{~F}_{7} \mathrm{O}$ ) auf $\mathrm{m} / \mathrm{z} 161$ kommt die Summenformel $\mathrm{C}_{4} \mathrm{H}_{2} \mathrm{~F}_{5} \mathrm{O}$ zu, während m/z 150 durch $\mathrm{C}_{3} \mathrm{~F}_{6}$ erklärt werden kann. Für die Messung des Umsatzes des Alkohols $\mathrm{CF}_{3} \mathrm{CF}_{2} \mathrm{CF}_{2} \mathrm{CH}_{2} \mathrm{OH}$ bei Zugabe von Fluor-Atomen scheint daher das Fragment mit der höchsten Zahl an H-Atomen auf m/z 161 am besten geeignet.

Die Kinetik-Messungen wurden in der in Kap. 3.1.1 (S. 16) beschriebenen Apparatur unter Einsatz des Strömungsreaktors aus Abbildung 5 (S. 17) durchgeführt. Die Ausführung und Auswertung nach der Relativmethode ist in Kap. 3.5.1 (S. 50) beschrieben.

Methan $\left(\mathrm{M}_{\mathrm{r}}=16\right)$ diente als Referenzsubstanz. Der Umsatz des Alkohols $\mathrm{CF}_{3} \mathrm{CF}_{2} \mathrm{CF}_{2} \mathrm{CH}_{2} \mathrm{OH}$ konnte anhand der Intensitätsänderung seines Fragment-Peaks m/z 161 und der von Methan anhand seines Molekül-Peaks m/z 16 bestimmt werden. 
$\vartheta=-15^{\circ} \mathrm{C} ; \phi_{\mathrm{He}}=735 \mathrm{SCCM} ; \phi_{\mathrm{CF} 3 \mathrm{CF} 2 \mathrm{CF} 2 \mathrm{CH} 2 \mathrm{OH}}=3,8 \mathrm{SCCM} ; \phi_{\mathrm{CH} 4}=3,7 \mathrm{SCCM} ; \mathrm{p}_{(\mathrm{StrR})}=127 \mathrm{~Pa} ;$ $\mathrm{s}=3,1 \mathrm{~cm} ; \mathrm{IP}=28 \mathrm{eV}$; Zählzeit $=10 \mathrm{~s}$

\begin{tabular}{|r|r|r|r|r|r|r|r|}
\hline \multicolumn{1}{|c|}{$\mathbf{I}(\mathbf{1 6 1})$} & $\Delta \mathbf{I}(\mathbf{1 6 1})$ & $\begin{array}{c}\mathbf{I}(\mathbf{1 6 1}) \\
\text { +F / w.E. }\end{array}$ & $\begin{array}{c}\Delta \mathbf{I}(\mathbf{1 6 1}) \\
\text { +F / w.E. }\end{array}$ & $\begin{array}{c}\mathbf{I}(\mathbf{1 6}) \\
\text {-F / w.E. }\end{array}$ & $\begin{array}{c}\Delta \mathbf{I}(\mathbf{1 6}) \\
\text {-F / w.E. }\end{array}$ & $\begin{array}{c}\mathbf{I}(\mathbf{1 6}) \\
\text { +F / w.E. }\end{array}$ & $\begin{array}{c}\Delta \mathbf{I}(\mathbf{1 6}) \\
\text { +F / w.E. }\end{array}$ \\
\hline 4650 & 50,87 & 4036 & 43,69 & 101300 & 386,2 & 73490 & 335,1 \\
\hline 4718 & 55,75 & 4235 & 64,3 & 91610 & 2859 & 68970 & 847 \\
\hline 4804 & 22,9 & 3843 & 54,64 & 101300 & 345,2 & 69840 & 445,9 \\
\hline 4695 & 43,31 & 3915 & 25,93 & 99300 & 558,7 & 68260 & 780,9 \\
\hline 4705 & 73,14 & 4276 & 90,29 & 103600 & 369,1 & 86990 & 224,6 \\
\hline 4634 & 49,19 & 4350 & 42,08 & 102300 & 1582 & 82550 & 581,8 \\
\hline 4748 & 56,86 & 4365 & 23,88 & 102600 & 764,4 & 87060 & 546,1 \\
\hline
\end{tabular}

Tabelle 42: Meßwerte Kinetik CF3CF2CF2CH2OH + F rel. zu CH4; $258 \mathrm{~K}$

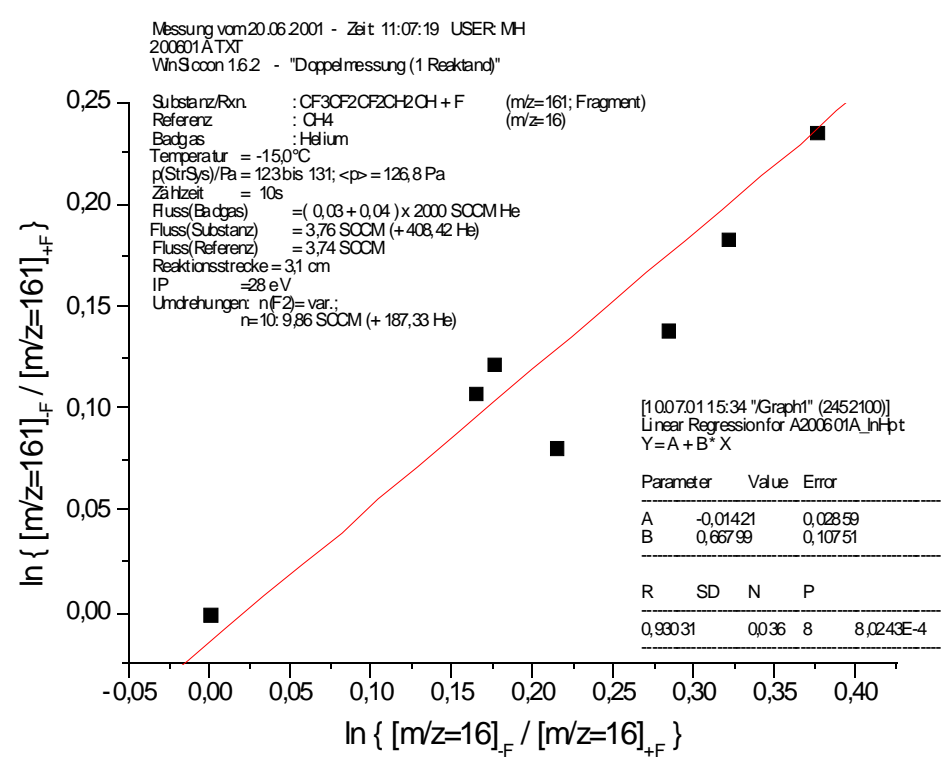

Abbildung 162: Kinetik CF3CF2CF2CH2OH + F rel. zu CH4; $258 \mathrm{~K}$

Aus der Steigung der Auftragung in Abbildung 162 erhält man den relativen

Geschwindigkeitskoeffizienten $\mathrm{k}_{\mathrm{rel}}=(0,67 \pm 0,11)$. 
$\vartheta=+25^{\circ} \mathrm{C} ; \phi_{\mathrm{He}}=679 \mathrm{SCCM} ; \phi_{\mathrm{CF} 3 \mathrm{CF} 2 \mathrm{CF} 2 \mathrm{CH} 2 \mathrm{OH}}=3,8 \mathrm{SCCM} ; \phi_{\mathrm{CH} 4}=3,7 \mathrm{SCCM} ; \mathrm{p}_{(\mathrm{StrR})}=134 \mathrm{~Pa} ;$ $\mathrm{s}=2,6 \mathrm{~cm} ; \mathrm{IP}=29,5 \mathrm{eV}$; Zählzeit = $10 \mathrm{~s}$

\begin{tabular}{|c|c|c|c|c|c|c|c|}
\hline $\begin{array}{c}\text { I(161) } \\
-F / \text { w.E. }\end{array}$ & $\begin{array}{l}\Delta \mathrm{I}(\mathbf{1 6 1}) \\
\text {-F / w.E. }\end{array}$ & $\begin{array}{c}\text { I(161) } \\
+\mathrm{F} / \mathrm{w.E} . \\
\end{array}$ & $\begin{array}{r}\Delta \mathrm{I}(161) \\
+\mathrm{F} / \mathrm{w.E} .\end{array}$ & $\begin{array}{c}\text { I(16) } \\
-F / \text { w.E. }\end{array}$ & $\begin{array}{c}\Delta \mathrm{I}(16) \\
\text {-F / w.E. }\end{array}$ & $\begin{array}{c}\mathrm{I}(16) \\
+\mathrm{F} / \mathrm{w.E} .\end{array}$ & $\begin{array}{c}\Delta \mathrm{I}(16) \\
+\mathrm{F} / \mathrm{w.E} .\end{array}$ \\
\hline 8701 & 36,03 & 7700 & 149,8 & 129400 & 728,6 & 102900 & 350 \\
\hline 8531 & 98,21 & 7316 & 65,67 & 121300 & 1161 & 94100 & 1630 \\
\hline 8267 & 122,3 & 7381 & 87,43 & 122600 & 1841 & 103000 & 1195 \\
\hline 7970 & 112,7 & 7277 & 34,94 & 126600 & 1050 & 103300 & 1030 \\
\hline 8166 & 96,51 & 7623 & 66,93 & 129600 & 1050 & 111000 & 534,6 \\
\hline 8187 & 113,3 & 7619 & 129,8 & 130700 & 451,8 & 111000 & 756,1 \\
\hline 8087 & 84,48 & 7740 & 49 & 131300 & 451,8 & 120600 & 728,6 \\
\hline 8069 & 84,1 & 7803 & 103,2 & 133300 & 451,8 & 121600 & 728,6 \\
\hline 7846 & 54,86 & 7800 & 50,54 & 131100 & 833,2 & 128700 & 880,8 \\
\hline 7979 & 84,59 & 7877 & 68,05 & 134900 & 405,7 & 130600 & 499,7 \\
\hline
\end{tabular}

Tabelle 43: Meßwerte Kinetik CF3CF2CF2CH2OH + F rel. zu CH4; $298 \mathrm{~K}$

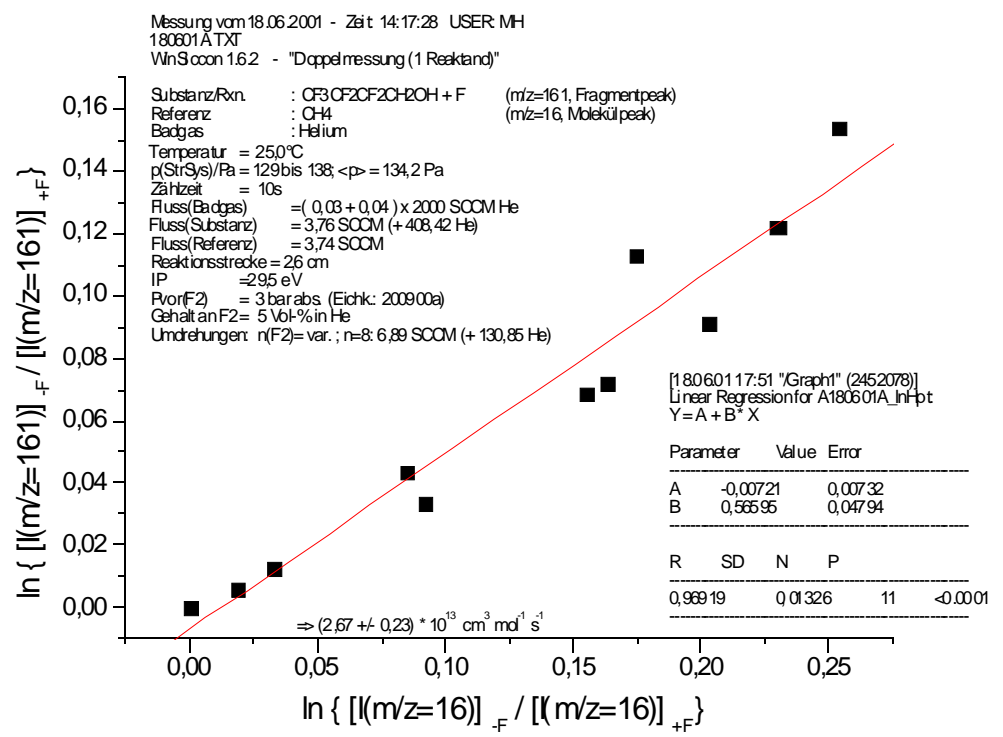

Abbildung 163: Kinetik CF3CF2CF2CH2OH + F rel. zu CH4; $298 \mathrm{~K}$

Aus der Steigung der Auftragung in Abbildung 163 erhält man den relativen

Geschwindigkeitskoeffizienten $\mathrm{k}_{\text {rel }}=(0,57 \pm 0,05)$. 
$\vartheta=+65{ }^{\circ} \mathrm{C} ; \phi_{\mathrm{He}}=736 \mathrm{SCCM} ; \phi_{\mathrm{CF} 3 \mathrm{CF} 2 \mathrm{CF} 2 \mathrm{CH} 2 \mathrm{OH}}=3,8 \mathrm{SCCM} ; \phi_{\mathrm{CH} 4}=3,7 \mathrm{SCCM} ; \mathrm{p}_{(\mathrm{StrR})}=140 \mathrm{~Pa} ;$ $\mathrm{s}=3,0 \mathrm{~cm} ; \mathrm{IP}=28 \mathrm{eV}$; Zählzeit $=5 \mathrm{~s}$

\begin{tabular}{|c|c|c|c|c|c|c|c|}
\hline $\begin{array}{c}\text { I(161) } \\
- \text {-F / w.E. }\end{array}$ & $\begin{array}{c}\Delta \mathbf{I}(\mathbf{1 6 1}) \\
-\mathrm{F} / \mathrm{w} . \mathrm{E} .\end{array}$ & $\begin{array}{c}\mathrm{I}(161) \\
+\mathrm{F} / \mathrm{w.E} .\end{array}$ & $\begin{array}{c}\Delta \mathrm{I}(161) \\
+\mathrm{F} / \mathrm{w} . \mathrm{E} .\end{array}$ & $\begin{array}{c}\mathbf{I}(16) \\
-\mathrm{F} / \mathrm{w.E} .\end{array}$ & $\begin{array}{c}\Delta \mathbf{I}(\mathbf{1 6}) \\
-\mathrm{F} / \mathrm{w} . \mathbf{E} .\end{array}$ & $\begin{array}{c}\mathrm{I}(16) \\
+\mathrm{F} / \mathrm{w.E.}\end{array}$ & $\begin{array}{c}\Delta \mathrm{I}(16) \\
+\mathrm{F} / \mathrm{w.E} .\end{array}$ \\
\hline 2423 & 56,9 & 2184 & 67,82 & 42280 & 796,4 & 34530 & 385,7 \\
\hline 2173 & 51,01 & 2003 & 67,22 & 43340 & 117,7 & 36430 & 130,7 \\
\hline 2115 & 136,3 & 2037 & 54,75 & 42130 & 317,9 & 36790 & 159,6 \\
\hline 1935 & 53,15 & 1845 & 61,6 & 42900 & 196,4 & 37970 & 165,2 \\
\hline 1925 & 61,93 & 1783 & 53,81 & 41410 & 366,7 & 36260 & 330,4 \\
\hline 1872 & 53,99 & 1840 & 60,55 & 41340 & 250,3 & 38740 & 263,6 \\
\hline 1624 & 63,62 & 1602 & 59,78 & 42790 & 402,1 & 40320 & 120,5 \\
\hline 1886 & 64,89 & 1845 & 71,96 & 40990 & 622,5 & 37300 & 354,9 \\
\hline 1882 & 58,43 & 1822 & 65,49 & 44010 & 334,3 & 39710 & 108,9 \\
\hline 1855 & 58,63 & 1777 & 58,26 & 44380 & 253,9 & 38620 & 133,9 \\
\hline 1883 & 54,56 & 1729 & 54,48 & 42300 & 544,3 & 35410 & 737,7 \\
\hline
\end{tabular}

Tabelle 44: : Meßwerte Kinetik CF3CF2CF2CH2OH + F rel. zu CH4; $338 \mathrm{~K}$

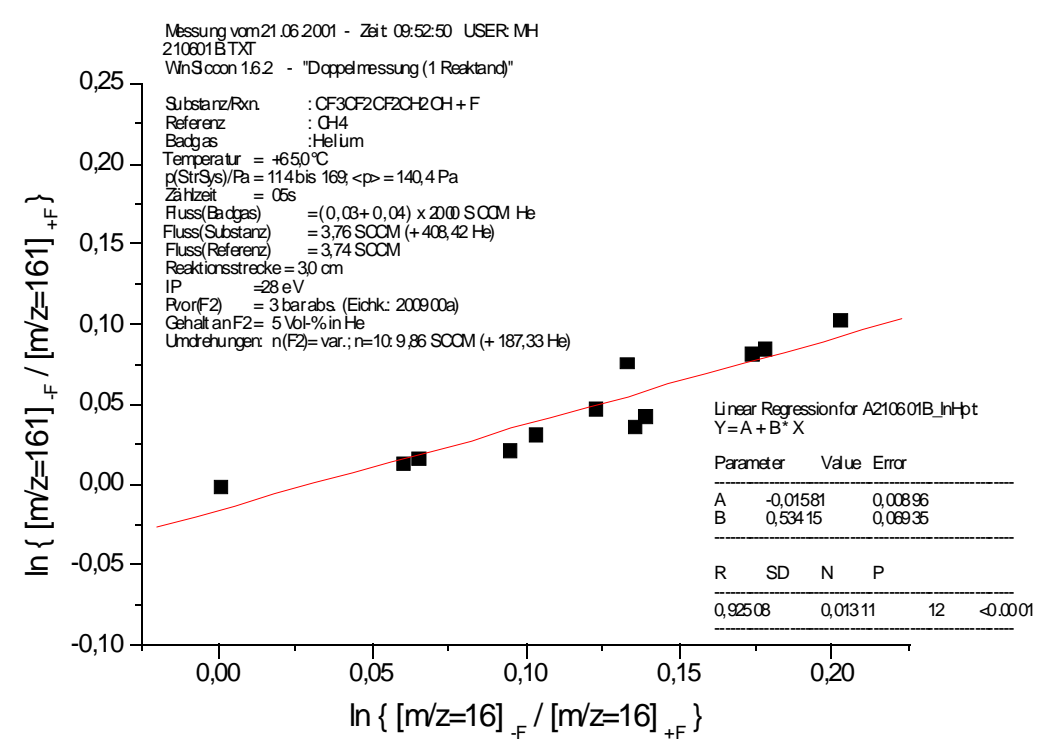

Abbildung 164: Kinetik CF3CF2CF2CH2OH + F rel. zu CH4; $338 \mathrm{~K}$

Aus der Steigung der Auftragung in Abbildung 164 erhält man den relativen

Geschwindigkeitskoeffizienten $\mathrm{k}_{\mathrm{rel}}=(0,53 \pm 0,07)$. 
Aus den Ergebnissen der Abbildungen 162 bis 164 kann eine Arrhenius-Auftragung gemäß Gleichung (11) (S. 10) angefertigt und mit den Geschwindigkeitskoeffizienten der bekannten Referenzreaktion ${ }^{140} \mathrm{k}_{\mathrm{ref}}(\mathrm{T})$ die absoluten Geschwindigkeitskoeffizienten berechnet werden.

$\mathrm{k}_{\mathrm{ref}}(\mathrm{T})=(1,81) \cdot 10^{14} \cdot \exp \{-(3,33) \mathrm{kJ} / \mathrm{mol} /(\mathrm{R} \cdot \mathrm{T})\} \mathrm{cm}^{3} /(\mathrm{mol} \cdot \mathrm{s})$

Die Ergebnisse sind in Tabelle 45 eingetragen, die Auftragung ist in Abbildung $165 \mathrm{zu}$ sehen.

\begin{tabular}{|c|c|c|c|c|c|c|}
\hline $\begin{array}{l}\vartheta / \\
{ }^{\circ} \mathbf{C}\end{array}$ & $\mathbf{T} / \mathrm{K}$ & $\mathbf{T}^{-1} / \mathbf{K}^{-1}$ & $\mathbf{k}_{\text {rel }}$ & $\ln \left\{k_{\text {rel }}\right\}$ & $\begin{array}{c}\mathbf{k}_{\mathrm{CH} 2 \mathrm{FCH} 2 \mathrm{OH}+\mathrm{F}} / \\
\mathbf{c m}^{3} /(\mathbf{m o l ~ s})\end{array}$ & $\begin{array}{c}\ln \left\{\mathbf{k}_{\mathrm{CH} 2 \mathrm{FCH} 2 \mathrm{OH}+\mathrm{F}} /\right. \\
\left.\left(\mathbf{c m}^{3} /(\mathbf{m o l} \mathbf{s})\right)\right\}\end{array}$ \\
\hline-15 & 258,15 & $3,870 \mathrm{E}-03$ & 0,6680 & $-0,4035$ & $2,56 \mathrm{E}+13$ & 30,8745 \\
\hline 25 & 298,15 & $3,350 \mathrm{E}-03$ & 0,5660 & $-0,5693$ & $2,67 \mathrm{E}+13$ & 30,9169 \\
\hline 65 & 338,15 & $2,960 \mathrm{E}-03$ & 0,5342 & $-0,6271$ & $2,96 \mathrm{E}+13$ & 31,0180 \\
\hline
\end{tabular}

Tabelle 45: $k(T)$ sowie Werte der Arrhenius-Auftragung f. CF3CF2CF2CH2OH $+\mathrm{F}$

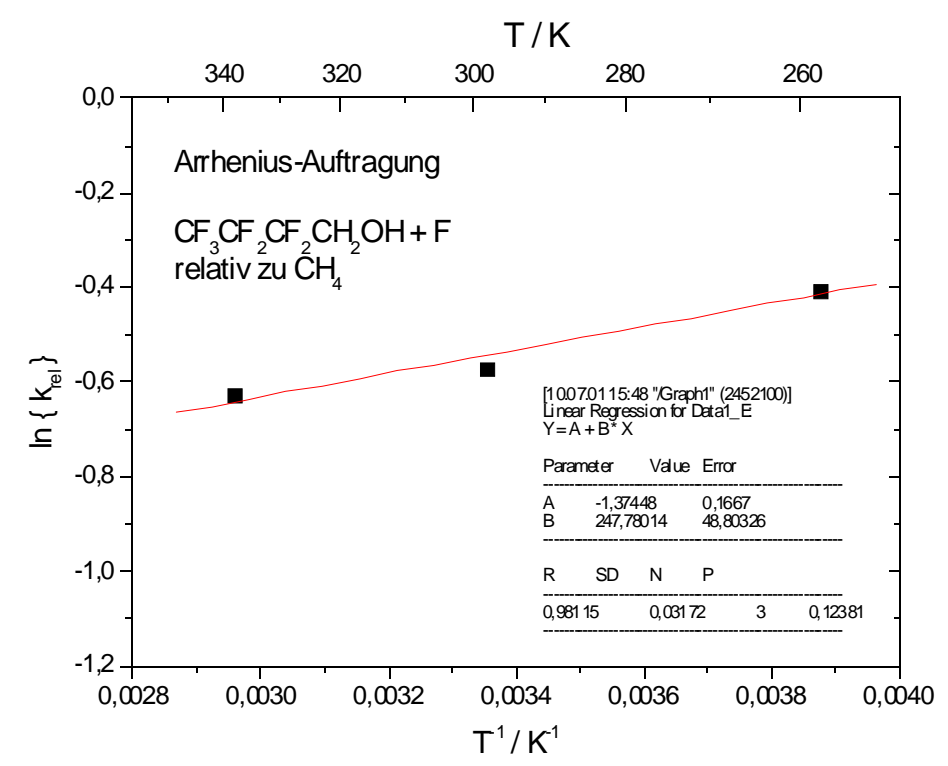

Abbildung 165: Arrhenius-Auftragung f. CF3CF2CF2CH2OH + F rel. zu CH4

Die Aktivierungsenergie der hier untersuchten Reaktion erhält man durch Vergleich der Steigung aus Abbildung 165 mit dem Ausdruck in Gleichung (27) (S. 116):

$\mathrm{E}_{\mathrm{a}}=\mathrm{E}_{\mathrm{a}, \text { Referenz }}-($ Steigung $) \cdot \mathrm{R}=(3330-(247,78 \cdot 8,314)) \mathrm{J} / \mathrm{mol}=+1269,96 \mathrm{~J} / \mathrm{mol}$.

Bezüglich des präexponentiellen Faktors wird der Achsenabschnitt aus Abbildung 165 herangezogen:

$A=\exp ($ Achsenabschnitt $) \cdot A_{\text {Referenz }}=\exp (-1,37448) \cdot 1,81 \cdot 10^{14} \mathrm{~cm}^{3} \mathrm{~mol}^{-1} \mathrm{~s}^{-1}$ 


$$
=4,58 \cdot 10^{13} \mathrm{~cm}^{3} \mathrm{~mol}^{-1} \mathrm{~s}^{-1} .
$$

Im Ergebnis beträgt der gesuchte Geschwindigkeitskoeffizient damit:

$$
\begin{aligned}
& \mathbf{k}(\mathbf{T})=(4,6 \pm 0,8) \cdot 10^{13} \cdot \exp \{-(1,27 \pm 0,41) \mathrm{kJ} / \mathrm{mol} /(\mathbf{R} \cdot \mathbf{T})\} \mathbf{c m}^{3} /(\mathbf{m o l} \cdot \mathbf{s}) \\
& \text { für: } \mathrm{CF}_{3} \mathrm{CF}_{2} \mathrm{CF}_{2} \mathrm{CH}_{2} \mathrm{OH}+\mathrm{F} \rightarrow \text { Produkte }
\end{aligned}
$$

Mit $\mathrm{T}=298 \mathrm{~K}$ errechnet man daraus: $\mathbf{k}(298 \mathrm{~K})=(\mathbf{2 , 7 4} \pm \mathbf{0 , 6 4}) \cdot 10^{13} \mathbf{c m}^{\mathbf{3}} \mathbf{m o l}^{-1} \mathbf{s}^{-1}$.

Weil die Reaktion im Rahmen ihrer Fehlergrenzen kaum eine Temperaturabhängigkeit zeigt, wird mit den absoluten Geschwindigkeitskoeffizienten aus Tabelle 45 zudem eine Auftragung gemäß Gleichung (13) (S. 11) angefertigt ( Abbildung 166).

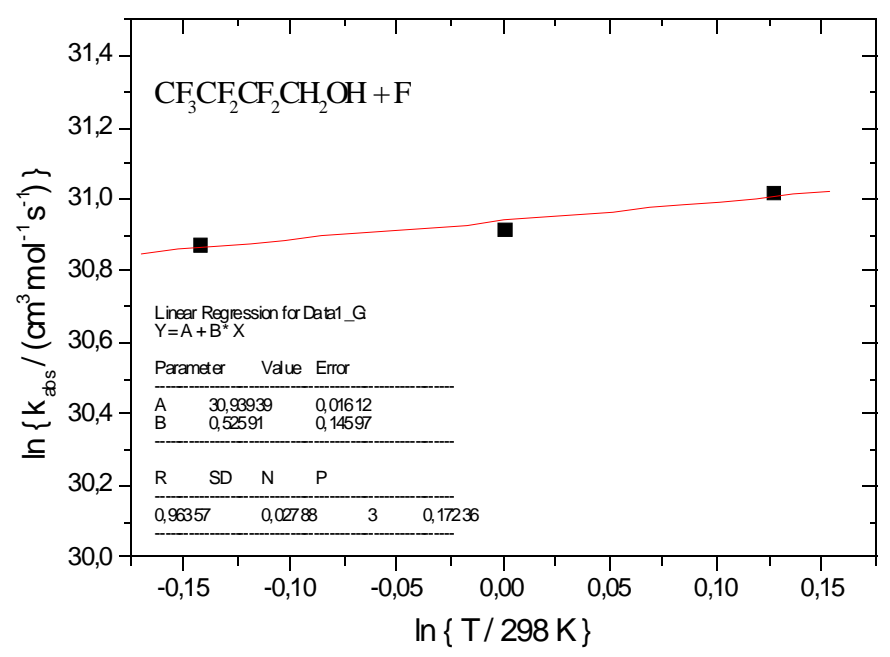

Abbildung 166: $\ln (k)$ vs. $\ln (T)$ f. CF3CF2CF2CH2OH + F

Aus den Ergebnissen der Abbildung 166 berechnet man:

$$
\begin{aligned}
& \mathbf{k}(\mathbf{T})=(2,73 \pm \mathbf{0 , 0 4}) \cdot 10^{13} \cdot(\mathrm{T} / 298 \mathrm{~K})^{(0,53 \pm 0,15)} \mathbf{c m}^{3} /(\mathbf{m o l} \cdot \mathbf{s}) \\
& \text { für: } \mathrm{CF}_{3} \mathrm{CF}_{2} \mathrm{CF}_{2} \mathrm{CH}_{2} \mathrm{OH}+\mathrm{F} \rightarrow \text { Produkte }
\end{aligned}
$$

Mit $\mathrm{T}=298 \mathrm{~K}$ errechnet man daraus: $\mathbf{k}(298 \mathrm{~K})=(\mathbf{2 , 7 3} \pm \mathbf{0 , 0 4}) \cdot \mathbf{1 0}^{\mathbf{1 3}} \mathbf{c m}^{\mathbf{3}} \mathbf{m o l}^{-1} \mathrm{~s}^{-\mathbf{1}}$. 


\subsubsection{Kinetik der Reaktion des erzeugten Radikals mit molekularem Sauerstoff}

Die Messungen wurden in der in Kap. 3.1.1 (S. 16) beschriebenen Apparatur unter Einsatz des Strömungsreaktors aus Abbildung 5 (S. 17) durchgeführt.

Das Dichlormethyl-Radikal diente als Referenzsubstanz. Der Umsatz des Alkohol-Radikals konnte anhand der Intensitätsänderung seines Molekül-Peaks m/z 199 und der des Dichlormethyl-Radikals anhand seines Molekül-Peaks m/z 83 oder 85 bestimmt werden.

Die Zuordnung des Radikals zu dem Peak auf m/z 199 wurde bereits oben im Rahmen der Erläuterungen zu Abb. 161 (S. 183) gemacht.

Die Messungen wurden umsatzkorrigiert nach der Relativmethode durchgeführt, wobei zur Messung des Umsatzes des Alkohols bei Zugabe der Fluor-Atome die Intensitätsänderung auf $\mathrm{m} / \mathrm{z} 161$, und für den des Dichlormethans diejenige auf m/z 84 oder 86 diente; die genaue Ausführung und Auswertung ist in Kap. 3.5.1 (S. 50) beschrieben.

Es konnte oben bei der Diskussion um Abb. 161 (S. 183) begründet werden, daß der Fragment-Peak m/z 161 des Alkohols zur Messung seines Umsatzes geeignet ist.

$\vartheta=-15^{\circ} \mathrm{C} ; \phi_{\mathrm{He}}=786 \mathrm{SCCM} ; \phi_{\mathrm{CF} 3 \mathrm{CF} 2 \mathrm{CF} 2 \mathrm{CH} 2 \mathrm{OH}}=3,8 \mathrm{SCCM} ; \phi_{\mathrm{CH} 2 \mathrm{Cl} 2}=8,9 \mathrm{SCCM}$;

$\mathrm{p}_{(\mathrm{StrR})}=135 \mathrm{~Pa} ; \mathrm{s}=4,1 \mathrm{~cm} ; \mathrm{IP}=28 \mathrm{eV} ;$ Zählzeit $=10 \mathrm{~s}$

\begin{tabular}{|c|c|c|c|c|c|c|c|}
\hline $\begin{array}{c}\text { I(199) } \\
-\mathrm{O}_{2} / \text { w.E. }\end{array}$ & $\begin{array}{c}\Delta \mathrm{I}(\mathbf{1 9 9}) \\
-\mathrm{O}_{2} / \text { w.E. }\end{array}$ & $\begin{array}{c}\text { I(199) } \\
+\mathrm{O}_{2} / \text { w.E. }\end{array}$ & $\begin{array}{c}\Delta \mathbf{I}(\mathbf{1 9 9}) \\
+\mathbf{O}_{2} / \text { w.E. }\end{array}$ & $\begin{array}{c}\mathrm{I}(\mathbf{8 5}) \\
-\mathrm{O}_{2} / \mathbf{w . E .}\end{array}$ & $\begin{array}{c}\Delta \mathbf{I}(\mathbf{8 5}) \\
-\mathrm{O}_{2} / \text { w.E. }\end{array}$ & $\begin{array}{c}\mathrm{I}(\mathbf{8 5}) \\
+\mathrm{O}_{2} / \text { w.E. }\end{array}$ & $\begin{aligned} & \Delta \mathrm{I}(\mathbf{8 5}) \\
+ & \mathbf{O}_{2} / \mathbf{w} . \mathbf{E} .\end{aligned}$ \\
\hline 2007 & 37,21 & 1256 & 37,02 & 4227 & 158,6 & 3597 & 116,8 \\
\hline 1876 & 46,47 & 1158 & 47,03 & 4814 & 113,1 & 3774 & 94,52 \\
\hline 1901 & 44,95 & 1114 & 42,65 & 4630 & 181 & 3830 & 161,1 \\
\hline 1917 & 63,12 & 1239 & 53,63 & 5468 & 302,6 & 4368 & 141,6 \\
\hline 2087 & 68,96 & 1449 & 48,68 & 5662 & 185,9 & 4602 & 139,9 \\
\hline 2174 & 69,77 & 1444 & 48,7 & 5860 & 110,7 & 4690 & 126,7 \\
\hline 2291 & 51,39 & 1661 & 55,74 & 5790 & 122,2 & 5080 & 127 \\
\hline 2325 & 45,09 & 1652 & 59,15 & 5603 & 172,3 & 4963 & 1044 \\
\hline 1907 & 271,9 & 1306 & 82,35 & 7198 & 330,3 & 5788 & 274,3 \\
\hline 2204 & 57,52 & 1715 & 65,08 & 5923 & 183,5 & 5093 & 151,8 \\
\hline 2252 & 47,15 & 1988 & 59,71 & 5438 & 142,3 & 5068 & 229,7 \\
\hline 2259 & 42,15 & 2022 & 53,34 & 5635 & 161,2 & 5225 & 173,9 \\
\hline
\end{tabular}

Tabelle 46: Meßwerte Kinetik $\{\mathrm{CF} 3 \mathrm{CF} 2 \mathrm{CF} 2 \mathrm{CH} 2 \mathrm{OH}+\mathrm{F}\}+\{\mathrm{O} 2\}$ rel. zu CH2Cl2; $258 \mathrm{~K}$ 


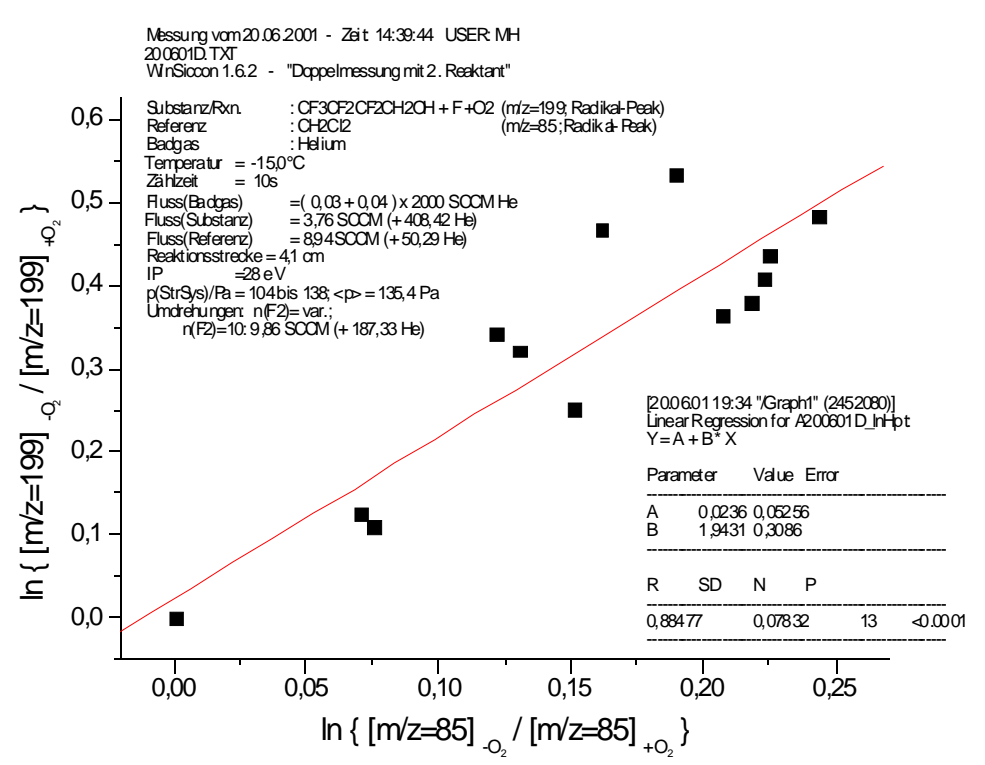

Abbildung 167: $\{\mathrm{CF} 3 \mathrm{CF} 2 \mathrm{CF} 2 \mathrm{CH} 2 \mathrm{OH}+\mathrm{F}\}+\{\mathrm{O} 2\}$ rel. zu CH2Cl2; $258 \mathrm{~K}$

Aus der Steigung der Auftragung in Abbildung 167 erhält man den relativen

Geschwindigkeitskoeffizienten $\mathrm{k}_{\mathrm{rel}}=(1,94 \pm 0,31)$.

$\vartheta=+25^{\circ} \mathrm{C} ; \phi_{\mathrm{He}}=786 \mathrm{SCCM} ; \phi_{\mathrm{CF} 3 \mathrm{CF} 2 \mathrm{CF} 2 \mathrm{CH} 2 \mathrm{OH}}=3,8 \mathrm{SCCM} ; \phi_{\mathrm{CH} 2 \mathrm{Cl} 2}=8,9 \mathrm{SCCM} ;$

$\mathrm{p}_{(\mathrm{StrR})}=144 \mathrm{~Pa} ; \mathrm{s}=4,1 \mathrm{~cm} ; \mathrm{IP}=28 \mathrm{eV} ;$ Zählzeit $=10 \mathrm{~s}$

\begin{tabular}{|c|c|c|c|c|c|c|c|}
\hline $\begin{array}{c}\mathbf{I}(199) \\
-\mathrm{O}_{2} / \text { w.E. }\end{array}$ & $\begin{array}{c}\Delta \mathrm{I}(199) \\
-\mathrm{O}_{2} / \text { w.E. }\end{array}$ & $\begin{array}{c}\text { I(199) } \\
+\mathrm{O}_{2} / \text { w.E. }\end{array}$ & $\begin{array}{c}\Delta \mathrm{I}(199) \\
+\mathrm{O}_{2} / \text { w.E. }\end{array}$ & $\begin{array}{c}\mathbf{I}(\mathbf{8 5}) \\
-\mathrm{O}_{2} / \mathrm{w.E} .\end{array}$ & $\begin{array}{c}\Delta \mathrm{I}(\mathbf{8 5}) \\
-\mathrm{O}_{2} / \mathrm{w.E.}\end{array}$ & $\begin{array}{c}\mathrm{I}(85) \\
+\mathrm{O}_{2} / \mathrm{w} . \mathrm{E} .\end{array}$ & $\begin{array}{c}\Delta \mathrm{I}(\mathbf{8 5}) \\
+\mathrm{O}_{2} / \mathrm{w} . \mathrm{E} .\end{array}$ \\
\hline 1815 & 48,52 & 1334 & 54,44 & 5476 & 215,9 & 4654 & 204,2 \\
\hline 1625 & 53,2 & 1238 & 57,44 & 5640 & 200,9 & 4963 & 215,9 \\
\hline 1749 & 44,42 & 1351 & 48,84 & 5267 & 214,3 & 4711 & 210,4 \\
\hline 1758 & 53,1 & 1373 & 43,96 & 5622 & 183,6 & 4944 & 176,7 \\
\hline 1734 & 82,02 & 1503 & 55,94 & 5423 & 219,1 & 4990 & 224,7 \\
\hline 1764 & 55,68 & 1489 & 61,19 & 5793 & 286,1 & 5333 & 311,2 \\
\hline 1386 & 52,34 & 1240 & 49,87 & 5227 & 179,5 & 4957 & 185 \\
\hline 1361 & 48,19 & 1203 & 49,99 & 4943 & 179,5 & 4733 & 165,9 \\
\hline 1454 & 44,13 & 1334 & 38,05 & 4993 & 187,5 & 4813 & 167,7 \\
\hline
\end{tabular}

Tabelle 47: Meßwerte Kinetik $\{\mathrm{CF} 3 \mathrm{CF} 2 \mathrm{CF} 2 \mathrm{CH} 2 \mathrm{OH}+\mathrm{F}\}+\{\mathrm{O} 2\}$ rel. zu CH2Cl2; $298 \mathrm{~K}$ 


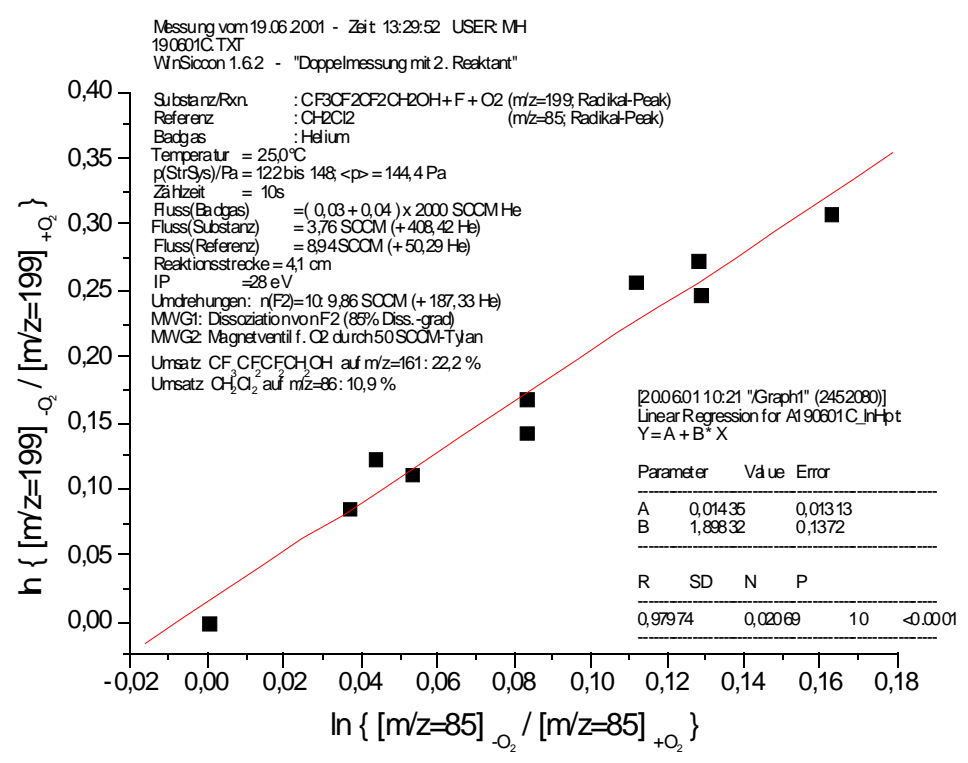

Abbildung 168: $\{\mathrm{CF} 3 \mathrm{CF} 2 \mathrm{CF} 2 \mathrm{CH} 2 \mathrm{OH}+\mathrm{F}\}+\{\mathrm{O} 2\}$ rel. zu CH2Cl2; $298 \mathrm{~K}$

Aus der Steigung der Auftragung in Abbildung 168 erhält man den relativen

Geschwindigkeitskoeffizienten $\mathrm{k}_{\mathrm{rel}}=(1,90 \pm 0,14)$.

$\vartheta=+25,7{ }^{\circ} \mathrm{C} ; \phi_{\mathrm{He}}=1378 \mathrm{SCCM} ; \phi_{\mathrm{CF} 3 \mathrm{CF} 2 \mathrm{CF} 2 \mathrm{CH} 2 \mathrm{OH}}=8,3 \mathrm{SCCM} ; \phi_{\mathrm{CH} 2 \mathrm{Cl} 2}=20,9 \mathrm{SCCM}$;

$\mathrm{p}_{\text {(StrR) }}=175 \mathrm{~Pa} ; \mathrm{s}=4,2 \mathrm{~cm} ; \mathrm{IP}=24,5 \mathrm{eV} ;$ Zählzeit $=5 \mathrm{~s}$

\begin{tabular}{|c|c|c|c|c|c|c|c|}
\hline $\begin{array}{c}\text { I(199) } \\
-\mathrm{O}_{2} / \text { w.E. }\end{array}$ & $\begin{array}{c}\Delta \mathrm{I}(\mathbf{1 9 9}) \\
-\mathrm{O}_{2} / \text { w.E. }\end{array}$ & $\begin{array}{c}\mathrm{I}(199) \\
+\mathrm{O}_{2} / \text { w.E. }\end{array}$ & $\begin{array}{c}\Delta \mathbf{I}(199) \\
+\mathbf{O}_{2} / \text { w.E. }\end{array}$ & $\begin{array}{c}\mathbf{I}(\mathbf{8 3}) \\
-\mathrm{O}_{2} / \mathbf{w . E .}\end{array}$ & $\begin{array}{c}\Delta \mathrm{I}(\mathbf{I 3 3}) \\
-\mathrm{O}_{2} / \mathbf{w . E .}\end{array}$ & $\begin{array}{c}\mathrm{I}(83) \\
+\mathrm{O}_{2} / \text { w.E. }\end{array}$ & $\begin{array}{c}\Delta \mathrm{I}(\mathbf{8 3}) \\
+\mathbf{O}_{2} / \mathbf{w} . \mathbf{E} .\end{array}$ \\
\hline 790,6 & 40,16 & 561,6 & 28,28 & 1905 & 110,8 & 1595 & 130 \\
\hline 720,7 & 35,4 & 534,1 & 33,34 & 1949 & 109,3 & 1626 & 122,8 \\
\hline 755,6 & 36,37 & 500,5 & 31,26 & 2000 & 130,1 & 1759 & 103,9 \\
\hline 498,6 & 36,42 & 368,9 & 26,61 & 2194 & 105 & 1804 & 104,3 \\
\hline 749,7 & 40,91 & 496,1 & 32,43 & 2172 & 130,5 & 1798 & 126,3 \\
\hline 733,1 & 39,73 & 422,4 & 30,29 & 2208 & 155,3 & 1628 & 138,9 \\
\hline 724,3 & 47,18 & 407,6 & 37,51 & 1943 & 124,5 & 1331 & 145,4 \\
\hline 655,2 & 100,6 & 372,2 & 111,1 & 2031 & 108,6 & 1479 & 113,2 \\
\hline 682,4 & 42,23 & 330,3 & 33,23 & 2279 & 113 & 1665 & 100,5 \\
\hline 674,7 & 32,58 & 322,9 & 29,15 & 2389 & 114,3 & 1761 & 138,2 \\
\hline 643,1 & 38,64 & 625,1 & 24,74 & 1951 & 111,1 & 1891 & 118,4 \\
\hline
\end{tabular}

Tabelle 48: Meßwerte Kinetik $\{\mathrm{CF} 3 \mathrm{CF} 2 \mathrm{CF} 2 \mathrm{CH} 2 \mathrm{OH}+\mathrm{F}\}+\{\mathrm{O} 2\}$ rel. zu CH2Cl2; $299 \mathrm{~K}$ 


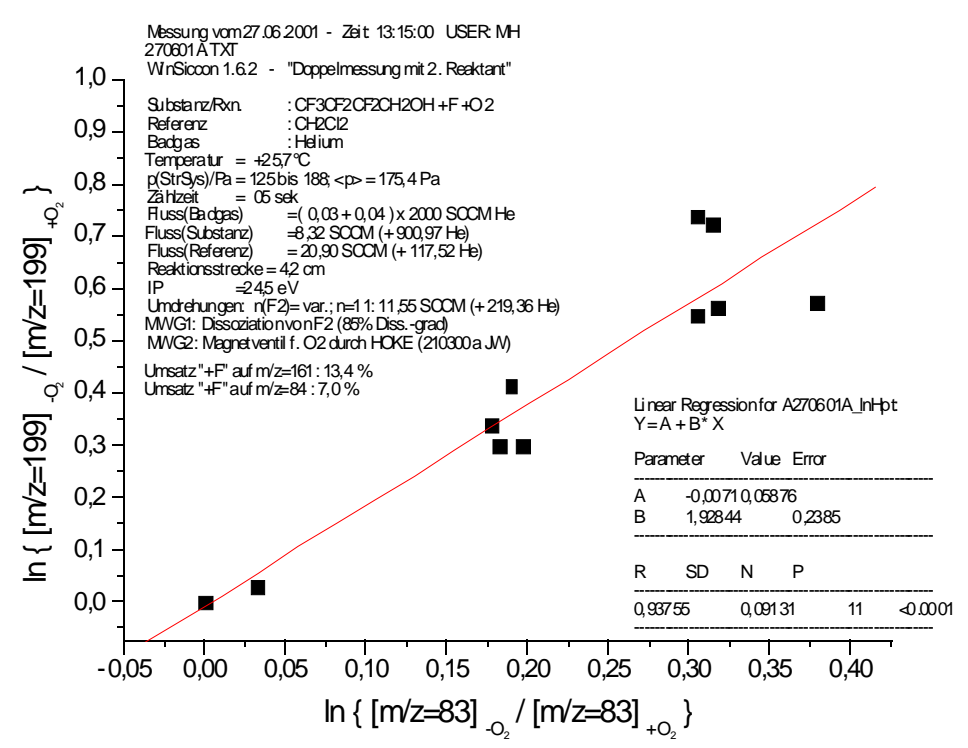

Abbildung 169: $\{$ CF3CF2CF2CH2OH $+F\}+\{O 2\}$ rel. zu CH2Cl2; $299 \mathrm{~K}$

Aus der Steigung der Auftragung in Abbildung 169 erhält man den relativen

Geschwindigkeitskoeffizienten $\mathrm{k}_{\mathrm{rel}}=(1,93 \pm 0,24)$.

$\vartheta=+65,0^{\circ} \mathrm{C} ; \phi_{\mathrm{He}}=1378 \mathrm{SCCM} ; \phi_{\text {СF3Сन2СF2CH2OH }}=8,3 \mathrm{SCCM} ; \phi_{\mathrm{CH} 2 \mathrm{Cl} 2}=20,9 \mathrm{SCCM}$

$\mathrm{p}_{\text {(StrR })}=205 \mathrm{~Pa} ; \mathrm{s}=4,2 \mathrm{~cm} ; \mathrm{IP}=24,5 \mathrm{eV} ;$ Zählzeit $=5 \mathrm{~s}$

\begin{tabular}{|c|c|c|c|c|c|c|c|}
\hline $\begin{array}{c}I(199) \\
-O_{2} / \text { w.E. }\end{array}$ & $\begin{array}{c}\Delta \mathrm{I}(199) \\
-\mathrm{O}_{2} / \mathrm{w.E.}\end{array}$ & $\begin{array}{c}\mathrm{I}(199) \\
+\mathrm{O}_{2} / \text { w.E. }\end{array}$ & $\begin{array}{c}\Delta \mathrm{I}(199) \\
+\mathrm{O}_{2} / \text { w.E. }\end{array}$ & $\begin{array}{c}\mathbf{I}(\mathbf{8 3 )} \\
-\mathrm{O}_{2} / \mathrm{w.E.}\end{array}$ & $\begin{array}{c}\Delta \mathrm{I}(\mathbf{8 3}) \\
-\mathrm{O}_{2} / \mathrm{w.E} .\end{array}$ & $\begin{array}{c}\mathrm{I}(83) \\
+\mathrm{O}_{2} / \mathrm{w} . \mathrm{E} .\end{array}$ & $\begin{array}{c}\Delta \mathrm{I}(\mathbf{8 3}) \\
+\mathrm{O}_{2} / \text { w.E. }\end{array}$ \\
\hline 877,3 & 34,56 & 401,3 & 31,82 & 2318 & 98,31 & 1767 & 102,5 \\
\hline 1105 & 39,02 & 497,6 & 23,17 & 2323 & 100,1 & 1685 & 85,41 \\
\hline 1096 & 37,75 & 511,9 & 27,63 & 2477 & 89,66 & 1846 & 72,17 \\
\hline 1085 & 34,04 & 555 & 30,35 & 2391 & 93,56 & 1827 & 76,81 \\
\hline 1040 & 39,77 & 597,9 & 35,07 & 2389 & 97,43 & 2021 & 49,02 \\
\hline 1133 & 40,62 & 633,8 & 39,91 & 2448 & 78,34 & 1903 & 61,34 \\
\hline 1133 & 47,3 & 682,7 & 30,71 & 2330 & 83,26 & 1774 & 91,15 \\
\hline 1072 & 41,15 & 652,9 & 27,39 & 2519 & 106,6 & 1965 & 144,6 \\
\hline 1125 & 42,79 & 812,3 & 33,19 & 2027 & 108,7 & 1798 & 121,8 \\
\hline 1142 & 35,86 & 939,4 & 30,01 & 2182 & 59,97 & 1992 & 63,55 \\
\hline 1093 & 36,55 & 447,4 & 29,44 & 2516 & 95,01 & 1698 & 129,1 \\
\hline
\end{tabular}

Tabelle 49: Meßwerte Kinetik $\{\mathrm{CF} 3 \mathrm{CF} 2 \mathrm{CF} 2 \mathrm{CH} 2 \mathrm{OH}+\mathrm{F}\}+\{\mathrm{O} 2\}$ rel. zu CH2Cl2; $338 \mathrm{~K}$ 


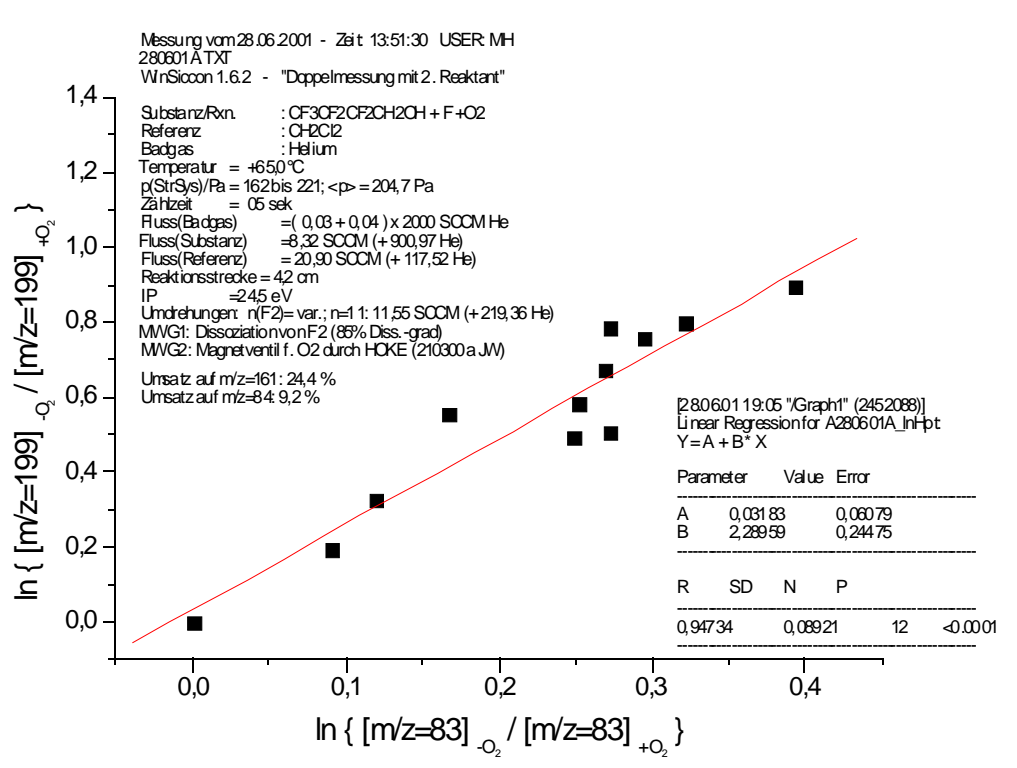

Abbildung 170: $\{\mathrm{CF} 3 \mathrm{CF} 2 \mathrm{CF} 2 \mathrm{CH} 2 \mathrm{OH}+\mathrm{F}\}+\{\mathrm{O} 2\}$ rel. zu CH2Cl2; $338 \mathrm{~K}$

Aus der Steigung der Auftragung in Abbildung 170 erhält man den relativen

Geschwindigkeitskoeffizienten $\mathrm{k}_{\text {rel }}=(2,29 \pm 0,24)$.

$\vartheta=+85,0^{\circ} \mathrm{C} ; \phi_{\mathrm{He}}=895 \mathrm{SCCM} ; \phi_{\mathrm{CF} 3 \mathrm{CF} 2 \mathrm{CF} 2 \mathrm{CH} 2 \mathrm{OH}}=8,3 \mathrm{SCCM} ; \phi_{\mathrm{CH} 2 \mathrm{C} 2}=20,9 \mathrm{SCCM}$;

$\mathrm{p}_{(\mathrm{StrR})}=205 \mathrm{~Pa} ; \mathrm{s}=4,2 \mathrm{~cm} ; \mathrm{IP}=24,5 \mathrm{eV} ;$ Zählzeit $=5 \mathrm{~s}$

\begin{tabular}{|c|c|c|c|c|c|c|c|}
\hline $\begin{array}{c}\text { I(199) } \\
-\mathrm{O}_{2} / \text { w.E. }\end{array}$ & $\begin{array}{c}\Delta \mathrm{I}(\mathbf{1 9 9}) \\
-\mathrm{O}_{2} / \text { w.E. }\end{array}$ & $\begin{array}{c}\mathrm{I}(199) \\
+\mathrm{O}_{2} / \text { w.E. }\end{array}$ & $\begin{aligned} \Delta \mathrm{I}(199) \\
+\mathrm{O}_{2} / \text { w.E. }\end{aligned}$ & $\begin{array}{c}\mathbf{I}(\mathbf{8 3}) \\
-\mathbf{O}_{2} / \text { w.E. }\end{array}$ & $\begin{array}{c}\Delta \mathbf{I}(\mathbf{8 3}) \\
-\mathbf{O}_{2} / \mathbf{w . E .}\end{array}$ & $\begin{array}{c}\mathrm{I}(\mathbf{8 3}) \\
+\mathrm{O}_{2} / \text { w.E. }\end{array}$ & $\begin{array}{c}\Delta \mathbf{I}(\mathbf{8 3}) \\
+\mathbf{O}_{2} / \text { w.E. }\end{array}$ \\
\hline 882,8 & 32,49 & 400,5 & 26,86 & 3393 & 94,06 & 2345 & 88,63 \\
\hline 875,9 & 35,38 & 350,8 & 27,41 & 3319 & 100,3 & 2148 & 112,9 \\
\hline 905,6 & 36,09 & 389,7 & 29,58 & 3301 & 109,9 & 2493 & 115,3 \\
\hline 921,7 & 34,3 & 419,8 & 33,47 & 3394 & 111,5 & 2498 & 107,3 \\
\hline 936,8 & 38,04 & 459,9 & 26,1 & 3210 & 121,3 & 2511 & 112,9 \\
\hline 915,3 & 36,66 & 470 & 30,24 & 3320 & 104,5 & 2366 & 101,5 \\
\hline 975,7 & 34,1 & 575,4 & 36,47 & 3244 & 110 & 2625 & 83,5 \\
\hline 938,7 & 25,79 & 632,7 & 30,95 & 3160 & 104,6 & 2680 & 108,1 \\
\hline 912,7 & 34,09 & 678,7 & 31,89 & 3141 & 112,6 & 2805 & 119,4 \\
\hline 917,9 & 29,71 & 731,9 & 36,63 & 3104 & 90,59 & 2782 & 123,8 \\
\hline 898,7 & 35,48 & 809,7 & 29,53 & 3169 & 104,2 & 3019 & 100,9 \\
\hline
\end{tabular}

Tabelle 50: Meßwerte Kinetik $\{\mathrm{CF} 3 \mathrm{CF} 2 \mathrm{CF} 2 \mathrm{CH} 2 \mathrm{OH}+\mathrm{F}\}+\{\mathrm{O} 2\}$ rel. zu CH2Cl2; $358 \mathrm{~K}$ 


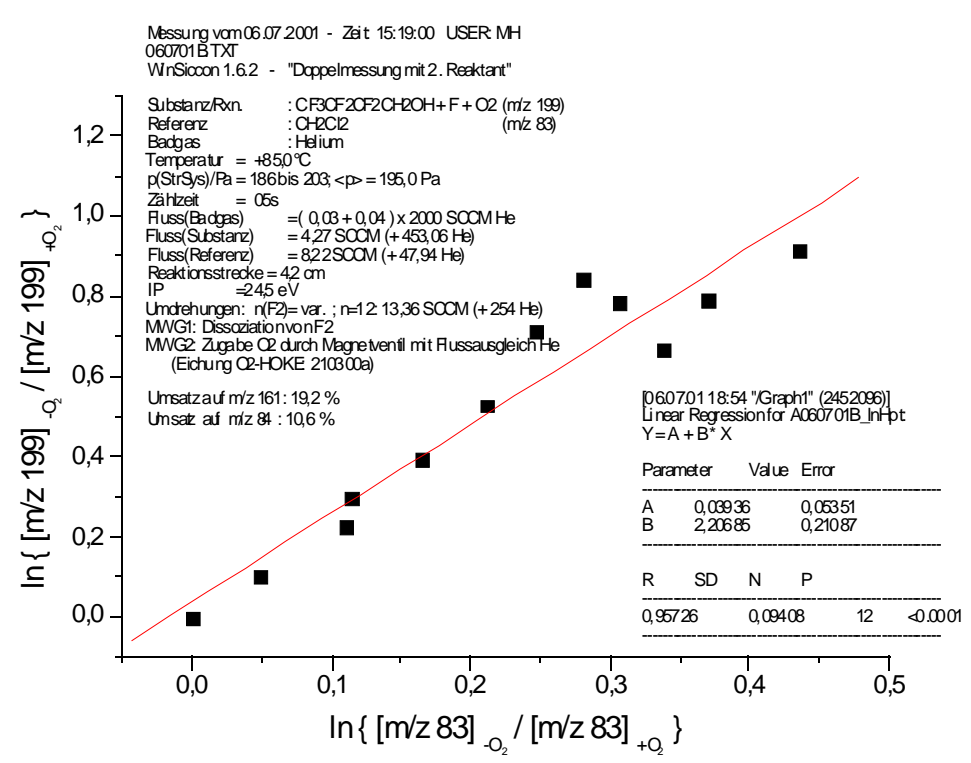

Abbildung 171: $\{$ CF3CF2CF2CH2OH + F $\}+\{O 2\}$ rel. zu CH2Cl2; $358 \mathrm{~K}$

Aus der Steigung der Auftragung in Abbildung 171 erhält man den relativen

Geschwindigkeitskoeffizienten $\mathrm{k}_{\mathrm{rel}}=(2,21 \pm 0,21)$.

Aus den ermittelten relativen Geschwindigkeitskoeffizienten der Abbildungen 167 bis 171 können nun mit der bekannten Temperaturabhängigkeit des Geschwindigkeitskoeffizienten für die Referenzreaktion ${ }^{141} \mathrm{k}_{\mathrm{ref}}(\mathrm{T})$ die absoluten Geschwindigkeitskoeffizienten $\mathrm{k}$ für die Reaktion des aus dem Alkohol erzeugten Radikals mit $\mathrm{O}_{2}$ berechnet werden (Tabelle 51).

$\mathrm{k}_{\mathrm{ref}}(\mathrm{T})=(1,56 \pm 0,08) \cdot 10^{17} \cdot(\mathrm{T} / 298 \mathrm{~K})^{-(6,7 \pm 0,7)} \cdot[\mathrm{He}] \mathrm{cm}^{6} /\left(\mathrm{mol}^{2} \cdot \mathrm{s}\right)$.

Ferner kann mit diesen Werten eine Arrhenius-Auftragung gemäß Gleichung (11) (S. 10) angefertigt werden, welche in Abbildung 172 zu sehen ist.

\begin{tabular}{|c|c|c|c|c|c|c|c|c|}
\hline $\begin{array}{l}\vartheta / \\
{ }^{\circ} \mathbf{C}\end{array}$ & $\mathbf{T} / \mathbf{K}$ & $\mathbf{T}^{-1} / \mathbf{K}^{-1}$ & $\mathbf{k}_{\text {rel }}$ & $\ln \left(k_{\text {rel }}\right)$ & $\begin{array}{c}\mathbf{k}_{\text {termolek. }} / \\
\left\{\mathrm{cm}^{6} /\right. \\
\left.\left(\mathrm{mol}^{2} \mathbf{s}\right)\right\}\end{array}$ & $\mathbf{p}_{\text {StrR }} / \mathbf{P a}$ & $\begin{array}{c}{[\mathrm{He}] /} \\
\mathrm{mol} / \mathrm{cm}^{3}\end{array}$ & $\begin{array}{c}\mathbf{k}_{\text {bimolek. }} / \\
\left\{\mathbf{c m}^{3} /\right. \\
(\mathrm{mol} \mathrm{s})\}\end{array}$ \\
\hline-15 & 258,15 & $3,87 \mathrm{E}-03$ & 1,9431 & 0,6643 & $4,08 \mathrm{E}+17$ & 135,4 & $6,31 \mathrm{E}-08$ & $2,57 \mathrm{E}+10$ \\
\hline 25 & 298,15 & $3,35 \mathrm{E}-03$ & 1,8983 & 0,6410 & $1,55 \mathrm{E}+017$ & 144,4 & $5,83 \mathrm{E}-08$ & $9,06 \mathrm{E}+09$ \\
\hline 26 & 299,15 & $3,34 \mathrm{E}-03$ & 1,9284 & 0,6567 & $1,52 \mathrm{E}+017$ & 175,4 & $7,05 \mathrm{E}-08$ & $1,07 \mathrm{E}+10$ \\
\hline 65 & 338,15 & $2,96 \mathrm{E}-03$ & 2,2896 & 0,8284 & $6,69 \mathrm{E}+016$ & 204,7 & $7,28 \mathrm{E}-08$ & $4,87 \mathrm{E}+09$ \\
\hline 85 & 358,15 & $2,79 \mathrm{E}-03$ & 2,2069 & 0,7916 & $4,55 \mathrm{E}+016$ & 195 & $6,55 \mathrm{E}-08$ & $2,98 \mathrm{E}+09$ \\
\hline
\end{tabular}

Tabelle 51: $k(T)$ sowie Werte f. Arrhenius-Auftr. f. $\{\mathrm{CF} 3 \mathrm{CF} 2 \mathrm{CF} 2 \mathrm{CH} 2 \mathrm{OH}+\mathrm{F}\}+\{\mathrm{O} 2\}$ 


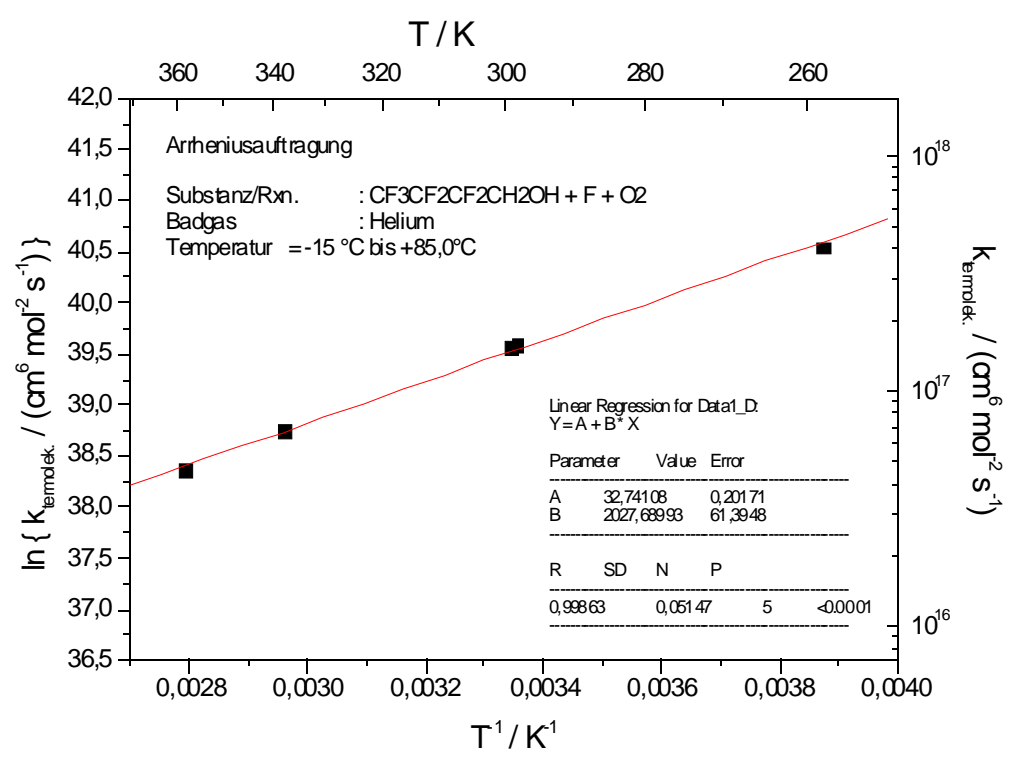

Abbildung 172: Arrhenius-Auftr. f. CF3CF2CF2CHOH +O2

Aus dem Achsenabschnitt $\mathrm{a}=32,74$ der Auftragung in Abbildung 172 kann der ArrheniusVorfaktor A berechnet werden: $A=\exp (32,74108)=1,66 E+14$. Aus der Steigung $b=2028 \mathrm{~K}$ läßt sich die Aktivierungsenergie bestimmen: $E_{a}=-8,314 \mathrm{~J} /(\mathrm{mol} \mathrm{K}) \cdot 2027,68993 \mathrm{~K}=-16,86$ kJ / mol. Damit ergibt sich als Arrhenius-Gleichung folgende Temperaturabhängigkeit für den Geschwindigkeitskoeffizienten:

$$
\begin{aligned}
& \mathbf{k}(\mathbf{T})=\mathbf{1 , 6 6} \cdot \mathbf{1 0}^{\mathbf{1 4}} \cdot \exp \{-(-16,86 \mathbf{k J} / \mathbf{m o l}) /(\mathbf{R} \cdot \mathbf{T})\} \cdot[\mathrm{He}] \mathbf{~ c m}^{6} /\left(\mathbf{m o l}^{2} \cdot \mathbf{s}\right) \\
& \text { für: }\left\{\text { Radikal aus der Reaktion } \mathrm{CF}_{3} \mathrm{CF}_{2} \mathrm{CF}_{2} \mathrm{CH}_{2} \mathrm{OH}+\mathrm{F}\right\}+\left\{\mathrm{O}_{2}\right\} \rightarrow \text { Produkte }
\end{aligned}
$$

Weil die hier untersuchten Reaktionen bei sehr niedrigem Druck ausgeführt wurden, könnte es sich um termolekulare Reaktionen im Sinne von Gleichung (28) (S. 124) handeln. Daher wird $\mathrm{k}(\mathrm{T})$ als Ergebnis hier termolekular angegeben.

Aus dem Druck im Reaktor und dem Gesamtgasfluß sowie den anderen angegebenen Reaktionsbedingungen können gemäß Gleichung (19) (S. 14) die Konzentration des Badgases Helium und damit die bimolekularen Geschwindigkeitskoeffizienten berechnet werden. Diese Werte sind als $\mathrm{k}_{\text {bimolek. }}$ in Tabelle 51 eingetragen. 
Weil Assoziationsreaktionen mit $\mathrm{O}_{2}$ üblicherweise ohne Energiebarriere ablaufen, sollte besser eine Auftragung $\ln (\mathrm{k})$ vs. $\ln (\mathrm{T})$ gemäß Gleichung (13) (S. 11) angefertigt werden (Abb. 173)

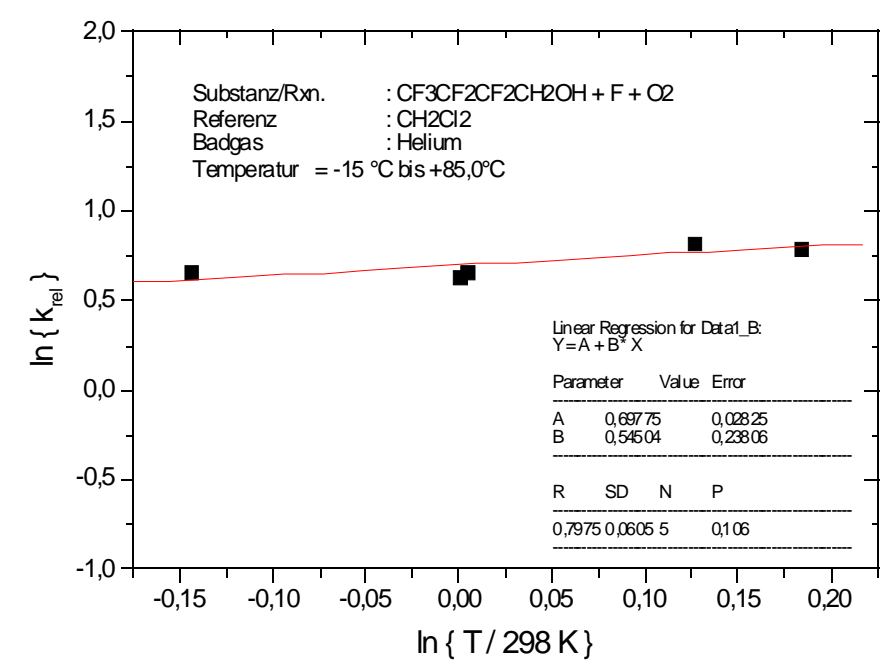

Abbildung 173: $\ln (k)$ vs. $\ln (T)$ f. CF3CF2CF2CHOH + O2 rel. zu CHCl2

Mittels Gleichung (29) (S. 126) kann nun aus dem Achsenabschnitt a der Abb. 173 der Vorfaktor A berechnet werden:

$\mathrm{A}=\mathrm{A}_{\mathrm{ref}} \cdot \exp (\mathrm{a})=1,56 \cdot 10^{17} \mathrm{~cm}^{6} /\left(\mathrm{mol}^{2} \cdot \mathrm{s}\right) \cdot \exp (0,69775)=3,13 \cdot 10^{17} \mathrm{~cm}^{6} /\left(\mathrm{mol}^{2} \cdot \mathrm{s}\right)$.

Den Parameter n kann man gemäß Gleichung (29) (S. 126) folgendermaßen aus der Steigung b der Abbildung 173 ermittlen:

$\mathrm{n}=\left(\mathrm{b}+\mathrm{n}_{\mathrm{ref}}\right)=(0,545+(-6,70))=-6,15$.

Somit ergibt sich als Temperaturabhängigkeit des Geschwindigkeitskoeffizienten:

$$
k(T)=(3,13 \pm 0,18) \cdot 10^{17} \cdot(T / 298 K)^{-(6,15 \pm 0,71)} \cdot[\mathrm{He}] \mathrm{cm}^{6} /\left(\mathrm{mol}^{2} \cdot \mathrm{s}\right)
$$

für: $\left\{\right.$ Radikal aus der Reaktion $\left.\mathrm{CF}_{3} \mathrm{CF}_{2} \mathrm{CF}_{2} \mathrm{CH}_{2} \mathrm{OH}+\mathrm{F}\right\}+\left\{\mathrm{O}_{2}\right\} \rightarrow$ Produkte

Mit $\mathrm{T}=298 \mathrm{~K}$ berechnet man daraus:

$$
\mathrm{k}(298 \mathrm{~K})=(3,13 \pm 0,18) \cdot 10^{17} \cdot[\mathrm{He}] \mathrm{cm}^{6} /\left(\mathrm{mol}^{2} \cdot \mathrm{s}\right)
$$




\subsubsection{Diskussion der Ergebnisse}

Der ermittelte Geschwindigkeitskoeffizient für die Reaktion des Alkohols $\mathrm{CF}_{3} \mathrm{CF}_{2} \mathrm{CF}_{2} \mathrm{CH}_{2} \mathrm{OH}$ mit Fluor-Atomen ist mit $\mathrm{k}(298 \mathrm{~K})=2,74 \cdot 10^{13} \mathrm{~cm}^{3} \mathrm{~mol}^{-1} \mathrm{~s}^{-1}$ der niedrigste aller bisher untersuchten strukturell ähnlichen fluorierten Alkohole. Für $\mathrm{CF}_{3} \mathrm{CH}_{2} \mathrm{OH}$ hatte sich $\mathrm{k}(298 \mathrm{~K})=6,27$. $10^{13} \mathrm{~cm}^{3} \mathrm{~mol}^{-1} \mathrm{~s}^{-1}$, für $\mathrm{CHF}_{2} \mathrm{CH}_{2} \mathrm{OH} \mathrm{k}(299 \mathrm{~K})=6,92 \cdot 10^{13} \mathrm{~cm}^{3} \mathrm{~mol}^{-1} \mathrm{~s}^{-1}$ und für $\mathrm{CH}_{2} \mathrm{FCH}_{2} \mathrm{OH}$ $\mathrm{k}(298 \mathrm{~K})=1,17 \cdot 10^{14} \mathrm{~cm}^{3} \mathrm{~mol}^{-1} \mathrm{~s}^{-1}$ ergeben. Dies kann als Indiz für die Herabsetzung der Geschwindigkeitskoeffizienten mit zunehmender Zahl Fluor-Substituenten angesehen werden. Es kann vermutlich aber nicht allein auf die Elektronegativität zurückgeführt werden, denn sie hat für $\mathrm{F}$ etwa den Wert ${ }^{142} 4$ und für die $\mathrm{CF}_{3}$-Gruppe etwa 3,35. Es ist anzunehmen, daß sie für die $\mathrm{CF}_{3} \mathrm{CF}_{2} \mathrm{CF}_{2}$-Gruppe ca. 3,4 bis 3,9 beträgt. Danach sollte der Geschwindigkeitskoeffizient zwischen dem des $\mathrm{CH}_{2} \mathrm{FCH}_{2} \mathrm{OH}$ und dem des Ethanols liegen. Tatsächlich liegt er aber wesentlich darunter, so daß hier auch die Größe des Substituenten einen hohen Einfluß hat. Der Angriff des F-Atoms auf den Alkohol ist vermutlich durch die große $\mathrm{CF}_{3} \mathrm{CF}_{2} \mathrm{CF}_{2}$-Gruppe nur aus bestimmten Vorzugsrichtungen möglich, was zu einer Herabsetzung des Geschwindigkeitskoeffizienten führt. Bei den anderen fluorierten Alkoholen wurde bereits eine nur schwache Temperaturabhängigkeit ermittelt. Für $\mathrm{CF}_{3} \mathrm{CF}_{2} \mathrm{CF}_{2} \mathrm{CH}_{2} \mathrm{OH}+\mathrm{F}$ wurde eine $\mathrm{T}^{0,5}$-Abhängigkeit festgestellt. Eine solche ergibt sich auch aus der Stoßtheorie (vgl. Gleichung (14), S. 11) für Reaktionen ohne Aktivierungsenergie. Die Abstraktionsreaktionen der fluorierten Alkohole mit F-Atomen scheinen also ohne nennenswerte Aktivierungsenergie zu verlaufen.

Bei der Reaktion des erzeugten Radikals mit molekularem Sauerstoff wurde ein Geschwindigkeitskoeffizient von $\mathrm{k}(298 \mathrm{~K})=1 \cdot 10^{10} \mathrm{~cm}^{3} \mathrm{~mol}^{-1} \mathrm{~s}^{-1}$ gefunden. Damit ist auch dies der kleinste Wert im Vergleich mit den übrigen hier untersuchten fluorierten Alkoholen. Für die analoge Reaktion mit $\mathrm{CF}_{3} \mathrm{CH}_{2} \mathrm{OH}$ wurde $\mathrm{k}(298 \mathrm{~K})=2,4 \cdot 10^{10} \mathrm{~cm}^{3} \mathrm{~mol}^{-1} \mathrm{~s}^{-1}$ bestimmt, für $\mathrm{CHF}_{2} \mathrm{CH}_{2} \mathrm{OH}$ $\mathrm{k}(298 \mathrm{~K})=2,3 \cdot 10^{11} \mathrm{~cm}^{3} \mathrm{~mol}^{-1} \mathrm{~s}^{-1}$ gefunden und für $\mathrm{CH}_{2} \mathrm{FCH}_{2} \mathrm{OH} \mathrm{k}(298 \mathrm{~K})=1,15 \cdot 10^{12} \mathrm{~cm}^{3}$ $\mathrm{mol}^{-1} \mathrm{~s}^{-1}$. Als Erklärung hierfür können die gleichen Argumente wie für die Abstraktionsreaktion herangezogen werden. Bezüglich der Temperaturabhängigkeit wurde eine negative Aktivierungsenergie bzw. ein negativer Parameter n gefunden, was für Assoziationsreaktionen mit $\mathrm{O}_{2}$ durchaus üblich ist; es läßt sich damit erklären, daß keine nennenswerte Energiebarriere für die Komplexbildung vorhanden ist und somit bei höherer Temperatur eine größere Überschußenergie im angeregten Komplex vorliegt, die zunehmend zu einer raschen Rückreaktion (Zerfall in die Edukte) führt, bevor eine Stoßdeaktivierung mit einem dritten Stoßpartner (Badgas) erfolgen kann.

142 Die Elektronegativitätswerte sind entnommen aus: Huheey, J. (1988), S. 158 und 169. 


\subsection{Untersuchungen von Methan-Derivaten}

\subsubsection{Das Methan $\left(\mathrm{CH}_{4}\right)$}

\subsubsection{Produkte der radikalerzeugenden Reaktion mit Fluor-Atomen}

Die Reaktion von Methan mit Fluor-Atomen führt zur Bildung des Methyl-Radikals und HF:<smiles>CCPC</smiles>

\subsubsection{Voruntersuchung: Nachweisempfindlichkeit von CO relativ zu Ar im EI-MS}

Um bei den späteren Analysen das giftige Kohlenmonoxid durch Argon ersetzen zu können, wurde in Abhängigkeit der EI-Elektronenenergie (IP) zunächst die massenspektrometrische Empfindlichkeit (vgl. dazu Kap. 2.2, S. 12) von CO relativ zu Ar gemessen: $\varepsilon(\mathrm{CO}) / \varepsilon(\mathrm{Ar})$.

Für den Ansatz $\mathrm{I}\left(\mathrm{P}_{\mathrm{i}}\right)=\varepsilon_{\mathrm{i}}\left[\mathrm{P}_{\mathrm{i}}\right]$ ergibt sich: $\varepsilon(\mathrm{CO}) / \varepsilon(\mathrm{Ar})=\mathrm{I}(\mathrm{CO}) / \mathrm{I}(\mathrm{Ar}) \cdot[\mathrm{Ar}] /[\mathrm{CO}]$

\begin{tabular}{|c|c|c|}
\hline IP / eV & $\frac{\mathrm{I}(\mathrm{CO})}{\mathrm{I}(\mathrm{Ar})} \cdot \frac{[\mathrm{Ar}]}{[\mathrm{CO}]}$ & $\varepsilon(\mathrm{CO}) / \varepsilon(\mathrm{Ar})$ \\
\hline 26,0 & $\frac{1,755 \cdot 10^{4} \text { w.E. }}{1,515 \cdot 10^{4} \text { w.E. }} \cdot \frac{4,82 \cdot 10^{-9} \mathrm{~mol} / \mathrm{cm}^{3}}{5,70 \cdot 10^{-9} \mathrm{~mol} / \mathrm{cm}^{3}}$ & 0,98 \\
\hline 24,0 & $\frac{5,8424 \cdot 10^{4} \text { w.E. }}{3,4413 \cdot 10^{4} \text { w.E. }} \cdot \frac{4,80 \cdot 10^{-9} \mathrm{~mol} / \mathrm{cm}^{3}}{5,73 \cdot 10^{-9} \mathrm{~mol} / \mathrm{cm}^{3}}$ & 1,42 \\
\hline 23,0 & $\frac{4,1182 \cdot 10^{4} \text { w.E. }}{1,9657 \cdot 10^{4} \text { w.E. }} \cdot \frac{4,82 \cdot 10^{-9} \mathrm{~mol} / \mathrm{cm}^{3}}{5,70 \cdot 10^{-9} \mathrm{~mol} / \mathrm{cm}^{3}}$ & 1,77 \\
\hline 22,5 & $\frac{3,306 \cdot 10^{4} \text { w.E. }}{1,3485 \cdot 10^{4} \text { w.E. }} \cdot \frac{4,82 \cdot 10^{-9} \mathrm{~mol} / \mathrm{cm}^{3}}{5,74 \cdot 10^{-9} \mathrm{~mol} / \mathrm{cm}^{3}}$ & 2,06 \\
\hline 22,0 & $\frac{2,389 \cdot 10^{4} \text { w.E. }}{5,805 \cdot 10^{3} \text { w.E. }} \cdot \frac{4,82 \cdot 10^{-9} \mathrm{~mol} / \mathrm{cm}^{3}}{5,74 \cdot 10^{-9} \mathrm{~mol} / \mathrm{cm}^{3}}$ & 3,46 \\
\hline 21,5 & $\frac{1,751 \cdot 10^{4} \text { w.E. }}{1,539 \cdot 10^{3} \text { w.E. } \cdot \frac{4,82 \cdot 10^{-9} \mathrm{~mol} / \mathrm{cm}^{3}}{5,74 \cdot 10^{-9} \mathrm{~mol} / \mathrm{cm}^{3}}}$ & 9,55 \\
\hline 17 & für $17 \mathrm{eV} \mathrm{siehe:} \mathrm{Beiderhase,} \mathrm{T.}(1995), \mathrm{S}^{3} \cdot 78$. & 49 \\
\hline
\end{tabular}

Tabelle 52: Massenspektrometrische Empfindlichkeit von CO rel. zu Ar

Die Ergebnisse der eigenen Messung sind umseitig nochmals grafisch dargestellt. 


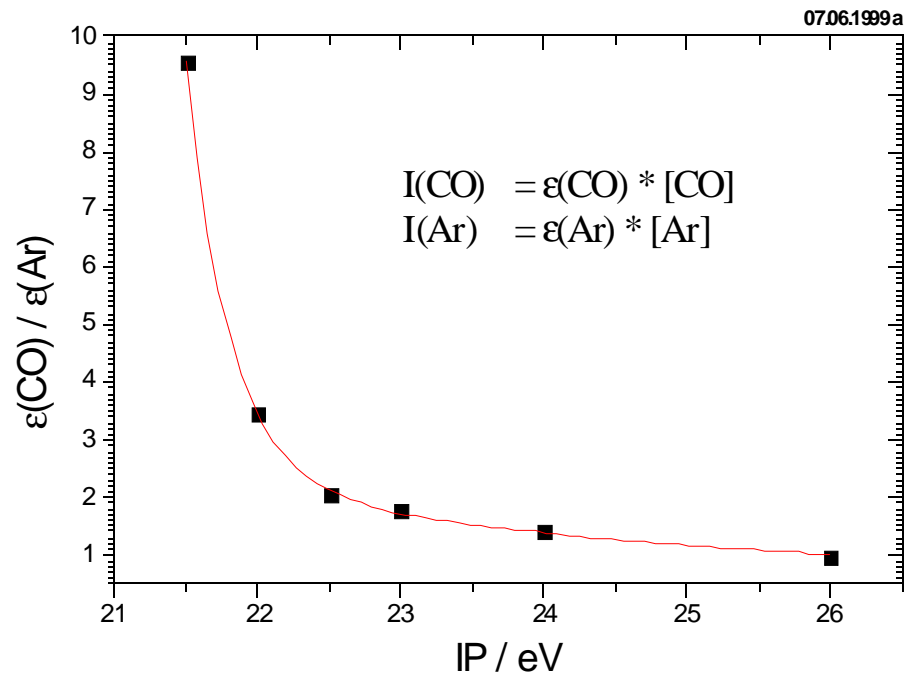

Abbildung 174: Massenspektrometrische Empfindlichkeit von CO rel. zu Ar

Die in Tabelle 52 eingetragenen Messungen wurden in der in Kap. 3.1.1 (S. 16) beschriebenen Apparatur unter Einsatz des Strömungsreaktors aus Abbildung 5 (S. 17) durchgeführt. Zur Ausführung vgl. Kap. 3.5.1 (S. 50). Der Gasfluß an Helium betrug 600 SCCM, der von CO etwa 66 SCCM und der von Ar etwa 55 SCCM (für CO und Ar wurde er gesondert für jeden Meßpunkt exakt ermittelt). Zur Bestimmung der Gasflüsse von CO und Ar kamen geeichte Flußmesser (TYLAN FC 260) zum Einsatz. Damit konnten dann mittels Gleichung (19) (S. 14) die Konzentrationen [CO] und [Ar] berechnet werden.

Mit den Angaben aus Tabelle 52 bzw. Abbildung 174 kann nun aus gemessenen Intensitäten I von $\operatorname{Ar}(\mathrm{m} / \mathrm{z} 40)$ und $\mathrm{CO}(\mathrm{m} / \mathrm{z} 28)$ bei bekannter Argon-Konzentration die Konzentration des Kohlenmonoxids CO ermittelt werden:

$$
[\mathrm{CO}]=\frac{\varepsilon(\mathrm{Ar})}{\varepsilon(\mathrm{CO})} \cdot \frac{\mathrm{I}(\mathrm{CO})}{\mathrm{I}(\mathrm{Ar})} \cdot[\mathrm{Ar}]
$$

\subsubsection{Produkte der Reaktion des erzeugten Radikals mit Sauerstoff-Atomen}

Die Messungen wurden in der in Kap. 3.1.1 (S. 16) beschriebenen Apparatur unter Einsatz des Strömungsreaktors aus Abbildung 5 (S. 17) durchgeführt; zur Ausführung vgl. Kap. 3.5.1 (S. 50). Das aus Methan unter Zugabe von Fluor-Atomen erzeugte Methyl-Radikal wurde mit Sauerstoff-Atomen zur Reaktion gebracht. Dabei zeigten sich Zunahmen auf m/z 28, 29 und 
30. Diese können durch die Bildung von Methanal (Formaldehyd, HCHO) erklärt werden, welches Fragmente auf diesen Massenzahlen aufweist.
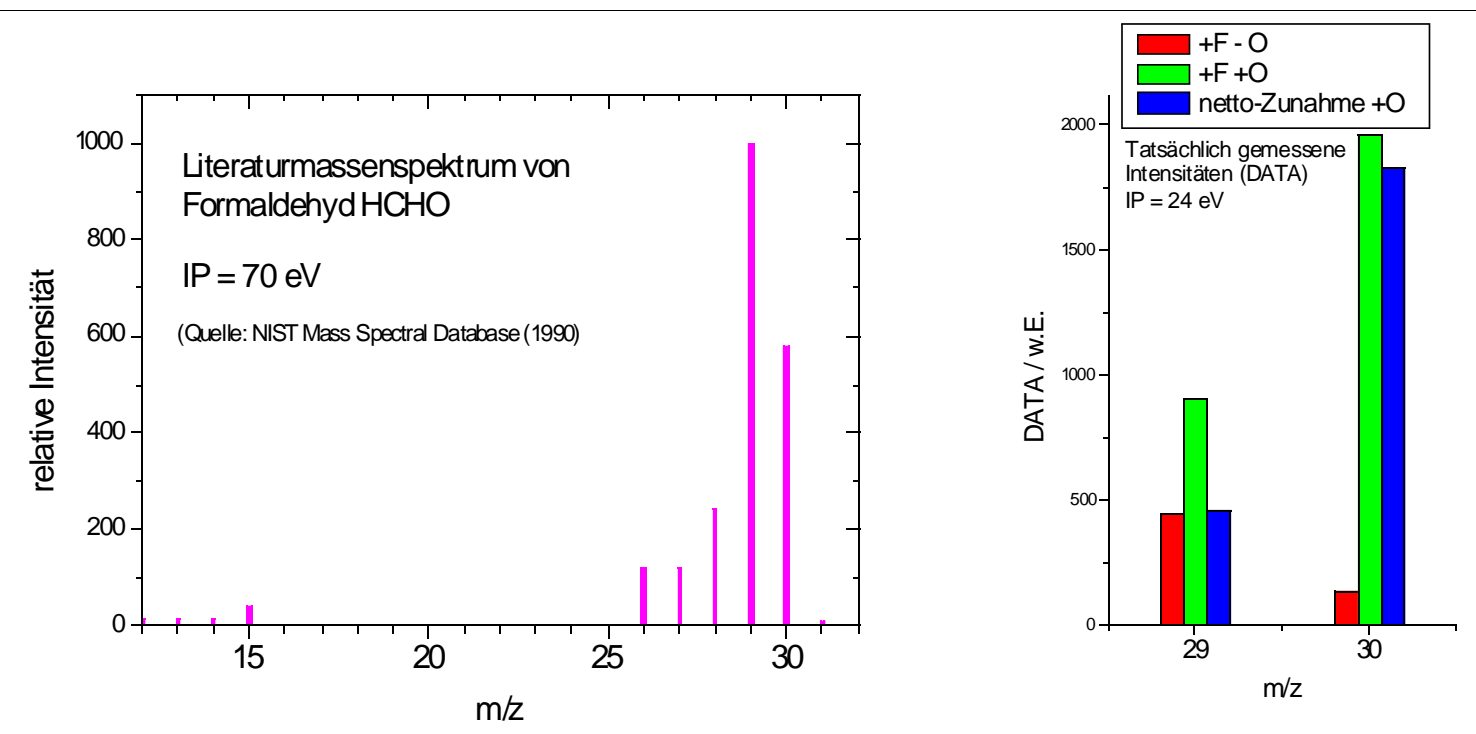

Abbildung 175: Literatur-EI-MS von HCHO sowie EI-MS für CH4+F+/- O

In dem Literaturspektrum von HCHO bei IP $=70 \mathrm{eV}$ (linke Grafik in Abb. 175) ist der höchste Peak das Fragment auf m/z 29, während die Intensität des Molekül-Peaks nur rund $60 \%$ davon beträgt. In dem hier gemessenen Spektrum bei IP $=24 \mathrm{eV}$ ist jedoch die Intensität auf m/z 30 deutlich höher (Faktor 4) als auf m/z 29 (vgl. blaue Säulen der rechten Grafik in Abb. 175). Weil die Intensität des Fragments auf $\mathrm{m} / \mathrm{z} 28$ bereits bei IP $=70 \mathrm{eV}$ lediglich $24 \%$ derjenigen auf m/z 29 beträgt, kann man davon ausgehen, daß sie auf m/z 28 bei IP $=24 \mathrm{eV}$ vernachlässigbar klein geworden ist. Unter der Voraussetzung, daß sich bei der Absenkung des IP von 70 auf $24 \mathrm{eV}$ die Intensität auf m/z 28 relativ zu m/z 30 gleichermaßen vermindert wie die auf $\mathrm{m} / \mathrm{z} 29$, resultiert für $\mathrm{m} / \mathrm{z} 28$ bei $\mathrm{IP}=24 \mathrm{eV}$ nur noch eine relative Intensität von weniger als $6 \%$ des Molekül-Peaks auf m/z 30 .

Weil aber auf m/z 28 eine viel höhere Zunahme beobachtet wurde, ist davon auszugehen, daß neben Methanal (HCHO) auch Kohlenmonoxid (CO) entsteht. Der genaue Anteil an CO soll nachfolgend bestimmt werden.

Dazu wurden Messungen bei einer Temperatur von 298 K, einem Druck von 202 Pa und Gasflüssen von $\phi_{\mathrm{He}}=600 \mathrm{SCCM}, \phi_{\mathrm{CH} 4}=26,0 \mathrm{SCCM}, \phi_{\mathrm{F} 2 / \mathrm{He}}=122,8 \mathrm{SCCM}$ und $\phi_{\mathrm{O} 2}=8 \mathrm{SCCM}$ (insgesamt also: $\phi_{\text {gesamt }}=756,8$ SCCM) ausgeführt. Statt Methan wurde später Argon zugegeben $\left(\phi_{\mathrm{Ar}}=26,0 \mathrm{SCCM}\right)$.

Damit errechnet man als Ausgangskonzentration für Methan: $[\mathrm{CH} 4]_{0}=2,80 \cdot 10^{-9} \mathrm{~mol} / \mathrm{cm}^{3}$ und für Argon $[\mathrm{Ar}]_{0}=2,80 \cdot 10^{-9} \mathrm{~mol} / \mathrm{cm}^{3}$. 
Auf dem Molekül-Peak von Methan (m/z 16) konnte nun der Umsatz bei Zugabe von FluorAtomen bestimmt werden.

\begin{tabular}{|l|l|l|l|l|l|}
\hline \multicolumn{2}{|l|}{ Umsatz von Methan auf m/z 16: } \\
\hline & Zustand MWE & DATA & Umsatz & & \\
\hline Messung1: & aus, d.h. ,,-F“ & $3,02 \mathrm{E}+004$ & & & \\
\hline & ein, d.h. „,F“ & $1,60 \mathrm{E}+004$ & $46,83 \%$ & & \\
\hline Messung2: & aus, d.h. ,-F“ & $3,05 \mathrm{E}+004$ & & Mittelwert: & $47,27 \%$ \\
\hline & ein, d.h. ,+F“ & $1,60 \mathrm{E}+004$ & $47,70 \%$ & & \\
\hline
\end{tabular}

Zur Durchführung der in Kap. 3.5.1 (S. 50) beschriebenen Umsatzkorrektur waren folgende Messungen auf dem Fragment-Peak des Methans m/z 15 erforderlich:

Intensität Fragment-Peak auf m/z 15 (MWE: ausgeschaltet):

\begin{tabular}{|l|l|l|l|l|}
\hline Messung1: & DATA (unkorr.) & $1,19 \mathrm{E}+004$ & => Korr.-wert: & 6264,48 \\
\hline Messung2: & DATA (unkorr.) & $1,33 \mathrm{E}+004$ & => Korr.-wert: & 6997,44 \\
\hline & & & Mittelwert: & 6630,96 \\
\hline
\end{tabular}

Nun mußte der Umsatz des Methyl-Radikals bei Zugabe von Sauerstoff-Atomen ermittelt werden (wegen großer Streuungen waren mehrere Messungen erforderlich):

\begin{tabular}{|c|c|c|c|c|}
\hline \multicolumn{5}{|c|}{ Umsatz auf Radikal-Peak m/z 15: } \\
\hline \multirow[t]{2}{*}{ Messung1: } & $\begin{array}{l}\text { DATA ,,-O“ } \\
\text { (unkorr.) }\end{array}$ & $9,93 \mathrm{E}+003$ & & \\
\hline & $\begin{array}{l}\text { DATA ,,+O“ } \\
\text { (unkorr.) }\end{array}$ & $8,64 \mathrm{E}+003$ & $\begin{array}{l}\text { Umsatz nach } \\
\text { Umsatzkorrektur }\end{array}$ & $39,08 \%$ \\
\hline \multirow[t]{2}{*}{ Messung2: } & $\begin{array}{l}\text { DATA ,-O“ } \\
\text { (unkorr.) }\end{array}$ & $9,43 E+003$ & & \\
\hline & $\begin{array}{l}\text { DATA ,,+O“ } \\
\text { (unkorr.) }\end{array}$ & $8,48 \mathrm{E}+003$ & $\begin{array}{l}\text { Umsatz nach } \\
\text { Umsatzkorrektur }\end{array}$ & $33,87 \%$ \\
\hline \multirow[t]{2}{*}{ Messung3: } & $\begin{array}{l}\text { DATA ,-O“ } \\
\text { (unkorr.) }\end{array}$ & $9,45 \mathrm{E}+003$ & & \\
\hline & $\begin{array}{l}\text { DATA ,,+O“ } \\
\text { (unkorr.) }\end{array}$ & $7,92 \mathrm{E}+003$ & $\begin{array}{l}\text { Umsatz nach } \\
\text { Umsatzkorrektur }\end{array}$ & $54,15 \%$ \\
\hline \multirow[t]{2}{*}{ Messung4: } & $\begin{array}{l}\text { DATA ,-O“" } \\
\text { (unkorr.) }\end{array}$ & $9,59 \mathrm{E}+003$ & & \\
\hline & $\begin{array}{l}\text { DATA ,,+O“ } \\
\text { (unkorr.) }\end{array}$ & $8,69 \mathrm{E}+003$ & $\begin{array}{l}\text { Umsatz nach } \\
\text { Umsatzkorrektur }\end{array}$ & $30,46 \%$ \\
\hline \multirow[t]{4}{*}{ Messung5: } & $\begin{array}{l}\text { DATA ,-O“ } \\
\text { (unkorr.) }\end{array}$ & $9,72 \mathrm{E}+003$ & & \\
\hline & $\begin{array}{l}\text { DATA ,,+O“ } \\
\text { (unkorr.) }\end{array}$ & $8,79 \mathrm{E}+003$ & $\begin{array}{l}\text { Umsatz nach } \\
\text { Umsatzkorrektur }\end{array}$ & $30,16 \%$ \\
\hline & & & Mittelwert: & $37,54 \%$ \\
\hline & & \multicolumn{2}{|c|}{$=>[\mathrm{CH} 3]_{\text {reagiert }} / \mathrm{mol} / \mathrm{cm} 3:$} & $4,97 \mathrm{E}-010$ \\
\hline
\end{tabular}


Die Konzentration der Methyl-Radikale, die mit Sauerstoff-Atomen reagiert haben, ergab sich aus der Ausgangskonzentration von Methan, dem Umsatz von Methan mit F-Atomen und dem Umsatz der Methyl-Radikale mit Sauerstoff-Atomen:

$[\mathrm{CH} 3]_{\text {reagiert }}=2,80 \cdot 10^{-9} \mathrm{~mol} / \mathrm{cm}^{3} \cdot(0,4727) \cdot(0,3754)=4,97 \cdot 10^{-10} \mathrm{~mol} / \mathrm{cm}^{3}$.

Nun erfolgte die eigentliche Messung auf dem Molekül-Peak von CO:

\begin{tabular}{|c|c|c|c|}
\hline \multicolumn{4}{|c|}{ Messung auf m/z 28 (DATA-Werte): } \\
\hline Messung-Nr. & $+\mathrm{F}-\mathrm{O}$ & $+\mathrm{F}+\mathrm{O}$ & Differenz \\
\hline 1 & $2,76 \mathrm{E}+002$ & $1,63 \mathrm{E}+003$ & 1355,8 \\
\hline 2 & $4,846 \mathrm{E}+02$ & $2,254 \mathrm{E}+03$ & 1769,4 \\
\hline 3 & $4,700 \mathrm{E}+02$ & $2,222 \mathrm{E}+03$ & 1752 \\
\hline 4 & $5,688 \mathrm{E}+02$ & $2,292 \mathrm{E}+03$ & 1723,2 \\
\hline & & Mittelwert: & 1650,1 \\
\hline & \multicolumn{2}{|c|}{.$/ .6 \%$ von $\mathrm{I}(30)=0,06 * 1823,8:$} & $-109,43$ \\
\hline & \multicolumn{2}{|c|}{ => effektive Zunahme durch CO: } & 1540,67 \\
\hline
\end{tabular}

Um aus den DATA-Werten die Konzentration [CO] berechnen zu können, waren nun noch die DATA-Werte für Argon erforderlich (vgl. Gleichung (30), S. 199):

\begin{tabular}{|r|r|}
\hline Messung I(Ar) auf $\mathrm{m} / \mathrm{z}$ 40: \\
\hline Messung-Nr. & DATA \\
\hline 1 & $1,17 \mathrm{E}+004$ \\
\hline 2 & $1,328 \mathrm{E}+04$ \\
\hline 3 & $1,360 \mathrm{E}+04$ \\
\hline 4 & $1,362 \mathrm{E}+04$ \\
\hline 5 & $1,348 \mathrm{E}+04$ \\
\hline 6 & $1,400 \mathrm{E}+04$ \\
\hline Mittelwert: & $1,328 \mathrm{E}+04$ \\
\hline
\end{tabular}

Mit dem Verhältnis der massenspektrometrischen Empfindlichkeiten $\varepsilon(\mathrm{CO}) / \varepsilon(\mathrm{Ar})=1,42$ aus Tabelle 52 (S. 198) bzw. Abbildung 174 (S. 199) kann man mittels Gleichung (30) (S. 199) die Konzentration an $\mathrm{CO}$ berechnen:

$$
[\mathrm{CO}]=\frac{1}{1,42} \cdot \frac{1540,67}{1,328 \cdot 10^{04}} \cdot 2,80 \cdot 10^{-09} \mathrm{~mol} / \mathrm{cm}^{3}=2,29 \cdot 10^{-10} \mathrm{~mol} / \mathrm{cm}^{3}
$$

Mit dem Verhältnis aus der entstandenen CO-Konzentration und der Konzentration der Methyl-Radikale, die insgesamt mit Sauerstoff-Atomen reagiert haben, läßt sich schlußendlich 
der Anteil $\alpha$ für den Reaktionskanal der CO-Bildung berechnen:

$$
\alpha(\mathrm{CO})=\frac{2,29 \cdot 10^{-10} \mathrm{~mol} / \mathrm{cm}^{3}}{4,97 \cdot 10^{-10} \mathrm{~mol} / \mathrm{cm}^{3}}=46 \mathrm{~mol} \%
$$

Aus den Standardabweichungen der einzelnen Messungen kann gemäß den Gesetzen der Fehlerfortpflanzung ein Fehler von $\pm 6 \%$ angegeben werden.

Als Endergebnis erhält man daher: $\alpha(\mathrm{CO})=(\mathbf{4 6} \pm \mathbf{6}) \mathbf{m o l}-\%$.

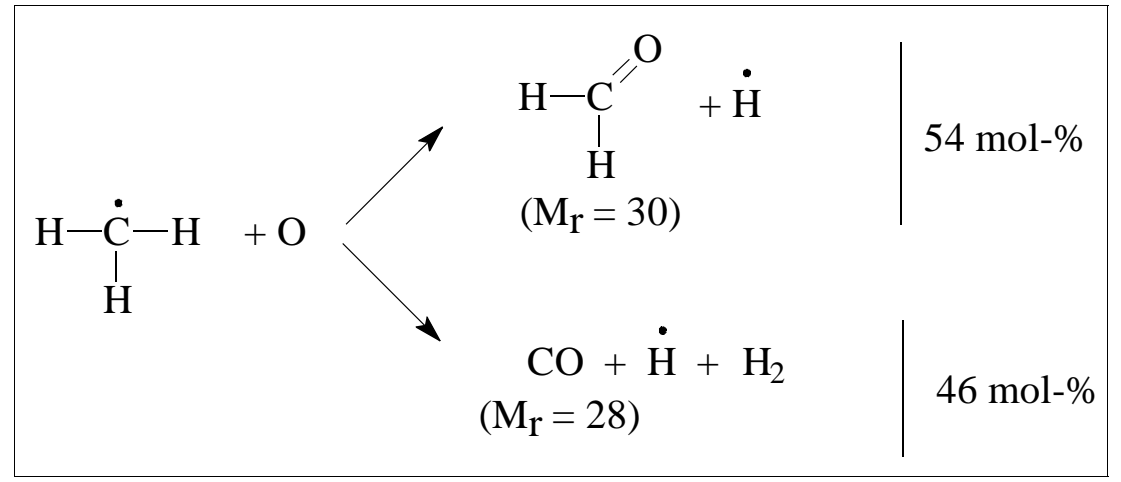

Abbildung 176: Reaktionskanäle f. $\mathrm{CH} 3+\mathrm{O}$

\subsubsection{Diskussion der Ergebnisse}

In der Literatur wird weitgehend davon ausgegangen, daß es für die Reaktion der MethylRadikale mit Sauerstoff-Atomen nur einen Reaktionskanal gibt, nämlich den zu Methanal $(\mathrm{HCHO})$ und Wasserstoff-Atomen ${ }^{143}$. Es lag nur ein Bericht mit einer davon abweichenden Meinung vor; dort wurde mittels FT-IR-Emissionsmessungen Kohlenmonoxid als Primärprodukt mit einem Anteil von $\alpha^{\text {Literatur }}(\mathrm{CO})=(40 \pm 10) \%$ ermittelt bzw. abgeleitet ${ }^{144}$.

Das hier erhaltene Ergebnis stimmt im Rahmen seiner Fehlergrenzen hervorragend mit dem der zuletzt vorgestellten (Minderheits-)Meinung in der Literatur überein.

Gestützt wird dieses Resultat zudem durch eine FT-IR-Messung (Absorption) unserer Arbeitsgruppe, bei der Iodmethan mit einem Excimer-LASER (193 nm) zu Methyl-Radikalen und gleichzeitig in Cophotolyse $\mathrm{SO}_{2}$ zu O-Atomen photolysiert (vgl. Kap. 3.5.5, S. 59) und die Produkte anhand authentischer Vergleichsspektren quantitativ bestimmt wurden. Hierbei ergab sich ein Kohlenmonoxid-Kanalanteil $\operatorname{von}^{145} \alpha(\mathrm{CO})=(45 \pm 2)$ mol-\%. Letzterer stimmt ausgezeichnet mit dem in der vorliegenden Arbeit ermittelten Wert überein.

143 Vgl. z.B.: Slagle, I.R.; Sarzynski, D.; Gutman, D. (1987).

144 Seakins, P.W.; Leone, S.R. (1992).

145 Zeuch, T. (voraussichtlich 2003). 


\subsubsection{Das Chlormethan $\left(\mathrm{CH}_{3} \mathrm{Cl}\right)$}

\subsubsection{Produkte der radikalerzeugenden Reaktion mit Fluor-Atomen}

Bei der Reaktion von Chlormethan mit Fluor-Atomen wurde nur der Abstraktionskanal gefunden, der zur Bildung des Chlormethyl-Radikals führt ${ }^{146}: \mathrm{CH}_{3} \mathrm{Cl}+\mathrm{F} \rightarrow \mathrm{CH}_{2} \mathrm{Cl}+\mathrm{HF}$

\subsubsection{Kinetik der radikalerzeugenden Reaktion mit Fluor-Atomen}

Die Reaktion wurde temperaturabhängig zwischen 253 und $358 \mathrm{~K}$ relativ zu Methan untersucht. Als Geschwindigkeitskoeffizient erhält $\operatorname{man}^{147}$ :

$$
\begin{aligned}
& \mathbf{k}=(4,33 \pm 0,88) \cdot 10^{13} \exp \{-(1,34 \pm 0,51) \mathrm{kJ} / \mathrm{mol} /(\mathrm{R} \cdot \mathrm{T})\} \mathrm{cm}^{3} \mathrm{~mol}^{-1} \mathrm{~s}^{-1} \\
& \text { für: } \mathrm{CH}_{3} \mathrm{Cl}+\mathrm{F} \rightarrow \text { Produkte }
\end{aligned}
$$

Bei $\mathrm{T}=298 \mathrm{~K}$ ergibt sich damit: $\mathrm{k}^{298 \mathrm{~K}}=2,52 \mathrm{E}+13 \mathrm{~cm}^{3} \mathrm{~mol}^{-1} \mathrm{~s}^{-1}$.

\subsubsection{Produkte der Reaktion des erzeugten Radikals mit Sauerstoff-Atomen}

Analog zur Bestimmung des Anteils an Kohlenmonoxid bei der Reaktion $\mathrm{CH}_{3}+\mathrm{O}$ (vgl. Kap. 4.4.1, S. 198) wird nachfolgend dieser Anteil für die Reaktion $\mathrm{CH}_{2} \mathrm{Cl}+\mathrm{O}$ bestimmt.

Dazu wurden Messungen bei einer Temperatur von 298 K, einem Druck von 189 Pa und Gasflüssen von $\phi_{\mathrm{He}}=600 \mathrm{SCCM}, \phi_{\mathrm{CH} 3 \mathrm{Cl}}=26,1 \mathrm{SCCM}, \phi_{\mathrm{F} 2 / \mathrm{He}}=95,9 \mathrm{SCCM}$ und $\phi_{\mathrm{O} 2}=5 \mathrm{SCCM}$ (insgesamt also: $\phi_{\text {gesant }}=727$ SCCM) ausgeführt. Methan wurde zur Eichung später durch Argon ersetzt $\left(\phi_{\mathrm{Ar}}=26,1 \mathrm{SCCM}\right)$.

Damit errechnet man als Ausgangskonzentration für Methan: $[\mathrm{CH} 4]_{0}=2,74 \cdot 10^{-9} \mathrm{~mol} / \mathrm{cm}^{3}$ und für Argon $[\mathrm{Ar}]_{0}=2,74 \cdot 10^{-9} \mathrm{~mol} / \mathrm{cm}^{3}$.

Auf dem Molekül-Peak von Chlormethan (m/z 50) konnte nun der Umsatz bei Zugabe von

\begin{tabular}{|c|c|c|c|c|c|}
\hline \multicolumn{6}{|c|}{ Umsatz von Chlormethan auf m/z 50: } \\
\hline & Zustand MWE & DATA & Umsatz & & \\
\hline \multirow[t]{2}{*}{ Messung1: } & aus, d.h. ,,-F“ & $6,25 \mathrm{E}+004$ & & & \\
\hline & ein, d.h. „,+F“ & $3,99 \mathrm{E}+004$ & $36,11 \%$ & & \\
\hline \multirow[t]{2}{*}{ Messung2: } & aus, d.h. ,,-F“ & $6,13 \mathrm{E}+004$ & & Mittelwert: & $35,93 \%$ \\
\hline & ein, d.h. ,,+F“ & $3,94 \mathrm{E}+004$ & $35,76 \%$ & & \\
\hline
\end{tabular}
Fluor-Atomen bestimmt werden. 
Zur Durchführung der in Kap. 3.5.1 (S. 50) beschriebenen Umsatzkorrektur waren folgende Messungen auf dem Fragment-Peak des Chlormethans m/z 49 erforderlich:

\begin{tabular}{|l|l|r|l|r|}
\hline \multicolumn{6}{|l|}{ Intensität Fragment-Peak auf m/z 49 (MWE: ausgeschaltet): } \\
\hline Messung1: & DATA (unkorr.) & $3,84 \mathrm{E}+003$ & => Korr.-wert: & 2456,99 \\
\hline Messung2: & DATA (unkorr.) & $3,77 \mathrm{E}+003$ & => Korr.-wert: & 2413,42 \\
\hline & & & Mittelwert: & 2435,21 \\
\hline
\end{tabular}

Nun mußte der Umsatz des Chlormethyl-Radikals bei Zugabe von Sauerstoff-Atomen ermittelt werden (wegen großer Streuungen waren mehrere Messungen erforderlich):

\begin{tabular}{|c|c|c|c|c|}
\hline \multicolumn{5}{|c|}{ Umsatz auf Radikal-Peak m/z 49: } \\
\hline \multirow[t]{2}{*}{ Messung1: } & $\begin{array}{l}\text { DATA ,,O“ } \\
\text { (unkorr.) }\end{array}$ & $3,29 \mathrm{E}+003$ & & \\
\hline & $\begin{array}{l}\text { DATA ,,+O“ } \\
\text { (unkorr.) }\end{array}$ & $2,61 \mathrm{E}+003$ & $\begin{array}{l}\text { Umsatz nach } \\
\text { Umsatzkorrektur }\end{array}$ & $80,09 \%$ \\
\hline \multirow[t]{2}{*}{ Messung2: } & $\begin{array}{l}\text { DATA ,-O“ } \\
\text { (unkorr.) }\end{array}$ & $3,20 \mathrm{E}+003$ & & \\
\hline & $\begin{array}{l}\text { DATA ,,+O“ } \\
\text { (unkorr.) }\end{array}$ & $2,66 \mathrm{E}+003$ & $\begin{array}{l}\text { Umsatz nach } \\
\text { Umsatzkorrektur }\end{array}$ & $70,42 \%$ \\
\hline \multirow[t]{2}{*}{ Messung3: } & $\begin{array}{l}\text { DATA ,-O“ } \\
\text { (unkorr.) }\end{array}$ & $3,38 \mathrm{E}+003$ & & \\
\hline & $\begin{array}{l}\text { DATA ,,+O“ } \\
\text { (unkorr.) }\end{array}$ & $2,62 \mathrm{E}+003$ & $\begin{array}{l}\text { Umsatz nach } \\
\text { Umsatzkorrektur }\end{array}$ & $80,87 \%$ \\
\hline \multirow[t]{2}{*}{ Messung4: } & $\begin{array}{l}\text { DATA ,-O“ } \\
\text { (unkorr.) }\end{array}$ & $3,33 \mathrm{E}+003$ & & \\
\hline & $\begin{array}{l}\text { DATA ,,+O“ } \\
\text { (unkorr.) }\end{array}$ & $2,64 \mathrm{E}+003$ & $\begin{array}{l}\text { Umsatz nach } \\
\text { Umsatzkorrektur }\end{array}$ & $77,75 \%$ \\
\hline \multirow[t]{2}{*}{ Messung5: } & $\begin{array}{l}\text { DATA ,-O“ } \\
\text { (unkorr.) }\end{array}$ & $3,23 \mathrm{E}+003$ & & \\
\hline & $\begin{array}{l}\text { DATA ,,+O“ } \\
\text { (unkorr.) }\end{array}$ & $2,66 \mathrm{E}+003$ & $\begin{array}{l}\text { Umsatz nach } \\
\text { Umsatzkorrektur }\end{array}$ & $72,42 \%$ \\
\hline \multirow[t]{4}{*}{ Messung6: } & $\begin{array}{l}\text { DATA ,,-O“ } \\
\text { (unkorr.) }\end{array}$ & $3,20 \mathrm{E}+003$ & & \\
\hline & $\begin{array}{l}\text { DATA ,,+O“ } \\
\text { (unkorr.) }\end{array}$ & $2,63 \mathrm{E}+003$ & $\begin{array}{l}\text { Umsatz nach } \\
\text { Umsatzkorrektur }\end{array}$ & $74,53 \%$ \\
\hline & & & Mittelwert: & $76,01 \%$ \\
\hline & & \multicolumn{2}{|c|}{$\Rightarrow[\mathrm{CH} 2 \mathrm{Cl}]_{\text {reagiert }} / \mathrm{mol} / \mathrm{cm} 3$ : } & 7,48E-010 \\
\hline
\end{tabular}

Die Konzentration der Chlormethyl-Radikale, die mit Sauerstoff-Atomen reagiert haben, ergab sich aus der Ausgangskonzentration von Chlormethan, dem Umsatz von Chlormethan mit FAtomen und dem Umsatz der Chlormethyl-Radikale mit Sauerstoff-Atomen:

$\left[\mathrm{CH}_{2} \mathrm{Cl}\right]_{\text {reagiert }}=2,74 \cdot 10^{-9} \mathrm{~mol} / \mathrm{cm}^{3} \cdot(0,3593) \cdot(0,7601)=7,48 \cdot 10^{-10} \mathrm{~mol} / \mathrm{cm}^{3}$. 
Nun erfolgte die eigentliche Messung auf dem Molekül-Peak von CO:

\begin{tabular}{|c|c|c|c|}
\hline \multicolumn{4}{|c|}{ Messung auf $\mathrm{m} / \mathrm{z} 28$ (DATA-Werte): } \\
\hline Messung-Nr. & $+\mathrm{F}-\mathrm{O}$ & $+\mathrm{F}+\mathrm{O}$ & Differenz \\
\hline 1 & $2,77 \mathrm{E}+003$ & $4,90 \mathrm{E}+002$ & 2278,5 \\
\hline 2 & $2,82 \mathrm{E}+003$ & $4,67 \mathrm{E}+002$ & 2355,2 \\
\hline 3 & $2,82 \mathrm{E}+003$ & $4,95 \mathrm{E}+002$ & 2320,2 \\
\hline & & Mittelwert: & 2317,97 \\
\hline & \multicolumn{2}{|c|}{ => effektive Zunahme durch CO: } & 2317,97 \\
\hline
\end{tabular}

Um aus den DATA-Werten die Konzentration [CO] berechnen zu können, waren nun noch die DATA-Werte für Argon erforderlich (vgl. Gleichung (30), S. 199):

\begin{tabular}{|r|r|}
\hline Messung I(Ar) auf $\mathrm{m} / \mathrm{z}$ 40: \\
\hline Messung-Nr. & DATA \\
\hline 1 & $1,362 \mathrm{E}+04$ \\
\hline 2 & $1,307 \mathrm{E}+04$ \\
\hline 3 & $1,362 \mathrm{E}+04$ \\
\hline 4 & $1,307 \mathrm{E}+04$ \\
\hline 5 & $1,408 \mathrm{E}+04$ \\
\hline 6 & $1,450 \mathrm{E}+04$ \\
\hline 7 & $1,448 \mathrm{E}+04$ \\
\hline 8 & $1,428 \mathrm{E}+04$ \\
\hline Mittelwert: & $1,384 \mathrm{E}+04$ \\
\hline
\end{tabular}

Mit dem Verhältnis der massenspektrometrischen Empfindlichkeiten $\varepsilon(\mathrm{CO}) / \varepsilon(\mathrm{Ar})=1,42$ aus Tabelle 52 (S. 198) bzw. Abbildung 174 (S. 199) kann man mittels Gleichung (30) (S. 199) die Konzentration an $\mathrm{CO}$ berechnen:

$$
[\mathrm{CO}]=\frac{1}{1,42} \cdot \frac{2317,97}{1,384 \cdot 10^{04}} \cdot 2,74 \cdot 10^{-09} \mathrm{~mol} / \mathrm{cm}^{3}=3,23 \cdot 10^{-10} \mathrm{~mol} / \mathrm{cm}^{3}
$$

Mit dem Verhältnis aus der entstandenen CO-Konzentration und der Konzentration der Methyl-Radikale, die insgesamt mit Sauerstoff-Atomen reagiert haben, läßt sich schlußendlich der Anteil $\alpha$ für den Reaktionskanal der CO-Bildung berechnen:

$$
\alpha(\mathrm{CO})=\frac{3,23 \cdot 10^{-10} \mathrm{~mol} / \mathrm{cm}^{3}}{7,48 \cdot 10^{-10} \mathrm{~mol} / \mathrm{cm}^{3}}=43 \mathrm{~mol} \%
$$

Aus den Standardabweichungen der einzelnen Messungen kann gemäß den Gesetzen der 
Fehlerfortpflanzung ein Fehler von $\pm 2 \%$ angegeben werden.

Als Endergebnis erhält man daher: $\alpha(\mathbf{C O})=(\mathbf{4 3} \pm \mathbf{2})$ mol-\%

Unter der Voraussetzung, daß für die Reaktion $\mathrm{CH}_{3}+\mathrm{O}$ nur ein Reaktionskanal existiert, der zu Methanal (HCHO) führt, war für die hier untersuchte Reaktion $\mathrm{CH}_{2} \mathrm{Cl}+\mathrm{O}$ ein Anteil von 35,5 mol-\% für den Kanalanteil zum Methanal ermittelt worden ${ }^{148}$. In Kapitel 4.4.1 (S. 198) wurde jedoch für $\mathrm{CH}_{3}+\mathrm{O}$ ein Anteil von 46 mol-\% für den Reaktionskanal bestimmt, der zu Kohlenmonoxid führt. Damit kann der Anteil für Methanal nur noch 54 mol-\% betragen. Berücksichtigt man diese neue Erkenntnis bei der Auswertung, dann erhält man für die Reaktion $\mathrm{CH}_{2} \mathrm{Cl}+\mathrm{O}$ einen Anteil von 19 mol-\% für den Kanalanteil, der zu Methanal führt.

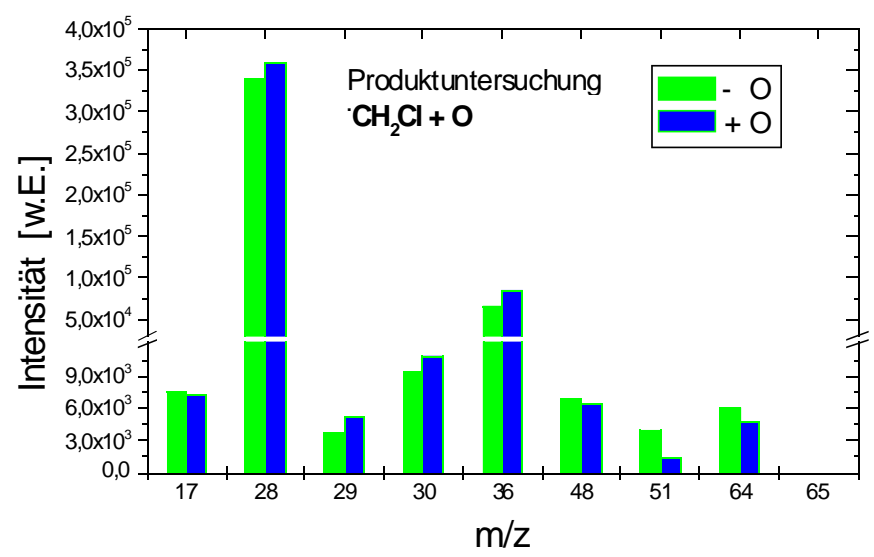

Abbildung 177: EI-MS Produktuntersuchung CH2Cl+O; $298 \mathrm{~K}$

Die Bedingungen während der Aufnahme des Produktspektrums ${ }^{149}$ in Abbildung 177 waren:

$\vartheta=25^{\circ} \mathrm{C} ; \phi_{\mathrm{He}}=15,83 \mathrm{~cm}^{3} / \mathrm{s} ; \phi_{\mathrm{CH} 3 \mathrm{Cl}}=0,42 \mathrm{~cm}^{3} / \mathrm{s} ; \mathrm{p}_{\mathrm{StrR}}=173 \mathrm{~Pa} ;$ Reaktionsstrecke $=7,5 \mathrm{~cm}$;

IP = 22,0 eV; Zählzeit = $20 \mathrm{~s}$; Mittelwert aus 6 Messungen gebildet.

Gemäß Abbildung 177 wurde keine Zunahme auf $\mathrm{m} / \mathrm{z}=64$ (ClCHO) beobachtet, so daß Reaktionskanal (1) der Abbildung 178 nicht direkt nachgewiesen werden konnte. Die Zunahmen auf $\mathrm{m} / \mathrm{z}=28(\mathrm{CO})$ und $36(\mathrm{HCl})$ könnten durch Kanal (2), aber auch durch die Folgereaktion (5) erklärt werden, so daß Kanal (1) zumindest nicht ausgeschlossen werden kann. Kanal (3) der Abbildung 178 wird durch Zunahme auf m/z = 30 (HCHO) bestätigt; Kanal (4) durch die Zunahme auf $\mathrm{m} / \mathrm{z}=29$ (HCO). Die Zunahme auf $\mathrm{m} / \mathrm{z}=29 \mathrm{kann}$ nicht nur durch ein Fragment des HCHO und/oder den Anteil des $\left({ }^{13} \mathrm{C}\right)$-Isotops im $\mathrm{CO}$ erklärt werden. Zur Bestätigung von Kanal (6) hätte auf Grund des ${ }^{37} \mathrm{Cl}$-Isotops eine Zunahme auf m/z 65 
vorliegen müssen, was jedoch nicht beobachtet wurde. Auch Kanal (7) konnte nicht gefunden werden, denn weder auf $\mathrm{m} / \mathrm{z}=17(\mathrm{OH})$ noch auf $\mathrm{m} / \mathrm{z}=48(\mathrm{CHCl})$ wurde eine Zunahme beobachtet.

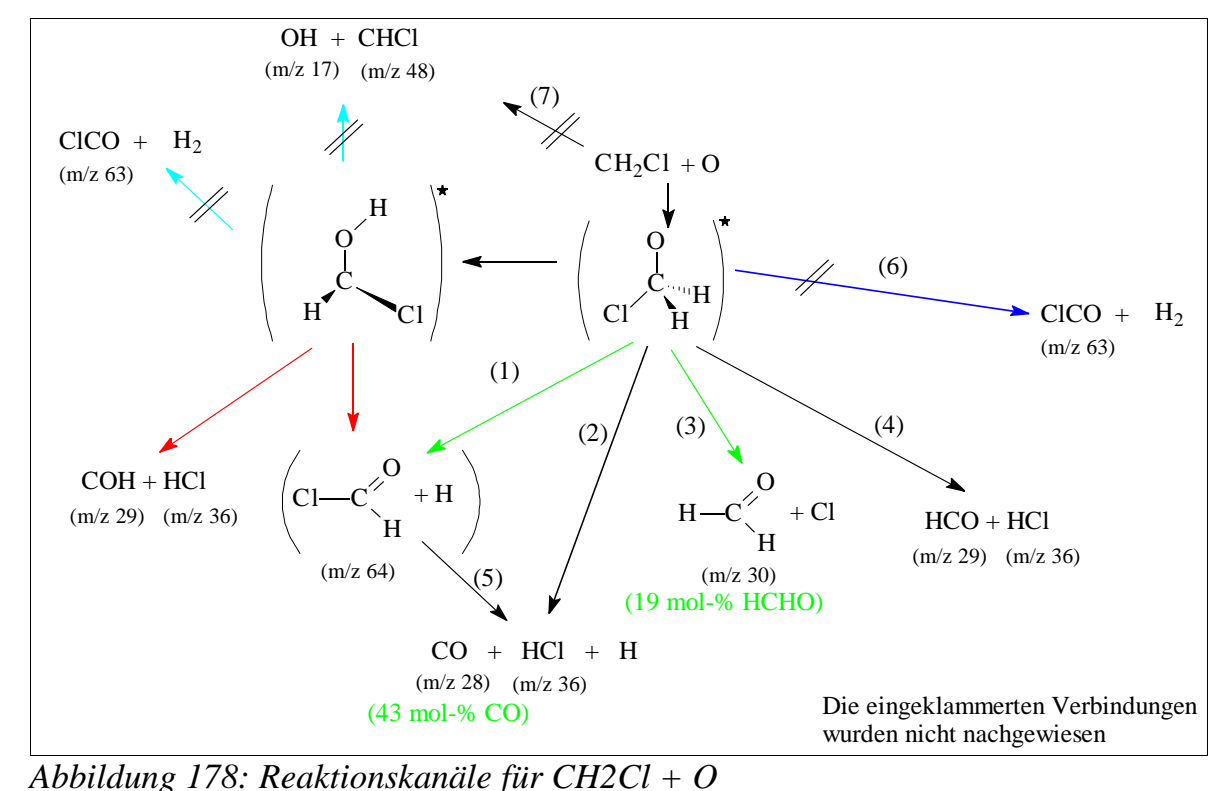

In nachstehendem Energiediagramm (Abbildung 179) sind die Standardbildungsenthalpien der Produkte für 298 K eingezeichnet. Darin erkennt man, daß die gefundenen Produktkanäle auch die thermodynamisch günstigsten sind. Zur Begründung, warum die Reaktionen zu den Produkten unmittelbar von dem gebildeten hochangeregten Komplex ausgehen, läßt sich sagen, daß die Stoßstabilsierung relativ zum Geschwindigkeitskoeffizienten $\mathrm{k}(\mathrm{E})$ des unimolekularen Zerfalls als unwahrscheinlich anzusehen ist $\mathrm{t}^{150}$.

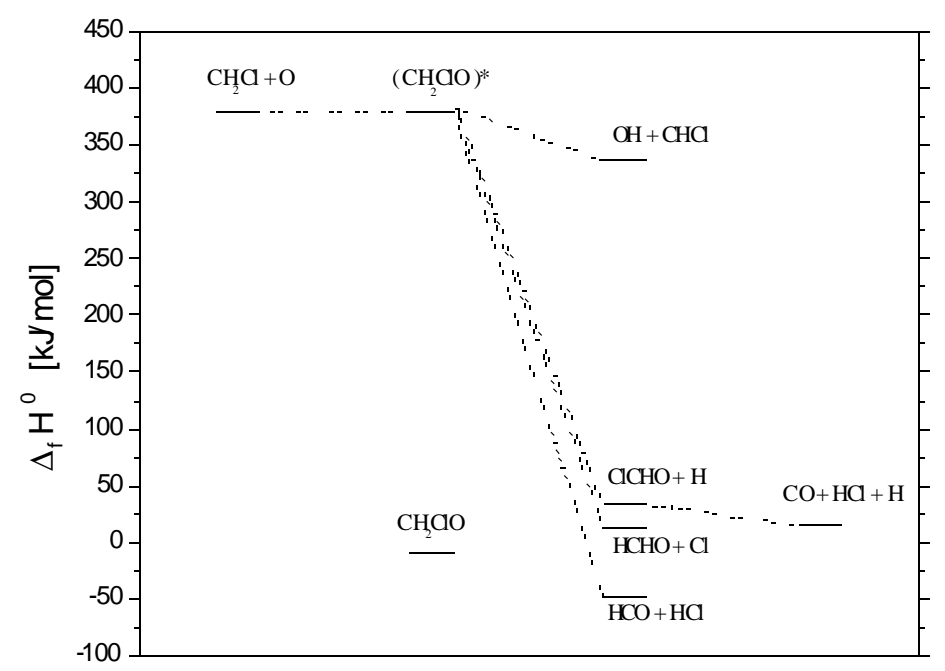

Abbildung 179: Energiediagramm der Reaktion $\mathrm{CH} 2 \mathrm{Cl}+\mathrm{O} ; 298 \mathrm{~K}$

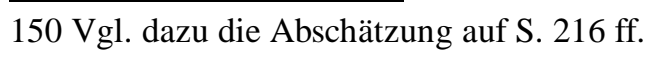




\subsubsection{Kinetik der Reaktion des erzeugten Radikals mit Sauerstoff-Atomen}

Die Reaktion wurde temperaturabhängig zwischen 258 und $358 \mathrm{~K}$ relativ $\mathrm{zu} \mathrm{CH}_{2} \mathrm{OCH}_{3}$ ausgeführt. Als Geschwindigkeitskoeffizient erhält man ${ }^{151}$ :

$$
\begin{aligned}
& \mathbf{k}(\mathbf{T})=(8,9 \pm \mathbf{1 , 8}) \cdot 10^{13} \exp \{-(-0,10 \pm 0,53) \mathrm{kJ} / \mathrm{mol} /(\mathbf{R} \cdot \mathbf{T})\} \mathrm{cm}^{3} \mathbf{m o l}^{-1} \mathrm{~s}^{-1} \\
& \text { für: } \mathrm{CH}_{2} \mathrm{Cl}+\mathrm{O}\left({ }^{3} \mathrm{P}\right) \rightarrow \text { Produkte }
\end{aligned}
$$

Für T $=298$ K ergibt sich damit: $\mathrm{k}^{298 \mathrm{~K}}=9,3 \mathrm{E}+13 \mathrm{~cm}^{3} /(\mathrm{mol} \mathrm{s})$.

\subsubsection{REMPI-Nachweis des erzeugten Radikals}

Es soll nun überprüft werden, ob das durch Zugabe von F-Atomen erzeugte Radikal auch mit dem REMPI-Verfahren (möglichst selektiv) zu ionisieren ist. Dieses Verfahren sowie die dafür eingesetzte Apparatur ist eingehend in Kap. 3.1.4 (S. 33 ff.) beschrieben. Die Meßwerte sind mit dem automatischen Wellenlängendurchlauf registriert worden (vgl. Kap. 3.5.4, S. 57), wobei zur Auswertung das Peakmaximum bei m/z 49 sowie 51 (bzw. den entsprechenden Flugzeiten) herangezogen wurde.

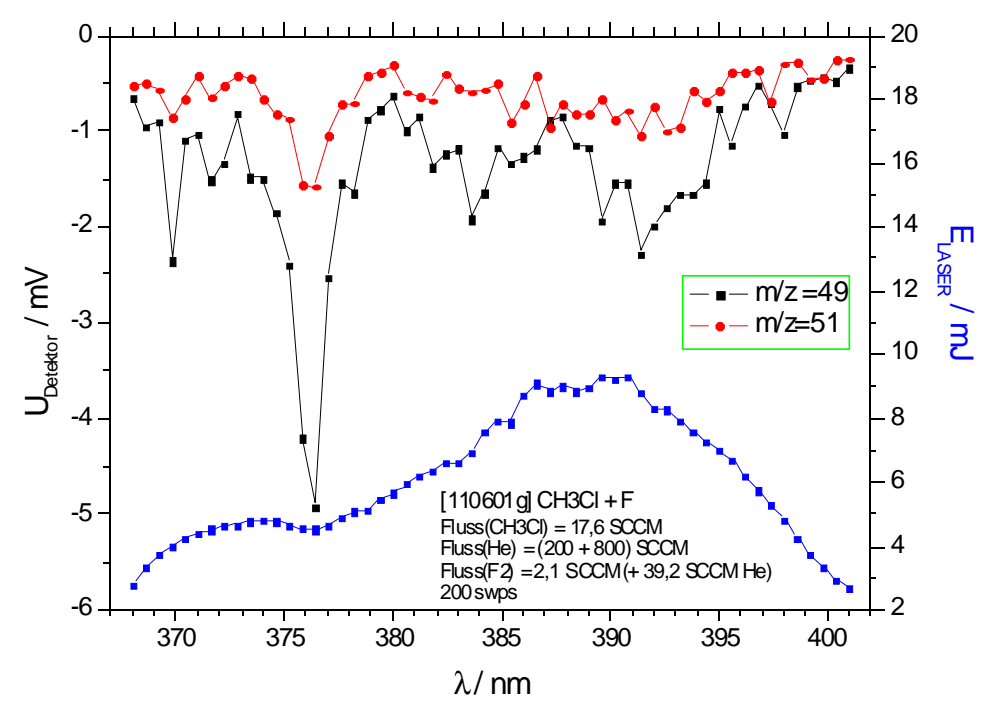

Abbildung 180: REMPI-Wellenlängendurchlauf 368-402 $\mathrm{nm} \mathrm{f.} \mathrm{CH} 3 \mathrm{Cl}+\mathrm{F}$

Das Radikal konnte gemäß Abb. 180 bei der Wellenlänge $\lambda=376,4$ nm nachgewiesen werden. In der Umgebung dieses Peaks wurde anschließend mit einer feineren Auflösung das Maximum (bzw. mathematisch gesehen: Minimum) genauer gesucht und bei $\lambda=\mathbf{3 7 6 , 0 2 5} \mathbf{n m}$ gefunden 
(vgl. Abbildung 181).

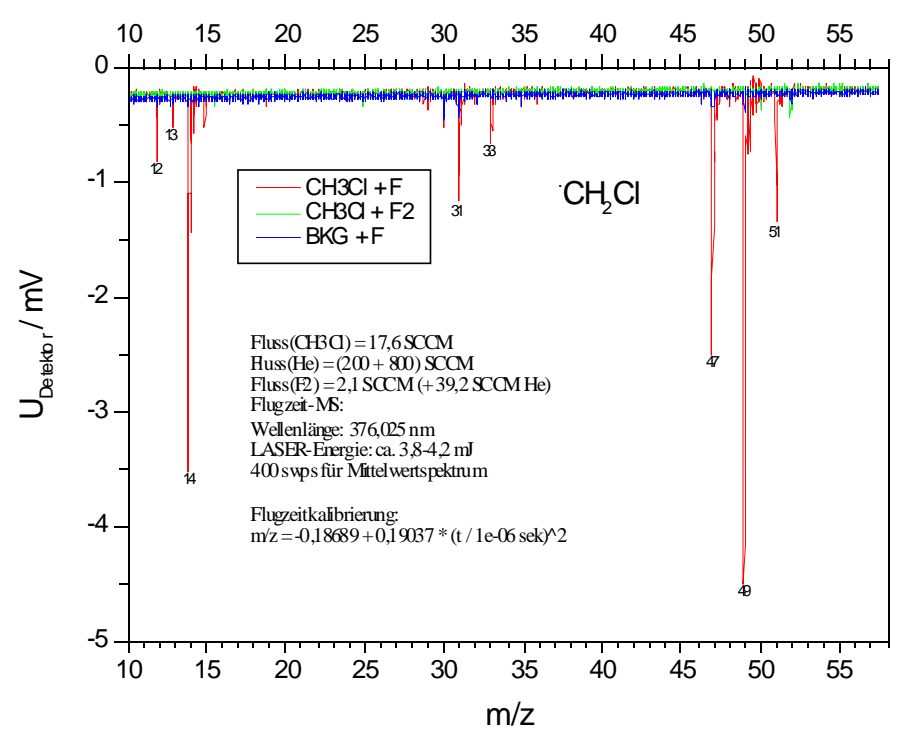

Abbildung 181: REMPI-Nachweis CH2Cl bei 376,025 nm

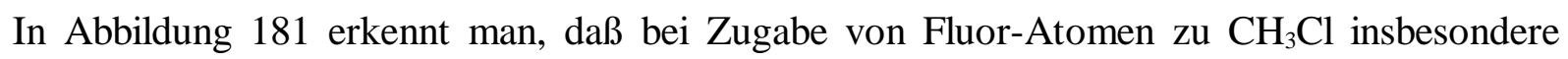
Peaks bei m/z 49 und 51 (d.h. der Masse des erzeugten $\mathrm{CH}_{2} \mathrm{Cl}$-Radikals) entstehen (rote Linie) und diese vollständig verschwinden, wenn der Zufluß von $\mathrm{CH}_{3} \mathrm{Cl}$ oder derjenige der FluorAtome abgeschaltet wird (blaue bzw. grüne Linie in Abb. 181).

\subsubsection{Diskussion der Ergebnisse}

In der analogen Reaktion $\mathrm{CH}_{2} \mathrm{~F}+\mathrm{O}$ wird der Formaldehyd-Anteil mit 9 mol-\% angegeben ${ }^{152}$, was deutlich geringer als die hier für $\mathrm{CH}_{2} \mathrm{Cl}+\mathrm{O}$ bestimmten 19 mol-\% ist; dennoch erscheint dieser Wert plausibel zu sein, weil die dazu notwendige C-F-Spaltung wesenlich mehr Energie erfordert als der hier notwendige C-Cl-Bindungsbruch ${ }^{153}$. Für $\mathrm{CH}_{2} \mathrm{~F}+\mathrm{O}$ wird mit 96 mol-\% $\mathrm{CO}$ als Hauptprodukt angegeben, welches auch für $\mathrm{CH}_{2} \mathrm{Cl}+\mathrm{O}$ (neben Formaldehyd) als Hauptprodukt mit 43 mol-\% identifiziert wurde und der themodynamisch günstigen HF- bzw. HCl-Eliminierung entspricht. In geringerem Maß wurde in beiden Reaktionen HCO nachgewiesen.

Qualitativ erhält man also eine recht gute Übereinstimmung bezüglich der Produkte beider Reaktionen.

152 Beiderhase, T. (1995); S. 77-80

153 Bezüglich der Bindungsstärken vergleiche: Lide, D. (1991); Abschnitt 9 S. 121.

Dort wird deutlich, daß die C-Cl-Bindung etwa 20-25\% schwächer als C-F ist. 
Es existiert auch eine theoretische Untersuchung der Reaktion $\mathrm{CH}_{2} \mathrm{Cl}+\mathrm{O}$, die auf der Methode G2MP2 basiert ${ }^{154}$.

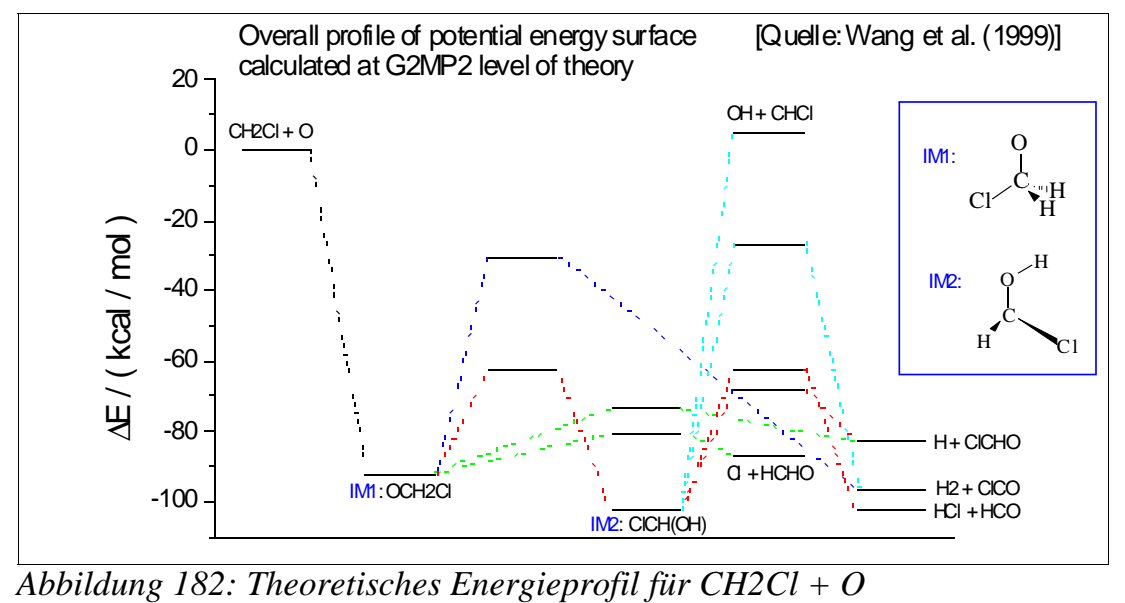

Die in Abbildung 182 nicht beschrifteten Energiestufen gehören zu Übergangszuständen ${ }^{155}$. Diese theoretischen Ergebnisse sind in Einklang mit den hier ermittelten experimentellen. Tatsächlich gefunden wurden nämlich nur die Produkte mit den geringsten Energiebarrieren; das sind diejenigen, die in Abbildung 182 auf dem roten oder grünen „Reaktionsweg“ erreichbar sind. Der grünen Linie folgend ist das günstigste Produkt Methanal (HCHO), das immerhin zu 19 mol-\% experimentell gefunden wurde. Das zweite Produkt auf dem „grünen“ Weg führt zu ClCHO. Es wurde im Experiment zwar nicht nachgewiesen, jedoch vermutlich deshalb, weil es sehr schnell zu $\mathrm{CO}+\mathrm{HCl}$ weiterzerfallen ist. Diesbezüglich lag eine theoretische Berechnung nicht vor. Das $\mathrm{ClCHO}$ kann auch über den „roten Weg“ erreicht werden. Da experimentell CO als Hauptprodukt identifiziert werden konnte, ist der Zerfall von $\mathrm{ClCHO}$ zu $\mathrm{CO}+\mathrm{HCl}$ sehr wahrscheinlich. Ebenfalls über einen rot skizzierten „Weg“ kann $\mathrm{HCl}+\mathrm{HCO}$ gebildet werden, welches auch im Experiment zu finden war. Die nur über die energetisch sehr ungünstigen blauen und cyanfarbenen Wege erreichbaren Produkte konnten experimentell nicht ermittelt werden.

Zur leichteren Vergleichbarkeit sind die „Reaktionswege“ oben in Abb. 182 in den gleichen Farben wie in Abb. 178 (S. 208) dargestellt. 


\subsubsection{Das Dichlormethan $\left(\mathrm{CH}_{2} \mathrm{Cl}_{2}\right)$}

\subsubsection{Produkte der radikalerzeugenden Reaktion mit Fluor-Atomen}

Bei der Reaktion von Dichlormethan mit Fluor-Atomen wurde nur der Abstraktionskanal gefunden, der zur Bildung des Dichlormethyl-Radikals führt ${ }^{156}: \mathrm{CH}_{2} \mathrm{Cl}_{2}+\mathrm{F} \rightarrow \mathrm{CHCl}_{2}+\mathrm{HF}$

\subsubsection{Kinetik der radikalerzeugenden Reaktion mit Fluor-Atomen}

Die Reaktion wurde temperaturabhängig zwischen 253 und $358 \mathrm{~K}$ relativ zu Methan untersucht. Als Geschwindigkeitskoeffizient erhält $\operatorname{man}^{157}$ :

$$
\begin{aligned}
& \mathbf{k}=(8,4 \pm \mathbf{1 , 0}) \cdot 10^{12} \exp \{-(-0,12 \pm 0,29) \mathrm{kJ} / \mathrm{mol} /(\mathbf{R} \cdot \mathbf{T})\} \mathrm{cm}^{3} \mathbf{m o l}^{-1} \mathrm{~s}^{-1} \\
& \text { für: } \mathrm{CH}_{2} \mathrm{Cl}_{2}+\mathrm{F} \rightarrow \text { Produkte }
\end{aligned}
$$

Für T $=298 \mathrm{~K}$ ergibt sich damit: $\mathrm{k}^{298 \mathrm{~K}}=8,8 \mathrm{E}+12 \mathrm{~cm}^{3} \mathrm{~mol}^{-1} \mathrm{~s}^{-1}$.

\subsubsection{Produkte der Reaktion des erzeugten Radikals mit Sauerstoff-Atomen}

Analog zur Bestimmung des Anteils an Kohlenmonoxid bei der Reaktion $\mathrm{CH}_{3}+\mathrm{O}$ (vgl. Kap. 4.4.1, S. 198) wird nachfolgend dieser Anteil für die Reaktion $\mathrm{CHCl}_{2}+\mathrm{O}$ bestimmt.

Dazu wurden Messungen bei einer Temperatur von 298 K, einem Druck von 224 Pa und Gasflüssen von $\phi_{\mathrm{He}}=902,6 \mathrm{SCCM}, \phi_{\mathrm{CH} 2 \mathrm{Cl} 2}=24,9 \mathrm{SCCM}, \phi_{\mathrm{F} 2}=4,8 \mathrm{SCCM}$ und $\phi_{\mathrm{O} 2}=12$ SCCM (insgesamt also: $\phi_{\text {gesant }}=944,3$ SCCM) ausgeführt. Später wurde zur Eichung noch Argon zugeführt ( $\phi_{\mathrm{Ar}}=55,6 \mathrm{SCCM}$, dann: $\left.\phi_{\text {gesant }}=999,9 \mathrm{SCCM}\right)$.

Damit errechnet man als Ausgangskonzentration für Methan: $[\mathrm{CH} 4]_{0}=2,38 \cdot 10^{-9} \mathrm{~mol} / \mathrm{cm}^{3}$ und für Argon $[\mathrm{Ar}]_{0}=5,12 \cdot 10^{-9} \mathrm{~mol} / \mathrm{cm}^{3}$.

Auf dem Molekül-Peak von Dichlormethan (m/z 84) konnte nun der Umsatz bei Zugabe von Fluor-Atomen bestimmt werden.

\begin{tabular}{|l|l|l|l|l|l|}
\hline Umsatz von Dichlormethan auf m/z 84: \\
\hline & Zustand MWE & DATA & Umsatz & & \\
\hline Messung1: & aus, d.h. ,-F“ & $4,750 \mathrm{E}+04$ & & & \\
\hline & ein, d.h. ,,FF“ & $2,977 \mathrm{E}+04$ & $37,32 \%$ & & \\
\hline
\end{tabular}

Zur Durchführung der in Kap. 3.5.1 (S. 50) beschriebenen Umsatzkorrektur waren folgende Messungen auf dem Fragment-Peak des Dichlormethans m/z 83 erforderlich: 
Intensität Fragment-Peak auf m/z 83 (MWE: ausgeschaltet):

\begin{tabular}{l|l|r|l} 
Messung1: & DATA (unkorr.) & 1508 => Korr.-wert:
\end{tabular}

945,25

Nun mußte der Umsatz des Dichlormethyl-Radikals bei Zugabe von Sauerstoff-Atomen ermittelt werden (wegen großer Streuungen waren mehrere Messungen erforderlich):

\begin{tabular}{|c|c|c|c|c|}
\hline \multicolumn{5}{|c|}{ Umsatz auf Radikal-Peak m/z 83: } \\
\hline \multirow[t]{2}{*}{ Messung1: } & $\begin{array}{l}\text { DATA ,,-O“ } \\
\text { (unkorr.) }\end{array}$ & $1,213 \mathrm{E}+03$ & & \\
\hline & $\begin{array}{l}\text { DATA ,,+O“ } \\
\text { (unkorr.) }\end{array}$ & $1,046 \mathrm{E}+03$ & $\begin{array}{l}\text { Umsatz nach } \\
\text { Umsatzkorrektur }\end{array}$ & $62,37 \%$ \\
\hline \multirow[t]{2}{*}{ Messung2: } & $\begin{array}{l}\text { DATA ,-O“ } \\
\text { (unkorr.) }\end{array}$ & $1,177 \mathrm{E}+03$ & & \\
\hline & $\begin{array}{l}\text { DATA ,,+O“ } \\
\text { (unkorr.) }\end{array}$ & $1,053 \mathrm{E}+03$ & $\begin{array}{l}\text { Umsatz nach } \\
\text { Umsatzkorrektur }\end{array}$ & $53,51 \%$ \\
\hline \multirow[t]{4}{*}{ Messung3: } & $\begin{array}{l}\text { DATA ,-O“ } \\
\text { (unkorr.) }\end{array}$ & $1,223 \mathrm{E}+03$ & & \\
\hline & $\begin{array}{l}\text { DATA ,,+O“ } \\
\text { (unkorr.) }\end{array}$ & $1,028 \mathrm{E}+03$ & $\begin{array}{l}\text { Umsatz nach } \\
\text { Umsatzkorrektur }\end{array}$ & $70,21 \%$ \\
\hline & & & Mittelwert: & $62,03 \%$ \\
\hline & & $\Rightarrow[\mathrm{CH}$ & $\mathrm{HCl} 2]_{\text {reagiert }} / \mathrm{mol} / \mathrm{cm} 3$ & $5,51 \mathrm{E}-10$ \\
\hline
\end{tabular}

Die Konzentration der Dichlormethyl-Radikale, die mit Sauerstoff-Atomen reagiert haben, ergab sich aus der Ausgangskonzentration von Dichlormethan, dem Umsatz von Dichlormethan mit F-Atomen und dem Umsatz der Dichlormethyl-Radikale mit SauerstoffAtomen:

$\left[\mathrm{CHCl}_{2}\right]_{\text {reagiert }}=2,38 \cdot 10^{-9} \mathrm{~mol} / \mathrm{cm}^{3} \cdot(0,3732) \cdot(0,6203)=5,51 \cdot 10^{-10} \mathrm{~mol} / \mathrm{cm}^{3}$.

Nun erfolgte die eigentliche Messung auf dem Molekül-Peak von CO:

\begin{tabular}{|r|r|r|r|}
\hline Messung auf m/z 28 (DATA-Werte): \\
\hline Messung-Nr. & $+\mathrm{F}-\mathrm{O}$ & $+\mathrm{F}+\mathrm{O}$ & Differenz \\
\hline 1 & $1,992 \mathrm{E}+03$ & $2,250 \mathrm{E}+02$ & 1767 \\
\hline 2 & $3,393 \mathrm{E}+03$ & $4,477 \mathrm{E}+02$ & 2945,3 \\
\hline 3 & $3,217 \mathrm{E}+03$ & $5,262 \mathrm{E}+02$ & 2690,8 \\
\hline 4 & $3,01 \mathrm{E}+003$ & $4,81 \mathrm{E}+002$ & 2524,2 \\
\hline & & Mittelwert: & 2481,83 \\
\hline & \multicolumn{2}{|c|}{ = effektive Zunahme durch CO: } & 2481,83 \\
\hline
\end{tabular}

Um aus den DATA-Werten die Konzentration [CO] berechnen zu können, waren nun noch die 
DATA-Werte für Argon erforderlich (vgl. Gleichung (30), S. 199):

\begin{tabular}{|r|r|}
\hline Messung I(Ar) auf $\mathrm{m} / \mathrm{z}$ 40: \\
\hline Messung-Nr. & DATA \\
\hline 1 & $2,382 \mathrm{E}+04$ \\
\hline 2 & $2,272 \mathrm{E}+04$ \\
\hline 3 & $2,277 \mathrm{E}+04$ \\
\hline 4 & $2,292 \mathrm{E}+04$ \\
\hline Mittelwert: & $2,306 \mathrm{E}+04$ \\
\hline
\end{tabular}

Mit dem Verhältnis der massenspektrometrischen Empfindlichkeiten $\varepsilon(\mathrm{CO}) / \varepsilon(\mathrm{Ar})=1,42$ aus Tabelle 52 (S. 198) bzw. Abbildung 174 (S. 199) kann man mittels Gleichung (30) (S. 199) die Konzentration an $\mathrm{CO}$ berechnen:

$$
[\mathrm{CO}]=\frac{1}{1,42} \cdot \frac{2481,83}{2,306 \cdot 10^{04}} \cdot 2,38 \cdot 10^{-09} \mathrm{~mol} / \mathrm{cm}^{3}=3,88 \cdot 10^{-10} \mathrm{~mol} / \mathrm{cm}^{3}
$$

Mit dem Verhältnis aus der entstandenen CO-Konzentration und der Konzentration der Methyl-Radikale, die insgesamt mit Sauerstoff-Atomen reagiert haben, läßt sich schlußendlich der Anteil $\alpha$ für den Reaktionskanal der CO-Bildung berechnen:

$$
\alpha(\mathrm{CO})=\frac{3,88 \cdot 10^{-10} \mathrm{~mol} / \mathrm{cm}^{3}}{5,51 \cdot 10^{-10} \mathrm{~mol} / \mathrm{cm}^{3}}=70 \mathrm{~mol} \%
$$

Aus den Standardabweichungen der einzelnen Messungen kann gemäß den Gesetzen der Fehlerfortpflanzung ein Fehler von $\pm 9 \%$ angegeben werden.

Als Endergebnis erhält man daher: $\alpha(\mathbf{C O})=(\mathbf{7 0} \pm 9)$ mol- $\%$.

Das qualitative Produktspektrum ${ }^{158}$ (Abb. 183) wurde mit der in Kapitel 3.1.1 (S. 16) beschriebenen Apparatur unter folgenden Versuchsbedingungen aufgenommen (zur Ausführung der Messungen siehe Kapitel 3.5.1, S. 50): $\vartheta=+25{ }^{\circ} \mathrm{C} ; \phi_{\mathrm{He}}=15,83 \mathrm{~cm}^{3} / \mathrm{s} ; \phi_{\mathrm{CH} 2 \mathrm{Cl} 2}$ $=0,96 \mathrm{~cm}^{3} / \mathrm{s} ; \mathrm{p}_{\mathrm{StrR}}=147 \mathrm{~Pa} ; \mathrm{s}=8,5 \mathrm{~cm} ; \mathrm{IP}=19,5 \mathrm{eV}$; Zählzeit $=20 \mathrm{~s}$; Mittelwert aus 3 Messungen

Es zeigt (Abb. 183) keine Zunahme auf m/z = 98 bzw. $100\left(\mathrm{COCl}_{2}\right)$, so daß Reaktionskanal (3) und/oder (4) in Abb. 184 unter den Versuchsbedingungen nicht gefunden wurde. Eine geringe Zunahme auf $\mathrm{m} / \mathrm{z}=64(\mathrm{ClCHO})$ bestätigt Kanal (1) und die starke Zunahme auf $\mathrm{m} / \mathrm{z}=63$ bzw. 65 (ClCO) spricht für die Anwesenheit von Kanal (2) und/oder (5). Die sehr starke 
Zunahme auf m/z = $28(\mathrm{CO}), 35(\mathrm{Cl})$ und 36 bzw. $38(\mathrm{HCl})$ könnte durch die Kanäle (7), (8) und/oder (9) erklärt werden.

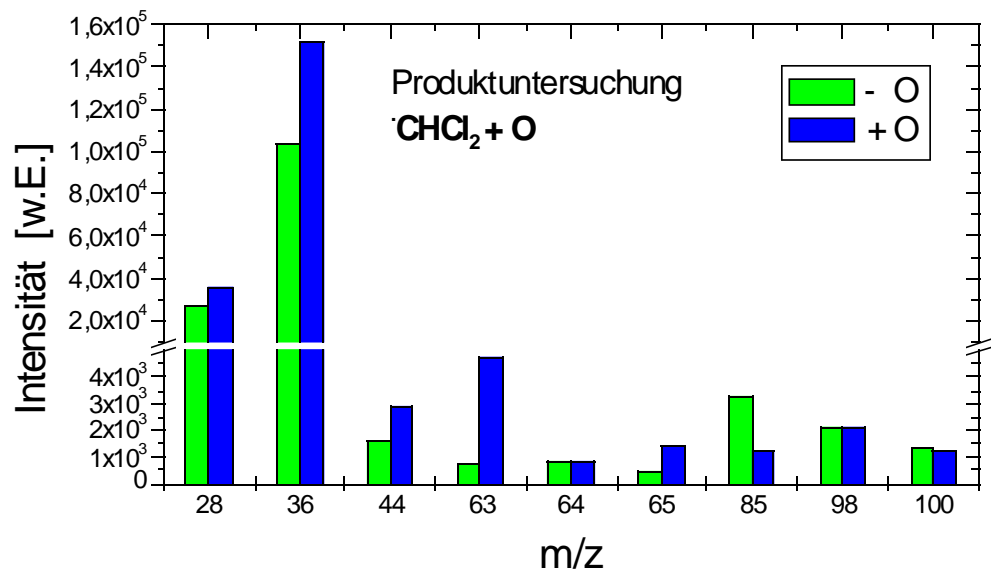

Abbildung 183: EI-MS Produktuntersuchung CHCl2 +O

Die Kanäle (10) und (11) werden nicht bestätigt, da auf $\mathrm{m} / \mathrm{z}=82\left(\mathrm{CCl}_{2}\right)$ keine Zunahme beobachtet werden konnte.

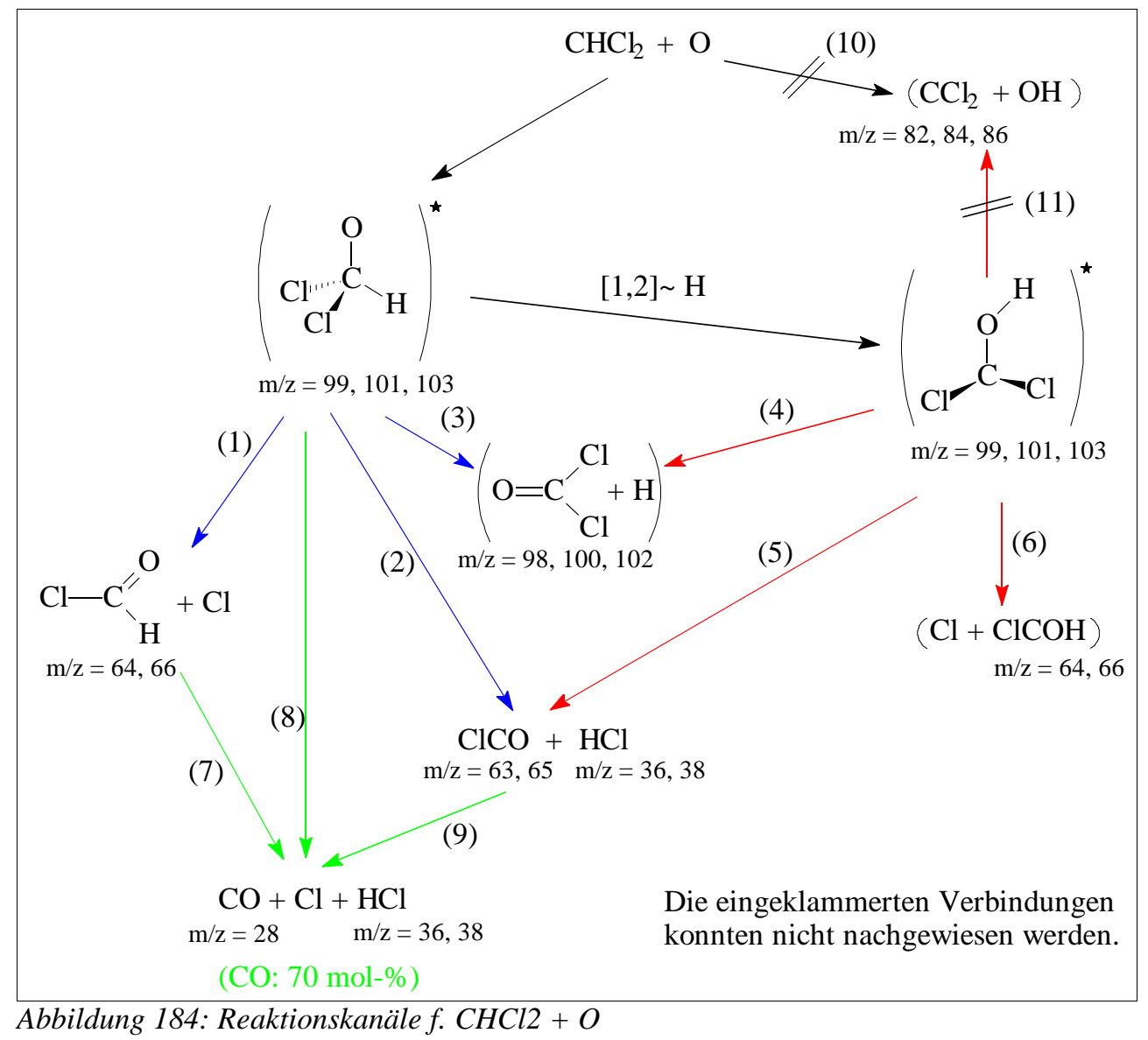

Die bei Verlängerung der Reaktionsstrecke von 3,0 auf 8,5 cm beobachtete Zunahme auf $\mathrm{m} / \mathrm{z}=44\left(\mathrm{CO}_{2}\right)$ kann durch die Folgereaktion $\mathrm{ClCO}+\mathrm{O} \rightarrow \mathrm{CO}_{2}+\mathrm{Cl}$ erklärt werden. 
In nachstehendem Energiediagramm (Abbildung 185) sind die Standardbildungsenthalpien der Produkte aus Abbildung 184 für 298 K eingezeichnet.

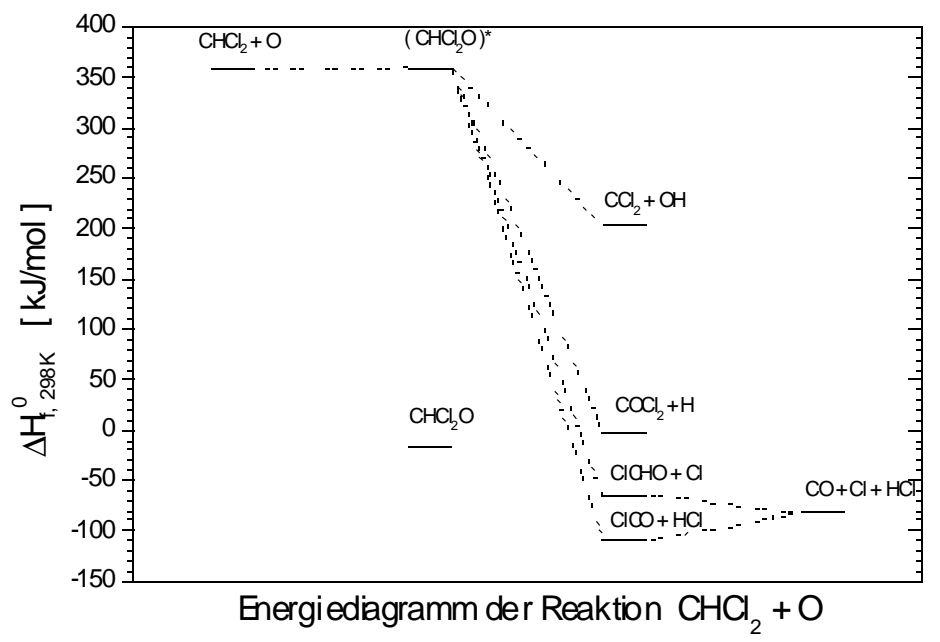

Abbildung 185: Standardbildungsenthalpien der Produkte aus $\mathrm{CHCl2}+\mathrm{O}$

Dort erkennt man, daß die gefundenen Reaktionsprodukte gleichzeitig die thermodynamisch günstigsten sind.

Der durch die Reaktion $\mathrm{CHCl}_{2}+\mathrm{O} \rightarrow\left(\mathrm{CHCl}_{2} \mathrm{O}\right)^{*}$ gebildete hoch angeregte Komplex liegt $375,4 \mathrm{~kJ} / \mathrm{mol}$ über seinem (berechneten) Grundzustand, was zu einem hohen Wert für den Geschwindigkeitskoeffizient des unimolekularen Zerfalls $\mathrm{k}(\mathrm{E})$ führt ${ }^{159}$. Deshalb ist die Lebensdauer solcher Komplexe in der Regel um Größenordnungen kleiner als die mittlere Zeit zwischen zwei Stößen bei dem gewählten Druck ${ }^{160,161}$. Bezüglich der Zahl der Stöße von $\left(\mathrm{CHCl}_{2} \mathrm{O}\right)^{*}$ mit Helium kann eine Abschätzung durch Berechnung der Stoßzahl gemäß Gleichung (14) (S. 11) gemacht werden; unter Verwendung der im Anhang abgeschätzten Lennard-Jones-Parameter für den angeregten Komplex erhält man bei $298 \mathrm{~K}: \mathrm{Z}_{\mathrm{AB}}=3,12 \mathrm{E}+14$ $\mathrm{cm}^{3} /(\mathrm{mol} \mathrm{s})$. Für einen Druck von $80 \mathrm{~Pa}$ ergibt das: $[\mathrm{He}] \cdot \mathrm{Z}_{\mathrm{AB}}=9,4 \mathrm{E}+06$ Stöße pro Sekunde; bei $220 \mathrm{~Pa}$ sind es 2,5E+07 Stöße pro Sekunde. Eine Abschätzung für k(E) soll hier nur nach der einfacheren Theorie erfolgen. Diese geht von s gleichen Oszillatoren mit identischer Schwingungsfrequenz $v$ im Molekül aus und berücksichtigt die Wahrscheinlichkeit dafür, daß von insgesamt v Quanten (Gesamtenergie im Molekül $=\mathrm{v} \cdot \mathrm{h} \cdot \mathrm{v})$ gerade $\mathrm{m}$ Quanten in der für den unimolekularen Zerfall kritischen Koordinate ,angesammelt“ werden. Dabei wird 
vorausgesetzt, daß sich die Energie innerhalb der Oszillatoren beliebig umverteilen kann ${ }^{162}$. Das allgemein angeregte Teilchen $A^{*}$ muß also erst $m$ Quanten in der „richtigen“ Bindung lokalisieren und kann dann als ,,speziell angeregtes“ Molekül $\mathrm{A}^{\neq}$zerfallen

$$
\mathrm{A}^{\natural} \stackrel{\mathrm{k}(\mathrm{E})}{\longrightarrow} \mathrm{A}^{\neq} \stackrel{\mathrm{k}^{\ddagger}}{\longrightarrow} \text { Produkte }
$$

Für die Wahrscheinlichkeit $\mathrm{P}^{\neq}$, daß von insgesamt v Quanten $\mathrm{m}$ in einer Bindung stecken gilt ${ }^{163}$ :

$$
\mathrm{P}^{\neq}=\frac{(\mathrm{v}-\mathrm{m}+\mathrm{s}-1) ! \mathrm{v} !}{(\mathrm{v}-\mathrm{m}) !(\mathrm{v}+\mathrm{s}-1) !}
$$

Unter der Annahme der Quasistationarität für $\left[\mathrm{A}^{\ddagger}\right]$ erhält man ${ }^{164}$ :

$$
\mathrm{k}(\mathrm{E})=\mathrm{k}^{\neq} \cdot \mathrm{P}^{\neq}
$$

Der Geschwindigkeitskoeffizient $\mathrm{k}^{\neq}$dürfte in der Größenordnung einer Schwingungsfrequenz liegen. Aus Infrarot-Spektren ist bekannt, daß die Wellenzahlen der Streckschwingungen C-H ca. $3000 \mathrm{~cm}^{-1}$; C-C ca. $1000 \mathrm{~cm}^{-1}$ und C-Cl ca $700 \mathrm{~cm}^{-1}$ betragen ${ }^{165}$.

Für die folgenden Berechnungen (Tabelle 53) wurde für $\mathrm{k}^{\star}$ die Frequenz der C-Cl-Schwingung $\left(2,05 \mathrm{E}+13 \mathrm{~s}^{-1}\right)$ verwendet und die Gesamtenergie im Molekül mit $\mathbf{E}=\mathbf{3 7 5 , 4} \mathbf{~ k J} / \mathbf{m o l}$ angenommen $(=>v=46$ Quanten $h v)$; für die Zahl der Oszillatoren in $\left(\mathrm{CHCl}_{2} \mathrm{O}\right)^{*}$ wird $\mathrm{s}=3 \cdot 5-6=9$ angesetzt:

\begin{tabular}{|c|c|c|c|}
\hline $\begin{array}{c}\text { Energiebarriere= m·h·v } \\
\mathrm{E}_{0}[\mathbf{J} / \mathbf{~ m o l}]\end{array}$ & $\mathbf{m}$ Quanten & $\mathbf{k}(\mathbf{E})[\mathbf{1} / \mathbf{s}]$ \\
\hline $3,59 \mathrm{E}+05$ & 44 & $4,32 \mathrm{E}-08$ & $8,85 \mathrm{E}+05$ \\
\hline $3,51 \mathrm{E}+05$ & 43 & $1,59 \mathrm{E}-07$ & $3,24 \mathrm{E}+06$ \\
\hline $3,43 \mathrm{E}+05$ & 42 & $4,76 \mathrm{E}-07$ & $9,73 \mathrm{E}+06$ \\
\hline $3,35 \mathrm{E}+05$ & 41 & $1,24 \mathrm{E}-06$ & $2,53 \mathrm{E}+07$ \\
\hline $3,26 \mathrm{E}+05$ & 40 & $2,89 \mathrm{E}-06$ & $5,90 \mathrm{E}+07$ \\
\hline $3,18 \mathrm{E}+05$ & 39 & $6,18 \mathrm{E}-06$ & $1,26 \mathrm{E}+08$ \\
\hline $3,10 \mathrm{E}+05$ & 38 & $1,24 \mathrm{E}-05$ & $2,53 \mathrm{E}+08$ \\
\hline $3,02 \mathrm{E}+05$ & 37 & $2,34 \mathrm{E}-05$ & $4,78 \mathrm{E}+08$ \\
\hline $2,94 \mathrm{E}+05$ & 36 & $4,21 \mathrm{E}-05$ & $8,60 \mathrm{E}+08$ \\
\hline $2,86 \mathrm{E}+05$ & 35 & $7,26 \mathrm{E}-05$ & $1,49 \mathrm{E}+09$ \\
\hline $2,77 \mathrm{E}+05$ & 34 & $1,21 \mathrm{E}-04$ & $2,48 \mathrm{E}+09$ \\
\hline $2,69 \mathrm{E}+05$ & 33 & $1,96 \mathrm{E}-04$ & $4,00 \mathrm{E}+09$ \\
\hline $2,61 \mathrm{E}+05$ & 32 & $3,07 \mathrm{E}-04$ & $6,29 \mathrm{E}+09$ \\
\hline $2,53 \mathrm{E}+05$ & 31 & $4,71 \mathrm{E}-04$ & $9,64 \mathrm{E}+09$ \\
\hline $2,45 \mathrm{E}+05$ & 30 & $7,07 \mathrm{E}-04$ & $1,45 \mathrm{E}+10$ \\
\hline
\end{tabular}

Tabelle 53: Abschätzung $k(E)$ für unimolekularen Zerfall von $(\mathrm{CHCl2O}) *$

162 In Pilling/Seakins (1995) S. 137 ist für die Energieumverteilung ein Geschwindigkeitskoeffizient von

$\approx 1 \mathrm{E}+12 \mathrm{~s}^{-1}$ angegeben, so daß diese Voraussetzung erfüllt sein sollte.

163 Pilling / Seakins (1995); S. 127; Gleichung E-22.

164 Pilling / Seakins (1995); S. 126-127; Abwandlung von Gleichung E-25.

165 Hesse / Meier / Zeeh (1995); S. 39. 
Die berechneten Werte der Tabelle 53 sind in Abb. 186 grafisch dargestellt.

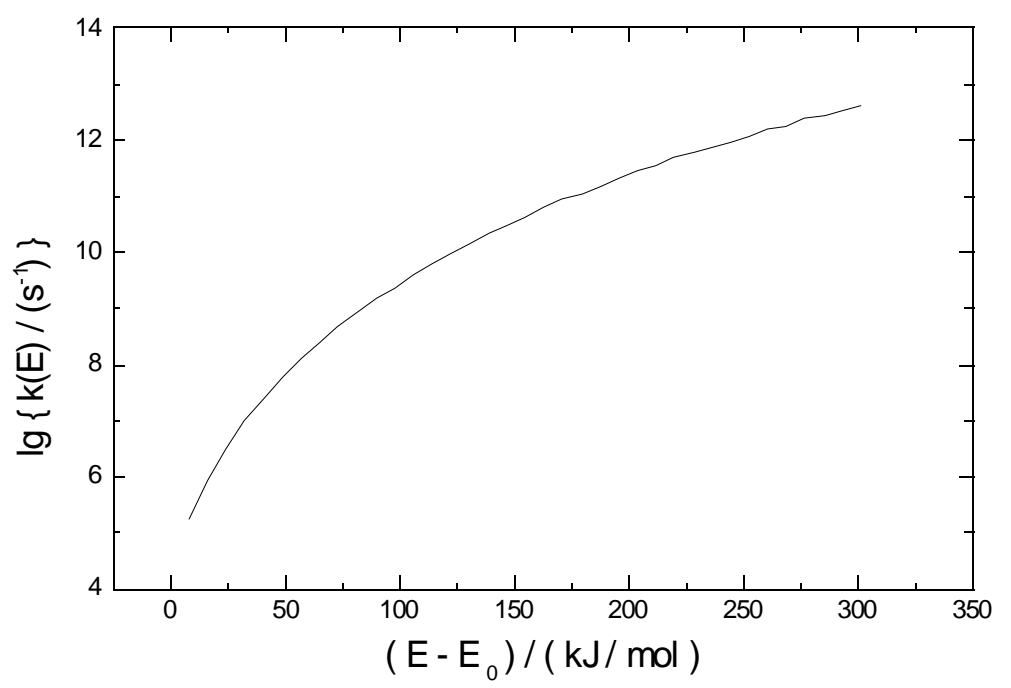

Abbildung 186: Abschätzung $k(E)$ für unimolekularen Zerfall von $(\mathrm{CHCl2O})^{*}$

Weil auch bei hohen Energiebarrieren $(\leq 277 \mathrm{~kJ} / \mathrm{mol})$ der Wert für $k(E)$ um den Faktor 100 größer als die Zahl der Stöße $\left(\leq 2,5 \mathrm{E}+07 \mathrm{~s}^{-1}\right)$ ist, kann auch unter der Annahme starker Stößße $e^{166}$ eine Stoßstabilisierung als unwahrscheinlich und somit das Energiediagramm in oben gezeichneter Form als gerechtfertigt angesehen werden. Im Rahmen der Diskussion der Ergebnisse ist in Abbildung 195 (S. 228) auch ein Energieprofil mit den berechneten Energiebarrieren für verschiedene Übergangszustände der Reaktion $\mathrm{CHCl}_{2}+\mathrm{O}$ dargestellt; diesem Profil kann man entnehmen, daß lediglich für zwei Reaktionskanäle (die beide zu $\mathrm{OH}+$ $\mathrm{CCl}_{2}$ führen) Energien von über $200 \mathrm{~kJ} / \mathrm{mol}$ (bezogen auf $\mathrm{OCHCl}_{2}$ im Grundzustand) aufzuwenden sind. Demnach ist für alle übrigen Kanäle eine Stoßstabilisierung vor dem unimolekularen Zerfall auszuschließen.

Zudem wurde im Experiment keine Zunahme auf der Masse des angeregten Komplexes beobachtet.

\subsubsection{Kinetik der Reaktion des erzeugten Radikals mit Sauerstoff-Atomen}

Die Reaktion wurde temperaturabhängig zwischen 258 und $358 \mathrm{~K}$ relativ zu $\mathrm{CH}_{3}$ ausgeführt. Als Geschwindigkeitskoeffizient erhält man ${ }^{167}$ :

$$
\begin{aligned}
& \mathbf{k}=(8,6 \pm 3,8) \cdot 10^{13} \exp \{-(1,7 \pm 1,1) \mathrm{kJ} / \mathrm{mol} /(\mathbf{R} \cdot \mathbf{T})\} \mathrm{cm}^{3} \mathbf{m o l}^{-1} \mathbf{s}^{-1} \\
& \text { für: } \mathrm{CHCl}_{2}+\mathrm{O} \rightarrow \text { Produkte }
\end{aligned}
$$

166 Jeder Stoß führt zur vollständigen Deaktivierung des angeregten Komplexes. 
Für T $=298 \mathrm{~K}$ ergibt sich damit: $\mathrm{k}^{298 \mathrm{~K}}=4,3 \mathrm{E}+13 \mathrm{~cm}^{3} /(\mathrm{mol} \mathrm{s})$.

\subsubsection{REMPI-Nachweis des erzeugten Radikals}

Es soll nun überprüft werden, ob das durch Zugabe von F-Atomen erzeugte Radikal auch mit dem REMPI-Verfahren (möglichst selektiv) zu ionisieren ist. Dieses Verfahren sowie die dafür eingesetzte Apparatur ist eingehend in Kap. 3.1.4 (S. 33 ff.) beschrieben. Die Meßwerte sind mit dem automatischen Wellenlängendurchlauf registriert worden (vgl. Kap. 3.5.4, S. 57), wobei zur Auswertung einmal das Peakmaximum und einmal das Peakintegral bei $\mathrm{m} / \mathrm{z} 83$ sowie 85 (bzw. den entsprechenden Flugzeiten) herangezogen wurde.
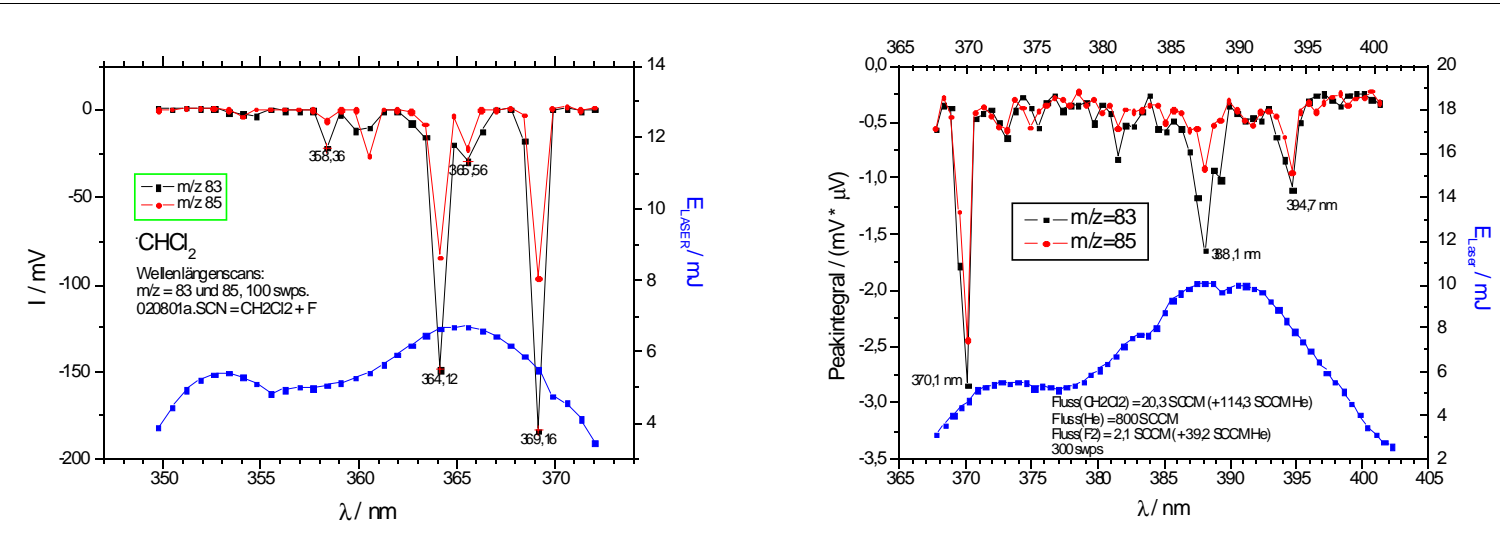

Abbildung 187: REMPI-Nachweis des CHCl2-Radikals bei verschiedenen Wellenlängen

Das Radikal konnte gemäß Abb. 187 bei den Wellenlängen $\lambda=364,1 ; 369,2 ; 370,1 ; 388,1$ und 394,7 nm nachgewiesen werden.
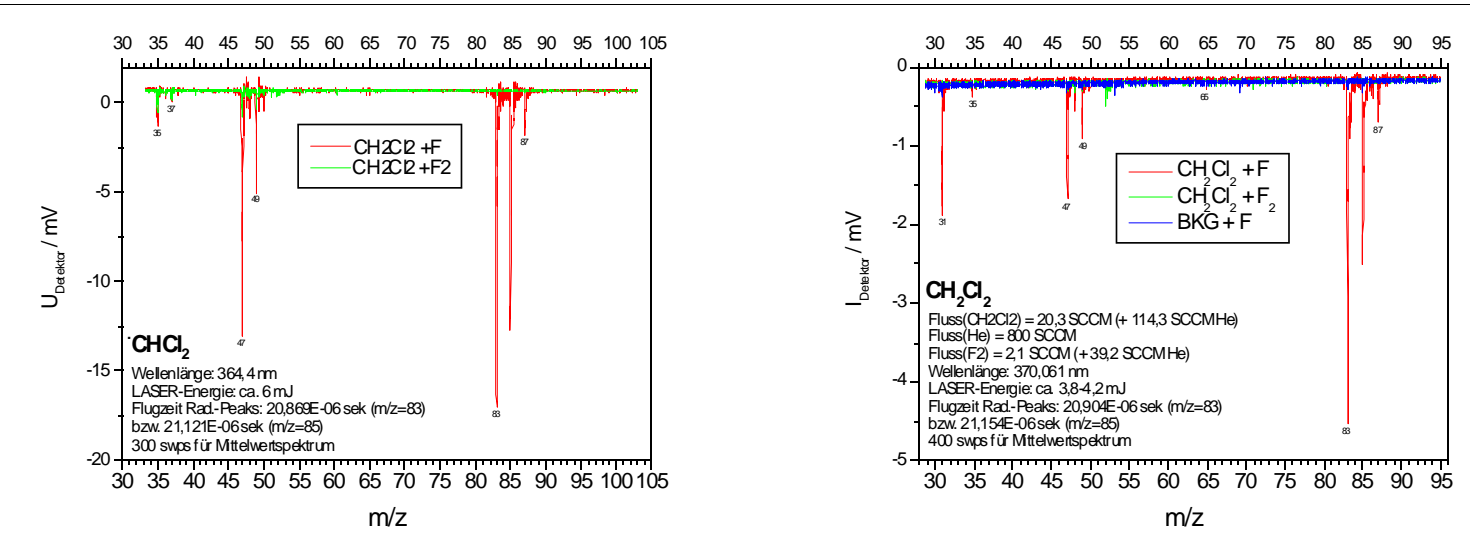

Abbildung 188: REMPI-Nachweis des CHCl2-Radikals bei verschiedenen Wellenlängen

In der Umgebung dieser Peaks wurde anschließend mit einer feineren Auflösung das Maximum (bzw. mathematisch gesehen: Minimum) genauer gesucht und bei $\lambda=\mathbf{3 6 4 , 4}$ sowie 370,061 nm gefunden (vgl. Abbildung 188). 
In Abbildung 188 erkennt man, daß bei Zugabe von Fluor-Atomen $\mathrm{zu} \mathrm{CH}_{2} \mathrm{Cl}_{2}$ insbesondere Peaks bei m/z 83 und 85 (d.h. der Masse des erzeugten $\mathrm{CH}_{2} \mathrm{Cl}$-Radikals) entstehen (rote Linie) und diese vollständig verschwinden, wenn der Zufluß von $\mathrm{CH}_{2} \mathrm{Cl}_{2}$ oder derjenige der FluorAtome abgeschaltet wird (blaue bzw. grüne Linie in Abb. 188).

Bei automatischen Wellenlängendurchläufen in den Bereichen 420-460 nm sowie 485-540 nm konnte kein nennenswertes Signal des $\mathrm{CHCl}_{2}$-Radikals gefunden werden.

\subsubsection{Kinetik der Reaktion des erzeugten Radikals mit Stickstoffmonoxid}

Die Messungen wurden in der in Kap. 3.1.4 (S. 33) beschriebenen Apparatur durchgeführt. Die genaue Ausführung und Auswertung ist in Kap. 3.5.4 (S. 57) beschrieben.

Das Fluormethyl-Radikal diente als Referenzsubstanz. Der Umsatz des Dichlormethyl-Radikals konnte anhand der Intensitätsänderung seines Molekül-Peaks m/z 83 und der des FluormethylRadikals anhand seines Molekül-Peaks m/z 33 bestimmt werden.

Im vorangegangenen Unterkapitel war als optimale Wellenlänge für den Nachweis des Dichlormethyl-Radikals $\lambda=370,06 \mathrm{~nm}$ ermittelt worden. Das als Referenz verwendete Fluormethyl-Radikal ${ }^{168}$ hat bei dieser Wellenlänge zwar nicht die maximale, aber eine für die durchzuführende Kinetik-Messung ausreichend hohe Intensität auf m/z 33.

Weil die Meßwerte aus unbekanntem Grund starken Streuungen unterlagen, mußten im Prinzip dieselben Messungen mehrfach ausgeführt werden. Als Ergebnis soll dann der Mittelwert aus allen Messungen verwendet werden.

Die Meßwerte und die daraus angefertigten doppelt-logarithmischen Auftragungen gemäß Gleichung (10) (S. 10) sind auf den folgenden Seiten dargestellt. 
$\vartheta=25{ }^{\circ} \mathrm{C} ; \phi_{\mathrm{He}}=379 \mathrm{SCCM} ; \phi_{\mathrm{CH} 2 \mathrm{Cl} 2}=19,7 \mathrm{SCCM} ; \phi_{\mathrm{CH} 3 \mathrm{~F}}=10,9 \mathrm{SCCM} ; \phi_{\mathrm{NO}}=186 . .457$ $\mathrm{SCCM} ; \mathrm{p}_{(\mathrm{StrR})}=186 . .196 \mathrm{~Pa} ; \mathrm{s}_{\mathrm{KW}+\mathrm{F}}=1 \mathrm{~cm} ; \mathrm{s}_{\text {Radikal }+\mathrm{NO}}=3 \mathrm{~cm} ; \mathrm{E}_{\mathrm{LASER}}=6,7 \mathrm{~mJ} ; \lambda_{\mathrm{LASER}}=370,06$ nm; Auswertung mit Mittelwert aus 600 einzelnen Flugzeitmassenspektren (= 600 swps).

\begin{tabular}{|c|c|c|c|}
\hline I(83) ,,-NO“ / w.E. & $\mathrm{I}(83),,+\mathrm{NO} “ /$ w.E. & $\mathrm{I}(33),,-\mathrm{NO} “ /$ w.E. & $\mathrm{I}(33),,+\mathrm{NO} “ /$ w.E. \\
\hline$-7,23 \mathrm{E}-002$ & $-6,44 \mathrm{E}-002$ & $-2,23 \mathrm{E}-001$ & $-1,69 \mathrm{E}-001$ \\
\hline$-7,32 \mathrm{E}-002$ & $-6,18 \mathrm{E}-002$ & $-2,05 \mathrm{E}-001$ & $-1,72 \mathrm{E}-001$ \\
\hline$-6,76 \mathrm{E}-002$ & $-6,39 \mathrm{E}-002$ & $-2,14 \mathrm{E}-001$ & $-1,71 \mathrm{E}-001$ \\
\hline$-7,31 \mathrm{E}-002$ & $-6,27 \mathrm{E}-002$ & $-2,13 \mathrm{E}-001$ & $-1,61 \mathrm{E}-001$ \\
\hline$-7,60 \mathrm{E}-002$ & $-4,78 \mathrm{E}-002$ & $-2,01 \mathrm{E}-001$ & $-1,40 \mathrm{E}-001$ \\
\hline$-6,68 \mathrm{E}-002$ & $-5,71 \mathrm{E}-002$ & $-2,07 \mathrm{E}-001$ & $-1,60 \mathrm{E}-001$ \\
\hline$-7,63 \mathrm{E}-002$ & $-5,29 \mathrm{E}-002$ & $-2,08 \mathrm{E}-001$ & $-1,53 \mathrm{E}-001$ \\
\hline$-7,34 \mathrm{E}-002$ & $-5,01 \mathrm{E}-002$ & $-2,08 \mathrm{E}-001$ & $-1,43 \mathrm{E}-001$ \\
\hline$-6,78 \mathrm{E}-002$ & $-5,73 \mathrm{E}-002$ & $-1,85 \mathrm{E}-001$ & $-1,49 \mathrm{E}-001$ \\
\hline$-5,83 \mathrm{E}-002$ & $-5,20 \mathrm{E}-002$ & $-2,04 \mathrm{E}-001$ & $-1,26 \mathrm{E}-001$ \\
\hline$-6,31 \mathrm{E}-002$ & $-5,00 \mathrm{E}-002$ & $-1,90 \mathrm{E}-001$ & $-1,36 \mathrm{E}-001$ \\
\hline$-5,72 \mathrm{E}-002$ & $-4,22 \mathrm{E}-002$ & $-1,85 \mathrm{E}-001$ & $-1,46 \mathrm{E}-001$ \\
\hline$-5,93 \mathrm{E}-002$ & $-4,68 \mathrm{E}-002$ & $-2,00 \mathrm{E}-001$ & $-1,37 \mathrm{E}-001$ \\
\hline
\end{tabular}

Tabelle 54: Meßwerte Kinetik CHCl2 + NO rel. zu CH2F; 298 K

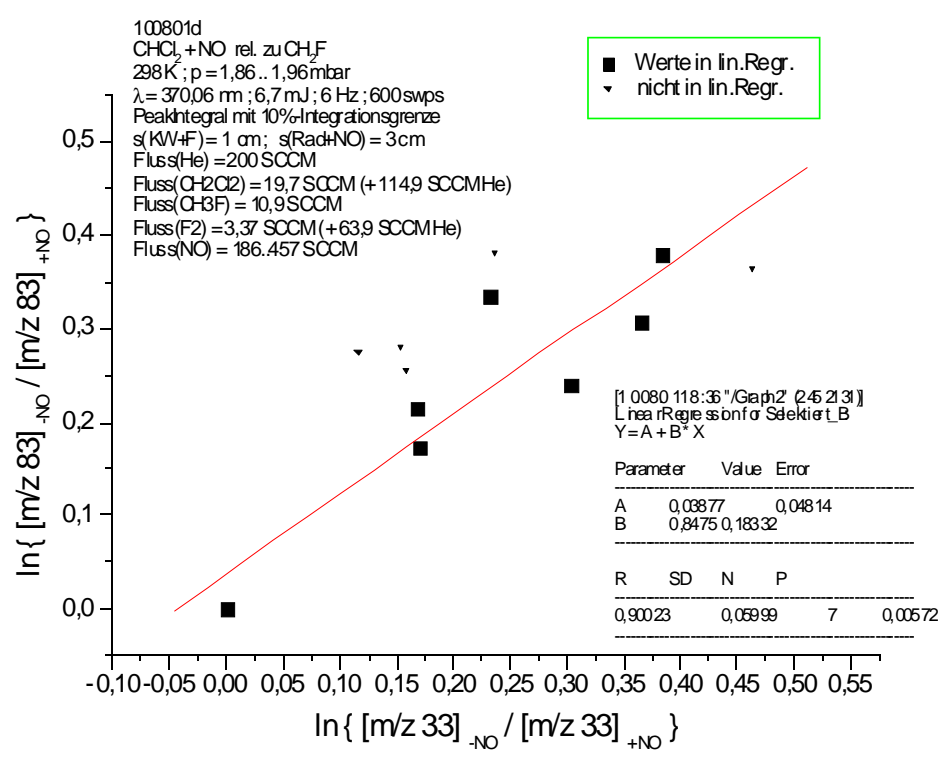

Abbildung 189: Kinetik CHCl2 + NO rel. zu CH2F; 298 K

Aus der Steigung erhält man: $\mathrm{k}_{\mathrm{rel}}=(0,85 \pm 0,18)$. 
$\vartheta=25{ }^{\circ} \mathrm{C} ; \phi_{\mathrm{He}}=379 \mathrm{SCCM} ; \phi_{\mathrm{CH} 2 \mathrm{Cl} 2}=19,7 \mathrm{SCCM} ; \phi_{\mathrm{CH} 3 \mathrm{~F}}=10,9 \mathrm{SCCM} ; \phi_{\mathrm{NO}}=186 . .457$

$\mathrm{SCCM} ; \mathrm{p}_{(\mathrm{StrR})}=186 . .196 \mathrm{~Pa} ; \mathrm{s}_{\mathrm{KW}+\mathrm{F}}=1 \mathrm{~cm} ; \mathrm{s}_{\text {Radikal }+\mathrm{NO}}=3 \mathrm{~cm} ; \mathrm{E}_{\mathrm{LASER}}=6,7 \mathrm{~mJ} ; \lambda_{\mathrm{LASER}}=370,06$ nm; Auswertung mit Mittelwert aus 600 einzelnen Flugzeitmassenspektren (=600 swps).

\begin{tabular}{|r|r|r|r|}
\hline $\mathbf{I}(\mathbf{8 3}), \mathbf{, - N O}$ “ $/ \mathbf{w . E .}$ & $\mathbf{I}(\mathbf{8 3}), \boldsymbol{, + N O}$ “ $/ \mathbf{w . E}$. & $\mathbf{I}(\mathbf{3 3}),,-\mathbf{N O} “ / \mathbf{w . E}$. & $\mathbf{I}(\mathbf{3 3}),,+\mathbf{N O}$ “ $\mathbf{w . E}$. \\
\hline$-7,23 \mathrm{E}-002$ & $-6,44 \mathrm{E}-002$ & $-2,23 \mathrm{E}-001$ & $-1,69 \mathrm{E}-001$ \\
\hline$-7,32 \mathrm{E}-002$ & $-6,18 \mathrm{E}-002$ & $-2,05 \mathrm{E}-001$ & $-1,72 \mathrm{E}-001$ \\
\hline$-6,76 \mathrm{E}-002$ & $-6,39 \mathrm{E}-002$ & $-2,14 \mathrm{E}-001$ & $-1,71 \mathrm{E}-001$ \\
\hline$-7,31 \mathrm{E}-002$ & $-6,27 \mathrm{E}-002$ & $-2,13 \mathrm{E}-001$ & $-1,61 \mathrm{E}-001$ \\
\hline$-7,60 \mathrm{E}-002$ & $-4,78 \mathrm{E}-002$ & $-2,01 \mathrm{E}-001$ & $-1,40 \mathrm{E}-001$ \\
\hline$-6,68 \mathrm{E}-002$ & $-5,71 \mathrm{E}-002$ & $-2,07 \mathrm{E}-001$ & $-1,60 \mathrm{E}-001$ \\
\hline$-7,63 \mathrm{E}-002$ & $-5,29 \mathrm{E}-002$ & $-2,08 \mathrm{E}-001$ & $-1,53 \mathrm{E}-001$ \\
\hline$-7,34 \mathrm{E}-002$ & $-5,01 \mathrm{E}-002$ & $-2,08 \mathrm{E}-001$ & $-1,43 \mathrm{E}-001$ \\
\hline$-6,78 \mathrm{E}-002$ & $-5,73 \mathrm{E}-002$ & $-1,85 \mathrm{E}-001$ & $-1,49 \mathrm{E}-001$ \\
\hline$-5,83 \mathrm{E}-002$ & $-5,20 \mathrm{E}-002$ & $-2,04 \mathrm{E}-001$ & $-1,26 \mathrm{E}-001$ \\
\hline$-6,31 \mathrm{E}-002$ & $-5,00 \mathrm{E}-002$ & $-1,90 \mathrm{E}-001$ & $-1,36 \mathrm{E}-001$ \\
\hline$-5,72 \mathrm{E}-002$ & $-4,22 \mathrm{E}-002$ & $-1,85 \mathrm{E}-001$ & $-1,46 \mathrm{E}-001$ \\
\hline$-5,93 \mathrm{E}-002$ & $-4,68 \mathrm{E}-002$ & $-2,00 \mathrm{E}-001$ & $-1,37 \mathrm{E}-001$ \\
\hline
\end{tabular}

Tabelle 55: Meßwerte Kinetik CHCl2 + NO rel. zu CH2F; 298 K

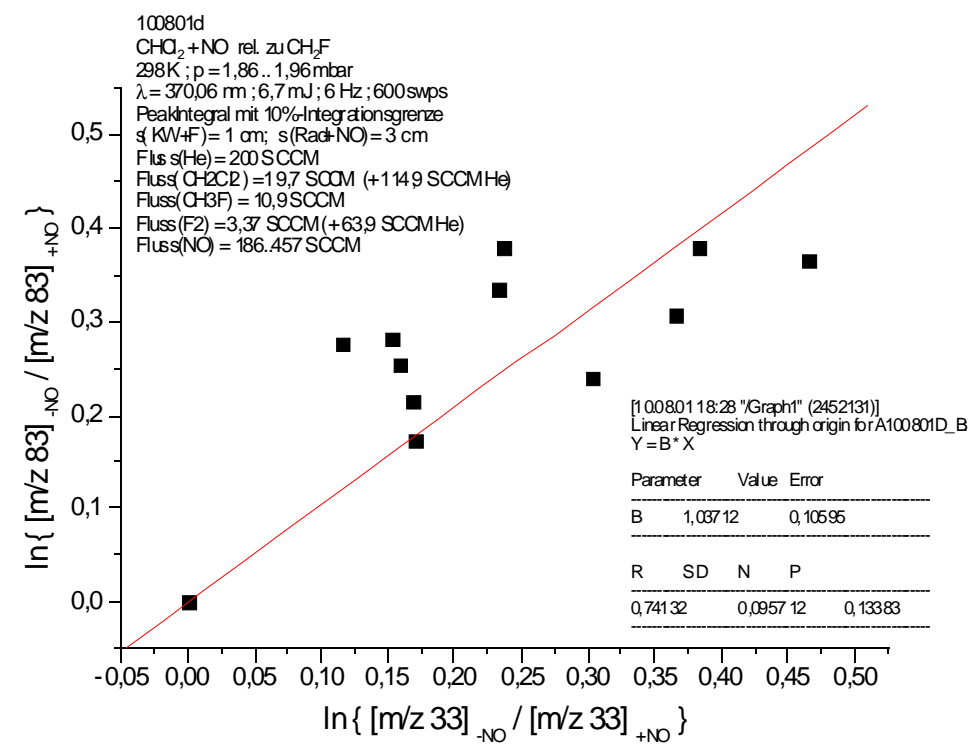

Abbildung 190: Kinetik CHCl2 + NO rel. zu CH2F; 298 K

Aus der Steigung erhält man: $\mathrm{k}_{\mathrm{rel}}=(1,04 \pm 0,11)$. 
$\vartheta=25{ }^{\circ} \mathrm{C} ; \phi_{\mathrm{He}}=432 \mathrm{SCCM} ; \phi_{\mathrm{CH} 2 \mathrm{Cl} 2}=25,2 \mathrm{SCCM} ; \phi_{\mathrm{CH} 3 \mathrm{~F}}=13,6 \mathrm{SCCM} ; \phi_{\mathrm{NO}}=186 . .457$ $\mathrm{SCCM} ; \mathrm{p}_{(\mathrm{StrR})}=218 . .236 \mathrm{~Pa} ; \mathrm{s}_{\mathrm{KW}+\mathrm{F}}=1 \mathrm{~cm} ; \mathrm{s}_{\text {Radikal }+\mathrm{NO}}=3 \mathrm{~cm} ; \mathrm{E}_{\mathrm{LASER}}=6,7 \mathrm{~mJ} ; \lambda_{\mathrm{LASER}}=370,06$ nm; Auswertung mit Mittelwert aus 600 einzelnen Flugzeitmassenspektren (= 600 swps).

\begin{tabular}{|r|r|r|r|}
\hline $\mathbf{I}(\mathbf{8 3}), \boldsymbol{, - N O}$ “ $/ \mathbf{w . E}$. & $\mathbf{I}(\mathbf{8 3}),,+\mathbf{N O}$ “ $/ \mathbf{w . E}$. & $\mathbf{I}(\mathbf{3 3}),,-\mathbf{N O}$ “ $/ \mathbf{w . E}$. & $\mathbf{I}(\mathbf{3 3}),,+\mathbf{N O}$ “ $/ \mathbf{w . E}$. \\
\hline$-9,58 \mathrm{E}-002$ & $-8,40 \mathrm{E}-002$ & $-3,26 \mathrm{E}-001$ & $-2,98 \mathrm{E}-001$ \\
\hline$-1,03 \mathrm{E}-001$ & $-8,83 \mathrm{E}-002$ & $-3,28 \mathrm{E}-001$ & $-2,84 \mathrm{E}-001$ \\
\hline$-1,03 \mathrm{E}-001$ & $-8,66 \mathrm{E}-002$ & $-3,23 \mathrm{E}-001$ & $-2,67 \mathrm{E}-001$ \\
\hline$-9,96 \mathrm{E}-002$ & $-7,49 \mathrm{E}-002$ & $-3,18 \mathrm{E}-001$ & $-2,49 \mathrm{E}-001$ \\
\hline$-9,14 \mathrm{E}-002$ & $-8,51 \mathrm{E}-002$ & $-3,17 \mathrm{E}-001$ & $-2,59 \mathrm{E}-001$ \\
\hline$-9,77 \mathrm{E}-002$ & $-7,44 \mathrm{E}-002$ & $-2,80 \mathrm{E}-001$ & $-2,38 \mathrm{E}-001$ \\
\hline$-9,61 \mathrm{E}-002$ & $-8,87 \mathrm{E}-002$ & $-3,06 \mathrm{E}-001$ & $-2,79 \mathrm{E}-001$ \\
\hline$-8,85 \mathrm{E}-002$ & $-8,32 \mathrm{E}-002$ & $-3,02 \mathrm{E}-001$ & $-2,68 \mathrm{E}-001$ \\
\hline$-1,04 \mathrm{E}-001$ & $-8,73 \mathrm{E}-002$ & $-3,11 \mathrm{E}-001$ & $-2,64 \mathrm{E}-001$ \\
\hline$-8,60 \mathrm{E}-002$ & $-7,91 \mathrm{E}-002$ & $-2,89 \mathrm{E}-001$ & $-2,32 \mathrm{E}-001$ \\
\hline$-9,18 \mathrm{E}-002$ & $-6,99 \mathrm{E}-002$ & $-2,81 \mathrm{E}-001$ & $-2,29 \mathrm{E}-001$ \\
\hline$-8,94 \mathrm{E}-002$ & $-7,27 \mathrm{E}-002$ & $-2,94 \mathrm{E}-001$ & $-2,16 \mathrm{E}-001$ \\
\hline$-8,43 \mathrm{E}-002$ & $-6,89 \mathrm{E}-002$ & $-2,87 \mathrm{E}-001$ & $-2,28 \mathrm{E}-001$ \\
\hline$-7,73 \mathrm{E}-002$ & $-7,01 \mathrm{E}-002$ & $-2,69 \mathrm{E}-001$ & $-2,10 \mathrm{E}-001$ \\
\hline$-8,41 \mathrm{E}-002$ & $-8,91 \mathrm{E}-002$ & $-2,92 \mathrm{E}-001$ & $-1,94 \mathrm{E}-001$ \\
\hline
\end{tabular}

Tabelle 56: Meßwerte Kinetik CHCl2 + NO rel. zu CH2F; 298 K

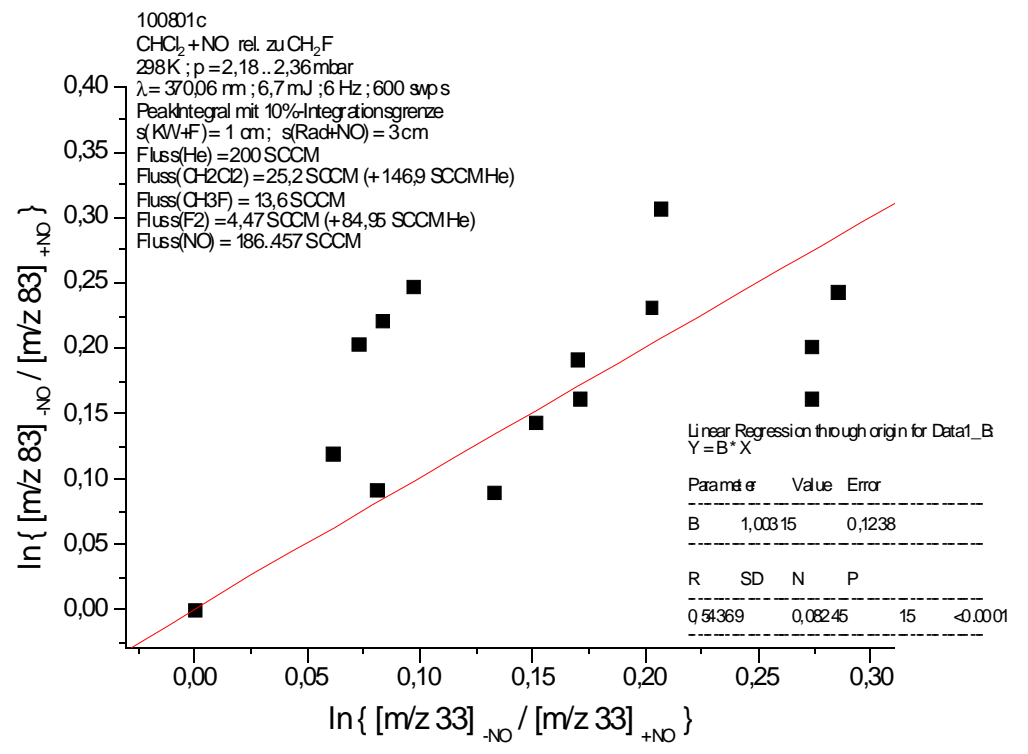

Abbildung 191: Kinetik CHCl2 + NO rel. zu CH2F; 298 K

Aus der Steigung erhält man: $\mathrm{k}_{\mathrm{rel}}=(1,00 \pm 0,12)$. 
$\vartheta=25{ }^{\circ} \mathrm{C} ; \phi_{\mathrm{He}}=432 \mathrm{SCCM} ; \phi_{\mathrm{CH} 2 \mathrm{Cl} 2}=25,2 \mathrm{SCCM} ; \phi_{\mathrm{CH} 3 \mathrm{~F}}=13,6 \mathrm{SCCM} ; \phi_{\mathrm{NO}}=186 . .457$

$\mathrm{SCCM} ; \mathrm{p}_{(\mathrm{StrR})}=218 . .236 \mathrm{~Pa} ; \mathrm{s}_{\mathrm{KW}+\mathrm{F}}=1 \mathrm{~cm} ; \mathrm{s}_{\text {Radikal }+\mathrm{NO}}=3 \mathrm{~cm} ; \mathrm{E}_{\mathrm{LASER}}=6,7 \mathrm{~mJ} ; \lambda_{\mathrm{LASER}}=370,06$ nm; Auswertung mit Mittelwert aus 600 einzelnen Flugzeitmassenspektren (=600 swps).

\begin{tabular}{|c|c|c|c|}
\hline I(83), ,-NO“ / w.E. & $\mathrm{I}(\mathbf{8 3}),,+\mathrm{NO} “ /$ w.E. & $\mathbf{I}(33),,-N O “ /$ w.E. & $\mathrm{I}(33),,+\mathrm{NO} “ 6 /$ w.E. \\
\hline$-9,58 \mathrm{E}-002$ & $-8,40 \mathrm{E}-002$ & $-3,26 \mathrm{E}-001$ & $-2,98 \mathrm{E}-001$ \\
\hline$-1,03 \mathrm{E}-001$ & $-8,83 \mathrm{E}-002$ & $-3,28 \mathrm{E}-001$ & $-2,84 \mathrm{E}-001$ \\
\hline$-1,03 \mathrm{E}-001$ & $-8,66 \mathrm{E}-002$ & $-3,23 \mathrm{E}-001$ & $-2,67 \mathrm{E}-001$ \\
\hline$-9,96 \mathrm{E}-002$ & $-7,49 \mathrm{E}-002$ & $-3,18 \mathrm{E}-001$ & $-2,49 \mathrm{E}-001$ \\
\hline$-9,14 \mathrm{E}-002$ & $-8,51 \mathrm{E}-002$ & $-3,17 \mathrm{E}-001$ & $-2,59 \mathrm{E}-001$ \\
\hline$-9,77 \mathrm{E}-002$ & $-7,44 \mathrm{E}-002$ & $-2,80 \mathrm{E}-001$ & $-2,38 \mathrm{E}-001$ \\
\hline$-9,61 \mathrm{E}-002$ & $-8,87 \mathrm{E}-002$ & $-3,06 \mathrm{E}-001$ & $-2,79 \mathrm{E}-001$ \\
\hline$-8,85 \mathrm{E}-002$ & $-8,32 \mathrm{E}-002$ & $-3,02 \mathrm{E}-001$ & $-2,68 \mathrm{E}-001$ \\
\hline$-1,04 \mathrm{E}-001$ & $-8,73 \mathrm{E}-002$ & $-3,11 \mathrm{E}-001$ & $-2,64 \mathrm{E}-001$ \\
\hline$-8,60 \mathrm{E}-002$ & $-7,91 \mathrm{E}-002$ & $-2,89 \mathrm{E}-001$ & $-2,32 \mathrm{E}-001$ \\
\hline$-9,18 \mathrm{E}-002$ & $-6,99 \mathrm{E}-002$ & $-2,81 \mathrm{E}-001$ & $-2,29 \mathrm{E}-001$ \\
\hline$-8,94 \mathrm{E}-002$ & $-7,27 \mathrm{E}-002$ & $-2,94 \mathrm{E}-001$ & $-2,16 \mathrm{E}-001$ \\
\hline$-8,43 \mathrm{E}-002$ & $-6,89 \mathrm{E}-002$ & $-2,87 \mathrm{E}-001$ & $-2,28 \mathrm{E}-001$ \\
\hline$-7,73 \mathrm{E}-002$ & $-7,01 \mathrm{E}-002$ & $-2,69 \mathrm{E}-001$ & $-2,10 \mathrm{E}-001$ \\
\hline$-8,41 \mathrm{E}-002$ & $-8,91 \mathrm{E}-002$ & $-2,92 \mathrm{E}-001$ & $-1,94 \mathrm{E}-001$ \\
\hline
\end{tabular}

Tabelle 57: Meßwerte Kinetik CHCl2 + NO rel. zu CH2F; $298 \mathrm{~K}$

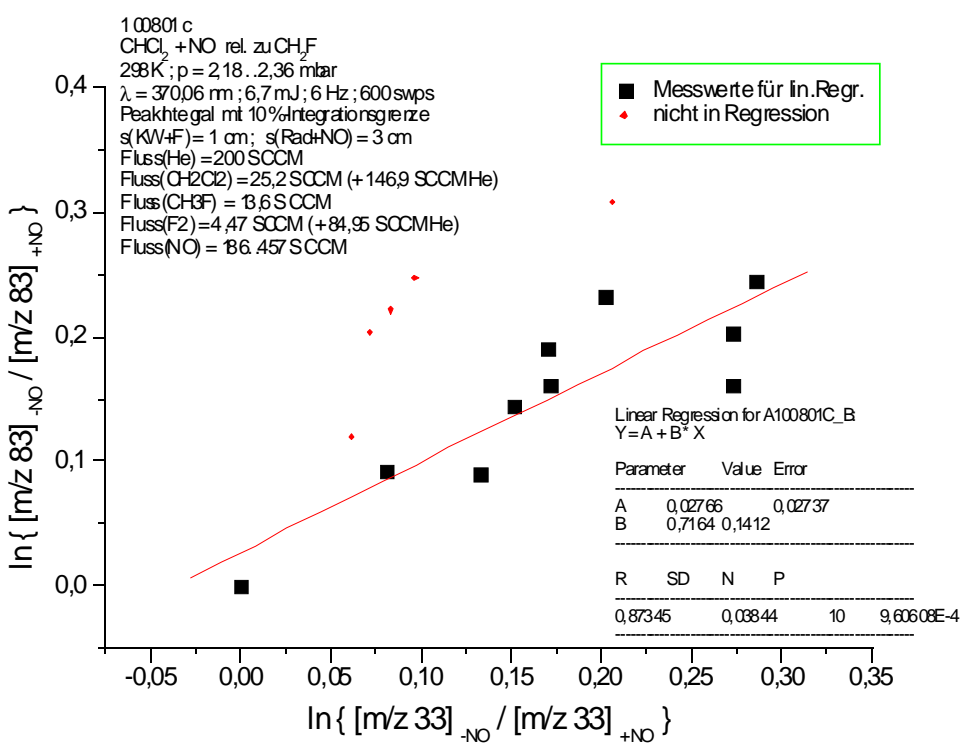

Abbildung 192: Kinetik CHCl2 + NO rel. zu CH2F; 298 K

Aus der Steigung erhält man: $\mathrm{k}_{\mathrm{rel}}=(0,72 \pm 0,14)$. 
$\vartheta=25{ }^{\circ} \mathrm{C} ; \phi_{\mathrm{He}}=432 \mathrm{SCCM} ; \phi_{\mathrm{CH} 2 \mathrm{Cl} 2}=25,2 \mathrm{SCCM} ; \phi_{\mathrm{CH} 3 \mathrm{~F}}=13,6 \mathrm{SCCM} ; \phi_{\mathrm{NO}}=186 . .457$ $\mathrm{SCCM} ; \mathrm{p}_{(\mathrm{StrR})}=218 . .236 \mathrm{~Pa} ; \mathrm{s}_{\mathrm{KW}+\mathrm{F}}=1 \mathrm{~cm} ; \mathrm{s}_{\text {Radikal }+\mathrm{NO}}=3 \mathrm{~cm} ; \mathrm{E}_{\mathrm{LASER}}=6,7 \mathrm{~mJ} ; \lambda_{\mathrm{LASER}}=370,06$ nm; Auswertung mit Mittelwert aus 600 einzelnen Flugzeitmassenspektren (= 600 swps).

\begin{tabular}{|r|r|r|r|}
\hline $\mathbf{I}(\mathbf{8 3}),,-\mathbf{N O}$ “ $\mathbf{w . E .}$ & $\mathbf{I}(\mathbf{8 3}),,+\mathbf{N O} “ / \mathbf{w . E}$. & $\mathbf{I}(\mathbf{3 3}),,-\mathbf{N O}$ “ $\mathbf{w . E}$. & $\mathbf{I}(\mathbf{3 3}),,+\mathbf{N O}$ “ $\mathbf{w . E .}$ \\
\hline$-7,15 \mathrm{E}-003$ & $-5,65 \mathrm{E}-003$ & $-1,65 \mathrm{E}-002$ & $-1,38 \mathrm{E}-002$ \\
\hline$-7,43 \mathrm{E}-003$ & $-4,20 \mathrm{E}-003$ & $-1,55 \mathrm{E}-002$ & $-1,28 \mathrm{E}-002$ \\
\hline$-5,92 \mathrm{E}-003$ & $-5,21 \mathrm{E}-003$ & $-1,68 \mathrm{E}-002$ & $-1,39 \mathrm{E}-002$ \\
\hline$-7,15 \mathrm{E}-003$ & $-4,20 \mathrm{E}-003$ & $-1,49 \mathrm{E}-002$ & $-1,26 \mathrm{E}-002$ \\
\hline$-6,44 \mathrm{E}-003$ & $-5,49 \mathrm{E}-003$ & $-1,59 \mathrm{E}-002$ & $-1,23 \mathrm{E}-002$ \\
\hline$-6,98 \mathrm{E}-003$ & $-4,08 \mathrm{E}-003$ & $-1,56 \mathrm{E}-002$ & $-1,10 \mathrm{E}-002$ \\
\hline$-6,21 \mathrm{E}-003$ & $-3,94 \mathrm{E}-003$ & $-1,73 \mathrm{E}-002$ & $-1,15 \mathrm{E}-002$ \\
\hline$-6,51 \mathrm{E}-003$ & $-4,69 \mathrm{E}-003$ & $-1,76 \mathrm{E}-002$ & $-1,13 \mathrm{E}-002$ \\
\hline$-6,99 \mathrm{E}-003$ & $-4,57 \mathrm{E}-003$ & $-1,59 \mathrm{E}-002$ & $-1,18 \mathrm{E}-002$ \\
\hline$-7,73 \mathrm{E}-003$ & $-3,50 \mathrm{E}-003$ & $-1,76 \mathrm{E}-002$ & $-1,09 \mathrm{E}-002$ \\
\hline
\end{tabular}

Tabelle 58: Meßwerte Kinetik $\mathrm{CHCl}+\mathrm{NO}$ rel. zu CH2F; $298 \mathrm{~K}$

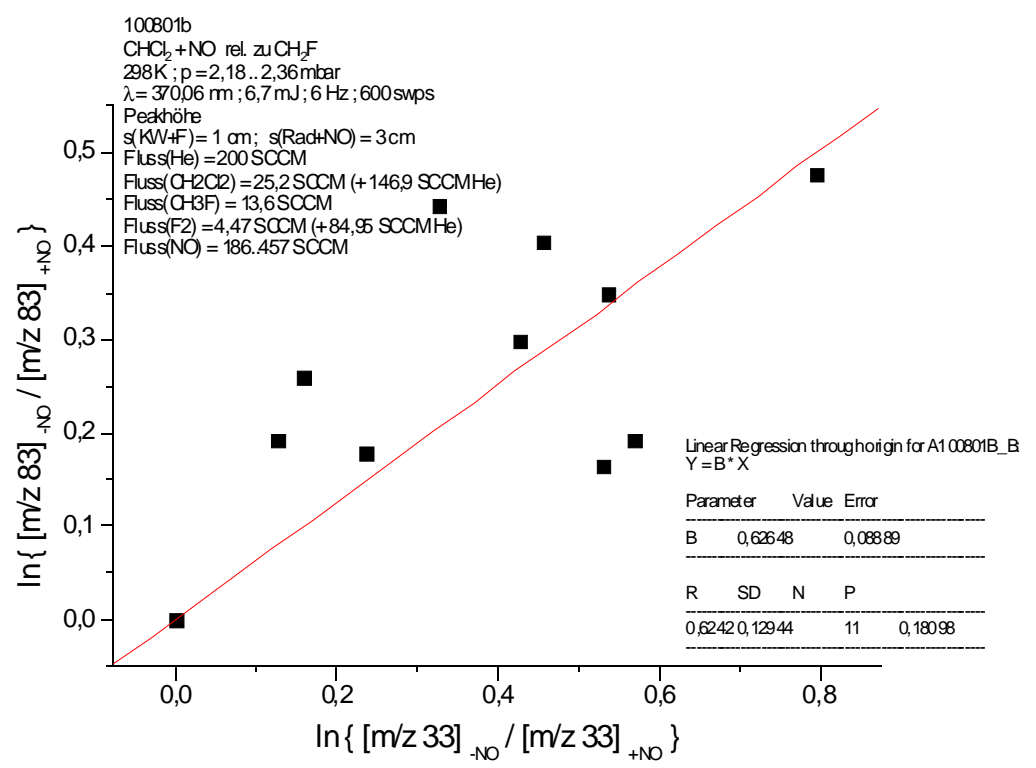

Abbildung 193: Kinetik CHCl2 + NO rel. zu CH2F; $298 \mathrm{~K}$

Aus der Steigung erhält man: $\mathrm{k}_{\mathrm{rel}}=(0,63 \pm 0,09)$. 
$\vartheta=25{ }^{\circ} \mathrm{C} ; \phi_{\mathrm{He}}=432 \mathrm{SCCM} ; \phi_{\mathrm{CH} 2 \mathrm{Cl} 2}=25,2 \mathrm{SCCM} ; \phi_{\mathrm{CH} 3 \mathrm{~F}}=13,6 \mathrm{SCCM} ; \phi_{\mathrm{NO}}=186 . .457$

$\mathrm{SCCM} ; \mathrm{p}_{(\mathrm{StrR})}=218 . .236 \mathrm{~Pa} ; \mathrm{s}_{\mathrm{KW}+\mathrm{F}}=1 \mathrm{~cm} ; \mathrm{s}_{\text {Radikal }+\mathrm{NO}}=3 \mathrm{~cm} ; \mathrm{E}_{\mathrm{LASER}}=6,7 \mathrm{~mJ} ; \lambda_{\mathrm{LASER}}=370,06$ nm; Auswertung mit Mittelwert aus 600 einzelnen Flugzeitmassenspektren (=600 swps).

\begin{tabular}{|c|c|c|c|}
\hline I(83) ,,-NO“ / w.E. & $\mathrm{I}(83),,+\mathrm{NO} “ /$ w.E. & $\mathbf{I}(\mathbf{3 3}),,-\mathrm{NO} “ /$ w.E. & $\mathrm{I}(33),,+\mathrm{NO}$ \\
\hline$-7,15 \mathrm{E}-003$ & $-5,65 \mathrm{E}-003$ & $-1,65 \mathrm{E}-002$ & $-1,38 \mathrm{E}-002$ \\
\hline$-7,43 \mathrm{E}-003$ & $-4,20 \mathrm{E}-003$ & $-1,55 \mathrm{E}-002$ & $-1,28 \mathrm{E}-002$ \\
\hline$-5,92 \mathrm{E}-003$ & $-5,21 \mathrm{E}-003$ & $-1,68 \mathrm{E}-002$ & $-1,39 \mathrm{E}-002$ \\
\hline$-7,15 \mathrm{E}-003$ & $-4,20 \mathrm{E}-003$ & $-1,49 \mathrm{E}-002$ & $-1,26 \mathrm{E}-002$ \\
\hline$-6,44 \mathrm{E}-003$ & $-5,49 \mathrm{E}-003$ & $-1,59 \mathrm{E}-002$ & $-1,23 \mathrm{E}-002$ \\
\hline$-6,98 \mathrm{E}-003$ & $-4,08 \mathrm{E}-003$ & $-1,56 \mathrm{E}-002$ & $-1,10 \mathrm{E}-002$ \\
\hline$-6,21 \mathrm{E}-003$ & $-3,94 \mathrm{E}-003$ & $-1,73 \mathrm{E}-002$ & $-1,15 \mathrm{E}-002$ \\
\hline$-6,51 \mathrm{E}-003$ & $-4,69 \mathrm{E}-003$ & $-1,76 \mathrm{E}-002$ & $-1,13 \mathrm{E}-002$ \\
\hline$-6,99 \mathrm{E}-003$ & $-4,57 \mathrm{E}-003$ & $-1,59 \mathrm{E}-002$ & $-1,18 \mathrm{E}-002$ \\
\hline$-7,73 \mathrm{E}-003$ & $-3,50 \mathrm{E}-003$ & $-1,76 \mathrm{E}-002$ & $-1,09 \mathrm{E}-002$ \\
\hline
\end{tabular}

Tabelle 59: Meßwerte Kinetik CHCl2 + NO rel. zu CH2F; $298 \mathrm{~K}$

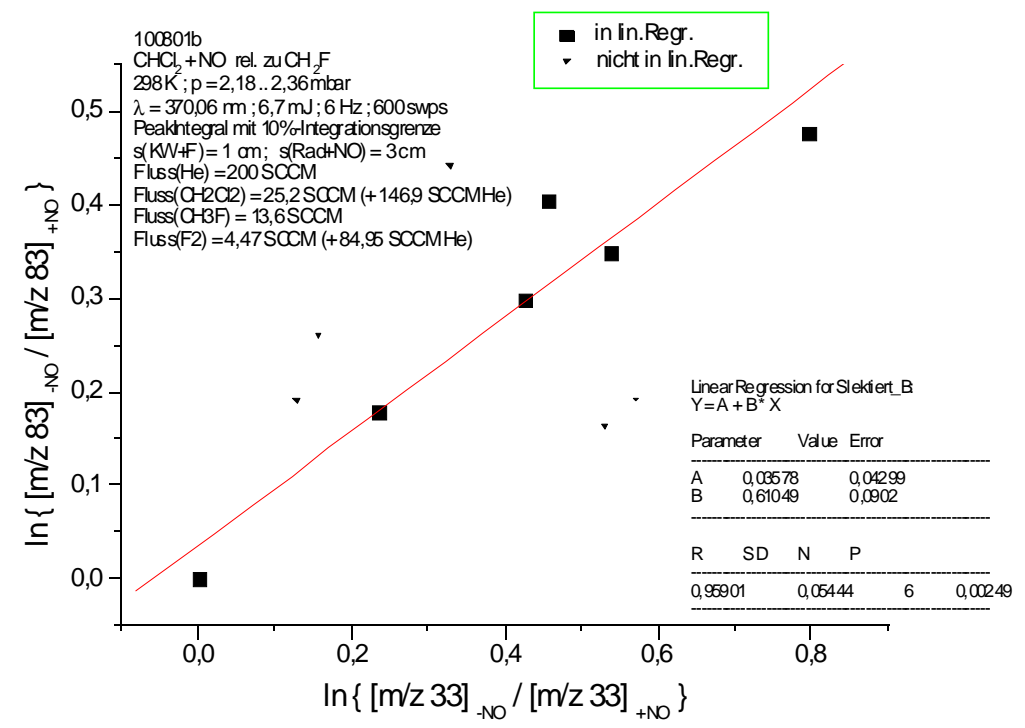

Abbildung 194: Kinetik CHCl2 + NO rel. zu CH2F; 298 K

Aus der Steigung erhält man: $\mathrm{k}_{\mathrm{rel}}=(0,61 \pm 0,09)$. 
Für das Gesamtergebnis soll der arithmetische Mittelwert ermittelt werden:

\begin{tabular}{|c|c|c|c|c|}
\hline Messung & $\mathbf{k}_{\text {rel }}$ & $\Delta \mathbf{k}_{\text {rel }}$ & $\mathbf{p} / \mathbf{m b a r}$ & {$[\mathbf{H e}] / \mathbf{~ m o l} / \mathbf{c m}^{\mathbf{3}}$} \\
\hline 1 & 0,8475 & 0,18 & 1,91 & $7,71 \mathrm{E}-08$ \\
\hline 2 & 1,0371 & 0,11 & 1,91 & $7,71 \mathrm{E}-08$ \\
\hline 3 & 0,7164 & 0,14 & 2,27 & $9,16 \mathrm{E}-08$ \\
\hline 4 & 1,0032 & 0,12 & 2,27 & $9,16 \mathrm{E}-08$ \\
\hline 5 & 0,6105 & 0,09 & 2,27 & $9,16 \mathrm{E}-08$ \\
\hline 6 & 0,6265 & 0,09 & 2,27 & $9,16 \mathrm{E}-08$ \\
\hline Mittelwert: & 0,81 & 0,19 & & $8,67 \mathrm{E}-08$ \\
\hline
\end{tabular}

Als Ergebnis bleibt damit festzuhalten: $\mathbf{k}_{\text {rel }}=(\mathbf{0 , 8 1} \pm \mathbf{0 , 1 9})$

Für die Referenzreaktion ist folgender Geschwindigkeitskoeffizient $\mathrm{k}_{\text {ref }}$ angegeben ${ }^{169}$ :

$\mathrm{k}_{\mathrm{ref}}(298 \mathrm{~K})=1,5 \cdot 10^{17} \mathrm{~cm}^{6} /\left(\mathrm{mol}^{2} \cdot \mathrm{s}\right)$.

Mit dem soeben berechneten Mittelwert erhält man für den Geschwindigkeitskoeffizienten der hier untersuchten Reaktion: $\mathrm{k}_{\text {termol. }}(298 \mathrm{~K})=(0,81 \pm 0,19) \cdot 1,5 \cdot 10^{17}[\mathrm{He}] \mathrm{cm}^{6} /\left(\mathrm{mol}^{2} \cdot \mathrm{s}\right)=$

$$
\begin{aligned}
& \mathbf{k}_{\text {termol. }}(\mathbf{2 9 8 K})=(\mathbf{1 , 2} \pm \mathbf{0 , 3}) \cdot \mathbf{1 0}^{17} \cdot[\mathrm{He}] \mathbf{c m}^{6} /\left(\mathbf{m o l}^{2} \cdot \mathbf{s}\right) \\
& \text { für } \mathrm{CHCl}_{2}+\mathrm{NO}+\mathrm{M} \rightarrow \text { Produkte }+\mathrm{M}^{*} \quad ; \mathrm{M} \text { : dritter Stoßpartner }(\mathrm{He})
\end{aligned}
$$

Mit der mittleren Helium-Konzentration errechnet man folgenden Wert für den Geschwindigkeitskoeffizienten der bimolekularen Reaktion:

$$
\mathrm{k}_{\text {bimolek. }}(298 \mathrm{~K})=1,0 \cdot 10^{10} \mathrm{~cm}^{3} /(\mathrm{mol} \cdot \mathrm{s}) ; \quad \text { für }[\mathrm{He}]=8,67 \cdot 10^{-08} \mathrm{~mol} / \mathrm{cm}^{3}
$$

\subsubsection{Diskussion der Ergebnisse}

Bezüglich der ermittelten Produkte aus der Reaktion des Dichlormethyl-Radikals mit Sauerstoff-Atomen bietet sich der Vergleich mit den Produkten der analogen Reaktion $\mathrm{CHF}_{2}$ $+\mathrm{O} \quad \mathrm{an}^{170}$. Das zum dort gefundenen Hauptprodukt $\mathrm{COF}_{2}$ analoge Produkt $\mathrm{COCl}_{2}$ wurde hier nicht nachgewiesen. Dafür wäre ein C-H-Bindungsbruch erforderlich; die C-H-Bindung ist aber etwas schwächer als die C-F-Bindung und erheblich stärker als die C-Cl-Bindung ${ }^{171}$, so

169 Beiderhase, T. (1995), S. 150.

170 Vgl. dazu: Beiderhase, T. (1995); S. 85-87.

171 Bezüglich der Bindungsstärken vergleiche: Lide, D. (1991); Abschnitt 9, S. 121.

Dort wird deutlich, daß die C-Cl-Bindung etwa 20-25\% schwächer als C-F und C-H etwa 3-8 \% schwächer als die C-F-Bindung ist. 
daß dieses Resultat plausibel erscheint. Die Produkte ClCHO bzw. FCHO werden bei beiden Reaktionen gebildet. Ebenso entstehen die HCl- bzw. HF-Eliminierungsprodukte ClCO bzw. FCO in beiden Reaktionen gleichermaßen. Auch die aus primären und sekundären Zerfallsreaktionen mögliche CO-Bildung wurde in beiden Reaktionen beobachtet.

Insgesamt korrelieren die Ergebnisse der Reaktionen $\mathrm{CHCl}_{2}+\mathrm{O}$ bzw. $\mathrm{CHF}_{2}+\mathrm{O}$ also recht gut und werden dadurch bestätigt.

Es existiert auch eine theoretische Untersuchung der Reaktion $\mathrm{CH}_{2} \mathrm{Cl}+\mathrm{O}$, die auf der Methode G2MP2 basiert (Abbildung 195) ${ }^{172}$.

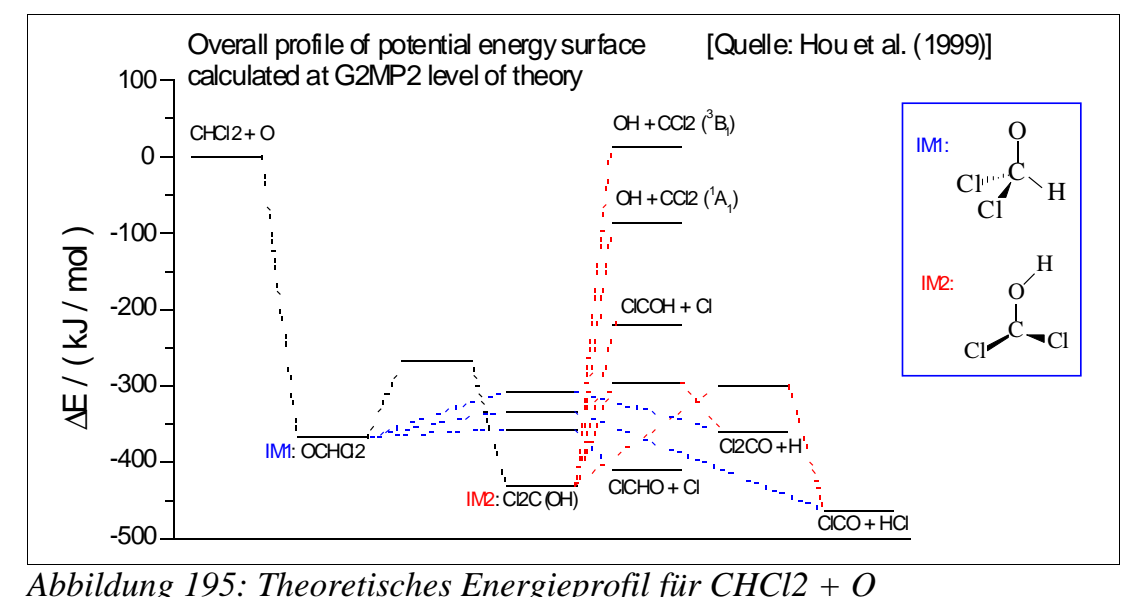

Die in Abb. 195 nicht beschrifteten Energiestufen gehören zu den entsprechenden Übergangszuständen der Reaktionskanäle. Die Farben der „Reaktionswege“ entsprechen denen in Abb. 184 (S. 215), wo die experimentell gefundenen Ergebnisse zusammengestellt sind.. Die gerechneten Kanäle stehen insofern in Einklang mit dem Experiment, als daß die energetisch ungünstigen (im wesentlichen die rot gezeichneten Wege in Abb. 195 bzw. Abb. 184) tatsächlich nicht nachgewiesen werden konnten. Allerdings ist experimentell CO mit 70 mol-\% das Hauptprodukt, welches bei den gerechneten Produktkanälen überhaupt nicht enthalten ist. Vermutlich stammt es aber aus den in Abb. 184 (S. 215) grün gezeichneten Folgereaktionen, was dann wieder in Übereinstimmung mit der Theorie wäre. Für weitere Aussagen müßten die G2MP2-Rechnungen noch um diese Kanäle (7) und (9), ggf. auch (8) erweitert werden.

Die einzig vorliegende publizierte Temperaturabhängigkeit des Geschwindigkeitskoeffizienten für die Reaktion $\mathrm{CHCl}_{2}+\mathrm{O} \rightarrow$ Produkte wurde im Temperaturbereich 302-900 K folgendermaßen bestimmt ${ }^{173}: \mathrm{k}_{\mathrm{Lit}}=(5,4 \pm 0,6) \cdot 10^{13} \cdot \exp \{-(-0,48 \pm 0,16) \mathrm{kJ} / \mathrm{mol} /(\mathrm{R} \cdot \mathrm{T})\} \mathrm{cm}^{3} /$ (mol-s). 
In der vorliegenden Arbeit ist angegeben:

$\mathrm{k}(\mathrm{T})=(8,9 \pm 1,8) \cdot 10^{13} \cdot \exp \{-(-0,10 \pm 0,53) \mathrm{kJ} / \mathrm{mol} /(\mathrm{R} \cdot \mathrm{T})\} \mathrm{cm}^{3} /(\mathrm{mol} \cdot \mathrm{s})$.

Die Aktivierungsenergien stimmen im Rahmen ihrer Fehlergrenzen gut überein, die Vorfaktoren sind zwar in der gleichen Gräßenordnung, weichen aber doch voneinander ab. Für $\mathrm{T}=298 \mathrm{~K}$ errechnet man aus der Literatur-Gleichung $\mathrm{k}_{\mathrm{Lit}}(298 \mathrm{~K})=6,6 \cdot 10^{13} \mathrm{~cm}^{3} /(\mathrm{mol} \cdot \mathrm{s})$ und in dieser Arbeit $\mathrm{k}(298 \mathrm{~K})=4,3 \cdot 10^{13} \mathrm{~cm}^{3} /(\mathrm{mol} \cdot \mathrm{s})$. Die Werte bei $298 \mathrm{~K}$ sind ähnlich, was die vorliegenden Ergebnisse stützt. In der genannten Literatur ${ }^{174}$ wurde $\mathrm{HCl}$ als Produkt der Reaktion $\mathrm{CHCl}_{2}+\mathrm{O}$ nachgewiesen, so wie in der vorliegenden Arbeit auch.

Bezüglich der Reaktion $\mathrm{CHCl}_{2}+\mathrm{NO} \rightarrow$ Produkte lagen keine Geschwindigkeitskoeffizienten vor, die in anderen Arbeitsgruppen bestimmt worden waren. Jedoch ist der gemessene Wert von $\mathrm{k}_{\text {bimolek. }}(298 \mathrm{~K})=1,0 \cdot 10^{10} \mathrm{~cm}^{3} /(\mathrm{mol} \cdot \mathrm{s})$ etwa der gleiche, wie bei den Reaktionen $\mathrm{CHF}_{2}+$ NO $\left(\mathrm{k}=2 \cdot 10^{10} \mathrm{~cm}^{3} /(\mathrm{mol} \cdot \mathrm{s})\right)$ und $\mathrm{CH}_{2} \mathrm{~F}+\mathrm{NO}\left(\mathrm{k}=1,3 \cdot 10^{10} \mathrm{~cm}^{3} /(\mathrm{mol} \cdot \mathrm{s})\right)$; da diese Moleküle strukturell sehr ähnlich sind, dürfte das hier gefundene Resultat in der richtigen Größenordnung sein. Für $\mathrm{CH}_{3}+\mathrm{NO}$ ist er bei vergleichbarer Helium-Konzentration deutlich höher ${ }^{175}\left(\mathrm{k}=7 \cdot 10^{10} \mathrm{~cm}^{3} /(\mathrm{mol} \cdot \mathrm{s})\right)$, was aber nicht verwundert, denn es wurde im Verlauf der Arbeit ja bei den Alkoholen und Ethern festgestellt, daß mit zunehmender Zahl an elektronegativen Substituenten der Geschwindigkeitskoeffizient bei den Assoziationsreaktionen abnimmt. 


\subsubsection{Das 1,1,1,2-Tetrafluorethan $\left(\mathrm{CF}_{3} \mathrm{CH}_{2} \mathrm{~F}\right)$}

\subsubsection{Produkte der radikalerzeugenden Reaktion mit Fluor-Atomen}

In den EI-Übersichtsmassenspektren der Abbildung 196, die mit der in Kapitel 3.1.1 (S. 16) beschriebenen Apparatur registriert wurden, zeigt das linke Spektrum $\mathrm{CF}_{3} \mathrm{CH}_{2} \mathrm{~F}+\mathrm{F}_{2}$ bei ausgeschalteter Mikrowellenentladung, während letztere bei dem rechten Spektrum eingeschaltet war $\left(\mathrm{CF}_{3} \mathrm{CH}_{2} \mathrm{~F}+\mathrm{F}\right)$. Die Durchführung der Messungen ist in Kapitel 3.5.1 (S. 50) beschrieben. Der nicht vollständig gezeigte Peak m/z 40 stammt vom Badgas Argon.

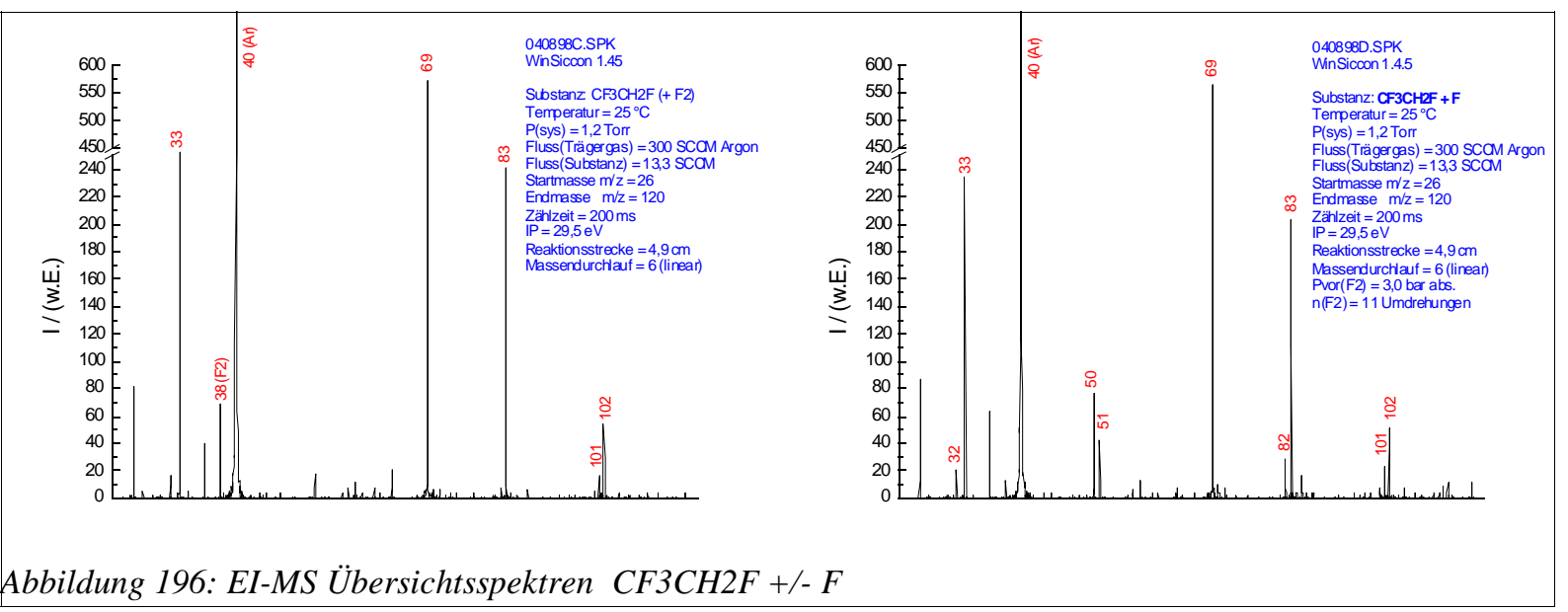

Es zeigte sich bei Zugabe von Fluor-Atomen eine Abnahme auf dem Molekül-Peak m/z 102 sowie Zunahmen insbesondere auf $\mathrm{m} / \mathrm{z}$ 101; 82; 51 und 50.

In Abb. 197 ist die Intensität auf m/z 20 in Abhängigkeit des Gasflusses von $\mathrm{CF}_{3} \mathrm{CH}_{2} \mathrm{~F}$ bei konstantem Gasfluß an Fluor-Atomen dargestellt. Man kann daraus schließen, daß $\mathrm{HF}$ aus der Reaktion $\mathrm{CF}_{3} \mathrm{CH}_{2} \mathrm{~F}+\mathrm{F}$ gebildet wird. Zusammen mit der Zunahme auf $\mathrm{m} / \mathrm{z}$ 101, d.h. dem Molekül-Peak des Radikals $\mathrm{CF}_{3} \mathrm{CHF}$, muß man davon ausgehen, daß folgende Reaktion abläuft:

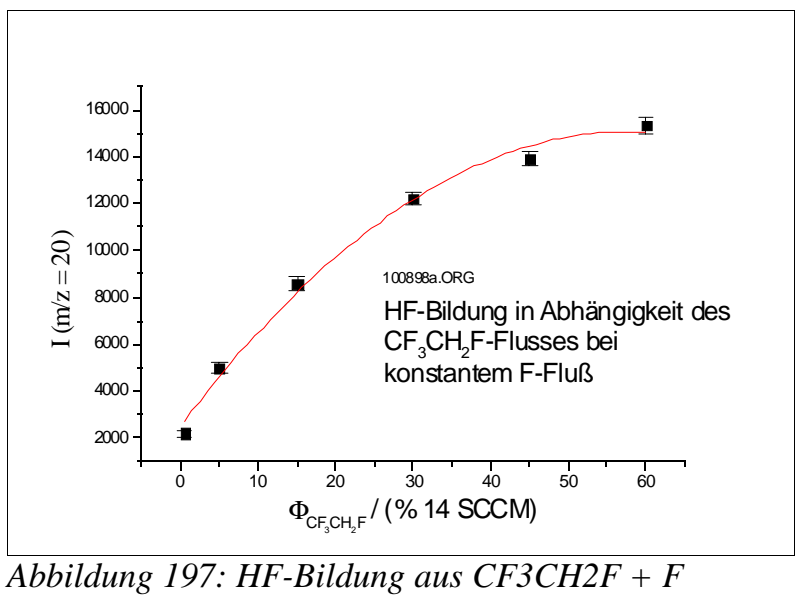

$\mathrm{CF}_{3} \mathrm{CH}_{2} \mathrm{~F}+\mathrm{F} \rightarrow \mathrm{CF}_{3} \mathrm{CHF}+\mathrm{HF}$

Gestützt wird diese Annahme durch die Zunahme auf m/z 82, bei der es sich um das Fragment $\mathrm{CF}_{2} \mathrm{CHF}$ des erzeugten Radikals handelt. 


\subsubsection{Kinetik der radikalerzeugenden Reaktion mit Fluor-Atomen}

Die Messungen wurden in der in Kap. 3.1.1 (S. 16) beschriebenen Apparatur unter Einsatz des Strömungsreaktors aus Abbildung 5 (S. 17) durchgeführt. Die Ausführung und Auswertung nach der Relativmethode ist in Kap. 3.5.1 (S. 50) beschrieben.

In einer ersten Messung diente Trichlormethan (Chloroform) als Referenzsubstanz. Der Umsatz von $\mathrm{CF}_{3} \mathrm{CH}_{2} \mathrm{~F}$ konnte anhand der Intensitätsänderung seines Molekül-Peaks m/z 102, der von Trichlormethan anhand seines Molekül-Peaks m/z 120 bestimmt werden.

$\vartheta=+25^{\circ} \mathrm{C} ; \phi_{\mathrm{Ar}}=573 \mathrm{SCCM} ; \phi_{\mathrm{CF} 3 \mathrm{CH} 2 \mathrm{~F}}=14,4 \mathrm{SCCM} ; \phi_{\mathrm{CHCl} 13}=17,1 \mathrm{SCCM} ; \mathrm{p}_{(\mathrm{StrR})}=160 \mathrm{~Pa} ;$ $\mathrm{s}=3,0 \mathrm{~cm} ; \mathrm{IP}=29,5 \mathrm{eV}$; Zählzeit = $10 \mathrm{~s}$

\begin{tabular}{|c|c|c|c|c|c|c|c|}
\hline $\begin{array}{c}\text { I(102) } \\
\text {-F / w.E. }\end{array}$ & $\begin{array}{c}\Delta \mathbf{I}(\mathbf{1 0 2}) \\
-\mathrm{F} / \mathrm{w.E} .\end{array}$ & $\begin{array}{c}\mathrm{I}(102) \\
+\mathrm{F} / \mathrm{w.E.}\end{array}$ & $\begin{array}{c}\Delta \mathbf{I}(102) \\
+\mathrm{F} / \mathrm{w.E} .\end{array}$ & $\begin{array}{c}\text { I(120) } \\
\text {-F / w.E. }\end{array}$ & $\begin{array}{c}\Delta \mathbf{I}(120) \\
-\mathbf{F} / \mathbf{w . E .}\end{array}$ & $\begin{array}{c}\mathrm{I}(120) \\
+\mathrm{F} / \mathrm{w.E.}\end{array}$ & $\begin{array}{r}\Delta \mathbf{I}(120) \\
+\mathrm{F} / \mathrm{w} . \mathrm{E} .\end{array}$ \\
\hline 9245 & 163,4 & 8950 & 168,8 & 15100 & 129,8 & 15030 & 301 \\
\hline 9128 & 150,5 & 8910 & 155,8 & 16930 & 352,6 & 16300 & 183,6 \\
\hline 9205 & 142,5 & 8385 & 247,2 & 17180 & 200,1 & 15800 & 290,3 \\
\hline 9093 & 219,3 & 8038 & 93,95 & 17630 & 238,5 & 15450 & 378,5 \\
\hline 8113 & 142,4 & 6253 & 158,1 & 17300 & 343,5 & 13600 & 129,8 \\
\hline 8488 & 188,7 & 6763 & 215 & 17980 & 200,1 & 14630 & 327,8 \\
\hline 8423 & 141,8 & 7430 & 185,9 & 18300 & 224,9 & 15780 & 79,5 \\
\hline 8475 & 187,9 & 7335 & 215,1 & 17700 & 129,8 & 15380 & 238,5 \\
\hline 8840 & 165,7 & 7523 & 254,9 & 18280 & 327,8 & 15430 & 200,1 \\
\hline
\end{tabular}

Tabelle 60: Meßwerte Kinetik CF3CH2F + F rel. zu CHCl3; $298 \mathrm{~K}$

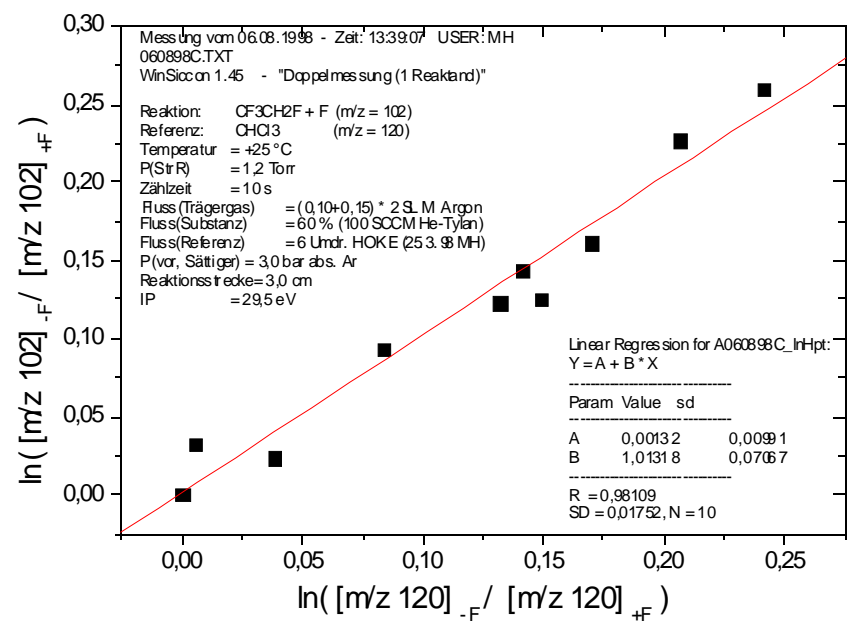

Abbildung 198: Kinetik CF3CH2F + F rel. zu CHCl3; $298 \mathrm{~K}$ 
Aus der Steigung der Auftragung in Abbildung 198 läßt sich der folgende relative Geschwindigkeitskoeffizient ermitteln:

$$
\mathrm{k}_{\text {rel }}=(1,01 \pm 0,07)
$$

Für die Referenzreaktion sind in der Literatur verschiedene Werte angegeben. Davon sollen die Verwendung finden, die unter ähnlichen Reaktionsbedingungen wie hier bestimmt wurden.

\begin{tabular}{|c|c|}
\hline $\mathbf{k}_{\text {referenz }}(\mathbf{2 9 8 K}) / \mathbf{c m}^{\mathbf{3}} /(\mathbf{m o l} \cdot \mathbf{s})$ & Literaturquelle \\
\hline $1,62 \cdot 10^{12}$ & Lit. $^{176}$ \\
\hline $3,73 \cdot 10^{12}$ & Lit. $^{177}$ \\
\hline $3,19 \cdot 10^{12}$ & Lit. $^{178}$ \\
\hline$(2,85 \pm 1,10) \cdot 10^{12}$ & Mittelwert $^{17}$ Standardabweichung \\
\hline
\end{tabular}

Mit dem Mittelwert der veröffentlichten Geschwindigkeitskoeffizienten der Referenzreaktion erhält man für die hier untersuchte Reaktion: $\mathrm{k}_{1}=\mathrm{k}_{\text {rel }} \cdot \mathrm{k}_{\text {referenz, Mittelwert }}$

$$
\begin{aligned}
& \mathbf{k}_{\mathbf{1}}(298 \mathrm{~K})=(2,9 \pm \mathbf{1 , 1}) \cdot \mathbf{1 0}^{12} \mathrm{~cm}^{3} /(\mathrm{mol} \cdot \mathbf{s}) \\
& \text { für } \mathrm{CF}_{3} \mathrm{CH}_{2} \mathrm{~F}+\mathrm{F} \rightarrow \text { Produkte }
\end{aligned}
$$

In den weiteren Messungen zu Bestimmung der Temperaturabhängigkeit des Geschwindigkeitskoeffizienten diente Dichlormethan als Referenzsubstanz. Als Badgas kam nun Helium statt Argon zum Einsatz.

Der Umsatz von $\mathrm{CF}_{3} \mathrm{CH}_{2} \mathrm{~F}$ konnte anhand der Intensitätsänderung seines Molekül-Peaks $\mathrm{m} / \mathrm{z}$ 102, der von Dichlormethan anhand seines Molekül-Peaks m/z 86 bestimmt werden.

Die Ergebnisse sind auf den nächsten Seiten dargestellt. 
$\vartheta=-15{ }^{\circ} \mathrm{C} ; \phi_{\mathrm{He}}=647 \mathrm{SCCM} ; \phi_{\mathrm{CF} 3 \mathrm{CH} 2 \mathrm{~F}}=8,4 \mathrm{SCCM} ; \phi_{\mathrm{CH} 2 \mathrm{Cl} 2}=11,8 \mathrm{SCCM} ; \mathrm{p}_{(\mathrm{StrR})}=160 \mathrm{~Pa} ;$ $\mathrm{s}=3,0 \mathrm{~cm} ; \mathrm{IP}=70 \mathrm{eV}$; Zählzeit $=10 \mathrm{~s}$

\begin{tabular}{|r|r|r|r|r|r|r|r|}
\hline $\begin{array}{c}\mathbf{I}(\mathbf{1 0 2}) \\
\text {-F / w.E. }\end{array}$ & $\begin{array}{c}\Delta \mathbf{I}(\mathbf{1 0 2}) \\
\mathbf{- F} / \mathbf{w . E} .\end{array}$ & $\begin{array}{c}\mathbf{I}(\mathbf{1 0 2}) \\
+\mathbf{F} / \mathbf{w . E} .\end{array}$ & $\begin{array}{c}\Delta \mathbf{I}(\mathbf{1 0 2}) \\
+\mathbf{+ F} / \mathbf{w . E} .\end{array}$ & $\begin{array}{c}\mathbf{I}(\mathbf{8 6}) \\
\text {-F / w.E. }\end{array}$ & $\begin{array}{c}\Delta \mathbf{I}(\mathbf{8 6}) \\
\mathbf{- F} / \mathbf{w . E} .\end{array}$ & $\begin{array}{c}\mathbf{I}(\mathbf{8 6}) \\
+\mathbf{F} / \mathbf{w . E} .\end{array}$ & $\begin{array}{c}\Delta \mathbf{I}(\mathbf{8 6}) \\
+\mathbf{F} / \mathbf{w . E} .\end{array}$ \\
\hline 27130 & 456,7 & 25730 & 352,6 & 134800 & 795 & 112500 & 918 \\
\hline 29750 & 275,4 & 29230 & 352,6 & 138800 & 2385 & 130500 & 918 \\
\hline 31080 & 352,6 & 29600 & 468,1 & 155000 & 0 & 137500 & 918 \\
\hline 31450 & 668,3 & 30280 & 200,1 & 154800 & 795 & 135500 & 2053 \\
\hline 32230 & 824,9 & 30130 & 397,5 & 164800 & 2001 & 138800 & 1522 \\
\hline 33200 & 343,5 & 30880 & 600,2 & 169800 & 2001 & 136800 & 795 \\
\hline 33200 & 410,5 & 31100 & 687 & 174500 & 918 & 138300 & 795 \\
\hline 35980 & 238,5 & 33000 & 410,5 & 174500 & 918 & 136500 & 1590 \\
\hline 36230 & 352,6 & 32730 & 525,3 & 185500 & 918 & 139500 & 2053 \\
\hline 36930 & 456,7 & 33850 & 378,5 & 188000 & 1298 & 142300 & 795 \\
\hline 37730 & 715,5 & 34150 & 304,5 & 194800 & 2001 & 142300 & 2385 \\
\hline
\end{tabular}

Tabelle 61: Meßwerte Kinetik CF3CH2F + F rel. zu CH2Cl2; $258 \mathrm{~K}$

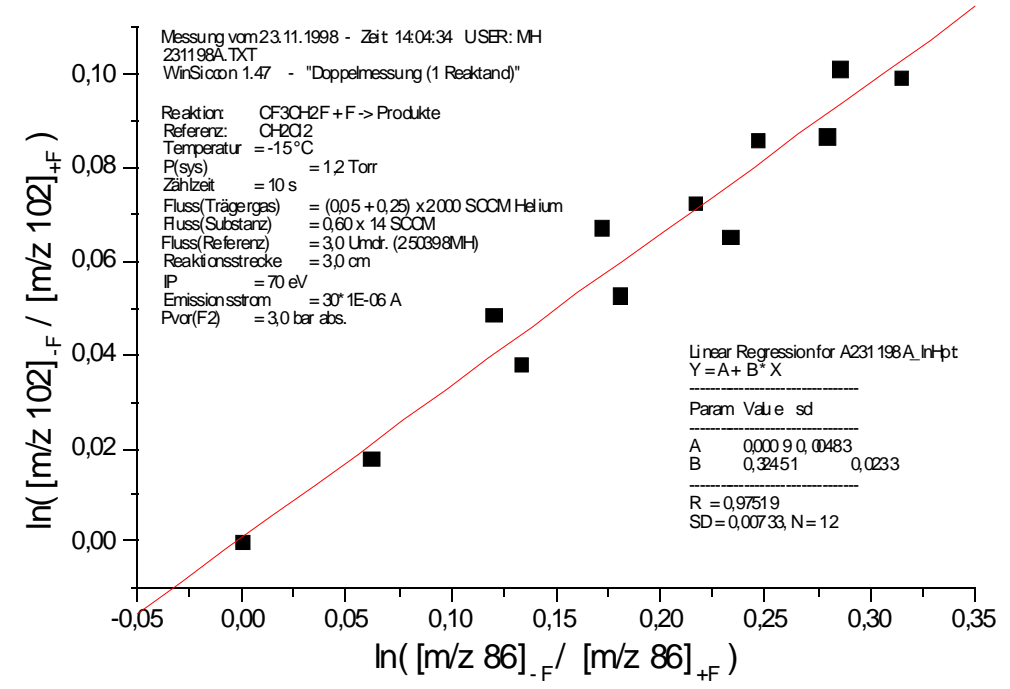

Abbildung 199: Kinetik CF3CH2F + F rel. zu CH2Cl2; $258 \mathrm{~K}$

Aus der Steigung der Auftragung in Abbildung 199 läßt sich der folgende relative Geschwindigkeitskoeffizient ermitteln: $\mathrm{k}_{\mathrm{rel}}=(0,32 \pm 0,02)$. 
$\vartheta=5{ }^{\circ} \mathrm{C} ; \phi_{\mathrm{He}}=647 \mathrm{SCCM} ; \phi_{\mathrm{CF} 3 \mathrm{CH} 2 \mathrm{~F}}=5,6 \mathrm{SCCM} ; \phi_{\mathrm{CH} 2 \mathrm{C} 22}=6,4 \mathrm{SCCM} ; \mathrm{p}_{(\mathrm{StrR})}=160 \mathrm{~Pa}$ $\mathrm{s}=3,0 \mathrm{~cm} ; \mathrm{IP}=70 \mathrm{eV} ;$ Zählzeit $=10 \mathrm{~s}$

\begin{tabular}{|c|c|c|c|c|c|c|c|}
\hline $\begin{array}{c}\text { I(102) } \\
- \text { F / w.E. }\end{array}$ & $\begin{array}{c}\Delta \mathbf{I}(102) \\
-\mathbf{F} / \mathbf{w . E} .\end{array}$ & $\begin{array}{c}\text { I(102) } \\
+\mathrm{F} / \text { w.E. }\end{array}$ & $\begin{array}{c}\Delta \mathbf{I}(\mathbf{1 0 2}) \\
+\mathbf{F} / \mathbf{w . E} .\end{array}$ & $\begin{array}{c}\mathbf{I}(\mathbf{8 6}) \\
-\mathbf{F} / \mathbf{w . E .}\end{array}$ & $\begin{array}{c}\Delta \mathbf{I}(\mathbf{8 6}) \\
-\mathbf{F} / \mathbf{w . E .}\end{array}$ & $\begin{array}{c}\mathbf{I}(\mathbf{8 6}) \\
+\mathrm{F} / \mathrm{w.E.}\end{array}$ & $\begin{array}{c}\Delta \mathbf{I}(\mathbf{8 6}) \\
+\mathbf{F} / \mathbf{w . E .}\end{array}$ \\
\hline 27630 & 352,6 & 26450 & 378,5 & 138800 & 795 & 123000 & 1298 \\
\hline 27200 & 565,9 & 25630 & 586 & 137800 & 795 & 115500 & 918 \\
\hline 28350 & 275,4 & 26930 & 375,7 & 137000 & 1298 & 113500 & 918 \\
\hline 27850 & 275,4 & 26080 & 152,2 & 136800 & 795 & 107500 & 918 \\
\hline 27500 & 535,3 & 25400 & 224,9 & 139500 & 1590 & 106500 & 918 \\
\hline 27680 & 152,2 & 25400 & 224,9 & 138500 & 1590 & 100000 & 0 \\
\hline 28330 & 152,2 & 25550 & 558,4 & 138300 & 795 & 100300 & 795 \\
\hline 28180 & 654 & 25230 & 352,6 & 138000 & 0 & 98500 & 745,8 \\
\hline 27980 & 375,7 & 25080 & 761,2 & 139800 & 2001 & 95730 & 824,9 \\
\hline 28150 & 615,8 & 27400 & 343,5 & 142800 & 1522 & 126300 & 2001 \\
\hline 29100 & 367,2 & 28600 & 259,6 & 144300 & 795 & 132500 & 1590 \\
\hline
\end{tabular}

Tabelle 62: Meßwerte Kinetik $\mathrm{CF} 3 \mathrm{CH} 2 \mathrm{~F}+\mathrm{F}$ rel. zu CH2Cl2; $278 \mathrm{~K}$

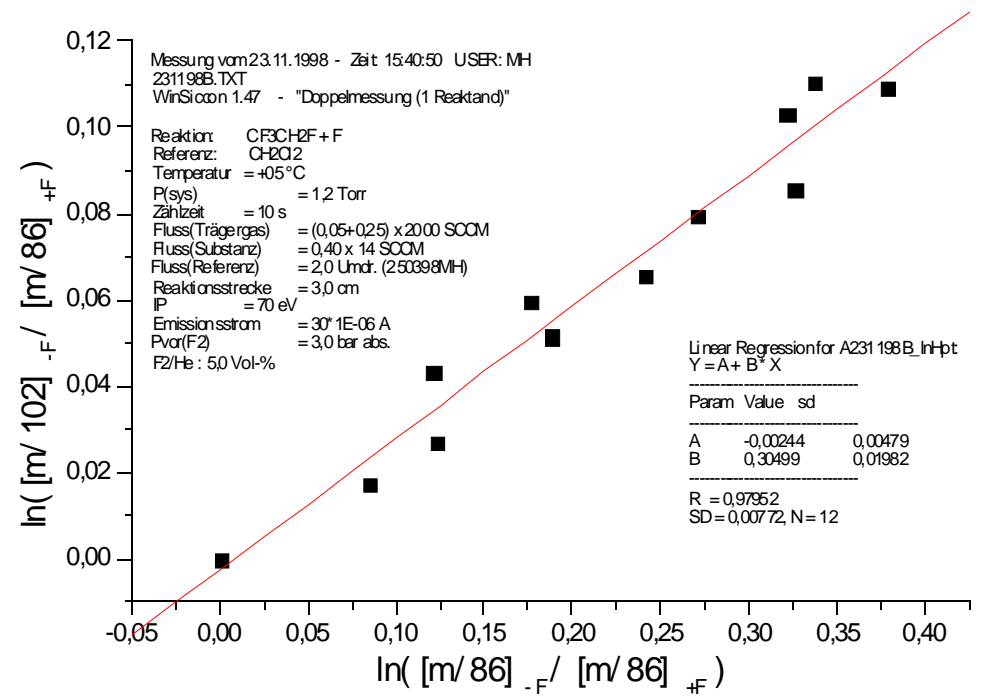

Abbildung 200: Kinetik CF3CH2F + F rel. zu CH2Cl2; $278 \mathrm{~K}$

Aus der Steigung der Auftragung in Abbildung 200 läßt sich der folgende relative Geschwindigkeitskoeffizient ermitteln: $\mathrm{k}_{\mathrm{rel}}=(0,30 \pm 0,02)$. 
$\vartheta=26^{\circ} \mathrm{C} ; \phi_{\mathrm{He}}=647 \mathrm{SCCM} ; \phi_{\mathrm{CF} 3 \mathrm{CH} 2 \mathrm{~F}}=8,4 \mathrm{SCCM} ; \phi_{\mathrm{CH} 2 \mathrm{Cl} 2}=11,8 \mathrm{SCCM} ; \mathrm{p}_{(\mathrm{StrR})}=173 \mathrm{~Pa} ;$ $\mathrm{s}=3,0 \mathrm{~cm} ; \mathrm{IP}=70 \mathrm{eV}$; Zählzeit $=10 \mathrm{~s}$

\begin{tabular}{|c|c|c|c|c|c|c|c|}
\hline $\begin{array}{c}\text { I(102) } \\
\text {-F / w.E. }\end{array}$ & $\begin{array}{c}\Delta \mathbf{I}(\mathbf{1 0 2}) \\
\text {-F / w.E. }\end{array}$ & $\begin{aligned} & I(102) \\
+ & F / w . E .\end{aligned}$ & $\begin{array}{c}\Delta \mathrm{I}(102) \\
+\mathrm{F} / \mathrm{w.E.}\end{array}$ & $\begin{array}{c}\mathbf{I}(\mathbf{8 6}) \\
-\mathrm{F} / \mathrm{w.E} .\end{array}$ & $\begin{array}{c}\Delta \mathbf{I}(\mathbf{8 6}) \\
\text {-F / w.E. }\end{array}$ & $\begin{array}{c}\mathrm{I}(86) \\
+\mathrm{F} / \mathrm{w.E} .\end{array}$ & $\begin{array}{c}\Delta \mathrm{I}(\mathbf{8 6}) \\
+\mathrm{F} / \mathrm{w} . \mathrm{E} .\end{array}$ \\
\hline 15860 & 166,8 & 14800 & 152,3 & 83980 & 1440 & 70040 & 417,9 \\
\hline 16500 & 124,3 & 14920 & 55,6 & 86220 & 1030 & 64840 & 324,2 \\
\hline 16800 & 129,8 & 14780 & 352,6 & 91350 & 477 & 65930 & 200,1 \\
\hline 17880 & 79,5 & 16080 & 238,5 & 93150 & 904,1 & 72950 & 1517 \\
\hline 17430 & 352,6 & 15480 & 238,5 & 96330 & 474,8 & 69030 & 437,9 \\
\hline 17550 & 527,3 & 13500 & 183,6 & 94980 & 556,5 & 45500 & 290,3 \\
\hline 18000 & 343,5 & 14700 & 183,6 & 100300 & 795 & 56530 & 474,8 \\
\hline 18150 & 205,3 & 15230 & 352,6 & 99300 & 810,7 & 59480 & 327,8 \\
\hline 18180 & 271,5 & 15050 & 205,3 & 98000 & 1265 & 59230 & 912,2 \\
\hline 17700 & 343,5 & 15280 & 200,1 & 99930 & 1370 & 62500 & 622,6 \\
\hline 18430 & 200,1 & 16300 & 259,6 & 101800 & 2001 & 72150 & 420,7 \\
\hline
\end{tabular}

Tabelle 63: Meßwerte Kinetik CF3CH2F + F rel. zu CH2Cl2; $299 \mathrm{~K}$

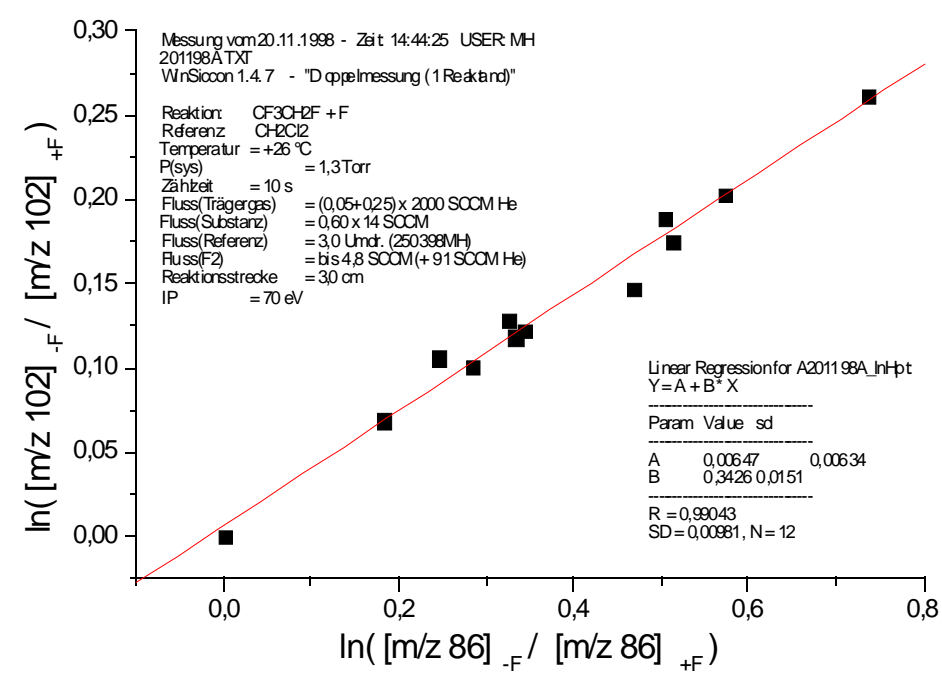

Abbildung 201: Kinetik CF3CH2F + F rel. zu CH2Cl2; 299 K

Aus der Steigung der Auftragung in Abbildung 201 läßt sich der folgende relative Geschwindigkeitskoeffizient ermitteln: $\mathrm{k}_{\mathrm{rel}}=(0,34 \pm 0,02)$. 
$\vartheta=45^{\circ} \mathrm{C} ; \phi_{\mathrm{He}}=647 \mathrm{SCCM} ; \phi_{\mathrm{CF} 3 \mathrm{CH} 2 \mathrm{~F}}=8,4 \mathrm{SCCM} ; \phi_{\mathrm{CH} 2 \mathrm{Cl} 2}=11,8 \mathrm{SCCM} ; \mathrm{p}_{(\mathrm{StrR})}=173 \mathrm{~Pa} ;$ $\mathrm{s}=3,0 \mathrm{~cm} ; \mathrm{IP}=70 \mathrm{eV}$; Zählzeit $=10 \mathrm{~s}$

\begin{tabular}{|c|c|c|c|c|c|c|c|}
\hline $\begin{array}{c}\text { I(102) } \\
-\mathrm{F} / \mathrm{w.E} .\end{array}$ & $\begin{array}{c}\Delta \mathrm{I}(102) \\
-\mathrm{F} / \mathrm{w.E} .\end{array}$ & $\begin{array}{c}\mathbf{I}(102) \\
+\mathrm{F} / \mathrm{w.E}\end{array}$ & $\begin{array}{c}\Delta \mathbf{I}(\mathbf{1 0 2}) \\
+\mathbf{F} / \text { w.E. }\end{array}$ & $\begin{array}{c}\mathbf{I}(\mathbf{8 6}) \\
-\mathrm{F} / \mathrm{w.E} .\end{array}$ & $\begin{array}{c}\Delta \mathbf{I}(\mathbf{8 6}) \\
-\mathbf{F} / \mathbf{w . E} .\end{array}$ & $\begin{array}{c}\mathbf{I}(\mathbf{8 6}) \\
+\mathrm{F} / \mathrm{w.E} .\end{array}$ & $\begin{array}{c}\Delta \mathbf{I}(\mathbf{8 6}) \\
+\mathbf{F} / \text { w.E. }\end{array}$ \\
\hline 20630 & 327,8 & 19580 & 301 & 122500 & 918 & 102500 & 2053 \\
\hline 21280 & 200,1 & 19800 & 389,5 & 123500 & 918 & 99280 & 600,2 \\
\hline 21130 & 200,1 & 18550 & 378,5 & 123000 & 1298 & 85950 & 558,4 \\
\hline 21280 & 352,6 & 18530 & 152,2 & 121500 & 918 & 85280 & 541,1 \\
\hline 21150 & 205,3 & 18630 & 397,5 & 122500 & 1590 & 86350 & 705,1 \\
\hline 20850 & 275,4 & 18330 & 152,2 & 123500 & 918 & 87350 & 304,5 \\
\hline 20400 & 290,3 & 18750 & 205,3 & 116500 & 1590 & 88580 & 1149 \\
\hline 20580 & 152,2 & 18880 & 301 & 117000 & 0 & 90550 & 2385 \\
\hline 20930 & 238,5 & 19250 & 159 & 125000 & 1298 & 97530 & 703,6 \\
\hline 21530 & 456,7 & 20850 & 205,3 & 125500 & 1590 & 114500 & 918 \\
\hline 21630 & 238,5 & 21300 & 449,7 & 127000 & 1298 & 123500 & 918 \\
\hline 21680 & 79,5 & 20950 & 205,3 & 126000 & 1298 & 108800 & 2385 \\
\hline 21600 & 224,9 & 20800 & 224,9 & 128300 & 795 & 110800 & 795 \\
\hline 21080 & 200,1 & 18730 & 327,8 & 126300 & 1522 & 90900 & 550,8 \\
\hline 21100 & 565,9 & 18750 & 331 & 125800 & 1522 & 91580 & 541,1 \\
\hline 20680 & 152,2 & 18750 & 159 & 125500 & 918 & 92300 & 980,1 \\
\hline 20480 & 301 & 17380 & 525,3 & 120000 & 2249 & 79250 & 717 \\
\hline
\end{tabular}

Tabelle 64: Meßwerte Kinetik CF3CH2F + F rel. zu CH2Cl2; $318 \mathrm{~K}$

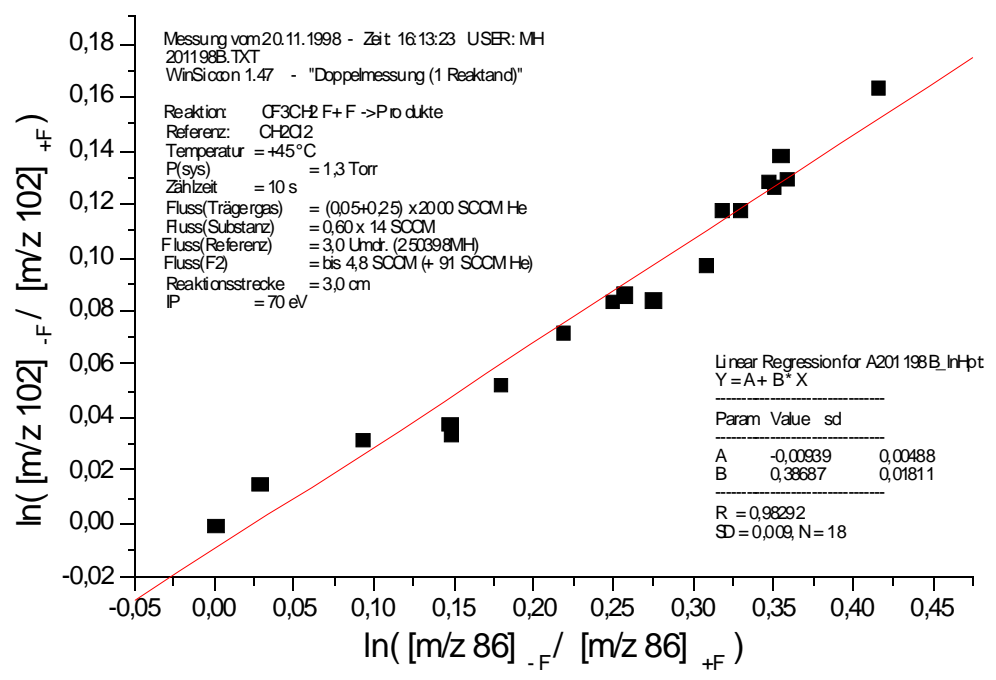

Abbildung 202: Kinetik CF3CH2F + F rel. zu CH2Cl2; $318 \mathrm{~K}$

Aus Abb. 202 erhält man: $k_{\text {rel }}=(0,39 \pm 0,02)$. 
$\vartheta=65^{\circ} \mathrm{C} ; \phi_{\mathrm{He}}=647 \mathrm{SCCM} ; \phi_{\mathrm{CF} 3 \mathrm{CH} 2 \mathrm{~F}}=5,6 \mathrm{SCCM} ; \phi_{\mathrm{CH} 2 \mathrm{Cl} 2}=6,4 \mathrm{SCCM} ; \mathrm{p}_{(\mathrm{StrR})}=173 \mathrm{~Pa} ;$ $\mathrm{s}=3,0 \mathrm{~cm} ; \mathrm{IP}=70 \mathrm{eV}$; Zählzeit $=10 \mathrm{~s}$

\begin{tabular}{|r|r|r|r|r|r|r|r|}
\hline $\begin{array}{c}\mathbf{I}(\mathbf{1 0 2}) \\
\text {-F / w.E. }\end{array}$ & $\begin{array}{c}\Delta \mathbf{I}(\mathbf{1 0 2}) \\
\mathbf{- F} / \mathbf{w . E} .\end{array}$ & $\begin{array}{c}\mathbf{I}(\mathbf{1 0 2}) \\
\text { +F / w.E. }\end{array}$ & $\begin{array}{c}\Delta \mathbf{I}(\mathbf{1 0 2}) \\
+\mathbf{F} / \mathbf{w . E} .\end{array}$ & $\begin{array}{c}\mathbf{I}(\mathbf{8 6}) \\
\text {-F / w.E. }\end{array}$ & $\begin{array}{c}\Delta \mathbf{I}(\mathbf{8 6}) \\
\text {-F / w.E. }\end{array}$ & $\begin{array}{c}\mathbf{I}(\mathbf{8 6}) \\
+\mathbf{F} / \mathbf{w . E} .\end{array}$ & $\begin{array}{c}\Delta \mathbf{I}(\mathbf{8 6}) \\
+\mathbf{F} / \mathbf{w . E} .\end{array}$ \\
\hline 14080 & 152,2 & 13800 & 129,8 & 80230 & 271,5 & 76130 & 418,2 \\
\hline 16430 & 397,5 & 16080 & 352,6 & 82000 & 908,8 & 75150 & 543,1 \\
\hline 16430 & 200,1 & 15850 & 304,5 & 82380 & 715,5 & 73980 & 824,9 \\
\hline 16080 & 238,5 & 15330 & 327,8 & 84130 & 1274 & 71530 & 271,5 \\
\hline 16350 & 331 & 15650 & 205,3 & 85250 & 494,4 & 71130 & 352,6 \\
\hline 17550 & 275,4 & 16380 & 271,5 & 85780 & 912,2 & 67250 & 275,4 \\
\hline 17930 & 271,5 & 16400 & 290,3 & 88530 & 761,2 & 68730 & 352,6 \\
\hline 17980 & 327,8 & 16380 & 200,1 & 91680 & 456,7 & 68200 & 389,5 \\
\hline 17980 & 152,2 & 16550 & 91,8 & 90350 & 668,3 & 67180 & 525,3 \\
\hline 18280 & 271,5 & 16650 & 304,5 & 91830 & 327,8 & 66900 & 389,5 \\
\hline
\end{tabular}

Tabelle 65: Meßwerte Kinetik CF3CH2F + F rel. zu CH2Cl2; $338 \mathrm{~K}$

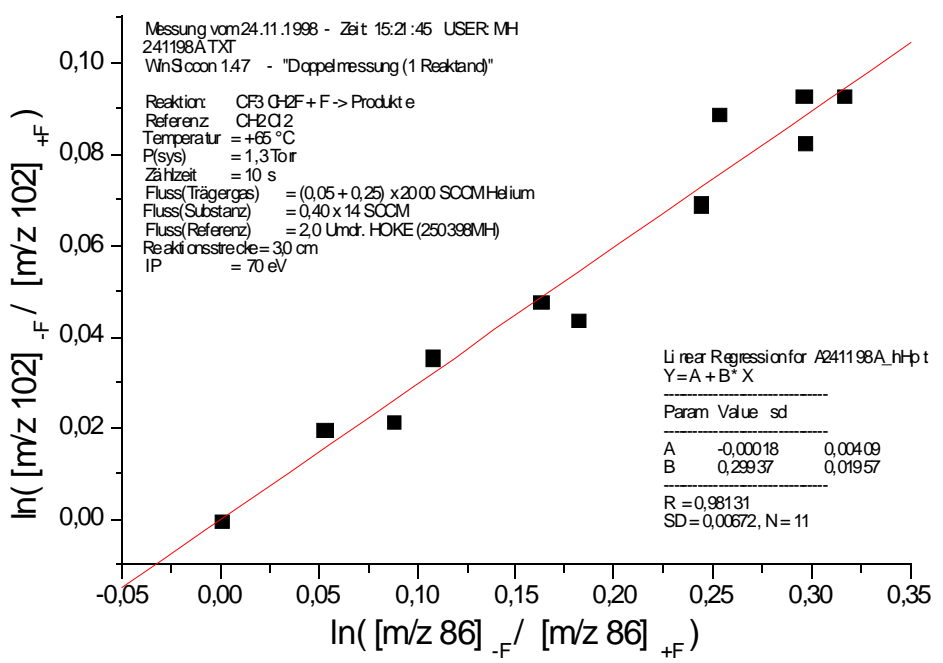

Abbildung 203: Kinetik CF3CH2F + F rel. zu CH2Cl2; $338 \mathrm{~K}$

Aus der Steigung der Auftragung in Abbildung 203 läßt sich der folgende relative Geschwindigkeitskoeffizient ermitteln: $\mathrm{k}_{\mathrm{rel}}=(0,30 \pm 0,02)$. 
$\vartheta=85^{\circ} \mathrm{C} ; \phi_{\mathrm{He}}=647 \mathrm{SCCM} ; \phi_{\mathrm{CF} 3 \mathrm{CH} 2 \mathrm{~F}}=5,6 \mathrm{SCCM} ; \phi_{\mathrm{CH} 2 \mathrm{Cl} 2}=6,4 \mathrm{SCCM} ; \mathrm{p}_{(\mathrm{StrR})}=173 \mathrm{~Pa} ;$ $\mathrm{s}=3,0 \mathrm{~cm} ; \mathrm{IP}=70 \mathrm{eV}$; Zählzeit $=10 \mathrm{~s}$

\begin{tabular}{|c|c|c|c|c|c|c|c|}
\hline $\begin{array}{c}\mathbf{I}(\mathbf{1 0 2}) \\
-\mathrm{F} / \mathrm{w.E}\end{array}$ & $\begin{array}{c}\Delta \mathrm{I}(\mathbf{1 0 2}) \\
-\mathrm{F} / \mathrm{w.E} .\end{array}$ & $\begin{array}{c}\mathrm{I}(\mathbf{1 0 2}) \\
+\mathrm{F} / \mathrm{w.E} .\end{array}$ & $\begin{array}{c}\Delta \mathrm{I}(102) \\
+\mathrm{F} / \mathrm{w} . \mathrm{E} .\end{array}$ & $\begin{array}{c}\mathbf{I}(\mathbf{8 6}) \\
-\mathbf{F} / \mathbf{w . E .}\end{array}$ & $\begin{array}{c}\Delta \mathrm{I}(\mathbf{8 6}) \\
-\mathrm{F} / \mathrm{w.E} .\end{array}$ & $\begin{array}{c}\mathbf{I}(\mathbf{8 6}) \\
+\mathbf{F} / \mathbf{w} . \mathbf{E} .\end{array}$ & $\begin{array}{c}\Delta \mathrm{I}(\mathbf{I 6 6}) \\
+\mathrm{F} / \mathrm{w.E} .\end{array}$ \\
\hline 17480 & 152,2 & 17230 & 271,5 & 98230 & 666,7 & 92100 & 594,9 \\
\hline 17850 & 91,8 & 17250 & 159 & 98050 & 587,8 & 90450 & 693,1 \\
\hline 17780 & 79,5 & 17130 & 200,1 & 97850 & 91,8 & 86780 & 627,7 \\
\hline 17850 & 159 & 17200 & 259,6 & 97630 & 456,7 & 84150 & 275,4 \\
\hline 17950 & 91,8 & 16930 & 301 & 99350 & 494,4 & 82500 & 789,7 \\
\hline 17980 & 271,5 & 16650 & 331 & 99080 & 271,5 & 79730 & 456,7 \\
\hline 17900 & 410,5 & 16580 & 152,2 & 100100 & 1000 & 78650 & 477 \\
\hline 18000 & 129,8 & 16580 & 352,6 & 99900 & 318 & 75500 & 745,8 \\
\hline 17900 & 259,6 & 16500 & 129,8 & 98980 & 1274 & 73900 & 768 \\
\hline 18130 & 152,2 & 16580 & 200,1 & 98000 & 1318 & 72300 & 290,3 \\
\hline 18130 & 200,1 & 16300 & 224,9 & 100500 & 918 & 72130 & 474,8 \\
\hline
\end{tabular}

Tabelle 66: Meßwerte Kinetik CF3CH2F + F rel. zu CH2Cl2; $358 \mathrm{~K}$

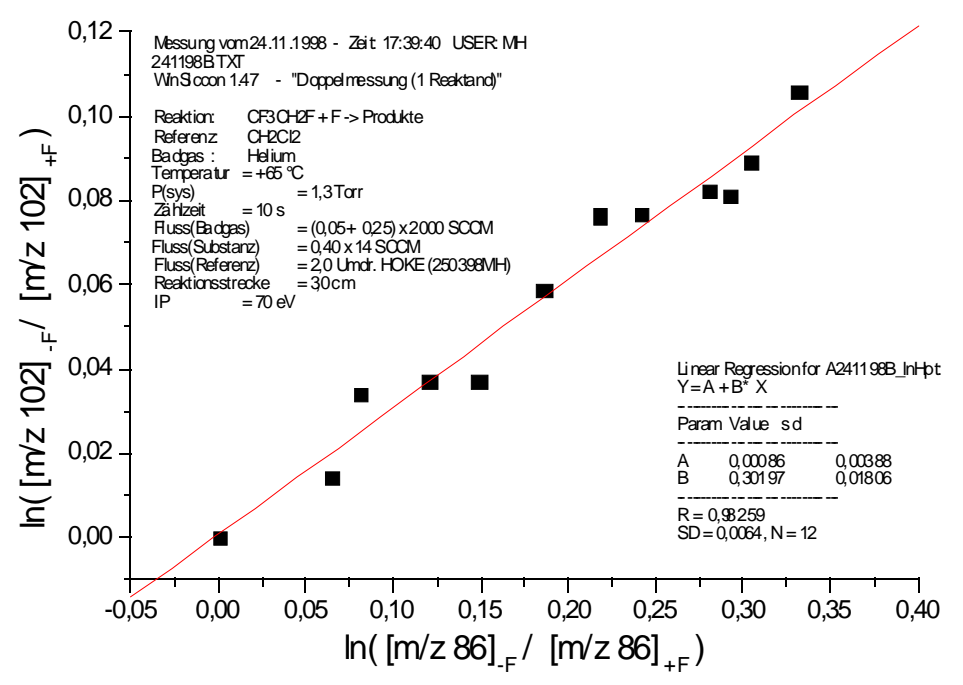

Abbildung 204: Kinetik CF3CH2F + F rel. zu CH2Cl2; $358 \mathrm{~K}$

Aus der Steigung der Auftragung in Abbildung 204 läßt sich der folgende relative Geschwindigkeitskoeffizient ermitteln: $\mathrm{k}_{\mathrm{rel}}=(0,30 \pm 0,02)$. 
Aus den ermittelten relativen Geschwindigkeitskoeffizienten der Abbildungen 199 bis 204 können nun mit der bekannten Temperaturabhängigkeit des Geschwindigkeitskoeffizienten für die Referenzreaktion ${ }^{179} \mathrm{k}_{\text {ref }}(\mathrm{T})$ die absoluten Geschwindigkeitskoeffizienten $\mathrm{k}_{2}$ für die Reaktion des Methylperfluorbutylethers mit F-Atomen berechnet werden (Tabelle 67).

$\mathrm{k}_{\mathrm{ref}}(\mathrm{T})=(8,4 \pm 1,0) \cdot 10^{12} \cdot \exp \{(0,12 \pm 0,29) \mathrm{kJ} / \mathrm{mol} /(\mathrm{R} \cdot \mathrm{T})\} \mathrm{cm}^{3} /(\mathrm{mol} \cdot \mathrm{s})$

Ferner kann mit diesen Werten eine Arrhenius-Auftragung gemäß Gleichung (11) (S. 10) vorgenommen werden.

\begin{tabular}{|r|r|r|r|r|r|}
\hline \multicolumn{1}{|c|}{$\vartheta /{ }^{\circ} \mathbf{C}$} & \multicolumn{1}{|c|}{$\mathbf{T}^{-1} / \mathbf{K}^{-1}$} & $\ln (\mathbf{T} / \mathbf{2 9 8} \mathbf{K})$ & \multicolumn{1}{c|}{$\mathbf{k}_{\text {rel }}$} & \multicolumn{1}{|c|}{$\mathbf{l n}\left(\mathbf{k}_{\text {rel }}\right)$} & $\begin{array}{c}\mathbf{k}_{2} / \\
\mathbf{c m}^{\mathbf{3}} /(\mathbf{m o l} \mathbf{~ s})\end{array}$ \\
\hline-15 & $3,870 \mathrm{E}-03$ & $-0,1436$ & 0,325 & $-1,1254$ & $2,88 \mathrm{E}+12$ \\
\hline 5 & $3,600 \mathrm{E}-03$ & $-0,0689$ & 0,305 & $-1,1875$ & $2,70 \mathrm{E}+12$ \\
\hline 15 & $3,470 \mathrm{E}-03$ & $-0,0336$ & 0,303 & $-1,1937$ & $2,68 \mathrm{E}+12$ \\
\hline 26 & $3,340 \mathrm{E}-03$ & 0,0039 & 0,343 & $-1,0712$ & $3,02 \mathrm{E}+12$ \\
\hline 45 & $3,140 \mathrm{E}-03$ & 0,0654 & 0,387 & $-0,9497$ & $3,40 \mathrm{E}+12$ \\
\hline 65 & $2,960 \mathrm{E}-03$ & 0,1264 & 0,299 & $-1,2061$ & $2,62 \mathrm{E}+12$ \\
\hline 85 & $2,790 \mathrm{E}-03$ & 0,1839 & 0,302 & $-1,1974$ & $2,64 \mathrm{E}+12$ \\
\hline
\end{tabular}

Tabelle 67: $k(T)$ sowie Werte f. Arrhenius-Auftr. f. CF3CH2F +F

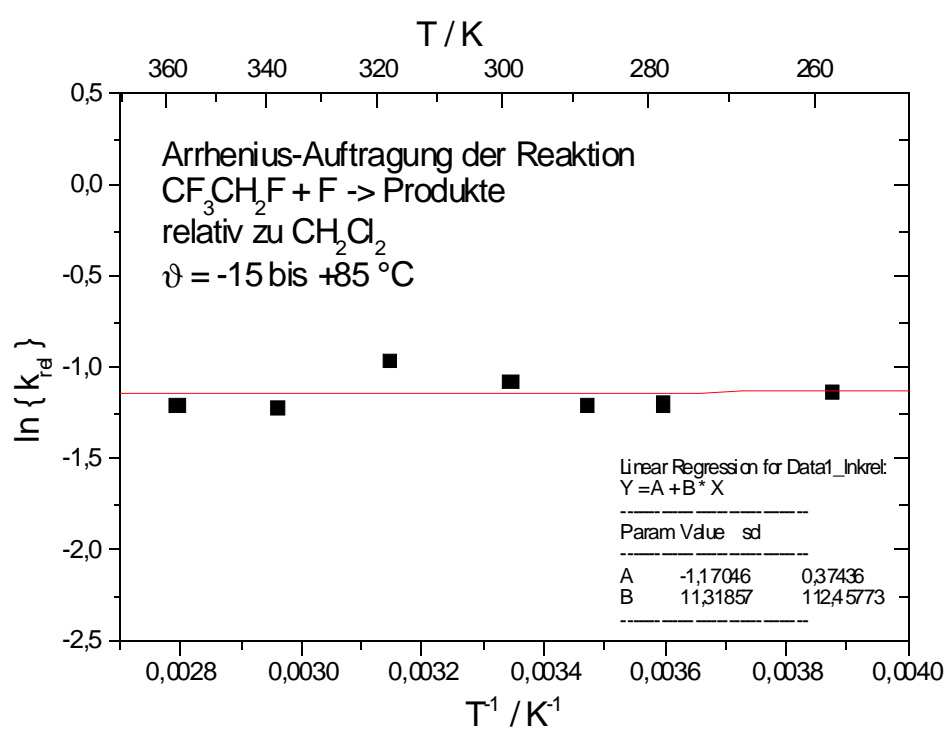

Abbildung 205: Arrhenius-Auftragung f. CF3CH2F +F

179 Hold, M. (1997); S. 115. Dieser Wert ist auch in Kap. 4.4.3 (S. 212) zu finden. 
Die Aktivierungsenergie der hier untersuchten Reaktion erhält man durch Vergleich der Steigung aus Abbildung 205 mit dem Ausdruck in Gleichung (27) (S. 116):

$E_{a}=E_{a, \text { Referenz }}-($ Steigung $) \cdot R=(-120-(11,319 \cdot 8,314)) \mathrm{J} / \mathrm{mol}=-214,1 \mathrm{~J} / \mathrm{mol}$.

Bezüglich des präexponentiellen Faktors wird der Achsenabschnitt aus Abbildung 95 herangezogen:

$\mathrm{A}=\exp ($ Achsenabschnitt $) \cdot \mathrm{A}_{\text {Referenz }}=\exp (-1,17046) \cdot 8,4 \cdot 10^{12} \mathrm{~cm}^{3} \mathrm{~mol}^{-1} \mathrm{~s}^{-1}$

$$
=2,6 \cdot 10^{12} \mathrm{~cm}^{3} \mathrm{~mol}^{-1} \mathrm{~s}^{-1} \text {. }
$$

Im Ergebnis beträgt der gesuchte Geschwindigkeitskoeffizient damit:

$$
\begin{aligned}
& \mathbf{k}_{2}(\mathbf{T})=(2,6 \pm 1,0) \cdot 10^{12} \cdot \exp \{-(-0,214 \pm 0,979) \mathrm{kJ} / \mathrm{mol} /(\mathbf{R} \cdot \mathbf{T})\} \mathrm{cm}^{3} /(\mathbf{m o l} \cdot \mathbf{s}) \\
& \text { für: } \mathrm{CF}_{3} \mathrm{CH}_{2} \mathrm{~F}+\mathrm{F} \rightarrow \text { Produkte }
\end{aligned}
$$

Mit $\mathrm{T}=298 \mathrm{~K}$ errechnet man daraus: $\mathbf{k}_{2}(298 \mathrm{~K})=(\mathbf{2 , 8} \pm \mathbf{1 , 6}) \cdot \mathbf{1 0}^{\mathbf{1 2}} \mathbf{c m}^{\mathbf{3}} \mathbf{m o l}^{-1} \mathbf{s}^{-1}$.

Mit einer Aktivierungsenergie von lediglich -214 J/mol ist der Geschwindigkeitskoeffizient für diese Reaktion praktisch temperaturunabhängig.

Auf Grund der geringen Aktivierungsenergie empfiehlt sich eher eine Auftragung gemäß Gleichung (13) (S. 11). Aus der logarithmierten Version dieser Gleichung läßt sich leicht der Parameter n ermitteln (Abb. 206).

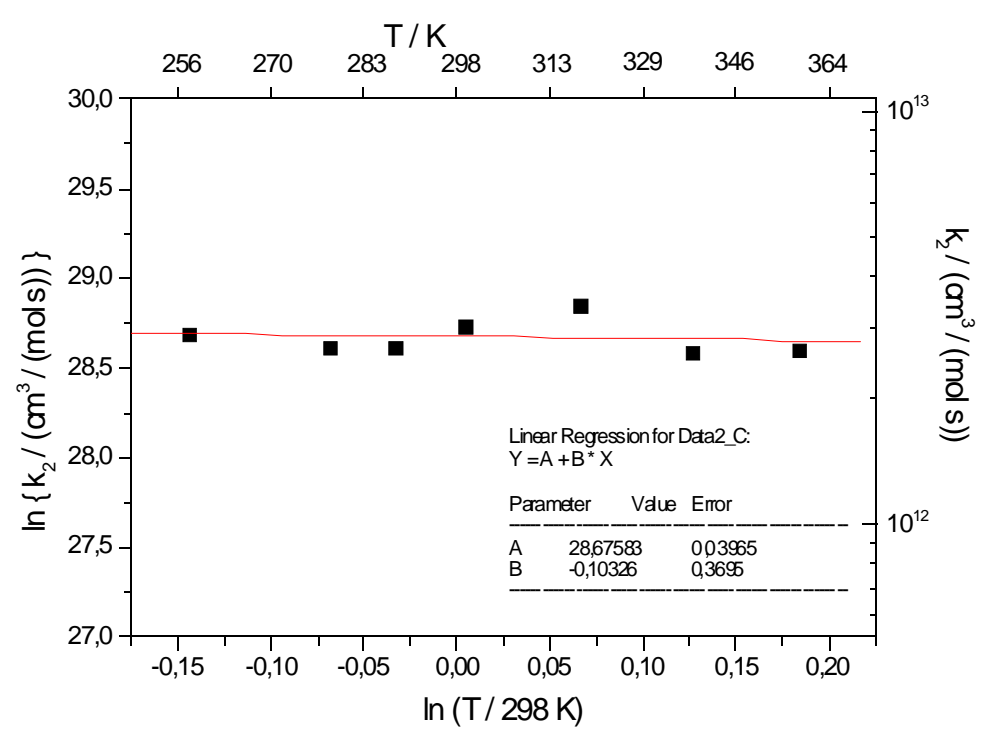

Abbildung 206: $\ln (k)$ vs. $\ln (T) f . C F 3 C H 2 F+F$

Der gesuchte Parameter $\mathrm{n}$ ist die Steigung b der Auftragung in Abbildung 206: $\mathrm{n}=(-0,10 \pm 0,37)$. 
Aus dem Achsenabschnitt a ergibt sich der Parameter A = exp(a):

$A=\exp (28,6758) \mathrm{cm}^{3} /(\mathrm{mol} \cdot \mathrm{s})=(9,39 \pm 0,03) \cdot 10^{12} \mathrm{~cm}^{3} /(\mathrm{mol} \cdot \mathrm{s})$.

Damit ergibt sich für den gesuchten Geschwindigkeitskoeffizienten:

$$
\mathrm{k}_{2}(\mathrm{~T})=(2,84 \pm 0,11) \cdot 10^{12} \cdot(\mathrm{T} / 298 \mathrm{~K})^{(-0,10 \pm 0,37)} \mathrm{cm}^{3} /(\mathrm{mol} \cdot \mathrm{s})
$$

für: $\mathrm{CF}_{3} \mathrm{CH}_{2} \mathrm{~F}+\mathrm{F} \rightarrow$ Produkte

Mit $\mathrm{T}=298 \mathrm{~K}$ errechnet man daraus: $\mathbf{k}_{2}(298 \mathrm{~K})=(\mathbf{2 , 8 4} \pm \mathbf{0 , 1 1}) \cdot \mathbf{1 0} \mathbf{c m}^{\mathbf{1 2}} \mathbf{m o l}^{-1} \mathrm{~s}^{-1}$.

\subsubsection{Kinetik der Kombinationsreaktion des erzeugten Radikals}

Die Messung wurde in der in Kap. 3.1.1 (S. 16) beschriebenen Apparatur unter Einsatz des Strömungsreaktors aus Abbildung 5 (S. 17) durchgeführt. Ihre Ausführung und Auswertung nach der Absolutmethode ist in Kap. 3.5.1 (S. 50) beschrieben. Das Maximum der Intensität des Radikal-Peaks m/z 101 lag bei s = 4,05 cm Reaktionsstrecke; diese wurde als Startpunkt für die Radikalkombination festgelegt.

$\vartheta=25^{\circ} \mathrm{C} ; \phi_{\mathrm{He}}=869 \mathrm{SCCM} ; \phi_{\mathrm{CF} 3 \mathrm{CH} 2 \mathrm{~F}}=22,4 \mathrm{SCCM} ; \phi_{\mathrm{F} 2}=1,8 \mathrm{SCCM} ; \mathrm{p}_{(\mathrm{StrR})}=140 \mathrm{~Pa} ;$ IP = 29,5 eV; Zählzeit $=10 \mathrm{~s}$

\begin{tabular}{|c|c|c|c|c|c|c|c|}
\hline $\begin{array}{l}\mathrm{s} / \\
\mathrm{cm}\end{array}$ & $\begin{array}{c}\left(\mathbf{t}-\mathbf{t}_{0}\right) / \\
\text { ms }\end{array}$ & $\begin{array}{c}\text { I(101) } \\
-\mathrm{F} / \mathrm{w.E} .\end{array}$ & $\begin{array}{l}\Delta \mathbf{I}(\mathbf{1 0 1}) \\
-\mathbf{F} / \mathbf{w . E .}\end{array}$ & $\begin{array}{c}\text { I(101) } \\
+\mathrm{F} / \mathrm{w.E.}\end{array}$ & $\begin{array}{c}\Delta \mathrm{I}(101) \\
+\mathrm{F} / \mathrm{w} . \mathrm{E} .\end{array}$ & $\begin{array}{l}\text { I(Differenz): } \\
,+F^{66} . / .,,-F^{66}\end{array}$ & $\begin{array}{c}{[\mathrm{I}(\text { Diff. })]_{\mathrm{t}=\mathbf{0}} /} \\
{[\mathrm{I}(\text { Diff. })]_{\mathrm{t}}}\end{array}$ \\
\hline 4,05 & 0,00 & 2980 & 35,11 & 5273 & 69,49 & 2293 & 1,00 \\
\hline 4,95 & 0,22 & 3057 & 98,12 & 5157 & 142,7 & 2100 & 1,09 \\
\hline 5,85 & 0,43 & 3068 & 56,47 & 5110 & 35,73 & 2042 & 1,12 \\
\hline 6,55 & 0,60 & 3063 & 96,08 & 5152 & 100,9 & 2089 & 1,10 \\
\hline 7,15 & 0,75 & 3153 & 72,89 & 5102 & 57,63 & 1949 & 1,18 \\
\hline 7,80 & 0,90 & 3217 & 72,59 & 5213 & 119,9 & 1996 & 1,15 \\
\hline
\end{tabular}

Tabelle 68: Meßwerte Kinetik CF3CHF + CF3CHF; $298 \mathrm{~K}$

Der Umsatz von $\mathrm{CF}_{3} \mathrm{CH}_{2} \mathrm{~F}$ konnte auf seinem Molekül-Peak m/z 102 gesmessen werden; er betrug $(5,6 \pm 2,5) \%$. Dieser Wert ist das arithmetische Mittel bzw. die Standardabweichung für viele Messungen bei den verschiedenen Reaktionsstrecken. Eine Korrelation mit letzterer konnte nicht festgestellt werden, daher wurde zur weiteren Auswertung der Mittelwert herangezogen. 
Aus den Reaktionsbedingungen, die oberhalb der Tabelle 68 angegeben sind, kann gemäß Gleichung (19) (S. 14) die Ausgangskonzentration von $\mathrm{CF}_{3} \mathrm{CH}_{2} \mathrm{~F}$ berechnet werden. Sie betrug $\left[\mathrm{CF}_{3} \mathrm{CH}_{2} \mathrm{~F}\right]_{0}=1,41 \cdot 10^{-09} \mathrm{~mol} / \mathrm{cm}^{3}$. Mit einem Umsatz von $5,6 \%$ ergibt sich daraus die Anfangskonzentration der $\mathrm{CF}_{3} \mathrm{CHF}-$ Radikale bei $\mathrm{t}=0:\left[\mathrm{CF}_{3} \mathrm{CHF}\right]_{0}=7,9 \cdot 10^{-11} \mathrm{~mol} / \mathrm{cm}^{3}$.

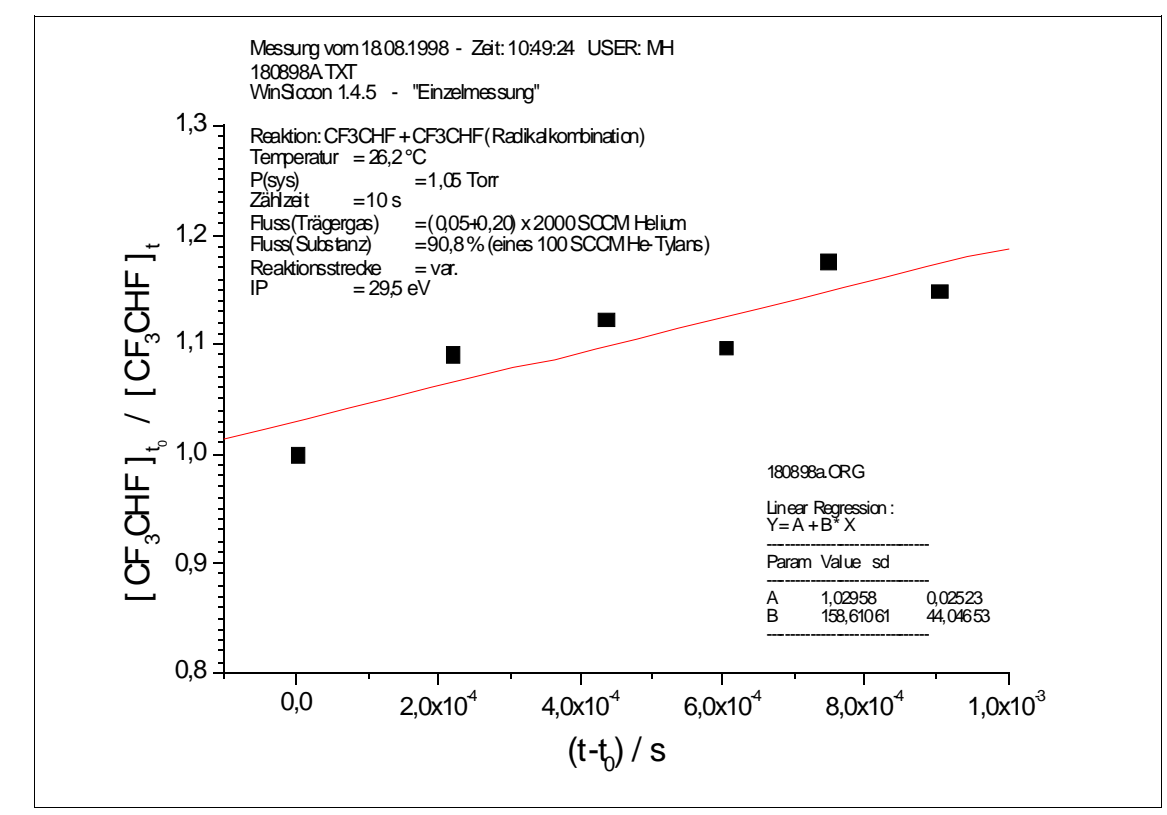

Abbildung 207: Kinetik CF3CHF + CF3CHF; $298 \mathrm{~K}$

Mit einer Steigung aus Abb. 207 von $b=(158,6 \pm 44,0) \mathrm{s}^{-1}$ kann man mittels Gleichung (8) (S. 9) nun den Geschwindigkeitskoeffizienten k für die Radikalkombination errechnen:

$$
\mathrm{k}=\frac{158,6 \frac{1}{\mathrm{~s}}}{2 \cdot 7,9 \cdot 10^{-11} \frac{\mathrm{mol}}{\mathrm{cm}^{3}}}=1,0 \cdot 10^{12} \frac{\mathrm{cm}^{3}}{\mathrm{~mol} \cdot \mathrm{s}}
$$

Unter Berücksichtigung der Standardabweichung des Umsatzes und derjenigen der Steigung in Abb. 207 erhält man nach den Gesetzen der Fehlerfortpflanzung einen Fehler von 5,3E11 $\mathrm{cm}^{3} /(\operatorname{mol~s})$.

Somit ergibt sich als Endergebnis:

$$
\mathrm{k}(298 \mathrm{~K})=(1,0 \pm 0,5) \cdot 10^{12} \mathrm{~cm}^{3} \mathrm{~mol}^{-1} \mathrm{~s}^{-1}
$$

für: $\mathrm{CF}_{3} \mathrm{CHF}+\mathrm{CF}_{3} \mathrm{CHF} \rightarrow$ Produkte 


\subsubsection{Produkte der Reaktion des erzeugten Radikals mit Sauerstoff-Atomen}

Um einen Überblick über die Änderungen im Massenspektrum zu erhalten, sind mit der in Kapitel 3.1.1 (S. 16) beschriebenen Apparatur unmittelbar nacheinander EI-Übersichtsspektren unter Zugabe und Abwesenheit von atomarem Sauerstoff (Fluor-Atome wurden stets gleichermaßen zugegeben) aufgenommen worden.

Es wurden anschließend gezielt in Einzelzählung (5 sek Zählzeit, IP = $22 \mathrm{eV}$ ) die m/z-Werte überprüft, welche im Übersichtsspektrum größere Veränderungen zeigten. Die Durchführung der Messungen ist in Kapitel 3.5.1 (S. 50) beschrieben. In Abbildung 208 sind die Reaktionsbedingungen und Ergebnisse dargestellt.
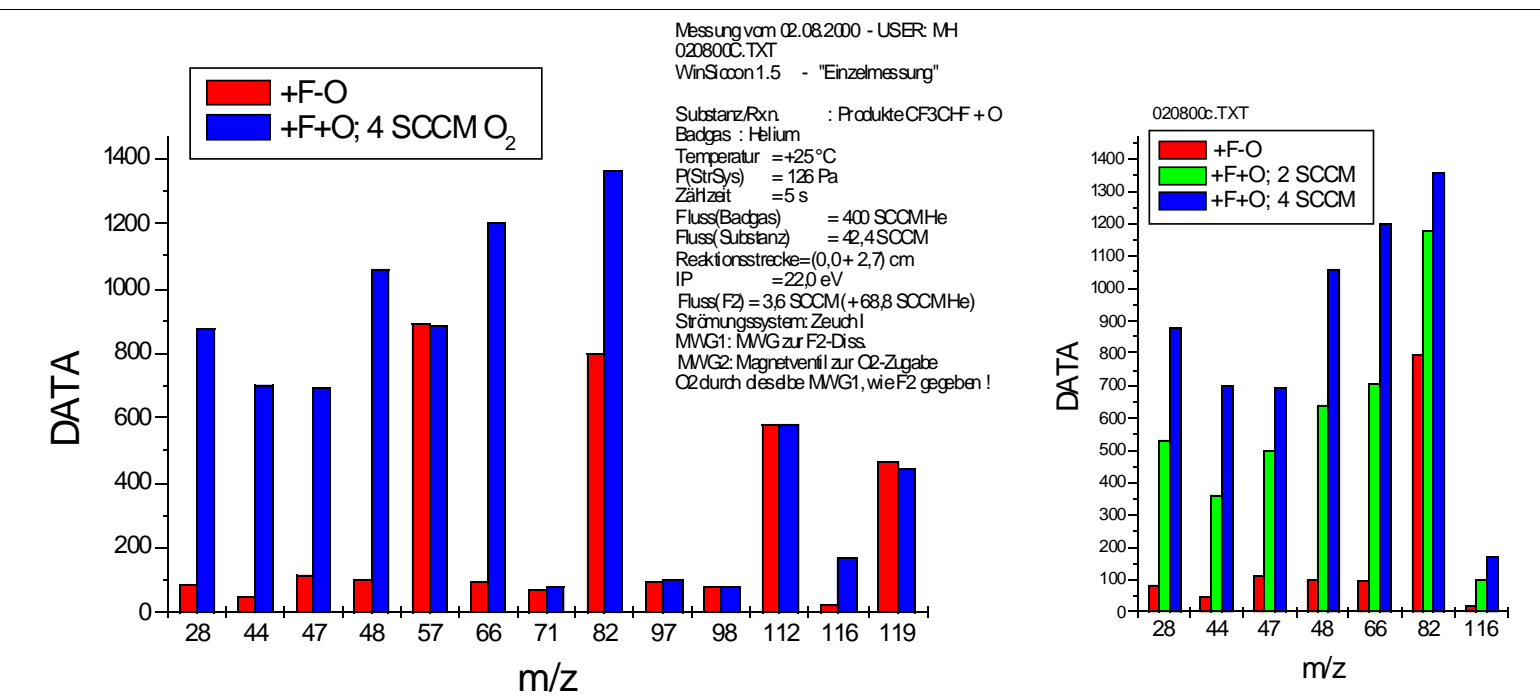

Abbildung 208: Einzelzählung mit EI-MS für CF3CH2F+F+/- $O$

Die beobachteten Zunahmen auf m/z 48; 66 und 116 lassen sich durch die drei in Abbildung 209 dargestellten Reaktionen erklären.

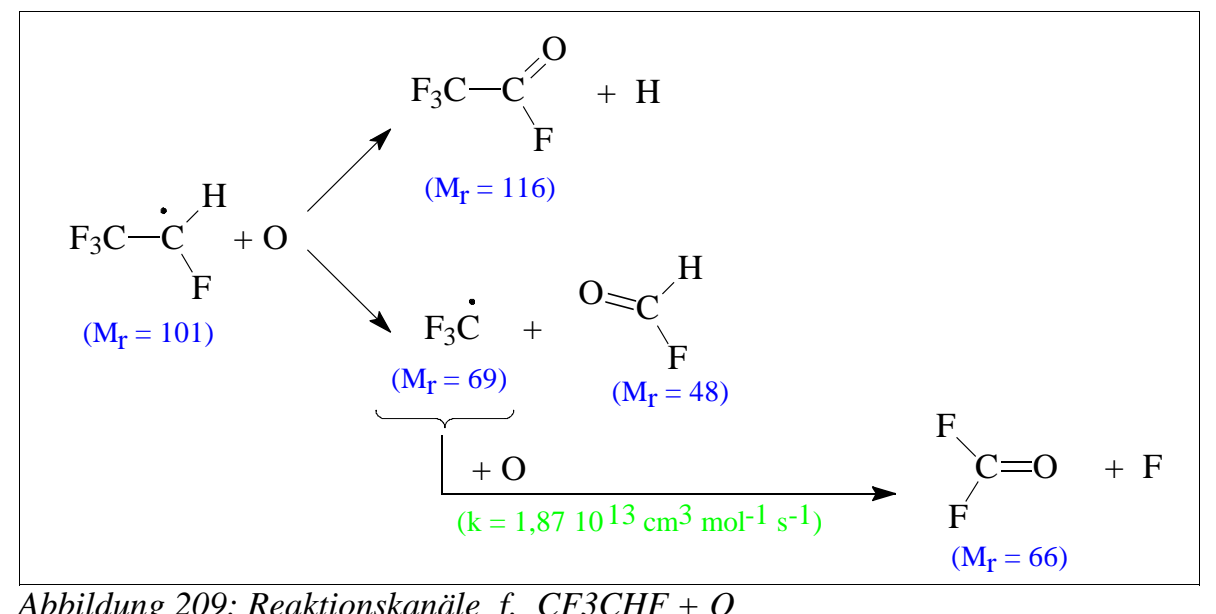

Die Zunahme auf m/z 28 spricht für die Bildung von $\mathrm{CO}$ und die auf $\mathrm{m} / \mathrm{z} 44$ für $\mathrm{CO}_{2}$. Bei m/z 
47 handelt es sich wahrscheinlich um COF, das jedoch auch als Fragment der vorgeschlagenen Produkte $\mathrm{F}_{2} \mathrm{CO}$, FCHO und $\mathrm{F} 3 \mathrm{CCFO}$ auftreten könnte. Andererseits wäre durch die schnelle Reaktion $\mathrm{COF}+\mathrm{O} \rightarrow \mathrm{CO}_{2}+\mathrm{F}$ die Zunahme auf $\mathrm{m} / \mathrm{z} 44$ erklärbar. $\mathrm{CO}$ könnte durch Zerfall des angeregten Alkoxy-Komplexes entstehen: $\left(\mathrm{CF}_{3} \mathrm{COHF}\right)^{*} \rightarrow \mathrm{CO}+\mathrm{HF}+\mathrm{CF}_{3}$. Auch die Folgereaktion ${ }^{180} \mathrm{CF}_{3}+\mathrm{O}$ ist mit $\mathrm{k}=1,87 \mathrm{E}+13 \mathrm{~cm}^{3} /(\mathrm{mol} \cdot \mathrm{s})$ sehr schnell.

\subsubsection{Kinetik der Reaktion des erzeugten Radikals mit Sauerstoff-Atomen}

Die Messungen wurden in der in Kap. 3.1.1 (S. 16) beschriebenen Apparatur unter Einsatz des Strömungsreaktors aus Abbildung 6 (S. 17) durchgeführt.

Das Methyl-Radikal diente als Referenzsubstanz. Der Umsatz des $\mathrm{CF}_{3} \mathrm{CHF}$-Radikals konnte anhand der Intensitätsänderung seines Molekül-Peaks m/z 101 und der des Methyl-Radikals anhand seines Molekül-Peaks m/z 15 bestimmt werden.

Die Messungen wurden umsatzkorrigiert nach der Relativmethode durchgeführt, wobei zur Messung des Umsatzes von $\mathrm{CF}_{3} \mathrm{CH}_{2} \mathrm{~F}$ bei Zugabe der Fluor-Atome die Intensitätsänderung auf m/z 102, und für den des Methans diejenige auf m/z 16 diente; die genaue Ausführung und Auswertung ist in Kap. 3.5.1 (S. 50) beschrieben.

$\vartheta=25{ }^{\circ} \mathrm{C} ; \phi_{\mathrm{He}}=469 \mathrm{SCCM} ; \phi_{\mathrm{CF} 3 \mathrm{CH} 2 \mathrm{~F}}=43,3 \mathrm{SCCM} ; \phi_{\mathrm{CH} 4}=2,0 \mathrm{SCCM} ; \mathrm{p}_{(\mathrm{StrR})}=111 \mathrm{~Pa} ;$ $\mathrm{S}_{\mathrm{CF} 3 \mathrm{CH} 2 \mathrm{~F}+\mathrm{F}}=1 \mathrm{~cm} ; \mathrm{S}_{\mathrm{CF} 3 \mathrm{CHF}+\mathrm{O}}=2,7 \mathrm{~cm} ; \mathrm{IP}=29,5 \mathrm{eV} ;$ Zählzeit $=5 \mathrm{~s}$

\begin{tabular}{|c|c|c|c|c|c|c|c|}
\hline $\begin{array}{c}\text { I(101) } \\
-\mathrm{O} / \text { w.E. }\end{array}$ & $\begin{array}{c}\Delta \mathbf{I}(101) \\
-\mathbf{O} / \mathbf{w . E} .\end{array}$ & $\begin{array}{c}\mathrm{I}(101) \\
+\mathrm{O} / \mathrm{w.E} .\end{array}$ & $\begin{array}{c}\Delta \mathrm{I}(101) \\
+\mathrm{O} / \mathrm{w} . \mathrm{E} .\end{array}$ & $\begin{array}{c}\mathbf{I}(15) \\
-\mathbf{O} / \mathbf{w . E .}\end{array}$ & $\begin{array}{c}\Delta \mathbf{I}(15) \\
-\mathbf{O} / \mathbf{w . E .}\end{array}$ & $\begin{array}{c}\mathbf{I}(15) \\
+\mathrm{O} / \mathrm{w} . \mathrm{E} .\end{array}$ & $\begin{array}{c}\Delta \mathrm{I}(15) \\
+\mathrm{O} / \mathrm{w.E.}\end{array}$ \\
\hline 2501 & 147,8 & 2047 & 155,5 & 1865 & 110 & 1188 & 135,2 \\
\hline 2414 & 102,7 & 2124 & 83,56 & 1950 & 155,3 & 1259 & 131,2 \\
\hline 2513 & 122 & 2307 & 76,22 & 1694 & 180,9 & 862,3 & 163 \\
\hline 2401 & 151,5 & 2198 & 174,6 & 1851 & 137 & 1111 & 140,7 \\
\hline 2610 & 183,1 & 1903 & 165,5 & 2037 & 126,5 & 707,3 & 153,3 \\
\hline 2558 & 124,9 & 1944 & 106,4 & 1993 & 161,8 & 1023 & 168,1 \\
\hline 2503 & 94,97 & 2128 & 146,4 & 1857 & 141,9 & 1127 & 156,8 \\
\hline 2104 & 74,01 & 1691 & 80,64 & 1871 & 160,8 & 1253 & 146,3 \\
\hline 1992 & 107,5 & 1815 & 100,1 & 1761 & 180,8 & 938,3 & 204 \\
\hline 2538 & 119,5 & 2385 & 172,7 & 561,2 & 192,7 & 364 & 195,7 \\
\hline 2552 & 177,1 & 1852 & 111,4 & 2001 & 139,3 & 725,2 & 147,1 \\
\hline
\end{tabular}

Tabelle 69: Meßwerte Kinetik CF3CHF + O rel. zu CH3; $298 \mathrm{~K}$

180 Ryan, K.R.; Plumb, I.C. (1982). 


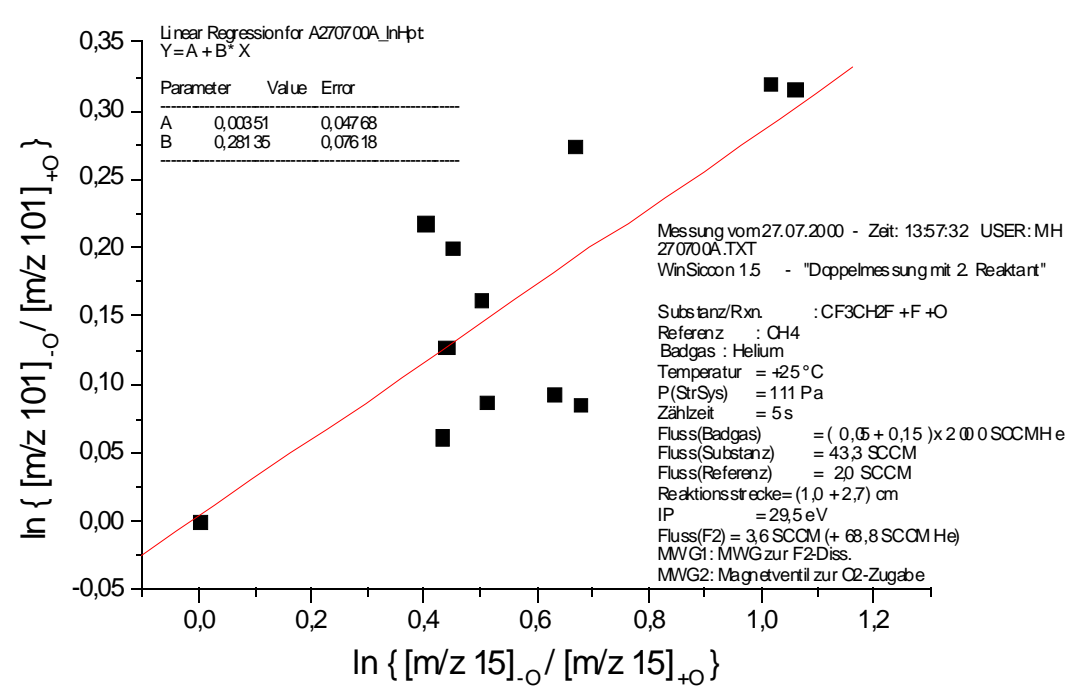

Abbildung 210: Kinetik CF3CHF + O rel. zu CH3; $298 \mathrm{~K}$

Aus der Steigung der Auftragung in Abbildung 210 läßt sich der folgende relative Geschwindigkeitskoeffizient ermitteln: $\mathrm{k}_{\mathrm{rel}}=(0,28 \pm 0,08)$.

Weil die Meßwerte relativ stark gestreut waren, wurde die Messung unter annähernd gleichen Bedingungen wiederholt, wobei sich ein identisches Resultat, aber eine wesentlich bessere Korrelation ergab (Abbildung 211).

$\vartheta=25{ }^{\circ} \mathrm{C} ; \phi_{\mathrm{He}}=450 \mathrm{SCCM} ; \phi_{\mathrm{CF} 3 \mathrm{CH} 2 \mathrm{~F}}=43,3 \mathrm{SCCM} ; \phi_{\mathrm{CH} 4}=2,0 \mathrm{SCCM} ; \mathrm{p}_{(\mathrm{StrR})}=108 \mathrm{~Pa} ;$ $\mathrm{S}_{\mathrm{CF} 3 \mathrm{CH} 2 \mathrm{~F}+\mathrm{F}}=0,5 \mathrm{~cm} ; \mathrm{S}_{\mathrm{CF} 3 \mathrm{CHF}+\mathrm{O}}=2,7 \mathrm{~cm} ; \mathrm{IP}=29,5 \mathrm{eV} ;$ Zählzeit $=5 \mathrm{~s}$

\begin{tabular}{|c|c|c|c|c|c|c|c|}
\hline $\begin{array}{c}\text { I(101) } \\
-\mathrm{O} / \text { w.E. }\end{array}$ & $\begin{array}{c}\Delta \mathbf{I}(\mathbf{1 0 1}) \\
-\mathrm{O} / \mathrm{w.E.}\end{array}$ & $\begin{array}{c}\mathrm{I}(\mathbf{1 0 1}) \\
+\mathrm{O} / \mathrm{w.E.}\end{array}$ & $\begin{array}{c}\Delta \mathbf{I}(\mathbf{1 0 1}) \\
+\mathrm{O} / \mathrm{w} . \mathrm{E} .\end{array}$ & $\begin{array}{c}I(15) \\
-O / \text { w.E. }\end{array}$ & $\begin{array}{c}\Delta \mathbf{I}(\mathbf{1 5}) \\
-\mathbf{O} / \mathbf{w . E .} .\end{array}$ & $\begin{array}{c}I(15) \\
+O / \text { w.E. }\end{array}$ & $\begin{array}{c}\Delta \mathbf{I}(\mathbf{1 5}) \\
+\mathrm{O} / \mathrm{w.E.}\end{array}$ \\
\hline 2174 & 136,3 & 2119 & 108,8 & 2761 & 141,4 & 2549 & 207,4 \\
\hline 2355 & 109,9 & 2217 & 133,8 & 2607 & 180,2 & 2207 & 156,6 \\
\hline 2327 & 140,6 & 2245 & 129,7 & 2632 & 192,5 & 2232 & 131,1 \\
\hline 2353 & 116,8 & 1959 & 129,2 & 2266 & 252,6 & 1266 & 167,4 \\
\hline 2250 & 144,1 & 2106 & 95,65 & 2607 & 166,8 & 2032 & 187,9 \\
\hline 2221 & 125,1 & 1974 & 103 & 2551 & 180,4 & 1851 & 169 \\
\hline 2383 & 97,55 & 2108 & 90,35 & 2629 & 198,6 & 1554 & 153,3 \\
\hline
\end{tabular}

Tabelle 70: Meßwerte Kinetik $\mathrm{CF} 3 \mathrm{CHF}+\mathrm{O}$ rel. zu CH3; $298 \mathrm{~K}$ 


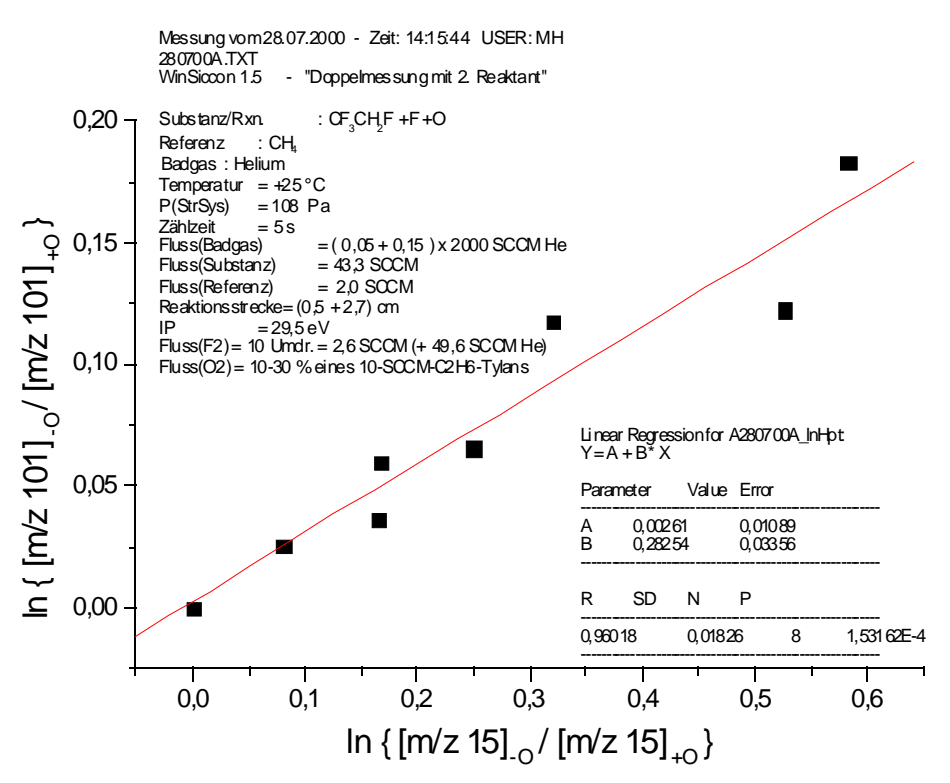

Abbildung 211: Kinetik CF3CHF + O rel. zu CH3; $298 \mathrm{~K}$

Aus der Steigung der Auftragung in Abbildung 211 läßt sich der folgende relative Geschwindigkeitskoeffizient ermitteln: $\mathrm{k}_{\mathrm{rel}}=(0,28 \pm 0,03)$.

Für den Geschwindigkeitskoeffizienten der Referenzreaktion ${ }^{181} \mathrm{k}_{\mathrm{ref}}$ ist angegeben: $\mathrm{k}_{\mathrm{ref}}(298 \mathrm{~K})=8,43 \cdot 10^{13} \mathrm{~cm}^{3} /(\mathrm{mol} \cdot \mathrm{s})$.

Damit erhält man den Geschwindigkeitskoeffizienten $\mathrm{k}$ für die hier untersuchte Reaktion gemäß $\mathrm{k}=\mathrm{k}_{\mathrm{rel}} \cdot \mathrm{k}_{\mathrm{ref}}$.

Das Endergebnis lautet somit:

$$
\begin{aligned}
& \mathbf{k}(298 \mathrm{~K})=(2,36 \pm \mathbf{0 , 2 5}) \cdot \mathbf{1 0}^{13} \mathrm{~cm}^{3} /(\mathbf{m o l} \cdot \mathbf{s}) \\
& \text { für } \mathrm{CF}_{3} \mathrm{CHF}+\mathrm{O} \rightarrow \text { Produkte }
\end{aligned}
$$

\subsubsection{Kinetik der Reaktion des erzeugten Radikals mit molekularem Sauerstoff}

Die Messungen wurden in der in Kap. 3.1.1 (S. 16) beschriebenen Apparatur unter Einsatz des Strömungsreaktors aus Abbildung 5 (S. 17) durchgeführt.

In einer ersten Messung diente das Methyl-Radikal als Referenzsubstanz. Sie wurde umsatzkorrigiert nach der Relativmethode durchgeführt, wobei zur Ermittlung des Umsatzes von $\mathrm{CF}_{3} \mathrm{CH}_{2} \mathrm{~F}$ bei Zugabe der Fluor-Atome die Intensitätsänderung auf $\mathrm{m} / \mathrm{z} 102$, und für den des Methans diejenige auf m/z 16 diente; die genaue Ausführung und Auswertung ist in 181 Atkinson, R.; et al. (1997). 
Kap. 3.5.1 (S. 50) beschrieben. Der Umsatz des $\mathrm{CF}_{3}$ CHF-Radikals konnte anhand der Intensitätsänderung seines Fragment-Peaks m/z 82 und der des Methyl-Radikals anhand seines Molekül-Peaks m/z 15 bestimmt werden.

$\vartheta=25^{\circ} \mathrm{C} ; \phi_{\mathrm{He}}=664 \mathrm{SCCM} ; \phi_{\mathrm{CF} 3 \mathrm{CH} 2 \mathrm{~F}}=23,0 \mathrm{SCCM} ; \phi_{\mathrm{CH} 4}=1,4 \mathrm{SCCM} ; \mathrm{p}_{(\mathrm{StrR})}=153 \mathrm{~Pa}$; $\mathrm{s}=3,0 \mathrm{~cm} ; \mathrm{IP}=29,5 \mathrm{eV}$; Zählzeit $=5 \mathrm{~s}$

\begin{tabular}{|c|c|c|c|c|c|c|c|}
\hline $\begin{array}{c}\mathbf{I}(\mathbf{8 2}) \\
-\mathrm{O}_{2} / \mathrm{w.E.}\end{array}$ & $\begin{array}{c}\Delta \mathbf{I}(\mathbf{8 2}) \\
-\mathbf{O}_{2} / \mathrm{w.E} .\end{array}$ & $\begin{array}{c}I(82) \\
+O_{2} / \text { w.E. }\end{array}$ & $\begin{array}{c}\Delta \mathrm{I}(\mathbf{8 2}) \\
+\mathrm{O}_{2} / \mathrm{w.E.}\end{array}$ & $\begin{array}{c}\mathbf{I}(15) \\
-\mathrm{O}_{2} / \mathrm{w} . \mathrm{E} .\end{array}$ & $\begin{array}{c}\Delta \mathbf{I}(15) \\
-\mathbf{O}_{2} / \mathbf{w . E} .\end{array}$ & $\begin{array}{c}I(15) \\
+O_{2} / \text { w.E. }\end{array}$ & $\begin{aligned} & \Delta I(15) \\
&+O_{2} / w . E .\end{aligned}$ \\
\hline 3135 & 171,8 & 2517 & 150,7 & 1935 & 317,3 & 1575 & 269,9 \\
\hline 2760 & 116,8 & 2040 & 113,7 & 2058 & 329,6 & 1318 & 297,5 \\
\hline 2485 & 109,7 & 1585 & 149,1 & 3203 & 330,3 & 2183 & 429,9 \\
\hline 2628 & 124,3 & 1778 & 146,6 & 2933 & 428,3 & 1933 & 390,5 \\
\hline 2682 & 145,9 & 1572 & 149 & 2033 & 348,7 & 1095 & 307,8 \\
\hline 2620 & 180,7 & 1742 & 166,2 & 2507 & 418,6 & 1567 & 348 \\
\hline 2499 & 114,6 & 1809 & 137,3 & 2567 & 369,6 & 1847 & 315,4 \\
\hline 2353 & 182,6 & 2075 & 135,2 & 1939 & 444 & 1559 & 418,9 \\
\hline 2441 & 132,3 & 2021 & 216,7 & 2279 & 502,8 & 2079 & 325,5 \\
\hline 2297 & 257,3 & 2045 & 132,6 & 2759 & 337,1 & 2279 & 337,1 \\
\hline 1951 & 165,4 & 1719 & 126 & 2719 & 363,6 & 2459 & 348,4 \\
\hline 2501 & 178,8 & 2095 & 119,7 & 907,1 & 171 & 777,1 & 182,8 \\
\hline 2755 & 131,7 & 2169 & 125,5 & 1390 & 277,2 & 1036 & 338,6 \\
\hline
\end{tabular}

Tabelle 71: Meßwerte Kinetik CF3CHF + O2 rel. zu CH3; $298 \mathrm{~K}$

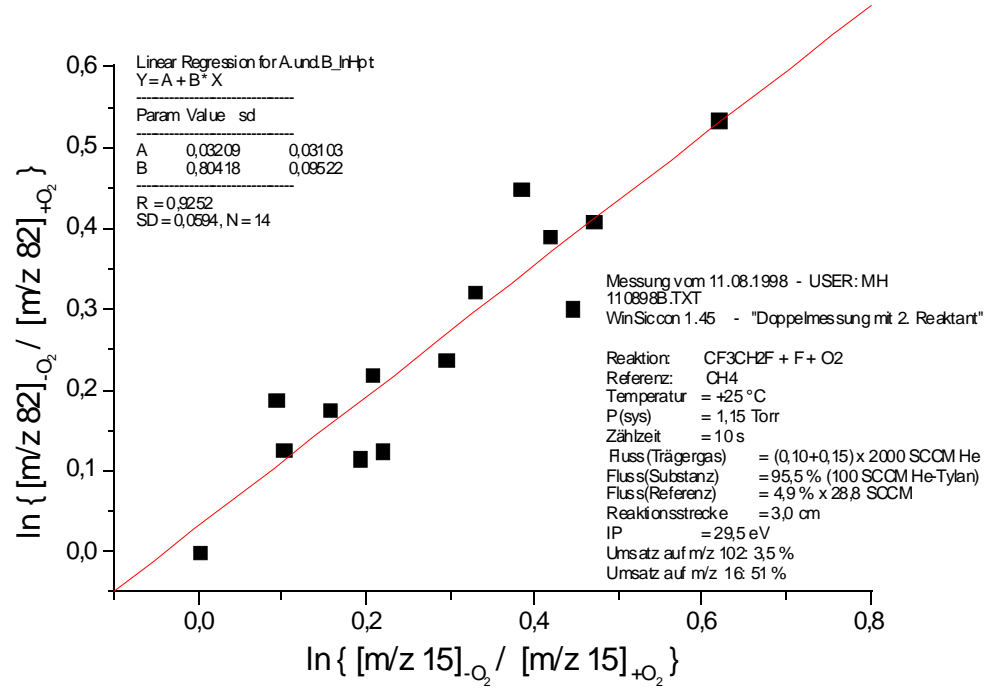

Abbildung 212: Kinetik CF3CHF + O2 rel. zu CH3; $298 \mathrm{~K}$

Aus der Steigung der Auftragung in Abbildung 212 läßt sich der folgende relative 
Geschwindigkeitskoeffizient ermitteln: $\mathrm{k}_{\mathrm{rel}}=(0,80 \pm 0,10)$.

Für den Geschwindigkeitskoeffizienten der Referenzreaktion ${ }^{182} \mathrm{k}_{\mathrm{ref}}$ ist angegeben:

$\mathrm{k}_{\mathrm{ref}}(295 \mathrm{~K})=(1,23 \pm 0,39) \cdot 10^{17} \cdot[\mathrm{He}] \mathrm{cm}^{6} /\left(\mathrm{mol}^{2} \cdot \mathrm{s}\right)$.

Damit erhält man den Geschwindigkeitskoeffizienten $\mathrm{k}_{1}$ für die hier untersuchte Reaktion gemäß $\mathrm{k}_{1}=\mathrm{k}_{\mathrm{rel}} \cdot \mathrm{k}_{\mathrm{ref}} \cdot$

Das Endergebnis aus der Relativmessung lautet somit:

$$
\begin{aligned}
& \mathbf{k}_{\mathbf{1}}(\mathbf{2 9 8 K})=(\mathbf{9 , 8} \pm \mathbf{3 , 4}) \cdot \mathbf{1 0}^{16} \cdot[\mathrm{He}] \mathrm{cm}^{6} /\left(\mathrm{mol}^{2} \cdot \mathbf{s}\right) \\
& \text { für: } \mathrm{CF}_{3} \mathrm{CHF}+\mathrm{O}_{2}+\mathrm{M} \rightarrow \text { Produkte } \quad ; \mathrm{M} \text { : dritter Stoßpartner }(\mathrm{He})
\end{aligned}
$$

Mit $[\mathrm{He}]=6,1 \cdot 10^{-8} \mathrm{~mol} \cdot \mathrm{cm}^{-3}$ errechnet man daraus: $\mathrm{k}_{1}(298 \mathrm{~K})=6,0 \cdot 10^{9} \mathrm{~cm}^{3} /(\mathrm{mol} \cdot \mathrm{s})$.

In einer zweiten Messung wurde die Kinetik der Reaktion $\mathrm{CF}_{3} \mathrm{CHF}+\mathrm{O}_{2}$ nach der Absolutmethode bestimmt, so wie es in Kap. 3.5.1 (S. 50) beschrieben ist.

Dazu wurde durch Variation der Reaktionsstrecke das Maximum auf dem Radikal-Fragment $\mathrm{m} / \mathrm{z} 82$ gesucht und ausgehend von diesem Punkt die Intensitäten auf m/z 82 in Abhängigkeit der (stetig erhöhten) Reaktionsstrecke gemessen. Der Fluß an $\mathrm{O}_{2}$ wurde so hoch gewählt, daß davon auszugehen war, die Bedingung zur Auswertung nach dem Geschwindigkeitsgesetz „pseudo-erster-Ordnung“ erfüllt zu haben. Dazu muß eine Auftragung gemäß Gleichung (4) (S. 8) angefertigt werden.

Die Umrechnung der Reaktionsstrecke in die -zeit erfolgte nach Gleichung (18) (S. 13) in Verbindung mit Gleichung (17).

Der Gasfluß von $\mathrm{O}_{2}$ konnte mit einem geeichten Flußmesser ermittelt und daraus die Konzentration $\left[\mathrm{O}_{2}\right]$ mittels Gleichung (19) (S. 14) berechnet werden: $\left[\mathrm{O}_{2}\right]=2,85 \cdot 10^{-8} \mathrm{~mol} / \mathrm{cm}^{3}$.

Der Umsatz von $\mathrm{CF}_{3} \mathrm{CH}_{2} \mathrm{~F}$ bei Zugabe von Fluor-Atomen betrug 8,8\% (gemessen auf dem Molekül-Peak m/z 102).

Die übrigen Reaktionsbedingungen sind umseitig vor der Tabelle 72 mit den Meßwerten vermerkt. 
$\vartheta=25{ }^{\circ} \mathrm{C} ; \phi_{\mathrm{He}}=951 \mathrm{SCCM} ; \phi_{\mathrm{CF} 3 \mathrm{CH} 2 \mathrm{~F}}=31,7 \mathrm{SCCM} ; \phi_{\mathrm{O} 2}=557,6 \mathrm{SCCM} ; \phi_{\mathrm{gesant}}=1566 \mathrm{SCCM} ;$ $\mathrm{p}_{(\mathrm{StrR})}=180 \mathrm{~Pa} ; \mathrm{IP}=29,5 \mathrm{eV} ;$ Zählzeit $=10 \mathrm{~s}$

\begin{tabular}{|r|r|r|r|}
\hline $\mathbf{s} / \mathbf{~ c m}$ & \multicolumn{1}{|c|}{$\mathbf{t}$ / } & $\mathbf{I}(\mathbf{8 2})$ / w.E. & $\ln \{\mathbf{I}(\mathbf{8 2})$ / w.E. $\}$ \\
\hline 6,2 & $1,21 \mathrm{E}-03$ & 1930 & 7,565 \\
\hline 7,2 & $1,40 \mathrm{E}-03$ & 1960 & 7,581 \\
\hline 8,1 & $1,58 \mathrm{E}-03$ & 1840 & 7,518 \\
\hline 9,1 & $1,77 \mathrm{E}-03$ & 1880 & 7,539 \\
\hline 9,7 & $1,89 \mathrm{E}-03$ & 1790 & 7,490 \\
\hline 10,5 & $2,05 \mathrm{E}-03$ & 1810 & 7,501 \\
\hline 11,4 & $2,21 \mathrm{E}-03$ & 1850 & 7,523 \\
\hline 12,5 & $2,44 \mathrm{E}-03$ & 1710 & 7,444 \\
\hline 13,5 & $2,63 \mathrm{E}-03$ & 1630 & 7,396 \\
\hline 14,4 & $2,80 \mathrm{E}-03$ & 1660 & 7,415 \\
\hline 15,0 & $2,92 \mathrm{E}-03$ & 1610 & 7,384 \\
\hline
\end{tabular}

Tabelle 72: Meßwerte Kinetik CF3CHF + O2 (Absolutmessung); $298 \mathrm{~K}$

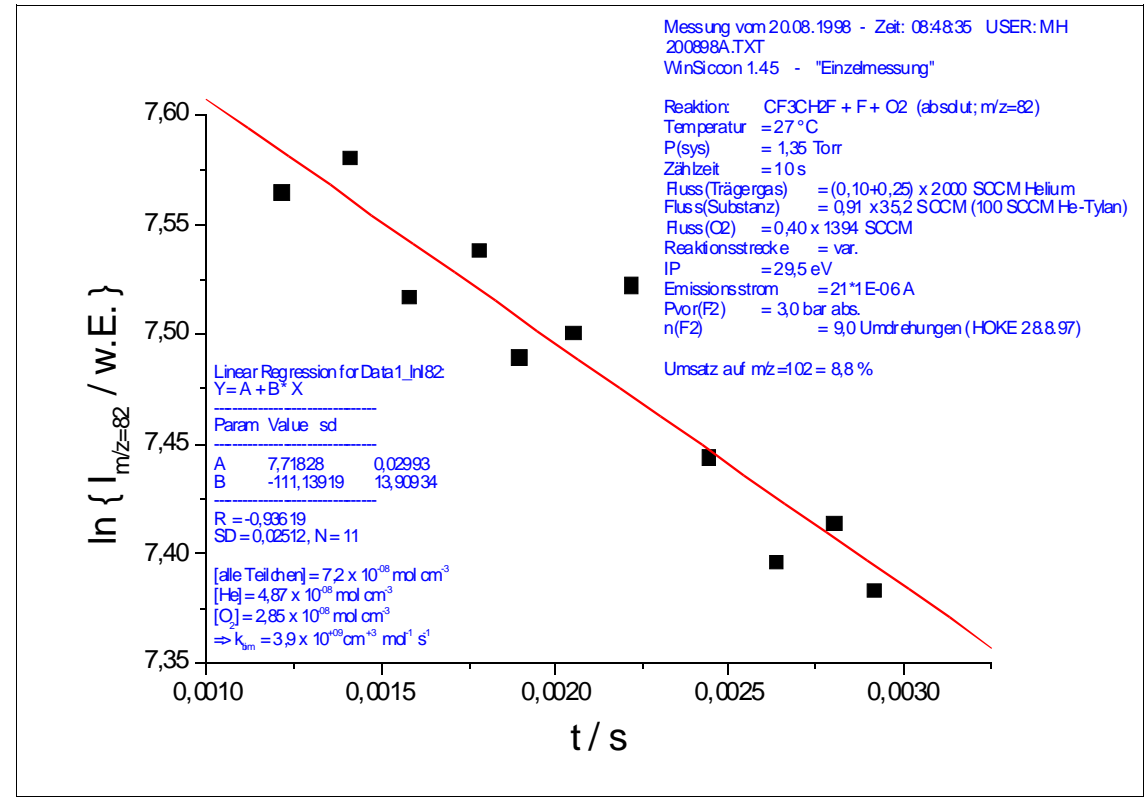

Abbildung 213: Kinetik CF3CHF + O2 (Absolutmessung); $298 \mathrm{~K}$

Aus der Steigung $b=(-111,1 \pm 13,9) \mathrm{s}^{-1}$ der Auftragung in Abb. 213 kann man mit der als konstant vorausgesetzten $\mathrm{O}_{2}$-Konzentration den Geschwindigkeitskoeffizienten $\mathrm{k}_{2}$ berechnen: $\mathrm{k}_{2}=-(-111,1 \pm 13,9) \mathrm{s}^{-1} /\left(2,85 \cdot 10^{-8} \mathrm{~mol} / \mathrm{cm}^{3}\right)=(3,9 \pm 0,5) \cdot 10^{9} \mathrm{~cm}^{3} \mathrm{~mol}^{-1} \mathrm{~s}^{-1}$.

Obwohl $\mathrm{O}_{2}$ in wirklich hohem Überschuß zugeführt wurde, ergab eine Simulationsrechnung, daß unter den gewählten Reaktionsbedingungen die Radikalkombination als Konkurrenz- 
reaktion nicht vollständig zurückgedrängt werden konnte (vgl. die schwarze und grüne Linie in Abbildung 214). Aus diesem Grund sollte mit dem Computerprogramm „RSF 3.0“ der Geschwindigkeitskoeffizient für die hier untersuchte Reaktion an die tatsächlichen Meßwerte angepaßt werden. Dem Programm wurden daher die gemessenen Radikal-Konzentrationen in Abhängigkeit der Reaktionszeit sowie die bekannten Geschwindigkeitskoeffizienten für die radikalerzeugende und die Kombinationsreaktion der Radikale mitgeteilt. Ferner mußten die bekannten Anfangskonzentrationen für $\mathrm{CF}_{3} \mathrm{CH}_{2} \mathrm{~F}, \mathrm{O}_{2}$ und die Fluor-Atome eingegeben werden.

Hieraus berechnete das Programm einen Wert von $\mathrm{k}_{2}=(4,4 \pm 0,6) \cdot 10^{9} \mathrm{~cm}^{3} \mathrm{~mol}^{-1} \mathrm{~s}^{-1}$.

Er unterscheidet sich im Rahmen der Fehlerschranken aber nicht von dem oben unmittelbar aus der Messung ermittelten.

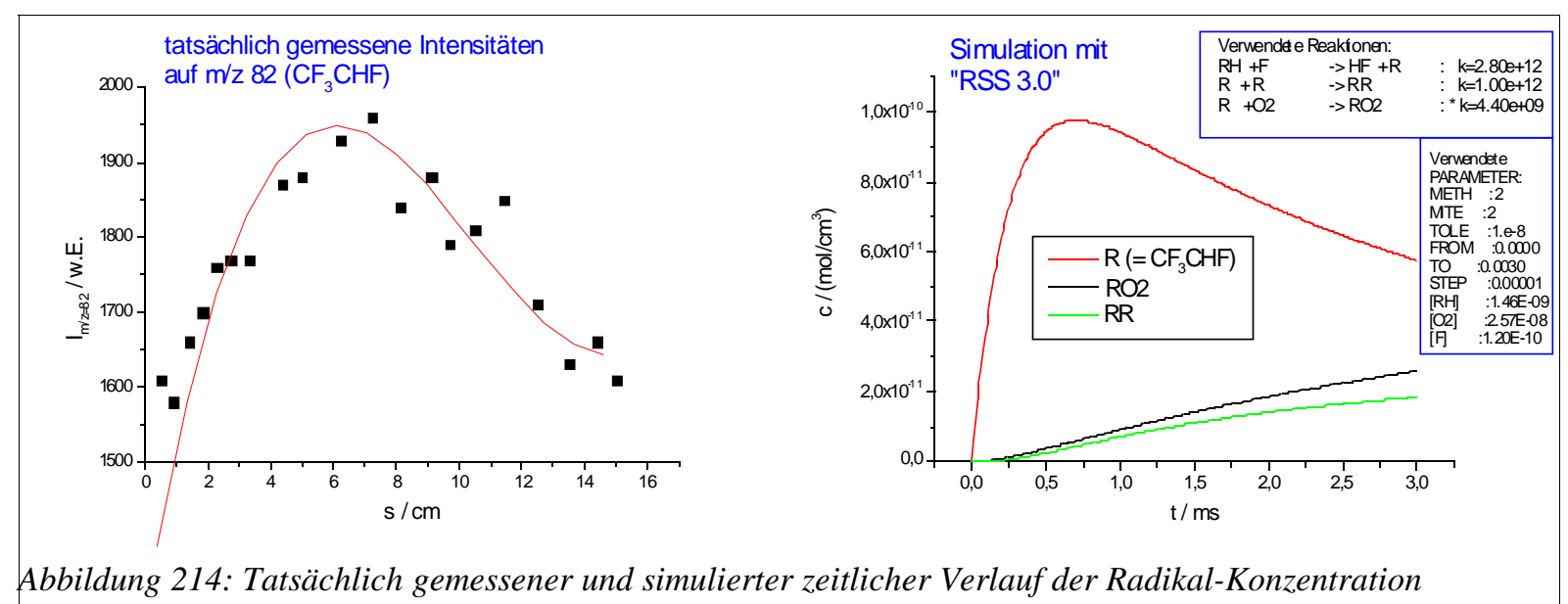

Für das Ergebnis aus der Absolutmessung kann der angepaßte Geschwindigkeitskoeffizient verwendet werden. Weil es sich um eine Assoziationsreaktion handelt, die wahrscheinlich im Niederdruckbereich ausgeführt wurde, soll er jedoch termolekular formuliert werden (vgl. Gleichung (28), S. 124). Die Konzentration der Stoßpartner M (im wesentlichen Helium) betrug $[\mathrm{M}]=7,2 \cdot 10^{-8} \mathrm{~mol} / \mathrm{cm}^{3}$; damit resultiert aus der Absolutmessung folgendes Endergebnis:

$$
\mathbf{k}_{2}(298 \mathrm{~K})=(6,1 \pm 0,8) \cdot 10^{16} \cdot[\mathrm{He}] \mathrm{cm}^{6} /\left(\mathrm{mol}^{2} \cdot \mathrm{s}\right)
$$

für: $\mathrm{CF}_{3} \mathrm{CHF}+\mathrm{O}_{2}+\mathrm{M} \rightarrow$ Produkte

; M: dritter Stoßpartner (He) 


\subsubsection{Diskussion der Ergebnisse}

Für die radikalerzeugende Reaktion $\mathrm{CF}_{3} \mathrm{CH}_{2} \mathrm{~F}+\mathrm{F} \rightarrow$ Produkte wurde die Temperaturabhängigkeit des Geschwindigkeitskoeffizienten $\mathrm{k}_{2}(\mathrm{~T})$ folgendermaßen bestimmt: $\mathrm{k}_{2}(\mathrm{~T})=(2,6 \pm 1,0) \cdot 10^{12} \cdot \exp \{-(-0,214 \pm 0,979) \mathrm{kJ} / \mathrm{mol} /(\mathrm{R} \cdot \mathrm{T})\} \mathrm{cm}^{3} /(\mathrm{mol} \cdot \mathrm{s})$.

Diese schwach negative Aktivierungsenergie (die im Rahmen der Fehlergrenzen beinahe Null ist) weicht ganz erheblich von zwei in der Literatur veröffentlichten Werten ab; dort wird für die Aktivierungsenergie zum einen ${ }^{183} \mathrm{E}_{\mathrm{a} \text {, Lit. }}=9,146 \mathrm{~kJ} / \mathrm{mol}$ und zum anderen ${ }^{184}$ 9,395 kJ/mol angegeben. Der erste genannte Literaturwert ist unter ähnlichen Reaktionsbedingungen, wie sie auch hier vorlagen, ermittelt worden, so daß dies als Ursache ausscheidet. Der hier aus $\mathrm{k}_{2}(\mathrm{~T})$ berechnete Wert für $298 \mathrm{~K}$ ist mit $\mathrm{k}_{2}(298 \mathrm{~K})=(2,8 \pm 1,6) \cdot 10^{12} \mathrm{~cm}^{3} \mathrm{~mol}^{-1} \mathrm{~s}^{-1}$ zudem höher, als die in der Literatur gefundenen Werte von 9,77 $\cdot 10^{11} \mathrm{~cm}^{3} \mathrm{~mol}^{-1} \mathrm{~s}^{-1}$ (Lit. ${ }^{183}$ ) und 1,33 $\cdot 10^{12}$ $\mathrm{cm}^{3} \mathrm{~mol}^{-1} \mathrm{~s}^{-1}$ (Lit. ${ }^{184}$ ). Es ist nicht auszuschließen, daß der Grund für die Abweichung in der für die Referenzreaktion verwendeten Temperaturabhängigkeit des Geschwindigkeitskoeffizienten zu suchen ist; der Vergleich mit anderen veröffentlichten Untersuchungen der Reaktion $\mathrm{CH}_{2} \mathrm{Cl}_{2}$ + F zeigte andererseits jedoch eine recht gute Übereinstimmung mit dem selbst ermittelten Ergebnis (insbesondere im Hinblick auf die Aktivierungsenergie ${ }^{185}$. Schließlich wird der hier für $\mathrm{CF}_{3} \mathrm{CH}_{2} \mathrm{~F}+\mathrm{F}$ bestimmte Wert aber auch durch die oben zuerst dargestellte Relativmessung mit Trichlormethan als Referenz gestützt, der mit $\mathrm{k}_{1}(298 \mathrm{~K})=(2,9 \pm 1,1) \cdot 10^{12} \mathrm{~cm}^{3} /(\mathrm{mol} \cdot \mathrm{s})$ praktisch ein identisches Ergebnis lieferte wie die Messung mit Dichlormethan als Referenz. Die beiden hier durchgeführten Analysen sind demnach konsistent und eine Begründung für die Abweichung zu den Literaturangaben kann nicht gegeben werden.

Bezüglich der Radikalkombinationsreaktion $\mathrm{CF}_{3} \mathrm{CHF}+\mathrm{CF}_{3} \mathrm{CHF}$ ist in dieser Arbeit bei $298 \mathrm{~K}$ als Geschwindigkeitskoeffizient $\mathrm{k}(298 \mathrm{~K})=(1,0 \pm 0,5) \cdot 10^{12} \mathrm{~cm}^{3} \mathrm{~mol}^{-1} \mathrm{~s}^{-1}$ bestimmt worden. Aus der Literatur lag der Wert $\mathrm{k}_{\mathrm{Lit}}(298 \mathrm{~K})=(6,0 \pm 1,8) \cdot 10^{12} \mathrm{~cm}^{3} \mathrm{~mol}^{-1} \mathrm{~s}^{-1} \operatorname{vor}^{186}$, der bei wesentlich höherem Druck (260-807 mbar) ermittelt wurde. Auf diesen Reaktionstyp sollte der unterschiedliche Druck aber eigentlich keinen Einfluß haben. Anhand der relativ hohen Fehler, die sowohl hier als auch bei der Literatur angegeben sind, erkennt man aber, daß die Messungen nicht leicht durchführbar sind und zudem eine Vernichtung von Radikalen durch die Wand immer in Betracht gezogen werden muß, die sehr gut die Abweichung der beiden 
Werte voneinander erklären kann. Die Einordnung von $\mathrm{CF}_{3} \mathrm{CH}_{2} \mathrm{~F}$ in das Kapitel der MethanDerivate erfolgte deshalb, weil die $\mathrm{CF}_{3}$-Gruppe als inerter Substituent aufgefaßt werden kann. Daher bietet sich auch der Vergleich mit dem strukturell ähnlichen $\mathrm{CH}_{2} \mathrm{~F}_{2}$ an. Dem F-Atom kommt eine Elektronegativität ${ }^{187}$ von etwa 3,9 zu, der $\mathrm{CF}_{3}$-Gruppe eine von ca. 3,35. Daher unterscheiden sich diese Systeme aus elektronischer Sicht nicht wesentlich. Für die Kombinationsreaktion der $\mathrm{CHF}_{2}$-Radikale ist ein Wert von $\mathrm{k}_{\mathrm{Lit}}(298 \mathrm{~K})=2,6 \cdot 10^{12} \mathrm{~cm}^{3} \mathrm{~mol}^{-1} \mathrm{~s}^{-1}$ bestimmt worden ${ }^{188}$. Im Vergleich dazu ist es kaum verständlich, daß $\mathrm{CF}_{3} \mathrm{CHF}$ mit der sterisch deutlich anspruchsvolleren $\mathrm{CF}_{3}$-Gruppe einen höheren Geschwindigkeitskoeffizienten haben soll (zumal sich dieser Effekt bei der Kombinationsreaktion quasi überproportional bemerkbar machen müßte, weil ja beide Reaktionspartner die große Gruppe tragen). Insofern erscheint der in dieser Arbeit gemessene Wert plausibler als derjenige in der Literatur.

Auch in Bezug auf die Produkte der Reaktion $\mathrm{CF}_{3} \mathrm{CHF}+\mathrm{O}$ kann ein Vergleich mit dem strukturell ähnlichen $\mathrm{CHF}_{2}$-Radikal vorgenommen werden. Bei letzterem wurden einige den hier gefundenen Produkten analoge Verbindungen nachgewiesen, so z.B. $\mathrm{F}_{2} \mathrm{CO}$ als Hauptprodukt (hier: $\mathrm{CF}_{3} \mathrm{CFO}$ ). Genau wie hier fanden sich $\mathrm{FCHO}, \mathrm{CO}$ und $\mathrm{CO}_{2}$ als Nebenund Folgeprodukte ${ }^{189}$.

Für die Reaktion $\mathrm{CF}_{3} \mathrm{CHF}+\mathrm{O}$ konnte $\mathrm{k}(298 \mathrm{~K})=(2,36 \pm 0,25) \cdot 10^{13} \mathrm{~cm}^{3} /(\mathrm{mol} \cdot \mathrm{s})$ in zwei unabhängigen Messungen bestimmt werden. Der Geschwindigkeitskoeffizient der analogen Reaktion mit dem strukturell und elektronisch ähnlichen $\mathrm{CHF}_{2}$-Radikal lautet ${ }^{190}: \mathrm{k}_{\mathrm{Vgl}} .(298 \mathrm{~K})=$ $4,8 \cdot 10^{13} \mathrm{~cm}^{3} /(\mathrm{mol} \cdot \mathrm{s})$. Aus den bereits erörterten Gründen sollte für $\mathrm{CF}_{3} \mathrm{CHF}$ ein ähnlicher (aus sterischen Gründen eher etwas geringerer) Wert wie für das $\mathrm{CHF}_{2}$-Radikal zu erwarten sein. Dieses Plausibilitätskriterium ist gut erfüllt. Direkte Vergleichswerte aus der Literatur lagen nicht vor.

Bei der Reaktion $\mathrm{CF}_{3} \mathrm{CHF}+\mathrm{O}_{2}+\mathrm{M} \rightarrow$ Produkte lautet das Endergebnis aus der Relativmessung $\mathrm{k}_{1}(298 \mathrm{~K})=(9,8 \pm 3,4) \cdot 10^{16} \cdot[\mathrm{He}] \mathrm{cm}^{6} /\left(\mathrm{mol}^{2} \cdot \mathrm{s}\right)$, woraus man für den herrschenden Druck mit $[\mathrm{He}]=6,1 \cdot 10^{-8} \mathrm{~mol} \cdot \mathrm{cm}^{-3}$ errechnen $\mathrm{kann}: \mathrm{k}_{1}(298 \mathrm{~K})=6,0 \cdot 10^{9}$

187 Die Elektronegativitätswerte sind entnommen aus: Huheey, J. (1988), S. 158 und 169.

188 Beiderhase, T. (1995), S. 52.

189 Beiderhase, T. (1995), S. 85 ff.

190 Beiderhase, T. (1995), S. 90. 
$\mathrm{cm}^{3} /(\mathrm{mol} \cdot \mathrm{s})$. Als Endergebnis aus der Absolutmessung wurde $\mathrm{k}_{2}(298 \mathrm{~K})=(6,1 \pm 0,8) \cdot 10^{16}$. [He] $\mathrm{cm}^{6} /\left(\mathrm{mol}^{2} \cdot \mathrm{s}\right)$ ermittelt. Die beiden Angaben stimmen innerhalb ihrer Fehlergrenzen überein und sind damit konsistent. Der Geschwindigkeitskoeffizient der analogen Reaktion mit dem strukturell und elektronisch ähnlichen $\mathrm{CHF}_{2}-\mathrm{Radikal}_{\text {lautet }}{ }^{191}: \mathrm{k}_{\mathrm{Vgl..}}(298 \mathrm{~K})=8,0 \cdot 10^{17} \cdot[\mathrm{He}]$ $\mathrm{cm}^{6} /\left(\mathrm{mol}^{2} \cdot \mathrm{s}\right)$. Ein relativ dazu etwas kleinerer Wert war für $\mathrm{CF}_{3} \mathrm{CHF}+\mathrm{O}_{2}$ nach den obigen Ausführungen zu erwarten, jedoch ist eine Erniedrigung fast um den Faktor 10 doch überraschend viel und kann hier nicht erklärt werden. $\mathrm{Zu}$ dem einzigen aus der Literatur vorliegenden Wert von ${ }^{192} \mathrm{k}_{\mathrm{Lit}}(298 \mathrm{~K})=1,26 \cdot 10^{12} \mathrm{~cm}^{3} /(\mathrm{mol} \cdot \mathrm{s})$ ergibt sich eine Abweichung von etwa Faktor 200. Der Literaturwert wurde zwar auch in Helium, allerdings bei einem Druck von 92-815 mbar ermittelt. Auch wenn ein größeres Molekül wie $\mathrm{CF}_{3} \mathrm{CHFO}_{2}$ den Hochdruckgrenzwert bei kleineren Drücken erreichen kann als z.B. $\mathrm{CHF}_{2} \mathrm{O}_{2}$, so ist dennoch wohl davon auszugehen, daß dieser bei den hier vorliegenden Reaktionsbedingungen mit einem Druck von 1-2 mbar offensichtlich noch nicht erreicht ist (vgl. dazu auch die Ausführungen zu Gleichung (28) auf S. 124). Insofern sind der Literaturwert und der hier ermittelte nicht miteinander vergleichbar, sondern sie ergänzen sich vielmehr. Im Vergleich zur analogen Reaktion des $\mathrm{CHF}_{2}$-Radikals und weil die hier durchgeführte Absolut- und die Relativmessung konsistent waren, erscheint das Ergebnis plausibel.

191 Beiderhase, T. (1995), S. 140.

192 Maricq, M.M.; Szente, J.J.; Kaiser, E.W. (1992). 


\subsubsection{Das lodethan $\left(\mathrm{CH}_{3} \mathrm{CH}_{2} \mathrm{l}\right)$}

\subsubsection{Erzeugung von Ethyl-Radikalen durch LASER-Photolyse}

In der in Kapitel 3.1.5 (S. 42) beschriebenen Multireflektionskugelzelle wurde Iodethan durch den Puls eines Excimer-LASERs in Ethyl-Radikale und Iod-Atome gespalten (vgl. auch Kapitel 3.5.5 (S. 59). Durch Messung der Energie hinter der Zelle einmal in evakuiertem Zustand und einmal mit der Iodethan-Füllung kann man ermitteln, wieviel Energie in der Zelle zur Bildung der Radikale verbleibt. Mit der bekannten Wellenlänge (193 nm) kann dann die Anzahl der Photonen berechnet werden. Als grobe Näherung kann die Zahl der Radikale gleich der Zahl der Photonen gesetzt werden. Mit den bekannten Abmessungen des LASER-Strahls und des IR-Strahlengangs kann das Nachweisvolumen errechnet und somit die Konzentration der Radikale im Nachweisvolumen angegeben werden.

\subsubsection{Kinetik der Reaktion des erzeugten Radikals mit molekularem Sauerstoff}

Iodethan und molekularer Sauerstoff (6 mbar) wurden gemeinsam in die in Kapitel 3.1.5 (S. 42) beschriebene Multireflektionskugelzelle gefüllt, um zeitaufgelöst die Kinetik des $\mathrm{CH}_{3} \mathrm{CH}_{2}$-Radikals mit $\mathrm{O}_{2}$ als Absorptionsmessung aufnehmen zu können. Die Problematik dieser zeitaufgelösten Step-Scan-Messungen ist bereits in Kapitel 3.1.5 beschrieben worden.

Es war die äquivalente Neufüllung der Zelle für jeden Interferogrammpunkt erforderlich. Um diesen Aufwand auf das Nötigste reduzieren zu können, wurden die zu erwartenden IRFrequenzen des $\mathrm{CH}_{3} \mathrm{CH}_{2} \mathrm{O}_{2}$-Komplexes (genauer: die der C-O Schwingung) nach der Methode „B3LYP“ vorausberechnet ${ }^{193}$. Auf dieser Grundlage konnte ein Filter in den IR-Strahlengang gebaut werden, der nur im Bereich der zu erwartenden Banden durchlässig ist (OCLI, USA; Typ WO8674-9; $\varnothing=1 \mathrm{~cm}$; max. Durchlaß bei $8674 \mathrm{~nm}$ bzw.1152,9 $\mathrm{cm}^{-1}$ ), was die Zahl der Interferogrammpunkte auf ca. 80-150 reduziert hat. Der Aufnahmebereich war dadurch auf 980 bis $1370 \mathrm{~cm}^{-1}$ festgelegt. Als spektrale Auflösung wurde $10 \mathrm{~cm}^{-1}$ gewählt; die Zeitauflösung betrug $5 \mu$ s (es wurden 15 Zeitscheiben registriert).

Die Ausführung und Auswertung der Messung ist in Kapitel 3.5.5 (S. 59) beschrieben.

Zur Auswertung wurde das Integral des Absorbanz-Spektrums im Bereich 1070 bis $1160 \mathrm{~cm}^{-1}$ herangezogen.

Mit der ersten Messung soll der zeitliche Verlauf der Banden aus der C-O Schwingung des Peroxo-Komplexes $\mathrm{CH}_{3} \mathrm{CH}_{2} \mathrm{O}_{2}$ dargestellt werden (Abbildung 215).

193 H. Sommnitz, Arbeitsgruppe Prof. Dr. Zellner, Univ. Essen, persönliche Mitteilung vom August 2000. 

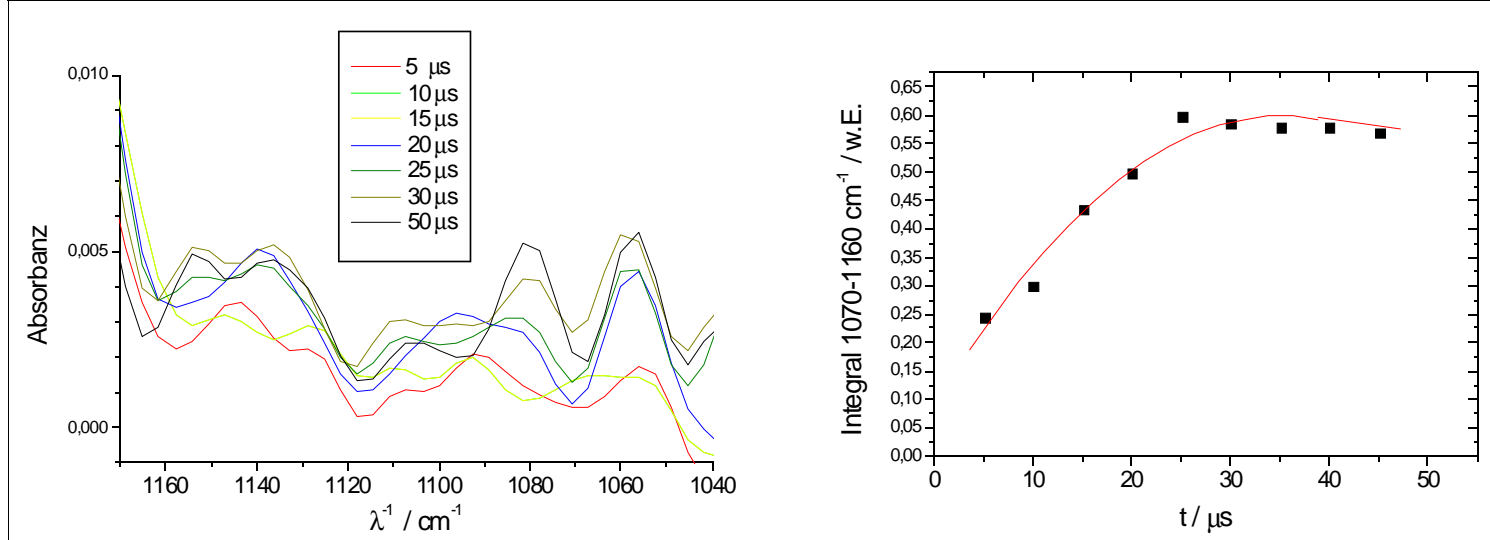

Abbildung 215: Zeitliche Entwicklung der Absorbanzf. CH3CH2OO (1040-1070 1/cm)

Um die Reproduzierbarkeit zu gewährleisten, wurde die Messung wiederholt (Abbildung 216).
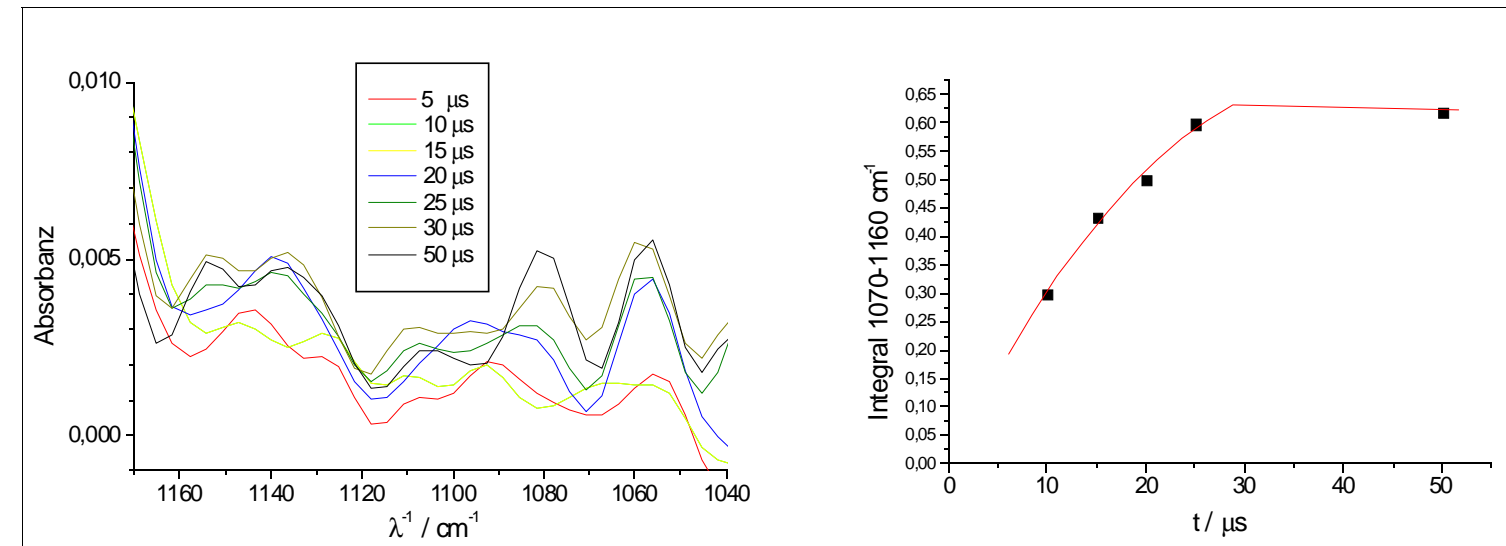

Abbildung 216: Zeitliche Entwicklung der Absorbanzf. CH3CH2OO (1040-1070 1/cm)

Aus den Meßwerten der Abb. 216 konnte mittels Gleichung (26) (S. 61) der Geschwindigkeitskoeffizient der Reaktion $\mathrm{CH}_{3} \mathrm{CH}_{2}+\mathrm{O}_{2} \rightarrow \mathrm{CH}_{3} \mathrm{CH}_{2} \mathrm{O}_{2}$ ermittelt werden. Die Berechnung der $\mathrm{O}_{2}$-Konzentration ist in Kapitel 3.5.5 (S. 59) beschrieben.

Aus der Steigung der Abb. 217 kann mit der bekannten $\mathrm{O}_{2}$-Konzentration das Ergebnis berechnet werden:

$\mathrm{k}(298 \mathrm{~K})=(1,7 \pm 0,5) \cdot 10^{12} \mathrm{~cm}^{3} /(\mathrm{mol} \cdot \mathrm{s})$

für $\mathrm{p}_{\mathrm{O} 2}=6$ mbar.

Die Auswertungen und Ergebnisse weiterer Messungen werden später veröffentlicht ${ }^{194}$.

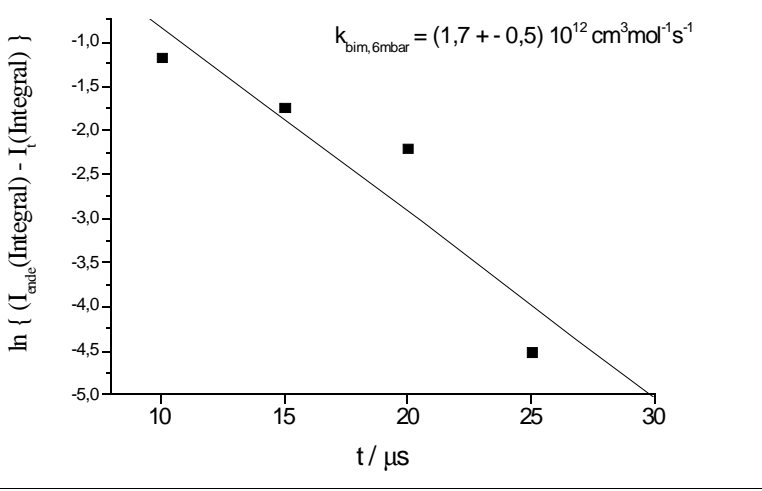

Abbildung 217: Kinetik $\mathrm{CH} 3 \mathrm{CH} 2+\mathrm{O} 2$ 


\subsubsection{Diskussion der Ergebnisse}

Vergleichswerte aus der Literatur, die nach dem selben Verfahren (FT-IR, Absorption) gemessen wurden, lagen nicht vor. Jedoch konnte ein Wert gefunden werden, der mittels „ultraviolet cavity ring-down spectroscopic detection“ bei ungefähr den gleichen Druckverhältnissen erhalten worden war $^{195}$; dort ist $\mathrm{k}(298 \mathrm{~K})=(1,63 \pm 0,18) \cdot 10^{12}$ $\mathrm{cm}^{3} /(\mathrm{mol} \cdot \mathrm{s})$ als Ergebnis vermerkt. Dieser Wert stimmt hervorragend mit dem hier erhaltenen $\mathrm{k}(298 \mathrm{~K})=(1,7 \pm 0,5) \cdot 10^{12} \mathrm{~cm}^{3} /(\mathrm{mol} \cdot \mathrm{s})$ überein.

Im Rahmen der vorliegenden Arbeit war der Aufbau der Apparatur und die Erstellung der dazu notwendigen Computersteuerung (vgl. Kap. 3.1.5, S. 42) vorrangiges Ziel. Mit der obigen Messung konnte gezeigt werden, daß die Methode und Apparatur funktionieren. Weitere zeitaufgelöste FT-IR-Messungen mit Ethyl-Radikalen wurden und werden im Rahmen einer weiteren Dissertation durchgeführt ${ }^{196}$.

Die Übertragung der Methode auf andere Substanzen ist eng verknüpft mit der Vorausberechnung der IR-Frequenzen, da nur so die entsprechenden Filter gezielt eingebaut werden können. Die vorliegende Messung hat gezeigt, daß mit dieser Methode voraussichtlich nur Radikale untersucht werden können, deren C-O Schwingung im Peroxo-Komplex in etwa die Intensität hat, die das Ethyl-Radikal aufweist. Bei geringeren Intensitäten könnte das Signal-/Rauschverhältnis eine sichere Auswertung verhindern. 


\section{Zusammenfassung}

\subsection{Allgemeines zur Messung der Geschwindigkeitskoeffizienten}

Die nachfolgend angegebenen Geschwindigkeitskoeffizienten wurden hautsächlich nach der Relativ- und nur in Einzelfällen nach der Absolutmethode bestimmt. Die Reaktionen erfolgten dazu in einem isothermen Strömungsreaktor mit Probennahme aus dem Molekularstrahl und massenspektrometrischem Nachweis. Der Reaktor konnte im Bereich $-20{ }^{\circ} \mathrm{C}$ bis $+90{ }^{\circ} \mathrm{C}$ temperiert werden; der Druck im Innern betrug üblicherweise 100 bis $250 \mathrm{~Pa}$. Die zugegebenen Fluor- und Sauerstoff-Atome wurden mit einer elektrodenlosen Mikrowellenentladung erzeugt.

Sofern nichts anderes angegeben ist, sind die Produktuntersuchungen ebenfalls mit dieser Anordnung durchgeführt worden.

Die Steuerung und Auswertung der Messungen erfolgte computergestützt durch das im Rahmen der vorliegenden Arbeit (weiter)entwickelte Programm „WinSiccon 1.7“.

\subsection{Ergebnisse der Analyse von 1-Ethinyl-1-methylcyclopropan}

Im ersten Teil der Untersuchung wurde der hochgespannte dreigliedrige Ring 1-Ethinyl-1methylcyclopropan in einem Strömungsreaktor mit Ofenheizung (vgl. Kap. 3.1.2, S. 26) bei Temperaturen von 280, 410, 530, 580, 632 und $680{ }^{\circ} \mathrm{C}$ thermisch aktiviert. Zur Vermeidung von Folgereaktionen bzw. intermolekularen Reaktionen betrug der He-Anteil stets 90-98 \% des gesamten Gasflusses. Die Reaktionszeiten lagen zwischen 50 und 300 ms, der Druck im Reaktor zwischen 10 und 30 mbar. Die Produkte des (wohl weitgehend unimolekularen) Zerfalls konnten in einer Glaskühlfalle bei $-196{ }^{\circ} \mathrm{C}$ ausgefroren und anschließend einer FT-IR, MS- und GC-MS-Analyse unterzogen werden. Dazu wurde im Rahmen dieser Arbeit eine GCMS-Kopplung aufgebaut und mit einem selbsterstellten Datenaufnahme- und Auswertungssystem versehen (vgl. Programmbeschreibung „GCMS-CP 1.4“). Erst bei $632{ }^{\circ} \mathrm{C}$ war das Edukt 1-Ethinyl-1-methylcyclopropan weitgehend umgesetzt.

Für die Identifizierung der meisten mutmaßlichen Produkte lagen weder authentische Spektren aus der Literatur vor, noch konnten diese Substanzen kommerziell beschafft werden. Daher wurden deren IR-Spektren mit dem Programm Gaussian nach der Methode „B3LYP/6-31G*“ berechnet. Obwohl solche berechneten Spektren nicht immer 100\%-ig mit den experimentell registrierten übereinstimmen, fanden sich insgesamt doch genügend Indizien für die Entstehung 
der in Abbildung 18 (S. 62) vorgeschlagenen Produkte. Die ausführliche Analyse der ProduktMischung aus der Pyrolyse bei $632{ }^{\circ} \mathrm{C}$ zeigt eine Zusammensetzung aus vier oder fünf Hauptund zwei Nebenprodukten sowie etwas Edukt. Verbindung 3 bzw. 4 der Abbildung 18 (S. 62) ist wahrscheinlich als Hauptprodukt und Verbindung 12 als Nebenprodukt anzusehen.

Unter der Voraussetzung, daß der Arrhenius-Vorfaktor (wie bei den strukturell ähnlichen Cycloropan-Derivaten) $10^{12} \mathrm{~s}^{-1}$ beträgt, kann die Aktivierungsenergie für den Zerfall des 1Ethinyl-1-methylcyclopropans auf $189 \mathrm{~kJ} / \mathrm{mol}$ geschätzt werden.

Im zweiten Teil der Untersuchung wurde quasi als Vorarbeit zur chemischen Aktivierung der Geschwindigkeitskoeffizient für die Reaktion von 1-Ethinyl-1-methylcyclopropan mit FluorAtomen nach der Relativmethode bestimmt. Diese Reaktion führt zu den in Abbildung 62 (S. 88) dargestellten Produkten, insbesondere also durch Abstraktion eines Wasserstoff-Atoms zu dem entsprechenden Radikal. Zur Messung der Kinetik fand ein Massenspektrometer mit Probennahme aus dem Molekularstrahl (vgl. Kap. 3.1.1, S. 16) Verwendung. Als Referenzsubstanz diente Methan. Das Ergebnis lautet: $\mathrm{k}=(8,0 \pm 0,5) \cdot 10^{13} \mathrm{~cm}^{3} /(\mathrm{mol} \cdot \mathrm{s})$.

Die im dritten Teil zu untersuchende chemische Aktivierung beruht auf der Reaktion des durch Abstraktion mit Fluor-Atomen erzeugten Radikals mit Wasserstoff-Atomen. Dadurch soll der ursprüngliche Kohlenwasserstoff zurückgebildet werden, jedoch ist durch diese chemische Aktivierung die Energie im Molekül nun deutlich erhöht und kann einen unimolekularen Zerfall bewirken. Im Zuge der Untersuchung stellte sich heraus, daß nicht nur bei der radikalerzeugenden Reaktion mit Fluor-Atomen, sondern auch bei der weiteren Reaktion mit den Wasserstoff-Atomen eine Vielzahl von Reaktionskanälen zur Verfügung stehen (vgl. Abbildungen 66 ff., S. 93 ff.). Es kommen derart viele potentielle Produkte in Betracht, daß selbst die FT-IR und GC-MS-Analysen keine sichere Zuordnung gestatten. Als weitere Komplikation kann nicht ausgeschlossen werden, daß sich der dreigliedrige Ring bereits unmittelbar nach der Radikalerzeugung und noch vor der nachgelagerten Reaktion mit Wasserstoff-Atomen öffnet (vgl. Abbildung 65, S. 92). Die gemessenen Spektren belegen, daß die chemische Aktivierung prinzipiell funktioniert und u.a. zu einem Allen als Produkt führt. Sie ist jedoch allenfalls mit begleitenden quantenchemischen Berechnungen interpretierbar, da nur hiermit eventuell eine Einschränkung der potentiellen Produktkanäle gemacht werden kann und eine gezielte experimentelle Überprüfung ermöglicht. 


\subsection{Ergebnisse der Analysen der teilfluorierten Ether}

\section{Bis(difluormethyl)ether}

Der Geschwindigkeitskoeffizient für die Reaktion von $\mathrm{CHF}_{2} \mathrm{OCHF}_{2}$ mit Fluor-Atomen wurde bei $298 \mathrm{~K}$ relativ zu $\mathrm{CHF}_{3}\left(\mathrm{k}_{\text {rel }}=(3,78 \pm 0,67)\right)$ sowie relativ zu $\mathrm{CF}_{3} \mathrm{CH}_{2} \mathrm{~F}\left(\mathrm{k}_{\text {rel }}=(0,20 \pm 0,03)\right)$ gemessen. Als Ergebnis erhielt man:

$\mathbf{k}(\mathbf{2 9 8 K})=(4,5 \pm \mathbf{1 , 6}) \cdot \mathbf{1 0}^{11} \mathrm{~cm}^{3} /(\mathbf{m o l} \cdot \mathbf{s}) \quad$; für: $\mathrm{CHF}_{2} \mathrm{OCHF}_{2}+\mathrm{F} \rightarrow$ Produkte

\section{Methylperfluorbutylether}

Aus Relativmessungen mit Dichlormethan als Referenzsubstanz konnte folgende Temperaturabhängigkeit des Geschwindigkeitskoeffizienten im Bereich -5 bis $+85{ }^{\circ} \mathrm{C}$ ermittelt werden:

$$
\begin{aligned}
& \mathbf{k}(\mathbf{T})=(9,39 \pm 0,03) \cdot 10^{12} \cdot(\mathrm{T} / 298 \mathrm{~K})^{(0,04 \pm 0,02)} \mathrm{cm}^{3} /(\mathbf{m o l} \cdot \mathbf{s}) . \\
& \text { für: } \mathrm{C}_{4} \mathrm{~F}_{9}-\mathrm{O}-\mathrm{CH}_{3}+\mathrm{F} \rightarrow \mathrm{C}_{4} \mathrm{~F}_{9}-\mathrm{O}-\mathrm{CH}_{2}+\mathrm{HF} .
\end{aligned}
$$

Aus Relativmessungen mit Dichlormethyl-Radikalen als Referenzsubstanz ließ sich folgende Temperaturabhängigkeit des Geschwindigkeitskoeffizienten im Bereich +25 bis $+85{ }^{\circ} \mathrm{C}$ bestimmen:

$$
\begin{aligned}
& \mathbf{k}(\mathbf{T})=(1,94 \pm \mathbf{0 , 1 0}) \cdot 10^{17} \cdot(\mathrm{T} / 298 \mathrm{~K})^{-(4,33 \pm 0,69)} \cdot[\mathrm{He}] \mathbf{c m}^{6} /\left(\mathbf{m o l}^{2} \cdot \mathbf{s}\right) \\
& \text { für: } \mathrm{C}_{4} \mathrm{~F}_{9}-\mathrm{O}-\mathrm{CH}_{2}+\mathrm{O}_{2}+\mathrm{M} \rightarrow \text { Produkte } ; \mathrm{M} \text { : dritter Stoßpartner }(\mathrm{He}) .
\end{aligned}
$$

Das $\mathrm{C}_{4} \mathrm{~F}_{9}-\mathrm{O}-\mathrm{CH}_{2}$-Radikal wurde bei $\lambda=\mathbf{3 8 7 , 8} \mathbf{~ n m}$ mit der REMPI-Methode im Flugzeitmassenspektrometer nachgewiesen.

\section{Ethylperfluorbutylether}

Das $\mathrm{C}_{4} \mathrm{~F}_{9}-\mathrm{O}-\mathrm{C}_{2} \mathrm{H}_{4}$-Radikal konnte bei $\lambda=386,3 ; 391,4$ und $469,3 \mathrm{~nm}$ mit der REMPIMethode im Flugzeitmassenspektrometer nachgewisen werden.

Ferner zeigte es einen Peak hoher Intensität auf m/z 263 im EI-Massenspektrometer. 


\subsection{Ergebnisse der Analysen der teilfluorierten Alkohole}

\section{$\underline{\text { 2,2,2-Trifluorethanol }}$}

In der Reaktion mit Fluor-Atomen gab es Hinweise auf die Bildung der in Abb. 109 (S. 132) dargestellten Produkte.

Aus Relativmessungen mit Methan als Referenzsubstanz konnte folgende Temperaturabhängigkeit des Geschwindigkeitskoeffizienten im Bereich -15 bis $+85{ }^{\circ} \mathrm{C}$ ermittelt werden:

$$
\begin{aligned}
& \mathbf{k}(\mathbf{T})=(6,27 \pm \mathbf{0 , 3 9}) \cdot 10^{13} \cdot(\mathbf{T} / 298 \mathrm{~K})^{-(0,58 \pm 0,53)} \mathrm{cm}^{3} /(\mathbf{m o l} \cdot \mathbf{s}) \\
& \text { für: } \mathrm{CF}_{3} \mathrm{CH}_{2} \mathrm{OH}+\mathrm{F} \rightarrow \text { Produkte }
\end{aligned}
$$

Als Produkt aus der Reaktion des mit Fluor-Atomen aus $\mathrm{CF}_{3} \mathrm{CH}_{2} \mathrm{OH}$ erzeugten Radikals mit $\mathrm{O}_{2}$ wurde $\mathrm{CF}_{3} \mathrm{CHO}$ nachgewiesen. Aus Relativmessungen mit Dichlormethyl-Radikalen als Referenzsubstanz ließ sich folgende Temperaturabhängigkeit des Geschwindigkeitskoeffizienten im Bereich -15 bis $+85^{\circ} \mathrm{C}$ bestimmen:

$$
k(T)=(4,65 \pm 0,30) \cdot 10^{17} \cdot(T / 298 K)^{-(3,75 \pm 0,75)} \cdot[\mathrm{He}] \mathrm{cm}^{6} /\left(\mathrm{mol}^{2} \cdot \mathrm{s}\right)
$$

für: $\left\{\right.$ Radikal aus der Reaktion $\left.\mathrm{CF}_{3} \mathrm{CH}_{2} \mathrm{OH}+\mathrm{F}\right\}+\left\{\mathrm{O}_{2}\right\} \rightarrow$ Produkte

Das erzeugte Radikal wurde bei $\lambda=\mathbf{3 8 8 , 6 0 1} \mathbf{~ n m}$ mit der REMPI-Methode im Flugzeitmassenspektrometer nachgewisen.

Aus einer Relativmessung mit Dichlormethyl-Radikalen als Referenzsubstanz konnte folgender Geschwindigkeitskoeffizient bestimmt werden:

$$
\begin{aligned}
& \mathbf{k}(298 \mathrm{~K})=(1,67 \pm \mathbf{0 , 3 0}) \cdot 10^{17} \cdot[\mathrm{He}] \mathbf{c m}^{6} /\left(\mathbf{m o l}^{2} \cdot \mathbf{s}\right) \\
& \text { für: }\left\{\text { Radikal aus der Reaktion } \mathrm{CF}_{3} \mathrm{CH}_{2} \mathrm{OH}+\mathrm{F}\right\}+\{\mathrm{NO}\} \rightarrow \text { Produkte }
\end{aligned}
$$

\section{2,2-Difluorethanol}

In der Reaktion mit Fluor-Atomen gab es Hinweise auf die Bildung der in Abb. 133 (S. 155) dargestellten Produkte.

Aus einer Relativmessung mit Methan als Referenzsubstanz konnte folgender Geschwindigkeitskoeffizient bestimmt werden:

$$
k(299 K)=(6,92 \pm 0,28) \cdot 10^{13} \mathrm{~cm}^{3} /(\mathrm{mol} \cdot \mathrm{s})
$$

für: $\mathrm{CHF}_{2} \mathrm{CH}_{2} \mathrm{OH}+\mathrm{F} \rightarrow$ Produkte 
Als Hauptprodukt der Reaktion des mit Fluor-Atomen aus $\mathrm{CHF}_{2} \mathrm{CH}_{2} \mathrm{OH}$ erzeugten Radikals mit $\mathrm{O}_{2}$ wurde $\mathrm{CHF}_{2} \mathrm{CHO}$ und als Nebenprodukt $\mathrm{HCHO}$ ermittelt.

Aus einer Relativmessung mit Chlormethyl-Radikalen als Referenzsubstanz ließ sich folgender Geschwindigkeitskoeffizient bestimmen:

$$
\begin{aligned}
& \mathbf{k}(300 K)=(3,18 \pm \mathbf{0 , 3 9}) \cdot 10^{18} \cdot[\mathrm{He}] \mathbf{c m}^{6} /\left(\mathbf{m o l}^{2} \cdot \mathbf{s}\right) \\
& \text { für: }\left\{\text { Radikal aus der Reaktion } \mathrm{CHF}_{2} \mathrm{CH}_{2} \mathrm{OH}+\mathrm{F}\right\}+\left\{\mathrm{O}_{2}\right\} \rightarrow \text { Produkte }
\end{aligned}
$$

\section{$\underline{\text { 2-Fluorethanol }}$}

Aus Relativmessungen mit Methan als Referenzsubstanz konnte folgende Temperaturabhängigkeit des Geschwindigkeitskoeffizienten im Bereich +5 bis $+85^{\circ} \mathrm{C}$ ermittelt werden:

$$
\begin{aligned}
& \mathbf{k}(\mathbf{T})=(1,17 \pm \mathbf{0 , 0 3}) \cdot 10^{14} \cdot(\mathbf{T} / 298 \mathrm{~K})^{-(0,20 \pm 0,23)} \mathbf{c m}^{3} /(\mathbf{m o l} \cdot \mathbf{s}) \\
& \text { für: } \mathrm{CH}_{2} \mathrm{FCH}_{2} \mathrm{OH}+\mathrm{F} \rightarrow \text { Produkte }
\end{aligned}
$$

Als Produkte der Reaktion des mit Fluor-Atomen aus $\mathrm{CH}_{2} \mathrm{FCH}_{2} \mathrm{OH}$ erzeugten Radikals mit $\mathrm{O}_{2}$ wurden mittels FT-IR- und MS-Analyse diejenigen in Abb. 149 (S. 170) identifiziert. Dazu mußte das IR-Spektrum von FCHO mit dem Programm Gaussian nach der Methode „B3LYP/6-31G*“ berechnet werden.

Aus Relativmessungen mit 1,4-Dioxanyl-Radikalen als Referenzsubstanz ließ sich folgende Temperaturabhängigkeit des Geschwindigkeitskoeffizienten im Bereich -15 bis $+85{ }^{\circ} \mathrm{C}$ bestimmen:

$$
\begin{aligned}
& \mathbf{k}(\mathbf{T})=(\mathbf{1 , 1 5} \pm \mathbf{0 , 0 4}) \cdot 10^{12} \cdot(\mathbf{T} / 298 \mathrm{~K})^{-(2,1 \pm 0,3)} \mathbf{c m}^{3} /(\mathbf{m o l} \cdot \mathbf{s}) \\
& \text { für: }\left\{\text { Radikal aus der Reaktion } \mathrm{CH}_{2} \mathrm{FCH} \mathrm{HH}_{2} \mathrm{OH}\right\}+\left\{\mathrm{O}_{2}\right\} \rightarrow \text { Produkte }
\end{aligned}
$$

\section{$\underline{2,2,3,3,4,4,4-H e p t a f l u o r b u t a n o l}$}

Aus Relativmessungen mit Methan als Referenzsubstanz konnte folgende Temperaturabhängigkeit des Geschwindigkeitskoeffizienten im Bereich -15 bis $+65{ }^{\circ} \mathrm{C}$ ermittelt werden:

$$
\begin{aligned}
& \mathbf{k}(\mathbf{T})=(2,73 \pm \mathbf{0 , 0 4}) \cdot 10^{13} \cdot(\mathbf{T} / 298 \mathrm{~K})^{(0,53 \pm 0,15)} \mathbf{c m}^{3} /(\mathbf{m o l} \cdot \mathbf{s}) \\
& \text { für: } \mathrm{CF}_{3} \mathrm{CF}_{2} \mathrm{CF}_{2} \mathrm{CH}_{2} \mathrm{OH}+\mathrm{F} \rightarrow \text { Produkte }
\end{aligned}
$$


Aus Relativmessungen mit Dichlormethyl-Radikalen als Referenzsubstanz ließ sich folgende Temperaturabhängigkeit des Geschwindigkeitskoeffizienten im Bereich -15 bis $+85{ }^{\circ} \mathrm{C}$ bestimmen:

$$
\begin{aligned}
& \mathbf{k}(\mathbf{T})=(\mathbf{3 , 1 3} \pm \mathbf{0 , 1 8}) \cdot 10^{17} \cdot(\mathrm{T} / 298 \mathrm{~K})^{-(6,15 \pm 0,71)} \cdot[\mathrm{He}] \mathbf{c m}^{6} /\left(\mathbf{m o l}^{2} \cdot \mathbf{s}\right) \\
& \text { für: }\left\{\text { Radikal aus der Reaktion } \mathrm{CF}_{3} \mathrm{CF}_{2} \mathrm{CF}_{2} \mathrm{CH}_{2} \mathrm{OH}+\mathrm{F}\right\}+\left\{\mathrm{O}_{2}\right\} \rightarrow \text { Produkte }
\end{aligned}
$$

\subsection{Ergebnisse der Analysen der Methan-Derivate}

\section{Methan}

Durch sorgfältige Messungen im Massenspektrometer bei niedriger EI-Elektronenenergie wurde herausgefunden, daß bei der Reaktion von Methyl-Radikalen mit atomarem Sauerstoff die Zunahme auf m/z 28 nicht nur durch ein Fragment des gebildeten Methanals (HCHO) erklärt werden kann, sondern auf die Entstehung von Kohlenstoffmonoxid zurückzuführen ist. Nach Eichmessungen konnte der Anteil des CO-Kanals quantifiziert werden; er beträgt: $\alpha(\mathrm{CO})=(\mathbf{4 6} \pm \mathbf{6}) \mathrm{mol}-\%$.

Somit bleibt für den Produktkanal, der zu Methanal führt, noch ein Anteil von 54 mol-\%.

\section{Chlormethan}

Im Temperaturbereich 253 bis $358 \mathrm{~K}$ erhielt man aus einer Relativmessung mit Methan als Referenzsubstanz folgende Temperaturabhängigkeit des Geschwindigkeitskoeffizienten ${ }^{197}$ :

$$
\begin{aligned}
& \mathrm{k}=(4,33 \pm 0,88) \cdot 10^{13} \exp \{-(1,34 \pm 0,51) \mathrm{kJ} / \mathrm{mol} /(\mathrm{R} \cdot \mathrm{T})\} \mathrm{cm}^{3} \mathrm{~mol}^{-1} \mathrm{~s}^{-1} \\
& \text { für: } \mathrm{CH}_{3} \mathrm{Cl}+\mathrm{F} \rightarrow \text { Produkte }
\end{aligned}
$$

Bei $\mathrm{T}=298 \mathrm{~K}$ ergibt sich damit: $\mathrm{k}(298 \mathrm{~K})=2,52 \cdot 10^{13} \mathrm{~cm}^{3} \mathrm{~mol}^{-1} \mathrm{~s}^{-1}$.

Qualitativ sind für die Reaktion $\mathrm{CH}_{2} \mathrm{Cl}+\mathrm{O}$ die in Abb. 178 (S. 208) dargestellten Produkte nachgewiesen worden. Zudem wurde der Anteil des gebildeten Kohlenmonoxids und Methanals quantitativ bestimmt:

$$
\alpha(\mathrm{CO})=(43 \pm 2) \text { mol- } \% \quad \text { bzw. } \quad \alpha(\mathrm{HCHO})=19 \mathrm{~mol}-\%
$$


Im Temperaturbereich 258 bis $358 \mathrm{~K}$ erhielt man aus einer Relativmessung mit $\mathrm{CH}_{2} \mathrm{OCH}_{3}$ als Referenzsubstanz folgende Temperaturabhängigkeit des Geschwindigkeitskoeffizienten ${ }^{198}$ :

$$
k(T)=(8,9 \pm 1,8) \cdot 10^{13} \exp \{-(-0,10 \pm 0,53) \mathrm{kJ} / \mathrm{mol} /(\mathrm{R} \cdot \mathrm{T})\} \mathrm{cm}^{3} \mathrm{~mol}^{-1} \mathrm{~s}^{-1}
$$

für: $\mathrm{CH}_{2} \mathrm{Cl}+\mathrm{O} \rightarrow$ Produkte

Für T $=298 \mathrm{~K}$ ergibt sich damit: $\mathrm{k}(298 \mathrm{~K})=9,3 \cdot 10^{13} \mathrm{~cm}^{3} /(\mathrm{mol} \cdot \mathrm{s})$.

Abschließend wurde das $\mathrm{CH}_{2} \mathrm{Cl}$-Radikal bei $\lambda=\mathbf{3 7 6 , 0 2 5} \mathbf{~ n m}$ mit der REMPI-Methode im Flugzeitmassenspektrometer nachgewiesen.

\section{Dichlormethan}

Im Temperaturbereich 253 bis $358 \mathrm{~K}$ erhielt man aus einer Relativmessung mit Methan als Referenzsubstanz folgende Temperaturabhängigkeit des Geschwindigkeitskoeffizienten ${ }^{199}$ :

$$
\begin{aligned}
& \mathbf{k}=(8,4 \pm 1,0) \cdot 10^{12} \exp \{-(-0,12 \pm 0,29) \mathbf{k J} / \mathbf{m o l} /(\mathbf{R} \cdot \mathbf{T})\} \mathbf{c m}^{3} \mathbf{m o l}^{-1} \mathbf{s}^{-1} \\
& \text { für: } \mathrm{CH}_{2} \mathrm{Cl}_{2}+\mathrm{F} \rightarrow \text { Produkte }
\end{aligned}
$$

Für T $=298 \mathrm{~K}$ ergibt sich damit: $\mathrm{k}(298 \mathrm{~K})=8,8 \cdot 10^{12} \mathrm{~cm}^{3} \mathrm{~mol}^{-1} \mathrm{~s}^{-1}$.

Qualitativ sind für die Reaktion $\mathrm{CHCl}_{2}+\mathrm{O}$ die in Abb. 184 (S. 215) dargestellten Produkte nachgewiesen worden. Zudem wurde der Anteil des gebildeten Kohlenmonoxids quantitativ bestimmt:

$$
\alpha(\mathrm{CO})=(70 \pm 9) \text { mol- } \%
$$

Im Temperaturbereich 258 bis $358 \mathrm{~K}$ ergab sich aus einer Relativmessung mit $\mathrm{CH}_{3}$ als Referenzsubstanz folgende Temperaturabhängigkeit des Geschwindigkeitskoeffizienten ${ }^{200}$ :

$$
\begin{aligned}
& \mathbf{k}=(8,6 \pm 3,8) \cdot 10^{13} \exp \{-(1,7 \pm 1,1) \mathrm{kJ} / \mathrm{mol} /(\mathrm{R} \cdot \mathbf{T})\} \mathrm{cm}^{3} \mathbf{m o l}^{-1} \mathrm{~s}^{-1} \\
& \text { für: } \mathrm{CHCl}_{2}+\mathrm{O} \rightarrow \text { Produkte }
\end{aligned}
$$

Für T $=298 \mathrm{~K}$ ergibt sich damit: $\mathrm{k}(298 \mathrm{~K})=4,3 \cdot 10^{13} \mathrm{~cm}^{3} /(\mathrm{mol} \mathrm{s})$.

Aus einer Relativmessung mit Fluormethyl-Radikalen als Referenzsubstanz ließ sich folgender Geschwindigkeitskoeffizient bestimmen:

$$
\begin{aligned}
& \mathbf{k}(\mathbf{2 9 8 K})=(\mathbf{1 , 2} \pm \mathbf{0 , 3}) \cdot \mathbf{1 0}^{17} \cdot[\mathrm{He}] \mathbf{c m}^{6} /\left(\mathbf{m o l}^{2} \cdot \mathbf{s}\right) \\
& \text { für } \mathrm{CHCl}_{2}+\mathrm{NO}+\mathrm{M} \rightarrow \text { Produkte }+\mathrm{M}^{*} \quad ; \mathrm{M}: \text { dritter Stoßpartner }(\mathrm{He})
\end{aligned}
$$


Abschließend wurde das $\mathrm{CH}_{2} \mathrm{Cl}$-Radikal bei $\lambda=\mathbf{3 6 4 , 4}$ sowie $370,061 \mathbf{n m}$ mit der REMPIMethode im Flugzeitmassenspektrometer nachgewiesen.

\section{$\underline{1,1,1,2-T e t r a f l u o r e t h a n}$}

Aus einer Relativmessung mit Trichlormethan als Referenzsubstanz konnte folgender Geschwindigkeitskoeffizient bestimmt werden:

$$
\begin{aligned}
& \mathbf{k}_{\mathbf{1}}(298 \mathrm{~K})=(2,9 \pm \mathbf{1 , 1}) \cdot \mathbf{1 0}^{\mathbf{1 2}} \mathrm{cm}^{3} /(\mathrm{mol} \cdot \mathbf{s}) \\
& \text { für } \mathrm{CF}_{3} \mathrm{CH}_{2} \mathrm{~F}+\mathrm{F} \rightarrow \text { Produkte }
\end{aligned}
$$

Aus Relativmessungen mit Dichlormethyl-Radikalen als Referenzsubstanz ließ sich folgende Temperaturabhängigkeit des Geschwindigkeitskoeffizienten im Bereich -15 bis $+85{ }^{\circ} \mathrm{C}$ ermitteln:

$$
\begin{aligned}
& \mathbf{k}_{2}(\mathbf{T})=(2,84 \pm \mathbf{0 , 1 1}) \cdot 10^{12} \cdot(\mathrm{T} / 298 \mathrm{~K})^{(-0,10 \pm 0,37)} \mathrm{cm}^{3} /(\mathbf{m o l} \cdot \mathbf{s}) \\
& \text { für: } \mathrm{CF}_{3} \mathrm{CH}_{2} \mathrm{~F}+\mathrm{F} \rightarrow \text { Produkte }
\end{aligned}
$$

In einer Absolutmessung wurde der Geschwindigkeitskoeffizient für die Kombinationsreaktion der Radikale bestimmt:

$$
\begin{aligned}
& \mathbf{k}(298 \mathrm{~K})=(1,0 \pm \mathbf{0 , 5}) \cdot \mathbf{1 0}^{12} \mathrm{~cm}^{3} \mathbf{m o l}^{-1} \mathrm{~s}^{-1} \\
& \text { für: } \mathrm{CF}_{3} \mathrm{CHF}+\mathrm{CF}_{3} \mathrm{CHF} \rightarrow \text { Produkte }
\end{aligned}
$$

Aus einer Relativmessung mit Methyl-Radikalen als Referenzsubstanz ergab sich folgender Geschwindigkeitskoeffizient:

$$
\begin{aligned}
& \mathbf{k}(298 \mathrm{~K})=(2,36 \pm \mathbf{0 , 2 5}) \cdot \mathbf{1 0}^{13} \mathrm{~cm}^{3} /(\mathrm{mol} \cdot \mathbf{s}) \\
& \text { für: } \mathrm{CF}_{3} \mathrm{CHF}+\mathrm{O} \rightarrow \text { Produkte }
\end{aligned}
$$

Mittels einer Relativmessung mit Methyl-Radikalen als Referenzsubstanz ließ sich ferner folgender Geschwindigkeitskoeffizient bestimmen:

$$
\begin{aligned}
& \mathbf{k}_{\mathbf{1}}(\mathbf{2 9 8 K})=(\mathbf{9 , 8} \pm \mathbf{3 , 4}) \cdot \mathbf{1 0}^{16} \cdot[\mathrm{He}] \mathrm{cm}^{6} /\left(\mathrm{mol}^{2} \cdot \mathbf{s}\right) \\
& \text { für: } \mathrm{CF}_{3} \mathrm{CHF}+\mathrm{O}_{2} \rightarrow \text { Produkte }
\end{aligned}
$$

Für die letzte Reaktion wurde zudem eine Absolutmessung durchgeführt und der Geschwindigkeitskoeffizient mit dem Programm „RSF 3.0“ an die Meßwerte angepaßt:

$$
k_{2}(298 \mathrm{~K})=(6,1 \pm 0,8) \cdot 10^{16} \cdot[\mathrm{He}] \mathrm{cm}^{6} /\left(\mathrm{mol}^{2} \cdot \mathrm{s}\right)
$$


für: $\mathrm{CF}_{3} \mathrm{CHF}+\mathrm{O}_{2} \rightarrow$ Produkte

\section{Iodethan}

Um zeitaufgelöste FT-IR-Absorptionsmessungen durchführen zu können, wurde ein geeignetes Computerprogramm zur automatischen Befüllung der Meß- und Reaktionszelle sowie zur Ansteuerung des Photolyse-Lasers und FT-IR-Spektrometers entwickelt („FFCP 1.2“).

Damit ließ sich der Geschwindigkeitskoeffizient für die Reaktion $\mathrm{CH}_{3} \mathrm{CH}_{2}+\mathrm{O}_{2} \rightarrow \mathrm{CH}_{3} \mathrm{CH}_{2} \mathrm{O}_{2}$ aus einer zeitaufgelösten Messung direkt bestimmen:

$$
\mathrm{k}(298 \mathrm{~K})=(1,7 \pm 0,5) \cdot 10^{12} \mathrm{~cm}^{3} /(\mathrm{mol} \cdot \mathrm{s}) \quad ; \text { für: } \mathrm{p}\left(\mathrm{O}_{2}\right)=6 \mathrm{mbar}
$$





\section{Anhang}

\section{A) Standardbildungsenthalpien}

Die in dieser Arbeit verwendeten Standardbildungsenthalpien für T = 298 K sind nachstehend in Tabelle 73 aufgeführt:

\begin{tabular}{|c|c|c|}
\hline Substanz & $\Delta \mathbf{H}_{\mathrm{f}, 298 \mathrm{~K}}{ }^{2}[\mathrm{~kJ} / \mathrm{mol}]$ & Literatur \\
\hline $\mathrm{CCl}_{2}$ & 108,8 & NIST Structures and Properties (1994) \\
\hline $\mathrm{CClF}$ & 249,4 & NIST Structures and Properties (1994) \\
\hline $\mathrm{CF}$ & $-221,8$ & NIST Structures and Properties (1994) \\
\hline $\mathrm{CH}_{2} \mathrm{Cl}$ & 129,7 & NIST Structures and Properties (1994) \\
\hline $\mathrm{CH}_{2} \mathrm{Cl}_{2}$ & $-95,8$ & NIST Structures and Properties (1994) \\
\hline $\mathrm{CH}_{2} \mathrm{ClF}$ & $-259,4$ & NIST Structures and Properties (1994) \\
\hline $\mathrm{CH}_{2} \mathrm{ClO}$ & $-10,0$ & MELIUS, C.F. (1996) \\
\hline $\mathrm{CH}_{3}$ & 145,6 & NIST Structures and Properties (1994) \\
\hline $\mathrm{CH}_{3} \mathrm{Cl}$ & $-82,0$ & NIST Structures and Properties (1994) \\
\hline $\mathrm{CH}_{3} \mathrm{~F}$ & $-246,9$ & NIST Structures and Properties (1994) \\
\hline $\mathrm{CHCl}$ & 297,1 & NIST Structures and Properties (1994) \\
\hline $\mathrm{CHCl}_{2}$ & 108,8 & NIST Structures and Properties (1994) \\
\hline $\mathrm{CHCl}_{2} \mathrm{O}$ & $-17,2$ & MELIUS, C.F.(1996) \\
\hline $\mathrm{Cl}$ & 121,3 & NIST Structures and Properties (1994) \\
\hline $\mathrm{ClCHO}$ & $-185,5$ & MELIUS, C.F. (1996) \\
\hline $\mathrm{ClCO}$ & $-16,6$ & NIST Structures and Properties (1994) \\
\hline $\mathrm{ClF}$ & $-50,2$ & NIST Structures and Properties (1994) \\
\hline $\mathrm{CO}$ & $-110,5$ & NIST Structures and Properties (1994) \\
\hline $\mathrm{COCl}_{2}$ & $-221,8$ & NIST Structures and Properties (1994) \\
\hline $\mathrm{F}$ & $-50,2$ & NIST Structures and Properties (1994) \\
\hline $\mathrm{H}$ & 218,0 & NIST Structures and Properties (1994) \\
\hline $\mathrm{HCHO}$ & $-108,8$ & NIST Structures and Properties (1994) \\
\hline $\mathrm{HCl}$ & $-92,5$ & NIST Structures and Properties (1994) \\
\hline $\mathrm{HCO}$ & 44,8 & NIST Structures and Properties (1994) \\
\hline $\mathrm{HF}$ & $-272,4$ & NIST Structures and Properties (1994) \\
\hline $\mathrm{O}$ & 249,4 & NIST Structures and Properties (1994) \\
\hline $\mathrm{OH}$ & 38,9 & NIST Structures and Properties (1994) \\
\hline
\end{tabular}

Tabelle 73: Standardbildungsenthalpien 


\section{B) Lennard-Jones-Parameter}

Außer den eingeklammerten sind alle Werte entnommen aus: Reid, R.C. et al. (1987).

\begin{tabular}{|l|c|c|}
\hline \multicolumn{1}{|c|}{ Substanz } & $\sigma\left[\mathbf{1 0}^{-\mathbf{1 0}} \mathbf{m}\right]$ & $\varepsilon / \mathbf{k}_{\mathbf{B}}[\mathbf{K}]$ \\
\hline $\mathrm{He}$ & 2,55 & 10,22 \\
\hline $\mathrm{CH}_{4}$ & 3,76 & 148,6 \\
\hline $\mathrm{CH}_{2} \mathrm{Cl}_{2}$ & 4,90 & 356,3 \\
\hline $\mathrm{CH}_{3} \mathrm{Cl}$ & 4,18 & 350 \\
\hline $\left.\mathrm{CH}_{2} \mathrm{ClO}\right)$ & 4,17 & 496,1 \\
\hline$\left(\mathrm{CHCl}_{2} \mathrm{O}\right)$ & 4,53 & 467,7 \\
\hline
\end{tabular}

Tabelle 74: Lennard-Jones-Parameter

Die eingeklammerten Werte wurden folgendermaßen abgeschätzt ${ }^{201}$ :

$$
\sigma=0,785 \cdot \mathrm{V}_{\mathrm{C}}^{\frac{1}{3}} \text { und } \frac{\epsilon}{\mathrm{k}_{\mathrm{B}}}=0,897 \mathrm{~T}_{\mathrm{C}}
$$

und die dafür benötigten kritischen Werte wurden aus Inkrementtabellen nach Jobak $\left(\mathrm{V}_{\mathrm{C}}\right)$ bzw. Fedor $\left(\mathrm{T}_{\mathrm{C}}\right)$ ermittelt ${ }^{202}$, wobei für den enthaltenen Sauerstoff näherungsweise das Inkrement ,-O-“ verwendet wurde:

$$
\mathrm{V}_{\mathrm{C}}=17,5+\sum \Delta_{\mathrm{V}} \quad \mathrm{T}_{\mathrm{C}}=535 \cdot \log \left(\sum \Delta_{\mathrm{T}}\right)
$$

\begin{tabular}{|c|c|c|c|c|}
\hline Inkrement & $\mathrm{CH}_{2}$ & $\mathrm{CH}-$ & $-\mathrm{Cl}$ & $-\mathrm{O}-$ \\
\hline$\Delta_{\mathbf{V}}$ & 56 & 41 & 58 & 18 \\
\hline$\Delta_{\mathbf{T}}$ & 0,45 & 1,34 & $\begin{array}{c}4,2 \text { (monosubst.) } \\
3,71 \text { (disubst.) }\end{array}$ & 1,56 \\
\hline
\end{tabular}

Mit diesen Werten erhält man:

\begin{tabular}{|c|c|c|}
\hline & $\mathbf{V}_{\mathbf{C}}\left[\mathbf{c m}^{3} / \mathbf{m o l}\right]$ & $\mathbf{T}_{\mathbf{C}}[\mathbf{K}]$ \\
\hline$\left(\mathbf{C H}_{2} \mathbf{C l O}\right)$ & 149,5 & 553,1 \\
\hline$\left(\mathbf{C H C l}_{2} \mathbf{O}\right)$ & 192,5 & 521,36 \\
\hline
\end{tabular}




\section{C) Fehlerrechnung}

(i) Mittlerer Fehler des Mittelwertes der Intensitäten $(\Delta \mathrm{I})$ :

Aus den $n$ einzelnen Meßwerten $I_{i}($ für $i=1$.. n) und dem arithmetischen Mittelwert I erhält man:

$$
\Delta I=\frac{t_{n-1, \alpha} \cdot \sqrt{\frac{1}{(n-1)} \sum_{i=1}^{n}\left(I_{i}-I\right)^{2}}}{\sqrt{n}}
$$

wobei $\mathrm{t}_{\mathrm{n}-1, \alpha}$ der Student'sche Faktor für das Konfidenzintervall zum Niveau 1- $\alpha$ ist; für letzteres wurde in der vorliegenden Arbeit der Wert „95 \%“verwendet.

(ii) Fehler der Geschwindigkeitskoeffizienten $(\Delta \mathrm{k})$ :

Mit der Standardabweichung des relativen Geschwindigkeitskoeffizienten $\left(\Delta \mathrm{k}_{\mathrm{rel}}\right)$ errechnet man den Fehler des absoluten Geschwindigkeitskoeffizienten gemäß

$$
\Delta \mathrm{k}=\sqrt{\left(\mathrm{k}_{\mathrm{ref}} \cdot \Delta \mathrm{k}_{\mathrm{rel}}\right)^{2}+\left(\mathrm{k}_{\mathrm{rel}} \cdot \Delta \mathrm{k}_{\mathrm{ref}}\right)^{2}}
$$

(iii) Fehler der präexponentiellen Faktoren $(\Delta \mathrm{A})$ :

Mit der Standardabweichung des Achsenabschnitts $(\Delta \mathrm{a})$ aus der Auftragung $\ln \left(\mathrm{k}_{\mathrm{rel}}\right)$ vs. $\mathrm{T}^{-1}$ erhält man:

$$
\Delta \mathrm{A}=\sqrt{\left(\mathrm{e}^{\mathrm{a}} \cdot \mathrm{A}_{\text {ref }} \cdot \Delta \mathrm{a}\right)^{2}+\left(\mathrm{e}^{\mathrm{a}} \cdot \Delta \mathrm{A}_{\text {ref }}\right)^{2}}
$$

(iv) Fehler der Aktivierungsenergien $\left(\Delta \mathrm{E}_{\mathrm{a}}\right)$ :

Mit der Standardabweichung der Steigung $(\Delta b)$ aus der Auftragung $\ln \left(\mathrm{k}_{\mathrm{rel}}\right)$ vs. $\mathrm{T}^{-1}$ erhält man:

$$
\Delta \mathrm{E}_{\mathrm{a}}=\sqrt{\left(\Delta \mathrm{E}_{\mathrm{a}, \text { ref. }}\right)^{2}+(-\mathrm{R} \cdot \Delta \mathrm{b})^{2}}
$$

(v) Fehler der Vorfaktoren $(\Delta \mathrm{A})$ :

Mit der Standardabweichung des Achsenabschnitts $(\Delta \mathrm{a})$ aus der Auftragung $\ln \left(\mathrm{k}_{\mathrm{rel}}\right)$ vs. $\ln (\mathrm{T})$ erhält man:

$$
\Delta \mathrm{A}=\sqrt{\left(\mathrm{e}^{\mathrm{a}} \cdot \mathrm{A}_{\text {ref }} \cdot \Delta \mathrm{a}\right)^{2}+\left(\mathrm{e}^{\mathrm{a}} \cdot \Delta \mathrm{A}_{\text {ref }}\right)^{2}}
$$

(vi) Fehler der Parameter $\mathrm{n}(\Delta \mathrm{n})$ :

Mit der Standardabweichung der Steigung $(\Delta \mathrm{b})$ aus der Auftragung $\ln \left(\mathrm{k}_{\mathrm{rel}}\right)$ vs. $\ln (\mathrm{T})$ erhält man: 


$$
\Delta \mathrm{n}=\sqrt{(\Delta \mathrm{b})^{2}+\left(\Delta \mathrm{n}_{\text {ref }}\right)^{2}}
$$

(vii) Fehler der Geschwindigkeitskoeffizienten aus einer Arrhenius-Gl. ( $\Delta \mathrm{k})$ :

Mit der Standardabweichung des präexponentiellen Faktors $(\Delta \mathrm{A})$ sowie der der Aktivierungsenergie $\left(\Delta \mathrm{E}_{\mathrm{a}}\right)$ folgt:

$$
\Delta \mathrm{k}=\sqrt{\left(\mathrm{e}^{\frac{-\mathrm{E}_{\mathrm{a}}}{\mathrm{R} \cdot \mathrm{T}}} \cdot \Delta \mathrm{A}\right)^{2}+\left(\mathrm{A} \cdot \frac{-1}{\mathrm{R} \cdot \mathrm{T}} \cdot \mathrm{e}^{\frac{-\mathrm{E}_{\mathrm{a}}}{\mathrm{R} \cdot \mathrm{T}}} \cdot \Delta \mathrm{E}_{\mathrm{a}}\right)^{2}}
$$

(viii) Fehler der Geschwindigkeitskoeffizienten der Radikal-Kombination $(\Delta \mathrm{k})$ :

Mit der Standardabweichung der Steigung $(\Delta b)$ der Auftragung gemäß Gleichung (8) (S. 9) sowie derjenigen der Radikalanfangskonzentration $\left(\Delta c:=\Delta[R]_{0}\right)$ folgt:

$$
\Delta \mathrm{k}=\sqrt{\left(\frac{1}{2 \cdot \mathrm{c}} \cdot \Delta \mathrm{b}\right)^{2}+\left(\frac{-\mathrm{b}}{2 \cdot \mathrm{c}^{2}} \cdot \Delta \mathrm{c}\right)^{2}} \quad \text { mit: } \mathrm{c}:=[\mathrm{R}]_{0}
$$




\section{Literaturverzeichnis}

Atkins, P.W.

Physikalische Chemie; 2. korrigierter Nachdruck der 1. Aufl.; VCH Verlag; Weinheim 1990.

Atkinson, D.B.; Hudgens, J.W.

Chemical kinetic studies using ultraviolet cavity ring-down spectroscopic detection: selfreaction of ethyl and ethylperoxy radicals and the reaction $\mathrm{O} 2+\mathrm{C} 2 \mathrm{H} 5=\mathrm{C} 2 \mathrm{H} 5 \mathrm{O} 2$;

J. Phys. Chem. A: 101, 3901-3909 (1997).

Atkinson, R.; Baulch, D.L.; Cox, R.A.; Hampson, R.F.,Jr.; Kerr, J.A.; Rossi, M.J.; Troe, J. Evaluated kinetic, photochemical and heterogeneous data for atmospheric chemistry: supplement V, IUPAC subcommittee on gas kinetic data evaluation for atmospheric chemistry; J. Phys. Chem. Ref. Data 26, 521-1011 (1997).

Bartels, M.; Heinemann, P.; Hoyermann, K.

The reactions of hydrogen atoms with cyclo- $\mathrm{C} 3 \mathrm{H} 5$ and cyclo- $\mathrm{C} 5 \mathrm{H} 9$ radicals: Rates and mechanisms at low pressure; Symp. Int. Combust. Proc. 20, 723 (1985).

Carey, F.A.; Sundberg, R.J.

Organische Chemie: Ein weiterführendes Lehrbuch; Hrsg. von G. Erker; VCH Verlag; Weinheim 1995.

Chesick, J.P.

The kinetics of thermal isomerization of methylcyclopropane; J. Am. Chem. Soc. 82, 3277-3284 (1960).

Dalacker, V.; Hopf, H.

Tetrahedron Lett.; 1974, 15.

DeMore, W.B.; Sander, S.P.; Golden, D.M.; Hampson, R.F.; Kurylo, M.J.; Howard, C.J.; Ravishankara, A.R.; Kolb, C.E.; Molina, M.J. ;

Chemical kinetics and photochemical data for use in stratospheric modeling. Evaluation number 12; JPL Publication 97-4, (1997).

Dhooge, P.; Glass, S.; Nimitz, J.

Nonflammable, Nonaqueous, Low Atmosheric Impact, High Performance Cleaning Solvents; Proceedings of the 4th Aerosspace Materials; Processes, and Environmental Technology Conference; Huntsville, AL; September 18-20, 2000

(http://www.etec-nm.com/library/ampet00e.htm).

Dietz, W.; Neusser, H.J.; Boesel, U.; Schlag, E.W.; Lin, S.N.

Chem. Phys. 66, 105 (1982).

Dorofeev, Y.; Hack, W.; Hold, M.; Hoyermann, K.; Morozov, I.; Vasiliev E.

Atmospheric reactions of 2,2,2-Trifluorethanol; to be published in Russian „Doclady

Physical Chemistry“ 2001.

Fügener, M.

Die Reaktion von Trichlorethen mit atomarem Fluor; Hausarbeit im Rahmen der Ersten Staatsprüfung für das Lehramt an Gymnasien; Univ. Göttingen 2001. 
Gieseking, T.

Massenspektrometrische Untersuchungen der Reaktionen von Fluoratomen mit ungesättigten Kohlenwasserstoffen in der Gasphase; Hausarbeit im Rahmen der Ersten Staatsprüfung für das Lehramt an Gymnasien; Univ. Göttingen 2001.

Heerdt, W.

Die Reaktion von Dichlorethen mit atomarem Fluor; Hausarbeit im Rahmen der Ersten Staatsprüfung für das Lehramt an Gymnasien; Univ. Göttingen 2001.

Heinemann, P.

Dissertation; Univ. Göttingen 1986.

Hellwig, J.

Dissertation; Univ. Göttingen 1999.

Hesse, M.; Meier, H.; Zeeh, B.

Spektroskopische Methoden in der organischen Chemie;

5. Auflage; Thieme Verlag; Stuttgart/New York 1995.

Hippler, H.; Troe, J.; Wendelken, H.J.

J. Chem. Phys.; 1983, 78(11), S. 6709-6717.

Hold, M.

Diplomarbeit; Univ. Göttingen 1997.

Hold, M.

Programmbeschreibung für GCMS-CP Version 1.4; Univ. Göttingen 2001;

(im Anschluß an diese Arbeit abgedruckt).

Hold, M.

Programmbeschreibung für WinSiccon Version 1.7; Univ. Göttingen 2001

(im Anschluß an diese Arbeit abgedruckt).

Hold, M.

Programmbeschreibung für FFCP Version 1.2; Univ. Göttingen 2001

(im Anschluß an diese Arbeit abgedruckt).

Hopf, H.; Wachholz, G.; Walsh, R.; de Meijere, A.; Teichmann

Chem. Ber.; 1989, 122, S. 377-382.

Hopf, H.; Wachholz, G.

Chem. Ber.; 1987, 120, S. 1259-1261.

Hou, H.; Wang, B.; Gu, Y.

Theoretical Investigation of the $\mathrm{O}(3 \mathrm{P})+\mathrm{CHX} 2(\mathrm{X}=\mathrm{F}, \mathrm{Cl})$ Reactions;

J. Phys. Chem. A 103, 8075-8081 (1999).

Howard, C.J.

J. Phys. Chem.; 1979, 83, S. 3. 
Hoyermann, K.; Nacke, F.

Elementary reactions of the methoxymethyl radical in the gas phase: $\mathrm{CH} 3 \mathrm{OCH} 3+\mathrm{F}$,

$\mathrm{CH} 2 \mathrm{OCH} 3+\mathrm{CH} 2 \mathrm{OCH} 3, \mathrm{CH} 2 \mathrm{OCH} 3+\mathrm{O} 2$ and $\mathrm{CH} 2 \mathrm{OCH} 3+\mathrm{O}$;

Symp. Int. Combust. Proc. 26, 505-512 (1996).

Hoyermann, K.

Primärprodukte von Elementarreaktionen aus der Kohlenwasserstoffoxidation,

Habilitation, Univ. Göttingen 1979.

Hoyermann, K.

in: Physical Chemistry, An Advanced Treatise, (Jost, W.; Ed.); Vol. VI B,

S. 931; Academic Press, 1975.

Huheey, J.

Anorganische Chemie - Prinzipien von Struktur und Reaktivität; deGruyter-Verlag,

Berlin / New York 1988.

Karger, G.

Chemie in unserer Zeit; 1993, 27, S. 174-175.

Kersten, C.

Dissertation; Univ. Göttingen 1999.

Khatoon, T.; Edelbuttel-Einhaus, J.; Hoyermann, K.; Wagner, H.Gg. Rates and mechanisms of the reactions of ethanol and propanol with fluorine and chlorine atoms; Ber. Bunsenges. Phys. Chem. 93, 626 (1989).

Leibnitz, E.; Struppe, H.

Handbuch der Gaschromatographie; 3. Aufl.; Verlag Geest\&Portig; Leipzig 1984.

Lide, D. (ed.)

Handbook of Chemistry and Physics; 72nd edition; CRC Press; Boca Raton 1991

Louis, F.; Sawerysyn, J.

Kinetics and products studies of reactions between fluorine atoms and $\mathrm{CHF} 3, \mathrm{CHClF} 2$, CHCl2F and CHCl3; J. Chem. Soc. Faraday Trans. 94, 1437-1445 (1998).

Louis, F.; Talhaoui, A.; Sawerysyn, J-P.; Rayez, M-T.; Rayez, J-C.

Rate coefficients for the gas phase reactions of CF3CH2F (HFC-134a) with chlorine and fluorine atoms: experimental and ab initio theoretical studies; J. Phys. Chem. A: 101, 8503-8507 (1997)

Lowry, T.; Richardson, K.

Mechanismen und Theorie in der Organischen Chemie; Verlag Chemie; Weinheim 1980.

March, J.

Advanced Organic Chemistry - Reactions, Mechanisms and Structure; 4th edition;

Wiley\&Sons; New York 1992.

Maricq, M.M.; Szente, J.J.; Hurley, M.D.; Wallington, T.J.

Atmospheric chemistry of HFC-134a: kinetic and mechanistic study of the CF3CFHO2 + HO2 reaction; J. Phys. Chem. 98, 8962-8970 (1994). 
Maricq, M.M.; Szente, J.J.; Kaiser, E.W.

A kinetic study of the CF3CFH recombination and $\mathrm{O} 2$ addition reactions;

Chem. Phys. Lett. 197, 149-156 (1992).

Mathew, L.; Warkentin, J.

The Cyclopropylmethyl Free Radical Clock. Calibration for the Range $30-89{ }^{\circ} \mathrm{C}$;

J. Am. Chem. Soc. 108, S. 7981-7984 (1986).

Melius, C.F.

BAC-MP4; Heats of formation and free energies; Sandia National Laboratories;

Livermore, California 94550, (1996).

Morozov, I; Nielsen, C.; Papagiannakopoulos, P.; Sidebottom, H.

IAFAEE: Impact of Alternative Fluorinated Alcohols and Ethers on the Environment - a laboratory and modelling study; A research project financed by the Commission of the European Communities under the Energy, Environment and Sustrainable Development Programme through contract EVK2-CT-1999-00009; Poster Lausanne 2000 (http://www.kjemei.uio.no/09_spekt/Atmosfaere/IAFAEE/).

Mourits, F.M.; Rummens, F.H.A.

Can. J. Chem. 55, 3007 (1977).

Nacke, F.

Dissertation; Univ. Göttingen 1998.

Naumann, K.

Chemie in unserer Zeit; 1993, 27, S. 32-41.

Neufield, P.D.

Janzen,A.R.; Aziz, R.A.; J. Chem. Phys., 1972, 57, S. 1100.

NIST

Standard Reference Database 25; Structures and Properties; Version 2.02; Jan. 1994

NIST

Standard Reference Database Number 69 - July 2001 Release, Chemistry WebBook

(http://webbook.nist.gov/chemistry).

NIST

Standard Reference Database Series 1a; NIST/EPA/MSDC Mass Spectral Database;

Version 3.01; June 1990.

Nottingham, W.C.; Rudolph, R.N.; Andrews, K.P.; Moore, J.H.; Tossell, J.A.

Flowtube reactor study of the association reactions of $\mathrm{CHCl} 2$ and $\mathrm{CCl} 3$ with $\mathrm{O} 2$ at low pressure; Int. J. Chem. Kinet. 26, 749-756 (1994).

Pilling, M.J.; Seakins, P.W.

Reaction Kinetics; Oxford University Press; Oxford 1995.

Plumb, I. C.; Ryan, K. R.

Kinetics of the Reactions of CH3 with O(3P) and O2 at $295 \mathrm{~K}$;

Int. J. Chem. Kinet. 14, 861 (1982). 
Pouchert, Ch.P. (ed.)

The Aldrich library of infrared spectra; 2. ed.; Milwaukee 1978.

Reid, R.C.; Prausnitz, J.M.; Poling, B.E.

The Properties of Gases and Liquids; $4^{\text {th }}$ Edition; McGraw-Hill; New York 1987.

Reid, R.C.; Prausnitz, J.M.; Sherwood, T.K

The Properties of Gases and Liquids;

$3^{\text {rd }}$ Edition; McGraw-Hill; New York 1977.

Rickborn, S.F.; Rogers, D.S.; Ring, M.A.; O'Neal, H.E.

Kinetic and product studies of the thermal decomposition of dimethylsilane in a singlepulse shock tube and in a stirred flow reactor; J. Phys. Chem. 90, 408-414 (1986)

Römpp Chemie Lexikon

9. Auflage; Thieme Verlag; Stuttgart/New York 1991.

Ryan, K.R.; Plumb, I.C.

Kinetics of the reactions of $\mathrm{CF} 3$ with $\mathrm{O}(3 \mathrm{P})$ and $\mathrm{O} 2$ at $295 \mathrm{~K}$;

J. Phys. Chem. 86, 4678 (1982).

Schrader, B. (ed.)

Infrared and Raman Spectroscopy; VCH Verlag; Weinheim 1995.

Seakins, P.W.; Leone, S.R.

A laser flash photolysis/time-resolved FTIR emission study of a new channel in the reaction of $\mathrm{CH} 3$ + O: production of $\mathrm{CO}(\mathrm{v})$; J. Phys. Chem. 96, 4478-4485 (1992).

Seeba, J.

Dissertation; Univ. Göttingen 1994.

Slagle, I.R.; Sarzynski, D.; Gutman, D.

Kinetics of the reaction between methyl radicals and oxygen atoms between 294 and

900K; J. Phys. Chem. 91, 4375 (1987).

Stoliarov, S.I.; Bencsura, A.; Shafir, E.; Knyazev, V.; Slagle, I.

Kinetics of the Reaction of the $\mathrm{CHCl} 2$ Radical with Oxygen Atoms;

J. Phys. Chem. A 105, 79-81 (2001).

Streitwieser, A.; Heathcock, C.

Organische Chemie; 2. Nachdr. der 1. Aufl.; VCH Verlag; Weinheim 1990.

Tröger, U.

Dissertation; Univ. Göttingen 1997.

Ullmann's Encyclopedia of industrial chemistry

Volume A 6; VCH Verlag; Weinheim 1986.

Vakhtin, A.B.; Petrov, A.K.

Intracavity absorption of $\mathrm{He}-\mathrm{Ne}$ laser radiation as a direct time-resolved probe of $\mathrm{R}+$ $\mathrm{NO}=\mathrm{RNO}$ reaction kinetics $(\mathrm{R}=\mathrm{C} 3 \mathrm{~F} 7, \mathrm{CF} 3, \mathrm{CH} 3)$; Spectrochim. Acta 46, 603 (1990). 
Wachholz, G.

Dissertation; Univ. Braunschweig 1987.

Wang, B.; Hou, H.; Gu, Y.

Theoretical Investigation of the Reaction of $\mathrm{O}(3 \mathrm{P})$ with $\mathrm{CH} 2 \mathrm{Cl}$;

J. Phys. Chem. A 103, 2060-2065 (1999).

Wedler, G

Lehrbuch der Physikalischen Chemie; 3. Aufl.; VCH Verlag; Weinheim 1987.

Wehmeyer, J.

Dissertation; Univ. Göttingen; Veröffentlichung voraussichtlich in 2002

Zellner, R.

Chemie in unserer Zeit; 1993, 27, S. 230-236.

Zellner, R.

Chemie in unserer Zeit; 1995, 29, S. 322-323.

Zeuch, T.

Dissertation; Univ. Göttingen; Veröffentlichung voraussichtlich in 2003.

Zeuch, T.

Experimentelle Untersuchungen der Reaktionen von Alkenen mit Sauerstoff und Fluor in der Gasphase; Hausarbeit im Rahmen der Ersten Staatsprüfung für das Lehramt an Gymnasien; Univ. Göttingen 2001.

Zhang, Z.; Saini, R.D.; Kurylo, M.J.; Huie, R.E.

Rate constants for the reactions of the hydroxyl radical with several partially fluorinated ethers; J. Phys. Chem. 96, 9301-9304 (1992). 




\section{Programmbeschreibung für}

\section{GCMS-CP}

Version 1.4

(GC-MS control program)

Steuerungs- und Auswertungsprogramm für die GC-MS-Kopplung am VARIAN MAT $\mathrm{CH} 7$ - Massenspektrometer

Programmierung:

Markus Hold, 2000-2001

Dokumentation:

Markus Hold, 2001 



\section{Inhaltsverzeichnis}

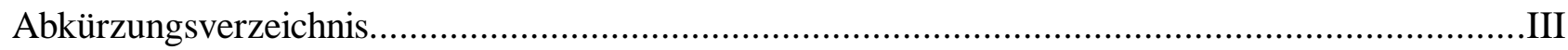

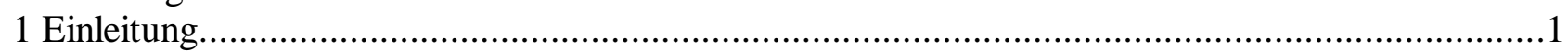

1.1 Zur Programmierung / Betriebssystemvoraussetzungen........................................... 1

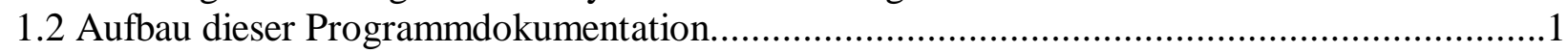

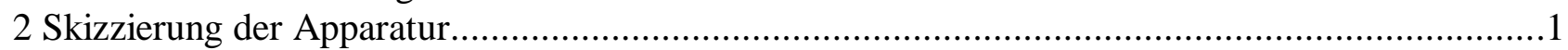

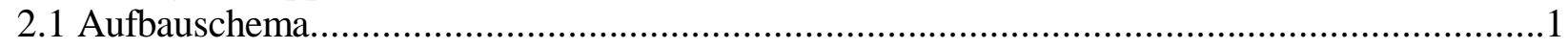

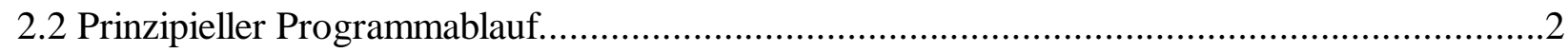

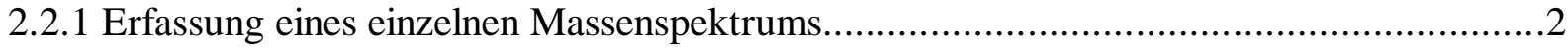

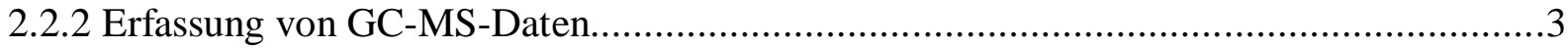

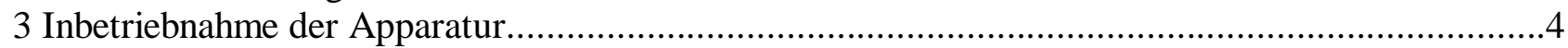

3.1 Erzeugung des Hochvakuums im MS.................................................................... 4

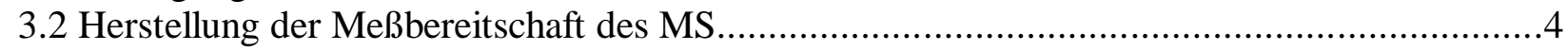

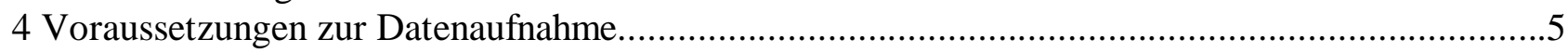

4.1 Installation der MCS-Karte und deren Software „MCS-32“..............................................5

4.2 Installation der AD-Karte und deren Software „Instacal“ .................................................5

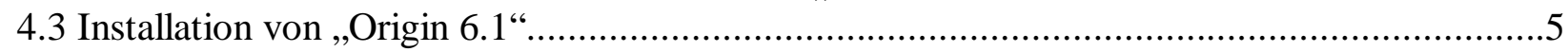

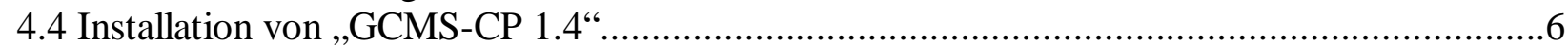

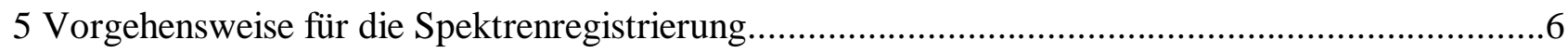

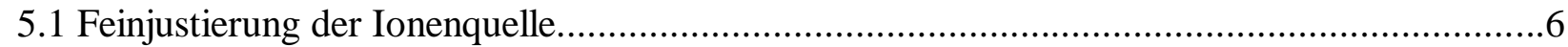

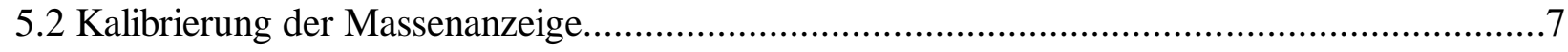

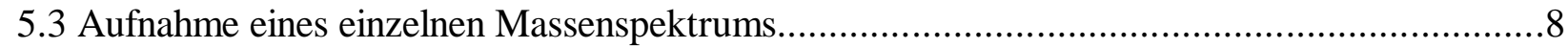

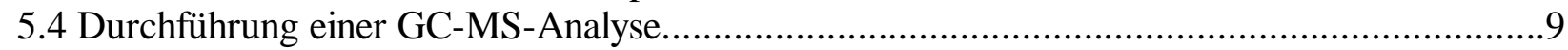

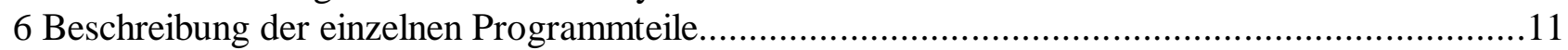

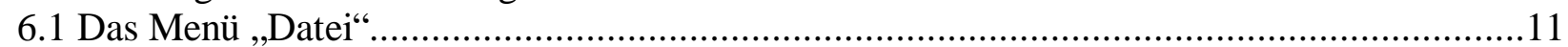

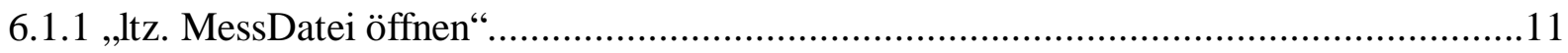

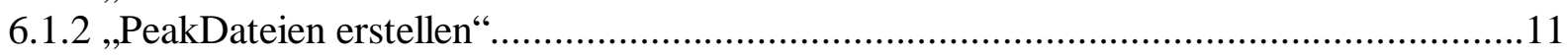

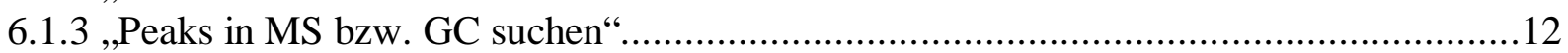

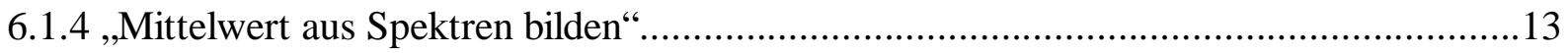

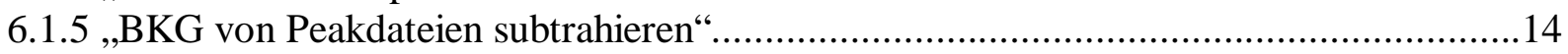

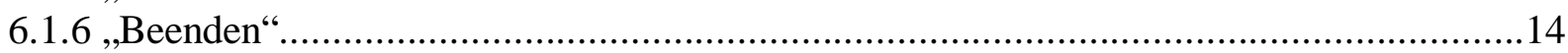

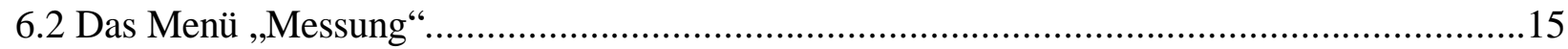

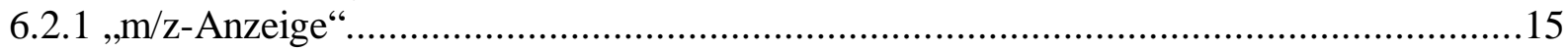

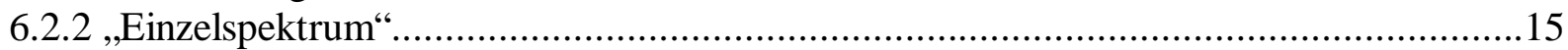

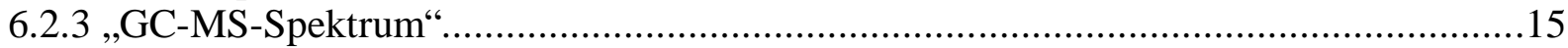

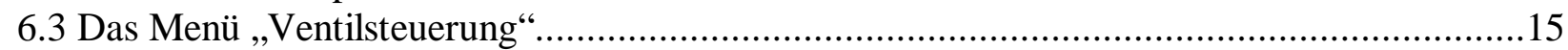

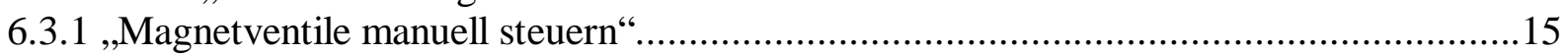

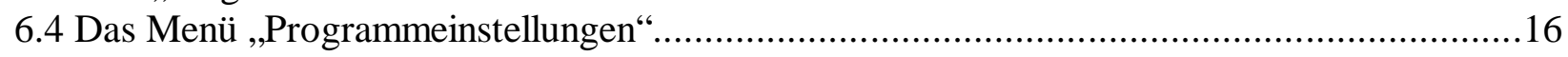

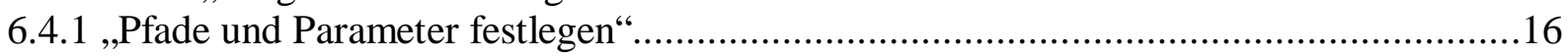

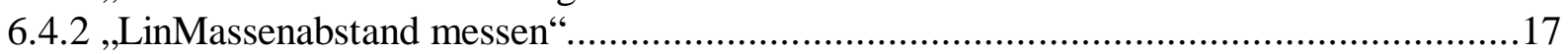

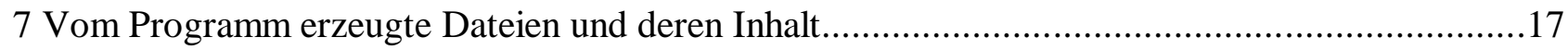

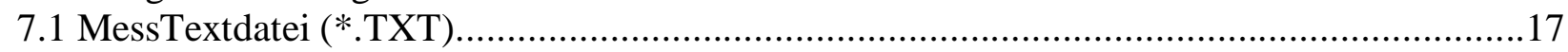

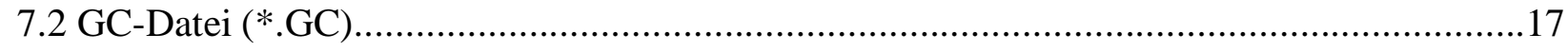

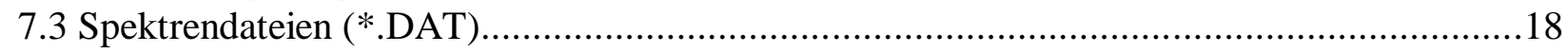

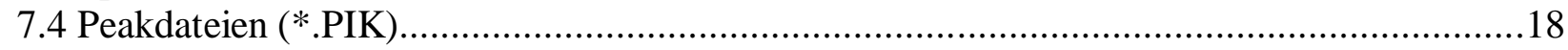

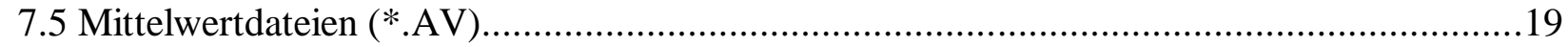

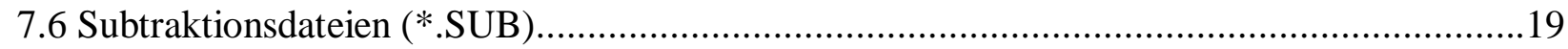

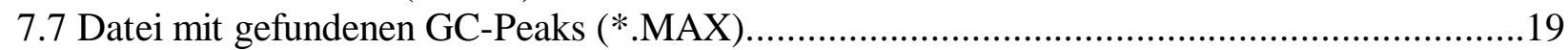

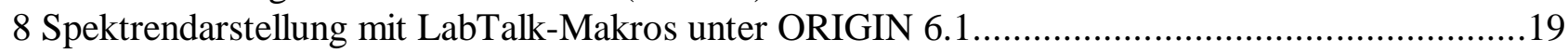




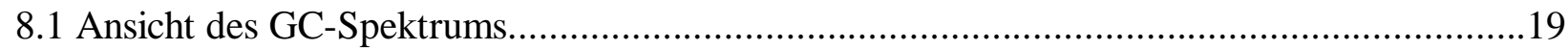

8.2 Ansicht ausgewählter MS-Spektren...................................................................21

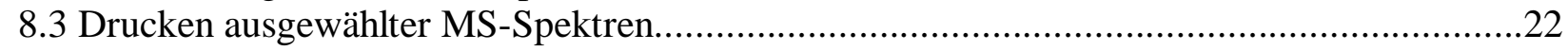

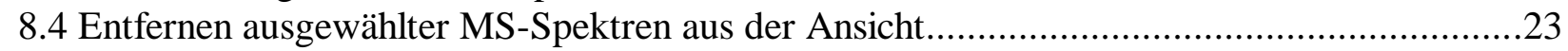

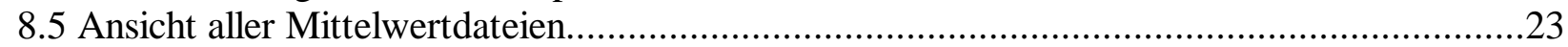

8.6 Ansicht aller MS-Spektren mit Peak im GC-Spektrum..............................................23

8.7 Skalierung der DATA-Achsen (Y-Achsen) aller MS-Spektren.....................................23

8.8 Automatische Skalierung der DATA-Achsen...........................................................24

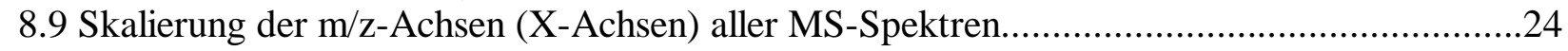

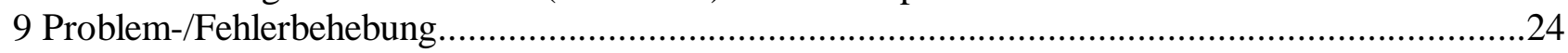

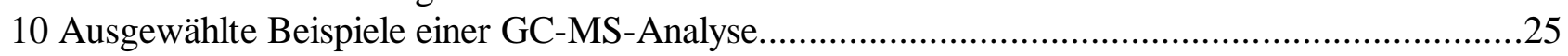

10.1 Analyse einer niedrig siedenden Erdölfraktion......................................................25

10.2 Analyse der Mischung 1,1,1,2-Tetrafluorethan/Propen/2-Buten.................................27

10.3 Analyse der Mischung 2-Chlor-2-methylpropan/1-Chlor-2-methylpropan........................28

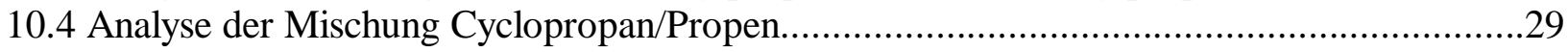

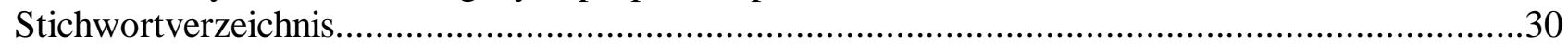




\section{Abkürzungsverzeichnis}

\begin{tabular}{|c|c|}
\hline AD-Karte & Analog-Digital-Wandler-Karte (ISA-Einsteckkarte für Computer) \\
\hline B & magnetische Flußdichte \\
\hline $\mathrm{BKG}$ & $\begin{array}{l}\text { background (Hintergrund im MS; das sind üblicherweise Peaks der Luft und } \\
\text { anderer Verunreinigungen im MS bei } \mathrm{m} / \mathrm{z} 17,18,28,32 \text { und } 40 \text {, ggf. 57) }\end{array}$ \\
\hline bzw. & beziehungsweise \\
\hline ca. & circa (= ungefähr, etwa) \\
\hline DATA-Wert & $\begin{array}{l}\text { Anzahl der NIM-Pulse, die von der MCS-Karte innerhalb der gewünschten } \\
\text { „Aufnahmezeit pro Kanal“ registriert wurden (= Anzahl der in dieser Zeit am SEV } \\
\left.\text { aufgetroffenen Ionen, falls } U_{\text {NIM-Puls }} \leq-800 \mathrm{mV}\right)\end{array}$ \\
\hline EI & elctron impact (= Elekronenstoßionisation) \\
\hline GC & Gaschromatograph \\
\hline GC-MS & $\begin{array}{l}\text { Ankopplung eines GC direkt an ein MS; dadurch erhält man für jede die GC-Säule } \\
\text { verlassende Fraktion ein Massenspektrum }\end{array}$ \\
\hline GCMS-CP & GC-MS-control-program \\
\hline ggf. & gegebenenfalls \\
\hline Kap. & Kapitel \\
\hline lt. & laut \\
\hline ltz. & letzte \\
\hline $\mathrm{m} / \mathrm{z}$ & $\begin{array}{l}\text { Verhältnis von Masse zur Ladung der im MS erzeugten Ionen; } \\
\text { in einem Sektorfeld-MS gelangen bei einem bestimmten B-Feld alle Ionen mit dem } \\
\text { der massenspektrometrischen Grundgleichung entsprechenden m/z-Verhältnis zum } \\
\text { Detektor; bei m/z } 20 \text { können z.B. } \mathrm{HF}^{+} \text {oder } \mathrm{Ar}^{2+} \text { detektiert werden. }\end{array}$ \\
\hline MCS-Karte & $\begin{array}{l}\text { multichannel-scaling-Karte (ISA-Einsteckkarte für Computer); } \\
\text { diese Karte kann in } 8192 \text { Kanälen jeweils die innerhalb einer vorgegebenen } \\
\text { Zählzeit pro Kanal ankommende Anzahl NIM-Pulse speichern. }\end{array}$ \\
\hline MS & Massenspektrometer \\
\hline NIM-Puls & $\begin{array}{l}\text { negativer Spannungspuls des um den Faktor } 200 \text { verstärkten SEV-Signals; } \\
\text { jedes am SEV auftreffende Ion erzeugt üblicherweise einen Puls in Höhe von ca. } \\
-800 \mathrm{mV} \text {. }\end{array}$ \\
\hline SEV & Sekundärelektronenvervielfacher \\
\hline$\Gamma \mathrm{TL}$ & Transistor-Transistor-Logik \\
\hline
\end{tabular}





\section{Einleitung}

\subsection{Zur Programmierung / Betriebssystemvoraussetzungen}

Das Programm „GCMS-CP“ ist in der Programmiersprache „Object-Pascal“ geschrieben und wurde als 32-Bit-Programm mit „Delphi 2.01“ der Firma Borland compiliert.

Es ist ausschließlich unter den 32-Bit-Betriebssystemen „Windows“ der Firma Microsoft lauffähig; erfolgreich getestet wurde es unter Windows 95, 98 und Windows NT 4.0, wobei letzteres empfohlen wird, weil es das vom Programm verwendete Multitasking am besten unterstützt.

\subsection{Aufbau dieser Programmdokumentation}

Nach einer Skizzierung des Apparaturaufbaus und einer kurzen Beschreibung der Inbetriebnahme des GC-MS-Gerätes, werden zunächst die Voraussetzungen für die Datenaufnahme (Installation der dazu notwendigen Meßkarten im Computer sowie der einzelnen Programme) beschrieben.

Das generelle Vorgehen zur Registrierung von Spektren mit „GCMS-CP“ wird in Kap. 5 (S. 6) dargestellt. Im Kap. 6 (S. 11) werden dann die einzelnen Programmteile ausführlich erklärt. Die Inhalte der vom Programm erzeugten Dateien sind in Kap. 7 (S. 17) erläutert.

Um die aufgenommenen Daten mühelos als Spektren darstellen und ausdrucken zu können, wurden für das Programm „Origin 6.1“ in dessen Programmiersprache „LabTalk“ einige Zusatzprogramme entwickelt, deren Verwendung in Kap. 8 (S. 19) beschrieben wird.

\section{Skizzierung der Apparatur}

\subsection{Aufbauschema}

Die nachstehende Abbildung skizziert den Aufbau der Apparatur.

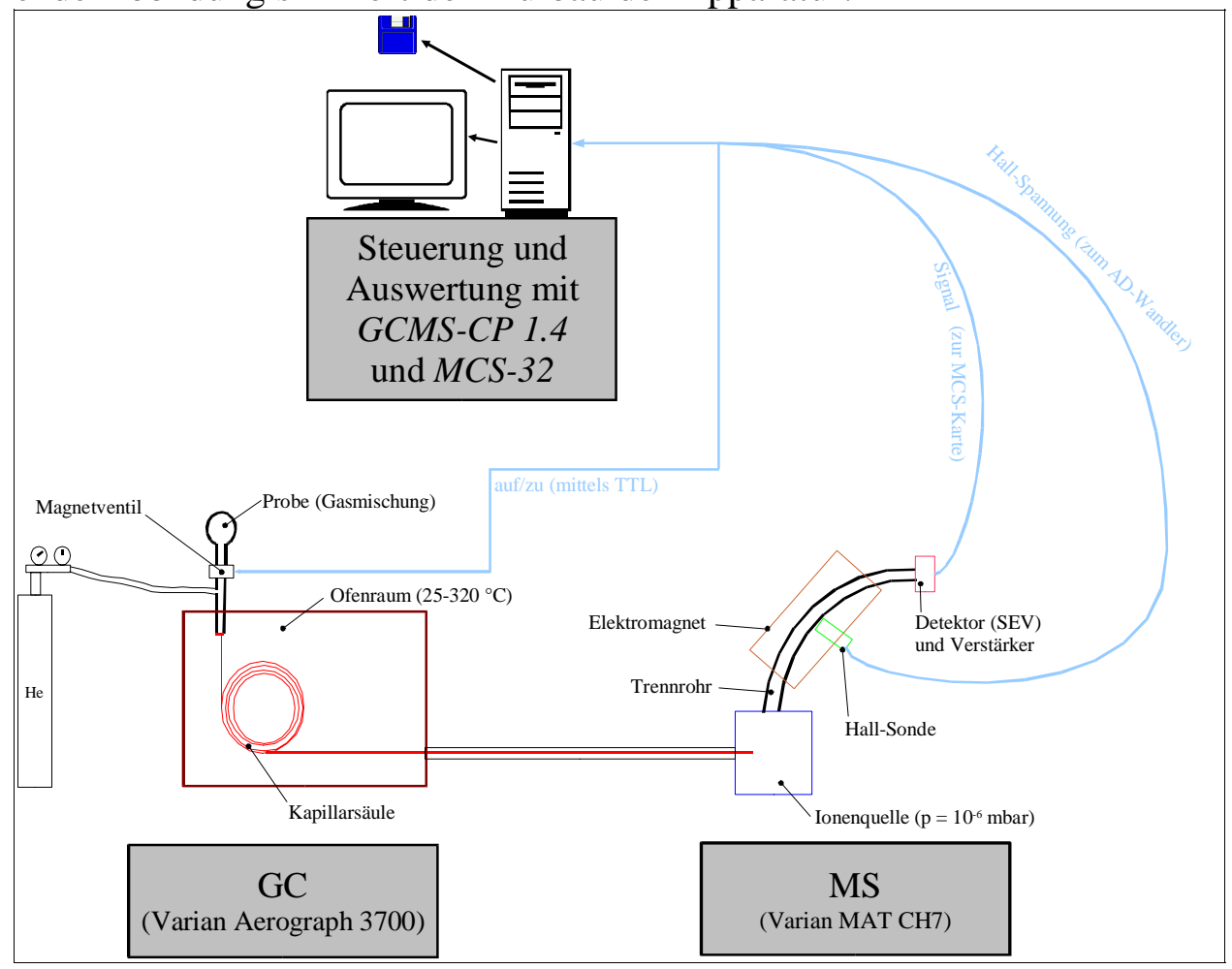


Zur Datenaufnahme wird zum einen die Spannung der Hall-Sonde über eine ADWandlerkarte (ComputerBoards; CIODAS802/16) in einem Personalcomputer (Pentium I) erfaßt, um daraus die Massenzahl zu ermitteln. Zum anderen wird das Signal, das ein am SEV auftreffendes Ion erzeugt, direkt an einen schnellen Vorverstärker (EG\&G ORTEC; VT120A) mit einer Eingangsimpedanz von $50 \Omega$ sowie einer Ansprechzeit kleiner als 1 ns geleitet und dort um den Faktor 200 verstärkt. Dadurch erzeugt ein einzelnes Ion einen NIM-Puls in einer Höhe von ca. $-800 \mathrm{mV}$. Die Pulse werden von einer $100 \mathrm{MHz}$-Zählerkarte (EG\&G ORTEC; multichannel scaling card: MCS-plus) jeweils für eine programmierte Zeit in einem einzelnen Kanal aufsummiert. Diese Zeit kann zwischen $2 \mu$ s und 30 min variiert werden. Insgesamt stehen bis zu 8192 Kanäle zur Erfassung eines Spektrums zur Verfügung. Die Programmierung der Zählerkarte erfolgt über ein mitgeliefertes Programm (EG\&G ORTEC; MCS-32 Operating Software Version 2). Dieses

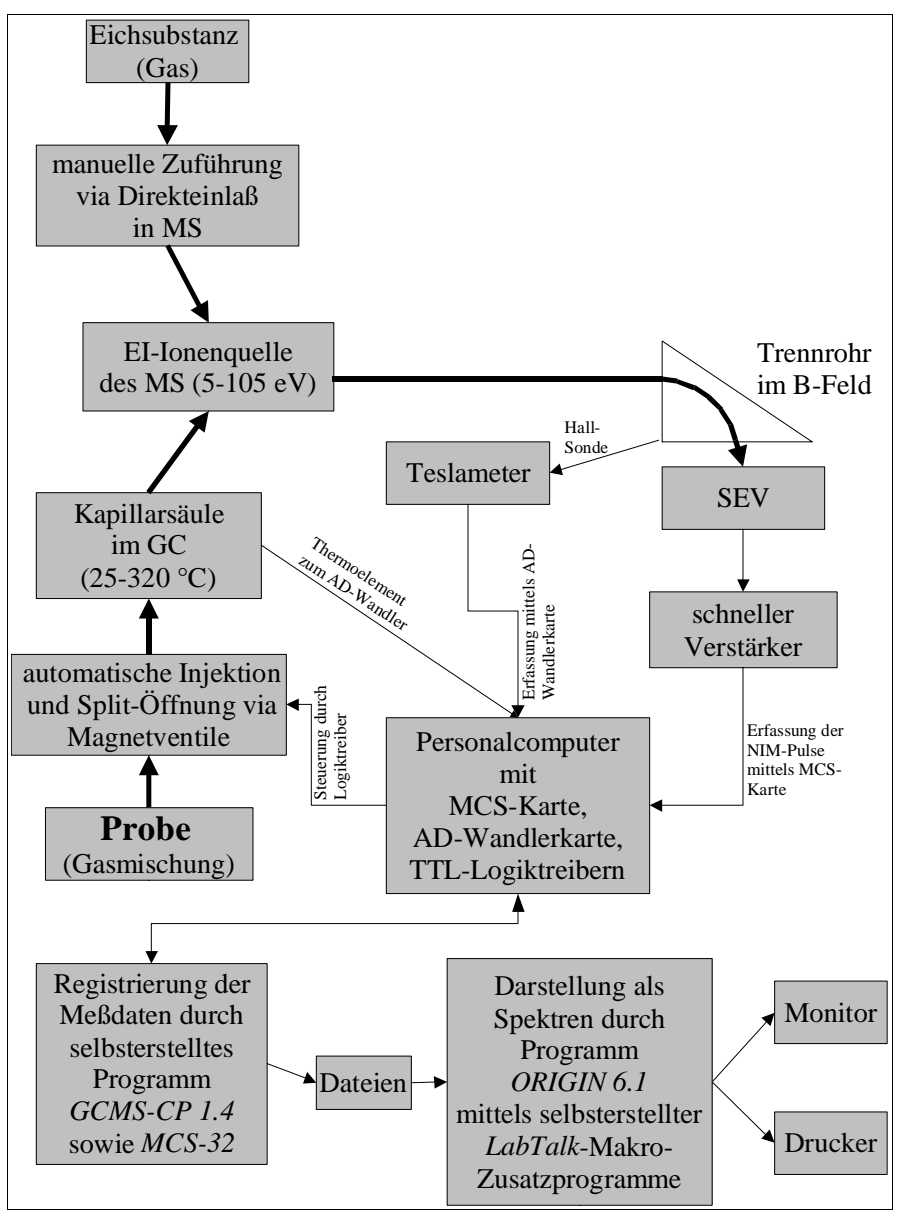
Programm wird seinerseits jedoch vom

Programm „GCMS-CP“ gesteuert, das auch die Errechnung der Massenzahl sowie die Injektion der (Gas-)Probe in das GC-MS-Gerät übernimmt. Ferner kann die Temperatur im GC-Ofen mittels eines Thermoelements ausgelesen und gespeichert werden.

\subsection{Prinzipieller Programmablauf}

\subsubsection{Erfassung eines einzelnen Massenspektrums}

Die Aufnahme einzelner Massenspektren kommt für reine Substanzen in Betracht, die über den Direkteinlaß in das MS gegeben werden (zur genauen Vorgehensweise siehe auch Kap. 5.3, S. 8).

Der Benutzer muß am MS den Magnetstrom auf eine m/z-Zahl unterhalb des zu registrierenden Bereichs einstellen und dann in dem Programm „GCMS-CP“ die Start- und Stop-m/z eingeben. Ferner hat er die Durchlaufgeschwindigkeit am MS einzustellen und diese auch im Programm zu wählen. Zudem muß er die Aufnahmezeit pro Kanal der MCS-Karte festlegen. Damit auch zwei unmittelbar benachbarte Peaks noch getrennt in verschiedenen Kanälen registriert werden können, ist diese Zeit (abhängig von der Durchlaufgeschwindigkeit) so festzulegen werden, daß mindestens ca. drei Kanäle pro durchlaufene $\mathrm{m} / \mathrm{z}$-Zahl verfügbar sind.

Wenn der Benutzer dem „GCMS-CP“-Programm den Befehl zur Aufnahme erteilt, beginnt es ständig die aktuelle m/z-Zahl zu ermitteln; d.h. das Programm wird zunächst nur in eine Aufnahmebereitschaft versetzt. Gleichzeitig startet es das „MCS-32“-Programm, teilt ihm die Aufnahmezeit pro Kanal und die Anzahl aufzunehmender Kanäle (die bis zum Erreichen der gewünschten Stop-m/z notwendig ist) mit und versetzt dann auch dieses zunächst nur in eine Aufnahmebereitschaft. 
Sobald der Benutzer nun am MS den Massendurchlauf startet, erkennt das Programm den Anstieg der m/z-Zahl und ab Erreichen der gewünschten Start-m/z beginnt es mit der tatsächlichen Aufnahme. Dazu gibt es einen kurzen 5V-TTL-Puls als Startsignal an die MCS-Karte, welche daraufhin mit der Zählung der ankommenden NIM-Pulse in die einzelnen Kanäle beginnt. Bei jedem Wechsel zwischen den einzelnen Kanälen gibt die MCS-Karte ihrerseits einen 5V-TTL-Puls aus, der als Triggersignal der AD-Karte dient. Bei jedem Triggersignal erfaßt die AD-Karte nun die HallSpannung.

Dieses aufwendig erscheinende Vorgehen mit den TTL-Pulsen hat den Vorteil, daß sowohl die ADals auch die MCS-Karte nur einmal vor Beginn der Messung von der Software Befehle zur Erzeugung der Meßbereitschaft entgegennehmen muß; danach aber können die Karten unabhängig von der Software und damit zeitlich echt parallel Daten aufnehmen. Alle so registrierten Daten werden zunächst auf der jeweiligen Karte selbst zwischengespeichert.

Erst am Ende der Messung, d.h. nach Erreichen der Stop-m/z, werden die erfaßten Rohdaten durch das „GCMS-CP“-Programm weiterverarbeitet. Die Hall-Spannung war zunächst nur als 16-Bit digitalisierter Wert gespeichert und muß daher in den analogen Spannungswert umgerechnet werden und erst danach kann aus dem m/z-Kalibrierungspolynom (siehe Kap. 5.2, S. 7 und Kap. 6.4.1, S. 16) der eigentliche $\mathrm{m} / \mathrm{z}$-Wert errechnet werden. Ebenso sind die Zählwerte der einzelnen Kanäle (im folgenden als DATA-Wert bezeichnet) zunächst nur als 32-Bit digitalisierte Werte gespeichert und müssen noch in den Dezimalwert umgewandelt werden. Schließlich werden die Ergebnisse dieser Konvertierungen in einer Datei (*.DAT) zusammengefaßt, so daß zu jedem DATA-Wert eines Kanals auch die zugehörige $\mathrm{m} / \mathrm{z}-$ Zahl angegeben ist.

\subsubsection{Erfassung von GC-MS-Daten}

Eine GC-MS-Analyse kommt für Substanz-Mischungen in Betracht. Diese werden in die Kapillarsäule des GC injiziert und dort getrennt. Die einzelnen Substanzen verlassen also nacheinander die Säule und gelangen direkt in die EI-Ionenquelle des MS.

Der „Detektor“ des GC ist in dieser Anordnung das MS. Deshalb müssen nach Injektion ständig in rascher Folge einzelne Massenspektren registriert werden, denn man weiß ja nicht, wann eine Substanz die Säule verläßt.

Aus diesem Grund wird am MS ein zyklischer Massendurchlauf mit schnellem Rücksprung eingestellt. Das MS fährt also den Magnetstrom beginnend bei der gewünschten $S t a r t-m / z$ bis zu einer eingestellten Stop-m/z hoch und fällt dann sehr rasch auf die Start-m/z zurück, um dann den Zyklus von neuem zu beginnen. Der Rücksprung ist dann besonders sehr schnell, wenn an dem MS die Option zur „Feld-Regelung“ am Einschub „BSR“ aktiviert ist.

Der Benutzer muß zunächst den eben beschriebenen zyklischer Massendurchlauf mit schnellem Rücksprung einschalten. Ferner muß er analog der im vorangegangenen Unterkapitel beschrieben Art dem ,GCMS-CP“-Programm die notwendigen Parameter mitteilen, wobei hier unbedingt auf exakte Übereinstimmung zwischen der am MS eingestellten und im Programm eingegebenen Stop$\mathrm{m} / \mathrm{z} \mathrm{zu}$ achten ist.

Wenn der Benutzer dem Programm den Startbefehl erteilt, öffnet es zunächst das Split-Ventil an der GC-Injektionskammer (um den Druck in der Kammer herabzusetzen), dann öffnet es ganz kurz das Ventil zur Injektion der Substanzmischung und schließt danach wieder das Split-Ventil.

Das Programm prüft nun, ob die m/z-Zahl unter die Start-m/z fällt und gibt beim nächsten Erreichen der Start-m/z das Signal zur Datenaufnahme. Dann werden automatisch in rascher Folge einzelne Massenspektren registriert (analog der im vorangegangenen Unterkapitel beschriebenen Art).

Das Problem ist jedoch, daß der Speicherplatz auf der AD- und MCS-Karte lediglich für ein 
einzelnes Spektrum ausreicht. Daher müssen zwischen den einzelnen Massenspektren die Rohdaten (d.h. die digitalisierten Werte) vom „MCS-32“- und vom „GCMS-CP“-Programm aus den Karten ausgelesen und auf der Festplatte zwischengespeichert werden. Dieser Vorgang erfordert jedoch etwas Rechenzeit, weil die Karten nicht echt-, sondern nur quasi-parallel ausgelesen werden können (deshalb ist das Betriebssystem Windows NT auch geeigneter, weil es das Multitasking wesentlich besser verwaltet). Da die Zeit für den Rücksprung von der Stop-m/z auf die Start-m/z alleine bei langsamen Computern für dieses Zwischenspeichern nicht ausreicht, muß man dem Programm eine zusätzliche empirisch zu bestimmende Verzögerungszeit (siehe Kap. 6.4.1, S. 16) zugestehen. Sie beträgt z.B. auf einem „80486-PC“ unter „Windows 95“ca. 1300 ms, auf einem „Pentium I“-PC unter „Windows NT 4.0 SP 1“ ohne aktive Netzwerkverbindungen ca. 300 ms. Bei dem derzeit eingesetzten „Pentium I / 166 MHz“-PC unter „Windows NT 4.0 SP 6“ mit aktiven Netzwerkverbindungen beträgt sie $500 \mathrm{~ms}$.

Erst am Ende der Datenaufnahme werden die Rohdaten der einzelnen Massenspektren in die eigentlichen Werte konvertiert und in fortlaufend numerierten Dateien gespeichert. Dabei wird auch die Summe der DATA-Werte über alle m/z-Werte in jedem einzelnen Massenspektrum ermittelt. Letztere wird für jedes Massenspektrum zusammen mit der Nummer dieses Spektrums und der Zeit seit Injektion in die Kapillarsäule sowie der Temperatur im GC-Ofen in einer separaten Datei (*.GC) gespeichert; sie stellt das GC-Spektrum dar (siehe auch Kap. 7, S. 17).

\section{Inbetriebnahme der Apparatur}

\subsection{Erzeugung des Hochvakuums im MS}

In den GC sollte die Kapillarsäule eingebaut und mittels eines Übergangsstücks an das MS gekoppelt werden (die Abdichtung zwischen Säule und Übergangsstück erfolgt mit Graphit-Pulver). Dabei endet die Säule unmittelbar vor der Ionenquelle des MS. Damit die Kapillarsäule nicht ins Vakuum „ausblutet“, muß sie während des Betriebs des MS ständig mit He durchspült werden.

Es empfiehlt sich folgendes Vorgehen beim Einschalten:

(a) He-Fluß durch die Kapillarsäule einstellen (ca. 1,2 bar abs. Vordruck), wobei der Zufluß in die Injektionskammer mit einem „Hoke“-Ventil (ca. 10 Umdrehungen) begrenzt werden sollte.

(b) Kühlwasser für die Turbomolekularpumpen und die Elektromagnete einschalten (ca. 0,8 bar).

(c) Die Vorpumpe zu den Turbomolekularpumpen starten und das MS damit vorevakuieren.

(d) Am MS-Einschub „BSG“ die Taste „Vorvakuum“ und die Taste „Hochvakuum“ drücken.

(e) Sobald die Drehzahl der Turbomolekularpumpen im grünen Bereich liegt, kann mit der Taste „Sicherheit“" am Einschub „BSG“ die Druckanzeige eingeschaltet werden.

Der Druck sollte einige Minuten nach dem Einschalten bei $5 \cdot 10^{-5}$ Torr liegen und innerhalb von $24 \mathrm{~h}$ auf ca. $2 \cdot 10^{-6}$ Torr sinken (auch trotz He-Fluß durch die Kapillarsäule in das MS).

Entgegen der Bedienungsanleitung zum MS wird mit der Taste „Vorvakuum“ nicht die Vorpumpe eingeschaltet, ferner wurde der Wasserdruckwächter überbrückt (d.h. außer Funktion gesetzt).

\subsection{Herstellung der Meßbereitschaft des MS}

Sobald das Hochvakuum im MS erzeugt wurde (der Druck muß kleiner $10^{-4}$ Torr sein), kann die Meßbereitschaft des MS hergestellt werden. Dazu sind folgende Schritte auszuführen:

(a) Spannungsversorgung für die externe Hall-Sonde einschalten. 
(b) Spannungsversorgung für den ORTEC-Verstärker einschalten.

(c) Am Einschub „BSG“ die Taste „Elektronik“ drücken

(d) Am Einschub „BSG“ die Taste „Ionenquelle“ drücken. Kurz danach muß am Einschub „BCT“ die Hochspannung von ca. $3 \mathrm{kV}$ angezeigt werden.

(e) Am Einschub „BCE“ mit dem Drehknopf „Kathodenstrom“ ganz langsam bis zum Anschlag den Strom durch die Glühkathode hochregeln. Die Anzeige sollte nun ca. 5 A anzeigen (evtl. muß zur Aktivierung der Anzeige der rote Knopf am Drehschalter „Emissionsstrom“ etwas bewegt werden $=>$ Wackelkontakt).

(f) Am Einschub „BSH“ den SEV einschalten.

Der Magnetstrom kann am Einschub „BSR“ geregelt werden.

\section{Voraussetzungen zur Datenaufnahme}

\subsection{Installation der MCS-Karte und deren Software „MCS-32“}

In den Computer muß die „MCS-plus“-Karte eingebaut sein. Dann ist das mitgelieferte Programm „MCS-32 v. 2“ gemäß Handbuch zu installieren, wobei der „MCS-plus“-Karte die Board-Nr. 1 zugewiesen werden muß. Das Installationsverzeichnis kann beliebig gewählt werden, jedoch sollte man es sich notieren, weil es später in dem Programm „GCMS-CP“ nochmals angegeben werden muß (siehe Kap. 6.4.1, S. 16). Der ORTEC-Verstärker „VT120A“ ist zwischen SEV-Ausgang und der Eingangs-BNC-Buchse für NIM-Pulse der „MCS-plus“-Karte einzubauen.

In das Installationsverzeichnis sollte zusätzlich die Datei „JUSTIEREN.JOB“ kopiert werden, die auf der „GCMS-CP“-Installationsdiskette gespeichert ist. Es kann nun eine Verknüpfung zu dem Programm „MCS32.EXE“ angelegt werden, in der das Programm mit dem Startparameter ,,-J Justieren.JOB“" gestartet wird; wenn z.B. „MCS32.EXE“ im Verzeichnis „C:IProgrammelMcs32l“ installiert ist, hat man bei der neu erstellten Verknüfung als Ziel einzugeben:

\section{C:IProgrammelMcs32\Mcs32.exe -J C:ProgrammelMcs32\Justieren.JOB}

\subsection{Installation der AD-Karte und deren Software „Instacal““}

In den Computer muß die ComputerBoards-AD-Wandlerkarte „CIO-DAS802/16“ eingebaut sein. Dann ist das mitgelieferte Programm „InstaCal Version 5.11“ gemäß Handbuch zu installieren, wobei der AD-Wandlerkarte unbedingt die Board-Nr. 0 zugewiesen werden muß. Das Installationsverzeichnis kann beliebig gewählt werden.

Der Ausgang „Ch A Out“ der MCS-Karte ist nun mit dem Eingang „IR Inp“ der AD-Karte zu verbinden. Ferner ist einer der 5V-TTL-Logik-Ausgänge (z.B. „DOut 1“) der AD-Karte mit dem Eingang „Start In“ der MCS-Karte zu verbinden; die Nr. des TTL-Ausgangs ist in dem Programm „GCMS-CP“ anzugeben (siehe Kap. 6.4.1, S. 16).

Die übrigen drei 5V-TTL-Logik-Ausgänge der AD-Karte sind mit den TTL-Schaltern für die Magnetventile zur Injektion, zur Split-Öffnung sowie zum Einlaß des Trägergases in die ProbenVorratskammer zu verbinden und die jeweilige DOut-Nr. im Programm „GCMS-CP“ anzugeben (siehe Kap. 6.4.1, S. 16).

\subsection{Installation von ,Origin 6.1“}

Das zur Spektrendarstellung verwendete Programm „Origin 6.1“ muß zunächst lt. Anweisung des 
Handbuches installiert werden. Das Installationsverzeichnis kann beliebig gewählt werden.

Zusätzlich ist an beliebig wählbarem Ort auf der Festplatte ein Verzeichnis für die zusätzlichen LabTalk-Makro-Skripte neu anzulegen. In dieses Verzeichnis sind alle auf der „GCMS-CP“Installationsdiskette im Verzeichnis „ORIGIN_SKRIPTE“ vorhandenen Dateien zu kopieren; im einzelnen sind dies: „GCMS_SKRIPT.OGS“, „GCMS_GC.OTP“, „GCMS_MS.OTP“, „GC_DATA_mit_Skript.OTW“.

In dem „Origin 6.1“-Installationsverzeichnis muß die vorhandene Datei „CUSTOM.OGS“ durch die auf der „GCMS-CP“-Installationsdiskette vorhandene gleichnamige Datei ersetzt (= überschrieben) werden.

Ferner muß von der „GCMS-CP“-Installationsdiskette in das „Origin 6.1“-Installationsverzeichnis die Datei „GCMS_IMP.INI“" kopiert werden. Jedoch muß diese Datei nun (z.B. mit dem WindowsEditor) geöffnet und der Pfad zu dem soeben selbst erstellten Verzeichnis mit den LabTalk-MakroSkripten eingegeben werden. Wenn diese z.B. in „C:IPROGRAMMElORIGIN_SKRIPTE“ gespeichert sind, muß die Datei „GCMS_IMP.INI“ auf folgenden Inhalt geändert werden:

[Pfade]

SKRIPTE=C:PROGRAMMEIORIGIN_SKRIPTE\

Es ist zu beachten, daß die Pfadangabe mit einem ,।“ enden muß.

\subsection{Installation von „GCMS-CP 1.4““}

An beliebig wählbarem Ort auf der Festplatte ist ein Vezeichnis für das Programm neu anzulegen. Dorthin sind von der „GCMS-CP“-Installationsdiskette die Dateien aus dem Verzeichnis „PROGRAMMDATEIEN“ zu kopieren. Es empfiehlt sich, zu der Programmdatei „GCMS_CP.EXE“ eine Verknüpfung im Windows-StartMenü oder auf dem Windows-Desktop anzulegen.

Nach dem ersten Start müssen nun im Programmteil „Programmeinstellungen / Pfade und Parameter festlegen“ die notwendigen Angaben zur Verkabelung und zu dem Verzeichnis des „MCS-32“Programms gemacht werden (siehe dazu Kap. 6.4.1, S. 16).

\section{Vorgehensweise für die Spektrenregistrierung}

\subsection{Feinjustierung der Ionenquelle}

Nachdem die Apparatur gemäß Kap. 3 (S. 4) in Betrieb genommen und die Meßbereitschaft hergestellt wurde, sollte nach einer Aufwärmzeit von mindestens 30 min zunächst die Feinjustierung der Ionenquelle vorgenommen werden.

Dazu startet man das „MCS-32“-Programm durch die im Kap. 4.1 (S. 5) erstellte Verknüpfung, wodurch automatisch die Zählung mit 200 ms pro Kanal gestartet wird. Es werden 260 Kanäle am Bildschirm angezeigt und das Programm fängt nach jedem Durchgang wieder bei Kanal 1 an zu zählen und überschreibt damit die vorherigen Messungen am Bildschirm. Die Skalierung in YRichtung kann automatisch durch Klick auf den entsprechenden Schalter am rechten Rand vorgenommen werden (siehe dazu auch das Handbuch zu „MCS-32“).

Ferner startet man das Programm „GCMS-CP“ und wählt dort im Menü „Messung“ den Programmteil ,m/z-Anzeige“ ( siehe dazu Kap. 6.2.1, S. 15).

Durch den Direkteinlaß muß eine gasförmige (Eich-)Substanz mit bekanntem Massenspektrum zugegeben werden. Am MS-Einschub „BSR“ wird nun der Magnetstrom so geregelt (vorher ggf. 
Feld-Regelung ausschalten), daß das „MCS-32“-Justierprogramm einen möglichst hohen Peak der Substanz anzeigt. Nun versucht man durch Variieren der Potentiale am MS-Einschub „BCT“ das Signal zu maximieren (vgl. dazu auch das Handbuch zum MS); dabei kann es notwendig sein, auch den Magnetstrom leicht nachzuregeln.

Es erscheint sinnvoll, die beiden Programme folgendermaßen am Bildschirm darzustellen (das Programm „GCMS-CP“ verkleinert sich bei Aktivierung der m/z-Anzeige automatisch):

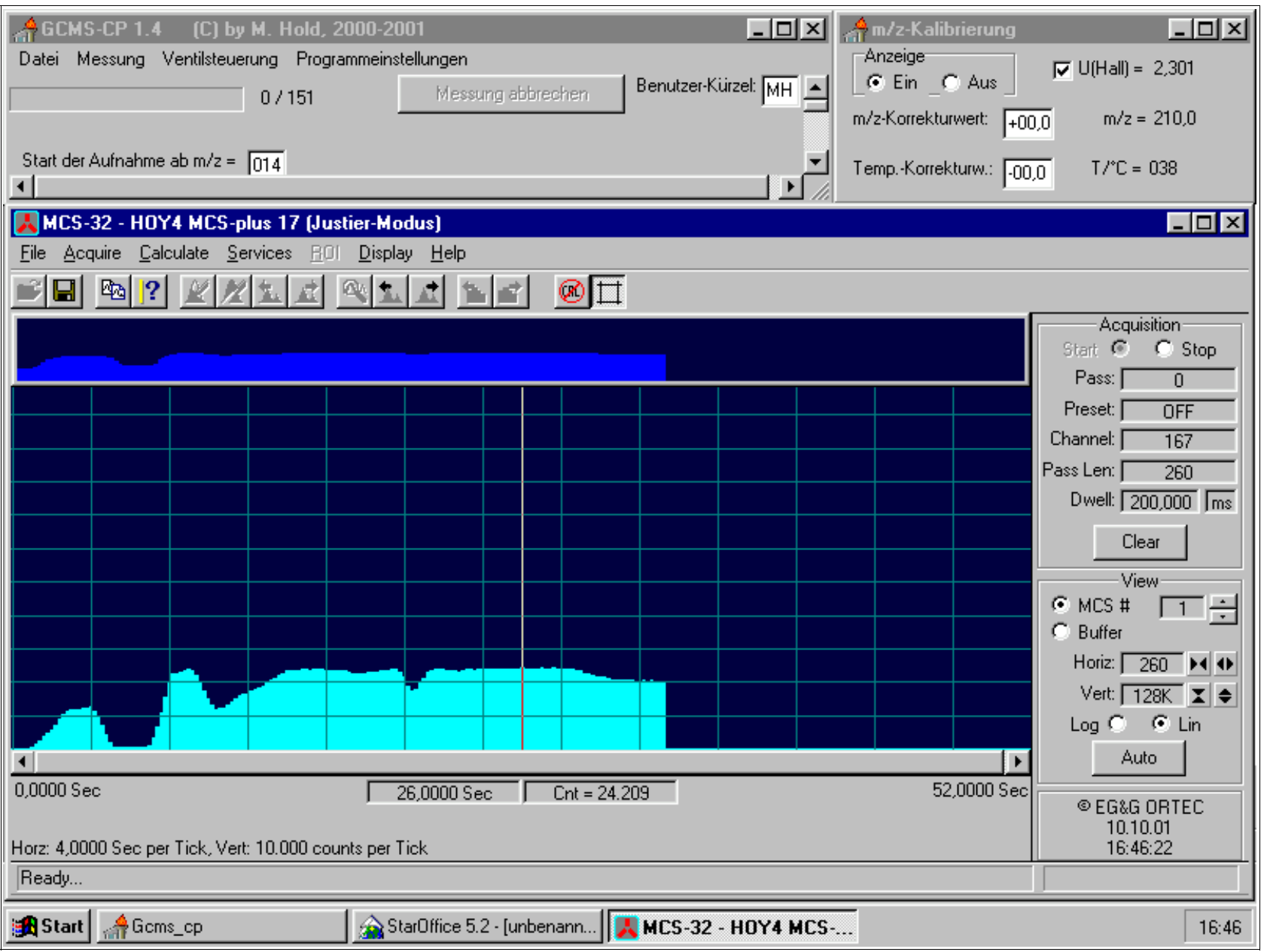

\subsection{Kalibrierung der Massenanzeige}

Unmittelbar nach Feinjustage der Ionenquelle sollte anhand der durch den Direkteinlaß zugeführten Eichsubstanz auch die Kalibrierung der externen Hall-Sonde überprüft werden. Man verwendet auch dazu die beiden im vorangegangenen Unterkapitel gestarteten Programme.

Durch Ändern des Magnetstroms am MS-Einschub „BSR“ (vorher ggf. Feld-Regelung ausschalten) stellt man nach und nach alle Peaks des Massenspektrums der Eichsubstanz ein und vergleicht die $\mathrm{m} / \mathrm{z}$-Anzeige mit dem wahren Wert (den man dem Literatur-Massenspektrum entnimmt).

Falls die Werte nicht gleich sind, notiert man sich für jeden Peak die gemessene Hall-Spannung (siehe Kap. 6.2.1, S. 15) und den wahren m/z-Wert. Mit diesen Wertepaaren wird nun z.B. mit dem Programm „Origin“ eine Auftragung $\mathrm{U}_{\text {Hall }} /$ Volt versus m/z gemacht und diese Werte durch einen Polynom-Fit (bis maximal 3. Ordnung) beschrieben. Die Fit-Parameter dieses Polynoms müssen dann im ,GCMS-CP“-Programm im Programmteil „Pfade und Parameter festlegen“ eingegeben werden (siehe dazu Kap. 6.4.1, S. 16). 
Die interne Hall-Sonde des MS, die zur Anzeige am MS herangezogen wird und die auch zur Magnetfeld-Regelung erforderlich ist, muß zwar nicht exakt kalibriert werden, sie sollte aber ungefähr mit der externen Sonde übereinstimmen (mindestens auf \pm 10 Massenzahlen genau).

\subsection{Aufnahme eines einzelnen Massenspektrums}

Um die automatische Speicherung in getrennten Verzeichnissen zu ermöglichen, sollte zunächst ein aus zwei Buchstaben bestehendes Benutzerkürzel eingegeben werden.

Zur Registrierung eines einzelnen Massenspektrums müssen dann die

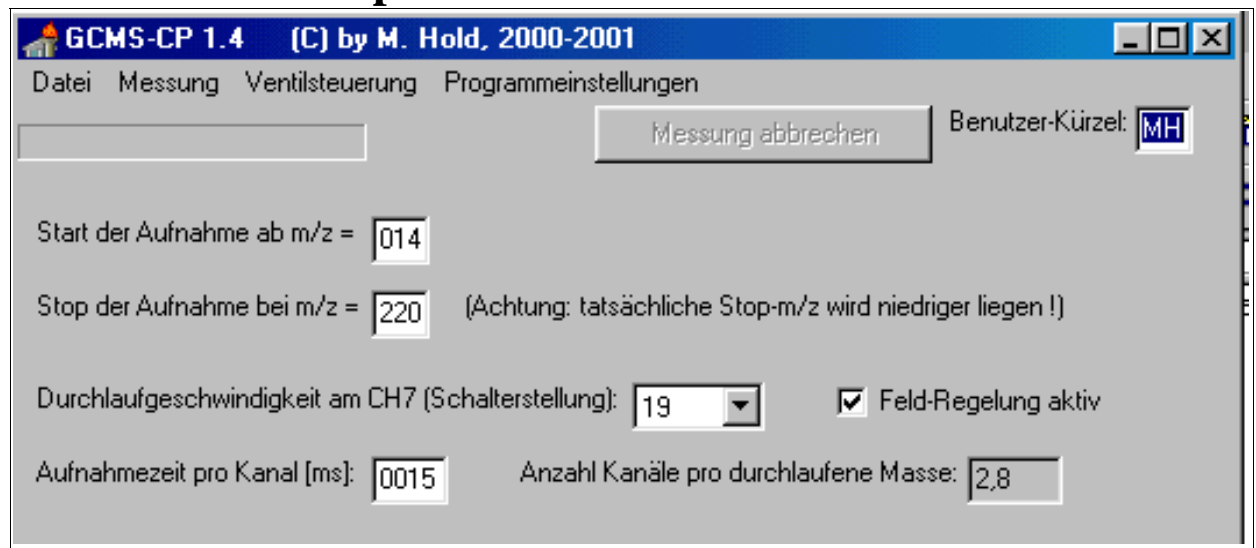
m/z-Zahl für Start und Stop der Aufnahme eingegeben werden. Es ist zu beachten, daß am MSEinschub „BSR“ die Rücksprung-Grenze für den Massendurchlauf mindestens gleich hoch wie die Stop-m/z-Zahl des Programms eingestellt wird.

Nun ist am MS-Einschub „BSR“ die Durchlaufgeschwindigkeit einzustellen und diese gleichermaßen im Programm auszuwählen. Außer zur Feinjustage der Ionenquelle und zur m/z-Kalibrierung sollte immer die Feld-Regelung am MS aktiviert werden; dazu muß der Magnetstrom auf Null geregelt und dann die entsprechende Taste am MS-Einschub „BSR“ gedrückt werden. Im Programm muß deshalb die entsprechende Auswahloption „Feld-Regelung aktiv“ gleichermaßen ausgewählt werden (Häkchen machen).

Abschließend ist die Aufnahmezeit pro Kanal so auszuwählen, daß üblicherweise mindestens ca. 3 Kanäle pro durchlaufene Masse zur Aufnahme bereitstehen; dieser Wert wird vom Programm berechnet und angezeigt.

Sobald obige Eingaben getätigt wurden, die Substanz über den Direkteinlaß zugeführt wird und das MS betriebsbereit ist, kann die Messung gestartet werden. Zunächst sollte die m/z-Anzeige aktiviert werden (siehe Kap. 6.2.1, S. 15). Dann muß im Menü „Messung“ der Programmteil

„Einzelspektrum“ gewählt werden. Es öffnet sich ein Fenster, in dem der Dateiname für die Ergebnisdatei eingegeben werden muß.

Das „GCMS-CP“-Programm startet nun quasi als von ihm ferngesteuertes Programm „MCS-32“. Es erscheint daher auch ein kleines ,Job Control“-Fenster des „MCS-32“-Programms, das den Status seines ,Jobs“ anzeigt; falls es stört, darf dieses Fenster ggf. mit der Maus zur Seite gezogen, aber nicht geschlossen oder abgebrochen werden.

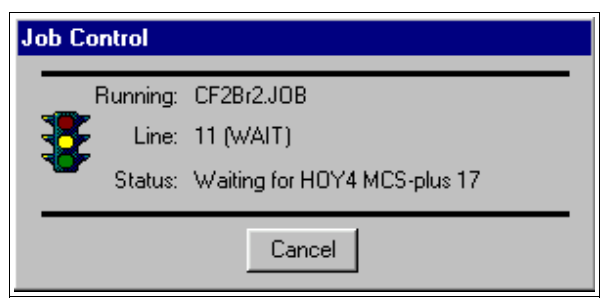

Das „GCMS-CP“-Programm fordert den Benutzer auf, mit dem Magnetstromregler den $\mathrm{m} / \mathrm{z}$-Wert so einzustellen, daß er unter der im Programm eingegebenen Start-m/z liegt (man sollte ca. 10 Massenzahlen darunter gehen); zunächst muß dieses Hinweisfenster mit $\mathrm{OK}$

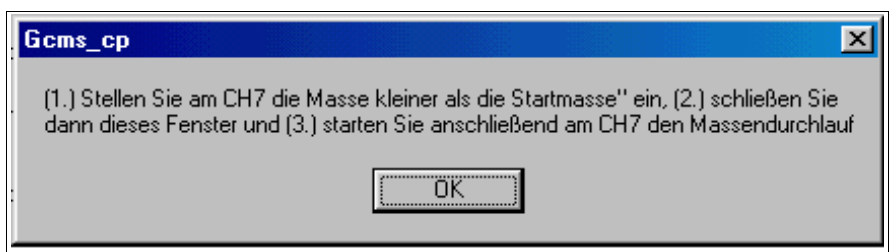
geschlossen und erst danach darf am MS-Einschub „BSR“ der Massendurchlauf gestartet werden. 
Nach Beendigung der Daten-Aufnahme erscheint eine entsprechende Meldung. Die Peak-Datei (vgl. Kap. 7, S. 17) kann sofort über das Menü „Datei“ im Programmteil „ltz. MessDatei öffnen“ als Text angezeigt werden.

\subsection{Durchführung einer GC-MS-Analyse}

Um die automatische

Speicherung in getrenn-

ten Verzeichnissen zu ermöglichen, sollte zunächst ein aus zwei Buchstaben bestehendes Benutzerkürzel eingegeben werden.

Zur Registrierung der einzelnen Massenspektren müssen dann die $\mathrm{m} / \mathrm{z}$-Zahl für Start und Stop der Aufnahme eingegeben werden. Es ist zu beachten, daß am MSEinschub „BSR“ die Rücksprung-Grenze für den Massendurchlauf exakt gleich hoch wie die Stopm/z-Zahl des Programms eingestellt wird (dazu nur die m/z-Anzeige des Programms verwenden !).

Nun ist am MS-Einschub „BSR“ die Durchlaufgeschwindigkeit einzugeben und diese gleichermaßen im Programm auszuwählen. Um einen schnellen Rücksprung von der Stop- auf die Start-m/z zu gewährleisten ist unbedingt die Feld-Regelung am MS zu aktivieren; dazu muß der Magnetstrom auf Null geregelt und dann die entsprechende Taste am MS-Einschub „BSR“ gedrückt werden. Im Programm muß deshalb die entsprechende Auswahloption „Feld-Regelung aktiv“ gleichermaßen ausgewählt werden (Häkchen machen).

Weiterhin ist die Aufnahmezeit pro Kanal so auszuwählen, daß üblicherweise mindestens ca. 3 Kanäle pro durchlaufene Masse zur Aufnahme bereitstehen; dieser Wert wird vom Programm berechnet und angezeigt.

Es kann auch eine Probenbezeichnung eingegebenen werden, die die analysierte Substanzmischung beschreibt. Zudem sollten die sonstigen Bedingungen bei der Analyse angegeben werden. Alle diese Angaben sind später in der

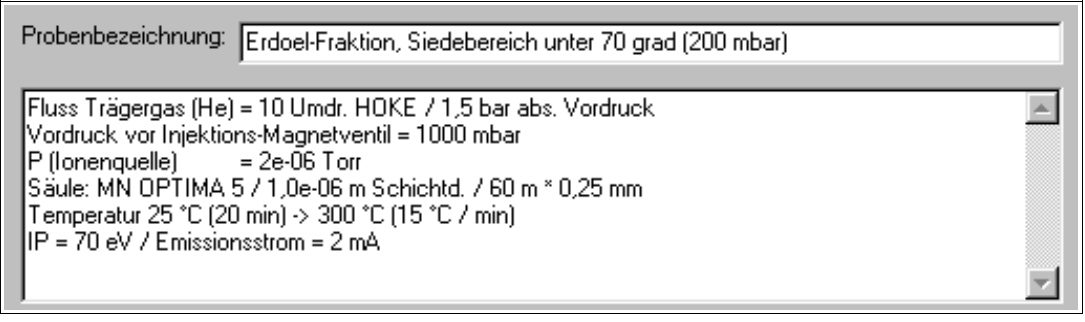
MeßtextDatei gespeichert.

Ferner muß die Gesamtaufnahmezeit angegeben werden, d.h. für wieviele Minuten das Programm nach der Injektion nun die Massenspektren registrieren soll. Anhand dieser Zeit berechnet das Programm die Anzahl der aufzunehmenden Massenspektren. Diese An-

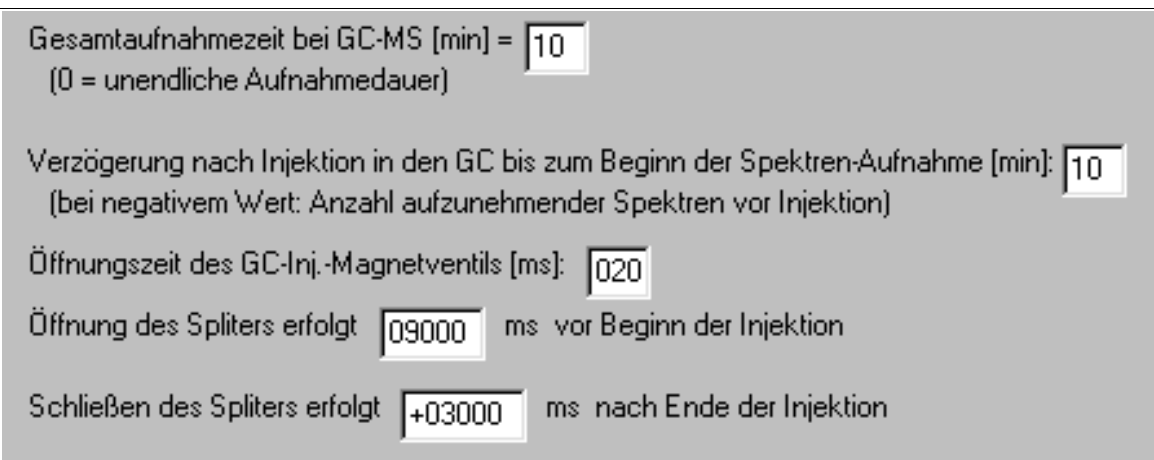
zahl darf jedoch nicht größer als 999 werden, wobei das Programm diese Vorgabe überwacht. Auch 
Kapitel 5 : Vorgehensweise für die Spektrenregistrierung

die gewünschte unendliche Aufnahmedauer durch Eingabe einer Gesamtaufnahmezeit von null Minuten bewirkt die Aufnahme von nur 999 Spektren. Die Anzahl der aufzunehmenden Spektren wird am oberen Fensterrand angezeigt.

Selbst das Trägergas He benötigt bereits einige Minuten für den Durchgang durch die Kapillarsäule, daher kann der Beginn der Spektrenregistrierung nach der Injektion auch um eine vorgegebene Anzahl Minuten verzögert werden.

Um einen möglichst scharfen GC-Peak zu erhalten, sollte die eigentliche Injektion, d.h. das Öffnen des entsprechenden Magnetventils nur für sehr kurze Zeit erfolgen. Bedingt durch die Trägheit des Ventils ist jedoch keine kürzere Zeit als 12-15 ms möglich.

Ebenfalls um einen möglichst scharfen GC-Peak zu erhalten, sollte das Split-Ventil der Injektionskammer bereits einige Sekunden vor der Injektion geöffnet werden, um den Druck in der Injektionskammer zu verringern und damit eine schnelle Injektion in die Kammer zu gewährleisten. Dieses Split-Ventil sollte auch während und einige Sekunden nach der Injektion noch geöffnet sein, damit möglichst nur eine kleine Substanzmenge, diese aber schnell, in die Kapillaräule gelangt und die Injektionskammer schnellstmöglich auch wieder nur mit He gefüllt wird. Daher sind dem Programm die gewünschten Zeiten einzugeben. Sofern man das Schließen des Split-Ventils vor der Injektion wünscht, muß der Wert mit negativem Vorzeichen eigegeben werden.

Sobald obige Eingaben getätigt wurden, wird die Substanz in das Vorratsgefäß eingefüllt und dieses mit He auf insgesamt ca. 1 bar abs. aufgefüllt (siehe dazu Kap. 6.3.1, S. 15).

Sofern das MS betriebsbereit ist (siehe dazu Kap. 3, S. 4), kann die Messung gestartet werden. Zunächst sollte die m/z-Anzeige aktiviert werden (siehe Kap. 6.2.1, S. 15). Dann muß im Menü „Messung“ der Programmteil „GC-MS-Spektrum“ gewählt werden. Es öffnet sich nun ein Fenster, in dem der Dateiname für die Ergebnisdatei eingegeben werden muß. Dieser Dateiname darf keine Sonderzeichen enthalten, daher werden diese ggf. automatisch durch ,_-" ersetzt.

Das „GCMS-CP“-Programm startet nun quasi als von ihm ferngesteuertes Programm „MCS-32“. Es erscheint daher auch ein kleines „Job Control“"-Fenster des „MCS-32“-Programms, das den Status seines ,Jobs“ anzeigt; falls es stört, darf dieses Fenster ggf. mit der Maus zur Seite gezogen, aber nicht geschlossen oder abgebrochen werden.

Das „GCMS-CP“-Programm fordert den Benutzer auf, (1.) am MS den ,zyklischen Massendurchlauf mit schnellem Rücksprung“ einzustellen, (2.) die gewünschte Stop-m/z (d.h. die Rücksprunggrenze) am MS einzustellen, (3.) mit dem Magnetstromregler den $\mathrm{m} / \mathrm{z}-$ Wert so einzustellen, daß er 10 Massenzahlen
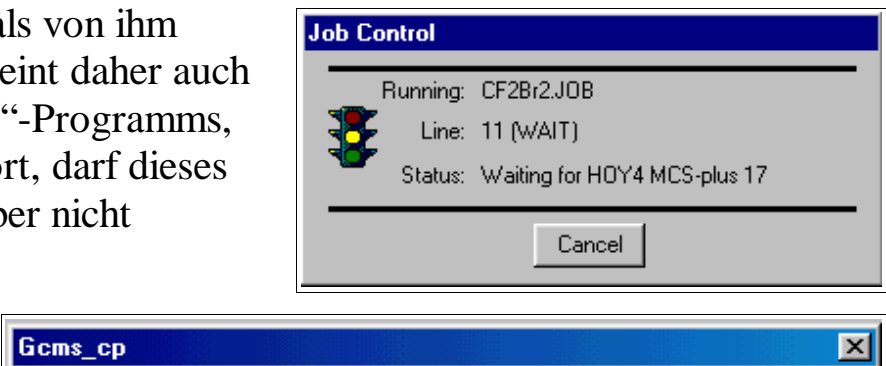

(1.) Stellen Sie am $\mathrm{CH} 7$ den "zyklischen Durchlaut mit schnellem Riucksprung" ein, (2.) Stellen Sie am CH7 die gewuinschte "Stop-Masse" ein, (3.) Regeln Sie am CH7 die Masse min. $10 \mathrm{M}$ unter die "Start-Masse" ein, (4.) Starten Sie dann den Massendurchlauf und (5.) Schließen Sie dann erst dieses Fenster mit OK

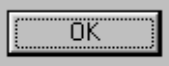
unter der im Programm eingegebenen Start-m/z liegt, (4.) den Massendurchlauf zu starten und (5.) dieses Hinweisfenster mit OK zu schließen.

Nach Beendigung der Daten-Aufnahme erscheint eine entsprechende Meldung.

Die GC-Datei (vgl. Kap. 7, S. 17) kann sofort nach der Aufnahme über das Menü „Datei“ im Programmteil ,ltz. MessDatei öffnen“ als Text angezeigt werden.

Die Aufnahme kann aber auch vorzeitig durch Klick auf den Schalter „Messung abbrechen“

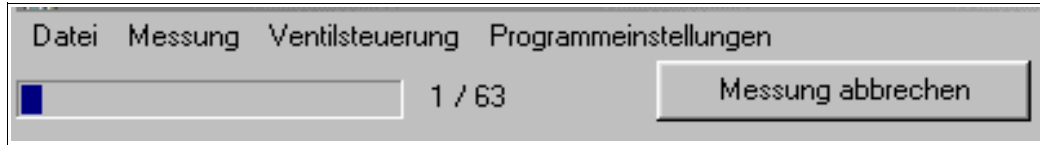


beendet werden. Die bis zu diesem Moment aufgenommenen Daten werden trotzdem konvertiert und gespeichert.

Allerdings ist das „MCS-32 Job Control“-Programm im Fall des vorzeitigen Abbruchs manuell durch Klick auf den Schalter „Cancel“ zu beenden. Es wird dringend empfohlen, hiernach auch das „GCMS-CP“-Programm zu beenden und das ganze Betriebssystem neu zu starten (bzw. bei „Windows NT“ sich als Benutzer ab- und wieder neu anzumelden), weil sonst einige

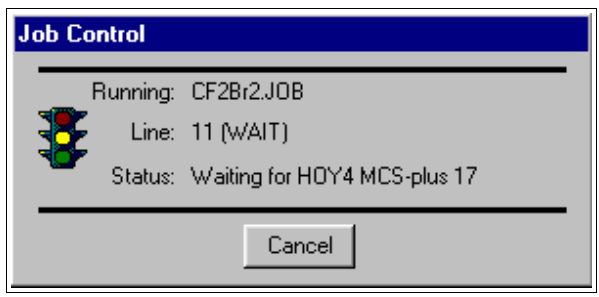
speicherresidente Programme nicht beseitigt werden und weitere Messungen behindern könnten. Das Programm gibt ggf. eine entsprechende Meldung aus.

\section{Beschreibung der einzelnen Programmteile}

\subsection{Das Menü „Datei““}

\subsection{1 ,Itz. MessDatei öffnen“6}

Mit diesem Programmteil wird das Windows-Programm „Editor“ gestartet, um damit die zuletzt aufgenommenen Daten anzeigen zu können.

Sofern unmittelbar zuvor ein Einzelspektrum aufgenommen wurde, zeigt das Programm automatisch die Peakdatei dieses Spektrums an. Falls zuvor eine GC-MS-Aufnahme erfolgte, wird hier automatisch der Inhalt der GC-Datei angezeigt.

\subsection{2 „PeakDateien erstellen“6}

Nach der automatischen Datenkonvertierung werden am Ende einer jeden Messung zunächst alle einzelnen Meßpunkte in der Spektrendatei (*.DAT) gespeichert. Diese bestehen aus dem jeweiligen $\mathrm{m} / \mathrm{z}$-Wert und dem jeweiligen DATA-Wert. Weil die Durchlaufgeschwindigkeit (und damit die Durchlaufzeit pro Kanal) üblicherweise relativ zur Aufnahmezeit pro Kanal langsam sein sollte, werden häufig DATA-Werte einer Masse auf mehrere Meßpunkte aufgeteilt. Dieser Programmteil soll nun solche Peaks erkennen und die aufgeteilten Meßpunkte zu einem einzigen zusammenführen; das Ergebnis wird separat in der Peakdatei (*.PIK) gespeichert.

Man kann dem Programm angeben, welcher DATAWert mindestens insgesamt für einen Peak überschritten sein muß, damit dieser als Peak gespeichert wird. Da Störungen aus der Umgebung hin und wieder einen DATA-Wert in Höhe von 1 ,einstreuen“, sollte man solch kleine Peaks möglichst gar nicht erst speichern;

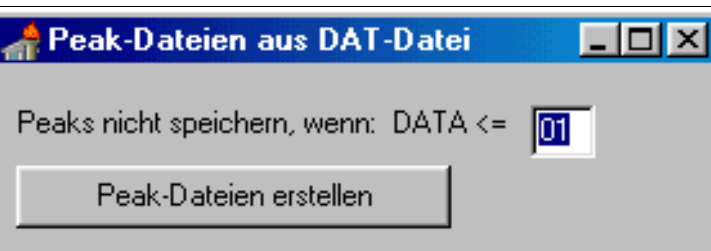
bei hohem Ionisierungspotential (70 eV) und längerer Aufnahmezeit pro Kanal (> $100 \mathrm{~ms})$ kann eventuell ein noch höherer Wert empfehlenswert sein.

Sobald man auf den Schalter „Peak-Dateien erstellen“ klickt, wird der Dateiname der GC-Datei (*GC) abgefragt, für deren Massenspektren die Peakdateien gewonnen werden sollen; die dann erstellten Peak-Dateien haben den gleichen Dateiname wie die Spektrendateien (*.DAT), jedoch die Dateiextension , ${ }^{*}$.PIK“.

Der Algorithmus zur Peak-Erkennung läßt sich knapp folgendermaßen beschreiben: Das Programm ließt von Beginn an die Spektrendatei. Sobald ein DATA-Wert größer Null auftaucht wird der Beginn eines Peaks angenommen. Nun ließt das Programm den nächsten Wert ein. Ist dieser Wert 
gleich Null, wird der zuvor begonnene Peak als beendet betrachtet und gespeichert. Ist der Wert aber ungleich Null, so prüft das Programm, ob der aktuelle Wert kleiner als der vorhergehende ist (negative Steigung) und zugleich kleiner als der nachfolgende Wert ist (positive Steigung); sind beide Kriterien erfüllt, wird der zuvor begonnene Peak beendet und gespeichert, der aktuelle Wert verworfen und mit dem nächsten Wert ein neuer Peak begonnen. Trafen keine der bisher genannten Kriterien zu, so prüft das Programm als nächstes, ob der aktuelle Wert kleiner als der vorhergehende ist (negative Steigung) und der nächste Wert Null ist; in diesem Fall wird der aktuelle Wert zum Peak hinzuaddiert und der Peak dann beendet und gespeichert. In allen anderen Fällen wird der aktuelle Wert einfach zum Peak hinzuaddiert und beim nächsten Wert wieder alle soeben beschriebenen Peak-Ende-Kriteien überprüft. Sofern jetzt der letzte Peak beendet und kein neuer begonnen wurde, startet der Algorithums wieder ganz von vorne.

Unter dem soeben erwähnten Verfahren ,zum Peak hinzuaddieren“ ist konkret folgendes zu verstehen: Der DATA-Wert aus der Spektrendatei (*.DAT) wird zum Peak-DATA-Wert hinzuaddiert und der Peak-m/z-Wert wird als gewichteter Mittelwert (gewichtet mit den DATAWerten) aus den einzelnen m/z-Werten der Spektrendatei berechnet; ein Peak-Wert in der Peakdatei (*.PIK) besteht damit aus der Summe aller zugehörigen DATA-Werte und dem gewichteten Mittelwert aller zugehörigen $\mathrm{m} / \mathrm{z}$-Werte. Die Peak-m/z-Werte werden dabei vom Programm auf eine Nachkommastelle gerundet.

\subsection{3 ,Peaks in MS bzw. GC suchen““}

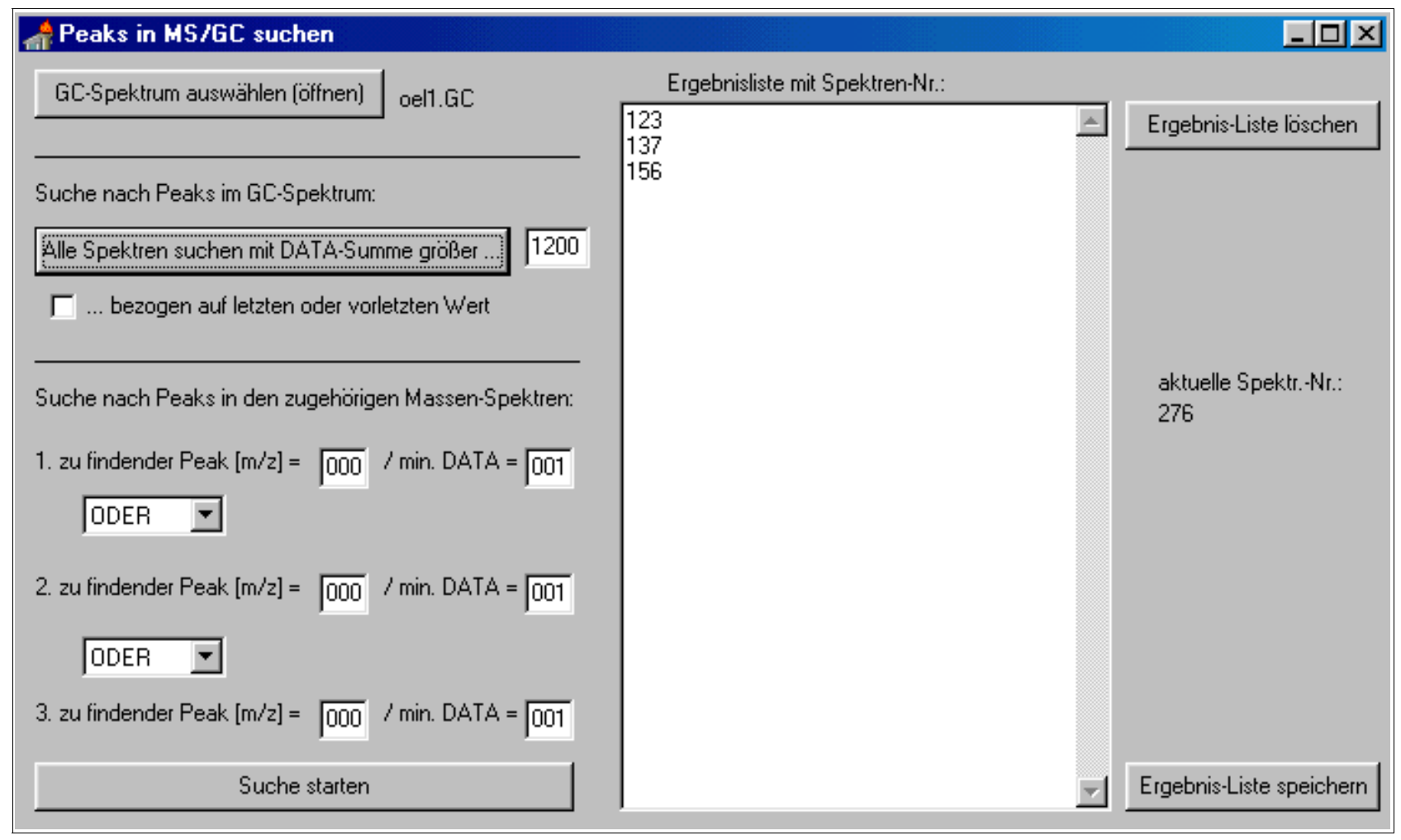

Mit diesem Programmteil können einerseits bestimmte Peaks in den einzelnen Massenspektren und andererseits Peaks im GC-Spektrum gesucht und so gespeichert werden, daß sie später sofort in „Origin 6.1“ importiert werden können.

Durch Klick auf den Schalter „GC-Spektrum auswählen (öffnen)“ wird zunächst der Dateiname der GC-Datei abgefragt, auf die sich die Suche GC-Spektrum auswählen [öffnen] beziehen soll. Er gilt auch für die Suche nach Peaks in den zu dieser GC-Datei gehörenden Massenspektren. 
In einem GC-Spektrum kann das Programm alle Peaks suchen, die eine eingegebene DATA-Summe übersteigen. Dazu ist lediglich diese DATA-Summe einzugeben und anschließend auf den Schalter „Alle Spektren suchen mit DATA-Summe größer ..." zu klicken. Die Nummern der gefundenen

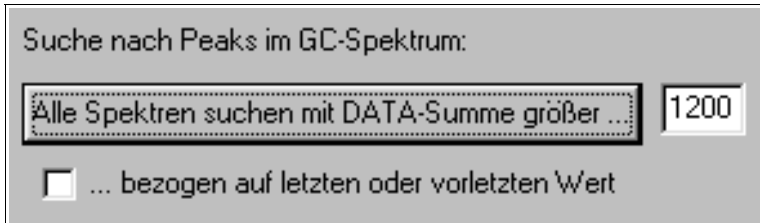
Massenspektren werden der Ergebnisliste hinzugefügt. Sofern die Option , ... bezogen auf letzten oder vorletzten Wert“ aktiviert ist, werden nur noch solche GC-Peaks in die Ergebnisliste aufgenommen, deren DATA-Summe um den eingegebenen Wert höher ist als diejenige des ersten oder zweiten unmittelbar vorausgehenden Meßpunktes. Diese Option ist sehr nützlich, falls die Grundlinie des GC-Spektrums im Verlauf der Aufnahme höher wird, als die ersten Peaks im Spektrum sind.

Sofern man bereits einen Verdacht bezüglich der zu analysierenden Substanzen hat, kann man auch gezielt in den Massenspektren (Peakdateien, *.PIK) nach bis zu drei Peaks suchen. Dazu ist der $\mathrm{m} / \mathrm{z}$-Wert sowie der mindestens im Massenspektrum registrierte DATA-Wert einzugeben. Es können drei solcher Werte durch ein logisches UND bzw. ODER verknüpft werden. Im nebenstehenden Beispiel werden also nur diejenigen Massenspektren gefunden, die sowohl einen Peak bei m/z 57 mit mindestens einem DATA-Wert von 50 als auch einen Peak bei $\mathrm{m} / \mathrm{z} 72$ mit mindestens einem DATA-Wert von 25 aufweisen. Nach einem Klick auf den Schalter, ,Suche

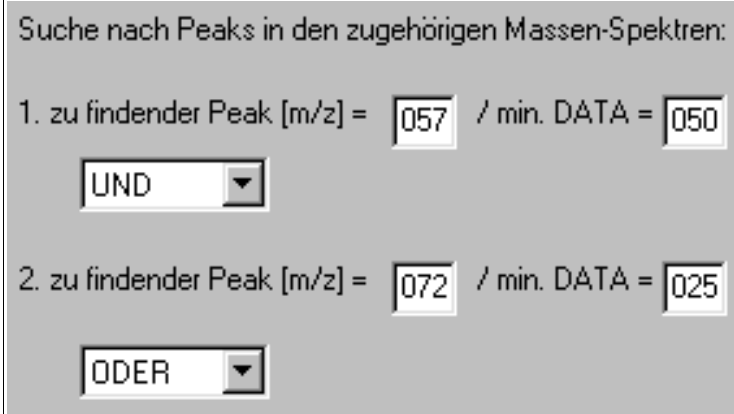

3. zu findender Peak $[\mathrm{m} / \mathrm{z}]=\sqrt{000} / \mathrm{min}$. DATA $=\sqrt{001}$ Suche starten starten“" werden alle Massenspektren der gewählten GC-MS-Analyse im Hinblick auf die Suchkriterien überprüft und die zutreffenden der Ergebnisliste hinzugefügt.

Neben der Nummer des gefundenen Massenspektrums werden auch die tatsächlich vorgefundenen DATA-Werte der gesuchten Peaks angegeben. In Anknüpfung an das Ergebnisliste mit Spektren-Nr.:
\begin{tabular}{|ccc|}
201 & (DATA1: 110 / DATA2: 25 / DATA3: 0] & (DATA \\
254 & (DATA1: $104 /$ DATA2: 26 / DATA3: 0) & \\
\hline
\end{tabular} vorangegangene Beispiel erfüllt also z.B. das Massenspektrum Nr. 201 die Suchkriterien, denn es enthält einen Peak bei m/z 57 mit einem DATA-Wert von 110 und einen Peak bei m/z 72 mit einem DATA-Wert von 25.

Durch Klick auf den Schalter „Ergebnisliste speichern“ wird diese Liste unter dem Name der GC-Datei, jedoch mit der Extension „,.MAX“ gespeichert. Das Programm „Origin 6.1“ kann nun die Spektren dieser Liste automatisch importieren und anzeigen (siehe Kap. 8.6, S. 23).

Grundsätzlich werden die Suchergebnisse der Ergebnisliste stets hinzugefügt, d.h. die vorher enthaltenen Ergebnisse werden nicht überschrieben, sondern ergänzt. Man kann manuell einzelne Spektren aus dieser Liste löschen, indem man in die Liste klickt und dann einfach wie bei einem Texteditor die Ziffern löscht. Man kann jedoch auch durch Klick auf den Schalter „Ergebnisliste löschen“ alle Einträge der Liste löschen.

\subsection{4 „Mittelwert aus Spektren bilden“}

Mit diesem Programmteil kann aus mehreren Massenspektren einer GC-MS-Analyse ein MittelwertSpektrum erzeugt werden. Dazu werden die $\mathrm{m} / \mathrm{z}$-Werte der einzelnen Spektren zunächst auf ganze 
Zahlen gerundet und dann das arithmetische Mittel der DATA-Werte zu diesem gerundeten m/zWert in den einzelnen Spektren berechnet und gespeichert.

Im Programm muß lediglich die Nummer des Massenspektrums (Peakdatei, *.PIK), mit dem die Mittelwertbildung starten und diejenige bei der sie enden soll eingegeben werden. Durch Klick auf den Schalter „Mittelwertbildung starten“ wird zunächst der Dateiname des GCSpektrums abgefragt, dann erfolgt die Berechnung und zum Schluß gibt das Programm den Dateiname aus, unter dem die Ergebnisdatei (mit der Dateiextension „*.AV“) gespeichert ist.

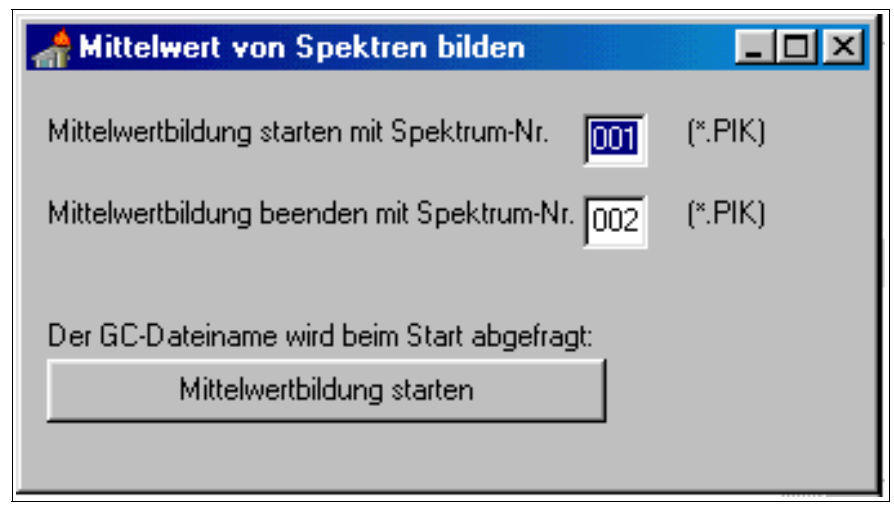

Die Mittelwertbildung ist sinnvoll bei der Hintergrund-Subtraktion, d.h. bei der Korrektur der Spektren um die im MS vorhandenen Verunreinigungen. Man kann z.B. den Mittelwert aus den Spektren bilden lassen, die aufgenommen wurden, bevor der erste GC-Peak auftrat und dann dieses Mittelwert-Spektrum von allen anderen als Hintergrund subtrahieren (siehe dazu unten Kap. 6.1.5).

Falls während der Registrierung die Grundlinie stark gestiegen ist, sind die Massenspektren von Peaks, die selbst kaum höher als diese hoch gestiegene Grundlinie sind, nur noch schwer zu interpretieren. In diesem Fall sollte der Mittelwert aus einigen Spektren der hohen Grundlinie unmittelbar vor auftreten des GC-Peaks erstellt und dieses Mittelwert-Spektrum als Hintergrund subtrahiert werden. Dadurch erhält man ein relativ reines Massenspektrum nur von der diesen Peak hervorrufenden Substanz.

\subsection{5 „BKG von Peakdateien subtrahieren“}

In diesem Programmteil kann eine MittelwertSpektrum-Datei (vgl. oben Kap. 6.1.4) von allen Massenspektren einer GC-MS-Analyse subtrahiert werden. Es kann auch eine Peakdatei subtrahiert werden.

Nach Klick auf den Schalter „Subtraktion starten“ wird zunächst der Dateiname der GC-Datei abgefragt, danach derjenige der zu subtrahierenden Mittelwert-Spektrum-Datei. Die Ergebnisse werden in den Subtraktionsdateien gespeichert, die den gleichen Name wie die Peakdateien, jedoch die Dateiextension „*.SUB“ haben. Sie haben das gleiche Dateiformat, wie die Peakdateien (siehe Kap. 7, S. 17).

Um überhaupt eine Subtraktion durchführen zu können, rundet das Programm in einem ersten Durchlauf alle $\mathrm{m} / \mathrm{z}$-Werte der Peakdatei (*.PIK) auf eine ganze Zahl; sofern dadurch mehrere Peaks zusammenfallen, werden deren DATA-Werte aufsummiert.

\subsection{6 „Beenden““}

Mit diesem Menüpunkt kann das Programm beendet werden. Es erfolgt eine Sicherheitsrückfrage. 


\subsection{Das Menü „Messung““}

\subsection{1, ,m/z-Anzeige ${ }^{66}$}

Die wichtigste Aufgabe dieses Programmteils ist die Anzeige der m/z-Zahl. Um die Anzeige zu starten, muß der Knopf „Ein“ angeklickt werden; während einer Datenaufnahme stellt das Programm die Anzeige automatisch aus, um keine Rechenzeit zu vergeuden.

Zusätzlich zur m/z-Zahl wird auch die Temperatur im GC-Ofen angezeigt. Sofern die entsprechende Option

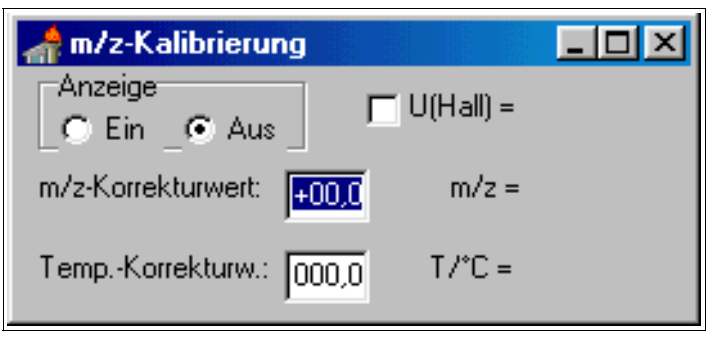
gewählt wurde, wird zudem die Spannung der Hall-Sonde angezeigt; dies ist wichtig, um für die m/zAnzeige eine Eichkurve bzw. einen Polynom-Fit erstellen zu können (vgl. dazu Kap. 5.2, S. 7).

Falls der m/z-Wert im gesamten Bereich lediglich um einen konstanten Wert verschoben ist, kann ein entsprechender Korrekturwert (positiv oder negativ) eingegeben werden, den das Programm bei der $\mathrm{m} / \mathrm{z}$-Anzeige sowie bei der Datenaufnahme berücksichtigt. Analog läßt sich die Temperatur-Anzeige für den GC-Ofen korrigieren.

\subsection{2 ,Einzelspektrum“6}

Dieser Menüpunkt startet die Aufnahme eines einzelnen Massenspektrums mit den im Hauptfenster eingegebenen Parametern. Zunächst wird der Dateiname abgefragt, unter dem das zu registrierende Spektrum gespeichert werden soll, dann erfolgt ein Hinweisfenster für das notwendige weitere Vorgehen. Erst nach Klick auf „OK“ in diesem Hinweisfenster beginnt die Datenaufnahme.

Die genaue Vorgehensweise ist bereits ausführlich oben in Kap. 5.3 (S. 8) beschrieben worden.

\subsection{3 „,GC-MS-Spektrum“}

Dieser Menüpunkt startet die Aufnahme eines GC-Spektrums (bzw. der zugrunde liegenden Massenspektren) mit den im Hauptfenster eingegebenen Parametern. Zunächst wird der Dateiname abgefragt, unter dem das zu registrierende Spektrum gespeichert werden soll, dann erfolgt ein Hinweisfenster für das notwendige weitere Vorgehen. Erst nach Klick auf „OK“ in diesem Hinweisfenster beginnt die Datenaufnahme.

Die genaue Vorgehensweise ist bereits ausführlich oben in Kap. 5.4 (S. 9) beschrieben worden.

\subsection{Das Menü „,Ventilsteuerung“}

\subsection{1 ,Magnetventile manuell steuern““}

Mit diesem Programmteil können das Splitund das Badgas-Magnetventil manuell gesteuert werden; dazu muß lediglich auf die entsprechenden Knöpfe ,auf“ bzw. ,zu“ geklickt werden.

Ein Klick auf den Schalter „,... für 15 ms“ öffnet das Badgas-Magnetventil für

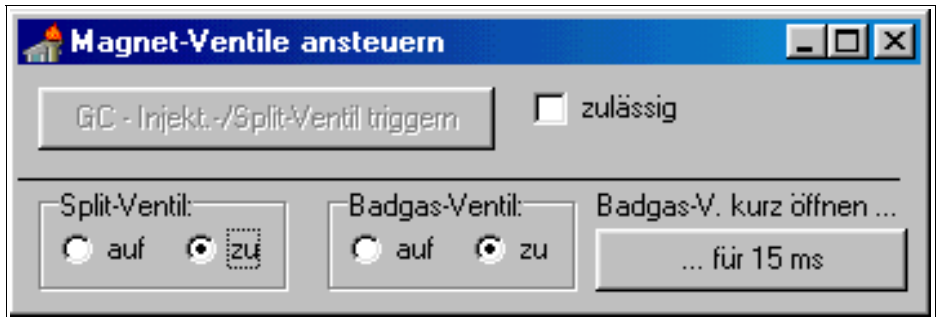


lediglich 15 ms; dies ist nützlich, um den Druck im Proben-Vorratsgefäß mit Badgas (Helium) auf ca. 1 bar abs. auffüllen zu können, ohne gleichzeitig die Substanz in Richtung Badgas-Druckflasche lange ausströmen lassen zu müssen.

Durch Wahl der Option ,zulässig“" wird auch der Schalter „GC-Injekt./Split-Ventil triggern“ aktivierbar. Damit werden die beiden Magnetventile mit den im Hauptfenster angegebenen Zeitparametern angesteuert;

술. Magnet-Ventile ansteuern

GC - Iniekt.-/Split-Ventil triggern zulässig es kann also die automatische Injektion mit Split ausgelöst werden, ohne eine Datenaufnahme zu starten. Man wird diese Option allenfalls für Test-Zwecke, nicht aber im Routinebetrieb benötigen.

\subsection{Das Menü „Programmeinstellungen““}

\subsection{1 „Pfade und Parameter festlegen“"}

In diesem Eingabefenster muß dem Programm die bei der Installation der AD-Karte vorgenommene Verkabelung (siehe Kap. 4.2, S. 5) mitgeteilt werden. Der Eingangskanal für die Spannung des Thermoelements muß zwangsläufig unmittelbar neben demjenigen für die Hall-Spannung liegen.

Auch ist der bei der Installation der MCS-Karte gewählte Installationspfad für das Programm „MCS32“ (vgl. Kap. 4.1, S. 5) einzugegeben; durch Klick auf den Schalter „... “ öffnet sich ein Dialogfenster, um diesen Pfad komfortabel auswählen zu können.

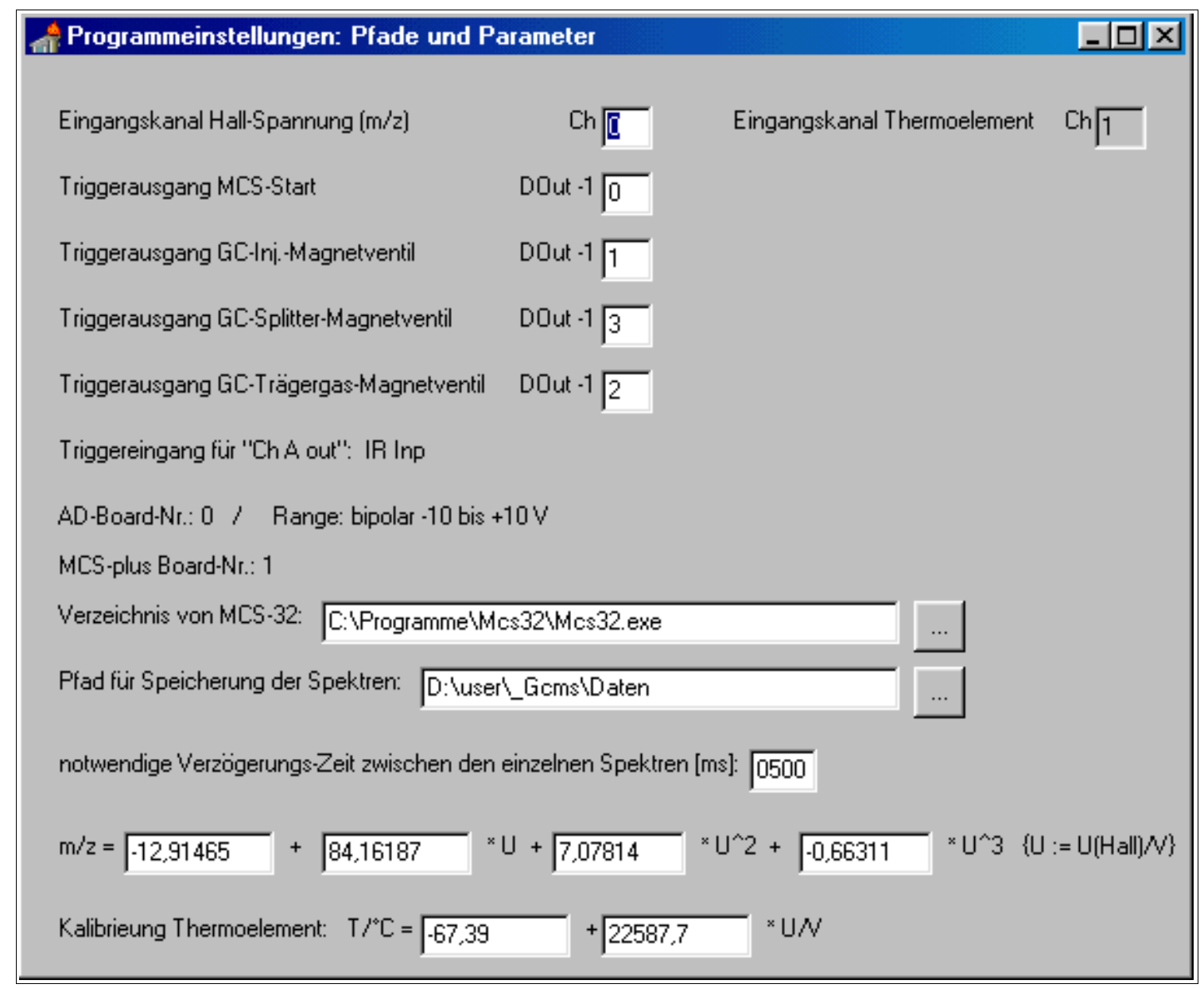

Ebenso sollte ein Pfad eingeben werden, in dem „GCMS-CP“ die Meßdaten speichert. Das Programm legt nämlich in diesem Verzeichnis automatisch ein Unterverzeichnis an, das den Name 
des Benutzerkürzels hat. Es legt standardmäßig in letzterem Verzeichnis für jede Messung ein neues Unterverzeichnis an, dessen Name aus dem aktuellen Datum (JJJJMMTT) und einer fortlaufenden alphabetischen Numerierung besteht; z.B. werden die Dateien der 2. Messung am 30.09.2001 des Benutzers MH im Verzeichnis „D:Iuser\_Gcms\Daten\MH\20010930B\“ gespeichert.

Ferner ist die „notwendige Verzögerungszeit zwischen den einzelnen Spektren“ einzugeben, die das Programm benötigt, um bei der GC-MS-Analyse die Daten eines Massenspektrums abzuspeichern, bevor wieder ein weiteres Spektrum aufgenommen werden kann (siehe dazu Kap. 2.2, S. 2 sowie Kap. 9, S. 24).

Unerläßlich für eine korrekte Datenaufnahme ist auch das Fit-Polynom zur Berechnung der m/z-Zahl aus der Hall-Spannung. Im Kap. 5.2 (S. 7) ist beschrieben, wie man dieses Polynom erhält.

Letztlich ist noch die Empfindlichkeit des Thermoelements einzugeben, damit das Programm aus der eingehenden Thermospannung die Temperatur im GC-Ofen berechnen kann.

\subsection{2 ,LinMassenabstand messen“"}

Dieser Programmteil wurde dazu verwendet, den Zeitabstand zwischen den benachbarten m/zWerten für die verschiedenen am MS einstellbaren Durchlaufgeschwindigkeiten zu bestimmen. Das Programm benötigt diesen Wert, um die Anzahl der aufzunehmenden MCS-Kanäle eines Massenspektrums sowie die Anzahl der aufzunehmenden Massenspektren für eine GC-Analyse berechnen zu können. Die so gemessenen Werte sind im Programmquellcode eingearbeitet.

Für den Routinebetrieb wird dieser Programmteil also nicht benötigt.

\section{Vom Programm erzeugte Dateien und deren Inhalt}

\subsection{MessTextdatei (*.TXT)}

In der MessTextdatei sind alle bei der GC-MS-Analyse eingestellten Parameter angegeben, so wie sie gemäß Kap. 5.4 (S. 9) eingegeben wurden.

Nachstehend ein Beispiel:

GCMS_CP 1.4 / Messung vom Dienstag, 24. Juli 2001 / 12:18 Uhr / User: MH

20010724B/oel1.TXT

Probenbezeichnung: Erdoel / Fraktion mit Sdp. < 353 K

Durchlaufgeschwindigkeit $\mathrm{v}=19$ (Magnet-Feld-Regelung)

AufnahmeZeit pro Kanal [ms] $=0015$

InjektionsZeit in GC [ms] = 015

Öffnung des Split vor Inj.-Start [ms] = 09000

Schließen des Split nach Inj.-Ende $[\mathrm{ms}]=+03000$

Fluss Trägergas $(\mathrm{He})=10 \mathrm{Umdr}$. HOKE / 1,5 bar abs. Vordruck

Vordruck vor Injektions-Magnetventil = $1000 \mathrm{mbar}$

$\mathrm{P}$ (Ionenquelle) = 2e-06 Torr

Säule: MN OPTIMA 5 / 1,0e-06 m Schichtd. / $60 \mathrm{~m} * 0,25 \mathrm{~mm}$

Temperatur ca. $25^{\circ} \mathrm{C}$

$\mathrm{IP}=70 \mathrm{eV} / 2 \mathrm{~mA}$

\subsection{GC-Datei (*.GC)}

Die GC-Datei enthält die Daten für das GC-Spektrum im ASCII-Format mit Tabulator-Zeichen 
(ASCII-Code Nr. 9) als Trennzeichen zwischen den Datenspalten. Diese Datenart kann problemlos in jede Tabellenkalkulation und auch in „Origin 6.1“ importiert werden.

Im einzelnen enthält die Datei folgende Datenspalten:

- die Nummer des zugehörigen Massenspektrums (fortlaufende Numerierung, beginnend mit 001)

- die Summe der DATA-Werte über alle m/z-Zahlen des Massenspektrums, mit Ausnahme derjenigen bei $\mathrm{m} / \mathrm{z}=17 ; 18 ; 28 ; 32$ sowie 40 (d.h. der üblicherweise im MS enthaltene Hintergrund wird nicht mitgezählt)

- die vergangene Zeit [in Sekunden] seit Injektion in die GC-Säule bzw. Injektionskammer

- die Summe der DATA-Werte über alle m/z-Zahlen des Massenspektrums (d.h. mit HintergrundSignalen des MS)

- die Temperatur $\left[\right.$ in $\left.{ }^{\circ} \mathrm{C}\right]$ im GC-Ofen

\subsection{Spektrendateien $(* . D A T)$}

Die Spektrendatei enthält alle Daten eines einzelnen Massenspektrums, die sich unmittelbar aus der Konvertierung der aufgenommenen Rohdaten ergeben (vgl. Kap. 2.2, S. 2). Auch sie werden im ASCII-Format mit Tabulator-Zeichen (ASCII-Code Nr. 9) als Trennzeichen zwischen den Datenspalten gespeichert.

Im einzelnen enthält die Datei folgende Datenspalten:

- die m/z-Zahl (auf zwei Nachkommastellen gerundet)

- den von der MCS-Karte registrierten DATA-Wert bei dieser m/z-Zahl

\subsection{Peakdateien $(* . P I K)$}

Die Peakdatei enthält alle Peaks eines einzelnen Massenspektrums, die sich aus der Anwendung des im Kap. 6.1.2 (S. 11) beschriebenen Peak-Such-Algorithmus auf die Spektrendatei ergeben. Die Ergebnisdaten werden ebenfalls im ASCII-Format mit Tabulator-Zeichen (ASCII-Code Nr. 9) als Trennzeichen zwischen den Datenspalten gespeichert.

Im einzelnen enthält die Datei folgende Datenspalten (vgl. dazu Kap. 6.1.2, S. 11):

- den gewichteten Mittelwert der m/z-Zahl (auf eine Nachkommastelle gerundet)

- die Summe der DATA-Werte im gesamten Peak

Nachstehend folgt ein Beispiel für die Auswirkung des Peak-Such-Algorithmus:

\begin{tabular}{|c|c|c|c|c|c|c|}
\hline \multicolumn{2}{|c|}{ Spektrendatei: } & \multirow{12}{*}{\multicolumn{2}{|c|}{$\rightarrow \quad$ wird zu }} & \multirow[t]{12}{*}{$\rightarrow$} & \multicolumn{2}{|c|}{ Peakdatei: } \\
\hline $\mathrm{m} / \mathrm{z}$ & DATA-Wert & & & & $\overline{\mathrm{m} / \mathrm{z}}$ & DATA-Summe \\
\hline 39,67 & 0 & & & & 40,1 & 26 \\
\hline 40,06 & 24 & & & & 41,1 & 92 \\
\hline 40,40 & 2 & & & & 42,2 & 82 \\
\hline 40,70 & 0 & & & & & \\
\hline 41,03 & 73 & & & & & \\
\hline 41,37 & 19 & & & & & \\
\hline 41,76 & 2 & & & & & \\
\hline 42,06 & 61 & & & & & \\
\hline 42,43 & 21 & & & & & \\
\hline 42,79 & 0 & & & & & \\
\hline
\end{tabular}




\subsection{Mittelwertdateien (*.AV)}

Die Mittelwertdatei enthält den Mittelwert aus mehreren Peakdateien, der sich nach Anwendung des im Kap. 6.1.4 (S. 13) beschriebenen Programmteils „Mittelwert aus Spektren bilden“ ergibt. Auch diese Ergebnisdaten werden im ASCII-Format mit Tabulator-Zeichen (ASCII-Code Nr. 9) als Trennzeichen zwischen den Datenspalten gespeichert.

Im einzelnen enthält die Datei folgende Datenspalten (vgl. dazu Kap. 6.1.4 (S. 13):

- die $\mathrm{m} / \mathrm{z}-$ Zahl (auf ganze Zahl gerundet)

- den Mittelwert (arithmetisches Mittel) der DATA-Summen der Peaks bei dieser gerundeten m/zZahl aus den einzelnen Peakdateien

\subsection{Subtraktionsdateien $(* . S U B)$}

Die Subtraktionsdateien enthalten die Differenz aus den einzelnen Peakdateien und einer ausgewählten Mittelwertdatei, die sich nach Anwendung des im Kap. 6.1.5 (S. 14) beschriebenen Programmteils „BKG von Peakdateien subtrahieren“ ergibt. Auch diese Ergebnisdaten werden im ASCII-Format mit Tabulator-Zeichen (ASCII-Code Nr. 9) als Trennzeichen zwischen den Datenspalten gespeichert.

Im einzelnen enthält die Datei folgende Datenspalten (vgl. dazu Kap. 6.1.5, S. 14):

- die $\mathrm{m} / \mathrm{z}-$ Zahl (auf ganze Zahl gerundet)

- die Differenz der DATA-Summen der Peaks in der Peakdatei und der Mittelwertdatei bei dieser gerundeten $\mathrm{m} / \mathrm{z}-\mathrm{Zahl}$

\subsection{Datei mit gefundenen GC-Peaks (*.MAX)}

Diese Datei enthält lediglich die Nummern der im Programmteil „Peaks in MS bzw. GC suchen“ gefundenen/ausgewählten Massenspektren (vgl. Kap. 6.1.3, S. 12). Auch sie wird im ASCII-Format erstellt.

Sie ist erforderlich, damit „Origin 6.1“ diese Massenspektren automatisch importieren und anzeigen kann (vgl. Kap. 8.6, S. 23).

\section{Spektrendarstellung mit LabTalk-Makros unter ORIGIN 6.1}

\subsection{Ansicht des GC-Spektrums}

Sobald „Origin 6.1“ und die zusätzlichen „LabTalk-Skripte“ gemäß der Anleitung in Kap. 4.3 (S. 5) installiert wurden, können die mit „GCMS-CP“ registrierten Spektren recht komfortabel ausgewählt und angezeigt/gedruckt werden.

Durch Klick auf den Schalter 喿 (der in den Origin-Handbüchern als „Custom Routine“Button bezeichnet wird) in der ,Standard toolbar" des Programms „Origin 6.1“ werden die speziell zur GC-MS-Auswertung erstellten
Verarbeitung von GC-HS-Rohdaten [by MH 2001] $x$

Nein $\quad$ Abbrechen


Skripte aufgerufen.

Zunächst erscheint das Fenster mit der Frage, ob GC-Daten importiert werden sollen. Wenn diese Frage bejaht wird, wird (nach Sicherheitsrückfrage zum Speichern) ggf. das aktuelle Projekt geschlossen und es erscheint ein „Öffnen“-Fenster zur Auswahl der zu öffnenden GC-Datei.

In diesem „Öffnen“-Fenster muß nun die anzuzeigende GC-Datei (vgl. dazu auch Kap. 7, S. 17) eingegeben werden; als Dateityp ist daher standardmäßig ,".GC““ vorgegeben.

Durch diese Auswahl wird aber nicht nur das eigentliche GC-Spektrum angezeigt, sondern quasi alle damit zusammenhängende Dateien (d.h. auch die zugehörigen Massenspektren) zur weiteren Bearbeitung in „Origin“ vorbereitet.

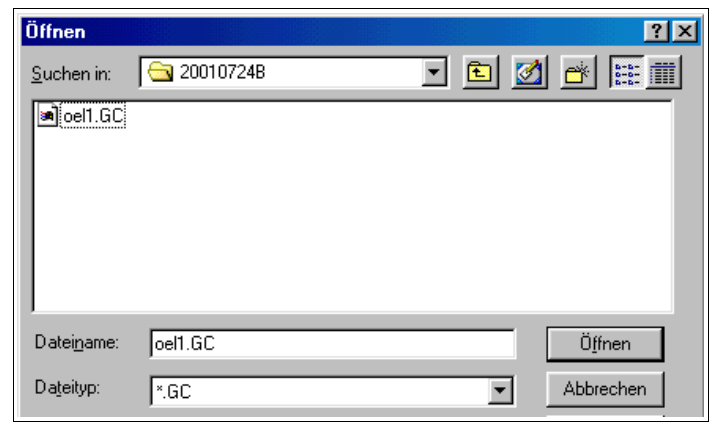

Unmittelbar nach dem Öffnen der GC-Datei sollte der Bildschirm folgendermaßen aussehen:

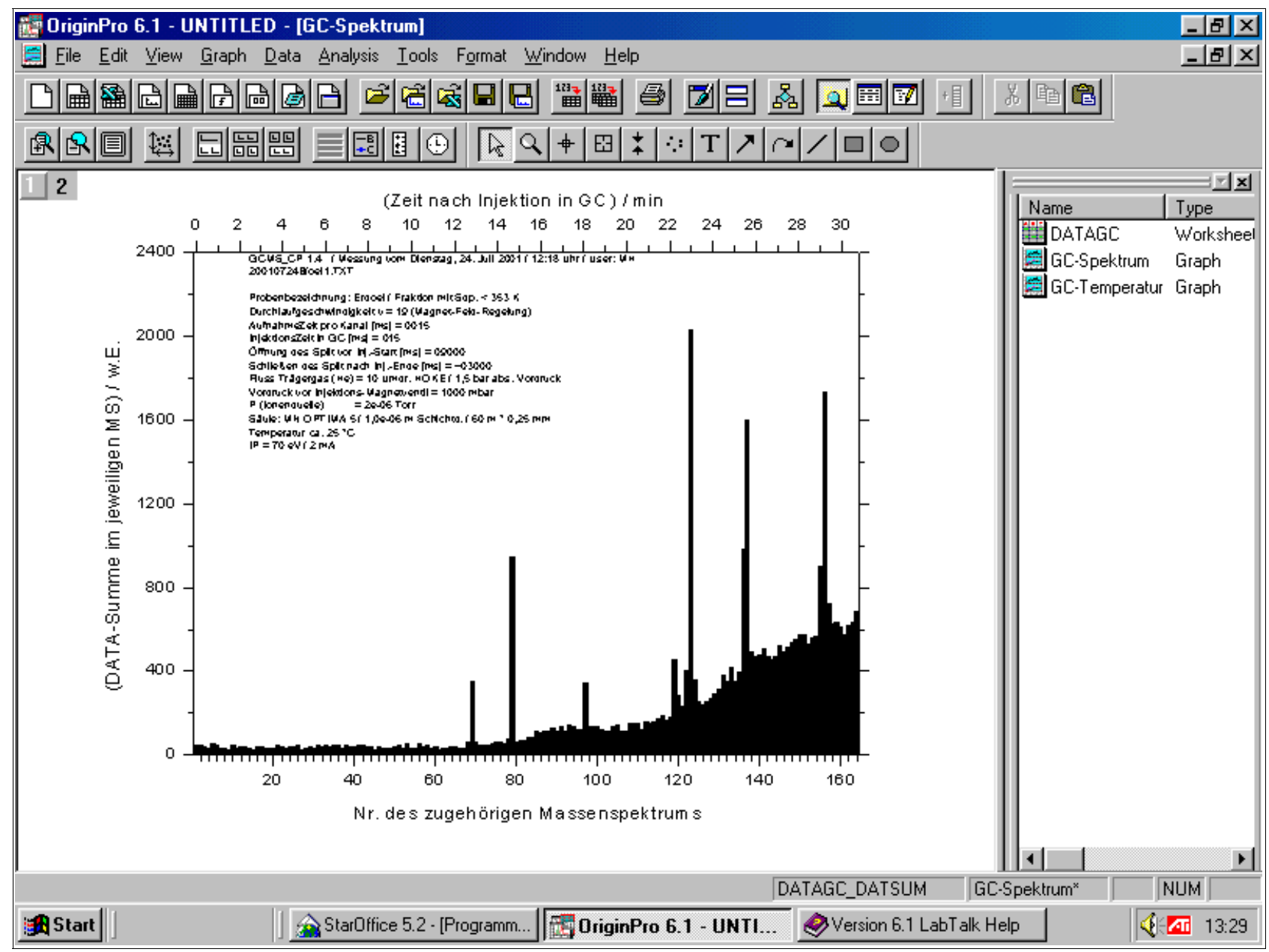

Man sieht also zunächst das GC-Spektrum am Bildschirm. Durch einen Klick auf 国 kann dieses Spektrum gedruckt werden.

Ein Doppelklick auf ⿶GC-Temperatur am rechten Bildschirmrand zeigt statt des GC-Spektrums den Temperaturverlauf im GC-Ofen am Bildschirm.

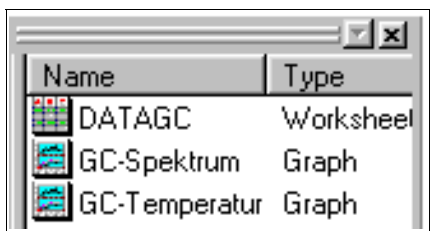




\subsection{Ansicht ausgewählter MS-Spektren}

Sobald ein GC-Spektrum gemäß der oben in Kap. 8.1 (S. 19) beschriebenen Weise geöffnet ist, kann auch durch Doppelklick auf 㽞DATAGC am rechten

Bildschirmrand das „OriginWorksheet" mit den GC-Daten geöffnet werden.

Dieses Worksheet enthält einerseits die Daten aus der GC-Datei

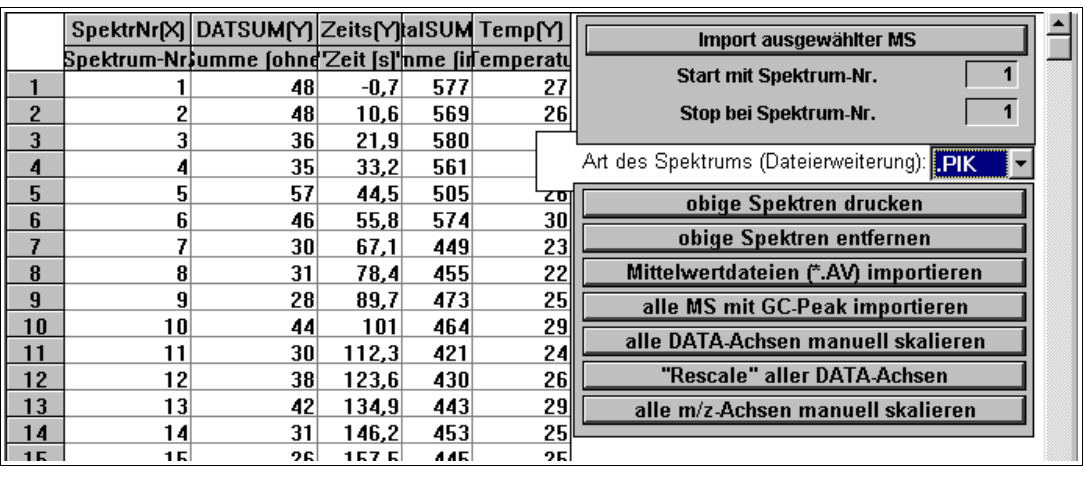
(vgl. Kap. 7, S. 17), andererseits aber auch einige Schalter zur weiteren Auswertung der Massenspektren.

Um nun einige der zum GC-Spektrum zugehörigen Massenspektren anzeigen zu können, muß zunächst ausgewählt werden, ob diese aus der ,*.DAT“Datei, der „*.PIK“-Datei oder der „*.SUB“"Datei (siehe dazu Kap. 7, S. 17) erzeugt werden sollen.

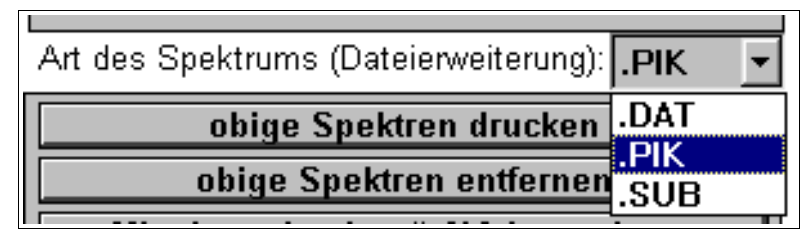
Die gewünschte „Art des Spektrums (Dateierweiterung)“ ist in der Auswahlbox entsprechend zu wählen.

In obigem GC-Spektrum war z.B. der erste Peak bei Massenspektrum Nr. 69 zu erkennen. Wenn man nun z.B. die Spektren Nr. 68 bis 69 ansehen möchte, dann sind diese Nummern in der nebenstehend abgebildeten Eingabebox einzugeben und

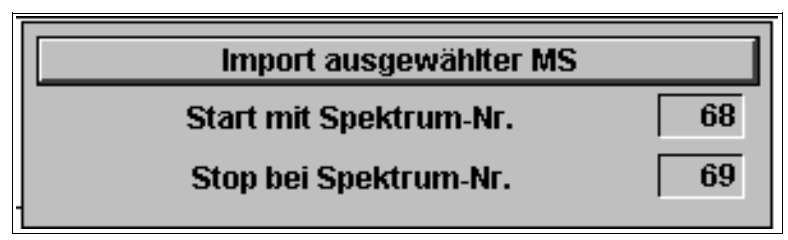
anschließend der Schalter „Import ausgewählter MS“ anzuklicken. 
„Origin“ erzeugt nun diese Massenspektren und zeigt das mit der höchsten Nr. automatisch am Bildschirm an:

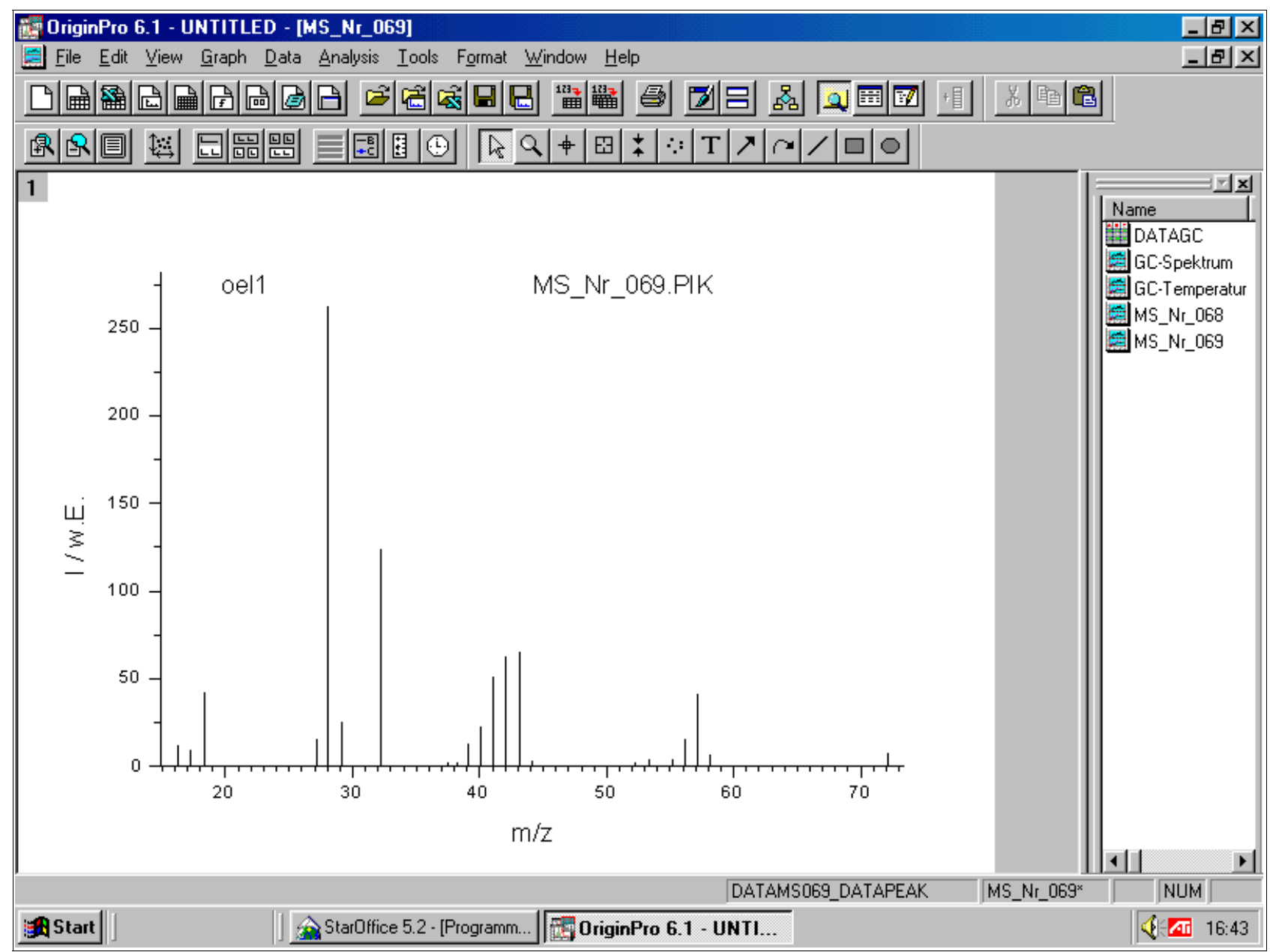

Um nun z.B. das Massenspektrum Nr. 68 anzuzeigen, ist am rechten Bildschirmrand einfach auf

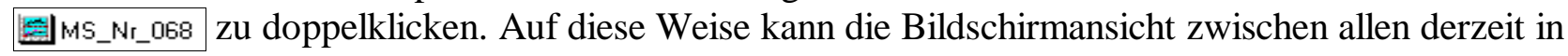
„Origin“ erzeugten Spektren schnell gewechselt werden.

Auch das Worksheet mit den Schaltern kann man durch Doppelklick auf um z.B. weitere Massenspektren zu importieren.

\subsection{Drucken ausgewählter MS-Spektren}

Natürlich kann jedes einzelne Spektrum (oder aber auch alle geöffneten Spektren) durch den Druckbefehl „File/Print ...“ des „Origin“Programms gedruckt werden.

Sollen jedoch nur ausgewählte Spektren gedruckt werden, muß durch Doppelklick auf 呣DATAGC am rechten Bildschirmrand zunächst das „Origin-Worksheet“ mit den Schaltern angezeigt werden (vgl.oben Kap. 8.2, S. 21).

Sollen z.B. die Massenspektren Nr. 68 bis 70 gedruckt werden, sind in der nebenstehend abgebildeten Eingabebox diese Nummern entsprechend einzugeben.

\begin{tabular}{|l|r|}
\hline $\begin{array}{l}\text { Start mit Spektrum-Nr. } \\
\text { Stop bei Spektrum-Nr. }\end{array}$ & $\mathbf{6 8}$ \\
\hline
\end{tabular}


Dann muß auf den Schalter obige Spektren drucken geklickt werden und es werden selektiv die Massenspektren Nr. 68 bis 70 gedruckt.

Diese Methode setzt jedoch voraus, daß die zu druckenden Spektren zuvor bereits erzeugt, d.h. sie in „Origin“ bereits importiert (vgl.oben Kap. 8.2, S. 21) wurden.

\subsection{Entfernen ausgewählter MS-Spektren aus der Ansicht}

Völlig analog zu der oben in Kap. 8.3 beschriebenen Methode lassen sich selektiv auch die zuvor in „Origin“ importierten Massenspektren wieder aus der Ansicht entfernen.

Jedoch ist nun auf den Schalter obige Spektren entfernen zu klicken.

Diese Art des Entfernens ist dem bloßen Löschen des „Graph-Windows“ vorzuziehen, weil so auch das (der Übersichtlichkeit halber nicht angezeigte) Massenspektren-"Worksheet" sowie das „Dataset“ gelöscht werden.

\subsection{Ansicht aller Mittelwertdateien}

Alle gemäß Kap. 6.1.4 (S. 13) erzeugten Mittelwertdateien können durch Klick auf den Mittelwertdateien (".AV) importieren nebenstehend abgebildeten Schalter als Spektren in „Origin“ importiert und angezeigt werden.

\subsection{Ansicht aller MS-Spektren mit Peak im GC-Spektrum}

Dieser Programmteil ermöglicht die wohl bequemste Art, sich zu allen GC-Peaks die zugehörigen Massenspektrum importieren und anzeigen zu lassen.

Zunächst läßt man sich gemäß der in Kap. 6.1.3 (S. 12) beschriebenen Art und Weise vom Programm „GCMS-CP“ alle Nummern der gesuchten Massenspektren ermitteln und speichert diese ab (,,*.MAX“-Datei).

Anschließend können durch einen Klick auf den nebenstehend abgebildeten Schalter alle ermittelten alle MS mit GC-Peak importieren Massenspektren in „Origin“ importiert und dann ggf. angezeigt/gedruckt werden.

\subsection{Skalierung der DATA-Achsen (Y-Achsen) aller MS-Spektren}

Durch Klick auf den nebenstehend abgebildeten Schalter können die DATA-Achsen (Y-Achsen) aller alle DATA-Achsen manuell skalieren bereits importierten Massenspektren auf eine einheitliche Höhe skaliert werden.

Es öffnet sich das nebenstehend abgebildete Fenster, in dem das Maximum der Y-Skalierung einzugeben ist; der Minimum-Wert ist automatisch Null. 
Nach Klick auf den Schalter „OK“ werden alle Spektren neu skaliert. Der Fortschritt dabei kann am linken unteren Rand des „Origin“-Fensters verfolgt werden (eine Zahl läuft von 1 bis 999 durch).

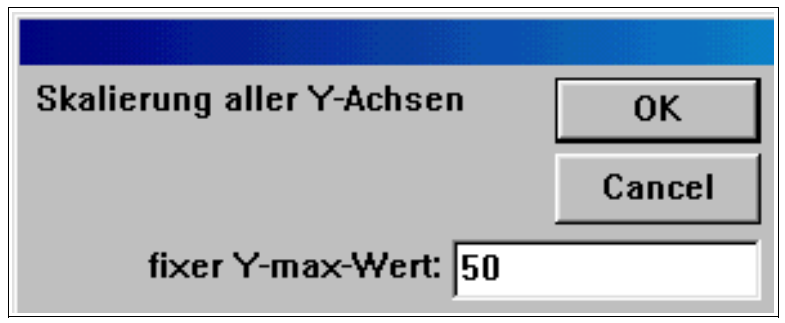

\subsection{Automatische Skalierung der DATA-Achsen}

Durch Klick auf den Schalter werden die DATAAchsen aller bereits importierten Massenspektren automatisch auf eine möglichst bildschirmausfüllende Größe skaliert. Diese Funktion bezieht sich leider nicht nur auf die DATA-Werte in dem angezeigten m/z-Bereich, sondern auf das gesamte gespeicherte Spektrum.

\subsection{Skalierung der m/z-Achsen (X-Achsen) aller MS-Spektren}

Durch Klick auf den nebenstehend abgebildeten Schalter können die m/z-Achsen (X-Achsen) aller alle $\mathrm{m} / \mathrm{z}$-Achsen manuell skalieren bereits importierten Massenspektren auf einen einheitlichen Bereich skaliert werden.

Es öffnet sich das nebenstehend abgebildete Fenster, in dem das Minimum und Maximum der m/zSkalierung (X-Achse) einzugeben ist.

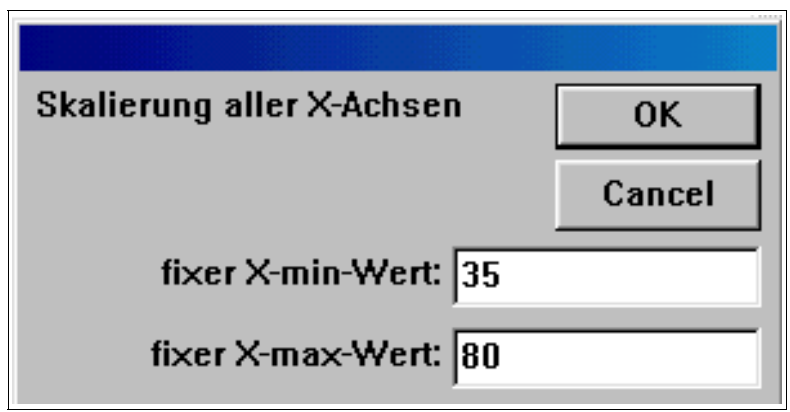

Nach Klick auf den Schalter „OK“ werden alle Spektren neu skaliert. Der Fortschritt dabei kann am linken unteren Rand des „Origin“-Fensters verfolgt werden (eine Zahl läuft von 1 bis 999 durch).

\section{Problem-/Fehlerbehebung}

Der einzige bisher aufgetretene Fehler betrifft die Fehlermeldung (die im Anschluß an eine GC-MS-Analyse während der Datenkonvertierung auftaucht), daß eine MCS-Datei nicht gefunden wurde. Hierfür ist eine nicht ausreichend hohe Verzögerungszeit zwischen der Aufnahme

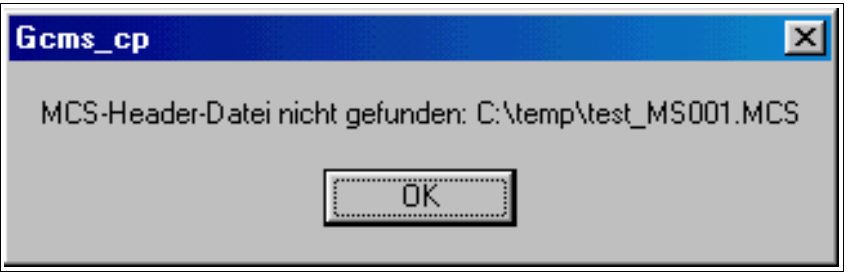
der Massenspektren verantwortlich, so daß das „MCS-32“ nicht genug Zeit hat, seine Rohdaten zwischenzuspeichern. Diese Messung ist komplett zu verwerfen, denn die Zuordnung der m/z-Werte zu den DATA-Werten ist dann falsch (auch wenn es nicht so scheint).

Abhilfe schafft nur eine Erhöhung der Verzögerungszeit (siehe Kap. 6.4.1, S. 16) oder ein schnellerer Computer. Während der GC-MS-Messung wird sowohl vom „GCMS-CP“- als auch vom „MCS-32“-Programm die Nummer des aktuell registrierten Spektrums angezeigt. Bei einer ausreichend hohen Verzögerungszeit stimmen diese beiden Nummern immer überein (wobei der Wechsel der Nummer beim „MCS-32“-Programm geringfügig länger dauert, weil dieses seine Rohdaten langsamer zwischenspeichert). 
Sollte die Nachweisempfindlichkeit nicht ausreichen, kann ggf. der Emissionsstrom am MS erhöht werden. Auch eine Erhöhung der SEV-Versorgungsspannung kann sinnvoll sein. Jedoch ist darauf zu achten, daß dadurch auch das Rauschen deutlich erhöht werden kann; die SEV-

Versorgungsspannung darf daher nur so hoch sein, daß die MCS-Karte bei m/z 0 keine NIM-Pulse registriert. In letzterem Fall ist es sinnvoller, das SEV-Ausgangssignal höher zu verstärken. Derzeit wird es um den Faktor 200 verstärkt, aber ein höherer Faktor wäre sicherlich besser.

\section{Ausgewählte Beispiele einer GC-MS-Analyse}

\subsection{Analyse einer niedrig siedenden Erdölfraktion}

Das nachstehende GC-Spektrum zeigt den Inhalt einer Erdölfraktion mit Siedebereich $50-70{ }^{\circ} \mathrm{C}$.

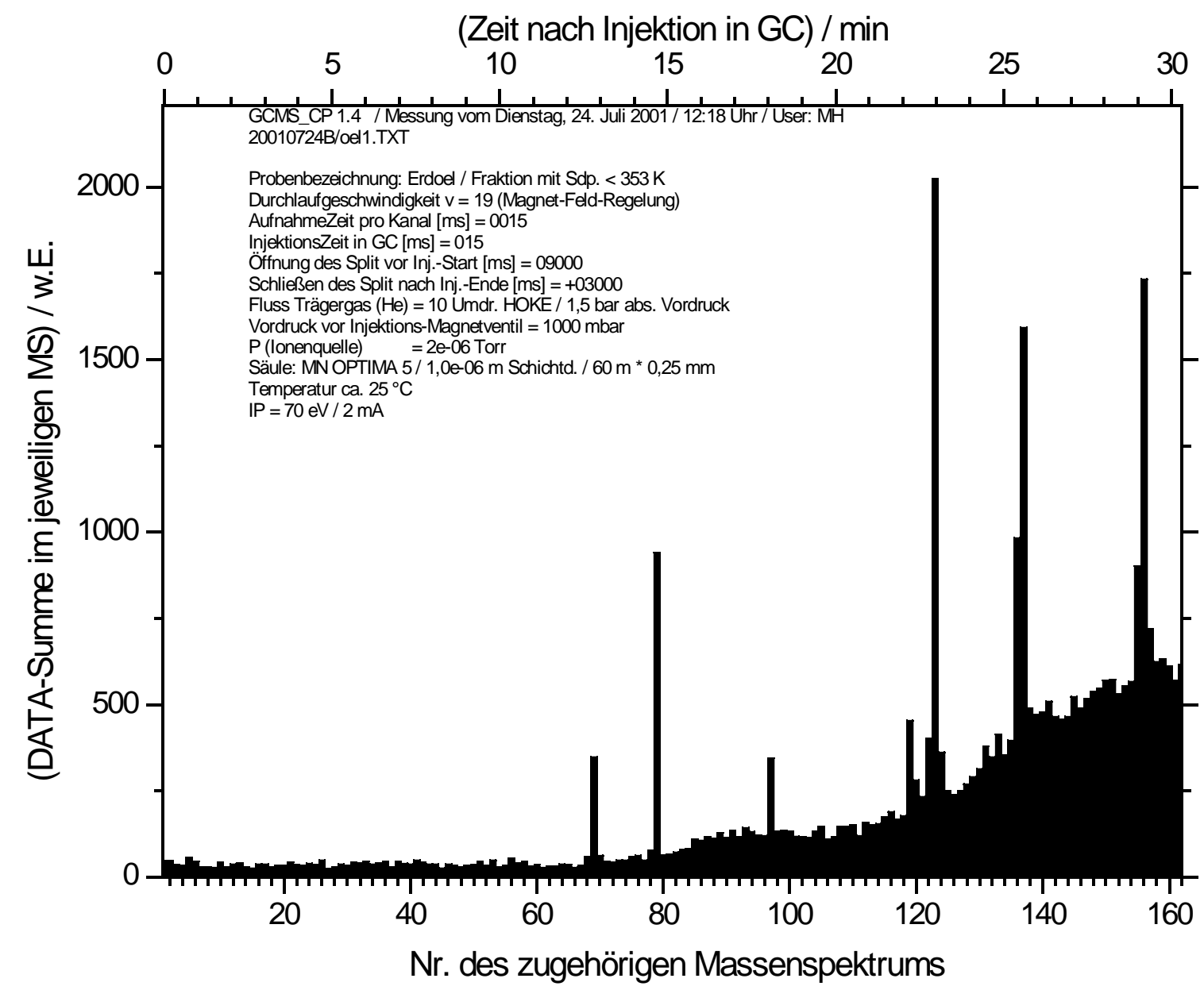

Man erkennt, daß diese Fraktion aus mindestens 7 Komponenten besteht.

Die Hauptkomponenten sind durch die Massenspektren Nr. 79, 123, 137 und 156 charakterisiert. 
Die erste Hauptkomponente dürfte gemäß Massenspektrum Nr. 79

\section{Pentan}

sein.

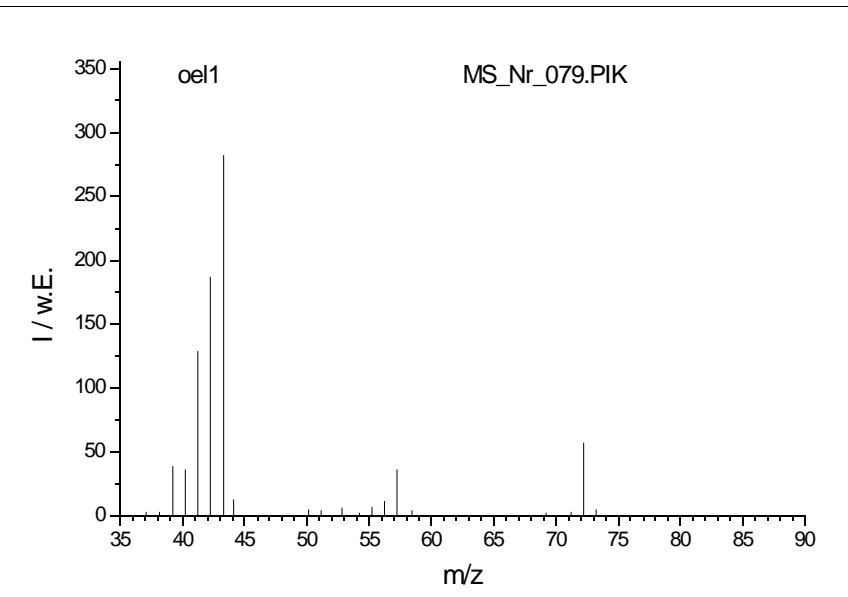

Die zweite Hauptkomponente dürfte gemäß Massenspektrum Nr. 123

2-Methylpentan

sein.

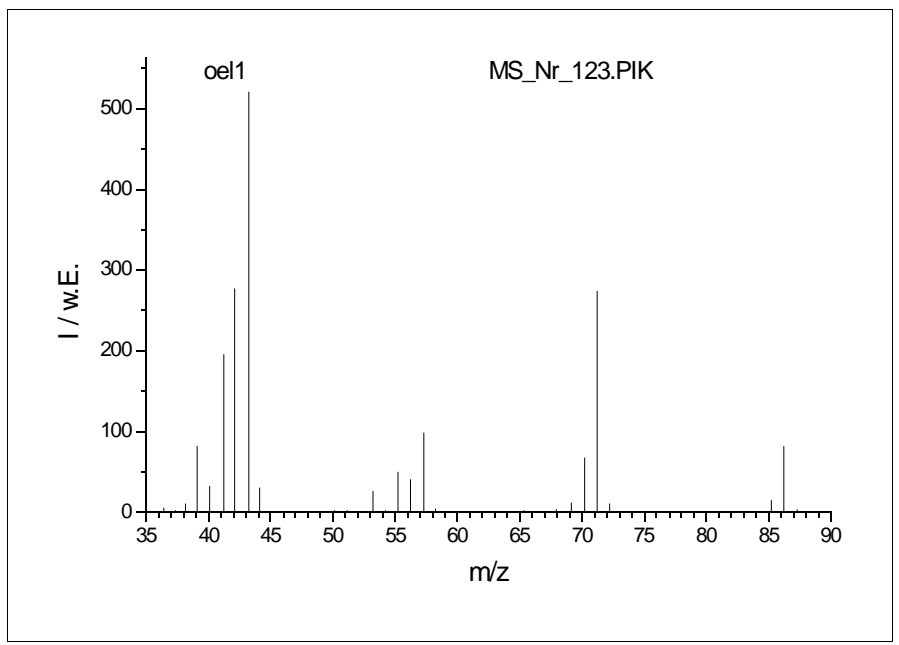

Die dritte Hauptkomponente dürfte gemäß Massenspektrum Nr. 137

3-Methylpentan

sein.

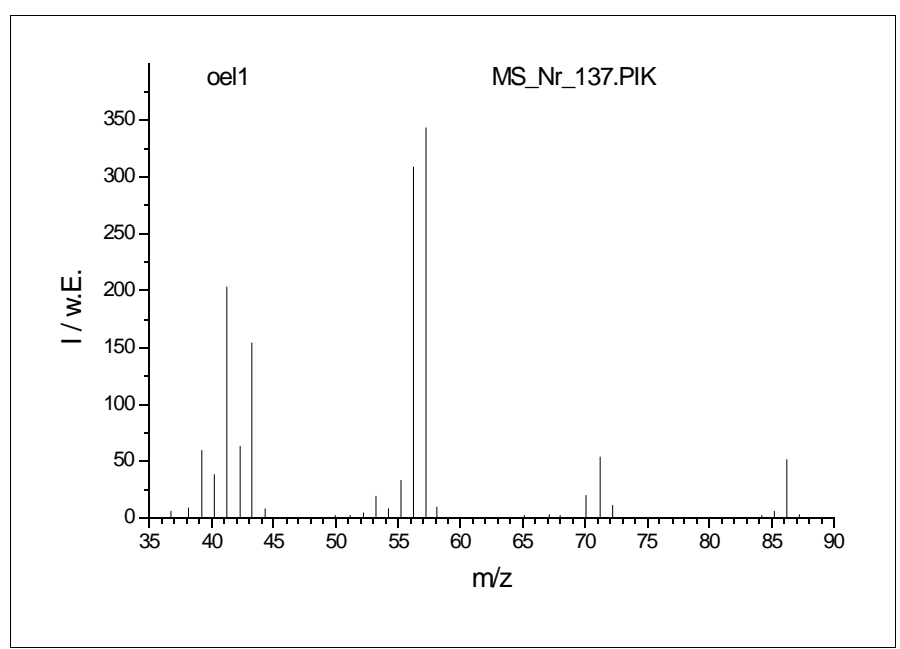




\subsection{Analyse der Mischung 1,1,1,2-Tetrafluorethan/Propen/2-Buten}

Das nebenstehende GC-Spektrum zeigt die vollständige Trennung von $\mathrm{CF}_{3} \mathrm{CH}_{2} \mathrm{~F}, \mathrm{CH}_{3} \mathrm{CH}=\mathrm{CH}_{2}$ und

$\mathrm{CH}_{3} \mathrm{CH}=\mathrm{CHCH}_{3}$.
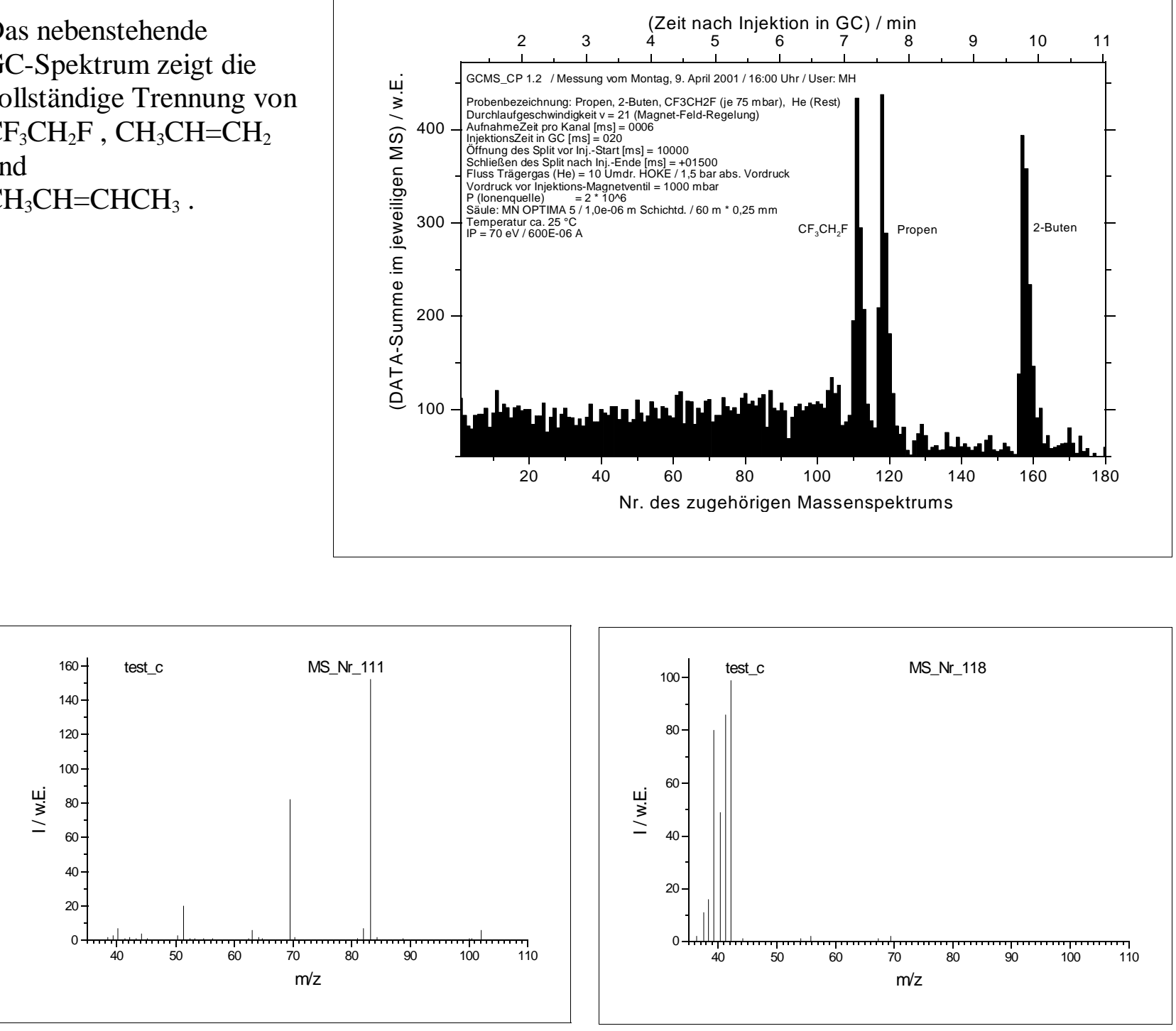

Aus den Massenspektren läßt sich leicht folgern, daß der erste GC-Peak (Massenspektrum Nr. 111) der Komponente 1,1,1,2-Tetrafluorethan zuzurechnen ist.

Der zweite GC-Peak (Massenspektrum Nr. 118) ist der Komponente Propen zuzurechnen.

Der dritte GC-Peak (Massenspektrum Nr. 157) stammt von der Komponente 2-Buten.

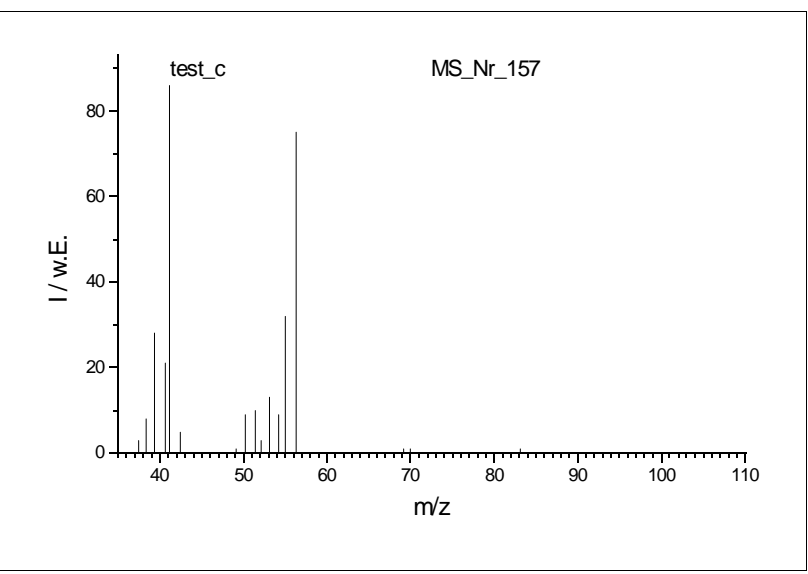




\subsection{Analyse der Mischung 2-Chlor-2-methylpropan/1-Chlor-2-methylpropan}

Das nebenstehende GC-Spektrum zeigt, daß die chlorierten

Kohlenwasserstoffe deutlich längere Retentionszeiten haben als die reinen Kohlenwasserstoffe, aber auch als die fluorierten.

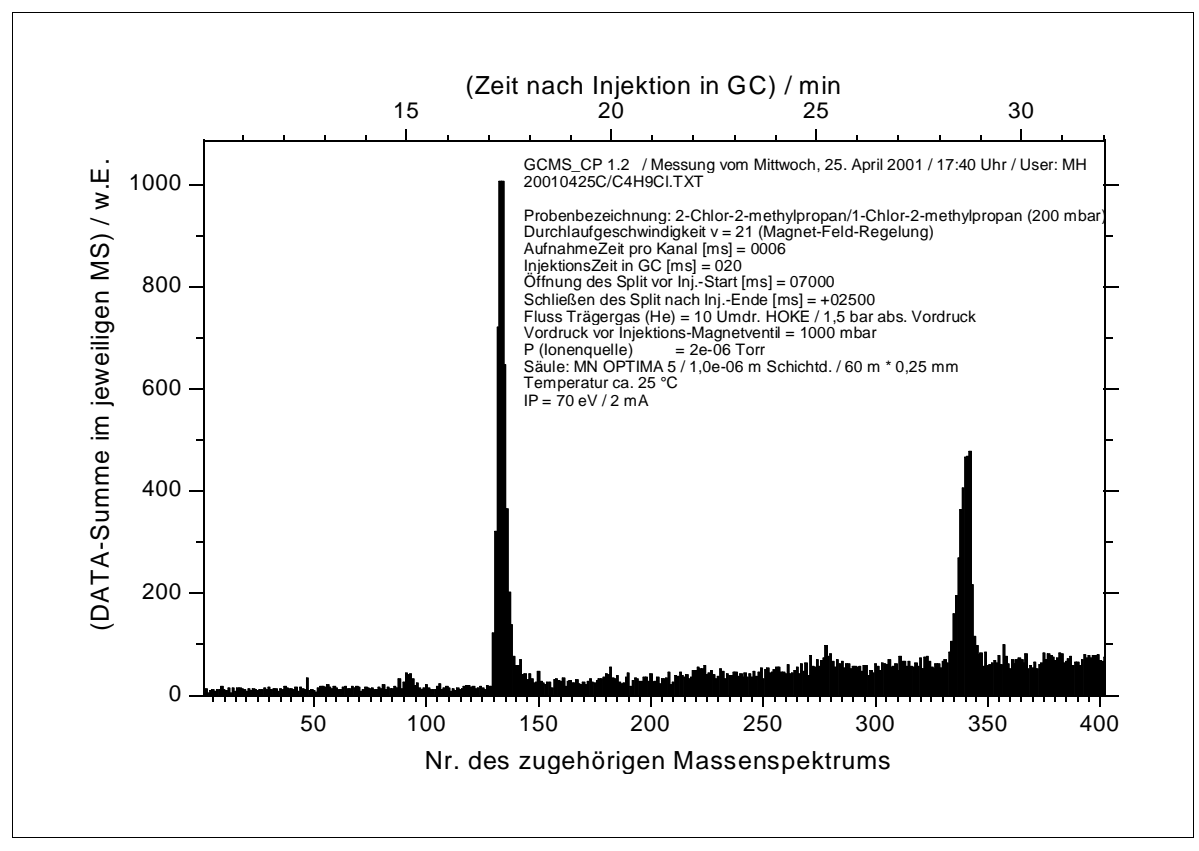

Aus den Massenspektren läßt sich leicht folgern, daß der erste GC-Peak (Massenspektrum Nr. 133) der Komponente 2-Chlor-2-methylpropan zuzurechnen ist.

Der zweite GC-Peak (Massenspektrum Nr. 342) stammt hingegen von der Komponente 1-Chlor-2-methylpropan.
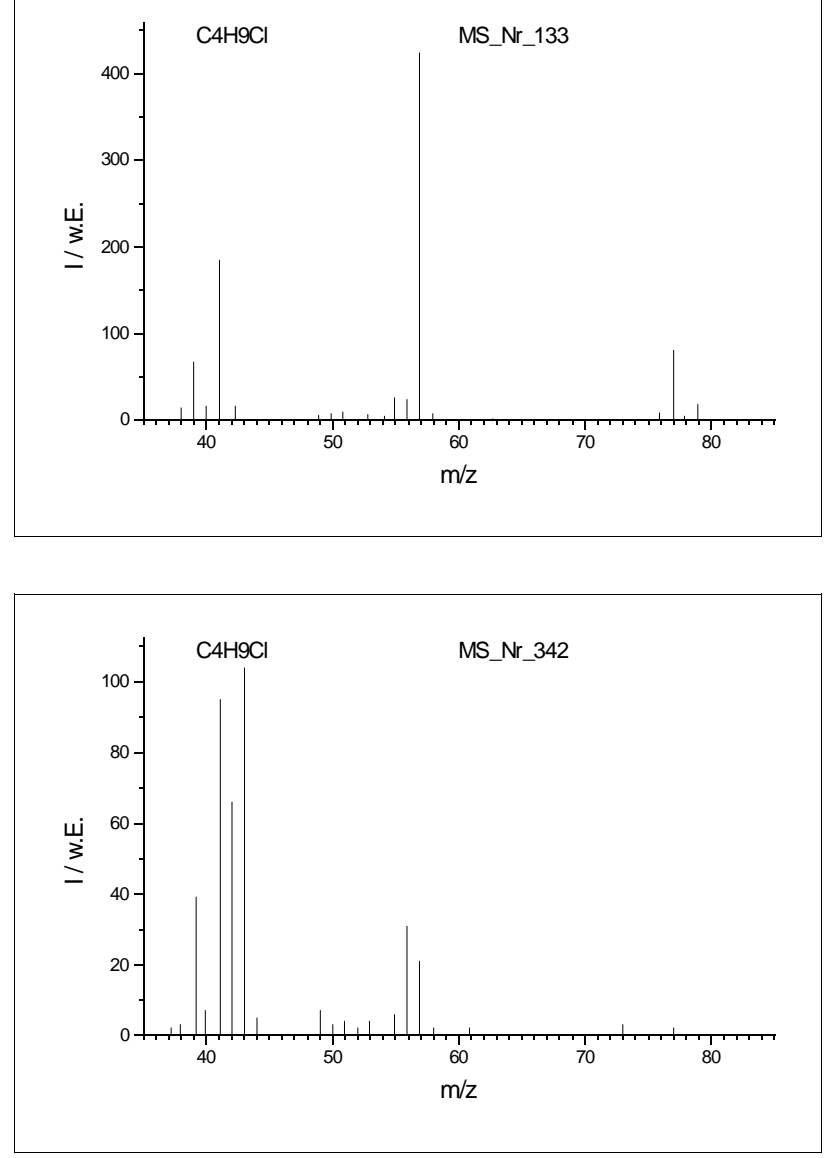


\subsection{Analyse der Mischung Cyclopropan/Propen}

Das nebenstehende GC-Spektrum zeigt, daß auch die entstehende Substanzmischung einer thermischen Isomerisierung von Cyclopropan gut mit der GC-MS-Kopplung analysiert werden kann.

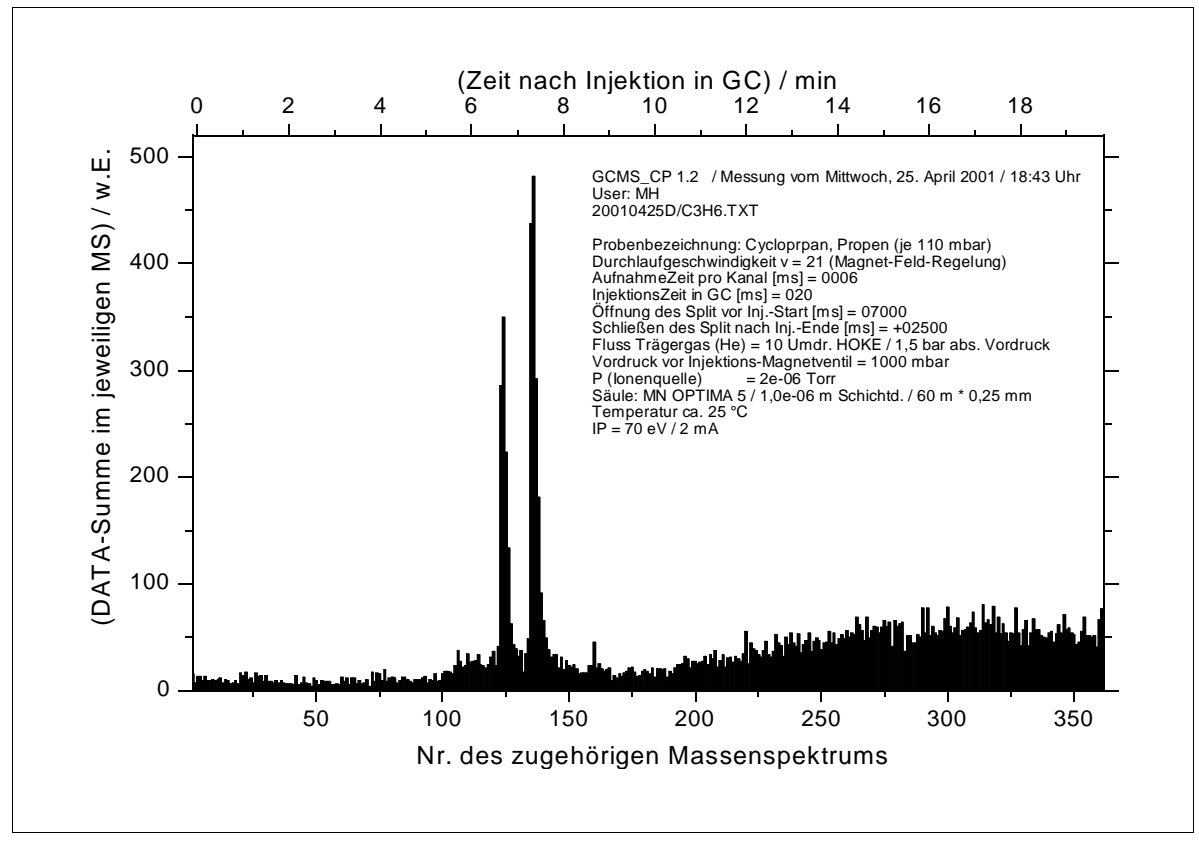

Aus den Massenspektren läßt sich folgern, daß der erste GC-Peak (Massenspektrum Nr. 124) der Komponente Propen zuzurechnen ist.

Der zweite GC-Peak (Massenspektrum Nr. 136) stammt hingegen von der Komponente Cyclopropan.
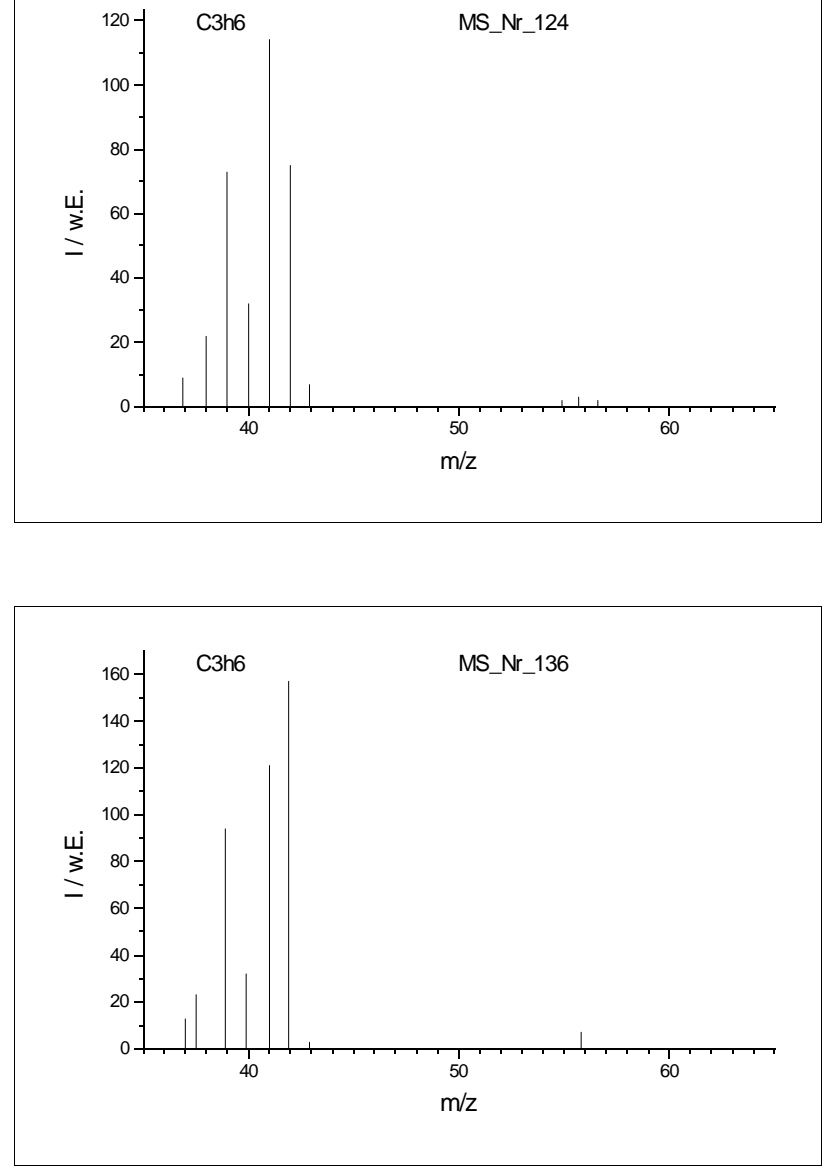


\section{Stichwortverzeichnis}

*.AV.

14, 18

*.DAT. .18

*.GC.

17,19

*.MAX $13,19,23$

*.PIK 11,18

*.SUB 19

*.TXT.

AD-Karte $2 f, 5,16$

Aufnahmezeit pro Kanal.

Badgas-Magnetventil. .15

Benutzerkürzels.

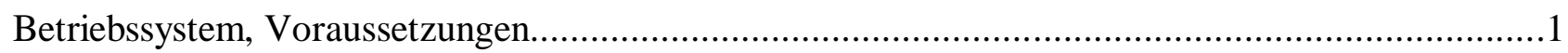

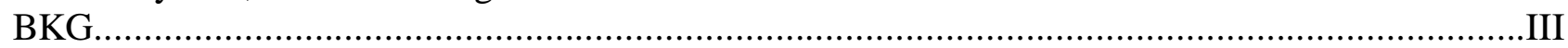

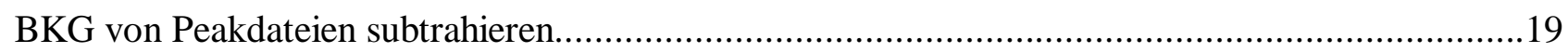

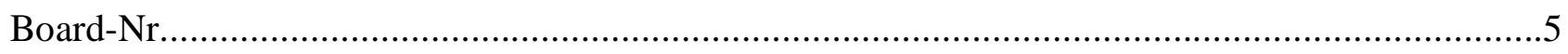

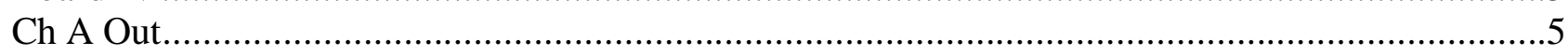

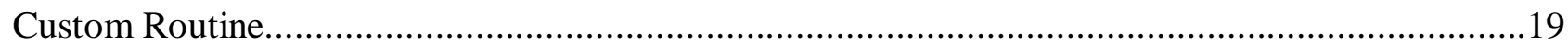

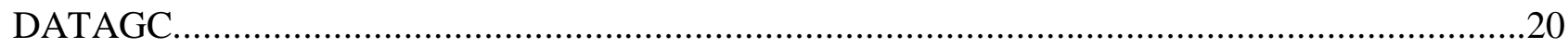

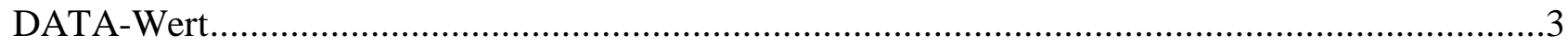

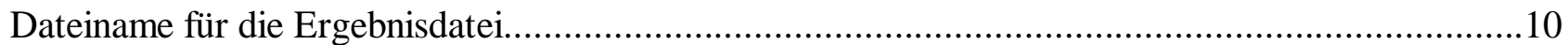

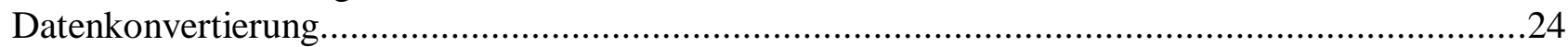

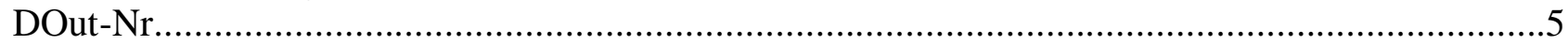

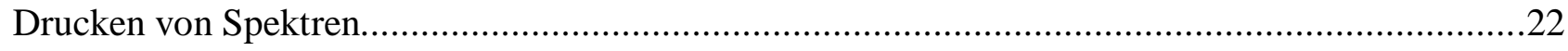

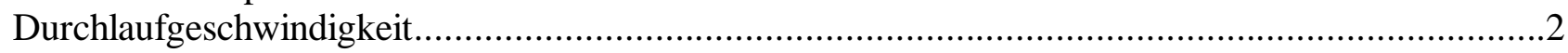

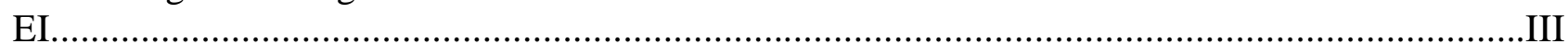

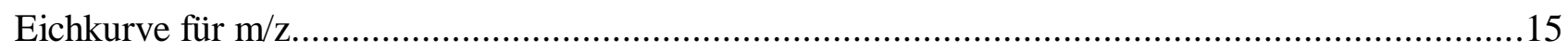

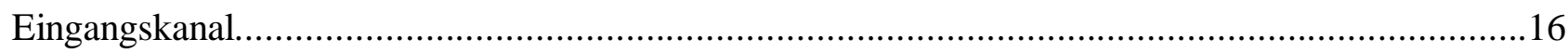

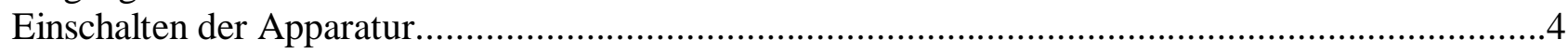

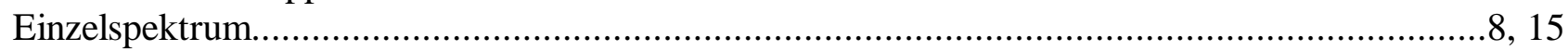

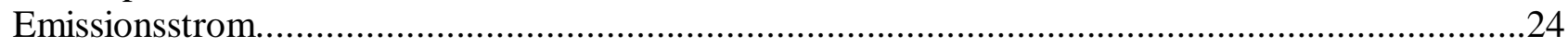

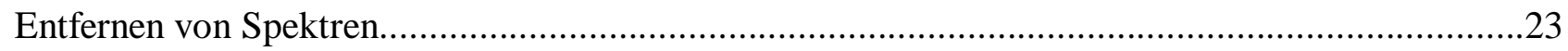

Feinjustierung, Ionenquelle..............................................................................................

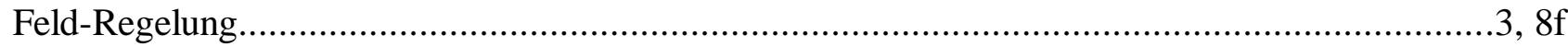

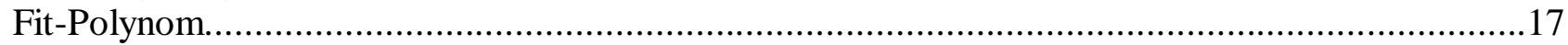

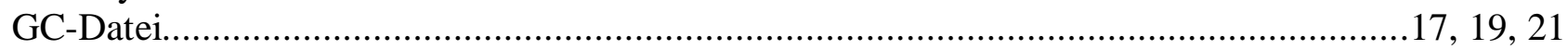

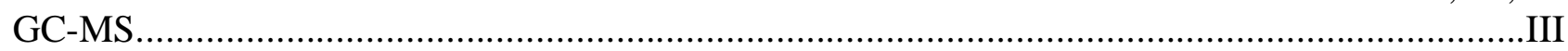

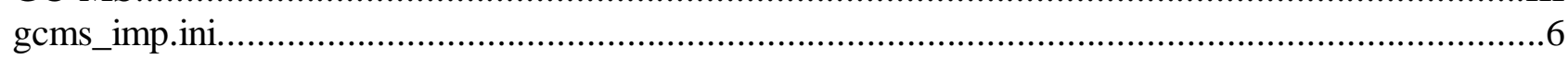

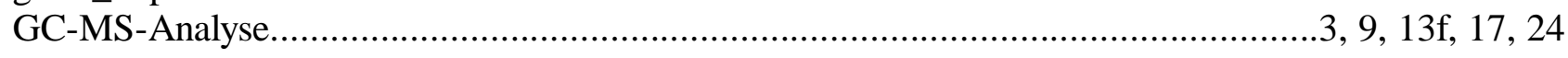

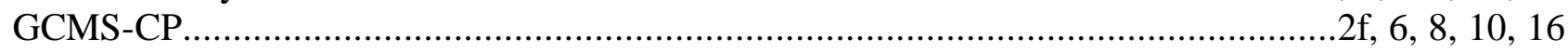

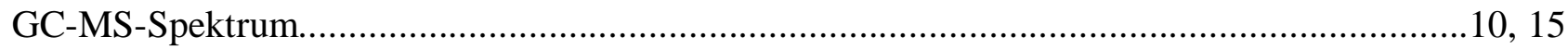

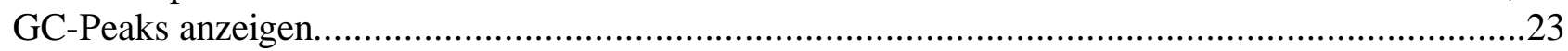

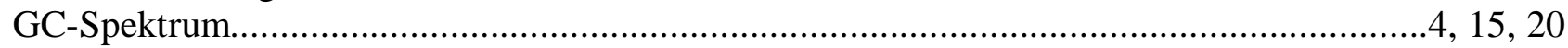

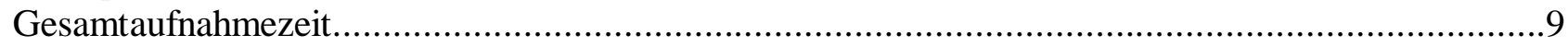

Hall-Sonde

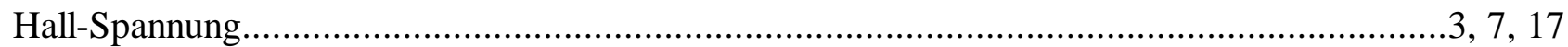

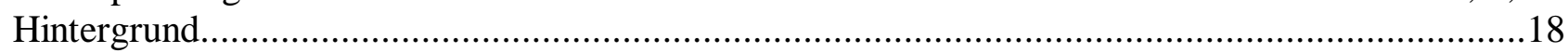

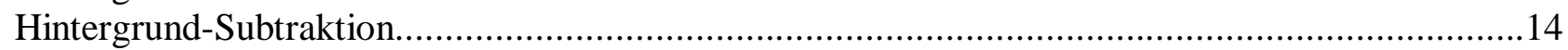

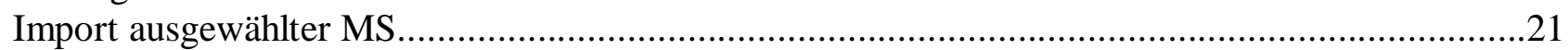


Inbetriebnahme der Apparatur.

Injektion...

Ionenquelle, Feinjustierung.

IR Inp.

Job Control......

Justierprogramm.

Justierung, Ionenquelle.

Kalibrierung der Hall-Sonde..

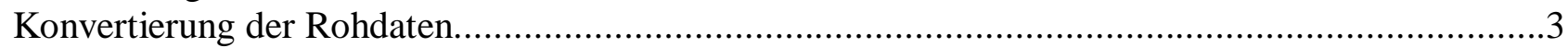

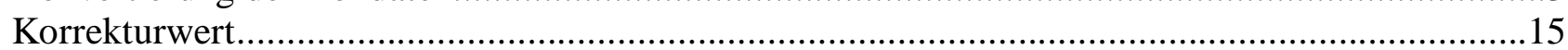

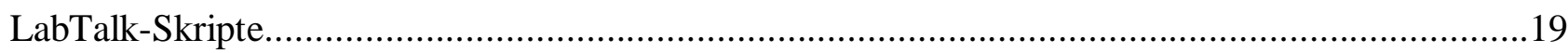

$\mathrm{m} / \mathrm{z}$.

Magnetventile manuell steuern.

Massenspektren.

MCS-32.

MCS-Karte.

Meßbereitschaft der Apparatur herstellen.

MessTextdatei

Messung abbrechen.

Mittelwertdatei.

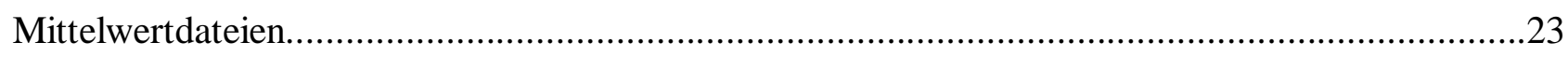

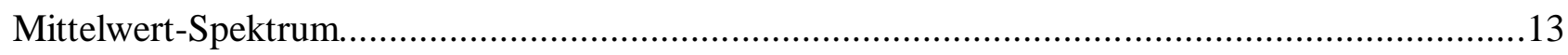

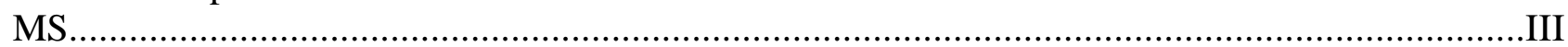

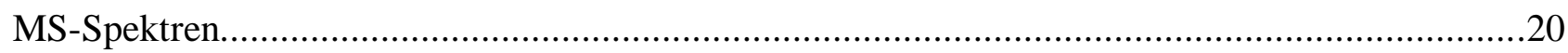

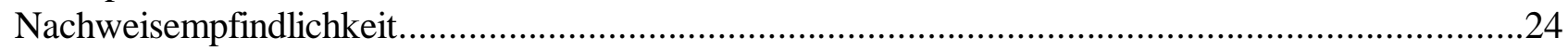

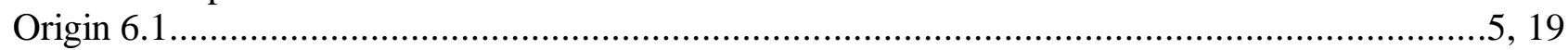

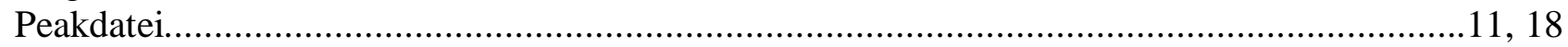

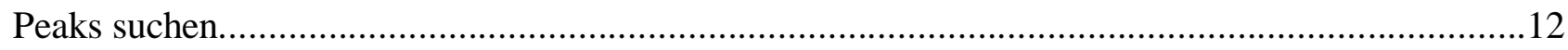

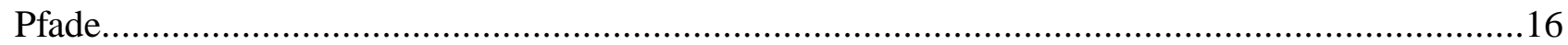

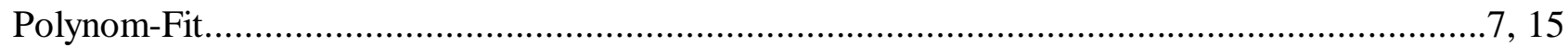

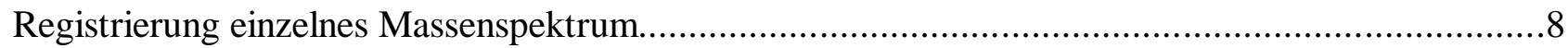

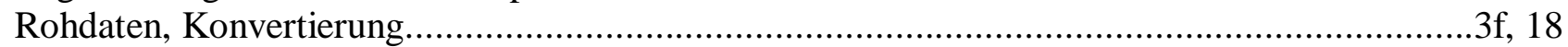

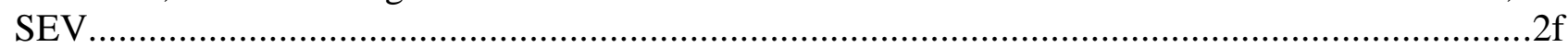

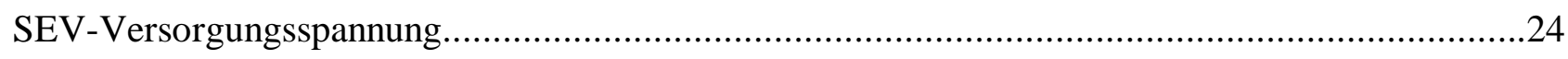

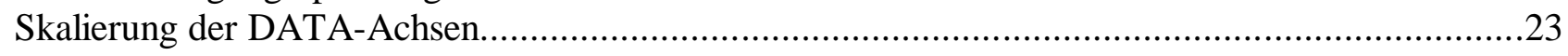

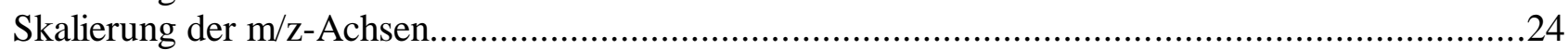

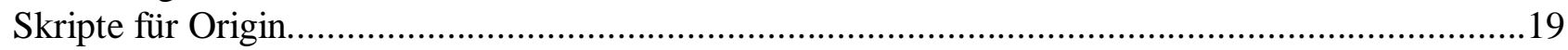

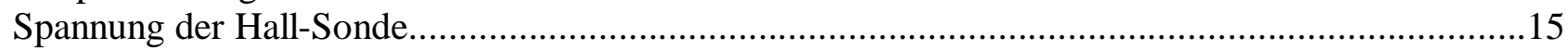

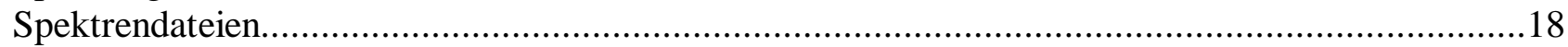

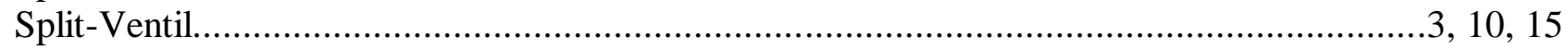

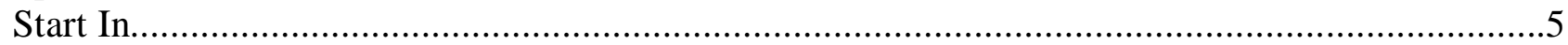

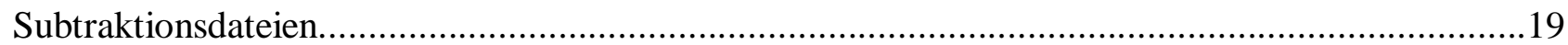

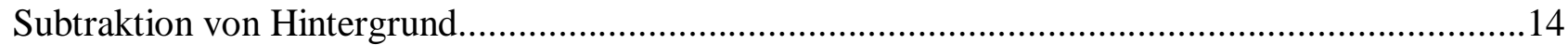

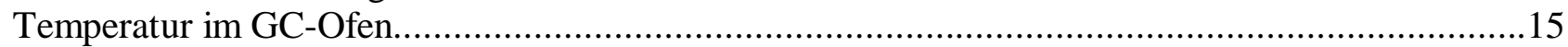

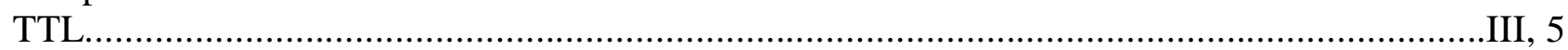

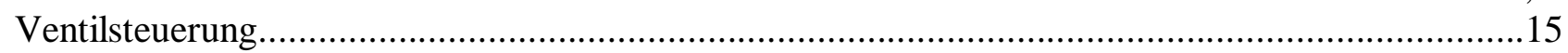

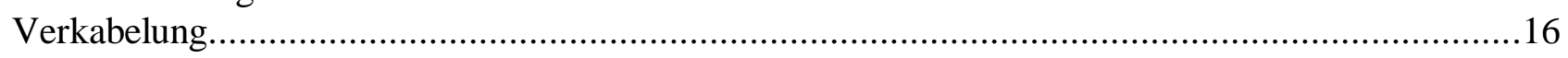

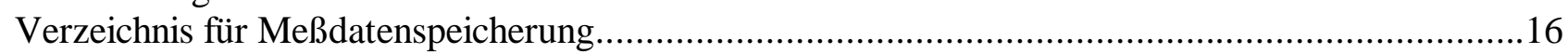

Verzögerungszeit zwischen einzelnen Spektren....................................................4, 16, 24

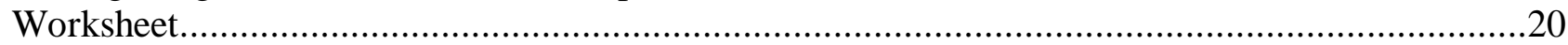






\section{Programmbeschreibung für}

\section{WINSICCON \\ Version 1.7}

(Windows Single Ion Counting Control Program)

Kontroll- und Auswertungsprogramm für Einzelionenzählung am VARIAN MAT CH5 - Massenspektrometer

Konzeption:

Frank Nacke, 1994

Jens Wehmeyer, 2001

Markus Hold, 1997-2001

Programmierung:

Frank Nacke, 1994

Markus Hold, 1997-2001

Dokumentation:

Markus Hold, 1997-2001 



\section{Inhaltsverzeichnis}

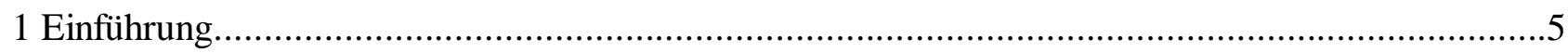

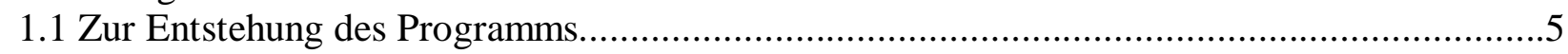

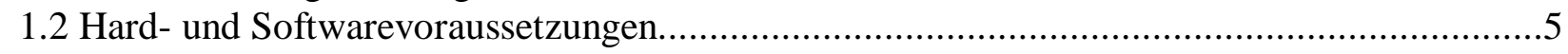

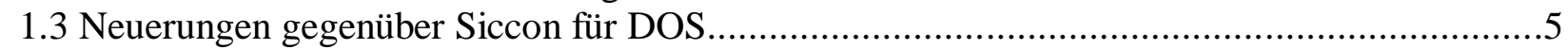

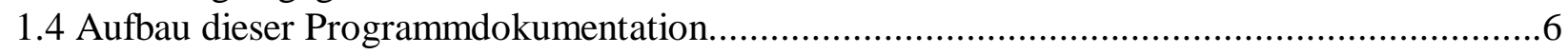

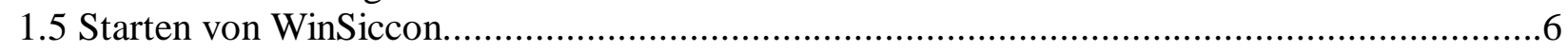

1.6 Typische Vorgehensweise bei der Analyse unbekannter Substanzen....................................6

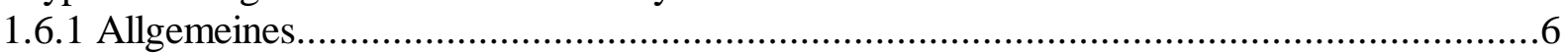

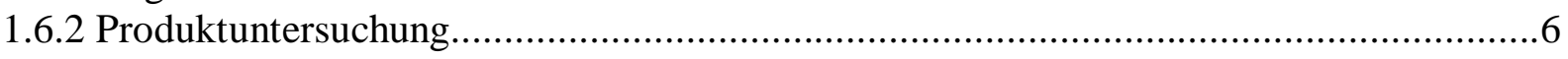

1.6.3 Bestimmung der Kinetik der radikalerzeugenden Reaktion......................................

1.6.4 Bestimmung der Kinetik der Reaktion „Radikal + 2. Reaktant“ “...............................

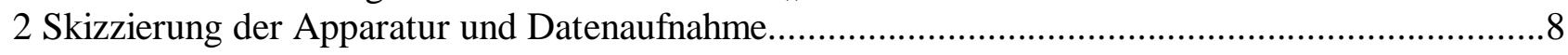

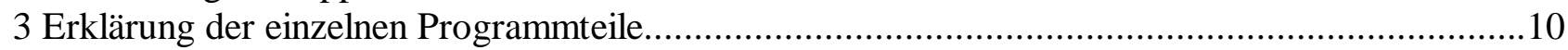

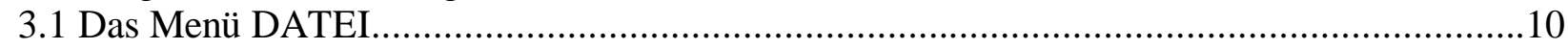

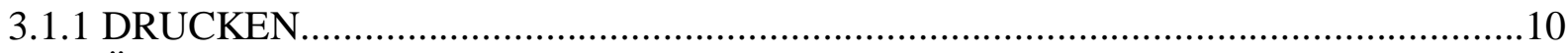

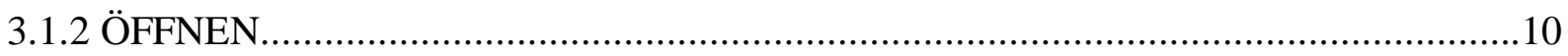

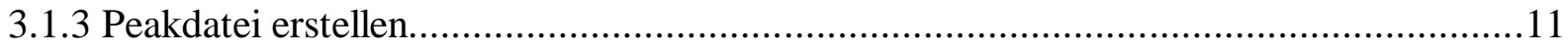

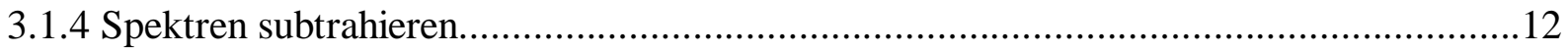

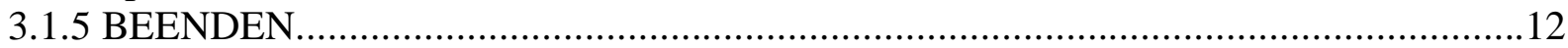

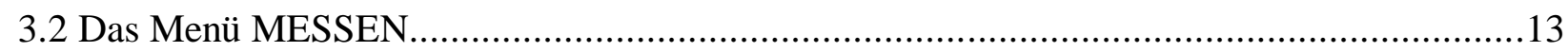

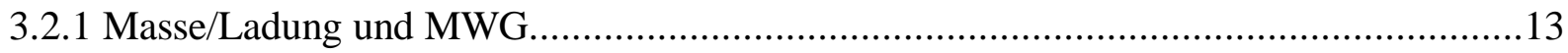

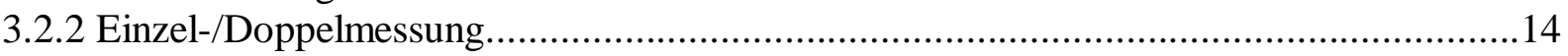

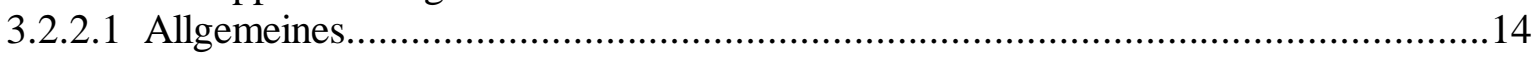

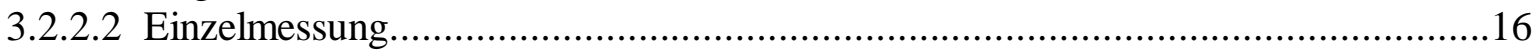

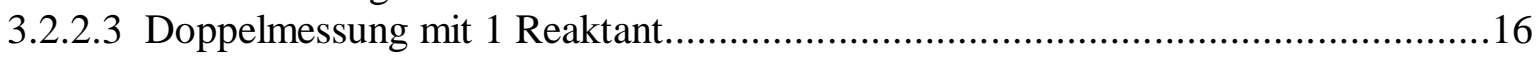

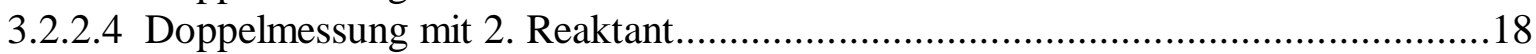

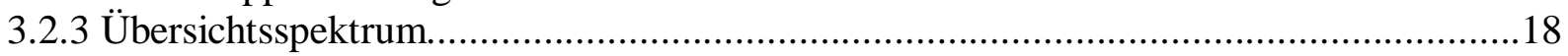

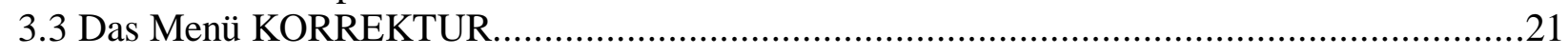

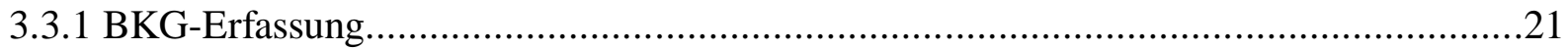

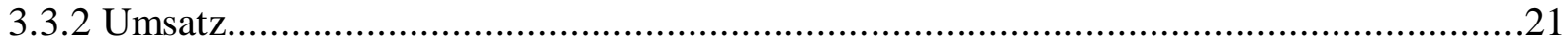

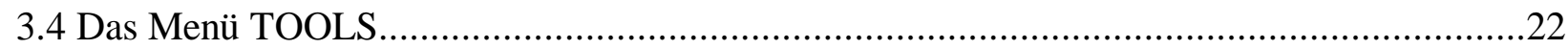

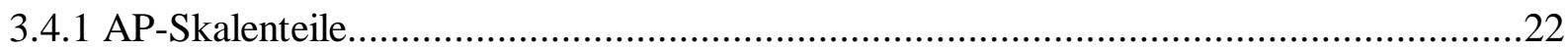

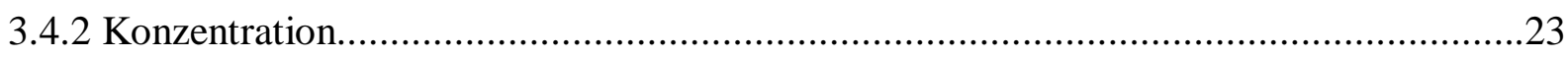

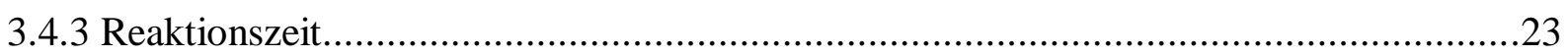

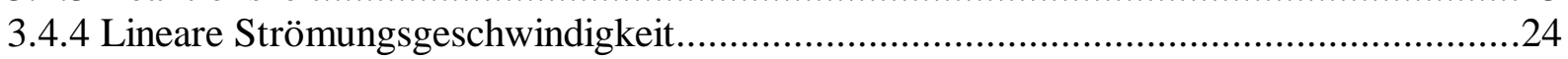

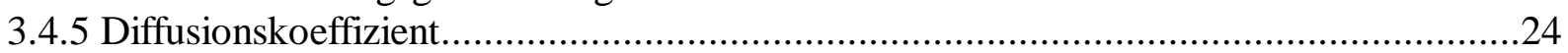

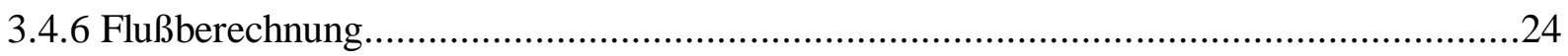

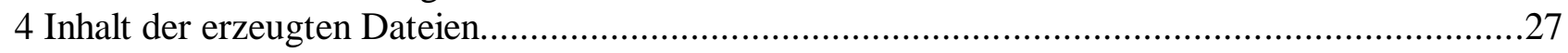

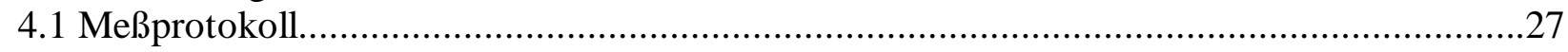

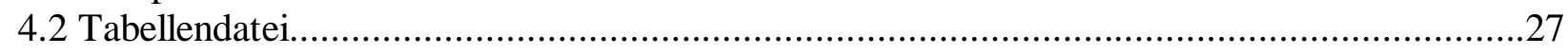

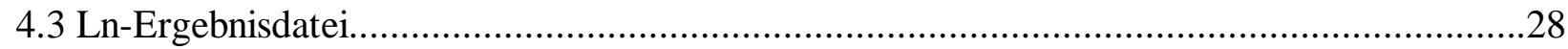

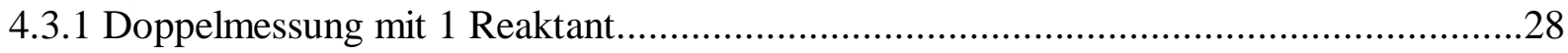

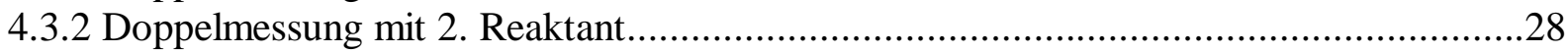

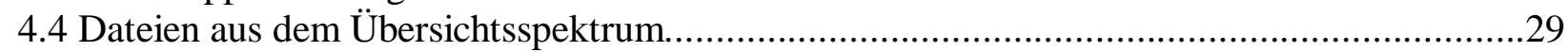

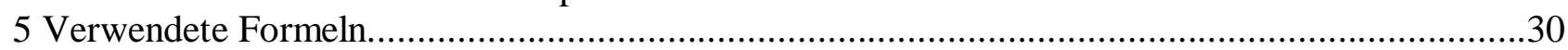

5.1 Berechnung der Logarithmen-Verhältnisse .......................................................... 30

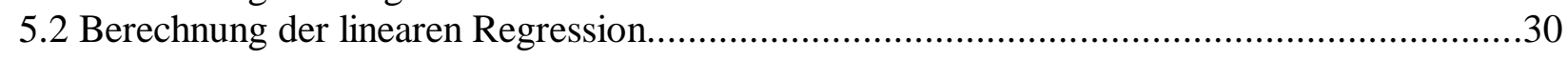

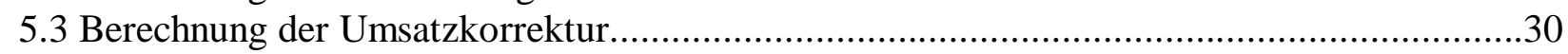

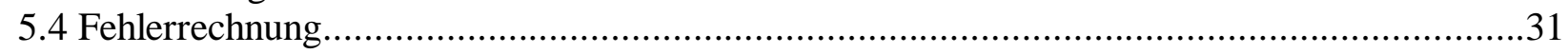




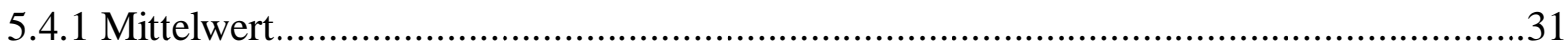

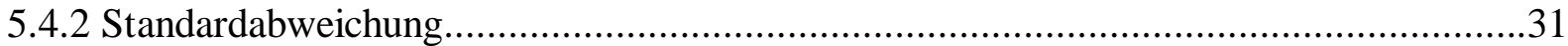

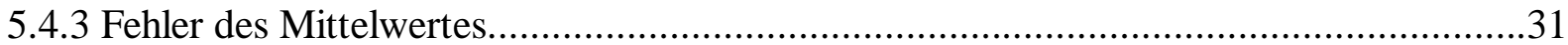

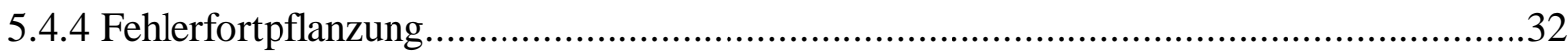

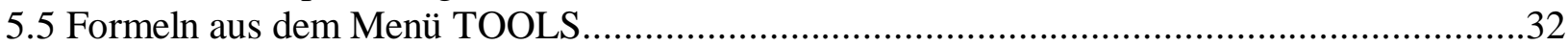

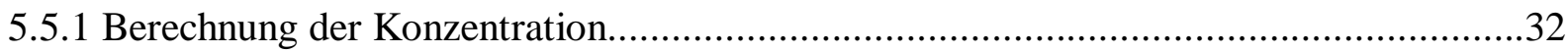

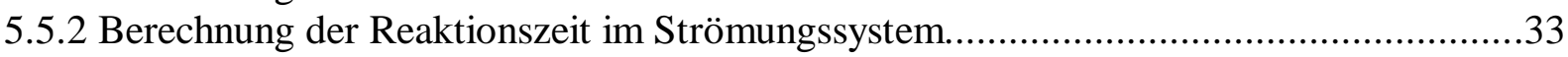

5.5.3 Berechnung der linearen Strömungsgeschwindigkeit.............................................33

5.5.4 Berechnung des Diffusionskoeffizienten nach Chapman-Enskog...............................33

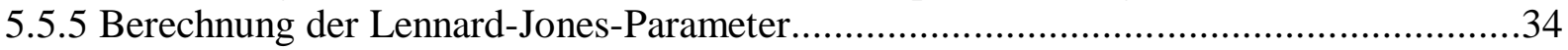

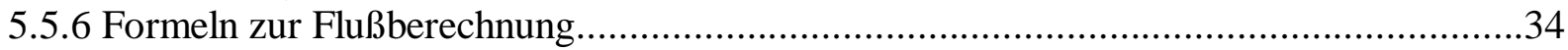

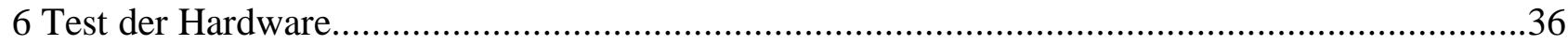

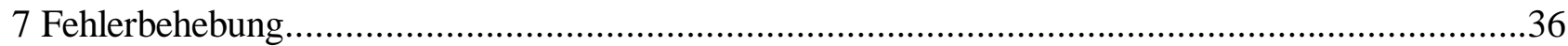

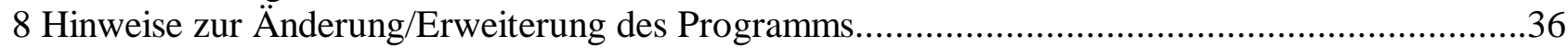

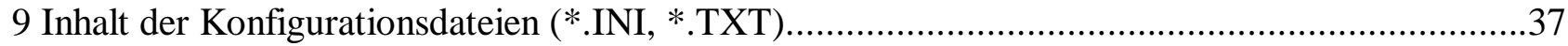

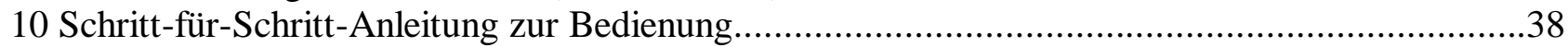

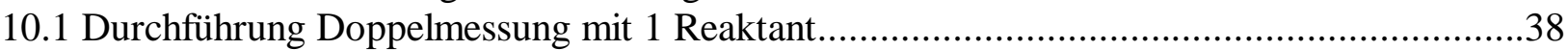

10.2 Durchführung (umsatzkorrigierter) Doppelmessung mit 2. Reaktant................................39

10.3 Messung mit Fragment der Haupt-Substanz auf Peak des Radikals der Referenz-Substanz. 40 


\section{Einführung}

\subsection{Zur Entstehung des Programms}

Die vorliegende Version des Programms WinSiccon ist eine Weiterentwicklung des Programms Siccon 1.1 für DOS, das 1994 von Frank Nacke erstellt wurde. Damals stand nur ein 80286Computer zur Verfügung, so daß eine Version für Windows nicht lauffähig gewesen wäre. Inzwischen wurden die Meßwerterfassungskarten jedoch in einen leistungsfähigeren Computer eingebaut, so daß nun auch ein schneller Programmablauf unter Windows möglich ist. Daher erfolgte ab 1997 die Portierung des Programms auf die grafische Benutzeroberfläche Windows 3.1, welche die Bedienung etwas erleichtert, da verschiedene Fenster nebeneinander gleichzeitig betrachtet werden können. Ferner wurden sehr viele neue Funktionen (siehe unten) hinzugefügt.

Beide Programme sind in der Computersprache Objekt Pascal geschrieben; das Programm WinSiccon wurde mit Borland Delphi 1.02 entwickelt und compiliert. Die Copyright-Vermerke im Programm sind Bestandteil der Lizenzvereinbarung mit der Firma Borland zur Weitergabe von compilierten Programmen; sie dienen also nicht (nur) der Selbstdarstellung der Autoren.

\subsection{Hard- und Softwarevoraussetzungen}

Das Programm setzt mindestens einen 80386-Computer mit numerischem Coprozessor (80x87) voraus und ist für den Einsatz unter Microsoft-Windows 3.1 bzw. 3.11 for Workgroups konzipiert.

Sofern der Adressraum der Meßwerterfassungskarten frei ist, sollte auch der Einsatz unter Win95 möglich sein; keinesfalls aber ist es unter dem Betriebssystemen Windows NT einsetzbar.

\subsection{Neuerungen gegenüber Siccon für DOS}

Die wichtigsten Erweiterungen sollen kurz stichpunktartig erwähnt werden:

- $\mathrm{m} / \mathrm{z}$-Anzeige kann als eigenes Fenster ständig angezeigt werden.

- Meßprotokoll kann vollständig am Bildschirm eingesehen werden.

- Meßtexte beliebiger Länge können vor jedem Meßzyklus eingegeben werden und erscheinen dann automatisch im Meßprotokoll.

- Wenn ein Meßprotokoll angelegt wird, stehen alle Meßwerte inkl. Fehler auch noch als Tabelle in einer ASCII-Datei zur weiteren Bearbeitung (z.B. in einer Tabellenkalkulation) zur Verfügung.

- Bei Messung gegen eine Referenzsubstanz werden die aufgenommenen Meßwertpaare unmittelbar nach jedem Meßzyklus grafisch dargestellt und auch eine lineare Regression angezeigt. Dies erfolgt sowohl bei den radikalerzeugenden Reaktionen als auch bei den Reaktionen der Radikale mit einem 2. Reaktant.

- Die Fehlerrechnung erfolgt nun für ein Konfidenzintervall, das den Mittelwert wahlweise mit 50, 68, 75, 95 oder $99 \%$ Wahrscheinlichkeit überstreicht.

- Evtl. vorhandene Hintergrundwerte einer best. m/z-Zahl können getrennt für die eigentliche Messung und die Referenz-Messung inkl. Fehler eingegeben werden und werden bei der Auswertung (DIFF-Werte) berücksichtigt; die erfaßten Fehler gehen über Fehlerfortpflanzung in die weiteren Berechnungen ein.

- Bei der Messung der Reaktion eines Radikals mit einem Reaktant kann bei Überlagerung mit Fragmenten aus den Muttermolekülen durch WinSiccon unterstützt umsatzkorrigiert gemessen werden. Die grafisch angezeigte lineare Regression erfolgt dann bereits unter Berücksichtigung dieser Umsatzkorrekturen und stellt daher unmittelbar das Ergebnis dar.

- Die Zugabe eines Reaktanten (in WinSiccon mit „2. Reaktant“ bezeichnet) zu erzeugten Radikalen kann auch computergesteuert mittels Magnetventil erfolgen. Wahlweise kann aber auch 
computergesteuert eine zweite Mikrowellenentladung gestartet werden.

- Die gesamten Ergebnisse werden sinnvoll aufbereitet in verschiedene Dateien geschrieben, die einen unmittelbaren Import und somit eine rasche (Nach-)Auswertung mittels Tabellenkalkulation ermöglichen.

- Es können Übersichtsspektren aufgenommen werden, wobei das Programm im Anschluß an die Registrierung die in den Rohmeßdaten erkennbar zusammengehörenden Peaks zu jeweils einem Meßpunkt (mittelerer m/z-Wert; aufsummierter DATA-Wert) zusammenfaßt und gesondert in einer Datei abspeichert. Diese „Peak-Dateien“ können nun problemlos voneinander subtrahiert werden, um automatisch ein Differenzspektrum zu erzeugen.

\subsection{Aufbau dieser Programmdokumentation}

Die Dokumentation erfolgt nach dem Aufbau des Programms, d.h. es werden die einzelnen Menüpunkte vorgestellt und die jeweiligen Programmteile erklärt.

\section{dif. WinSiccon 1.7 - [C] by F. Nacke [1994] und M. Hold [1997-2001]}

Datei Messen Karrektur Tools Hilfe

In einem weiteren Kapitel werden die in WinSiccon verwendeten Formeln angegeben.

\subsection{Starten von WinSiccon}

Nach dem Doppelklick auf das Programmsymbol im Programm-Manager startet WinSiccon und fragt zunächst zwingend ein Benutzer-Kürzel ab. Dieses maximal zwei Buchstaben lange Kürzel erscheint auf jedem Meßprotokoll und wird auch als Name für das Verzeichnis zum Speichern der Meßdaten sowie als Anfang für den Name eines Tagesunterverzeichnisses verwendet.

Um sicherzustellen, daß während einer Messung auch genug Rechenzeit zur Verfügung steht, wird dringend empfohlen WinSiccon als einziges Programm (neben dem Programm-Manager) zu starten und keinesfalls Anwendungen im Hintergrund laufen zu lassen! Beim Start überprüft WinSiccon diese Bedingungen und gibt ggf. folgende Fehlermeldung aus:

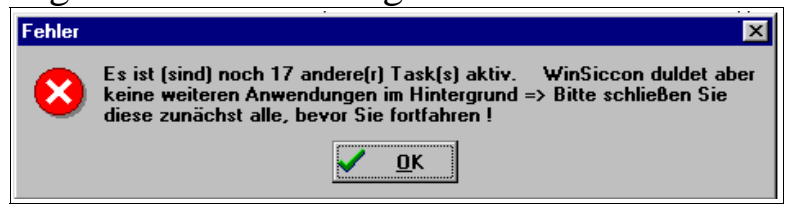

Im Falle dieser Fehlermeldung fragt das System nach einem Klick auf OK gleich, ob sie WinSiccon beenden wollen (was Sie in dem Falle auch tun sollten).

Nach dem Start des Massenspektrometers sollte ca. 30 min zum Warmlaufen der Elektronik abgewartet und dann zuerst die Kalibrierung der Hall-Sonde überprüft werden (vgl. Kap. 3.2.1, S.13).

\subsection{Typische Vorgehensweise bei der Analyse unbekannter Substanzen}

\subsubsection{Allgemeines}

Die Untersuchung einer unbekannten Substanz sollte damit beginnen, die Reaktionsprodukte der zu untersuchenden Reaktion zu ermitteln, zumindest aber die dadurch belegten Massen. Daraufhin kann die Geschwindigkeit der radikalerzeugenden Reaktion (z.B. Kohlenwasserstoff + F) und dann diejenige der Reaktion des Radikals mit einem weiteren Reaktanten (z.B. Radikal + O) bestimmt werden.

\subsubsection{Produktuntersuchung}

Zur Produktuntersuchung empfiehlt es sich, zunächst nachfolgend aufgeführte Übersichtsspektren 
aufzunehmen (vgl. Kap. 3.2.3, S. 18), wobei der He-Fluß jeweils so angepaßt werden muß, daß bei allen Spektren der gleiche Druck im Strömungssystem herrscht (zum Auffinden der Masse und zum Einschalten des MWG bzw. Magnetventils siehe Kap. 3.2.1, S. 13) :

(1.) nur Hintergrund, d.h. nur He-Fluß ohne irgendwelche Substanzen

(2.) nur zu untersuchende Substanz und evtl. $\mathrm{F}_{2}$ (aber ohne MWG !)

(3.) zu untersuchende Substanz mit F-Atomen (möglichst nur 10-20 \% Umsatz der Substanz)

(4.) zu untersuchende Substanz mit F-Atomen und zweitem Reaktant (z.B. O-Atomen)

(5.) zu untersuchende Substanz mit $F_{2}$ und zweitem Reaktant, aber ohne Mikrowellenentladung(en).

Im Anschluß daran sollte Spektrum (1) von (2), dann (2) von (3), dann (3) von (4) und zur Kontrolle auf Reaktion des zweiten Reaktanten mit der Substanz auch (2) von (5) subtrahiert werden (vgl. dazu Kap. 3.1.4, S. 12). Auf diese Art erhält man einen ersten Überblick, auf welchen m/z-Werten durch die jeweiligen Reaktionen $\mathrm{Zu}$ - oder Abnahmen entstehen.

Weil die Spektrenregistrierung relativ schnell erfolgt (Registrierungszeit pro Meßpunkt nur einige hundert ms), sind diese Meßwert jedoch gewissen Schwankungen unterlegen, so daß insbesondere bei nur geringen Änderungen im Differenzspektrum eine Nachmessung mit längerer Registrierungszeit (10-30 s) erfolgen muß. Dazu verwendet man z.B. den Programmteil Einzelmessung (vgl. Kap. , S. ), wo zudem jeder Meßpunkt noch wiederholt gemessen und als Mittelwert verwendet werden kann. Um das manuelle Zuschalten der MWG und/oder des zweiten Reaktanten zu sparen, kann diese Nachmessung aber auch mit den Programmteilen zur Doppelmessung durchgeführt werden (vgl. Kap. 3.2.2.3, S. 16 oder Kap. 3.2.2.4, S. 18).

Aus den Änderungen der m/z-Werte in den Differenzspektren können nun unter Berücksichtigung der Thermodynamik (Reaktionsethalpien) sinnvolle Reaktionsmechanismen abgeleitet werden. Durch Änderung des Ionisierungspotentials am Massenspektrometer kann die Fragmetierung beeinflußt werden, was evtl. zur Aufklärung der Reaktionsprodukte beitragen kann. Ergänzend können die (stabilen) Endprodukte aber auch in einer $\mathrm{N}_{2}(\mathrm{l})$-gekühlten Kühlfalle ausgefroren und dann einer IRund/oder GC-MS-Analyse unterzogen werden.

\subsubsection{Bestimmung der Kinetik der radikalerzeugenden Reaktion}

Die Radikalerzeugung läuft typischerweise über die Reaktion Kohlenwasserstoff + F-Atome, deren Kinetik am besten nach der Relativmethode bestimmt werden kann.

Aus der Produktuntersuchung ist ja hervorgegangen, welche $\mathrm{m} / \mathrm{z}$-Werte von der Reaktion betroffen sind. Die Relativsubstanz muß eine Molekülmasse haben, die nicht auf einem durch die Reaktion belegten $\mathrm{m} / \mathrm{z}$-Wert liegt und sollte in etwa den gleichen Geschwindigkeitskoeffizient aufweisen, wie die zu untersuchende Reaktion; man muß also ggf. verschiedene Relativsubstanzen ausprobieren, bis letztere Bedingung erfüllt ist. Ferner sollten die zu untersuchende Substanz und die Relativsubstanz etwa in gleicher Konzentration in den Strömungsreaktor gegeben werden

Einige allgemeine Hinweise zum Programmteil „Messen“ sind in Kap. 3.2.2.1 (S. 14) angegeben und die Besonderheiten bei einer „Doppelmessung mit 1 Reaktant“ sind in Kap. 3.2.2.3 (S. 16) beschrieben.

Zur Hilfe bei der konkreten Durchführung der Messung ist ferner eine Schritt-für-Schritt-Anleitung hinten im Kap. 10.1 (S. 38) abgedruckt.

\subsubsection{Bestimmung der Kinetik der Reaktion „Radikal + 2. Reaktant“}

Sofern der Geschwindigkeitskoeffizient der Reaktion „Radikal + 2. Reaktant“ relativ klein ist (etwa $10^{9}$ bis $10^{11} \mathrm{~cm}^{3} \mathrm{~mol}^{-1} \mathrm{~s}^{-1}$ ) kann die Reaktionskinitik nach der Absolutmethode bestimmt werden. Dazu muß der Programmteil „Einzelmessung“ (vgl. Kap. , S. ) verwendet werden, wobei dann die 
Reaktionsstrecke jeweils im Programm eingegeben werden kann und anschließend automatisch zusammen mit den gemessenen DATA-Werten und dem Druck im Strömungsreaktor in der Tabellendatei (*.TAB) gespeichert wird. Diese Tabellendatei kann dann leicht in eine Tabellenkalkulation importiert und dort weiterverarbeitet werden. Je nach Auswertungsmethode muß evtl. lediglich noch der Logarithmus der DATA-Werte gebildet und die Reaktionsstrecke in die Reaktionszeit (siehe dazu Kap. 3.4.3, S.23) umgerechnet werden. Zur Auswertung der Reaktion „,pseudo 1. Ordnung“ kann die erforderliche Konzentration des 2. Reaktanten vom Programm berechnet werden (siehe Kap. 3.4.2. S. 23).

Falls der Geschwindigkeitskoeffizient der Reaktion „Radikal + 2. Reaktant“ jedoch groß ist (etwa $10^{13}$ bis $10^{14} \mathrm{~cm}^{3} \mathrm{~mol}^{-1} \mathrm{~s}^{-1}$ ) sollte die Reaktionskinitik nach der Relativmethode bestimmt werden.

Aus der Produktuntersuchung ist ja hervorgegangen, welche $\mathrm{m} / \mathrm{z}$-Werte von der Reaktion betroffen sind. Die Radikalmasse (und bei umsatzkorrigierter Messung auch die Molekülmasse) der Relativsubstanz muß auf einer laut Produktuntersuchung nicht belegten Masse liegen und sollte in etwa den gleichen Geschwindigkeitskoeffizient aufweisen, wie die zu untersuchende Reaktion; man muß also ggf. verschiedene Relativsubstanzen ausprobieren, bis letztere Bedingung erfüllt ist. Ferner sollten das Radikal der zu untersuchenden Substanz und dasjenige der Relativsubstanz etwa in gleicher Konzentration in dem Strömungsreaktor erzeugt werden.

Einige allgemeine Hinweise zum Programmteil „Messen“ sind in Kap. 3.2.2.1 (S. 14) angegeben und die Besonderheiten bei einer „Doppelmessung mit 2. Reaktant“ sind in Kap. 3.2.2.4 (S. 18) beschrieben.

Zur Hilfe bei der konkreten Durchführung der Messung ist ferner eine Schritt-für-Schritt-Anleitung hinten im Kap. 10.2 (S. 39) abgedruckt.

\section{Skizzierung der Apparatur und Datenaufnahme}

Das vom SEV verstärkte Signal wird über eine dreiteilige Baugruppe (SSR Instruments; Model 1120 Amplifier/Diskriminator/Pulsformer) in einen Zweikanalzähler (SSR Instruments; Model 1110 Digital Syncronous Computer) gegeben. Die durch ein einzelnes Ion am SEV erzeugte Ladung fließt über einen $50 \Omega$ Widerstand schnell ab und der dabei erzeugte Spannungspuls wird im ersten Teil der Baugruppe (Amplifier) um den Faktor 2300 verstärkt; im zweiten Teil (Diskriminator) werden zur Störsignalunterdrückung alle Signale unter $50 \mu \mathrm{V}$,,ausgeschieden“ und im dritten Teil schließlich aus den verbleibenden Signalen ein normierter Puls geformt.

Diese normierten Pulse werden nun auf den Zweikanalzähler gegeben, der sie über eine voreingestellte Zeit aufsummiert; anschließend wird diese Summe dann an einen angeschlossenen Personal Computer weitergeleitet. Parallel dazu wird das Signal auch auf einen Vielkanalanalysator (Tracor Northern NS-575 A Digital Signal Analyser; $15 \mathrm{MHz}$ ) gegeben, auf dem es optisch verfolgt werden kann.

Da die Anzahl der pro Zeiteinheit auf den SEV auftreffenden Ionen etwa proportional der Konzentration der jeweiligen Substanz am Ort der Probennahme ist, führt eine Konzentrationsänderung durch Variation der Reaktionsparameter zu einer Intensitätsänderung des jeweiligen Signals, welche mittels Zweikanalzähler recht empfindlich nachgewiesen werden kann. Unter Umständen vereinzelt auftretende Störpulse, die oberhalb der Diskriminatorschwelle liegen, werden als einzelner Puls registriert und dürften gegenüber der Gesamtzahl der Pulse zu vernachlässigen sein.

Das für eine bestimmte Konzentration registrierte Signal ist stoffspezifisch und auch von experimentellen Parametern (Art der Probennahme, Einstellung der Ionenoptik) abhängig. Daher 
können (unter sonst gleichen Bedingungen) aus unterschiedlichen Signalen für ein und denselben Stoff zwar die relativen, jedoch nicht die absoluten Konzentrationen angegeben werden; letztere müßten durch eine Kalibrierung bestimmt werden. Für unterschiedliche Stoffe ist nicht einmal die Ermittlung der relativen Konzentrationen möglich, was insbesondere bei der Bestimmung von Produktverzweigungsverhältnissen zu beachten ist.

Der an die Apparatur angeschlossene Personal Computer (Typ: 80386) ist mit drei Schnittstellen (ISA-Karten der Firma „Appli Data“) ausgerüstet:

- PA110: Interface mit 64 digitalen optoentkoppelten Eingängen

- PA150: Interface mit 16 digitalen Ausgängen

- PA300: 12 Bit A/D-Wandler mit 16 Kanälen und 2 Logik-Treibern

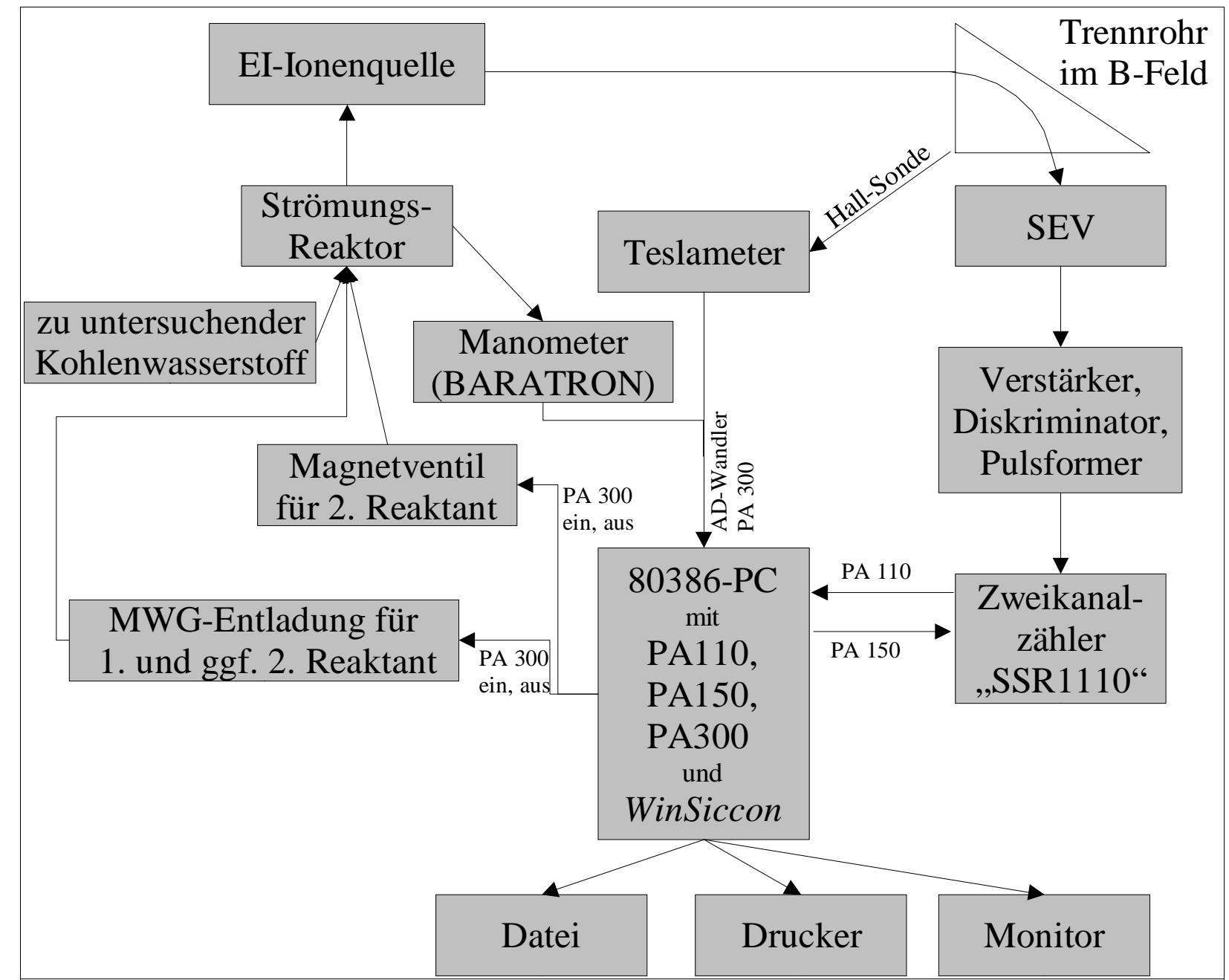

Über die PA150 wird ein Startimpuls gegeben, der den Zweikanalzähler veranlaßt, eine an letzterem voreingestellte Zeit alle Pulse zu zählen. Im Programm kann man die Anzahl der Messungen zwischen 1 und 10 beliebig vorgeben.

Über die PA110 werden die Kanäle DATA, BKG, SUM und DIFF des Zweikanalzählers im BCDFormat eingelesen, wobei der DATA-Wert unmittelbar das registrierte aufsummierte Signal angibt und BKG ein ggf. vorher eingelesenes Hintergrundsignal; SUM und DIFF sind die Summe bzw. Differenz aus DATA und BKG. Diese Werte werden sofort auf dem Bildschirm ausgegeben und auf die Festplatte geschrieben. Sofern man die Option, mehrfach hintereinander zu messen, nutzt, werden auch der arithmetische Mittelwert und der mittlere Fehler des Mittelwertes für ein vorgegebenes 
Konfidenzintervall berechnet und ausgegeben, so daß man starke Schwankungen sofort erkennen und eine Wiederholung der Messung durchführen kann; bei Relativmessungen werden zudem noch jeweils die gemessenen Logarithmenverhältnisse grafisch dargestellt und eine lineare Regression mit allen bisher gemessenen Werten durchgeführt.

Über die PA300 wird eine vom Teslameter ausgegebene Spannung digitalisiert (12 Bit) und in den Rechner eingelesen; das m/z-Verhältnis wird vom Programm über eine Eichkurve berechnet und angezeigt sowie für jeden Meßpunkt im Meßprotokoll gespeichert.

Die Manometer, die den Druck im Strömungsreaktor, im Zwischenvakuum und in der Ionenquelle messen, geben eine dem Druck proportionale Spannung aus. Diese Spannung wird ebenfalls von der PA300 digitalisiert (12 Bit) und in den Rechner eingelesen; der Druck wird vom Programm über eine Eichkurve berechnet und angezeigt sowie für jeden Meßpunkt im Meßprotokoll gespeichert.

Weiterhin kann mit dem 5 V-Signal des 1. Logik-Treibers der PA300 über einen Optokoppler der Mikrowellengenerator vom Programm automatisch ein- bzw. ausgeschaltet werden. Diese Funktion ermöglicht es, schnell hintereinander, mit einer dem Programm vorgegebenen Pause nach dem Einbzw. Ausschalten (um Anstiegs- bzw. Abfallflanken abzuschneiden), zu zählen und damit weitgehend konstante Versuchsbedingungen zu erlangen. Im Meßprotokoll wird automatisch der jeweilige Zustand (,ein“/,,aus“) des MWG sowie die Pause-Zeit eingetragen.

Mittels des 2. Logik-Treibers der PA300 kann entweder ein 2. Mikrowellengenerator oder aber ein TTL-Schalter und mit letzterem ein Magnetventil zur Zugabe eines weiteren Reaktanten vom Programm angesteuert werden. Auch hierbei können analog zum 1. Logik-Treiber Pausezeiten vor und nach dem Ein- bzw. Abschalten vorgegeben werden.

\section{Erklärung der einzelnen Programmteile}

\subsection{Das Menü DATEI}

\subsubsection{DRUCKEN}

Dieser Menüpunkt ist nur dann aktivierbar, wenn auch ein Meßprotokoll angezeigt wird. Nach dem üblichen Druck-Dialog kann letzteres ausgedruckt werden. Bei Verwendung eines Nadeldruckers ist es jedoch besser, das Programm EDITOR aus der Windows-Gruppe ZUBEHÖR zu verwenden, da dieses eine Drucker-Schriftart verwendet, welche wesentlich schönere Ausdrucke liefert. Die Schriftart des Meßprotokolls kann zwar mittels der rechten Maustaste geändert werden, jedoch ist der EDITOR leichter zu Handhaben und numeriert zudem noch die Seiten durch.

\subsection{2 ÖFFNEN}

Mit Hilfe dieses Menüs kann ein beliebiges Meßprotokoll zur Ansicht und zum Ausdrucken geöffnet werden. Hierzu wird automatisch der WINDOWS-EDITOR gestartet und die gewählte Datei dort geladen. Zwangsläufig wird zuvor das ggf. geöffnete Fenster „m/z-Anzeige“ geschlossen, weil das Programm (wie oben beschrieben) nicht multitaskingfähig ist. Dies bedeutet aber auch, daß der WINDOWS-EDITOR auf jeden Fall erst wieder beendet werden muß, bevor erneut das Fenster „m/z-Anzeige“ aufgerufen wird (sehr wichtig, um Programmabsturz zu vermeiden !!!!). 


\subsubsection{Peakdatei erstellen}

Bei der Registrierung von Übersichtsspektren werden in der Spektrendatei (*.DAT) jeweils nach der am SSR 1110 eingestellten Einzelmeßzeit (üblicherweise $200 \mathrm{~ms}$ bei der Durchlaufgeschwindigkeit 6) die Meßpunkte gespeichert. Diese bestehen aus der Zeit seit Start des Durchlaufs, dem jeweiligen DATA-Wert (= Summe der in der Einzelmeßzeit registrierten Ionnen) und dem jeweiligen m/z-Wert. Weil die Durchlaufgeschwindigkeit üblicherweise langsam ist, werden häufig Peaks einer Masse auf mehrere Meßpunkte aufgeteilt. Dieser Programmteil soll nun Peaks erkennen und die aufgeteilten Meßpunkte zu einem einzigen zusammenführen; das Ergebnis wird separat in der Peakdatei (*.PIK) gespeichert.

Man kann dem Programm angeben, welcher DATAWert mindestens insgesamt für einen Peak überschritten sein muß, damit dieser als Peak gespeichert wird. Da Störungen aus der Umgebung hin und wieder einen DATA-Wert in Höhe von 1 ,einstreuen“, sollte man solch kleine Peaks möglichst gar nicht erst speichern; bei

PeakDatei erstellen

Peak nicht speichern, wenn: DATA $<=01$

PeakDatei aus DAT-Datei erstellen hohem Ionisierungspotential $(70 \mathrm{eV})$ kann ein noch höherer Wert empfehlenswert sein.

Sobald man auf den Schalter „PeakDatei aus DAT-Datei erstellen“ klickt, wird der Dateiname der Spektrendatei (*.DAT) abgefragt, aus der die Peakdatei gewonnen werden soll; die dann erstellte PeakDatei hat den gleichen Dateiname, jedoch die Dateiextension *.PIK.

Der Algorithmus zur Peak-Erkennung läßt sich knapp folgendermaßen beschreiben: Das Programm ließt von Beginn an die Spektrendatei. Sobald ein DATA-Wert größer Null auftaucht wird der Beginn eines Peaks angenommen. Nun ließt das Programm den nächsten Wert ein. Ist dieser Wert gleich Null, wird der zuvor begonnene Peak als beendet betrachtet und gespeichert. Ist der Wert aber ungleich Null, so prüft das Programm, ob der aktuelle Wert kleiner als der vorhergehende ist (negative Steigung) und zugleich kleiner als der nachfolgende Wert ist (positive Steigung); sind beide Kriterien erfüllt, wird der zuvor begonnene Peak beendet und gespeichert, der aktuelle Wert verworfen und mit dem nächsten Wert ein neuer Peak begonnen. Trafen keine der bisher genannten Kriterien zu, so prüft das Programm als nächstes, ob der aktuelle Wert kleiner als der vorhergehende ist (negative Steigung) und der nächste Wert Null ist; in diesem Fall wird der aktuelle Wert zum Peak hinzuaddiert und der Peak dann beendet und gespeichert. In allen anderen Fällen wird der aktuelle Wert einfach zum Peak hinzuaddiert und beim nächsten Wert wieder alle soeben beschriebenen PeakEnde-Kriterien überprüft. Sofern jetzt der letzte Peak beendet und kein neuer begonnen wurde, startet der Algorithums wieder ganz von vorne.

Unter dem soeben erwähnten Verfahren ,zum Peak hinzuaddieren“ ist konkret folgendes zu verstehen: Der DATA-Wert aus der Spektrendatei (*.DAT) wird zum Peak-DATA-Wert hinzuaddiert und der Peak-m/z-Wert wird als gewichteter Mittelwert (gewichtet mit den DATAWerten) aus den einzelnen m/z-Werten der Spektrendatei berechnet; ein Peak-Wert in der Peakdatei (*.PIK) besteht damit aus der Summe aller zugehörigen DATA-Werte und dem gewichteten Mittelwert der m/z-Werte. Die Peak-m/z-Werte werden dabei vom Programm auf eine Nachkommastelle gerundet. 


\subsubsection{Spektren subtrahieren}

Sofern die Übersichtsspektren mit einer gut geeichten und nicht schwankenden Hall-Sonde aufgenommen wurden, können die Peakdateien (*.PIK) voneinander subtrahiert werden, z.B. um einen Hintergrund zu berücksichtigen.

Sie müssen jeweils durch Klick auf den Schalter „Öffnen“ die beiden voneinander zu subtrahierenden Peakdateien öffnen. Anschließend klicken Sie auf den Schalter

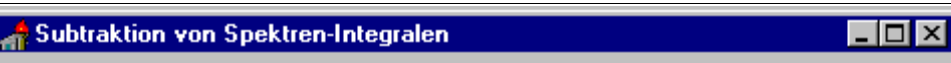

Name der Ausgangs-Peak-Datei:

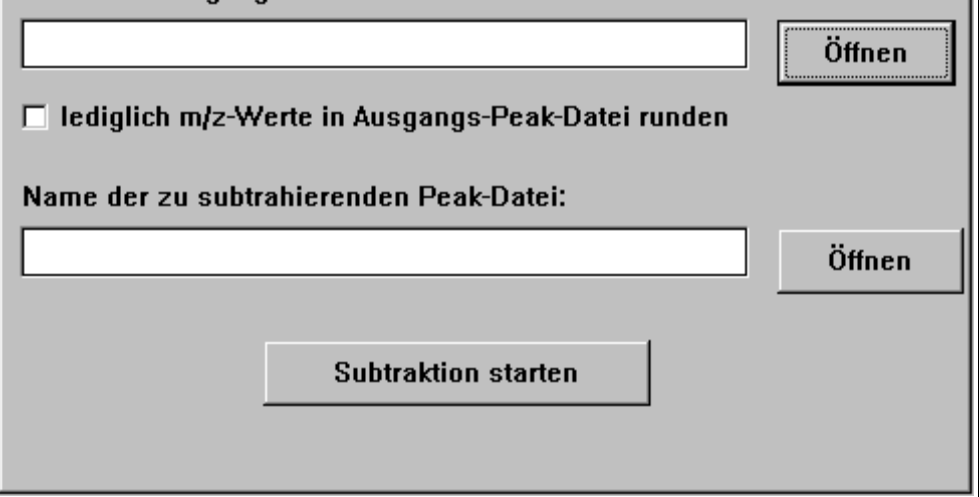
„Subtraktion starten“ und müssen einen Dateiname für die Ergebnisdatei

(Extension: *.SUB) angeben. Die Ergebnisdatei (*.SUB) hat das gleiche Format, wie die Peakdatei.

Sofern der Bereich der registrierten Massenzahlen in beiden Dateien unterschiedlich ist, berücksichtigt das Programm jeweils nur die Massenzahlen, die in beiden Dateien gleichermaßen vorhanden sind.

Um überhaupt eine Subtraktion durchführen zu können, rundet das Programm in einem ersten Durchlauf alle m/z-Werte der Peakdatei (*.PIK) auf eine ganze Zahl; sofern dadurch mehrere Peaks zusammenfallen, werden deren DATA-Werte aufsummiert.

Es ist auch möglich, statt einer Subtraktion nur die m/z-Werte einer Peakdatei auf ganze Zahlen zu runden. Dazu müssen Sie den Name der zu rundenden AusgangsPeakDatei angeben, dann die Option „lediglich $\mathrm{m} / z$-Werte der AusgangsPeakDatei runden" auswählen (durch Klick in die Check-Box) und dann auf ,Subtraktion starten“ klicken.

\subsubsection{BEENDEN}

Dieser Menüpunkt beendet das Programm nach einer entsprechenden Sicherheitsrückfrage.

Wird ein noch nicht gespeichertes Meßprotokoll-Fenster angezeigt, so muß dieses vorher geschlossen werden. Ein unbeabsichtigter Datenverlust wird dadurch vermieden. 


\subsection{Das Menü MESSEN}

\subsubsection{Masse/Ladung und MWG}

Die Spannung der Hall-Sonde wird nach einer Eichkurve in das zugehörige Masse-Ladungsverhältnis $\mathrm{m} / \mathrm{z}$ umgerechnet und in nebenstehendem „Anzeige“Fenster dargestellt. Wird die $\mathrm{m} / \mathrm{z}$-Zahl mit der ersten Nachkommastelle gewünscht, dann ist die entsprechende Option zu aktivieren (die Checkbox „Nachk.“ anklicken); da das Massenspektrometer jedoch z.Zt. nicht hochauflösend ist und außerdem der

\begin{tabular}{|c|c|}
\hline 슌 $\mathrm{m} / \mathrm{z}$ - Anzeige $-\square(\square$ & 슘 $\mathrm{m} / \mathrm{z}$ - Anzeige $\quad-\mid \square x$ \\
\hline \begin{tabular}{|ll} 
Anzeige & Kalibrier. \\
\end{tabular} & Anzeige Kalibrier. \\
\hline Г[ (Nachk.) & \\
\hline MWG1: $\subset$ An $C$ Aus & I U[Hall]: \\
\hline MWG2: $\triangle$ An & $\begin{array}{l}\text { Korrekturwert: }-0,0 \\
\text { [fiir mill }\end{array}$ \\
\hline $\begin{array}{l}\text { Гp(sys): } \\
\text { Г } p(Z V): \\
\text { Г }(\mid \mathrm{lQ}):\end{array}$ & \\
\hline
\end{tabular}
AD-Wandler nur auf 12 Bit genau digitalisiert, wird dadurch prinzipiell eine zu hohe Genauigkeit vorgetäuscht.

Die Eichkurve für die Hall-Sonde wurde mit Perfluorkerosin (PFK) als Eichsubstanz im Bereich bis zur Massenzahl m/z = 430 aufgenommen und damit ein Polynom 3. Grades als Fit-Kurve erstellt. Die Koeffizienten a, b1, b2, b3 dieses Polynoms sind in der Datei WIN_SIC.INI im Abschnitt ,[mz_Eich]“ gespeichert, so daß die Massenzahl gemäß $\mathrm{m} / \mathrm{z}=\mathrm{a}+\mathrm{b} 1 \cdot \mathrm{U}+\mathrm{b} 2 \cdot \mathrm{U}^{2}+\mathrm{b} 3 \cdot \mathrm{U}^{3}$ vom Programm berechnet werden kann.

Muß bei höheren Massenzahlen als 430 gearbeitet werden oder ist die Steigung der KalibrierungEichkurve nicht mehr korrekt, so ist diese Eichkurve natürlich entsprechend zu erweiteren bzw. neu zu erstellen. Dazu muß im Fenster ,m/z-Anzeige“ die Seite „Kalibrier.“ durch Klick in den Vordergrund gebracht werden und dort die Anzeige der Hall-Spannung „U(Hall)“ aktiviert werden. Dann wird die Eichsubstanz (z.B. PFK) in das Massenspektrometer gegeben und durch Ändern des Magnetstroms nacheinander die bekannten charakteristischen Peaks der Eichsubstanz am Tracor auf ihr Peakmaximum eingeregelt. Von jedem dieser Peaks kennt man aus der Literatur den entsprechenden $\mathrm{m} / \mathrm{z}$-Wert und man notiert jeweils den im ,m/z-Anzeige“-Fenster dazu angezeigten Wert der Hall-Spannung. Nun kann ein neues Fit-Polynom erstellt werden, indem die m/zLiteraturwerte gegen die gemessene Hall-Spannung aufgetragen werden und daraus ein Polynom maximal 3. Grades als Fit-Kurve erzeugt wird. Die Koeffizienten a, b1, b2, b3 des so erzeugten FitPolynoms sind in der Datei „WIN_SIC.INI“ unter dem Abschnitt ,[[mz_Eich]“ einzugeben; diese Datei befindet sich in dem Verzeichnis, in dem auch das Programm WinSiccon (WinSi170.EXE) selbst gespeichert ist.

Falls die Anzeige des m/z-Wertes im gesamten interessierenden m/z-Bereich stets um einen gleichen Betrag verschoben ist (z.B. tritt häufig bei Zuschalten des MWG eine Verschiebung um ca. 0,1 bis 0,3 ein), so kann dies durch Eingabe eines Korrekturwertes in das entsprechende Feld im , $m / z-$ Anzeige“-Fenster auf der Seite ,Kalibrier.“ temporär korrigiert werden.

Das Programm erwartet die Hall-Spannung am Eingangskanal Nr. 0 der PA300-AD-Karte.

Ferner werden im ,m/z-Anzeige“-Fenster auf der Seite „Anzeige“ die beiden TTL-Logik-Treiber der PA300-Karte angesteuert (z.B. um den Mikrowellengenerator [MWG ] manuell zu- bzw. abzuschalten); dazu ist nur der entsprechende Button anzuklicken, wobei MWG1 den Logik-Treiber Nr. 0 und MWG2 den Logik-Treiber Nr. 1 ansteuert.

Über diese Logik-Treiber kann auch ein Magnetventil zum Ab- und Einschalten eines Gas-Zuflusses angesteuert werden. Dazu ist ein TTL-Schalter erforderlich, der über den TTL-Puls $(+5 \mathrm{~V})$ des Logik-Treibers eine $220 \mathrm{~V}$-Spannung für das Öffnen des Magnetventils weitergibt.

Weiterhin ermöglicht dieses Fenster die Anzeige der aktuellen Drücke im Strömungssystem p(sys), im Zwischenvakuum p(ZV) sowie in der Ionenquelle p(IQ); dazu sind die entsprechenden Optionen 
zu aktivieren (Klick in die zugehörige Check-Box).

Die jeweiligen Druck-Meßgeräte liefern eine dem Druck proportionale Ausgangsspannung, die dann vom Programm zur Berechnung des Drucks verwendet wird. Das Programm erwartet die Spannnung des BARATRON-Manometers zur Messung von p(sys) am Eingangskanal Nr. 1 der PA300-ADKarte; die Spannung des PenningVac-PM41-Anzeigegerätes zur Messung von p(ZV) bzw. p(IQ) erwartet das Programm an dem Eingangskanal Nr. 2 bzw. Nr. 3 der PA300-AD-Karte.

Weil es verschiedene BARATRON-Geräte gibt, die zwar alle eine Ausgangsspannung von 0-10 V liefern, sich aber im Meßbereich (0-10 mbar, 0-100 mbar oder 0-1000 mbar) unterscheiden, muß dem Programm in der Datei „WIN_SIC.INI“ im Abschnitt „,[Baratron_Eich]“ der Umrechnungsfaktor des aktuell verwendeten Geräts mitgeteilt werden $(\mathrm{p}=$ Faktor $\cdot \mathrm{U})$. Der benötigte Faktor kann der nachstehenden Tabelle entnommen werden:

\begin{tabular}{|c|c|c|c|}
\hline BARATORN-Meßbereich & $0-10 \mathrm{mbar}$ & $0-100 \mathrm{mbar}$ & $0-1000 \mathrm{mbar}$ \\
\hline Faktor $=$ & 1 & 10 & 100 \\
\hline
\end{tabular}

Die Berechnung von p(ZV) bzw. p(IQ) erfolgt über ein Polynom 3. Grades, das die Ausgangsspannung des PM41-Anzeigegerätes in einen Druck (in Torr !) umrechnen muß und deren Koeffizienten a, b1, b2 und b3 in der Datei „WIN_SIC.INI“ im Abschnitt ,,[Penning_Eich]“ angegeben werden.

\subsubsection{Einzel-/Doppelmessung}

\subsubsection{Allgemeines}

Nach einem Hinweis auf die Luftkühlung für das Entladungsröhrchen erfolgt die Frage, ob ein Tagesunterverzeichnis angelegt werden soll. Verneint man, so werden die Daten standardmäßig in dem in der Datei „WIN_SIC.INI“ im Abschnitt „,[PFADE]“ angegebenen „HAUPTPFAD” gespeichert; in dem Datei-Speichern-Dialog kann jedoch jedes beliebige Verzeichnis und jeder beliebige Dateiname angegeben werden. Bejaht man die Frage, so wird in dem im HAUPTPFAD befindlichen Unterverzeichnis $\mathrm{BK}^{1}$ ein neues Unterverzeichnis mit einem Namen BKjjmmtt angelegt (BK $=$ Benutzerkürzel, $\mathrm{jj}=\mathrm{Jahr}, \mathrm{mm}=$ Monat, $\mathrm{tt}=$ Tag), wobei jjmmtt das Tagesdatum ist. Dadurch wird die Zuordnung der Daten zu einem Benutzer und auch eine Tagesdatensicherung auf Diskette erleichtert.

Standardmäßig wird ein Dateiname aus dem Tagesdatum und der laufenden Numerierung „,A“ bis , Z“ vorgeschlagen. Vor einem Überschreiben alter Dateien warnt das System den Benutzer.

Nach Auswahl des Dateinamens öffnet sich das „Messtext - Systemeinstellungen“-Fenster und es ist zunächst die gewünschte Wahrscheinlichkeit zur Berechnung des Konfidenzintervalls für die Fehlerrechnung anzugeben. Standardmäßig werden 95\% vorgeschlagen.

Ferner können in diesem Fenster die Systemeinstellungen, wie z.B. Temperatur, Druck, Gasflüsse etc. eingegeben werden, die für alle folgenden Meßzyklen gelten und automatisch am Anfang des Meßprotokolls (vgl. Kap. 4) eingefügt werden. Um etwas Schreibarbeit zu sparen, lädt das Programm automatisch den zuletzt eingebenen Meßtext. Durch Klick auf den Knopf „Messtext laden“ kann jedoch der Text aus der Datei „StdMess.TXT“ geladen werden. Diese Datei kann mit dem WINDOWS-EDITOR bearbeitet werden. Sobald das Fenster mit Klick auf „OK“ geschlossen wird, erhät der Anwender zudem Gelegenheit, den gerade eingegebenen Messtext auch für analoge

1 Das Verzeichnis BK muß zuvor bereits angelegt worden sein ! 
spätere Messungen abzuspeichern. Alle diese Dateien befinden sich separat für jeden Benutzer in einem Unterverzeichnis mit dem Name des beim Start angegebenen Benutzerkürzels; dieses Unterverzeichnis muß sich jedoch in dem Verzeichnis befinden, in dem auch WinSiccon (WINSI170.EXE) selbst gespeichert ist.

Durch Betätigung des Schalters „AP-Skt.ber.“ erscheint das Fenster, das im Menü „TOOLS“ unter „AP-Skalenteile“ erläutert wird (siehe dort). Damit kann das gewünschte Ionisierungspotential des Massenspektrometers in die entsprechenden Skalenteile seines Potentiometers umgerechnet werden.

Mit dem Schalter „Konz.-Berechng.“ erscheint das

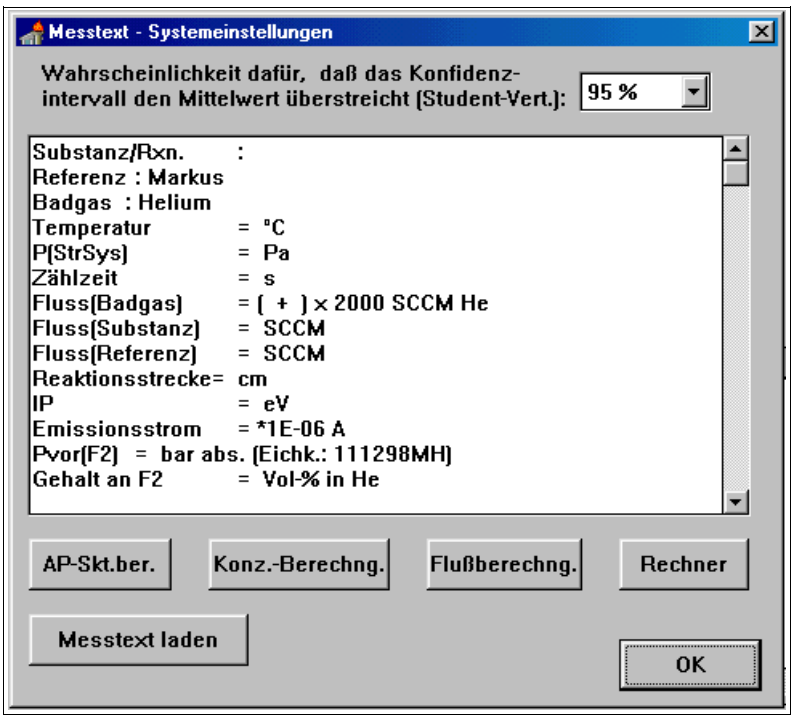

Fenster, das im Menü „TOOLS“ unter „Konz/Zeit/Geschw.“ erläutert wird (siehe Kapitel 3.4.2, S. 23); es können dort die Konzentration einer Spezies im Strömungsreaktor, die Reaktionszeit und die lineare Strömungsgeschwindigkeit errechnet werden. Die Ergebnisse können mit STRG-c in die Zwischenablage kopiert und dann in die Messtext-Systemeinstellungen über STRG-v eingefügt werden.

Über den Schalter „Flußberechnung“ wird das Fenster aufgerufen, in dem Gas-Flüsse durch TYLANflow-controller oder geeichte HOKE-Ventile berechnet werden können (Kap. 3.4.6, S. 24).

Über den Schalter „Rechner“ wird der zu Windows gehörende Taschenrechner am Bildschirm angezeigt. Nach dem erstmaligen Laden sollte er nur noch über ALT-TAB aufgerufen und nach Beendigung aller Rechnungen wieder geschlossen werden!

Das während der Messung ständig automatisch aktualisierte Meßprotokoll ist normalerweise schreibgeschützt; falls jedoch z.B. ein falscher Meßtext übernommen wurde, so kann in das Fenster „Messprotokoll“ hineingeklickt und über die rechte Maustaste ein kleines Kontextmenü geöffnet werden. Damit ist es möglich, den Änderungs-Modus zu aktivieren, in dem das Meßprotokoll geändert werden kann; nach der Änderung sollte sofort durch Drücken der ESC-Taste wieder der Änderungs-Modus beendet werden.

Die in der m/z-Anzeige angezeigten Druck-Werte und die Massenzahl werden vom Programm bei der Einzel- bzw. Relativmessung zu jedem Meßpunkt automatisch ins Meßprotokoll eingetragen. Nach Abschluß der Messung wird zudem der minimale, maximale und durchschnittliche Druck im Strömungsreaktor für die gesamte Messung in das zuletzt geöffnete Meßprotokoll geschrieben.

ACHTUNG: Während ein Meßzyklus läuft, sollten keinerlei Tasten gedrückt oder mit der Maus irgendwo hingeklickt werden; das Programm sollte zwar eigentlich dadurch nicht gestört werden, aber es muß ja nicht unbedingt ein Fehler provoziert werden. Ein Abbruch ist nicht möglich.

Ferner ist zu beachten, daß der ,,SSR-1110“ nur eine Bandbreite von $85 \mathrm{MHz}$ hat, d.h. bei $10 \mathrm{~s}$ Zählzeit können maximal $850 \cdot 10^{6}$ Counts pro Meßpunkt erfaßt werden; um jedoch ganz sicher zu sein, daß keine Sättigung vorliegt, sollte darauf geachtet werden, daß die Signalintensitäten nur $1 \%$ dieses Wertes erreichen, zumal die Sättigung langsam ansteigt und nicht abrupt einsetzt. 


\subsubsection{Einzelmessung}

Es erscheint das Fenster „Einzelmessung“ und das Fenster „Meßprotokoll““.

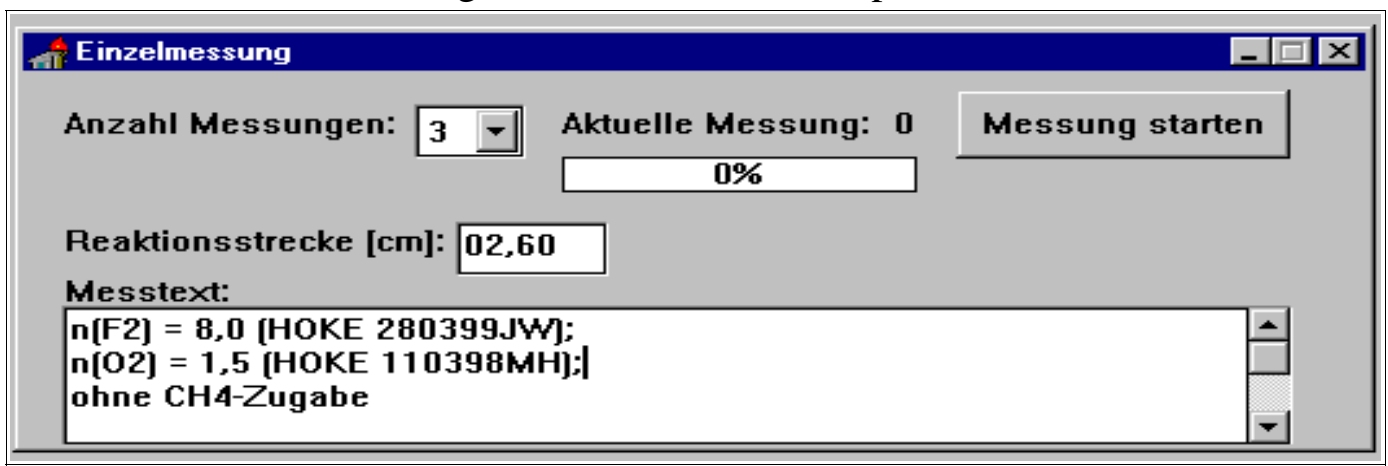

Im Fenster Einzelmessung muß nun die Anzahl der Messungen (= Anzahl Wiederholungen) gewählt werden und es kann beliebig langer Meßtext individuell für den nun folgenden Meßzyklus eingegeben werden (z.B. die aktuelle Dosierung = Öffnung des Hoke-Ventils von Fluor), der dann automatisch ins Meßprotokoll übernommen wird. Die eingegebene Reaktionsstrecke wird sowohl ins Meßprotokoll als auch in die Tabellendatei (*.TAB) übernommen, was zur Auswertung von Absolutmessungen sehr nützlich ist.

Durch Drücken des Schalters „Messung starten“ wird ein Meßzyklus gestartet und die Meßwerte laufend ins Meßprotokoll eingetragen; die DATA, BKG und DIFF-Werte werden dabei vom ,SSR 1110“-Computer (Einzelionenzähler) abgefragt. Wenn die eingestellte Anzahl Messungen durchgeführt wurde, erfolgt die Berechnung und Ausgabe der Mittelwerte (in $<>$ gesetzt) mit den zugehörigen mittleren Fehlern des Mittelwertes zum gewählten Konfidenzintervall; die \%-Angabe in Klammern ist der relative Fehler (mittlerer Fehler des Mittelwertes dividiert durch den Mittelwert).

Ist ein Hintergrund zu berücksichtigen, so muß dieser am „SSR-1110“ über den BKGSUB-Mode erfaßt (vgl. Handbuch zu diesem Gerät) oder separat gemessen und später bei der Auswertung in der Tabellenkalkulation berücksichtigt werden.

Das während der Messung ständig automatisch aktualisierte Meßprotokoll ist normalerweise schreibgeschützt; falls jedoch

\begin{tabular}{|c|c|}
\hline Messprotokoll & $-9 \square$ \\
\hline $\begin{array}{l}m / z=0 / \text { MWG1: AUS / MWG2: AUS } \\
\text { Reaktionsstrecke } s=02,60 \mathrm{~cm} \\
n[F 2]=8 \\
n[02]=1,5 \\
\text { ohne CH4-Zugabe }\end{array}$ & \\
\hline $\begin{array}{lcc}\text { DATA: } & \text { BKG: } & \text { DIFF: } \\
1,67 E+08 & 1,67 E+08 & 1,67 E+08 \\
1,67 E+08 & 1,67 E+08 & 1,67 E+08 \\
\langle D A T A\rangle=1,665 E+08 \pm 0,000 E+00[0,00 \%] \\
\langle B K G\rangle=1,665 E+08 \pm 0,000 E+00[0,00 \%] \\
\langle\text { DIFF }\rangle=1,665 E+08 \pm 0,000 E+00[0,00 \%]\end{array}$ & \\
\hline \begin{tabular}{|l|}
11 \\
\end{tabular} & D \\
\hline
\end{tabular}
z.B. ein falscher Meßtext übernommen wurde, so kann in das Fenster „Messprotokoll“ hineingeklickt und über die rechte Maustaste ein kleines Kontextmenü geöffnet werden. Damit ist es möglich, den Änderungs-Modus zu aktivieren, in dem das Meßprotokoll geändert werden kann; nach der Änderung sollte sofort durch Drücken der ESC-Taste wieder der Änderungs-Modus beendet werden.

\subsubsection{Doppelmessung mit 1 Reaktant}

Es gelten hier die gleichen Ausführungen wie zum Punkt „Einzelmessung“, mit der Erweiterung, daß eine Hintergrund-Korrektur auch durch WinSiccon erfolgen kann und hier auch muß (vgl. dazu die Erläuterungen zum Menü KORREKTUR im Kapitel 3.3.1, S. 21). Dies ist notwendig, falls bereits durch Verunreinigungen im Massenspektrometer (ohne Substanzzugabe) ein Peak auf derjenigen $\mathrm{m} / \mathrm{z}$-Zahl erscheint, auf der hier die Kinetik gemessen werden soll. 
Bei einer Doppelmessung wird ein Meßzyklus ohne Mikrowellenentladung durchgeführt, dann die im Feld „MWG-Pause“ angegebene Zeit gewartet und sofort anschließend ein Meßzyklus mit eingeschalteter Mikrowellenentladung durchgeführt. Das Einschalten

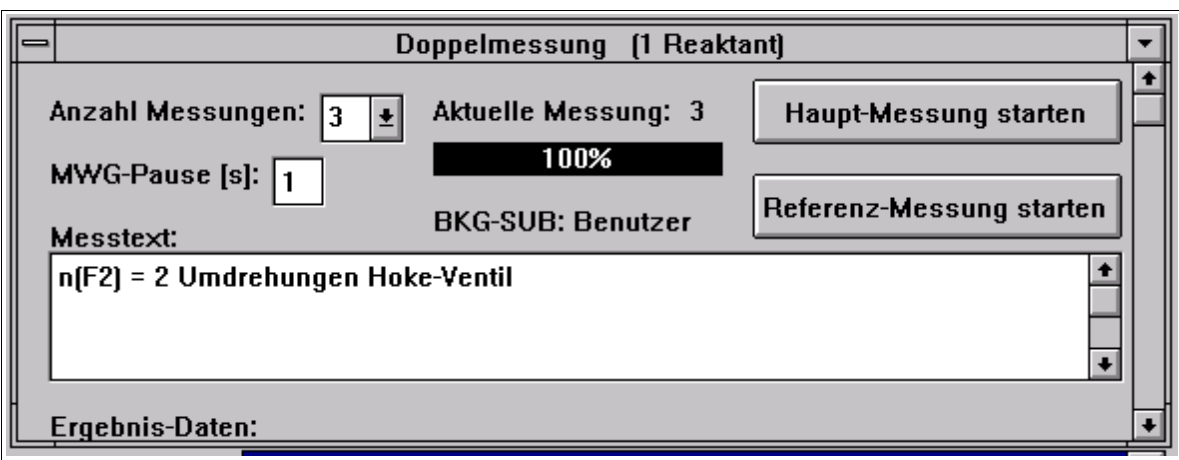
des MWG1 erfolgt automatisch durch das Programm und wird im „m/z-Fenster“ angezeigt.

Häufig werden Kinetik-Messungen am CH5 gegen eine Referenz-Substanz durchgeführt; WinSiccon unterstützt dieses Verfahren, indem die eigentliche Messung (= Hauptmessung) und die Referenzmessung durch zwei verschiedene Schalter gestartet und dadurch zusammenhängende Meßwert-Paare gebildet werden können. Haupt- oder Referenzmessung können in beliebiger Reihenfolge und beliebig oft durchgeführt werden. Erst wenn jeweils jede Meßart mindestens einmal durchgeführt wurde, wird ein Meßwertpaar gebildet.

Man stellt z.B. m/z für die Hauptmessung ein, führt einen Doppel-Meßzyklus ( ,Hauptmessung starten“) durch, stellt dann m/z für die Referenz ein und führt erneut einen Doppel-Meßzyklus (diesmal aber mit „Referenzmessung starten“) durch.

Die beiden so erhaltenen Meßwertpaare werden nun grafisch angezeigt und auch eine Regressionsgerade erzeugt. Der größte Punkt in der Grafik zeigt das zuletzt hinzugekommene Wertepaar an.

Der Anwender kann nun entscheiden, ob dieser Punkt gespeichert werden soll oder kann beliebig oft die Haupt- oder Referenzmessung wiederholen (z.B. wenn ein Einzelwert aufgrund von Flußschwankungen zu sehr fehlerbehaftet ist).

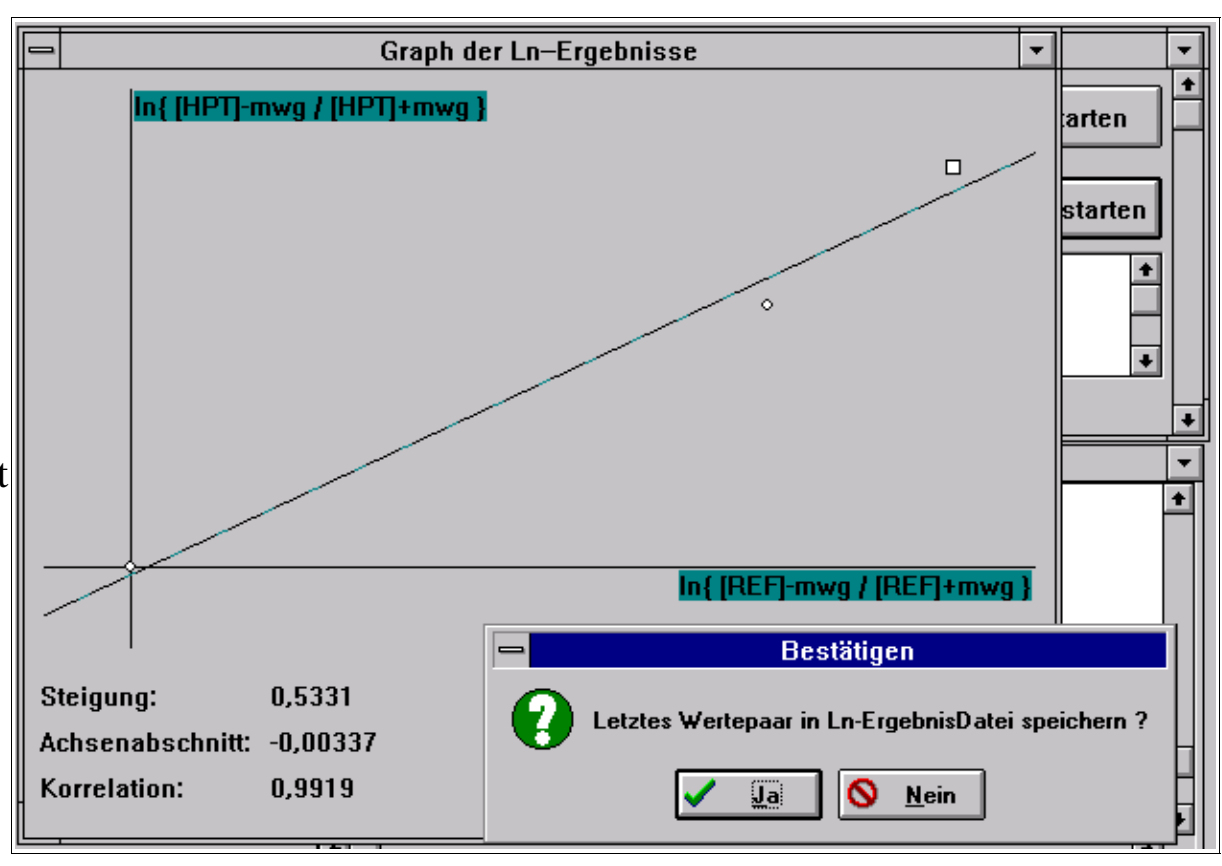

Die Entscheidung einen Punkt zu speichern oder nicht hat keinen Einfluß auf das Meßprotokoll, welches immer alle Werte aufzeichnet, sondern nur auf die Ln-Ergebnisdatei (*.DAT), in der alle Daten eines grafisch angezeigten Meßwertpaares (Einzelwerte, Fehler und Ln-Werte) eingetragen werden. Es handelt sich dabei um eine ASCII-Datei mit TAB-Zeichen (ASCII \#9) als Datentrenner, die direkt in Tabellenkalkulationsprogramme eingelesen werden kann (vgl. Kap. 4, S. 27).

Die Aufnahme eines Meßwertpaares kann jeweils beliebig mit der Haupt- oder Referenzmessung begonnen werden; dadurch wird die Anzahl der Wechsel der Massenzahlen minimiert.

Soll keine Referenzmessung durchgeführt werden, so ist ausschließlich der „Hauptmessung starten“- 
Schalter zu betätigen und es erfolgt nie eine grafische Darstellung und Eintragung der ln-Werte.

\subsubsection{Doppelmessung mit 2. Reaktant}

Mit diesem Programmteil kann der Umsatz eines erzeugten Radikals mit einem 2. Reaktant gemessen werden; ggf. sogar BKG- und/oder umsatzkorrigiert.

Der MWG1 wird automatisch eingeschaltet, sobald auf „Haupt-Messung starten“ oder „ReferenzMessung starten“ gedrückt wird, um die Radikalerzeugung zu starten.

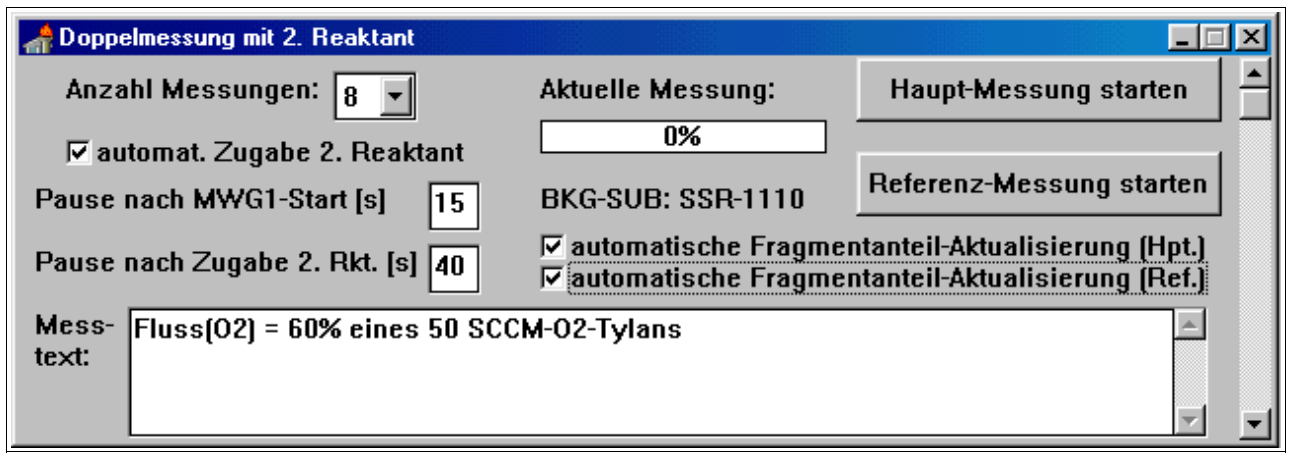

Sofern der 2. Reaktant automatisch zugegeben werden kann (mit 2. MWG oder Magnetventil = MWG2), sind noch die Zeiten für die Pausen einzugeben, die zwischen dem automatischen Start von MWG1 bzw. MWG2 und dem Beginn der Einzelionenzählung liegen sollen; standardmäßig sind $15 \mathrm{~s}$ bzw. 40 s eingestellt. Falls keine automatische Zugabe des 2. Reaktant erfolgen kann, erscheint nach Drücken des Start-Schalters eine Meldung, daß der 2. Reaktant (noch) nicht zugegeben werden darf und erst nach Bestätigung dieser Meldung (OK-Schalter) wird ein Meßzyklus gestartet; dann erscheint erneut eine Meldung, daß nun auch der 2. Reaktant zugeben werden muß und nach Bestätigung dieser Meldung wird ein weiterer Meßzyklus durchgeführt. Sobald sowohl Haupt- als auch Referenzmessung für eine bestimmte Konzentration des 2. Reaktanten erfolgt sind, wird das Ergebnis grafisch angezeigt und man kann entscheiden, ob es in der Ln-Ergebnisdatei (*.DAT) (vgl. Kapitel 4, S. 27) gespeichert werden soll; verneint man die Frage, so können sowohl die Haupt- als auch die Referenzmessung beliebig oft wiederholt werden, wobei dann die Frage jeweils erneut erscheint.

Bezüglich der BKG-Korrektur gelten die gleichen Ausführungen wie für die Doppelmessung mit 1 Reaktant; eine Beschreibung findet sich unten im Kapitel 3.3.1 (S. 21).

Häufig sind die Peaks der Radikale noch durch Fragmente des Muttermoleküls überlagert und aus diesem Grund ermöglicht WinSiccon eine automatische Korrektur dieser überlagerten Signale. Um dies zu nutzen, muß vor(!) den eigentlichen Messungen im Menü „Korrektur“ der Punkt „Umsatz“ gewählt werden und die dort geforderten Messungen durchgeführt werden. Eine genaue Beschreibung findet man unten im Kapitel 3.3.2 (S. 21). Weil man nach einem Wechsel der Massenzahlen oft nicht mehr dieselbe Intensität einregeln kann, wie zum Zeitpunkt der Umsatzkorrektur-Messungen, bietet das Programm für die Haupt- und Referenzmessung jeweils die Option (Check-Box), automatisch eine Aktualisierung des Fragmentanteils durchzuführen. Bei dieser Option wird vor der eigentlichen Messung auf dem Radikal-Peak automatisch MWG1 ausgeschaltet und erneut das reine Fragment $I^{\text {Fragment }}$ gemessen, das dann mit dem gespeicherten Umsatz auf dem Mutter-Peak zur Berechnung von $\mathrm{I}^{\mathrm{k} o r r}$ verwendet wird. Es wird empfohlen diese Option immer zu nutzen.

\subsection{3 Übersichtsspektrum}

Bei Aufruf dieses Programms erfolgt zunächst der Hinweis, am CH5 eine lineare Durchlaufge- 
schwindigkeit von max. „6“ zu wählen und „,N $\geq 20 \mathrm{E}+04$ “ einzustellen. Letztere Einstellung bezieht sich auf die Zählzeit am „SSR-1110“-Computer, wobei diese 200 ms nur als Vorschlag betrachtet werden sollten. Im Einzelfall kann eine andere Zählzeit durchaus sinnvoll sein. Dabei kann man als Faustregel beachten, daß eine Verdopplung der Zählzeit mit einer Verringerung der Durchlaufgeschwindigkeit um eine Einheit einhergehen sollte (z.B. lin. Durchlauf von ,5“ für „,N=40E+04“)

Da zwischen den einzelnen Zählungen eine durch die Elektronik bedingte mittlere „Totzeit“ von 17,2 ms (min. 11 und max. $64 \mathrm{~ms}$ ) liegt, sollte die Durchlaufgeschwindigkeit auf keinen Fall viel schneller als ,6“ eingestellt werden. Bei exponentiellem Durchlauf kann man mit der Geschwindigkeit „9“ noch reproduzierbare Werte erlangen. Während einer Messung werden noch die DATA-Werte der vorhergehenden Messung berechnet, der m/z-Wert gelesen, die Zeit gemessen und alle diese Werte in der Datei gespeichert; dieser Vorgang dauert mindestens $80 \mathrm{~ms}$. Aus diesem Grund darf die Zählzeit niemals kleiner als 200 ms $(\mathrm{N}=20 \mathrm{E}+04)$ gewählt werden !

Auch wenn bei der Erstellung dieses Programms viele Tests durchgeführt wurden und auch sehr gute Übereinstimmungen mit den Literatur-Massenspektren vorhanden waren, sei dennoch ausdrücklich darauf hingewiesen, daß die erwähnte Totzeit von 17,2 ms eine Verfälschung der Peakhöhe bewirken kann und die Spektren nur als Übersichtsspektren verwendet werden sollten! Dieses Problem läßt sich durch Verwendung der Peak-Datei (*.PIK) zumindest vermindern; daher wird im Anschluß an die Messung stets automatisch eine Peakdatei erstellt (vgl. dazu Kap. 3.1.3, S.11). Zur Sicherheit sollten die relevanten Peaks aber anschließend einzeln mit längerer Zählzeit (einige s) nochmals genau gemessen werden.

Um nun ein Spektrum aufzunehmen, ist am CH5 eine $\mathrm{m} / \mathrm{z}-$ Zahl einzustellen, die etwas unter der gewünschten Startmasse liegt, dann sind die Startmasse und Endmasse in die entsprechenden Felder einzutragen. In dem großen Texteingabefeld können beliebige Angaben zum aufzunehmenden Massenspektrum gemacht werden, die dann in einer separaten Datei mit gleichem Dateinamen (siehe unten), jedoch der Dateiextension ,*.TXT“" abgespeichert werden; falls bereits eine Textdatei mit diesem Name existiert, wird eine Warnung ausgegeben. Es ist deshalb besser, die Spektren immer in einem separaten Verzeichnis zu speichern (siehe weiter unten).

Das Programm lädt automatisch den Meßtext der letzten Messung; dieser

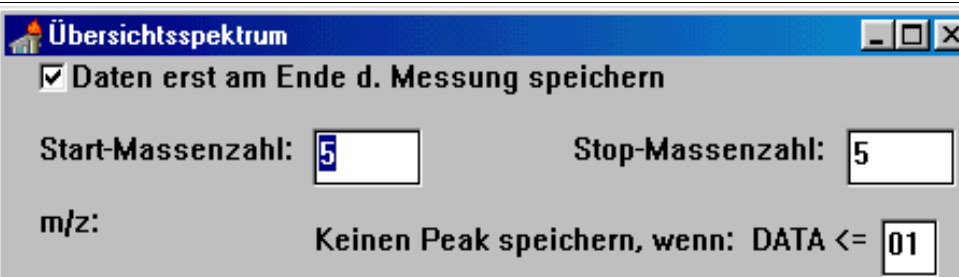

Messtext fuir Spektrum [Systemeinstellungen]:

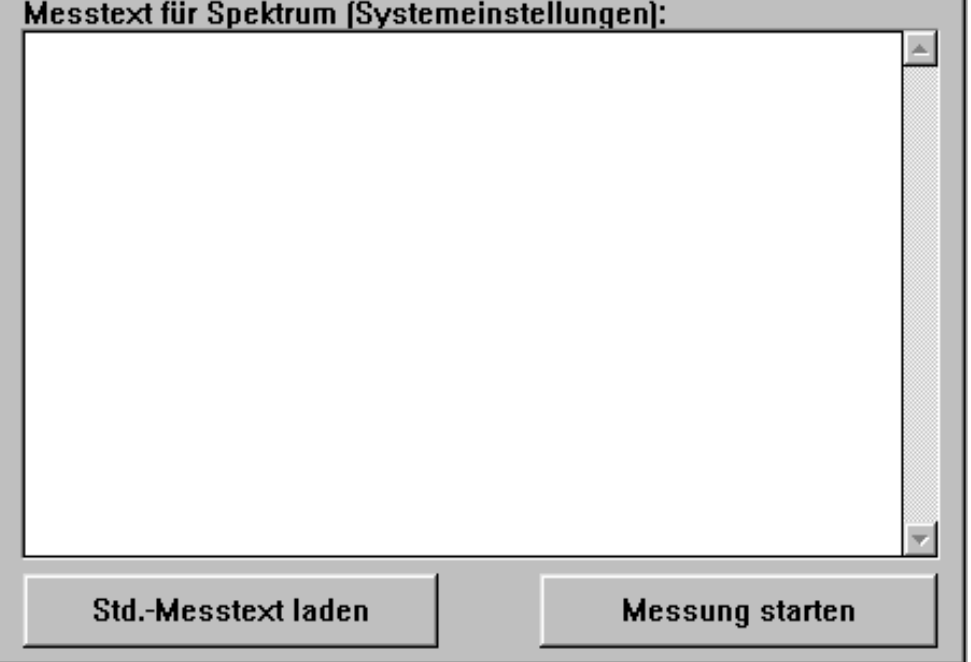

kann jedoch durch Klick auf den Schalter „Std.-Messtext laden“ mit einem Text aus der Datei „StdSpekt.TXT“ überschrieben werden, die im Programmverzeichnis (d.h. in dem Verzeichnis, in dem auch das Programm WinSiccon selbst gespeichert ist) gespeichert sein muß.

Zum Start der Messung ist auf „Messung starten“ zu klicken.

Es muß dann zuerst ein Dateiname eingegeben werden, unter dem das Spektrum gespeichert werden soll; die Dateiextension ist immer ,*.DAT“, der Datenpfad wird dem Eintrag „SPEKTRUMPFAD“ im Abschnitt ,[PFADE]“ der Datei „WIN_SIC.INI“ entnommen, kann aber beliebig geändert 
werden.

Sobald das nun folgende Fenster geschlossen wird, ist am CH5 der Massendurchlauf zu starten und ab der gewählten Start-Massenzahl beginnt WinSiccon automatisch die Meßwerte zu registrieren, bis die eingegebene Stop-Massenzahl erreicht ist. Ein Abbruch ist durch Rückdrehen der Magnetfeldstärke auf NULL möglich. Zum Schluß ertönt kurz ein Signal und es erscheint die Meldung, den Massendurchlauf am CH5 jetzt zu beenden.

Nach Abschluß der Spektrenregistrierung sollte am „SSR-1110“ wieder eine gebräuchliche Zählzeit eingestellt werden (z.B. N=10E+06), sofern nicht anschließend ein weiteres Spektrum registriert werden soll.

Als Ergebnis speichert WinSiccon die seit dem Start verstrichene Zeit, den DATA-Wert vom „SSR1110 “ und den $\mathrm{m} / \mathrm{z}$-Wert.

Da die gemessenen m/z-Werte gewissen Schwankungen unterliegen, empfiehlt sich eine Auftragung der DATA-Werte gegen die Zeit als Liniengraph und die anschließende manuelle Zuweisung (chemischer Sachverstand !) von m/z-Zahlenwerten zu den Peaks.

Falls die Hall-Sonde jedoch sehr gut geeicht ist und auch keinen Schwankungen während der Spektrenregistrierung unterlag, kann auch die Auftragung aus der Peakdatei (*.PIK) erfolgen, in der jeweils zu einem Massenzahl-Mittelwert die gesamte DATA-Summe gespeichert ist; diese Auftragung der DATA-Summe vs. m/z-Mittelwert sollte sinnvollerweise als Säulendiagramm erfolgen. Die Beschreinung der Erstellung einer PeakDatei findet sich im Kap. 3.1.3 (S.11).

Es ist zu beachten, daß der ,SSR-1110“ nur eine Bandbreite von $85 \mathrm{MHz}$ hat, d.h. bei $200 \mathrm{~ms}$ Zählzeit können maximal $1,7 \cdot 10^{7}$ Counts pro Meßpunkt erfaßt werden; um jedoch ganz sicher zu sein, daß keine Sättigung vorliegt, sollte darauf geachtet werden, daß die Signalintensitäten nur $1 \%$ dieses Wertes erreichen.

Als Beispiel sei das Spektrum von tert-Butyliodid $\left(\mathrm{M}_{\mathrm{r}}=184\right)$ abgebildet:
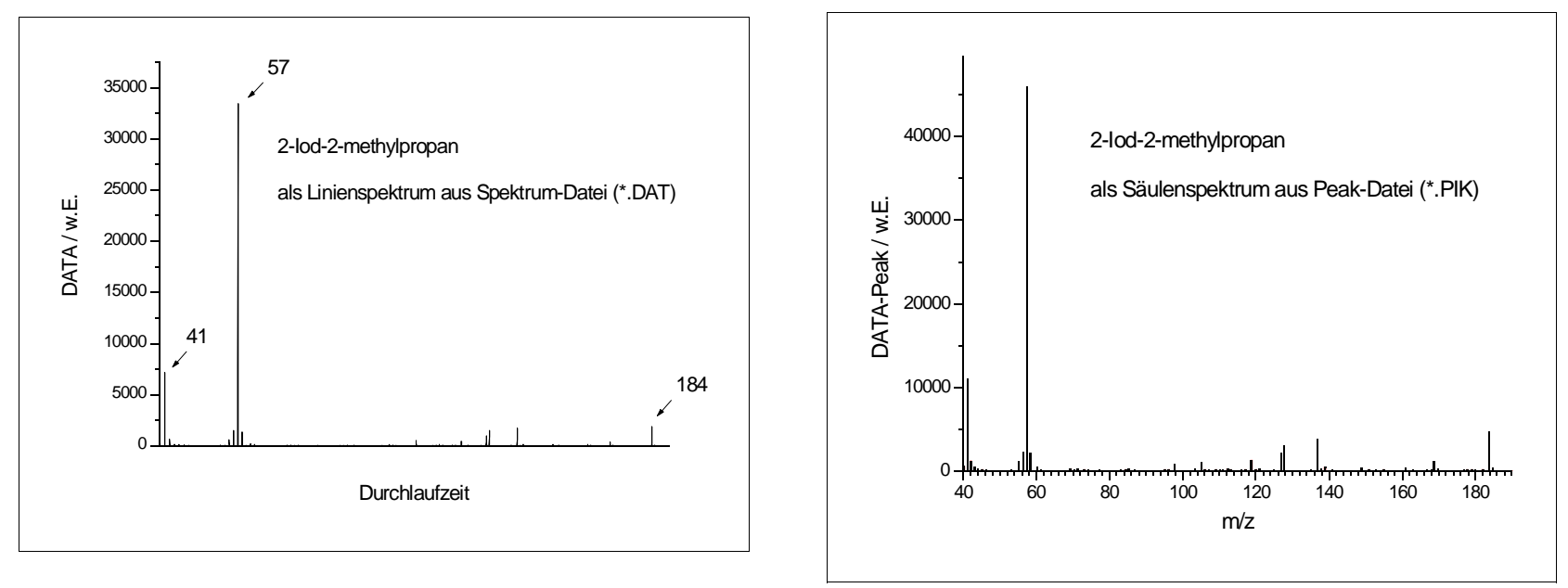


\subsection{Das Menü KORREKTUR}

\subsubsection{BKG-Erfassung}

Dieser Menüpunkt ist ausschließlich im Doppelmessung-Modus zugänglich und ermöglicht ggf. vorhandene Hintergrund-Signale (BKG), die auch meßbar sind, wenn ausschließlich Trägergas (He) fließt, von den eigentlichen Meßwerten zu subtrahieren.

Man stellt dazu den $\mathrm{m} / \mathrm{z}-$ Wert der zu korrigierenden Masse ein und kann dann durch Drücken des Schalters „HptMessung“ bzw. „Ref.-

Messung" den Hintergrund für die Haupt- bzw.

Referenzmessung messen.

Dabei werden die

Einstellungen aus dem „DoppelMessung“-Fenster (Anzahl Messungen, MWG-

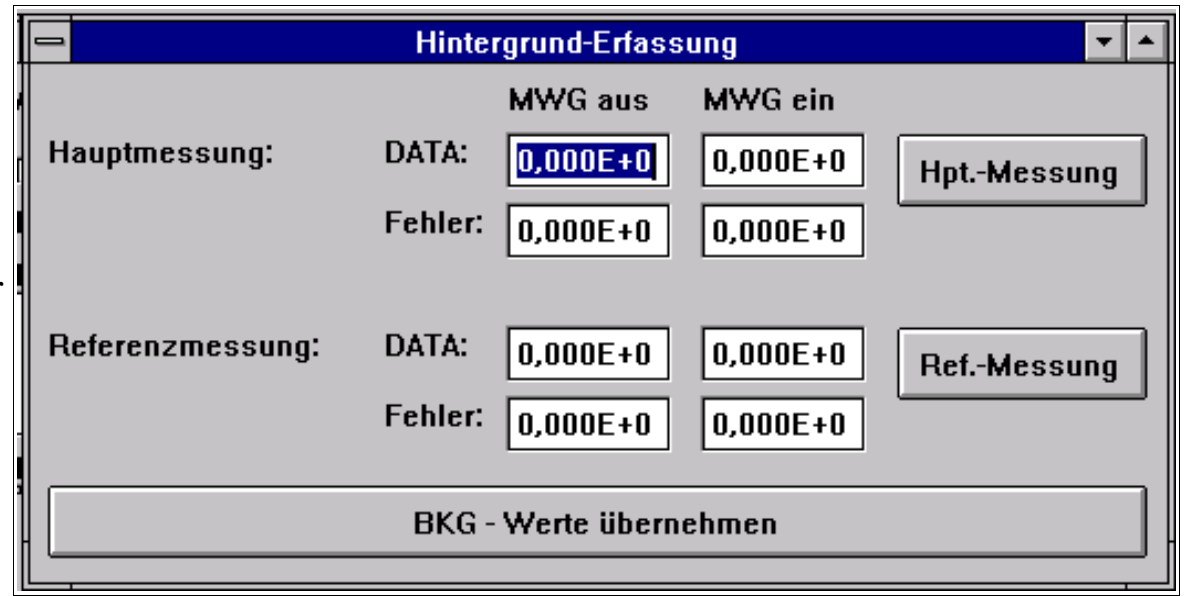
Pause, Konfidenzintervall) berücksichtigt. Man kann jedoch die BKG-Werte auch manuell eintragen, wenn sie aus einer anderen Messung (des gleichen Tages !!) bereits bekannt sind.

Beim Schließen des Fensters erfolgt nochmals eine Rückfrage, ob die eingegebenen Werte zukünftig verwendet werden sollen. Bejaht man diese Frage, so werden bei den künftig gemessenen DATAWerten die eingegebenen BKG-Werte subtrahiert und daraus die DIFF-Werte berechnet. Der Fehler der DIFF-Werte ergibt sich dann aus der Fehlerfortpflanzung von den BKG-Werte-Fehlern und den DATA-Werte-Fehlern. Verneint man hingegen die Frage, so werden nur die ggf. im „SSR-1110“Computer vorhandenen BKG-Werte benutzt; eine Fehlerfortpflanzungsrechnung kann damit jedoch nicht erfolgen.

Im „DoppelMessung“-Fenster wird der jeweilige Modus angezeigt ( „BKG-SUB: ...“)

Solange dieses Fenster angezeigt wird erfolgt keine Aktualisierung der m/z-Anzeige und es sind keine anderen Programmteile aufrufbar!

\subsubsection{Umsatz}

Dieser Menüpunkt ist lediglich für die „Doppelmessung mit 2. Reaktant“ wählbar. Es soll die automatische Korrektur von Radikal-Peaks, die mit Fragment-Peaks des Muttermoleküls überlagert sind, ermöglicht werden.

Falls erforderlich, muß zunächst eine BKG-Korrektur (vgl. Kap. 3.3.1) für die Muttermolekül-Signale durchgeführt werden. Erst dann darf im Fenster „Umsatzkorrektur“ die „Ermittlung des Umsatzes auf dem Peak des Muttermoleküls“ für die Haupt- und/oder Referenzmessung durch Klick auf den entsprechenden Schalter durchgeführt werden. 
Falls erforderlich, muß nun eine BKG-Korrektur für die jeweiligen Radikal-Peaks erfolgen. Nur sofern bei der „Doppelmessung mit 2. Reaktant“ nicht die Option zur ,automatischen FragmentanteilAktualisierung " gewählt wurde, muß jetzt im Fenster „Umsatzkorrektur“ die „Ermittlung der Intensität des Fragment-Peaks" für die Haupt- und/oder

Referenzmessung durch Klick auf den entsprechenden Schalter erfolgen; ansonsten genügt die bereits erfolgte Messung des „Umsatzes auf dem Peak des Muttermoleküls" und dieser Programmteil kann beendet werden.

Nach Verlassen des Fensters mit dem Schalter ,Werte übernehmen" können nun die eigentlichen Messungen im Fenster „Doppelmessung mit 2. Reaktant“ erfolgen.

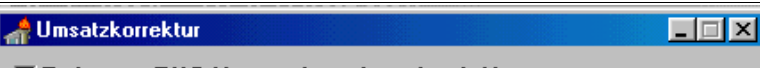

ГReferenz-BKG-Umsatzkorrektur durch Hauptmessung

Ermittlung des Umsatzes auf dem Peak des Muttermolekïls: MWG-Pause [s]: 15

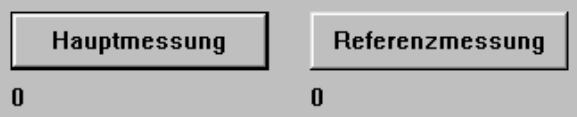

Ermittlung der Intensität des Fragment-Peaks [ I $\{-\mathrm{F}-0\}$ ] :

[sofern nicht automatische Fragmentanteil-Aktualisierung]

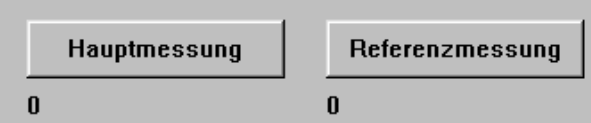

Werte ibernehmen

Die Berechnung der Umsatzkorrektur ist in Kapitel 5.3 (S. 30) genauer erläutert.

Es ist zu beachten, daß (nur) in der Ln-Ergebnisdatei (*.DAT) nun die umsatzkorrigierten DIFFWerte $\left(\mathrm{I}^{\mathrm{kor}}\right)$ gespeichert werden; aus diesen sind auch die dort gespeicherten LogarithmenVerhältnisse berechnet, so daß die Steigung der Auftragung von „lnHpt“ gegen „,lnRef“ unmittelbar die gesuchte Größe k / $\mathrm{k}_{\text {Ref }}$ liefert.

Im Messprotokoll sind zwar die gemessenen DIFF-Werte unverändert, jedoch die Abnahmen bereits umsatzkorrigiert angegeben; ferner sind dort alle für die Berechnung der Umsatzkorrektur verwendeten Werte (Faktoren) gespeichert.

Für den (seltenen) Fall, daß der Referenz-Radikal-Peak von einem Fragment der Haupt-Substanz überlagert ist, kann, sofern sonst kein BKG vorliegt(!), dieser Fragment-Anteil als BKG für Referenz (MWG1: Aus) eingeben werden. Wird nun im Fenster „Umsatzkorrektur“ die Option „ReferenzBKG-Umsatzkorrektur durch Hauptmesung“ aktiviert, dann wird bei der Messung des Umsatzes auf der Muttermasse der Hauptsubstanz auch der BGK-Wert für die Referenz-Substanz (MWG1: Ein) ausgerechnet:

Wird im späteren $\mathrm{I}^{\mathrm{BKG}}(\operatorname{Ref})_{+\mathrm{MWG}}=\mathrm{I}^{\mathrm{BKG}}(\operatorname{Ref})_{-\mathrm{MWG}} \cdot \mathrm{I}^{\text {Mutterpeak }}(\mathrm{Hpt})_{+\mathrm{MWG}} / \mathrm{I}^{\text {Mutterpeak }}(\mathrm{Hpt})_{-\mathrm{MWG}}$ Verlauf der Messungen nochmals die Intensität des Fragmentes auf dem Radikal-Peak der Haupt(!)Substanz aktualisiert, dann werden auch automatisch beide BKG-Werte der Referenz-Substanz prozentual geändert (= aktualisiert).

\subsection{Das Menü TOOLS}

\subsubsection{AP-Skalenteile}

Dieses Hilfsprogramm ermöglicht es, die im AP-Modus benötigten Skalenteile am Potentiometer auszurechnen, die für eine bestimmte Ionisierungsenergie notwendig sind.

Es können Werte zwischen IP = 4,5 eV (0 Skalenteile) und 29,5 eV (1000 Skalenteile) eingestellt werden. 
Die Berechnung erfolgt unmittelbar bei Eingabe des Wertes automatisch.

Dieses Unterfenster muß erst geschlossen werden, bevor man in WinSiccon andere Funktionen nutzen kann.

\subsubsection{Konzentration}

Für die Berechnung der Konzentration, der Reaktionszeit und der linearen Strömungsgeschwindigkeit wird nur ein Fenster verwendet, auf dem zunächst oben angeklickt werden muß, welcher Wert zu berechnen ist; abhängig davon werden dann alle notwendigen Angaben abgefragt, indem man sich mit der TAB-Taste weiterbewegt. Nachdem alle Werte eingegeben worden sind, kann mit dem entsprechenden Schalter „Berechnen“ der gewünschte Wert berechnet werden.

Bitte wählen Sie unbedingt die korrekten Einheiten zu den von Ihnen eingegebenen Zahlenwerten (eine Umrechnung der Zahlenwerte bei Änderung der Einheit erfolgt nicht !) und beachten Sie in welchen Einheiten die Ergebnisse angegeben sind. Insbesondere ist $\mathrm{zu}$ beachten, daß sämtliche Gasflüsse bezogen auf den Standarddruck von 1 atm und die Standardtemperatur von 273,15 K einzugeben sind; so wie sie von den TYLAN FC 260 auch geregelt werden.

Es werden für die Berechnungen ideale Bedingungen vorausgesetzt, die ggf. von der Wirklichkeit abweichen können (vgl. dazu auch die Formeln für die

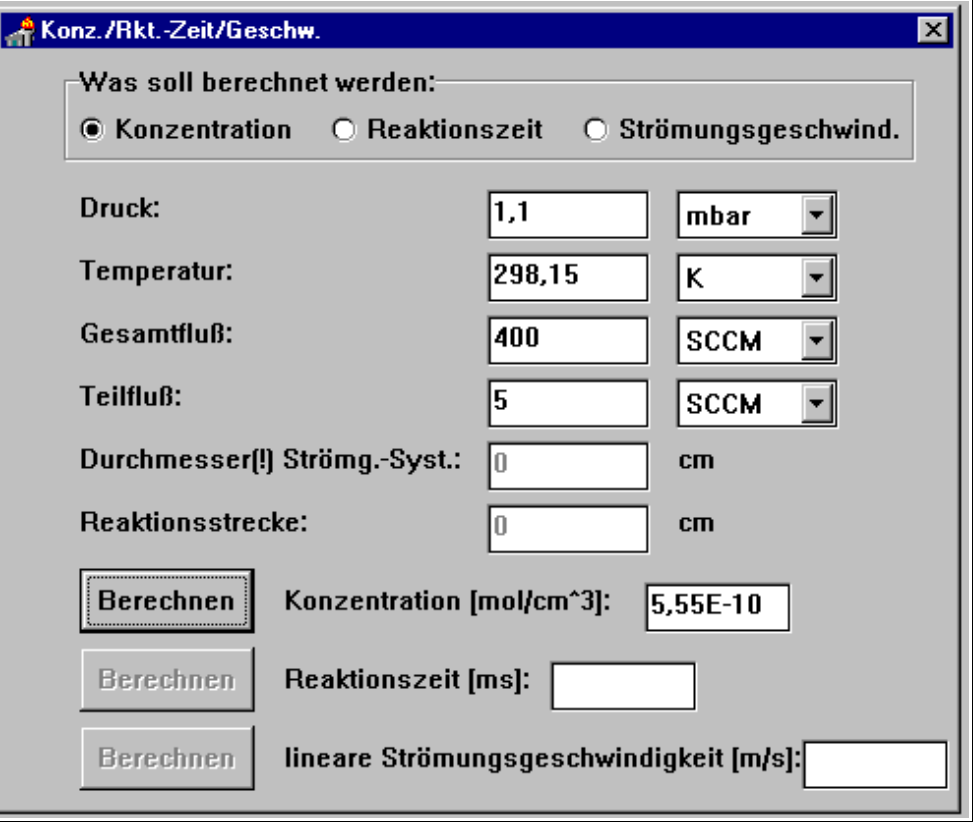
Berechnungen in den Kapiteln 5.5.1 ff., S. 32 ff.).

Zur Berechnung der Konzentration benötigt man folgende Angaben:

- Druck [in: Torr, mbar oder Pa]

- Temperatur [in: ${ }^{\circ} \mathrm{C}$ oder $\mathrm{K}$ ]

- Gesamtfluß aller Gase im Strömungssystem [in: $\mathrm{cm}^{3} / \mathrm{s}=\mathrm{SCCS}$ oder $\mathrm{cm}^{3} / \mathrm{min}=\mathrm{SCCM}$ ]

- Teilfluß desjenigen Gases, dessen Konzentration berechnet werden soll [SCCS oder SCCM]. Bei Flüssigkeiten, die über einen Sättiger zugegeben werden, kann nährungsweise das Verhältnis aus dem Dampfdruck der Flüssigkeit (für die Temperatur im Sättiger) und aus dem absoluten Gesamtdruck im Sättiger mit dem Volumenfluß des Dosierventils multipliziert werden. Letzteren ermittelt man am besten über eine Eichkurve des Dosierventils mit der jeweiligen Gasmischung oder nährungsweise mit dem reinen Trägergas. Es ist dabei zu beachten, daß das Programm den Volumenfluß bezogen auf $273 \mathrm{~K}$ und $1 \mathrm{~atm}$ als Eingabe erwartet. Letzteres kann vorteilhaft mit dem Programmteil „Flußberechnung“ im Menü „TOOLS“ (Kap. 3.4.6, S. 24) berechnet werden.

\subsubsection{Reaktionszeit}

Eine allgemeine Beschreibung dieses Fensters befindet sich im Kapitel 3.4.2 .

Zur Berechnung der Reaktionszeit unter Annahme idealer Bedingungen benötigt man folgende Angaben:

- Druck [in: Torr, mbar oder Pa] 
- Temperatur [in: ${ }^{\circ} \mathrm{C}$ oder $\mathrm{K}$ ]

- Gesamtfluß [in: SCCS oder SCCM]

- Durchmesser (nicht Radius!) des Strömungssystems [cm]

- Reaktionsstrecke (Sondenstellung) [cm]

\subsubsection{Lineare Strömungsgeschwindigkeit}

Eine allgemeine Beschreibung dieses Fensters befindet sich im Kapitel 3.4.2 .

Zur Berechnung der linearen Strömungsgeschwindigkeit unter Annahme idealer Bedingungen benötigt man folgende Angaben:

- Druck [in: Torr, mbar oder Pa]

- Temperatur [in: ${ }^{\circ} \mathrm{C}$ oder $\mathrm{K}$ ]

- Gesamtfluß [in: SCCS oder SCCM]

- Durchmesser (nicht Radius !) des Strömungssystems [cm]

\subsubsection{Diffusionskoeffizient}

In diesem Programmteil kann der

Diffusionskoeffizient nach CHAPMAN-ENSKOG berechnet werden (vgl. Formeln in Kapitel 5.5.4).

Dazu werden folgende Angaben benötigt:

- Druck $\mathrm{p}$ [Torr]

- Temperatur T [K]

- Molmasse M [g/mol]

- Lennard-J.-Länge $\sigma\left[10^{-10} \mathrm{~m}\right]$

- Lennard-Jones-Energie $\varepsilon$ dividiert durch die

\begin{tabular}{|c|c|c|c|}
\hline \multicolumn{4}{|c|}{ Binärer Diffusionskoeffizient nach Chapman-Enskog: } \\
\hline \multirow{2}{*}{$\begin{array}{l}\text { Druck [Torr]: } \\
\text { Temperatur [K]: }\end{array}$} & 0 & \multirow[b]{2}{*}{ L-J ber. } & \multirow[b]{3}{*}{ Trägergas } \\
\hline & 0 & & \\
\hline & Substanz & \\
\hline \multicolumn{2}{|c|}{ Lennard-Jones-Länge SIGMA [1E-10 m]: } & $\mathbf{0}$ & 2,551 \\
\hline \multicolumn{2}{|c|}{ Lennard-Jones-Energie EPSILON / k [K]: } & $\mathbf{0}$ & 10,22 \\
\hline \multicolumn{2}{|c|}{ Molmasse [g/mol]: } & $\mathbf{0}$ & 4,00260 \\
\hline Berechnen & \multicolumn{2}{|c|}{ Binärer Diffusionskoeffizient: } & $\mathrm{cm}^{\wedge} 2 / \mathrm{s}$ \\
\hline
\end{tabular}

Boltzmann-Konstante: $\left(\varepsilon / \mathrm{k}_{\mathrm{B}}[\mathrm{K}]\right)$

Diese Angaben sind auf der linken Seite für die eigentliche Substanz einzugeben und auf der rechten Seite für das jeweilige Trägergas; da oft Helium Verwendung findet, sind die vorgegebenen Werte in der Spalte Trägergas diejenigen von Helium.

Sofern die Lennard-Jones-Parameter nicht Tabellenwerken zu entnehmen sind, können sie grob (!) über folgende kritische Werte (Physikalische Werte am Kritischen Punkt) abgeschätzt werden:

Kritisches Volumen $\mathrm{V}_{\mathrm{c}}\left[\mathrm{cm}^{3} / \mathrm{mol}\right]\left(=\mathrm{M} / \varrho_{\mathrm{c}}\right)$

- Kritische Temperatur $\mathrm{T}_{c}[\mathrm{~K}]$

- Kritische Kompressibilität $\mathrm{Z}_{\mathrm{c}}$ [dimensionslos]

Um die Lennard-Jones-Parameter vom Programm abschätzen zu lassen, ist der Schalter „L-J ber.“ zu drücken; es öffnet sich dann ein Fenster, welches die kritischen Werte abfragt und nach Drücken des „OK“-Schalters werden die Lennard-Jones-Parameter automatisch berechnet und eingefügt.

\subsubsection{Flußberechnung}

In diesem Programmteil können die Gasflüsse von geeichten HOKE-Ventilen berechnent werden; auch wenn die Zugabe über einen Sättiger erfolgt. Ferner lassen sich die Gasflüsse berechnen, die bei Verwendung von TYLAN-flow-controllern fließen. 
Üblicherweise ergibt die Auftragung der Quadratwurzel des Flusses durch ein HOKEVentil vs. n (die Anzahl Umdrehungen am Ventil) eine Gerade. In diesem Fall können die Parameter a

(Achsenabschnitt) und $b$ (Steigung) der

Regressionsgeraden in die entsprechenden Felder eingetragen werden und nach Eingabe der Anzahl Umdrehungen $n$ erhält man durch Klick auf den Schalter „Fluss berechnen“ den Fluss durch das HOKE-Ventil in der

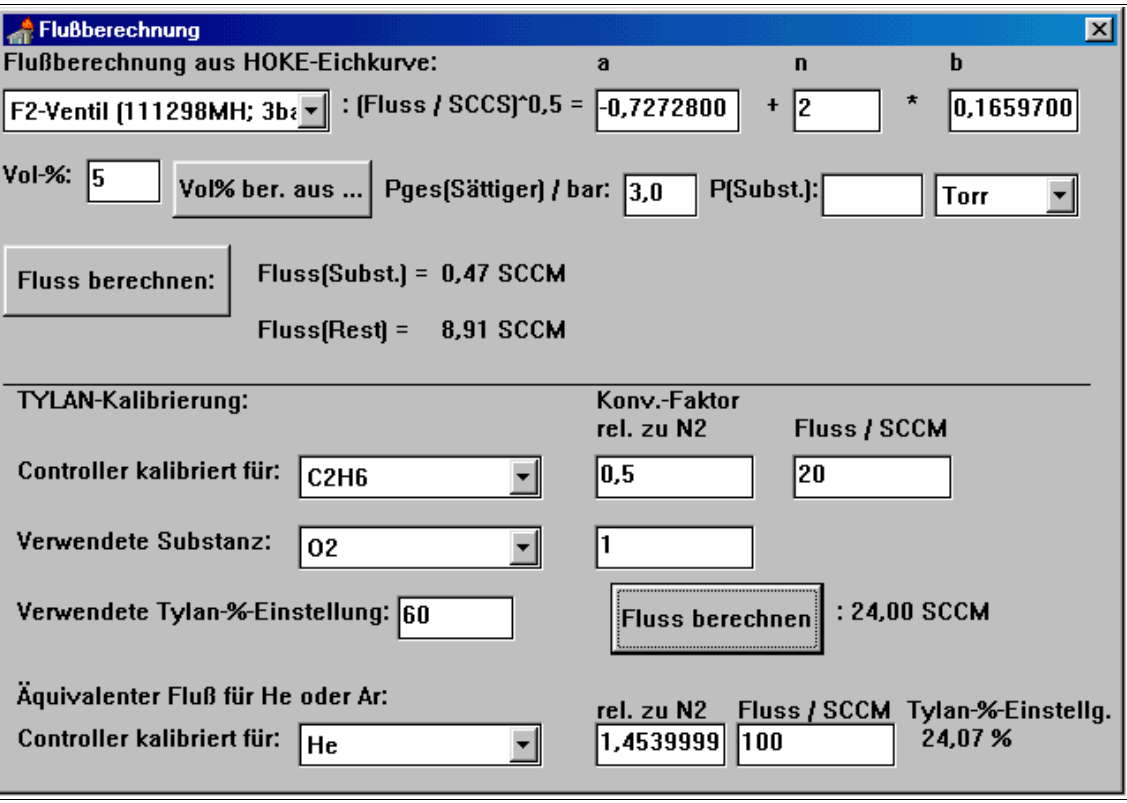

Einheit SCCM. Durch Eingabe einer Vol-\%-Zahl in das entsprechende Feld wird nur der Fluss mit diesem Faktor multipliziert (z.B. wenn man eine nur 5\%-ige $\mathrm{F}_{2}-/ \mathrm{He}-\mathrm{Mischung}$ hat, würde man 5 Vol- $\%$ eingeben, um den reinen $\mathrm{F}_{2}$-Fluß zu berechnen; die restlichen 95 Vol-\% entfallen dann auf den He Fluß). Die berechneten Werte werden automatisch in die Zwischenablage kopiert und können somit mittels der Tasten STRG + v nun z.B. in die „Messtext-Systemeinstellungen“ kopiert werden.

Die Eingabe der Vol-\%-Zahl ist auch erforderlich, falls Substanzen durch einen Sättiger zugegeben werden, weil sich diese Substanz nur zu einem bestimmten Vol-\%Anteil in dem Trägergas befindet.

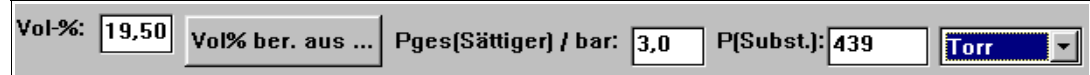
Dieser Anteil kann berechnet werden, indem der Gesamtdruck im Sättiger (meist 3,0 bar abs.) und der Dampfdruck der Flüssigkeit (bei der Temperatur im Sättiger !) in die jeweiligen Felder eingetragen wird und dann auf den Schalter „Vol-\% ber. aus ..." geklickt wird. Dadurch wird der Vol-\%-Anteil der Substanz in das Feld Vol-\% eingetragen und man kann den Fluß berechnen.

In der Datei „HOKE.TXT“ im Programmverzeichnis können die Koeffizienten a und b der Regressionsgerade gespeichert werden und dann später im Programm ausgewählt werden. Dazu muß diese Datei mit dem Windows-Editor geöffnet werden und eine neue Zeile in folgendem Format eingefügt werden: ,,a b Name_HOKE_Ventil“, z.B.: „,0.0012 0.20561 F2-Ventil(020399MH)““ Als Dezimaltrennzeichen des Achsenabschnitts a und der Steigung b ist (ausnahmsweise) der Punkt $\mathrm{zu}$ verwenden und die Werte sind jeweils durch ein Leerzeichen zu trennen.

Sofern ein TYLAN-flow-controller verwendet wird, ist dieser nur auf eine bestimmte Gasart geeicht, z.B. $20 \mathrm{SCCM} \mathrm{C}_{2} \mathrm{H}_{6}$. Die eingestellte \%-Zahl an dem TYLAN-Steuergerät bezieht sich auf diesen Wert. Will man jedoch ein anderes Gas (z.B. $\mathrm{O}_{2}$ ) verwenden, so ist eine Umrechnung erforderlich, die den Konversionsfaktor relativ zu $\mathrm{N}_{2}$ benötigt (vgl. die Tabellen im TYLAN-Handbuch). Sind diese Wert bekannt, so können sie in die entsprechenden Felder eingetragen werden und durch Klick auf den Schalter „Fluss berechnen“ wird der Fluß unter Berücksichtigung der \%-Einstellung am Steuergerät berechnet (in der Einheit SCCM). Der berechnete Wert wird automatisch in die Zwischenablage kopiert und kann somit mittels der Tasten STRG + v nun z.B. in die „MesstextSystemeinstellungen“" kopiert werden.

Wird eine Doppelmessung mit 2. Reaktant durchgeführt, bei der relativ große Mengen des 2. Reaktanten zugegeben werden müssen (z.B. bei $\mathrm{O}_{2}$ oder NO), empfiehlt es sich, einen Druckausgleich mit Badgas (He oder Ar) vorzunehmen. Sobald der Fluß des 2. Reaktanten abgestellt 
wird, sollte die dadurch fehlende Gasmenge durch Badgas ersetzt werden. Daher berechnet das Programm die für diese äquivalente Badgasmenge erforderliche Tylan-\%-Einstellung automatisch, so daß man bei Variation der Menge des 2. Reaktanten sofort auch die äquivalente Badgasmenge mittels TYLAN nachregulieren kann.

In der Datei „TYLAN.TXT“ im Programmverzeichnis kann der Konversionsfaktor relativ zu N $\mathrm{N}_{2}$ gespeichert und dann später im Programm ausgewählt werden. Dazu muß diese Datei mit dem Windows-Editor geöffnet werden und eine neue Zeile in folgendem Format eingefügt werden: „Konv_Faktor Gas_Name“, z.B.: „1.45 Ar““

Als Dezimaltrennzeichen ist (ausnahmsweise) der Punkt zu verwenden und die Werte sind durch ein Leerzeichen zu trennen. 


\section{Inhalt der erzeugten Dateien}

\subsection{Meßprotokoll}

Wie in Kapitel 3.2.2.1 (S. 14) bereits erläutert wurde, wird das Meßprotokoll üblicherweise in einem Tagesunterverzeichnis, dessen Name aus dem Benutzerkürzel und dem Tagesdatum besteht, abgespeichert. Das Tagesunterverzeichnis wird seinerseits in dem in der Datei „WIN_SIC.INI“ angegebenen HAUPTPFAD und dort im Unterverzeichnis $B K$ (=Benutzerkürzel) angelegt.

Das Meßprotokoll ist eine ASCII-Datei mit der Dateiextension *.TX*; da sie im ANSI-Zeichensatz gespeichert wird, kommt es beim Einlesen in DOS-Programme zu nicht korrekter Darstellung von Umlauten und Sonderzeichen. In Windows-Programmen ist eine Bearbeitung jedoch problemlos möglich.

Da auch unter Windows 3.11 nur begrenzter Speicherplatz zur Verfügung steht, können im Meßprotokoll theoretisch maximal $64 \mathrm{~KB}$ Text gespeichert werden; in der Praxis sind je nach Arbeitsumgebung sogar nur 40-50 KB möglich. Wenn der Speicherplatz erschöpft ist, speichert WinSiccon automatisch den aktuellen Inhalt in eine Datei mit der Extension ,"*.TXi“, wobei i eine beginnend mit 1 fortlaufend numerierte Zahl ist; das zweite zwischengespeicherte Meßprotokoll erhält also z.B. die Extension ,*.TX2“. Das aktuell am Bildschirm angezeigte und somit zuletzt gespeicherte Meßprotokoll hat immer die normale Extension ,*.TXT“.

Am Anfang des Protokolls wird der Dateiname, das Benutzerkürzel und die verwendete Versionsnummer von WinSiccon gespeichert, dann folgen die Eintragungen aus dem Fenster „Messtext - Systemeinstellungen“.

Anschließend wird jeder Meßzyklus fortlaufend numeriert und die jeweiligen DATA, BKG und DIFF-Werte eingetragen. Am Ende eines Meßzyklusses werden die Mittelwerte (〈DATA $\rangle$, etc.) und der mittlere Fehler des Mittelwertes $( \pm$...), sowie in Klammern der relative Fehler abgespeichert; ferner werden die angezeigten Drücke $\mathrm{p}(\mathrm{sys}), \mathrm{p}(\mathrm{ZV}), \mathrm{p}(\mathrm{IQ})$ jeweils gespeichert.

Im Doppelmessung-Modus wird ferner nach jedem Doppel-Meßzyklus noch der Umsatz der Reaktion ausgegeben. Dieser ist ggf. umsatzkorrigiert berechnet (vgl. Kap. 3.3.2, S.21).

Das Meßprotokoll kann am besten mittels des Windows-Editors ausgedruckt werden (vgl. dazu auch Kap.3.1.1, S. 10).

\subsection{Tabellendatei}

Sofern vor Beginn einer Messung bereits ein Meßprotokoll-Dateiname vergeben wurde, wird in dem Verzeichnis, in dem auch das Meßprotokoll gespeichert wird, eine Datei mit der Extension „*.TAB““ angelegt. Es handelt sich auch hier um eine ASCII-Datei, in der tabellarisch durch TABULATORZeichen (ASCII \#9) getrennt folgende Werte gespeichert sind:

- Meßzyklus-Nummer laut Meßprotokoll

- $\mathrm{m} / \mathrm{z}$-Verhältnis

- Zustand MWG1 ( „AUS“ oder „EIN“ )

- Zustand MWG2 ( „AUS“ oder ,EIN“ )

- Mittelwert <DATA>

- mittlerer Fehler des Mittelwertes <DATA> gemäß gewünschtem Konfidenzniveau (Student-Fkt.)

- Mittelwert <BKG>

- mittlerer Fehler des Mittelwertes $<B K G>$ gemäß gewünschtem Konfidenzniveau (Student-Faktor)

Um die Auswertung einer Absolutmessung zu erleichtern, werden (nur) bei einer Einzelmessung zusätzlich noch folgende Werte gespeichert: 
- Druck im Strömungssystem p(StrSys) $[\mathrm{Pa}]$

- Reaktionsstrecke s [cm]

\subsection{Ln-Ergebnisdatei}

\subsubsection{Doppelmessung mit 1 Reaktant}

Sofern der Doppelmessung-Modus Verwendung findet, wird in dem Verzeichnis, in dem auch das Meßprotokoll gespeichert wird, eine Datei mit der Extension „*.DAT“ angelegt. Es handelt sich auch hier um eine ASCII-Datei, in der tabellarisch, durch TABULATOR-Zeichen (ASCII \#9) getrennt, folgende Werte gespeichert werden:

- $\ln \left\{[\operatorname{Ref}]_{-\mathrm{MWG}} /[\operatorname{Ref}]_{+\mathrm{MWG}}\right\} ;$ vgl. Kapitel 5.1 (S. 30)

- $\ln \left\{[\mathrm{Hpt}]_{-\mathrm{MWG}} /[\mathrm{Hpt}]_{+\mathrm{MWG}}\right\} ; \operatorname{vgl}$. Kapitel 5.1

- Fehler von $\ln \left\{[\operatorname{Ref}]_{-\mathrm{MWG}} /[\operatorname{Ref}]_{+\mathrm{MWG}}\right\}$; vgl. Kapitel 5.4.4 (S. 32)

- Fehler von $\ln \left\{[\mathrm{Hpt}]_{-\mathrm{MWG}} /[\mathrm{Hpt}]_{+\mathrm{MWG}}\right\}$; vgl. Kapitel 5.4.4

- Meßzyklus-Nummer der Hauptmessung laut Meßprotokoll

- $\mathrm{m} / \mathrm{z}$-Wert der Hauptmessung

- Mittelwert $\left\langle\right.$ DIFF ${ }_{- \text {MwG }}$ von der Hauptmessung ohne MWG-Entladung

- mittlerer Fehler des Mittelwertes d $\langle\mathrm{DIFF}\rangle_{\text {-MWG }}$ von der Hauptmessung

- Mittelwert $\left\langle\right.$ DIFF ${ }_{+ \text {MWG }}$ von der Hauptmessung mit MWG-Entladung

- mittlerer Fehler des Mittelwertes d $\langle\mathrm{DIFF}\rangle_{+\mathrm{MWG}}$ von der Hauptmessung

- Mittelwert $\langle\mathrm{DIFF}\rangle_{{ }_{\text {MWG }}}$ von der Referenzmessung ohne MWG-Entladung

- mittlerer Fehler des Mittelwertes d $\left\langle\right.$ DIFF ${ }_{\text {-MwG }}$ von der Referenzmessung

- Mittelwert $\langle\text { DIFF }\rangle_{+ \text {MWG }}$ von der Referenzmessung mit MWG-Entladung

- mittlerer Fehler des Mittelwertes $d\left\langle\right.$ DIFF ${ }_{+ \text {MWG }}$ von der Referenzmessung

- $\mathrm{m} / \mathrm{z}$-Wert der Referenzmessung

- Meßzyklus-Nummer der Referenzmessung laut Meßprotokoll

Dieser Datei-Aufbau eignet sich bestens sowohl für eine grafische Auftragung mit Fehlerbalken (es sind die Werte 1-4 zu verwenden) als auch für die Übernahme in die Meßwerttabellen (dafür sind die Werte 6-15 zu verwenden).

\subsubsection{Doppelmessung mit 2. Reaktant}

In dem Verzeichnis, in dem auch das Meßprotokoll gespeichert wird, wird eine Datei mit der Extension ,*.DAT“ angelegt. Es handelt sich um eine ASCII-Datei, in der tabellarisch, durch TABULATOR-Zeichen (ASCII \#9) getrennt, folgende Werte gespeichert werden:

- $\ln \left\{\mathrm{I}^{\mathrm{korr}}(\mathrm{Ref})_{-2 \text {. Reaktant }} / \mathrm{I}^{\mathrm{korr}}(\mathrm{Ref})_{+2 \text {. Reaktant }}\right\} ; \operatorname{vgl.~Kapitel} 5.3$ (S. 30)

- $\ln \left\{\mathrm{I}^{\mathrm{korr}}(\mathrm{Hpt})_{\text {-2. Reaktant }} / \mathrm{I}^{\mathrm{korr}}(\mathrm{Hpt})_{+2 \text {. Reaktant }}\right\} ; \operatorname{vgl}$. Kapitel 5.3

- Fehler von $\ln \left\{\mathrm{I}^{\mathrm{korr}}(\mathrm{Ref})_{\text {-2. Reaktant }} / \mathrm{I}^{\mathrm{korr}}(\mathrm{Ref})_{+2 \text {. Reaktant }}\right\}$; vgl. Kapitel 5.4.4 (S. 32)

- Fehler von $\ln \left\{\mathrm{I}^{\mathrm{korr}}(\mathrm{Hpt})_{-2 \text {. Reaktant }} / \mathrm{I}^{\mathrm{korr}}(\mathrm{Hpt})_{+2 \text {. Reaktant }}\right\}$; vgl. Kapitel 5.4.4

- Meßzyklus-Nummer der Hauptmessung laut Meßprotokoll

$\mathrm{m} / \mathrm{z}$-Wert der Hauptmessung

- Berechnete Intensität $\mathrm{I}^{\mathrm{korr}}(\mathrm{Hpt})_{-2 \text {. Reaktant }}$ von der Hauptmessung ohne 2. Reaktant; vgl. Kap. 5.3

- Fehler von $\mathrm{I}^{\mathrm{korr}}(\mathrm{Hpt})_{-2 \text {. Reaktant }}$ 
- Berechnete Intensität $\mathrm{I}^{\mathrm{korr}}(\mathrm{Hpt})_{+ \text {2. Reaktant }}$ von der Hauptmessung mit 2. Reaktant; vgl. Kap. 5.3

- Fehler von $\mathrm{I}^{\mathrm{korr}}(\mathrm{Hpt})_{+2 \text {. Reaktant }}$

- Berechnete Intensität $I^{\text {korr }}\left(\right.$ Ref) ${ }_{\text {2. Reaktant }}$ von der Referenzmessung ohne 2. Reaktant; vgl. Kap. 5.3

- Fehler von I ${ }^{\text {korr }}\left(\right.$ Ref) ${ }_{2 \text {. Reaktant }}$

- Berechnete Intensität $\mathrm{I}^{\mathrm{kor}}(\mathrm{Ref})_{+2 \text {. Reaktant }}$ von der Referenzmessung mit 2. Reaktant; vgl. Kap. 5.3

- Fehler von $\mathrm{I}^{\mathrm{korr}}(\mathrm{Hpt})_{\text {-2. Reaktant }}$

$\mathrm{m} / \mathrm{z}$-Wert der Referenzmessung

- Meßzyklus-Nummer der Referenzmessung laut Meßprotokoll

\subsection{Dateien aus dem Übersichtsspektrum}

Üblicherweise sind die Spektrendaten in dem Verzeichnis gespeichert, das im Eintrag „SPEKTRUMPFAD“ in der Datei „WIN_SIC.INI“ angegeben ist. Neben der Textdatei (*.TXT), die den Meßtext enthält, legt das Programm noch zwei ASCII-Dateien, nämlich die Spektrendatei (*.DAT) und die Peakdatei (*.PIK), an.

In der , *.DAT“-Datei sind folgende Werte, getrennt durch TABULATOR-Zeichen (ASCII \#9), gespeichert (vgl. dazu auch Kap. 3.2.3, S. 18):

- Zeit seit dem Start der Messung [s]

- DATA-Wert [w.E.]

- $\mathrm{m} / \mathrm{z}-\mathrm{Wert}$

In der „*.PIK“-Datei sind folgende Werte, getrennt durch TABULATOR-Zeichen (ASCII \#9), gespeichert (vgl. dazu auch Kap. 3.1.3, S. 11):

- $\mathrm{m} / \mathrm{z}-$ Wert (als mit den DATA-Werten gewichteter Mittelwert eines Peaks)

- Peak-Wert (= Summe der DATA-Werte in einem Peak)

Optional können Peak-Dateien voneinander subtrahiert werden, um Differenzspektren zu erzeugen (vgl. dazu Kap. 3.1.4, S. 12). Diese Subtraktionsdateien werden mit der Extension „*.SUB“ gespeichert und enthalten folgende durch Tabulator-Zeichen (ASCII 9) getrennte Werte:

m/z-Wert (gerundet auf ganze Zahl)

- Differenz der Peak-Werte 


\section{Verwendete Formeln}

\subsection{Berechnung der Logarithmen-Verhältnisse}

Im Doppelmessung-Modus werden, sofern man die Option gegen eine Referenz zu messen ausnutzt, automatisch die folgenden Logarithmen berechnet:

$$
\ln H p t=\ln \left\{\frac{[\mathrm{Hpt}]_{-M W G}}{[\mathrm{Hpt}]_{+M W G}}\right\} \quad \ln \operatorname{Ref}=\ln \left\{\frac{[\mathrm{Ref}]_{-M W G}}{[\operatorname{Ref}]_{+M W G}}\right\}
$$

Dabei bedeutet [Hpt] die Konzentration (vgl. unten) der Hauptsubstanz; ,-MWG“ deutet an, daß die Messung ohne Reaktion stattgefunden hat, , ,+MWG“ entsprechend mit Reaktion. Analog wird auch lnRef berechnet und diese beiden Werte sowohl grafisch dargestellt, als auch in der ln-Ergebnisdatei (*.DAT) gespeichert.

Da es sich ja um Relativmessungen handelt, ist es nicht notwendig, die Konzentrationen direkt auszurechnen; daher werden für [Hpt] einfach die gemessenen Mittelwerte <DIFF> eingesetzt und die Fehler gemäß unten stehender Formel aus den Fehlern dieser DIFF-Mittelwerte nach den Gesetzen der Fehlerfortpflanzung berechnet. Analog wird mit [Ref] verfahren.

\subsection{Berechnung der linearen Regression}

Die in der grafischen Darstellung im Doppelmessung-Modus ausgegebene Regressionsgerade wird nach der Methode der kleinsten Quadratsumme berechnet.

Die Formeln dazu sind in jedem Lehrbuch der Statistik abgedruckt.

\subsection{Berechnung der Umsatzkorrektur}

Bsp.: Bei der Messung von $\mathrm{CH}_{3}+\mathrm{O} \rightarrow$ Produkte ist bei höherer Ionisierungsenergie oft auf $\mathrm{m} / \mathrm{z}$ $=15(\mathrm{CH} 3)$ ein Fragment des Muttermoleküls $\left(\mathrm{CH}_{4}\right)$ zu beklagen.

Im Menüpunkt KORREKTUR /

UMSATZ (vgl. Kap. 3.3.2, S. 21) wird mit dem Schalter „Ermittlung des

Umsatzes auf dem Peak des

Muttermoleküls" das Verhältnis von $\mathrm{I}(16+\mathrm{F}-\mathrm{O}) \mathrm{zu} \mathrm{I}(16-\mathrm{F}-\mathrm{O})$ auf $\mathrm{m} / \mathrm{z} 16$ bestimmt, d.h. die relative Abnahme des Mutterionen-Peaks. Der reine

Fragmentanteil auf $\mathrm{m} / \mathrm{z} 15$ wird natürlich in gleicher Höhe vermindert; wenn man also (hypothetisch) annimmt, das Radikal selbst würde auf $\mathrm{m} / \mathrm{z} 15$ kein Signal zeigen, würde man die in nebenstehender Grafik grün gezeichneten Peaks erwarten, die beide bezogen auf die schwarzen

Peaks um den selben Prozentsatz (im diesem Beispiel: $30 \%$ ) vermindert sind. Tatsächlich wird auf $\mathrm{m} / \mathrm{z} 15$ aber der rote Peak gemessen. Die Differenz aus dem grünen hypothetischen und dem roten tatsächlichen Wert auf m/z 15 ergibt die reine Signalintensität des Radikals. 
Der tatsächliche Wert I Iragment (15-F-O) wird entweder im Menüpunkt KORREKTUR / UMSATZ (vgl. Kap. 3.3.2, S. 21) mit dem Schalter „Ermittlung der Intensität des Fragment-Peaks“ gemessen oder automatisch bei Option zur ,automatischen Fragmentanteil-Korrektur“ (vgl. Kap. 3.2.2.4S. 18), so daß dann vom Programm eine automatische Umsatzkorrektur-Berechnung wie folgt vorgenommen werden kann:

$$
\begin{aligned}
& \mathrm{I}^{\text {korr }}\left(\mathrm{CH}_{3}\right)_{-\mathrm{O}}=\mathrm{I}^{\text {korr }}(15+\mathrm{F}-\mathrm{O})=\mathrm{I}^{\text {mess }}(15+\mathrm{F}-\mathrm{O})-\mathrm{I}^{\text {Fragment }}(15-\mathrm{F}-\mathrm{O}) \frac{\mathrm{I}^{\text {Mutter }}(16+\mathrm{F}-\mathrm{O})}{\mathrm{I}^{\text {Mutter }}(16-\mathrm{F}-\mathrm{O})} \\
& \mathrm{I}^{\text {korr }}\left(\mathrm{CH}_{3}\right)_{+\mathrm{O}}=\mathrm{I}^{\text {korr }}(15+\mathrm{F}+\mathrm{O})=\mathrm{I}^{\text {mess }}(15+\mathrm{F}+\mathrm{O})-\mathrm{I}^{\text {Fragment }}(15-\mathrm{F}-\mathrm{O}) \frac{\mathrm{I}^{\text {Mutter }}(16+\mathrm{F}-\mathrm{O})}{\mathrm{I}^{\text {Mutter }}(16-\mathrm{F}-\mathrm{O})}
\end{aligned}
$$

Die Zahlen (z.B. 15) deuten den m/z-Wert an, $+F$ bedeutet, daß unter Zugabe von Fluor-Atomen gemessen wurde; die übrigen Symbole lauten analog. ${ }^{\text {mess }}$ ist der tatsächlich gemessene, d.h. mit dem Fragment-Peak überlagerte Radikal-Peak. Hiervon ist der hypothetisch ohne Radikalbildung entsthende Fragment-Anteil zu subtrahieren, um den ,netto-Radikal-Peak“ ${ }^{\text {korr }}$ zu erhalten.

$\mathrm{I}^{\mathrm{korr}}$ ist die für die weitere Auswertung (Berechnung des umsatzkorrigierten Umsatzes; Bildung der Logarithmen-Verhältnisse; Anzeige des Graphs mit Regression) verwendete umsatzkorrigierte Intensität des Radikal-Peaks.

\subsection{Fehlerrechnung}

\begin{tabular}{|c|c|c|}
\hline$\langle x\rangle=\frac{1}{n} \sum_{i=1}^{n} x_{i}$ & $\begin{array}{l}\langle x> \\
x_{i}\end{array}$ & $\begin{array}{l}\text { : Mittelwert der Meßgröße x } \\
\text { : i-ter Einzel-Meßwert }\end{array}$ \\
\hline
\end{tabular}

\subsubsection{Mittelwert}

\subsubsection{Standardabweichung}

$s=\sqrt{\frac{1}{(n-1)} \sum_{i=1}^{n}\left(x_{i}-\langle x\rangle\right)^{2}}$

\subsubsection{Fehler des Mittelwertes}

Die im Meßprotokoll angegebenen Fehler der Mittelwerte wurden nach nebenstehender Formel berechnet:

Dabei ist $\mathrm{t}(\mathrm{n}-1, \alpha)$ der Student'sche Faktor mit dem das berechnete Konfidenzintervall $[\langle\mathrm{x}\rangle-\Delta\langle\mathrm{x}\rangle,\langle\mathrm{x}\rangle+\Delta\langle\mathrm{x}\rangle]$ bei einer sehr großen $\Delta\langle x\rangle=\frac{s \cdot t(n-1, \alpha)}{\sqrt{n}}$ Zahl von Meßreihen den zu messenden Mittelwert x mit der Wahrscheinlichkeit 1- $\alpha$ überstreicht.

Standardmäßig rechnet das Programm mit einem Wert von 1- $\alpha=95 \%$.

Es können jedoch auch die Werte $1-\alpha=50 \%, 68 \%, 75 \%, 90 \%$ oder $99 \%$ oder <keine> berechnet 
werden. Der verwendete Wert ist im Meßprotokoll angegeben.

Wird im „Messtext - Systemeinstellungen“-Fenster der Wert <keine> ausgewählt, so rechnet das Programm mit einem Studentfaktor $\mathrm{t}=1$, unabhängig von der Zahl $\mathrm{n}$; der angegebene Fehler ist dann die Standardabweichung dividiert durch die Quadratwurzel von n.

\subsubsection{Fehlerfortpflanzung}

Für eine allgemeine Funktion $\mathrm{z}=\mathrm{f}(\mathrm{x}, \mathrm{y})$ wird die Fehlerfortpflanzung gemäß nachstehender Formel berechnet.

$$
d z=\sqrt{\left(\frac{\partial z}{\partial x}\right)^{2} d x^{2}+\left(\frac{\partial z}{\partial y}\right)^{2} d y^{2}}
$$

Anwendung findet dieses Prinzip bei der Fehlerfortpflanzung der im Doppelmessung-Modus eingegebenen BKG-Werte auf den daraus berechneten Fehler des DIFF-Mittelwertes:

$$
d\langle D I F F\rangle=\sqrt{d\langle D A T A\rangle^{2}+d\langle B K G\rangle^{2}}
$$

Analog wird auch die Fehlerfortpflanzung bei der Umsatzkorrektur gemäß Kapitel 5.3 (S. 30) berechnet.

Ferner wird es bei der Fehlerfortpflanzung der aus den einzelnen DIFF-Mittelwerten berechneten Logarithmen-Verhältnisse (vgl. Kap.5.1, S. 30) benutzt:

$$
d \operatorname{lnHpt}=\sqrt{\left(\frac{d[H p t]_{-M W G}}{[H p t]_{-M W G}}\right)^{2}+\left(\frac{d[H p t]_{+M W G}}{[H p t]_{+M W G}}\right)^{2}}
$$

\subsection{Formeln aus dem Menü TOOLS}

\subsubsection{Berechnung der Konzentration}

Hierbei sind ...

$\left[\mathrm{c}_{\mathrm{i}}\right] \quad$ : Konzentration der Substanz i im Strömungs-

$$
\left[c_{i}\right]=\frac{\Phi_{i}}{\Phi_{\text {ges }}} \frac{p}{T R}=\frac{\Phi_{i}}{\Phi_{\text {ges }}} \frac{p T_{0}}{T p_{0} V_{0}}
$$

Reakt.; Einheit: Stoffmenge pro Volumen

$\Phi_{\mathrm{i}} \quad$ : Gasfluß der Substanz i bei einem Druck von 1 atm und einer Temperatur von $0{ }^{\circ} \mathrm{C}$; Einheit ist Volumen pro Zeit

$\Phi_{\text {ges }} \quad: \sum \Phi_{\mathrm{i}}$; gesamter Gasfluß bei einem Druck von 1 atm und einer Temperatur von $0{ }^{\circ} \mathrm{C}$

p : : Druck im Strömungssystem

T : absolute Temperatur im Strömungssystem; die Einheit ist $K$

$\mathrm{R} \quad$ : allg. Gaskonstante $\left(\mathrm{R}=8,314 \mathrm{~J} \mathrm{~mol}^{-1} \mathrm{~K}^{-1}\right)$

$\mathrm{T}_{0}=273,15 \mathrm{~K} ; \mathrm{p}_{0}=760$ Torr $; \mathrm{V}_{0}=22413,6 \mathrm{~cm}^{3} / \mathrm{mol}$ 


\subsubsection{Berechnung der Reaktionszeit im Strömungssystem}

$$
\mathrm{t}=\pi \mathrm{r}^{2} \frac{\mathrm{s}}{\Phi_{\text {ges }}} \frac{\mathrm{T}_{0} \mathrm{p}}{\mathrm{p}_{0} \mathrm{~T}}
$$

Zusätzlich zu den Erläuterungen in Kapitel 5.5.1 gilt:

t $\quad$ : Reaktionszeit im Strömungssystem

r : Radius des Strömungssystems

s : : Reaktionsstrecke (Sondenstellung)

\subsubsection{Berechnung der linearen Strömungsgeschwindigkeit}

$$
\mathrm{v}=\frac{\Phi_{\text {ges }}}{\pi \mathrm{r}^{2}} \frac{\mathrm{T} \mathrm{p}_{0}}{\mathrm{~T}_{0} \mathrm{p}}
$$

Bezüglich der Symbole vgl. Kapitel 5.5.1 f. .

\subsubsection{Berechnung des Diffusionskoeffizienten nach Chapman-Enskog}

Der binäre Diffusionskoeffizient berechnet sich nach Chapman-Enskog ${ }^{2}$ gemäß der folgenden Formel:

$$
\mathrm{D}_{\mathrm{AB}}=2,6610^{-3} \frac{\sqrt{\mathrm{T}^{3}}}{\mathrm{p} \sqrt{\mathrm{M}_{\mathrm{AB}}} \sigma_{\mathrm{AB}}^{2} \Omega_{\mathrm{D}}}
$$

mit:

$$
M_{A B}=\frac{2}{\frac{1}{M_{A}}+\frac{1}{M_{B}}}
$$

und

$$
\sigma_{\mathrm{AB}}=\frac{\sigma_{\mathrm{A}}+\sigma_{\mathrm{B}}}{2}
$$

$\mathrm{D}_{\mathrm{AB}} \quad$ : binärer Diffusionskoeffizient $\left[\mathrm{cm}^{2} / \mathrm{s}\right]$

T : Temperatur $[\mathrm{K}]$

p : Druck [bar]

$\mathrm{M}_{\mathrm{A}}, \mathrm{M}_{\mathrm{B}}$ : Molmasse von Trägergas A bzw. Gas B [g/mol]

$\sigma_{\mathrm{A}}, \sigma_{\mathrm{B}} \quad$ : Lennard-Jones-Länge von Substanz A bzw. B $\left[10^{-10} \mathrm{~m}\right]$

$\Omega_{\mathrm{D}} \quad$ : Diffusions-Stoßintegral [dimensionslos]

Für $\Omega_{\mathrm{D}}$ wird folgende Nährung ${ }^{3}$ verwendet: 


$$
\Omega_{\mathrm{D}}=\frac{1,06036}{\mathrm{~T}^{\prime 0,15610}}+\frac{0,19300}{\exp \left(0,47635 \mathrm{~T}^{\prime}\right)}+\frac{1,03587}{\exp \left(1,52996 \mathrm{~T}^{\prime}\right)}+\frac{1,76474}{\exp \left(3,89411 \mathrm{~T}^{\prime}\right)}
$$

mit:

$$
\mathrm{T}^{\prime}=\frac{\mathrm{k}_{\mathrm{B}} \mathrm{T}}{\epsilon_{\mathrm{AB}}}
$$

und

$$
\epsilon_{\mathrm{AB}}=\sqrt{\epsilon_{\mathrm{A}} \epsilon_{\mathrm{B}}}
$$

$\mathrm{k}_{\mathrm{B}} \quad$ : Boltzmann-Konstante

$\mathrm{T} \quad$ : Temperatur $[\mathrm{K}]$

$\varepsilon_{\mathrm{A}}, \varepsilon_{\mathrm{B}}:$ Lennard-Jones-Energie von Substanz A bzw. B [J]

\subsubsection{Berechnung der Lennard-Jones-Parameter}

ACHTUNG: Die auf diese Weise abgeschätzten Lennard-Jones-Parameter weichen oft ganz erheblich von der Wirklichkeit ab, so daß diese Berechnung nur als sehr grobe Nährung zu verstehen ist und es auf jeden Fall vorzuziehen ist, die korrekten Parameter aus Tabellen zu entnehmen !

Im übrigen sollte auch in der Original-Literatur ${ }^{4}$ nachgelesen werden, ob die angegebene Formel für den interessierenden Stoff überhaupt geeignet ist.

$$
\sigma=0,687 \sqrt[3]{\mathrm{V}_{\mathrm{c}}}
$$

$$
\frac{\epsilon}{\mathrm{k}_{\mathrm{B}}}=0,404 \mathrm{k}_{\mathrm{B}} \mathrm{T}_{\mathrm{c}} \mathrm{Z}_{\mathrm{c}}^{-0,53}
$$

mit:

$\mathrm{V}_{\mathrm{c}} \quad:$ Kritisches Volumen $\left[\mathrm{cm}^{3} / \mathrm{mol}\right]$

$\mathrm{T}_{\mathrm{c}} \quad:$ Kritische Temperatur $[\mathrm{K}]$

$\mathrm{Z}_{\mathrm{c}}$ : Kritische Kompressibilität [dimensionslos]

$\mathrm{k}_{\mathrm{B}} \quad$ : Boltzmann-Konstante

\subsubsection{Formeln zur Flußberechnung}

Der Fluß einer Substanz i durch ein HOKE-Ventil wird folgendermaßen berechnet:

$$
\begin{array}{ll}
\frac{\phi_{i}}{S C C M}=60 \cdot\left(\frac{\text { Vol- } \%}{100}\right) \cdot(a+n \cdot b)^{2} & \text { mit: a = Achsenabschnitt, } \mathrm{b}=\text { Steigung der Regr. } \\
\mathrm{n}=\text { Anzahl Umdrehungen am HOKE-Ventil } \\
\text { Vol- } \%=\text { Volumen-\% der Substanz i im Gasstrom }
\end{array}
$$

Dabei wird davon ausgegangen, daß die Parameter a und b einer linearen Regression aus der Auftragung der Quadratwurzel des Flusses [in SCCS] gegen die Zahl der Umdrehungen n des HOKE-Ventils entnommen wurden und diese Auftragung tatsächlich eine Gerade ergeben hat.

Die Volumen-\% der Substanz i im Gasstrom, die durch einen Sättiger zugeführt wird, berechnet das Programm folgendermaßen:

4 HALKIADAKIS E.A.; BOWREY R.G.; Chem. Eng. Sci. 30, 53 (1975) 


$$
\begin{aligned}
& (\text { Vol- } \%)=\frac{p_{\text {Substanzi }}}{p_{\text {ges., Sătiger }}} \cdot 100 \quad \text { mit: } \quad \text { pubstanz }=\begin{array}{l}
\text { Dampfdruck der flüssigen Substanz i } \\
\text { im Sättiger bei der dortigen Temperatur; }
\end{array} \\
& \mathrm{p}_{\text {ges, } \text { Sattiger }}=\text { Gesamtdruck (absolut) im Sättiger }
\end{aligned}
$$

Der Fluß durch die TYLAN-flow-controller wird folgendermaßen berechnet:

$$
\frac{\phi_{\text {Substanz }}}{S C C M}=(\% \text {-Einstellung }) \cdot \frac{\phi_{\text {Kalibriert }}}{S C C M} \cdot \frac{N_{2} \text {-Konv.-Faktor(Substanz) }}{\left.N_{2} \text {-Konv.-Faktor(Kalibriert }\right)}
$$

mit: $\quad$ (\%-Einstellung): $\quad$ Einstellung am Regelgerät, wieviel \% des kalibrierten Wertes zugegeben werden

$\phi_{\text {kalibriert: }} \quad$ Standard-Fluß, für den der TYLAN bei $100 \%$ kalibriert wurde

$\mathrm{N}_{2}$-Konv.-Faktor: Konversionsfaktor relativ zu N $\mathrm{N}_{2}$ (vgl. TYLAN-Handbuch) 


\section{Test der Hardware}

Um sicherzustellen, daß keine Hardware-Fehler vorliegen, sollten gelegentlich die im

Einzelionenzähler „SSR 1110“ implementierten Testprogramme (vgl. dafür die Dokumentation zu diesem Gerät) eingestellt und dann mit WinSiccon im Einzelmessung-Modus diese Werte überprüft werden.

Falls in WinSiccon andere Werte als die im Testprogramm eingestellten angezeigt werden, so ist wahrscheinlich ein defekter Stecker oder ein defektes Kabel dafür verantwortlich. Mit dem im Programmverzeichnis von WinSiccon gespeicherten Programm „BIT.EXE“ kann dann jedes einzelne übertragene Bit angezeigt und mit dem Sollwert aus dem Testprogramm verglichen werden. Mit „BIT.EXE“ können auch (Soll-)Dezimalzahlen in Dualzahlen ungerechnet werden, so daß die bitweise Überprüfung erleichtert wird.

\section{Fehlerbehebung}

Der einzige bisher bekannte Fehler taucht (ganz selten) bei der Berechnung der Standardabweichung auf, wenn mehrmals hintereinander ausschließlich der gleiche DATA-Wert gemessen wurde. Dies ist leider kein vermeidbarer Fehler von WinSiccon, sondern es handelt sich um ein Problem mit der Gleitkomma-Arithmetik und liegt daran, daß bei dieser Berechnung erst die Änderung einer signifikanten Nachkommastelle eintritt, die die Kapazität des aufzunehmenden Registers überschreitet und somit nicht mehr berechnet werden kann; es erfolgt dann normalerweise ein „Runtime-Error“, der eine Fortsetzung des Programms verhindert. Da die Fehlerberechung bei dieser Art Messung jedoch keine so große Genauigkeit erfordert, fängt WinSiccon den Runtime-Error ab und setzt die Standardabweichung einfach auf Null (was in diesem Fall ja korrekt ist). Damit ermöglicht es eine unbeeinträchtigte Fortsetzung der Messungen. Die unmittelbar letzte Messung kann aber auch einfach wiederholt werden, wobei der Fehler dann meistens nicht mehr auftritt.

Da ein Programm niemals fehlerfrei sein wird, sollte bei Auftreten eines Fehlers möglichst eine genaue Beschreibung der Umstände erfolgen, die ihn verursacht haben (In welchem Programmteil trat er auf; was wurde unmittelbar vorher gemacht, etc.), damit eine Korrektur des Programms vorgenommen werden kann.

\section{Hinweise zur Änderung/Erweiterung des Programms}

Wegen der zeitkritischen Messungen bei der Aufnahme von Übersichtsspektren wurde in diesem Programmteil auf eine strukturierte Programmierung (Aufteilung in Unterprogramme) verzichtet und der gesamte Quellcode sequenziell eingegeben, wodurch schnellstes Laufzeitverhalten resultiert. Jedoch sind dadurch viele Anweisungen mehrfach enthalten und auch die Anweisungen aus den Einzel- und Relativmessungs-Routinen sind dort nochmals vorhanden. Sofern das Programm also geändert werden soll, muß dies in allen Programmteilen erfolgen und anschließend nochmals auf Konsistenz überprüft werden ! 


\section{Inhalt der Konfigurationsdateien (*.INI, *.TXT)}

Die Datei WIN_SIC.INI hat z.Zt. folgenden Aufbau und Inhalt:

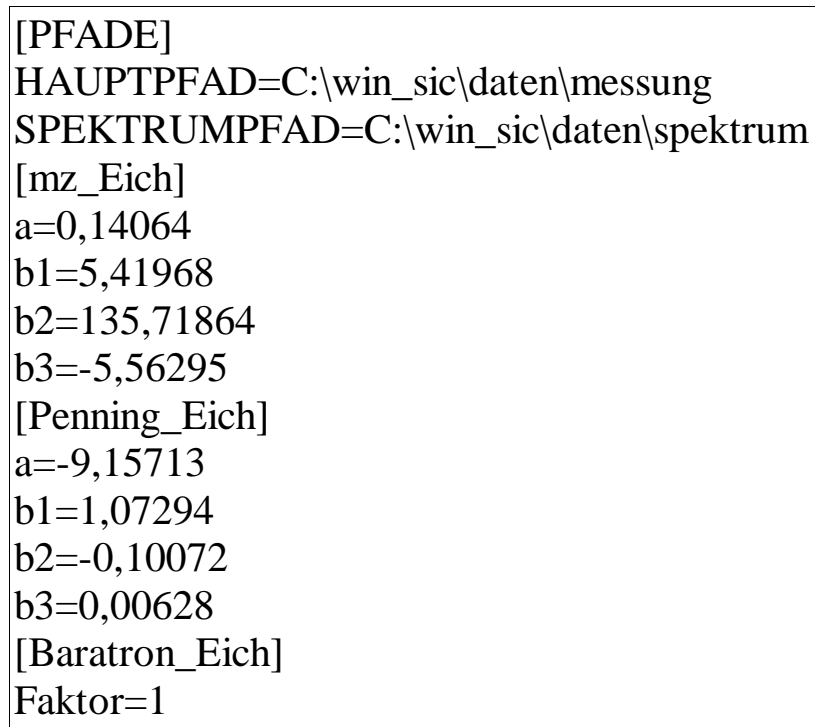

Die Datei HOKE.TXT hat z.Zt. folgenden Aufbau und Inhalt:

-0.72728 0.16597 F2-Ventil (111298MH; 3bar) $0.205610 .26265 \mathrm{KW}-$ Ventil (250398MH; 3bar) $0.385290 .24264 \mathrm{KW}-$ Ventil (290897B; 3bar)

Die Datei TYLAN.TXT hat z.Zt. folgenden Aufbau und Inhalt:

$0.50 \mathrm{C} 2 \mathrm{H} 6$

$0.72 \mathrm{CH} 4$

$1.00 \mathrm{O} 2$

$1.454 \mathrm{He}$

$1.45 \mathrm{Ar}$ 


\section{Schritt-für-Schritt-Anleitung zur Bedienung}

\subsection{Durchführung Doppelmessung mit 1 Reaktant}

1. Im Menü „MESSEN“das Programm „Masse/Ladung und MWG“ starten

2. Im Menü „MESSEN“ das Programm „Doppelmessung (1 Reaktant)“ starten

3. Die Frage nach dem Anlegen eines Meßprotokolls mit „JA“ beantworten

4. Ggf. die Frage nach dem Anlegen eines Tagesunterverzeichnisses mit „JA“ beantworten

5. Den vorgeschlagenen Dateinamen bestätigen oder nach eigenem Wunsch ändern

6. In dem Fenster „Messtext-Systemeinstellungen“ das Konfidenzintervall für die Fehlerrechnung wählen und falls im Messprotokoll gewünscht auch die Systemeinstellungen angeben

7. In dem Fenster „Doppelmessung (1 Reaktant)“ nun die Anzahl der Messungen wählen

8. Die MWG-Pause angeben

9. Falls ein Hintergrund zu berücksichtigen ist, diesen im Menü „Korrektur“ unter „BKG-Erfassung“ eingeben oder automatisch messen lassen (dazu vorher entsprechend $\mathrm{m} / \mathrm{z}$ am CH5 einstellen); jeweils für die Haupt- und Referenzmessung

10.m/z für die Referenzsubstanz einstellen

11.Falls gewünscht einen individuellen Messtext für die unmittelbar nächste Messung eingeben

12.Den „Referenz-Messung starten“-Schalter drücken

13.Ggf. Schritte 10-12 wiederholen, falls Fehler zu groß o.ä.

14.m/z für die (Haupt-)Substanz einstellen

15.Falls gewünscht einen individuellen Messtext für die unmittelbar nächste Messung eingeben

16.Den „Haupt-Messung starten“-Schalter drücken

17.Bei Ansicht der Grafik nun entscheiden, ob das Wertepaar gespeichert werden soll oder nicht; falls es nicht gespeichert werden soll, können die Schritte 10-12 oder 14-16 beliebig wiederholt werden.

18.Abwechselnd die Schritte 14-17 und 10-13 bzw. die Schritte 10-13 und 14-17 bei anderen Umsätzen (d.h. anderen F-Konzentrationen) wiederholen, bis die Meßreihe vollendet ist

19.Das Fenster „Doppelmessung (1 Reaktant)“ über das Systemmenü schließen und die Frage nach dem Speichern des Protokolls mit „JA“ beantworten

20.Das Messprotokoll in den Windows-Editor laden und mit diesem drucken

21.Die Ln-Ergebnisdatei in z.B. ORIGIN als ASCII-Datei importieren und den Plot mit oder ohne Fehlerbalken erstellen 


\subsection{Durchführung (umsatzkorrigierter) Doppelmessung mit 2. Reaktant}

1. Im Menü „MESSEN“das Programm „Masse/Ladung und MWG“ starten

2. Im Menü „MESSEN“ das Programm „Doppelmessung mit 2. Reaktant“ starten

3. Die Frage nach dem Anlegen eines Meßprotokolls mit „JA“ beantworten

4. Ggf. die Frage nach dem Anlegen eines Tagesunterverzeichnisses mit „JA“ beantworten

5. Den vorgeschlagenen Dateinamen bestätigen oder nach eigenem Wunsch ändern

6. In dem Fenster „Messtext-Systemeinstellungen“ das Konfidenzintervall für die Fehlerrechnung wählen und falls im Messprotokoll gewünscht auch die Systemeinstellungen angeben

7. In dem Fenster „Doppelmessung mit 2. Reaktant“" nun die Anzahl der Messungen wählen

8. Falls eine automatische Zugabe des 2. Reaktant möglich ist, nun die Pause [s] eingeben, die WinSiccon nach der Zugabe des 2. Reaktant verstreichen läßt, bevor es die Einzelionenzählung beginnt

9. Falls ein Hintergrund für den Molekülionen-Peak (Mutter-Peak) zu berücksichtigen ist, diesen im Menü „Korrektur“ unter „BKG-Erfassung“ eingeben oder automatisch messen lassen (dazu vorher entsprechend $\mathrm{m} / \mathrm{z}$ am CH5 einstellen); jeweils für die Haupt- und Referenzmessung

10.Falls erforderlich, nun im Menü „Korrektur“ unter „Umsatz“ die Umsätze auf den MuttermolekülPeaks jeweils für die Haupt- und Referenzmessung ermitteln (dazu vorher entsprechend $\mathrm{m} / \mathrm{z}$ am CH5 einstellen)

11.Falls ein Hintergrund für den Radikal-Peak zu berücksichtigen ist, diesen im Menü „Korrektur“ unter „BKG-Erfassung“ eingeben oder automatisch messen lassen (dazu vorher entsprechend m/z am CH5 einstellen); jeweils für die Haupt- und Referenzmessung

12.Falls erforderlich, nun im Menü „Korrektur“ unter „Umsatz“ die Intensitäten der Fragment-Peaks (auf den Massen der Radikale) jeweils für die Haupt- und Referenzmessung ermitteln (dazu vorher entsprechend $\mathrm{m} / \mathrm{z}$ am CH5 einstellen); dies kann unterbleiben, sofern die Option zur ,automatischen Fragmentanteil-Aktualisierung“ gewählt wird, da diese Messungen dann später automatisch durchgeführt werden.

13.m/z für die Referenzsubstanz einstellen

14.Falls gewünscht einen individuellen Messtext für die unmittelbar nächste Messung eingeben

15.Den „Referenz-Messung starten“-Schalter drücken (MWG1 startet nun) und bei manueller Zugabe des 2. Reaktanten eine angemessene Zeit warten, bis das nächste Meldungsfenster ("Bitte den 2. Reaktanten noch nicht zugeben") bestätigt und dadurch die Einzelionenzählung gestartet wird

16.Bei manueller Zugabe des 2. Reaktanten erscheint dann die Meldung ,Jetzt bitte auch den 2. Reaktanten zugeben“; sobald dies geschehen und das Signal konstant ist, kann diese Meldung mit „OK“ bestätigt werden, wodurch die Einzelionenzählung gestartet wird

17.Ggf. Schritte 15-16 wiederholen, falls Fehler zu groß o.ä.

18.m/z für die (Haupt-)Substanz einstellen

19.Falls gewünscht einen individuellen Messtext für die unmittelbar nächste Messung eingeben

20.Den „Haupt-Messung starten“-Schalter drücken (MWG1 startet nun) und bei manueller Zugabe des 2. Reaktanten eine angemessene Zeit warten, bis das nächste Meldungsfenster ("Bitte den 2. Reaktanten noch nicht zugeben") bestätigt und dadurch die Einzelionenzählung gestartet wird 
21.Bei manueller Zugabe des 2. Reaktanten erscheint dann die Meldung „Jetzt bitte auch den 2. Reaktanten zugeben"; sobald dies geschehen und das Signal konstant ist, kann diese Meldung mit „OK“ bestätigt werden, wodurch die Einzelionenzählung gestartet wird

22.Bei Ansicht der Grafik nun entscheiden, ob das Wertepaar gespeichert werden soll oder nicht; falls es nicht gespeichert werden soll, können die Schritte 13-16 oder 18-21 beliebig wiederholt werden.

23.Bei stärkeren Änderungen der Signalintensitäten kann eine Wiederholung von Schritt 12 erforderlich sein (?)

24.Die Schritte 13-23 wiederholen, bis die Meßreihe vollendet ist

25.Das Fenster „Doppelmessung (1 Reaktant)“ über das Systemmenü schließen und die Frage nach dem Speichern des Protokolls mit „JA“ beantworten

26.Das Messprotokoll in den Windows-Editor laden und mit diesem drucken

27.Die Ln-Ergebnisdatei in z.B. ORIGIN als ASCII-Datei importieren, dann den Plot mit oder ohne Fehlerbalken erstellen und drucken

\subsection{Messung mit Fragment der Haupt-Substanz auf Peak des Radikals der Referenz-Substanz}

1. Ggf. BKG für Radikal-Peak der Hauptmessung mit reinem Trägergas aufnehmen

2. BKG für Referenz (Fragment der Hauptsubstanz) auf der Masse des Radikal-Peaks der Referenz für MWG1=aus mit Fluß von Trägergas + Hauptsubstanz aufnehmen

3. Diesen BKG (noch) nicht verwenden

4. Nun auch Gasfluß der Referenz-Substanz einschalten

5. Umsatz der Hauptsubstanz auf dem Mutter-Peak messen (dadurch wird auch der BKG-Wert des Radikal-Peaks der Referenz für MWG1=ein berechnet)

6. Umsatz der Referenzsubstanz auf dem Mutter-Peak messen

7. Nun die gespeicherten BKG-Werte verwenden

8. Messung des Radikal-Peaks der Haupt-Substanz für MWG1=aus

9. Messung des Radikal-Peaks der Referenz für MWG1=aus 


\section{Stichwortverzeichnis}

ACHTUNG

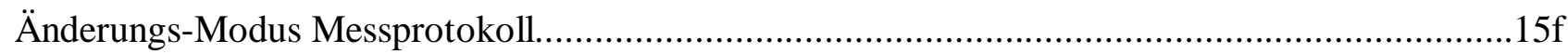

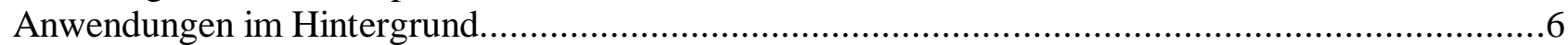

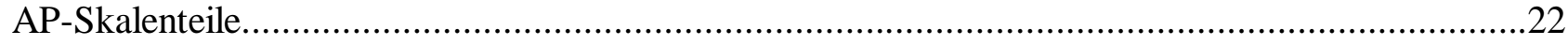

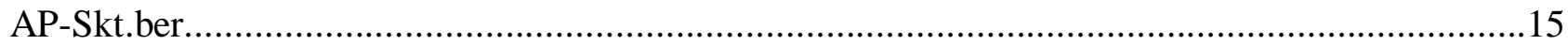

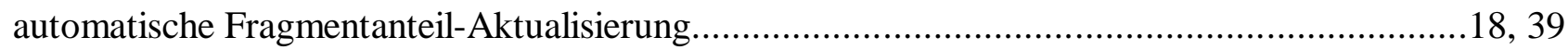

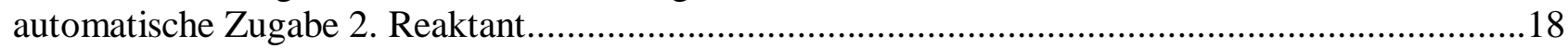

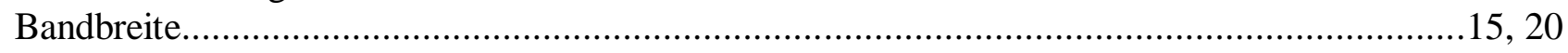

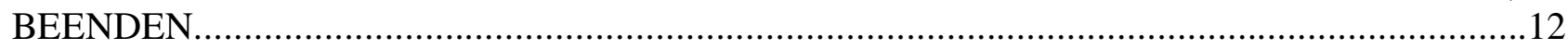

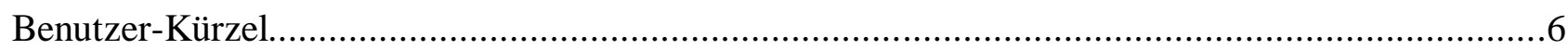

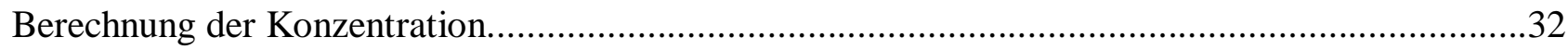

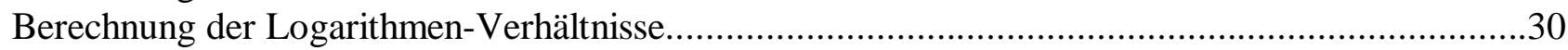

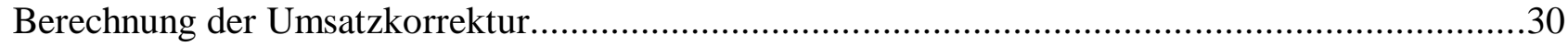

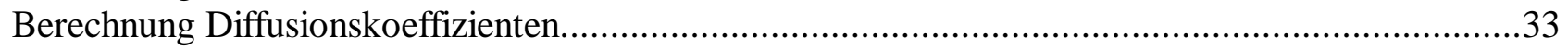

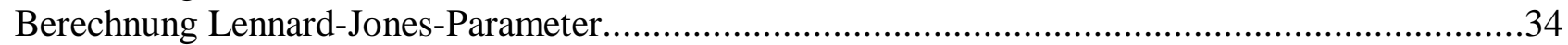

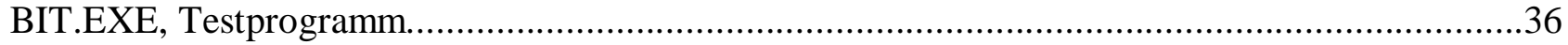

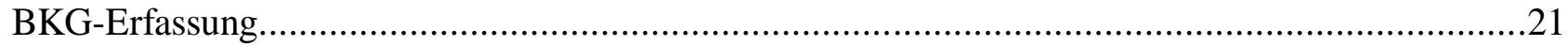

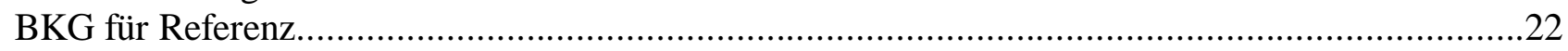

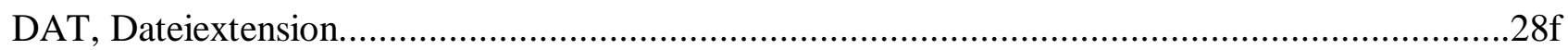

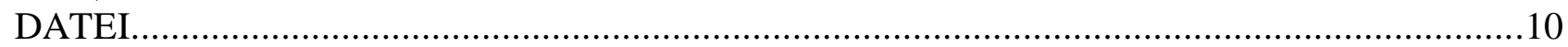

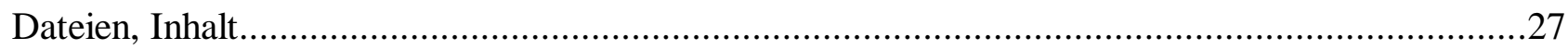

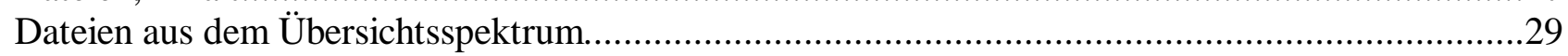

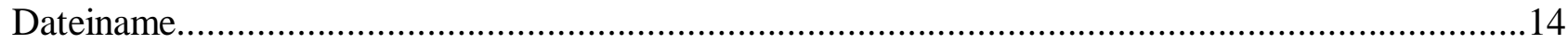

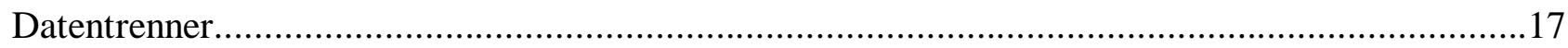

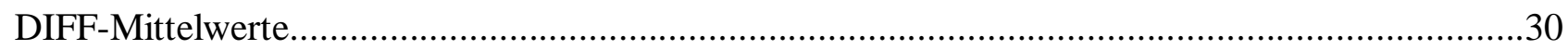

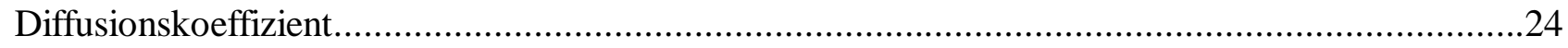

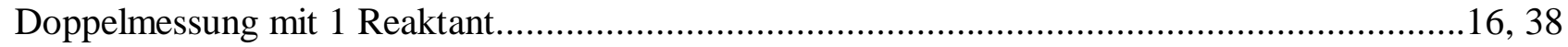

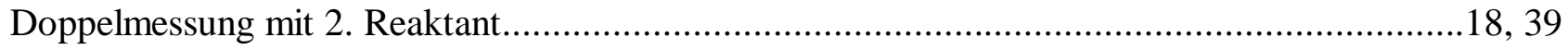

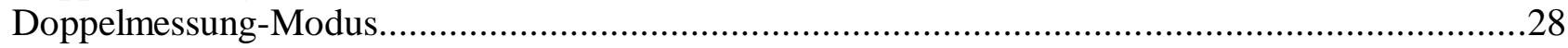

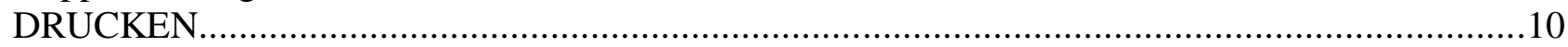

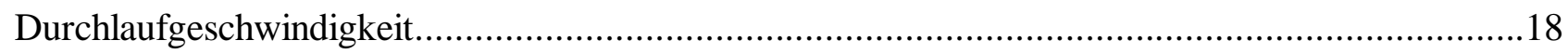

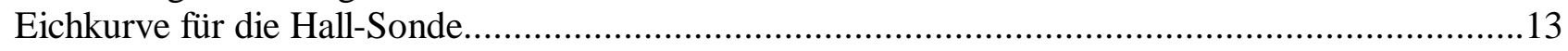

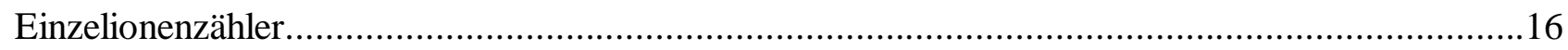

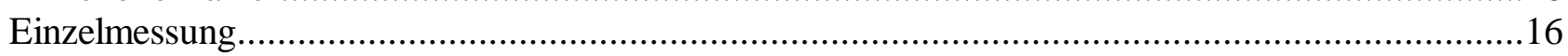

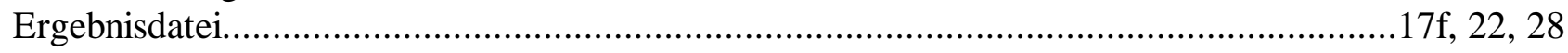

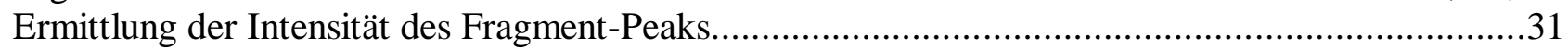

Ermittlung des Umsatzes auf dem Peak des Muttermoleküls...................................................30

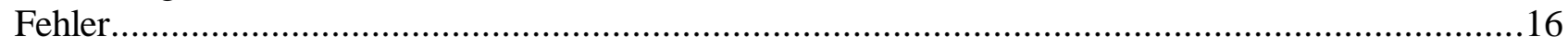

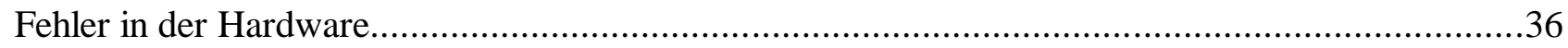

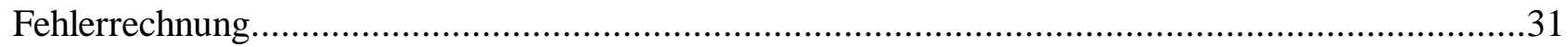

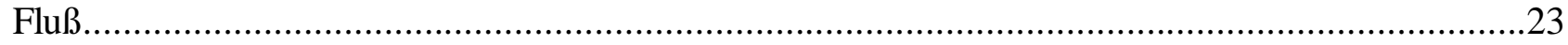

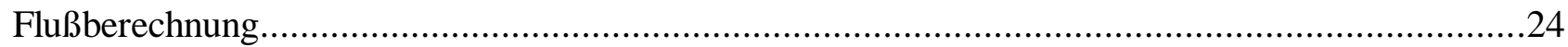

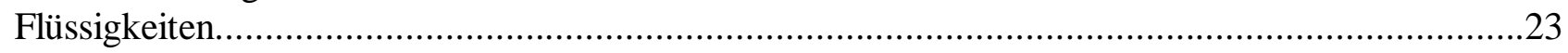

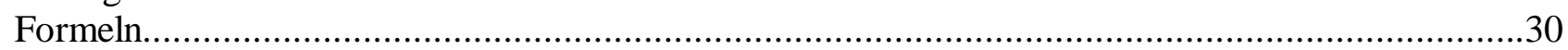

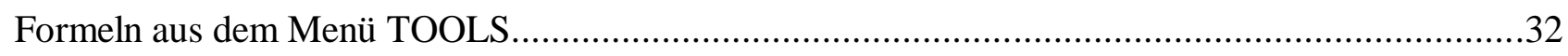

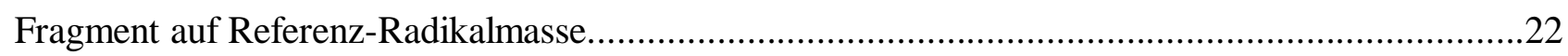

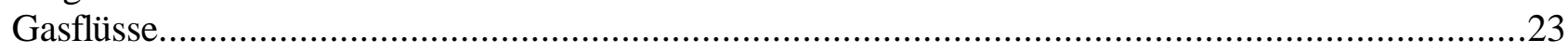

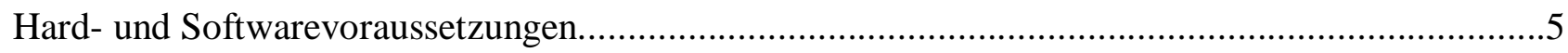




\section{Programmbeschreibung für WinSiccon 1.7}

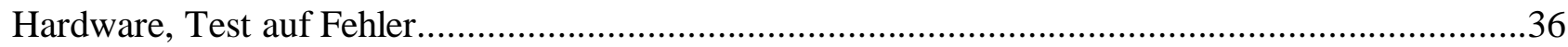

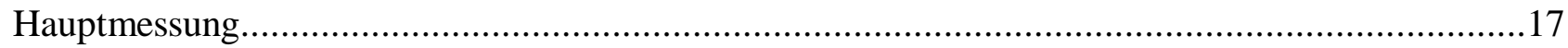

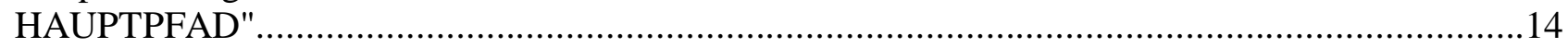

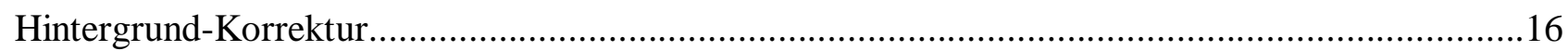

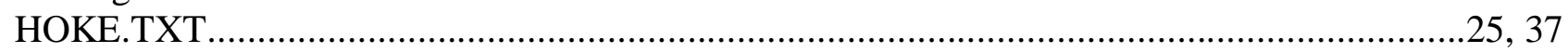

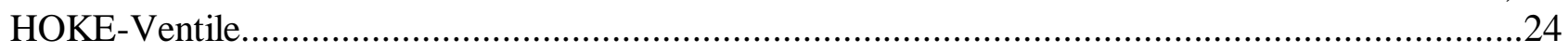

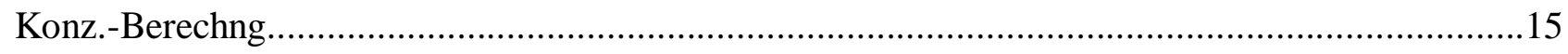

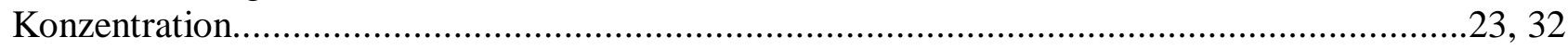

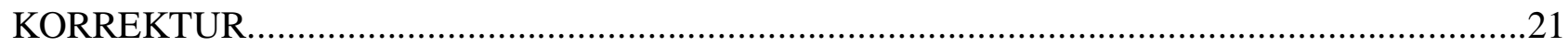

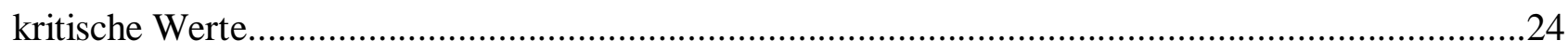

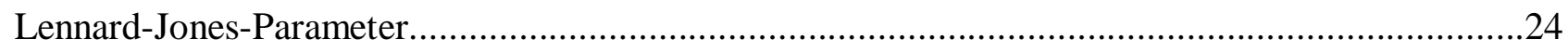

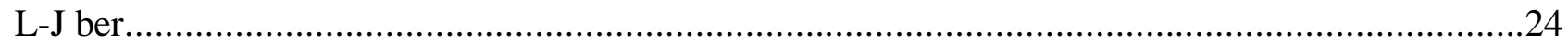

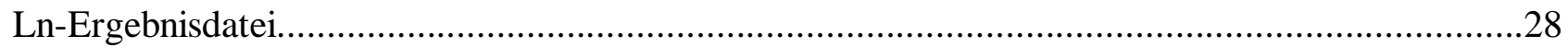

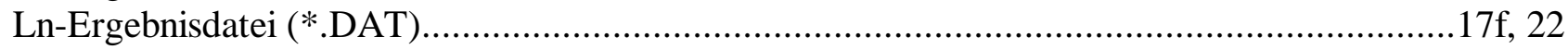

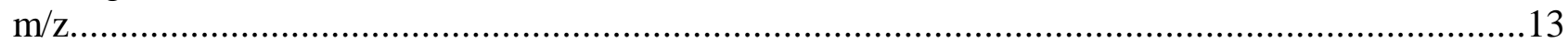

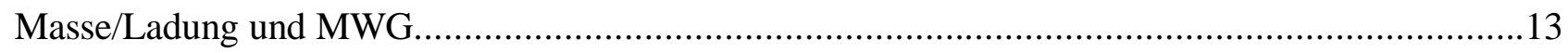

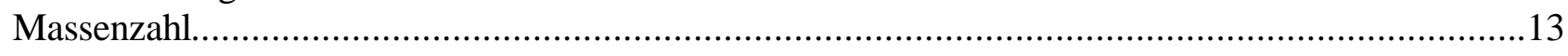

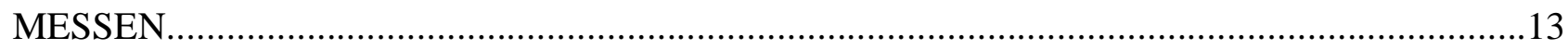

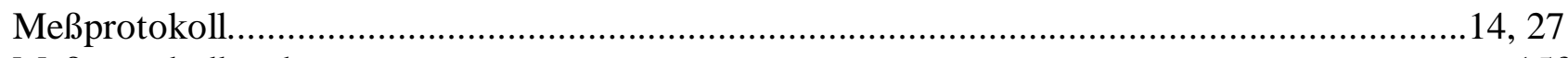

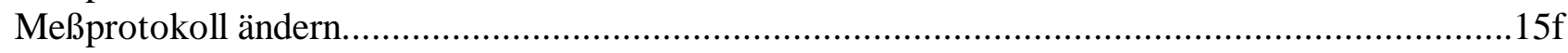

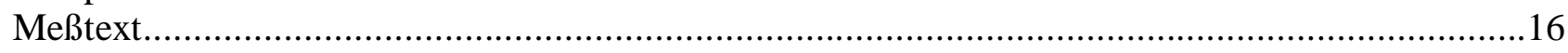

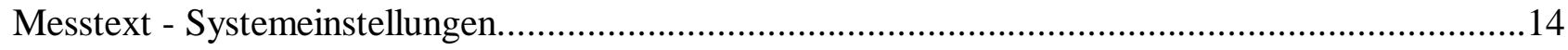

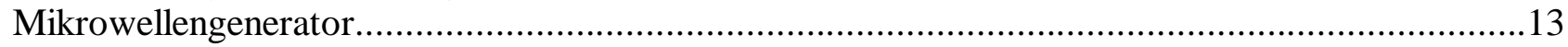

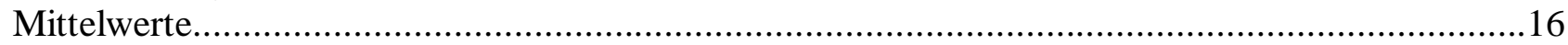

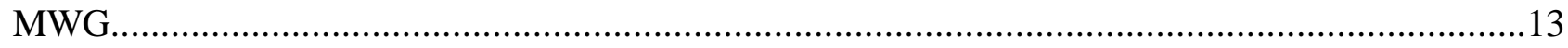

MWG-Pause

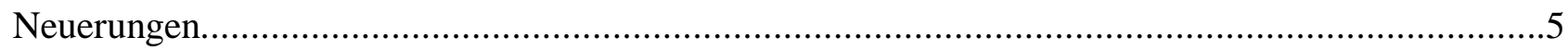

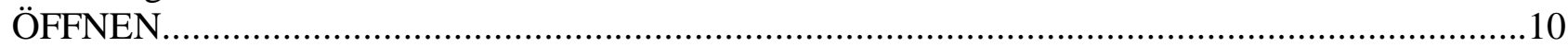

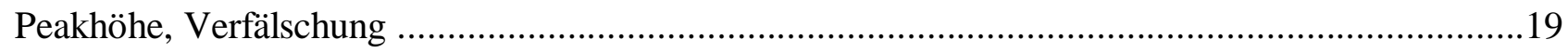

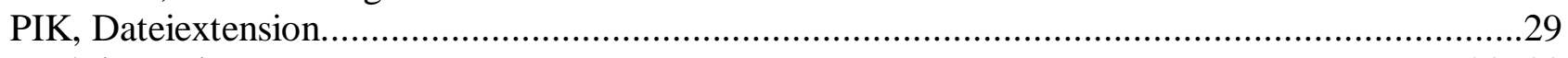

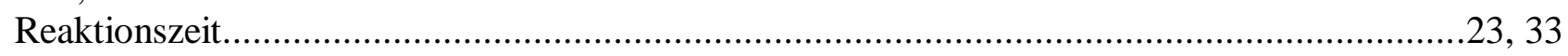

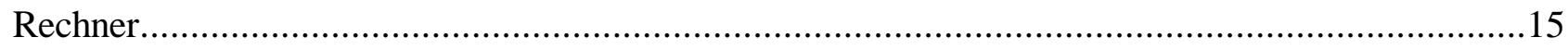

Referenz-BKG-Umsatzkorrektur durch Hauptmesung......................................................22

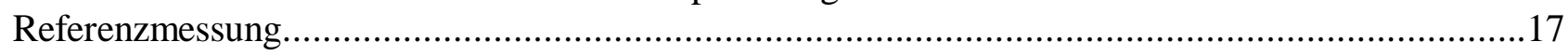

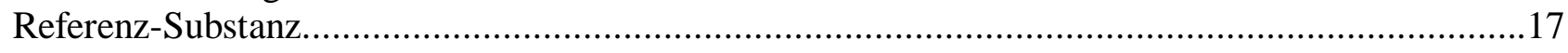

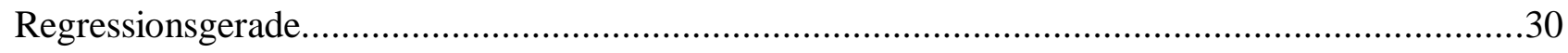

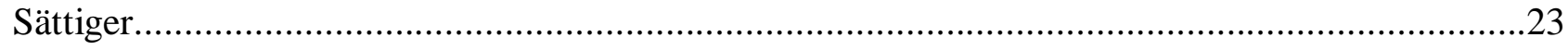

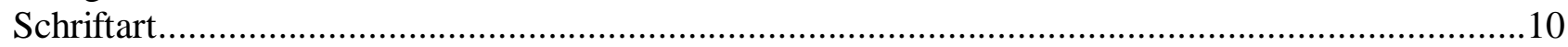

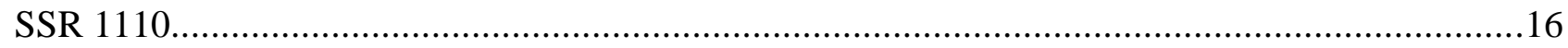

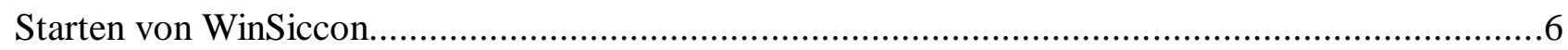

StdMess.TXT“"

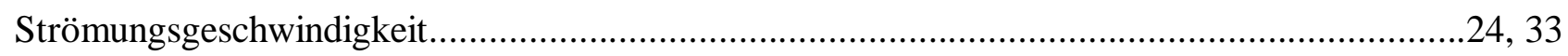

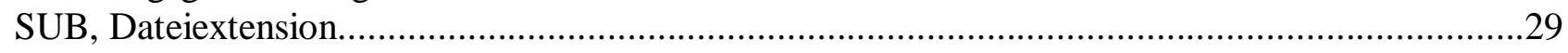

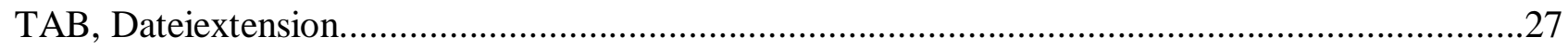

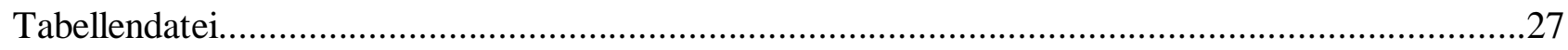

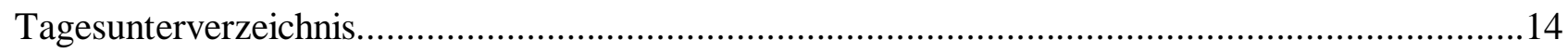

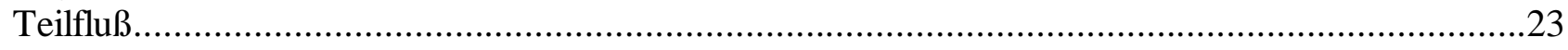

Testprogramme

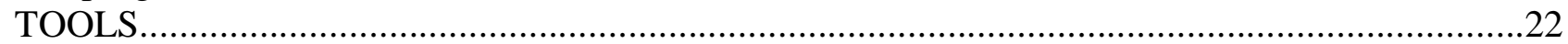

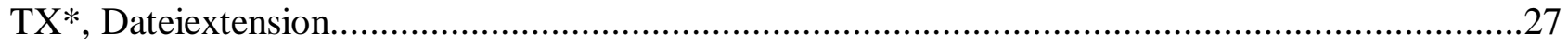


TYLAN.TXT.

26,37

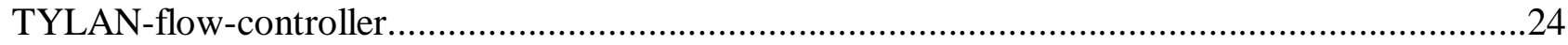

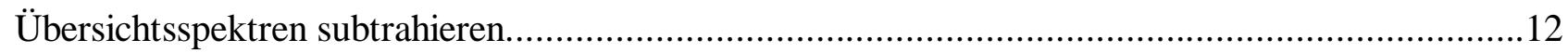

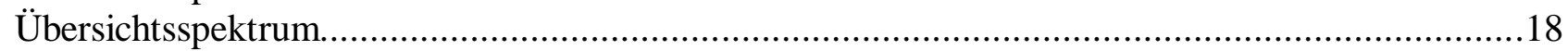

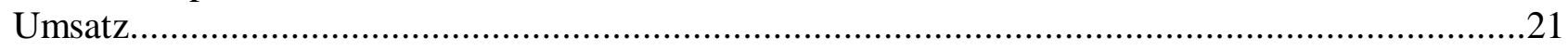

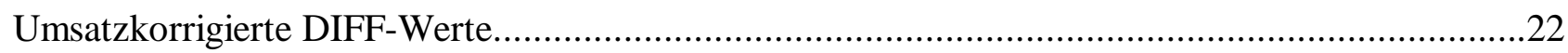

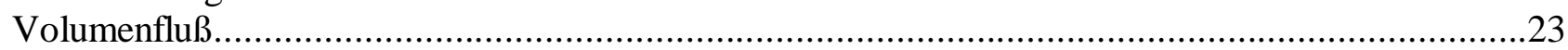

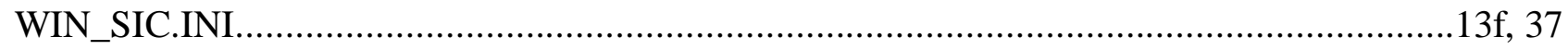

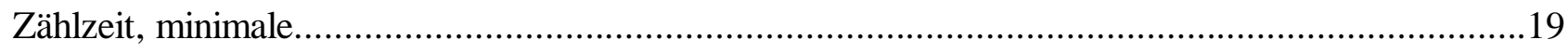

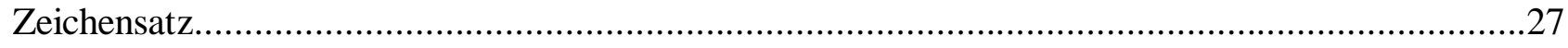

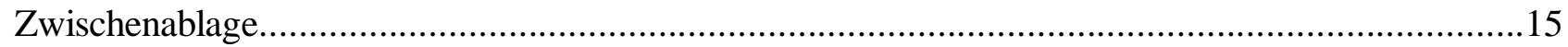






\title{
Programmbeschreibung für
}

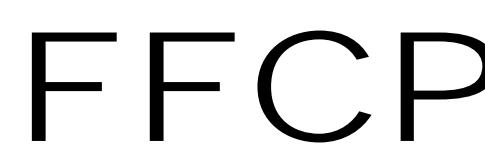

Version 1.2

(FT-IR Fill Control Program)

\author{
Steuerungsprogramm für \\ die automatische Befüllung \\ der Kugelzelle am FT-IR-Spektrometer \\ sowie für die Triggerung \\ des LASERs und der "Step-Scan“-Aufnahme
}

Konzeption:

Thomas Zeuch, 2000

Markus Hold, 2000-2001

Programmierung und Dokumentation:

Markus Hold, 2000-2001 



\section{Inhaltsverzeichnis}

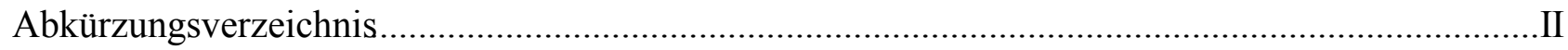

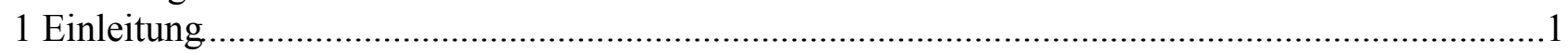

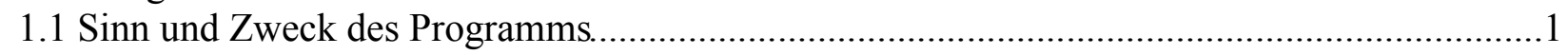

1.2 Zur Programmierung / Betriebssystemvoraussetzungen ...............................................

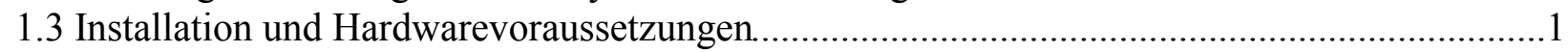

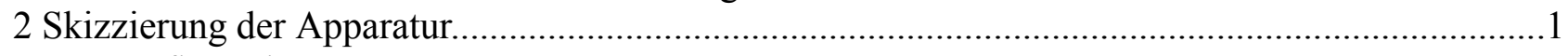

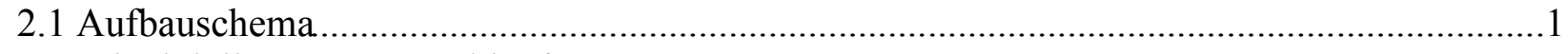

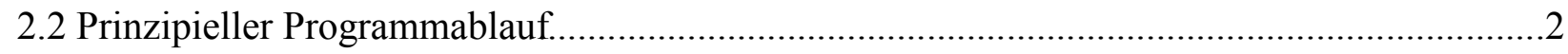

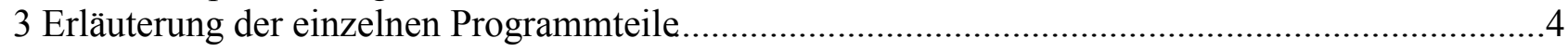

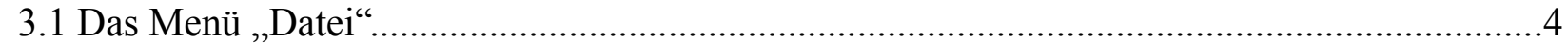

3.1.1 „Protokoll anlegen“

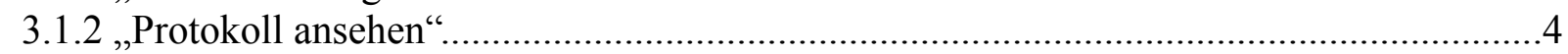

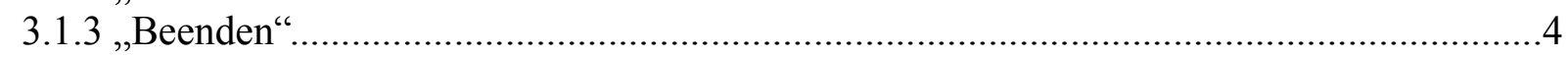

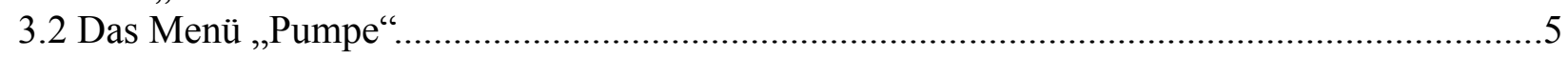

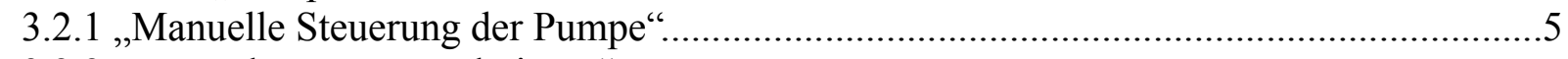

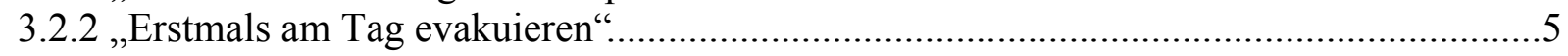

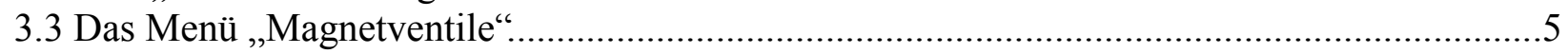

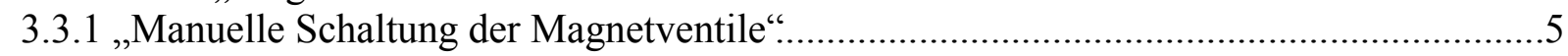

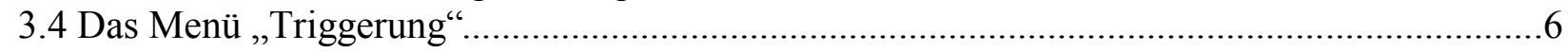

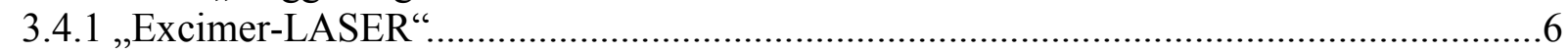

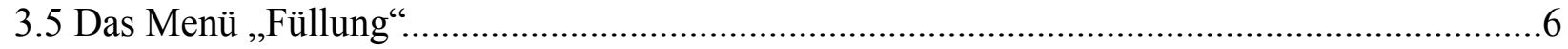

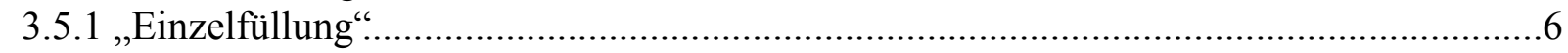

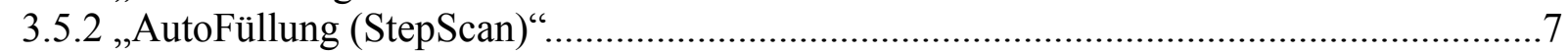

3.5.3 „Letzte Füllung (Badgas) am Tag“ .....................................................................

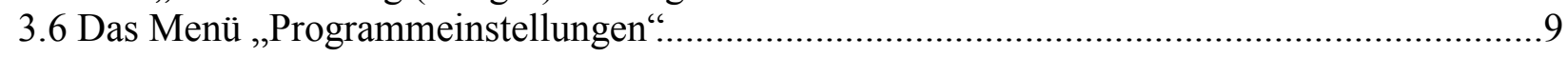

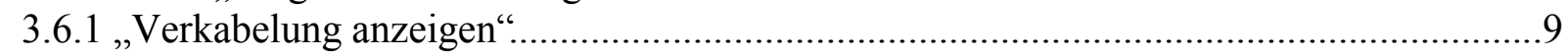

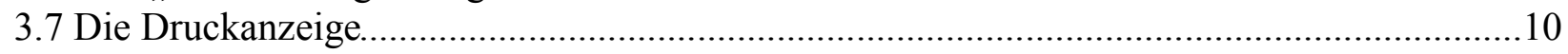

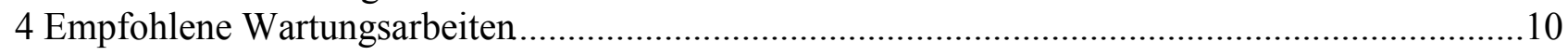

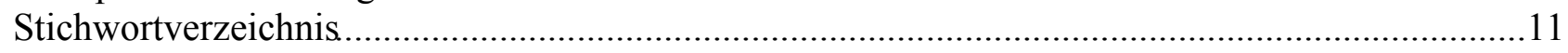




\section{Abkürzungsverzeichnis}

$\begin{array}{ll}\text { AD-Karte } & \text { Analog-Digital-Wandler-Karte (ISA-Einsteckkarte für Computer) } \\ \text { bzw. } & \text { beziehungsweise } \\ \text { ca. } & \text { circa (= ungefähr, etwa) } \\ \text { ggf. } & \text { gegebenenfalls } \\ \text { Kap. } & \text { Kapitel } \\ \text { lt. } & \text { laut } \\ \text { ltz. } & \text { letzte } \\ \text { TTL } & \text { Transistor-Transistor-Logik }\end{array}$




\section{Einleitung}

\subsection{Sinn und Zweck des Programms}

Das Programm „FFCP 1.2“ wurde vorrangig entwickelt, um zeitaufgelöste Messungen im sogenannten ,Step-Scan“-Modus mit dem FT-IR-Spektrometer durchführen zu können.

Dazu ist es notwendig, entsprechend der gewünschten Auflösung eine bestimmte Anzahl Interferogrammpunkte zu registrieren und somit auch die gleiche Anzahl absolut identischer Experimente in der Kugel-Reaktionszelle am FT-IR-Spektrometer durchzuführen. Daher muß diese Reaktionszelle für jedes einzelne Experiment mit genau der gleichen Konzentration der Reaktanten gefüllt, dann die Reaktion durch einen in Bezug auf die Zeitverzögerung und Energie jedesmal gleichen LASER-Puls initiiert und schließlich durch einenTrigger-TTL-Puls jedesmal in gleicher Weise (in Bezug auf die Zeitverzögerung) die „Step-Scan“-Messung gestartet werden; anschließend ist die Reaktionsmischung abzupumpen und der Vorgang für das nächste Experiment erneut zu starten.

Quasi als Nebenprodukt dieser Zielvorgaben ermöglicht das Programm auch das automatische Einfüllen eines bestimmten Reaktanten in die Kugelzelle bis zu einem vorgegebenen Druck, die Triggerung des LASERs (und ggf. der FT-IR-Software) sowie die Steuerung der Pumpe.

\subsection{Zur Programmierung / Betriebssystemvoraussetzungen}

Das Programm „GCMS-CP“ ist in der Programmiersprache „Object-Pascal“" geschrieben und wurde als 32-Bit-Programm mit „Delphi 2.01“ der Firma Borland compiliert.

Es ist ausschließlich unter den 32-Bit-Betriebssystemen „Windows“ (Windows 95 und 98 sowie Windows NT 4.0) der Firma Microsoft lauffähig; getestet wurde es unter Windows 95.

\subsection{Installation und Hardwarevoraussetzungen}

Das Programm „FFCP“ benötigt eine AD-Karte der Firma „ComputerBoards“ vom Typ: „CIODAS802/16“ und eine parallele Schnittstelle „LPT1“. Erstere muß durch das mit der Karte mitgelieferte Programm „InstaCal“ als Board-Nr. 0 installiert sein.

Das Programm kann in jedes beliebige Verzeichnis kopiert und dort gestartet werden. Es muß lediglich auch die Datei ,FFCP.INI“ dorthin kopiert werden und in ihr im Abschnitt „Pfade]“ der „ProtokollPfad“ eingetragen werden, d.h. der Pfad, in dem die Meßprotokolle gespeichert werden

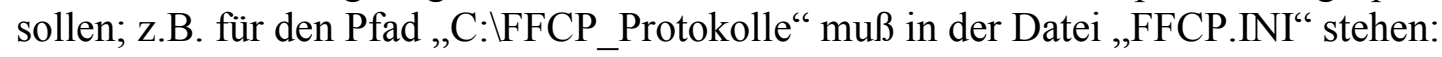

\section{Skizzierung der Apparatur}

\subsection{Aufbauschema}

Für die Aufnahme der IR-Spektren steht ein FT-IR-Spektrometer (BRUKER; Typ: IFS 66) zur Verfügung. Die Fourier-Transformation wird dabei unmittelbar nach der Registrierung des Interferogramms auf einem Personalcomputer (Pentium III mit OS/2) durch die mitgelieferte Software (BRUKER, OPUS 3.0.4) durchgeführt. Mit diesem Gerät ist eine sehr hohe Auflösung (bis zu $\left.0,12 \mathrm{~cm}^{-1}\right)$ möglich.

Um eine hohe Nachweisempfindlichkeit in einem kleinen Volumen zu ermöglichen, ist in der 
Meßzelle eine Spiegelanordnung nach White (drei goldbeschichtete Spiegel, die im Abstand ihrer Brennweite [ $f=200]$ voneinander entfernt sind) eingebaut. Es werden bis zu 40 Reflexionen und somit ein Lichtweg des IR-Strahls von ca. 8 m Länge erreicht.

Das Gerät kann bis zu 60 Interferogrammpunkte pro Sekunde aufnehmen.

Mit dem MCT-Detektor sind zeitaufgelöste Messungen bis $5 \mu$ s möglich.

Die Reaktanten werden als Gase mit Hilfe von Magnetventilen, die vom Programm „FFCP“ gesteuert werden, in die Reaktionszelle eingefüllt, wobei der Zufluß ggf. durch ein Dosierventil (HOKE, Mikro-Mite) vor dem Magnetventil vermindert wird.

Das Evakuieren bzw. Abpumpen der Reaktionszelle erfolgt durch eine Ölpumpe (Brandt RD 15), die vom Programm „FFCP“ gesteuert werden kann; zwischen Zelle und Pumpe ist ein Magnetventil eingebaut, das ebenfalls vom Programm steuerbar ist.

Der Druck in der Zelle wird durch mehrere Druckmeßgeräte (BARATRON; 0-10 mbar, 0-100 mbar sowie 0-1000 mbar) vom Programm „FFCP“ eingelesen.

Mit einem Excimer-LASER (Compex 102, Lambda Physik; bis zu 120 mJ pro Puls) wird die Reaktion initiiert.

Die nachstehende Abbildung skizziert den Aufbau der Apparatur.

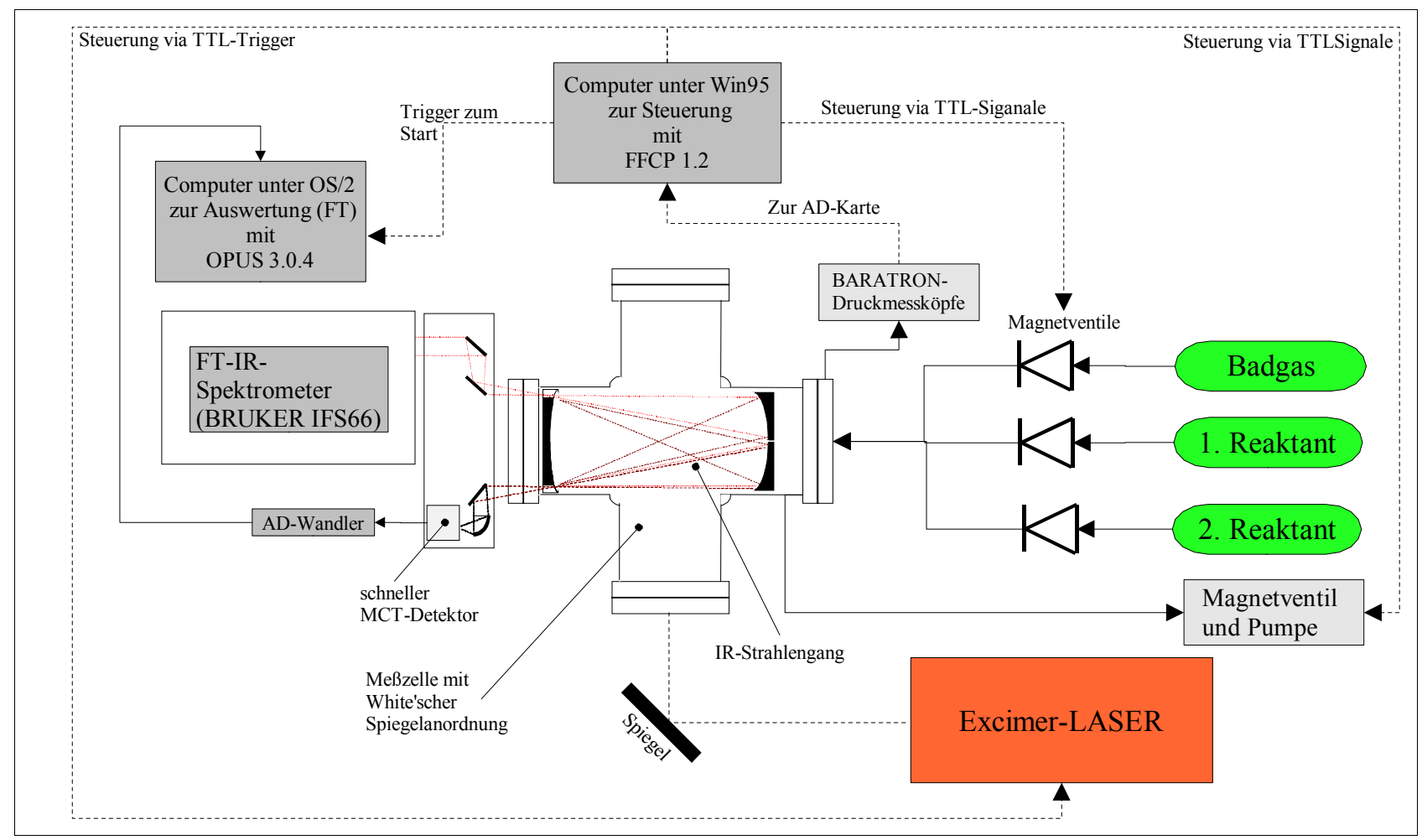

\subsection{Prinzipieller Programmablauf}

Zur Vorbereitung einer Messung müssen die beiden Reaktanten und das Badgas mit einem geeigneten Vordruck bzw. einer geeigneten Einstellung desDosierventils an die Zelle angeschlossen werden. Ferner muß das Programm „Opus“ im ,Step-Scan“-Modus mit den gewünschten Parametern (insbesondere externer Triggerung) gestartet und in Aufnahmebereitschaft versetzt werden. 
Nun übernimmt das Programm „FFCP“ die weitere Steuerung/Kontrolle.

Bei der automatischen „Step-Scan“-Messung wird zunächst die Pumpe gestartet, dann erst nach einer kurzen Verzögerungszeit (um eine Verunreinigung der Zelle zu vermeiden) das Magnetventil zwischen Pumpe und Reaktionszelle geöffnet und die Zelle so lange evakuiert, bis der Druck unter einen vom Benutzer vorgegebenen Wert gesunken ist. Dann schließt dieses Magnetventil und die Pumpe wird abgeschaltet (um Vibrationen während der Messung zu vermeiden).

Jetzt wird das entsprechende Magnetventil geöffnet, um den 1. Reaktant in die Zelle einzufüllen. Dabei wird ständig der Druck gemessen und das Magnetventil bei Erreichen des gewünschten Fülldruckes geschlossen. Die Druckmessung erfolgt dabei automatisch mit dem Druckmeßgerät, das die höchst mögliche Genauigkeit bietet. Auf diese Weise werden nacheinander die drei Gase in der gewünschten Menge eingefüllt.

Nach Füllung der Zelle wartet das Programm eine vorgegebene Zeit bis zur vollständigen Durchmischung und gibt dann einen Trigger-TTL-Puls zum Start zeitgleich sowohl an den LASER als auch an „Opus“. Sofern mehrere Photolysen derselben Füllung gewünscht sind, wartet das Programm eine vorgegebene Zeit und gibt dann erneut die Trigger-Pulse an den LASER und „Opus“.

Danach wird die Reaktionsmischung abgepumpt und die Füllprozedur startet wie oben beschrieben von neuem. 


\section{Erläuterung der einzelnen Programmteile}

\subsection{Das Menü „Datei““}

\subsection{1 „Protokoll anlegen“}

Mit diesem Programmteil kann ein Protokoll angelegt werden, in dem alle vom Programm ausgeführten Aktionen automatisch eingetragen werden.

Voraussetzung ist allerdings, daß in dem in der Datei „FFCP.INI“" angegebenen Verzeichnis „ProtokollPfad“ (vgl. dazu Kap. 1.3, S. 1) ein weiteres Verzeichnis mit dem Name

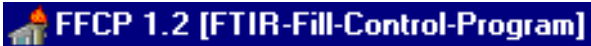
Datei Pumpe Magnetventile Triggerung

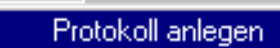

Protokoll ansehen

Programm beenden des Benutzerkürzels angelegt worden ist.

Das Programm fragt zunächst den Dateiname ab, unter dem das Protokoll als Textdatei gespeichert werden soll.

Dann können zusätzliche Erläuterungen zum Meßprotokoll abgegeben werden. Sinnvoll ist die Angabe der Zuordnung, welche Substanz unter welcher Stoff-Nr. eingefüllt wird.

Auch die Temperatur und die durchzuführende Reaktion sollte hier angeben werden.

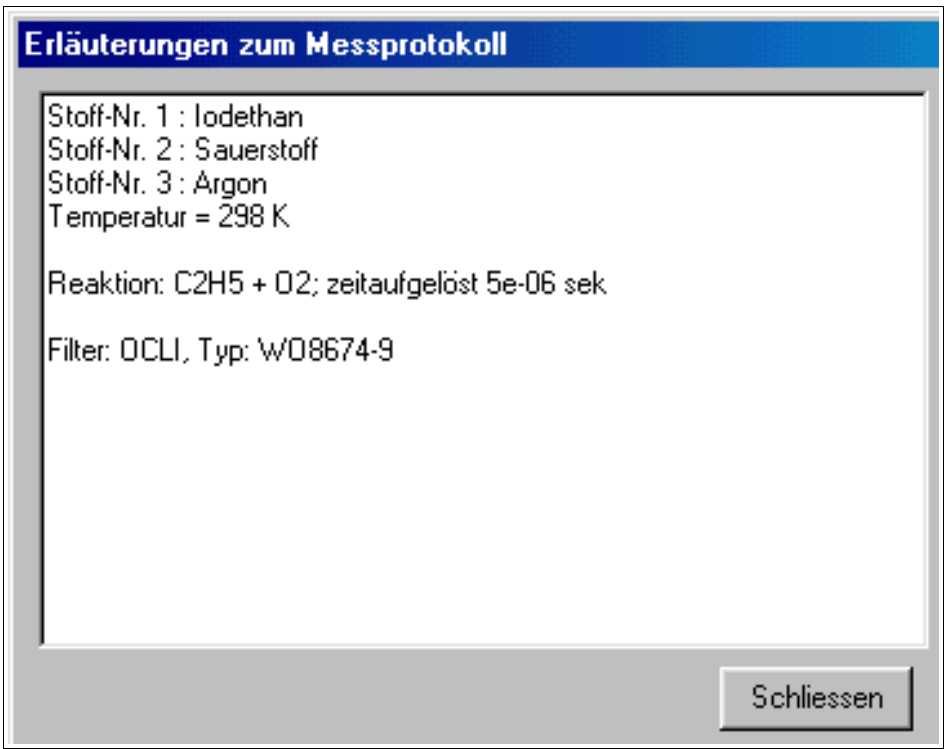

Sofern bereits zuvor ein Protokoll angelegt worden war, fragt das Programm, ob dieses geschlossen und ein neues angelegt werden soll.

Bei einer automatischen „Step-Scan“-Messung wird zwangsweise ein Protokoll angelegt, bei allen anderen Programmteilen kann dies optional erfolgen.

\subsection{2 „Protokoll ansehen“}

Bei Wahl dieses Programmteils wird der zu „Windows“ gehörende „Editor“ gestartet und in diesem das aktuelle Meßprotokoll angezeigt.

Das Protokoll wird dadurch nicht geschlossen, d.h. die weiteren Aktionen des Programms werden weiter angefügt, sind aber erst dann im „Editor“ sichtbar, wenn erneut dieser Programmteil „Protokoll ansehen“ gewählt wird.

\subsection{3 „Beenden $“$}

Mit diesem Programmteil wird das Programm nach Sicherheitsrückfrage beendet. 


\subsection{Das Menü „Pumpe“}

\subsection{1 „Manuelle Steuerung der Pumpe“}

Mit diesem Programmteil kann die Pumpe zum Evakuieren der Zelle manuell ein- und ausgeschaltet werden.

Bei Klick auf den Auswahlschalter „Pumpe einschalten“ wird zuerst die Pumpe eingeschaltet und erst nach 2 sek Wartezeit das Magnetventil zwischen Pumpe und Zelle geöffnet, damit kein Gas in die Zelle zurücksteigen kann.

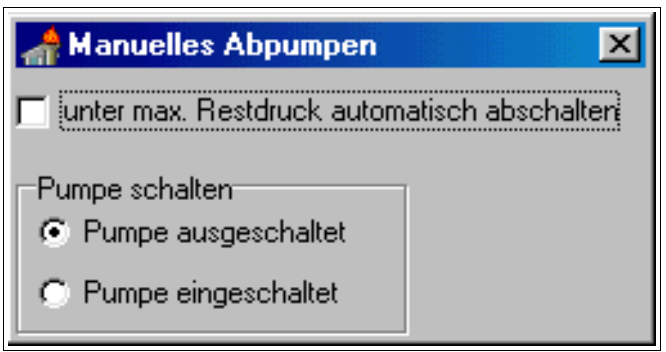

Im Meßprotokoll wird eingetragen, daß die Pumpe

geschaltet wurde und zudem der Druck in der Zelle vor dem Einschalten protokolliert.

Sofern die Option ,unter max. Restdruck automatisch abschalten" gewählt ist, erfolgt automatisch der Abschaltvorgang, sobald der im Hauptfenster eingegebene Restdruck unterschritten ist (dieser Wert muß größer als 0,02 mbar sein, sonst muß der Benutzer die Pumpe manuell abschalten). Dabei wird zunächst das Magnetventil zwischen Pumpe und Zelle geschlossen und erst nach 0,5 sek Wartezeit die Pumpe abgeschaltet.

\subsection{2 „Erstmals am Tag evakuieren“}

Sofern in der Zelle Atmosphärendruck oder höherer Druck herrscht (so wie es bei einer Befüllung mit Badgas zum Abschalten der Apparatur über Nacht üblich ist), gibt es Probleme beim Öffnen des Magnetventils, wenn die Pumpe bereits läuft.

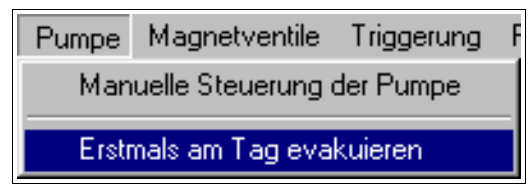

Deshalb wird mit diesem Programmteil zuerst das Magnetventil geöffnet und dann erst die Pumpe eingeschaltet.

Man benötigt diesen Information Programmteil meist nur zur ersten Evakuierung am Tag, da die Zelle sonst überwiegend mit geringerem Druck gefüllt ist.

\subsection{Das Menü „Magnetventile“}

\subsection{1 „Manuelle Schaltung der Magnetventile“}

\begin{tabular}{|c|c|}
\hline \hline Magnetventile Triggerung Füllung Progran \\
\hline Manuelle Schaltung der Magnetventile \\
\hline
\end{tabular}

Mit diesem Programmteil können die einzelnen Magnetventile, die zum Einlaß der drei Gase in die Zelle dienen, geöffnet und geschlossen werden.

Dazu ist einfach auf den entsprechenden Auswahlschalter zu klicken.

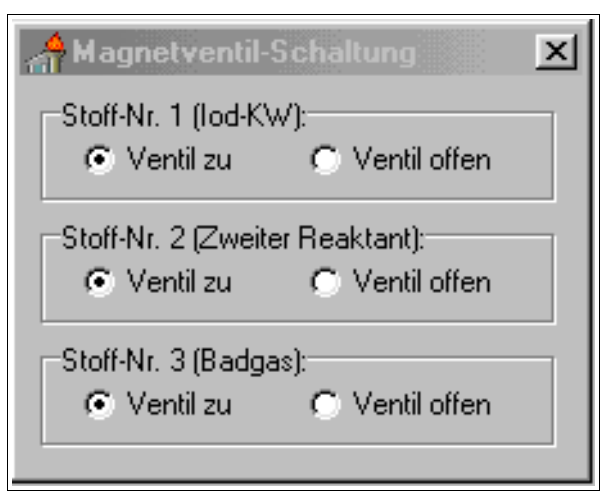




\subsection{Das Menü „,Triggerung“}

\subsection{1 ,Excimer-LASER ${ }^{6}$}

Triggerung Fiillung $\mathrm{PI}$

Excimer-LASER
Mit diesem Programmteil können der Excimer-LASER und ggf. das FT-IRProgramm „Opus“ getriggert werden.

Es muß die Frequenz (zwischen 0,1 und $20 \mathrm{~Hz}$ ) gewählt werden und es ist die Anzahl der Trigger-Pulse anzugeben.

Nach Klick auf den Schalter „Start des LASERs“ beginnt das Programm, die gewünschten Trigger-Pulse auszugeben. Bei Wahl der entsprechenden Option wird absolut zeitgleich mit dem LASER auch die Software „Opus“ getriggert.

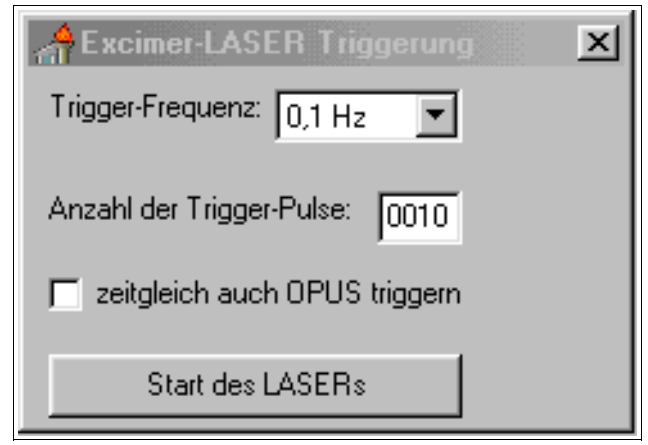

\subsection{Das Menü „Füllung““}

\subsection{1 „Einzelfüllung“6}

\begin{tabular}{|c|c|}
\hline Füllung Programmeinstellungen & Mit diesem Programmteil \\
\hline AutoFilllung (StepScan) & \\
\hline Einzel-Füllung & einem gewünschten Druck \\
\hline Letzte Füllung (Badgas) am Tag & in die Zelle einfüllen. \\
\hline
\end{tabular}

1.] lod-Kohlenwasserstoff: Einzufillender Druck [mbar]: $\overline{001,20}$ Fülldruck bis 1. Stop [\%]: $\overline{090}$

Dazu muß im Hauptformular (das ist das Fenster, das unmittelbar nach dem Start des Programms angezeigt wird) lediglich der gewünschte einzufüllende Druck angegebenen werden, dann im Fenster „EinzelFüllung“ dieser Stoff ausgewählt und schließlich auf den Schalter „Füllung durchführen“ geklickt werden.

Sofern der Vordruck sehr hoch ist, wird wahrscheinlich ein höherer Druck als gewünscht erreicht; dann kann es ratsam sein, die \%-Angabe für den „Fülldruck bis 1. Stop“ auf unter 100\% abzusenken.

Nach Starten des EinzelFüllung-Programms ermittelt das Programm zuerst den aktuellen Druck in der Zelle, wählt einen für den Enddruck geeignetenBARATRON-Druckmesser und öffnet dann das Einlaß-Magnetventil für den benötigten Reaktanten. Nun mißt das Programm ständig den aktuellen Druck in der Zelle und schließt das Magnetventil, sobald die geforderte \%-Zahl des Druckes des einzufüllenden Reaktanten erreicht ist. Für das oben abgebildete Beispiel möge der Druck vor dem Einfüllen 2,00 mbar betragen haben; dann würde nach Programmstart für den ersten Reaktanten das Magnetventil so lange geöffnet, bis ein Druck von $(2,00+90 \% \cdot 1,20)$ mbar $=3,08$ mbar gemessen wird. Nun wartet das Programm 1,5 sek und mißt abermals den Druck (der nun höher sein kann, weil entweder der Reaktant nur langsam von der Wand desorbiert oder die Druckmessung zuvor zu träge war). Falls der gewünschte Enddruck noch nicht erreicht wurde, wird folgende Prozedur so lange wiederholt, bis er erreicht ist: das Einlaß-Magnetventil wird erneut für $20 \mathrm{~ms}$ geöffnet, danach wird 2,5 sek gewartet und dann erst der Druck gemessen.

Es empfiehlt sich, die Reaktanten so langsam einströmen zu lassen, daß man auch bei 100\% 
„Fülldruck bis 1. Stop“ den gewünschten Enddruck nicht überschreitet (ggf. muß ein HOKEDosierventil eingesetzt werden).

In das Meßprotokoll wird die durch das Einfüllen tatsächlich hervorgerufene Druckdifferenz eingetragen.

\subsection{2, „AutoFüllung (StepScan)““}

\begin{tabular}{|c|}
\hline \hline Füllung Programmeinstellungen \\
\hline AutoFiillung (StepScan) \\
\hline Einzel-Füllung \\
\hline Letzte Fuillung (Badgas) am Tag \\
\hline
\end{tabular}

Dieser

Programmteil

dient der wiederholten äquivalenten

Befüllung der

Zelle zur Durchführung von zeitaufgelösten Step-Scan-Messungen in Absorption.

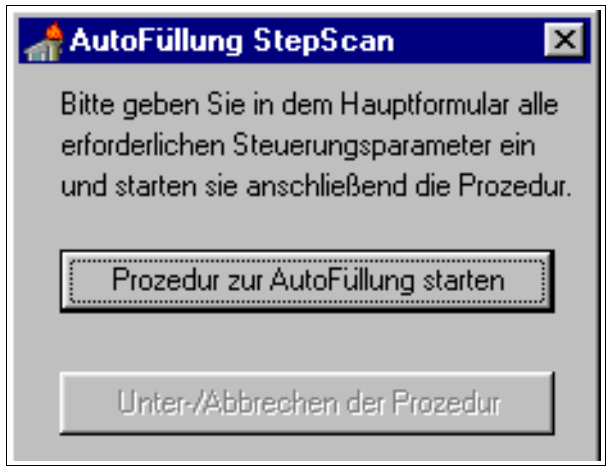

Es ist erforderlich, daß im Hauptformular alle nebenstehenden Parameter eingegeben werden.

Zunächst ist der Restdruck einzugeben, bis zu dem automatisch abgepumpt werden soll; dies entspricht dem oben in Kap. 3.2.1 (S. 5) beschriebenen Vorgehen.

Dann sind die Mengen (d.h. Drücke) der einzufüllenden Reaktanten anzugeben; der Vorgang der Befüllung mit den einzelnen Reaktanten entspricht demoben in Kap. 3.5.1 (S. 6) Beschriebenen. Wenn ein „einzufüllender Druck“" von Null eingegeben wird, wird dieser Reaktant nicht eingefüllt.

Es sollte eine „Wartezeit nach der Befüllung“ eingegeben werden, damit sich die Reaktionsmischung zunächst vollsändig durchmischen kann.

Ebenso ist es sinnvoll, eine „Wartezeit zwischen den einzelnerPhotolysen“ derselben Mischung einzugeben, damit die gebildeten Produkte vor der nächsten Photolyse zunächst aus der Nachweiszone heraus diffundieren können.

Auch ist die Anzahl der Photolysen anzugeben, die bei derselben Füllung gemacht werden sollen. Man muß jedoch darauf achten, daß die gebildeten Produkte nicht die Messung verfälschen, indem sie selbst im relevanten Bereich absorbieren oder aber diese Produkte ihrerseits bei der nächsten Photolyse in Folgeprodukte zerfallen, die im relevanten Bereich absorbieren.

Im Programm „Opus“ wird nach Eingabe der gewünschten Auflösung und des aufzunehmenden 
Wellenlängenbereichs die Anzahl der Interferogrammpunkte berechnet. Man sollte diese Zahl so gering wie nur irgend möglich halten (durch Wahl der für das Experiment geringstmöglichen Auflösung sowie Einsatz eines Filters, der nur im für das Experiment erforderlichen Bereich durchlässig ist), denn für jeden Interferogrammpunkt muß ein äquivalentes Experiment durchgeführt und somit die Zelle entsprechend oft befüllt werden.

Es empfiehlt sich, die „Gesamtzahl der Füllungen“ gleich der Anzahl der Interferogrammpunkte und die „Anzahl der Photolysen pro Füllung“ gleich der Wiederholung der Messung in „Opus“ zu wählen.

Damit ein ggf. angestauter Vordruck nicht zu einer fehlerhaften Befüllung der ersten Füllungen führt, sollten etwa 2-3 Füllungen vor der ersten Photolyse und damit vor Beginn der Messungen durchgeführt werden. Die Gesamtzahl der Füllungen ist dann aber um diese Anzahl zu erhöhen.

Der prinzipielle Programmablauf wurde bereitsoben in Kap. 2.2 (S. 2) beschrieben.

Prozedur zur AutoFüillung starten

Unter-/Abbrechen der Prozedur
Der Programmteil wird durch Klick auf den Schalter „Prozedur zur AutoFüllung starten" gestartet.

Er kann durch Klick auf den Schalter „Unter-/Abbrechen der Prozedur" unterbrochen oder vorzeitig beendet werden.

Die Unterbrechung kann z.B. erforderlich sein, um $\mathrm{N}_{2}(1)$ in den Detektor nachzufüllen. Zur Fortsetzung muß dann lediglich die nebenstehende Frage mit „Nein“ beantwortet werden. Das Programm beendet vor deren Anzeige immer erst den aktuellen Füll-Zyklus.

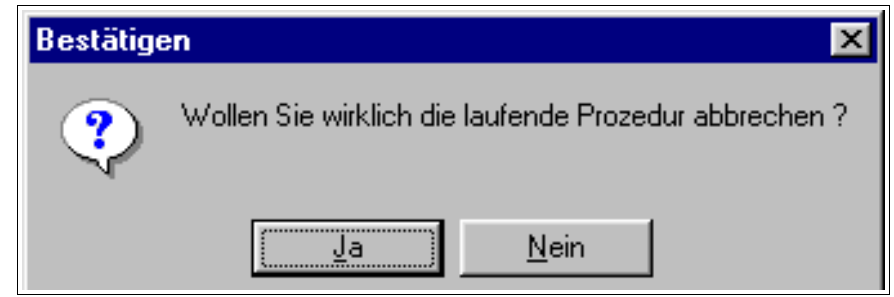

\subsection{3 „Letzte Füllung (Badgas) am Tag“6}

\begin{tabular}{|l|}
\hline Füllung Programmeinstellungen \\
\hline AutoFiullung (StepScan) \\
\hline Einzel-Füllung \\
\hline Letzte Fïllung (Badgas) am Tag \\
\hline
\end{tabular}

Mit diesem Programmteil kann die Zelle mit ca. 1 bar Badgas befüllt werden, um ein Eindringen von Luftfeuchtigkeit und anderen Verunreinigungen bei längerer Standzeit (auch über Nacht) zu vermindern.

Es erfolgt der Hinweis, daß mit diesem

Programmteil die Zelle zunächst evakuiert wird, dann das Badgas eingefüllt und erst danach das Ventil zwischen

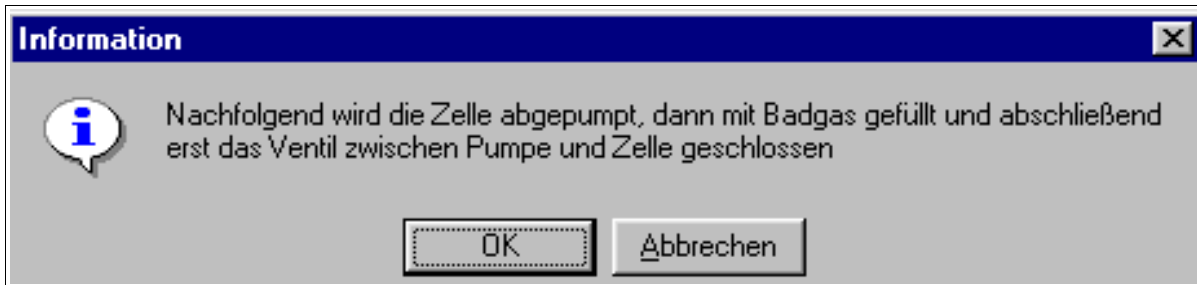
Pumpe und Zelle geschlossen wird. Diese Reihenfolge ist wichtig, weil es sonst Probleme gibt, die Pumpe am nächsten Tag zu starten und das Ventil zu öffnen.

Zuerst wird vom Programm die Pumpe gestartet und es folgt die nebenstehende Meldung, die mit Klick auf „OK“ bestätigt werden muß, um die Pumpe auszuschalten.

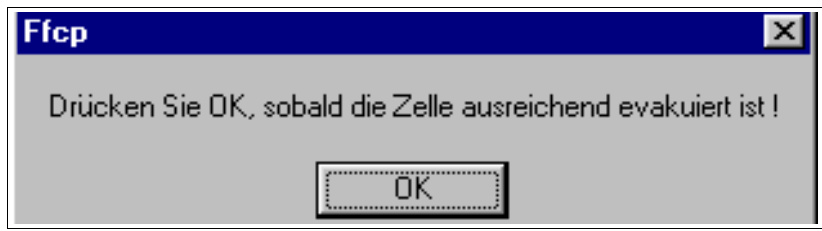

Das Programm bietet nun die Möglichkeit, die Zelle automatisch mit Badgas zu befüllen. Bejaht 
man diese Möglichkeit, öffnet das Programm das Magnetventil für das Badgas, füllt 990 mbar davon ein und schließt das Einlaß-Magnetventil wieder; dann erst schließt es das Magnetventil zwischen Pumpe und Zelle.

Wird die Möglichkeit zur automatischen Befüllung hingegen verneint, muß der Benutzer die Zelle selbst füllen; durch Bestätigung der nebenstehenden Meldung mit Klick auf „OK“ wird dann das Magnetventil zwischen Pumpe und Zelle

Soll die Zelle jetzt automatisch mit Badgas gefuilt werden ? (dann schließen Sie nun die entsprechende Leitung an !]

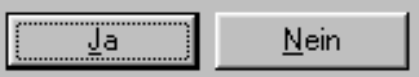
geschlossen.

\subsection{Das Menü „Programmeinstellungen“}

\subsection{1 „Verkabelung anzeigen“}

\section{Programmeinstellungen}

Verkabelung anzeigen

Das Programm setzt voraus, daß die AD-Karte „CIO-DAS802/16“ der Firma ComputerBoards eingebaut ist und als Board-Nr. 0 installiert wurde.

In diesem Programmteil wird angezeigt, an welchen TTLAusgängen der AD-Karte die jeweiligen Magnetventile bzw. der Schalter für die Pumpe anzuschließen sind sowie an welchen TTL-Ausgängen der LPT1-Schnittstelle das Magnetventil zwischen Pumpe und Zelle bzw. die TriggerAusgänge für den Photolyse-LASER und das „Opus“-Programm anzuschließen ist.

Ferner ist angegeben, an welchem AD-Eingangskanal der AD-Karte das Signal der BARATRONDruckmeßgeräte angeschlossen werden muß.

Vom Benutzer einzugeben ist, welche BARATRON-Meßgeräte tatsächlich an der Apparatur vorhanden sind (Häkchen in Auswahl-Box setzen) und welche Port-Adresse die parallele Schnittstelle im Computer hat.

\begin{tabular}{|c|c|}
\hline A Verkabelung Anzeig & [Programmkonstanten] \\
\hline AD-Board-Nr.: 0 / Rang & unipolar 0 bis $+10 \mathrm{~V}$ \\
\hline 1.) lod-Kohlenwasserstoff: & Magnetventil an DigitalOut2 (Bit \# 1) \\
\hline 2.) Zweiter Reaktant: & Magnetventil an DigitalOut3 (Bit \# 2) \\
\hline 3.] Badgas [z.B. Argon] & Magnetventil an DigitalOut4 (Bit \# 3) \\
\hline automatisches A.bpumpen: & Pumpe $\quad$ : DigitalOut1 (Bit \# 0) \\
\hline & Magnetventil: Pin \# 6 von LPT1 \\
\hline $\begin{array}{l}\text { Trigger-Ausgang für LASER } \\
\text { Trigger-Ausgang für OPUS }\end{array}$ & $\begin{array}{l}\text { Pin \# } 2 \text { von LPT1 } \\
\text { Pin \# } 4 \text { von LPT1 }\end{array}$ \\
\hline 10 mbar BARATRON: AL & Eingang 5 (Channel \# 4) / Г Gerät ist angeschlossen \\
\hline 100 mbar BARATRON: AL & Eingang 4 (Channel \# 3) / $\sqrt{\checkmark}$ Gerät ist angeschlossen \\
\hline 1000 mbar BARATRON: AL & Eingang 2 (Channel \# 1) / $/$ Gerät ist angeschlossen \\
\hline Port-Adresse LPT1: $\longdiv { \$ 3 7 }$ & \\
\hline
\end{tabular}

Füllen Sie die Zelle nun manuell mit Gas [erst dann OK !]

\section{$\mathrm{OK}$}




\subsection{Die Druckanzeige}

Der Benutzer kann den vom BARATRON gemessenen Druck in der Zelle auch am Bildschirm anzeigen lassen.

Dazu muß lediglich auf „Anzeige ein“ geklickt werden. Das Programm sucht automatisch das BARATRON mit der höchsten Genauigkeit; das gewählte Gerät wird angezeigt. Der Benutzer kann das BARATRON jedoch nicht selbst wählen.

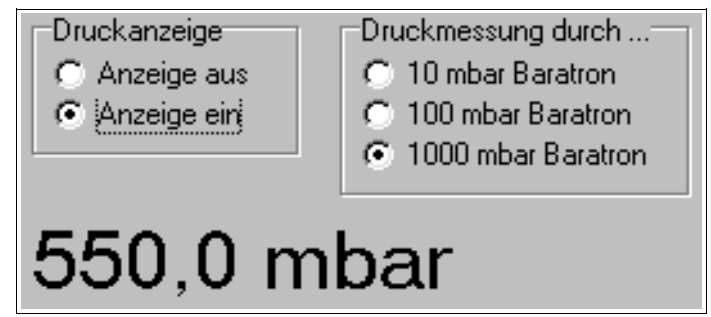

Falls es bei der AutoFüllung für Step-Scan trotz angemessener Vordrücke der Reaktanten zu Fehlfüllungen kommt, sollte dieDruckanzeige während der Messung abgeschaltet werden; dadurch wird das Problem meist behoben.

\section{Empfohlene Wartungsarbeiten}

In regelmäßigen Abständen sollten die angeschlossenen BARATRON-Druckaufnehmer kalibriert werden (vgl. Handbuch zu diesen Geräten).

Weil im „FFCP“-Programm der Druckaufnehmer nicht selbst ausgewählt werden kann, muß dazu ein anderes Spannungsmeßgerät (Meßbereich 0-10 V) verwendet werden. Alternativ kann jedoch auch der Programmteil „Test Analog“ des mit der AD-Karte gelieferten Programms „InstaCal“ dazu herangezogen werden. Alle angeschlossenen Druckaufnehmer sollten nach Kalibrierung den gleichen Druck anzeigen, wobei die ausgegebene Spannung beim 0-1000 mbar-Gerät noch mit dem Faktor 100 und diejenige des 0-100 mbar-Gerät noch mit dem Faktor 10 multipliziert werden muß. 


\section{Stichwortverzeichnis}

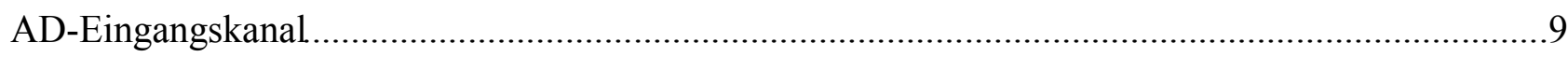

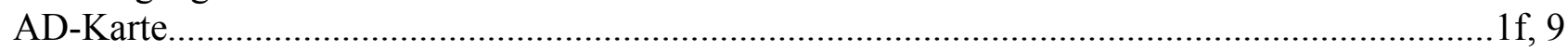

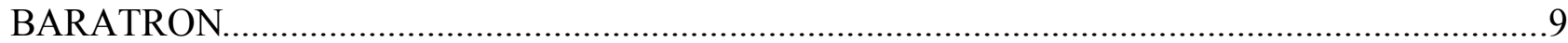

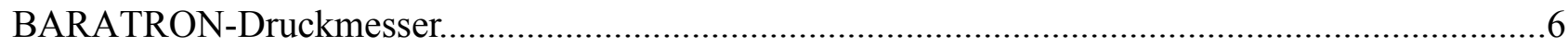

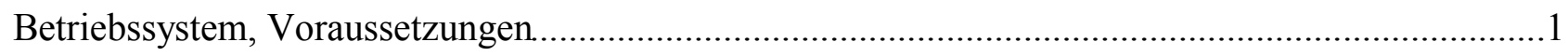

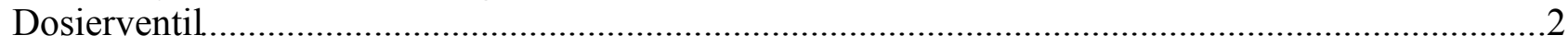

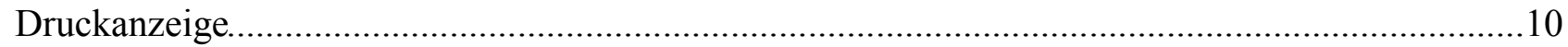

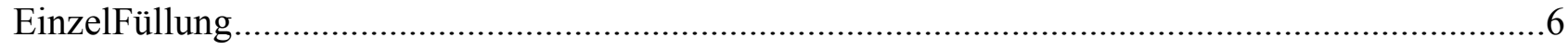

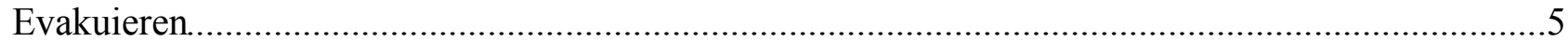

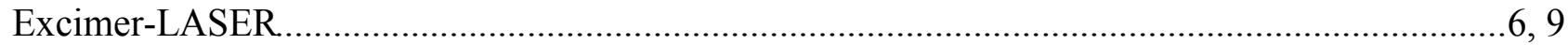

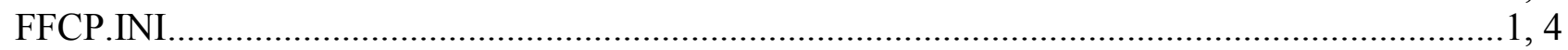

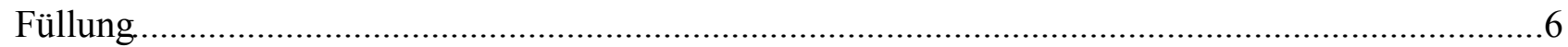

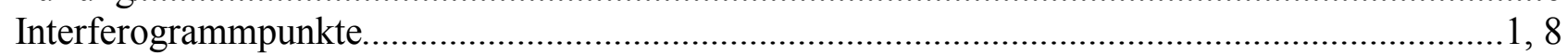

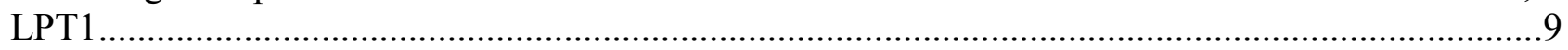

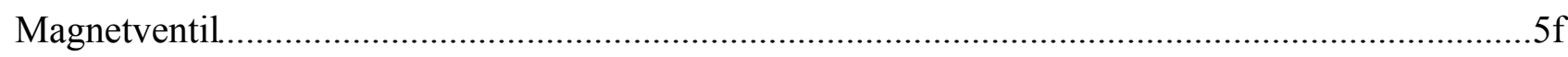

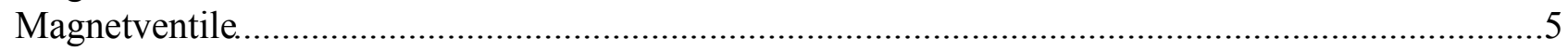

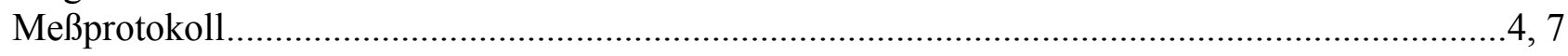

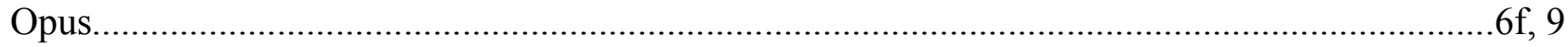

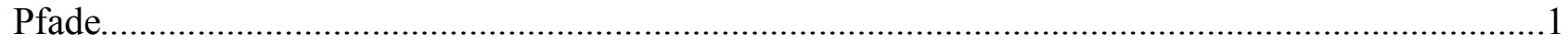

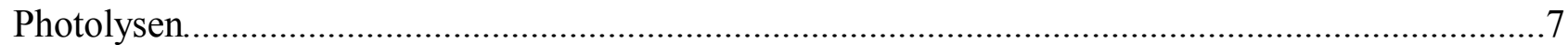

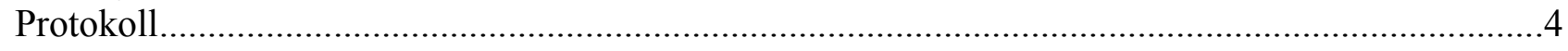

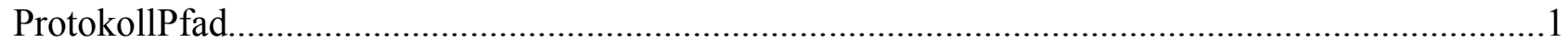

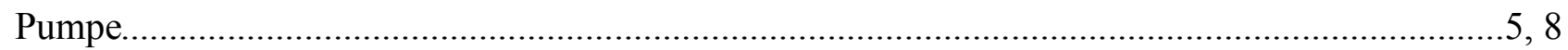

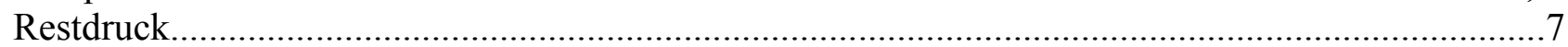

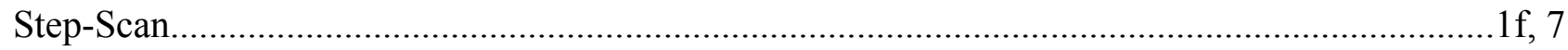

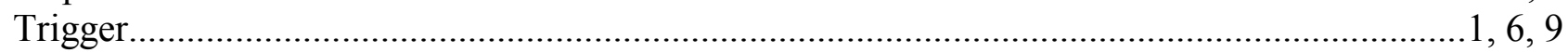

TTL

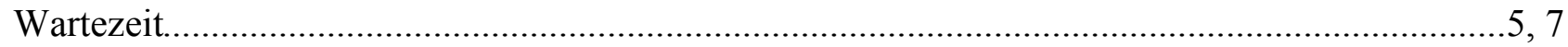






\section{Lebenslauf}

Am 19.12.1969 wurde ich als Kind des Werbekaufmanns Friedrich Hold und dessen Ehefrau, der Großhandelskauffrau Irmgard Hold, in Kassel geboren und besitze die deutsche Staatsangehörigkeit.

Von 1976 bis 1980 besuchte ich die Grundschule in Obervellmar, danach von 1980 bis 1986 die Gesamtschule Ahnatal in Vellmar. Anschließend besuchte ich von 1986 bis 1989 die Goetheschule in Kassel und erwarb dort im Mai 1989 die allgemeine Hochschulreife.

Meinen Wehrdienst leistete ich von Juni 1989 bis August 1990 als Fernmeldeaufklärer „HFSprechfunk Russisch“ im FmBtl 320 in Frankenberg/Eder.

Zum Wintersemester 1990/1991 immatrikulierte ich mich an der Universität Göttingen, um dort das Studium der Chemie aufzunehmen. Zum Wintersemester 1991/1992 immatrikulierte ich mich ferner an der FernUniversität/Gesamthochschule in Hagen im Studienfach Wirtschaftswissenschaften/Betriebswirtschaftslehre.

Im September 1993 bestand ich die Diplom-Vorprüfung im integrierten Studiengang Wirtschaftswissenschaften und im Februar 1995 diejenige im Studiengang Chemie.

Meine Diplomarbeit im Studienfach Chemie mit dem Titel „Erzeugung und Reaktionen von Chlormethyl- und Dichlormethylradikalen sowie von Radikalen teilfluorierter Ether in der Gasphase" fertigte ich unter Leitung von Prof. Dr. K. Hoyermann im Institut für Physikalische Chemie der Universität Göttingen an. Nach dem Bestehen der Diplom-Hauptprüfung Chemie war ich dort seit November 1997 in zwei Teilprojekten des Sonderforschungsbereichs 357 (,Molekulare Mechanismen unimolekularer Prozesse“) beschäftigt und konnte in diesem Rahmen auch die vorliegende Dissertation erstellen.

Von November bis Dezember 2000 hatte ich einen Forschungsaufenthalt an der Akademie der Wissenschaften (Semenov-Institut) in Moskau.

Im Studienfach Wirtschaftswissenschaften/Betriebswirtschaftslehre fertigte ich meine Diplomarbeit mit dem Titel „Die Haftung des GmbH-Geschäftsführers“ unter Leitung von Prof. Dr. E. von Olshausen am Lehrgebiet Unternehmensrecht der FernUniversität Hagen an und bestand anschließend im November 1999 die Diplomprüfung I sowie im September 2001 die Diplomprüfung II (Dipl.-Kaufmann). 
